
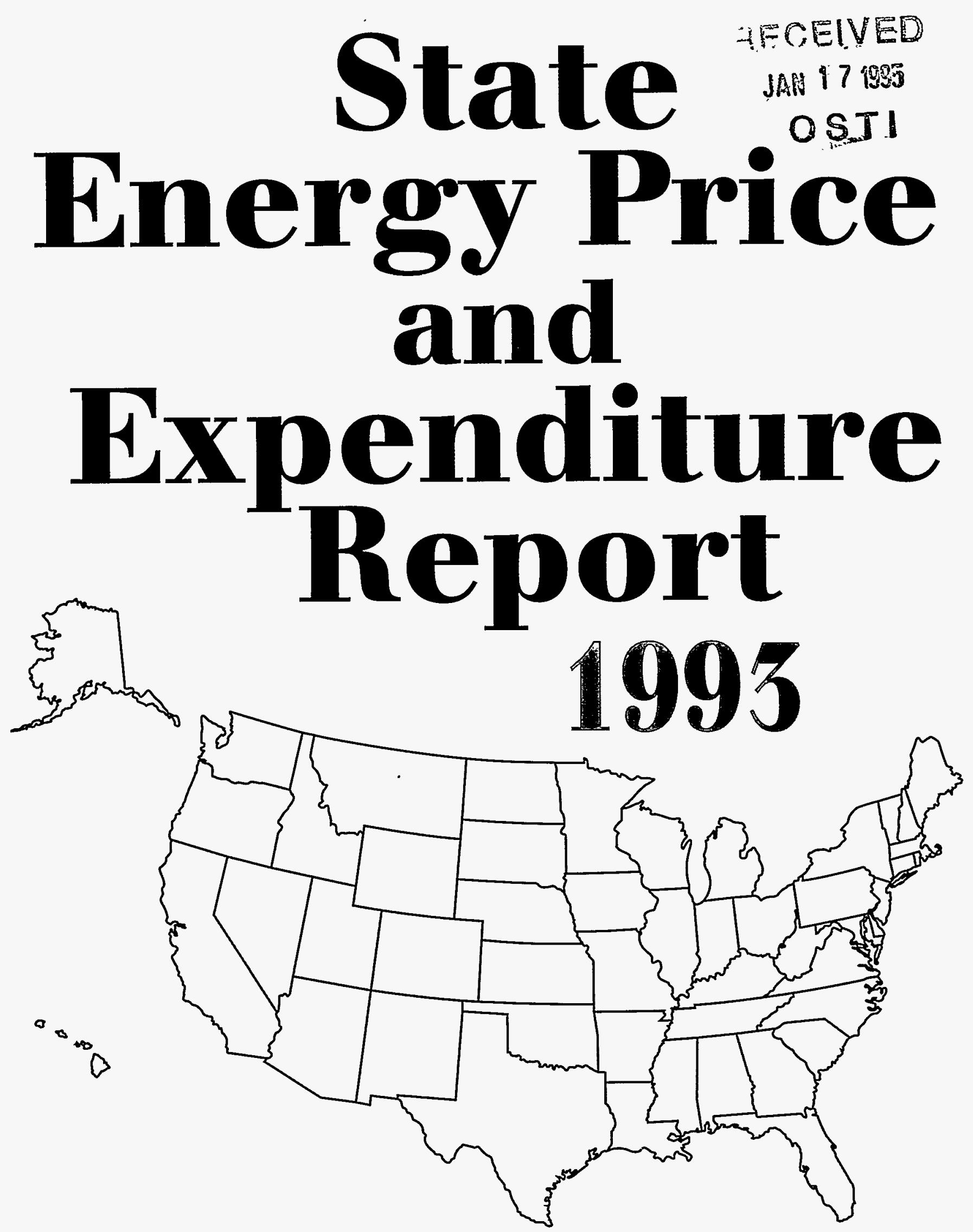

EIA

Energy Information Administration 


\section{State Energy Price and Expenditure Report 1993}

The State Energy Price and Expenditure Report 1993 presents energy price and expenditure estimates for the 50 States, the District of Columbia, and the United States for selected years. The estimates are presented by energy source (e.g., petroleum, natural gas, coal, and electricity) and by major consuming sector. This publication is an update of the State Energy Price and Expenditure Report 1992 (published December 1994).

Publication of this report is in keeping with responsibilities given the Energy Information Administration (EIA) in Public Law 95-91 (Department of Energy Organization Act), which states, in part, in Section 205(a)(2) that:

The Administrator shall be responsible for carrying out a central, comprehensive, and unified energy data and information program which will collect, evaluate, assemble, analyze and disseminate data and information....

The State Energy Price and Expenditure Report is a part of EIA's historical integrated energy data program and is intended to provide historical energy information to Members of Congress, Federal and State agencies, and the general public.

\section{Contacts}

The State Energy Price and Expenditure Report is prepared by the Energy Information Administration under the direction of W. Calvin Kilgore, Director of the Office of Energy Markets and End Use, 202-5861617; Lynda T. Carlson, Director of the Energy End Use and Integrated Statistics Division, 202-586-1112; and Katherine E. Seiferlein, Chief of the Integrated Statistics Branch, 202-586-5692.

Questions concerning the contents of the State Energy Price and Expenditure Report may be referred to:

Julia Hutchins, 202-586-5138, jhutchin@.eia.doe.gov Thomas Leckey, 202-586-9413, tleckey@eia.doe.gov Roy Stanley, 202-586-5839, rstanley@eia.doe.gov Raymond Boyer, 202-586-1680, rboyer@eia.doe.gov The Division fax number is 202-586-0018.

Released for printing: December 22, 1995 GPO Stock No.: 061-003-00948-4

\section{Ordering Information}

This publication and other EIA publications may be purchased from the Superintendent of Documents, U.S. Government Printing Office.

Telephone and fax orders should be directed to:

Superintendent of Documents

U.S. Government Printing Office

Main Order Desk

202-512-1800

Fax: 202-512-2250

8 a.m. to $4: 30$ p.m., eastern time, $M-F$

All mail orders should be directed to:

U.S. Government Printing Office

P.O. Box 371954

Pittsburgh, PA $15250-7954$

Complimentary subscriptions and single issues are available to certain groups of subscribers, such as public and academic libraries; Federal, State, local, and foreign governments; EIA survey respondents; and the media. For further information and for answers to questions on energy statistics, please contact EIA's National Energy Information Center at:

National Energy Information Center, EI-231

Energy Information Administration

Forrestal Building, Room 1F-048

Washington, DC 20585

202-586-8800

Internet E-Mail: infoctr@eia.doe.gov

Fax: 202-586-0727

TTY: For people who are deaf or hard of hearing: 202-586-1181

9 a.m. to 5 p.m., eastern time, $M-F$

\section{Electronic Access}

Some EIA publications are available on Internet at:

World Wide Web Site: http/www.eia.doe.gov Gopher Site: gopher://gopher.eia.doe.gov FTP Site: ftp://ftp.eia.doe.gov

Data from the State Energy Data System and the State Energy Price and Expenditure Data System are also available on personal computer diskettes and a CD-ROM. For information on diskettes, see the inside back cover of this report. For information on the CD-ROM, call 202-586-8800. 


\title{
State Energy Price and Expenditure Report 1993
}

\section{December 1995}

\author{
Energy Information Administration \\ Office of Energy Markets and End Use \\ U.S. Department of Energy \\ Washington, DC 20585
}

This report was prepared by the Energy Information Administration, the independent statistical and analytical agency within the Department of Energy. The information contained herein should not be construed as advocating or reflecting any policy position of the Department of Energy or any other organization. 


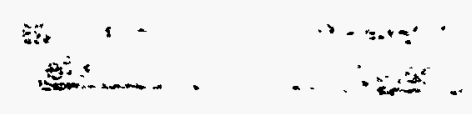




\section{Contents}

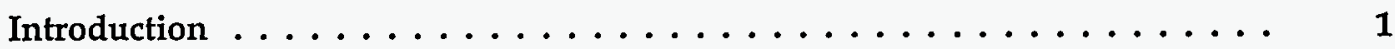

State Rankings . . . . . . . . . . . . . . . . . . . 5

Statistical Tables

United States . . . . . . . . . . . . . . . . . . . . . . . . 17

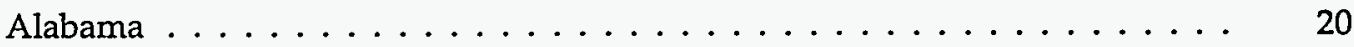

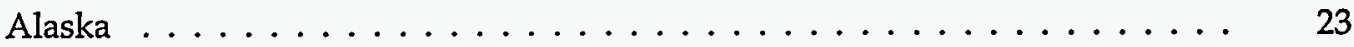

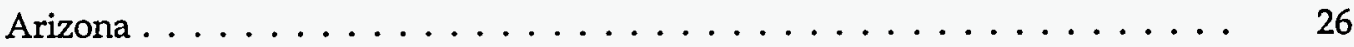

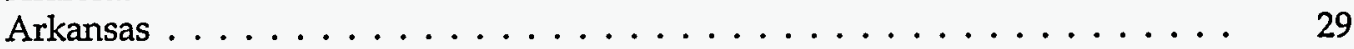

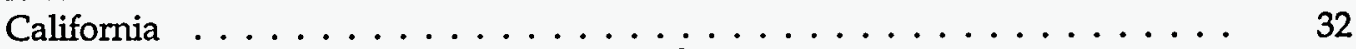

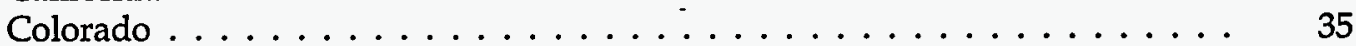

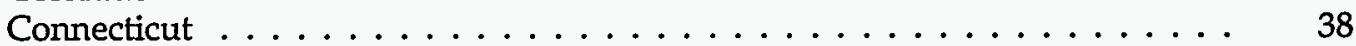

Delaware . . . . . . . . . . . . . . . . . . . . 41

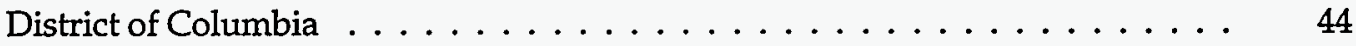

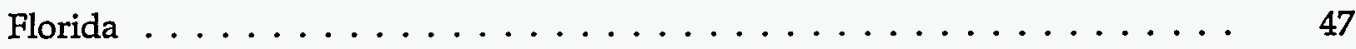

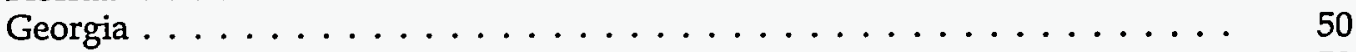

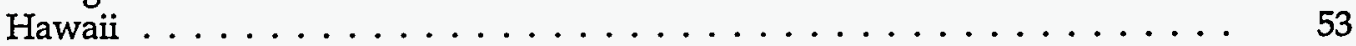

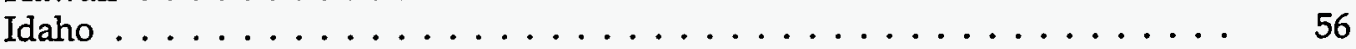

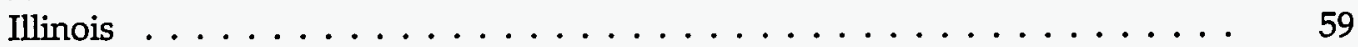

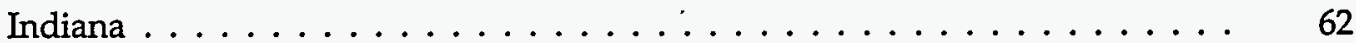

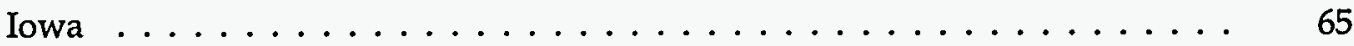

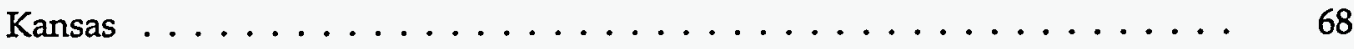

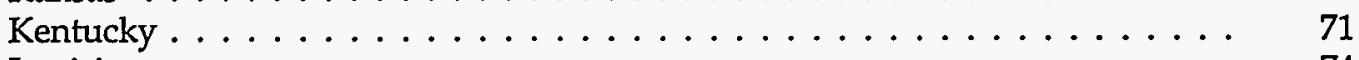

Louisiana . . . . . . . . . . . . . . . . . . 74

Maine ............................ 77

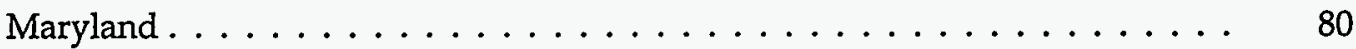

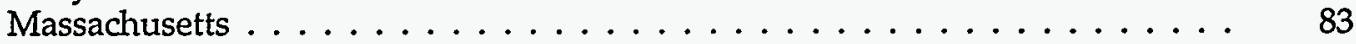

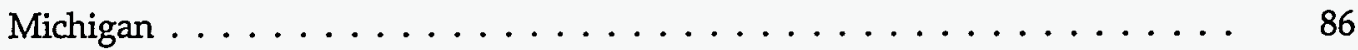

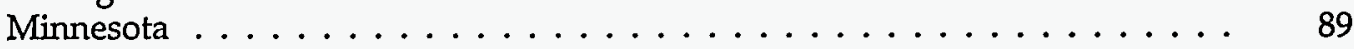

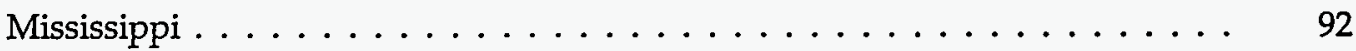

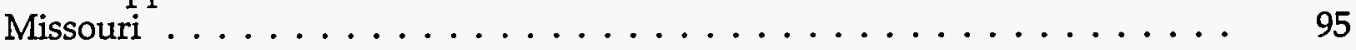

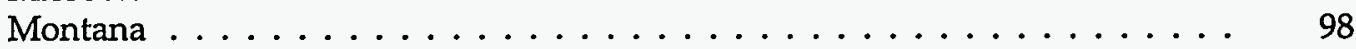

Nebraska . . . . . . . . . . . . . . . . . . . . 101

Nevada . . . . . . . . . . . . . . . . . . . . . . . . . 104

New Hampshire . . . . . . . . . . . . . . . . . . . 107

New Jersey . . . . . . . . . . . . . . . . . . . . . 110

New Mexico . . . . . . . . . . . . . . . . . . . . . . . . . . . 113

New York . . . . . . . . . . . . . . . . . . . . . . . . 116

North Carolina . . . . . . . . . . . . . . . . . . . . . 119

North Dakota . . . . . . . . . . . . . . . . . . . . . . . . . . 122

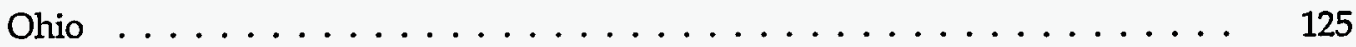

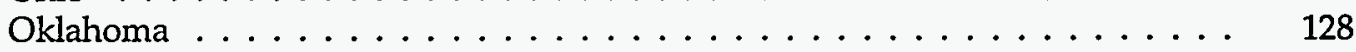

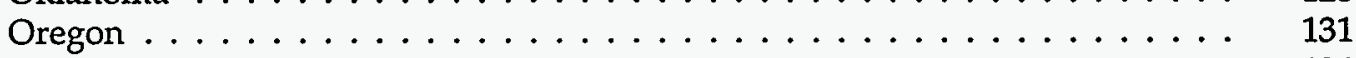

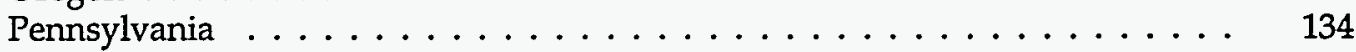

Rhode Island . . . . . . . . . . . . . . . . . . . . . . . . . 137

South Carolina ......................... 140 
Statistical Tables (continued)

South Dakota . . . . . . . . . . . . . . . . . . . . . . . . . . 143

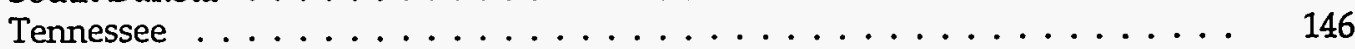

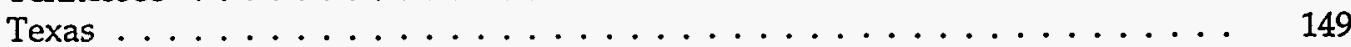

Utah . . . . . . . . . . . . . . . . . . . . 152

Vermont ........................... 155

Virginia . . . . . . . . . . . . . . . . . . . . . . 158

Washington . . . . . . . . . . . . . . . 161

West Virginia . . . . . . . . . . . . . . . . . . . . . 164

Wisconsin . . . . . . . . . . . . . . . . . . 167

Wyoming $\ldots \ldots \ldots \ldots \ldots \ldots \ldots \ldots \ldots \ldots \ldots \ldots \ldots \ldots \ldots$

Appendices

A. Documentation . . . . . . . . . . . . . . . . . 175

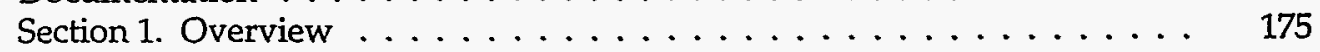

Section 2. Coal . . . . . . . . . . . . . . . . . . . 181

Coking Coal . . . . . . . . . . . . . . . . . . . 181

Steam Coal . . . . . . . . . . . . . . . . . . . . . . . . . 182

Coal Coke, Imports and Exports . . . . . . . . . . . . . . . 188

Section 3. Natural Gas . . . . . . . . . . . . . . . . . . . . . . 189

Section 4. Petroleum . . . . . . . . . . . . . . . . . . . 193

Asphalt and Road Oil . . . . . . . . . . . . . . . . . . 193

Aviation Gasoline . . . . . . . . . . . . . . . . . . . . . . . . 194

Distillate Fuel . . . . . . . . . . . . . . . . . . . . . . . . . . . 194

Heavy Oil (Utilities) . . . . . . . . . . . . . . . . . . 209

Jet Fuel . . . . . . . . . . . . . . . . . . . . . 209

Kerosene . . . . . . . . . . . . . . . . . . 211

Light Oil (Utilities) . . . . . . . . . . . . . . . . . . . . . . 214

Liquefied Petroleum Gases . . . . . . . . . . . . . . . . 214

Lubricants . . . . . . . . . . . . . . . . . . 220

Motor Gasoline . . . . . . . . . . . . . . . . . . . . . 221

Petroleum Coke (Utilities) . . . . . . . . . . . . . . . . 229

Residual Fuel . . . . . . . . . . . . . . . . . . 230

Other Petroleum . . . . . . . . . . . . . . . . 238

Section 5. Electricity . . . . . . . . . . . . . . . . 243

Electricity Consumed by End-Use Sectors . . . . . . . . . . . . 243

Nuclear Fuel for Generation of Electricity . . . . . . . . . . . . . 244

Biomass Fuels for Generation of Electricity . . . . . . . . . . . . 246

Section 6. Consumption Adjustments for Calculating Expenditures . . . . 249

B. Metric and Other Physical Conversion Factors . . . . . . . . . . . . 255

C. Summary of Changes Since the State Energy Price and Expenditure Report 1992261

Glossary ............................ 267 


\section{Introduction}

The State Energy Price and Expenditure Report (SEPER) presents energy price and expenditure estimates individually for the 50 States and the District of Columbia and in aggregate for the United States. The price and expenditure estimates are provided by energy source and economic sector and are published for the years 1970,1975, 1980, 1985, and 1990 through 1993. Data for all years, 1970 through 1993, are available on personal computer diskettes.

Consumption estimates used to calculate expenditures and the documentation for those estimates are taken from the State Energy Data Report 1993, Consumption Estimates (SEDR), published in July 1995. Expenditures are calculated by multiplying the price estimates by the consumption estimates, adjusted to remove process fuel and intermediate product consumption. All expenditures are consumer expenditures; that is, they represent estimates of money directly spent by consumers to purchase energy, generally including taxes (see box on page 2).

The documentation in Appendix A describes how the price estimates are developed, including sources of data, methods of estimation, and conversion factors applied. Appendix B provides metric and other physical conversion factors for measures used in energy analyses. This report is an update of the State Energy Price and Expenditure Report 1992, published in December 1994. Changes from the last report are summarized in Appendix C.

Although all prices and expenditures in this report are expressed in nominal dollars, a supplement to this report planned for early 1996, entitled "Additional Measures of Energy Consumption, Expenditures, and Prices," will provide energy expenditure estimates in constant 1992 dollars and fixed-weight energy price indices in both nominal and real terms, along with an explanation of the methodology used to develop them. The supplement will also include State estimates of energy expenditures and energy expenditures per capita, both in constant 1992 dollars and fixed-weight price indices.
Reliable data for State-level prices rarely exist, especially as series that are consistent over a long period. Estimates and assumptions are applied to fill data gaps and to maintain consistent definitions in the data series over time. SEPER incorporates the most consistent series and procedures possible. Users of this report (and of the personal computer diskettes) should recognize the limitations imposed on the system due to changing and inadequate data sources. Estimates often are based on a variety of surrogate measures that are selected on the basis of availability, applicability as indicators, continuity over time, and consistency among the various energy commodities. Original source documents for data used in SEPER (cited in the documentation) include descriptions of collection methodologies, universes, imputation or adjustment techniques (if any), and errors associated with the individual processes. Due to the numerous collection forms and procedures associated with these reports, it is not possible to develop a meaningful numerical estimate of the overall errors of the material published in SEPER.

It is also important to note that, even within a State, a single average price may have limited meaning in that it represents a consumption-weighted average over a whole State. For example, urban and rural electricity prices can vary significantly from a State's weighted average, and prices in one region of a State may differ from those in another because of access to less expensive hydroelectricity. Differences within a State may be greater than differences among adjacent States. Thus, the principal value of the estimates in this report lies in general comparisons among the States, interstate comparisons for a given year, and the analysis of trends over several years.

The five economic sectors used in SEPER correspond to those used in SEDR as follows:

- Residential Sector-The residential sector is considered to consist of all private residences, whether occupied or vacant, owned or rented, including single-family homes, multifamily housing units, and mobile homes. Secondary

Note: Throughout this report, the term "State" includes the District of Columbia. 
homes, such as summer homes, are also included. Institutional housing, such as school dormitories, hospitals, and military barracks generally are not included in the residential sector; they are included in the commercial sector.

- Commercial Sector-The commercial sector, as defined economically, consists of business establishments that are not engaged in transportation or in manufacturing or other types of industrial activity (agriculture, mining, or construction). Commercial establishments include hotels, motels, restaurants, wholesale businesses, retail stores, laundries, and other service enterprises; religious and nonprofit organizations; health, social, and educational institutions; and Federal, State, and local governments. Street lights, pumps, bridges, and public services are also included if the establishment operating them is considered commercial.

\section{Taxes in SEPER}

While the objective is to provide price estimates that include all taxes, the data sources used in $S E P E R$ do not treat taxes uniformly. In general, where taxes are included in the source data, they are also included in SEPER. Where taxes are not included but can be separately estimated, they are generally added. In many cases, States and even some localities provide tax exemptions for various kinds of activities or classes of end users, and readers of this report should be careful to consider these exceptions when making detailed comparisons. The Energy Information Administration (EIA) is continuing to analyze these cases to see if a better representation can be made. A comprehensive and detailed study of taxes in EIA data is available in the report End-Use Taxes: Current EIA Practices, DOE/EIA-0583 (Washington, DC, August 1994). The status of tax data in this edition of SEPER is summarized below and described more fully in the Appendix A documentation for each energy source and sector.

\section{End-Use Sectors}

Coal. All steam coal and coking coal prices include taxes in all years. Appropriately, coal imports and exports in the industrial sector do not include end-user taxes.

Natural Gas. Natural gas prices are intended to include all Federal, State, and local taxes, surcharges, and adjustments billed to consumers. However, sales and other taxes itemized directly on customers' bills are frequently not reported as revenues and, therefore, are not included in calculating the prices.
Petroleum. Motor gasoline and diesel fuel prices include excise and other per-gallon taxes but do not include general sales taxes due to wide variation at the local level. Residual fuel oil, distillate fuel oil, and kerosene prices include sales taxes in all years. Residential liquefied petroleum gas prices include taxes in all years, but commercial, industrial, and transportation sector prices do not include taxes from 1985 forward. Jet fuel, aviation gasoline, asphalt and road oil, lubricants and other petroleum products do not include taxes. Other petroleum products are miscellaneous products, petrochemical feedstocks (naphtha, other oils, and still gas), industrial petroleum coke, special naphthas, and waxes.

Electricity. Taxes paid directly by electric utilities (rather than end users) are considered operating costs and are passed on to the end users as part of the price. Depending on jurisdiction, taxes collected from end users and turned over directly to a government authority are not generally included in the reported revenues and, therefore, are not included in calculating the prices.

\section{Electric Utility Sector}

Coal, natural gas, petroleum coke, nuclear, and biomass fuels prices include all taxes, transportation, and handling costs. There are no direct fuel costs (or taxes) for hydroelectric, geothermal, centralized solar, or wind energy. Capital, operation, and maintenance costs and related taxes associated with these energy sources are included indirectly because electricity prices reflect their presence in the rate base. 
- Industrial Sector-The industrial sector comprises manufacturing industries, which make up the largest part of the sector, along with mining, construction, agriculture, fisheries, and forestry. Establishments in the sector range from steel mills, to small farms, to companies assembling electronic components.

- Transportation Sector-The transportation sector consists of private and public vehicles that move people and commodities. Included are automobiles, trucks, buses, motorcycles, railroads and railways (including streetcars), aircraft, ships, barges, and natural gas pipelines.

- Electric Utility Sector-The electric utility sector consists of privately and publicly owned establishments that generate, transmit, distribute, or sell electricity primarily for use by the public and meet the definition of an electric utility. Nonutility power producers are not included in the electric utility sector.

Although end-use allocations of energy consumption and expenditures follow those guidelines as closely as possible, some data are collected by using different classifications. For example, electric utilities often classify commercial and industrial users by the quantity of electricity purchases rather than by the business activity of the purchaser. Agricultural use of natural gas is collected and reported in the commercial sector, rather than in the industrial sector. Since agricultural use of natural gas cannot be identified separately, it remains in the commercial sector in this report. Another example is master-metered condominiums, apartments, and buildings with a combination of residential and commercial units. In many cases, billing and metering practices cause residential energy usage of electricity, natural gas, or fuel oil to be included in the commercial sector. In those cases, there is no basis for separating residential from commercial use. Readers are advised to consult the $S E D R$ documentation for specific assumptions regarding the consumption estimates.
Where prices for an energy source and sector are not available, comparable prices are substituted. For example, the transportation sector motor gasoline prices are also applied to the commercial and industrial sectors. In some cases, the average of adjacent States' prices is assigned to a missing State price. The documentation elaborates on these price assumptions. There are no direct fuel costs for hydroelectric, geothermal, centralized solar, or wind energy. Capital costs associated with these energy sources frequently are extensive and are included indirectly because the prices charged to the consumer for electricity reflect their presence in the rate base.

Except where specified, it is not generally possible to describe the prices in this report as "wholesale" or "retail." The prices paid in each consuming sector are actually a combination of both sets of prices depending on a number of closely interrelated factors, and the data reflect the combination of prices actually paid by each sector. Almost all residential sector prices are close to retail prices, reflecting the relatively small quantities of individual purchases and the increased costs of extensive, diffuse, and multilayered distribution systems. Similarly, in the transportation sector almost everyone pays the same retail-like price for motor gasoline, regardless of volume purchased or location of purchase. Conversely, residual fuel oil prices in the transportation sector are certainly more wholesale-like as a result of large deliveries to bulk facilities in major ports. In the same manner, most large industrial and many large commercial expenditures can be thought of as near wholesale, frequently involving direct access to a producer or bulk distribution facility for very large quantities. Many smaller industrial and commercial facilities pay something much closer to retail prices as a result of the small quantities involved and their institutional distance from primary suppliers. Notable exceptions to these relationships include natural gas and electric utilities, which typically establish fixed rates for each of several classes of service depending on representative quantities, service factors, and distribution expenses. 


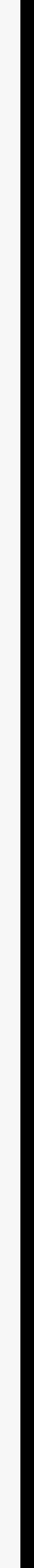


State Rankings 


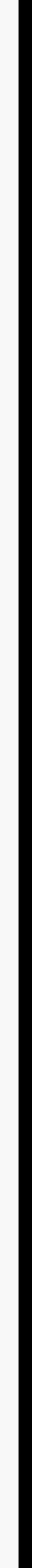


Table 1. Energy Prices

by State, 1993

(Dollars per Million Btu)

\begin{tabular}{|c|c|c|}
\hline Rank & State & Price \\
\hline 1 & Arizona & 11.90 \\
\hline 2 & District of Columbia & 11.80 \\
\hline 3 & Connecticut & 11.73 \\
\hline 4 & New Hampshire & 11.58 \\
\hline 5 & Hawaii & 11.22 \\
\hline 6 & Vermont & 11.12 \\
\hline 7 & New York & 11.05 \\
\hline 8 & Florida & 10.57 \\
\hline 9 & Massachusetts & 10.52 \\
\hline 10 & Maryland & 10.05 \\
\hline 11 & North Carolina & 9.92 \\
\hline 12 & Rhode Island & 9.75 \\
\hline 13 & California & 9.60 \\
\hline 14 & Maine & 9.39 \\
\hline 15 & New Mexico & 9.12 \\
\hline 16 & Virginia & 9.10 \\
\hline 17 & Nevada & 8.92 \\
\hline 18 & New Jersey & 8.87 \\
\hline 19 & Georgia & 8.83 \\
\hline 20 & Delaware & 8.78 \\
\hline 21 & Arkansas & 8.76 \\
\hline 22 & Illinois & 8.69 \\
\hline 23 & Pennsylvania & 8.63 \\
\hline 24 & Ohio & 8.58 \\
\hline 25 & South Carolina & 8.57 \\
\hline 26 & Oregon & 8.51 \\
\hline \multirow[t]{2}{*}{27} & Missouri & 8.47 \\
\hline & United States & 8.42 \\
\hline 28 & South Dakota & 8.37 \\
\hline 29 & Mississippi & 8.29 \\
\hline 30 & Tennessee & 8.22 \\
\hline 31 & Wisconsin & 8.18 \\
\hline 32 & Minnesota & 8.08 \\
\hline 33 & Nebraska & 8.04 \\
\hline 34 & Michigan & 8.00 \\
\hline 35 & Idaho & 7.99 \\
\hline 36 & Colorado & 7.96 \\
\hline 37 & Montana & 7.82 \\
\hline 38 & Alabama & 7.81 \\
\hline 39 & lowa & 7.66 \\
\hline 40 & Kentucky & 7.61 \\
\hline 41 & Kansas & 7.47 \\
\hline 42 & Oklahoma & 7.31 \\
\hline 43 & Washington & 7.30 \\
\hline 44 & Utah & 6.93 \\
\hline 45 & West Virginia & 6.84 \\
\hline 46 & Indiana & 6.69 \\
\hline 47 & Alaska & 6.56 \\
\hline 48 & Texas & 6.46 \\
\hline 49 & North Dakota & 6.37 \\
\hline 50 & Wyoming & 6.11 \\
\hline 51 & Louisiana & 5.55 \\
\hline
\end{tabular}

Note: Rankings are based on unrounded data. Source: State Energy Price and Expenditure Data System 1993.
Table 2. Energy Expenditures

by State, 1993

(Million Dollars)

\begin{tabular}{|c|c|c|}
\hline Rank & State & Expenditure \\
\hline 1 & California & 48,816 \\
\hline 2 & Texas & 45,490 \\
\hline 3 & New York & 30,368 \\
\hline 4 & Pennsylvania & 22,984 \\
\hline 5 & Illinois & 22,605 \\
\hline 6 & Ohio & 22,495 \\
\hline 7 & Florida & 21,316 \\
\hline 8 & Michigan & 17,538 \\
\hline 9 & New Jersey & 16,408 \\
\hline 10 & Georgia & 13,833 \\
\hline 11 & North Carolina & 13,621 \\
\hline 12 & Louisiana & 13,095 \\
\hline 13 & Indiana & 12,318 \\
\hline 14 & Virginia & 11,816 \\
\hline 15 & Massachusetts & 11,327 \\
\hline 16 & Tennessee & 9,892 \\
\hline 17 & Missouri & 9,806 \\
\hline 18 & Alabama & 8,746 \\
\hline 19 & Wisconsin & 8,669 \\
\hline 20 & Washington & 8,628 \\
\hline 21 & Maryland & 8,583 \\
\hline 22 & Minnesota & 8,286 \\
\hline 23 & Kentucky & 7,757 \\
\hline 24 & Arizona & 7,380 \\
\hline 25 & South Carolina & 7,095 \\
\hline 26 & Connecticut & 6,607 \\
\hline 27 & Oklahoma & 6,427 \\
\hline 28 & Colorado & 5,872 \\
\hline 29 & lowa & 5,561 \\
\hline 30 & Kansas & 5,397 \\
\hline 31 & Oregon & 5,366 \\
\hline 32 & Mississippi & 5,110 \\
\hline 33 & Arkansas & 5,095 \\
\hline 34 & West Virginia & 3,927 \\
\hline 35 & New Mexico & 3,192 \\
\hline 36 & Nebraska & 3,175 \\
\hline 37 & Utah & 2,922 \\
\hline 38 & Nevada & 2,746 \\
\hline 39 & Maine & 2,669 \\
\hline 40 & New Hampshire & 2,091 \\
\hline 41 & Idaho & 2,081 \\
\hline 42 & Hawaii & 1,975 \\
\hline 43 & Rhode Island & 1,926 \\
\hline 44 & Montana & 1,851 \\
\hline 45 & Alaska & 1,845 \\
\hline 46 & Wyoming & 1,667 \\
\hline 47 & North Dakota & 1,634 \\
\hline 48 & Delaware & 1,494 \\
\hline 49 & South Dakota & 1,391 \\
\hline 50 & District of Columbia & 1,222 \\
\hline \multirow[t]{2}{*}{51} & Vermont & 1,167 \\
\hline & United States & ${ }^{a} 493,337$ \\
\hline
\end{tabular}

\footnotetext{
${ }^{\mathrm{a}}$ Includes $\$ 56$ million for coal coke net imports, which is not allocated to the States.

Note: Rankings are based on unrounded data.

Source: State Energy Price and Expenditure Data System 1993.
} 
Table 3. Energy Expenditures per Capita by State, 1993 (Dollars)

\begin{tabular}{|c|c|c|}
\hline Rank & State & Expenditure \\
\hline 1 & Wyoming & 3,546 \\
\hline 2 & Alaska & 3,085 \\
\hline 3 & Louisiana & 3,052 \\
\hline 4 & North Dakota & 2,564 \\
\hline 5 & Texas & 2,524 \\
\hline 6 & Montana & 2,201 \\
\hline 7 & West Virginia & 2,160 \\
\hline 8 & Indiana & 2,159 \\
\hline 9 & Maine & 2,152 \\
\hline 10 & Delaware & 2,141 \\
\hline 11 & Kansas & 2,129 \\
\hline 12 & District of Columbia & 2,109 \\
\hline 13 & Arkansas & 2,100 \\
\hline 14 & Alabama & 2,092 \\
\hline 15 & New Jersey & 2,088 \\
\hline 16 & Kentucky & 2,044 \\
\hline 17 & Ohio & 2,034 \\
\hline 18 & Vermont & 2,026 \\
\hline 19 & Connecticut & 2,015 \\
\hline 20 & Georgia & 2,004 \\
\hline 21 & Oklahoma & 1,988 \\
\hline 22 & Nevada & 1,987 \\
\hline 23 & New Mexico & 1,975 \\
\hline 24 & lowa & 1,971 \\
\hline 25 & Nebraska & 1,968 \\
\hline 26 & North Carolina & 1,959 \\
\hline 27 & South Carolina & 1,955 \\
\hline 28 & South Dakota & 1,943 \\
\hline 29 & Tennessee & 1,942 \\
\hline 30 & Mississippi & 1,936 \\
\hline 31 & Illinois & 1,934 \\
\hline \multirow[t]{2}{*}{32} & Rhode Island & 1,926 \\
\hline & United States & 1,914 \\
\hline 33 & Pennsylvania & 1,911 \\
\hline 34 & Idaho & 1,892 \\
\hline 35 & Massachusetts & 1,882 \\
\hline 36 & Missouri & 1,873 \\
\hline 37 & Arizona & 1,871 \\
\hline 38 & New Hampshire & 1,861 \\
\hline 39 & Michigan & 1,854 \\
\hline 40 & Minnesota & 1,831 \\
\hline 41 & Virginia & 1,825 \\
\hline 42 & Oregon & 1,768 \\
\hline 43 & Maryland & 1,731 \\
\hline 44 & Wisconsin & 1,719 \\
\hline 45 & Hawaii & 1,693 \\
\hline 46 & New York & 1,673 \\
\hline 47 & Colorado & 1,648 \\
\hline 48 & Washington & 1,641 \\
\hline 49 & Utah & 1,571 \\
\hline 50 & California & 1,564 \\
\hline 51 & Florida & 1,553 \\
\hline
\end{tabular}

Note: Rankings are based on unrounded data. Source: State Energy Price and Expenditure Data System 1993.
Table 4. Motor Gasoline Expenditures per Capita by State, 1993

(Dollars)

\begin{tabular}{|c|c|c|}
\hline Rank & State & Expenditure \\
\hline 1 & Wyoming & 734 \\
\hline 2 & Montana & 675 \\
\hline 3 & North Dakota & 671 \\
\hline 4 & New Mexico & 671 \\
\hline 5 & South Dakota & 634 \\
\hline 6 & Vermont & 605 \\
\hline 7 & Oregon & 594 \\
\hline 8 & Idaho & 590 \\
\hline 9 & Maine & 584 \\
\hline 10 & Nevada & 584 \\
\hline 11 & Arkansas & 582 \\
\hline 12 & Alabama & 579 \\
\hline 13 & Delaware & 578 \\
\hline 14 & Tennessee & 570 \\
\hline 15 & Minnesota & 567 \\
\hline 16 & Arizona & 563 \\
\hline 17 & Oklahoma & 561 \\
\hline 18 & Mississippi & 561 \\
\hline 19 & West Virginia & 560 \\
\hline 20 & Kentucky & 555 \\
\hline 21 & Texas & 554 \\
\hline 22 & Georgia & 553 \\
\hline 23 & Colorado & 551 \\
\hline 24 & New Hampshire & 550 \\
\hline 25 & North Carolina & 550 \\
\hline 26 & Connecticut & 549 \\
\hline 27 & Nebraska & 548 \\
\hline 28 & Virginia & 548 \\
\hline 29 & Missouri & 543 \\
\hline 30 & Washington & 540 \\
\hline 31 & Alaska & 538 \\
\hline 32 & South Carolina & 534 \\
\hline 33 & Louisiana & 530 \\
\hline 34 & lowa & 528 \\
\hline 35 & Kansas & 506 \\
\hline \multirow[t]{2}{*}{36} & Indiana & 504 \\
\hline & United States & 504 \\
\hline 37 & Florida & 499 \\
\hline 38 & Ohio & 494 \\
\hline 39 & Maryland & 493 \\
\hline 40 & Michigan & 490 \\
\hline 41 & California & 485 \\
\hline 42 & Wisconsin & 482 \\
\hline 43 & Utah & 480 \\
\hline 44 & Massachusetts & 476 \\
\hline 45 & Hawaii & 463 \\
\hline 46 & Rhode Island & 458 \\
\hline 47 & Pennsylvania & 445 \\
\hline 48 & Illinois & 435 \\
\hline 49 & New Jersey & 428 \\
\hline 50 & District of Columbia & 365 \\
\hline 51 & New York & 354 \\
\hline
\end{tabular}

Note: Rankings are based on unrounded data. Source: State Energy Price and Expenditure Data System 1993. 
Table 5. Motor Gasoline Prices

by State, 1993

(Dollars per Million Btu)

\begin{tabular}{|c|c|c|}
\hline Rank & State & Price \\
\hline 1 & Hawaii & 11.35 \\
\hline 2 & Connecticut & 10.35 \\
\hline 3 & Alaska & 10.24 \\
\hline 4 & Oregon & 10.24 \\
\hline 5 & New Mexico & 10.13 \\
\hline 6 & Colorado & 9.87 \\
\hline 7 & West Virginia & 9.87 \\
\hline 8 & Arizona & 9.83 \\
\hline 9 & Montana & 9.83 \\
\hline 10 & Rhode Island & 9.83 \\
\hline 11 & Massachusetts & 9.73 \\
\hline 12 & Idaho & 9.67 \\
\hline 13 & District of Columbia & 9.62 \\
\hline 14 & North Dakota & 9.60 \\
\hline 15 & Maine & 9.59 \\
\hline 16 & Minnesota & 9.51 \\
\hline 17 & Nevada & 9.47 \\
\hline 18 & New Hampshire & 9.42 \\
\hline 19 & Washington & 9.42 \\
\hline 20 & Louisiana & 9.39 \\
\hline 21 & Maryland & 9.39 \\
\hline 22 & Vermont & 9.35 \\
\hline 23 & California & 9.34 \\
\hline 24 & Nebraska & 9.33 \\
\hline 25 & New York & 9.30 \\
\hline 26 & Pennsyivania & 9.27 \\
\hline 27 & Delaware & 9.24 \\
\hline 28 & Texas & 9.16 \\
\hline 29 & Virginia & 9.15 \\
\hline 30 & New Jersey & 9.09 \\
\hline \multirow[t]{2}{*}{31} & Ohio & 9.07 \\
\hline & United States & 9.07 \\
\hline 32 & South Dakota & 9.03 \\
\hline 33 & Tennessee & 9.03 \\
\hline 34 & Utah & 9.02 \\
\hline 35 & Wisconsin & 8.97 \\
\hline 36 & North Carolina & 8.93 \\
\hline 37 & Alabama & 8.87 \\
\hline 38 & Mississippi & 8.83 \\
\hline 39 & Illinois & 8.83 \\
\hline 40 & Arkansas & 8.82 \\
\hline 41 & Kentucky & 8.77 \\
\hline 42 & Florida & 8.68 \\
\hline 43 & lowa & 8.68 \\
\hline 44 & Wyoming & 8.68 \\
\hline 45 & Kansas & 8.57 \\
\hline 46 & Oklahoma & 8.47 \\
\hline 47 & Indiana & 8.36 \\
\hline 48 & Michigan & 8.35 \\
\hline 49 & South Carolina & 8.20 \\
\hline 50 & Missouri & 8.18 \\
\hline 51 & Georgia & 7.81 \\
\hline
\end{tabular}

Note: Rankings are based on unrounded data.

Source: State Energy Price and Expenditure Data System 1993.
Table 6. Motor Gasoline Expenditures

by State, 1993

(Million Dollars)

\begin{tabular}{|c|c|c|}
\hline Rank & State & Expenditure \\
\hline 1 & California & 15,140 \\
\hline 2 & Texas & 9,981 \\
\hline 3 & Florida & 6,846 \\
\hline 4 & New York & 6,431 \\
\hline 5 & Ohio & 5,469 \\
\hline 6 & Pennsylvania & 5,354 \\
\hline 7 & Illinois & 5,080 \\
\hline 8 & Michigan & 4,640 \\
\hline 9 & North Carolina & 3,821 \\
\hline 10 & Georgia & 3,816 \\
\hline 11 & Virginia & 3,546 \\
\hline 12 & New Jersey & 3,364 \\
\hline 13 & Tennessee & 2,904 \\
\hline 14 & Indiana & 2,878 \\
\hline 15 & Massachusetts & 2,865 \\
\hline 16 & Missouri & 2,840 \\
\hline 17 & Washington & 2,838 \\
\hline 18 & Minnesota & 2,566 \\
\hline 19 & Maryland & 2,445 \\
\hline 20 & Wisconsin & 2,434 \\
\hline 21 & Alabama & 2,421 \\
\hline 22 & Louisiana & 2,273 \\
\hline 23 & Arizona & 2,222 \\
\hline 24 & Kentucky & 2,108 \\
\hline 25 & Colorado & 1,964 \\
\hline 26 & South Carolina & 1,940 \\
\hline 27 & Oklahoma & 1,815 \\
\hline 28 & Oregon & 1,803 \\
\hline 29 & Connecticut & 1,799 \\
\hline 30 & lowa & 1,490 \\
\hline 31 & Mississippi & 1,480 \\
\hline 32 & Arkansas & 1,411 \\
\hline 33 & Kansas & 1,282 \\
\hline 34 & New Mexico & 1,085 \\
\hline 35 & West Virginia & 1,017 \\
\hline 36 & Utah & 892 \\
\hline 37 & Nebraska & 884 \\
\hline 38 & Nevada & 807 \\
\hline 39 & Maine & 724 \\
\hline 40 & Idaho & 649 \\
\hline 41 & New Hampshire & 618 \\
\hline 42 & Montana & 568 \\
\hline 43 & Hawaii & 540 \\
\hline 44 & Rhode Island & 458 \\
\hline 45 & South Dakota & 454 \\
\hline 46 & North Dakota & 428 \\
\hline 47 & Delaware & 403 \\
\hline 48 & Vermont & 348 \\
\hline 49 & Wyoming & 345 \\
\hline 50 & Alaska & 321 \\
\hline \multirow[t]{2}{*}{51} & District of Columbia & 211 \\
\hline & United States & 130,021 \\
\hline
\end{tabular}

Note: Rankings are based on unrounded data.

Source: State Energy Price and Expenditure Data System 1993. 
Table 7. Petroleum Prices

by State, 1993

(Dollars per Million Btu)

\begin{tabular}{|c|c|c|}
\hline Rank & State & Price \\
\hline 1 & Arizona & 8.72 \\
\hline 2 & Vermont & 8.39 \\
\hline 3 & Idaho & 8.39 \\
\hline 4 & Colorado & 8.07 \\
\hline 5 & Ohio & 8.06 \\
\hline 6 & Wisconsin & 8.03 \\
\hline 7 & Oregon & 8.02 \\
\hline 8 & Arkansas & 8.02 \\
\hline 9 & District of Columbia & 7.97 \\
\hline 10 & Rhode Island & 7.96 \\
\hline 11 & New Mexico & 7.91 \\
\hline 12 & Illinois & 7.88 \\
\hline 13 & Nebraska & 7.88 \\
\hline 14 & Minnesota & 7.82 \\
\hline 15 & Connecticut & 7.80 \\
\hline 16 & Montana & 7.77 \\
\hline 17 & Michigan & 7.77 \\
\hline 18 & Nevada & 7.68 \\
\hline 19 & North Carolina & 7.67 \\
\hline 20 & Kentucky & 7.64 \\
\hline 21 & Tennessee & 7.64 \\
\hline 22 & Pennsylvania & 7.63 \\
\hline 23 & lowa & 7.63 \\
\hline 24 & California & 7.59 \\
\hline 25 & South Dakota & 7.58 \\
\hline 26 & North Dakota & 7.54 \\
\hline 27 & Maryland & 7.53 \\
\hline 28 & Virginia & 7.50 \\
\hline 29 & West Virginia & 7.47 \\
\hline 30 & Utah & 7.43 \\
\hline 31 & Alabama & 7.41 \\
\hline 32 & Wyoming & 7.38 \\
\hline 33 & Oklahoma & 7.20 \\
\hline 34 & New Hampshire & 7.18 \\
\hline 35 & New York & 7.15 \\
\hline 36 & Missouri & 7.14 \\
\hline \multirow[t]{2}{*}{37} & Kansas & 7.10 \\
\hline & United States & 7.09 \\
\hline 38 & South Carolina & 7.04 \\
\hline 39 & Massachusetts & 7.04 \\
\hline 40 & Indiana & 6.89 \\
\hline 41 & Georgia & 6.84 \\
\hline 42 & Alaska & 6.77 \\
\hline 43 & Washington & 6.73 \\
\hline 44 & Mississippi & 6.56 \\
\hline 45 & Maine & 6.54 \\
\hline 46 & New Jersey & 6.44 \\
\hline 47 & Florida & 6.28 \\
\hline 48 & Delaware & 6.20 \\
\hline 49 & Hawaij & 6.05 \\
\hline 50 & Texas & 5.79 \\
\hline 51 & Louisiana & 5.42 \\
\hline
\end{tabular}

Note: Rankings are based on unrounded data.

Source: State Energy Price and Expenditure Data System 1993.
Table 8. Petroleum Expenditures

by State, 1993

(Million Dollars)

\begin{tabular}{|c|c|c|}
\hline Rank & State & Expenditure \\
\hline 1 & Texas & 23,576 \\
\hline 2 & California & 22,256 \\
\hline 3 & New York & 11,335 \\
\hline 4 & Florida & 10,255 \\
\hline 5 & Pennsylvania & 9,472 \\
\hline 6 & Ohio & 8,832 \\
\hline 7 & Illinois & 8,280 \\
\hline 8 & Michigan & 7,339 \\
\hline 9 & New Jersey & 7,155 \\
\hline 10 & Louisiana & 6,601 \\
\hline 11 & Georgia & 6,039 \\
\hline 12 & North Carolina & 6,011 \\
\hline 13 & Virginia & 5,572 \\
\hline 14 & Indiana & 5,369 \\
\hline 15 & Massachusetts & 5,216 \\
\hline 16 & Missouri & 4,750 \\
\hline 17 & Washington & 4,612 \\
\hline 18 & Tennessee & 4,580 \\
\hline 19 & Minnesota & 4,165 \\
\hline 20 & Wisconsin & 4,010 \\
\hline 21 & Maryland & 3,951 \\
\hline 22 & Alabama & 3,889 \\
\hline 23 & Kentucky & 3,885 \\
\hline 24 & Arizona & 3,284 \\
\hline 25 & Connecticut & 3,121 \\
\hline 26 & Oklahoma & 3,053 \\
\hline 27 & Colorado & 2,955 \\
\hline 28 & South Carolina & 2,925 \\
\hline 29 & Oregon & 2,865 \\
\hline 30 & Mississippi & 2,584 \\
\hline 31 & Kansas & 2,518 \\
\hline 32 & lowa & 2,505 \\
\hline 33 & Arkansas & 2,329 \\
\hline 34 & West Virginia & 2,010 \\
\hline 35 & New Mexico & 1,809 \\
\hline 36 & Nebraska & 1,619 \\
\hline 37 & Maine & 1,542 \\
\hline 38 & Utah & 1,463 \\
\hline 39 & Nevada & 1,389 \\
\hline 40 & Hawaii & 1,262 \\
\hline 41 & Alaska & 1,197 \\
\hline 42 & Idaho & 1,111 \\
\hline 43 & Montana & 1,090 \\
\hline 44 & New Hampshire & 1,052 \\
\hline 45 & Wyoming & 848 \\
\hline 46 & North Dakota & 827 \\
\hline 47 & South Dakota & 823 \\
\hline 48 & Rhode Island & 784 \\
\hline 49 & Delaware & 755 \\
\hline 50 & Vermont & 679 \\
\hline \multirow[t]{2}{*}{51} & District of Columbia & 293 \\
\hline & United States & 225,842 \\
\hline
\end{tabular}

Note: Rankings are based on unrounded data.

Source: State Energy Price and Expenditure Data System 1993. 
Table 9. Natural Gas Prices

by State, 1993

(Dollars per Million Btu)

\begin{tabular}{|c|c|c|}
\hline Rank & State & Price \\
\hline 1 & Hawaii & ${ }^{\mathrm{a}} 13.05$ \\
\hline 2 & District of Columbia & 7.01 \\
\hline 3 & Connecticut & 6.99 \\
\hline 4 & New Hampshire & 6.56 \\
\hline 5 & Massachusetts & 6.10 \\
\hline 6 & Maine & 6.06 \\
\hline 7 & New York & 6.00 \\
\hline 8 & Rhode Island & 5.97 \\
\hline 9 & Maryland & 5.44 \\
\hline 10 & Pennsylvania & 5.33 \\
\hline 11 & Georgia & 5.18 \\
\hline 12 & Virginia & 5.14 \\
\hline 13 & New Jersey & 5.07 \\
\hline 14 & Ohio & 5.03 \\
\hline 15 & Vermont & 4.99 \\
\hline 16 & Illinois & 4.97 \\
\hline 17 & Wisconsin & 4.92 \\
\hline 18 & Missouri & 4.88 \\
\hline 19 & Arizona & 4.86 \\
\hline 20 & North Carolina & 4.79 \\
\hline 21 & South Dakota & 4.60 \\
\hline 22 & Indiana & 4.52 \\
\hline 23 & lowa & 4.49 \\
\hline 24 & Tennessee & 4.49 \\
\hline 25 & North Dakota & 4.41 \\
\hline 26 & Michigan & 4.39 \\
\hline 27 & Minnesota & 4.36 \\
\hline 28 & Alabama & 4.34 \\
\hline 29 & West Virginia & 4.33 \\
\hline 30 & Kentucky & 4.30 \\
\hline 31 & Nebraska & 4.25 \\
\hline 32 & Montana & 4.21 \\
\hline \multirow[t]{2}{*}{33} & South Carolina & 4.19 \\
\hline & United States & 4.16 \\
\hline 34 & Oregon & 4.14 \\
\hline 35 & California & 4.11 \\
\hline 36 & Nevada & 4.05 \\
\hline 37 & Delaware & 4.04 \\
\hline 38 & Utah & 3.99 \\
\hline 39 & Washington & 3.97 \\
\hline 40 & New Mexico & 3.91 \\
\hline 41 & Arkansas & 3.78 \\
\hline 42 & Idaho & 3.77 \\
\hline 43 & Colorado & 3.75 \\
\hline 44 & Wyoming & 3.70 \\
\hline 45 & Kansas & 3.59 \\
\hline 46 & Florida & 3.38 \\
\hline 47 & Mississippi & 3.31 \\
\hline 48 & Oklahoma & 3.17 \\
\hline 49 & Texas & 2.79 \\
\hline 50 & Louisiana & 2.50 \\
\hline 51 & Alaska & 1.87 \\
\hline
\end{tabular}

a Based on small quantities of liquefied natural gas.

Note: Rankings are based on unrounded data.

Source: State Energy Price and Expenditure Data System 1993.
Table 10. Natural Gas Expenditures

by State, 1993

(Million Dollars)

\begin{tabular}{|c|c|c|}
\hline Rank & State & Expenditure \\
\hline 1 & Texas & 8,848 \\
\hline 2 & California & 7,759 \\
\hline 3 & New York & 5,790 \\
\hline 4 & Illinois & 5,101 \\
\hline 5 & Ohio & 4,222 \\
\hline 6 & Michigan & 3,882 \\
\hline 7 & Pennsylvania & 3,540 \\
\hline 8 & Louisiana & 2,965 \\
\hline 9 & New Jersey & 2,842 \\
\hline 10 & Indiana & 2,281 \\
\hline 11 & Massachusetts & 1,964 \\
\hline 12 & Georgia & 1,788 \\
\hline 13 & Wisconsin & 1,681 \\
\hline 14 & Oklahoma & 1,430 \\
\hline 15 & Minnesota & 1,355 \\
\hline 16 & Missouri & 1,323 \\
\hline 17 & Florida & 1,178 \\
\hline 18 & Virginia & 1,116 \\
\hline 19 & lowa & 1,084 \\
\hline 20 & Tennessee & 1,068 \\
\hline 21 & Alabama & 1,063 \\
\hline 22 & Kansas & 1,036 \\
\hline 23 & Maryland & 996 \\
\hline 24 & Colorado & 928 \\
\hline 25 & North Carolina & 891 \\
\hline 26 & Kentucky & 799 \\
\hline 27 & Connecticut & 797 \\
\hline 28 & Washington & 771 \\
\hline 29 & Arkansas & 762 \\
\hline 30 & South Carolina & 599 \\
\hline 31 & Mississippi & 589 \\
\hline 32 & Oregon & 560 \\
\hline 33 & Utah & 513 \\
\hline 34 & Nebraska & 512 \\
\hline 35 & West Virginia & 501 \\
\hline 36 & Arizona & 487 \\
\hline 37 & Rhode Island & 463 \\
\hline 38 & New Mexico & 388 \\
\hline 39 & Nevada & 346 \\
\hline 40 & Wyoming & 281 \\
\hline 41 & District of Columbia & 232 \\
\hline 42 & Alaska & 213 \\
\hline 43 & Idaho & 205 \\
\hline 44 & Montana & 198 \\
\hline 45 & Delaware & 171 \\
\hline 46 & South Dakota & 132 \\
\hline 47 & North Dakota & 125 \\
\hline 48 & New Hampshire & 110 \\
\hline 49 & Hawaii & 37 \\
\hline 50 & Vermont & 36 \\
\hline \multirow[t]{2}{*}{51} & Maine & 31 \\
\hline & United States & 75,982 \\
\hline
\end{tabular}

Note: Rankings are based on unrounded data.

Source: State Energy Price and Expenditure Data System 1993. 
Table 11. Coal Prices

by State, 1993

(Dollars per Million Btu)

\begin{tabular}{|c|c|c|}
\hline Rank & State & Price \\
\hline 1 & Alaska & 4.77 \\
\hline 2 & Vermont & 3.62 \\
\hline 3 & Rhode Island & 3.31 \\
\hline 4 & Maine & 2.36 \\
\hline 5 & District of Columbia & 1.96 \\
\hline 6 & California & 1.90 \\
\hline 7 & Idaho & 1.79 \\
\hline 8 & Georgia & 1.78 \\
\hline 9 & Florida & 1.78 \\
\hline 10 & Connecticut & 1.75 \\
\hline 11 & New Jersey & 1.75 \\
\hline 12 & Alabama & 1.75 \\
\hline 13 & Hawaii & 1.74 \\
\hline 14 & Massachusetts & 1.71 \\
\hline 15 & Arkansas & 1.71 \\
\hline 16 & North Carolina & 1.71 \\
\hline 17 & Illinois & 1.67 \\
\hline 18 & Delaware & 1.67 \\
\hline 19 & New Hampshire & 1.66 \\
\hline 20 & Mississippi & 1.64 \\
\hline 21 & South Carolina & 1.60 \\
\hline 22 & Louisiana & 1.59 \\
\hline 23 & Maryland & 1.58 \\
\hline 24 & New York & 1.56 \\
\hline 25 & Michigan & 1.55 \\
\hline 26 & Virginia & 1.54 \\
\hline 27 & Pennsyivania & 1.50 \\
\hline 28 & Nevada & 1.47 \\
\hline 29 & Washington & 1.45 \\
\hline \multirow[t]{2}{*}{30} & Ohio & 1.43 \\
\hline & United States & 1.43 \\
\hline 31 & West Virginia & 1.43 \\
\hline 32 & Texas & 1.42 \\
\hline 33 & Arizona & 1.38 \\
\hline 34 & Indiana & 1.37 \\
\hline 35 & New Mexico & 1.37 \\
\hline 36 & Tennessee & 1.28 \\
\hline 37 & Wisconsin & 1.27 \\
\hline 38 & Oklahoma & 1.25 \\
\hline 39 & Missouri & 1.25 \\
\hline 40 & Utah & 1.24 \\
\hline 41 & Kentucky & 1.22 \\
\hline 42 & Minnesota & 1.21 \\
\hline 43 & South Dakota & 1.20 \\
\hline 44 & Oregon & 1.20 \\
\hline 45 & North Dakota & 1.18 \\
\hline 46 & Colorado & 1.11 \\
\hline 47 & lowa & 1.07 \\
\hline 48 & Kansas & 1.03 \\
\hline 49 & Wyoming & 0.83 \\
\hline 50 & Nebraska & 0.79 \\
\hline 51 & Montana & 0.73 \\
\hline
\end{tabular}

Note: Rankings are based on unrounded data. Source: State Energy Price and Expenditure Data System 1993.
Table 12. Coal Expenditures

by State, 1993

(Million Dollars)

\begin{tabular}{|c|c|c|}
\hline Rank & State & Expenditure \\
\hline 1 & Pennsylvania & 2,116 \\
\hline 2 & Ohio & 2,051 \\
\hline 3 & Texas & 2,008 \\
\hline 4 & Indiana & 1,805 \\
\hline 5 & Alabama & 1,414 \\
\hline 6 & Illinois & 1,360 \\
\hline 7 & Georgia & 1,175 \\
\hline 8 & Florida & 1,158 \\
\hline 9 & West Virginia & 1,145 \\
\hline 10 & Kentucky & 1,129 \\
\hline 11 & Michigan & 1,101 \\
\hline 12 & North Carolina & 1,096 \\
\hline 13 & Tennessee & 880 \\
\hline 14 & Missouri & 582 \\
\hline 15 & Arizona & 536 \\
\hline 16 & Virginia & 533 \\
\hline 17 & South Carolina & 528 \\
\hline 18 & Wisconsin & 514 \\
\hline 19 & New York & 479 \\
\hline 20 & North Dakota & 471 \\
\hline 21 & Utah & 459 \\
\hline 22 & Oklahoma & 414 \\
\hline 23 & Maryland & 413 \\
\hline 24 & Minnesota & 392 \\
\hline 25 & Wyoming & 386 \\
\hline 26 & Colorado & 375 \\
\hline 27 & New Mexico & 370 \\
\hline 28 & lowa & 364 \\
\hline 29 & Louisiana & 354 \\
\hline 30 & Arkansas & 342 \\
\hline 31 & Kansas & 310 \\
\hline 32 & Nevada & 253 \\
\hline 33 & Massachusetts & 169 \\
\hline 34 & Mississippi & 163 \\
\hline 35 & Washington & 142 \\
\hline 36 & Nebraska & 131 \\
\hline 37 & Montana & 115 \\
\hline 38 & New Jersey & 110 \\
\hline 39 & California & 108 \\
\hline 40 & Delaware & 106 \\
\hline 41 & Alaska & 65 \\
\hline 42 & New Hampshire & 62 \\
\hline 43 & Oregon & 44 \\
\hline 44 & South Dakota & 41 \\
\hline 45 & Connecticut & 36 \\
\hline 46 & Maine & 26 \\
\hline 47 & Idaho & 17 \\
\hline 48 & Hawaii & 3 \\
\hline 49 & District of Columbia & 3 \\
\hline 50 & Vermont & 1 \\
\hline \multirow[t]{2}{*}{51} & Rhode Island & (s) \\
\hline & United States & 27,857 \\
\hline
\end{tabular}

(s)=Value less than 0.5 million dollars.

Note: Rankings are based on unrounded data.

Source: State Energy Price and Expenditure Data System 1993. 
Table 13. Electricity Prices

by State, 1993

(Dollars per Million Btu)

\begin{tabular}{|c|c|c|}
\hline Rank & State & Price \\
\hline 1 & New Hampshire & 31.81 \\
\hline 2 & New York & 31.43 \\
\hline 3 & Hawaii & 31.34 \\
\hline 4 & Rhode Island & 30.52 \\
\hline 5 & Connecticut & 30.07 \\
\hline 6 & Alaska & 29.72 \\
\hline 7 & New Jersey & 29.31 \\
\hline 8 & Massachusetts & 29.24 \\
\hline 9 & California & 28.51 \\
\hline 10 & Maine & 26.68 \\
\hline 11 & Vermont & 26.47 \\
\hline 12 & Arizona & 24.05 \\
\hline 13 & Pennsylvania & 23.26 \\
\hline 14 & Illinois & 22.76 \\
\hline 15 & New Mexico & 21.40 \\
\hline 16 & Florida & 21.09 \\
\hline 17 & Michigan & 20.98 \\
\hline 18 & Delaware & 20.53 \\
\hline \multirow[t]{2}{*}{19} & Maryland & 20.40 \\
\hline & United States & 20.38 \\
\hline 20 & District of Columbia & 19.83 \\
\hline 21 & Georgia & 19.71 \\
\hline 22 & Arkansas & 19.60 \\
\hline 23 & North Carolina & 19.44 \\
\hline 24 & Kansas & 19.39 \\
\hline 25 & Texas & 18.93 \\
\hline 26 & Louisiana & 18.56 \\
\hline 27 & Missouri & 18.55 \\
\hline 28 & Virginia & 18.30 \\
\hline 29 & Ohio & 18.30 \\
\hline 30 & Mississippi & 18.28 \\
\hline 31 & South Dakota & 18.17 \\
\hline 32 & Colorado & 17.78 \\
\hline 33 & Oklahoma & 17.51 \\
\hline 34 & lowa & 17.49 \\
\hline 35 & Nevada & 17.28 \\
\hline 36 & North Dakota & 17.10 \\
\hline 37 & Alabama & 16.78 \\
\hline 38 & South Carolina & 16.53 \\
\hline 39 & Minnesota & 16.46 \\
\hline 40 & Nebraska & 16.24 \\
\hline 41 & Wisconsin & 16.24 \\
\hline 42 & Utah & 15.69 \\
\hline 43 & West Virginia & 15.34 \\
\hline 44 & Tennessee & 15.33 \\
\hline 45 & Indiana & 15.19 \\
\hline 46 & Oregon & 12.97 \\
\hline 47 & Montana & 12.87 \\
\hline 48 & Kentucky & 12.70 \\
\hline 49 & Wyoming & 12.55 \\
\hline 50 & Idaho & 11.71 \\
\hline 51 & Washington & 10.77 \\
\hline
\end{tabular}

Note: Rankings are based on unrounded data. Source: State Energy Price and Expenditure Data System 1993.
Table 14. Electricity Expenditures

by State, 1993

(Million Dollars)

\begin{tabular}{|c|c|c|}
\hline Rank & State & Expenditure \\
\hline 1 & California & 20,165 \\
\hline 2 & Texas & 15,644 \\
\hline 3 & New York & 13,961 \\
\hline 4 & Florida & 10,994 \\
\hline 5 & Pennsylvania & 9,430 \\
\hline 6 & Ohio & 9,163 \\
\hline 7 & Illinois & 9,065 \\
\hline 8 & North Carolina & 6,618 \\
\hline 9 & New Jersey & 6,524 \\
\hline 10 & Michigan & 6,213 \\
\hline 11 & Georgia & 5,948 \\
\hline 12 & Virginia & 5,053 \\
\hline 13 & Massachusetts & 4,518 \\
\hline 14 & Indiana & 4,194 \\
\hline 15 & Louisiana & 4,128 \\
\hline 16 & Tennessee & 4,115 \\
\hline 17 & Maryland & 3,749 \\
\hline 18 & Missouri & 3,710 \\
\hline 19 & Arizona & 3,644 \\
\hline 20 & Alabama & 3,566 \\
\hline 21 & South Carolina & 3,471 \\
\hline 22 & Washington & 3,249 \\
\hline 23 & Wisconsin & 2,914 \\
\hline 24 & Kentucky & 2,913 \\
\hline 25 & Connecticut & 2,795 \\
\hline 26 & Minnesota & 2,728 \\
\hline 27 & Oklahoma & 2,403 \\
\hline 28 & Mississippi & 2,087 \\
\hline 29 & Arkansas & 2,041 \\
\hline 30 & Colorado & 1,979 \\
\hline 31 & Oregon & 1,973 \\
\hline 32 & lowa & 1,916 \\
\hline 33 & Kansas & 1,891 \\
\hline 34 & West Virginia & 1,266 \\
\hline 35 & Maine & 1,088 \\
\hline 36 & Nevada & 1,066 \\
\hline 37 & New Mexico & 1,058 \\
\hline 38 & Nebraska & 1,039 \\
\hline 39 & New Hampshire & 951 \\
\hline 40 & Hawaii & 903 \\
\hline 41 & Utah & 887 \\
\hline 42 & Idaho & 748 \\
\hline 43 & District of Columbia & 702 \\
\hline 44 & Rhode Island & 682 \\
\hline 45 & Delaware & 633 \\
\hline 46 & Montana & 555 \\
\hline 47 & Wyoming & 494 \\
\hline 48 & Vermont & 453 \\
\hline 49 & Alaska & 441 \\
\hline 50 & North Dakota & 431 \\
\hline \multirow[t]{2}{*}{51} & South Dakota & 428 \\
\hline & United States & 196,585 \\
\hline
\end{tabular}

Note: Rankings are based on unrounded data.

Source: State Energy Price and Expenditure Data System 1993. 


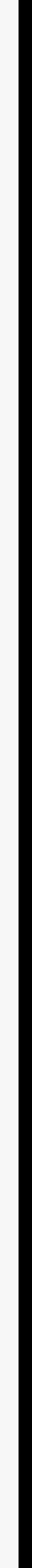


Statistical Tables 



\begin{tabular}{|c|c|c|c|c|c|c|c|c|}
\hline Energy Source & 1970 & 1975 & 1980 & 1985 & 1990 & 1991 & 1992 & 1993 \\
\hline & \multicolumn{8}{|c|}{ Prices in Dollars per Million Btu } \\
\hline 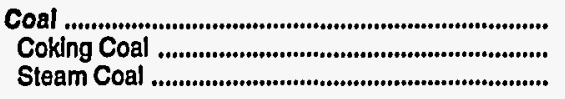 & $\begin{array}{r}0.37 \\
.45 \\
.35\end{array}$ & $\begin{array}{r}1.03 \\
1.65 \\
.90\end{array}$ & $\begin{array}{l}1.47 \\
2.10 \\
1.38\end{array}$ & $\begin{array}{l}1.70 \\
2.03 \\
1.67\end{array}$ & $\begin{array}{l}1.49 \\
1.79 \\
1.48\end{array}$ & $\begin{array}{l}1.49 \\
1.83 \\
1.47\end{array}$ & $\begin{array}{r}A_{1.46} \\
1.83 \\
1.44\end{array}$ & $\begin{array}{l}1.43 \\
1.79 \\
1.41\end{array}$ \\
\hline 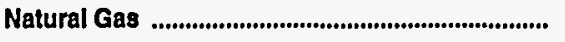 & .59 & 1.18 & 2.86 & 4.61 & 3.85 & 3.78 & 3.89 & 4.16 \\
\hline 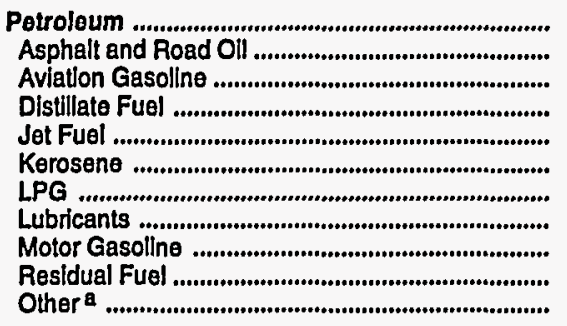 & $\begin{array}{r}1.71 \\
.68 \\
2.17 \\
1.16 \\
.73 \\
1.19 \\
1.46 \\
5.08 \\
2.85 \\
.42 \\
.95\end{array}$ & $\begin{array}{l}3.33 \\
1.89 \\
3.45 \\
2.60 \\
2.05 \\
2.73 \\
2.97 \\
7.48 \\
4.65 \\
1.93 \\
2.43\end{array}$ & $\begin{array}{r}7.35 \\
3.68 \\
9.02 \\
6.70 \\
6.36 \\
6.97 \\
5.64 \\
14.36 \\
9.84 \\
3.88 \\
6.57\end{array}$ & $\begin{array}{r}7.60 \\
4.77 \\
9.99 \\
7.16 \\
5.91 \\
8.17 \\
6.33 \\
17.61 \\
9.01 \\
4.30 \\
7.13\end{array}$ & $\begin{array}{r}A_{7.45} \\
3.02 \\
9.32 \\
7.67 \\
5.68 \\
8.26 \\
6.51 \\
R_{14.60} \\
9.12 \\
3.16 \\
R_{5.75}\end{array}$ & $\begin{array}{r}R_{7.18} \\
3.14 \\
8.71 \\
7.27 \\
4.83 \\
7.83 \\
6.54 \\
R_{16.79} \\
8.93 \\
2.62 \\
R_{5.15}\end{array}$ & $\begin{array}{r}7.06 \\
2.50 \\
8.54 \\
7.08 \\
4.52 \\
7.25 \\
5.95 \\
R_{19.43} \\
8.96 \\
R_{2.27} \\
R_{4.93}\end{array}$ & $\begin{array}{r}7.09 \\
2.90 \\
8.24 \\
7.07 \\
4.29 \\
6.98 \\
5.88 \\
18.94 \\
9.07 \\
2.25 \\
4.47\end{array}$ \\
\hline 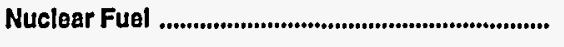 & .18 & 24 & .43 & .71 & .67 & .63 & .59 & .56 \\
\hline Blomass Fuels at Uttlitles ....................................... & .65 & .92 & 1.74 & .79 & .52 & .55 & P. .55 & .53 \\
\hline 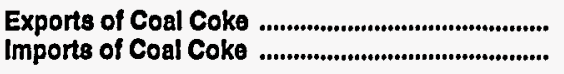 & $\begin{array}{r}1.27 \\
.93\end{array}$ & $\begin{array}{l}2.37 \\
3.47\end{array}$ & $\begin{array}{l}2.54 \\
3.19\end{array}$ & $\begin{array}{l}2.76 \\
2.99\end{array}$ & $\begin{array}{l}3.53 \\
3.80\end{array}$ & $\begin{array}{l}2.74 \\
3.40\end{array}$ & $\begin{array}{l}2.78 \\
3.32\end{array}$ & $\begin{array}{l}2.88 \\
3.04\end{array}$ \\
\hline Primary Energy — Five Sectors b ....................... & 1.08 & 2.19 & 4.58 & 4.92 & 4.52 & $R_{4.35}$ & 4.32 & 4.38 \\
\hline 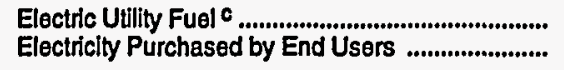 & $\begin{array}{r}.32 \\
4.99\end{array}$ & .96 & $\begin{array}{r}1.75 \\
13.95\end{array}$ & $\begin{array}{r}1.85 \\
19.05\end{array}$ & $\begin{array}{r}1.46 \\
19.33\end{array}$ & $\begin{array}{r}1.37 \\
19.85\end{array}$ & $\begin{array}{r}1.34 \\
20.06\end{array}$ & $\begin{array}{r}1.35 \\
20.38\end{array}$ \\
\hline \multirow[t]{2}{*}{ 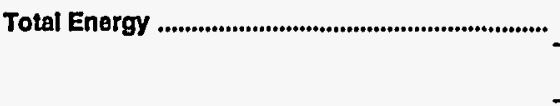 } & 1.65 & 3.33 & 6.91 & 8.42 & $\mathbf{R}_{8.38}$ & $R_{8.33}$ & $\mathrm{~B}_{8.26}$ & 8.42 \\
\hline & \multicolumn{8}{|c|}{ Expenditures in Millions of Dollars } \\
\hline 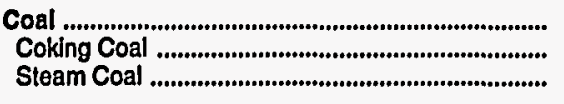 & $\begin{array}{l}4,594 \\
1,175 \\
3,419\end{array}$ & $\begin{array}{r}13,047 \\
3,692 \\
9,356\end{array}$ & $\begin{array}{r}22,648 \\
3,753 \\
18,895\end{array}$ & $\begin{array}{r}29,719 \\
2,228 \\
27,491\end{array}$ & $\begin{array}{r}A_{28,381} \\
1,862 \\
A_{26,519}\end{array}$ & $\begin{array}{r}R_{27,866} \\
1,660 \\
R_{26,207}\end{array}$ & $\begin{array}{r}R_{27,417} \\
1,587 \\
R_{25,830}\end{array}$ & $\begin{array}{r}27,857 \\
1,505 \\
26,352\end{array}$ \\
\hline 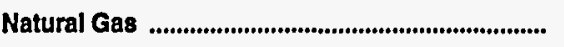 & 10,891 & 20,061 & 51,061 & 72,938 & 64,102 & 64,697 & 68,401 & 75,982 \\
\hline 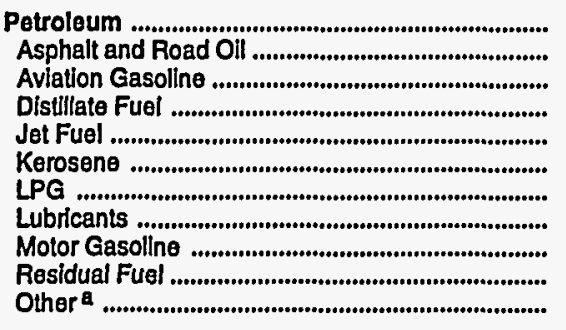 & $\begin{array}{r}48,088 \\
731 \\
218 \\
6,253 \\
1,441 \\
648 \\
2,446 \\
1,532 \\
31,596 \\
2,046 \\
1,177\end{array}$ & $\begin{array}{r}103,858 \\
1,914 \\
245 \\
15,680 \\
4,193 \\
897 \\
5,231 \\
2,277 \\
59,446 \\
10,374 \\
3,602\end{array}$ & $\begin{array}{r}238,408 \\
3,543 \\
580 \\
40,797 \\
13,923 \\
2,291 \\
10,926 \\
5,081 \\
124,408 \\
21,573 \\
15,286\end{array}$ & $\begin{array}{r}223,196 \\
4,916 \\
503 \\
43,611 \\
14,747 \\
1,927 \\
13,108 \\
5,671 \\
118,044 \\
11,495 \\
9,175\end{array}$ & $\begin{array}{r}R_{234,826} \\
3,529 \\
419 \\
49,266 \\
17,784 \\
727 \\
13,187 \\
R_{5,289} \\
126,472 \\
8,707 \\
R_{9,445}\end{array}$ & $\begin{array}{r}R_{222,298} \\
3,382 \\
363 \\
45,105 \\
14,609 \\
751 \\
14,372 \\
R_{5,444} \\
123,051 \\
6,787 \\
R_{8,434}\end{array}$ & $\begin{array}{r}R_{221,701} \\
2,755 \\
351 \\
R_{44,919} \\
13,559 \\
623 \\
R_{13,628} \\
R_{6,422} \\
125,158 \\
R_{5,576} \\
R_{8,711}\end{array}$ & $\begin{array}{r}225,842 \\
3,336 \\
316 \\
45,675 \\
13,002 \\
717 \\
13,212 \\
6,374 \\
130,021 \\
5,450 \\
7,739\end{array}$ \\
\hline Nuclear Fuel ........................................................ & 44 & 448 & 1,189 & 2,930 & 4,142 & 4,172 & 3,878 & 3,658 \\
\hline Blomass Fuels at Utilities ...................................... & 2 & 2 & 8 & 11 & 11 & 12 & $R_{12}$ & 11 \\
\hline 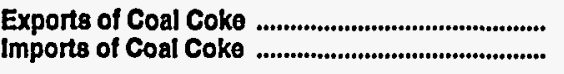 & $\begin{array}{r}-78 \\
4\end{array}$ & $\begin{array}{r}-75 \\
156\end{array}$ & $\begin{array}{r}-130 \\
52\end{array}$ & $\begin{array}{r}-77 \\
43\end{array}$ & $\begin{array}{r}-50 \\
72\end{array}$ & $\begin{array}{r}-50 \\
93\end{array}$ & $\frac{-44}{143}$ & $\begin{array}{l}-60 \\
116\end{array}$ \\
\hline Primary Energy - Flve Sectors b ....................... & 63,544 & 137,499 & 313,237 & 328,760 & $R_{331,485}$ & $R_{319,087}$ & $R_{321,507}$ & 333,405 \\
\hline 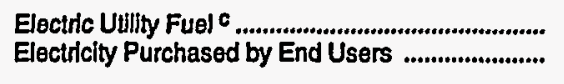 & $\begin{array}{r}-4,316 \\
23,351\end{array}$ & $\begin{array}{r}-16,396 \\
50,680\end{array}$ & $\begin{array}{l}\mathbf{3 7}, 435 \\
98,098\end{array}$ & $\begin{array}{r}-42,558 \\
149,242\end{array}$ & $\begin{array}{r}38,443 \\
176,742\end{array}$ & $\begin{array}{r}-36,501 \\
184,822\end{array}$ & $\begin{array}{r}A_{-35,764} \\
186,956\end{array}$ & $\begin{array}{r}36,653 \\
196,585\end{array}$ \\
\hline Total Energy & 82,579 & 171,782 & 373,900 & 435,444 & $R_{469,785}$ & $R_{467,408}$ & $A_{472,699}$ & 493,337 \\
\hline
\end{tabular}

ancludes petroleum coke used at electric utilities.

b Biomass fuels are not included, except those consumed at electric utilities and those added to motor gasoline.

- There are no direct fuel costs for hydroelectric, geothermal, wind, photovoltalc, or solar thermal energy.
$\mathrm{R}=$ Revised data.

Note: Expenditure totals may not equal sum of components due to independent rounding.

Sources: Data sources, estimation procedures, and assumptions are described in Appendix A. 


\section{U Energy Price Estimates by Sector, United States 1970, 1975, 1980, 1985, and 1990-1993}

(Dollars per Million Btu)

\begin{tabular}{|c|}
\hline Sector and Energy Source \\
\hline 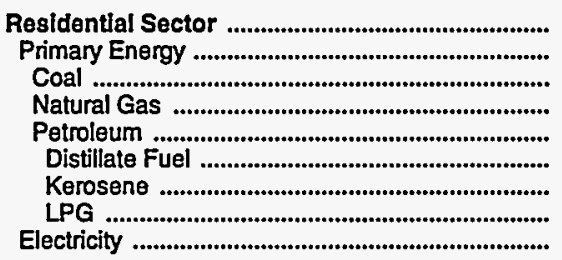 \\
\hline 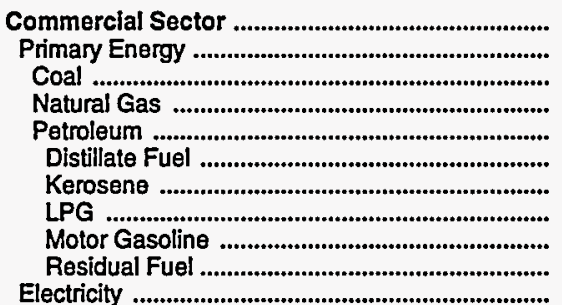 \\
\hline
\end{tabular}

Industrial Sector .

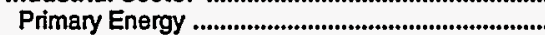
Coal

Coking Coal

.

Natural Gas

Petroleum

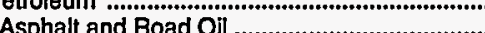

Distillate Fuel .........................................................

Kerosene ...

LPG

Lubricants

(n)

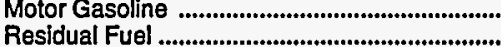

Other

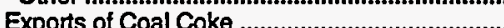

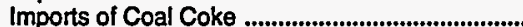

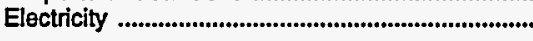

Transportation Sector ................................................

Primary Energy ..............................................................

Coal

Petroleum .....................................................................

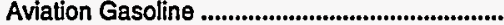

Distillate Fuel .......................................................

Jet Fue

LPG

Lubricants .........

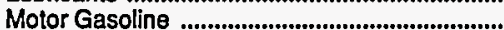

Residual Fuel

Electricity

Total Energy ...

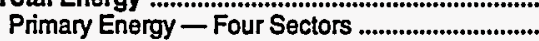

Electricity

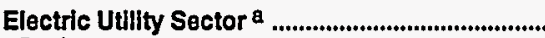

Coal

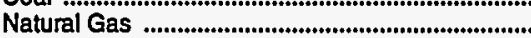

Petroleum

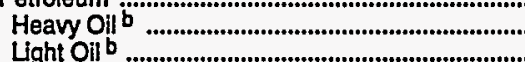

Light Oil b

Petroleum Coke ...........................................................

Nuclear Fuel

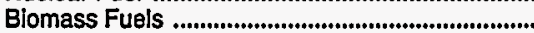

Primary Energy - Flve Sectors ${ }^{c}$

\begin{tabular}{l|l}
1970 & 197 \\
2.12 & 3. \\
1.24 & 2.1 \\
1.13 & 2.47 \\
1.06 & 1 \\
1.56 & 3. \\
1.39 & 2.74 \\
1.54 & 3 \\
2.12 & 4. \\
6.51 & 10 \\
&
\end{tabular}

1975

1985

1990

1991

1992

1993

1.97

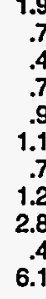

.79
.45
.75
.90
1.10
.77
1.24
2.86
.45
6.10

1.32

2.39

2.42

2.32

2.60

4.66

1.91

10.11

$.83 \quad 2.20$

$\begin{array}{ll}.83 & 1.60 \\ .45 & 1.50\end{array}$

$.45 \quad 1.65$

$.44 \quad 1.28$

$\begin{array}{lr}.38 & .95 \\ .96 & 2.41\end{array}$

$.68 \quad 1.89$

$.72 \quad 2.23$

$.77 \quad 2.34$

$\begin{array}{ll}1.10 & 2.51 \\ 5.08 & 7.49\end{array}$

$\begin{array}{ll}5.08 & 7.48 \\ 2.86 & 4.65\end{array}$

$\begin{array}{rr}2.86 & 4.65 \\ .46 & 1.91\end{array}$

$\begin{array}{ll}.46 & 1.91 \\ 1.96 & 2.43\end{array}$

$\begin{array}{ll}1.27 & 2.37\end{array}$

$.93 \quad 3.47$

$\begin{array}{ll}2.99 & 6.07\end{array}$

$2.31 \quad 4.02$

$2.31 \quad 4.02$

$.41 \quad 1.26$

$\begin{array}{ll}2.31 & 4.02 \\ 2.17 & 3.45\end{array}$

$1.31 \quad 2.80$

$.73 \quad 2.05$

$1.11 \quad 2.51$

$2.85 \quad 4.64$

4.64
1.72

11.72

3.33

2.65
8.61

1.30

4.99

$\begin{array}{lll}.32 & .96 & 1.75\end{array}$

.31

.28

.42

.41

.57
.29

.18

.65

1.08

\subsection{8}

4.02

1.55

3.32

5.64
6.45

6.46

5.15

9.77

4.12
16.06

\subsection{1}

3.77
1.87

2.10

1.56

2.52

5.59

3.68

5.54

6.30

5.18
14.36

14.36

9.82

3.69
6.58

254

3.10

10.81

8.61

8.61
8.60

$8 . \overline{0}$

9.02

7.19

6.36

$\begin{array}{r}5.20 \\ \hline\end{array}$

14.36

9.84

3.31
14.71

\subsection{1}

5.85

13.95

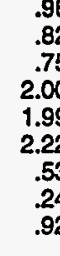

2.19

$7.55 \quad 11.14$

4.56

2.90

3.60

7.26

7.02

8.32

7.92
15.71
11.14

6.48
3.25

3.25
5.94

8.27

7.92

8.77

9.10
21.66

\subsection{1}

5.52
1.80

5.34

6.33

6.35

6.94

8.59

9.01

4.50
21.31

6.09

4.49
1.89

1.89
2.03

1.81

3.87

6.20

4.77

6.10

6.96

5.66

17.61

9.07

4.24
7.16

2.76

14.57

\subsection{6}

8.25

$8 . \overline{25}$

9.99

7.50

5.91

8.64

17.61

9.01
4.36

4.36
19.74

$\begin{array}{r}8.42 \\ 6.53 \\ \hline\end{array}$

19.05

1.85

1.65
3.43

4.35

4.24

5.89

1.27

.71
.79

4.92

\subsection{4}

6.31

${ }^{R_{3.02}}$

5.63

8.89

8.01

8.86

10.94

22.96

12.03

4.93
1.61

1.61

4.70

5.97

6.10

6.75

8.61

9.15

3.41

21.20

5.40

$R_{3.70}$

1.69
1.79

1.63

2.95

R 5.37

3.02

5.68

6.5

5.37

R 14.60

9.15

3.10

$\mathrm{P}_{5.83}$

3.53

3.80

13.92

R $_{8.27}$

${ }^{\text {R } 8.26}$

A $_{8.26}$

9.32

8.46

5.68

8.46

R $_{14.60}$

9.12

2.98

23.49

$R_{8.38}$

6.24

19.33

1.46

1.46
1.45
2.32

3.41

3.30

5.62

.83

.67

.52

4.52

12.34
6.28

6.28

3.07
5.66

8.68

7.65

8.44

10.94

23.57

12.21

4.80

1.56

4.69

5.47 


\begin{tabular}{|c|c|c|c|c|c|c|c|c|}
\hline Sector and Energy Source & 1970 & 1975 & 1980 & 1985 & 1990 & 1991 & 1992 & 1993 \\
\hline 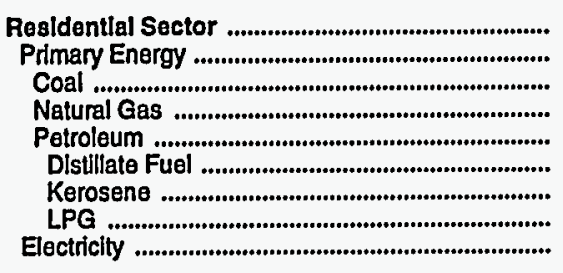 & $\begin{array}{r}20,083 \\
9,731 \\
173 \\
5,272 \\
4,286 \\
2,603 \\
459 \\
1,225 \\
10,352\end{array}$ & $\begin{array}{r}36,844 \\
16,201 \\
209 \\
8,410 \\
7,582 \\
4,954 \\
504 \\
2,124 \\
20,644\end{array}$ & $\begin{array}{r}68,825 \\
30,367 \\
175 \\
17,497 \\
12,695 \\
9,234 \\
887 \\
2,575 \\
38,458\end{array}$ & $\begin{array}{r}98,307 \\
39,636 \\
225 \\
27,136 \\
12,274 \\
7,910 \\
1,391 \\
2,974 \\
58,672\end{array}$ & $\begin{array}{r}R_{109,266} \\
P_{36,890} \\
R_{187} \\
25,442 \\
11,262 \\
6,703 \\
567 \\
3,992 \\
72,376\end{array}$ & $\begin{array}{r}R_{114,738} \\
R_{37,913} \\
R_{173} \\
26,508 \\
11,232 \\
6,361 \\
611 \\
4,260 \\
76,825\end{array}$ & $\begin{array}{r}R_{115,148} \\
R_{38,298} \\
R_{163} \\
27,600 \\
R_{10,534} \\
R_{6,054} \\
507 \\
3,974 \\
76,850\end{array}$ & $\begin{array}{r}124,430 \\
41,614 \\
170 \\
30,535 \\
10,910 \\
6,245 \\
572 \\
4,093 \\
82,815\end{array}$ \\
\hline 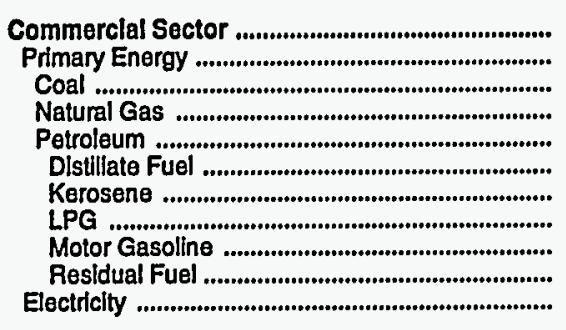 & $\begin{array}{r}10,668 \\
3,333 \\
98 \\
1,844 \\
1,391 \\
646 \\
47 \\
127 \\
247 \\
323 \\
7,335\end{array}$ & $\begin{array}{r}22,835 \\
6,680 \\
162 \\
3,385 \\
3,133 \\
1,423 \\
114 \\
242 \\
415 \\
939 \\
16,155\end{array}$ & $\begin{array}{r}46,881 \\
16,260 \\
135 \\
8,858 \\
7,267 \\
3,337 \\
262 \\
296 \\
1,046 \\
2,325 \\
30,621\end{array}$ & $\begin{array}{r}70,263 \\
20,139 \\
193 \\
13,368 \\
6,579 \\
3,965 \\
228 \\
496 \\
866 \\
1,025 \\
50,124\end{array}$ & $\begin{array}{r}78,922 \\
18,244 \\
150 \\
12,683 \\
5,412 \\
2,971 \\
80 \\
555 \\
1,011 \\
796 \\
60,678\end{array}$ & $\begin{array}{r}R_{B 1,482} \\
18,021 \\
132 \\
13,178 \\
4,711 \\
2,697 \\
73 \\
620 \\
764 \\
557 \\
63,462\end{array}$ & $\begin{array}{r}R_{82,408} \\
R_{18,120} \\
R_{144} \\
13,690 \\
R_{4,286} \\
R_{2,423} \\
62 \\
568 \\
721 \\
512 \\
R_{64,288}\end{array}$ & $\begin{array}{r}86,736 \\
19,034 \\
153 \\
15,069 \\
3,812 \\
2,361 \\
75 \\
617 \\
278 \\
481 \\
67,702\end{array}$ \\
\hline 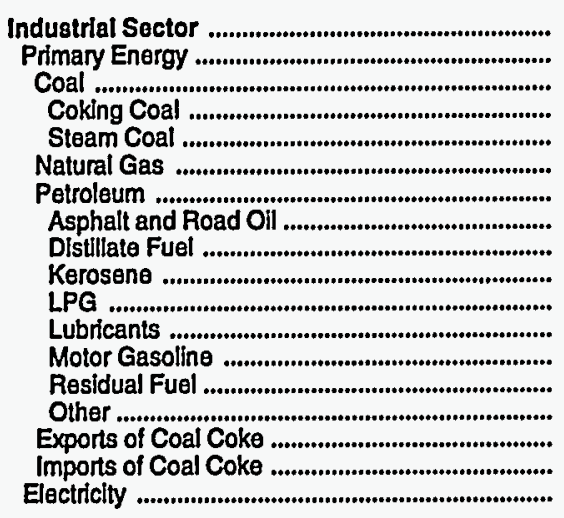 & $\begin{array}{r}16,458 \\
10,834 \\
2,082 \\
1,175 \\
907 \\
2,625 \\
6,202 \\
731 \\
866 \\
142 \\
1,046 \\
786 \\
824 \\
635 \\
1,171 \\
-78 \\
4 \\
5,624\end{array}$ & $\begin{array}{r}41,169 \\
27,409 \\
5,498 \\
3,692 \\
1,806 \\
5,844 \\
15,986 \\
1,914 \\
2,907 \\
278 \\
2,760 \\
1,119 \\
1,039 \\
2,367 \\
3,601 \\
-75 \\
156 \\
13,760\end{array}$ & $\begin{array}{r}94,520 \\
65,657 \\
5,888 \\
3,753 \\
2,135 \\
16,350 \\
43,497 \\
3,543 \\
7,232 \\
1,143 \\
7,967 \\
2,613 \\
1,553 \\
4,175 \\
15,271 \\
-130 \\
52 \\
28,863\end{array}$ & $\begin{array}{r}105,723 \\
65,533 \\
5,244 \\
2,228 \\
3,016 \\
21,615 \\
38,708 \\
4,916 \\
7,208 \\
308 \\
9,399 \\
2,916 \\
1,977 \\
2,817 \\
9,166 \\
-77 \\
43 \\
40,190\end{array}$ & $\begin{array}{r}R_{101,057} \\
R_{57,700} \\
4,649 \\
1,862 \\
2,787 \\
19,348 \\
R_{33,681} \\
3,529 \\
6,698 \\
81 \\
8,456 \\
R_{2,720} \\
1,685 \\
1,087 \\
R_{9,425} \\
-50 \\
72 \\
43,358\end{array}$ & $\begin{array}{r}A_{99,767} \\
R_{55,563} \\
R_{4,398} \\
1,660 \\
R_{2,738} \\
18,912 \\
R_{32,210} \\
3,382 \\
5,841 \\
67 \\
9,317 \\
R_{2,800} \\
1,729 \\
658 \\
R_{8,416} \\
-50 \\
93 \\
44,204\end{array}$ & $\begin{array}{r}R_{102,259} \\
R_{56,786} \\
R_{4,246} \\
1,587 \\
R_{2,659} \\
20,553 \\
R_{31,887} \\
2,755 \\
R_{5,608} \\
54 \\
R_{8,934} \\
R_{3,303} \\
1,737 \\
804 \\
R_{8,692} \\
444 \\
143 \\
45,473\end{array}$ & $\begin{array}{r}103,853 \\
58,129 \\
4,139 \\
1,505 \\
2,634 \\
23,366 \\
30,567 \\
3,336 \\
5,246 \\
70 \\
8,343 \\
3,278 \\
1,627 \\
941 \\
7,726 \\
-60 \\
116 \\
45,724\end{array}$ \\
\hline 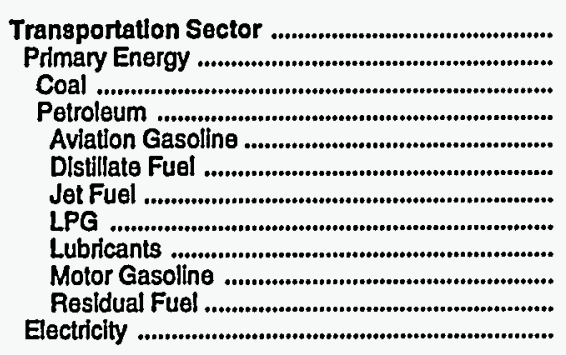 & $\begin{array}{r}35,370 \\
35,330 \\
3 \\
35,327 \\
218 \\
2,058 \\
1,441 \\
49 \\
745 \\
30,525 \\
291 \\
40\end{array}$ & $\begin{array}{r}70,934 \\
70,814 \\
1 \\
70,813 \\
245 \\
5,938 \\
4,150 \\
105 \\
1,158 \\
57,992 \\
1,226 \\
120\end{array}$ & $\begin{array}{r}163,674 \\
163,517 \\
- \\
163,517 \\
580 \\
20,090 \\
13,856 \\
88 \\
2,468 \\
121,809 \\
4,626 \\
156\end{array}$ & $\begin{array}{r}161,150 \\
160,894 \\
- \\
160,894 \\
503 \\
24,027 \\
14,747 \\
239 \\
2,754 \\
115,201 \\
3,422 \\
256\end{array}$ & $\begin{array}{r}R_{180,540} \\
R_{180,209} \\
- \\
R_{180,209} \\
419 \\
32,409 \\
17,784 \\
185 \\
R_{2,569} \\
123,775 \\
3,067 \\
331\end{array}$ & $\begin{array}{r}R_{171,421} \\
R_{171,089} \\
- \\
R_{171,089} \\
363 \\
29,818 \\
14,609 \\
175 \\
R_{2,644} \\
120,557 \\
2,922 \\
331\end{array}$ & $\begin{array}{r}R_{172,884} \\
R_{172,539} \\
- \\
R_{172,539} \\
351 \\
30,512 \\
13,559 \\
153 \\
R_{3,120} \\
122,700 \\
R_{2,145} \\
R_{345}\end{array}$ & $\begin{array}{r}178,318 \\
177,976 \\
- \\
177,976 \\
316 \\
31,479 \\
13,002 \\
160 \\
3,096 \\
128,116 \\
1,806 \\
343\end{array}$ \\
\hline 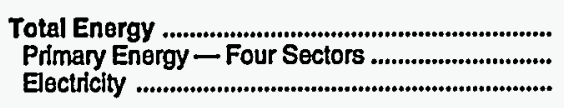 & $\begin{array}{l}82,579 \\
59,228 \\
23,351\end{array}$ & $\begin{array}{r}171,782 \\
121,103 \\
50,680\end{array}$ & $\begin{array}{r}373,900 \\
275,802 \\
98,098\end{array}$ & $\begin{array}{l}435,444 \\
286,202 \\
149,242\end{array}$ & $\begin{array}{r}R_{469,785} \\
R_{293,043} \\
176,742\end{array}$ & $\begin{array}{r}A_{467,408} \\
R_{282,586} \\
184,822\end{array}$ & $\begin{array}{r}R_{472,699} \\
R_{285,743} \\
186,956\end{array}$ & $\begin{array}{l}493,337 \\
296,752 \\
196,585\end{array}$ \\
\hline 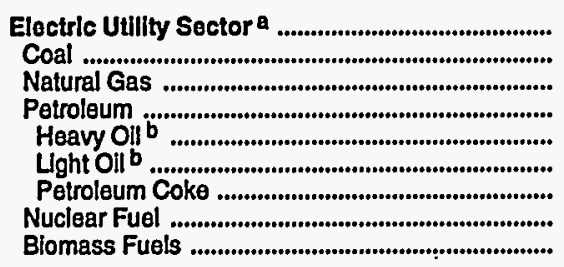 & $\begin{array}{r}4,316 \\
2,237 \\
1,151 \\
882 \\
797 \\
80 \\
6 \\
44 \\
2\end{array}$ & $\begin{array}{r}16,396 \\
7,178 \\
2,422 \\
6,345 \\
5,842 \\
502 \\
1 \\
448 \\
2\end{array}$ & $\begin{array}{r}37,435 \\
16,450 \\
8,357 \\
11,432 \\
10,446 \\
972 \\
14 \\
1,189 \\
8\end{array}$ & $\begin{array}{r}42,558 \\
24,056 \\
10,819 \\
4,742 \\
4,232 \\
502 \\
9 \\
2,930 \\
11\end{array}$ & $\begin{array}{r}38,443 \\
23,396 \\
6,631 \\
4,263 \\
3,757 \\
485 \\
20 \\
4,142 \\
11\end{array}$ & $\begin{array}{r}36,501 \\
23,164 \\
6,099 \\
3,055 \\
2,649 \\
387 \\
18 \\
4,172 \\
12\end{array}$ & $\begin{array}{r}\text { P }_{35,764} \\
22,863 \\
6,558 \\
2,454 \\
2,114 \\
321 \\
19 \\
3,878 \\
R_{12}\end{array}$ & $\begin{array}{r}36,653 \\
23,395 \\
7,012 \\
2,578 \\
2,222 \\
343 \\
13 \\
3,658 \\
11\end{array}$ \\
\hline Primary Enorgy - Flve Sectors ${ }^{\circ} \ldots . . . . . . . . . . . . . . . . . . .$. & 63,544 & 137,499 & 313,237 & 328,760 & $\mathrm{R}_{331,485}$ & $R_{319,087}$ & $R_{321,507}$ & 333,405 \\
\hline
\end{tabular}

a There are no direct fuel costs for hydroelectric, geothermal, wind, photovoltalc, or solar thermal energy.

b Heavy oil includes fuel oil nos. 4, 5, and 6 , and residual fuel oils. Light oll includes fuel nos. 1 and 2, kerosene, and jet fuel.

- Biomass fuels are not included, except those consumed at electric utilities and those added to motor gasoline.

-No consumption, Including cases where adjustments were made. See explanation of adjustments in Section 6 of Appendix $A$

Note: Totals may not equal sum of components due to independent rounding.

Sources: Data sources, estimation procedures, and assumptions are described in Appendix A.

$R=$ Revised data. 
A Energy Price and Expenditure Estimates by Source, Alabama 1970, 1975, 1980, 1985, and 1990-1993

\begin{tabular}{|c|c|c|c|c|c|c|c|c|}
\hline Energy Sourcé & 1970 & 1975 & 1980 & 1985 & 1990 & 1991 & 1992 & 1993 \\
\hline & \multicolumn{8}{|c|}{ Prices in Dollars per Million Btu } \\
\hline $\begin{array}{l}\text { Coal } \\
\text { Coking Coal } \\
\text { Steam Coal }\end{array}$ & $\begin{array}{r}0.32 \\
.42 \\
.26\end{array}$ & $\begin{array}{r}1.10 \\
1.50 \\
.94\end{array}$ & $\begin{array}{l}1.70 \\
1.96 \\
1.63\end{array}$ & $\begin{array}{l}2.01 \\
2.02 \\
2.00\end{array}$ & $\begin{array}{l}1.83 \\
1.83 \\
1.83\end{array}$ & $\begin{array}{l}1.80 \\
1.80 \\
1.79\end{array}$ & $\begin{array}{l}1.72 \\
1.78 \\
1.71\end{array}$ & $\begin{array}{l}1.75 \\
1.77 \\
1.75\end{array}$ \\
\hline Natural Gas & .52 & .96 & 2.90 & 4.73 & 4.07 & 4.08 & 4.06 & 4.34 \\
\hline $\begin{array}{l}\text { Petroleum } \\
\text { Asphalt and Road Oil } \\
\text { Aviation Gasoline } \\
\text { Distillate Fuel } \\
\text { Jet Fuel } \\
\text { Kerosene } \\
\text { LPG } \\
\text { Lubricants } \\
\text { Motor Gasoline } \\
\text { Residual Fuel } \\
\text { Other }{ }^{a}\end{array}$ & $\begin{array}{r}2.09 \\
.65 \\
2.17 \\
1.10 \\
.73 \\
.90 \\
2.00 \\
5.08 \\
2.82 \\
.41 \\
.61\end{array}$ & $\begin{array}{l}3.31 \\
1.85 \\
3.45 \\
2.60 \\
2.03 \\
2.45 \\
3.83 \\
7.48 \\
4.26 \\
1.59 \\
1.85\end{array}$ & $\begin{array}{r}7.86 \\
3.02 \\
9.02 \\
6.58 \\
6.39 \\
6.42 \\
6.62 \\
14.36 \\
9.89 \\
2.99 \\
5.80\end{array}$ & $\begin{array}{r}7.96 \\
4.72 \\
9.99 \\
6.66 \\
6.17 \\
8.63 \\
6.98 \\
17.61 \\
9.15 \\
3.80 \\
7.07\end{array}$ & $\begin{array}{r}\text { R } 7.81 \\
2.94 \\
9.32 \\
7.55 \\
5.99 \\
7.62 \\
10.13 \\
\text { R } 14.60 \\
8.96 \\
2.18 \\
\text { R } 6.12\end{array}$ & $\begin{array}{r}R 7.49 \\
3.31 \\
8.71 \\
7.09 \\
5.03 \\
7.01 \\
11.24 \\
R 16.79 \\
8.69 \\
1.74 \\
R \mathbf{5 . 3 1}\end{array}$ & $\begin{array}{r}R_{7.26} \\
2.03 \\
8.54 \\
6.55 \\
4.73 \\
4.72 \\
10.23 \\
\mathrm{R}_{19.43} \\
8.67 \\
1.68 \\
\mathrm{R}_{5.09}\end{array}$ & $\begin{array}{r}7.41 \\
2.38 \\
8.24 \\
6.68 \\
4.41 \\
5.14 \\
9.77 \\
18.94 \\
8.87 \\
1.66 \\
4.58\end{array}$ \\
\hline 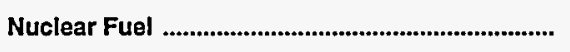 & - & .14 & .33 & .77 & .56 & .71 & .75 & .67 \\
\hline 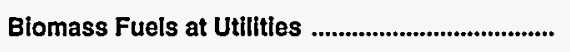 & - & - & - & - & - & - & - & - \\
\hline Primary Energy - Five Sectors ${ }^{b}$ & .84 & 1.82 & 3.37 & 3.99 & $R_{4.04}$ & 3.79 & ${ }^{\mathrm{A}_{3.59}}$ & 3.67 \\
\hline 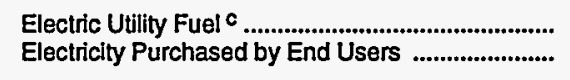 & $\begin{array}{r}.26 \\
3.51\end{array}$ & $\begin{array}{r}.88 \\
6.87\end{array}$ & $\begin{array}{r}1.17 \\
12.52\end{array}$ & $\begin{array}{r}1.74 \\
16.59\end{array}$ & $\begin{array}{r}1.60 \\
16.46\end{array}$ & $\begin{array}{r}1.56 \\
16.69\end{array}$ & $\begin{array}{r}1.48 \\
16.47\end{array}$ & $\begin{array}{r}1.53 \\
16.78\end{array}$ \\
\hline \multirow[t]{2}{*}{ 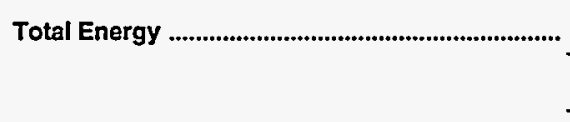 } & 1.37 & 2.84 & 6.41 & 7.90 & ${ }^{R} 7.88$ & ${ }^{\mathrm{R}} 7.78$ & $\mathrm{R}_{7.47}$ & 7.81 \\
\hline & \multicolumn{8}{|c|}{ Expenditures in Millions of Dollars } \\
\hline $\begin{array}{l}\text { Coal } \\
\text { Coking Coal } \\
\text { Steam Coal }\end{array}$ & $\begin{array}{r}215.3 \\
99.4 \\
115.9\end{array}$ & $\begin{array}{l}700.9 \\
269.2 \\
431.7\end{array}$ & $\begin{array}{r}1,121.0 \\
254.7 \\
866.3\end{array}$ & $\begin{array}{r}1,328.5 \\
156.1 \\
1,172.4\end{array}$ & $\begin{array}{r}1,238.1 \\
160.8 \\
1,077.2\end{array}$ & $\begin{array}{r}1,292.2 \\
153.0 \\
1,139.2\end{array}$ & $\begin{array}{r}1,327.5 \\
157.6 \\
1,170.0\end{array}$ & $\begin{array}{r}1,414.5 \\
152.3 \\
1,262.2\end{array}$ \\
\hline 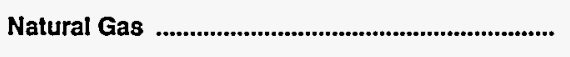 & 143.2 & 227.1 & 676.5 & 923.7 & 841.6 & 871.7 & 932.1 & $1,062.9$ \\
\hline $\begin{array}{l}\text { Petroleum } \\
\text { Asphalt and Road Oil } \\
\text { Aviation Gasoline } \\
\text { Distillate Fuel } \\
\text { Jet Fuel } \\
\text { Kerosene } \\
\text { LPG } \\
\text { Lubricants } \\
\text { Motor Gasoline } \\
\text { Otheridual Fuel }\end{array}$ & $\begin{array}{r}731.8 \\
13.7 \\
3.8 \\
54.6 \\
7.2 \\
6.7 \\
57.0 \\
25.0 \\
547.6 \\
8.0 \\
8.2\end{array}$ & $\begin{array}{r}1,595.1 \\
33.2 \\
4.3 \\
221.6 \\
19.1 \\
9.4 \\
91.9 \\
47.6 \\
1,010.7 \\
127.4 \\
29.9\end{array}$ & $\begin{array}{r}3,495.8 \\
62.8 \\
11.3 \\
579.2 \\
72.3 \\
45.6 \\
116.3 \\
86.4 \\
2,301.3 \\
135.2 \\
85.3\end{array}$ & $\begin{array}{r}3,353.6 \\
117.7 \\
8.7 \\
631.8 \\
121.6 \\
5.3 \\
91.7 \\
96.4 \\
2,090.3 \\
53.6 \\
136.6\end{array}$ & \begin{tabular}{r|}
$R_{4,018.7}$ \\
84.3 \\
5.4 \\
$1,118.7$ \\
63.1 \\
2.8 \\
152.7 \\
${ }^{R} 90.0$ \\
$2,303.4$ \\
52.6 \\
$R_{145.7}$
\end{tabular} & $\begin{array}{r}\mathrm{R}_{3,850.9} \\
116.0 \\
4.8 \\
987.1 \\
63.6 \\
3.8 \\
154.6 \\
\mathrm{R}_{92.6} \\
2,260.4 \\
38.5 \\
\mathrm{R}_{129.4}\end{array}$ & $\begin{array}{r}\mathrm{A}_{3,794.7} \\
66.6 \\
4.6 \\
931.9 \\
55.4 \\
2.2 \\
147.0 \\
\mathrm{R}_{109.2} \\
2,312.9 \\
\mathrm{R}_{39.0} \\
\mathrm{R}_{126.0}\end{array}$ & $\begin{array}{r}3,889.2 \\
78.6 \\
4.3 \\
894.9 \\
48.5 \\
2.3 \\
177.3 \\
108.4 \\
2,421.4 \\
40.5 \\
113.1\end{array}$ \\
\hline 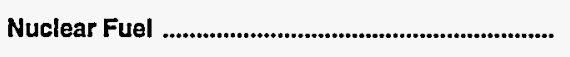 & - & 4.2 & 85.2 & 118.7 & 71.7 & 120.9 & 154.4 & 127.7 \\
\hline 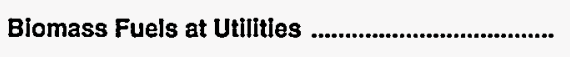 & - & - & - & - & - & - & - & - \\
\hline Primary Energy - Five Sectors ${ }^{b}$............................ & $1,090.4$ & $2,527.2$ & $5,378.4$ & $5,724.5$ & $R_{6,170.1}$ & ${ }_{6,135.7}$ & $\mathrm{~F}_{6,208.8}$ & $6,494.4$ \\
\hline Electric Utility Fuel c & $\begin{array}{r}-103.4 \\
411.6\end{array}$ & $\begin{array}{r}-385.8 \\
940.2\end{array}$ & $\begin{array}{r}-849.4 \\
2,120.5\end{array}$ & $\begin{array}{r}-1,174.9 \\
2,735.9\end{array}$ & $\begin{array}{r}-1,066.3 \\
3,236.6\end{array}$ & $\begin{array}{r}-1,172.3 \\
3,356.8\end{array}$ & $\begin{aligned}-1,206.8 \\
\mathrm{R}_{3,362.2}\end{aligned}$ & $\begin{array}{r}-1,315.1 \\
3,566.3\end{array}$ \\
\hline 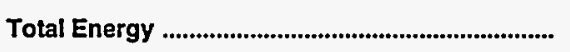 & $1,398.6$ & $3,081.5$ & $6,649.6$ & $7,285.5$ & $\mathrm{R}_{8,340.4}$ & ${ }^{\mathrm{B}} 8,320.2$ & $\mathrm{~F}_{8,364.1}$ & $8,745.5$ \\
\hline
\end{tabular}

a Includes petroleum coke used at electric utilities.

b Biomass fuels are not included, except those consumed at electric utilities and those added to motor gasoline.

c There are no direct fuel costs for hydroelectric, geothermal, wind, photovoltaic, or solar thermal energy.

R=Revised data.

-No consumption, including cases where adjustments were made. See explanation of adjustments in Section 6 of Appendix A.

Note: Expenditure totals may not equal sum of components due to Independent rounding.

Sources: Data sources, estimation procedures, and assumptions are described in Appendix A. 


\begin{tabular}{|c|c|c|c|c|c|c|c|c|}
\hline Sector and Energy Source & 1970 & 1975 & 1980 & 1985 & 1990 & 1991 & 1992 & 1993 \\
\hline 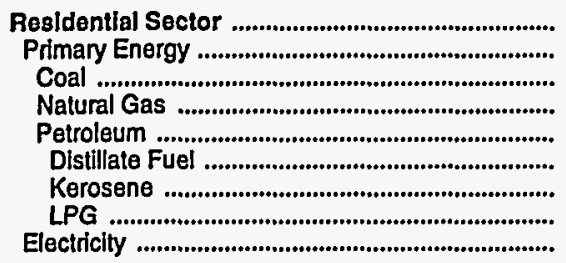 & $\begin{array}{r}2.45 \\
1.37 \\
.81 \\
1.10 \\
2.17 \\
1.24 \\
1.62 \\
2.22 \\
4.62\end{array}$ & $\begin{array}{l}4.48 \\
2.13 \\
1.82 \\
1.52 \\
4.22 \\
2.53 \\
3.31 \\
4.32 \\
8.05\end{array}$ & $\begin{array}{r}9.06 \\
4.53 \\
2.97 \\
3.91 \\
7.89 \\
6.83 \\
9.13 \\
7.75 \\
14.44\end{array}$ & $\begin{array}{r}12.82 \\
6.46 \\
3.19 \\
6.18 \\
8.49 \\
7.68 \\
8.90 \\
8.49 \\
18.74\end{array}$ & $\begin{array}{r}13.84 \\
7.12 \\
2.70 \\
6.38 \\
10.91 \\
6.70 \\
7.76 \\
11.05 \\
19.32\end{array}$ & $\begin{array}{r}14.40 \\
7.68 \\
2.81 \\
6.86 \\
12.16 \\
6.16 \\
7.15 \\
12.45 \\
19.61\end{array}$ & $\begin{array}{r}13.92 \\
7.11 \\
2.69 \\
6.56 \\
10.94 \\
5.52 \\
6.40 \\
11.08 \\
19.59\end{array}$ & $\begin{array}{r}14.29 \\
7.40 \\
2.73 \\
6.89 \\
10.05 \\
5.38 \\
6.24 \\
10.18 \\
20.00\end{array}$ \\
\hline 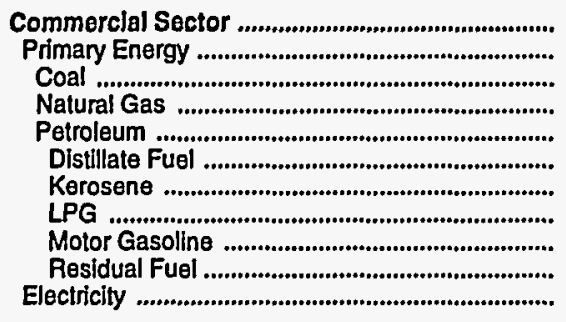 & $\begin{array}{r}1.98 \\
.75 \\
.28 \\
.58 \\
1.53 \\
.97 \\
.75 \\
1.58 \\
2.82 \\
.38 \\
5.39\end{array}$ & $\begin{array}{l}3.97 \\
1.46 \\
1.07 \\
1.04 \\
2.96 \\
2.22 \\
2.24 \\
3.07 \\
4.26 \\
1.69 \\
8.98\end{array}$ & $\begin{array}{r}8.44 \\
3.78 \\
1.73 \\
3.27 \\
6.61 \\
6.22 \\
5.91 \\
5.28 \\
9.89 \\
3.39 \\
16.19\end{array}$ & $\begin{array}{r}11.47 \\
5.40 \\
1.86 \\
5.27 \\
6.17 \\
6.76 \\
8.06 \\
4.97 \\
9.15 \\
4.02 \\
20.01\end{array}$ & $\begin{array}{r}12.34 \\
5.24 \\
1.65 \\
5.28 \\
5.63 \\
5.96 \\
7.41 \\
8.47 \\
8.96 \\
2.65 \\
19.52\end{array}$ & $\begin{array}{r}13.34 \\
5.64 \\
1.64 \\
5.59 \\
5.87 \\
5.54 \\
6.77 \\
9.38 \\
8.69 \\
2.13 \\
19.82\end{array}$ & $\begin{array}{r}12.97 \\
5.28 \\
1.61 \\
5.55 \\
5.03 \\
3.63 \\
3.76 \\
9.16 \\
8.67 \\
-\overline{1} \\
19.91\end{array}$ & $\begin{array}{r}13.49 \\
5.69 \\
1.59 \\
6.01 \\
4.92 \\
3.30 \\
3.85 \\
9.24 \\
8.87 \\
-\overline{14}\end{array}$ \\
\hline 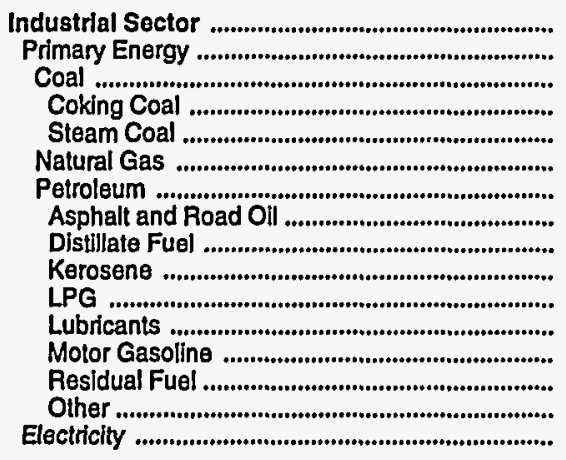 & $\begin{array}{r}.63 \\
.44 \\
.40 \\
.42 \\
.28 \\
.32 \\
.92 \\
.65 \\
.69 \\
.75 \\
1.58 \\
5.08 \\
2.82 \\
.51 \\
.72 \\
2.24\end{array}$ & $\begin{array}{l}1.86 \\
1.36 \\
1.39 \\
1.50 \\
1.07 \\
.73 \\
2.10 \\
1.85 \\
2.04 \\
2.24 \\
3.07 \\
7.48 \\
4.26 \\
1.74 \\
1.85 \\
5.40\end{array}$ & $\begin{array}{r}3.99 \\
2.69 \\
1.89 \\
1.96 \\
1.73 \\
2.46 \\
4.66 \\
3.02 \\
5.28 \\
5.91 \\
5.28 \\
14.36 \\
9.89 \\
3.05 \\
5.80 \\
10.29\end{array}$ & $\begin{array}{r}5.58 \\
3.77 \\
1.95 \\
2.02 \\
1.86 \\
4.09 \\
6.62 \\
4.72 \\
7.02 \\
8.06 \\
4.97 \\
17.61 \\
9.15 \\
4.02 \\
7.07 \\
13.60\end{array}$ & $\begin{array}{r}5.04 \\
3.28 \\
1.76 \\
1.83 \\
1.65 \\
3.07 \\
R_{5.69} \\
2.94 \\
6.45 \\
7.41 \\
8.47 \\
R_{14.60} \\
8.96 \\
2.65 \\
R_{6.12} \\
12.72\end{array}$ & $\begin{array}{r}A_{4.91} \\
R_{3.10} \\
1.74 \\
1.80 \\
1.64 \\
2.92 \\
R_{5.33} \\
3.31 \\
5.90 \\
6.77 \\
9.38 \\
R_{16.79} \\
8.69 \\
2.13 \\
R_{5.31} \\
12.81\end{array}$ & $\begin{array}{r}R_{4.55} \\
R_{2.76} \\
1.70 \\
1.78 \\
1.61 \\
2.99 \\
R_{4} 4.16 \\
2.03 \\
3.27 \\
3.76 \\
9.16 \\
R_{19.43} \\
8.67 \\
2.13 \\
\mathrm{R}_{5.09} \\
12.57\end{array}$ & $\begin{array}{r}4.82 \\
2.96 \\
1.70 \\
1.77 \\
1.59 \\
3.27 \\
4.24 \\
2.38 \\
3.35 \\
3.85 \\
9.24 \\
18.94 \\
8.87 \\
2.08 \\
4.58 \\
12.72\end{array}$ \\
\hline 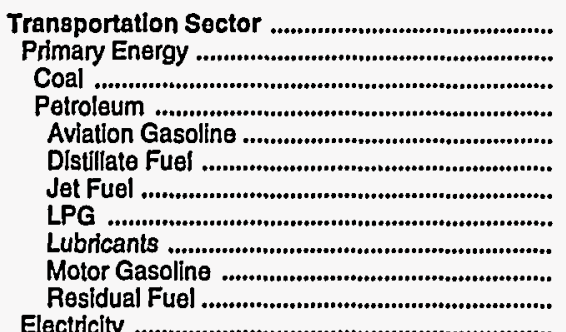 & $\begin{array}{r}2.45 \\
2.45 \\
.28 \\
2.46 \\
2.17 \\
1.33 \\
.73 \\
1.58 \\
5.08 \\
2.82 \\
.34 \\
-\end{array}$ & $\begin{array}{l}3.67 \\
3.67 \\
1.07 \\
3.67 \\
3.45 \\
2.92 \\
2.03 \\
3.07 \\
7.48 \\
4.26 \\
1.47\end{array}$ & $\begin{array}{r}8.78 \\
8.78 \\
- \\
8.78 \\
9.02 \\
6.99 \\
6.39 \\
5.28 \\
14.36 \\
9.89 \\
2.93\end{array}$ & $\begin{array}{r}8.34 \\
8.34 \\
- \\
8.34 \\
9.99 \\
6.54 \\
6.17 \\
4.97 \\
17.61 \\
9.15 \\
3.72 \\
-\end{array}$ & $\begin{array}{r}R_{8.37} \\
R_{8.37} \\
-\bar{r} \\
R_{8.37} \\
9.32 \\
8.09 \\
5.99 \\
8.47 \\
R_{14.60} \\
8.96 \\
2.02\end{array}$ & $\begin{array}{r}R_{7.98} \\
R_{7.98} \\
R_{7.98} \\
8.71 \\
7.57 \\
5.03 \\
9.38 \\
R_{16.79} \\
8.69 \\
1.71\end{array}$ & $\begin{array}{r}R_{8.00} \\
R_{8.00} \\
-\overline{8} \\
R_{8.00} \\
8.54 \\
7.72 \\
4.73 \\
9.16 \\
R_{19.43} \\
8.67 \\
R_{1.65}\end{array}$ & $\begin{array}{r}8.18 \\
8.18 \\
- \\
8.18 \\
8.24 \\
7.76 \\
4.41 \\
9.24 \\
18.94 \\
8.87 \\
1.58\end{array}$ \\
\hline 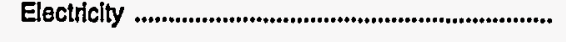 & - & - & - & - & - & - & - & - \\
\hline 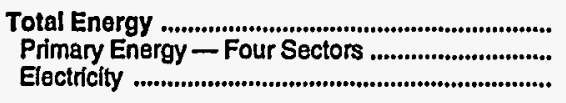 & $\begin{array}{l}1.37 \\
1.09 \\
3.51\end{array}$ & $\begin{array}{l}2.84 \\
2.26 \\
6.87\end{array}$ & $\begin{array}{r}6.41 \\
5.22 \\
12.52\end{array}$ & $\begin{array}{r}7.90 \\
6.01 \\
16.59\end{array}$ & $\begin{array}{r}R_{7.88} \\
R_{5.92} \\
16.46\end{array}$ & $\begin{array}{r}R_{7.78} \\
R_{5.72} \\
16.69\end{array}$ & $\begin{array}{r}R_{7.47} \\
R_{5.46} \\
16.47\end{array}$ & $\begin{array}{r}7.81 \\
5.71 \\
16.78\end{array}$ \\
\hline 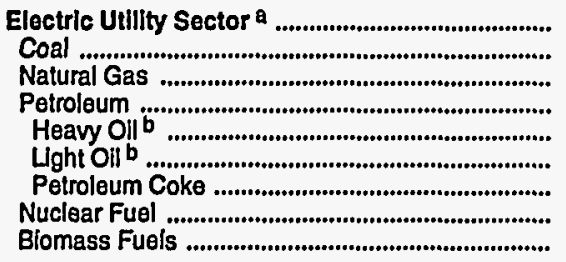 & $\begin{array}{r}.26 \\
.26 \\
.26 \\
.20 \\
-81 \\
.17 \\
- \\
-\end{array}$ & $\begin{array}{r}.88 \\
.92 \\
1.08 \\
2.08 \\
1.69 \\
2.16 \\
-14 \\
-\end{array}$ & $\begin{array}{r}1.17 \\
1.61 \\
2.62 \\
6.35 \\
- \\
6.35 \\
- \\
.33 \\
-\end{array}$ & $\begin{array}{r}1.74 \\
2.02 \\
3.17 \\
6.00 \\
- \\
6.00 \\
- \\
.77 \\
-\end{array}$ & $\begin{array}{r}1.60 \\
1.84 \\
2.16 \\
5.57 \\
- \\
5.57 \\
- \\
.56 \\
-\end{array}$ & $\begin{array}{r}1.56 \\
1.81 \\
1.87 \\
5.12 \\
-\overline{5} \\
5.12 \\
-\overline{11} \\
-\end{array}$ & $\begin{array}{r}1.48 \\
1.73 \\
2.23 \\
4.60 \\
\overline{4} \\
4.60 \\
\overline{-} \\
.75 \\
-\end{array}$ & $\begin{array}{r}1.53 \\
1.76 \\
2.60 \\
4.25 \\
\overline{-} \\
4.25 \\
\overline{-} \\
.67 \\
-\end{array}$ \\
\hline Primary Energy - Five Sectors c ........................ & .84 & 1.82 & 3.37 & 3.99 & $P_{4.04}$ & 3.79 & $\mathrm{R}_{3.59}$ & 3.67 \\
\hline
\end{tabular}

a There are no direct fuel costs for hydroelectric, geothermal, wind, photovoltaic, or solar thermal energy.

b Heavy oil includes fual oil nos. 4,5 , and 6 , and residual fuel oils. Light oil includes fuel oil nos. 1 and 2 , kerosene, and jet fuel.

- Blomass fuels are not included, except those consumed at electric

$R=$ Revised data.

-No consumption, including cases where adjustments were made. See explanation of adjustments in Section 6 of Appendix A.

Sources: Data sources, estimation procedures, and assumptions are describod in Appendix A.

utilities and those added to motor gasoline. 
A Energy Expenditure Estimates by Sector, Alabama

L 1970, 1975, 1980, 1985, and 1990-1993

A (Million Dollars)

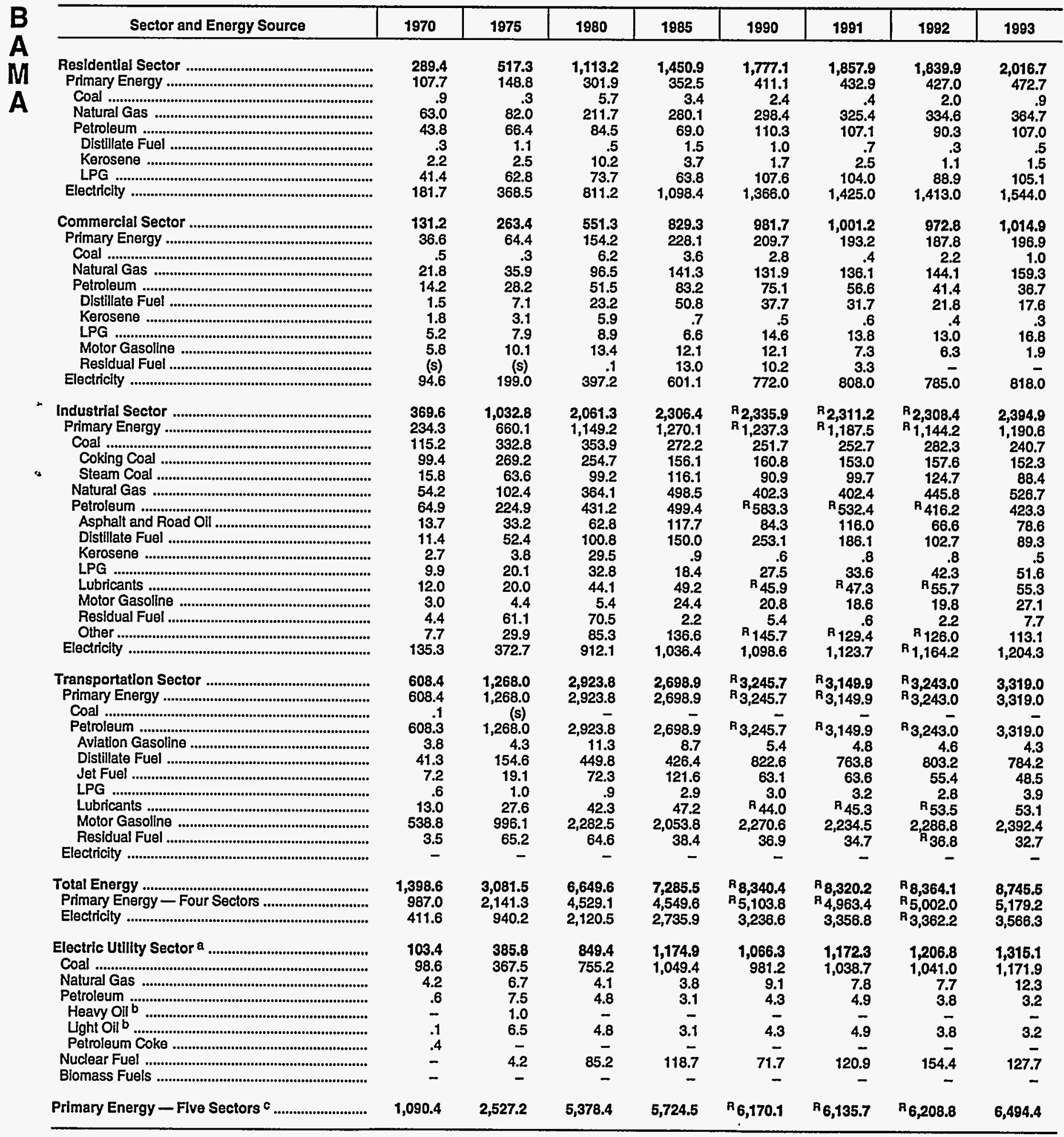

a There are no direct fuel costs for hydroelectric, geothermal, wind, photovoltaic, or solar themal energy.

b Heavy oll includes fuel oil nos. 4,5 , and 6 , and residual fuel oils. Light oil includes fuel nos. 1 and 2 , kerosene, and jet fuel.

c Biomass fuels are not included, except those consumed at electric utilities and those added to motor gasoline.

$\mathbf{R}=$ Revised data.

$\rightarrow$ No consumption, including cases where adjustments were made. See explanation of adjustments in Section 6 of Appendlx A.

(s) =Value less than 0.05 million dollars.

Note: Totals may not equal sum of components due to independent rounding.

Sources: Data sources, estimation procedures, and assumptions are described in Appendix A. 
Energy Price and Expenditure Estimates by Source, Alaska 1970, 1975, 1980, 1985, and 1990-1993

\begin{tabular}{|c|c|c|c|c|c|c|c|c|}
\hline Energy Source & 1970 & 1975 & 1980 & 1985 & 1990 & 1991 & 1992 & 1993 \\
\hline & \multicolumn{8}{|c|}{ Prices In Dollars per Million Btu } \\
\hline 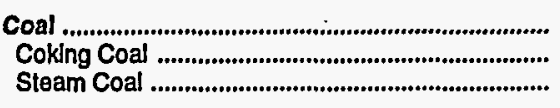 & $\overrightarrow{0.92}$ & $1 . \overline{1.40}$ & $\begin{array}{r}1.91 \\
1.91\end{array}$ & $\begin{array}{r}3.65 \\
3.65\end{array}$ & $\begin{array}{r}3.79 \\
3.79\end{array}$ & $\frac{4.02}{4.02}$ & $\begin{array}{l}R_{4.16} \\
R_{4.16}\end{array}$ & $\begin{array}{r}4.77 \\
-\overline{4}\end{array}$ \\
\hline Natural Gas ............................................................. & .67 & .89 & .62 & 1.23 & 1.96 & 1.83 & 1.75 & 1.87 \\
\hline 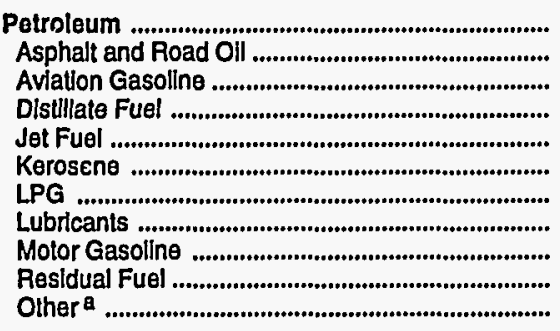 & $\begin{array}{r}1.33 \\
.57 \\
2.17 \\
1.15 \\
.73 \\
1.25 \\
2.09 \\
5.08 \\
3.18 \\
1.37 \\
.43\end{array}$ & $\begin{array}{l}3.00 \\
1.80 \\
3.45 \\
2.88 \\
2.04 \\
3.18 \\
3.80 \\
7.48 \\
5.15 \\
2.34 \\
1.31\end{array}$ & $\begin{array}{r}7.06 \\
3.62 \\
9.02 \\
6.82 \\
6.21 \\
7.29 \\
6.88 \\
14.36 \\
10.20 \\
4.07 \\
4.04\end{array}$ & $\begin{array}{r}7.04 \\
4.47 \\
9.99 \\
7.67 \\
6.07 \\
8.05 \\
13.62 \\
17.61 \\
9.83 \\
4.66 \\
2.79\end{array}$ & $\begin{array}{r}7.59 \\
3.14 \\
9.32 \\
8.46 \\
6.17 \\
9.05 \\
15.10 \\
814.60 \\
10.03 \\
5.26 \\
-\end{array}$ & $\begin{array}{r}6.77 \\
3.28 \\
8.71 \\
7.74 \\
5.50 \\
8.68 \\
15.64 \\
16.79 \\
8.99 \\
3.33 \\
-\end{array}$ & $\begin{array}{r}6.80 \\
2.80 \\
8.54 \\
7.44 \\
5.15 \\
7.47 \\
14.68 \\
R 19.43 \\
9.95 \\
R_{3.23} \\
-\end{array}$ & $\begin{array}{r}6.77 \\
2.95 \\
8.24 \\
7.50 \\
4.96 \\
7.51 \\
14.56 \\
18.94 \\
10.24 \\
3.08 \\
-\end{array}$ \\
\hline Nuclear Fuel & - & - & - & - & - & - & - & - \\
\hline 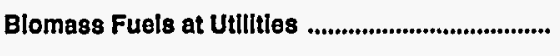 & - & - & - & - & - & - & - & - \\
\hline Prlmary Energy - Flve Sectors $b$...................... & 1.12 & 2.21 & 4.04 & 4.71 & $\mathrm{R}_{5.51}$ & 4.86 & $R_{4.78}$ & 4.85 \\
\hline $\begin{array}{l}\text { Electric Utility Fuel } \mathrm{C} \\
\text { Electriclty Purchased by End Users }\end{array}$ & 9.66 & 9.95 & $\begin{array}{r}1.25 \\
15.09\end{array}$ & $\begin{array}{r}1.71 \\
24.52\end{array}$ & $\begin{array}{r}2.37 \\
27.80\end{array}$ & $\begin{array}{r}1.81 \\
28.75\end{array}$ & $\begin{array}{r}1.91 \\
29.22\end{array}$ & $\begin{array}{r}1.89 \\
29.72\end{array}$ \\
\hline \multirow[t]{2}{*}{ 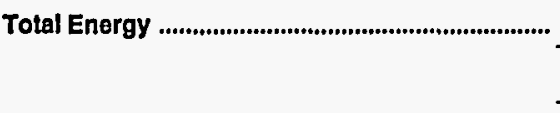 } & 1.39 & 2.71 & 5.06 & 5.99 & 7.01 & 6.46 & ${ }^{R} 6.37$ & 6.56 \\
\hline & \multicolumn{8}{|c|}{ Expenditures in Millions of Dollars } \\
\hline 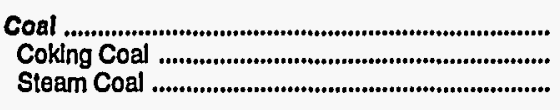 & $\begin{array}{c}12.1 \\
12.1\end{array}$ & $\frac{21.4}{21.4}$ & $\begin{array}{l}8.2 \\
\overline{8.2}\end{array}$ & $\begin{array}{c}42.3 \\
-\overline{42.3}\end{array}$ & $\begin{array}{c}47.0 \\
- \\
47.0\end{array}$ & $\begin{array}{c}51.0 \\
- \\
51.0\end{array}$ & $\begin{array}{c}R_{52.1} \\
- \\
R_{52.1}\end{array}$ & $\begin{array}{c}65.0 \\
- \\
65.0\end{array}$ \\
\hline 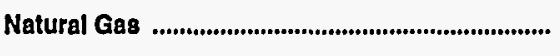 & 26.2 & 54.5 & 64.5 & 162.4 & 223.7 & 212.4 & 210.6 & 213.5 \\
\hline 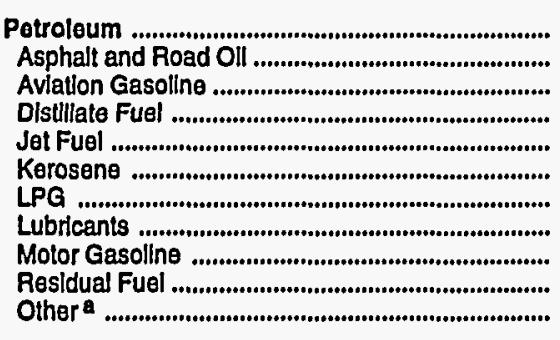 & $\begin{array}{r}122.7 \\
1.0 \\
5.1 \\
33.3 \\
27.5 \\
.2 \\
1.1 \\
1.9 \\
43.8 \\
8.7 \\
.1\end{array}$ & $\begin{array}{r}354.0 \\
3.8 \\
8.1 \\
116.6 \\
85.0 \\
2.2 \\
2.5 \\
6.6 \\
113.0 \\
15.7 \\
.5\end{array}$ & $\begin{array}{r}853.7 \\
7.4 \\
22.7 \\
264.0 \\
335.7 \\
.8 \\
4.2 \\
10.0 \\
196.9 \\
9.4 \\
2.5\end{array}$ & $\begin{array}{r}1,428.2 \\
14.4 \\
24.7 \\
462.1 \\
520.3 \\
.3 \\
15.2 \\
11.2 \\
291.2 \\
84.4 \\
4.5\end{array}$ & $\begin{array}{r}R_{1,554.7} \\
5.6 \\
23.1 \\
570.9 \\
604.3 \\
.1 \\
20.6 \\
R 10.4 \\
306.6 \\
13.0 \\
-\end{array}$ & $\begin{array}{r}R_{1,284.8} \\
5.6 \\
27.2 \\
441.4 \\
528.3 \\
.4 \\
22.3 \\
R 10.7 \\
238.4 \\
10.5 \\
-\end{array}$ & $\begin{array}{r}R_{1,256.2} \\
4.9 \\
19.8 \\
450.3 \\
426.9 \\
.1 \\
20.6 \\
R_{12.6} \\
307.3 \\
R_{13.6} \\
-\end{array}$ & $\begin{array}{r}1,196.8 \\
.8 \\
17.1 \\
407.9 \\
412.5 \\
.2 \\
12.3 \\
12.5 \\
321.4 \\
12.0 \\
-\end{array}$ \\
\hline 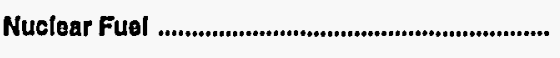 & - & - & - & - & - & - & - & - \\
\hline Blomass Fuels at Utititles ........................................ & - & - & - & - & - & - & - & - \\
\hline Prlmary Enorgy — Flve Sectors b ...................... & 161.0 & 429.9 & 926.4 & $1,632.9$ & $R_{1,825.3}$ & $R_{1,548.2}$ & $A_{1,518.9}$ & $1,475.3$ \\
\hline 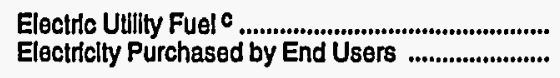 & $\begin{array}{r}-9.9 \\
33.9\end{array}$ & $\begin{array}{r}-27.0 \\
65.9\end{array}$ & $\begin{array}{r}-48.3 \\
129.5\end{array}$ & $\begin{array}{r}-77.0 \\
331.5\end{array}$ & $\begin{array}{r}-102.1 \\
400.9\end{array}$ & $\begin{array}{r}-73.6 \\
414.8\end{array}$ & $\begin{array}{r}-72.1 \\
429.9\end{array}$ & $\begin{array}{l}-71.2 \\
440.7\end{array}$ \\
\hline 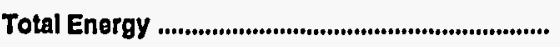 & 185.0 & 468.9 & $1,007.7$ & $1,887.3$ & $R_{2,124.1}$ & ${ }^{B_{1,889.4}}$ & ${ }^{A_{1,676.7}}$ & $1,844.7$ \\
\hline
\end{tabular}

a Includes petroleum coke used at electric utillties.

b Blomass fuels are not included, except those consumed at electric uitlitles and those added to motor gasoline.

c There are no direct fuel costs for hydroelectric, geothermal, wind, photovoltalc, or solar thermal energy.

$\mathrm{R}=$ Rovised data.
-No consumption, including cases where adjustments were made. Se日 explanation of adjustments in Section 6 of Appendix A.

Note: Expenditure totals may not equal sum of components due to independent rounding.

Sources: Data sources, estimation procedures, and assumptions are described in Appendix A. 
A Energy Price Estimates by Sector, Alaska

L 1970, 1975, 1980, 1985, and 1990-1993

A (Dollars per Million Btu)

\begin{tabular}{|c|c|c|c|c|c|c|c|c|}
\hline Sector and Energy Source & 1970 & 1975 & 1980 & 1985 & 1990 & 1991 & 1892 & 1993 \\
\hline $\begin{array}{l}\text { Resldentlal Sector } \\
\text { Primary Energy } \\
\text { Coal } \\
\text { Natural Gas } \\
\text { Petroleum } \\
\text { Distillate Fuel } \\
\text { Kerosene } \\
\text { LPG }\end{array}$ & $\begin{array}{l}2.34 \\
1.49 \\
2.47 \\
1.51 \\
1.46 \\
1.40 \\
1.61 \\
2.93 \\
9.29\end{array}$ & $\begin{array}{r}3.27 \\
2.26 \\
2.87 \\
1.62 \\
2.90 \\
2.80 \\
3.23 \\
6.07 \\
10.16\end{array}$ & $\begin{array}{r}6.67 \\
4.31 \\
1 . \overline{3} \\
7.21 \\
7.05 \\
12 . \overline{-} \\
16.18\end{array}$ & $\begin{array}{r}9.18 \\
5.20 \\
7.75 \\
2.79 \\
8.32 \\
7.81 \\
8.99 \\
13.97 \\
25.96\end{array}$ & $\begin{array}{r}10.35 \\
6.36 \\
7.96 \\
4.00 \\
8.78 \\
7.94 \\
9.14 \\
16.66 \\
29.64\end{array}$ & $\begin{array}{r}10.78 \\
6.62 \\
10.99 \\
4.17 \\
8.63 \\
7.57 \\
8.71 \\
17.04 \\
31.26\end{array}$ & $\begin{array}{r}10.10 \\
5.72 \\
7.92 \\
3.78 \\
7.77 \\
6.78 \\
7.80 \\
15.84 \\
31.81\end{array}$ & $\begin{array}{r}10.60 \\
5.68 \\
7.96 \\
3.99 \\
7.68 \\
6.93 \\
7.98 \\
15.70 \\
32.74\end{array}$ \\
\hline $\begin{array}{l}\text { Commercial Sector } \\
\text { Primary Energy } \\
\text { Coal } \\
\text { Natural Gas } \\
\text { Petroleum } \\
\text { Distillate Fuel } \\
\text { Kerosene } \\
\text { LPG } \\
\text { Motor Gasoline } \\
\text { Eesidual Fuel }\end{array}$ & $\begin{array}{r}1.67 \\
1.08 \\
1.01 \\
.68 \\
1.66 \\
1.21 \\
1 . \overline{10} \\
3.18 \\
1.49 \\
9.46\end{array}$ & $\begin{array}{r}2.60 \\
1.81 \\
1.57 \\
.97 \\
3.21 \\
2.60 \\
-\overline{2} \\
2.32 \\
5.15 \\
2.52 \\
10.83\end{array}$ & $\begin{array}{r}4.16 \\
2.54 \\
\overline{-} \\
1.06 \\
7.69 \\
6.75 \\
-\overline{-} \\
3.96 \\
10.20 \\
4.31 \\
18.02\end{array}$ & $\begin{array}{r}7.15 \\
3.66 \\
3.37 \\
2.35 \\
7.69 \\
7.01 \\
7.89 \\
13.07 \\
9.83 \\
24.36\end{array}$ & $\begin{array}{r}8.05 \\
3.78 \\
2.76 \\
2.77 \\
7.32 \\
7.16 \\
7.97 \\
9.03 \\
10.03 \\
27.34\end{array}$ & $\begin{array}{r}8.19 \\
3.66 \\
2.17 \\
2.88 \\
7.43 \\
7.24 \\
7.63 \\
9.29 \\
8.99 \\
27.87\end{array}$ & $\begin{array}{r}R_{8.14} \\
R_{3} .76 \\
R_{3.94} \\
2.63 \\
6.46 \\
6.26 \\
7.08 \\
9.26 \\
9.95 \\
28.71\end{array}$ & $\begin{array}{r}8.66 \\
3.98 \\
5.21 \\
2.80 \\
6.26 \\
6.19 \\
7.45 \\
9.21 \\
10.24 \\
28.73\end{array}$ \\
\hline $\begin{array}{l}\text { Industrial Sector } \\
\text { Primary Energy } \\
\text { Coal } \\
\text { Coking Coal } \\
\text { Steam Coal } \\
\text { Natural Gas } \\
\text { Petroleum } \\
\text { Asphalt and Road Oil } \\
\text { Distillate Fuel } \\
\text { Kerosene } \\
\text { LPG } \\
\text { Motor Gasoline } \\
\text { Residual Fuel } \\
\text { Other } \\
\text { Electricity }\end{array}$ & $\begin{array}{r}.75 \\
.70 \\
1.01 \\
-\overline{1.01} \\
.43 \\
.75 \\
.57 \\
.66 \\
.76 \\
1.10 \\
5.08 \\
3.18 \\
.36 \\
.43 \\
5.36\end{array}$ & $\begin{array}{r}1.84 \\
1.66 \\
1.57 \\
- \\
1.57 \\
.81 \\
2.65 \\
1.80 \\
2.68 \\
3.02 \\
2.32 \\
7.48 \\
5.15 \\
1.85 \\
1.31 \\
6.79\end{array}$ & $\begin{array}{r}1.91 \\
1.61 \\
- \\
- \\
- \\
.39 \\
5.96 \\
3.62 \\
6.27 \\
7.29 \\
3.96 \\
14.36 \\
10.20 \\
3.59 \\
4.04 \\
10.32\end{array}$ & $\begin{array}{r}2.61 \\
2.38 \\
- \\
- \\
- \\
.71 \\
5.68 \\
4.47 \\
6.98 \\
7.89 \\
13.07 \\
17.61 \\
9.83 \\
4.55 \\
2.79 \\
19.13\end{array}$ & $\begin{array}{r}2.86 \\
2.35 \\
- \\
- \\
\overline{-} \\
1.28 \\
{ }^{8} 6.47 \\
3.14 \\
7.05 \\
7.97 \\
9.03 \\
R 14.60 \\
10.03 \\
3.33 \\
22.99\end{array}$ & $\begin{array}{r}2.79 \\
\mathrm{R}_{2.31} \\
- \\
\overline{-} \\
\overline{1.18} \\
\mathrm{R} 6.17 \\
3.28 \\
6.75 \\
7.63 \\
9.29 \\
\mathrm{~A}_{16.79} \\
8.99 \\
2.96 \\
23.90\end{array}$ & $\begin{array}{r}R_{2.60} \\
2.13 \\
\overline{-} \\
\overline{-} \\
1.18 \\
R_{5.73} \\
2.80 \\
6.27 \\
7.08 \\
9.26 \\
R_{19.43} \\
9.95 \\
2.70 \\
22.68\end{array}$ & $\begin{array}{r}2.70 \\
2.16 \\
5.21 \\
-2 \overline{5} \\
5.21 \\
1.30 \\
6.25 \\
2.95 \\
6.59 \\
7.45 \\
9.21 \\
18.94 \\
10.24 \\
2.56 \\
-5 \\
23.98\end{array}$ \\
\hline 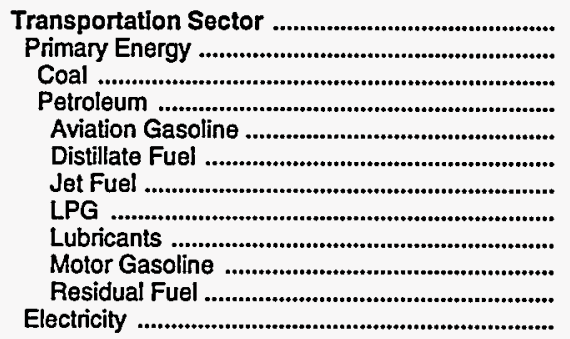 & $\begin{array}{r}1.39 \\
1.39 \\
1.01 \\
1.39 \\
2.17 \\
1.46 \\
.73 \\
1.10 \\
5.08 \\
3.18 \\
1.11 \\
-\end{array}$ & $\begin{array}{r}3.06 \\
.06 \\
1.57 \\
3.06 \\
3.45 \\
3.13 \\
2.04 \\
7 . \overline{7} \\
5.48 \\
2.14 \\
-\end{array}$ & $\begin{array}{r}7.31 \\
7.31 \\
- \\
7.31 \\
9.02 \\
7.39 \\
6.21 \\
3.96 \\
14.36 \\
10.20 \\
- \\
-\end{array}$ & $\begin{array}{r}7.27 \\
7.27 \\
- \\
7.27 \\
9.99 \\
8.00 \\
6.07 \\
13.07 \\
17.61 \\
9.83 \\
4.55 \\
-\end{array}$ & $\begin{array}{r}7.56 \\
7.56 \\
7 . \overline{6} \\
9.32 \\
9.03 \\
6.17 \\
9.03 \\
{ }_{1} 14.60 \\
10.03 \\
5.00 \\
-\end{array}$ & $\begin{array}{r}6.69 \\
6.69 \\
-\overline{6.69} \\
8.71 \\
8.35 \\
5.50 \\
9.29 \\
16.79 \\
8.99 \\
2.96 \\
-\end{array}$ & $\begin{array}{r}\mathrm{A}_{6.87} \\
\mathrm{~A}_{6.87} \\
\mathrm{R}_{6.87} \\
8.54 \\
8.49 \\
5.15 \\
9.26 \\
\mathrm{~A}_{19.43} \\
9.95 \\
\mathrm{R}_{3.24} \\
-\end{array}$ & $\begin{array}{r}6.84 \\
6.84 \\
- \\
6.84 \\
8.24 \\
8.43 \\
4.96 \\
9.21 \\
18.94 \\
10.24 \\
2.88 \\
-\end{array}$ \\
\hline $\begin{array}{l}\text { Total Energy } \\
\text { Primary Energy - Four Sectors } \\
\text { Electricity }\end{array}$ & $\begin{array}{l}1.39 \\
1.17 \\
9.02\end{array}$ & $\begin{array}{l}2.71 \\
2.42 \\
9.61\end{array}$ & $\begin{array}{r}5.06 \\
4.61 \\
15.09\end{array}$ & $\begin{array}{r}5.99 \\
5.16 \\
24.52\end{array}$ & $\begin{array}{r}7.01 \\
5.97 \\
27.80\end{array}$ & $\begin{array}{r}6.46 \\
R 5.31 \\
28.75\end{array}$ & $\begin{array}{l}{ }_{6}{ }_{6.37} \\
\text { R }_{5.17} \\
29.22\end{array}$ & $\begin{array}{r}6.56 \\
5.27 \\
29.72\end{array}$ \\
\hline $\begin{array}{l}\text { Electric Utility Sector }{ }^{\mathrm{a}} \\
\text { Coal } \\
\text { Natural Gas } \\
\text { Petroleum } \\
\text { Heavy Oil }{ }^{b} \\
\text { Light Oil }\end{array}$ & $\begin{array}{r}.66 \\
.68 \\
.37 \\
1.68 \\
1.35 \\
1.68\end{array}$ & $\begin{array}{l}.95 \\
.96 \\
.51 \\
3.10 \\
2.67 \\
3.11\end{array}$ & $\begin{array}{r}1.25 \\
1.91 \\
.48 \\
4.90 \\
4.08 \\
5.48\end{array}$ & $\begin{array}{r}1.71 \\
1.80 \\
.92 \\
6.12 \\
5.18 \\
7.06\end{array}$ & $\begin{array}{r}2.37 \\
2.46 \\
1.58 \\
9.27 \\
6.38 \\
10.36\end{array}$ & $\begin{array}{l}1.81 \\
1.94 \\
1.16 \\
6.14 \\
3.73 \\
7.31\end{array}$ & $\begin{array}{l}1.91 \\
1.99 \\
1.18 \\
6.57 \\
3.97 \\
7.25\end{array}$ & $\begin{array}{l}1.89 \\
2.11 \\
1.25 \\
5.18 \\
3.48 \\
6.22\end{array}$ \\
\hline $\begin{array}{l}\text { Petroleum Coke } \\
\text { Nuclear Fuel } \\
\text { Biomass Fuels }\end{array}$ & $\bar{z}$ & $\bar{z}$ & $\overline{-}$ & $\bar{z}$ & $\bar{z}$ & $\bar{z}$ & $\overline{-}$ & $\bar{z}$ \\
\hline 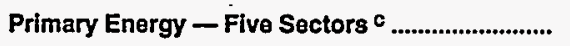 & 1.12 & 2.21 & 4.04 & 4.71 & $\mathrm{R}_{5.51}$ & 4.86 & $\mathrm{R}_{4.78}$ & 4.85 \\
\hline
\end{tabular}

a There are no direct fuel costs for hydroelectric, geothermal, wind, phofovoltaic, or solar themal energy.

beavy oil includes fuel oil nos. 4, 5, and 6, and residual fuel oils. Light oil includes fuel oil nos. 1 and 2 , kerosene, and jet fuel.

c Biomass fuels are not included, except those consumed at electric utilities and those added to motor gasoline.

$R=$ Revised data

-No consumption, including cases where adjustments were made. See explanation of adjustments in Section 6 of Appendix A.

Sources: Data sources, estimation procedures, and assumptions are described in Appendix A. 


\begin{tabular}{|c|c|c|c|c|c|c|c|c|}
\hline Sector and Energy Source & 1970 & 1975 & 1980 & 1985 & 1990 & 1991 & 1992 & 1993 \\
\hline 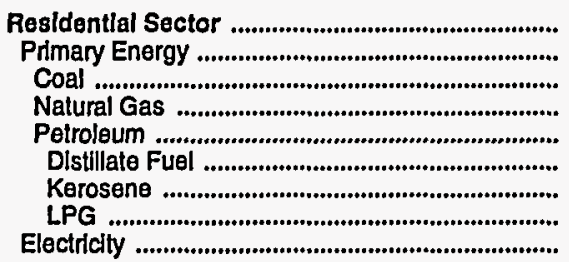 & $\begin{array}{r}38.6 \\
21.9 \\
.4 \\
.4 \\
9.4 \\
12.1 \\
11.1 \\
.2 \\
.9 \\
16.7\end{array}$ & $\begin{array}{r}77.9 \\
46.8 \\
.3 \\
16.9 \\
29.6 \\
26.4 \\
1.7 \\
1.5 \\
31.1\end{array}$ & $\begin{array}{r}124.8 \\
64.5 \\
-\overline{1} \\
13.8 \\
50.8 \\
48.2 \\
\overline{2.6} \\
60.3\end{array}$ & $\begin{array}{r}273.6 \\
125.3 \\
18.7 \\
37.3 \\
69.3 \\
59.6 \\
.1 \\
.9 .6 \\
148.3\end{array}$ & $\begin{array}{r}342.4 \\
174.4 \\
21.7 \\
53.7 \\
98.9 \\
80.7 \\
.1 \\
18.1 \\
168.0\end{array}$ & $\begin{array}{r}349.0 \\
178.0 \\
30.6 \\
56.7 \\
90.7 \\
70.4 \\
.4 \\
19.9 \\
171.0\end{array}$ & $\begin{array}{r}336.7 \\
158.7 \\
22.5 \\
54.4 \\
81.7 \\
63.4 \\
(s) \\
18.3 \\
178.0\end{array}$ & $\begin{array}{r}324.1 \\
142.1 \\
24.8 \\
54.9 \\
62.5 \\
51.5 \\
(\mathrm{~s}) \\
10.9 \\
182.0\end{array}$ \\
\hline 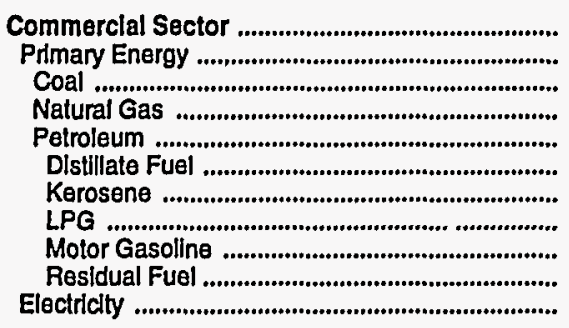 & $\begin{array}{r}39.0 \\
23.5 \\
.3 \\
8.6 \\
14.7 \\
3.0 \\
- \\
.1 \\
4.1 \\
7.5 \\
15.4\end{array}$ & $\begin{array}{r}66.4 \\
42.1 \\
.3 \\
14.0 \\
27.8 \\
7.6 \\
-1 \\
11.2 \\
8.9 \\
24.3\end{array}$ & $\begin{array}{r}99.0 \\
54.2 \\
- \\
17.5 \\
36.8 \\
22.7 \\
\overline{.1} \\
13.8 \\
.1 \\
44.8\end{array}$ & $\begin{array}{r}274.3 \\
116.6 \\
15.1 \\
48.1 \\
53.4 \\
37.8 \\
.1 \\
1.6 \\
13.8 \\
157.7\end{array}$ & $\begin{array}{r}323.4 \\
124.3 \\
14.0 \\
56.9 \\
53.5 \\
49.0 \\
(\mathrm{~s}) \\
1.7 \\
2.7 \\
199.0\end{array}$ & $\begin{array}{r}326.8 \\
118.8 \\
11.2 \\
60.4 \\
47.1 \\
41.1 \\
(s) \\
1.9 \\
4.1 \\
208.0\end{array}$ & $\begin{array}{r}\mathrm{P}_{347.1} \\
\mathrm{~F}_{132.1} \\
\mathrm{P}_{20.8} \\
56.2 \\
55.1 \\
50.2 \\
(\mathrm{~s}) \\
1.9 \\
3.0 \\
\overline{215.0}\end{array}$ & $\begin{array}{r}351.0 \\
130.9 \\
30.1 \\
55.6 \\
45.2 \\
43.6 \\
(s) \\
1.1 \\
.4 \\
220.1\end{array}$ \\
\hline 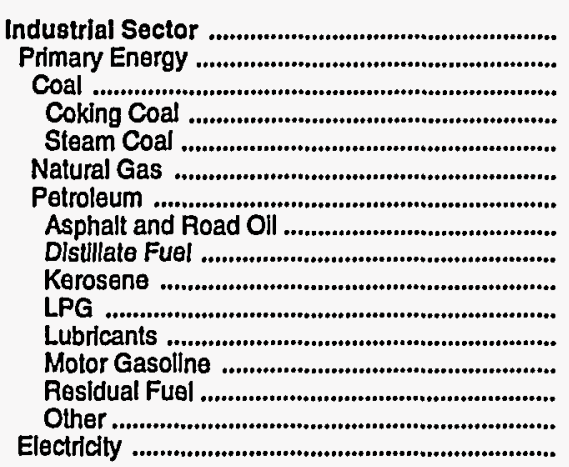 & $\begin{array}{r}25.7 \\
23.9 \\
8.6 \\
- \\
8.6 \\
5.1 \\
10.2 \\
1.0 \\
6.9 \\
.1 \\
.2 \\
(5) \\
1.8 \\
.1 \\
.1 \\
1.7\end{array}$ & $\begin{array}{r}81.2 \\
70.7 \\
16.5 \\
- \\
16.5 \\
13.5 \\
40.7 \\
3.8 \\
30.8 \\
.5 \\
.8 \\
1.1 \\
2.9 \\
.3 \\
.5\end{array}$ & $\begin{array}{r}128.0 \\
103.5 \\
- \\
- \\
\overline{-} \\
19.5 \\
84.0 \\
7.4 \\
64.0 \\
.8 \\
1.3 \\
1.8 \\
5.9 \\
.3 \\
2.5 \\
24.5\end{array}$ & $\begin{array}{r}255.4 \\
230.0 \\
- \\
- \\
- \\
45.2 \\
184.8 \\
14.4 \\
71.2 \\
.2 \\
3.3 \\
2.0 \\
21.0 \\
68.3 \\
4.5 \\
25.5\end{array}$ & $\begin{array}{r}R_{169.7} \\
R_{135.8} \\
- \\
- \\
- \\
58.5 \\
R_{77.3} \\
5.6 \\
64.7 \\
(\mathrm{~s}) \\
.6 \\
R_{1.9} \\
2.9 \\
1.7 \\
33.9\end{array}$ & $\begin{array}{r}R_{185.3} \\
R_{149.6} \\
- \\
- \\
- \\
59.1 \\
R_{90.5} \\
5.6 \\
76.3 \\
(\mathrm{~s}) \\
.3 \\
R_{1.9} \\
2.7 \\
3.5 \\
- \\
35.8\end{array}$ & $\begin{array}{r}R_{188.2} \\
R_{151.3} \\
- \\
- \\
- \\
65.9 \\
R_{85.4} \\
4.9 \\
71.4 \\
(\mathrm{~s}) \\
.3 \\
R_{2.3} \\
3.0 \\
3.5 \\
- \\
36.9\end{array}$ & $\begin{array}{r}175.1 \\
136.5 \\
.2 \\
-.2 \\
67.9 \\
68.4 \\
.8 \\
59.7 \\
.2 \\
.2 \\
2.3 \\
2.2 \\
3.2 \\
-2 \\
38.7\end{array}$ \\
\hline 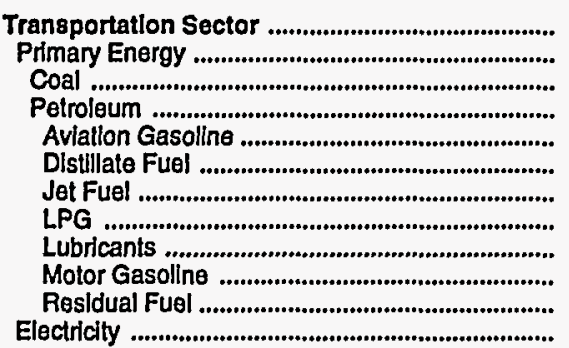 & $\begin{array}{r}81.8 \\
81.8 \\
(s) \\
81.7 \\
5.1 \\
8.5 \\
27.5 \\
(5) \\
1.8 \\
37.9 \\
.9 \\
-\end{array}$ & $\begin{array}{r}243.3 \\
243.3 \\
(\mathrm{~s}) \\
243.3 \\
8.1 \\
39.3 \\
85.0 \\
- \\
5.5 \\
98.9 \\
6.5 \\
-\end{array}$ & $\begin{array}{r}655.9 \\
655.9 \\
- \\
655.9 \\
22.7 \\
112.1 \\
335.7 \\
.1 \\
8.2 \\
177.1 \\
- \\
-\end{array}$ & $\begin{array}{r}1,084.0 \\
1,084.0 \\
- \\
1,084.0 \\
24.7 \\
272.2 \\
520.3 \\
.7 \\
9.1 \\
256.4 \\
.5 \\
-\end{array}$ & $\begin{array}{r}R_{1,288.7} \\
R_{1,288.7} \\
- \\
R_{1,288.7} \\
23.1 \\
347.1 \\
604.3 \\
.2 \\
R_{8.5} \\
301.0 \\
4.4 \\
-\end{array}$ & $\begin{array}{r}R_{1,028.3} \\
R_{1,028.3} \\
- \\
R_{1,028.3} \\
27.2 \\
231.0 \\
528.3 \\
.1 \\
R_{8.8} \\
231.6 \\
1.4 \\
-\end{array}$ & $\begin{array}{r}R_{1,004.7} \\
R_{1,004.7} \\
- \\
R_{1,004.7} \\
19.8 \\
239.7 \\
426.9 \\
.1 \\
R_{10.4} \\
301.3 \\
R_{6.4} \\
-\end{array}$ & $\begin{array}{r}994.5 \\
994.5 \\
- \\
994.5 \\
17.1 \\
233.6 \\
412.5 \\
.1 \\
10.3 \\
318.9 \\
2.2 \\
-\end{array}$ \\
\hline $\begin{array}{l}\text { Total Energy } \\
\text { Primary Energy - Four Sectors } \\
\text { Electricity }\end{array}$ & $\begin{array}{r}185.0 \\
151.2 \\
33.9\end{array}$ & $\begin{array}{r}468.9 \\
403.0 \\
65.9\end{array}$ & $\begin{array}{r}1,007.7 \\
878.2 \\
129.5\end{array}$ & $\begin{array}{r}1,887.3 \\
1,555.9 \\
331.5\end{array}$ & $\begin{array}{r}F_{2,124.1} \\
F_{1,723.2} \\
400.9\end{array}$ & $\begin{array}{r}R_{1,889.4} \\
R_{1,474.7} \\
414.8\end{array}$ & $\begin{array}{r}F_{1}, 876.7 \\
F_{1,446.8} \\
429.9\end{array}$ & $\begin{array}{r}1,844.7 \\
1,404.0 \\
440.7\end{array}$ \\
\hline 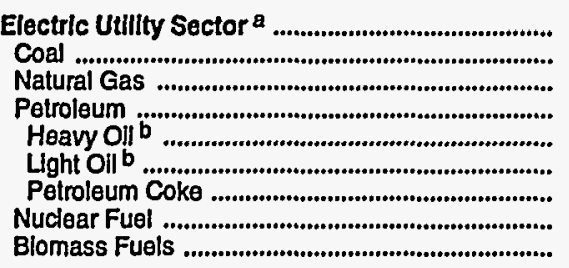 & $\begin{array}{l}9.9 \\
2.9 \\
3.1 \\
3.9 \\
(s) \\
3.9 \\
= \\
=\end{array}$ & $\begin{array}{r}27.0 \\
4.3 \\
10.1 \\
12.6 \\
(s) \\
12.6 \\
= \\
=\end{array}$ & $\begin{array}{r}48.3 \\
8.2 \\
13.8 \\
26.3 \\
9.1 \\
17.2 \\
- \\
- \\
-\end{array}$ & $\begin{array}{r}77.0 \\
8.4 \\
31.8 \\
36.8 \\
15.5 \\
21.3 \\
- \\
-\end{array}$ & $\begin{array}{r}102.1 \\
11.3 \\
54.6 \\
36.2 \\
6.9 \\
29.4 \\
= \\
-\end{array}$ & $\begin{array}{r}73.6 \\
9.1 \\
36.3 \\
28.2 \\
5.6 \\
22.6 \\
- \\
-\end{array}$ & $\begin{array}{r}72.1 \\
8.7 \\
34.0 \\
29.3 \\
3.7 \\
25.7 \\
= \\
-\end{array}$ & $\begin{array}{r}71.2 \\
9.9 \\
35.1 \\
26.2 \\
6.7 \\
19.5 \\
= \\
=\end{array}$ \\
\hline Primary Energy — Flve Sectors ${ }^{c}$ & 161.0 & 429.9 & 926.4 & $1,632.9$ & $R_{1,825.3}$ & ${ }^{A_{1,548.2}}$ & $A_{1,518.9}$ & $1,475.3$ \\
\hline
\end{tabular}

a There are no direct fuel costs for hydroelectric, geothermal, wind, pholovoltalc, or solar thermal energy.

b Heavy oll Includes fuel oll nos. 4, 5, and 6, and residual fuel oils. Light oil includes fuel nos. 1 and 2, kerosene, and Jet fuel.

- Biomass fuels are not included, except those consumed at electric utilities and those added to motor gasoline.

$R=$ Revised data. $\rightarrow$ No consumption, including cases where adjustments were made. See explanation of adjustments in Section 6 of Appendix A.

$(s)=V a l u e$ less than 0.05 million dollars.

Note: Totals may not equal sum of components due to independent rounding.

Sources: Data sources, estimation procedures, and assumptions are described in Appendix A. 


\section{A Energy Price and Expenditure Estimates by Source, Arizona}

R 1970, 1975, 1980, 1985, and 1990-1993

\begin{tabular}{|c|c|c|c|c|c|c|c|c|}
\hline Energy Source & 1970 & 1975 & 1980 & 1985 & 1990 & 1991 & 1992 & 1993 \\
\hline & \multicolumn{8}{|c|}{ Prices in Dollars per Million Btu } \\
\hline Coal & 0.21 & 0.23 & 1.01 & 1.36 & 1.45 & 1.43 & 1.40 & 1.38 \\
\hline Coking Coal & - & - & - & - & - & - & - & - \\
\hline 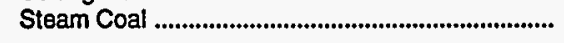 & .21 & .23 & 1.01 & 1.36 & 1.45 & 1.43 & 1.40 & 1.38 \\
\hline Natural Gas & .54 & 1.01 & 2.86 & 4.92 & 4.52 & 4.58 & 4.57 & 4.86 \\
\hline Petroleum ........................................................................ & 1.96 & 3.45 & 8.14 & 8.08 & $\mathrm{~A}_{8.39}$ & $\mathrm{R}_{7.80}$ & 8.11 $_{8.11}$ & B.72 \\
\hline 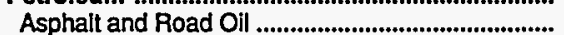 & .60 & 1.87 & 3.63 & 4.76 & 2.71 & 3.34 & 2.99 & 2.95 \\
\hline Aviation Gasoline .................................................. & 2.17 & 3.45 & 9.02 & 9.99 & 9.32 & 8.71 & 8.54 & 8.24 \\
\hline 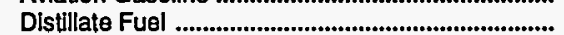 & 1.10 & 2.49 & 6.57 & 6.45 & 8.23 & 7.78 & 7.55 & 8.43 \\
\hline Jet Fuel & .76 & 2.12 & 6.59 & 6.20 & 6.04 & 5.03 & 4.72 & 4.69 \\
\hline 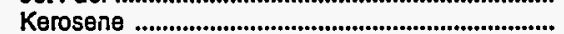 & 1.63 & 3.19 & 5.45 & 5.42 & 8.14 & 7.37 & 11.09 & 8.92 \\
\hline LPG & 2.08 & 4.06 & 6.85 & 9.98 & 11.74 & 12.23 & 10.49 & 10.34 \\
\hline Lubricants ......................................................... & 5.08 & 7.48 & 14.36 & 17.61 & $R_{14.60}$ & $R_{16.79}$ & $R_{19.43}$ & 18.94 \\
\hline 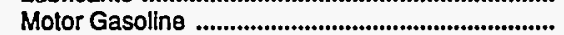 & 2.80 & 4.62 & 9.68 & 9.06 & 9.22 & 8.57 & 9.23 & 9.83 \\
\hline 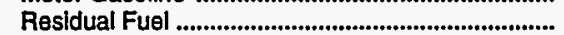 & .48 & 2.08 & 3.92 & 3.79 & 3.31 & 2.65 & 2.53 & 2.43 \\
\hline Other ${ }^{\mathrm{a}}$ & - & 1.31 & 4.04 & - & - & - & - & - \\
\hline 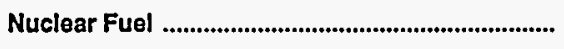 & - & - & - & .65 & .72 & .70 & .55 & .58 \\
\hline 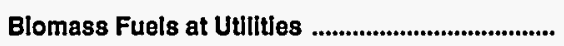 & - & - & - & - & - & - & - & - \\
\hline 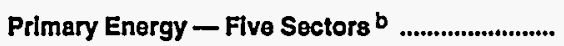 & 1.29 & 2.25 & 4.49 & 4.58 & 3.98 & 3.64 & $R_{3.68}$ & 4.03 \\
\hline 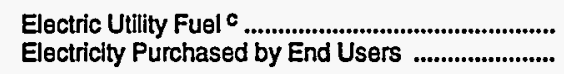 & $\begin{array}{r}.33 \\
5.32\end{array}$ & $\begin{array}{r}.84 \\
9.65\end{array}$ & $\begin{array}{r}1.35 \\
15.68\end{array}$ & $\begin{array}{r}1.60 \\
21.15\end{array}$ & $\begin{array}{r}1.21 \\
22.82\end{array}$ & $\begin{array}{r}1.13 \\
23.09\end{array}$ & $\begin{array}{r}1.08 \\
23.94\end{array}$ & $\begin{array}{r}1.12 \\
24.05\end{array}$ \\
\hline \multirow[t]{2}{*}{ 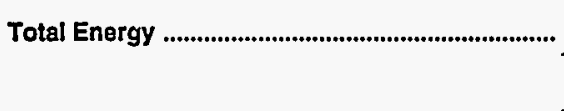 } & 1.97 & 3.87 & 8.42 & 10.03 & $8_{11.31}$ & 11.02 & $R_{11.56}$ & 11.90 \\
\hline & \multicolumn{8}{|c|}{ Expenditures In Millions of Dollars } \\
\hline 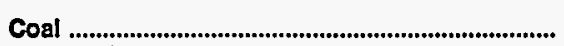 & 1.8 & 21.1 & 247.0 & 466.2 & 498.5 & 497.6 & $\mathbf{5 1 5 . 3}$ & 536.1 \\
\hline 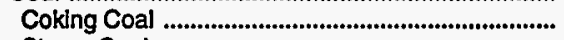 & - & - & - & - & - & - & - & - \\
\hline 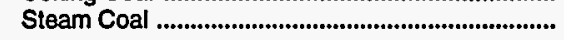 & 1.8 & 21.1 & 247.0 & 466.2 & 498.5 & 497.6 & 515.3 & 536.1 \\
\hline Natural Gas & 96.8 & 148.4 & 434.0 & 580.6 & 464.2 & 468.8 & 492.8 & 486.6 \\
\hline Petroleum & 417.7 & $1,056.8$ & $2,448.1$ & $2,563.0$ & $R_{2,922.3}$ & $R_{2,747.8}$ & $R_{2,928.3}$ & $3,283.9$ \\
\hline 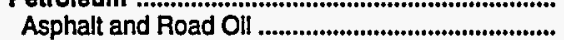 & 14.6 & 28.9 & 49.6 & 81.0 & 42.6 & 48.3 & 59.1 & 45.5 \\
\hline 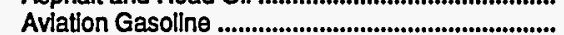 & 4.7 & 6.2 & 12.8 & 9.3 & 9.1 & 8.3 & 6.8 & 5.3 \\
\hline Dlstillate Fuel ................................................ & 31.3 & 147.1 & 412.0 & 382.6 & 577.1 & 469.4 & 496.4 & 665.1 \\
\hline Jet Fuel & 27.5 & 82.9 & 289.7 & 244.4 & 285.9 & 270.2 & 219.1 & 207.4 \\
\hline Kerosene & 1.5 & 3.8 & 2.3 & .5 & .9 & 1.5 & .2 & .2 \\
\hline LPG & 10.2 & 16.9 & 40.0 & 61.9 & 57.8 & 63.5 & 63.6 & 68.7 \\
\hline 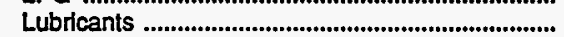 & 10.6 & 21.4 & 53.2 & 59.4 & $\mathrm{R}_{55.4}$ & $R_{57.0}$ & ${ }^{R_{67.3}}$ & 66.8 \\
\hline Motor Gasoline & 316.9 & 671.9 & $1,555.4$ & $1,719.7$ & $1,892.9$ & $1,827.1$ & $2,014.6$ & $2,222.0$ \\
\hline 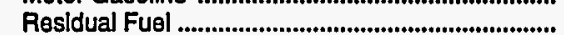 & .3 & 77.7 & 33.0 & 4.2 & .5 & 2.4 & 1.2 & 2.9 \\
\hline Other a & 0 & (s) & .1 & - & - & - & - & - \\
\hline Nuclear Fuel & - & - & - & 8.0 & 158.1 & 188.9 & 148.6 & 136.0 \\
\hline 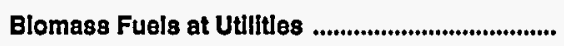 & - & - & - & - & - & - & - & - \\
\hline Prlmary Energy — Flve Sectors b ........................... & 516.4 & $1,226.3$ & $3,129.0$ & $3,617.8$ & $8_{4,043.1}$ & $\mathrm{R}_{3,903.2}$ & $R_{4,086.1}$ & $4,442.6$ \\
\hline 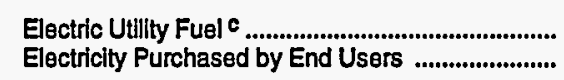 & $\begin{array}{r}-23.5 \\
250.1\end{array}$ & $\begin{array}{r}-129.6 \\
697.1\end{array}$ & $\begin{array}{r}-398.7 \\
1,431.6\end{array}$ & $\begin{array}{r}-580.5 \\
2,381.4\end{array}$ & $\begin{array}{r}-696.2 \\
3,181.4\end{array}$ & $\begin{array}{r}-711.4 \\
3,249.2\end{array}$ & $\begin{array}{r}-713.0 \\
3,516.7\end{array}$ & $\begin{array}{r}-708.9 \\
3,644.0\end{array}$ \\
\hline 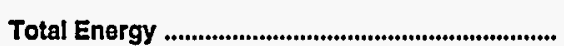 & 743.0 & $1,793.8$ & $4,161.9$ & $5,418.7$ & ${ }^{R_{6,528,4}}$ & $R_{6,441.0}$ & $R_{6,889,8}$ & $7,379.7$ \\
\hline
\end{tabular}

a Includes petroleum coke used at electric utilities.

b Blomass fuels are not included, except those consumed at electric

explanation of adjustments in Section 6 of Appendix $A$.

(s)=Value less than 0.05 million dollars.

utilittles and those added to motor gasoline.

c There are no direct fuel costs for hydroelectric, geothermal, wind, photovoltalc, or solar thermal energy.

$R=$ Revised data.

-No consumption, including cases where adjustments were made. See

Note: Expenditure totals may not equal sum of components due to independent rounding.

Sources: Data sources, estimation procedures, and assumptions are described in Appendix A. 


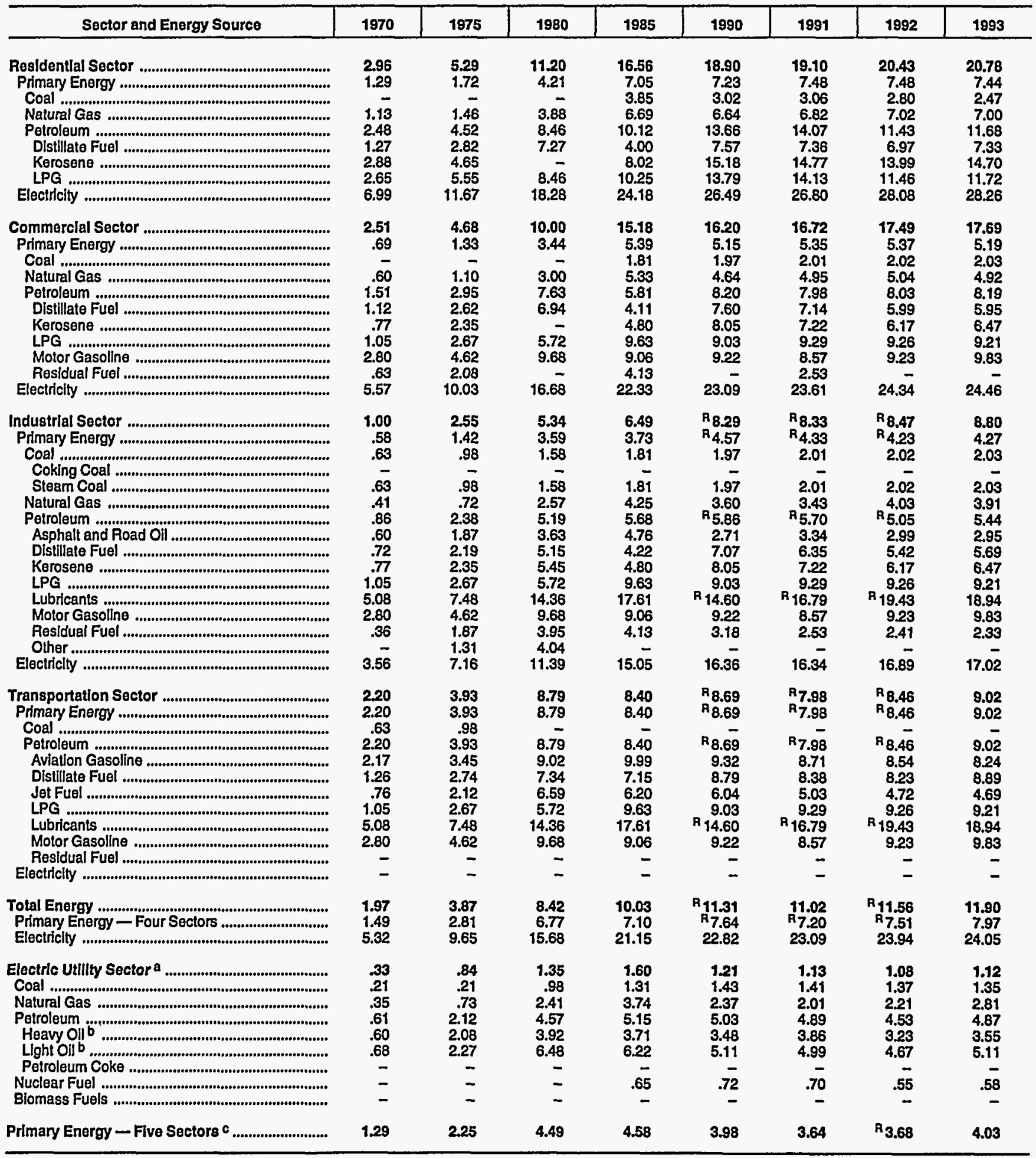

a There are no direct fuel costs for hydroelectric, geothermal, wind, photovoltalc, or solar thermal energy.

Heavy oll includes fuel oil nos. 4, 5, and 6, and residual fuel oils. Ught

$R=$ Revised data.

-No consumption, including cases where adjustments were made. Seo explanation of adjustments in Section 6 of Appendix A.

Sources: Data sources, estimation procedures, and assumptions are oil includes fuel oll nos. 1 and 2, kerosene, and jet fuel

c Blomass fuels are not included, except those consumed at electric described in Appendix A. utilitiles and those added to motor gasoline. 
A Energy Expenditure Estimates by Sector, Arizona
R $1970,1975,1980,1985$, and 1990-1993

(Million Dollars)

\begin{tabular}{|c|c|c|c|c|c|c|c|c|}
\hline Sector and Energy Source & 1970 & 1975 & 1980 & 1985 & 1990 & 1991 & 1992 & 1993 \\
\hline $\begin{array}{l}\text { Residential Sector } \\
\text { Primary Energy } \\
\text { Coal ... } \\
\text { Natural Gas } \\
\text { Petroleum } \\
\text { Distillate Fuel } \\
\text { Kerosene } \\
\text { LPG }\end{array}$ & $\begin{array}{r}149.1 \\
45.8 \\
- \\
35.6 \\
10.2 \\
.7 \\
1.1 \\
8.4 \\
103.3\end{array}$ & $\begin{array}{r}359.2 \\
75.0 \\
- \\
58.2 \\
16.8 \\
3.6 \\
2.0 \\
11.2 \\
284.3\end{array}$ & $\begin{array}{r}741.3 \\
140.1 \\
-\overline{-} \\
119.6 \\
20.5 \\
.1 \\
\overline{-} \\
20.4 \\
601.2\end{array}$ & $\begin{array}{r}1,246.7 \\
236.2 \\
(s) \\
200.5 \\
35.7 \\
.3 \\
.1 \\
35.3 \\
1,010.5\end{array}$ & $\begin{array}{r}1,636.7 \\
246.8 \\
(s) \\
207.7 \\
39.1 \\
.5 \\
(s) \\
38.6 \\
1,390.0\end{array}$ & $\begin{array}{r}1,694.0 \\
264.0 \\
(s) \\
219.2 \\
44.8 \\
.2 \\
.1 \\
.1 \\
44.5 \\
1,430.0\end{array}$ & $\begin{array}{r}1,800.0 \\
244.9 \\
.1 \\
.1 \\
205.5 \\
39.3 \\
.2 \\
.2 \\
.2 \\
39.0 \\
1,555.0\end{array}$ & $\begin{array}{r}1,849.0 \\
238.0 \\
(\mathrm{~s}) \\
202.8 \\
35.2 \\
.2 \\
.1 \\
34.9 \\
1,611.0\end{array}$ \\
\hline 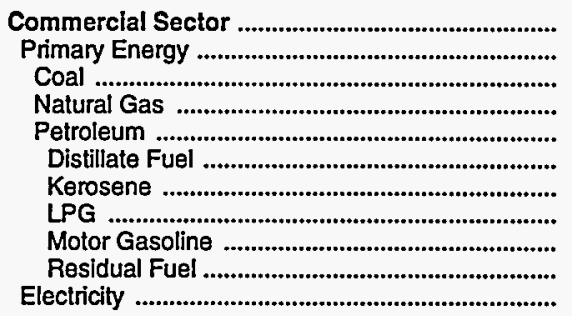 & $\begin{array}{r}107.8 \\
18.7 \\
-\overrightarrow{1} \\
14.3 \\
4.3 \\
1.4 \\
.1 \\
.6 \\
2.2 \\
.1 \\
89.1\end{array}$ & $\begin{array}{r}296.9 \\
51.7 \\
- \\
37.8 \\
13.9 \\
7.4 \\
.2 \\
.9 \\
4.3 \\
1.1 \\
245.1\end{array}$ & $\begin{array}{r}628.4 \\
109.1 \\
86.2 \\
22.9 \\
11.3 \\
-\overline{-} \\
2.4 \\
9.1 \\
\overline{-1} \\
519.3\end{array}$ & $\begin{array}{r}1,101.9 \\
165.3 \\
(s) \\
141.3 \\
24.0 \\
11.4 \\
.1 \\
5.9 \\
6.7 \\
(s) \\
936.7\end{array}$ & $\begin{array}{r}1,440.6 \\
175.6 \\
(s) \\
136.0 \\
39.5 \\
22.6 \\
.1 \\
4.5 \\
12.4 \\
- \\
1,265.0\end{array}$ & $\begin{array}{r}1,447.9 \\
174.9 \\
(s) \\
140.1 \\
34.8 \\
12.6 \\
.1 \\
5.2 \\
16.7 \\
.2 \\
1,273.0\end{array}$ & $\begin{array}{r}1,528.6 \\
169.6 \\
.1 \\
141.1 \\
28.4 \\
7.9 \\
(\mathrm{~s}) \\
5.6 \\
14.9 \\
1,359.0\end{array}$ & $\begin{array}{r}1,555.3 \\
160.3 \\
(\mathrm{~s}) \\
139.7 \\
20.6 \\
5.8 \\
(\mathrm{~s}) \\
4.8 \\
9.9 \\
- \\
1,395.0\end{array}$ \\
\hline $\begin{array}{l}\text { Industrial Sector } \\
\text { Primary Energy } \\
\text { Coal } \\
\text { Coking Coal } \\
\text { Steam Coal } \\
\text { Natural Gas } \\
\text { Petroleum } \\
\text { Asphalt and Road Oil } \\
\text { Distillate Fuel } \\
\text { Kerosene } \\
\text { LPG } \\
\text { Mubricants } \\
\text { Retor Gasoline } \\
\text { Other } \\
\text { Electricity Fuly }\end{array}$ & $\begin{array}{r}115.2 \\
57.4 \\
.1 \\
- \\
.1 \\
25.2 \\
32.1 \\
14.6 \\
5.8 \\
.4 \\
1.0 \\
3.6 \\
6.7 \\
.1 \\
- \\
57.8\end{array}$ & $\begin{array}{r}304.3 \\
136.7 \\
2.6 \\
- \\
2.6 \\
38.5 \\
95.6 \\
28.9 \\
39.6 \\
1.6 \\
4.3 \\
9.3 \\
10.7 \\
1.2 \\
(s) \\
167.7\end{array}$ & $\begin{array}{r}650.4 \\
339.3 \\
20.6 \\
- \\
20.6 \\
101.5 \\
217.1 \\
49.6 \\
107.1 \\
2.3 \\
15.5 \\
23.0 \\
15.7 \\
3.8 \\
.1 \\
311.1\end{array}$ & $\begin{array}{r}767.9 \\
333.6 \\
70.2 \\
- \\
70.2 \\
73.4 \\
190.1 \\
81.0 \\
45.5 \\
.3 \\
17.5 \\
25.7 \\
19.2 \\
.8 \\
\overline{8} \\
434.2\end{array}$ & $\begin{array}{r}R 845.5 \\
R_{319.1} \\
26.1 \\
- \\
26.1 \\
61.0 \\
R_{232.0} \\
42.6 \\
127.2 \\
.8 \\
12.9 \\
R^{24.0} \\
24.2 \\
.3 \\
- \\
526.4\end{array}$ & $\begin{array}{r}R_{8} 836.3 \\
R_{290.1} \\
27.6 \\
-\overline{-} \\
27.6 \\
61.6 \\
R_{200.9} \\
48.3 \\
96.2 \\
1.4 \\
11.9 \\
\text { R } 24.7 \\
16.6 \\
1.9 \\
-\overline{5} \\
546.2\end{array}$ & $\begin{array}{r}\text { A } 902.7 \\
\text { R }_{300.0} \\
25.9 \\
- \\
25.9 \\
75.7 \\
\mathrm{R}_{198.4} \\
59.1 \\
75.2 \\
(\mathrm{~s}) \\
17.2 \\
\mathrm{R} 29.1 \\
16.8 \\
1.0 \\
- \\
602.7\end{array}$ & $\begin{array}{r}928.4 \\
290.4 \\
27.3 \\
\overline{-} \\
27.3 \\
85.1 \\
178.0 \\
45.5 \\
56.5 \\
(5) \\
27.0 \\
28.9 \\
17.5 \\
2.6 \\
- \\
638.0\end{array}$ \\
\hline 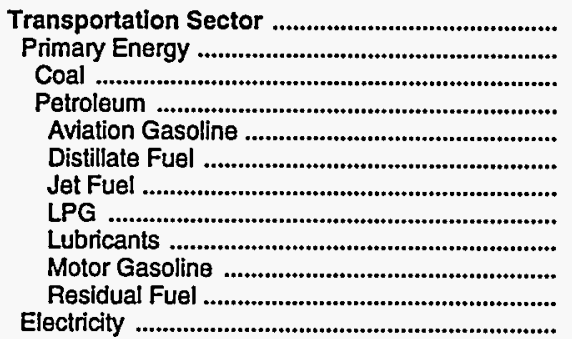 & $\begin{array}{r}370.9 \\
370.9 \\
(s) \\
370.9 \\
4.7 \\
23.4 \\
27.5 \\
.2 \\
7.1 \\
308.1 \\
-\end{array}$ & $\begin{array}{r}833.4 \\
833.4 \\
(\mathrm{~s}) \\
833.4 \\
6.2 \\
75.8 \\
81.7 \\
.5 \\
12.1 \\
656.9 \\
- \\
-\end{array}$ & $\begin{array}{r}2,141.9 \\
2,141.9 \\
- \\
2,141.9 \\
12.8 \\
277.0 \\
289.7 \\
1.6 \\
30.2 \\
1,530.5 \\
- \\
-\end{array}$ & $\begin{array}{r}2,302.2 \\
2,302.2 \\
- \\
2,302.2 \\
9.3 \\
317.7 \\
244.4 \\
3.2 \\
33.7 \\
1,693.9 \\
-\end{array}$ & $\begin{array}{r}R_{2,605.5} \\
R_{2,605.5} \\
- \\
R_{2,605.5} \\
9.1 \\
420.9 \\
285.9 \\
1.8 \\
1.8 \\
1,856.3 \\
- \\
-\end{array}$ & $\begin{array}{r}R_{2,462.8} \\
R_{2,462.8} \\
- \\
R_{2,462.8} \\
8.3 \\
356.2 \\
270.2 \\
1.9 \\
A_{32.4} \\
1,793.8 \\
-\end{array}$ & $\begin{array}{r}R_{2,658.6} \\
R_{2,658.6} \\
- \\
R_{2,658.6} \\
6.8 \\
409.7 \\
219.1 \\
1.9 \\
R_{38.2} \\
1,982.9 \\
- \\
-\end{array}$ & $\begin{array}{r}3,046.9 \\
3,046.9 \\
-\bar{r} \\
3,046.9 \\
5.3 \\
599.7 \\
207.4 \\
1.9 \\
37.9 \\
2,194.7 \\
- \\
-\end{array}$ \\
\hline 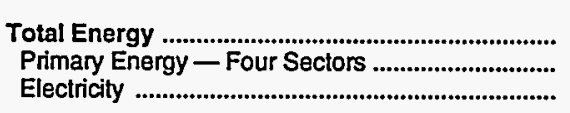 & $\begin{array}{l}743.0 \\
492.9 \\
250.1\end{array}$ & $\begin{array}{r}1,793.8 \\
1,096.7 \\
697.1\end{array}$ & $\begin{array}{l}4,161.9 \\
2,730.3 \\
1,431.6\end{array}$ & $\begin{array}{l}5,418.7 \\
3,037.3 \\
2,381.4\end{array}$ & $\begin{array}{r}\text { P }_{6,528.4} \\
\text { R }_{3,347.0} \\
3,181.4\end{array}$ & $\begin{array}{r}R_{6,441.0} \\
R_{3,191.8} \\
3,249.2\end{array}$ & $\begin{array}{r}R_{6,889.8} \\
R_{3,373.1} \\
3,516.7\end{array}$ & $\begin{array}{l}7,379.7 \\
3,735.7 \\
3,644.0\end{array}$ \\
\hline $\begin{array}{l}\text { Electric Utillty Sector }{ }^{\mathrm{a}} \\
\text { Coal } \\
\text { Natural Gas } \\
\text { Petroleum } \\
\text { Heavy Oil } \mathrm{b} \\
\text { Light Oil } \mathrm{b} \text {. } \\
\text { Pelroleum Coke } \\
\text { Nuclear Fuel } \\
\text { Biomass Fuels }\end{array}$ & $\begin{array}{r}23.5 \\
1.8 \\
21.7 \\
.1 \\
.1 \\
(s) \\
- \\
- \\
-\end{array}$ & $\begin{array}{r}129.6 \\
18.5 \\
13.9 \\
97.2 \\
75.4 \\
21.8 \\
- \\
- \\
-\end{array}$ & $\begin{array}{r}398.7 \\
226.3 \\
126.7 \\
45.7 \\
29.2 \\
16.5 \\
= \\
=\end{array}$ & $\begin{array}{r}580.5 \\
396.0 \\
165.5 \\
11.0 \\
3.4 \\
7.7 \\
\overline{8.0} \\
-\end{array}$ & $\begin{array}{r}696.2 \\
472.4 \\
59.5 \\
6.2 \\
.2 \\
6.0 \\
- \\
158.1 \\
-\end{array}$ & $\begin{array}{r}711.4 \\
470.0 \\
47.9 \\
4.5 \\
.3 \\
4.2 \\
- \\
188.9 \\
-\end{array}$ & $\begin{array}{r}713.0 \\
489.2 \\
70.6 \\
3.6 \\
.2 \\
3.3 \\
- \\
149.6 \\
-\end{array}$ & $\begin{array}{r}706.9 \\
508.7 \\
59.0 \\
3.2 \\
.4 \\
2.8 \\
- \\
136.0 \\
-\end{array}$ \\
\hline 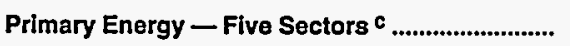 & 516.4 & 1,226.3 & $3,129.0$ & $3,617.8$ & $R_{4,043.1}$ & $\mathrm{~A}_{3,903.2}$ & $R_{4,086.1}$ & $4,442.6$ \\
\hline
\end{tabular}

a There are no direct fuel costs for hydroelectric, geothermal, wind,

-No consumption, including cases where adjustments were made. See photovollaic, or solar themal energy.

beavy oil includes fuel oil nos. 4, 5, and 6, and residual fuel oils. Light explanation of adjustments in Section 6 of Appendix A.

$(s)=$ Value less than 0.05 million dollars.

Note: Totals may not equal sum of components due to independent rounding.

Sources: Data sources, estimation procedures, and assumptlons are described in Appendix A.

c Biomass fuels are not included, except those consumed at electric utilities and those added to motor gasoline.

$\mathrm{R}=$ Revised data. 


\begin{tabular}{|c|c|c|c|c|c|c|c|c|}
\hline Enorgy Source & 1970 & 1975 & 1980 & 1985 & 1990 & 1991 & 1992 & 1993 \\
\hline & \multicolumn{8}{|c|}{ Prices In Doliars per Million Btu } \\
\hline 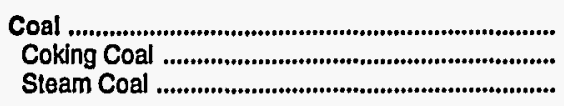 & $\begin{array}{l}- \\
-\end{array}$ & $\frac{1.22}{1.22}$ & $\frac{1.44}{1.44}$ & $\begin{array}{r}1.60 \\
1.60\end{array}$ & $\frac{1.62}{1.62}$ & $\begin{array}{r}1.61 \\
1.61\end{array}$ & $\begin{array}{r}1.66 \\
-\overline{1} \\
1.66\end{array}$ & $\begin{array}{r}1.71 \\
1.71\end{array}$ \\
\hline Natural Gas & .38 & .79 & 2.27 & 3.83 & 3.27 & 3.36 & 3.44 & 3.78 \\
\hline 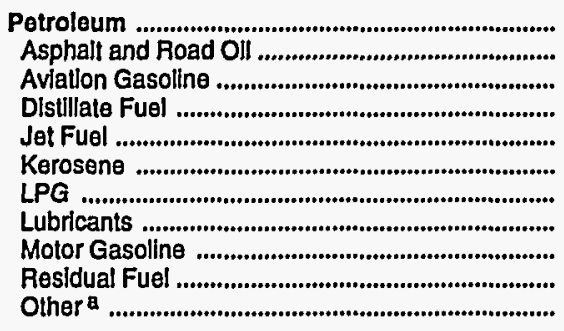 & $\begin{array}{r}1.97 \\
.68 \\
2.17 \\
.98 \\
.72 \\
.88 \\
1.63 \\
5.08 \\
2.74 \\
.43 \\
.86\end{array}$ & $\begin{array}{l}3.31 \\
1.81 \\
3.45 \\
2.39 \\
2.01 \\
2.41 \\
3.12 \\
7.48 \\
4.60 \\
1.72 \\
2.32\end{array}$ & $\begin{array}{r}7.57 \\
3.58 \\
9.02 \\
6.04 \\
6.34 \\
5.51 \\
6.97 \\
14.36 \\
9.93 \\
3.23 \\
6.16\end{array}$ & $\begin{array}{r}8.29 \\
4.21 \\
9.99 \\
7.92 \\
5.96 \\
11.00 \\
8.54 \\
17.61 \\
8.80 \\
4.01 \\
7.81\end{array}$ & $\begin{array}{r}\mathbf{R}_{\mathbf{8 . 4 5}} \\
\mathbf{3 . 0 1} \\
9.32 \\
7.69 \\
5.90 \\
8.42 \\
10.15 \\
\mathrm{~A}_{14.60} \\
8.86 \\
2.55 \\
\mathbf{R}_{\mathbf{8 . 3 8}}\end{array}$ & $\begin{array}{r}R_{8.39} \\
3.22 \\
8.71 \\
7.43 \\
5.01 \\
7.80 \\
11.20 \\
R_{16.79} \\
8.81 \\
2.30 \\
R_{8.10}\end{array}$ & $\begin{array}{r}R_{7.85} \\
2.23 \\
8.54 \\
6.21 \\
4.52 \\
4.09 \\
10.13 \\
R_{19.43} \\
8.69 \\
2.00 \\
R_{9.61}\end{array}$ & $\begin{array}{r}8.02 \\
2.70 \\
8.24 \\
6.74 \\
4.45 \\
5.47 \\
9.56 \\
18.94 \\
8.82 \\
2.08 \\
8.51\end{array}$ \\
\hline 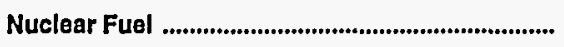 & - & 24 & .54 & .77 & .73 & .70 & .64 & .56 \\
\hline Blomass Fuels at Utilitles ..................................... & - & - & - & - & - & - & - & - \\
\hline Prlmary Energy - Five Sectors ${ }^{b}$ & 1.02 & 2.11 & 4.38 & 4.38 & 4.19 & 4.10 & $R_{4.05}$ & 4.20 \\
\hline 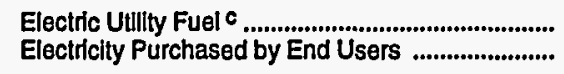 & $\begin{array}{r}26 \\
4.78\end{array}$ & $\begin{array}{r}.72 \\
7.80\end{array}$ & $\begin{array}{r}1.46 \\
12.77\end{array}$ & $\begin{array}{r}1.36 \\
18.24\end{array}$ & $\begin{array}{r}1.32 \\
19.78\end{array}$ & $\begin{array}{r}1.27 \\
19.76\end{array}$ & $\begin{array}{r}1.31 \\
19.81\end{array}$ & $\begin{array}{r}1.28 \\
19.60\end{array}$ \\
\hline \multirow[t]{2}{*}{ 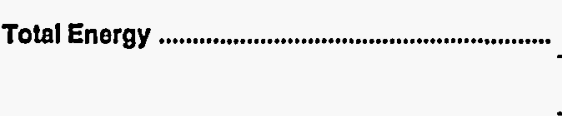 } & 1.52 & 2.98 & 6.68 & B.41 & ${ }^{R} 8.75$ & ${ }^{R_{8.91}}$ & ${ }^{8} 8.57$ & B.76 \\
\hline & \multicolumn{8}{|c|}{ Expenditures in Millions of Dollars } \\
\hline 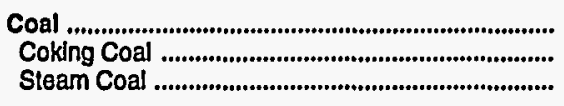 & $\overline{-}$ & $\begin{array}{l}1.1 \\
\overline{1.1}\end{array}$ & $\begin{array}{c}52.6 \\
- \\
52.6\end{array}$ & $\begin{array}{c}351.1 \\
- \\
351.1\end{array}$ & $\begin{array}{c}344.9 \\
-\overline{4} .9\end{array}$ & $\begin{array}{c}346.9 \\
- \\
346.9\end{array}$ & $\begin{array}{c}366.3 \\
- \\
366.3\end{array}$ & $\begin{array}{c}342.5 \\
- \\
342.5\end{array}$ \\
\hline Natural Gas & 133.8 & 185.8 & 581.7 & 636.9 & 665.3 & 635.2 & 675.5 & 762.3 \\
\hline 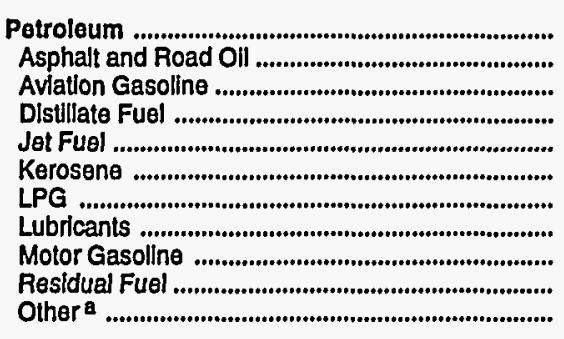 & $\begin{array}{r}469.3 \\
9.4 \\
3.2 \\
31.1 \\
8.5 \\
4.1 \\
62.4 \\
16.4 \\
323.7 \\
2.4 \\
8.0\end{array}$ & $\begin{array}{r}1,130.8 \\
27.4 \\
4.4 \\
133.2 \\
21.7 \\
9.4 \\
109.4 \\
28.0 \\
666.5 \\
97.6 \\
33.2\end{array}$ & $\begin{array}{r}2,318.9 \\
65.9 \\
12.5 \\
376.2 \\
70.0 \\
17.8 \\
123.7 \\
61.0 \\
1,381.9 \\
100.3 \\
109.6\end{array}$ & $\begin{array}{r}2,302.5 \\
35.3 \\
4.4 \\
687.9 \\
65.7 \\
9.7 \\
112.9 \\
68.0 \\
1,235.5 \\
17.0 \\
65.9\end{array}$ & $\begin{array}{r}R_{2,289.7} \\
9.9 \\
5.9 \\
638.6 \\
54.5 \\
1.8 \\
127.3 \\
R_{63.5} \\
1,341.8 \\
2.7 \\
R_{43.6}\end{array}$ & $\begin{array}{r}R_{2,235.1} \\
11.4 \\
6.3 \\
582.9 \\
48.8 \\
1.6 \\
133.8 \\
R_{65.3} \\
1,342.2 \\
1.1 \\
R_{41.6}\end{array}$ & $\begin{array}{r}\text { R }_{2,189.8} \\
17.4 \\
6.6 \\
554.5 \\
28.0 \\
.5 \\
110.5 \\
\mathrm{R}_{77.1} \\
1,342.6 \\
.2 \\
\mathrm{R}_{52.5}\end{array}$ & $\begin{array}{r}2,329.1 \\
26.1 \\
5.6 \\
615.0 \\
25.2 \\
.9 \\
119.7 \\
76.5 \\
1,411.2 \\
2.3 \\
46.7\end{array}$ \\
\hline Nuclear Fual & - & 12.7 & 46.0 & 82.8 & 88.3 & 95.4 & 77.7 & 81.5 \\
\hline 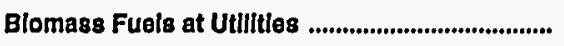 & - & - & - & - & - & - & - & - \\
\hline Primary Energy - Flve Sectors b ....................... & 603.0 & $1,330.3$ & $2,999.2$ & $3,373.2$ & $R_{3,388.2}$ & $R_{3,312.6}$ & $A_{3,309.3}$ & $3,515.3$ \\
\hline $\begin{array}{l}\text { Electrlc Utility Fuel c .................................................. } \\
\text { Electrlclty Purchased by End Users ................... }\end{array}$ & $\begin{array}{r}-29.3 \\
217.4\end{array}$ & $\begin{array}{r}-82.2 \\
480.4\end{array}$ & $\begin{array}{r}-286.3 \\
1,149.8\end{array}$ & $\begin{array}{r}-451.4 \\
1,440.1\end{array}$ & $\begin{array}{r}-476.2 \\
1,789.8\end{array}$ & $\begin{array}{r}-473.8 \\
1,859.8\end{array}$ & $\begin{array}{r}-475.8 \\
1,863.5\end{array}$ & $\begin{array}{r}-460.9 \\
2,040.7\end{array}$ \\
\hline 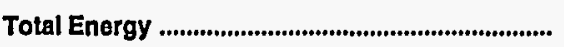 & 791.2 & $1,728.5$ & $3,862.7$ & $4,361.9$ & $R_{4,701.8}$ & $R_{4,698.6}$ & $R_{4,696.9}$ & $5,095.2$ \\
\hline
\end{tabular}

Coal

Potroleum

Aviatlon Gasolin

thillate Fuel

Residual Fuel

Blomass Fuels at Utilitles

Primary Energy - Five Sectors ${ }^{b}$

Electric Utillty Fuel

Tolal Energy

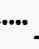

a Includes petroleum coke used at electric utilities.

b Blomass fuels are not included, except those consumed at electric utllitles and those added to motor gasoline.

c There are no direct fuel costs for hydroelectric, geothermal, wind, photovoltalc, or solar thermal energy.

$R=$ Rovised data.
-No consumption, including cases where adjustments were made. Seo explanation of adjustments in Sectlon 6 of Appendix A.

Note: Expenditure totals may not equal sum of components due to Independent rounding.

Sources: Data sources, estimation procedures, and assumptions are described in Appendlx A. 
A Energy Price Estimates by Sector, Arkansas

R 1970, 1975, 1980, 1985, and 1990-1993

K (Dollars per Million Btu)

\begin{tabular}{|c|c|c|c|c|c|c|c|c|}
\hline Sector and Energy Source & 1970 & 1975 & 1980 & 1985 & 1990 & 1991 & 1992 & 1993 \\
\hline 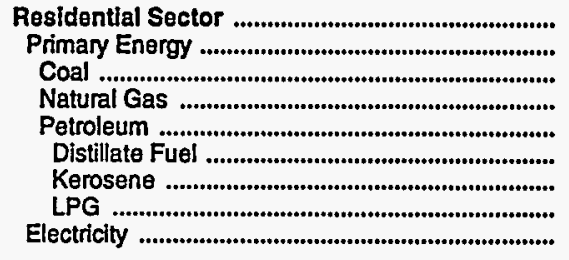 & $\begin{array}{r}1.91 \\
1.07 \\
-7 \\
.75 \\
1.81 \\
.93 \\
1.40 \\
1.84 \\
6.82\end{array}$ & $\begin{array}{l}3.90 \\
1.82 \\
1 . \overline{12} \\
3.44 \\
2.40 \\
2.80 \\
3.51 \\
9.35\end{array}$ & $\begin{array}{r}8.14 \\
3.45 \\
2.97 \\
2.49 \\
8.54 \\
6.54 \\
8.77 \\
15.58\end{array}$ & $\begin{array}{r}11.53 \\
5.01 \\
3.19 \\
4.35 \\
8.52 \\
10.33 \\
11.07 \\
8.46 \\
21.91\end{array}$ & $\begin{array}{r}13.67 \\
5.90 \\
2.70 \\
5.06 \\
10.74 \\
7.69 \\
8.24 \\
10.78 \\
23.65\end{array}$ & $\begin{array}{r}13.75 \\
5.83 \\
2.81 \\
4.90 \\
12.09 \\
7.11 \\
7.62 \\
12.15 \\
23.76\end{array}$ & $\begin{array}{r}13.90 \\
5.73 \\
2.69 \\
5.07 \\
10.54 \\
4.99 \\
5.34 \\
10.66 \\
24.28\end{array}$ & $\begin{array}{r}13.84 \\
5.85 \\
2.73 \\
5.31 \\
9.87 \\
5.68 \\
6.09 \\
9.91 \\
24.25\end{array}$ \\
\hline 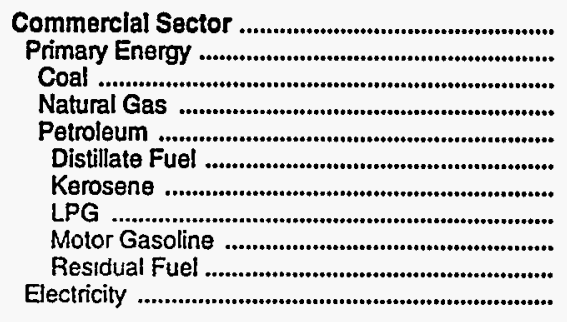 & $\begin{array}{r}1.58 \\
.64 \\
.52 \\
. .37 \\
.86 \\
.77 \\
1.23 \\
2.74 \\
.42 \\
6.07\end{array}$ & $\begin{array}{r}3.08 \\
1.25 \\
. \overline{-90} \\
2.23 \\
2.29 \\
2.32 \\
2.64 \\
4.60 \\
1.75 \\
8.60\end{array}$ & $\begin{array}{l}6.73 \\
2.80 \\
1.89 \\
2.29 \\
5.24 \\
6.25 \\
5.51 \\
5.54 \\
9.93 \\
3.33 \\
14.74\end{array}$ & $\begin{array}{r}10.20 \\
5.34 \\
2.12 \\
4.06 \\
9.10 \\
9.09 \\
10.98 \\
8.65 \\
8.80 \\
19.0 \overline{6}\end{array}$ & $\begin{array}{r}11.64 \\
4.94 \\
1.99 \\
4.43 \\
7.86 \\
6.84 \\
8.62 \\
9.42 \\
8.86 \\
20.40\end{array}$ & $\begin{array}{r}11.59 \\
4.70 \\
1.86 \\
4.28 \\
7.86 \\
6.39 \\
7.92 \\
10.22 \\
8.81 \\
20.32\end{array}$ & $\begin{array}{r}11.67 \\
4.49 \\
1.86 \\
4.35 \\
5.53 \\
3.28 \\
3.44 \\
9.62 \\
8.69 \\
2.00 \\
20.72\end{array}$ & $\begin{array}{r}11.41 \\
4.52 \\
1.88 \\
4.37 \\
5.75 \\
4.06 \\
5.14 \\
9.21 \\
8.82 \\
2.08 \\
20.58\end{array}$ \\
\hline 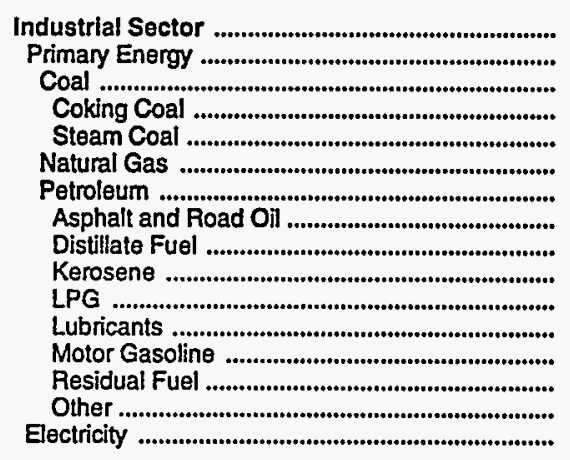 & $\begin{array}{r}.69 \\
.46 \\
- \\
- \\
. \overline{.} \\
.98 \\
.68 \\
.67 \\
.77 \\
1.23 \\
5.08 \\
2.74 \\
.45 \\
.86 \\
2.78\end{array}$ & $\begin{array}{l}1.63 \\
1.29 \\
1.22 \\
1 . \overline{2} \\
.68 \\
2.18 \\
1.81 \\
2.09 \\
2.32 \\
2.64 \\
7.48 \\
4.60 \\
1.63 \\
2.32 \\
5.18\end{array}$ & $\begin{array}{r}4.17 \\
3.26 \\
1.89 \\
- \\
1.89 \\
2.24 \\
4.95 \\
3.58 \\
4.87 \\
5.51 \\
5.54 \\
14.36 \\
9.93 \\
2.95 \\
6.16 \\
9.15\end{array}$ & $\begin{array}{r}6.73 \\
5.48 \\
2.12 \\
2.12 \\
2.12 \\
3.65 \\
8.32 \\
4.21 \\
9.44 \\
10.98 \\
8.65 \\
17.61 \\
8.80 \\
4.01 \\
7.81 \\
13.74\end{array}$ & $\begin{array}{r}R_{5} .95 \\
R_{4.06} \\
1.99 \\
- \\
1.99 \\
2.86 \\
\text { R7.66 } \\
3.01 \\
7.41 \\
8.62 \\
9.42 \\
\text { A } 14.60 \\
8.86 \\
2.54 \\
R_{8.38} \\
14.93\end{array}$ & $\begin{array}{r}R_{6.23} \\
R_{4.10} \\
1.86 \\
- \\
1.86 \\
3.01 \\
R_{7.62} \\
3.22 \\
6.80 \\
7.92 \\
10.22 \\
R^{16.79} \\
8.81 \\
2.30 \\
R_{8.10} \\
14.80\end{array}$ & $\begin{array}{r}A_{5} .66 \\
R_{3.63} \\
1.86 \\
-\overline{1} \\
1.86 \\
3.11 \\
{ }^{3} 5.03 \\
2.23 \\
2.96 \\
3.44 \\
9.62 \\
\text { P }_{19.43} \\
8.69 \\
2.00 \\
R_{9.61} \\
14.72\end{array}$ & $\begin{array}{r}5.94 \\
3.91 \\
1.88 \\
- \\
1.88 \\
3.27 \\
5.68 \\
2.70 \\
4.42 \\
5.14 \\
9.21 \\
18.94 \\
8.82 \\
2.08 \\
8.51 \\
14.20\end{array}$ \\
\hline 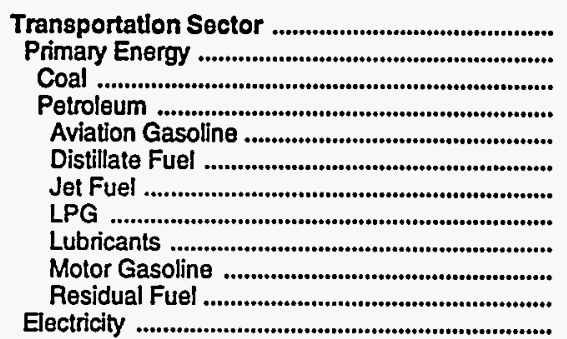 & $\begin{array}{r}2.38 \\
2.38 \\
-\overline{-} \\
2.38 \\
2.17 \\
1.16 \\
.72 \\
1.23 \\
5.08 \\
2.74 \\
.40 \\
-\end{array}$ & $\begin{array}{l}4.06 \\
4.06 \\
1.22 \\
4.06 \\
3.45 \\
2.53 \\
2.01 \\
2.64 \\
7.48 \\
4.60 \\
1.57\end{array}$ & $\begin{array}{r}9.11 \\
9.11 \\
9 . \overline{11} \\
9.02 \\
6.70 \\
6.34 \\
5.54 \\
14.36 \\
9.93 \\
= \\
-\end{array}$ & $\begin{array}{r}8.24 \\
8.24 \\
8 . \overline{-} \\
9.99 \\
6.56 \\
5.96 \\
8.65 \\
17.61 \\
8.80 \\
-\end{array}$ & $\begin{array}{r}R_{8.54} \\
R_{8.54} \\
R_{8.54} \\
9.32 \\
7.87 \\
5.90 \\
9.42 \\
R_{14.60} \\
8.86 \\
-\end{array}$ & $\begin{array}{r}R_{8.42} \\
R_{8.42} \\
R_{8.42} \\
8.71 \\
7.64 \\
5.01 \\
10.22 \\
A_{16.79} \\
8.81 \\
-\end{array}$ & $\begin{array}{r}\mathrm{R}_{8.42} \\
\mathrm{R}_{8.42} \\
\mathrm{R}_{8 . \overline{42}} \\
8.54 \\
7.69 \\
4.52 \\
9.62 \\
\mathrm{R}_{19.43} \\
8.69 \\
- \\
-\end{array}$ & $\begin{array}{r}8.49 \\
8.49 \\
- \\
8.49 \\
8.24 \\
7.65 \\
4.45 \\
9.21 \\
18.94 \\
8.82 \\
=\end{array}$ \\
\hline Electricity & - & - & - & - & & & - & - \\
\hline 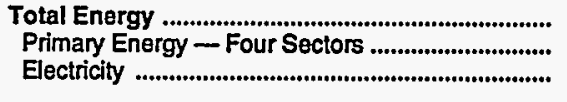 & $\begin{array}{l}1.52 \\
1.20 \\
4.78\end{array}$ & $\begin{array}{l}2.98 \\
2.41 \\
7.80\end{array}$ & $\begin{array}{r}6.68 \\
5.56 \\
12.77\end{array}$ & $\begin{array}{r}8.41 \\
6.64 \\
18.24\end{array}$ & $\begin{array}{l}R_{8.75} \\
R_{6.52} \\
19.78\end{array}$ & $\begin{array}{l}R_{8.91} \\
R_{6.55} \\
19.76\end{array}$ & $\begin{array}{l}R_{8.57} \\
R_{6.24} \\
19.81\end{array}$ & $\begin{array}{r}8.76 \\
6.40 \\
19.60\end{array}$ \\
\hline 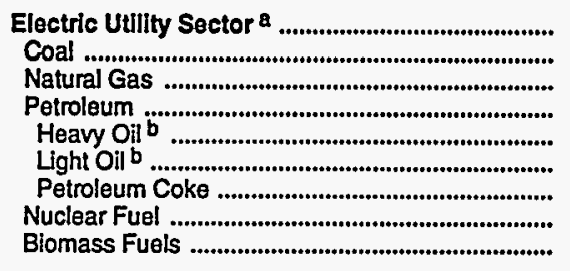 & $\begin{array}{l}.26 \\
.25 \\
.42 \\
.42 \\
.46 \\
= \\
-\end{array}$ & $\begin{array}{r}.72 \\
.61 \\
1.79 \\
1.78 \\
2.22 \\
.24 \\
-\end{array}$ & $\begin{array}{r}1.46 \\
1.34 \\
2.16 \\
3.39 \\
3.34 \\
4.34 \\
.54 \\
-\end{array}$ & $\begin{array}{l}1.36 \\
1.58 \\
2.82 \\
4.99 \\
3.84 \\
5.86 \\
-7 \\
-\end{array}$ & $\begin{array}{l}1.32 \\
1.61 \\
1.54 \\
4.72 \\
2.75 \\
4.94 \\
\overline{.73} \\
-\end{array}$ & $\begin{array}{l}1.27 \\
1.60 \\
1.41 \\
5.61 \\
2.72 \\
5.64 \\
.70 \\
-\end{array}$ & $\begin{array}{r}1.31 \\
1.65 \\
1.53 \\
4.81 \\
2.16 \\
4.81 \\
.64 \\
-\end{array}$ & $\begin{array}{r}1.28 \\
1.70 \\
2.21 \\
4.47 \\
2.07 \\
4.58 \\
-56 \\
.56\end{array}$ \\
\hline Primary Energy - Five Sectors c ............................ & 1.02 & 2.11 & 4.38 & 4.38 & 4.19 & 4.10 & A 4.05 & 4.20 \\
\hline
\end{tabular}

a There are no direct fuel costs for hydroelectric, geothermal, wind, photovoltaic, or solar thermal energy.

b Heavy oil includes fuel oil nos. 4, 5, and 6, and residual tuel oils. Light oil includes fuel oil nos. 1 and 2, kerosene, and jet fuel.

Biomass fuels are not included, except those consumed at electric utilities and those added to motor gasoline.

R=Revised data.

- No consumption, including cases where adjustments were made. Seo explanation of adjustments in Section 6 of Appendix A.

Sources: Data sources, estimation procedures, and assumptions are described in Appendix A. 


\begin{tabular}{|c|c|c|c|c|c|c|c|c|}
\hline Sector and Energy Source & 1970 & 1975 & 1980 & 1985 & 1990 & 1991 & 1992 & 1993 \\
\hline 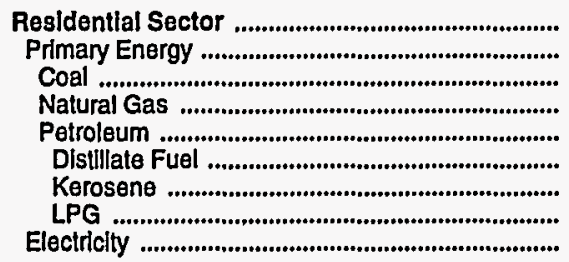 & $\begin{array}{r}192.8 \\
92.3 \\
- \\
45.1 \\
47.2 \\
.4 \\
1.2 \\
45.6 \\
100.5\end{array}$ & $\begin{array}{r}373.2 \\
125.8 \\
- \\
54.2 \\
71.7 \\
2.2 \\
2.0 \\
67.4 \\
247.4\end{array}$ & $\begin{array}{r}734.6 \\
190.8 \\
.1 \\
115.9 \\
74.8 \\
5.8 \\
-\overline{0} \\
69.0 \\
543.7\end{array}$ & $\begin{array}{r}911.3 \\
243.5 \\
(s) \\
177.9 \\
65.5 \\
(s) \\
1.9 \\
63.5 \\
667.9\end{array}$ & $\begin{array}{r}1,125.1 \\
273.1 \\
(s) \\
199.9 \\
73.3 \\
(s) \\
.9 \\
72.3 \\
852.0\end{array}$ & $\begin{array}{r}1,168.5 \\
276.5 \\
(s) \\
202.4 \\
74.1 \\
(s) \\
.6 \\
73.5 \\
892.0\end{array}$ & $\begin{array}{r}1,124.9 \\
259.8 \\
.1 \\
201.3 \\
58.5 \\
.4 \\
.4 \\
.2 \\
57.9 \\
865.0\end{array}$ & $\begin{array}{r}1,279.4 \\
306.4 \\
(s) \\
245.0 \\
61.4 \\
(s) \\
.3 \\
61.0 \\
973.0\end{array}$ \\
\hline 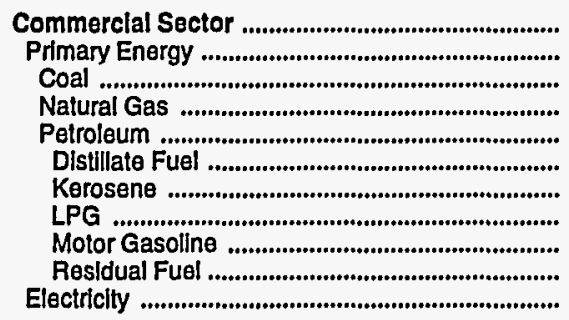 & $\begin{array}{r}87.1 \\
29.3 \\
-\overline{2} \\
20.6 \\
8.7 \\
.2 \\
.4 \\
5.4 \\
2.6 \\
.1 \\
57.8\end{array}$ & $\begin{array}{r}184.7 \\
56.2 \\
29.7 \\
26.5 \\
1.2 \\
1.0 \\
8.9 \\
3.5 \\
11.9 \\
128.6\end{array}$ & $\begin{array}{r}371.3 \\
103.5 \\
.2 \\
69.9 \\
33.5 \\
4.1 \\
4.1 \\
7.7 \\
8.5 \\
9.2 \\
267.8\end{array}$ & $\begin{array}{r}575.1 \\
194.7 \\
(s) \\
110.5 \\
84.2 \\
62.0 \\
5.2 \\
11.5 \\
5.5 \\
- \\
380.4\end{array}$ & $\begin{array}{r}612.4 \\
147.4 \\
(s) \\
112.1 \\
35.3 \\
17.5 \\
.1 \\
11.2 \\
6.6 \\
-\overline{1} \\
465.0\end{array}$ & $\begin{array}{r}620.5 \\
140.5 \\
(\mathrm{~s}) \\
113.0 \\
27.4 \\
12.7 \\
.1 \\
10.9 \\
3.7 \\
480.0\end{array}$ & $\begin{array}{r}608.7 \\
130.8 \\
(s) \\
110.9 \\
19.8 \\
7.2 \\
.1 \\
9.2 \\
3.3 \\
.1 \\
478.0\end{array}$ & $\begin{array}{r}661.8 \\
149.8 \\
(s) \\
128.2 \\
21.6 \\
10.1 \\
.2 \\
10.0 \\
1.3 \\
(s) \\
512.0\end{array}$ \\
\hline 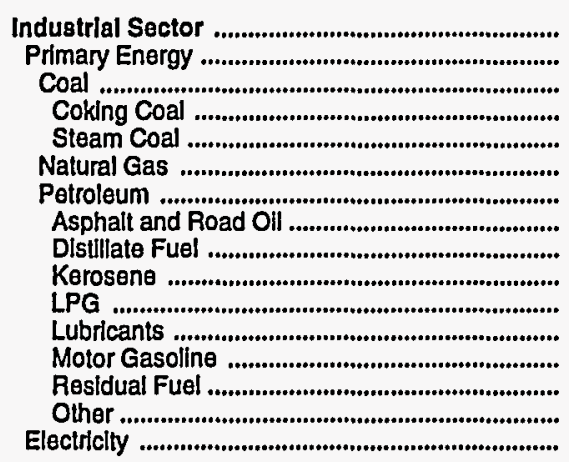 & $\begin{array}{r}147.4 \\
88.3 \\
- \\
- \\
- \\
40.7 \\
47.7 \\
9.4 \\
7.7 \\
2.6 \\
8.2 \\
7.1 \\
4.2 \\
.5 \\
8.0 \\
59.1\end{array}$ & $\begin{array}{r}370.4 \\
265.9 \\
1.1 \\
- \\
1.1 \\
82.3 \\
182.6 \\
27.4 \\
34.5 \\
6.3 \\
26.4 \\
14.0 \\
4.1 \\
36.7 \\
33.2 \\
104.4\end{array}$ & $\begin{array}{r}1,000.4 \\
662.1 \\
12.0 \\
12.0 \\
12.0 \\
265.8 \\
384.4 \\
65.9 \\
100.5 \\
13.7 \\
42.8 \\
23.3 \\
2.7 \\
25.9 \\
109.6 \\
338.3\end{array}$ & $\begin{array}{r}1,264.4 \\
872.6 \\
17.0 \\
-\overline{17.0} \\
314.5 \\
541.1 \\
35.3 \\
331.9 \\
2.6 \\
33.4 \\
26.0 \\
29.1 \\
16.8 \\
65.9 \\
391.8\end{array}$ & $\begin{array}{r}A_{1,082.6} \\
R_{609.8} \\
11.6 \\
- \\
11.6 \\
303.0 \\
R_{295.1} \\
9.9 \\
153.8 \\
.8 \\
40.9 \\
R_{24.3} \\
19.3 \\
2.5 \\
R_{43.6} \\
472.8\end{array}$ & $\begin{array}{r}\mathrm{R}_{1,033.7} \\
\mathrm{R}_{545.9} \\
12.8 \\
-\overline{12.8} \\
12.8 \\
279.7 \\
\mathrm{R}_{253.4} \\
11.4 \\
105.9 \\
.9 \\
46.5 \\
\mathrm{R}_{25.0} \\
21.0 \\
1.1 \\
\mathrm{R}_{41.6} \\
487.9\end{array}$ & $\begin{array}{r}R_{1}, 091.0 \\
R_{570.5} \\
13.2 \\
- \\
13.2 \\
320.8 \\
R_{236.5} \\
17.4 \\
75.5 \\
.2 \\
41.3 \\
R_{29.5} \\
20.0 \\
.2 \\
{ }_{52.5} \\
520.5\end{array}$ & $\begin{array}{r}1,178.2 \\
622.4 \\
14.6 \\
-\overline{14.6} \\
341.0 \\
266.9 \\
26.1 \\
97.6 \\
.4 \\
46.4 \\
29.3 \\
18.2 \\
2.2 \\
46.7 \\
555.8\end{array}$ \\
\hline 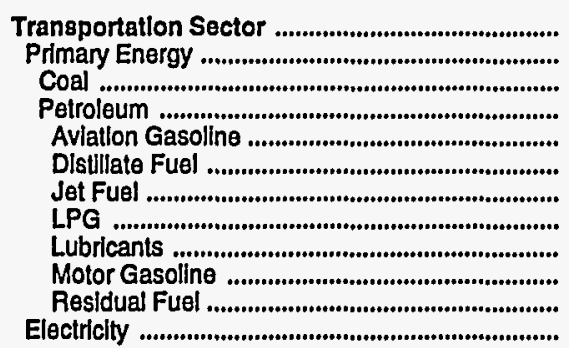 & $\begin{array}{r}363.9 \\
363.9 \\
-\overline{3} \\
363.9 \\
3.2 \\
22.8 \\
8.5 \\
3.2 \\
9.2 \\
316.9 \\
\text { (s) }\end{array}$ & $\begin{array}{r}800.2 \\
800.2 \\
(s) \\
800.2 \\
4.4 \\
94.4 \\
21.7 \\
6.7 \\
14.0 \\
658.9 \\
.1 \\
-1\end{array}$ & $\begin{array}{r}1,756.4 \\
1,756.4 \\
- \\
1,756.4 \\
12.5 \\
261.3 \\
70.0 \\
4.2 \\
37.6 \\
1,370.7 \\
- \\
-\end{array}$ & $\begin{array}{r}1,611.1 \\
1,611.1 \\
-\overline{1,611.1} \\
4.4 \\
293.5 \\
65.7 \\
4.6 \\
42.0 \\
1,200.9 \\
- \\
-\end{array}$ & $\begin{array}{r}R_{1,881.6} \\
R_{1,881.6} \\
- \\
R_{1,881.6} \\
5.9 \\
463.2 \\
54.5 \\
2.9 \\
R_{39.2} \\
1,316.0 \\
-\end{array}$ & $\begin{array}{r}R_{1}, 875.9 \\
R_{1,875.9} \\
- \\
R_{1,875.9} \\
6.3 \\
460.0 \\
48.8 \\
2.9 \\
R_{40.3} \\
1,317.5 \\
-\end{array}$ & $\begin{array}{r}R_{1,872.3} \\
R_{1,872.3} \\
- \\
R_{1,872.3} \\
6.6 \\
468.8 \\
28.0 \\
2.2 \\
R_{47.6} \\
1,319.3 \\
- \\
-\end{array}$ & $\begin{array}{r}1,975.8 \\
1,975.8 \\
- \\
1,975.8 \\
5.6 \\
503.9 \\
25.2 \\
2.3 \\
47.2 \\
1,391.7 \\
- \\
-\end{array}$ \\
\hline 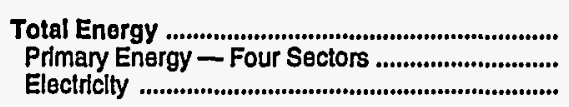 & $\begin{array}{l}791.2 \\
573.8 \\
217.4\end{array}$ & $\begin{array}{r}1,728.5 \\
1,248.1 \\
480.4\end{array}$ & $\begin{array}{l}3,862.7 \\
2,712.9 \\
1,149.8\end{array}$ & $\begin{array}{l}4,361.9 \\
2,921.8 \\
1,440.1\end{array}$ & $\begin{array}{r}R_{4,701.8} \\
R_{2,912.0} \\
1,789.8\end{array}$ & $\begin{array}{r}R_{4,698.6} \\
R_{2,838.7} \\
1,859.8\end{array}$ & $\begin{array}{r}\mathrm{A}_{4} 4,696.9 \\
\mathrm{P}_{2,833.4} \\
1,863.5\end{array}$ & $\begin{array}{l}5,095.2 \\
3,054.4 \\
2,040.7\end{array}$ \\
\hline 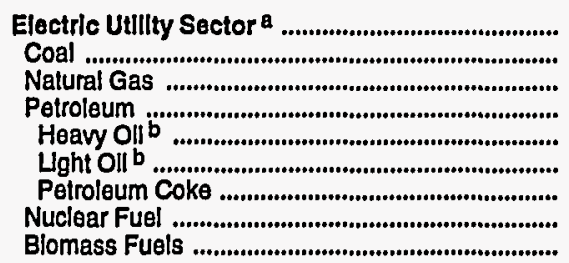 & $\begin{array}{r}29.3 \\
27.4 \\
1.9 \\
1.8 \\
(s) \\
= \\
-\end{array}$ & $\begin{array}{r}82.2 \\
19 . \overline{7} \\
49.8 \\
49.0 \\
.8 \\
\overline{1} . \overline{7} \\
-\end{array}$ & $\begin{array}{r}286.3 \\
40.3 \\
130.1 \\
69.8 \\
65.3 \\
4.5 \\
-\overline{4} \\
46.0 \\
\end{array}$ & $\begin{array}{r}451.4 \\
334.0 \\
34.0 \\
.6 \\
.2 \\
.4 \\
\overline{-} \\
82.8 \\
-\end{array}$ & $\begin{array}{r}476.2 \\
333.3 \\
50.3 \\
4.3 \\
.3 \\
4.0 \\
-\overline{88.3} \\
-\end{array}$ & $\begin{array}{r}473.8 \\
334.2 \\
40.1 \\
4.2 \\
(s) \\
4.2 \\
- \\
95.4 \\
-\end{array}$ & $\begin{array}{r}475.8 \\
353.1 \\
42.4 \\
2.7 \\
(s) \\
2.7 \\
- \\
77.7 \\
-\end{array}$ & $\begin{array}{r}460.9 \\
327.9 \\
48.1 \\
3.4 \\
.1 \\
3.4 \\
- \\
81.5 \\
-\end{array}$ \\
\hline 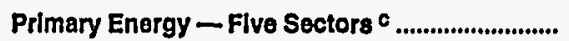 & 603.0 & $1,330.3$ & $2,999.2$ & $3,373.2$ & $R_{3,388.2}$ & $\mathrm{R}_{3,312.6}$ & $\mathrm{~A}_{3,309.3}$ & $3,515.3$ \\
\hline
\end{tabular}

a There are no direct fuel costs for hydroelectric, geothermal, wind, photovoltalc, or solar thermal energy.

t Heavy oll Includes fuel oll nos. 4, 5, and 6, and residual fuel olls. Light oll Includes fuel nos. 1 and 2, kerosene, and jet fuel.

- Blomass fuals are not included, except those consumed at electric utllities and those added to motor gasoline.

$R=$ Povised data.
-No consumption, Including cases where adjustments were made. See explanation of adjustments in Section 6 of Appendix A.

(s) =Value less than 0.05 million dollars.

Note: Totals may not equal sum of components due to independent rounding.

Sources: Data sources, estimation procedures, and assumptions are described in Appendix A. 
C Energy Price and Expenditure Estimates by Source, California

A $1970,1975,1980,1985$, and 1990-1993

\begin{tabular}{|c|c|c|c|c|c|c|c|c|}
\hline Energy Source & 1970 & 1975 & 1980 & 1985 & 1990 & 1991 & 1992 & 1993 \\
\hline & \multicolumn{8}{|c|}{ Prices in Dollars per Million Btu } \\
\hline 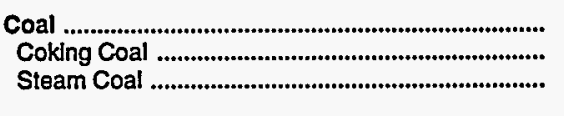 & $\begin{array}{r}0.45 \\
.43 \\
.83\end{array}$ & $\begin{array}{r}1.32 \\
1.38 \\
.92\end{array}$ & $\begin{array}{l}1.91 \\
1.97 \\
1.82\end{array}$ & $2 . \overline{-}$ & $\begin{array}{r}2.01 \\
2 . \overline{01}\end{array}$ & 1.99 & $\frac{1.83}{1.83}$ & $1 . \overline{0}$ \\
\hline 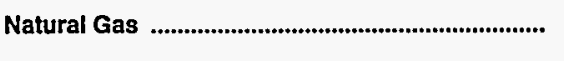 & .56 & 1.25 & 3.54 & 5.01 & 4.31 & 4.49 & 4.11 & 4.11 \\
\hline $\begin{array}{l}\text { Petroleum } \\
\text { Asphalt and Road Oil } \\
\text { Aviation Gasoline } \\
\text { Distillate Fuel } \\
\text { Jet Fuel } \\
\text { Kerosene } \\
\text { LPG }\end{array}$ & $\begin{array}{r}1.78 \\
.49 \\
2.17 \\
1.26 \\
.73 \\
1.08 \\
1.84 \\
5.08 \\
2.80 \\
.38 \\
1.04\end{array}$ & $\begin{array}{l}3.53 \\
1.62 \\
3.45 \\
2.97 \\
2.04 \\
2.76 \\
3.08 \\
7.48 \\
4.84 \\
2.38 \\
2.27\end{array}$ & $\begin{array}{r}7.37 \\
3.78 \\
9.02 \\
6.62 \\
6.21 \\
6.44 \\
6.09 \\
14.36 \\
10.19 \\
4.49 \\
6.28\end{array}$ & $\begin{array}{r}7.39 \\
4.78 \\
9.99 \\
6.48 \\
6.01 \\
6.50 \\
9.35 \\
17.61 \\
8.68 \\
4.75 \\
7.43\end{array}$ & $\begin{array}{r}R_{7.20} \\
3.13 \\
9.32 \\
7.39 \\
5.76 \\
8.42 \\
10.21 \\
\mathrm{R}_{14.60} \\
8.57 \\
3.66 \\
\mathrm{R} 5.78\end{array}$ & $\begin{array}{r}R_{6.88} \\
3.18 \\
8.71 \\
7.25 \\
4.80 \\
7.93 \\
10.95 \\
R_{16.79} \\
8.18 \\
2.57 \\
R_{5.70}\end{array}$ & $\begin{array}{r}\mathrm{R} 7.55 \\
2.74 \\
8.54 \\
\mathrm{R} 7.67 \\
4.53 \\
\mathrm{R} 8.84 \\
10.64 \\
\mathrm{R} 19.43 \\
9.19 \\
\mathrm{R} \\
\mathrm{R} 1.86 \\
\mathrm{R} 5.49\end{array}$ & $\begin{array}{r}7.59 \\
2.60 \\
8.24 \\
7.98 \\
4.50 \\
9.82 \\
11.40 \\
18.94 \\
9.34 \\
2.03 \\
4.92\end{array}$ \\
\hline 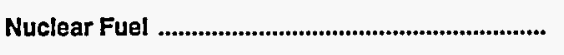 & .19 & .21 & .49 & .96 & .72 & .67 & .55 & .44 \\
\hline 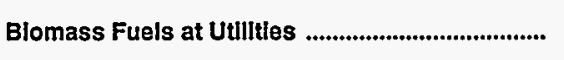 & .65 & .92 & 1.74 & .79 & (d) & $(d)$ & (d) & $(d)$ \\
\hline Primary Energy - Flve Sectors ${ }^{b}$............................... & 1.20 & 2.61 & 5.95 & 6.19 & $R_{5.78}$ & $\mathrm{R}_{5.59}$ & 5.75 & 5.80 \\
\hline 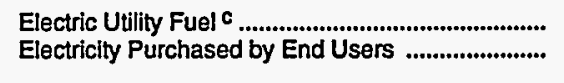 & $\begin{array}{r}.34 \\
4.76\end{array}$ & $\begin{array}{l}1.82 \\
8.71\end{array}$ & $\begin{array}{r}3.99 \\
17.16\end{array}$ & $\begin{array}{r}3.70 \\
22.90\end{array}$ & $\begin{array}{r}2.17 \\
25.98\end{array}$ & $\begin{array}{r}1.95 \\
27.70\end{array}$ & $\begin{array}{r}1.87 \\
28.39\end{array}$ & $\begin{array}{r}1.93 \\
28.51\end{array}$ \\
\hline \multirow[t]{2}{*}{ 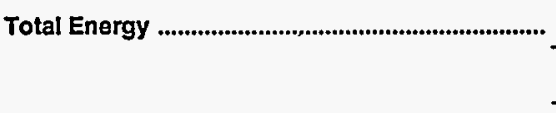 } & 1.73 & 3.47 & 7.71 & 8.91 & $\mathrm{R}_{9.04}$ & 9.15 & R9.66 & 9.60 \\
\hline & \multicolumn{8}{|c|}{ Expenditures In Mililons of Dollars } \\
\hline 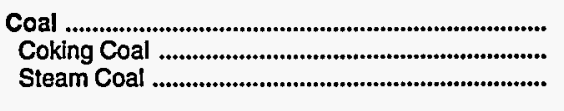 & $\begin{array}{r}27.9 \\
25.6 \\
2.3\end{array}$ & $\begin{array}{r}74.6 \\
67.7 \\
6.9\end{array}$ & $\begin{array}{r}126.7 \\
79.8 \\
46.8\end{array}$ & $\begin{array}{c}104.1 \\
- \\
104.1\end{array}$ & $\begin{array}{l}131.1 \\
- \\
131.1\end{array}$ & $\begin{array}{c}127.4 \\
\overline{-} \\
127.4\end{array}$ & $\begin{array}{l}118.8 \\
11 \overline{8.8}\end{array}$ & $\begin{array}{l}108.0 \\
108.0\end{array}$ \\
\hline Natural Gas & $1,126.7$ & $2,148.2$ & $-6,063.2$ & $9,251.8$ & $7,830.2$ & $8,242.8$ & $7,897.4$ & $7,758.8$ \\
\hline $\begin{array}{l}\text { Petroleum } \\
\text { Asphalt and Road OII } \\
\text { Aviation Gasoline } \\
\text { Distillate Fuel } \\
\text { Jet Fuel } \\
\text { Kerosene } \\
\text { LPG }\end{array}$ & $\begin{array}{r}4,210.9 \\
39.0 \\
23.9 \\
283.0 \\
242.7 \\
6.1 \\
99.5 \\
122.3 \\
3,149.1 \\
161.1 \\
84.0\end{array}$ & $\begin{array}{r}9,939.3 \\
141.0 \\
28.5 \\
719.4 \\
716.0 \\
31.8 \\
169.8 \\
164.9 \\
6,137.9 \\
1,628.1 \\
202.0\end{array}$ & $\begin{array}{r}24,321.3 \\
462.2 \\
13.0 \\
2,390.8 \\
2,199.3 \\
77.3 \\
365.8 \\
427.3 \\
13,579.1 \\
4,131.7 \\
674.9\end{array}$ & \begin{tabular}{r|}
$21,232.6$ \\
439.0 \\
68.3 \\
$2,729.9$ \\
$2,257.8$ \\
33.8 \\
585.7 \\
476.9 \\
$12,192.3$ \\
$1,953.0$ \\
496.0
\end{tabular} & $\begin{array}{r}R_{23,632.7} \\
308.8 \\
52.0 \\
3,548.4 \\
3,081.3 \\
6.9 \\
615.7 \\
R_{444.8} \\
13,699.5 \\
1,479.4 \\
R_{395.9}\end{array}$ & $\begin{array}{r}R_{20,907.6} \\
301.0 \\
48.0 \\
3,181.4 \\
2,438.9 \\
6.3 \\
573.4 \\
R_{457.8} \\
12,829.8 \\
727.3 \\
R_{343.7}\end{array}$ & $\begin{array}{r}\mathrm{A}_{22,657.6} \\
246.7 \\
45.7 \\
\mathrm{P}_{3,001.8} \\
2,219.3 \\
3.8 \\
556.0 \\
\mathrm{R}_{540.1} \\
15,248.9 \\
\mathrm{R}_{398.9} \\
\mathrm{P}_{396.5}\end{array}$ & $\begin{array}{r}22,256.4 \\
214.7 \\
34.1 \\
2,743.0 \\
2,273.1 \\
7.3 \\
475.5 \\
536.0 \\
15,140.2 \\
473.2 \\
359.3\end{array}$ \\
\hline 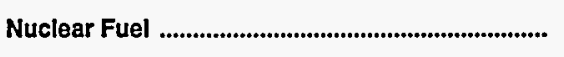 & 6.7 & 14.4 & 26.1 & 204.0 & 251.9 & 226.9 & 207.7 & 147.8 \\
\hline 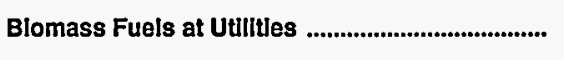 & .3 & .2 & .4 & (8) & $(d)$ & (d) & (d) & $(d)$ \\
\hline Primary Energy —Five Sectors b ............................ & $5,372.4$ & $12,176.6$ & $30,537.6$ & $30,792.6$ & $R_{31,846.0}$ & $\mathrm{R}_{29,504.7}$ & ${ }^{\mathrm{R}} 30,881.5$ & $30,270.9$ \\
\hline 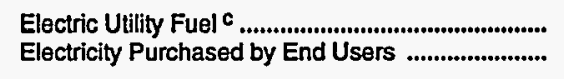 & $\begin{array}{r}-282.1 \\
1,886.7\end{array}$ & $\begin{array}{r}-1,553.7 \\
4,328.6\end{array}$ & $\begin{array}{r}-4,018.4 \\
9,560.0\end{array}$ & $\begin{array}{r}-3,498.6 \\
14,143.4\end{array}$ & $\begin{array}{r}-1,882.4 \\
18,417.8\end{array}$ & $\begin{array}{r}-1,572,2 \\
19,412.5\end{array}$ & $\begin{array}{r}-1,802.4 \\
20,377.5\end{array}$ & $\begin{array}{r}-1,620.3 \\
20,165.2\end{array}$ \\
\hline 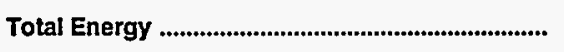 & $6,977.0$ & $14,951.5$ & $36,079.3$ & $41,437.4$ & $R_{48,381.5}$ & $R_{47,345,0}$ & ${ }^{R} 49,456.6$ & $48,815.8$ \\
\hline
\end{tabular}

a Includes petroleum coke used at electric utilities.

b Biomass fuels are not included, except those consumed at electric utilities and those added to motor gasoline.

c There are no direct fuel costs for hydroelectric, geothermal, wind, photovoltaic, or solar thermal energy.

Utilities used biomass fuels at no charge or recelved a fee for accepting them.

$R=$ Revised data.

-No consumption, including cases where adjustments were made. Se日 explanation of adjustments in Section 6 of Appendix A.

(s)=Value less than 0.05 million dollars.

Note: Expenditure totals may not equal sum of components due to independent rounding.

Sources: Data sources, estimation procedures, and assumptlons are described in Appendix A. 
Energy Price Estimates by Sector, California 1970, 1975, 1980, 1985, and 1990-1993

(Dollars per Million Btu)

\begin{tabular}{|c|c|c|c|c|c|c|c|c|}
\hline Sector and Energy Source & 1970 & 1975 & 1980 & 1985 & 1990 & 1991 & 1992 & 1993 \\
\hline 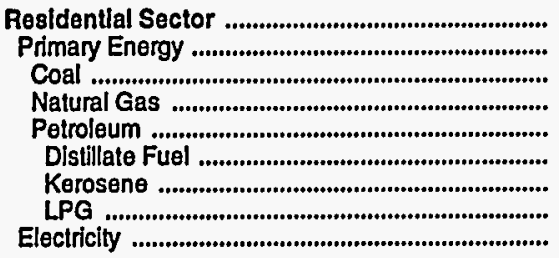 & $\begin{array}{r}1.92 \\
.99 \\
1.31 \\
.93 \\
2.49 \\
1.27 \\
2.57 \\
2.67 \\
6.53\end{array}$ & $\begin{array}{r}3.20 \\
1.55 \\
-\overline{1} \\
1.49 \\
4.17 \\
2.80 \\
5.08 \\
4.45 \\
10.68\end{array}$ & $\begin{array}{r}6.76 \\
3.53 \\
5.13 \\
3.37 \\
8.14 \\
6.92 \\
13.04 \\
8.15 \\
17.18\end{array}$ & $\begin{array}{r}10.02 \\
5.61 \\
4.54 \\
5.51 \\
8.53 \\
5.25 \\
9.18 \\
8.66 \\
22.80\end{array}$ & $\begin{array}{r}12.67 \\
5.86 \\
3.77 \\
5.60 \\
12.00 \\
5.70 \\
9.96 \\
12.45 \\
29.26\end{array}$ & $\begin{array}{r}13.74 \\
6.41 \\
5.21 \\
6.11 \\
12.39 \\
5.60 \\
9.78 \\
12.75 \\
31.61\end{array}$ & $\begin{array}{r}14.32 \\
6.07 \\
3.76 \\
5.81 \\
12.99 \\
R_{7.04} \\
R_{12.31} \\
13.39 \\
32.46\end{array}$ & $\begin{array}{r}14.29 \\
6.28 \\
3.77 \\
6.00 \\
13.89 \\
7.40 \\
12.94 \\
14.24 \\
33.12\end{array}$ \\
\hline 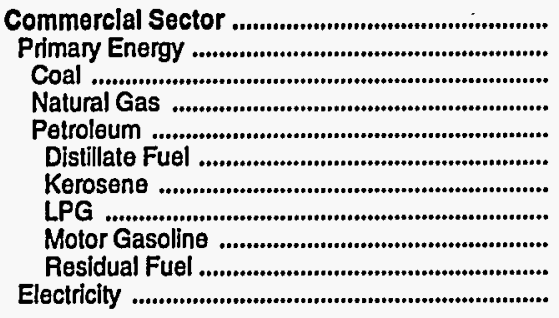 & $\begin{array}{r}2.09 \\
.71 \\
.63 \\
.69 \\
.76 \\
1.12 \\
.78 \\
1.37 \\
2.80 \\
.40 \\
5.02\end{array}$ & $\begin{array}{r}4.36 \\
1.48 \\
1.22 \\
2.93 \\
2.60 \\
2.50 \\
2.77 \\
4.84 \\
2.45 \\
8.73\end{array}$ & $\begin{array}{r}9.58 \\
4.30 \\
1.82 \\
3.82 \\
6.02 \\
6.60 \\
6.38 \\
5.21 \\
10.19 \\
4.90 \\
17.99\end{array}$ & $\begin{array}{r}15.05 \\
6.42 \\
2.28 \\
6.39 \\
6.71 \\
5.40 \\
6.27 \\
9.65 \\
8.68 \\
3.93 \\
23.61\end{array}$ & $\begin{array}{r}15.08 \\
5.14 \\
2.00 \\
4.96 \\
6.27 \\
5.72 \\
6.02 \\
9.03 \\
8.57 \\
3.00 \\
26.31\end{array}$ & $\begin{array}{r}15.96 \\
5.43 \\
1.97 \\
5.36 \\
6.01 \\
5.42 \\
5.46 \\
9.29 \\
8.18 \\
2.24 \\
28.12\end{array}$ & $\begin{array}{r}16.82 \\
5.19 \\
1.83 \\
5.01 \\
R_{7.51} \\
R_{6.05} \\
R_{6.19} \\
9.26 \\
9.19 \\
R_{2.25} \\
R_{29.06}\end{array}$ & $\begin{array}{r}17.95 \\
5.84 \\
1.86 \\
5.81 \\
7.03 \\
6.01 \\
6.49 \\
9.21 \\
9.34 \\
2.14 \\
29.27\end{array}$ \\
\hline 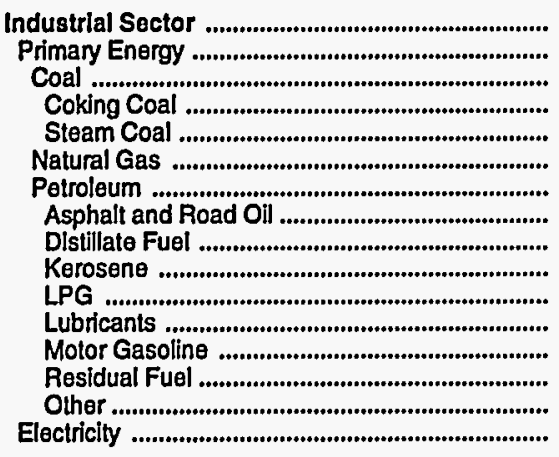 & $\begin{array}{r}.87 \\
.57 \\
.43 \\
.43 \\
.63 \\
.38 \\
.92 \\
.49 \\
.68 \\
.78 \\
1.37 \\
5.08 \\
2.80 \\
.35 \\
1.04 \\
2.90\end{array}$ & $\begin{array}{r}2.24 \\
1.52 \\
1.32 \\
1.38 \\
.92 \\
1.05 \\
2.26 \\
1.62 \\
2.21 \\
2.50 \\
2.77 \\
7.48 \\
4.84 \\
1.66 \\
2.27 \\
6.70\end{array}$ & $\begin{array}{r}6.20 \\
4.34 \\
1.91 \\
1.97 \\
1.82 \\
3.64 \\
5.22 \\
3.78 \\
5.49 \\
6.38 \\
5.21 \\
14.36 \\
10.19 \\
3.16 \\
6.28 \\
16.04\end{array}$ & $\begin{array}{r}7.93 \\
5.19 \\
2.28 \\
- \\
2.28 \\
4.54 \\
6.04 \\
4.78 \\
5.54 \\
6.27 \\
9.65 \\
17.61 \\
8.68 \\
3.93 \\
7.43 \\
22.00\end{array}$ & $\begin{array}{r}R_{7.08} \\
R_{4.34} \\
2.00 \\
- \\
2.00 \\
3.79 \\
R_{5.59} \\
3.13 \\
5.33 \\
6.02 \\
9.03 \\
R_{14.60} \\
8.57 \\
3.00 \\
R_{5.78} \\
21.35\end{array}$ & $\begin{array}{r}R_{7.19} \\
R_{4.22} \\
1.97 \\
- \\
1.97 \\
3.86 \\
R_{5.39} \\
3.18 \\
4.82 \\
5.46 \\
9.29 \\
R_{16.79} \\
8.18 \\
2.24 \\
R_{5.70} \\
22.22\end{array}$ & $\begin{array}{r}A_{7.18} \\
A_{4.14} \\
1.83 \\
- \\
1.83 \\
3.57 \\
R_{5.72} \\
2.74 \\
R_{5.47} \\
R_{6.19} \\
9.26 \\
R_{19.43} \\
9.19 \\
R_{2.25} \\
R_{5.49} \\
22.24\end{array}$ & $\begin{array}{r}6.34 \\
3.42 \\
1.86 \\
- \\
1.86 \\
2.72 \\
5.48 \\
2.60 \\
5.74 \\
6.49 \\
9.21 \\
18.94 \\
9.34 \\
2.14 \\
4.92 \\
21.50\end{array}$ \\
\hline 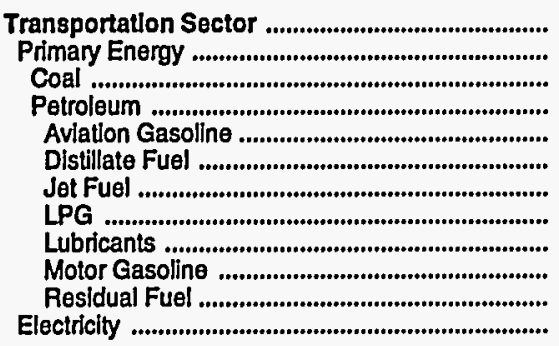 & $\begin{array}{r}2.07 \\
2.07 \\
.63 \\
2.07 \\
2.17 \\
1.42 \\
.73 \\
1.37 \\
5.08 \\
2.80 \\
.36 \\
2.88\end{array}$ & $\begin{array}{r}4.02 \\
4.02 \\
.92 \\
4.02 \\
3.45 \\
3.22 \\
2.04 \\
2.77 \\
7.48 \\
4.84 \\
2.12 \\
4.34\end{array}$ & $\begin{array}{r}8.22 \\
8.22 \\
-\overline{-} \\
8.22 \\
9.02 \\
7.07 \\
6.21 \\
5.21 \\
14.36 \\
10.19 \\
4.14 \\
11.39\end{array}$ & $\begin{array}{r}7.68 \\
7.67 \\
- \\
7.67 \\
9.99 \\
6.90 \\
6.01 \\
9.65 \\
17.61 \\
8.68 \\
5.02 \\
18.29\end{array}$ & $\begin{array}{r}R_{7.43} \\
7.42 \\
- \\
7.42 \\
9.32 \\
8.21 \\
5.76 \\
9.03 \\
R_{14.60} \\
8.57 \\
3.59 \\
13.25\end{array}$ & $\begin{array}{r}R_{7.02} \\
R_{7.01} \\
- \\
R_{7.01} \\
8.71 \\
8.02 \\
4.80 \\
9.29 \\
R_{16.79} \\
8.18 \\
2.58 \\
14.80\end{array}$ & $\begin{array}{r}R_{7.72} \\
R_{7.72} \\
- \\
R_{7.72} \\
8.54 \\
8.19 \\
4.53 \\
9.26 \\
\text { A }_{19.43} \\
9.19 \\
R_{1.84} \\
16.23\end{array}$ & $\begin{array}{r}7.80 \\
7.79 \\
- \\
7.79 \\
8.24 \\
8.46 \\
4.50 \\
9.21 \\
18.94 \\
9.34 \\
2.00 \\
14.78\end{array}$ \\
\hline 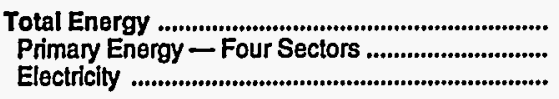 & $\begin{array}{l}1.73 \\
1.40 \\
4.76\end{array}$ & $\begin{array}{l}3.47 \\
2.79 \\
8.71\end{array}$ & $\begin{array}{r}7.71 \\
6.43 \\
17.16\end{array}$ & $\begin{array}{r}8.91 \\
6.77 \\
22.90\end{array}$ & $\begin{array}{r}R_{9.04} \\
R_{6.45} \\
25.98\end{array}$ & $\begin{array}{r}9.15 \\
R_{6.25} \\
27.70\end{array}$ & $\begin{array}{r}R_{9.66} \\
6.60 \\
28.39\end{array}$ & $\begin{array}{r}9.60 \\
6.55 \\
28.51\end{array}$ \\
\hline 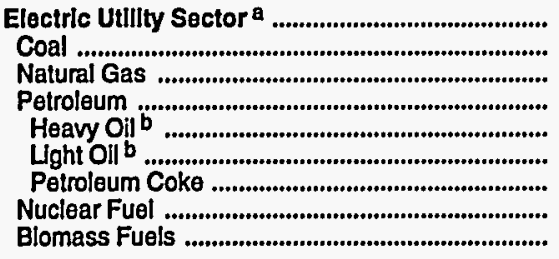 & $\begin{array}{r}.34 \\
. \\
.33 \\
.40 \\
.40 \\
.36 \\
-19 \\
.65\end{array}$ & $\begin{array}{r}1.82 \\
1.05 \\
2.50 \\
2.50 \\
2.43 \\
-21 \\
.92\end{array}$ & $\begin{array}{r}3.99 \\
- \\
3.53 \\
5.06 \\
5.03 \\
5.84 \\
- \\
.49 \\
1.74\end{array}$ & $\begin{array}{r}3.70 \\
- \\
4.47 \\
5.33 \\
5.31 \\
5.69 \\
- \\
.96 \\
.79\end{array}$ & $\begin{array}{r}2.17 \\
-\overline{3} \\
3.03 \\
4.36 \\
4.36 \\
4.57 \\
-\overline{72} \\
. \bar{d})\end{array}$ & $\begin{array}{r}1.95 \\
- \\
2.87 \\
3.23 \\
3.06 \\
4.90 \\
- \\
.67 \\
\left({ }^{d}\right)\end{array}$ & $\begin{array}{r}1.87 \\
- \\
2.72 \\
2.64 \\
2.18 \\
4.57 \\
- \\
.55 \\
(\mathrm{~d})\end{array}$ & $\begin{array}{r}1.93 \\
\overline{-} \\
2.96 \\
2.40 \\
2.31 \\
5.39 \\
\overline{-} \\
. \bar{d}\end{array}$ \\
\hline Primary Energy — Five Sectors c ........................ & 1.20 & 2.61 & 5.95 & 6.19 & $\mathrm{R}_{\mathbf{5 . 7 8}}$ & $R_{5.59}$ & $\mathbf{5 . 7 5}$ & 5.80 \\
\hline
\end{tabular}

a There are no direct fuel costs for hydroelectric, geothermal, wind, photovoltaic, or solar thermal energy.

beavy oil includes fuel oil nos. 4,5 , and 6 , and residual fuel oils. Light them.

$R=$ Revised data.

-No consumption, including cases where adjustments were made. See oll includes fuel oil nos. 1 and 2 , kerosene, and jet fuel.

$c$ Biomass fuels are not included, except those consumed at electric explanation of adjustments in Section 6 of Appendix A.

Sources: Data sources, estimation procedures, and assumptions are utilifies and those added to motor gasoline.

Utilities used blomass fuels at no charge or received a fee for accepting described in Appendix A. 


\begin{tabular}{|c|c|c|c|c|c|c|c|c|}
\hline Sector and Energy Source & 1970 & 1975 & 1980 & 1985 & 1990 & 1991 & 1982 & 1993 \\
\hline 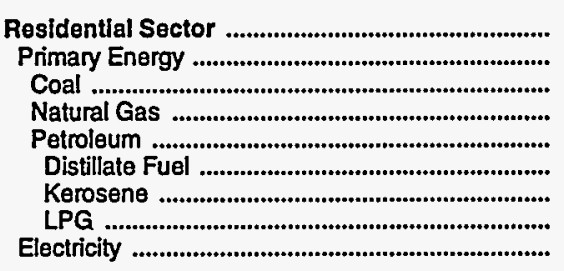 & $\begin{array}{r}1,401.1 \\
603.5 \\
1.1 \\
544.3 \\
58.2 \\
3.7 \\
2.4 \\
52.1 \\
797.6\end{array}$ & $\begin{array}{r}2,665.4 \\
1,052.7 \\
- \\
993.8 \\
58.9 \\
8.0 \\
6.1 \\
44.7 \\
1,612.8\end{array}$ & $\begin{array}{r}5,063.6 \\
2,014.1 \\
.2 \\
1,861.6 \\
152.4 \\
3.8 \\
1.3 \\
147.2 \\
3,049.5\end{array}$ & $\begin{array}{r}7,666.2 \\
3,193.4 \\
2.0 \\
3,016.1 \\
175.3 \\
4.5 \\
3.8 \\
167.0 \\
4,472.8\end{array}$ & $\begin{array}{r}9,892.5 \\
3,246.5 \\
.8 \\
2,973.8 \\
271.9 \\
7.5 \\
5.0 \\
259.4 \\
6,646.0\end{array}$ & $\begin{array}{r}10,642.7 \\
3,522.7 \\
1.9 \\
3,189.5 \\
331.3 \\
6.5 \\
4.4 \\
320.4 \\
7,120.0\end{array}$ & $\begin{array}{r}\mathrm{R}_{10,650.4} \\
\mathrm{R}_{3,106.4} \\
(\mathrm{~s}) \\
2,862.8 \\
243.6 \\
\mathrm{R}_{8.2} \\
2.3 \\
233.1 \\
7,544.0\end{array}$ & $\begin{array}{r}11,008.4 \\
3,395.5 \\
4.4 \\
3,121.0 \\
270.1 \\
6.7 \\
4.9 \\
258.5 \\
7,613.0\end{array}$ \\
\hline 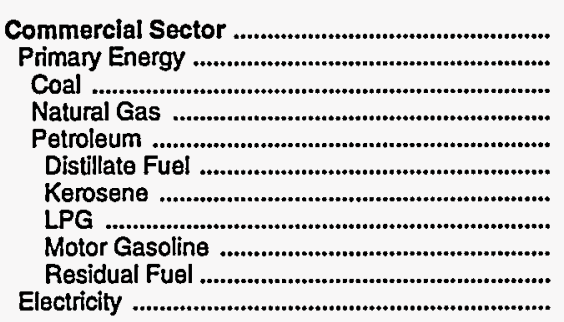 & $\begin{array}{r}905.1 \\
208.7 \\
1.0 \\
152.9 \\
54.8 \\
4.3 \\
2.3 \\
4.7 \\
21.8 \\
21.8 \\
696.3\end{array}$ & $\begin{array}{r}2,165.1 \\
442.1 \\
-. \\
309.6 \\
132.6 \\
9.8 \\
9.2 \\
4.9 \\
41.2 \\
67.4 \\
1,722.9\end{array}$ & $\begin{array}{r}5,377.9 \\
1,482.7 \\
.1 \\
1,027.9 \\
454.6 \\
124.0 \\
8.0 \\
16.6 \\
96.1 \\
209.9 \\
3,895.2\end{array}$ & $\begin{array}{r}7,528.4 \\
1,598.4 \\
1.8 \\
1,359.7 \\
236.9 \\
110.5 \\
12.5 \\
32.8 \\
80.2 \\
.9 \\
5,930.0\end{array}$ & $\begin{array}{r}9,683.0 \\
1,750.3 \\
.8 \\
1,459.7 \\
289.9 \\
152.8 \\
.6 \\
33.2 \\
86.3 \\
16.9 \\
7,932.7\end{array}$ & $\begin{array}{r}10,113.1 \\
1,847.1 \\
1.3 \\
1,581.9 \\
263.9 \\
140.5 \\
.7 \\
41.2 \\
70.7 \\
10.7 \\
8,266.0\end{array}$ & $\begin{array}{r}\mathrm{R}_{10,355.4} \\
\mathrm{P}_{1,639.7} \\
(\mathrm{~s}) \\
1,467.9 \\
\mathrm{~A}_{171.7} \\
\mathrm{P}_{70.2} \\
.7 \\
28.4 \\
71.8 \\
.6 \\
\mathrm{R}_{8,715.7}\end{array}$ & $\begin{array}{r}10,264.7 \\
1,613.7 \\
4.0 \\
1,510.7 \\
99.0 \\
55.7 \\
.7 \\
29.5 \\
12.8 \\
.2 \\
8,651.0\end{array}$ \\
\hline 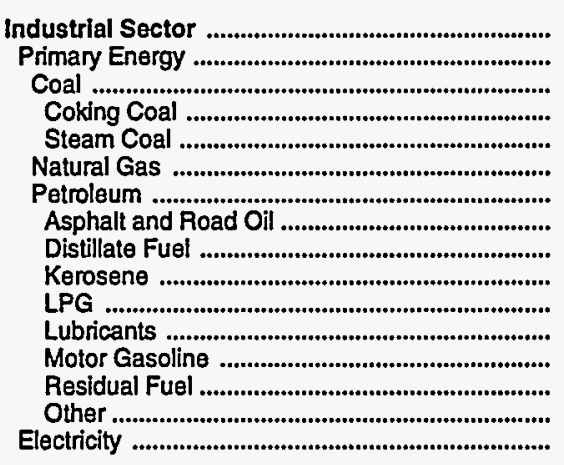 & $\begin{array}{r}920.6 \\
528.3 \\
25.8 \\
25.6 \\
.2 \\
209.3 \\
293.2 \\
39.0 \\
31.2 \\
1.5 \\
41.1 \\
46.6 \\
28.6 \\
21.3 \\
84.0 \\
392.2\end{array}$ & $\begin{array}{r}2,357.8 \\
1,368.8 \\
74.6 \\
67.7 \\
6.9 \\
539.6 \\
754.7 \\
141.0 \\
126.2 \\
16.5 \\
116.1 \\
56.6 \\
34.0 \\
62.4 \\
202.0 \\
988.9\end{array}$ & $\begin{array}{r}6,347.4 \\
3,739.6 \\
126.4 \\
79.8 \\
46.5 \\
1,248.7 \\
2,364.5 \\
462.2 \\
489.1 \\
67.9 \\
191.9 \\
183.1 \\
90.9 \\
204.4 \\
674.9 \\
2,607.7\end{array}$ & $\begin{array}{r}8,227.1 \\
4,501.7 \\
100.3 \\
-\overline{10} \\
100.3 \\
1,745.8 \\
2,655.6 \\
439.0 \\
586.9 \\
17.4 \\
343.2 \\
204.4 \\
139.8 \\
428.9 \\
496.0 \\
3,725.4\end{array}$ & $\begin{array}{r}R_{7,869.6} \\
R_{4,042.8} \\
129.6 \\
- \\
129.6 \\
1,967.7 \\
R_{1,945.5} \\
308.8 \\
590.6 \\
1.3 \\
292.6 \\
R_{190.6} \\
141.6 \\
24.1 \\
R_{395.9} \\
3,826.8\end{array}$ & $\begin{array}{r}R_{7,868.6} \\
R_{3,857.1} \\
124.2 \\
- \\
124.2 \\
2,147.0 \\
R_{1,585.8} \\
301.0 \\
400.2 \\
1.1 \\
186.3 \\
R_{196.2} \\
140.5 \\
16.8 \\
R_{343.7} \\
4,011.5\end{array}$ & $\begin{array}{r}\mathrm{R}_{7,876.7} \\
\mathrm{P}_{3,777.2} \\
118.8 \\
- \\
118.8 \\
1,981.9 \\
\mathrm{P}_{1,676.5} \\
246.7 \\
\mathrm{P}_{350.9} \\
.8 \\
272.6 \\
\text { P }_{231.5} \\
159.3 \\
\mathrm{R}_{18.4} \\
\mathrm{P}_{396.5} \\
4,099.5\end{array}$ & $\begin{array}{r}7,094.7 \\
3,209.5 \\
99.6 \\
- \\
99.6 \\
1,704.7 \\
1,405.2 \\
214.7 \\
290.1 \\
1.6 \\
165.8 \\
229.7 \\
130.7 \\
13.4 \\
359.3 \\
3,885.2\end{array}$ \\
\hline 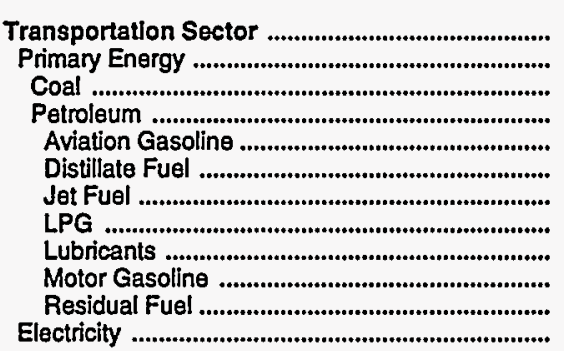 & $\begin{array}{r}3,750.3 \\
3,749.8 \\
.1 \\
3,749.7 \\
23.9 \\
243.7 \\
242.7 \\
1.6 \\
75.7 \\
3,098.8 \\
63.3 \\
.5\end{array}$ & $\begin{array}{r}7,763.2 \\
7,759.2 \\
(s) \\
7,759.2 \\
28.5 \\
573.4 \\
714.6 \\
4.0 \\
108.3 \\
6,062.6 \\
267.8 \\
4.0\end{array}$ & $\begin{array}{r}19,290.4 \\
19,282.8 \\
- \\
19,282.8 \\
13.0 \\
1,720.6 \\
2,166.4 \\
10.0 \\
244.2 \\
13,392.0 \\
1,736.6 \\
7.6\end{array}$ & $\begin{array}{r}18,015.7 \\
18,000.4 \\
18,000.4 \\
68.3 \\
2,017.9 \\
2,257.8 \\
42.6 \\
272.5 \\
11,972.3 \\
1,369.0 \\
15.2\end{array}$ & $\begin{array}{r}\mathrm{R}_{20,936.4} \\
\mathrm{R}_{20,924.0} \\
\mathrm{R}_{20,924.0} \\
52.0 \\
2,792.5 \\
3,081.3 \\
30.5 \\
\mathrm{R}_{254.2} \\
13,471.6 \\
1,241.9 \\
12.4\end{array}$ & $\begin{array}{r}R_{18,720.5} \\
R_{18,705.6} \\
- \\
R_{18,705.6} \\
48.0 \\
2,631.2 \\
2,438.9 \\
25.5 \\
R_{261.6} \\
12,618.6 \\
681.8 \\
14.9\end{array}$ & $\begin{array}{r}R_{20,574.0} \\
R_{20,555.8} \\
- \\
R_{20,555.8} \\
45.7 \\
2,569.2 \\
2,219.3 \\
21.9 \\
R_{308.6} \\
15,017.9 \\
R_{373.3} \\
R_{18.2}\end{array}$ & $\begin{array}{r}20,448.0 \\
20,432.0 \\
- \\
20,432.0 \\
34.1 \\
2,387.2 \\
2,273.1 \\
21.8 \\
306.3 \\
14,996.7 \\
412.8 \\
16.0\end{array}$ \\
\hline 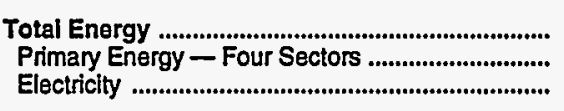 & $\begin{array}{l}6,977.0 \\
5,090.3 \\
1,886.7\end{array}$ & $\begin{array}{r}14,951.5 \\
10,622.9 \\
4,328.6\end{array}$ & $\begin{array}{r}36,079.3 \\
26,519.2 \\
9,560.0\end{array}$ & $\begin{array}{l}41,437.4 \\
27,294.0 \\
14,143.4\end{array}$ & $\begin{array}{r}\mathrm{F}_{48,381.5} \\
\mathrm{~F}_{29,963.6} \\
18,417.8\end{array}$ & $\begin{array}{r}R_{47,345.0} \\
\mathrm{R}_{27,932.5} \\
19,412.5\end{array}$ & $\begin{array}{r}\mathrm{R}_{4} 49,456.6 \\
\mathrm{R}_{29,079.1} \\
20,377.5\end{array}$ & $\begin{array}{l}48,815.8 \\
28,650.6 \\
20,165.2\end{array}$ \\
\hline 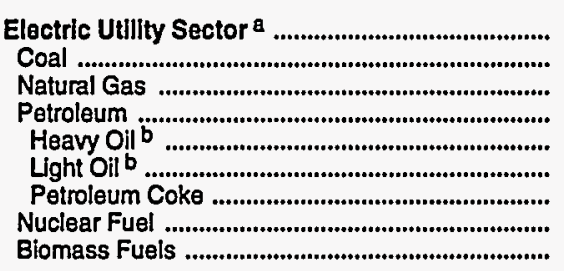 & $\begin{array}{r}282.1 \\
- \\
220.1 \\
54.9 \\
54.7 \\
.2 \\
-\overline{6} \\
.3\end{array}$ & $\begin{array}{r}1,553.7 \\
-\overline{3} \\
305.2 \\
1,234.0 \\
1,230.5 \\
3.4 \\
\overline{-4} \\
14.4 \\
.2\end{array}$ & $\begin{array}{r}4,018.4 \\
1,925.0 \\
2,067.0 \\
1,980.8 \\
86.2 \\
- \\
26.1 \\
.4\end{array}$ & $\begin{array}{r}3,498.6 \\
- \\
3,130.1 \\
164.4 \\
154.2 \\
10.2 \\
\overline{-} \\
204.0 \\
\text { (s) }\end{array}$ & $\begin{array}{r}1,882.4 \\
\overline{-} \\
1,429.0 \\
201.4 \\
196.4 \\
5.0 \\
\overline{251.9} \\
(\mathrm{~d})\end{array}$ & $\begin{array}{r}1,572.2 \\
\overline{1} \\
1,324.3 \\
20.9 \\
18.0 \\
3.0 \\
\overline{2} \\
226.9\end{array}$ & $\begin{array}{r}1,802.4 \\
-\overline{-} \\
1,584.8 \\
9.9 \\
6.6 \\
3.3 \\
-\overline{0} \\
207.7\end{array}$ & $\begin{array}{r}1,620.3 \\
-\overline{-} \\
1,422.4 \\
50.2 \\
46.8 \\
3.4 \\
\overline{1} \\
147.8\end{array}$ \\
\hline Primary Energy — Five Sectors ${ }^{c}$............................... & $5,372.4$ & $12,176.6$ & $30,537.6$ & $30,792.6$ & P $31,846.0^{-0}$ & ${ }_{2} 29,504.7$ & ${ }^{\mathrm{A}} 30,881.5$ & $30,270.9$ \\
\hline
\end{tabular}

a There are no direct fuel costs for hydroelectric, geothermal, wind, photovoltaic, or solar thermal energy.

b Heavy oil includes fuel oil nos. 4, 5, and 6, and residual fuel oils. Light oil includes fuel nos. 1 and 2 , kerosene, and jet fuel.

c Blomass fuels are not included, except those consumed at electric utilities and those added to motor gasoline.

Utilities used blomass fuels at no charge or recelved a fee for accepting them.
$R=$ Revised data

-No consumption, including cases where adjustments were made. See explanation of adjustments in Section 6 of Appendix A.

(s) =Value less than 0.05 million dollars.

Note: Totals may not equal sum of components due to independent rounding.

Sources: Data sources, estimation procedures, and assumptions are described in Appendix A. 


\begin{tabular}{|c|c|c|c|c|c|c|c|c|}
\hline Energy Source & 1970 & 1975 & 1880 & 1985 & 1990 & 1991 & 1992 & 1993 \\
\hline & \multicolumn{8}{|c|}{ Prices In Dollare per Million Btu } \\
\hline 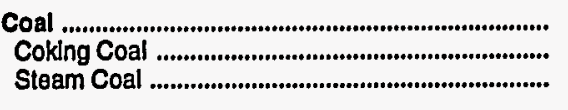 & $\begin{array}{r}0.33 \\
.43 \\
.30\end{array}$ & $\begin{array}{r}0.68 \\
1.38 \\
.53\end{array}$ & $\begin{array}{r}1.00 \\
1.97 \\
.89\end{array}$ & 1.17 & 1.07 & 1.10 & 1.11 & 1.11 \\
\hline 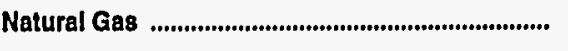 & .48 & .98 & 2.98 & 4.71 & 3.92 & 3.74 & 3.68 & 3.75 \\
\hline 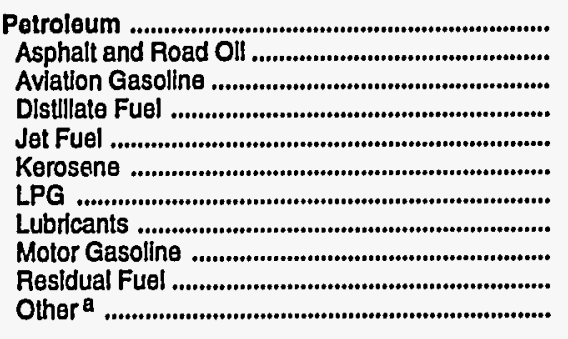 & $\begin{array}{r}1.88 \\
.58 \\
2.17 \\
1.04 \\
.76 \\
.98 \\
1.60 \\
5.08 \\
2.72 \\
.44 \\
1.09\end{array}$ & $\begin{array}{l}3.54 \\
2.01 \\
3.45 \\
2.30 \\
2.12 \\
2.22 \\
3.02 \\
7.48 \\
4.67 \\
1.59 \\
1.98\end{array}$ & $\begin{array}{r}7.93 \\
3.40 \\
9.02 \\
6.45 \\
6.59 \\
5.78 \\
5.88 \\
14.36 \\
9.36 \\
3.88 \\
7.39\end{array}$ & $\begin{array}{r}8.00 \\
4.73 \\
9.99 \\
6.26 \\
5.94 \\
7.69 \\
6.39 \\
17.61 \\
9.28 \\
3.80 \\
6.78\end{array}$ & $\begin{array}{r}R_{8.08} \\
2.59 \\
9.32 \\
7.70 \\
5.59 \\
6.99 \\
6.55 \\
R_{14.60} \\
9.29 \\
2.64 \\
3.98\end{array}$ & $\begin{array}{r}\boldsymbol{R}_{7.09} \\
3.24 \\
8.71 \\
7.33 \\
4.87 \\
7.06 \\
7.91 \\
\text { A } 16.79 \\
9.23 \\
3.86 \\
3.63\end{array}$ & $\begin{array}{r}R_{7.95} \\
2.89 \\
8.54 \\
6.81 \\
4.47 \\
6.02 \\
8.03 \\
R 19.43 \\
9.56 \\
3.22 \\
3.12\end{array}$ & $\begin{array}{r}8.07 \\
2.87 \\
8.24 \\
7.21 \\
4.25 \\
5.85 \\
6.94 \\
18.94 \\
9.87 \\
2.04 \\
3.01\end{array}$ \\
\hline 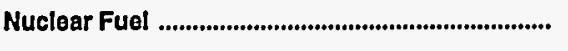 & - & - & 21 & - & - & - & - & - \\
\hline Blomass Fuels at Utilities ....................................... & - & - & - & .79 & .80 & .80 & - & - \\
\hline Primary Energy - Five Sectors b ....................... & 1.03 & 2.02 & 4.33 & 4.72 & A 4.38 & $R_{4.38}$ & $R_{4.36}$ & 4.47 \\
\hline $\begin{array}{l}\text { Electric Utllity Fuel c ................................................ } \\
\text { Electriclty Purchased by End Users ..................... }\end{array}$ & $\begin{array}{r}25 \\
6.09\end{array}$ & $\begin{array}{r}.60 \\
7.95\end{array}$ & $\begin{array}{r}1.12 \\
12.94\end{array}$ & $\begin{array}{r}1.21 \\
17.88\end{array}$ & $\begin{array}{r}1.08 \\
17.31\end{array}$ & $\begin{array}{r}1.11 \\
17.49\end{array}$ & $\begin{array}{r}1.11 \\
17.69\end{array}$ & $\begin{array}{r}1.12 \\
17.78\end{array}$ \\
\hline \multirow[t]{2}{*}{ 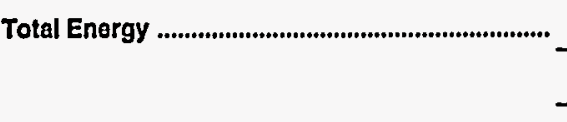 } & 1.52 & 2.92 & 6.49 & 8.20 & 8.09 & $\mathbf{R}_{7.94}$ & 7.98 & 7.96 \\
\hline & \multicolumn{8}{|c|}{ Expenditures in Militons of Dollars } \\
\hline 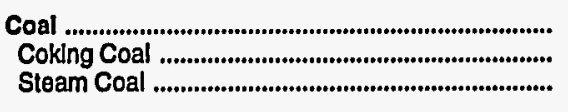 & $\begin{array}{l}38.3 \\
12.0 \\
26.3\end{array}$ & $\begin{array}{r}108.4 \\
39.5 \\
69.0\end{array}$ & $\begin{array}{r}248.2 \\
50.2 \\
197.9\end{array}$ & $\begin{array}{c}349.9 \\
- \\
349.9\end{array}$ & $\begin{array}{c}353.3 \\
- \\
353.3\end{array}$ & $\begin{array}{c}355.4 \\
- \\
355.4\end{array}$ & $\begin{array}{c}369.0 \\
\overrightarrow{369.0}\end{array}$ & $\begin{array}{c}374.7 \\
- \\
374.7\end{array}$ \\
\hline 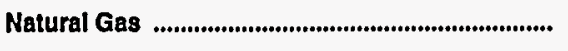 & 128.2 & 262.9 & 706.8 & 931.2 & 820.8 & 854.2 & 823.3 & 927.8 \\
\hline 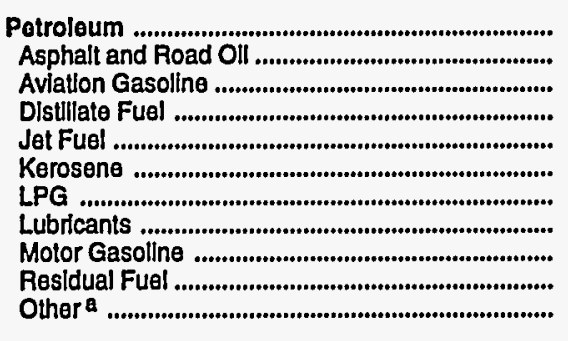 & $\begin{array}{r}503.5 \\
12.3 \\
3.7 \\
30.9 \\
32.0 \\
4.5 \\
27.5 \\
13.0 \\
372.5 \\
3.9 \\
3.1\end{array}$ & $\begin{array}{r}1,139.9 \\
29.8 \\
4.6 \\
118.1 \\
85.7 \\
3.5 \\
55.7 \\
20.8 \\
782.3 \\
32.7 \\
6.7\end{array}$ & $\begin{array}{r}2,577.0 \\
51.6 \\
12.1 \\
422.1 \\
175.9 \\
13.5 \\
83.3 \\
55.8 \\
1,685.6 \\
43.6 \\
33.5\end{array}$ & $\begin{array}{r}2,598.7 \\
97.5 \\
7.1 \\
348.2 \\
264.1 \\
4.0 \\
51.3 \\
62.3 \\
1,742.4 \\
3.7 \\
18.0\end{array}$ & $\begin{array}{r}A_{2,584.3} \\
55.9 \\
7.8 \\
465.0 \\
193.0 \\
2.0 \\
70.5 \\
A_{58.1} \\
1,725.8 \\
(8) \\
6.2\end{array}$ & $\begin{array}{r}A_{2,651.4} \\
66.8 \\
6.8 \\
504.2 \\
179.5 \\
2.0 \\
96.1 \\
P_{59.8} \\
1,730.1 \\
1.1 \\
5.0\end{array}$ & $\begin{array}{r}A_{2,711.2} \\
61.1 \\
5.9 \\
492.6 \\
186.0 \\
1.7 \\
88.7 \\
R 70.5 \\
1,798.2 \\
.7 \\
5.6\end{array}$ & $\begin{array}{r}2,955.3 \\
65.1 \\
5.1 \\
542.6 \\
215.6 \\
1.8 \\
85.5 \\
70.0 \\
1,964.4 \\
.1 \\
5.2\end{array}$ \\
\hline Nuclear Fuel ........................................................ & - & - & 1.5 & - & - & - & - & $\infty$ \\
\hline Blomass Fuels at Uttlltles ....................................... & - & - & - & (8) & (a) & (a) & - & - \\
\hline Primary Enorgy - Flve Sectors b .......................... & 670.0 & 1,5112 & $3,533.4$ & $3,879.8$ & $A_{3,758.4}$ & $\mathrm{~A}_{3,861.0}$ & $\mathrm{~A}_{3,903.5}$ & $4,257.8$ \\
\hline 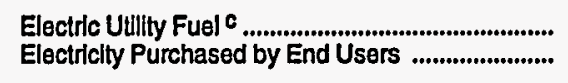 & $\begin{array}{r}-30.6 \\
222.3\end{array}$ & $\begin{array}{r}-105.4 \\
426.0\end{array}$ & $\begin{array}{r}-272.5 \\
918.2\end{array}$ & $\begin{array}{r}-342.6 \\
1,608.3\end{array}$ & $\begin{array}{r}-344.7 \\
1,800.4\end{array}$ & $\begin{array}{r}-345.8 \\
1,858.4\end{array}$ & $\begin{array}{r}-357.3 \\
1,902.3\end{array}$ & $\begin{array}{r}364.0 \\
1,978.7\end{array}$ \\
\hline 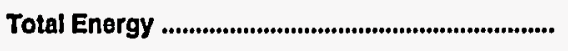 & 861.7 & $1,831.8$ & $4,179.0$ & $5,145.4$ & $A_{5,214.0}$ & ${ }^{n_{5,373.6}}$ & ${ }^{A_{5,448.5}}$ & $5,872.4$ \\
\hline
\end{tabular}

a Includes petroleum coke used at electric utilities.

b Blomass fuels are not included, except those consumed at electric utilities and those added to motor gasoline.

c There are no direct fuel costs for hydroelectric, geothermal, wind, photovoltalc, or solar thermal energy.

$R=$ Revised data.

- No consumption, including cases where adjustments were made. See explanation of adjustments in Section 6 of Appendix A.

(s) =Value less than 0.05 million dollars.

Note: Expenditure totals may not equal sum of components due to independent rounding.

Sources: Data sources, estimation procodures, and assumptions are described in Appendix A. 
C Energy Price Estimates by Sector, Colorado

1970, 1975, 1980, 1985, and 1990-1993

(Dollars per Million Btu)

\begin{tabular}{|c|c|c|c|c|c|c|c|c|}
\hline Sector and Energy Source & 1970 & 1975 & 1980 & 1985 & 1990 & 1991 & 1992 & 1993 \\
\hline 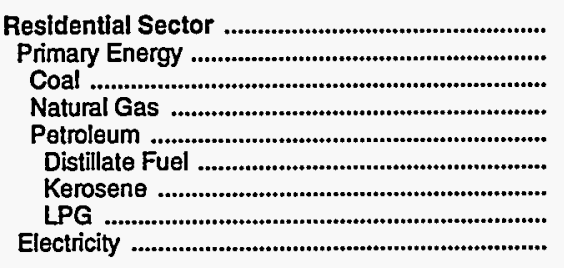 & $\begin{array}{r}1.71 \\
.88 \\
.90 \\
.74 \\
1.74 \\
1.28 \\
1.51 \\
1.79 \\
7.73\end{array}$ & $\begin{array}{l}2.76 \\
1.53 \\
1.58 \\
1.29 \\
3.26 \\
2.84 \\
2.96 \\
3.33 \\
9.94\end{array}$ & $\begin{array}{r}5.72 \\
3.53 \\
2.54 \\
3.26 \\
7.31 \\
6.96 \\
7.98 \\
7.32 \\
15.00\end{array}$ & $\begin{array}{r}8.76 \\
5.18 \\
2.83 \\
5.11 \\
6.71 \\
6.91 \\
9.20 \\
6.55 \\
20.28\end{array}$ & $\begin{array}{r}8.69 \\
4.70 \\
2.41 \\
4.55 \\
7.02 \\
6.19 \\
8.24 \\
7.02 \\
20.57\end{array}$ & $\begin{array}{r}8.53 \\
4.64 \\
2.36 \\
4.46 \\
7.30 \\
5.90 \\
7.85 \\
7.32 \\
20.72\end{array}$ & $\begin{array}{r}8.78 \\
4.64 \\
2.43 \\
4.46 \\
7.56 \\
4.82 \\
6.42 \\
7.66 \\
21.11\end{array}$ & $\begin{array}{r}8.58 \\
4.56 \\
2.16 \\
4.47 \\
6.10 \\
4.75 \\
6.32 \\
6.13 \\
21.21\end{array}$ \\
\hline 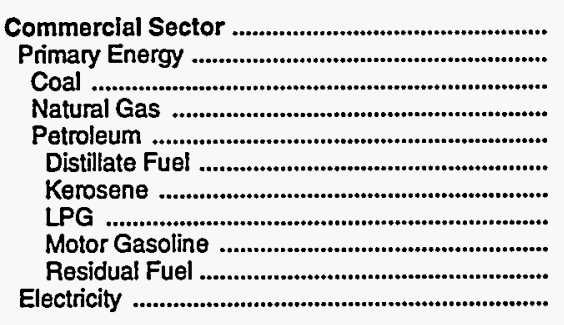 & $\begin{array}{r}1.66 \\
.62 \\
.39 \\
.59 \\
1.28 \\
1.06 \\
.89 \\
1.18 \\
2.72 \\
.38 \\
5.97\end{array}$ & $\begin{array}{r}2.73 \\
1.20 \\
.81 \\
1.10 \\
2.73 \\
2.49 \\
2.11 \\
2.59 \\
4.67 \\
1.93 \\
7.95\end{array}$ & $\begin{array}{r}6.09 \\
3.26 \\
1.20 \\
3.03 \\
7.08 \\
6.48 \\
5.65 \\
4.79 \\
9.36 \\
4.35 \\
14.37\end{array}$ & $\begin{array}{r}9.48 \\
4.64 \\
1.31 \\
4.61 \\
6.16 \\
5.45 \\
5.98 \\
6.12 \\
9.28 \\
4.07 \\
18.34\end{array}$ & $\begin{array}{l}9.27 \\
4.11 \\
1.29 \\
3.97 \\
6.36 \\
4.95 \\
5.98 \\
5.93 \\
9.29 \\
-16.89\end{array}$ & $\begin{array}{l}9.15 \\
4.11 \\
1.39 \\
3.92 \\
6.48 \\
4.30 \\
6.35 \\
8.68 \\
9.23 \\
17.07\end{array}$ & $\begin{array}{r}9.29 \\
4.02 \\
1.51 \\
3.91 \\
5.43 \\
4.04 \\
4.98 \\
8.50 \\
9.56 \\
1.75 \\
17.20\end{array}$ & $\begin{array}{r}9.36 \\
4.06 \\
1.37 \\
4.00 \\
5.08 \\
4.19 \\
4.99 \\
7.82 \\
9.87 \\
2.04 \\
17.36\end{array}$ \\
\hline 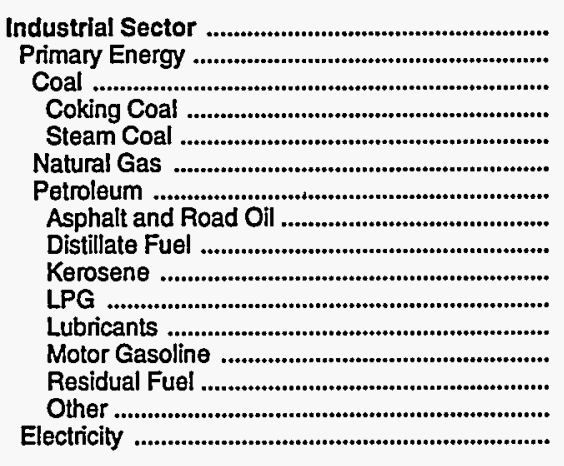 & $\begin{array}{r}.65 \\
.53 \\
.42 \\
.43 \\
.39 \\
.29 \\
.97 \\
.58 \\
.83 \\
.89 \\
1.18 \\
5.08 \\
2.72 \\
.47 \\
1.09 \\
3.50\end{array}$ & $\begin{array}{r}1.74 \\
1.40 \\
1.17 \\
1.38 \\
.81 \\
.72 \\
2.19 \\
2.01 \\
1.96 \\
2.11 \\
2.59 \\
7.48 \\
4.67 \\
1.43 \\
1.98 \\
5.55\end{array}$ & $\begin{array}{r}4.20 \\
3.44 \\
1.66 \\
1.97 \\
1.20 \\
2.65 \\
5.17 \\
3.40 \\
5.33 \\
5.65 \\
4.79 \\
14.36 \\
9.36 \\
3.82 \\
.7 .39 \\
9.40\end{array}$ & $\begin{array}{r}5.65 \\
4.34 \\
1.31 \\
-\overline{-} \\
1.31 \\
4.01 \\
5.80 \\
4.73 \\
5.25 \\
5.98 \\
6.12 \\
17.61 \\
9.28 \\
4.07 \\
6.78 \\
12.67\end{array}$ & $\begin{array}{r}R_{4} .95 \\
R_{3.30} \\
1.29 \\
\overline{-} \\
1.29 \\
2.77 \\
R_{4.49} \\
2.59 \\
5.26 \\
5.98 \\
5.93 \\
R_{14.60} \\
9.29 \\
2.46 \\
3.98 \\
13.17\end{array}$ & $\begin{array}{r}\mathrm{R}_{5.02} \\
\mathrm{R}_{3.45} \\
1.39 \\
1 . \overline{39} \\
1.29 \\
2.27 \\
\mathrm{R}_{5.29} \\
3.24 \\
5.58 \\
6.35 \\
8.68 \\
\mathrm{R}_{16.79} \\
9.23 \\
2.26 \\
3.63 \\
13.38\end{array}$ & $\begin{array}{r}R_{4.75} \\
\mathrm{R}_{3.19} \\
1.51 \\
-\overline{1.51} \\
1.15 \\
\mathrm{R} 4.64 \\
2.89 \\
4.38 \\
4.98 \\
8.50 \\
\mathrm{R}_{19.43} \\
9.56 \\
1.75 \\
3.12 \\
13.44\end{array}$ & $\begin{array}{r}4.62 \\
3.14 \\
1.37 \\
- \\
1.37 \\
2.32 \\
4.63 \\
2.87 \\
4.39 \\
4.99 \\
7.82 \\
18.94 \\
9.87 \\
2.04 \\
3.01 \\
13.27\end{array}$ \\
\hline 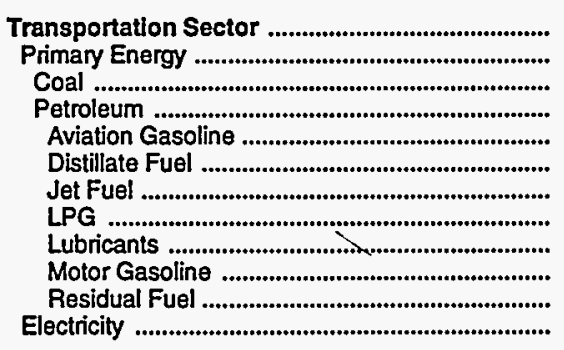 & $\begin{array}{r}2.17 \\
2.17 \\
.39 \\
2.17 \\
2.17 \\
1.20 \\
.76 \\
1.18 \\
5.08 \\
2.72 \\
.38 \\
-\end{array}$ & $\begin{array}{r}3.99 \\
3.99 \\
.81 \\
3.99 \\
3.45 \\
2.49 \\
2.12 \\
2.59 \\
7.48 \\
4.67 \\
1.86 \\
-\end{array}$ & $\begin{array}{r}8.75 \\
8.75 \\
- \\
8.75 \\
9.02 \\
7.13 \\
6.59 \\
4.79 \\
14.36 \\
9.36 \\
- \\
-\end{array}$ & $\begin{array}{r}8.43 \\
8.43 \\
-\overline{8} \\
8.43 \\
9.99 \\
6.70 \\
5.94 \\
6.12 \\
17.61 \\
9.28 \\
3.79 \\
-\end{array}$ & $\begin{array}{r}R_{8.77} \\
R_{8.77} \\
- \\
R 8.77 \\
9.32 \\
8.80 \\
5.59 \\
5.93 \\
R_{14.60} \\
9.29 \\
- \\
-\end{array}$ & $\begin{array}{r}\mathrm{R}_{8.56} \\
\mathrm{R}_{8.56} \\
\mathrm{R}_{8.56} \\
8.71 \\
8.39 \\
4.87 \\
8.68 \\
\mathrm{R}_{16.79} \\
9.23 \\
- \\
-\end{array}$ & $\begin{array}{r}R_{8.71} \\
R_{8.71} \\
- \\
R_{8.71} \\
8.54 \\
8.62 \\
4.47 \\
8.50 \\
R_{19.43} \\
9.56 \\
- \\
-\end{array}$ & $\begin{array}{r}8.79 \\
8.79 \\
- \\
8.79 \\
8.24 \\
8.70 \\
4.25 \\
7.82 \\
18.94 \\
9.87 \\
-\end{array}$ \\
\hline 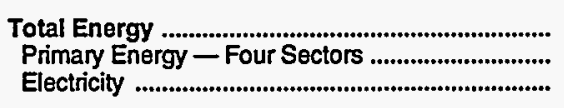 & $\begin{array}{l}1.52 \\
1.20 \\
6.09\end{array}$ & $\begin{array}{l}2.92 \\
2.45 \\
7.95\end{array}$ & $\begin{array}{r}6.49 \\
5.69 \\
12.94\end{array}$ & $\begin{array}{r}8.20 \\
6.58 \\
17.88\end{array}$ & $\begin{array}{r}8.09 \\
6.32 \\
17.31\end{array}$ & $\begin{array}{l}{ }_{1}^{R} 7.94 \\
R_{6.16} \\
17.49\end{array}$ & $\begin{array}{r}7.98 \\
\text { ค } 6.17 \\
17.69\end{array}$ & $\begin{array}{r}7.96 \\
6.22 \\
17.78\end{array}$ \\
\hline 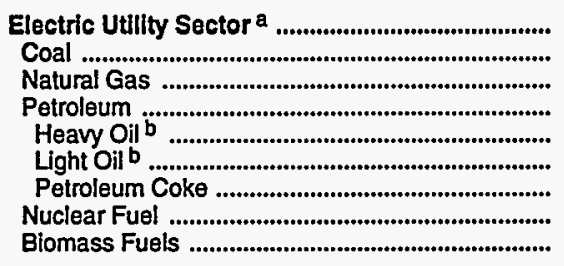 & $\begin{array}{l}.25 \\
.26 \\
.24 \\
.37 \\
.36 \\
.45 \\
- \\
-\end{array}$ & $\begin{array}{r}.60 \\
.48 \\
.59 \\
2.18 \\
1.94 \\
2.56 \\
- \\
- \\
-\end{array}$ & $\begin{array}{r}1.12 \\
.86 \\
2.64 \\
5.65 \\
4.38 \\
6.50 \\
. \overline{21} \\
-\end{array}$ & $\begin{array}{r}1.21 \\
1.15 \\
3.53 \\
5.79 \\
4.00 \\
5.92 \\
= \\
.79\end{array}$ & $\begin{array}{r}1.08 \\
1.06 \\
2.17 \\
5.34 \\
3.09 \\
5.35 \\
- \\
.80\end{array}$ & $\begin{array}{l}1.11 \\
1.09 \\
2.15 \\
4.39 \\
3.86 \\
5.13 \\
\overline{-} \\
.80\end{array}$ & $\begin{array}{l}1.11 \\
1.09 \\
2.14 \\
4.08 \\
3.23 \\
4.79 \\
= \\
=\end{array}$ & $\begin{array}{r}1.12 \\
1.09 \\
2.50 \\
4.81 \\
4 . \overline{-} \\
\overline{-} \\
\overline{-}\end{array}$ \\
\hline Primary Energy — Five Sectors ${ }^{c} \ldots \ldots . . . . . . . . . . . . . . . . . . .$. & 1.03 & 2.02 & 4.33 & 4.72 & $R_{4.38}$ & $R_{4.38}$ & $\mathbf{R}_{4.36}$ & 4.47 \\
\hline
\end{tabular}

a There are no direct fuel costs for hydroelectric, geothermal, wind, photovoltaic, or solar thermal energy.

b Heavy oil includes fuel oil nos. 4, 5, and 6, and residual fuel oils. Light

R=Revised data.

-No consumption, including cases where adjustments were made. See explanation of adjustments in Section 6 of Appendix A.

Sources: Data sources, estimation procedures, and assumptions are described in Appendix A.

oil includes fuel oil nos. 1 and 2 , kerosene, and jet fuel.

Biomass fuels are not included, except those consumed at electric utilities and those added to motor gasoline. 
Energy Expenditure Estimates by Sector, Colorado 1970, 1975, 1980, 1985, and 1990-1993

(Million Dollars)

\begin{tabular}{|c|c|c|c|c|c|c|c|c|}
\hline Sector and Energy Source & 1970 & 1975 & 1980 & 1985 & 1990 & 1991 & 1992 & 1993 \\
\hline $\begin{array}{l}\text { Residential Sector } \\
\text { Primary Energy } \\
\text { Coal } \\
\text { Natural Gas } \\
\text { Petroleum } \\
\text { Distlllate Fuel } \\
\text { Kerosene } \\
\text { LPG }\end{array}$ & $\begin{array}{r}185.9 \\
84.1 \\
1.6 \\
59.4 \\
23.1 \\
1.3 \\
1.0 \\
20.9 \\
101.8\end{array}$ & $\begin{array}{r}330.9 \\
156.5 \\
.2 \\
115.6 \\
40.7 \\
4.7 \\
.6 \\
35.4 \\
174.4\end{array}$ & $\begin{array}{r}684.1 \\
341.6 \\
1.9 \\
290.6 \\
49.1 \\
3.2 \\
1.0 \\
44.9 \\
342.5\end{array}$ & $\begin{array}{r}1,116.1 \\
502.9 \\
3.3 \\
459.9 \\
39.6 \\
4.3 \\
2.6 \\
32.8 \\
613.3\end{array}$ & $\begin{array}{r}1,153.3 \\
466.3 \\
1.0 \\
420.1 \\
45.2 \\
1.0 \\
1.0 \\
43.2 \\
687.0\end{array}$ & $\begin{array}{r}1,214.7 \\
500.7 \\
1.2 \\
447.2 \\
52.3 \\
.9 \\
1.1 \\
50.3 \\
714.0\end{array}$ & $\begin{array}{r}1,217.4 \\
481.4 \\
1.0 \\
431.4 \\
48.9 \\
.6 \\
1.3 \\
47.0 \\
736.0\end{array}$ & $\begin{array}{r}1,292.8 \\
521.8 \\
.6 \\
480.0 \\
41.2 \\
.9 \\
1.2 \\
39.1 \\
71.0\end{array}$ \\
\hline 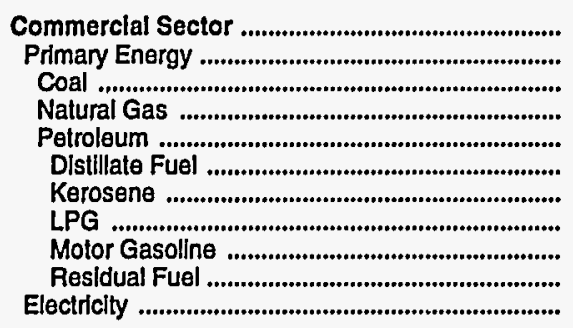 & $\begin{array}{r}134.4 \\
40.9 \\
1.3 \\
33.7 \\
5.9 \\
.9 \\
.7 \\
2.4 \\
1.8 \\
.1 \\
93.5\end{array}$ & $\begin{array}{r}258.5 \\
88.2 \\
.2 \\
75.5 \\
12.4 \\
3.4 \\
.6 \\
4.9 \\
2.7 \\
.9 \\
170.3\end{array}$ & $\begin{array}{r}594.0 \\
237.1 \\
1.7 \\
201.9 \\
33.6 \\
12.8 \\
.2 \\
5.2 \\
15.4 \\
.1 \\
356.8\end{array}$ & $\begin{array}{r}1,129.0 \\
356.8 \\
2.8 \\
317.8 \\
36.2 \\
21.6 \\
.5 \\
5.4 \\
8.6 \\
(s) \\
772.2\end{array}$ & $\begin{array}{r}1,128.8 \\
297.8 \\
1.0 \\
264.5 \\
32.2 \\
12.6 \\
.3 \\
6.4 \\
12.9 \\
-\overline{8} \\
831.0\end{array}$ & $\begin{array}{r}1,172.8 \\
321.8 \\
1.3 \\
278.6 \\
42.0 \\
14.8 \\
.4 \\
10.5 \\
16.3 \\
\overline{-1} \\
850.9\end{array}$ & $\begin{array}{r}1,170.0 \\
304.1 \\
1.2 \\
265.8 \\
37.1 \\
19.6 \\
.2 \\
9.2 \\
8.1 \\
(\mathrm{~s}) \\
866.0\end{array}$ & $\begin{array}{r}1,224.6 \\
319.6 \\
.7 \\
289.5 \\
29.3 \\
18.5 \\
.2 \\
8.8 \\
1.8 \\
(\mathrm{~s}) \\
905.0\end{array}$ \\
\hline 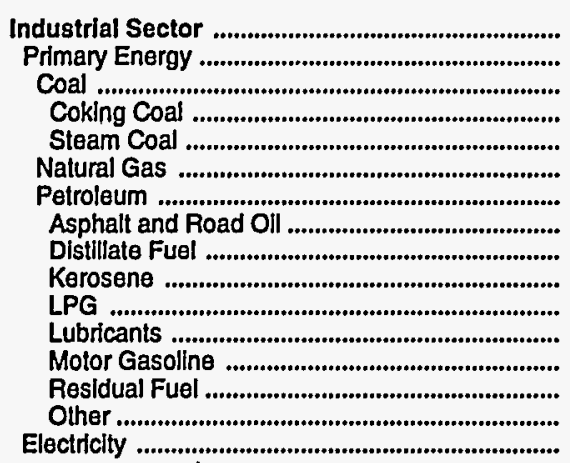 & $\begin{array}{r}121.5 \\
94.6 \\
17.4 \\
12.0 \\
5.4 \\
23.1 \\
54.1 \\
12.3 \\
10.1 \\
2.8 \\
3.6 \\
4.2 \\
14.8 \\
3.0 \\
3.1 \\
26.9\end{array}$ & $\begin{array}{r}314.6 \\
233.2 \\
53.4 \\
39.5 \\
14.0 \\
40.9 \\
138.9 \\
29.8 \\
38.6 \\
2.3 \\
13.6 \\
7.1 \\
21.1 \\
19.8 \\
6.7 \\
81.3\end{array}$ & $\begin{array}{r}769.0 \\
550.2 \\
71.3 \\
50.2 \\
21.1 \\
131.6 \\
347.2 \\
51.6 \\
123.7 \\
12.3 \\
32.4 \\
20.8 \\
34.2 \\
38.8 \\
33.5 \\
218.8\end{array}$ & $\begin{array}{r}631.1 \\
408.4 \\
22.4 \\
-\overline{1} \\
22.4 \\
136.3 \\
249.6 \\
97.5 \\
70.2 \\
.9 \\
11.6 \\
23.2 \\
28.3 \\
(s) \\
18.0 \\
222.7\end{array}$ & $\begin{array}{r}R_{632.1} \\
R_{349.8} \\
19.8 \\
-\overline{1} \\
19.8 \\
124.4 \\
R_{205.6} \\
55.9 \\
82.2 \\
.6 \\
19.2 \\
R_{21.6} \\
19.8 \\
(s) \\
6.2 \\
282.3\end{array}$ & $\begin{array}{r}R_{697.6} \\
R_{404.2} \\
21.6 \\
- \\
21.6 \\
116.1 \\
R_{266.5} \\
66.8 \\
114.8 \\
.6 \\
32.7 \\
R 22.2 \\
24.4 \\
(\mathrm{~s}) \\
5.0 \\
293.4\end{array}$ & $\begin{array}{r}R_{697.3} \\
R_{397.0} \\
22.3 \\
- \\
22.3 \\
115.3 \\
R_{259.3} \\
61.1 \\
110.9 \\
.2 \\
30.5 \\
R 26.2 \\
24.8 \\
(5) \\
5.6 \\
300.4\end{array}$ & $\begin{array}{r}721.9 \\
419.2 \\
22.3 \\
- \\
22.3 \\
146.0 \\
250.8 \\
65.1 \\
92.7 \\
.3 \\
35.3 \\
26.0 \\
26.1 \\
.1 \\
5.2 \\
302.7\end{array}$ \\
\hline $\begin{array}{l}\text { Transportation Sector } \\
\text { Primary Energy } \\
\text { Coal } \\
\text { Petroleum } \\
\text { Avlatlon Gasoline } \\
\text { DIstlllate Fuel } \\
\text { Jet Fuel } \\
\text { LPG } \\
\text { Lubricants } \\
\text { Molor Gasollne } \\
\text { Residual Fuel }\end{array}$ & $\begin{array}{r}419.9 \\
419.9 \\
(\mathrm{~s}) \\
419.8 \\
3.7 \\
18.6 \\
32.0 \\
.6 \\
8.8 \\
356.0 \\
.2 \\
-\end{array}$ & $\begin{array}{r}927.9 \\
927.9 \\
(\mathrm{~s}) \\
927.9 \\
4.6 \\
62.3 \\
85.7 \\
1.8 \\
13.7 \\
758.5 \\
1.2 \\
-\end{array}$ & $\begin{array}{r}2,131.9 \\
2,131.9 \\
- \\
2,131.9 \\
12.1 \\
272.1 \\
175.9 \\
.8 \\
35.1 \\
1,636.1 \\
- \\
-\end{array}$ & $\begin{array}{r}2,269.2 \\
2,269.2 \\
- \\
2,269.2 \\
7.1 \\
248.2 \\
264.1 \\
1.5 \\
39.1 \\
1,705.6 \\
3.5 \\
-\end{array}$ & $\begin{array}{r}R_{2,299.8} \\
R_{2,299.8} \\
- \\
R_{2,299.8} \\
7.8 \\
367.7 \\
193.0 \\
1.6 \\
R_{36.5} \\
1,693.2 \\
- \\
-\end{array}$ & $\begin{array}{r}R_{2,288.5} \\
R_{2,288.5} \\
- \\
R_{2,288.5} \\
6.8 \\
372.6 \\
179.5 \\
2.6 \\
R_{37.6} \\
1,689.4 \\
- \\
-\end{array}$ & $\begin{array}{r}R_{2,363.8} \\
R_{2,363.8} \\
- \\
R_{2,363.8} \\
5.9 \\
360.2 \\
186.0 \\
2.1 \\
R_{44.3} \\
1,765.3 \\
- \\
-\end{array}$ & $\begin{array}{r}2,633.2 \\
2,633.2 \\
- \\
2,633.2 \\
5.1 \\
429.7 \\
215.6 \\
2.4 \\
44.0 \\
1,936.4 \\
- \\
-\end{array}$ \\
\hline 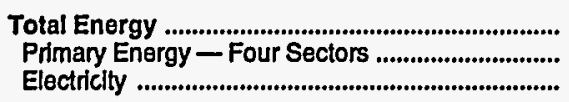 & $\begin{array}{l}861.7 \\
639.4 \\
222.3\end{array}$ & $\begin{array}{r}1,831.8 \\
1,405.8 \\
426.0\end{array}$ & $\begin{array}{r}4,179.0 \\
3,260.9 \\
918.2\end{array}$ & $\begin{array}{l}5,145.4 \\
3,537.1 \\
1,608.3\end{array}$ & $\begin{array}{r}R_{5,214.0} \\
R_{3,413.7} \\
1,800.4\end{array}$ & $\begin{array}{r}\mathrm{R}_{5,373.6} \\
\mathrm{R}_{3,515.2} \\
1,858.4\end{array}$ & $\begin{array}{r}\text { R }_{5,448.5} \\
\text { ค }_{3,546.2} \\
\mathbf{1}, 902.3\end{array}$ & $\begin{array}{l}5,872.4 \\
3,893.7 \\
1,978.7\end{array}$ \\
\hline 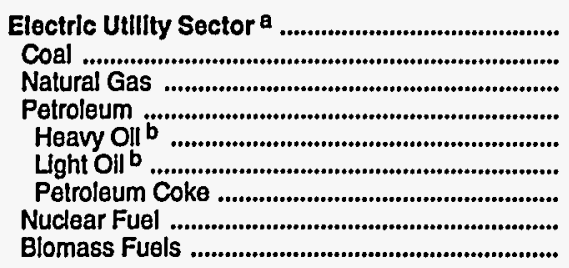 & $\begin{array}{r}30.6 \\
18.0 \\
12.0 \\
.6 \\
.6 \\
.1 \\
- \\
- \\
-\end{array}$ & $\begin{array}{r}105.4 \\
54.5 \\
30.9 \\
20.0 \\
10.8 \\
9.2 \\
- \\
=\end{array}$ & $\begin{array}{r}272.5 \\
173.3 \\
82.7 \\
15.1 \\
4.7 \\
10.3 \\
\overline{1.5} \\
-\end{array}$ & $\begin{array}{r}342.6 \\
321.3 \\
17.2 \\
4.1 \\
.2 \\
3.9 \\
- \\
-\overline{(s)}\end{array}$ & $\begin{array}{r}344.7 \\
331.4 \\
11.8 \\
1.6 \\
(s) \\
1.6 \\
- \\
-\end{array}$ & $\begin{array}{r}345.8 \\
331.4 \\
12.2 \\
2.2 \\
1.1 \\
1.1 \\
- \\
\overline{(s)}\end{array}$ & $\begin{array}{r}357.3 \\
344.5 \\
10.7 \\
2.1 \\
.7 \\
1.3 \\
- \\
- \\
-\end{array}$ & $\begin{array}{r}364.0 \\
351.0 \\
12.3 \\
.8 \\
\overline{.8} \\
\overline{-} \\
\overline{-}\end{array}$ \\
\hline Primary Energy - Five Sectors c ......................... & 670.0 & $1,511.2$ & 3,533.4 & $3,879.8$ & $R_{3,758.4}$ & $R_{3,861.0}$ & $A_{3,903.5}$ & $4,257.8$ \\
\hline
\end{tabular}

a There are no direct fuel costs for hydroelectric, geothermal, wind, photovoltalc, or solar themal energy.

b Heavy oil includes fuel oll nos. 4, 5, and 6, and residual fuel oils. Light oll includes fuel nos. 1 and 2, kerosene, and jet fuel.

c Blomass fuels are not included, except those consumed at electric utllitles and those added to motor gasoline.

$R=$ Revised data. $\rightarrow$ No consumption, Inciuding cases where adjustments were made. See explanation of adjustments in Section 6 of Appendix A.

$(s)=$ Value less than 0.05 million dollars.

Note: Totals may not equal sum of components due to independent rounding.

Sources: Data sources, estimation procedures, and assumptions are described in Appendix A. 
C Energy Price and Expenditure Estimates by Source, Connecticut

O 1970, 1975, 1980, 1985, and 1990-1993

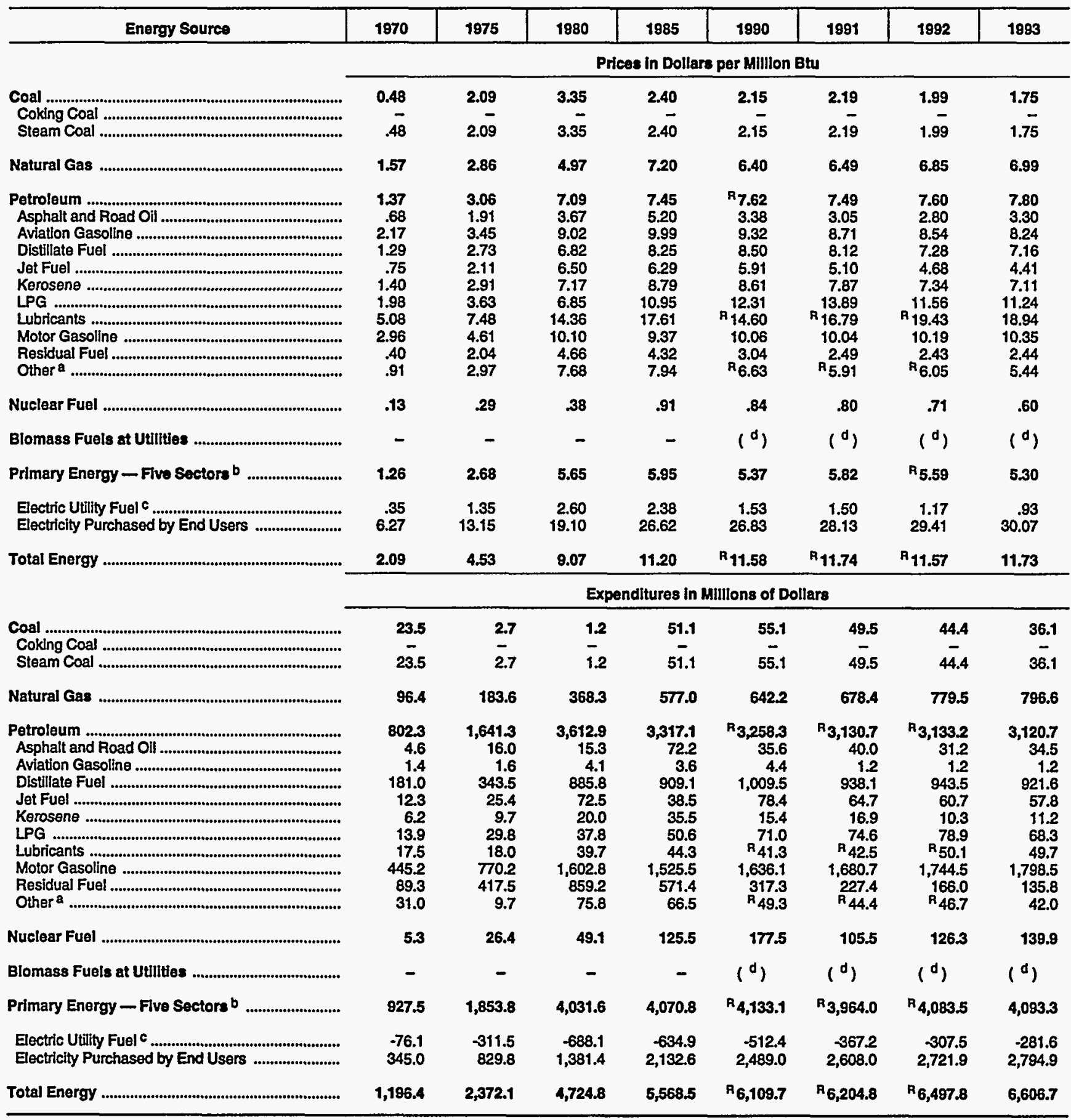

a Includes petroleum coke used at electric utilities.

b Blomass fuels are not Included, except those consumed at electric utilities and those added to motor gasoline.

c There are no direct fuel costs for hydroelectric, geothermal, wind, pholovoltaic, or solar thermal energy.

d Utilities used biomass fuels at no charge or recelved a fee for accepting

$R=$ Revised data

$\rightarrow$ No consumption, including cases where adjustments were made. See explanation of adjustments in Section 6 of Appendlx A.

Note: Expenditure totals may not equal sum of components due to independent rounding.

Sources: Data sources, estimation procedures, and assumptions are described in Appendix A. 
Energy Price Estimates by Sector, Connecticut

$1970,1975,1980,1985$, and 1990-1993

(Dollars per Million Btu)

\begin{tabular}{|c|c|c|c|c|c|c|c|c|}
\hline Sector and Energy Source & 1970 & 1975 & 1980 & 1985 & 1990 & 1991 & 1992 & 1993 \\
\hline $\begin{array}{l}\text { Residentlal Sector } \\
\text { Primary Energy } \\
\text { Coal } \\
\text { Natural Gas } \\
\text { Petroleum } \\
\text { Distillate Fuel } \\
\text { Kerosene } \\
\text { EPG }\end{array}$ & $\begin{array}{l}2.47 \\
1.62 \\
1.30 \\
1.88 \\
1.53 \\
1.48 \\
1.70 \\
2.70 \\
7.21\end{array}$ & $\begin{array}{r}5.14 \\
3.03 \\
2.62 \\
3.28 \\
2.93 \\
2.84 \\
3.16 \\
5.01 \\
14.49\end{array}$ & $\begin{array}{r}9.39 \\
6.73 \\
4.47 \\
5.72 \\
7.14 \\
7.07 \\
8.15 \\
9.21 \\
20.27\end{array}$ & $\begin{array}{r}13.42 \\
8.60 \\
4.39 \\
8.88 \\
8.48 \\
8.37 \\
8.98 \\
10.41 \\
29.24\end{array}$ & $\begin{array}{r}13.66 \\
8.61 \\
4.37 \\
8.31 \\
8.78 \\
8.55 \\
9.18 \\
13.60 \\
29.32\end{array}$ & $\begin{array}{r}14.06 \\
8.55 \\
4.08 \\
8.48 \\
8.61 \\
8.27 \\
8.88 \\
14.99 \\
30.79\end{array}$ & $\begin{array}{r}13.30 \\
7.92 \\
4.17 \\
8.72 \\
7.52 \\
7.24 \\
7.77 \\
12.43 \\
32.45\end{array}$ & $\begin{array}{r}13.48 \\
7.90 \\
3.96 \\
9.18 \\
7.25 \\
7.02 \\
7.54 \\
11.98 \\
33.38\end{array}$ \\
\hline 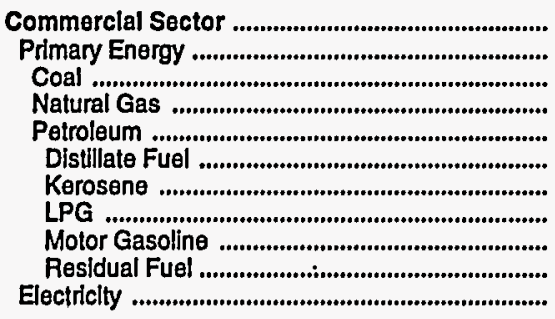 & $\begin{array}{r}2.60 \\
1.13 \\
.79 \\
1.45 \\
1.00 \\
1.09 \\
.79 \\
1.42 \\
2.96 \\
.42 \\
7.15\end{array}$ & $\begin{array}{r}5.93 \\
2.53 \\
2.00 \\
2.64 \\
2.47 \\
2.44 \\
2.67 \\
2.89 \\
4.61 \\
1.97 \\
13.70\end{array}$ & $\begin{array}{r}10.31 \\
5.43 \\
1.67 \\
4.67 \\
6.06 \\
6.37 \\
6.29 \\
5.31 \\
10.10 \\
4.59 \\
19.84\end{array}$ & $\begin{array}{r}13.55 \\
6.54 \\
2.38 \\
6.59 \\
6.54 \\
7.27 \\
7.70 \\
11.48 \\
9.37 \\
4.68 \\
27.30\end{array}$ & $\begin{array}{r}14.39 \\
6.12 \\
2.63 \\
6.10 \\
6.16 \\
6.87 \\
7.68 \\
10.80 \\
10.06 \\
3.25 \\
27.09\end{array}$ & $\begin{array}{r}15.49 \\
6.64 \\
2.66 \\
6.69 \\
6.60 \\
6.46 \\
7.02 \\
11.94 \\
10.04 \\
2.69 \\
28.21\end{array}$ & $\begin{array}{r}15.04 \\
6.65 \\
2.66 \\
7.00 \\
6.35 \\
5.59 \\
5.75 \\
9.96 \\
10.19 \\
2.53 \\
29.30\end{array}$ & $\begin{array}{r}15.77 \\
6.82 \\
2.34 \\
6.84 \\
6.83 \\
5.47 \\
5.77 \\
10.02 \\
10.35 \\
2.66 \\
29.83\end{array}$ \\
\hline 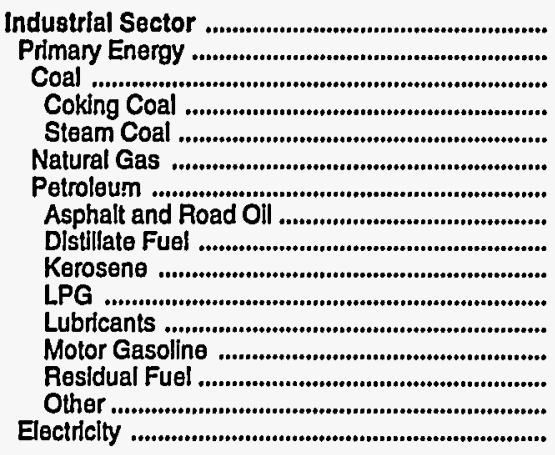 & $\begin{array}{r}1.06 \\
.72 \\
.79 \\
-\overline{19} \\
.79 \\
.69 \\
.68 \\
.73 \\
.79 \\
1.42 \\
5.08 \\
2.96 \\
.43 \\
.91 \\
4.27\end{array}$ & $\begin{array}{r}3.45 \\
2.29 \\
2.00 \\
-\overline{2.00} \\
2.24 \\
2.30 \\
1.91 \\
2.41 \\
2.67 \\
2.89 \\
7.48 \\
4.61 \\
2.12 \\
2.97 \\
10.51\end{array}$ & $\begin{array}{r}7.04 \\
5.13 \\
- \\
- \\
- \\
4.08 \\
5.40 \\
3.67 \\
5.75 \\
6.29 \\
5.31 \\
14.36 \\
10.10 \\
4.55 \\
7.68 \\
16.60\end{array}$ & $\begin{array}{r}9.88 \\
6.10 \\
2.38 \\
- \\
2.38 \\
5.38 \\
6.41 \\
5.20 \\
6.72 \\
7.70 \\
11.48 \\
17.61 \\
9.37 \\
4.68 \\
7.94 \\
21.93\end{array}$ & $\begin{array}{r}R_{9.34} \\
R_{5.19} \\
2.63 \\
- \\
2.63 \\
4.65 \\
R_{5.56} \\
3.38 \\
6.70 \\
7.68 \\
10.80 \\
R_{14.60} \\
10.06 \\
3.25 \\
R_{6.63} \\
22.15\end{array}$ & $\begin{array}{r}R_{8.91} \\
R_{4.89} \\
2.66 \\
- \\
2.66 \\
4.69 \\
R_{5.07} \\
3.05 \\
6.13 \\
7.02 \\
11.94 \\
R_{16.79} \\
10.04 \\
2.69 \\
R_{5.91} \\
23.21\end{array}$ & $\begin{array}{r}R_{8.89} \\
R_{4.82} \\
2.66 \\
-\overline{2.66} \\
4.79 \\
R_{4.88} \\
2.80 \\
5.02 \\
5.75 \\
9.96 \\
R_{19.43} \\
10.19 \\
2.53 \\
R_{6.05} \\
24.09\end{array}$ & $\begin{array}{r}8.71 \\
4.72 \\
2.34 \\
-\overline{2} \\
2.34 \\
4.64 \\
4.84 \\
3.30 \\
5.04 \\
5.77 \\
10.02 \\
18.94 \\
10.35 \\
2.66 \\
5.44 \\
24.30\end{array}$ \\
\hline 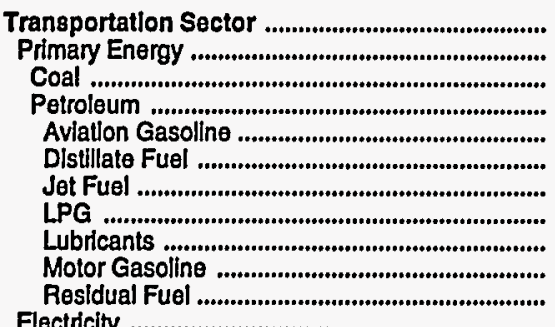 & $\begin{array}{r}2.63 \\
2.63 \\
.79 \\
2.63 \\
2.17 \\
1.39 \\
.75 \\
1.42 \\
5.08 \\
2.96 \\
.38\end{array}$ & $\begin{array}{l}4.30 \\
4.30 \\
2.00 \\
4.30 \\
3.45 \\
2.90 \\
2.09 \\
2.89 \\
7.48 \\
4.61 \\
1.72\end{array}$ & $\begin{array}{r}9.69 \\
9.69 \\
-\overline{9} \\
9.69 \\
9.02 \\
7.40 \\
6.51 \\
5.31 \\
14.36 \\
10.10 \\
3.88\end{array}$ & $\begin{array}{r}9.28 \\
9.28 \\
- \\
9.28 \\
9.99 \\
9.19 \\
6.29 \\
11.48 \\
17.61 \\
9.37 \\
4.06\end{array}$ & $\begin{array}{r}R_{9.76} \\
R_{9.76} \\
- \\
R_{9.76} \\
9.32 \\
9.74 \\
5.91 \\
10.80 \\
R_{14.60} \\
10.06 \\
2.74\end{array}$ & $\begin{array}{r}R_{9.68} \\
R_{9.68} \\
R_{9.68} \\
8.71 \\
9.42 \\
5.10 \\
11.94 \\
R_{16.79} \\
10.04 \\
2.29\end{array}$ & $\begin{array}{r}R_{9.71} \\
R_{9.71} \\
R_{9.71} \\
8.54 \\
8.90 \\
4.68 \\
9.96 \\
R_{19.43} \\
10.19 \\
R_{2.21}\end{array}$ & $\begin{array}{r}9.82 \\
9.82 \\
- \\
9.82 \\
8.24 \\
8.85 \\
4.41 \\
10.02 \\
18.94 \\
10.35 \\
2.28\end{array}$ \\
\hline 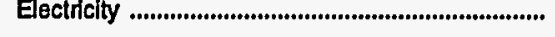 & - & - & - & - & - & - & - & - \\
\hline 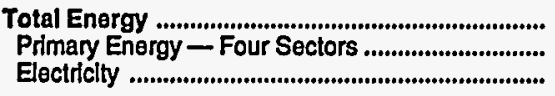 & $\begin{array}{l}2.09 \\
1.64 \\
6.27\end{array}$ & $\begin{array}{r}4.53 \\
3.35 \\
13.15\end{array}$ & $\begin{array}{r}9.07 \\
7.45 \\
19.10\end{array}$ & $\begin{array}{r}11.20 \\
8.23 \\
26.62\end{array}$ & $\begin{array}{r}R_{11.58} \\
8.32 \\
26.83\end{array}$ & $\begin{array}{r}A_{11.74} \\
R_{8.25} \\
28.13\end{array}$ & $\begin{array}{r}R_{11.57} \\
R_{8.05} \\
29.41\end{array}$ & $\begin{array}{r}11.73 \\
8.11 \\
30.07\end{array}$ \\
\hline 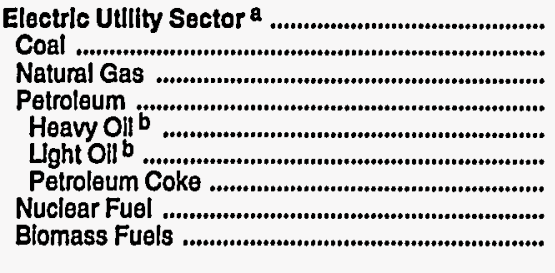 & $\begin{array}{r}.35 \\
.45 \\
.34 \\
.38 \\
.38 \\
.37 \\
-\overline{13} \\
-\end{array}$ & $\begin{array}{r}1.35 \\
1.24 \\
1.36 \\
2.02 \\
2.02 \\
2.36 \\
-\overline{29} \\
-\end{array}$ & $\begin{array}{r}2.60 \\
- \\
- \\
4.71 \\
4.70 \\
6.13 \\
- \\
.38 \\
-\end{array}$ & $\begin{array}{r}2.38 \\
2.35 \\
3.39 \\
4.25 \\
4.24 \\
5.88 \\
- \\
.91 \\
-\end{array}$ & $\begin{array}{r}1.53 \\
2.13 \\
2.70 \\
3.02 \\
3.01 \\
5.67 \\
\overline{(04}\end{array}$ & $\begin{array}{r}1.50 \\
2.17 \\
2.09 \\
2.49 \\
2.47 \\
4.92 \\
-\overline{80}\end{array}$ & $\begin{array}{r}1.17 \\
1.95 \\
2.66 \\
2.42 \\
2.40 \\
4.82 \\
-\overline{10} \\
.71\end{array}$ & $\begin{array}{r}.93 \\
1.70 \\
3.78 \\
2.40 \\
2.39 \\
4.12 \\
\overline{60} \\
.60\end{array}$ \\
\hline Primary Energy - Flve Sectors c ........................ & 1.26 & 2.68 & 5.65 & 5.95 & 5.37 & 5.82 & 85.59 & 5.30 \\
\hline
\end{tabular}

a There are no direct fuel costs for hydroelectric, geothermal, wind, photovoltaic, or solar thermal energy.

Heavy oll includes fuel oll nos. 4,5 , and 6 , and residual fuel oils. Light oll includes fuel oll nos. 1 and 2 , kerosene, and jet fuel.

c Biomass fuels are not included except those consumed at electric utillties and those added to motor gasoline.

Utilitites used blomass fuels at no charge or received a fee for accepting

them.

$R=$ Revised data.

-No consumption, including cases where adjustments were made. Soe explanation of adjustments in Section 6 of Appendix A.

Sources: Data sources, estimation procedures, and assumptions are described in Appendix A. 
N (Million Dollars)

\begin{tabular}{|c|c|c|c|c|c|c|c|c|}
\hline Sector and Energy Source & 1970 & 1975 & 1980 & 1985 & 1990 & 1991 & 1992 & 1993 \\
\hline 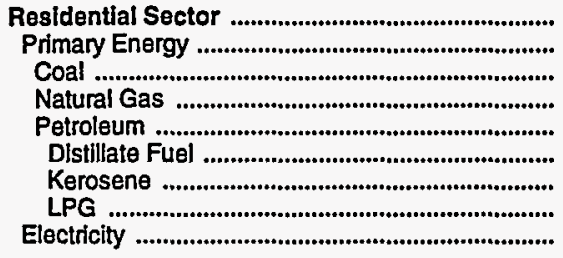 & $\begin{array}{r}353.6 \\
196.4 \\
.8 \\
59.6 \\
136.0 \\
122.7 \\
5.1 \\
8.2 \\
157.3\end{array}$ & $\begin{array}{r}708.8 \\
340.6 \\
.8 \\
105.8 \\
234.0 \\
214.5 \\
5.2 \\
14.3 \\
368.2\end{array}$ & $\begin{array}{r}1,342.0 \\
773.7 \\
1.0 \\
187.4 \\
585.3 \\
554.3 \\
10.8 \\
20.1 \\
568.4\end{array}$ & \begin{tabular}{r|}
$1,694.5$ \\
832.6 \\
2.2 \\
299.8 \\
530.6 \\
475.8 \\
30.8 \\
24.0 \\
861.9
\end{tabular} & $\begin{array}{r}1,981.6 \\
943.6 \\
.8 \\
321.3 \\
621.5 \\
569.1 \\
10.2 \\
42.2 \\
1,038.0\end{array}$ & $\begin{array}{r}2,024.2 \\
927.3 \\
.8 \\
325.0 \\
601.5 \\
541.2 \\
8.8 \\
51.5 \\
1,096.9\end{array}$ & $\begin{array}{r}2,172.8 \\
1,010.8 \\
1.0 \\
379.9 \\
630.0 \\
566.4 \\
8.6 \\
55.0 \\
1,162.0\end{array}$ & $\begin{array}{r}2,225.2 \\
1,018.3 \\
.7 \\
398.1 \\
619.5 \\
565.1 \\
9.0 \\
45.4 \\
1,207.0\end{array}$ \\
\hline 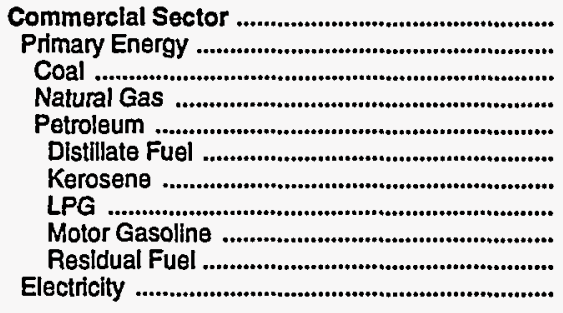 & $\begin{array}{r}169.6 \\
56.1 \\
.3 \\
21.3 \\
34.4 \\
29.5 \\
.1 \\
.8 \\
1.5 \\
2.6 \\
113.5\end{array}$ & $\begin{array}{r}398.3 \\
117.9 \\
.4 \\
42.3 \\
75.2 \\
59.7 \\
.2 \\
1.5 \\
5.8 \\
8.1 \\
280.4\end{array}$ & $\begin{array}{r}731.2 \\
254.8 \\
.2 \\
96.1 \\
158.4 \\
107.8 \\
.2 \\
.2 \\
2.0 \\
14.6 \\
33.8 \\
476.4\end{array}$ & $\begin{array}{r}1,195.1 \\
381.8 \\
.8 \\
166.9 \\
214.1 \\
150.2 \\
2.8 \\
4.7 \\
7.0 \\
49.4 \\
813.3\end{array}$ & $\begin{array}{r}1,333.1 \\
343.1 \\
.3 \\
185.3 \\
157.5 \\
117.2 \\
2.2 \\
5.9 \\
10.7 \\
21.4 \\
990.0\end{array}$ & $\begin{array}{r}1,405.2 \\
355.2 \\
.3 \\
185.2 \\
169.6 \\
112.2 \\
6.7 \\
7.2 \\
34.6 \\
9.0 \\
1,050.0\end{array}$ & $\begin{array}{r}1,504.1 \\
419.2 \\
.7 \\
214.8 \\
203.7 \\
95.9 \\
1.5 \\
7.8 \\
84.3 \\
14.2 \\
1,084.9\end{array}$ & $\begin{array}{r}1,527.9 \\
404.0 \\
.3 \\
220.6 \\
183.1 \\
81.7 \\
1.5 \\
6.7 \\
86.3 \\
6.9 \\
1,123.9\end{array}$ \\
\hline 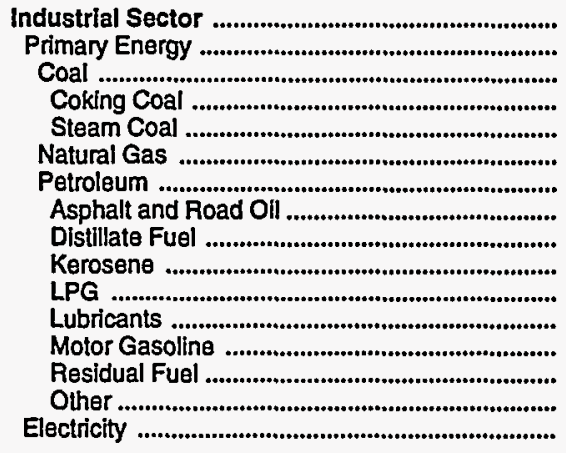 & $\begin{array}{r}193.4 \\
119.2 \\
2.7 \\
-\overline{2.7} \\
15.3 \\
101.1 \\
4.6 \\
8.3 \\
1.0 \\
4.8 \\
10.2 \\
4.2 \\
37.0 \\
31.0 \\
74.3\end{array}$ & $\begin{array}{r}420.1 \\
239.0 \\
1.4 \\
- \\
1.4 \\
34.9 \\
202.7 \\
16.0 \\
27.2 \\
4.3 \\
13.8 \\
9.1 \\
9.9 \\
121.7 \\
9.7 \\
181.2\end{array}$ & $\begin{array}{r}857.8 \\
521.2 \\
- \\
- \\
- \\
84.7 \\
436.4 \\
15.3 \\
108.4 \\
9.0 \\
15.3 \\
18.1 \\
3.5 \\
191.1 \\
75.8 \\
336.6\end{array}$ & $\begin{array}{r}861.9 \\
404.6 \\
.2 \\
- \\
.2 \\
105.0 \\
299.4 \\
72.2 \\
42.0 \\
1.9 \\
20.7 \\
20.2 \\
11.1 \\
64.8 \\
66.5 \\
457.4\end{array}$ & $\begin{array}{r}R 794.1 \\
R_{333.2} \\
.1 \\
- \\
.1 \\
122.2 \\
R_{210.9} \\
35.6 \\
39.8 \\
2.9 \\
21.4 \\
R_{18.9} \\
13.8 \\
29.3 \\
R 49.3 \\
461.0\end{array}$ & $\begin{array}{r}R_{806.7} \\
R_{345.7} \\
.2 \\
- \\
.2 \\
158.1 \\
R_{187.4} \\
40.0 \\
38.5 \\
1.5 \\
14.1 \\
R_{19.4} \\
12.6 \\
16.8 \\
R_{44.4} \\
461.0\end{array}$ & $\begin{array}{r}R_{830.5} \\
R_{355.5} \\
.8 \\
-.8 \\
179.0 \\
R_{175.7} \\
31.2 \\
27.3 \\
.3 \\
15.0 \\
R 22.9 \\
12.9 \\
19.5 \\
\text { R } 46.7 \\
475.0\end{array}$ & $\begin{array}{r}815.3 \\
351.3 \\
1.7 \\
- \\
1.7 \\
175.7 \\
173.8 \\
34.5 \\
24.1 \\
.8 \\
15.0 \\
22.7 \\
10.7 \\
24.1 \\
42.0 \\
464.0\end{array}$ \\
\hline $\begin{array}{l}\text { Transportation Sector } \\
\text { Primary Energy } \\
\text { Coal } \\
\text { Petroleum . } \\
\text { Aviation Gasoline } \\
\text { Distillate Fuel } \\
\text { Jet Fuel } \\
\text { LPG } \\
\text { Mubricants } \\
\text { Retor Gasolke } \\
\text { Electricity }\end{array}$ & $\begin{array}{r}479.8 \\
479.8 \\
(s) \\
479.8 \\
1.4 \\
18.3 \\
12.3 \\
.1 \\
7.3 \\
439.6 \\
.9\end{array}$ & $\begin{array}{r}844.8 \\
844.8 \\
(s) \\
844.8 \\
1.6 \\
40.5 \\
23.8 \\
.3 \\
8.9 \\
763.5 \\
6.3 \\
-\end{array}$ & $\begin{array}{r}1,793.8 \\
1,793.8 \\
- \\
1,793.8 \\
4.1 \\
111.2 \\
70.7 \\
.3 \\
21.5 \\
1,584.7 \\
1.3 \\
\end{array}$ & $\begin{array}{r}1,817.0 \\
1,817.0 \\
- \\
1,817.0 \\
3.6 \\
238.2 \\
38.5 \\
1.3 \\
24.0 \\
1,507.4 \\
3.9 \\
-\end{array}$ & $\begin{array}{r}R_{2,000.9} \\
R_{2,000.9} \\
- \\
R_{2,000.9} \\
4.4 \\
281.2 \\
78.4 \\
1.4 \\
R_{22.4} \\
1,611.6 \\
1.5 \\
-\end{array}$ & $\begin{array}{r}R_{1,968.7} \\
R_{1,968.7} \\
- \\
R_{1,968.7} \\
1.2 \\
243.0 \\
64.7 \\
1.7 \\
R_{23.1} \\
1,633.5 \\
1.3 \\
-\end{array}$ & $\begin{array}{r}R_{1,990.4} \\
R_{1,990.4} \\
- \\
R_{1,990.4} \\
1.2 \\
252.1 \\
60.7 \\
1.2 \\
R_{27.2} \\
1,647.3 \\
.6 \\
-\end{array}$ & $\begin{array}{r}2,038.2 \\
2,038.2 \\
- \\
2,038.2 \\
1.2 \\
248.9 \\
57.8 \\
1.2 \\
27.0 \\
1,701.6 \\
.5 \\
-\end{array}$ \\
\hline 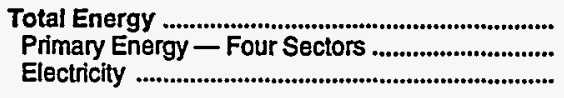 & $\begin{array}{r}1,196.4 \\
851.4 \\
345.0\end{array}$ & $\begin{array}{r}2,372.1 \\
1,542.3 \\
829.8\end{array}$ & $\begin{array}{l}4,724.8 \\
3,343.4 \\
1,381.4\end{array}$ & $\begin{array}{l}5,568.5 \\
3,435.9 \\
2,132.6\end{array}$ & $\begin{array}{r}R_{6,109.7} \\
R_{3,620.7} \\
2,489.0\end{array}$ & $\begin{array}{r}R_{6,204.8} \\
R_{3,596.8} \\
2,608.0\end{array}$ & $\begin{array}{r}R_{6,497.8} \\
R_{3,775.9} \\
2,721.9\end{array}$ & $\begin{array}{l}6,606.7 \\
3,811.8 \\
2,794.9\end{array}$ \\
\hline 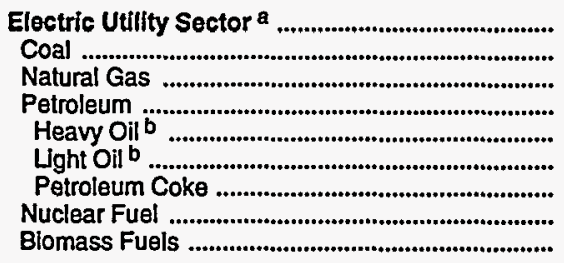 & $\begin{array}{r}76.1 \\
19.7 \\
.1 \\
51.0 \\
48.8 \\
2.2 \\
-\overline{5.3} \\
-\end{array}$ & $\begin{array}{r}311.5 \\
.1 \\
.5 \\
284.6 \\
281.4 \\
3.1 \\
- \\
26.4 \\
-\end{array}$ & $\begin{array}{r}688.1 \\
- \\
- \\
639.0 \\
633.0 \\
6.0 \\
- \\
49.1 \\
-\end{array}$ & $\begin{array}{r}634.9 \\
47.8 \\
5.4 \\
456.1 \\
453.2 \\
2.9 \\
- \\
125.5 \\
-\end{array}$ & $\begin{array}{r}512.4 \\
54.0 \\
13.5 \\
267.4 \\
265.2 \\
2.3 \\
- \\
177.5 \\
\left({ }^{d}\right)\end{array}$ & $\begin{array}{r}367.2 \\
48.2 \\
10.2 \\
203.4 \\
200.3 \\
3.1 \\
- \\
105.5 \\
(d)\end{array}$ & $\begin{array}{r}307.5 \\
41.9 \\
5.8 \\
133.5 \\
131.7 \\
1.8 \\
- \\
126.3 \\
(d)\end{array}$ & $\begin{array}{r}281.6 \\
33.4 \\
2.2 \\
106.1 \\
104.3 \\
1.8 \\
-\bar{c} \\
139.9\end{array}$ \\
\hline Primary Energy - Five Sectors c ........................ & 927.5 & $1,853.8$ & $4,031.6$ & $4,070.8$ & $R_{4,133.1}$ & $R_{3,964.0}$ & $R_{4,083.5}$ & $4,093.3$ \\
\hline
\end{tabular}

a There are no direct fuel costs for hydroelectric, geothermal, wind, photovoltaic, or solar thermal energy.

beavy oil includes fuel oil nos. 4, 5, and 6, and residual fuel olls. Light oll includes fuel nos. 1 and 2, kerosene, and jet fuel.

c Biomass fuels are not included, except those consumed at electric utilities and those added to motor gasoline.

$\checkmark$ Utilities used biomass fuels at no charge or recelved a fee for accepting them.
R=Revised data.

- No consumption, including cases where adjustments were made. See explanation of adjustments in Section 6 of Appendix A.

$(s)=$ Value less than 0.05 million dollars.

Note: Totals may not equal sum of components due to independent rounding.

Sources: Data sources, estimation procedures, and assumptions are described in Appendix A. 


\begin{tabular}{|c|c|c|c|c|c|c|c|c|}
\hline Energy Source & 1970 & 1975 & 1980 & 1985 & 1980 & 1991 & 1992 & 1993 \\
\hline & \multicolumn{8}{|c|}{ Prices In Dollars per Million Btu } \\
\hline $\begin{array}{l}\text { Coal } \\
\text { Coking Coal } \\
\text { Steam Coal }\end{array}$ &.$\overline{39}$ & $\frac{1.16}{1.16}$ & $1.5 \overline{7}$ & $1 . \overline{-}$ & $\frac{1.76}{1.76}$ & $\frac{1.75}{1.75}$ & $\frac{1.70}{1.70}$ & $\frac{1.67}{1.67}$ \\
\hline 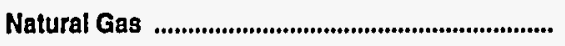 & .91 & 1.80 & 3.37 & 4.87 & 3.82 & 3.43 & 3.82 & 4.04 \\
\hline 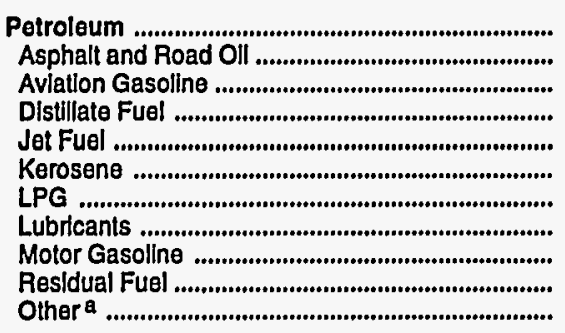 & $\begin{array}{r}1.27 \\
.66 \\
2.17 \\
1.16 \\
.73 \\
1.26 \\
1.25 \\
5.08 \\
2.86 \\
.45 \\
.35\end{array}$ & $\begin{array}{l}2.75 \\
1.76 \\
3.45 \\
2.53 \\
2.03 \\
3.14 \\
3.60 \\
7.48 \\
4.54 \\
1.92 \\
1.68\end{array}$ & $\begin{array}{r}6.00 \\
3.58 \\
9.02 \\
6.77 \\
6.46 \\
8.36 \\
5.20 \\
14.36 \\
9.60 \\
4.23 \\
5.84\end{array}$ & $\begin{array}{r}7.39 \\
4.97 \\
9.99 \\
7.58 \\
6.63 \\
8.65 \\
10.65 \\
17.61 \\
9.39 \\
4.16 \\
4.94\end{array}$ & $\begin{array}{r}{ }^{R} 6.92 \\
2.90 \\
9.32 \\
7.58 \\
6.33 \\
8.68 \\
12.14 \\
R_{14.60} \\
10.26 \\
2.71 \\
\mathrm{R}_{2.71}\end{array}$ & $\begin{array}{r}6.30 \\
3.06 \\
8.71 \\
7.08 \\
5.51 \\
8.11 \\
13.06 \\
\mathrm{R} \\
16.79 \\
9.51 \\
2.25 \\
\mathrm{R}_{2.56}\end{array}$ & $\begin{array}{r}6.04 \\
2.24 \\
8.54 \\
6.76 \\
5.14 \\
7.70 \\
712.05 \\
R_{19.43} \\
9.16 \\
R_{2.28} \\
\mathrm{R}_{2.18}\end{array}$ & $\begin{array}{r}6.20 \\
3.02 \\
8.24 \\
6.69 \\
4.88 \\
6.86 \\
11.59 \\
18.94 \\
9.24 \\
2.24 \\
4.29\end{array}$ \\
\hline Nuclear Fuel ......................................................................... & - & - & - & - & - & - & - & - \\
\hline 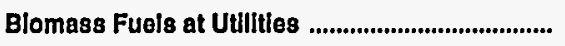 & - & - & - & - & - & - & - & - \\
\hline Primary Energy - Flve Sectors b ............................ & 1.06 & 2.48 & 5.13 & 5.16 & 4.92 & 4.59 & 4.64 & 4.54 \\
\hline 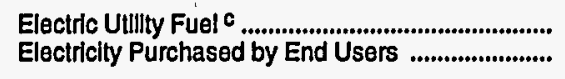 & $\begin{array}{r}.39 \\
4.94\end{array}$ & $\begin{array}{r}1.63 \\
11.69\end{array}$ & $\begin{array}{r}3.35 \\
18.84\end{array}$ & $\begin{array}{r}2.48 \\
21.42\end{array}$ & $\begin{array}{r}1.97 \\
19.00\end{array}$ & $\begin{array}{r}1.92 \\
19.70\end{array}$ & $\begin{array}{r}1.85 \\
19.71\end{array}$ & $\begin{array}{r}1.93 \\
20.53\end{array}$ \\
\hline \multirow[t]{2}{*}{ Total Energy } & 1.69 & 3.89 & 7.28 & 9.16 & 9.09 & 8.85 & ${ }^{R} 8.81$ & 8.78 \\
\hline & \multicolumn{8}{|c|}{ Expenditures in Millions of Dollars } \\
\hline $\begin{array}{l}\text { Coal } \\
\text { Coking Coal } \\
\text { Steam Coal }\end{array}$ & $\frac{14.5}{14.5}$ & $\begin{array}{l}26.6 \\
\overline{26.6}\end{array}$ & $\begin{array}{l}44.1 \\
44.1\end{array}$ & $\begin{array}{l}133.3 \\
133.3\end{array}$ & $\begin{array}{l}104.9 \\
104.9\end{array}$ & $\begin{array}{l}99.1 \\
99.1\end{array}$ & $\begin{array}{l}78.4 \\
\overline{78.4}\end{array}$ & $\begin{array}{l}105.8 \\
\overline{-} \\
105.8\end{array}$ \\
\hline 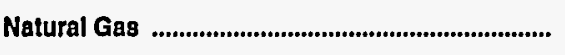 & 24.4 & 34.0 & 102.9 & 188.6 & 150.9 & 145.9 & 153.5 & 170.6 \\
\hline 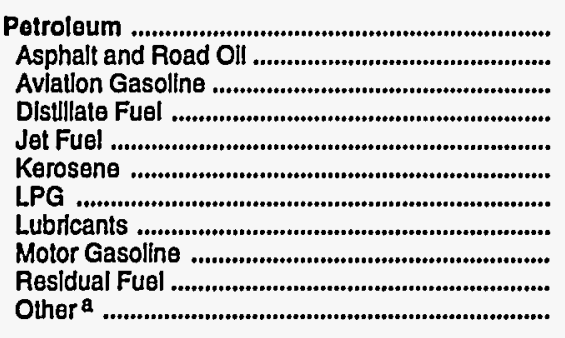 & $\begin{array}{r}173.0 \\
2.3 \\
.2 \\
29.1 \\
8.1 \\
3.1 \\
10.6 \\
3.3 \\
93.8 \\
18.6 \\
3.8\end{array}$ & $\begin{array}{r}435.9 \\
7.6 \\
.3 \\
62.2 \\
18.0 \\
4.9 \\
34.9 \\
3.7 \\
168.4 \\
123.3 \\
12.5\end{array}$ & $\begin{array}{r}1,059.8 \\
8.3 \\
.5 \\
146.5 \\
54.6 \\
14.3 \\
56.7 \\
12.1 \\
333.5 \\
335.5 \\
97.8\end{array}$ & $\begin{array}{r}818.0 \\
27.3 \\
.8 \\
150.8 \\
56.0 \\
34.6 \\
37.9 \\
13.5 \\
372.5 \\
92.7 \\
32.1\end{array}$ & $\begin{array}{r}\mathrm{R} 798.8 \\
10.3 \\
3.6 \\
142.0 \\
44.4 \\
7.8 \\
44.7 \\
\mathrm{R}_{12.6} \\
429.3 \\
63.4 \\
\mathrm{R} \\
40.7\end{array}$ & $\begin{array}{r}\mathrm{R} 778.2 \\
2.9 \\
.8 \\
141.1 \\
70.9 \\
8.6 \\
51.3 \\
\mathrm{R}_{13.0} \\
389.3 \\
67.1 \\
\mathrm{R}_{33.5}\end{array}$ & $\begin{array}{r}\mathrm{R} 724.9 \\
1.2 \\
.8 \\
127.4 \\
40.1 \\
6.5 \\
40.2 \\
415.3 \\
\mathrm{R}_{15} \\
392.5 \\
67.3 \\
\mathrm{R}_{33.6}\end{array}$ & $\begin{array}{r}755.4 \\
2.2 \\
2.1 \\
138.8 \\
37.8 \\
5.6 \\
41.9 \\
15.2 \\
403.4 \\
85.3 \\
23.1\end{array}$ \\
\hline 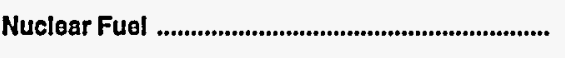 & - & - & - & - & - & - & - & - \\
\hline 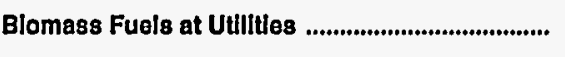 & - & - & - & - & - & - & - & - \\
\hline Primary Energy — Flve Sectors b .......................... & 211.8 & 496.5 & 1,206.8 & $1,139.9$ & $R_{1,054.7}$ & $R_{1,023.3}$ & ${ }^{\text {R }} 956.8$ & $1,031.8$ \\
\hline Electric Uitllty Fuel c ................................................... & $\begin{array}{r}-23.1 \\
75.7\end{array}$ & $\begin{array}{r}-106.3 \\
202.1\end{array}$ & $\begin{array}{r}-239.3 \\
368.7\end{array}$ & $\begin{array}{r}-229.9 \\
457.9\end{array}$ & $\begin{array}{r}-171.0 \\
533.6\end{array}$ & $\begin{array}{r}-175.9 \\
567.9\end{array}$ & $\begin{array}{r}-145.2 \\
568.1\end{array}$ & $\begin{array}{r}-170.3 \\
632.7\end{array}$ \\
\hline Total Energy & 264.4 & 592.2 & 1,336.2 & $1,367.9$ & $R_{1,417.3}$ & $R_{1,415.3}$ & ${ }^{R} \mathbf{1 , 3 7 9 . 7}$ & $1,494.1$ \\
\hline
\end{tabular}

a Includes petroleum coke used at electric utilities.

b Biomass fuels are not included, except those consumed at electric utilitles and those added to motor gasoline.

c There are no direct fuel costs for hydroelectric, geothermal, wind, photovoltalc, or solar thermal energy.

$R=$ Revised data.
-No consumption, including cases where adjustments were made. Seo explanation of adjustments in Section 6 of Appendix A.

Note: Expenditure totals may not equal sum of components due to independent rounding.

Sources: Data sources, estimation procedures, and assumptions are described in Appendix A. 
(Dollars per Million Btu)

\begin{tabular}{|c|c|c|c|c|c|c|c|c|}
\hline Sector and Energy Source & 1970 & 1975 & 1980 & 1985 & 1990 & 1991 & 1992 & 1993 \\
\hline 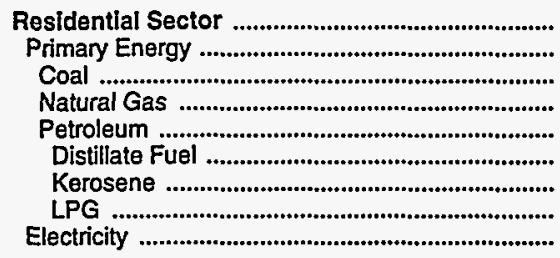 & $\begin{array}{l}2.39 \\
1.52 \\
1.13 \\
1.55 \\
1.51 \\
1.42 \\
1.34 \\
2.40 \\
7.53\end{array}$ & $\begin{array}{r}5.15 \\
2.78 \\
2.73 \\
2.39 \\
2.99 \\
2.71 \\
3.37 \\
4.73 \\
13.93\end{array}$ & $\begin{array}{r}10.19 \\
6.05 \\
3.38 \\
4.16 \\
7.34 \\
6.88 \\
8.55 \\
8.53 \\
21.76\end{array}$ & $\begin{array}{r}12.67 \\
7.86 \\
3.76 \\
6.91 \\
8.33 \\
7.54 \\
8.80 \\
10.37 \\
27.29\end{array}$ & $\begin{array}{r}13.78 \\
7.68 \\
3.75 \\
6.04 \\
9.19 \\
7.63 \\
8.90 \\
13.54 \\
24.65\end{array}$ & $\begin{array}{r}\mathbf{1 4 . 0 3} \\
7.57 \\
3.60 \\
5.72 \\
9.13 \\
7.19 \\
8.39 \\
14.48 \\
25.22\end{array}$ & $\begin{array}{r}13.57 \\
7.20 \\
3.74 \\
5.93 \\
8.39 \\
6.66 \\
7.77 \\
13.31 \\
25.35\end{array}$ & $\begin{array}{r}14.10 \\
7.26 \\
3.65 \\
6.47 \\
8.12 \\
6.48 \\
7.56 \\
12.71 \\
26.38\end{array}$ \\
\hline 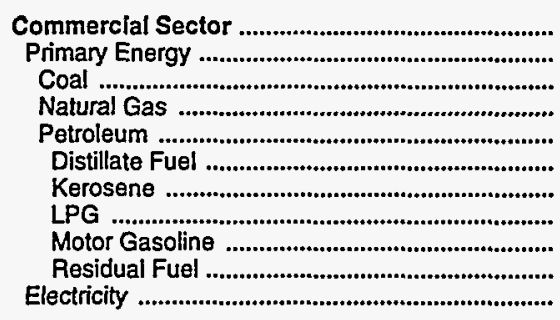 & $\begin{array}{r}1.55 \\
.76 \\
.28 \\
1.22 \\
.68 \\
1.12 \\
.85 \\
.99 \\
2.86 \\
.46 \\
6.56\end{array}$ & $\begin{array}{r}4.54 \\
2.11 \\
1.20 \\
1.87 \\
2.17 \\
2.39 \\
2.36 \\
3.40 \\
4.54 \\
1.95 \\
12.76\end{array}$ & $\begin{array}{r}6.60 \\
4.47 \\
1.20 \\
3.92 \\
4.54 \\
6.30 \\
6.36 \\
4.72 \\
9.60 \\
4.24 \\
20.78\end{array}$ & $\begin{array}{r}14.10 \\
6.57 \\
1.33 \\
6.30 \\
6.99 \\
6.57 \\
6.93 \\
11.06 \\
9.39 \\
4.35 \\
22.97\end{array}$ & $\begin{array}{r}12.81 \\
5.23 \\
1.22 \\
5.04 \\
5.82 \\
6.11 \\
6.44 \\
10.33 \\
10.26 \\
3.13 \\
20.48\end{array}$ & $\begin{array}{r}13.11 \\
5.16 \\
1.35 \\
4.69 \\
6.07 \\
5.52 \\
5.93 \\
11.09 \\
9.51 \\
2.38 \\
20.88\end{array}$ & $\begin{array}{r}13.04 \\
4.99 \\
1.32 \\
4.78 \\
5.35 \\
5.00 \\
5.32 \\
9.50 \\
9.16 \\
2.43 \\
20.88\end{array}$ & $\begin{array}{r}12.73 \\
4.67 \\
1.27 \\
5.27 \\
4.49 \\
4.82 \\
4.87 \\
9.32 \\
9.24 \\
2.35 \\
21.59\end{array}$ \\
\hline $\begin{array}{l}\text { Industrial Sector } \\
\text { Primary Energy } \\
\text { Coal } \\
\text { Coking Coal Coal } \\
\text { Steam Coal } \\
\text { Natural Gas } \\
\text { Petroleum } \\
\text { Asphalt and Road Oil } \\
\text { Distillate Fuel } \\
\text { Kerosene } \\
\text { LPG }\end{array}$ & $\begin{array}{r}1.02 \\
.66 \\
.28 \\
. \\
.28 \\
.57 \\
.69 \\
.66 \\
.78 \\
.85 \\
.99 \\
5.08 \\
2.86 \\
.46 \\
.50 \\
3.10\end{array}$ & $\begin{array}{l}3.14 \\
2.14 \\
1.20 \\
1.20 \\
1.37 \\
2.31 \\
1.76 \\
2.19 \\
2.36 \\
3.40 \\
7.48 \\
4.54 \\
1.87 \\
1.96 \\
9.25\end{array}$ & $\begin{array}{r}5.69 \\
4.36 \\
1.20 \\
-\overline{1} \\
1.20 \\
2.72 \\
5.23 \\
3.58 \\
5.71 \\
6.36 \\
4.72 \\
14.36 \\
9.60 \\
4.19 \\
6.16 \\
15.28\end{array}$ & $\begin{array}{r}6.66 \\
4.73 \\
1.33 \\
1.33 \\
4.38 \\
6.16 \\
4.97 \\
6.20 \\
6.93 \\
11.06 \\
17.61 \\
9.39 \\
4.35 \\
6.71 \\
16.15\end{array}$ & $\begin{array}{r}5.84 \\
3.83 \\
1.22 \\
1.22 \\
1.22 \\
3.39 \\
R 4.98 \\
2.90 \\
5.76 \\
6.44 \\
10.33 \\
R 14.60 \\
10.26 \\
3.13 \\
R 5.05 \\
13.26\end{array}$ & 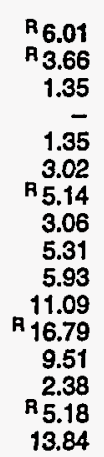 & $\begin{array}{r}R_{5} 5.87 \\
R_{3.53} \\
1.32, \\
- \\
1.32 \\
3.14 \\
R_{4.49} \\
2.24 \\
4.76 \\
5.32 \\
9.50 \\
\text { R }^{2} 9.43 \\
9.16 \\
2.43 \\
\mathrm{R}_{4.93} \\
13.81\end{array}$ & $\begin{array}{r}5.70 \\
3.38 \\
1.27 \\
- \\
1.27 \\
3.28 \\
3.97 \\
3.02 \\
4.36 \\
4.87 \\
9.32 \\
18.94 \\
9.24 \\
2.35 \\
4.29 \\
14.32\end{array}$ \\
\hline 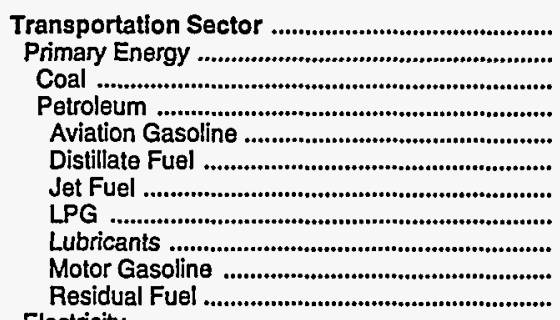 & $\begin{array}{r}2.13 \\
2.13 \\
.28 \\
2.13 \\
2.17 \\
1.24 \\
.73 \\
.99 \\
5.08 \\
2.86 \\
.42\end{array}$ & $\begin{array}{l}3.74 \\
3.74 \\
1.20 \\
3.74 \\
3.45 \\
2.81 \\
2.03 \\
3.40 \\
7.48 \\
4.54 \\
1.72\end{array}$ & $\begin{array}{r}8.41 \\
8.41 \\
8 . \overline{41} \\
9.02 \\
7.72 \\
6.46 \\
4.72 \\
14.36 \\
9.60 \\
3.93\end{array}$ & $\begin{array}{r}8.78 \\
8.78 \\
8.78 \\
9.99 \\
8.52 \\
6.63 \\
11.06 \\
17.61 \\
9.39 \\
3.99\end{array}$ & $\begin{array}{r}8.92 \\
8.92 \\
8.92 \\
9.32 \\
8.71 \\
6.33 \\
10.33 \\
\mathrm{R} 14.60 \\
10.26 \\
2.33\end{array}$ & $\begin{array}{r}R_{7.79} \\
R_{7.79} \\
\text { R } 7.79 \\
8.71 \\
8.26 \\
5.51 \\
11.09 \\
R_{16.79} \\
9.51 \\
2.04\end{array}$ & $\begin{array}{r}R_{7.88} \\
R_{7.88} \\
R_{7.88} \\
8.54 \\
7.97 \\
5.14 \\
9.50 \\
\mathrm{R}_{19.43} \\
9.16 \\
2.06\end{array}$ & $\begin{array}{r}7.85 \\
7.85 \\
- \\
7.85 \\
8.24 \\
7.92 \\
4.88 \\
9.32 \\
18.94 \\
9.24 \\
2.02\end{array}$ \\
\hline 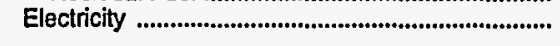 & - & - & - & - & - & $2 ., 4$ & - & 2.02 \\
\hline 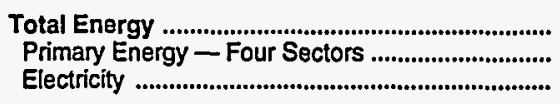 & $\begin{array}{l}1.69 \\
1.34 \\
4.94\end{array}$ & $\begin{array}{r}3.89 \\
2.89 \\
11.69\end{array}$ & $\begin{array}{r}7.28 \\
5.90 \\
18.84\end{array}$ & $\begin{array}{r}9.16 \\
7.11 \\
21.42\end{array}$ & $\begin{array}{r}9.09 \\
R 6.92 \\
19.00\end{array}$ & $\begin{array}{r}8.85 \\
\mathrm{R}_{6.47} \\
19.70\end{array}$ & $\begin{array}{l}R_{8.81} \\
R_{6.35} \\
19.71\end{array}$ & $\begin{array}{r}8.78 \\
6.19 \\
20.53\end{array}$ \\
\hline 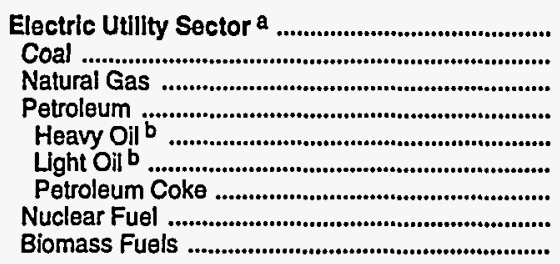 & $\begin{array}{l}.39 \\
.39 \\
.37 \\
.40 \\
.46 \\
.47 \\
.29 \\
- \\
-\end{array}$ & $\begin{array}{l}1.63 \\
1.15 \\
1.02 \\
1.92 \\
1.97 \\
2.18 \\
.49 \\
-\end{array}$ & $\begin{array}{r}3.35 \\
1.64 \\
3.47 \\
4.33 \\
4.27 \\
6.21 \\
4.32 \\
- \\
-\end{array}$ & $\begin{array}{l}2.48 \\
1.91 \\
3.88 \\
3.86 \\
4.13 \\
5.51 \\
1.27 \\
- \\
-\end{array}$ & $\begin{array}{r}1.97 \\
1.82 \\
2.58 \\
2.05 \\
2.71 \\
4.58 \\
.90 \\
- \\
-\end{array}$ & $\begin{array}{r}1.92 \\
1.78 \\
2.37 \\
1.92 \\
2.31 \\
4.62 \\
.84 \\
=\end{array}$ & $\begin{array}{r}1.85 \\
1.73 \\
2.60 \\
1.80 \\
2.32 \\
4.44 \\
.78 \\
- \\
-\end{array}$ & $\begin{array}{r}1.93 \\
1.69 \\
2.61 \\
2.30 \\
2.26 \\
3.96 \\
= \\
-\end{array}$ \\
\hline Primary Energy - Five Sectors ${ }^{c} \ldots \ldots \ldots \ldots . . . . . . . . . . . . . .$. & 1.06 & 2.48 & 5.13 & 5.16 & 4.92 & 4.59 & 4.64 & 4.54 \\
\hline
\end{tabular}

a There are no direct fuel costs for hydroelectric, geothermal, wind, photovoltaic, or solar thermal energy.

b Heavy oil includes fuel oil nos. 4, 5, and 6, and residual fuel oils. Light oil includes fuel oil nos. 1 and 2 , kerosene, and jet fuel.

$c$ Biomass fuels are not included, except those consumed at electric utilities and those added to motor gasoline.
$R=$ Revised data.

-No consumption, Including cases where adjustments were made. Seo explanation of adjustments in Section 6 of Appendix $A$.

Sources: Data sources, estimation procedures, and assumptions are described in Appendlx A. 
Energy Expenditure Estimates by Sector, Delaware $1970,1975,1980,1985$, and 1990-1993

(Million Dollars)

\begin{tabular}{|c|c|c|c|c|c|c|c|c|}
\hline Sector and Energy Source & 1970 & 1975 & 1980 & 1985 & 1990 & 1891 & 1992 & 1993 \\
\hline 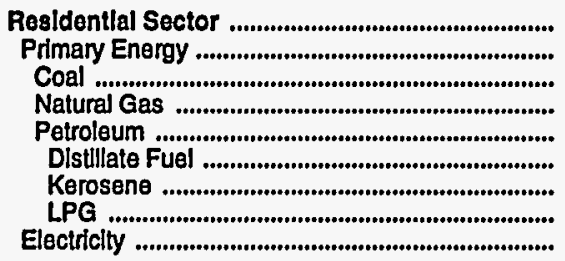 & $\begin{array}{r}65.9 \\
35.9 \\
.1 \\
12.4 \\
23.4 \\
16.8 \\
2.8 \\
3.8 \\
30.0\end{array}$ & $\begin{array}{r}135.5 \\
57.5 \\
2.2 \\
16.9 \\
40.4 \\
29.4 \\
4.1 \\
6.9 \\
77.9\end{array}$ & $\begin{array}{r}246.2 \\
107.7 \\
.2 \\
29.7 \\
77.8 \\
52.7 \\
13.3 \\
11.7 \\
138.6\end{array}$ & $\begin{array}{r}336.2 \\
157.1 \\
.2 \\
43.9 \\
113.0 \\
58.5 \\
32.4 \\
22.1 \\
179.1\end{array}$ & $\begin{array}{r}346.7 \\
123.7 \\
.7 \\
44.6 \\
78.4 \\
43.0 \\
7.3 \\
28.1 \\
223.0\end{array}$ & $\begin{array}{r}369.3 \\
126.2 \\
.6 \\
42.1 \\
83.5 \\
42.6 \\
7.9 \\
33.0 \\
243.0\end{array}$ & $\begin{array}{r}367.8 \\
126.8 \\
(s) \\
50.2 \\
76.5 \\
40.4 \\
6.3 \\
29.8 \\
241.0\end{array}$ & $\begin{array}{r}409.3 \\
135.3 \\
1.5 \\
55.6 \\
78.2 \\
42.9 \\
4.5 \\
30.8 \\
274.0\end{array}$ \\
\hline 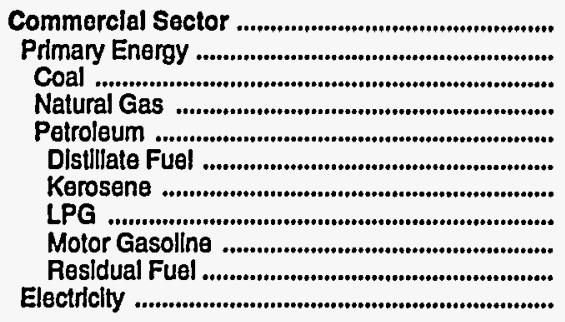 & $\begin{array}{r}34.4 \\
14.5 \\
(s) \\
3.5 \\
11.0 \\
5.1 \\
.2 \\
.3 \\
.4 \\
5.0 \\
19.9\end{array}$ & $\begin{array}{r}90.5 \\
32.5 \\
(\mathrm{~s}) \\
5.6 \\
26.8 \\
10.0 \\
.4 \\
.9 \\
.8 \\
14.7 \\
58.0\end{array}$ & $\begin{array}{r}261.3 \\
154.0 \\
.1 \\
13.1 \\
140.8 \\
23.3 \\
.3 \\
1.1 \\
2.3 \\
113.8 \\
107.3\end{array}$ & $\begin{array}{r}177.9 \\
44.9 \\
.1 \\
22.0 \\
22.8 \\
12.8 \\
2.0 \\
4.2 \\
1.9 \\
1.9 \\
133.0\end{array}$ & $\begin{array}{r}207.7 \\
42.7 \\
.4 \\
20.7 \\
21.6 \\
12.0 \\
.4 \\
3.8 \\
1.9 \\
3.6 \\
165.0\end{array}$ & $\begin{array}{r}218.4 \\
42.4 \\
.4 \\
20.5 \\
21.5 \\
14.1 \\
.4 \\
4.5 \\
1.7 \\
.8 \\
176.0\end{array}$ & $\begin{array}{r}219.5 \\
41.5 \\
(s) \\
24.5 \\
17.0 \\
10.2 \\
(s) \\
3.8 \\
1.7 \\
1.4 \\
178.0\end{array}$ & $\begin{array}{r}242.5 \\
46.5 \\
1.0 \\
28.4 \\
17.2 \\
9.3 \\
.2 \\
4.0 \\
.4 \\
3.3 \\
196.0\end{array}$ \\
\hline 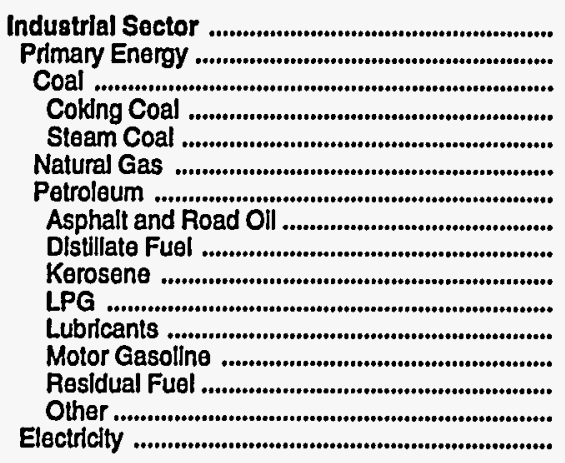 & $\begin{array}{r}57.1 \\
31.3 \\
.2 \\
- \\
.2 \\
7.0 \\
24.1 \\
2.3 \\
3.6 \\
.1 \\
6.5 \\
1.3 \\
1.4 \\
7.3 \\
1.6 \\
25.7\end{array}$ & $\begin{array}{r}160.3 \\
94.2 \\
.8 \\
.8 \\
.8 \\
9.5 \\
83.9 \\
7.6 \\
12.7 \\
.4 \\
26.7 \\
1.4 \\
1.5 \\
21.7 \\
11.8 \\
66.1\end{array}$ & $\begin{array}{r}375.0 \\
252.1 \\
5.4 \\
- \\
5.4 \\
34.8 \\
212.0 \\
8.3 \\
20.5 \\
.6 \\
43.6 \\
6.6 \\
1.8 \\
45.1 \\
85.6 \\
122.9\end{array}$ & $\begin{array}{r}355.5 \\
209.8 \\
7.0 \\
- \\
7.0 \\
93.5 \\
109.3 \\
27.3 \\
15.0 \\
.2 \\
11.4 \\
7.3 \\
2.7 \\
16.1 \\
29.4 \\
145.7\end{array}$ & 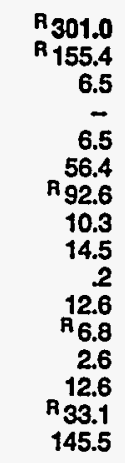 & $\begin{array}{r}R_{280.4} \\
R_{131.5} \\
7.0 \\
- \\
7.0 \\
47.5 \\
R 77.0 \\
2.9 \\
13.6 \\
.3 \\
13.5 \\
R 7.0 \\
2.5 \\
10.3 \\
R_{26.8} \\
148.9\end{array}$ & $\begin{array}{r}\mathrm{R}_{228.4} \\
\mathrm{R}_{129.3} \\
4.7 \\
- \\
4.7 \\
56.1 \\
\mathrm{P}_{68.5} \\
1.2 \\
9.5 \\
.1 \\
6.5 \\
\mathrm{R}^{6.3} \\
2.5 \\
14.9 \\
\mathrm{P}_{25.7} \\
149.1\end{array}$ & $\begin{array}{r}305.9 \\
143.2 \\
5.5 \\
- \\
5.5 \\
63.3 \\
74.4 \\
2.2 \\
9.2 \\
.8 \\
7.0 \\
8.2 \\
3.1 \\
20.7 \\
23.1 \\
162.7\end{array}$ \\
\hline 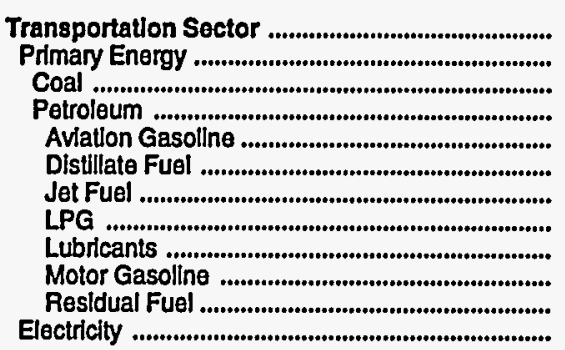 & $\begin{array}{r}107.0 \\
107.0 \\
(s) \\
107.0 \\
.2 \\
2.8 \\
8.1 \\
.1 \\
2.1 \\
92.1 \\
1.8 \\
-\end{array}$ & $\begin{array}{r}206.0 \\
206.0 \\
(s) \\
206.0 \\
.3 \\
8.4 \\
18.0 \\
.5 \\
2.3 \\
166.2 \\
10.4 \\
-\end{array}$ & $\begin{array}{r}453.7 \\
453.7 \\
453.7 \\
.5 \\
43.3 \\
54.6 \\
.2 \\
5.5 \\
329.4 \\
20.1 \\
-\end{array}$ & $\begin{array}{r}498.3 \\
498.3 \\
- \\
498.3 \\
.8 \\
61.3 \\
56.0 \\
2 \\
6.2 \\
367.9 \\
5.8 \\
-\end{array}$ & $\begin{array}{r}\text { R } 561.9 \\
\text { R } 561.9 \\
- \\
R_{561.9} \\
3.6 \\
69.6 \\
44.4 \\
2 \\
2 \\
R_{5.8} \\
424.9 \\
13.4 \\
-\end{array}$ & $\begin{array}{r}\text { R } 547.4 \\
\text { R } 547.4 \\
\text { R } 547.4 \\
.8 \\
67.6 \\
70.9 \\
.3 \\
.3 \\
38.9 \\
385.0 \\
16.9 \\
-\end{array}$ & $\begin{array}{r}\mathrm{R}_{514.0} \\
\mathrm{R}_{514.0} \\
\mathrm{R}_{514.0} \\
.8 \\
64.1 \\
40.1 \\
2 \\
2 \\
\mathrm{R}_{7.0} \\
388.4 \\
\mathrm{R}_{13.4} \\
-\end{array}$ & $\begin{array}{r}536.4 \\
536.4 \\
- \\
536.4 \\
2.1 \\
75.0 \\
37.8 \\
.2 \\
7.0 \\
399.9 \\
14.5 \\
-\end{array}$ \\
\hline 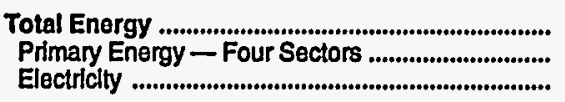 & $\begin{array}{r}264.4 \\
188.8 \\
75.7\end{array}$ & $\begin{array}{l}592.2 \\
390.2 \\
202.1\end{array}$ & $\begin{array}{r}1,336.2 \\
967.5 \\
368.7\end{array}$ & $\begin{array}{r}1,367.9 \\
910.0 \\
457.9\end{array}$ & $\begin{array}{r}\text { A }_{1,417.3} \\
\text { R }_{883.7} \\
533.6\end{array}$ & $\begin{array}{r}\mathrm{R}_{1,415.3} \\
\mathrm{R}_{847.4} \\
567.9\end{array}$ & $\begin{array}{r}R_{1,379.7} \\
R_{811.6} \\
568.1\end{array}$ & $\begin{array}{r}1,494.1 \\
861.5 \\
632.7\end{array}$ \\
\hline 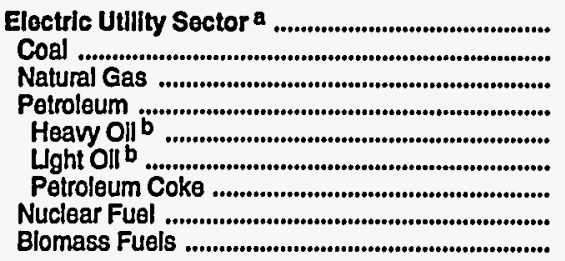 & $\begin{array}{r}23.1 \\
14.2 \\
1.4 \\
7.5 \\
4.5 \\
.8 \\
2.2 \\
= \\
-\end{array}$ & $\begin{array}{r}106.3 \\
25.6 \\
1.9 \\
78.8 \\
76.4 \\
1.7 \\
.7 \\
- \\
-\end{array}$ & $\begin{array}{r}239.3 \\
38.5 \\
25.3 \\
175.6 \\
156.5 \\
6.8 \\
12.2 \\
= \\
-\end{array}$ & $\begin{array}{r}229.9 \\
125.9 \\
29.3 \\
74.7 \\
68.8 \\
3.2 \\
2.7 \\
-\end{array}$ & $\begin{array}{r}171.0 \\
97.3 \\
29.3 \\
44.4 \\
33.9 \\
2.9 \\
7.6 \\
-\end{array}$ & $\begin{array}{r}175.9 \\
91.1 \\
35.8 \\
49.0 \\
39.1 \\
3.2 \\
6.7 \\
- \\
-\end{array}$ & $\begin{array}{r}145.2 \\
73.7 \\
22.6 \\
48.8 \\
37.6 \\
3.3 \\
7.9 \\
= \\
-\end{array}$ & $\begin{array}{r}170.3 \\
97.9 \\
23.4 \\
49.1 \\
46.7 \\
2.4 \\
= \\
-\end{array}$ \\
\hline Primary Energy - Flve Sector ${ }^{\circ} \ldots$ & 211.8 & 496.5 & $1,206.8$ & $1,139.9$ & $B_{1,054.7}$ & $A_{1,023.3}$ & R 956.8 & $1,031.8$ \\
\hline
\end{tabular}

a There are no direct fuel costs for hydroelectric, geothermal, wind, pholovoltalc, or solar themal energy.

Heavy oil includes fuel oll nos. 4,5 , and 6 , and residual fuel olls. Light ofl includes fuel nos. 1 and 2, kerosene, and jet fuel.

- Blomass fuels are not included, except those consumed at electric utllities and those added to motor gasoline.

$R=$ Rovised date.

-No consumption, Including cases where adjustments were made. See explanation of adjustments in Section 6 of Appendix A.

$(s)=$ Value less than 0.05 million dollars.

Note: Totals may not equal sum of components due to Independent rounding.

Sources: Data sources, estimation procodures, and assumptions are described in Appendix A. 


\section{Energy Price and Expenditure Estimates by Source, District of Columbia $1970,1975,1980,1985$, and $1990-1993$}

\begin{tabular}{|c|c|c|c|c|c|c|c|c|}
\hline Energy Source & 1970 & 1975 & 1980 & 1985 & 1990 & 1991 & 1992 & 1993 \\
\hline & \multicolumn{8}{|c|}{ Prices in Dollars per Million Btu } \\
\hline Coal & 0.29 & 1.32 & 1.76 & 2.01 & 1.96 & 1.96 & 1.95 & 1.96 \\
\hline Coking Coal & - & - & - & - & - & - & - & - \\
\hline 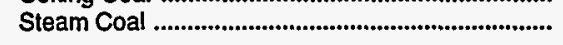 & .29 & 1.32 & 1.76 & 2.01 & 1.96 & 1.96 & 1.95 & 1.96 \\
\hline 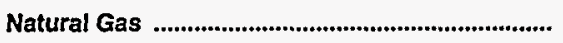 & 1.27 & 2.13 & 4.36 & $\mathbf{7 . 3 0}$ & 6.40 & 6.08 & 6.46 & 7.01 \\
\hline Petroleum & 1.19 & 3.30 & 7.86 & 8.74 & $\mathrm{R}_{8.78}$ & $R_{8.51}$ & $\mathrm{R}_{8.39}$ & 7.97 \\
\hline Asphalt and Road Oil .............................................. & $\begin{array}{r}.67 \\
-\end{array}$ & 1.80 & $\begin{array}{r}3.58 \\
-\end{array}$ & $\begin{array}{r}4.95 \\
-\end{array}$ & 2.94 & $\begin{array}{r}3.10 \\
-\end{array}$ & 2.35 & $\begin{array}{l}2.87 \\
8.24\end{array}$ \\
\hline Distillate Fuel & 1.09 & 2.61 & 7.18 & 7.95 & 8.26 & 7.21 & $6 . \overline{77}$ & $\begin{array}{l}8.24 \\
6.47\end{array}$ \\
\hline Jet Fuel & .73 & - & 6.46 & 5.80 & 5.47 & - & - & 4.16 \\
\hline 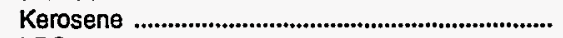 & 1.41 & 2.74 & 8.50 & 7.72 & 6.21 & 7.46 & 7.20 & 7.20 \\
\hline LPG & 1.49 & 3.37 & 6.00 & 11.49 & 10.89 & 11.78 & 10.16 & 10.25 \\
\hline 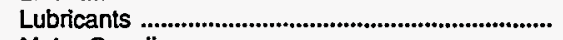 & 5.08 & 7.48 & 14.36 & 17.61 & $R_{14.60}$ & $R_{16.79}$ & $R_{19.43}$ & 18.94 \\
\hline Motor Gasoline & 2.86 & 4.85 & 9.97 & 10.19 & 10.66 & 10.14 & 9.73 & 9.62 \\
\hline 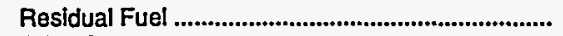 & .50 & 1.97 & 4.46 & 4.36 & 3.22 & 2.61 & 2.79 & 2.42 \\
\hline Other $^{\mathrm{a}}$ & - & - & - & - & - & - & - & - \\
\hline 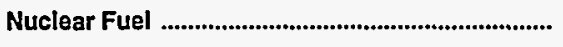 & - & - & - & - & - & - & - & - \\
\hline 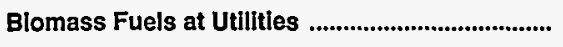 & - & - & - & - & - & - & - & - \\
\hline Primary Energy - Five Sectors ${ }^{b} \ldots \ldots \ldots . . . . . . . . . .$. & 1.06 & 2.85 & 6.36 & 7.83 & 7.59 & $\mathrm{R}_{7.23}$ & ${ }^{R} 7.33$ & 7.42 \\
\hline 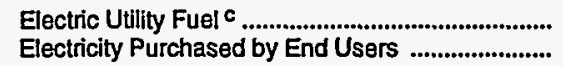 & $\begin{array}{r}.43 \\
5.39\end{array}$ & $\begin{array}{r}1.92 \\
10.74\end{array}$ & $\begin{array}{r}4.59 \\
14.92\end{array}$ & $\begin{array}{r}4.24 \\
20.89\end{array}$ & $\begin{array}{r}3.12 \\
17.38\end{array}$ & $\begin{array}{r}2.46 \\
18.34\end{array}$ & $\begin{array}{r}2.81 \\
18.97\end{array}$ & $\begin{array}{r}2.31 \\
19.83\end{array}$ \\
\hline \multirow[t]{2}{*}{ Total Energy } & 1.72 & 4.33 & 8.75 & 11.67 & $R_{11.28}$ & 11.27 & $R_{11.37}$ & 11.81 \\
\hline & \multicolumn{8}{|c|}{ Expenditures In Millions of Dollars } \\
\hline 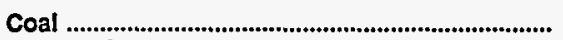 & 8.4 & 13.4 & 5.8 & 7.0 & 3.4 & 3.2 & 2.4 & 2.5 \\
\hline 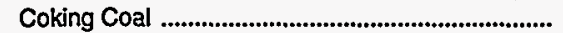 & - & - & - & - & - & - & - & - \\
\hline 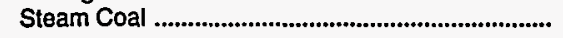 & 8.4 & 13.4 & 5.8 & 7.0 & 3.4 & 3.2 & 2.4 & 2.5 \\
\hline Natural Gas & 33.5 & 55.7 & 121.8 & 211.5 & 184.5 & 188.5 & 212.5 & 231.7 \\
\hline 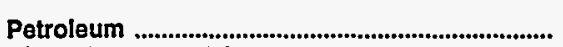 & 154.1 & 250.8 & 374.9 & 337.1 & $A_{326.6}$ & $\mathrm{R}_{296.8}$ & $\mathrm{~A}_{282.8}$ & 293.1 \\
\hline 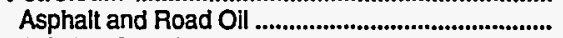 & .1 & 2 & .4 & .9 & .6 & .5 & .3 & .5 \\
\hline 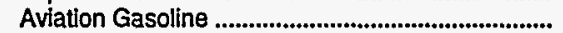 & - & - & - & - & - & - & - & .1 \\
\hline 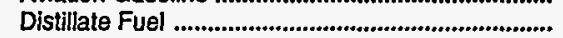 & 31.4 & 48.1 & 95.6 & 103.2 & 74.0 & 65.1 & 61.2 & 61.5 \\
\hline 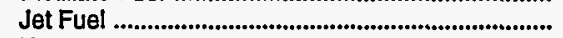 & (s) & - & 12.1 & .2 & .2 & - & - & 2.4 \\
\hline 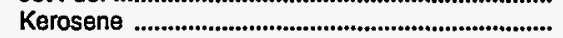 & .4 & 1.7 & 12.9 & 3.0 & .4 & .3 & .3 & .4 \\
\hline LPG & (s) & .1 & .1 & .2 & .2 & .2 & .3 & 2 \\
\hline 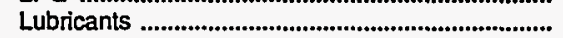 & 1.7 & 2.7 & 5.3 & 5.9 & $A_{5.5}$ & ${ }^{A} 5.7$ & $\mathrm{R}_{6.7}$ & 6.7 \\
\hline Motor Gasoline & 85.4 & 146.4 & 203.3 & 203.4 & 225.1 & 214.2 & 205.7 & 211.5 \\
\hline 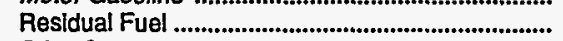 & 35.1 & 51.6 & 45.2 & 20.3 & 20.7 & 10.9 & 8.3 & 9.9 \\
\hline Other ${ }^{a}$ & - & - & - & - & - & - & - & - \\
\hline Nuclear Fuel & - & - & - & - & - & - & - & - \\
\hline Blomass Fuels at Utilitles .................................... & - & - & - & - & - & - & - & - \\
\hline Primary Energy - Flve Sectors ${ }^{b}$........................ & 195.9 & 319.9 & 502.4 & 555.6 & $\mathbf{A}_{514.6}$ & $R_{488.5}$ & $P_{497.8}$ & 527.3 \\
\hline 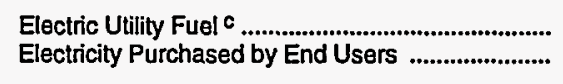 & $\begin{array}{r}-18.0 \\
99.2\end{array}$ & $\begin{array}{r}-31.7 \\
212.3\end{array}$ & $\begin{array}{r}-45.1 \\
356.5\end{array}$ & $\begin{array}{r}-8.3 \\
585.3\end{array}$ & $\begin{array}{l}-17.0 \\
584.0\end{array}$ & $\begin{array}{r}-7.6 \\
638.0\end{array}$ & $\begin{array}{r}-4.4 \\
650.0\end{array}$ & $\begin{array}{r}-6.9 \\
702.0\end{array}$ \\
\hline 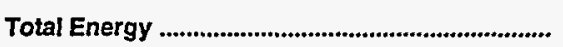 & 277.2 & 500.5 & 813.8 & $1,132.6$ & $R_{1,081.6}$ & $R_{1,118.9}$ & $R_{1,143.4}$ & $1,222.4$ \\
\hline
\end{tabular}

\footnotetext{
Includes petroleum coke used at electric utilities.

b Blomass fuels are not included, except those consumed at electric utilities and those added to motor gasoline.

c There are no direct fuel costs for hydroelectric, geothermal, wind, photovoltaic, or solar thermal energy.

$R=$ Revised data.

-No consumption, including cases where adjustments were made. Seo
}

explanation of adjustments in Sectlon 6 of Appendix A.

$(s)=$ Value less than 0.05 million dollars.

Note: Expenditure totals may not equal sum of components due to independent rounding.

Sources: Data sources, estimation procedures, and assumptions are described in Appendix A. 
Energy Price Estimates by Sector, District of Columbia

1970, 1975, 1980, 1985, and 1990-1993

(Dollars per Million Btu)

\begin{tabular}{|c|c|c|c|c|c|c|c|c|}
\hline Sector and Energy Source & 1970 & 1975 & 1980 & 1985 & 1990 & 1991 & 1992 & 1993 \\
\hline 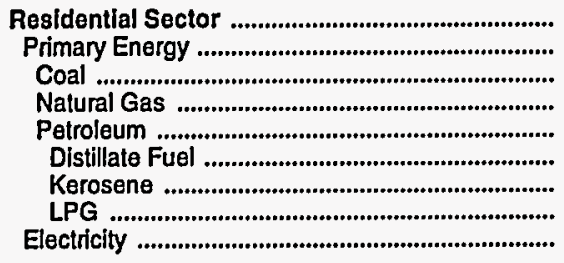 & $\begin{array}{l}2.01 \\
1.42 \\
1.05 \\
1.43 \\
1.42 \\
1.42 \\
1.50 \\
2.57 \\
7.02\end{array}$ & $\begin{array}{r}3.79 \\
2.44 \\
1.75 \\
2.30 \\
2.71 \\
2.71 \\
3.37 \\
4.61 \\
12.65\end{array}$ & $\begin{array}{r}7.12 \\
5.15 \\
3.18 \\
4.56 \\
7.41 \\
7.40 \\
8.55 \\
9.81 \\
17.32\end{array}$ & $\begin{array}{r}9.77 \\
7.67 \\
3.28 \\
7.80 \\
8.74 \\
8.74 \\
8.88 \\
13.53 \\
20.31\end{array}$ & $\begin{array}{r}9.54 \\
7.05 \\
3.36 \\
7.12 \\
8.26 \\
8.24 \\
8.37 \\
12.58 \\
17.82\end{array}$ & $\begin{array}{r}9.96 \\
6.98 \\
3.09 \\
7.03 \\
8.60 \\
8.58 \\
8.71 \\
13.46 \\
19.29\end{array}$ & $\begin{array}{r}10.06 \\
7.48 \\
3.10 \\
7.56 \\
8.11 \\
8.08 \\
8.21 \\
13.03 \\
19.30\end{array}$ & $\begin{array}{r}11.16 \\
8.14 \\
3.23 \\
8.28 \\
8.02 \\
7.99 \\
8.12 \\
13.36 \\
20.97\end{array}$ \\
\hline 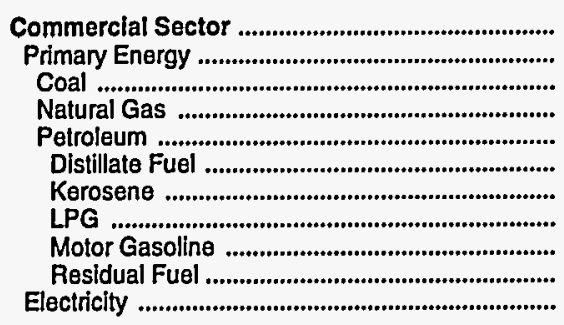 & $\begin{array}{r}1.40 \\
.71 \\
.11 \\
1.09 \\
.61 \\
1.12 \\
1.33 \\
1.03 \\
2.86 \\
.46 \\
6.86\end{array}$ & $\begin{array}{r}4.63 \\
2.11 \\
1.25 \\
1.96 \\
2.28 \\
2.39 \\
2.70 \\
2.75 \\
4.85 \\
2.02 \\
12.49\end{array}$ & $\begin{array}{r}8.62 \\
4.45 \\
1.19 \\
4.21 \\
6.60 \\
6.55 \\
8.50 \\
5.13 \\
9.97 \\
4.43 \\
18.41\end{array}$ & $\begin{array}{r}12.94 \\
5.98 \\
1.33 \\
6.62 \\
6.42 \\
6.73 \\
7.51 \\
10.88 \\
10.19 \\
5.16 \\
22.82\end{array}$ & $\begin{array}{r}11.78 \\
5.54 \\
1.21 \\
5.59 \\
6.43 \\
7.11 \\
5.25 \\
10.33 \\
10.66 \\
3.91 \\
18.53\end{array}$ & $\begin{array}{r}11.65 \\
5.02 \\
1.34 \\
5.14 \\
5.44 \\
6.02 \\
6.21 \\
11.09 \\
10.14 \\
3.38 \\
19.42\end{array}$ & $\begin{array}{r}\mathrm{R}_{11.92} \\
5.01 \\
1.32 \\
5.32 \\
4.62 \\
5.15 \\
6.37 \\
9.50 \\
9.73 \\
3.11 \\
\mathrm{R} \\
20.16\end{array}$ & $\begin{array}{r}12.33 \\
5.31 \\
1.27 \\
5.71 \\
4.78 \\
5.11 \\
6.19 \\
9.32 \\
9.62 \\
2.92 \\
20.84\end{array}$ \\
\hline & 1.19 & 4.29 & 10.20 & 17.08 & $\begin{array}{r}\mathrm{R}_{14.73} \\
\mathrm{R}_{8.69}\end{array}$ & $\begin{array}{r}R_{15.58} \\
R_{8.46}\end{array}$ & $\begin{array}{r}16.20 \\
R 709\end{array}$ & 16.84 \\
\hline Primary Energy & $\begin{array}{l}.49 \\
.11\end{array}$ & $\begin{array}{l}1.63 \\
1.25\end{array}$ & $\begin{array}{l}6.16 \\
1.20\end{array}$ & 8.31 & ${ }^{\mathrm{R}} 8.69$ & ${ }^{\mathrm{R}} 8.46$ & $\begin{array}{r}{ }^{\mathrm{A}} 7.99 \\
-\end{array}$ & $\begin{array}{r}7.22 \\
-\end{array}$ \\
\hline Coking Coal & - & - & - & - & - & - & - & - \\
\hline 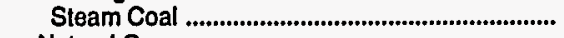 & .11 & 1.25 & 1.20 & - & - & - & - & - \\
\hline 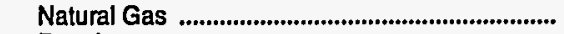 & .67 & 1.36 & 2.45 & - & - & - & - & - \\
\hline Petroleum & .66 & 2.08 & 7.60 & 8.31 & R 8.69 & ${ }^{\mathrm{R}} 8.46$ & R7.99 & 7.22 \\
\hline $\begin{array}{l}\text { Asphalt and Road Oil } \\
\text { Distillate Fuel }\end{array}$ & .67 & $\begin{array}{l}1.80 \\
2.50\end{array}$ & $\begin{array}{l}3.58 \\
7.63\end{array}$ & $\begin{array}{l}4.95 \\
6.73\end{array}$ & $\begin{array}{l}2.94 \\
6.14\end{array}$ & $\begin{array}{l}3.10 \\
5.56\end{array}$ & $\begin{array}{l}2.35 \\
5.70\end{array}$ & 2.87 \\
\hline $\begin{array}{l}\text { Distillate Fuel } \\
\text { Kerosene }\end{array}$ & $\begin{array}{l}1.22 \\
1.33\end{array}$ & $\begin{array}{l}2.50 \\
2.70\end{array}$ & 8.50 & 7.51 & 0.14 & 6.21 & 5.70 & $\begin{array}{c}5.54 \\
-\end{array}$ \\
\hline Rer & 1.03 & 2.75 & 5.13 & 10.88 & 10.33 & 11.09 & 9.50 & 9.32 \\
\hline 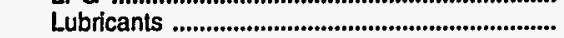 & 5.08 & 7.48 & 14.36 & 17.61 & $\mathrm{P}_{14.60}$ & R 16.79 & ${ }^{A} 19.43$ & 18.94 \\
\hline 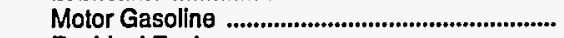 & - & - & - & 10.19 & 10.66 & 10.14 & 9.73 & 9.62 \\
\hline 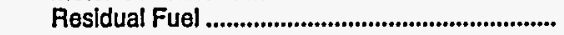 & .59 & 1.82 & 3.97 & 5.16 & 3.91 & 3.38 & 3.11 & - \\
\hline Other & - & - & $\overline{-}$ & $-\bar{c}$ & $-\overline{-}$ & - & $\bar{s}$ & $\overline{0}$ \\
\hline 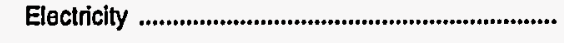 & 3.80 & 8.42 & 11.65 & 17.86 & 15.17 & 15.94 & 16.68 & 17.33 \\
\hline $\begin{array}{c}\text { Transportation Sector } \\
\text { Primary Energy }\end{array}$ & $\begin{array}{l}2.74 \\
2.74\end{array}$ & $\begin{array}{l}4.43 \\
4.43\end{array}$ & $\begin{array}{l}9.44 \\
9.40\end{array}$ & $\begin{array}{l}9.85 \\
9.68\end{array}$ & $\begin{array}{l}A_{10.55} \\
A_{10.45}\end{array}$ & $\begin{array}{l}\text { R } 9.99 \\
\text { R } 9.86\end{array}$ & $\begin{array}{l}\text { R9.64 } \\
\text { R9.50 }\end{array}$ & $\begin{array}{l}9.54 \\
9.39\end{array}$ \\
\hline Coal & .11 & 1.25 & & - & & - & - & - \\
\hline Petroleum & 2.74 & 4.43 & 9.40 & 9.68 & ${ }^{A} 10.45$ & R 9.86 & ${ }^{8} 9.50$ & 9.39 \\
\hline 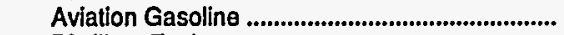 & - & - & - & $\overrightarrow{-}$ & - & - & - & 8.24 \\
\hline 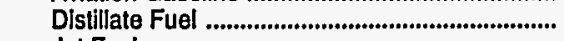 & 1.32 & 2.81 & 7.70 & 8.78 & 9.33 & 8.05 & 7.83 & 8.00 \\
\hline Jet Fuel & .73 & $=$ & 6.46 & 5.80 & 5.47 & & - & 4.16 \\
\hline LPG & 1.03 & 2.75 & 5.13 & 10.88 & 10.33 & 11.09 & 9.50 & 9.32 \\
\hline 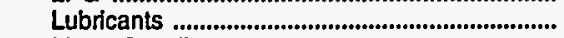 & 5.08 & 7.48 & 14.36 & 17.61 & $\mathrm{~A}_{14.60}$ & ${ }^{\mathrm{A}} 16.79$ & $R_{19.43}$ & 18.94 \\
\hline 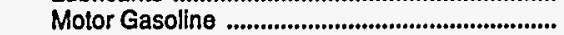 & 2.86 & 4.85 & 9.97 & 10.19 & 10.66 & 10.14 & 9.73 & 9.62 \\
\hline 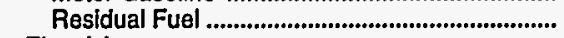 & .45 & 1.81 & 4.20 & 3.75 & 2.88 & - & 2.27 & - \\
\hline 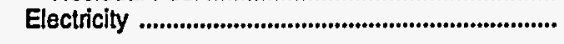 & - & - & 12.62 & 20.73 & 16.54 & 18.09 & 18.05 & 18.64 \\
\hline 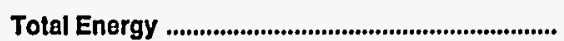 & 1.72 & 4.33 & 8.75 & 11.67 & $\mathrm{~A}_{11.28}$ & 11.27 & R 11.37 & 11.81 \\
\hline 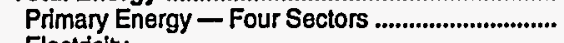 & 1.25 & 3.01 & 6.62 & 7.93 & 7.98 & ค 7.46 & $\mathrm{R}_{7.44}$ & 7.64 \\
\hline 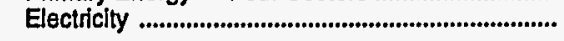 & 5.39 & 10.74 & 14.92 & 20.89 & 17.38 & 18.34 & 18.97 & 19.83 \\
\hline Electric Utility Sector $\mathrm{a}$.................................................. & .43 & 1.92 & 4.59 & 4.24 & 3.12 & 2.46 & 2.81 & 2.31 \\
\hline 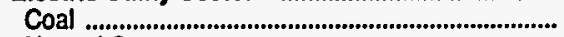 & .39 & 1.50 & - & - & - & - & - & - \\
\hline Natural Gas ..................................................................... & - & - & - & - & - & - & - & - \\
\hline Petroleum & .47 & 2.01 & 4.59 & 4.24 & 3.12 & 2.46 & 2.81 & 2.31 \\
\hline 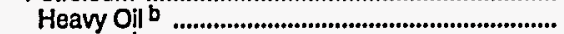 & .47 & 2.01 & 4.49 & 3.94 & 3.02 & 2.22 & 2.38 & 2.19 \\
\hline Ught Oil $\mathrm{b}$ & .46 & 2.11 & 5.95 & 5.43 & 4.29 & 4.56 & 4.45 & 3.98 \\
\hline Petroleum Coke ................................................................... & - & - & - & - & - & - & - & - \\
\hline 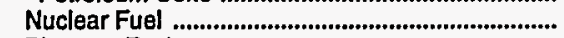 & - & - & - & - & - & - & - & - \\
\hline 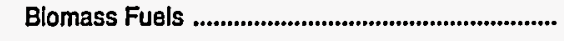 & - & - & - & - & - & - & - & - \\
\hline Primary Energy — Five Sectors ${ }^{c}$.............................. & 1.06 & 2.85 & 6.36 & 7.83 & 7.59 & $\mathrm{R}_{7.23}$ & ${ }^{R} 7.33$ & 7.42 \\
\hline
\end{tabular}

a There are no direct fuel costs for hydroelectric, geothermal, wind, photovoltalc, or solar thermal energy.

beavy ofl includes fuel oil nos. 4, 5, and 6, and residual fuel oils. Light oil includes fuel oil nos. 1 and 2 , kerosene, and jet fuel.

c Biomass fuels are not included, except those consumed at electric utilities and those added to motor gasoline.

$R=$ Revised data.

-No consumption, including cases where adjustments were made. See explanation of adjustments in Section 6 of Appendix A.

Sources: Data sources, estimation procedures, and assumptions are described in Appendix A. 
D Energy Expenditure Estimates by Sector, District of Columbia 1970, 1975, 1980, 1985, and 1990-1993

S (Million Dollars)

\begin{tabular}{|c|c|c|c|c|c|c|c|c|}
\hline Sector and Energy Source & 1970 & 1975 & 1980 & 1985 & 1990 & 1991 & 1992 & 1893 \\
\hline $\begin{array}{l}\text { Residentlal Sector } \\
\text { Primary Energy } \\
\text { Coal } \\
\text { Natural Gas } \\
\text { Petroleum } \\
\text { Distillate Fuel } \\
\text { Kerosene } \\
\text { LPG }\end{array}$ & $\begin{array}{r}54.0 \\
34.1 \\
.4 \\
20.2 \\
13.6 \\
13.4 \\
.2 \\
.2 \\
(s) \\
19.9\end{array}$ & $\begin{array}{r}88.6 \\
49.4 \\
.2 \\
30.7 \\
18.5 \\
18.3 \\
.1 \\
(s) \\
39.2\end{array}$ & $\begin{array}{r}162.5 \\
98.3 \\
3.0 \\
62.8 \\
32.6 \\
32.3 \\
.2 \\
(s) \\
64.1\end{array}$ & $\begin{array}{r}246.6 \\
161.2 \\
4.0 \\
131.4 \\
25.8 \\
25.2 \\
.5 \\
(\mathrm{~s}) \\
85.4\end{array}$ & $\begin{array}{r}208.1 \\
118.1 \\
2.0 \\
108.7 \\
7.4 \\
7.2 \\
.2 \\
(s) \\
90.0\end{array}$ & $\begin{array}{r}222.4 \\
118.4 \\
1.8 \\
108.1 \\
8.5 \\
8.2 \\
.2 \\
.1 \\
104.0\end{array}$ & $\begin{array}{r}233.8 \\
135.8 \\
1.4 \\
126.2 \\
8.2 \\
8.0 \\
.2 \\
.1 \\
.1\end{array}$ & $\begin{array}{r}264.7 \\
147.7 \\
1.5 \\
138.4 \\
7.9 \\
7.6 \\
.2 \\
.1 \\
117.0\end{array}$ \\
\hline $\begin{array}{l}\text { Commerclal Sector } \\
\text { Primary Energy } \\
\text { Coal } \\
\text { Natural Gas } \\
\text { Petroleum } \\
\text { Distillate Fuel } \\
\text { Kerosene } \\
\text { LPG } \\
\text { Motor Gasoline } \\
\text { Residual Fuel }\end{array}$ & $\begin{array}{r}82.6 \\
37.4 \\
.1 \\
12.9 \\
24.3 \\
8.5 \\
.1 \\
(s) \\
1.0 \\
14.8 \\
45.3\end{array}$ & $\begin{array}{r}153.4 \\
53.1 \\
.3 \\
24.4 \\
28.4 \\
13.0 \\
.1 \\
(s) \\
2.0 \\
13.4 \\
100.4\end{array}$ & $\begin{array}{r}242.5 \\
88.0 \\
2.1 \\
58.0 \\
27.9 \\
24.7 \\
(s) \\
(s) \\
2.1 \\
1.0 \\
154.6\end{array}$ & $\begin{array}{r}462.6 \\
125.5 \\
3.0 \\
80.1 \\
42.5 \\
29.4 \\
2.3 \\
(\mathrm{~s}) \\
1.5 \\
9.3 \\
337.0\end{array}$ & $\begin{array}{r}440.7 \\
107.6 \\
1.4 \\
75.9 \\
30.4 \\
20.8 \\
.2 \\
(s) \\
4.0 \\
5.4 \\
333.1\end{array}$ & $\begin{array}{r}469.5 \\
109.2 \\
1.4 \\
80.4 \\
27.3 \\
20.6 \\
.1 \\
(s) \\
1.9 \\
4.7 \\
360.4\end{array}$ & $\begin{array}{r}{ }^{8} 484.8 \\
110.8 \\
1.1 \\
86.3 \\
23.4 \\
16.5 \\
.2 \\
(\mathrm{~s}) \\
1.5 \\
5.3 \\
\text { R } 374.0\end{array}$ & $\begin{array}{r}524.6 \\
123.8 \\
1.1 \\
93.3 \\
29.4 \\
23.8 \\
.2 \\
(8) \\
1.6 \\
3.8 \\
401.0\end{array}$ \\
\hline $\begin{array}{l}\text { Industrlal Sector } \\
\text { Primary Energy }\end{array}$ & $\begin{array}{l}50.6 \\
16.6\end{array}$ & 94.4 & $\begin{array}{r}158.6 \\
25.2\end{array}$ & $\begin{array}{r}160.8 \\
6.4\end{array}$ & $\begin{array}{r}160.4 \\
6.4\end{array}$ & $\begin{array}{r}170.4 \\
4.4\end{array}$ & $\begin{array}{r}174.7 \\
4.7\end{array}$ & $\begin{array}{r}179.8 \\
3.8\end{array}$ \\
\hline Coal & 1.1 & 8.7 & .7 & - & - & - & - & - \\
\hline 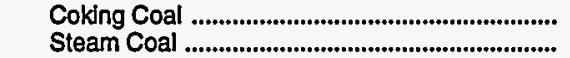 & $\overline{1.1}$ & $\overline{8.7}$ & $\overline{7}$ & $\overline{-}$ & $\overline{-}$ & $\overline{-}$ & 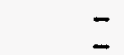 & $\overline{-}$ \\
\hline & .3 & .6 & .9 & - & - & $\overline{-}$ & $\overline{-}$ & $\overline{-}$ \\
\hline Petroleum & $\begin{array}{r}15.2 \\
.1\end{array}$ & $\begin{array}{r}12.4 \\
.2\end{array}$ & $\begin{array}{r}23.6 \\
.4\end{array}$ & $\begin{array}{r}6.4 \\
.9\end{array}$ & $\begin{array}{r}6.4 \\
.6\end{array}$ & $\begin{array}{r}4.4 \\
.5\end{array}$ & $\begin{array}{r}4.7 \\
.3\end{array}$ & $\begin{array}{r}3.8 \\
.5\end{array}$ \\
\hline 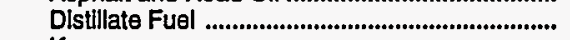 & 2.7 & 2.2 & 8.5 & 1.4 & .1 & .1 & .4 & .5 \\
\hline & .1 & 1.5 & 12.6 & .1 & - & (s) & - & - \\
\hline LPG & (s) & (s) & .1 & .1 & ค. .1 & R. .1 & $\mathrm{R} . .2$ & .1 \\
\hline Motor Gasoline & $\ddot{-}$ & $\ddot{-}$ & - & 3.1 & 5.0 & 3.1 & 3.0 & 1.8 \\
\hline 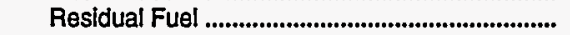 & 12.2 & 7.9 & 1.3 & (s) & (s) & (s) & (s) & - \\
\hline 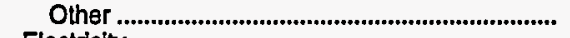 & - & - & 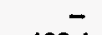 & & 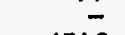 & $-\overrightarrow{1}$ & $-\overline{0}$ & $\overline{0}$ \\
\hline Electricity & 34.1 & 72.7 & 133.4 & 154.4 & 154.0 & 166.0 & 170.0 & 176.0 \\
\hline $\begin{array}{l}\text { Transportatlon Sector } \\
\text { Primary Energy }\end{array}$ & $\begin{array}{l}89.9 \\
89.9\end{array}$ & $\begin{array}{l}164.0 \\
164.0\end{array}$ & $\begin{array}{l}250.2 \\
245.8\end{array}$ & $\begin{array}{l}262.6 \\
254.2\end{array}$ & $\begin{array}{l}R_{272.4} \\
R_{265.4}\end{array}$ & $\begin{array}{l}R_{256.6} \\
R_{248.9}\end{array}$ & $\begin{array}{l}R_{250.0} \\
A_{242.0}\end{array}$ & $\begin{array}{l}253.0 \\
245.1\end{array}$ \\
\hline Coal & $\begin{array}{c}(\mathbf{s}) \\
89.9\end{array}$ & $\begin{array}{r}(s) \\
164.0\end{array}$ & $24 \overline{5}$ & $254 \overline{2}$ & $\mathrm{R}_{265.4}$ & $\mathrm{~A}_{248 . \overline{9}}$ & $\mathrm{R}_{242.0}$ & $24 \overline{1}$ \\
\hline 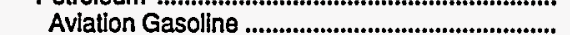 & - & - & - & - & 200 & - & - & .1 \\
\hline 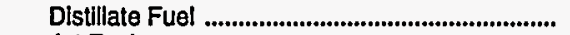 & 3.8 & 13.4 & 26.3 & 45.1 & 44.1 & 34.7 & 34.8 & 28.7 \\
\hline $\begin{array}{l}\text { Jat Fuel } \\
\text { LPG }\end{array}$ & $\begin{array}{l}(s) \\
(s)\end{array}$ & $\overline{(s)}$ & $\begin{array}{c}12.1 \\
(s)\end{array}$ & $\stackrel{.2}{(s)}$ & $\stackrel{.2}{(s)}$ & $\overline{(s)}$ & $\overline{(s)}$ & $\begin{array}{l}2.4 \\
(s)\end{array}$ \\
\hline $\begin{array}{l}\text { Lubricants } \\
\text { Motor Gasoline }\end{array}$ & $\begin{array}{r}1.6 \\
84.4\end{array}$ & $\begin{array}{r}2.1 \\
144.4\end{array}$ & $\begin{array}{r}4.7 \\
201.2\end{array}$ & $\begin{array}{r}5.2 \\
198.8\end{array}$ & $\begin{array}{r}R 4.9 \\
216.2\end{array}$ & $\begin{array}{l}85.0 \\
209.2\end{array}$ & $\begin{array}{r}R_{5.9} \\
201.2\end{array}$ & $\begin{array}{r}5.9 \\
208.0\end{array}$ \\
\hline Residual Fuel & $(s)$ & 4.0 & 1.6 & 4.8 & .1 & - & .1 & - \\
\hline 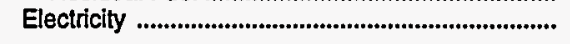 & - & - & 4.4 & 8.4 & 6.9 & 7.6 & R 8.0 & 7.9 \\
\hline 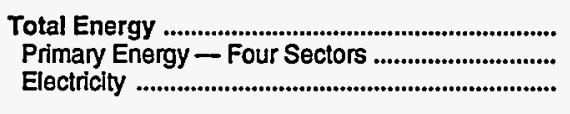 & $\begin{array}{r}277.2 \\
178.0 \\
99.2\end{array}$ & $\begin{array}{l}500.5 \\
288.2 \\
212.3\end{array}$ & $\begin{array}{l}813.8 \\
457.3 \\
356.5\end{array}$ & $\begin{array}{r}1,132.6 \\
547.3 \\
585.3\end{array}$ & $\begin{array}{r}R_{1}, 081.6 \\
R_{497.6} \\
584.0\end{array}$ & $\begin{array}{r}R_{1,118.9} \\
R_{480.9} \\
638.0\end{array}$ & $\begin{array}{r}R_{1,143.4} \\
R_{493.4} \\
650.0\end{array}$ & $\begin{array}{r}1,222.4 \\
520.4 \\
702.0\end{array}$ \\
\hline 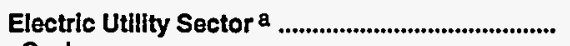 & 18.0 & 31.7 & 45.1 & 8.3 & 17.0 & 7.6 & 4.4 & 6.9 \\
\hline Coal & 6.8 & 4.2 & - & - & - & - & - & - \\
\hline Natural Gas & - & - & - & - & - & - & - & - \\
\hline Petroleum & 11.2 & 27.5 & 45.1 & 8.3 & 17.0 & 7.6 & 4.4 & 6.9 \\
\hline Heavy Oilb & 8.1 & 26.4 & 41.3 & 6.2 & 15.2 & 6.2 & 2.9 & 6.1 \\
\hline 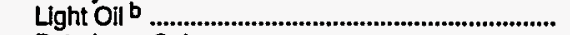 & 3.1 & 1.1 & 3.8 & 2.1 & 1.8 & 1.4 & 1.5 & .8 \\
\hline 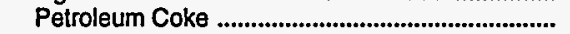 & - & - & - & - & - & - & - & - \\
\hline 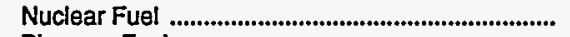 & - & - & - & - & - & - & - & - \\
\hline 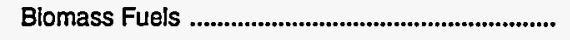 & - & - & - & - & - & - & - & - \\
\hline 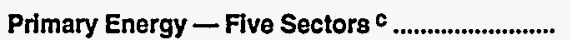 & 195.9 & 319.9 & 502.4 & 555.6 & $\mathbf{R}_{514.6}$ & $R_{488.5}$ & $P_{497.8}$ & 527.3 \\
\hline
\end{tabular}

a There are no direct fuel costs for hydroelectric, geothermal, wind, photovoltaic, or solar thermal energy.

b Heavy oil includes fuel oil nos. 4,5 , and 6 , and residual fuel oils. Light oll includes fuel nos. 1 and 2 , kerosene, and jet fuel.

c Biomass fuels are not included, except those consumed at electric utilitiles and those added to motor gasoline.

$R=$ Revised data.

- No consumption, including cases where adjustments were made. See explanation of adjustments in Section 6 of Appendix A.

(s) $=$ Value less than 0.05 million dollars.

Note: Totals may not equal sum of components due to Independent rounding.

Sources: Data sources, estimation procedures, and assumptions are described in Appendix A. 


\begin{tabular}{|c|c|c|c|c|c|c|c|c|}
\hline Energy Source & 1970 & 1975 & 1980 & 1985 & 1990 & 1991 & 1992 & 1993 \\
\hline & \multicolumn{8}{|c|}{ Prices In Dollars per Million Btu } \\
\hline 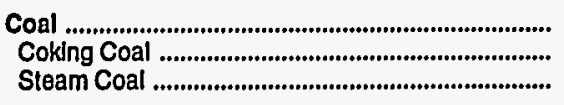 & $\begin{array}{r}0.31 \\
.31\end{array}$ & $\begin{array}{r}1.01 \\
1.01\end{array}$ & $\begin{array}{r}1.80 \\
1.80\end{array}$ & 2.12 & $\begin{array}{r}1.85 \\
1.85\end{array}$ & $\begin{array}{r}1.86 \\
1.86\end{array}$ & 1.83 & $\frac{1.78}{1.78}$ \\
\hline 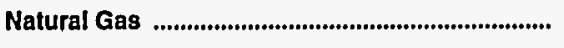 & .49 & 1.00 & 2.19 & 3.73 & 3.21 & 2.84 & 3.00 & 3.38 \\
\hline 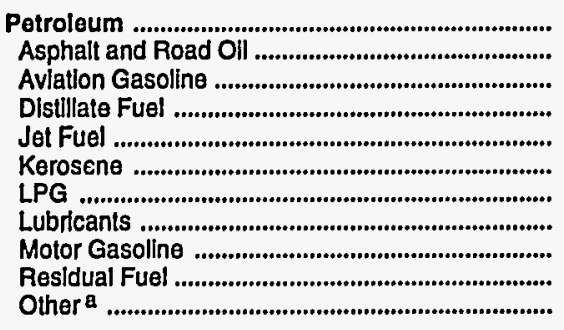 & $\begin{array}{r}1.53 \\
.67 \\
2.17 \\
1.08 \\
.73 \\
1.28 \\
2.61 \\
5.08 \\
2.81 \\
.33 \\
1.35\end{array}$ & $\begin{array}{l}3.02 \\
1.78 \\
3.45 \\
2.53 \\
2.03 \\
3.12 \\
5.14 \\
7.48 \\
4.39 \\
1.84 \\
3.11\end{array}$ & $\begin{array}{r}6.66 \\
3.57 \\
9.02 \\
6.91 \\
6.46 \\
8.45 \\
7.48 \\
14.36 \\
9.80 \\
3.61 \\
7.97\end{array}$ & $\begin{array}{r}7.46 \\
4.99 \\
9.99 \\
6.78 \\
5.90 \\
7.46 \\
10.91 \\
17.61 \\
9.03 \\
3.90 \\
8.30\end{array}$ & $\begin{array}{r}R_{6.96} \\
2.91 \\
9.32 \\
7.86 \\
5.64 \\
10.50 \\
11.70 \\
R_{14.60} \\
8.85 \\
2.92 \\
R_{6.62}\end{array}$ & $\begin{array}{r}\mathbf{R}_{6.41} \\
3.06 \\
8.71 \\
7.27 \\
4.93 \\
11.47 \\
12.19 \\
R_{16.79} \\
8.48 \\
2.20 \\
R_{5.85}\end{array}$ & $\begin{array}{r}6.42 \\
2.25 \\
8.54 \\
7.58 \\
4.56 \\
14.47 \\
14.15 \\
R 19.43 \\
8.29 \\
2.36 \\
R 5.84\end{array}$ & $\begin{array}{r}6.28 \\
2.86 \\
8.24 \\
6.88 \\
4.23 \\
8.74 \\
14.90 \\
18.94 \\
8.68 \\
2.16 \\
5.32\end{array}$ \\
\hline 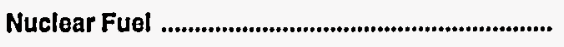 & - & .17 & .35 & .65 & .64 & .66 & .62 & .63 \\
\hline Blomass Fuels at Utilities .......................................... & - & - & - & - & - & - & - & - \\
\hline Primary Energy - Five Sectors $b$ & 1.20 & 2.44 & 5.12 & 5.17 & $\mathrm{P}_{4.82}$ & $\mathrm{~F}_{4.44}$ & 4.39 & 4.39 \\
\hline $\begin{array}{l}\text { Electric Utility Fuel c ................................................ } \\
\text { Electricity Purchased by End Users ...................... }\end{array}$ & $\begin{array}{r}.33 \\
5.67\end{array}$ & $\begin{array}{r}1.35 \\
10.46\end{array}$ & $\begin{array}{r}2.40 \\
16.24\end{array}$ & $\begin{array}{r}2.21 \\
22.59\end{array}$ & $\begin{array}{r}1.98 \\
20.62\end{array}$ & $\begin{array}{r}1.81 \\
20.99\end{array}$ & $\begin{array}{r}1.79 \\
20.49\end{array}$ & $\begin{array}{r}1.73 \\
21.09\end{array}$ \\
\hline \multirow[t]{2}{*}{ 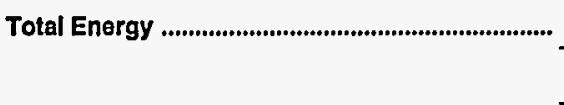 } & 2.35 & 4.62 & 8.84 & 10.84 & 10.54 & $R_{10.58}$ & $A_{10.28}$ & 10.57 \\
\hline & \multicolumn{8}{|c|}{ Expendltures in Millions of Dollars } \\
\hline 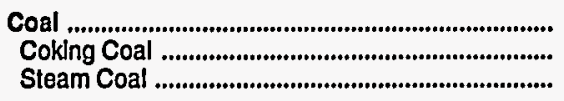 & $\begin{array}{c}35.8 \\
- \\
35.8\end{array}$ & $\begin{array}{c}135.0 \\
\overline{135.0}\end{array}$ & $\begin{array}{c}405.4 \\
- \\
405.4\end{array}$ & $\begin{array}{c}999.9 \\
- \\
999.9\end{array}$ & $\begin{array}{c}1,155.6 \\
- \\
1,15 \overline{5.6}\end{array}$ & $\begin{array}{c}1,194.6 \\
- \\
1,194.6\end{array}$ & $\begin{array}{c}1,191.7 \\
- \\
1,191.7\end{array}$ & $\begin{array}{c}1,158.2 \\
- \\
1,158.2\end{array}$ \\
\hline Natural Gas .................................................................... & 170.1 & 283.6 & 693.8 & $1,081.0$ & $1,081.4$ & $1,004.3$ & $1,077.9$ & $1,177.6$ \\
\hline 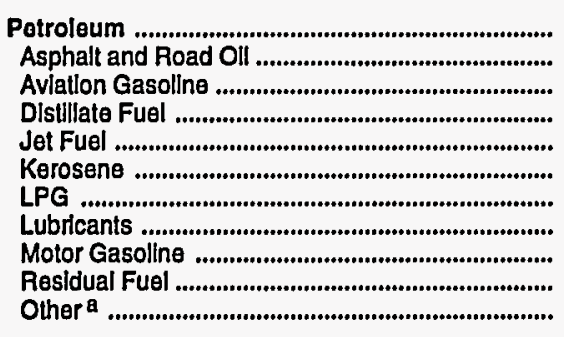 & $\begin{array}{r}1,632.1 \\
18.0 \\
34.4 \\
98.0 \\
96.6 \\
26.6 \\
77.1 \\
33.6 \\
1,125.2 \\
112.8 \\
9.8\end{array}$ & $\begin{array}{r}4,168.9 \\
43.3 \\
33.4 \\
343.6 \\
275.6 \\
15.5 \\
142.5 \\
54.0 \\
2,319.6 \\
915.2 \\
26.1\end{array}$ & $\begin{array}{r}11,051.3 \\
106.4 \\
61.0 \\
1,183.7 \\
1,302.3 \\
45.6 \\
294.6 \\
122.7 \\
5,627.4 \\
2,193.5 \\
114.0\end{array}$ & $\begin{array}{r}9,831.1 \\
220.8 \\
42.4 \\
1,198.8 \\
762.5 \\
107.1 \\
388.1 \\
137.0 \\
5,947.5 \\
911.5 \\
115.5\end{array}$ & $\begin{array}{r}R_{10,928.7} \\
131.3 \\
38.0 \\
1,574.3 \\
1,013.5 \\
19.6 \\
328.3 \\
R_{127.7} \\
6,581.4 \\
1,001.8 \\
R_{112.6}\end{array}$ & $\begin{array}{r}R_{9,922.7} \\
148.5 \\
31.3 \\
1,328.7 \\
693.5 \\
15.4 \\
350.5 \\
R_{131.5} \\
6,297.2 \\
826.2 \\
A_{99.9}\end{array}$ & $\begin{array}{r}R_{10,105.9} \\
103.7 \\
25.6 \\
1,531.7 \\
626.4 \\
25.6 \\
409.7 \\
R_{155.1} \\
6,237.3 \\
R_{888.0} \\
R_{102.9}\end{array}$ & $\begin{array}{r}10,254.9 \\
158.2 \\
21.9 \\
945.9 \\
635.8 \\
14.1 \\
433.7 \\
153.9 \\
6,846.3 \\
951.5 \\
93.7\end{array}$ \\
\hline 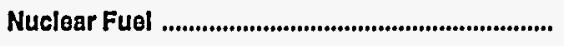 & - & 15.8 & 63.8 & 165.1 & 149.2 & 146.2 & 166.0 & 174.8 \\
\hline Blomass Fuels at Utilities ..................................... & - & - & - & - & - & - & - & - \\
\hline Primary Energy - Five Sectors b ....................... & $1,838.1$ & $4,603.2$ & $12,214.3$ & $12,077.1$ & $R_{13,314.8}$ & $R_{12,267.8}$ & $R_{12,541.5}$ & $12,765.6$ \\
\hline 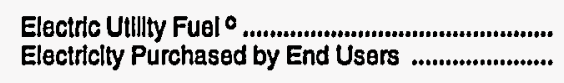 & $\begin{array}{r}-196.0 \\
971.7\end{array}$ & $\begin{array}{r}-1,114.2 \\
2,532.9\end{array}$ & $\begin{array}{r}-2,439.2 \\
5,029.8\end{array}$ & $\begin{array}{r}-2,244.7 \\
8,548.0\end{array}$ & $\begin{array}{r}-2,516.8 \\
10,098.0\end{array}$ & $\begin{array}{r}-2,424.6 \\
10,479.0\end{array}$ & $\begin{array}{r}-2,452.6 \\
10,280.0\end{array}$ & $\begin{array}{r}-2,443.6 \\
10,994.0\end{array}$ \\
\hline Total Enorgy. & $2,613.8$ & $6,021.9$ & $14,804.8$ & $18,380.3$ & $R_{20,896.0}$ & $R_{20,322.2}$ & $\mathrm{R}_{20,368.9}$ & $21,316.0$ \\
\hline
\end{tabular}

a Includes petroleum coke used at electric utllitles.

b Blomass fuels are not included, except those consumed at electric utllitles and those added to motor gasoline.

- There are no direct fuel costs for hydroelectric, geothermal, wind, photovoltalc, or solar thermal energy.

$R=$ Revised data.
-No consumptlon, including cases where adjustments were made. See explanation of adjustments in Section 6 of Appendix A.

Note: Expenditure totals may not equal sum of components due to Independent rounding.

Sources: Data sources, estimation procedures, and assumptions are described In Appendix A. 
F Energy Price Estimates by Sector, Florida
$1970,1975,1980,1985$, and 1990-1993

L $1970,1975,1980,1985$

O (Dollars per Million Btu)

\begin{tabular}{|c|c|c|c|c|c|c|c|c|}
\hline Sector and Energy Source & 1970 & 1975 & 1980 & 1985 & 1990 & 1991 & 1992 & 1993 \\
\hline 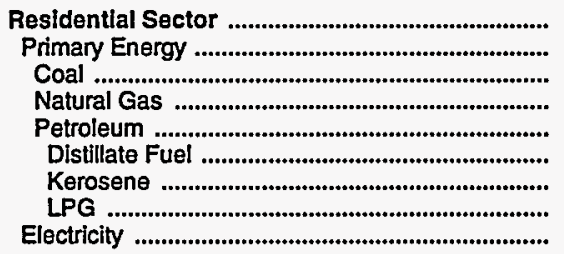 & $\begin{array}{l}4.60 \\
2.37 \\
2 . \overline{2} \\
2.35 \\
1.25 \\
1.63 \\
3.11 \\
6.10\end{array}$ & $\begin{array}{r}9.04 \\
4.19 \\
-\overline{2} \\
2.54 \\
5.10 \\
2.62 \\
3.27 \\
6.32 \\
10.92\end{array}$ & $\begin{array}{r}14.66 \\
7.48 \\
3.12 \\
4.49 \\
9.24 \\
6.92 \\
8.92 \\
10.34 \\
16.74\end{array}$ & $\begin{array}{r}21.57 \\
8.81 \\
3.31 \\
6.72 \\
10.04 \\
6.73 \\
9.34 \\
10.70 \\
24.73\end{array}$ & $\begin{array}{r}21.25 \\
10.49 \\
3.10 \\
7.79 \\
12.38 \\
9.59 \\
13.30 \\
12.55 \\
22.78\end{array}$ & $\begin{array}{r}21.65 \\
10.91 \\
2.94 \\
8.18 \\
12.75 \\
8.95 \\
12.40 \\
13.05 \\
23.18\end{array}$ & $\begin{array}{r}21.38 \\
12.66 \\
2.92 \\
8.25 \\
15.87 \\
11.11 \\
15.40 \\
16.37 \\
22.71\end{array}$ & $\begin{array}{r}22.11 \\
13.10 \\
3.10 \\
8.57 \\
16.63 \\
7.05 \\
9.77 \\
18.07 \\
23.41\end{array}$ \\
\hline 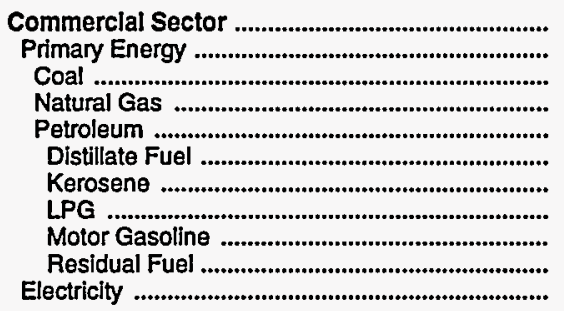 & $\begin{array}{r}3.53 \\
1.07 \\
-.99 \\
1.23 \\
.98 \\
.61 \\
1.27 \\
2.81 \\
.33 \\
6.24\end{array}$ & $\begin{array}{r}7.13 \\
2.03 \\
-\overline{1} \\
1.58 \\
2.52 \\
2.26 \\
2.38 \\
2.51 \\
4.39 \\
1.85 \\
11.44\end{array}$ & $\begin{array}{r}12.27 \\
4.68 \\
1.77 \\
3.21 \\
6.24 \\
6.30 \\
6.41 \\
5.46 \\
9.80 \\
3.71 \\
17.38\end{array}$ & $\begin{array}{r}15.73 \\
5.62 \\
2.04 \\
4.80 \\
6.27 \\
5.79 \\
6.49 \\
11.24 \\
9.03 \\
4.08 \\
22.03\end{array}$ & $\begin{array}{r}15.25 \\
5.55 \\
1.89 \\
4.64 \\
6.36 \\
7.29 \\
8.04 \\
10.15 \\
8.85 \\
3.09 \\
19.57\end{array}$ & $\begin{array}{r}15.41 \\
4.92 \\
1.89 \\
4.48 \\
5.40 \\
5.88 \\
7.13 \\
10.59 \\
8.48 \\
2.37 \\
19.85\end{array}$ & $\begin{array}{r}15.09 \\
5.07 \\
1.93 \\
4.53 \\
5.77 \\
6.46 \\
7.90 \\
10.04 \\
8.29 \\
2.49 \\
19.33\end{array}$ & $\begin{array}{r}16.04 \\
5.31 \\
1.94 \\
5.29 \\
5.38 \\
4.69 \\
5.34 \\
9.60 \\
8.68 \\
2.30 \\
19.66\end{array}$ \\
\hline $\begin{array}{l}\text { Industrial Sector } \\
\text { Primary Energy } \\
\text { Coal f... } \\
\text { Coking Coal } \\
\text { Steam Coal } \\
\text { Natural Gas } \\
\text { Petroleum } \\
\text { Asphalt and Road Oil . } \\
\text { Distillate Fuel } \\
\text { Kerosene } \\
\text { LPG }\end{array}$ & $\begin{array}{r}.93 \\
.55 \\
- \\
- \\
- \\
.37 \\
.69 \\
.67 \\
.56 \\
.61 \\
1.27 \\
5.08 \\
2.81 \\
.37 \\
1.35 \\
3.56\end{array}$ & $\begin{array}{r}2.71 \\
1.64 \\
.53 \\
. \\
.53 \\
.95 \\
2.18 \\
1.78 \\
2.20 \\
2.38 \\
2.51 \\
7.48 \\
4.39 \\
1.75 \\
3.11 \\
7.57\end{array}$ & $\begin{array}{r}5.48 \\
3.88 \\
1.77 \\
1 . \\
1.77 \\
2.61 \\
4.71 \\
3.57 \\
5.75 \\
6.41 \\
5.46 \\
14.36 \\
9.80 \\
3.44 \\
.7 .97 \\
13.38\end{array}$ & $\begin{array}{r}7.10 \\
4.99 \\
2.04 \\
- \\
2.04 \\
3.71 \\
6.11 \\
4.99 \\
5.81 \\
6.49 \\
11.24 \\
17.61 \\
9.03 \\
4.08 \\
8.30 \\
16.63\end{array}$ & $\begin{array}{r}\mathrm{R}_{6.13} \\
\mathrm{R}_{4.07} \\
1.89 \\
- \\
1.89 \\
3.89 \\
\mathrm{R}_{5.24} \\
2.91 \\
7.20 \\
8.04 \\
10.15 \\
\mathrm{R}_{14.60} \\
8.85 \\
3.09 \\
\mathrm{R}_{6.62} \\
14.90\end{array}$ & $\begin{array}{r}R_{5} 5.97 \\
R_{3.75} \\
1.89 \\
- \\
1.89 \\
2.83 \\
R_{4.95} \\
3.06 \\
6.38 \\
7.13 \\
10.59 \\
\text { R }_{16.79} \\
8.48 \\
2.37 \\
R_{5.85} \\
15.22\end{array}$ & $\begin{array}{r}\mathrm{R}_{5.70} \\
\mathrm{R}_{3.69} \\
1.93 \\
- \\
1.93 \\
2.93 \\
\mathrm{R}_{4.72} \\
2.25 \\
7.07 \\
7.90 \\
10.04 \\
\mathrm{R} 19.43 \\
8.29 \\
2.49 \\
\mathrm{R} 5.84 \\
14.71\end{array}$ & $\begin{array}{r}5.60 \\
3.71 \\
1.94 \\
1 . \overline{ } \\
1.94 \\
3.49 \\
4.26 \\
2.86 \\
4.78 \\
5.34 \\
9.60 \\
18.94 \\
8.68 \\
2.30 \\
5.32 \\
15.43\end{array}$ \\
\hline 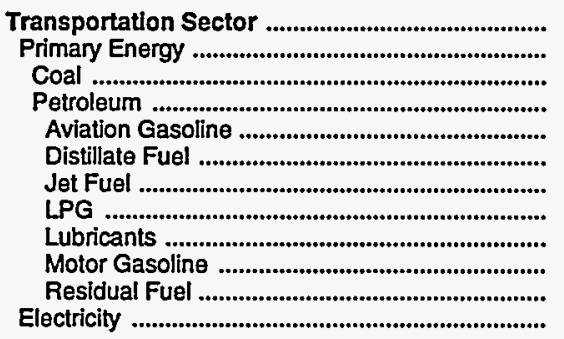 & $\begin{array}{r}2.19 \\
2.19 \\
2.19 \\
2.17 \\
1.44 \\
.73 \\
1.27 \\
5.08 \\
2.81 \\
.29 \\
-\end{array}$ & $\begin{array}{l}3.79 \\
3.79 \\
.53 \\
3.79 \\
3.45 \\
2.89 \\
2.03 \\
2.51 \\
7.48 \\
4.39 \\
1.60 \\
-\end{array}$ & $\begin{array}{r}8.39 \\
8.39 \\
- \\
8.39 \\
9.02 \\
7.72 \\
6.46 \\
5.46 \\
14.36 \\
9.80 \\
3.14 \\
-\end{array}$ & $\begin{array}{r}8.19 \\
8.19 \\
\overline{-} \\
8.19 \\
9.99 \\
7.24 \\
5.90 \\
11.24 \\
17.61 \\
9.03 \\
3.76 \\
22.04\end{array}$ & $\begin{array}{r}R 7.94 \\
7.93 \\
-\overline{7} \\
7.93 \\
9.32 \\
8.21 \\
5.64 \\
10.15 \\
14.60 \\
8.85 \\
2.56 \\
20.05\end{array}$ & $\begin{array}{r}R 7.64 \\
R 7.64 \\
- \\
R 7.64 \\
8.71 \\
7.75 \\
4.93 \\
10.59 \\
R \quad 16.79 \\
8.48 \\
1.95 \\
20.07\end{array}$ & $\begin{array}{r}R_{7.46} \\
R_{7.46} \\
- \\
R_{7.46} \\
8.54 \\
7.91 \\
4.56 \\
10.04 \\
R_{19.43} \\
8.29 \\
R_{2.12} \\
19.94\end{array}$ & $\begin{array}{r}7.62 \\
7.62 \\
- \\
7.62 \\
8.24 \\
8.20 \\
4.23 \\
9.60 \\
18.94 \\
8.68 \\
1.96 \\
20.37\end{array}$ \\
\hline 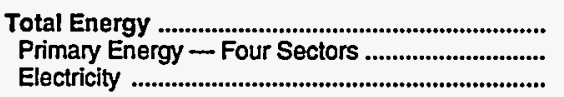 & $\begin{array}{l}2.35 \\
1.74 \\
5.67\end{array}$ & $\begin{array}{r}4.62 \\
3.28 \\
10.46\end{array}$ & $\begin{array}{r}8.84 \\
7.16 \\
16.24\end{array}$ & $\begin{array}{r}10.84 \\
7.46 \\
22.59\end{array}$ & $\begin{array}{r}10.54 \\
7.23 \\
20.62\end{array}$ & $\begin{aligned} R_{1} 10.58 \\
R_{6.92} \\
20.99\end{aligned}$ & $\begin{array}{r}R_{10.28} \\
R_{6.82} \\
20.49\end{array}$ & $\begin{array}{r}10.57 \\
6.90 \\
21.09\end{array}$ \\
\hline 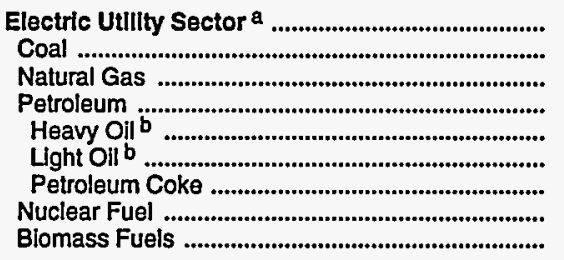 & $\begin{array}{l}.33 \\
.31 \\
.35 \\
.33 \\
.33 \\
.36 \\
- \\
- \\
-\end{array}$ & $\begin{array}{r}1.35 \\
1.01 \\
.72 \\
1.88 \\
1.85 \\
2.21 \\
.17 \\
-\end{array}$ & $\begin{array}{r}2.40 \\
1.80 \\
1.53 \\
3.80 \\
3.72 \\
5.76 \\
- \\
.35 \\
-\end{array}$ & $\begin{array}{r}2.21 \\
2.12 \\
3.25 \\
3.96 \\
3.87 \\
5.71 \\
- \\
.65 \\
-\end{array}$ & $\begin{array}{r}1.98 \\
1.85 \\
2.53 \\
3.08 \\
2.99 \\
5.09 \\
- \\
.64 \\
-\end{array}$ & $\begin{array}{l}1.81 \\
1.86 \\
2.14 \\
2.31 \\
2.23 \\
4.63 \\
\overline{-6} \\
-\end{array}$ & $\begin{array}{r}1.79 \\
1.82 \\
2.28 \\
2.46 \\
2.40 \\
4.46 \\
\overline{-62} \\
-\end{array}$ & $\begin{array}{l}1.73 \\
1.77 \\
2.34 \\
2.24 \\
2.19 \\
4.18 \\
.63 \\
-\end{array}$ \\
\hline Primary Energy — Flve Sectors $c^{c} \ldots . . . . . . . . . . . . . . . . . .$. & 1.20 & 2.44 & 5.12 & 5.17 & $R_{4.82}$ & $R_{4.44}$ & 4.39 & 4.39 \\
\hline
\end{tabular}

a There are no direct fuel costs for hydroelectric, geothermal, wind, photovoltaic, or solar thermal energy.

b Heavy oil includes fuel oll nos. 4, 5, and 6, and residual fuel oils. Light oil includes fuel oll nos. 1 and 2, kerosene, and jet fuel.

c Biomass fuals are not included, except those consumed at electric

R=Revised data.

- No consumption, including cases where adjustments were made. Seo explanation of adjustments in Section 6 of Appendix A.

Sources: Data sources, estimation procedures, and assumptions are utilities and those added to motor gasoline. 
(Million Dollars)

\begin{tabular}{|c|c|c|c|c|c|c|c|c|}
\hline Sector and Energy Source & 970 & 1975 & 1980 & 1985 & 1990 & 1991 & 1992 & 1993 \\
\hline 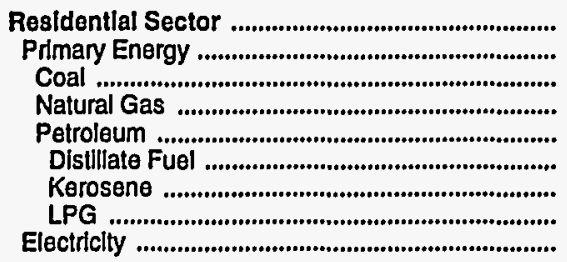 & $\begin{array}{r}645.6 \\
133.6 \\
-\overline{3} \\
37.0 \\
96.6 \\
7.4 \\
22.3 \\
66.9 \\
512.1\end{array}$ & $\begin{array}{r}1,488.1 \\
192.8 \\
-\overline{4} \\
151.7 \\
16.7 \\
13.4 \\
121.0 \\
1,295.3\end{array}$ & $\begin{array}{r}2,884.5 \\
329.5 \\
.3 \\
72.7 \\
256.5 \\
49.0 \\
39.1 \\
168.4 \\
2,555.0\end{array}$ & $\begin{array}{r}4,970.0 \\
403.2 \\
3.2 \\
100.9 \\
299.1 \\
22.3 \\
45.7 \\
231.1 \\
4,566.8\end{array}$ & $\begin{array}{r}5,888.6 \\
361.7 \\
.1 \\
109.9 \\
251.6 \\
13.1 \\
11.6 \\
227.0 \\
5,527.0\end{array}$ & $\begin{array}{r}6,144.5 \\
385.5 \\
(s) \\
115.9 \\
269.6 \\
12.4 \\
13.7 \\
243.5 \\
5,759.0\end{array}$ & $\begin{array}{r}6,153.6 \\
482.6 \\
.4 \\
130.6 \\
351.6 \\
20.0 \\
23.9 \\
307.7 \\
5,671.0\end{array}$ & $\begin{array}{r}6,631.5 \\
494.5 \\
.4 \\
139.6 \\
354.5 \\
13.1 \\
12.1 \\
329.3 \\
6,137.0\end{array}$ \\
\hline 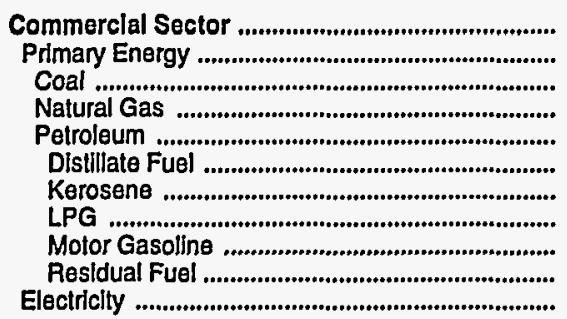 & $\begin{array}{r}411.3 \\
65.3 \\
24.9 \\
40.4 \\
11.7 \\
.5 \\
4.8 \\
20.4 \\
3.1 \\
345.9\end{array}$ & $\begin{array}{r}1,028.4 \\
134.2 \\
-\tilde{5} \\
80.9 \\
80.3 \\
29.3 \\
.5 \\
8.5 \\
23.9 \\
18.0 \\
894.1\end{array}$ & $\begin{array}{r}1,921.0 \\
294.8 \\
.3 \\
103.6 \\
190.9 \\
70.7 \\
1.0 \\
15.7 \\
69.0 \\
34.4 \\
1,626.2\end{array}$ & $\begin{array}{r}3,595.6 \\
492.3 \\
3.6 \\
163.4 \\
325.3 \\
123.3 \\
38.5 \\
42.8 \\
64.9 \\
55.7 \\
3,103.3\end{array}$ & $\begin{array}{r}4,195.0 \\
470.7 \\
.1 \\
183.0 \\
287.5 \\
137.6 \\
5.7 \\
32.4 \\
65.3 \\
46.6 \\
3,724.3\end{array}$ & $\begin{array}{r}4,266.5 \\
405.3 \\
(s) \\
193.2 \\
212.1 \\
102.7 \\
1.2 \\
34.9 \\
41.3 \\
32.0 \\
3,861.2\end{array}$ & $\begin{array}{r}R_{4,198.0} \\
419.7 \\
.5 \\
207.8 \\
211.3 \\
112.9 \\
1.3 \\
33.3 \\
35.6 \\
28.2 \\
3,778.3\end{array}$ & $\begin{array}{r}4,360.2 \\
363.9 \\
.5 \\
240.5 \\
122.9 \\
84.0 \\
1.6 \\
30.9 \\
4.4 \\
2.1 \\
3,996.4\end{array}$ \\
\hline 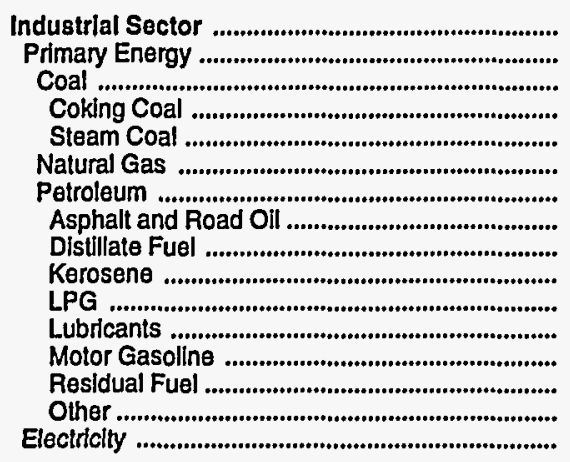 & $\begin{array}{r}235.4 \\
121.7 \\
- \\
- \\
- \\
35.8 \\
85.8 \\
18.0 \\
14.7 \\
3.9 \\
4.4 \\
12.9 \\
3.0 \\
19.1 \\
9.8 \\
113.7\end{array}$ & $\begin{array}{r}680.0 \\
336.6 \\
.3 \\
- \\
.3 \\
85.1 \\
251.2 \\
43.3 \\
60.0 \\
1.6 \\
11.5 \\
25.7 \\
2.1 \\
81.0 \\
26.1 \\
343.4\end{array}$ & $\begin{array}{r}2,059.8 \\
1,211.1 \\
30.2 \\
-\overline{3} \\
30.2 \\
259.6 \\
921.3 \\
106.4 \\
236.8 \\
5.5 \\
107.2 \\
52.6 \\
4.5 \\
294.2 \\
114.0 \\
848.6\end{array}$ & $\begin{array}{r}2,059.8 \\
1,183.1 \\
45.4 \\
- \\
45.4 \\
272.5 \\
865.3 \\
220.8 \\
154.0 \\
22.8 \\
98.3 \\
58.7 \\
48.5 \\
146.6 \\
115.5 \\
876.6\end{array}$ & $\begin{array}{r}R_{1,826.3} \\
R_{982.3} \\
57.0 \\
- \\
57.0 \\
304.1 \\
R_{621.2} \\
131.3 \\
146.4 \\
2.3 \\
61.1 \\
R_{54.8} \\
49.4 \\
63.4 \\
R_{112.6} \\
844.0\end{array}$ & $\begin{array}{r}R_{1}, 736.2 \\
880.2 \\
53.8 \\
- \\
53.8 \\
259.4 \\
R_{567.1} \\
148.5 \\
114.5 \\
.5 \\
65.3 \\
R_{56.4} \\
43.0 \\
39.0 \\
R_{99.9} \\
856.0\end{array}$ & $\begin{array}{r}\text { R }_{1,757.8} \\
\text { R }_{929.8} \\
64.4 \\
- \\
64.4 \\
273.1 \\
\text { R }_{592.2} \\
103.7 \\
149.1 \\
.4 \\
62.6 \\
\text { R } 66.5 \\
42.7 \\
64.5 \\
\text { P }_{102.9} \\
828.0\end{array}$ & $\begin{array}{r}1,929.1 \\
1,071.1 \\
63.1 \\
- \\
63.1 \\
385.7 \\
622.4 \\
158.2 \\
116.0 \\
.4 \\
67.9 \\
66.0 \\
44.2 \\
76.1 \\
93.7 \\
858.0\end{array}$ \\
\hline 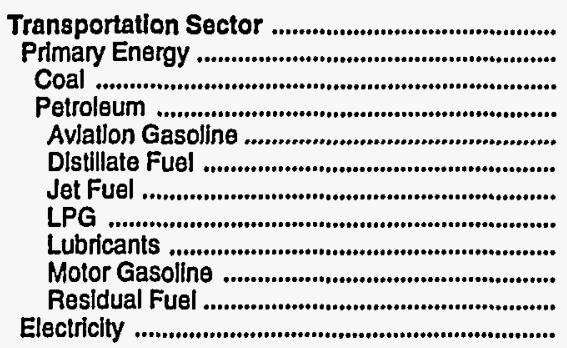 & $\begin{array}{r}1,321.5 \\
1,321.5 \\
- \\
1,321.5 \\
34.4 \\
63.0 \\
96.6 \\
.9 \\
20.6 \\
1,101.8 \\
4.2 \\
-\end{array}$ & $\begin{array}{r}2,825.4 \\
2,825.4 \\
(\mathrm{~s}) \\
2,825.4 \\
33.4 \\
171.1 \\
275.3 \\
1.6 \\
28.2 \\
2,293.5 \\
22.3 \\
-\end{array}$ & $\begin{array}{r}7,939.6 \\
7,939.6 \\
- \\
7,939.6 \\
61.0 \\
719.9 \\
1,302.3 \\
3.2 \\
70.1 \\
5,553.9 \\
229.2 \\
-\end{array}$ & $\begin{array}{r}7,755.0 \\
7,753.7 \\
- \\
7,753.7 \\
42.4 \\
857.8 \\
762.5 \\
15.8 \\
78.2 \\
5,834.1 \\
162.9 \\
1.3\end{array}$ & $\begin{array}{r}\text { R }_{8,986.1} \\
\text { F } 8,983.4_{-} \\
\text {R } 8,983.4 \\
38.0 \\
1,221.9 \\
1,013.5 \\
7.9 \\
\text { R } 73.0 \\
6,466.7 \\
162.4 \\
2.7\end{array}$ & $\begin{array}{r}R_{8,175.0} \\
R_{8,172.2} \\
- \\
R_{8,172.2} \\
31.3 \\
1,050.3 \\
693.5 \\
6.8 \\
R_{75.1} \\
6,212.9 \\
102.2 \\
2.8\end{array}$ & $\begin{array}{r}{ }^{R} 8,259.5 \\
\mathrm{~A}_{8,256.8} \\
- \\
\mathrm{A}_{8,256.8} \\
25.6 \\
1,212.7 \\
626.4 \\
6.1 \\
\mathrm{R}_{88.6} \\
6,159.0 \\
\mathrm{R}_{138.4} \\
2.7\end{array}$ & $\begin{array}{r}8,395.1 \\
8,392.5 \\
- \\
8,392.5 \\
21.9 \\
698.2 \\
635.8 \\
5.6 \\
87.9 \\
6,797.7 \\
145.3 \\
2.6\end{array}$ \\
\hline 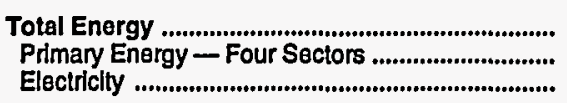 & $\begin{array}{r}2,613.8 \\
1,642.1 \\
971.7\end{array}$ & $\begin{array}{l}6,021.9 \\
3,489.0 \\
2,532.9\end{array}$ & $\begin{array}{r}14,804.8 \\
9,775.0 \\
5,029.8\end{array}$ & $\begin{array}{r}18,380.3 \\
9,832.3 \\
8,548.0\end{array}$ & $\begin{array}{r}R_{20,896.0} \\
R_{10,798.0} \\
10,098.0\end{array}$ & $\begin{array}{r}\mathrm{R}_{20,322.2} \\
\mathrm{R}_{9,843.2} \\
10,479.0\end{array}$ & $\begin{array}{r}A_{20,368.9} \\
A_{10,088.9} \\
10,280.0\end{array}$ & $\begin{array}{l}21,316.0 \\
10,322.0 \\
10,994.0\end{array}$ \\
\hline 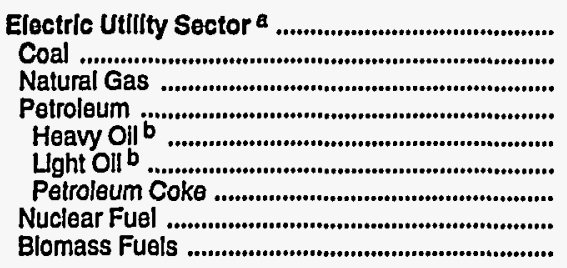 & $\begin{array}{r}196.0 \\
35.8 \\
72.4 \\
87.8 \\
86.5 \\
1.3 \\
= \\
=\end{array}$ & $\begin{array}{r}1,114.2 \\
134.7 \\
102.9 \\
860.8 \\
794.0 \\
66.8 \\
- \\
15.8 \\
-\end{array}$ & $\begin{array}{r}2,439.2 \\
374.6 \\
257.9 \\
1,743.0 \\
1,635.7 \\
107.3 \\
\overrightarrow{63.8} \\
-\end{array}$ & $\begin{array}{r}2,244.7 \\
947.7 \\
544.2 \\
587.7 \\
546.2 \\
41.5 \\
165.1 \\
-\end{array}$ & $\begin{array}{r}2,516.8 \\
1,098.4 \\
484.4 \\
784.9 \\
729.4 \\
55.4 \\
149.2 \\
-\end{array}$ & $\begin{array}{r}2,424.6 \\
1,140.8 \\
435.8 \\
701.8 \\
653.0 \\
48.8 \\
- \\
146.2 \\
-\end{array}$ & $\begin{array}{r}2,452.6 \\
1,126.4 \\
466.3 \\
693.9 \\
656.9 \\
37.0 \\
- \\
166.0 \\
-\end{array}$ & $\begin{array}{r}2,443.6 \\
1,094.2 \\
411.9 \\
762.7 \\
728.1 \\
34.6 \\
-\overline{174.8} \\
-\end{array}$ \\
\hline 1ergy - Flve Sectors c ............................ & 838.1 & $4,603.2$ & $12,214.3$ & $12,077.1$ & $R_{13,314.8}$ & $R_{12,267.8}$ & $R_{12,541.5}$ & $12,765.6$ \\
\hline
\end{tabular}

a There are no direct fuel costs for hydroelectric, geothermal, wind, photovoltalc, or solar thermal energy.

b Heavy oll includes fuel oll nos. 4, 5, and 6, and residual fuel olls. Light oll Includes fuel nos. 1 and 2, kerosene, and jet fuel.

- Blomass fuels are not Included, except those consumed at electric utillties and those added to motor gasoline.

$R=$ Revised data.
-No consumption, including cases where adjustments were made. See explanation of adjustments in Section 6 of Appendix A.

$(s)=$ Value less than 0.05 million dollars.

Note: Totals may not equal sum of components due to independent rounding.

Sources: Data sources, estlmation procedures, and assumptions are described in Appendix A.

360.2

84.0

2.1

928.1

63.1

63.1

(3).

16.0

67.9

4.2

.

395.1

-

.

5.6

2.6

11.9

- 


\section{G Energy Price and Expenditure Estimates by Source, Georgia \\ E 1970, 1975, 1980, 1985, and 1990-1993}

\begin{tabular}{|c|c|c|c|c|c|c|c|c|}
\hline Energy Source & 1970 & 1975 & 1980 & 1985 & 1990 & 1991 & 1992 & 1993 \\
\hline & \multicolumn{8}{|c|}{ Prices in Dollars per Million Btu } \\
\hline Coal & 0.39 & 0.95 & 1.50 & 1.87 & 1.79 & 1.80 & 1.80 & 1.78 \\
\hline 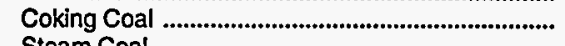 & - & - & - & - & - & $\overline{0}$ & - & - \\
\hline 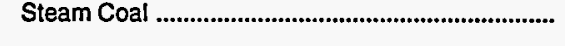 & .39 & .95 & 1.50 & 1.87 & 1.79 & 1.80 & 1.80 & 1.78 \\
\hline Natural Gas & .58 & 1.02 & 3.06 & 5.25 & 4.80 & 4.65 & 4.69 & 5.18 \\
\hline Petroleum ...... & 1.94 & 3.65 & 8.02 & 7.56 & R7.34 & R7.17 & $R_{6.81}$ & 6.84 \\
\hline 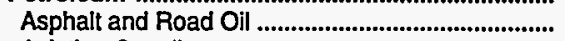 & .76 & 1.74 & 3.72 & 4.97 & 2.86 & 3.18 & 2.38 & 2.63 \\
\hline 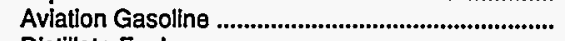 & 2.17 . & 3.45 & 9.02 & 9.99 & 9.32 & 8.71 & 8.54 & 8.24 \\
\hline 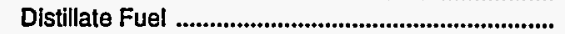 & 1.06 & 2.71 & 7.00 & 6.68 & 7.16 & 6.74 & 6.39 & 6.35 \\
\hline Jet Fuel & .73 & 2.03 & 6.46 & 5.66 & 5.45 & 4.61 & 4.39 & 4.13 \\
\hline 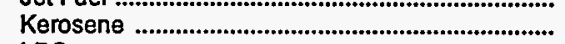 & .85 & 2.38 & 6.51 & 9.35 & 7.60 & 7.05 & 7.03 & 6.86 \\
\hline LPG & 2.01 & 3.58 & 6.40 & 9.48 & 10.16 & 10.58 & 8.93 & 8.75 \\
\hline 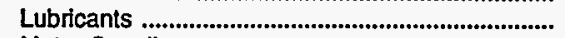 & 5.08 & 7.48 & 14.36 & 17.61 & $R_{14.60}$ & $\mathrm{R}_{16.79}$ & $R_{19.43}$ & 18.94 \\
\hline Motor Gasoline ........................................................................ & 2.80 & 4.73 & 9.91 & 8.76 & 8.24 & 7.95 & 7.66 & 7.81 \\
\hline 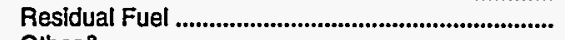 & .38 & 1.70 & 3.27 & 4.13 & 2.52 & 2.11 & 2.55 & 2.17 \\
\hline Other ${ }^{a}$ & 2.53 & 3.33 & 8.56 & 9.22 & ${ }^{\mathrm{R}} 7.52$ & ${ }^{R} 7.05$ & $R_{7.98}$ & 7.02 \\
\hline 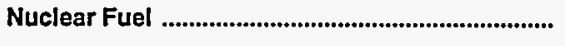 & - & .13 & .45 & .72 & .87 & .73 & .59 & .54 \\
\hline Biomass Fuels at Utilities & - & - & - & - & - & - & - & - \\
\hline Primary Energy - Five Sectors ${ }^{b}$ & 1.24 & 2.28 & 4.55 & 4.63 & P $_{4.27}$ & 4.17 & 4.05 & 4.21 \\
\hline 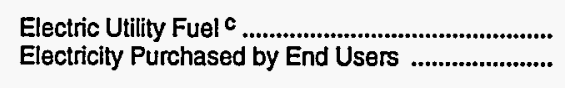 & .35 & .91 & $\begin{array}{r}1.38 \\
12.75\end{array}$ & $\begin{array}{r}1.73 \\
17.09\end{array}$ & $\begin{array}{r}1.53 \\
19.25\end{array}$ & $\begin{array}{r}1.46 \\
19.28\end{array}$ & $\begin{array}{r}1.39 \\
19.60\end{array}$ & $\begin{array}{r}1.40 \\
19.71\end{array}$ \\
\hline \multirow[t]{2}{*}{ Total Energy ................................................................ } & 1.86 & 3.68 & 7.34 & 8.48 & ${ }^{\mathrm{R}} 8.84$ & ${ }^{R} 8.76$ & $R_{8.65}$ & 8.83 \\
\hline & \multicolumn{8}{|c|}{ Expenditures in Millions of Dollars } \\
\hline Coal & 75.7 & 295.9 & 784.3 & $1,359.4$ & $1,282.3$ & $1,161.0$ & $1,107.8$ & $1,175.2$ \\
\hline Coking Coal & $\overline{75.7}$ & 295.9 & 784.3 & $1,35 \overline{9} .4$ & $1,28 \overline{2} .3$ & $1,161.0$ & $1,10 \overline{7.8}$ & $1,17 \overline{5} .2$ \\
\hline Natural Gas ............... & 195.4 & 336.1 & 970.9 & $1,467.5$ & $1,466.3$ & $1,471.1$ & 1.575 .5 & 1.788 .5 \\
\hline Petroleum & $1,070.5$ & $2,396.2$ & $5,638.0$ & $5,799.6$ & $R_{6,025.0}$ & $R_{5,614.1}$ & $A_{5,438.8}$ & $6,039.0$ \\
\hline 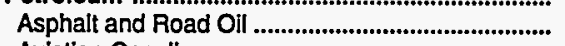 & 19.7 & 48.4 & 118.2 & 151.2 & 121.2 & 109.6 & 77.3 & $\begin{array}{r}0,009.0 \\
93.0\end{array}$ \\
\hline 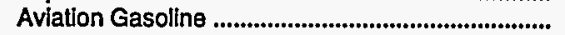 & 6.6 & & 17.6 & 10.7 & 9.2 & 8.0 & 7.2 & 7.0 \\
\hline 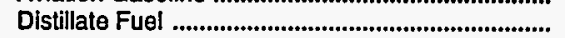 & 79.1 & 254.0 & 792.6 & 924.0 & $1,188.7$ & $1,057.5$ & $1,011.8$ & $1,155.0$ \\
\hline 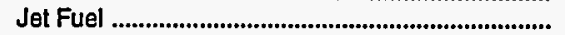 & 42.8 & 147.4 & 598.1 & 518.0 & 567.9 & 375.5 & 307.3 & 354.0 \\
\hline 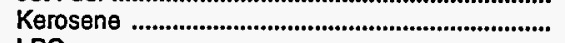 & 2.2 & 3.3 & 20.4 & 19.5 & 8.5 & 7.8 & & \\
\hline 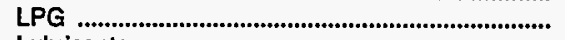 & 56.5 & 108.2 & 175.0 & 231.5 & 216.3 & 254.9 & 230.7 & 235.9 \\
\hline 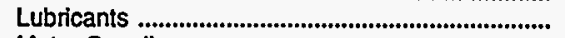 & 31.5 & 51.1 & 108.8 & 121.4 & $R_{113.3}$ & $R_{116.6}$ & ${ }^{R_{137.5}}$ & 136.5 \\
\hline 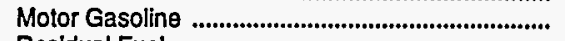 & 795.3 & $1,628.9$ & $3,409.4$ & $3,356.1$ & $3,580.3$ & $3,493.8$ & $3,376.8$ & $3,816.5$ \\
\hline $\begin{array}{l}\text { Residual Fuel } \\
\text { Other }\end{array}$ & $\begin{array}{l}24.5 \\
12.4\end{array}$ & $\begin{array}{r}115.5 \\
32.3\end{array}$ & $\begin{array}{l}185.0 \\
212.9\end{array}$ & $\begin{array}{l}285.0 \\
182.0\end{array}$ & $\begin{array}{r}51.4 \\
R_{168.1}\end{array}$ & $\begin{array}{r}32.8 \\
\mathbf{R}_{157.8}\end{array}$ & 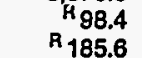 & $\begin{array}{r}67.9 \\
164.5\end{array}$ \\
\hline 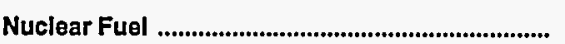 & - & 4.3 & 41.7 & 79.4 & 230.0 & 205.1 & 176.5 & 156.8 \\
\hline 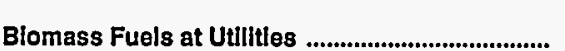 & - & - & - & _ & - & - & - & - \\
\hline & & & & & & & & \\
\hline Primary Energy －Flve Sectors b ............................ & $1,341.6$ & $3,032.5$ & $7,434.9$ & $8,705.8$ & $\mathrm{R}_{9,003.6}$ & ${ }^{n} 8,451.4$ & ${ }^{\circ} 8,298.7$ & $9,159.5$ \\
\hline $\begin{array}{l}\text { Electric Utillty Fuel }{ }^{c} \\
\text { Electricity Purchased by End Users }\end{array}$ & $\begin{array}{l}-88.1 \\
491.7\end{array}$ & $\begin{array}{r}-372.6 \\
1,265.9\end{array}$ & $\begin{array}{r}-837.7 \\
2,227.3\end{array}$ & $\begin{array}{r}-1,379.9 \\
3,690.2\end{array}$ & $\begin{array}{r}-1,425.9 \\
5,254.7\end{array}$ & $\begin{array}{r}-1,280.3 \\
5,317.3\end{array}$ & $\begin{array}{r}-1,212.4 \\
5,529.5\end{array}$ & $\begin{array}{r}-1,274.5 \\
5,947.8\end{array}$ \\
\hline Total Energy & $1,745.3$ & $3,925.8$ & $8,824.5$ & $11,016.0$ & $R_{12,832.4}$ & $R_{12,488.4}$ & $R_{12,615.7}$ & $13,832.8$ \\
\hline
\end{tabular}

a Includes petroleum coke used at electric utilitles.

b Biomass fuels are not included, except those consumed at electric utilitiles and those added to motor gasoline.

c There are no direct fuel costs for hydroelectric, geothermal, wind, photovoltalc, or solar thermal energy.

$R=$ Revised data.

-No consumption, including cases where adjustments were made. See explanation of adjustments in Section 6 of Appendlx A.

Note: Expenditure totals may not equal sum of components due to Independent rounding.

Sources: Data sources, estimation procedures, and assumptlons are describod in Appendix A. 
(Dollars per Million Btu)

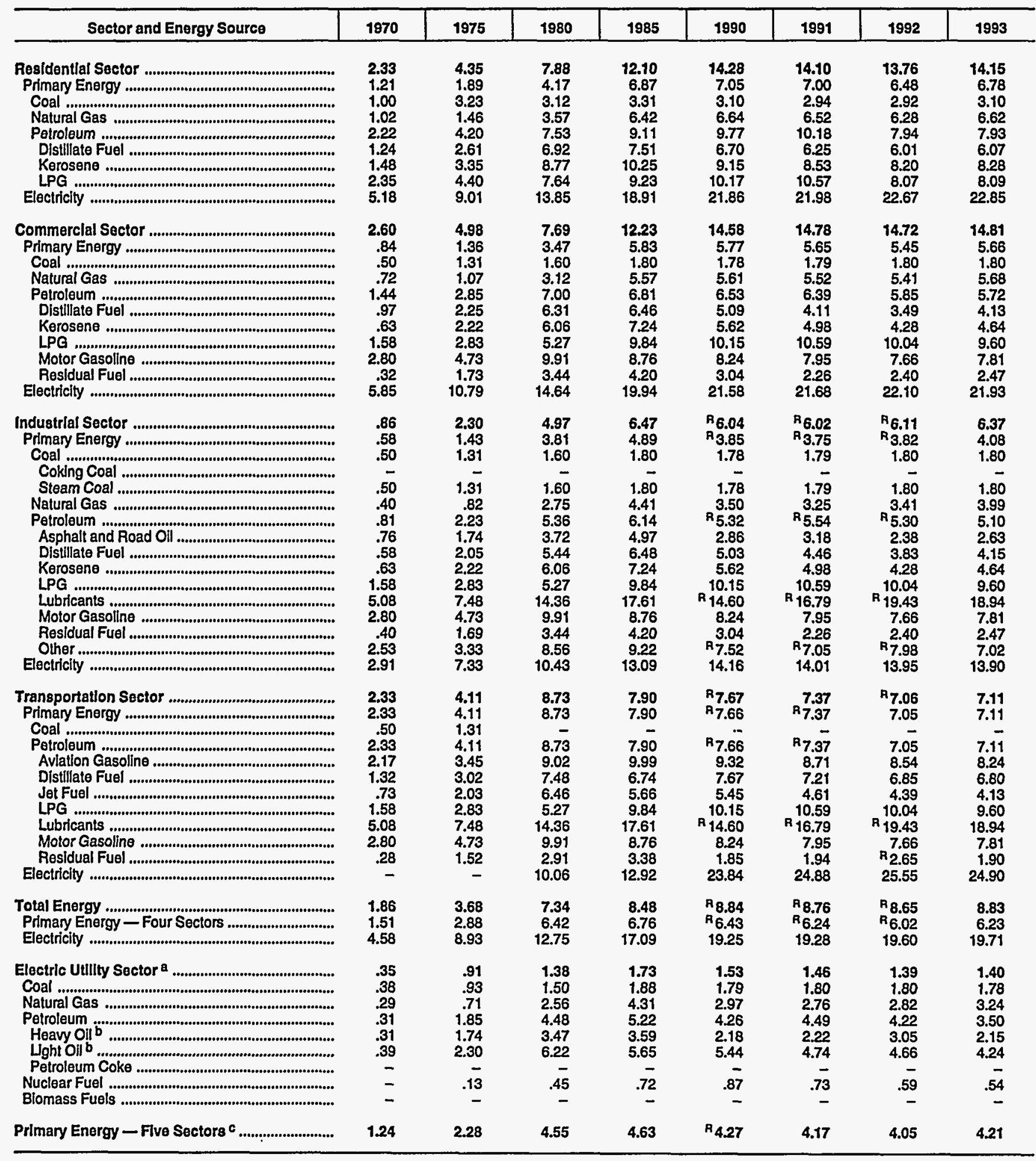

a There are no direct fuel costs for hydroelectric, geothermal, wind, photovoltalc, or solar thermal energy.

Heavy oil includes fuel oil nos. 4, 5, and 6, and residual fuel oils. Light oil includes fuel oll nos. 1 and 2, kerosene, and jet fuel.

c Blomass fuels are not included, except those consumed at electric utilitles and those added to motor gasoline.

$R=$ Revised data.

-No consumption, including cases where adjustments were made. See explanation of adjustments in Section 6 of Appendix A.

Sources: Data sources, estimation procedures, and assumptions are described in Appendix A. 
G Energy Expenditure Estimates by Sector, Georgia

E 1970, 1975, 1980, 1985, and 1990-1993

O (Million Dollars)

\begin{tabular}{|c|c|c|c|c|c|c|c|c|}
\hline Sector and Energy Source & 1970 & 1975 & 1980 & 1985 & 1990 & 1991 & 1992 & 1993 \\
\hline $\begin{array}{l}\text { Residential Sector } \\
\text { Primary Energy } \\
\text { Coal } \\
\text { Nalural Gas } \\
\text { Petroleum } \\
\text { Distillate Fuel } \\
\text { Kerosene }\end{array}$ & $\begin{array}{r}353.0 \\
132.3 \\
1.1 \\
91.6 \\
39.7 \\
1.8 \\
1.0 \\
36.9 \\
220.7\end{array}$ & $\begin{array}{r}706.7 \\
200.7 \\
1.4 \\
130.5 \\
68.9 \\
4.5 \\
.7 \\
63.7 \\
505.9\end{array}$ & $\begin{array}{r}1,406.8 \\
460.2 \\
.6 \\
332.0 \\
127.6 \\
23.3 \\
4.5 \\
99.7 \\
946.6\end{array}$ & $\begin{array}{r}2,234.3 \\
717.9 \\
1.1 \\
555.0 \\
161.8 \\
15.5 \\
15.0 \\
131.3 \\
1,516.4\end{array}$ & $\begin{array}{r}2,990.0 \\
757.0 \\
.6 \\
615.6 \\
140.8 \\
9.8 \\
5.8 \\
125.3 \\
2,233.0\end{array}$ & $\begin{array}{r}3,063.3 \\
799.3 \\
.2 \\
647.6 \\
151.5 \\
6.5 \\
5.5 \\
139.5 \\
2,264.0\end{array}$ & $\begin{array}{r}3,187.6 \\
826.7 \\
1.0 \\
696.9 \\
128.8 \\
6.2 \\
5.2 \\
5.1 \\
117.5 \\
2,361.0\end{array}$ & $\begin{array}{r}3,564.1 \\
924.1 \\
.6 \\
786.5 \\
137.1 \\
8.3 \\
6.4 \\
122.3 \\
2,640.0\end{array}$ \\
\hline $\begin{array}{l}\text { Commercial Sector } \\
\text { Primary Energy } \\
\text { Coal } \\
\text { Natural Gas } \\
\text { Petroleum } \\
\text { Distillate Fuel } \\
\text { Kerosene } \\
\text { LPG } \\
\text { Motor Gasoline } \\
\text { Residual Fuel }\end{array}$ & $\begin{array}{r}206.7 \\
43.5 \\
1.0 \\
28.6 \\
13.9 \\
4.0 \\
.1 \\
4.4 \\
5.1 \\
.2 \\
163.1\end{array}$ & $\begin{array}{r}497.0 \\
83.8 \\
1.0 \\
54.2 \\
28.6 \\
11.2 \\
.1 \\
7.2 \\
9.2 \\
.9 \\
413.2\end{array}$ & $\begin{array}{r}830.4 \\
232.9 \\
.6 \\
189.1 \\
43.2 \\
11.6 \\
.4 \\
12.1 \\
18.9 \\
.2 \\
597.5\end{array}$ & $\begin{array}{r}1,565.1 \\
407.6 \\
1.1 \\
295.1 \\
111.4 \\
58.2 \\
1.9 \\
24.7 \\
14.2 \\
12.4 \\
1,157.5\end{array}$ & $\begin{array}{r}2,117.8 \\
371.1 \\
.6 \\
285.0 \\
85.5 \\
37.7 \\
2.0 \\
22.1 \\
22.4 \\
1.3 \\
1,746.7\end{array}$ & $\begin{array}{r}2,133.1 \\
350.5 \\
.2 \\
289.4 \\
60.9 \\
20.6 \\
1.5 \\
24.7 \\
13.8 \\
.3 \\
1,782.6\end{array}$ & $\begin{array}{r}2,220.2 \\
364.7 \\
1.1 \\
298.9 \\
64.6 \\
21.1 \\
.9 \\
25.8 \\
16.7 \\
.1 \\
1,855.6\end{array}$ & $\begin{array}{r}2,352.3 \\
393.4 \\
.6 \\
335.4 \\
57.3 \\
27.3 \\
1.7 \\
25.6 \\
2.6 \\
.1 \\
1,958.9\end{array}$ \\
\hline 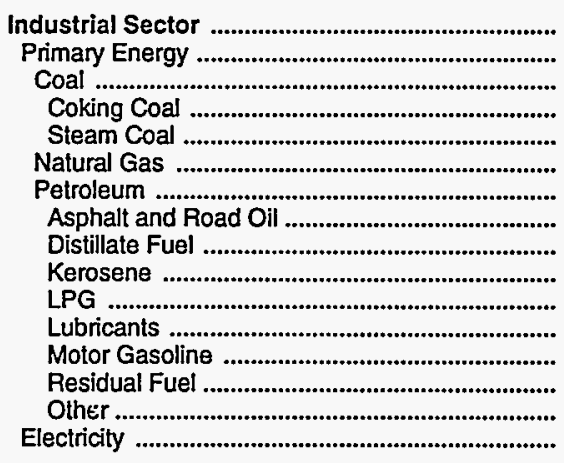 & $\begin{array}{r}270.4 \\
162.5 \\
6.0 \\
- \\
6.0 \\
58.0 \\
98.6 \\
19.7 \\
13.5 \\
1.1 \\
14.5 \\
14.6 \\
1.8 \\
21.0 \\
12.4 \\
107.9\end{array}$ & $\begin{array}{r}739.3 \\
392.5 \\
13.3 \\
- \\
13.3 \\
122.1 \\
257.1 \\
48.4 \\
42.2 \\
2.5 \\
36.2 \\
27.7 \\
1.5 \\
66.2 \\
32.3 \\
346.8\end{array}$ & $\begin{array}{r}1,855.6 \\
1,173.0 \\
26.5 \\
- \\
26.5 \\
440.0 \\
706.5 \\
118.2 \\
126.4 \\
15.4 \\
61.7 \\
55.0 \\
1.4 \\
115.4 \\
212.9 \\
682.6\end{array}$ & $\begin{array}{r}2,604.7 \\
1,590.8 \\
69.5 \\
- \\
69.5 \\
613.4 \\
907.9 \\
151.2 \\
135.3 \\
2.7 \\
67.9 \\
61.4 \\
57.5 \\
249.9 \\
182.0 \\
1,013.9\end{array}$ & $\begin{array}{r}R_{2,548.4} \\
R_{1,278.7} \\
99.5 \\
- \\
99.5 \\
559.8 \\
R_{619.4} \\
121.2 \\
118.4 \\
.7 \\
65.1 \\
R_{57.3} \\
55.4 \\
33.1 \\
R_{168.1} \\
1,269.7\end{array}$ & $\begin{array}{r}R_{2,459.3} \\
R_{1,194.0} \\
93.4 \\
- \\
93.4 \\
531.8 \\
R_{568.8} \\
109.6 \\
88.2 \\
.8 \\
86.4 \\
R_{59.0} \\
49.0 \\
18.0 \\
R_{157.8} \\
1,265.4\end{array}$ & $\begin{array}{r}R_{2,531.3} \\
\text { R }_{1,223.8} \\
79.9 \\
- \\
79.9 \\
576.4 \\
R_{567.6} \\
77.3 \\
61.6 \\
.2 \\
83.4 \\
R^{6} 69.6 \\
49.2 \\
40.7 \\
R_{185.6} \\
1,307.5\end{array}$ & $\begin{array}{r}2,644.5 \\
1,300.8 \\
76.9 \\
- \\
76.9 \\
656.6 \\
567.3 \\
93.0 \\
92.2 \\
.6 \\
83.9 \\
69.0 \\
29.2 \\
34.8 \\
164.5 \\
1,343.8\end{array}$ \\
\hline 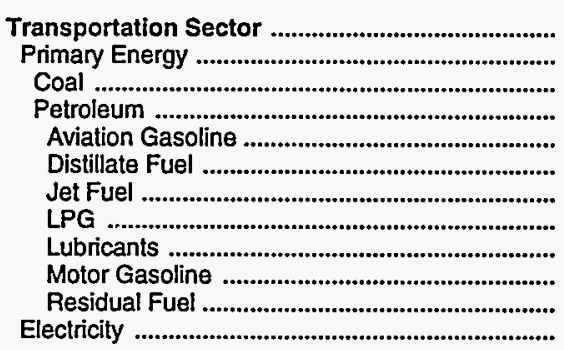 & $\begin{array}{r}915.2 \\
915.2 \\
(s) \\
915.2 \\
6.6 \\
59.6 \\
42.8 \\
.6 \\
16.9 \\
788.3 \\
.3 \\
-\end{array}$ & $\begin{array}{r}1,982.9 \\
1,982.9 \\
(s) \\
1,982.8 \\
6.9 \\
181.7 \\
147.4 \\
1.1 \\
23.4 \\
1,618.2 \\
4.1 \\
-\end{array}$ & $\begin{array}{r}4,731.6 \\
4,731.1 \\
- \\
4,731.1 \\
17.6 \\
616.2 \\
598.1 \\
1.5 \\
53.8 \\
3,389.1 \\
54.8 \\
.5\end{array}$ & $\begin{array}{r}4,612.0 \\
4,609.6 \\
- \\
4,609.6 \\
10.7 \\
707.4 \\
518.0 \\
7.5 \\
60.0 \\
3,284.4 \\
21.5 \\
2.4\end{array}$ & $\begin{array}{r}R_{5,176.2} \\
R_{5,170.8} \\
- \\
R_{5,170.8} \\
9.2 \\
1,015.9 \\
567.9 \\
3.9 \\
R_{56.0} \\
3,502.5 \\
15.4 \\
5.3\end{array}$ & $\begin{array}{r}R_{4,832.8} \\
R_{4,827.4} \\
- \\
R_{4,827.4} \\
8.0 \\
936.8 \\
375.5 \\
4.3 \\
R_{57.6} \\
3,431.1 \\
14.2 \\
5.4\end{array}$ & $\begin{array}{r}\text { R }_{4,676.5} \\
\text { R }_{4,671.1} \\
- \\
\text { R }_{4,671.1} \\
7.2 \\
917.4 \\
307.3 \\
4.0 \\
\text { R } 68.0 \\
3,310.9 \\
\text { ค } 56.3 \\
5.4\end{array}$ & $\begin{array}{r}5,271.8 \\
5,266.8 \\
- \\
5,266.8 \\
7.0 \\
1,018.9 \\
354.0 \\
4.1 \\
67.5 \\
3,784.6 \\
30.7 \\
5.1\end{array}$ \\
\hline $\begin{array}{l}\text { Total Energy . . } \\
\text { Primary Energy - Four Sectors } \\
\text { Electricity . . }\end{array}$ & $\begin{array}{r}1,745.3 \\
1,253.6 \\
491.7\end{array}$ & $\begin{array}{l}3,925.8 \\
2,659.9 \\
1,265.9\end{array}$ & $\begin{array}{l}8,824.5 \\
6,597.2 \\
2,227.3\end{array}$ & $\begin{array}{r}11,016.0 \\
7,325.8 \\
3,690.2\end{array}$ & $\begin{array}{r}R_{12,832.4} \\
R_{7,577.7} \\
5,254.7\end{array}$ & $\begin{array}{r}R_{12,488.4} \\
R_{7,171.1} \\
5,317.3\end{array}$ & $\begin{array}{r}\mathrm{R}_{12,615.7} \\
\mathrm{R}_{7,086.3} \\
5,529.5\end{array}$ & $\begin{array}{r}13,832.8 \\
7,885.0 \\
5,947.8\end{array}$ \\
\hline $\begin{array}{l}\text { Electric Utility Sector a } \\
\text { Coal } \\
\text { Natural Gas } \\
\text { Petroleum } \\
\text { Heavy Oil }{ }^{\mathrm{b}} \\
\text { Light Oil } \\
\text { Petroleum Coke } \\
\text { Nuclear Fuel } \\
\text { Biomass Fuels }\end{array}$ & $\begin{array}{r}88.1 \\
67.7 \\
17.3 \\
3.1 \\
3.0 \\
.1 \\
- \\
- \\
-\end{array}$ & $\begin{array}{r}372.6 \\
280.1 \\
29.3 \\
58.7 \\
44.3 \\
14.4 \\
\overrightarrow{4.3} \\
-\end{array}$ & $\begin{array}{r}837.7 \\
756.7 \\
9.7 \\
29.7 \\
14.6 \\
15.1 \\
\overline{-} \\
41.7 \\
-\end{array}$ & $\begin{array}{r}1,379.9 \\
1,287.7 \\
3.9 \\
9.0 \\
1.3 \\
7.7 \\
- \\
79.4 \\
-\end{array}$ & $\begin{array}{r}1,425.9 \\
1,181.5 \\
5.9 \\
8.5 \\
1.6 \\
6.9 \\
-\overline{230.0} \\
-\end{array}$ & $\begin{array}{r}1,280.3 \\
1,067.1 \\
2.4 \\
5.6 \\
.3 \\
5.3 \\
- \\
205.1 \\
-\end{array}$ & $\begin{array}{r}1,212.4 \\
1,025.8 \\
3.4 \\
6.7 \\
1.3 \\
5.4 \\
- \\
176.5 \\
-\end{array}$ & $\begin{array}{r}1,274.5 \\
1,097.1 \\
10.0 \\
10.6 \\
2.3 \\
8.3 \\
\overrightarrow{156.8} \\
-\end{array}$ \\
\hline 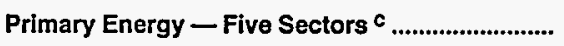 & $1,341.6$ & $3,032.5$ & $7,434.9$ & $8,705.8$ & R $9,003.6$ & ${ }^{R} 8,451.4$ & $R_{8,298.7}$ & $9,159.5$ \\
\hline
\end{tabular}

a There are no direct fuel costs for hydroelectric, geothermal, wind, photovoltaic, or solar thermal energy.

beavy oil includes fuel oil nos. 4, 5, and 6, and residual fuel oils. Light oil includes fuel nos. 1 and 2, kerosene, and jel fuel.

c Biomass fuels are not included, except those consumed at electric utilities and those added to motor gasoline.

$R=$ Revised data.

-No consumption, including cases where adjustments were made. See explanation of adjustments in Section 6 of Appendix A.

$(s)=V a l u e$ less than 0.05 million dollars.

Note: Totals may not equal sum of components due to Independent rounding.

Sources: Data sources, estimation procedures, and assumptions are described in Appendix A. 


\begin{tabular}{|c|c|c|c|c|c|c|c|c|}
\hline Energy Source & 1970 & 1975 & 1980 & 1985 & 1990 & 1991 & 1992 & 1993 \\
\hline & \multicolumn{8}{|c|}{ Prices in Dollars per Millton Btu } \\
\hline 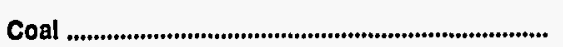 & - & - & - & 2.15 & 1.82 & 1.80 & 1.70 & 1.74 \\
\hline 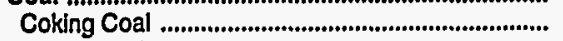 & - & - & - & - & - & - & - & - \\
\hline 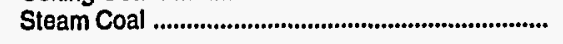 & - & - & - & 2.15 & 1.82 & 1.80 & 1.70 & 1.74 \\
\hline 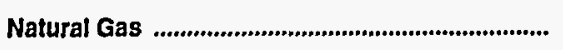 & - & - & 13.06 & 14.20 & 12.24 & 14.16 & 13.33 & 13.05 \\
\hline 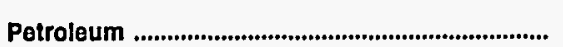 & 1.09 & 2.53 & 6.17 & 6.79 & ${ }^{\mathrm{R}} 6.51$ & 5.94 & 5.63 & 6.05 \\
\hline 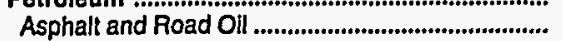 & .56 & 1.77 & 3.61 & 4.47 & 3.15 & 3.29 & 2.81 & 2.94 \\
\hline 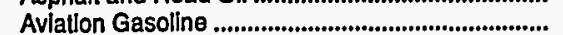 & 2.17 & 3.45 & 9.02 & 9.99 & 9.32 & 8.71 & 8.54 & 8.24 \\
\hline 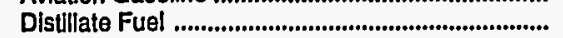 & 1.04 & 2.30 & 6.58 & 7.88 & 8.09 & 7.97 & 7.32 & 7.41 \\
\hline 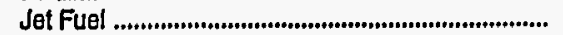 & .73 & 2.04 & 6.21 & 6.21 & 5.99 & 5.17 & 4.90 & 4.79 \\
\hline 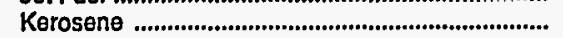 & .85 & 2.50 & 6.38 & 6.86 & 6.77 & 6.22 & 6.30 & 6.51 \\
\hline 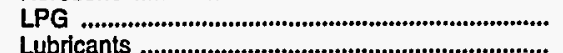 & $\begin{array}{l}2.53 \\
5.08\end{array}$ & $\begin{array}{l}3.77 \\
7.48\end{array}$ & $\begin{array}{r}6.32 \\
14.36\end{array}$ & $\begin{array}{l}13.79 \\
17.61\end{array}$ & $R_{14.60}^{15.56}$ & $\begin{aligned} 15.41 \\
R \\
16.79\end{aligned}$ & $\begin{array}{r}14.70 \\
R_{19.43}\end{array}$ & $\begin{array}{l}10.58 \\
18.94\end{array}$ \\
\hline Motor Gasollne & 3.32 & 5.44 & 10.81 & 11.14 & 11.71 & 10.40 & 10.95 & 11.35 \\
\hline 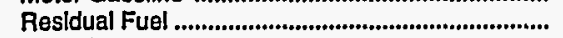 & .40 & 1.59 & 3.80 & 4.81 & 4.01 & 3.21 & $A_{2.82}$ & 2.99 \\
\hline Other ${ }^{a}$ & .51 & 1.39 & 2.49 & 2.51 & 2.02 & 1.72 & 1.29 & 1.06 \\
\hline 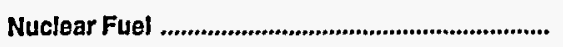 & - & - & - & - & - & - & - & - \\
\hline 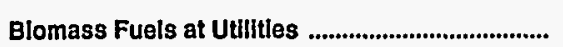 & .65 & .92 & - & .79 & (d) & - & - & - \\
\hline Prlmary Energy — Flve Sectors ${ }^{b}$............................. & 1.09 & 2.53 & 6.25 & 6.85 & 6.55 & 6.02 & 5.70 & 6.11 \\
\hline 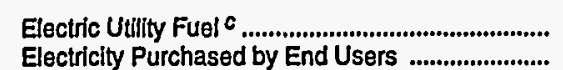 & $\begin{array}{r}.41 \\
6.98\end{array}$ & $\begin{array}{r}1.58 \\
12.80\end{array}$ & $\begin{array}{r}3.97 \\
22.01\end{array}$ & $\begin{array}{r}4.94 \\
29.81\end{array}$ & $\begin{array}{r}4.33 \\
26.53\end{array}$ & $\begin{array}{r}3.44 \\
27.14\end{array}$ & $\begin{array}{r}3.26 \\
27.81\end{array}$ & $\begin{array}{r}3.49 \\
31.34\end{array}$ \\
\hline \multirow[t]{2}{*}{ 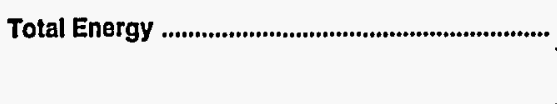 } & 1.73 & 3.95 & 8.71 & 10.30 & 10.03 & R9.96 & 9.78 & 11.22 \\
\hline & \multicolumn{8}{|c|}{ Expenditures in Millions of Dollars } \\
\hline Coal & _ & _ & - & 2.4 & 1.3 & 1.7 & 2.0 & 3.1 \\
\hline 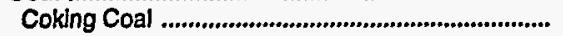 & - & - & - & - & - & - & - & - \\
\hline 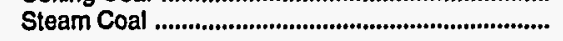 & - & - & - & 2.4 & 1.3 & 1.7 & 2.0 & 3.1 \\
\hline 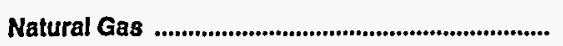 & - & - & 39.4 & 38.1 & 36.5 & 41.2 & 38.5 & 37.2 \\
\hline Petroleum. & 207.0 & 522.2 & $1,499.6$ & $1,547.4$ & $R_{1,743.2}$ & $R_{1,488.9}$ & $R_{1,367.6}$ & $1,261.9$ \\
\hline 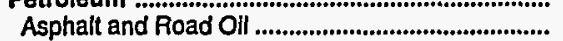 & 1.4 & 4.4 & 6.8 & 9.1 & 8.0 & 8.4 & 8.0 & 8.7 \\
\hline Avlation Gasoline ................................................... & 1.5 & 2.0 & 9.1 & 7.8 & 12.8 & 11.5 & 10.5 & 8.3 \\
\hline 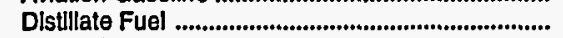 & 9.9 & 25.6 & 228.7 & 211.5 & 321.4 & 335.8 & 238.1 & 208.4 \\
\hline 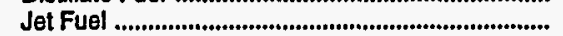 & 58.4 & 170.3 & 492.4 & 462.1 & 425.3 & 323.4 & 276.6 & 241.2 \\
\hline Kerosene ........................................................................ & .7 & 1.1 & .3 & .1 & (s) & (s) & (s) & (s) \\
\hline LPG & 8.5 & 10.3 & 31.1 & 6.5 & 9.8 & 10.8 & 31.6 & 18.9 \\
\hline Lubricants .................................................... & 2.2 & 4.7 & 8.2 & 9.1 & $R_{8.5}$ & R. 8.8 & $F_{10.4}$ & 10.3 \\
\hline Motor Gasoline & 99.2 & 193.5 & 410.7 & 444.3 & 530.3 & 490.0 & 510.1 & 539.8 \\
\hline 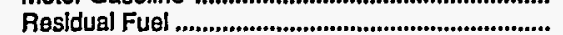 & 24.7 & 108.5 & 308.6 & 395.4 & 425.7 & 299.3 & $R_{281.2}$ & 225.5 \\
\hline Other $\mathrm{a}$ & .5 & 1.7 & 3.7 & 1.5 & 1.4 & .9 & 1.0 & .9 \\
\hline 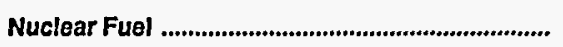 & - & - & - & - & - & - & - & - \\
\hline 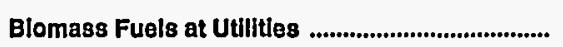 & .2 & 2 & - & .2 & $(0)$ & - & - & - \\
\hline Prlmary Energy — Flve Sectors b ............................ & 207.2 & 522.4 & $1,538.9$ & 1,588.1 & $R_{1,781.0}$ & $\mathrm{R}_{1, \mathbf{5 3 1 . 7}}$ & $R_{1,408.1}$ & $1,302.2$ \\
\hline 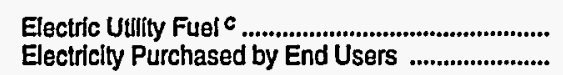 & $\begin{array}{r}-17.4 \\
87.4\end{array}$ & $\begin{array}{r}-92.4 \\
225.3\end{array}$ & $\begin{array}{r}-275.8 \\
456.9\end{array}$ & $\begin{array}{r}-342.5 \\
654.7\end{array}$ & $\begin{array}{l}-371.8 \\
732.1\end{array}$ & $\begin{array}{r}-271.5 \\
768.9\end{array}$ & $\begin{array}{r}-242.9 \\
803.0\end{array}$ & $\begin{array}{r}-230.3 \\
902.7\end{array}$ \\
\hline Total Energy & 277.2 & 655.3 & $1,720.0$ & $1,900.4$ & $R_{2,141.2}$ & $R_{2,029.1}$ & $R_{1,968.2}$ & $1,974.6$ \\
\hline
\end{tabular}

a Includes petroleum coke used at electric utilities.

b Blomass fuels are not included, except those consumed at electric utilities and those added to motor gasoline.

There are no direct fuel costs for hydroelectric, geothermal, wind, photovoltalc, or solar thermal energy.

Utilltes used blomass fuels at no charge or received a fee for accepting them.

$R=$ Revlsed data.
-No consumption, including cases where adjustments were made. See explanation of adjustments in Section 6 of Appendix A.

$(s)=$ Value less than 0.05 million dollars.

Note: Expenditure totals may not equal sum of components due to independent rounding.

Sources: Data sources, estimation procedures, and assumptions are described in Appendix A. 
H Energy Price Estimates by Sector, Hawaii

A 1970, 1975, 1980, 1985, and 1990-1993

W (Dollars per Million Btu)

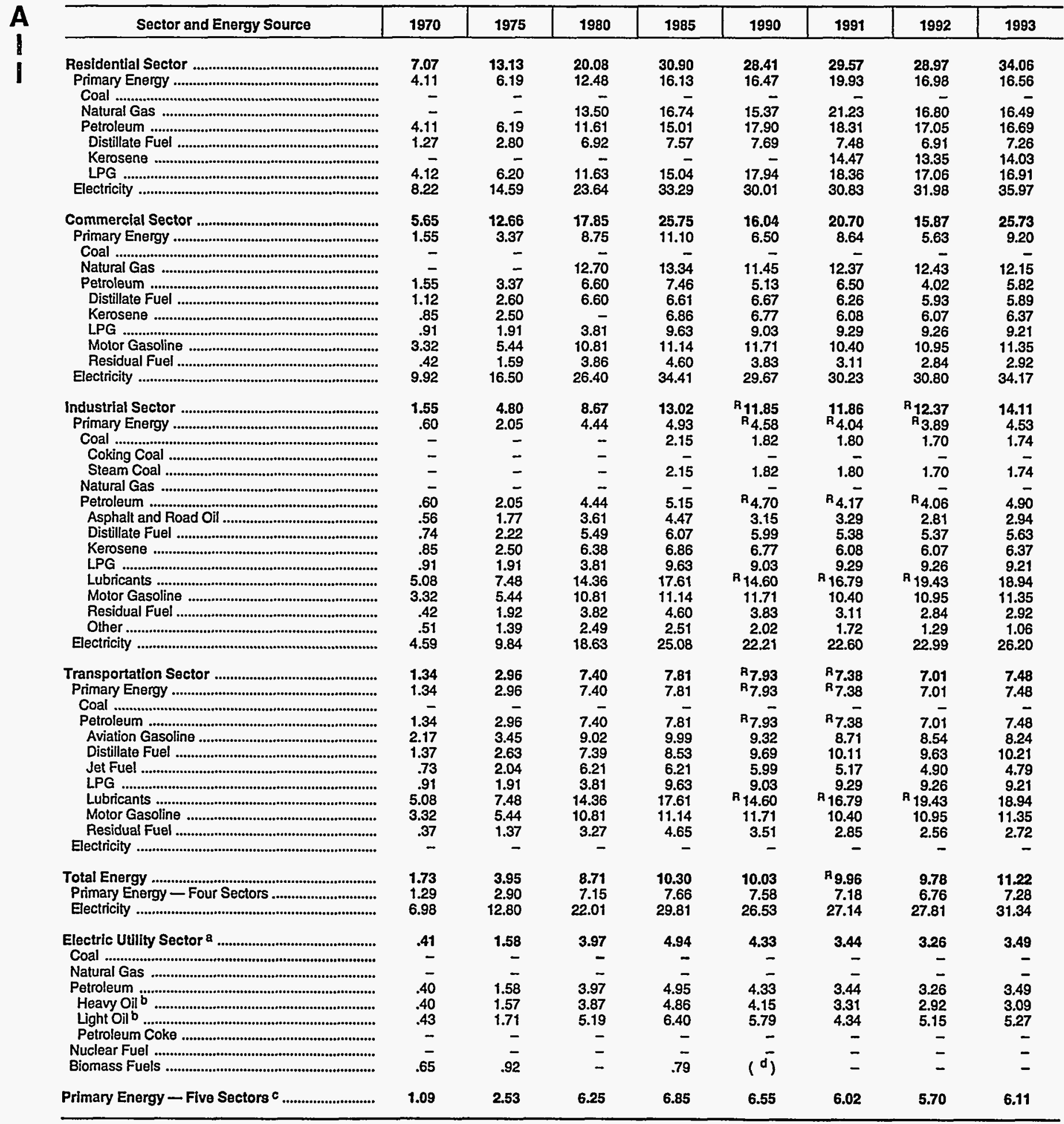

a There are no direct fuel costs for hydroelectric, geothermal, wind, photovoltaic, or solar thermal energy.

Heavy oil includes fuel oil nos. 4, 5, and 6, and residual fuel oils. Light oil includes fuel oil nos. 1 and 2, kerosene, and jet fuel.

c Biomass fuels are not included, except those consumed at electric utilities and those added to motor gasoline.

d Utilities used biomass fuels at no charge or received a fee for accepting

them.

$R=$ Revised data.

-No consumption, including cases where adjustments were made. See explanation of adjustments in Section 6 of Appendix A.

Sources: Data sources, estimation procedures, and assumptions are described in Appendix A. 
(Million Dollars)

\begin{tabular}{|c|c|c|c|c|c|c|c|c|}
\hline Sector and Energy Source & 1970 & 1975 & 1980 & 1985 & 1990 & 1991 & 1992 & 1993 \\
\hline 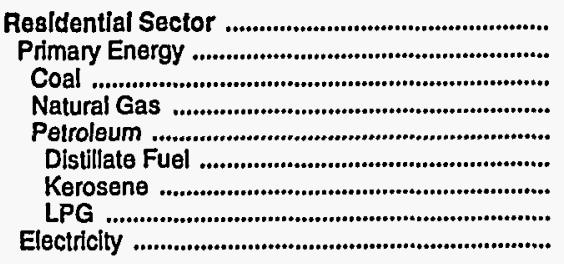 & $\begin{array}{r}43.0 \\
7.0 \\
- \\
- \\
7.0 \\
(s) \\
\overline{7.0} \\
36.0\end{array}$ & $\begin{array}{r}90.1 \\
7.4 \\
- \\
- \\
7.4 \\
(s) \\
- \\
7.4 \\
82.8\end{array}$ & $\begin{array}{r}185.3 \\
36.8 \\
- \\
18.4 \\
18.4 \\
(s) \\
-\overline{-} \\
18.4 \\
148.5\end{array}$ & $\begin{array}{r}230.2 \\
16.8 \\
-\overline{1} \\
11.3 \\
5.5 \\
(\mathrm{~s}) \\
\overline{5.5} \\
213.4\end{array}$ & $\begin{array}{r}255.6 \\
17.6 \\
- \\
9.3 \\
8.3 \\
(s) \\
- \\
8.3 \\
238.0\end{array}$ & $\begin{array}{r}273.2 \\
21.2 \\
- \\
12.5 \\
8.7 \\
(s) \\
(s) \\
8.7 \\
252.0\end{array}$ & $\begin{array}{r}301.5 \\
35.5 \\
-\overrightarrow{9} \\
9.9 \\
25.5 \\
(s) \\
(s) \\
25.5 \\
266.0\end{array}$ & $\begin{array}{r}318.1 \\
15.2 \\
-\overline{9} \\
5.8 \\
.1 \\
(\mathrm{~s}) \\
5.3 \\
302.9\end{array}$ \\
\hline 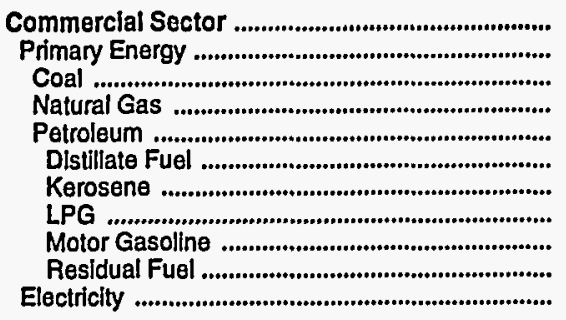 & $\begin{array}{r}30.3 \\
4.2 \\
- \\
- \\
4.2 \\
1.1 \\
.4 \\
.3 \\
2.3 \\
.1 \\
26.1\end{array}$ & $\begin{array}{r}67.7 \\
5.3 \\
- \\
- \\
5.3 \\
1.3 \\
.6 \\
.4 \\
2.8 \\
.2 \\
62.5\end{array}$ & $\begin{array}{r}172.7 \\
41.0 \\
- \\
21.0 \\
20.0 \\
15.3 \\
- \\
1.1 \\
3.1 \\
.6 \\
131.7\end{array}$ & $\begin{array}{r}225.4 \\
36.1 \\
- \\
26.8 \\
9.3 \\
5.2 \\
.1 \\
.6 \\
2.8 \\
.6 \\
189.3\end{array}$ & $\begin{array}{r}299.5 \\
71.4 \\
- \\
27.2 \\
44.2 \\
19.7 \\
(s) \\
.7 \\
3.6 \\
20.1 \\
228.1\end{array}$ & $\begin{array}{r}297.8 \\
54.9 \\
- \\
28.7 \\
26.2 \\
22.3 \\
(s) \\
.8 \\
2.7 \\
.4 \\
242.9\end{array}$ & $\begin{array}{r}321.7 \\
67.7 \\
- \\
28.6 \\
39.1 \\
15.1 \\
(\mathrm{~s}) \\
2.4 \\
2.6 \\
19.0 \\
254.0\end{array}$ & $\begin{array}{r}320.8 \\
38.8 \\
- \\
27.4 \\
11.4 \\
9.6 \\
(s) \\
.5 \\
.6 \\
.6 \\
282.1\end{array}$ \\
\hline 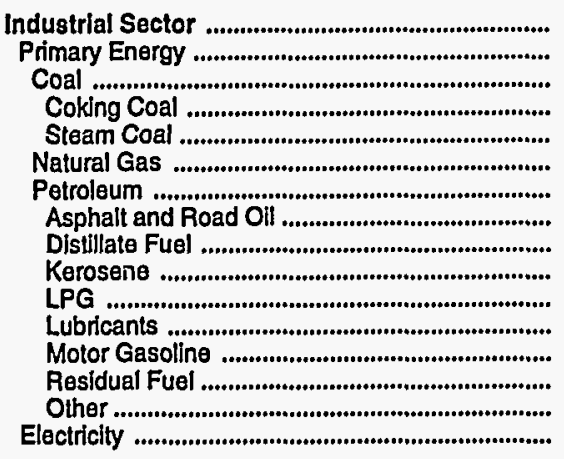 & $\begin{array}{r}36.0 \\
10.7 \\
- \\
- \\
- \\
- \\
10.7 \\
1.4 \\
2.8 \\
.3 \\
1.2 \\
.1 \\
.9 \\
3.5 \\
.5 \\
25.3\end{array}$ & $\begin{array}{r}110.9 \\
30.8 \\
- \\
- \\
- \\
- \\
30.8 \\
4.4 \\
7.3 \\
.4 \\
2.4 \\
1.3 \\
1.5 \\
11.7 \\
1.7 \\
80.1\end{array}$ & $\begin{array}{r}275.7 \\
99.1 \\
- \\
- \\
- \\
- \\
99.1 \\
6.8 \\
43.0 \\
.3 \\
11.3 \\
1.7 \\
2.8 \\
29.4 \\
3.7 \\
176.7\end{array}$ & $\begin{array}{r}325.7 \\
73.8 \\
2.4 \\
- \\
2.4 \\
- \\
71.3 \\
9.1 \\
16.5 \\
(\mathrm{~s}) \\
.2 \\
1.9 \\
6.1 \\
36.0 \\
1.5 \\
252.0\end{array}$ & $\begin{array}{r}R_{344.3} \\
R_{78.3} \\
1.3 \\
- \\
1.3 \\
- \\
R_{77.0} \\
8.0 \\
28.2 \\
(s) \\
.4 \\
R_{1.8} \\
8.2 \\
29.1 \\
1.4 \\
266.0\end{array}$ & $\begin{array}{r}\mathrm{R}_{341.3} \\
\mathrm{R}_{67.2} \\
1.7 \\
- \\
1.7 \\
- \\
\mathrm{R}_{65.6} \\
8.4 \\
21.5 \\
(\mathrm{~s}) \\
.9 \\
\mathrm{R}_{1.8} \\
8.2 \\
23.9 \\
.9 \\
274.0\end{array}$ & $\begin{array}{r}R_{342.8} \\
R_{59.9} \\
2.0 \\
- \\
2.0 \\
- \\
R_{57.9} \\
8.0 \\
18.7 \\
(s) \\
2.4 \\
R_{2.2} \\
8.7 \\
16.8 \\
1.0 \\
282.9\end{array}$ & $\begin{array}{r}387.0 \\
69.3 \\
3.1 \\
- \\
3.1 \\
- \\
66.1 \\
8.7 \\
14.6 \\
(s) \\
12.7 \\
2.2 \\
14.4 \\
12.7 \\
.9 \\
317.7\end{array}$ \\
\hline 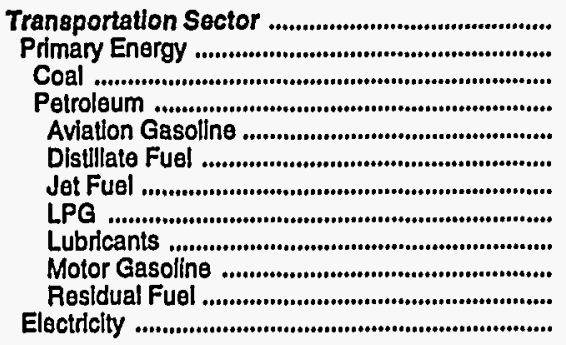 & $\begin{array}{r}167.9 \\
167.9 \\
- \\
167.9 \\
1.5 \\
5.7 \\
58.4 \\
.1 \\
2.1 \\
96.0 \\
4.1 \\
-\end{array}$ & $\begin{array}{r}386.6 \\
386.6 \\
- \\
386.6 \\
2.0 \\
12.7 \\
170.3 \\
.2 \\
3.4 \\
189.2 \\
8.7 \\
-\end{array}$ & $\begin{array}{r}1,086.3 \\
1,086.3 \\
- \\
1,086.3 \\
9.1 \\
143.5 \\
492.4 \\
.4 \\
6.5 \\
404.9 \\
29.7 \\
-\end{array}$ & $\begin{array}{r}1,119.0 \\
1,119.0 \\
- \\
1,119.0 \\
7.8 \\
161.7 \\
462.1 \\
.2 \\
7.2 \\
435.4 \\
44.6 \\
-\end{array}$ & $\begin{array}{r}R_{1,241.8} \\
A_{1,241.8} \\
- \\
R_{1,241.8} \\
12.8 \\
218.5 \\
425.3 \\
.4 \\
A_{6.7} \\
518.5 \\
59.5 \\
-\end{array}$ & $\begin{array}{r}R_{1,116.9} \\
R_{1,116.9} \\
- \\
R_{1,116.9} \\
11.5 \\
248.7 \\
323.4 \\
.5 \\
R_{6.9} \\
479.1 \\
46.7 \\
-\end{array}$ & $\begin{array}{r}R_{1,002.1} \\
R_{1,002.1} \\
- \\
R_{1,002.1} \\
10.5 \\
145.7 \\
276.6 \\
1.2 \\
R_{8.2} \\
498.8 \\
R_{61.0} \\
-\end{array}$ & $\begin{array}{r}948.6 \\
948.6 \\
-\overline{-} \\
948.6 \\
8.3 \\
120.0 \\
241.2 \\
.3 \\
8.1 \\
524.7 \\
46.0 \\
-\end{array}$ \\
\hline 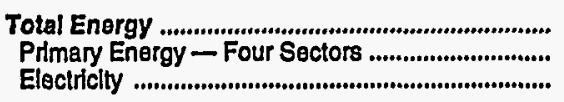 & $\begin{array}{r}277.2 \\
189.8 \\
87.4\end{array}$ & $\begin{array}{l}655.3 \\
430.0 \\
225.3\end{array}$ & $\begin{array}{r}1,720.0 \\
1,263.2 \\
456.9\end{array}$ & $\begin{array}{r}1,900.4 \\
1,245.7 \\
654.7\end{array}$ & $\begin{array}{r}R_{2,141.2} \\
R_{1,409.1} \\
732.1\end{array}$ & $\begin{array}{r}R_{2,029.1} \\
R_{1,260.2} \\
768.9\end{array}$ & $\begin{array}{r}R_{1,968.2} \\
A_{1,165.2} \\
803.0\end{array}$ & $\begin{array}{r}1,974.6 \\
1,071.8 \\
902.7\end{array}$ \\
\hline 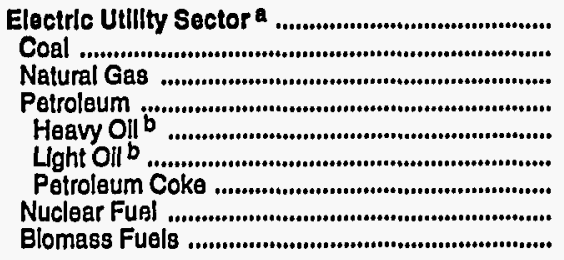 & $\begin{array}{r}17.4 \\
\overline{-} \\
17.2 \\
17.0 \\
.2 \\
\overline{2} \\
\overline{.2}\end{array}$ & $\begin{array}{r}92.4 \\
\overline{-} \\
92 . \overline{2} \\
87.9 \\
4.3 \\
\overline{-} \\
. \overline{2}\end{array}$ & $\begin{array}{r}275.8 \\
- \\
275.8 \\
248.9 \\
26.8 \\
- \\
- \\
-\end{array}$ & $\begin{array}{c}342.5 \\
- \\
- \\
342.3 \\
314.2 \\
28.0 \\
- \\
- \\
.2\end{array}$ & $\begin{array}{r}371.8 \\
- \\
- \\
371.8 \\
316.8 \\
55.0 \\
- \\
- \\
(\mathrm{d})\end{array}$ & $\begin{array}{r}271.5 \\
- \\
- \\
271.5 \\
228.3 \\
43.2 \\
- \\
- \\
-\end{array}$ & $\begin{array}{r}242.9 \\
- \\
- \\
242.9 \\
184.3 \\
58.6 \\
- \\
- \\
-\end{array}$ & $\begin{array}{r}230.3 \\
- \\
- \\
230.3 \\
166.2 \\
64.2 \\
= \\
=\end{array}$ \\
\hline 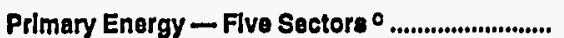 & 207.2 & 522.4 & $1,538.8$ & $1,588.1$ & $R_{1,781.0}$ & $R_{1,531.7}$ & $A_{1,408.1}$ & $1,302.2$ \\
\hline
\end{tabular}

a There are no direct fuel costs for hydroelectric, geothermal, wind, photovoltalc, or solar thermal energy.

Heavy oll includes fuel oll nos. 4, 5, and 6, and residual fuel oils. Light oll includes fuel nos. 1 and 2, kerosene, and jet fuel.

- Blomass fuels are not included, except those consumed at electric utllttles and those added to motor gasoline. them.
$R=$ Revised data.

-No consumption, Inciuding cases where adjustments were mado. Soo explanation of adjustments in Section 6 of Appendix A.

(s)=Value less than 0.05 million dollars.

Note: Totals may not equal sum of components due to independent rounding.

Sources: Data sources, estimation procedures, and assumptions are described in Appendix A. 
I Energy Price and Expenditure Estimates by Source, Idaho

D $1970,1975,1980,1985$, and 1990-1993

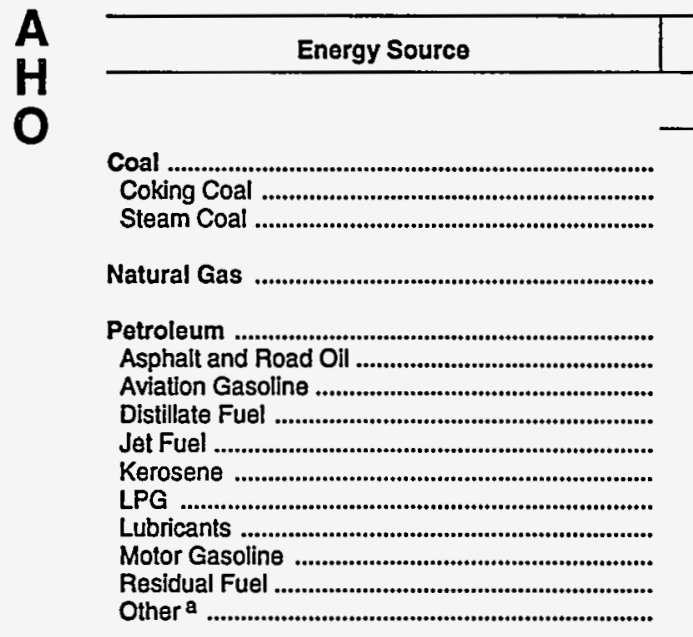

Nuclear Fuel

Blomass Fuels at Utilitles

Primary Energy - Five Sectors b

\begin{tabular}{l|l|}
1970 & 1975 \\
\hline
\end{tabular}

\begin{tabular}{l|l}
1980 & 1985 \\
\hline
\end{tabular}

1990

1991

1993

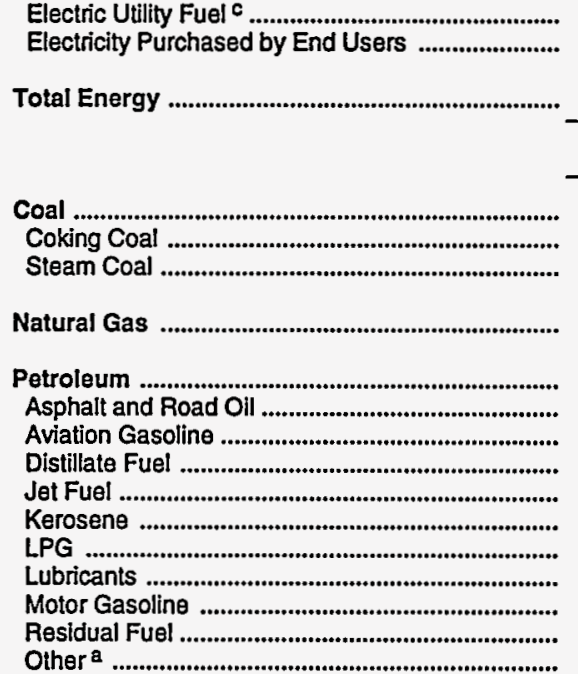

.

$-$

\begin{tabular}{rrrrrrrrr}
$\ldots .$. & - & - & - & - & - & - & - & - \\
$\ldots$. & 1.50 & 2.80 & 6.57 & 7.23 & ${ }^{R_{6.57}}$ & 6.49 & ${ }^{R_{6.65}}$ & 6.78 \\
$\ldots$ & .35 & 1.89 & 3.87 & 5.60 & 5.38 & 5.04 & 4.83 & 4.99 \\
$\ldots$ & 2.95 & 4.11 & 7.39 & 10.66 & 11.15 & 11.27 & 11.35 & 11.71 \\
$\ldots$ & 1.77 & 3.03 & 6.75 & 8.16 & ${ }^{R} 7.78$ & ${ }^{8} 7.68$ & 7.89 & 7.99 \\
\hline
\end{tabular}

Expenditures in Millions of Dollars

\begin{tabular}{|c|c|c|c|c|c|c|c|}
\hline 1.77 & 3.03 & 6.75 & 8.16 & ${ }^{R} 7.78$ & ${ }^{8} 7.68$ & 7.89 & 7.99 \\
\hline \multicolumn{8}{|c|}{ Expenditures in Millions of Dollars } \\
\hline 4.7 & 13.1 & 17.1 & 15.1 & 17.7 & 22.9 & 18.1 & 17.5 \\
\hline- & - & - & - & - & - & - & - \\
\hline 4.7 & 13.1 & 17.1 & 15.1 & 17.7 & 22.9 & 18.1 & 17.5 \\
\hline 29.5 & 84.6 & 182.6 & 192.9 & 142.4 & 173.8 & 170.1 & 205.2 \\
\hline 202.2 & 456.2 & 913.5 & 880.6 & R 969.1 & $R_{1,018.2}$ & $A_{1,007.6}$ & $1,110.6$ \\
\hline 4.5 & 11.0 & 19.2 & 20.0 & 22.4 & 21.5 & 28.5 & 28.8 \\
\hline 1.7 & 2.1 & 7.4 & 4.0 & 1.9 & 1.7 & (s) & 2.6 \\
\hline 32.9 & 112.3 & 215.6 & 247.7 & 319.4 & 361.4 & 307.5 & 352.8 \\
\hline 3.9 & 11.0 & 44.9 & 40.7 & 38.1 & 28.9 & 29.1 & 32.1 \\
\hline 1.1 & 2.1 & - & .3 & .5 & .2 & .1 & .1 \\
\hline 9.6 & 16.7 & 23.3 & 26.5 & 22.3 & 30.3 & 24.3 & 24.8 \\
\hline 4.7 & 7.4 & 15.8 & 17.7 & $R_{16.5}$ & R 17.0 & $\mathrm{H}_{20.0}$ & 19.9 \\
\hline 142.8 & 285.0 & 570.0 & 521.6 & 547.2 & 556.4 & 597.7 & 648.8 \\
\hline .6 & 8.6 & 17.1 & 2.0 & .7 & .6 & .2 & .7 \\
\hline .4 & - & - & - & - & - & - & - \\
\hline- & - & - & - & - & - & - & - \\
\hline- & - & - & $\rightarrow$ & - & - & - & - \\
\hline 236.4 & 553.9 & $1,113.1$ & $1,088.6$ & $R_{1,129.2}$ & $R_{1,214.9}$ & $A_{1,195.9}$ & $1,333.2$ \\
\hline (s) & -.1 & -.2 & -.2 & (s) & (s) & (s) & (s) \\
\hline 105.8 & 175.4 & 345.9 & 596.4 & 685.0 & 694.0 & 736.0 & 748.0 \\
\hline 342.2 & 729.2 & $1,458.8$ & $1,684.9$ & $R_{1,814.2}$ & $R_{1,908,9}$ & $\mathrm{~A}_{1,931,8}$ & $2,081.2$ \\
\hline
\end{tabular}

Nuclear Fuel.

Biomass Fuels at Utilities

Primary Energy - Flve Sectors ${ }^{b}$

Electric Utility Fuel c

Electricity Purchased by End Users .........................

Total Energy

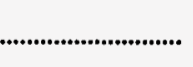

a Inciudes petroleum coke used at electric utilities.

b Blomass fuels are not included, except those consumed at electric explanation of adjustments in Sectlon 6 of Appendix A.

$(s)=$ Value less than 0.05 million dollars.

utilities and those added to motor gasoline.

$c$ There are no direct fuel costs for hydroelectric, geothermal, wind, photovoltaic, or solar thermal energy.

$\mathrm{R}=$ Revised data.

Note: Expenditure totals may not equal sum of components due to independent rounding.

Sources: Data sources, estimation procedures, and assumptions are

- No consumption, including cases where adjustments were made. See described in Appendix A. 


\begin{tabular}{|c|c|c|c|c|c|c|c|c|}
\hline Sector and Energy Source & 1970 & 1975 & 1980 & 1985 & 1990 & 1991 & 1992 & 1993 \\
\hline 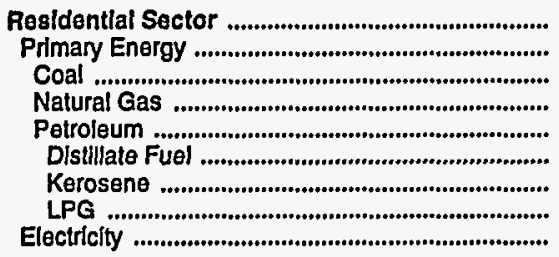 & $\begin{array}{r}2.58 \\
1.54 \\
.99 \\
1.31 \\
1.91 \\
1.40 \\
2 . \overline{-} \\
4.81\end{array}$ & $\begin{array}{r}3.43 \\
2.45 \\
1.78 \\
2.07 \\
3.25 \\
2.82 \\
- \\
4.17 \\
5.27\end{array}$ & $\begin{array}{r}7.14 \\
5.28 \\
2.56 \\
4.73 \\
6.97 \\
6.60 \\
7.85 \\
8.54\end{array}$ & $\begin{array}{r}10.30 \\
6.91 \\
1.97 \\
6.57 \\
7.84 \\
7.29 \\
12.00 \\
9.55 \\
12.60\end{array}$ & $\begin{array}{r}10.83 \\
5.95 \\
1.55 \\
4.91 \\
8.58 \\
7.37 \\
12.14 \\
11.73 \\
14.27\end{array}$ & $\begin{array}{r}10.61 \\
6.04 \\
1.49 \\
5.02 \\
8.45 \\
7.20 \\
11.86 \\
12.24 \\
14.33\end{array}$ & $\begin{array}{r}10.72 \\
5.78 \\
1.57 \\
5.08 \\
7.73 \\
6.49 \\
10.69 \\
11.55 \\
14.45\end{array}$ & $\begin{array}{r}10.57 \\
5.79 \\
1.29 \\
5.18 \\
7.76 \\
6.53 \\
10.75 \\
11.50 \\
14.64\end{array}$ \\
\hline 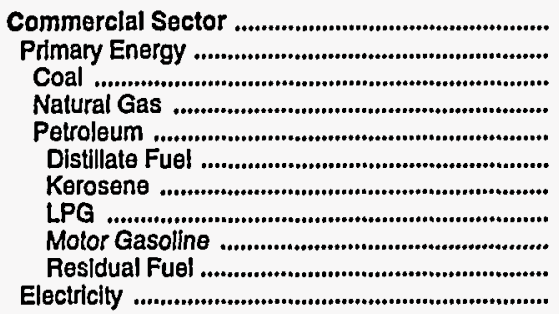 & $\begin{array}{r}2.12 \\
.95 \\
.50 \\
.96 \\
1.35 \\
1.21 \\
.82 \\
1.55 \\
2.81 \\
4.10\end{array}$ & $\begin{array}{r}2.91 \\
1.66 \\
.87 \\
1.47 \\
3.01 \\
2.62 \\
2.59 \\
3.25 \\
4.81 \\
4.88\end{array}$ & $\begin{array}{l}6.49 \\
4.53 \\
1.70 \\
4.36 \\
5.65 \\
6.41 \\
- \\
5.72 \\
9.79 \\
4.63 \\
8.33\end{array}$ & $\begin{array}{r}9.16 \\
5.70 \\
1.69 \\
5.42 \\
7.37 \\
6.82 \\
7.00 \\
9.38 \\
9.31 \\
3.67 \\
12.10\end{array}$ & $\begin{array}{r}9.23 \\
4.61 \\
1.77 \\
4.07 \\
6.96 \\
6.24 \\
6.53 \\
8.30 \\
9.15 \\
2.51 \\
12.54\end{array}$ & $\begin{array}{r}9.01 \\
4.98 \\
1.88 \\
4.28 \\
7.18 \\
5.66 \\
6.93 \\
8.68 \\
9.13 \\
2.31 \\
12.54\end{array}$ & $\begin{array}{r}9.43 \\
5.01 \\
1.90 \\
4.27 \\
7.12 \\
5.54 \\
6.77 \\
8.82 \\
9.52 \\
1.78 \\
12.66\end{array}$ & $\begin{array}{r}9.30 \\
4.65 \\
1.80 \\
4.48 \\
6.08 \\
5.73 \\
7.66 \\
8.79 \\
9.67 \\
2.93 \\
13.00\end{array}$ \\
\hline 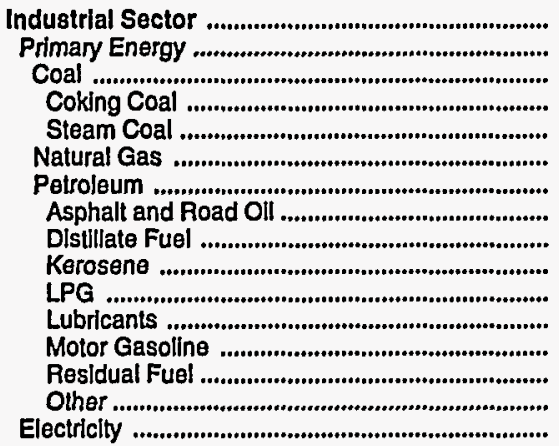 & $\begin{array}{r}.96 \\
.69 \\
.50 \\
- \\
.50 \\
.42 \\
.96 \\
.60 \\
.77 \\
.82 \\
1.55 \\
5.08 \\
2.81 \\
.34 \\
4.14 \\
1.84\end{array}$ & $\begin{array}{r}1.97 \\
1.81 \\
.87 \\
\overline{-} \\
.87 \\
1.11 \\
2.60 \\
1.88 \\
2.40 \\
2.59 \\
3.25 \\
7.48 \\
4.81 \\
2.01 \\
2.70\end{array}$ & $\begin{array}{r}4.53 \\
4.30 \\
1.70 \\
- \\
1.70 \\
3.58 \\
6.02 \\
3.64 \\
6.02 \\
- \\
5.72 \\
14.36 \\
9.79 \\
3.76 \\
-\overline{5} \\
5.44\end{array}$ & $\begin{array}{r}5.68 \\
4.80 \\
1.69 \\
- \\
1.69 \\
4.32 \\
6.60 \\
4.78 \\
6.15 \\
7.00 \\
9.38 \\
17.61 \\
9.31 \\
3.67 \\
7.69\end{array}$ & $\begin{array}{r}4.82 \\
3.65 \\
1.77 \\
- \\
1.77 \\
2.65 \\
R \mathbf{5 . 1 4} \\
2.64 \\
5.74 \\
6.53 \\
8.30 \\
\mathrm{R} \\
14.60 \\
9.15 \\
2.51 \\
-\overline{1} \\
7.69\end{array}$ & $\begin{array}{r}4.99 \\
R 4.10 \\
1.88 \\
- \\
1.88 \\
2.85 \\
R 5.89 \\
3.28 \\
6.10 \\
6.93 \\
8.68 \\
16.79 \\
9.13 \\
2.31 \\
- \\
7.68\end{array}$ & $\begin{array}{r}5.02 \\
\mathrm{R} 3.82 \\
1.90 \\
-\overline{1.90} \\
2.88 \\
\mathrm{R} 5.37 \\
2.93 \\
5.95 \\
6.77 \\
8.82 \\
\mathrm{~A} 19.43 \\
9.52 \\
1.78 \\
-\overline{0} \\
8.00\end{array}$ & $\begin{array}{r}5.05 \\
3.85 \\
1.80 \\
- \\
1.80 \\
2.91 \\
5.61 \\
2.83 \\
6.73 \\
7.66 \\
8.79 \\
18.94 \\
9.67 \\
2.93 \\
8.24\end{array}$ \\
\hline 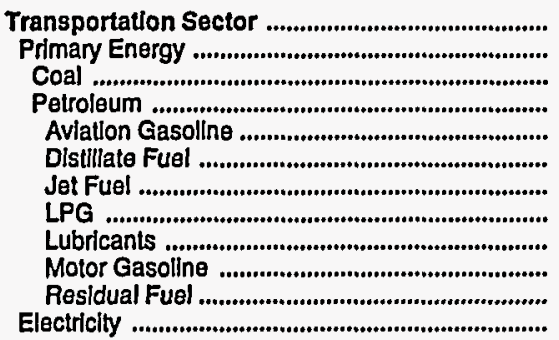 & $\begin{array}{r}2.47 \\
2.47 \\
.50 \\
2.47 \\
2.17 \\
1.31 \\
.76 \\
1.55 \\
5.08 \\
2.81 \\
.39 \\
-\end{array}$ & $\begin{array}{r}4.25 \\
4.25 \\
.87 \\
4.25 \\
3.45 \\
2.68 \\
2.12 \\
3.25 \\
7.48 \\
4.81 \\
- \\
-\end{array}$ & $\begin{array}{r}8.98 \\
8.98 \\
- \\
8.98 \\
9.02 \\
6.95 \\
6.59 \\
5.72 \\
14.36 \\
9.79 \\
- \\
-\end{array}$ & $\begin{array}{r}9.05 \\
9.05 \\
- \\
9.05 \\
9.99 \\
8.70 \\
6.68 \\
9.38 \\
17.61 \\
9.31 \\
- \\
-\end{array}$ & $\begin{array}{r}R_{9.01} \\
R_{9.01} \\
-\bar{r} \\
R_{9.01} \\
9.32 \\
9.27 \\
6.07 \\
8.30 \\
R_{14.60} \\
9.15 \\
- \\
-\end{array}$ & $\begin{array}{r}R_{8.87} \\
R_{8.87} \\
- \\
R_{8.87} \\
8.71 \\
8.74 \\
5.50 \\
8.68 \\
R_{16.79} \\
9.13 \\
- \\
-\end{array}$ & $\begin{array}{r}R_{9.13} \\
R_{9.13} \\
{ }_{9.13} \\
8.54 \\
8.59 \\
5.44 \\
8.82 \\
A_{19.43} \\
9.52 \\
- \\
-\end{array}$ & $\begin{array}{r}9.23 \\
9.23 \\
- \\
9.23 \\
8.24 \\
8.70 \\
5.42 \\
8.79 \\
18.94 \\
9.67 \\
- \\
-\end{array}$ \\
\hline 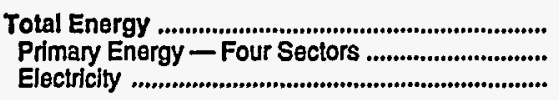 & $\begin{array}{l}1.77 \\
1.50 \\
2.95\end{array}$ & $\begin{array}{l}3.03 \\
2.80 \\
4.11\end{array}$ & $\begin{array}{l}6.75 \\
6.57 \\
7.39\end{array}$ & $\begin{array}{r}8.16 \\
7.23 \\
10.66\end{array}$ & $\begin{array}{r}A_{7.78} \\
R_{6.57} \\
11.15\end{array}$ & $\begin{array}{r}R_{7.68} \\
6.49 \\
11.27\end{array}$ & $\begin{array}{r}7.89 \\
R_{6.65} \\
11.35\end{array}$ & $\begin{array}{r}7.99 \\
6.78 \\
11.71\end{array}$ \\
\hline 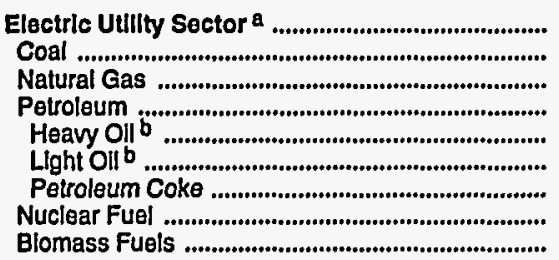 & $\begin{array}{c}35 \\
\overline{-} \\
\overline{35} \\
\overline{35} \\
\overline{-} \\
\overline{-}\end{array}$ & $\begin{array}{r}1.89 \\
-\overline{-} \\
2.20 \\
2 . \overline{0} \\
- \\
- \\
-\end{array}$ & $\begin{array}{r}3.87 \\
-\overline{76} \\
3.763 \\
- \\
6.39 \\
- \\
- \\
-\end{array}$ & $\begin{array}{r}5.60 \\
-\overline{5} \\
5.44 \\
6.07 \\
- \\
6.07 \\
- \\
- \\
-\end{array}$ & $\begin{array}{r}5.38 \\
- \\
\overline{-} \\
5.38 \\
\overline{-} \\
5.38 \\
- \\
- \\
-\end{array}$ & $\begin{array}{r}5.04 \\
- \\
\overline{5} \\
5.04 \\
\overline{-} \\
5.04 \\
- \\
-\end{array}$ & $\begin{array}{r}4.83 \\
- \\
\overline{-} \\
4.83 \\
\overline{-} \\
4.83 \\
- \\
- \\
-\end{array}$ & $\begin{array}{r}4.99 \\
- \\
\overline{-} \\
4.99 \\
\overline{-} \\
4.99 \\
- \\
-\end{array}$ \\
\hline Primary Energy - Flve Sectors c ........................ & 1.50 & 2.80 & 6.57 & 7.23 & $R_{6.57}$ & 6.49 & $R_{6.65}$ & 6.78 \\
\hline
\end{tabular}

a There are no direct fuel costs for hydroelectric, geothermal, wind, photovoltalc, or solar thermal energy.

Heavy oll includes fuel oll nos. 4, 5, and 6, and residual fuel olls. Light all includes fuel oll nos. 1 and 2, kerosene, and jet fuel.

c Blomass fuels are not Included, except those consumed at electric uttllties and those added to motor gasoline.
$R=$ Revised data.

- No consumption, Includjng cases where adjustments were made. See explanation of adjustments in Section 6 of Appendix A.

Sources: Data sources, estimation procedures, and assumptions are described in Appendix A. 
I Energy Expenditure Estimates by Sector, Idaho

D $1970,1975,1980,1985$, and 1990-1993

A (Million Dollars)

$\mathbf{H}$

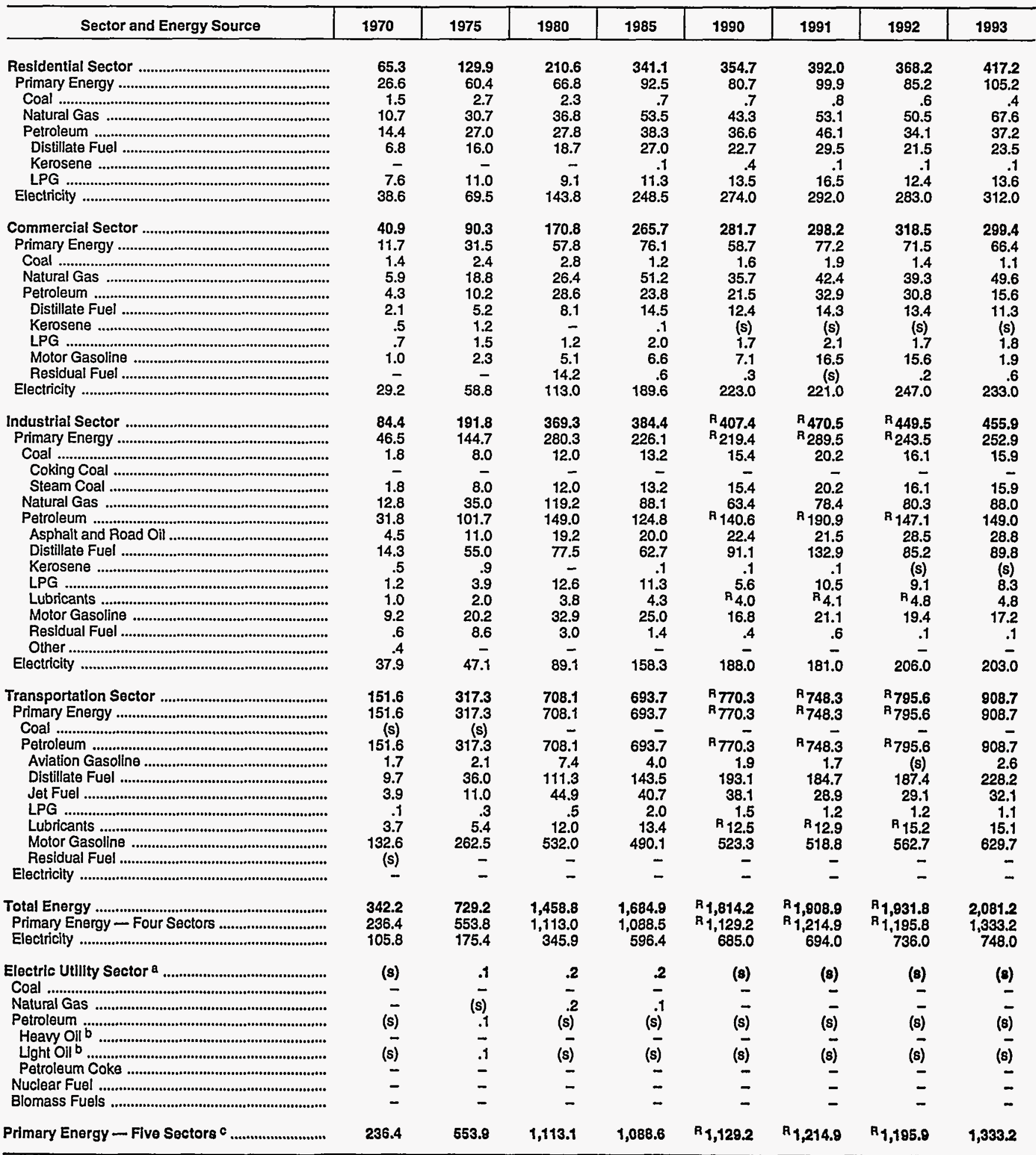

a There are no direct fuel costs for hydroelectric, geothermal, wind, photovoltaic, or solar thermal energy.

Heavy oll Includes fuel oil nos. 4, 5, and 6, and residual fuel olls. Light oll includes fuel nos. 1 and 2 , kerosene, and jet fuel.

c Blomass fuels are not Included, except those consumed at electric uttllties and those added to motor gasoline.

$R=$ Revised data.

-No consumption, Including cases where adjustments were made. See explanation of adjustments in Section 6 of Appendlx $A$.

$(s)=$ Value less than 0.05 million dollars.

Note: Totals may not equal sum of components due to independent rounding.

Sources: Data sources, estimation procedures, and assumptions are described in Appendlx A. 


\begin{tabular}{|c|c|c|c|c|c|c|c|c|}
\hline Energy Source & 1970 & 1975 & 1980 & 1985 & 1990 & 1991 & 1992 & 1993 \\
\hline & \multicolumn{8}{|c|}{ Prices in Dollars per Million Btu } \\
\hline 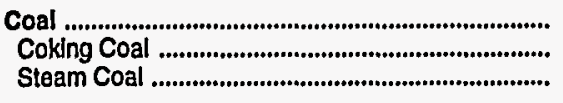 & $\begin{array}{r}0.36 \\
.42 \\
.35\end{array}$ & $\begin{array}{r}0.89 \\
1.49 \\
.82\end{array}$ & $\begin{array}{l}1.64 \\
1.93 \\
1.63\end{array}$ & $\begin{array}{l}2.12 \\
2.08 \\
2.12\end{array}$ & $\begin{array}{l}1.72 \\
1.84 \\
1.70\end{array}$ & $\begin{array}{l}1.70 \\
1.99 \\
1.67\end{array}$ & $\begin{array}{l}1.70 \\
2.00 \\
1.67\end{array}$ & $\begin{array}{l}1.67 \\
1.95 \\
1.65\end{array}$ \\
\hline 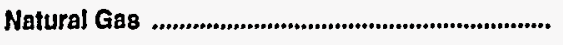 & .72 & 1.38 & 3.33 & 5.00 & 4.57 & 4.40 & 4.50 & 4.97 \\
\hline 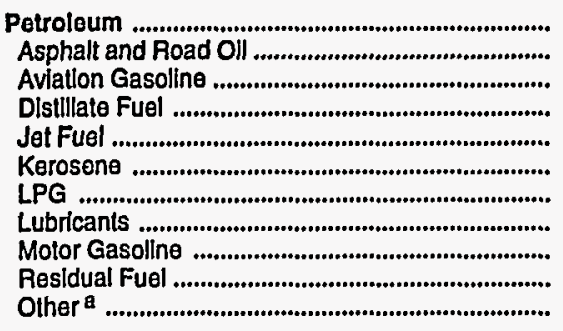 & $\begin{array}{r}1.86 \\
.64 \\
2.17 \\
1.11 \\
.74 \\
1.13 \\
1.39 \\
5.08 \\
3.05 \\
.60 \\
1.29\end{array}$ & $\begin{array}{l}3.39 \\
1.99 \\
3.45 \\
2.58 \\
2.09 \\
2.82 \\
2.68 \\
7.48 \\
4.73 \\
1.68 \\
2.36\end{array}$ & $\begin{array}{r}7.67 \\
3.83 \\
9.02 \\
6.88 \\
6.38 \\
6.67 \\
5.16 \\
14.36 \\
9.81 \\
4.92 \\
6.78\end{array}$ & $\begin{array}{r}8.49 \\
4.91 \\
9.99 \\
7.62 \\
6.00 \\
8.49 \\
8.75 \\
17.61 \\
9.03 \\
5.22 \\
7.79\end{array}$ & $\begin{array}{r}\mathbf{A}_{\mathbf{8 . 5 0}} \\
3.20 \\
9.32 \\
8.17 \\
5.84 \\
8.36 \\
9.11 \\
\mathrm{R}_{14.60} \\
9.35 \\
3.00 \\
\mathrm{R}_{6.41}\end{array}$ & $\begin{array}{r}R_{8.06} \\
3.38 \\
8.71 \\
7.43 \\
4.75 \\
7.68 \\
8.15 \\
R_{16.79} \\
9.13 \\
2.71 \\
R_{5.92}\end{array}$ & $\begin{array}{r}R_{7.84} \\
3.02 \\
8.54 \\
7.29 \\
4.44 \\
7.16 \\
7.77 \\
R_{19.43} \\
8.79 \\
2.80 \\
R_{6.19}\end{array}$ & $\begin{array}{r}7.88 \\
3.31 \\
8.24 \\
7.29 \\
4.12 \\
6.58 \\
8.28 \\
18.94 \\
8.83 \\
2.69 \\
5.47\end{array}$ \\
\hline 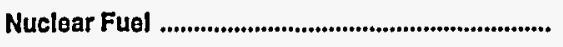 & .15 & .18 & .33 & .64 & .57 & .49 & .52 & .52 \\
\hline 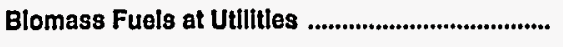 & .65 & - & - & - & - & - & 1.11 & - \\
\hline Primary Energy - Flve Sectors b ........................ & 1.08 & 2.00 & 4.32 & 4.83 & 4.34 & 4.01 & 4.03 & 4.07 \\
\hline 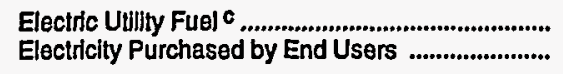 & $\begin{array}{r}.32 \\
5.98\end{array}$ & $\begin{array}{r}.69 \\
9.35\end{array}$ & $\begin{array}{r}1.60 \\
15.33\end{array}$ & $\begin{array}{r}1.67 \\
21.07\end{array}$ & $\begin{array}{r}1.12 \\
22.02\end{array}$ & $\begin{array}{r}1.06 \\
22.41\end{array}$ & $\begin{array}{r}1.04 \\
22.61\end{array}$ & $\begin{array}{r}1.07 \\
22.76\end{array}$ \\
\hline \multirow[t]{2}{*}{ 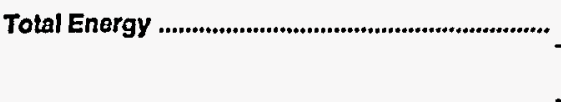 } & 1.69 & 3.15 & 6.69 & 8.45 & $R_{8.68}$ & $\mathrm{R}_{8.54}$ & $R_{8.42}$ & 8.69 \\
\hline & \multicolumn{8}{|c|}{ Expenditures in Millions of Dollars } \\
\hline 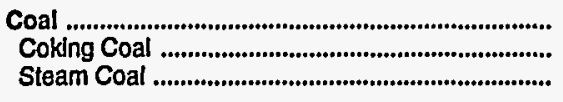 & $\begin{array}{r}329.4 \\
41.6 \\
287.8\end{array}$ & $\begin{array}{l}750.5 \\
120.7 \\
629.8\end{array}$ & $\begin{array}{r}1,388.1 \\
93.7 \\
1,294.5\end{array}$ & $\begin{array}{r}1,720.0 \\
131.6 \\
1,588.3\end{array}$ & $\begin{array}{r}1,283.1 \\
116.4 \\
1,166.8\end{array}$ & $\begin{array}{r}1,285.6 \\
119.4 \\
1,166.2\end{array}$ & $\begin{array}{r}1,180.0 \\
124.4 \\
1,055.7\end{array}$ & $\begin{array}{r}1,359.9 \\
113.0 \\
1,246.9\end{array}$ \\
\hline 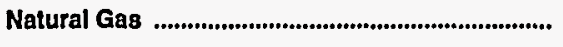 & 831.7 & $1,512.9$ & $3,601.8$ & $4,873.0$ & $4,271.0$ & $4,307.8$ & $4,433.0$ & $5,100.5$ \\
\hline 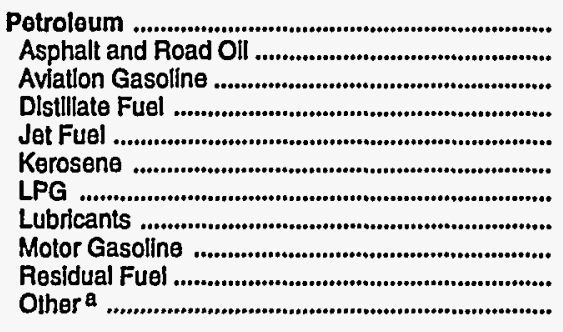 & $\begin{array}{r}2,593.2 \\
53.8 \\
2.9 \\
287.9 \\
95.2 \\
22.9 \\
148.4 \\
100.3 \\
1,715.3 \\
89.2 \\
77.2\end{array}$ & $\begin{array}{r}5,050.2 \\
135.0 \\
1.4 \\
770.9 \\
292.9 \\
41.9 \\
329.8 \\
141.6 \\
2,945.6 \\
223.0 \\
168.1\end{array}$ & $\begin{array}{r}10,370.9 \\
205.7 \\
6.0 \\
1,464.6 \\
710.2 \\
22.9 \\
702.6 \\
302.4 \\
5,622.7 \\
764.2 \\
569.7\end{array}$ & $\begin{array}{r}8,843.8 \\
244.5 \\
10.7 \\
1,427.6 \\
92.2 \\
36.3 \\
837.5 \\
337.5 \\
5,272.2 \\
157.5 \\
427.8\end{array}$ & $\begin{array}{r}A_{9,511.0} \\
176.9 \\
7.7 \\
2,024.7 \\
130.1 \\
8.2 \\
398.6 \\
R_{314.8} \\
5,913.3 \\
59.1 \\
R_{477.6}\end{array}$ & $\begin{array}{r}R_{\mathbf{B}, 167.4} \\
177.5 \\
7.7 \\
1,564.0 \\
172.6 \\
8.8 \\
420.0 \\
R_{324.0} \\
5,004.9 \\
53.1 \\
R_{434.6}\end{array}$ & $\begin{array}{r}R_{8,090.4} \\
186.1 \\
7.6 \\
1,543.7 \\
185.8 \\
5.8 \\
346.3 \\
R_{382.2} \\
4,907.5 \\
39.0 \\
R_{486.3}\end{array}$ & $\begin{array}{r}8,279.6 \\
138.7 \\
9.6 \\
1,629.1 \\
213.6 \\
6.6 \\
351.4 \\
379.3 \\
5,079.8 \\
35.3 \\
436.4\end{array}$ \\
\hline Nuclear Fuel ............................................................. & $4.1^{\circ}$ & 45.2 & 99.4 & 270.5 & 436.4 & 381.5 & 409.9 & 437.8 \\
\hline Blomass Fuels at Utilities ...................................... & (8) & - & - & - & - & - & .1 & - \\
\hline Primary Energy - Flve Sectors b ....................... & $3,758.5$ & $7,358.8$ & $15,460.2$ & $15,707.3$ & $R_{15,501.5}$ & $\mathrm{R}_{14,142.3}$ & $R_{14,113.4}$ & $15,177.8$ \\
\hline 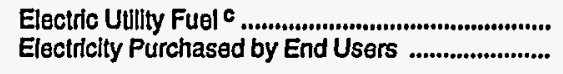 & $\begin{array}{r}-254.5 \\
1,417.5\end{array}$ & $\begin{array}{r}-689.6 \\
2,644.9\end{array}$ & $\begin{array}{r}-1,794.2 \\
4,948.6\end{array}$ & $\begin{array}{r}-1,856.1 \\
7,063.0\end{array}$ & $\begin{array}{r}-1,548.3 \\
8,307.1\end{array}$ & $\begin{array}{r}-1,486.4 \\
8,856.5\end{array}$ & $\begin{array}{r}-1,410.0 \\
8,598.5\end{array}$ & $\begin{array}{r}-1,637.6 \\
9,064.6\end{array}$ \\
\hline Total Energy & $4,921.4$ & $9,314.1$ & $18,614.6$ & $20,914.2$ & $R_{22,260.3}$ & $A_{21,512,4}$ & $R_{21,301.8}$ & $22,604.8$ \\
\hline
\end{tabular}

a Includes petroleum coke used at electric utilities.

b Biomass fuels are not included, except those consumed at electric utilitiles and those added to motor gasoline.

c There are no direct fuel costs for hydroelectric, geothermal, wind, photovoltalc, or solar themal energy.

$R=$ Revised data.

-No consumption, Including cases where adjustments were made. See explanation of adjustments in Section 6 of Appendix A.

(s)=Value less than 0.05 million dollars.

Note: Expenditure totals may not equal sum of components due to independent rounding.

Sources: Data sources, estimation procedures, and assumptions are described in Appendix A. 
I Energy Price Estimates by Sector, Illinois

L $\quad 1970,1975,1980,1985$, and 1990-1993

(Dollars per Million Btu)

\begin{tabular}{|c|c|c|c|c|c|c|c|c|}
\hline Sector and Energy Source & 1970 & 1975 & 1980 & 1985 & 1990 & 1991 & 1992 & 1998 \\
\hline $\begin{array}{l}\text { Residential Sector } \\
\text { Primary Energy } \\
\text { Coal ... } \\
\text { Natural Gas } \\
\text { Petroleum } \\
\text { Distillate Fuel } \\
\text { Kerosene } \\
\text { LPG }\end{array}$ & $\begin{array}{l}1.91 \\
1.11 \\
1.03 \\
1.02 \\
1.48 \\
1.21 \\
1.65 \\
2.02 \\
7.97\end{array}$ & $\begin{array}{r}3.06 \\
1.83 \\
2.11 \\
1.57 \\
2.95 \\
2.57 \\
3.18 \\
3.72 \\
11.41\end{array}$ & $\begin{array}{r}6.05 \\
3.77 \\
2.15 \\
3.53 \\
7.02 \\
6.91 \\
8.71 \\
7.07 \\
17.78\end{array}$ & $\begin{array}{r}9.05 \\
5.47 \\
2.34 \\
5.34 \\
7.76 \\
7.38 \\
9.07 \\
7.82 \\
26.42\end{array}$ & $\begin{array}{r}9.65 \\
5.05 \\
2.26 \\
4.95 \\
7.74 \\
7.36 \\
9.05 \\
7.90 \\
29.07\end{array}$ & $\begin{array}{r}9.67 \\
4.94 \\
2.19 \\
4.86 \\
7.10 \\
7.10 \\
8.73 \\
7.02 \\
28.92\end{array}$ & $\begin{array}{r}9.58 \\
5.08 \\
2.16 \\
5.00 \\
7.35 \\
6.71 \\
8.25 \\
7.61 \\
30.17\end{array}$ & $\begin{array}{r}10.05 \\
5.46 \\
2.26 \\
5.41 \\
7.30 \\
6.47 \\
7.95 \\
7.54 \\
30.14\end{array}$ \\
\hline 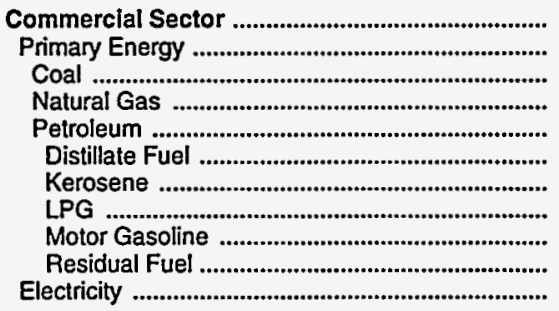 & $\begin{array}{r}1.90 \\
.73 \\
.46 \\
.73 \\
.85 \\
1.04 \\
.82 \\
1.11 \\
3.05 \\
.60 \\
6.61\end{array}$ & $\begin{array}{r}3.62 \\
1.43 \\
1.19 \\
1.28 \\
2.01 \\
2.39 \\
2.51 \\
2.29 \\
4.73 \\
1.36 \\
10.38\end{array}$ & $\begin{array}{r}7.37 \\
3.68 \\
1.71 \\
3.27 \\
6.41 \\
6.49 \\
5.93 \\
4.93 \\
9.81 \\
5.51 \\
16.70\end{array}$ & $\begin{array}{r}10.24 \\
4.98 \\
1.71 \\
4.84 \\
6.46 \\
6.11 \\
6.70 \\
8.89 \\
9.03 \\
4.14 \\
22.36\end{array}$ & $\begin{array}{r}11.18 \\
4.63 \\
1.39 \\
4.54 \\
6.61 \\
5.67 \\
7.39 \\
9.55 \\
9.35 \\
2.29 \\
22.18\end{array}$ & $\begin{array}{r}11.64 \\
4.53 \\
1.41 \\
4.47 \\
6.09 \\
4.91 \\
6.27 \\
8.57 \\
9.13 \\
2.63 \\
22.68\end{array}$ & $\begin{array}{r}11.54 \\
4.58 \\
1.28 \\
4.57 \\
5.70 \\
4.70 \\
6.35 \\
7.83 \\
8.79 \\
2.80 \\
\text { R } 23.06\end{array}$ & $\begin{array}{r}11.92 \\
4.96 \\
1.28 \\
4.99 \\
5.37 \\
4.54 \\
5.43 \\
8.65 \\
8.83 \\
2.86 \\
22.93\end{array}$ \\
\hline 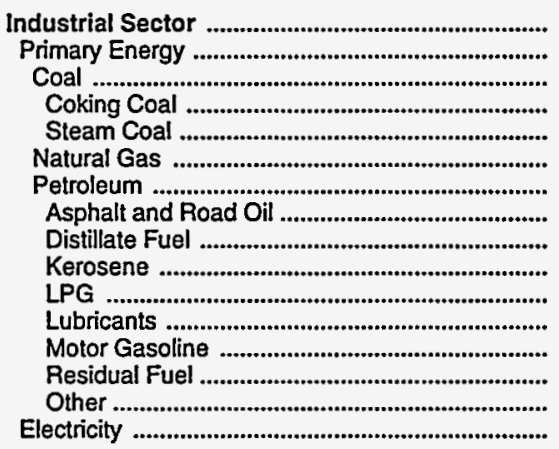 & $\begin{array}{r}.95 \\
.74 \\
.44 \\
.42 \\
.46 \\
.49 \\
1.15 \\
.64 \\
.76 \\
.82 \\
1.11 \\
5.08 \\
3.05 \\
.59 \\
1.29 \\
3.56\end{array}$ & $\begin{array}{l}2.23 \\
1.78 \\
1.33 \\
1.49 \\
1.19 \\
1.19 \\
2.52 \\
1.99 \\
2.33 \\
2.51 \\
2.29 \\
7.48 \\
4.73 \\
2.14 \\
2.36 \\
6.43\end{array}$ & $\begin{array}{r}4.94 \\
4.04 \\
1.79 \\
1.93 \\
1.71 \\
3.10 \\
5.58 \\
3.83 \\
5.37 \\
5.93 \\
4.93 \\
14.36 \\
9.81 \\
3.78 \\
.6 .78 \\
11.82\end{array}$ & $\begin{array}{r}6.69 \\
5.14 \\
1.88 \\
2.08 \\
1.71 \\
4.57 \\
7.62 \\
4.91 \\
6.05 \\
6.70 \\
8.89 \\
17.61 \\
9.03 \\
4.14 \\
7.79 \\
15.35\end{array}$ & $\begin{array}{r}R_{6.24} \\
R_{4.31} \\
1.58 \\
1.84 \\
1.39 \\
4.01 \\
R_{6.46} \\
3.20 \\
6.67 \\
7.39 \\
9.55 \\
R_{14.60} \\
9.35 \\
2.29 \\
R_{6.41} \\
15.83\end{array}$ & $\begin{array}{r}R_{6.04} \\
R_{4.07} \\
1.63 \\
1.99 \\
1.41 \\
3.70 \\
R 6.25 \\
3.38 \\
5.65 \\
6.27 \\
8.57 \\
R 16.79 \\
9.13 \\
2.63 \\
R 5.92 \\
16.09\end{array}$ & $\begin{array}{r}R_{6.09} \\
R_{4.08} \\
1.59 \\
2.00 \\
1.28 \\
3.68 \\
R_{6.13} \\
3.02 \\
5.73 \\
6.35 \\
7.83 \\
R_{19.43} \\
8.79 \\
2.80 \\
R_{6.19} \\
16.04\end{array}$ & $\begin{array}{r}6.26 \\
4.29 \\
1.54 \\
1.95 \\
1.28 \\
4.35 \\
6.14 \\
3.31 \\
4.90 \\
5.43 \\
8.65 \\
18.94 \\
8.83 \\
2.86 \\
5.47 \\
15.96\end{array}$ \\
\hline $\begin{array}{l}\text { Transportation Sector } \\
\text { Primary Energy } \\
\text { Coal foum } \\
\text { Petroleum } \\
\text { Aviation Gasoline } \\
\text { Distillate Fuel } \\
\text { Jet Fuel } \\
\text { LPG } \\
\text { Lubricants } \\
\text { Retor Gasoline } \\
\text { Electricityal Fuel }\end{array}$ & $\begin{array}{r}2.47 \\
2.47 \\
.46 \\
2.47 \\
2.17 \\
1.39 \\
.74 \\
1.11 \\
5.08 \\
3.05 \\
.57 \\
4.08\end{array}$ & $\begin{array}{l}4.07 \\
4.06 \\
1.19 \\
4.06 \\
3.45 \\
2.84 \\
2.08 \\
2.29 \\
7.48 \\
4.73 \\
1.61 \\
6.11\end{array}$ & $\begin{array}{r}9.00 \\
8.99 \\
- \\
8.99 \\
9.02 \\
7.45 \\
6.38 \\
4.93 \\
14.36 \\
9.81 \\
5.32 \\
11.82\end{array}$ & $\begin{array}{r}9.00 \\
8.98 \\
- \\
8.98 \\
9.99 \\
8.52 \\
6.00 \\
8.89 \\
17.61 \\
9.03 \\
5.88 \\
19.14\end{array}$ & $\begin{array}{r}R_{9.19} \\
R_{9.18} \\
- \\
R_{9.18} \\
9.32 \\
8.73 \\
5.84 \\
9.55 \\
R_{14.60} \\
9.35 \\
3.11 \\
19.64\end{array}$ & $\begin{array}{r}R_{8.83} \\
R_{8.82} \\
- \\
R_{8.82} \\
8.71 \\
8.21 \\
4.75 \\
8.57 \\
R_{16.79} \\
9.13 \\
2.39 \\
20.04\end{array}$ & $\begin{array}{r}\text { R }_{8.54} \\
\text { R }_{8.53} \\
- \\
\text { R }_{8.53} \\
8.54 \\
8.08 \\
4.44 \\
7.83 \\
\text { R }_{19.43} \\
8.79 \\
\text { A }_{2.50} \\
20.33\end{array}$ & $\begin{array}{r}8.51 \\
8.49 \\
- \\
8.49 \\
8.24 \\
8.16 \\
4.12 \\
8.65 \\
18.94 \\
8.83 \\
2.39 \\
20.59\end{array}$ \\
\hline 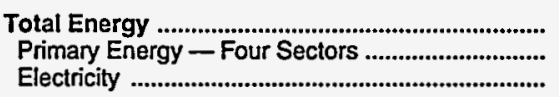 & $\begin{array}{l}1.69 \\
1.31 \\
5.98\end{array}$ & $\begin{array}{l}3.15 \\
2.49 \\
9.35\end{array}$ & $\begin{array}{r}6.69 \\
5.56 \\
15.33\end{array}$ & $\begin{array}{r}8.45 \\
6.48 \\
21.07\end{array}$ & $\begin{array}{l}A_{8.68} \\
R_{6.38} \\
22.02\end{array}$ & $\begin{array}{l}A_{8.54} \\
A_{5.96} \\
22.41\end{array}$ & $\begin{array}{l}R_{8.42} \\
R_{5.91} \\
22.61\end{array}$ & $\begin{array}{r}8.69 \\
6.15 \\
22.76\end{array}$ \\
\hline 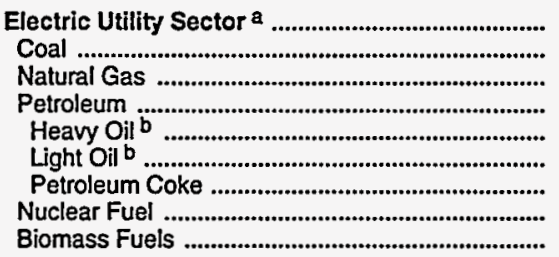 & $\begin{array}{l}.32 \\
.30 \\
.35 \\
.63 \\
.60 \\
.67 \\
-15 \\
.65\end{array}$ & $\begin{array}{r}.69 \\
.75 \\
1.13 \\
1.63 \\
1.35 \\
2.21 \\
. \\
.18 \\
-\end{array}$ & $\begin{array}{r}1.60 \\
1.62 \\
3.19 \\
5.64 \\
5.60 \\
6.38 \\
- \\
.33 \\
-\end{array}$ & $\begin{array}{r}1.67 \\
2.18 \\
5.19 \\
6.03 \\
6.03 \\
6.05 \\
- \\
.64 \\
-\end{array}$ & $\begin{array}{r}1.12 \\
1.75 \\
2.67 \\
3.99 \\
3.63 \\
5.26 \\
- \\
.57 \\
-\end{array}$ & $\begin{array}{r}1.06 \\
1.71 \\
2.10 \\
3.03 \\
2.73 \\
4.72 \\
-\overline{49} \\
-\end{array}$ & $\begin{array}{r}1.04 \\
1.74 \\
2.20 \\
3.06 \\
2.81 \\
4.44 \\
- \\
.52 \\
1.11\end{array}$ & $\begin{array}{r}1.07 \\
1.70 \\
2.44 \\
2.97 \\
2.66 \\
4.19 \\
- \\
.52 \\
-\end{array}$ \\
\hline 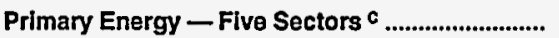 & 1.08 & 2.00 & 4.32 & 4.83 & 4.34 & 4.01 & 4.03 & 4.07 \\
\hline
\end{tabular}

a There are no direct fuel costs for hydroelectric, geothermal, wind, photovoltaic, or solar thermal energy.

b Heavy oil inciudes fuel oil nos. 4,5 , and 6 , and residual fuel oils. Light oil includes fuel oil nos. 1 and 2 , kerosene, and jet fuel.

c Biomass fuels are not included, except those consumed at electric utilities and those added to motor gasoline.

$\mathrm{R}=$ Revised data.

- No consumption, including cases where adjustments were made. See explanation of adjustments in Section 6 of Appendix A.

Sources: Data sources, estimation procedures, and assumptions are described in Appendix A 
Energy Expenditure Estimates by Sector, Illinois 1970, 1975, 1980, 1985, and 1990-1993

(Million Dollars)

\begin{tabular}{|c|c|c|c|c|c|c|c|c|}
\hline Sector and Energy Source & 1970 & 1975 & 1980 & 1985 & 1990 & 1991 & 1992 & 1993 \\
\hline 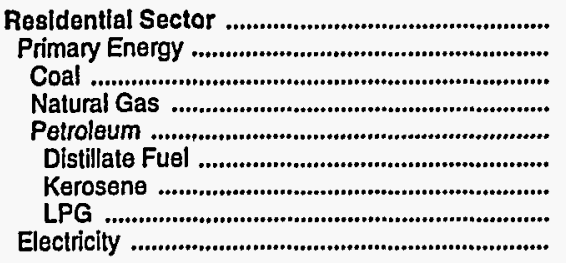 & $\begin{array}{r}1,252.7 \\
639.8 \\
18.2 \\
459.4 \\
162.2 \\
84.1 \\
12.5 \\
65.7 \\
612.9\end{array}$ & $\begin{array}{r}2,144.7 \\
1,118.4 \\
12.7 \\
772.0 \\
333.7 \\
185.3 \\
22.1 \\
126.3 \\
1,026.4\end{array}$ & $\begin{array}{r}3,801.3 \\
1,985.7 \\
3.1 \\
1,728.1 \\
254.4 \\
141.3 \\
7.9 \\
105.2 \\
1,815.6\end{array}$ & $\begin{array}{r}5,412.9 \\
2,710.7 \\
4.9 \\
2,480.4 \\
225.4 \\
97.1 \\
29.2 \\
99.1 \\
2,702.2\end{array}$ & $\begin{array}{r}5,650.6 \\
2,390.6 \\
4.7 \\
2,237.3 \\
148.5 \\
51.5 \\
5.2 \\
91.8 \\
3,260.0\end{array}$ & $\begin{array}{r}6,017.8 \\
2,468.8 \\
4.5 \\
2,311.5 \\
152.8 \\
50.8 \\
5.8 \\
96.3 \\
3,549.0\end{array}$ & $\begin{array}{r}5,899.4 \\
2,567.4 \\
4.9 \\
2,419.6 \\
142.9 \\
39.1 \\
2.8 \\
101.0 \\
3,332.0\end{array}$ & $\begin{array}{r}6,497.8 \\
2,875.9 \\
4.7 \\
2,734.1 \\
137.1 \\
27.9 \\
3.6 \\
105.5 \\
3,622.0\end{array}$ \\
\hline 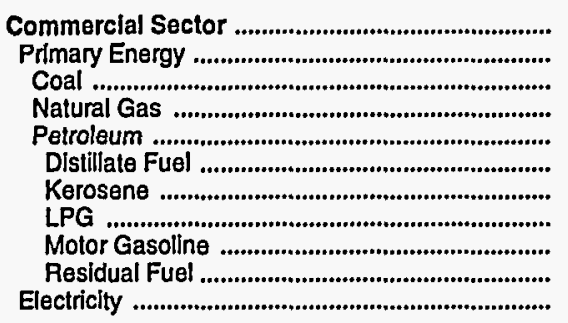 & $\begin{array}{r}733.8 \\
226.9 \\
15.2 \\
144.9 \\
66.8 \\
22.9 \\
.2 \\
6.4 \\
8.5 \\
28.8 \\
506.9\end{array}$ & $\begin{array}{r}1,419.3 \\
424.6 \\
13.4 \\
283.2 \\
128.0 \\
54.4 \\
.7 \\
13.7 \\
16.8 \\
42.4 \\
994.7\end{array}$ & $\begin{array}{r}2,802.2 \\
1,002.3 \\
4.5 \\
761.8 \\
236.0 \\
79.4 \\
.5 \\
12.9 \\
51.9 \\
91.1 \\
1,799.9\end{array}$ & $\begin{array}{r}3,768.8 \\
1,280.5 \\
6.7 \\
1,073.9 \\
200.0 \\
141.5 \\
3.6 \\
19.9 \\
26.1 \\
8.9 \\
2,488.3\end{array}$ & $\begin{array}{r}3,992.1 \\
1,036.8 \\
5.4 \\
929.3 \\
102.1 \\
51.1 \\
1.1 \\
19.6 \\
27.4 \\
3.0 \\
2,955.3\end{array}$ & $\begin{array}{r}4,138.8 \\
979.5 \\
5.3 \\
884.0 \\
90.3 \\
48.3 \\
1.4 \\
20.7 \\
19.1 \\
.6 \\
3,159.2\end{array}$ & $\begin{array}{r}R_{4,070.0} \\
1,008.3 \\
5.4 \\
915.9 \\
87.0 \\
49.4 \\
1.2 \\
18.3 \\
17.3 \\
.8 \\
R_{3,061.7}\end{array}$ & $\begin{array}{r}4,407.5 \\
1,123.2 \\
4.9 \\
1,036.2 \\
82.1 \\
52.7 \\
1.0 \\
21.4 \\
6.1 \\
1.0 \\
3,284.3\end{array}$ \\
\hline 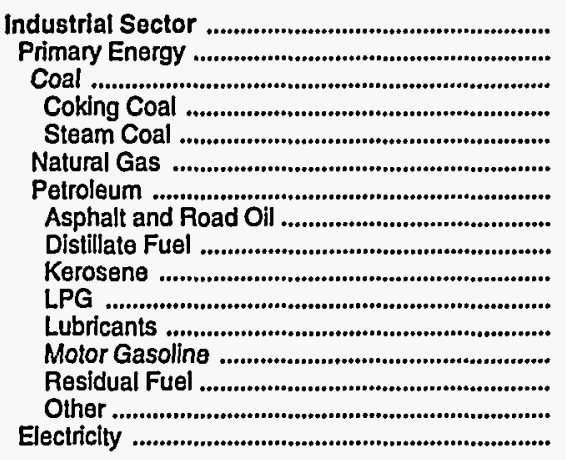 & $\begin{array}{r}1,057.8 \\
763.5 \\
115.5 \\
41.6 \\
73.9 \\
179.9 \\
468.1 \\
53.8 \\
47.4 \\
10.2 \\
74.2 \\
62.1 \\
96.4 \\
46.8 \\
77.2 \\
294.3\end{array}$ & $\begin{array}{r}2,224.5 \\
1,606.3 \\
230.2 \\
120.7 \\
109.5 \\
418.0 \\
958.1 \\
135.0 \\
150.9 \\
19.2 \\
185.6 \\
75.7 \\
106.5 \\
117.0 \\
168.1 \\
618.3\end{array}$ & $\begin{array}{r}4,777.0 \\
3,454.9 \\
228.7 \\
93.7 \\
135.1 \\
1,049.4 \\
2,176.7 \\
205.7 \\
240.0 \\
14.4 \\
581.2 \\
170.6 \\
180.7 \\
214.4 \\
569.7 \\
1,322.1\end{array}$ & $\begin{array}{r}5,325.8 \\
3,476.0 \\
266.9 \\
131.6 \\
135.2 \\
1,287.4 \\
1,921.8 \\
244.5 \\
224.0 \\
3.5 \\
705.0 \\
190.4 \\
82.5 \\
44.3 \\
427.8 \\
1,849.8\end{array}$ & $\begin{array}{r}A_{4,870.9} \\
R_{2,802.9} \\
237.9 \\
116.4 \\
121.6 \\
1,079.6 \\
R_{1,485.4} \\
176.9 \\
295.8 \\
2.0 \\
275.8 \\
A_{177.6} \\
61.7 \\
18.0 \\
R_{477.6} \\
2,068.0\end{array}$ & $\begin{array}{r}R_{4,879.5} \\
R_{2,755.9} \\
255.8 \\
119.4 \\
136.4 \\
1,084.8 \\
R_{1,415.4} \\
177.5 \\
252.6 \\
1.7 \\
293.4 \\
R_{182.7} \\
64.4 \\
8.5 \\
R_{434.6} \\
2,123.6\end{array}$ & $\begin{array}{r}R_{4,941.5} \\
R_{2,761.1} \\
233.6 \\
124.4 \\
109.2 \\
1,076.7 \\
R_{1,450.8} \\
186.1 \\
283.1 \\
1.7 \\
217.9 \\
R_{215.6} \\
56.0 \\
4.1 \\
R_{486.3} \\
2,180.5\end{array}$ & $\begin{array}{r}4,942.6 \\
2,808.0 \\
229.3 \\
113.0 \\
116.4 \\
1,290.4 \\
1,288.2 \\
138.7 \\
201.7 \\
2.0 \\
215.7 \\
214.0 \\
73.7 \\
6.1 \\
436.4 \\
2,134.6\end{array}$ \\
\hline 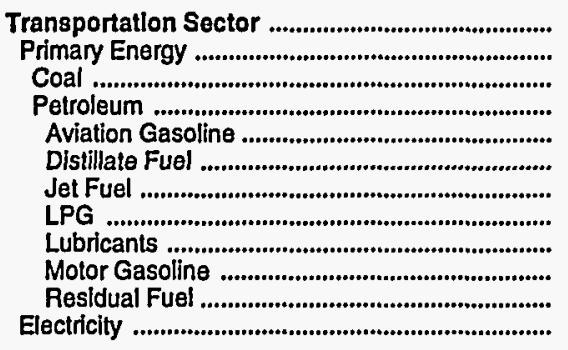 & $\begin{array}{r}1,877.0 \\
1,873.7 \\
.2 \\
1,873.5 \\
2.9 \\
123.2 \\
95.2 \\
2.2 \\
38.2 \\
1,610.4 \\
1.5 \\
3.3\end{array}$ & $\begin{array}{r}3,525.5 \\
3,519.9 \\
(s) \\
3,519.9 \\
1.4 \\
338.4 \\
285.7 \\
4.1 \\
65.9 \\
2,822.2 \\
2.2 \\
5.5\end{array}$ & $\begin{array}{r}7,234.1 \\
7,223.2 \\
- \\
7,223.2 \\
6.0 \\
978.7 \\
704.0 \\
3.2 \\
131.8 \\
5,390.1 \\
9.4 \\
10.9\end{array}$ & $\begin{array}{r}6,406.7 \\
6,383.9 \\
- \\
6,383.9 \\
10.7 \\
949.7 \\
92.2 \\
13.5 \\
147.1 \\
5,163.7 \\
6.9 \\
22.7\end{array}$ & $\begin{array}{r}\text { R } 7,746.7 \\
\text { A } 7,723.0 \\
- \\
\text { R }_{7,723.0} \\
7.7 \\
1,611.3 \\
130.1 \\
11.4 \\
\text { A }_{137.3} \\
5,824.2 \\
1.0 \\
23.7\end{array}$ & $\begin{array}{r}R_{6,476.3} \\
R_{6,451.6} \\
- \\
R_{6,451.6} \\
7.7 \\
1,198.8 \\
172.6 \\
9.6 \\
R_{141.3} \\
4,921.4 \\
.2 \\
24.7\end{array}$ & $\begin{array}{r}R_{6,390.9} \\
R_{6,366.6} \\
-\vec{n}_{6,366.6} \\
7.6 \\
1,162.7 \\
185.8 \\
9.1 \\
R_{166.7} \\
4,834.3 \\
.5 \\
R_{24.3}\end{array}$ & $\begin{array}{r}6,756.9 \\
6,733.1 \\
- \\
6,733.1 \\
9.6 \\
1,335.3 \\
213.6 \\
8.7 \\
165.4 \\
4,999.9 \\
.6 \\
23.7\end{array}$ \\
\hline 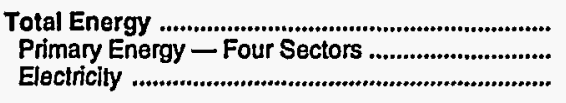 & $\begin{array}{l}4,921.4 \\
3,504.0 \\
1,417.5\end{array}$ & $\begin{array}{l}9,314.1 \\
6,669.2 \\
2,644.9\end{array}$ & $\begin{array}{r}18,614.6 \\
13,666.0 \\
4,948.6\end{array}$ & $\begin{array}{r}20,914.2 \\
13,851.2 \\
7,063.0\end{array}$ & $\begin{array}{r}A_{22,260.3} \\
R_{13,953.1} \\
8,307.1\end{array}$ & $\begin{array}{r}R_{21}, 512.4 \\
R_{12,655.8} \\
8,856.5\end{array}$ & $\begin{array}{r}R_{21,301.8} \\
R_{12,703.4} \\
8,598.5\end{array}$ & $\begin{array}{r}22,604.8 \\
13,540.2 \\
9,064.6\end{array}$ \\
\hline 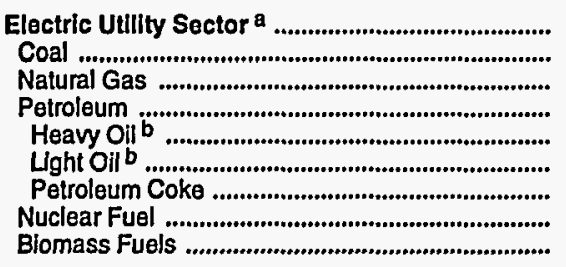 & $\begin{array}{r}254.5 \\
180.2 \\
47.7 \\
22.5 \\
12.2 \\
10.3 \\
- \\
4.1 \\
\text { (s) }\end{array}$ & $\begin{array}{r}689.6 \\
494.2 \\
39.8 \\
110.5 \\
61.4 \\
49.1 \\
- \\
45.2 \\
-\end{array}$ & $\begin{array}{r}1,794.2 \\
1,151.8 \\
62.5 \\
480.6 \\
449.3 \\
31.3 \\
- \\
99.4 \\
-\end{array}$ & $\begin{array}{r}1,856.1 \\
1,441.6 \\
31.3 \\
112.7 \\
97.4 \\
15.4 \\
- \\
270.5 \\
-\end{array}$ & $\begin{array}{r}1,548.3 \\
1,035.1 \\
24.8 \\
52.1 \\
37.0 \\
15.0 \\
- \\
436.4 \\
-\end{array}$ & $\begin{array}{r}1,486.4 \\
1,020.0 \\
27.6 \\
57.3 \\
43.7 \\
13.6 \\
- \\
381.5 \\
-\end{array}$ & $\begin{array}{r}1,410.0 \\
936.2 \\
20.8 \\
43.1 \\
33.7 \\
9.5 \\
- \\
409.9 \\
.1\end{array}$ & $\begin{array}{r}1,637.6 \\
1,121.0 \\
39.8 \\
39.0 \\
27.6 \\
11.4 \\
- \\
437.8 \\
-\end{array}$ \\
\hline Prlmary Energy - Flve Sectors ${ }^{c} \ldots \ldots \ldots \ldots \ldots \ldots \ldots$ & $3,758.5$ & $7,358.8$ & $15,460.2$ & $15,707.3$ & $R_{15,501.5}$ & $R_{14,142.3}$ & $R_{14,113.4}$ & $15,177.8$ \\
\hline
\end{tabular}

a There are no direct fuel costs for hydroelectric, geothermal, wind, photovoltaic, or solar thermal energy.

b Heavy oil includes fuel oll nos. 4, 5, and 6, and residual fuel oils. Light oil includes fuel nos. 1 and 2, kerosene, and jet fuel.

- Blomass fuels are not included, except those consumed at electric utilitites and those added to motor gasoline.

$R=$ Revised data.

-No consumption, including cases where adjustments were made. See explanation of adjustments in Section 6 of Appendix A.

(s)=Value less than 0.05 million dollars.

Note: Totals may not equal sum of components due to independent rounding.

Sources: Data sources, estimation procedures, and assumptions are described in Appendix A. 
I Energy Price and Expenditure Estimates by Source, Indiana

N 1970, 1975, 1980, 1985, and 1990-1993

\begin{tabular}{|c|c|c|c|c|c|c|c|c|}
\hline Energy Source & 1970 & 1975 & 1980 & 1985 & 1990 & 1891 & 1992 & 1993 \\
\hline & \multicolumn{8}{|c|}{ Prices in Dollars per Million Btu } \\
\hline 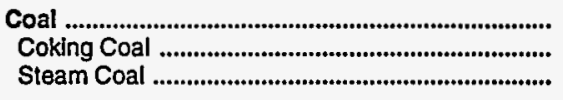 & $\begin{array}{r}0.36 \\
.44 \\
.32\end{array}$ & $\begin{array}{r}1.09 \\
1.76 \\
.73\end{array}$ & $\begin{array}{l}1.53 \\
2.13 \\
1.31\end{array}$ & $\begin{array}{l}1.77 \\
2.24 \\
1.64\end{array}$ & $\begin{array}{l}1.46 \\
1.84 \\
1.37\end{array}$ & $\begin{array}{l}1.46 \\
1.99 \\
1.36\end{array}$ & $\begin{array}{l}1.42 \\
2.00 \\
1.32\end{array}$ & $\begin{array}{l}1.37 \\
1.95 \\
1.28\end{array}$ \\
\hline 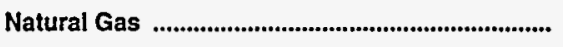 & .68 & 1.16 & 2.88 & 4.71 & 4.26 & 4.25 & 4.18 & 4.52 \\
\hline $\begin{array}{l}\text { Petroleum } \\
\text { Asphalt and Road Oll } \\
\text { Avlation Gasoline } \\
\text { Distillate Fuel } \\
\text { Jet Fuel } \\
\text { Kerosene } \\
\text { LPG } \\
\text { Lubricants } \\
\text { Metor Gasoline } \\
\text { Residual Fuel } \\
\text { Other }{ }^{a}\end{array}$ & $\begin{array}{r}2.01 \\
.76 \\
2.17 \\
1.03 \\
.74 \\
1.48 \\
1.86 \\
5.08 \\
2.98 \\
.57 \\
.77\end{array}$ & $\begin{array}{l}3.51 \\
2.05 \\
3.45 \\
2.49 \\
2.08 \\
3.01 \\
3.34 \\
7.48 \\
4.75 \\
1.81 \\
1.94\end{array}$ & $\begin{array}{r}7.78 \\
3.72 \\
9.02 \\
6.85 \\
6.38 \\
7.94 \\
6.08 \\
14.36 \\
10.00 \\
3.63 \\
4.63\end{array}$ & $\begin{array}{r}7.87 \\
4.81 \\
9.99 \\
7.66 \\
5.81 \\
8.03 \\
8.71 \\
17.61 \\
8.85 \\
4.40 \\
6.17\end{array}$ & $\begin{array}{r}R_{7.41} \\
3.14 \\
9.32 \\
7.59 \\
5.62 \\
8.32 \\
9.64 \\
R_{14.60} \\
8.74 \\
2.66 \\
R_{4.24}\end{array}$ & $\begin{array}{r}R_{7.23} \\
3.14 \\
8.71 \\
7.14 \\
4.76 \\
7.65 \\
9.37 \\
\text { P }_{16.79} \\
8.60 \\
2.37 \\
R_{4.15}\end{array}$ & $\begin{array}{r}R_{6.93} \\
2.62 \\
8.54 \\
6.80 \\
4.41 \\
6.56 \\
8.93 \\
R_{19.43} \\
8.28 \\
2.46 \\
R_{3.72}\end{array}$ & $\begin{array}{r}6.89 \\
3.07 \\
8.24 \\
6.75 \\
4.10 \\
6.65 \\
9.13 \\
18.94 \\
8.36 \\
2.20 \\
3.57\end{array}$ \\
\hline 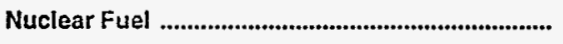 & - & - & - & - & - & - & - & - \\
\hline 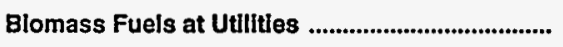 & - & - & - & - & - & - & - & - \\
\hline Primary Energy - Five Sectors $b$ & .93 & 1.90 & 3.67 & 4.12 & $\mathrm{R}_{3.73}$ & $\mathrm{R}_{3.66}$ & ค $_{3.56}$ & 3.63 \\
\hline 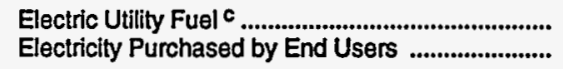 &. .26 & $\begin{array}{r}.62 \\
7.08\end{array}$ & $\begin{array}{r}1.30 \\
12.32\end{array}$ & $\begin{array}{r}1.66 \\
16.95\end{array}$ & $\begin{array}{r}1.38 \\
15.75\end{array}$ & $\begin{array}{r}1.36 \\
15.64\end{array}$ & $\begin{array}{r}1.32 \\
15.59\end{array}$ & $\begin{array}{r}1.28 \\
15.19\end{array}$ \\
\hline \multirow[t]{2}{*}{ Total Energy } & 1.43 & 2.82 & 5.64 & 6.94 & ${ }^{R_{6.72}}$ & ${ }^{P_{6.74}}$ & ${ }^{R_{6.62}}$ & 6.69 \\
\hline & \multicolumn{8}{|c|}{ Expenditures in Mlllions of Dollars } \\
\hline 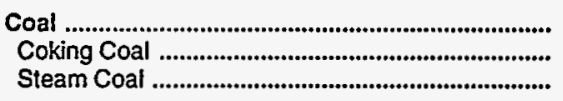 & $\begin{array}{l}364.5 \\
151.8 \\
212.7\end{array}$ & $\begin{array}{r}1,155.2 \\
651.7 \\
503.5\end{array}$ & $\begin{array}{r}1,775.9 \\
684.0 \\
1,091.9\end{array}$ & $\begin{array}{r}2,108.1 \\
560.1 \\
1,548.0\end{array}$ & $\begin{array}{r}1,983.5 \\
437.9 \\
1,545.6\end{array}$ & $\begin{array}{r}1,958.1 \\
438.9 \\
1,519.2\end{array}$ & $\begin{array}{r}1,844.5 \\
384.2 \\
1,460.2\end{array}$ & $\begin{array}{r}1,805.1 \\
344.6 \\
1,460.4\end{array}$ \\
\hline Natural Gas & 359.0 & 532.0 & $1,343.1$ & $1,995.4$ & $1,875.7$ & $1,903.3$ & $1,976.6$ & $2,280.5$ \\
\hline $\begin{array}{l}\text { Petroleum } \\
\text { Asphalt and Road Oil } \\
\text { Aviation Gasoline } \\
\text { Distillate Fuel } \\
\text { Jet Fuel } \\
\text { Kerosene } \\
\text { LPG } \\
\text { Motor Gasoline } \\
\text { Residual Fuel } \\
\text { Other }{ }^{2}\end{array}$ & $\begin{array}{r}1,314.0 \\
30.7 \\
4.0 \\
176.3 \\
10.6 \\
17.9 \\
62.9 \\
48.8 \\
921.2 \\
14.2 \\
27.4\end{array}$ & $\begin{array}{r}2,643.4 \\
82.5 \\
3.8 \\
473.9 \\
30.4 \\
14.3 \\
150.9 \\
72.8 \\
1,614.2 \\
120.0 \\
80.6\end{array}$ & $\begin{array}{r}5,383.4 \\
127.5 \\
11.8 \\
1,227.3 \\
76.5 \\
29.7 \\
174.3 \\
155.7 \\
3,162.9 \\
261.7 \\
155.9\end{array}$ & $\begin{array}{r}5,348.7 \\
170.2 \\
19.8 \\
1,373.4 \\
507.4 \\
33.3 \\
153.5 \\
173.8 \\
2,694.3 \\
57.9 \\
165.1\end{array}$ & $\begin{array}{r}R_{5,743.5} \\
178.1 \\
14.2 \\
1,447.3 \\
569.3 \\
17.4 \\
325.3 \\
R_{162.1} \\
2,827.0 \\
47.7 \\
R_{155.0}\end{array}$ & $\begin{array}{r}A_{5,407.4} \\
146.8 \\
13.3 \\
1,347.8 \\
463.5 \\
17.6 \\
316.9 \\
R_{166.9} \\
2,769.6 \\
31.0 \\
R_{134.1}\end{array}$ & $\begin{array}{r}R_{5,094.3} \\
108.0 \\
10.9 \\
1,266.2 \\
399.7 \\
11.1 \\
226.0 \\
R_{196.8} \\
2,697.2 \\
40.9 \\
R_{137.4}\end{array}$ & $\begin{array}{r}5,369.0 \\
193.6 \\
8.4 \\
1,302.0 \\
380.3 \\
13.1 \\
251.4 \\
195.4 \\
2,878.0 \\
27.7 \\
119.3\end{array}$ \\
\hline 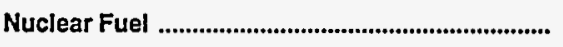 & - & - & - & - & - & - & - & - \\
\hline 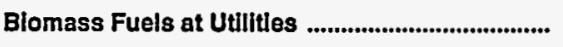 & - & - & - & - & - & - & - & - \\
\hline Primary Energy - Five Sectors b ........................... & $2,037.6$ & $4,330.6$ & $8,502.3$ & $9,452.2$ & $R_{9,602.7}$ & $R_{9,268.9}$ & $A_{6,915.4}$ & $9,454.6$ \\
\hline $\begin{array}{l}\text { Electric Utility Fuel c } \\
\text { Electricity Purchased by End Users }\end{array}$ & $\begin{array}{r}-136.5 \\
657.3\end{array}$ & $\begin{array}{r}-372.6 \\
1,252.3\end{array}$ & $\begin{array}{r}-951.4 \\
2,524.5\end{array}$ & $\begin{array}{r}-1,359.6 \\
3,647.8\end{array}$ & $\begin{array}{r}-1,404.9 \\
3,927.0\end{array}$ & $\begin{array}{r}-1,391.5 \\
4,062.7\end{array}$ & $\begin{array}{r}-1,336.4 \\
4,042.4\end{array}$ & $\begin{array}{r}-1,330.6 \\
4,193.9\end{array}$ \\
\hline Total Energy & $2,558.3$ & $5,210.2$ & $10,075.4$ & $11,740.5$ & $R_{12,124.8}$ & $R_{11,940.0}$ & $R_{11,621.4}$ & $12,317.9$ \\
\hline
\end{tabular}

\footnotetext{
a Includes petroleum coke used at electric utilities.

b Biomass fuels are not included, except those consumed at electric utilities and those added to motor gasoline.

c There are no direct fuel costs for hydroelectric, geothemal, wind, photovoltaic, or solar thermal energy.

$\mathrm{R}=$ Revised data.

-No consumpllon, including cases where adjustments were made. See explanation of adjustments in Section 6 of Appendix A.

Note: Expenditure totals may not equal sum of components due to independent rounding.

Sources: Data sources, estimation procedures, and assumptions are described in Appendix A.
} 
$\forall$ xipuedd $\forall$ ul pequosep ede suondwnsse pus 'sejnperojd uopewnse 'sejnos घlea :sejnos

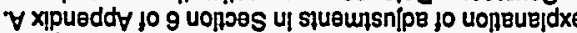

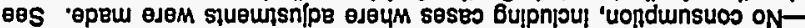
ввер pөspлөу =y

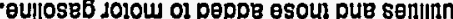

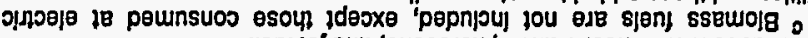
'jent lel pus 'euesojey 'z pue $\downarrow$ 'sou jIo jenj sepnjou $\| 0$

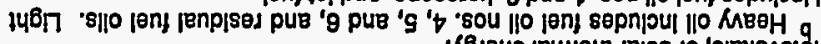

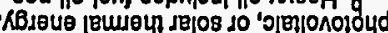

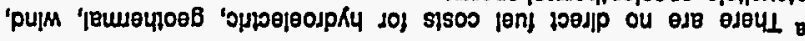

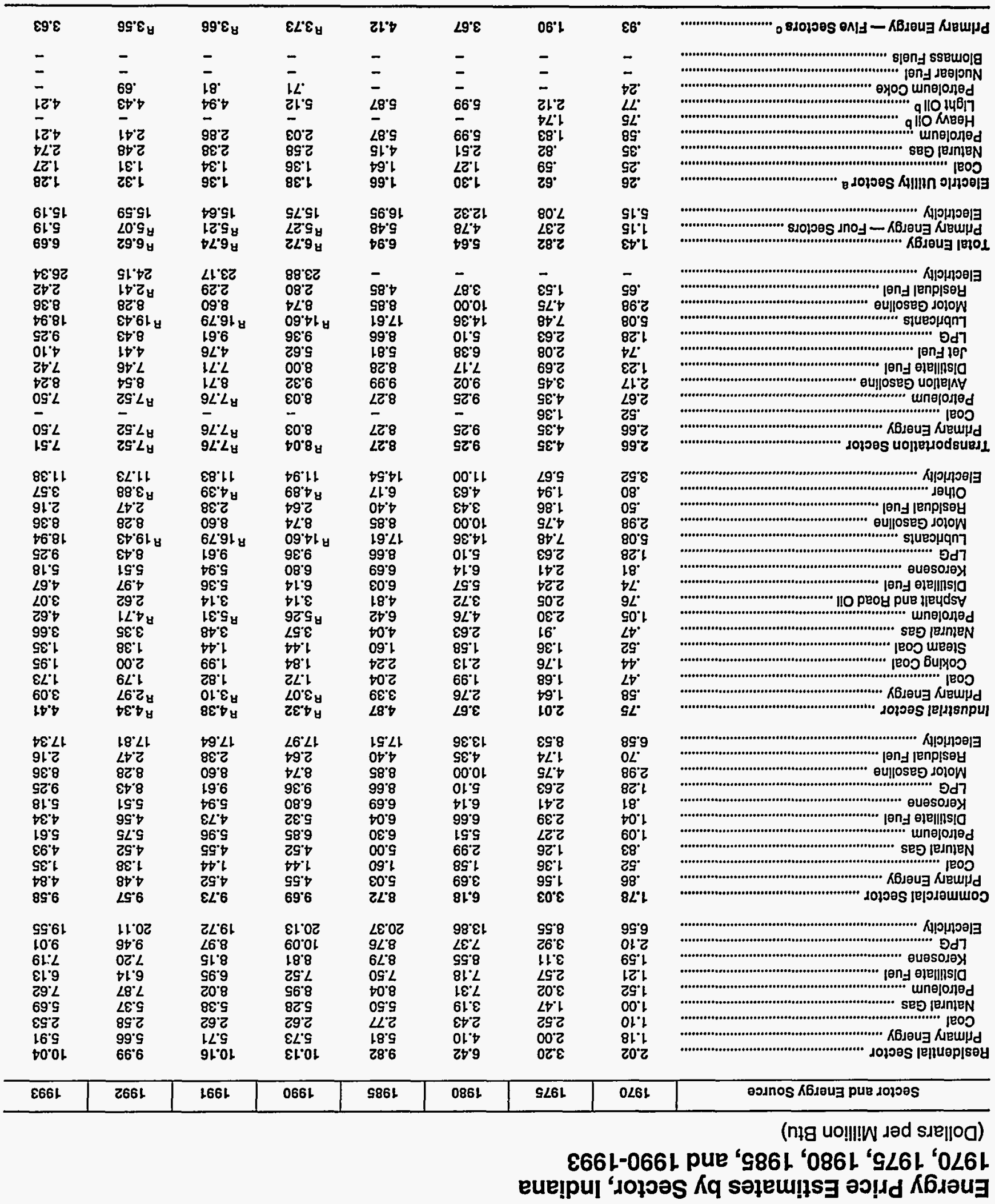


I Energy Expenditure Estimates by Sector, Indiana

N $1970,1975,1980,1985$, and 1990-1993

(Million Dollars)

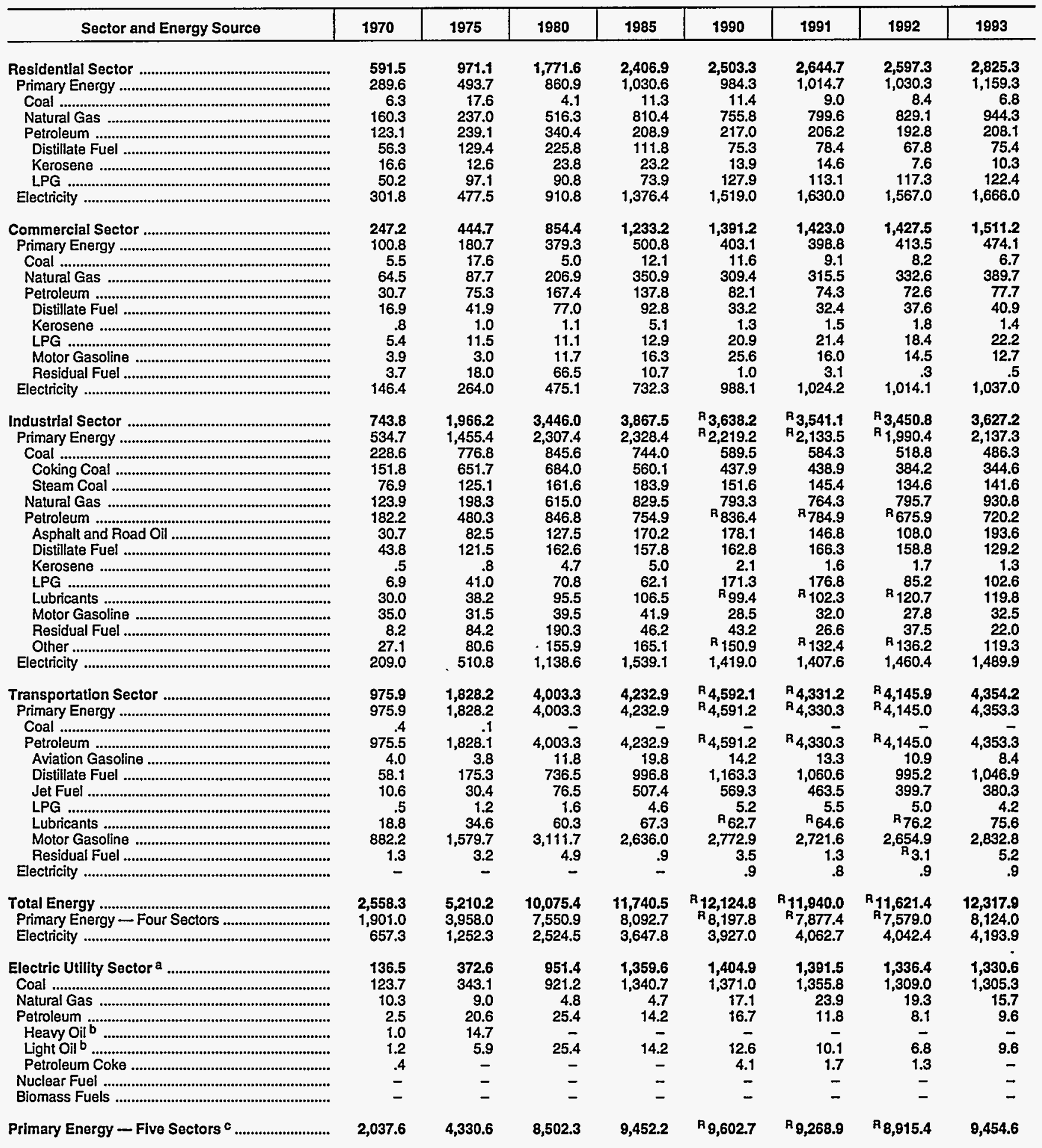

a There are no direct fuel costs for hydroelectric, geothermal, wind, photovoltaic, or solar thermal energy.

beavy oil includes fuel oil nos. 4, 5, and 6, and residual fuel oils. Light oil includes fuel nos. 1 and 2 , kerosene, and jet fuel.

c Biomass fuels are not included, except those consumed at electric utilities and those added to motor gasoline.

$R=$ Revised data.
-No consumption, including cases where adjustments were made. See explanation of adjustments in Section 6 of Appendix A.

Note: Totals may not equal sum of components due to Independent rounding.

Sources: Data sources, estimation procedures, and assumptions are described in Appendix A. 


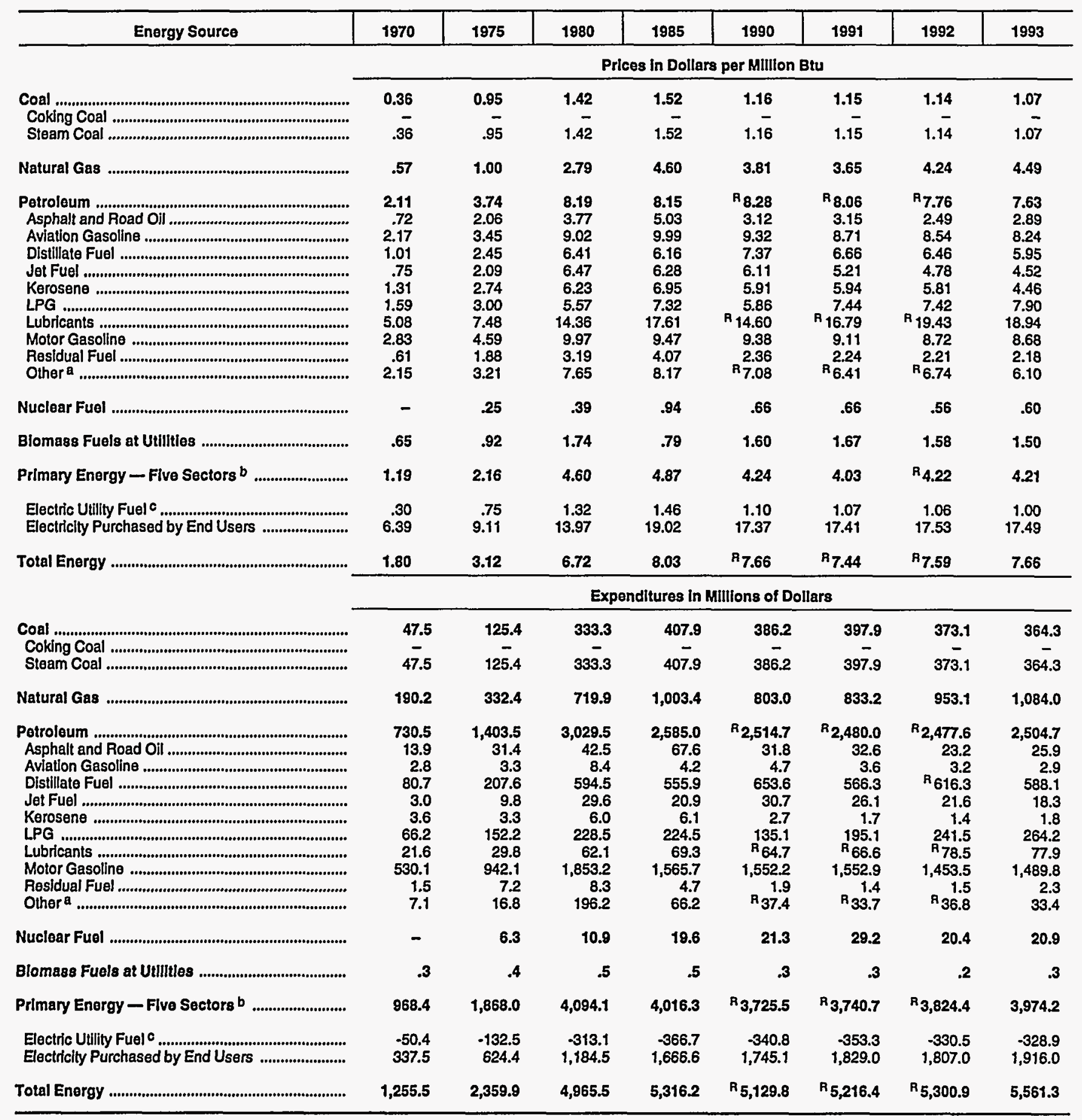

a includes petroloum coke used at electric utilities.

b Blomass fuels are not included, except those consumed at electric utilities and those added to motor gasoline.

- There are no direct fuel costs for hydroelectric, geothermal, wind, photovoltalc, or solar thermal energy.

$R=$ Revised data.

$\rightarrow$ No consumption, inciuding cases where adjustments were made. See explanation of adjustments in Section 6 of Appendix A.

Note: Expenditure totals may not equal sum of components due to independent rounding.

Sources: Data sources, estimation procedures, and assumptions are described in Appendix A. 
I Energy Price Estimates by Sector, lowa

o 1970, 1975, 1980, 1985, and 1990-1993

W (Dollars per Million Btu)

A

\begin{tabular}{|c|c|c|c|c|c|c|c|c|}
\hline Sector and Energy Source & 1970 & 1975 & 1980 & 1985 & 1990 & 1991 & 1992 & 1993 \\
\hline 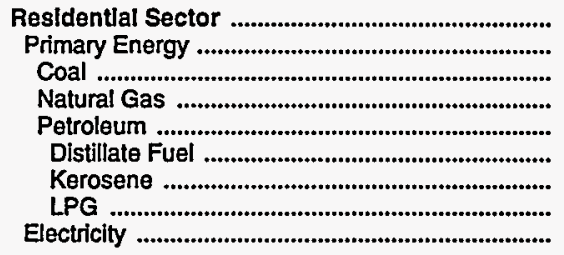 & $\begin{array}{r}2.06 \\
1.16 \\
1.27 \\
.96 \\
1.63 \\
1.22 \\
1.57 \\
1.85 \\
7.75\end{array}$ & $\begin{array}{r}3.44 \\
1.94 \\
3.69 \\
1.42 \\
3.25 \\
2.56 \\
2.99 \\
3.55 \\
10.46\end{array}$ & $\begin{array}{r}6.87 \\
4.09 \\
3.31 \\
3.18 \\
6.84 \\
6.79 \\
8.10 \\
6.86 \\
16.13\end{array}$ & $\begin{array}{r}9.65 \\
5.38 \\
3.41 \\
5.33 \\
5.81 \\
5.94 \\
7.33 \\
5.62 \\
22.53\end{array}$ & $\begin{array}{r}10.29 \\
5.19 \\
2.41 \\
4.96 \\
6.73 \\
5.73 \\
7.07 \\
7.19 \\
22.89\end{array}$ & $\begin{array}{r}9.90 \\
4.96 \\
2.32 \\
4.77 \\
6.08 \\
5.32 \\
6.57 \\
6.39 \\
22.74\end{array}$ & $\begin{array}{r}10.39 \\
5.43 \\
2.25 \\
5.21 \\
6.51 \\
5.38 \\
\mathrm{R} \\
6.63 \\
6.92 \\
23.50\end{array}$ & $\begin{array}{r}10.37 \\
5.56 \\
2.41 \\
5.46 \\
6.06 \\
4.34 \\
5.35 \\
6.64 \\
23.49\end{array}$ \\
\hline 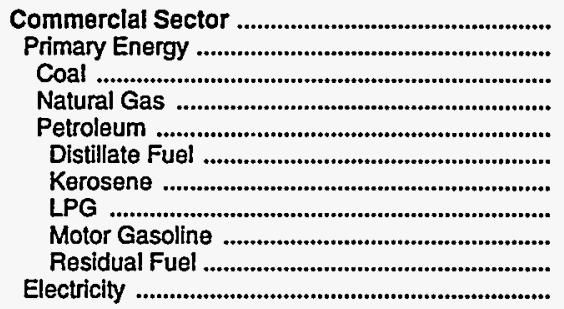 & $\begin{array}{r}1.80 \\
.77 \\
.41 \\
.68 \\
1.30 \\
1.05 \\
.81 \\
1.16 \\
2.83 \\
.66 \\
7.68\end{array}$ & $\begin{array}{r}2.94 \\
1.29 \\
1.24 \\
1.05 \\
2.71 \\
2.40 \\
2.30 \\
2.46 \\
4.59 \\
1.69 \\
10.55\end{array}$ & $\begin{array}{r}6.33 \\
3.38 \\
1.59 \\
2.84 \\
6.57 \\
6.44 \\
5.52 \\
4.88 \\
9.97 \\
3.80 \\
15.93\end{array}$ & $\begin{array}{r}9.24 \\
4.84 \\
1.66 \\
4.80 \\
6.28 \\
5.10 \\
5.86 \\
8.25 \\
9.47 \\
4.07 \\
21.88\end{array}$ & $\begin{array}{r}8.58 \\
3.93 \\
1.34 \\
4.00 \\
5.10 \\
4.32 \\
5.41 \\
4.85 \\
9.38 \\
2.36 \\
18.33\end{array}$ & $\begin{array}{r}8.63 \\
4.28 \\
1.31 \\
3.96 \\
7.01 \\
3.78 \\
4.70 \\
8.34 \\
9.11 \\
2.24 \\
18.24\end{array}$ & $\begin{array}{r}9.11 \\
4.59 \\
1.32 \\
4.25 \\
6.76 \\
4.07 \\
5.05 \\
7.73 \\
8.72 \\
2.21 \\
18.64\end{array}$ & $\begin{array}{r}9.30 \\
4.82 \\
1.39 \\
4.51 \\
7.25 \\
3.17 \\
3.69 \\
8.84 \\
8.68 \\
2.18 \\
18.44\end{array}$ \\
\hline 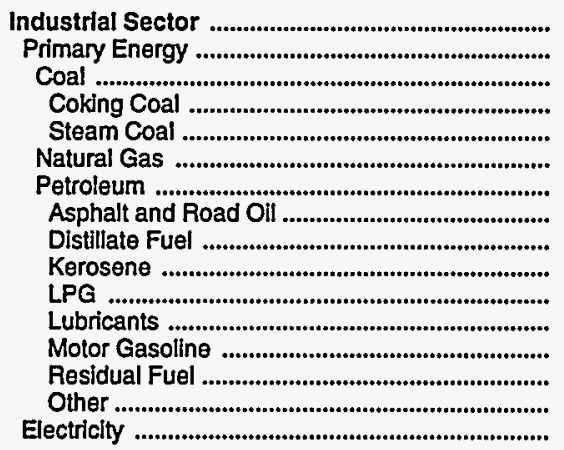 & $\begin{array}{r}1.04 \\
.83 \\
.41 \\
\overline{.41} \\
.36 \\
.48 \\
.72 \\
.75 \\
.81 \\
1.16 \\
5.08 \\
2.83 \\
.57 \\
2.15 \\
3.87\end{array}$ & $\begin{array}{r}2.01 \\
1.61 \\
1.24 \\
\overline{-} \\
1.24 \\
.77 \\
2.85 \\
2.06 \\
2.15 \\
2.30 \\
2.46 \\
7.48 \\
4.59 \\
1.92 \\
3.21 \\
6.31\end{array}$ & $\begin{array}{r}4.69 \\
3.96 \\
1.59 \\
- \\
1.59 \\
2.51 \\
6.27 \\
3.77 \\
5.28 \\
5.52 \\
4.88 \\
14.36 \\
9.97 \\
2.88 \\
7.65 \\
10.47\end{array}$ & $\begin{array}{r}5.86 \\
4.64 \\
1.66 \\
- \\
1.66 \\
3.87 \\
6.85 \\
5.03 \\
5.32 \\
5.86 \\
8.25 \\
17.61 \\
9.47 \\
4.07 \\
8.17 \\
13.50\end{array}$ & $\begin{array}{r}4.55 \\
3.18 \\
1.34 \\
- \\
1.34 \\
2.85 \\
R 5.39 \\
3.12 \\
4.92 \\
5.41 \\
4.85 \\
R 14.60 \\
9.38 \\
2.36 \\
R 7.08 \\
11.65\end{array}$ & $\begin{array}{r}R_{4.47} \\
3.14 \\
1.31 \\
- \\
1.31 \\
2.63 \\
R_{5.71} \\
3.15 \\
4.27 \\
4.70 \\
8.34 \\
R_{16.79} \\
9.11 \\
2.24 \\
R_{6.41} \\
11.76\end{array}$ & $\begin{array}{r}4.97 \\
R 3.75 \\
1.32 \\
- \\
1.32 \\
3.54 \\
\mathrm{~A}_{5.72} \\
2.49 \\
\mathrm{R}_{4.58} \\
5.05 \\
7.73 \\
\mathrm{R} \\
19.43 \\
8.72 \\
2.21 \\
\mathrm{~A} 6.74 \\
11.76\end{array}$ & $\begin{array}{r}4.95 \\
3.72 \\
1.39 \\
- \\
1.39 \\
3.76 \\
5.26 \\
2.89 \\
3.35 \\
3.69 \\
8.84 \\
18.94 \\
8.68 \\
2.18 \\
6.10 \\
11.50\end{array}$ \\
\hline 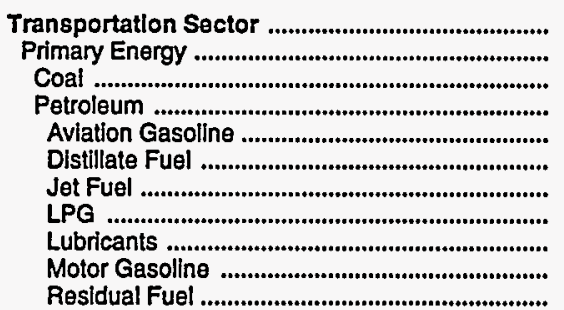 & $\begin{array}{r}2.60 \\
2.60 \\
.41 \\
2.60 \\
2.17 \\
1.27 \\
.75 \\
1.16 \\
5.08 \\
2.83 \\
.66\end{array}$ & $\begin{array}{r}4.24 \\
4.24 \\
1.24 \\
4.24 \\
3.45 \\
2.65 \\
2.09 \\
2.46 \\
7.48 \\
4.59 \\
-\end{array}$ & $\begin{array}{r}9.34 \\
9.34 \\
- \\
9.34 \\
9.02 \\
6.97 \\
6.47 \\
4.88 \\
14.36 \\
9.97 \\
-\end{array}$ & $\begin{array}{r}8.95 \\
8.95 \\
- \\
8.95 \\
9.99 \\
6.85 \\
6.28 \\
8.25 \\
17.61 \\
9.47 \\
-\end{array}$ & $\begin{array}{r}R_{9.22} \\
R_{9.22} \\
R_{9.22} \\
9.32 \\
8.74 \\
6.11 \\
4.85 \\
R_{14.60} \\
9.38 \\
1.82\end{array}$ & $\begin{array}{r}\mathrm{P}_{8.94} \\
\mathrm{P}_{8.94} \\
\mathrm{R}_{8.94} \\
8.71 \\
8.32 \\
5.21 \\
8.34 \\
\mathrm{R}_{16.79} \\
9.11 \\
-\end{array}$ & $\begin{array}{r}R_{8.63} \\
R_{8.63} \\
- \\
R_{8.63} \\
8.54 \\
8.05 \\
4.78 \\
7.73 \\
A_{19.43} \\
8.72 \\
-\end{array}$ & $\begin{array}{r}8.55 \\
8.55 \\
- \\
8.55 \\
8.24 \\
7.89 \\
4.52 \\
8.84 \\
18.94 \\
8.68 \\
-\end{array}$ \\
\hline 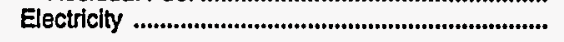 & - & - & - & - & - & - & - & - \\
\hline 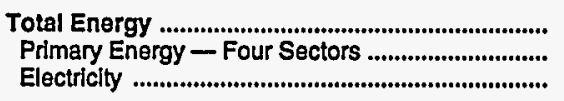 & $\begin{array}{l}1.80 \\
1.42 \\
6.39\end{array}$ & $\begin{array}{l}3.12 \\
2.52 \\
9.11\end{array}$ & $\begin{array}{r}6.72 \\
5.78 \\
13.97\end{array}$ & $\begin{array}{r}8.03 \\
6.36 \\
19.02\end{array}$ & $\begin{array}{r}R_{7.66} \\
P_{5.95} \\
17.37\end{array}$ & $\begin{array}{l}R_{7.44} \\
R_{5.68} \\
17.41\end{array}$ & $\begin{array}{l}R_{7.59} \\
R_{5.87} \\
17.53\end{array}$ & $\begin{array}{r}7.66 \\
5.91 \\
17.49\end{array}$ \\
\hline 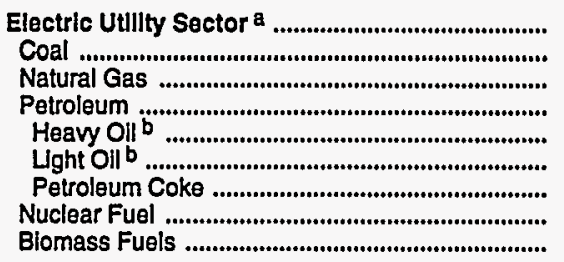 & $\begin{array}{l}.30 \\
.32 \\
.27 \\
.74 \\
.70 \\
.75 \\
- \\
-65\end{array}$ & $\begin{array}{r}.75 \\
.85 \\
.68 \\
2.05 \\
1.93 \\
2.11 \\
-25 \\
.92\end{array}$ & $\begin{array}{r}1.32 \\
1.39 \\
2.41 \\
5.41 \\
3.78 \\
6.06 \\
-\overline{39} \\
1.74\end{array}$ & $\begin{array}{r}1.46 \\
1.48 \\
3.61 \\
5.88 \\
3.99 \\
5.93 \\
-\overline{.94} \\
.79\end{array}$ & $\begin{array}{r}1.10 \\
1.12 \\
3.05 \\
5.18 \\
-\overline{6.18} \\
-\overline{66} \\
1.60\end{array}$ & $\begin{array}{r}1.07 \\
1.10 \\
2.69 \\
4.38 \\
\overline{-38} \\
4.38 \\
\overline{66} \\
1.67\end{array}$ & $\begin{array}{r}1.06 \\
1.10 \\
3.07 \\
4.24 \\
-\overline{4} \\
4.24 \\
- \\
.56 \\
1.58\end{array}$ & $\begin{array}{r}1.00 \\
1.01 \\
3.10 \\
4.08 \\
\overline{-} \\
4.08 \\
\overline{6} \\
.60 \\
1.60\end{array}$ \\
\hline Primary Energy - Flve Sectors ${ }^{C} \ldots . . . . . . . . . . . . . . . . . .$. & 1.18 & 2.16 & 4.60 & 4.87 & 4.24 & 4.03 & $R_{4.22}$ & 4.21 \\
\hline
\end{tabular}

a There are no direct fuel costs for hydroelectric, geothermal, wind, photovoltalc, or solar thermal energy.

beavy oil includes fuel oil nos. 4, 5, and 6, and residual fuel oils. Light oil includes fuel oll nos. 1 and 2 , kerosene, and let fuel.

c Blomass fuels are not included, except those consumed at electric utiltites and those added to motor gasoline.

$R=$ Revised data

- No consumption, including cases where adjustments were made. See explanation of adjustments in Section 6 of Appendix A.

Sources: Data sources, estimation procodures, and assumptions are described in Appendlx $A$. 
(Million Dollars)

\begin{tabular}{|c|c|c|c|c|c|c|c|c|}
\hline Sector and Energy Sourco & 1970 & 1975 & 1980 & 1985 & 1990 & 1991 & 1992 & 1993 \\
\hline 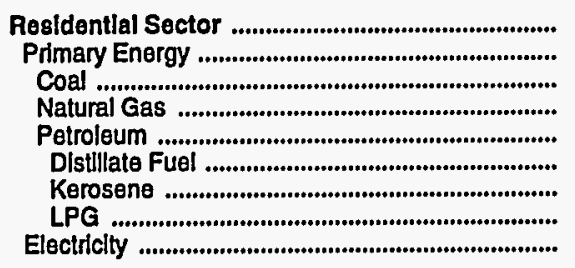 & $\begin{array}{r}332.2 \\
160.9 \\
1.6 \\
92.9 \\
66.4 \\
15.8 \\
2.9 \\
47.6 \\
171.3\end{array}$ & $\begin{array}{r}554.3 \\
256.7 \\
3.3 \\
134.7 \\
118.8 \\
26.9 \\
2.3 \\
89.5 \\
297.5\end{array}$ & $\begin{array}{r}1,020.5 \\
467.9 \\
2.1 \\
271.2 \\
194.6 \\
94.5 \\
2.2 \\
98.0 \\
552.6\end{array}$ & $\begin{array}{r}1,303.6 \\
546.3 \\
7.1 \\
424.1 \\
115.1 \\
49.7 \\
4.8 \\
60.6 \\
757.4\end{array}$ & $\begin{array}{r}1,281.3 \\
460.3 \\
5.0 \\
356.2 \\
99.1 \\
26.6 \\
1.0 \\
71.5 \\
821.0\end{array}$ & $\begin{array}{r}1,355.7 \\
489.7 \\
4.4 \\
379.0 \\
106.3 \\
27.5 \\
1.3 \\
77.6 \\
866.0\end{array}$ & $\begin{array}{r}1,328.3 \\
503.3 \\
1.2 \\
391.6 \\
110.4 \\
24.4 \\
.8 \\
85.3 \\
825.0\end{array}$ & $\begin{array}{r}1,464.3 \\
574.3 \\
1.5 \\
456.4 \\
116.4 \\
20.7 \\
1.0 \\
94.7 \\
890.0\end{array}$ \\
\hline 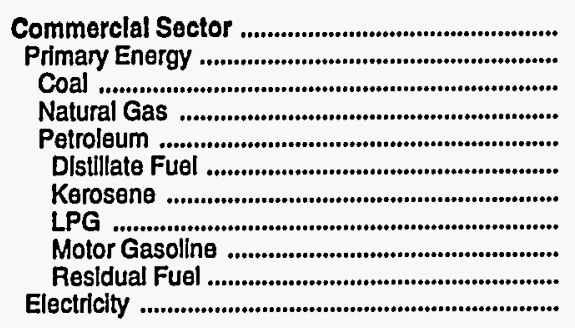 & $\begin{array}{r}151.3 \\
55.5 \\
1.0 \\
39.4 \\
15.1 \\
5.5 \\
.1 \\
5.3 \\
4.0 \\
.3 \\
95.8\end{array}$ & $\begin{array}{r}287.6 \\
103.3 \\
2.0 \\
71.1 \\
30.2 \\
10.1 \\
.1 \\
11.0 \\
7.8 \\
1.2 \\
184.3\end{array}$ & $\begin{array}{r}505.7 \\
206.7 \\
1.9 \\
144.0 \\
60.8 \\
28.2 \\
.2 \\
12.3 \\
18.3 \\
1.9 \\
299.0\end{array}$ & $\begin{array}{r}769.7 \\
298.9 \\
6.4 \\
231.3 \\
61.2 \\
33.4 \\
.2 \\
15.7 \\
11.8 \\
(s) \\
470.8\end{array}$ & $\begin{array}{r}682.8 \\
211.8 \\
5.1 \\
177.1 \\
29.5 \\
12.5 \\
1.2 \\
8.5 \\
6.9 \\
.5 \\
471.0\end{array}$ & $\begin{array}{r}749.6 \\
255.8 \\
4.6 \\
186.0 \\
65.2 \\
12.4 \\
.1 \\
17.9 \\
34.8 \\
.1 \\
494.0\end{array}$ & $\begin{array}{r}751.7 \\
256.7 \\
1.3 \\
196.8 \\
58.6 \\
11.6 \\
.1 \\
16.8 \\
29.6 \\
.5 \\
495.0\end{array}$ & $\begin{array}{r}823.8 \\
286.9 \\
1.4 \\
227.4 \\
58.1 \\
6.6 \\
.1 \\
22.2 \\
29.0 \\
.1 \\
537.0\end{array}$ \\
\hline $\begin{array}{l}\text { Industrlal Sector } \\
\text { Primary Energy } \\
\text { Coal } \\
\text { Coking Coal } \\
\text { Steam Coal } \\
\text { Natural Gas } \\
\text { Petroleum } \\
\text { Asphalt and Road Oll } \\
\text { Distllate Fuel } \\
\text { Kerosene } \\
\text { LPG }\end{array}$ & $\begin{array}{r}272.8 \\
202.4 \\
17.8 \\
- \\
17.8 \\
36.3 \\
148.2 \\
13.9 \\
25.8 \\
.7 \\
13.0 \\
6.8 \\
80.0 \\
.9 \\
7.1 \\
70.5\end{array}$ & $\begin{array}{r}533.1 \\
390.5 \\
35.1 \\
-\overline{-} \\
35.1 \\
94.6 \\
260.8 \\
31.4 \\
58.6 \\
.9 \\
51.2 \\
7.0 \\
91.5 \\
3.4 \\
16.8 \\
142.6\end{array}$ & $\begin{array}{r}1,335.6 \\
1,002.7 \\
51.6 \\
- \\
51.6 \\
288.2 \\
662.9 \\
42.5 \\
144.4 \\
3.7 \\
117.6 \\
16.7 \\
136.7 \\
5.0 \\
196.2 \\
332.9\end{array}$ & $\begin{array}{r}1,374.5 \\
936.1 \\
59.1 \\
- \\
59.1 \\
340.2 \\
536.8 \\
67.6 \\
148.4 \\
1.1 \\
145.5 \\
18.6 \\
84.8 \\
4.6 \\
66.2 \\
438.4\end{array}$ & $\begin{array}{r}R_{1,097.3} \\
R_{644.3} \\
71.3 \\
- \\
71.3 \\
259.0 \\
R_{314.0} \\
31.8 \\
118.5 \\
.6 \\
54.3 \\
R_{17.4} \\
52.5 \\
1.4 \\
R_{37.4} \\
453.0\end{array}$ & $\begin{array}{r}R_{1,159.2} \\
R_{690.2} \\
77.9 \\
-\overline{7} .9 \\
758.2 \\
258.2 \\
\text { R } 35.1 \\
32.6 \\
114.6 \\
.4 \\
98.2 \\
\text { R } 17.9 \\
55.5 \\
1.2 \\
R_{33.7} \\
469.0\end{array}$ & $\begin{array}{r}{ }^{R} 1,349.6 \\
R_{862.6} \\
69.9 \\
- \\
69.9 \\
357.7 \\
R_{435.0} \\
23.2 \\
R_{166.1} \\
.5 \\
138.2 \\
R_{21.1} \\
48.2 \\
1.0 \\
{ }^{R} 36.8 \\
487.0\end{array}$ & $\begin{array}{r}1,331.1 \\
842.1 \\
70.0 \\
-\overline{-} \\
70.0 \\
386.8 \\
385.3 \\
25.9 \\
120.2 \\
.7 \\
145.5 \\
21.0 \\
36.4 \\
2.2 \\
33.4 \\
489.0\end{array}$ \\
\hline $\begin{array}{l}\text { Transportation Sector } \\
\text { Prmary Energy } \\
\text { Coal } \\
\text { Petroleum } \\
\text { Aviation Gasoline } \\
\text { Dlstillate Fuel } \\
\text { Jet Fuel } \\
\text { LPG } \\
\text { Lubricants } \\
\text { Motor Gasoline } \\
\text { Residual Fuel }\end{array}$ & $\begin{array}{r}499.2 \\
499.2 \\
(s) \\
499.1 \\
2.8 \\
32.2 \\
3.0 \\
.3 \\
14.8 \\
446.0 \\
.1\end{array}$ & $\begin{array}{r}984.9 \\
984.9 \\
(\mathrm{~s}) \\
984.9 \\
3.3 \\
105.7 \\
9.8 \\
.5 \\
22.7 \\
842.8 \\
-\end{array}$ & \begin{tabular}{r|}
$2,103.8$ \\
$2,103.8$ \\
- \\
$2,103.8$ \\
8.4 \\
321.6 \\
29.6 \\
.6 \\
45.4 \\
$1,698.2$ \\
-
\end{tabular} & $\begin{array}{r}1,868.4 \\
1,868.4 \\
- \\
1,868.4 \\
4.2 \\
320.9 \\
20.9 \\
2.7 \\
50.7 \\
1,469.1 \\
-\end{array}$ & $\begin{array}{r}R_{2,068.4} \\
R_{2,068.4} \\
- \\
R_{2,068.4} \\
4.7 \\
492.3 \\
30.7 \\
.7 \\
R_{47.3} \\
1,492.7 \\
\text { (s) }\end{array}$ & $\begin{array}{r}R_{1,951.6} \\
R_{1,951.6} \\
- \\
R_{1,951.6} \\
3.6 \\
409.1 \\
26.1 \\
1.5 \\
R_{48.7} \\
1,462.6 \\
-\end{array}$ & $\begin{array}{r}R_{1,871.3} \\
R_{1,871.3} \\
R_{1,871.3} \\
3.2 \\
412.1 \\
21.6 \\
1.3 \\
R_{57.4} \\
1,375.7 \\
-\end{array}$ & $\begin{array}{r}1,942.0 \\
1,942.0 \\
- \\
1,942.0 \\
2.9 \\
437.7 \\
18.3 \\
1.7 \\
57.0 \\
1,424.4 \\
-\end{array}$ \\
\hline 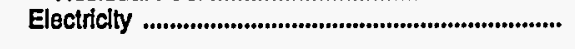 & - & - & - & - & - & - & - & - \\
\hline 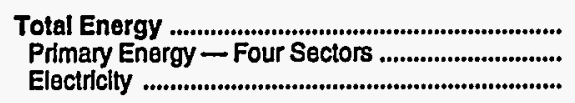 & $\begin{array}{r}1,255.5 \\
918.0 \\
337.5\end{array}$ & $\begin{array}{r}2,359.9 \\
1,735.4 \\
624.4\end{array}$ & $\begin{array}{l}4,965.5 \\
3,781.0 \\
1,184.5\end{array}$ & $\begin{array}{l}5,316.2 \\
3,649.7 \\
1,666.6\end{array}$ & $\begin{array}{r}R_{5,129.8} \\
R_{3,384.7} \\
1,745.1\end{array}$ & $\begin{array}{r}\mathrm{R}_{5,216.4} \\
{ }^{\mathrm{R}} 3,387.3 \\
1,829.0\end{array}$ & $\begin{array}{r}R_{5,300.9} \\
R_{3,493.8} \\
1,807.0\end{array}$ & $\begin{array}{l}5,561.3 \\
3,645.3 \\
1,916.0\end{array}$ \\
\hline 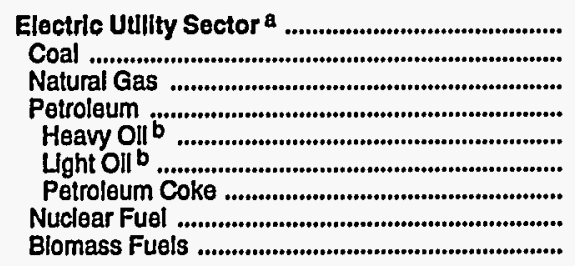 & $\begin{array}{r}50.4 \\
27.0 \\
21.5 \\
1.6 \\
.2 \\
1.4 \\
- \\
\overline{.3}\end{array}$ & $\begin{array}{r}132.5 \\
85.0 \\
32.0 \\
8.8 \\
2.6 \\
6.2 \\
-\overline{6} \\
6.3 \\
.4\end{array}$ & $\begin{array}{r}313.1 \\
277.7 \\
16.6 \\
7.4 \\
1.5 \\
5.9 \\
. \overline{1} \\
10.9 \\
.5\end{array}$ & $\begin{array}{r}366.7 \\
335.3 \\
7.7 \\
3.6 \\
.1 \\
3.5 \\
19.6 \\
.5\end{array}$ & $\begin{array}{r}340.8 \\
304.8 \\
10.7 \\
3.7 \\
\overline{3.7} \\
\overline{2} \\
21.3 \\
.3\end{array}$ & $\begin{array}{r}353.3 \\
311.1 \\
9.9 \\
2.8 \\
\overline{2.8} \\
-\overline{2} \\
29.2 \\
.3\end{array}$ & $\begin{array}{r}330.5 \\
300.7 \\
7.0 \\
2.2 \\
-\overline{2} \\
2.2 \\
-\overline{2} \\
20.4 \\
.2\end{array}$ & $\begin{array}{r}328.9 \\
291.4 \\
13.4 \\
2.9 \\
\overline{-} \\
2.9 \\
\overline{-} \\
20.9 \\
.3\end{array}$ \\
\hline Primary Energy - Five Sectors c ............................ & 868.4 & $1,868.0$ & $4,094.1$ & $4,016.3$ & $R_{3,725.5}$ & $R_{3,740.7}$ & $R_{3,824,4}$ & $3,974.2$ \\
\hline
\end{tabular}

a There are no direct fuel costs for hydroelectric, geothermal, wind, photovoltalc, or solar thermal energy.

b Heavy oll includes fuel oil nos. 4, 5, and 6, and residual fuel oils. Light oll includes fuel nos. 1 and 2, kerosene, and jet fual.

- Blomass fuels are not included, except those consumed at electric utilitites and those added to motor gasoline.

$R=$ Revised data.
-No consumption, including cases where adjustments were made. See explanation of adjustments in Section 6 of Appendix A.

$(s)=$ Value less than 0.05 million dollars.

Note: Totals may not equal sum of components due to independent rounding.

Sources: Data sources, estimation procedures, and assumptions are described in Appendix A. 
K Energy Price and Expenditure Estimates by Source, Kansas

A $1970,1975,1980,1985$, and 1990-1993

\begin{tabular}{|c|c|c|c|c|c|c|c|c|}
\hline Energy Source & 1970 & 1975 & 1980 & 1985 & 1990 & 1991 & 1992 & 1993 \\
\hline & \multicolumn{8}{|c|}{ Prices in Dollars per Million Btu } \\
\hline 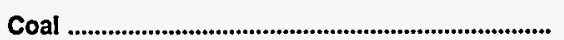 & 0.34 & 0.68 & 1.08 & 1.41 & 1.24 & 1.23 & 1.18 & 1.03 \\
\hline 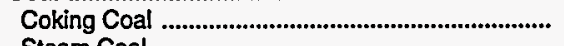 & - & - & - & - & - & - & - & $\overline{1}$ \\
\hline Steam Coal & .34 & .68 & 1.08 & 1.41 & 1.24 & 1.23 & 1.18 & 1.03 \\
\hline Natural Gas & .39 & .67 & 2.14 & 3.58 & 3.30 & 3.11 & 3.38 & 3.59 \\
\hline 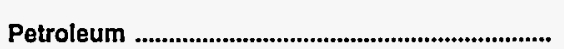 & 1.97 & 3.42 & 7.39 & 7.17 & ${ }^{R_{7.26}}$ & $R_{6.99}$ & $\mathrm{R}_{7.39}$ & 7.10 \\
\hline Asphalt and Road Oil & .74 & 2.10 & 4.02 & 5.22 & 2.94 & 2.98 & 2.54 & 3.16 \\
\hline Aviation Gasoline .................................................... & 2.17 & 3.45 & 9.02 & 9.99 & 9.32 & 8.71 & 8.54 & 8.24 \\
\hline 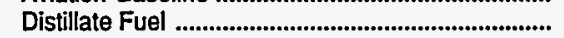 & .99 & 2.43 & 6.52 & 6.42 & 7.50 & 6.85 & ${ }^{R_{6.82}}$ & 7.04 \\
\hline Jet Fuel & .75 & 2.09 & 6.47 & 5.94 & 5.57 & 4.81 & 4.36 & 4.19 \\
\hline 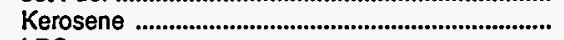 & .96 & 2.61 & 5.25 & 6.88 & 6.43 & 5.76 & 6.03 & 7.68 \\
\hline 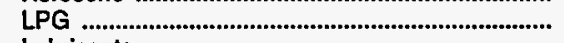 & 1.27 & 2.65 & 4.41 & 4.28 & 4.40 & 3.79 & 7.74 & 4.57 \\
\hline 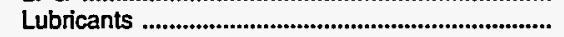 & 5.08 & 7.48 & 14.36 & 17.61 & $R_{14.60}$ & $\mathrm{P}_{16.79}$ & $R_{19.43}$ & 18.94 \\
\hline Motor Gasoline ...................................................................... & 2.64 & 4.50 & 9.27 & 9.28 & 8.90 & 8.72 & 8.49 & 8.57 \\
\hline Residual Fuel & .47 & 1.60 & 3.24 & 3.91 & 2.10 & 1.47 & 2.20 & 2.07 \\
\hline Other $\mathrm{a}^{2}$ & .83 & 1.76 & 3.95 & 6.62 & ${ }^{R} 6.45$ & ${ }^{8} 6.31$ & ${ }^{R} 6.97$ & 6.42 \\
\hline 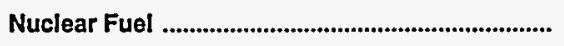 & - & - & - & .84 & .30 & .31 & .29 & .33 \\
\hline 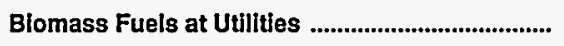 & - & - & - & - & - & - & - & - \\
\hline 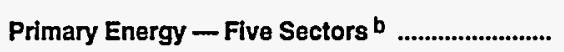 & .98 & 1.89 & 3.88 & 4.29 & P 3.95 & $\mathrm{R}_{3.80}$ & F 4.02 & 3.78 \\
\hline $\begin{array}{l}\text { Electric Utility Fuel c } \\
\text { Electricity Purchased by End Users }\end{array}$ & $\begin{array}{r}.30 \\
5.74\end{array}$ & $\begin{array}{r}.72 \\
7.72\end{array}$ & $\begin{array}{r}1.38 \\
13.75\end{array}$ & $\begin{array}{r}1.44 \\
19.07\end{array}$ & $\begin{array}{r}1.08 \\
19.31\end{array}$ & $\begin{array}{r}1.12 \\
19.30\end{array}$ & $\begin{array}{r}.99 \\
19.27\end{array}$ & $\begin{array}{r}.95 \\
19.39\end{array}$ \\
\hline \multirow[t]{2}{*}{ Total Energy } & 1.54 & 2.86 & 5.93 & 7.21 & $\mathrm{R}_{7.53}$ & $\mathrm{~B}_{7.31}$ & ${ }^{8} 7.53$ & 7.47 \\
\hline & \multicolumn{8}{|c|}{ Expenditures In Millions of Doliars } \\
\hline 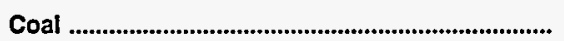 & 3.7 & 42.5 & 207.0 & 365.8 & 338.3 & 329.6 & 300.3 & 310.0 \\
\hline 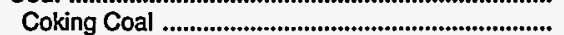 & - & - & - & - & - & - & - & - \\
\hline 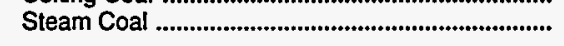 & 3.7 & 42.5 & 207.0 & 365.8 & 338.3 & 329.6 & 300.3 & 310.0 \\
\hline 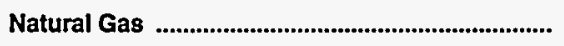 & 175.6 & 248.1 & 808.1 & 960.1 & 871.6 & 889.5 & 875.1 & $1,035.9$ \\
\hline Petroleum & 535.3 & $1,162.8$ & $2,507.7$ & $2,703.5$ & $R_{2,664.9}$ & $\mathrm{~B}_{2,427.3}$ & $\mathrm{P}_{2,681.4}$ & $2,517.8$ \\
\hline 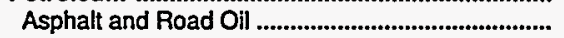 & 10.7 & 30.1 & 80.5 & 58.9 & 75.7 & 73.5 & 62.7 & 76.2 \\
\hline Aviation Gasoline .................................................... & 3.6 & 3.1 & 10.1 & 6.9 & 6.4 & 5.5 & 6.1 & 6.3 \\
\hline Distillate Fuel ............................................................... & 43.3 & 159.8 & 560.3 & 561.8 & 723.0 & 627.2 & 602.2 & 666.3 \\
\hline Jet Fuel & 6.4 & 15.0 & 89.3 & 147.6 & 115.4 & 88.2 & 101.0 & 84.7 \\
\hline 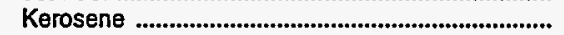 & 1.7 & 1.5 & 14.6 & 2.2 & 1.0 & .8 & 1.1 & 1.6 \\
\hline 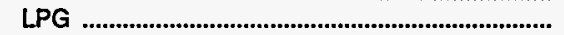 & 37.1 & 83.9 & 131.0 & 369.0 & 237.6 & 177.3 & 461.5 & 205.4 \\
\hline 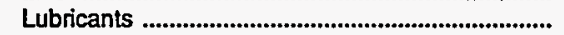 & 20.2 & 35.1 & 88.1 & 98.3 & $\overline{\mathrm{A}}_{91.7}$ & ${ }^{R} 94.3$ & $A_{111.3}$ & 110.5 \\
\hline 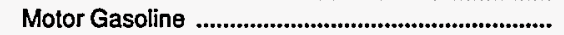 & 399.6 & 756.2 & $1,440.7$ & $1,375.2$ & $1,330.7$ & $1,284.5$ & $1,241.2$ & $1,281.9$ \\
\hline Residual Fuel & $\begin{array}{r}1.5 \\
11.2\end{array}$ & $\begin{array}{l}49.8 \\
28.3\end{array}$ & $\begin{array}{l}17.9 \\
75.3\end{array}$ & $\begin{array}{r}1.3 \\
82.2\end{array}$ & $\begin{array}{r}2.4 \\
R_{81.1}\end{array}$ & $\begin{array}{r}.8 \\
R_{75.3}\end{array}$ & $\begin{array}{r}1.7 \\
\mathrm{R}\end{array}$ & $\begin{array}{r}3.4 \\
81.6\end{array}$ \\
\hline & & & & & & & & \\
\hline 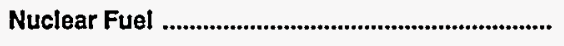 & - & - & - & 34.9 & 25.3 & 19.4 & 26.7 & 28.0 \\
\hline 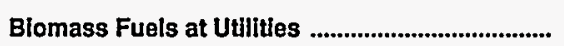 & - & - & - & - & - & - & - & - \\
\hline Primary Energy - Five Sectors ${ }^{b}$ & 714.6 & $1,453.4$ & 3,522.7 & $4,064.3$ & $\mathrm{R}_{3,900.1}$ & $\mathrm{R}_{\mathbf{3}, \mathbf{6 6 5 . 8}}$ & ${ }^{8} 3,883.5$ & $3,891.8$ \\
\hline $\begin{array}{l}\text { Electric Utility Fuel c } \\
\text { Electricity Purchased by End Users }\end{array}$ & $\begin{array}{r}-53.9 \\
259.0\end{array}$ & $\begin{array}{r}-159.5 \\
444.0\end{array}$ & $\begin{array}{r}-394.3 \\
986.7\end{array}$ & $\begin{array}{r}-453.4 \\
1,520.6\end{array}$ & $\begin{array}{r}-410.7 \\
1,774.8\end{array}$ & $\begin{array}{r}-408.0 \\
1,839.4\end{array}$ & $\begin{array}{r}-351.7 \\
1,765.3\end{array}$ & $\begin{array}{r}-385.2 \\
1,890.8\end{array}$ \\
\hline Total Energy ................................................................. & 919.7 & $1,737.9$ & $4,115.0$ & $5,131.5$ & $R_{5,264.1}$ & $R_{5,097.3}$ & ${ }^{8}$,297.1 & $5,397.3$ \\
\hline
\end{tabular}

a Includes petroleum coke used at electric utilities.

b Biomass fuels are not included, except those consumed at electric utilities and those added to motor gasoline.

c There are no direct fuel costs for hydroelectric, geothermal, wind, photovoltaic, or solar themal energy.

$R=$ Revised data.
-No consumption, including cases where adjustments were made. Seo explanation of adjustments in Section 6 of Appendix A.

Note: Expenditure totals may not equal sum of components due to independent rounding.

Sources: Data sources, estimation procedures, and assumptions are described in Appendix A. 


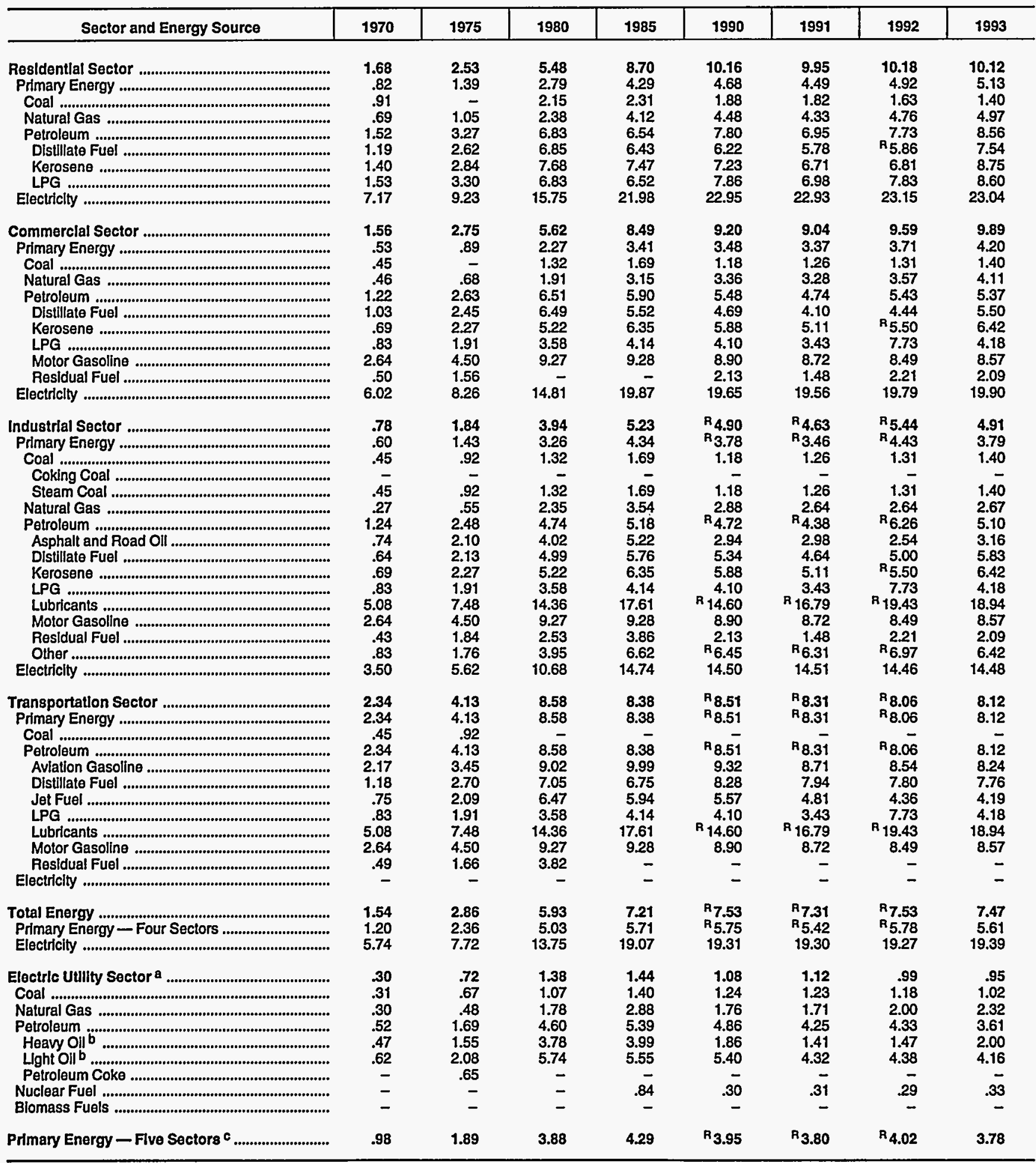

a There are no direct fuel costs for hydroelectric, geothermal, wind, photovoltalc, or solar thermal energy.

Heavy oil includes fuel oil nos. 4,5 , and 6 , and residual fuel oils. Light oll Includes fuel oil nos. 1 and 2, kerosene, and jet fuel.

c Blomass fuels are not included, except those consumed at electric utillties and those added to motor gasoline.
R=Revised data

-No consumption, including cases where adjustments were made. See explanation of adjustments in Section 6 of Appendix A.

Sources: Data sources, estimation procedures, and assumptions are described in Appendix $A$. 


\section{K Energy Expenditure Estimates by Sector, Kansas}

A $1970,1975,1980,1985$, and 1990-1993

N (Million Dollars)

\begin{tabular}{|c|c|c|c|c|c|c|c|c|}
\hline Sector and Energy Source & 1970 & 1975 & 1980 & 1985 & 1990 & 1991 & 1992 & 1993 \\
\hline 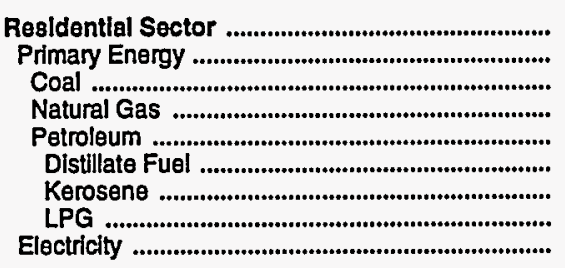 & $\begin{array}{r}226.7 \\
95.9 \\
.1 \\
66.7 \\
29.2 \\
.4 \\
.9 \\
27.9 \\
130.8\end{array}$ & $\begin{array}{r}338.9 \\
159.5 \\
101.2 \\
58.3 \\
1.5 \\
1.0 \\
55.9 \\
179.4\end{array}$ & $\begin{array}{r}646.6 \\
260.4 \\
.1 \\
201.9 \\
58.4 \\
6.0 \\
.2 \\
52.2 \\
386.2\end{array}$ & $\begin{array}{r}975.4 \\
360.8 \\
(\mathrm{~s}) \\
322.7 \\
38.1 \\
2.5 \\
1.1 \\
34.5 \\
614.6\end{array}$ & $\begin{array}{r}1,099.5 \\
354.6 \\
(s) \\
319.5 \\
35.0 \\
.9 \\
.5 \\
33.7 \\
745.0\end{array}$ & $\begin{array}{r}1,138.9 \\
361.8 \\
(s) \\
327.7 \\
34.1 \\
.8 \\
.4 \\
32.9 \\
777.0\end{array}$ & $\begin{array}{r}1,069.2 \\
368.2 \\
(8) \\
336.2 \\
32.1 \\
1.0 \\
.5 \\
30.6 \\
701.0\end{array}$ & $\begin{array}{r}1,238.1 \\
453.1 \\
.3 \\
416.8 \\
36.0 \\
1.2 \\
1.0 \\
33.8 \\
785.0\end{array}$ \\
\hline 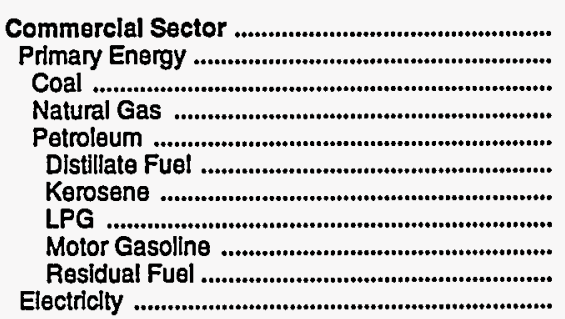 & $\begin{array}{r}112.0 \\
30.6 \\
.1 \\
23.9 \\
6.6 \\
.7 \\
.1 \\
2.7 \\
3.0 \\
.1 \\
81.4\end{array}$ & $\begin{array}{r}208.4 \\
50.3 \\
- \\
34.7 \\
15.6 \\
3.0 \\
.2 \\
5.7 \\
6.3 \\
.4 \\
158.1\end{array}$ & $\begin{array}{r}488.0 \\
144.1 \\
.1 \\
111.7 \\
32.3 \\
13.6 \\
.3 \\
4.8 \\
13.6 \\
-\overline{3} \\
343.9\end{array}$ & $\begin{array}{r}767.6 \\
213.4 \\
(\mathrm{~s}) \\
178.0 \\
35.3 \\
22.5 \\
.4 \\
3.9 \\
8.6 \\
\overline{4} \\
554.2\end{array}$ & $\begin{array}{r}847.2 \\
207.2 \\
(\mathrm{~s}) \\
188.3 \\
18.9 \\
7.7 \\
.2 \\
3.1 \\
7.5 \\
.4 \\
640.0\end{array}$ & $\begin{array}{r}874.8 \\
211.8 \\
(s) \\
194.5 \\
17.4 \\
8.7 \\
.1 \\
2.9 \\
5.7 \\
.1 \\
663.0\end{array}$ & $\begin{array}{r}872.1 \\
214.1 \\
(\mathrm{~s}) \\
190.5 \\
23.6 \\
13.0 \\
.1 \\
5.3 \\
4.9 \\
.3 \\
658.0\end{array}$ & $\begin{array}{r}841.6 \\
254.6 \\
.5 \\
227.5 \\
26.7 \\
20.7 \\
.2 \\
2.9 \\
2.5 \\
.4 \\
687.0\end{array}$ \\
\hline 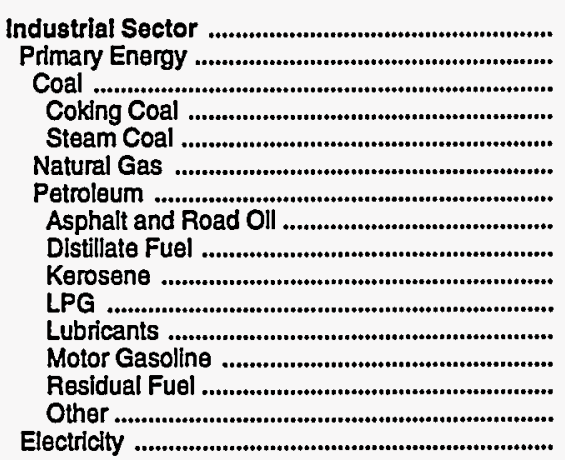 & $\begin{array}{r}165.8 \\
118.9 \\
1.0 \\
- \\
1.0 \\
35.5 \\
82.4 \\
10.7 \\
9.4 \\
.6 \\
5.4 \\
6.4 \\
38.5 \\
.2 \\
11.2 \\
46.8\end{array}$ & $\begin{array}{r}360.2 \\
253.7 \\
2.5 \\
- \\
2.5 \\
51.5 \\
199.6 \\
30.1 \\
43.8 \\
.3 \\
19.7 \\
11.5 \\
56.8 \\
9.0 \\
28.3 \\
106.5\end{array}$ & $\begin{array}{r}1,031.4 \\
774.8 \\
9.4 \\
- \\
9.4 \\
322.0 \\
443.3 \\
80.5 \\
101.0 \\
14.1 \\
72.5 \\
35.5 \\
58.3 \\
6.1 \\
75.3 \\
256.6\end{array}$ & $\begin{array}{r}1,459.5 \\
1,107.7 \\
13.1 \\
-. \\
13.1 \\
400.3 \\
694.2 \\
58.9 \\
130.8 \\
.7 \\
329.2 \\
39.6 \\
51.9 \\
.8 \\
82.2 \\
351.8\end{array}$ & $\begin{array}{r}\mathrm{R}_{1,262.4} \\
\mathrm{R}_{872.5} \\
4.5 \\
- \\
4.5 \\
316.4 \\
\mathrm{R}_{551.6} \\
75.7 \\
121.5 \\
.3 \\
198.7 \\
\mathrm{R}_{37.0} \\
35.5 \\
1.8 \\
\mathrm{R}_{81.1} \\
389.8\end{array}$ & $\begin{array}{r}R_{1,197.8} \\
R_{798.4} \\
4.6 \\
- \\
4.6 \\
307.6 \\
R_{486.2} \\
73.5 \\
123.6 \\
.3 \\
140.2 \\
R_{38.1} \\
34.6 \\
.7 \\
R_{75.3} \\
399.4\end{array}$ & $\begin{array}{r}R_{1}, 518.8 \\
R 1,113.5 \\
5.1 \\
- \\
5.1 \\
321.3 \\
R 787.1 \\
62.7 \\
R_{132.2} \\
.5 \\
422.8 \\
R_{44.9} \\
30.1 \\
1.4 \\
\text { R } 92.6 \\
406.3\end{array}$ & $\begin{array}{r}1,351.4 \\
932.7 \\
4.5 \\
- \\
4.5 \\
342.7 \\
585.4 \\
76.2 \\
172.9 \\
.4 \\
167.1 \\
44.6 \\
40.2 \\
2.5 \\
81.6 \\
418.7\end{array}$ \\
\hline 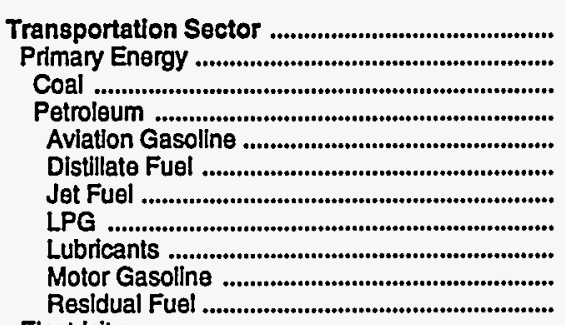 & $\begin{array}{r}415.3 \\
415.3 \\
(s) \\
415.3 \\
3.6 \\
32.2 \\
6.4 \\
1.1 \\
13.8 \\
358.2 \\
(s)\end{array}$ & $\begin{array}{r}830.4 \\
830.4 \\
(s) \\
830.4 \\
3.1 \\
92.9 \\
15.0 \\
2.6 \\
23.6 \\
693.1 \\
.2\end{array}$ & $\begin{array}{r}1,949.1 \\
1,949.1 \\
- \\
1,949.1 \\
10.1 \\
426.9 \\
89.3 \\
1.5 \\
52.5 \\
1,368.8 \\
\text { (s) }\end{array}$ & $\begin{array}{r}1,929.0 \\
1,929.0 \\
- \\
1,929.0 \\
6.9 \\
399.8 \\
147.6 \\
1.4 \\
58.6 \\
1,314.7 \\
-\end{array}$ & $\begin{array}{r}\mathrm{F}_{2,055.0} \\
\mathrm{~A}_{2,055.0} \\
\text { R }_{2,055.0} \\
6.4 \\
588.7 \\
115.4 \\
2.1 \\
\mathrm{R}_{54.7} \\
1,287.6 \\
-\end{array}$ & $\begin{array}{r}R_{1,885.8} \\
R_{1,885.8} \\
R_{1,885.8} \\
5.5 \\
490.3 \\
88.2 \\
1.3 \\
R_{56.3} \\
1,244.2 \\
-\end{array}$ & $\begin{array}{r}F_{1,836.0} \\
R_{1,836.0} \\
R_{1,836.0} \\
6.1 \\
453.4 \\
101.0 \\
2.8 \\
2 F_{66.4} \\
1,206.2 \\
-\end{array}$ & $\begin{array}{r}1,866.1 \\
1,866.1 \\
-\overline{1} \\
1,866.1 \\
6.3 \\
468.6 \\
84.7 \\
1.5 \\
65.9 \\
1,239.2 \\
-\end{array}$ \\
\hline 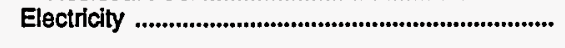 & $\because$ & - & - & - & - & - & - & - \\
\hline 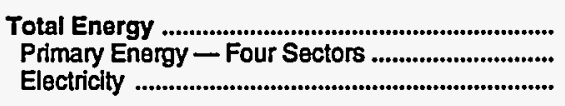 & $\begin{array}{l}919.7 \\
660.7 \\
259.0\end{array}$ & $\begin{array}{r}1,737.9 \\
1,293.9 \\
444.0\end{array}$ & $\begin{array}{r}4,115.0 \\
3,128.4 \\
986.7\end{array}$ & $\begin{array}{l}\mathbf{5 , 1 3 1 . 5} \\
3,610.9 \\
1,520.6\end{array}$ & $\begin{array}{r}R_{5,264.1} \\
R_{3,489.3} \\
1,774.8\end{array}$ & $\begin{array}{r}\text { A }_{5,097.3} \\
\text { P }_{3,257.9} \\
1,839.4\end{array}$ & $\begin{array}{r}R_{5,297.1} \\
R_{3,531.9} \\
1,765.3\end{array}$ & $\begin{array}{l}5,397.3 \\
3,506.5 \\
1,890.8\end{array}$ \\
\hline 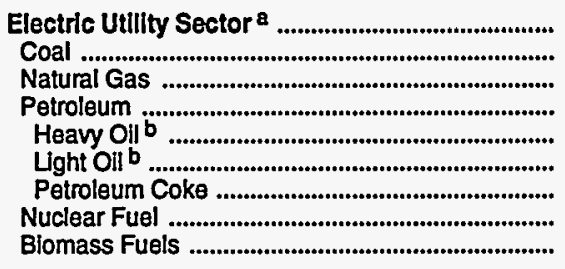 & $\begin{array}{r}53.9 \\
2.6 \\
49.5 \\
1.8 \\
1.1 \\
.6 \\
= \\
=\end{array}$ & $\begin{array}{r}159.5 \\
39.9 \\
60.6 \\
58.9 \\
40.3 \\
18.6 \\
\text { (s) } \\
-\end{array}$ & $\begin{array}{r}394.3 \\
197.4 \\
172.4 \\
24.5 \\
11.7 \\
12.8 \\
- \\
-\end{array}$ & $\begin{array}{r}453.4 \\
352.6 \\
59.1 \\
6.8 \\
.5 \\
6.3 \\
-\overline{34.9} \\
-\end{array}$ & $\begin{array}{r}410.7 \\
333.8 \\
47.3 \\
4.3 \\
.3 \\
4.1 \\
- \\
25.3 \\
-\end{array}$ & $\begin{array}{r}408.0 \\
325.0 \\
59.7 \\
3.9 \\
(8) \\
3.8 \\
- \\
19.4 \\
-\end{array}$ & $\begin{array}{r}351.7 \\
295.2 \\
27.1 \\
2.6 \\
(8) \\
2.6 \\
-\overline{2} \\
26.7 \\
-\end{array}$ & $\begin{array}{r}385.2 \\
304.7 \\
48.9 \\
3.6 \\
.5 \\
3.1 \\
. \overline{2} \\
28.0 \\
-\end{array}$ \\
\hline Primary Energy - Five Sectors ${ }^{c}$............................. & 714.6 & $1,453.4$ & $3,522.7$ & $4,064.3$ & $R_{3,900.1}$ & $\mathrm{R}_{3,665.8}$ & $R_{3,883.5}$ & $3,891.8$ \\
\hline
\end{tabular}

a There are no direct fuel costs for hydroelectric, geothermal, wind, photovoltaic, or solar thermal energy.

beavy oll includes fuel oil nos. 4, 5, and 6, and residual fuel oils. Light oil includes fuel nos. 1 and 2, kerosene, and jet fuel.

- Biomass fuels are not Included, except those consumed at electric utilities and those added to motor gasoline.

$R=$ Revised data.
-No consumption, including cases where adjustments were made. See explanation of adjustments in Section 6 of Appendix A.

$(s)=$ Value less than 0.05 million dollars.

Note: Totals may not equal sum of components due to independent rounding.

Sources: Data sources, estimation procedures, and assumptlons are described in Appendix A. 


\begin{tabular}{|c|c|c|c|c|c|c|c|c|}
\hline Energy Source & 1970 & 1975 & 1980 & 1985 & 1990 & 1991 & 1992 & 1993 \\
\hline & \multicolumn{8}{|c|}{ Prices in Dollars per Million Btu } \\
\hline 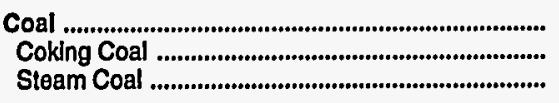 & $\begin{array}{r}0.26 \\
.38 \\
.25\end{array}$ & $\begin{array}{r}0.75 \\
1.60 \\
.70\end{array}$ & $\begin{array}{l}1.37 \\
1.81 \\
1.35\end{array}$ & $\begin{array}{l}1.48 \\
1.93 \\
1.46\end{array}$ & $\begin{array}{l}1.27 \\
1.80 \\
1.24\end{array}$ & $\begin{array}{l}1.24 \\
1.72 \\
1.23\end{array}$ & $\begin{array}{l}1.22 \\
1.74 \\
1.20\end{array}$ & $\begin{array}{l}1.22 \\
1.68 \\
1.21\end{array}$ \\
\hline 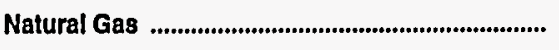 & .65 & 1.02 & 2.85 & 4.77 & 4.12 & 3.90 & 3.92 & 4.30 \\
\hline 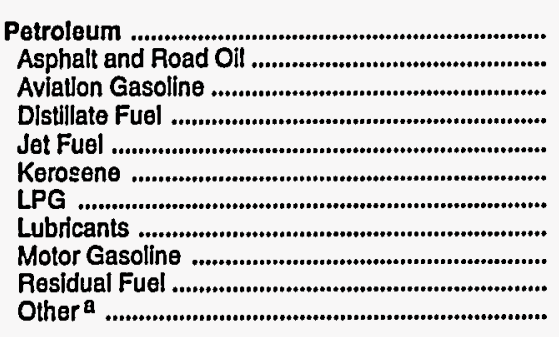 & $\begin{array}{r}2.12 \\
.67 \\
2.17 \\
1.21 \\
.73 \\
1.42 \\
1.90 \\
5.08 \\
2.93 \\
.56 \\
1.07\end{array}$ & $\begin{array}{l}3.81 \\
1.80 \\
3.45 \\
2.58 \\
2.03 \\
2.94 \\
3.60 \\
7.48 \\
4.69 \\
2.06 \\
2.49\end{array}$ & $\begin{array}{r}7.82 \\
3.56 \\
9.02 \\
6.41 \\
6.39 \\
7.55 \\
5.85 \\
14.36 \\
9.65 \\
3.64 \\
6.83\end{array}$ & $\begin{array}{r}7.87 \\
4.74 \\
9.99 \\
6.81 \\
6.17 \\
8.05 \\
6.58 \\
17.61 \\
8.80 \\
4.89 \\
7.08\end{array}$ & $\begin{array}{r}R_{7.97} \\
2.94 \\
9.32 \\
7.53 \\
5.82 \\
6.97 \\
6.91 \\
R_{14.60} \\
9.25 \\
3.61 \\
R_{5.12}\end{array}$ & $\begin{array}{r}R_{7.61} \\
3.30 \\
8.71 \\
6.95 \\
4.92 \\
6.41 \\
6.45 \\
R_{16.79} \\
8.94 \\
3.12 \\
R_{4.83}\end{array}$ & $\begin{array}{r}R_{7.37} \\
2.03 \\
8.54 \\
6.94 \\
4.61 \\
6.70 \\
6.52 \\
R_{19.43} \\
8.65 \\
2.71 \\
R_{4.23}\end{array}$ & $\begin{array}{r}7.64 \\
2.36 \\
8.24 \\
7.07 \\
4.36 \\
7.13 \\
9.72 \\
18.94 \\
8.77 \\
2.76 \\
4.13\end{array}$ \\
\hline 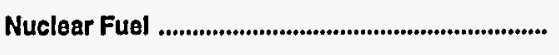 & - & - & - & - & - & - & - & - \\
\hline Blomass Fuels at Utilitles ..................................... & - & - & - & - & - & $\rightarrow$ & - & - \\
\hline Primary Energy - Flve Sectors b ..................... & .94 & 1.88 & 4.00 & 3.97 & 3.78 & $R_{3.63}$ & $\mathbf{R}_{3.60}$ & 3.60 \\
\hline 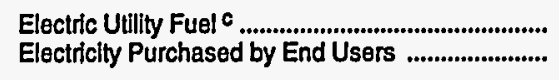 & $\frac{.22}{3.37}$ & $\begin{array}{r}.64 \\
5.32\end{array}$ & $\begin{array}{r}1.32 \\
10.07\end{array}$ & $\begin{array}{r}1.43 \\
14.84\end{array}$ & $\begin{array}{r}1.20 \\
13.17\end{array}$ & $\begin{array}{r}1.18 \\
12.96\end{array}$ & $\begin{array}{r}1.17 \\
12.33\end{array}$ & $\begin{array}{r}1.17 \\
12.70\end{array}$ \\
\hline \multirow[t]{2}{*}{ Total Energy ............................................................ } & 1.66 & 3.27 & 6.74 & 8.03 & 7.86 & ${ }^{R 7.70}$ & $R_{7.45}$ & 7.61 \\
\hline & \multicolumn{8}{|c|}{ Expenditures in Milllons of Dollars } \\
\hline 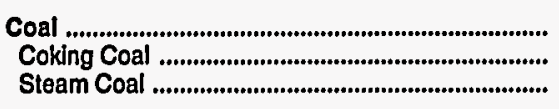 & $\begin{array}{r}138.9 \\
16.4 \\
122.4\end{array}$ & $\begin{array}{r}421.0 \\
52.1 \\
368.8\end{array}$ & $\begin{array}{r}878.8 \\
44.0 \\
834.8\end{array}$ & $\begin{array}{r}1,061.1 \\
60.5 \\
1,000.6\end{array}$ & $\begin{array}{r}1,018.6 \\
56.9 \\
961.8\end{array}$ & $\begin{array}{r}998.3 \\
39.4 \\
958.9\end{array}$ & $\begin{array}{r}991.1 \\
52.7 \\
938.4\end{array}$ & $\begin{array}{r}1,129.2 \\
52.9 \\
1,076.3\end{array}$ \\
\hline Natural Gas ................................................................ & 136.7 & 185.7 & 511.8 & 722.4 & 656.4 & 659.4 & 696.2 & 798.9 \\
\hline 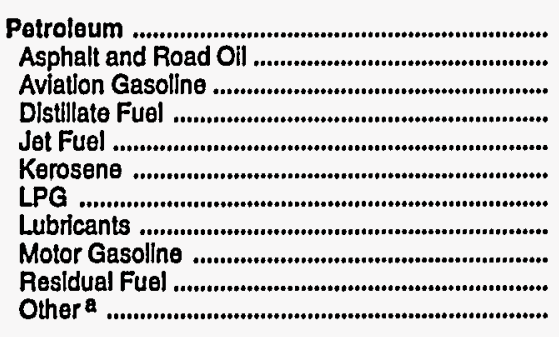 & $\begin{array}{r}760.8 \\
13.8 \\
3.6 \\
58.0 \\
12.6 \\
24.9 \\
67.5 \\
26.0 \\
517.3 \\
3.2 \\
33.8\end{array}$ & $\begin{array}{r}1,554.6 \\
31.3 \\
2.2 \\
164.1 \\
24.6 \\
26.3 \\
143.7 \\
47.6 \\
1,005.6 \\
11.1 \\
98.2\end{array}$ & $\begin{array}{r}3,870.9 \\
47.8 \\
5.1 \\
855.7 \\
104.4 \\
124.6 \\
216.4 \\
92.0 \\
2,019.1 \\
20.9 \\
385.0\end{array}$ & $\begin{array}{r}3,341.5 \\
58.9 \\
3.3 \\
863.2 \\
119.3 \\
68.8 \\
129.0 \\
102.7 \\
1,845.7 \\
9.5 \\
141.1\end{array}$ & $\begin{array}{r}A_{3,747.6} \\
59.1 \\
2.4 \\
1,026.1 \\
188.2 \\
22.4 \\
149.4 \\
R_{95.8} \\
2,079.8 \\
8.9 \\
R_{115.6}\end{array}$ & $\begin{array}{r}R_{3,588.1} \\
61.3 \\
2.3 \\
917.5 \\
177.3 \\
20.0 \\
153.7 \\
R_{98.6} \\
2,054.4 \\
5.4 \\
R_{97.6}\end{array}$ & $\begin{array}{r}8_{3,666.1} \\
34.1 \\
2.4 \\
R_{1,035.1} \\
179.7 \\
19.2 \\
149.7 \\
R_{116.3} \\
2,013.4 \\
4.5 \\
A_{111.8}\end{array}$ & $\begin{array}{r}3,885.1 \\
40.0 \\
1.7 \\
1,151.3 \\
140.8 \\
24.7 \\
199.3 \\
115.4 \\
2,107.5 \\
3.7 \\
100.7\end{array}$ \\
\hline 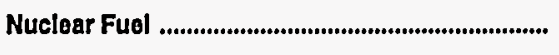 & - & - & - & - & - & - & - & - \\
\hline Blomass Fuele at Utilitles ..................................... & - & - & - & - & - & - & - & - \\
\hline 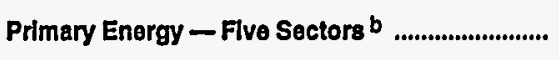 & $1,036.3$ & $2,161.3$ & $5,261.6$ & $5,125.0$ & $A_{5,422.7}$ & $R_{5,245.8}$ & $A_{5,353.4}$ & $5,813.2$ \\
\hline 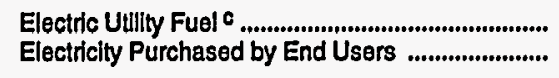 & $\begin{array}{r}-90.6 \\
354.9\end{array}$ & $\begin{array}{r}-309.8 \\
852.2\end{array}$ & $\begin{array}{r}-743.7 \\
1,698.6\end{array}$ & $\begin{array}{r}-883.4 \\
2,528.3\end{array}$ & $\begin{array}{r}-859.2 \\
2,707.4\end{array}$ & $\begin{array}{r}-862.0 \\
2,800.1\end{array}$ & $\begin{array}{r}-862.7 \\
A_{2,780.8}\end{array}$ & $\begin{array}{r}.969 .0 \\
2,912.6\end{array}$ \\
\hline 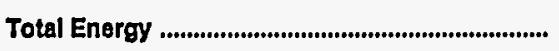 & $1,300.6$ & $2,703.7$ & $6,216.4$ & $6,769.9$ & $R_{7,270.8}$ & $R_{7,183.9}$ & $\mathrm{~A}_{7,271.5}$ & $7,756.8$ \\
\hline
\end{tabular}

\footnotetext{
a Includes petroleum coke used at electric utitities.

Blomass fuels are not included, except those consumed at electric utillties and those added to motor gasolline.

There are no direct fuel costs for hydroelectric, geothermal, wind, photovoltalc, or solar thermal energy.

RaRevlsed data.
}

-No consumption, including cases where adjustments were made. See explanation of adjustments in Section 6 of Appendix A.

Note: Expenditure totals may not equal sum of components due to independent rounding.

Sources: Data sources, estimation procedures, and assumptions are described in Appendix A. 
K Energy Price Estimates by Sector, Kentucky

E 1970, 1975, 1980, 1985, and 1990-1993

N (Dollars per Million Btu)

\begin{tabular}{|c|c|c|c|c|c|c|c|c|}
\hline Sector and Energy Source & 1970 & 1975 & 1980 & 1985 & 1990 & 1991 & 1992 & 1993 \\
\hline $\begin{array}{l}\text { Resldentlal Sector } \\
\text { Primary Energy } \\
\text { Coal . } \\
\text { Natural Gas } \\
\text { Petroleum } \\
\text { Distillate Fuel } \\
\text { Kerosene }\end{array}$ & $\begin{array}{r}1.87 \\
1.08 \\
.86 \\
.81 \\
1.98 \\
1.19 \\
1.73 \\
2.36 \\
5.85\end{array}$ & $\begin{array}{l}3.21 \\
1.77 \\
1.91 \\
1.22 \\
3.69 \\
2.49 \\
3.13 \\
4.16 \\
7.83\end{array}$ & $\begin{array}{r}6.84 \\
4.13 \\
2.30 \\
3.00 \\
8.10 \\
6.89 \\
8.52 \\
8.31 \\
12.91\end{array}$ & $\begin{array}{r}10.10 \\
5.75 \\
2.45 \\
5.15 \\
8.62 \\
7.67 \\
8.19 \\
9.78 \\
17.06\end{array}$ & $\begin{array}{r}10.48 \\
5.52 \\
2.25 \\
4.74 \\
9.59 \\
6.76 \\
7.22 \\
11.86 \\
16.70\end{array}$ & $\begin{array}{r}10.42 \\
5.33 \\
2.12 \\
4.65 \\
8.70 \\
6.27 \\
6.70 \\
10.54 \\
16.65\end{array}$ & $\begin{array}{r}10.23 \\
5.40 \\
2.09 \\
4.74 \\
8.97 \\
6.42 \\
6.86 \\
11.12 \\
16.69\end{array}$ & $\begin{array}{r}10.36 \\
5.61 \\
2.19 \\
5.01 \\
8.90 \\
7.09 \\
7.58 \\
10.22 \\
16.71\end{array}$ \\
\hline 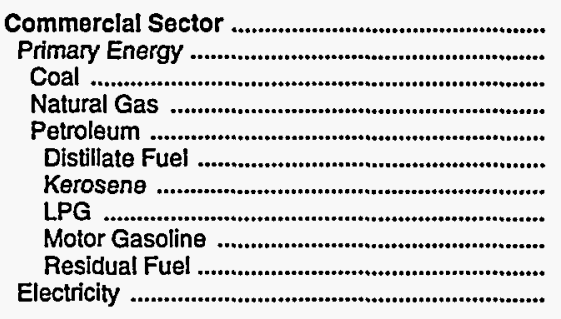 & $\begin{array}{r}1.51 \\
.75 \\
.44 \\
.66 \\
1.34 \\
1.02 \\
.79 \\
1.65 \\
2.93 \\
.78 \\
5.48\end{array}$ & $\begin{array}{l}2.55 \\
1.43 \\
1.30 \\
1.05 \\
2.88 \\
2.29 \\
2.53 \\
3.29 \\
4.69 \\
1.69 \\
5.26\end{array}$ & $\begin{array}{r}5.96 \\
4.01 \\
1.75 \\
2.89 \\
6.53 \\
6.49 \\
6.08 \\
5.22 \\
9.65 \\
4.12 \\
10.42\end{array}$ & $\begin{array}{r}7.95 \\
5.18 \\
1.88 \\
4.95 \\
6.88 \\
6.58 \\
7.89 \\
5.26 \\
8.80 \\
4.89 \\
12.34\end{array}$ & $\begin{array}{r}9.74 \\
4.58 \\
1.86 \\
4.35 \\
6.37 \\
5.09 \\
6.66 \\
4.73 \\
9.25 \\
3.61 \\
15.33\end{array}$ & $\begin{array}{l}9.56 \\
4.30 \\
1.87 \\
4.24 \\
5.52 \\
4.45 \\
5.78 \\
4.47 \\
8.94 \\
15 . \overline{-}\end{array}$ & $\begin{array}{r}9.23 \\
4.27 \\
1.76 \\
4.22 \\
5.51 \\
4.86 \\
6.29 \\
4.36 \\
8.65 \\
15.09\end{array}$ & $\begin{array}{r}9.45 \\
4.57 \\
1.70 \\
4.60 \\
6.42 \\
5.18 \\
6.30 \\
9.38 \\
8.77 \\
2.76 \\
15.11\end{array}$ \\
\hline 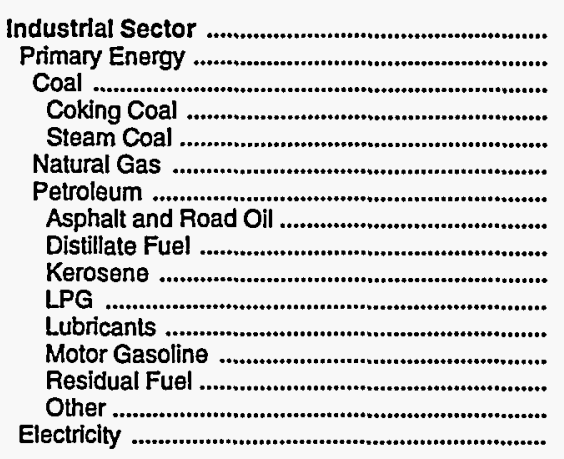 & $\begin{array}{r}1.00 \\
.70 \\
.42 \\
.38 \\
.44 \\
.48 \\
1.17 \\
.67 \\
.73 \\
.79 \\
1.65 \\
5.08 \\
2.93 \\
.44 \\
1.07 \\
2.16\end{array}$ & $\begin{array}{l}2.64 \\
1.81 \\
1.44 \\
1.60 \\
1.30 \\
.75 \\
2.67 \\
1.80 \\
2.31 \\
2.53 \\
3.29 \\
7.48 \\
4.69 \\
2.11 \\
2.49 \\
4.56\end{array}$ & $\begin{array}{r}5.23 \\
4.10 \\
1.77 \\
1.81 \\
1.75 \\
2.66 \\
5.92 \\
3.56 \\
5.43 \\
6.08 \\
5.22 \\
14.36 \\
9.65 \\
3.58 \\
.6 .83 \\
8.63\end{array}$ & $\begin{array}{r}7.07 \\
4.30 \\
1.90 \\
1.93 \\
1.88 \\
4.25 \\
6.84 \\
4.74 \\
6.87 \\
7.89 \\
5.26 \\
17.61 \\
8.80 \\
4.89 \\
7.08 \\
14.51\end{array}$ & $\begin{array}{r}R_{5} 5.68 \\
\text { P }_{3.62} \\
1.84 \\
1.80 \\
1.86 \\
3.47 \\
R_{5} .31 \\
2.94 \\
5.79 \\
6.66 \\
4.73 \\
\mathrm{~A}_{14.60} \\
9.25 \\
3.61 \\
\mathrm{~A}_{5.12} \\
10.49\end{array}$ & $\begin{array}{r}R_{5} .51 \\
R_{3.48} \\
1.82 \\
1.72 \\
1.87 \\
3.08 \\
R_{5.09} \\
3.30 \\
5.03 \\
5.78 \\
4.47 \\
R_{16.79} \\
8.94 \\
3.12 \\
R_{4.83} \\
9.93\end{array}$ & $\begin{array}{r}\mathrm{R}_{5} .38 \\
\mathrm{P}_{3.45} \\
1.75 \\
1.74 \\
1.76 \\
3.05 \\
\mathrm{P}_{4.94} \\
2.03 \\
\mathrm{R}_{5.47} \\
6.29 \\
4.36 \\
\mathrm{R}_{19.43} \\
8.65 \\
2.71 \\
\mathrm{R}_{4.23} \\
9.25\end{array}$ & $\begin{array}{r}5.54 \\
3.62 \\
1.70 \\
1.68 \\
1.70 \\
3.49 \\
5.61 \\
2.36 \\
5.49 \\
6.30 \\
9.38 \\
18.94 \\
8.77 \\
2.76 \\
4.13 \\
9.66\end{array}$ \\
\hline 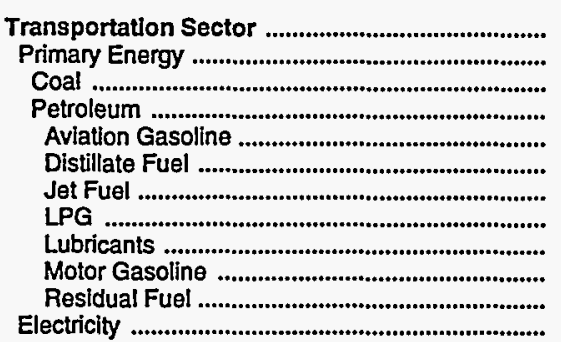 & $\begin{array}{r}2.58 \\
2.58 \\
.44 \\
2.58 \\
2.17 \\
1.45 \\
.73 \\
1.65 \\
5.08 \\
2.93 \\
.77 \\
-\end{array}$ & $\begin{array}{l}4.34 \\
4.34 \\
1.30 \\
4.34 \\
3.45 \\
2.78 \\
2.03 \\
3.29 \\
7.48 \\
4.69 \\
1.46\end{array}$ & $\begin{array}{r}8.82 \\
8.82 \\
8.82 \\
9.02 \\
6.86 \\
6.39 \\
5.22 \\
14.36 \\
9.65 \\
3.94 \\
-\end{array}$ & $\begin{array}{r}8.19 \\
8.19 \\
- \\
8.19 \\
9.99 \\
6.78 \\
6.17 \\
5.26 \\
17.61 \\
8.80 \\
- \\
-\end{array}$ & $\begin{array}{r}R_{8.70} \\
R_{8.70} \\
- \\
R_{8.70} \\
9.32 \\
8.21 \\
5.82 \\
4.73 \\
14.60 \\
9.25 \\
- \\
-\end{array}$ & $\begin{array}{r}\text { R }_{8.28} \\
\text { R } 8.28 \\
\text { R }_{8.28} \\
8.71 \\
7.75 \\
4.92 \\
4.47 \\
\text { R }_{16.79} \\
8.94 \\
-\end{array}$ & $\begin{array}{r}R_{8.01} \\
R_{8.01} \\
R_{8.01} \\
8.54 \\
7.56 \\
4.61 \\
4.36 \\
R_{19.43} \\
8.65 \\
- \\
-\end{array}$ & $\begin{array}{r}8.11 \\
8.11 \\
-\overline{11} \\
8.11 \\
8.24 \\
7.56 \\
4.36 \\
9.38 \\
18.94 \\
8.77 \\
-\end{array}$ \\
\hline 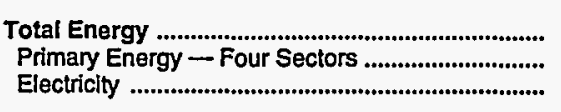 & $\begin{array}{l}1.66 \\
1.39 \\
3.37\end{array}$ & $\begin{array}{l}3.27 \\
2.77 \\
5.32\end{array}$ & $\begin{array}{r}6.74 \\
5.99 \\
10.07\end{array}$ & $\begin{array}{r}6.03 \\
6.30 \\
14.84\end{array}$ & $\begin{array}{r}7.86 \\
\mathbf{R} 6.35 \\
13.17\end{array}$ & $\begin{array}{l}R_{7.70} \\
R_{6.11} \\
12.96\end{array}$ & $\begin{array}{l}R_{7.45} \\
{ }^{R} 5.98 \\
12.33\end{array}$ & $\begin{array}{r}7.61 \\
6.13 \\
12.70\end{array}$ \\
\hline 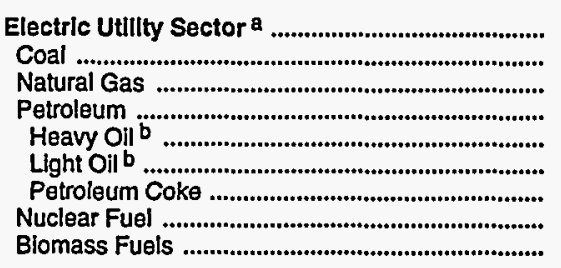 & $\begin{array}{r}.22 \\
.21 \\
.29 \\
.88 \\
.87 \\
1.12 \\
= \\
=\end{array}$ & $\begin{array}{r}.64 \\
.64 \\
.68 \\
1.72 \\
1.69 \\
2.25 \\
- \\
= \\
-\end{array}$ & $\begin{array}{l}1.32 \\
1.31 \\
2.16 \\
6.54 \\
-\overline{6} \\
6.54 \\
= \\
=\end{array}$ & $\begin{array}{r}1.43 \\
1.41 \\
3.54 \\
5.80 \\
5.80 \\
= \\
-\end{array}$ & $\begin{array}{r}1.20 \\
1.19 \\
2.98 \\
5.75 \\
-\overline{5.75} \\
= \\
=\end{array}$ & $\begin{array}{r}1.18 \\
1.18 \\
2.60 \\
5.05 \\
- \\
5.05 \\
= \\
=\end{array}$ & $\begin{array}{r}1.17 \\
1.16 \\
2.72 \\
4.79 \\
\overline{4} \\
4.79 \\
= \\
=\end{array}$ & $\begin{array}{r}1.17 \\
1.17 \\
3.01 \\
4.38 \\
4 . \overline{8} \\
= \\
=\end{array}$ \\
\hline 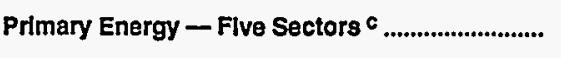 & .94 & 1.88 & 4.00 & 3.97 & 3.78 & $\mathrm{P}_{3.63}$ & A $_{3.60}$ & 3.60 \\
\hline
\end{tabular}

a There are no direct fuel costs for hydroelectric, geothermal, wind, photovoltaic, or solar thermal energy.

beavy oil includes fuel oil nos. 4,5 , and 6 , and residual fuel oils. Light oll includes fuel oil nos. 1 and 2, kerosene, and jet fuel.

c Biomass fuels are not included, except those consumed at electric

R=Revised data.

-No consumption, including cases where adjustments were made. See explanation of adjustments in Section 6 of Appendix A.

Sources: Data sources, estimation procedures, and assumptions are utilities and those added to motor gasoline. 


\begin{tabular}{|c|c|c|c|c|c|c|c|c|}
\hline Sector and Energy Source & 1970 & 1975 & 1980 & 1985 & 1990 & 1991 & 1992 & 1993 \\
\hline 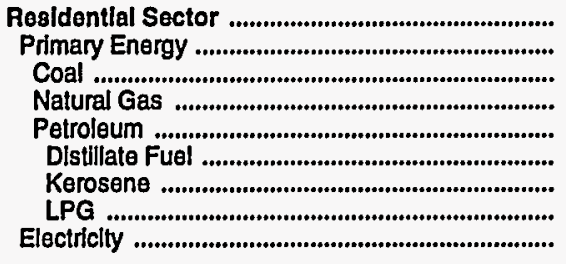 & $\begin{array}{r}268.1 \\
128.6 \\
3.8 \\
71.6 \\
53.1 \\
2.8 \\
20.4 \\
30.0 \\
139.6\end{array}$ & $\begin{array}{r}441.0 \\
185.0 \\
4.6 \\
97.1 \\
83.2 \\
6.4 \\
19.0 \\
57.8 \\
256.0\end{array}$ & $\begin{array}{r}986.8 \\
410.9 \\
5.6 \\
224.9 \\
180.4 \\
32.9 \\
84.6 \\
63.0 \\
575.9\end{array}$ & $\begin{array}{r}1,301.7 \\
455.5 \\
5.2 \\
318.9 \\
131.4 \\
36.8 \\
38.7 \\
55.9 \\
846.2\end{array}$ & $\begin{array}{r}1,354.2 \\
396.3 \\
2.9 \\
276.4 \\
117.0 \\
25.4 \\
13.1 \\
78.5 \\
958.0\end{array}$ & $\begin{array}{r}1,474.0 \\
415.0 \\
3.4 \\
289.6 \\
122.0 \\
25.7 \\
14.4 \\
81.9 \\
1,059.0\end{array}$ & $\begin{array}{r}1,451.6 \\
438.6 \\
3.9 \\
310.2 \\
124.6 \\
28.8 \\
14.2 \\
81.6 \\
1,013.0\end{array}$ & $\begin{array}{r}1,588.1 \\
492.0 \\
5.1 \\
351.3 \\
135.7 \\
32.2 \\
17.0 \\
86.5 \\
1,096.0\end{array}$ \\
\hline 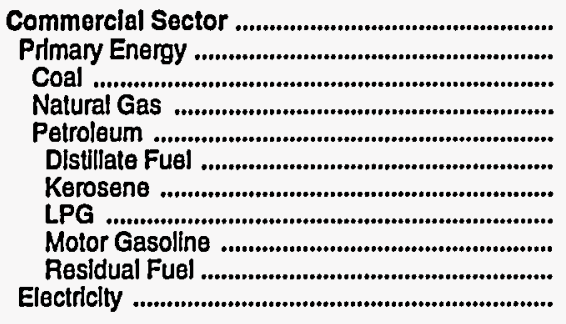 & $\begin{array}{r}111.2 \\
46.5 \\
3.5 \\
28.3 \\
14.6 \\
5.0 \\
1.8 \\
3.7 \\
4.1 \\
.1 \\
64.8\end{array}$ & $\begin{array}{r}193.0 \\
76.6 \\
5.6 \\
40.8 \\
30.2 \\
12.2 \\
3.0 \\
8.1 \\
6.8 \\
.1 \\
116.4\end{array}$ & $\begin{array}{r}563.7 \\
263.8 \\
7.7 \\
114.9 \\
141.1 \\
99.6 \\
21.4 \\
7.0 \\
12.7 \\
.5 \\
299.9\end{array}$ & $\begin{array}{r}663.3 \\
264.6 \\
7.4 \\
172.1 \\
85.1 \\
58.3 \\
4.1 \\
5.3 \\
17.5 \\
(5) \\
398.7\end{array}$ & $\begin{array}{r}812.2 \\
198.2 \\
4.5 \\
143.8 \\
50.0 \\
19.4 \\
3.6 \\
5.5 \\
21.5 \\
(\mathrm{~s}) \\
614.0\end{array}$ & $\begin{array}{r}852.2 \\
198.3 \\
5.6 \\
149.6 \\
43.0 \\
18.6 \\
3.3 \\
6.1 \\
15.0 \\
- \\
654.0\end{array}$ & $\begin{array}{r}837.5 \\
209.5 \\
6.0 \\
158.3 \\
45.2 \\
24.9 \\
2.1 \\
5.6 \\
12.6 \\
- \\
628.0\end{array}$ & $\begin{array}{r}878.2 \\
228.2 \\
7.3 \\
182.3 \\
38.6 \\
20.0 \\
2.8 \\
14.0 \\
1.8 \\
(\mathrm{~s}) \\
650.0\end{array}$ \\
\hline 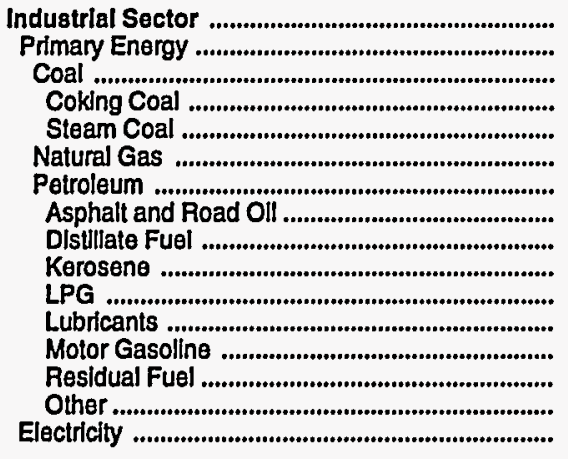 & $\begin{array}{r}341.1 \\
190.6 \\
44.0 \\
16.4 \\
27.5 \\
34.3 \\
112.3 \\
13.8 \\
8.9 \\
2.7 \\
33.5 \\
14.6 \\
3.2 \\
1.8 \\
33.8 \\
150.5\end{array}$ & $\begin{array}{r}923.2 \\
443.4 \\
102.3 \\
52.1 \\
50.1 \\
47.5 \\
293.6 \\
31.3 \\
44.7 \\
4.2 \\
77.0 \\
23.5 \\
4.8 \\
9.9 \\
98.2 \\
479.9\end{array}$ & $\begin{array}{r}1,994.8 \\
1,172.0 \\
134.6 \\
44.0 \\
90.6 \\
167.8 \\
869.6 \\
47.8 \\
203.6 \\
18.6 \\
146.2 \\
46.9 \\
4.5 \\
17.1 \\
385.0 \\
822.8\end{array}$ & $\begin{array}{r}2,306.0 \\
1,022.6 \\
178.3 \\
60.5 \\
117.9 \\
227.4 \\
617.0 \\
58.9 \\
224.3 \\
26.0 \\
65.9 \\
52.4 \\
39.0 \\
9.5 \\
141.1 \\
1,283.4\end{array}$ & $\begin{array}{r}R_{2,050.0} \\
R_{914.6} \\
160.0 \\
56.9 \\
103.2 \\
235.4 \\
R_{519.2} \\
59.1 \\
175.7 \\
5.7 \\
64.3 \\
R_{48.8} \\
41.0 \\
8.9 \\
R_{115.6} \\
1,135.4\end{array}$ & $\begin{array}{r}\mathbf{A}_{1}, 916.6 \\
\mathbf{R}_{829.5} \\
134.6 \\
39.4 \\
95.2 \\
219.6 \\
\mathbf{R}_{475.4} \\
61.3 \\
153.1 \\
2.4 \\
64.8 \\
\text { P }_{50.3} \\
40.6 \\
5.4 \\
\mathbf{R}_{97.6} \\
1,087.1\end{array}$ & $\begin{array}{r}R_{1,989.2} \\
R_{849.3} \\
124.8 \\
52.7 \\
72.1 \\
226.9 \\
R_{497.6} \\
34.1 \\
R_{184.4} \\
2.9 \\
61.5 \\
R_{59.3} \\
39.1 \\
4.5 \\
R_{111.8} \\
R_{1,139.8}\end{array}$ & $\begin{array}{r}2,105.8 \\
939.2 \\
154.0 \\
52.9 \\
101.2 \\
264.6 \\
520.6 \\
40.0 \\
167.6 \\
4.9 \\
96.9 \\
58.9 \\
48.0 \\
3.7 \\
100.7 \\
1,166.6\end{array}$ \\
\hline 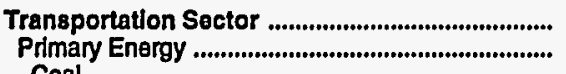 & $\begin{array}{l}580.1 \\
580.1\end{array}$ & $\begin{array}{l}1,146.5 \\
1,146.5\end{array}$ & $\begin{array}{l}2,671.1 \\
2,671.1\end{array}$ & $\begin{array}{l}2,498.9 \\
2,498.9\end{array}$ & $\begin{array}{l}R_{3,054.4} \\
R_{3,054.4}\end{array}$ & $\begin{array}{l}R_{2,941.0} \\
R_{2,941.0}\end{array}$ & $\begin{array}{l}R_{2,993.3} \\
R_{2,993.3}\end{array}$ & $\begin{array}{l}3,184.7 \\
3,184.7\end{array}$ \\
\hline 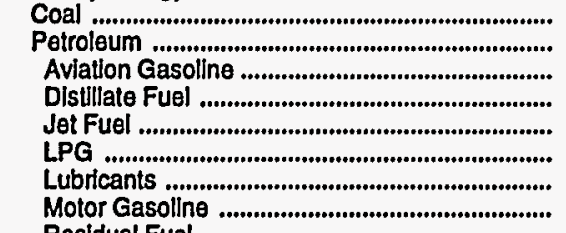 & $\begin{array}{r}580.0 \\
3.6 \\
41.4 \\
12.6 \\
.3 \\
11.4 \\
510.0\end{array}$ & $\begin{array}{r}(s) \\
1,146.5 \\
2.2 \\
100.8 \\
24.6 \\
.8 \\
24.0 \\
994.0\end{array}$ & $\begin{array}{r}- \\
2,671.1 \\
5.1 \\
511.0 \\
104.4 \\
.2 \\
45.1 \\
2,002.0\end{array}$ & $\begin{array}{r}- \\
2,498.9 \\
3.3 \\
534.7 \\
119.3 \\
1.9 \\
50.4 \\
1,789.3\end{array}$ & $\begin{array}{r}R_{3,054.4} \\
2.4 \\
798.4 \\
188.2 \\
1.1 \\
R_{47.0} \\
2,017.3\end{array}$ & $\begin{array}{r}R_{2,941.0} \\
2.3 \\
713.4 \\
177.3 \\
.8 \\
R_{48.3} \\
1,998.9\end{array}$ & $\begin{array}{r}R_{2,993.3} \\
2.4 \\
791.6 \\
179.7 \\
.9 \\
R_{57.0} \\
1,961.7\end{array}$ & $\begin{array}{r}- \\
3,184.7 \\
1.7 \\
926.1 \\
140.8 \\
1.9 \\
56.6 \\
2,057.7\end{array}$ \\
\hline 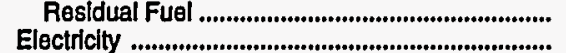 & $\stackrel{.7}{-}$ & (s) & $\begin{array}{l}3.4 \\
-\end{array}$ & $\overline{-}$ & $\overline{-}$ & $\overline{-}$ & $\overline{-}$ & - \\
\hline 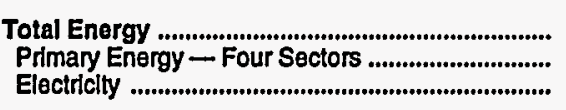 & $\begin{array}{r}1,300.6 \\
945.7 \\
354.9\end{array}$ & $\begin{array}{r}2,703.7 \\
1,851.5 \\
852.2\end{array}$ & $\begin{array}{l}6,216.4 \\
4,517.8 \\
1,698.6\end{array}$ & $\begin{array}{l}6,769.9 \\
4,241.6 \\
2,528.3\end{array}$ & $\begin{array}{r}R_{7,270.8} \\
R_{4,563.5} \\
2,707.4\end{array}$ & $\begin{array}{r}R_{7,183.9} \\
R_{4,383.8} \\
2,800.1\end{array}$ & $\begin{array}{l}R_{7,271.5} \\
R_{4,490.7} \\
R_{2,780.8}\end{array}$ & $\begin{array}{l}7,756.8 \\
4,844.2 \\
2,912.6\end{array}$ \\
\hline 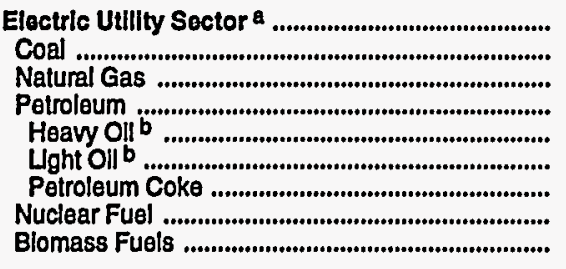 & $\begin{array}{r}90.6 \\
87.4 \\
2.5 \\
.7 \\
.7 \\
(8) \\
- \\
-\end{array}$ & $\begin{array}{r}309.8 \\
308.4 \\
.2 \\
1.2 \\
1.1 \\
.1 \\
= \\
- \\
-\end{array}$ & $\begin{array}{r}743.7 \\
730.9 \\
4.2 \\
8.6 \\
- \\
8.6 \\
- \\
- \\
-\end{array}$ & $\begin{array}{r}883.4 \\
870.2 \\
4.1 \\
9.1 \\
-\overline{9} \\
- \\
= \\
-\end{array}$ & $\begin{array}{r}859.2 \\
851.3 \\
.9 \\
7.1 \\
\overline{7.1} \\
- \\
- \\
-\end{array}$ & $\begin{array}{r}862.0 \\
854.7 \\
.6 \\
6.7 \\
- \\
6.7 \\
- \\
- \\
-\end{array}$ & $\begin{array}{r}862.7 \\
856.5 \\
.7 \\
5.5 \\
- \\
5.5 \\
- \\
- \\
-\end{array}$ & $\begin{array}{r}969.0 \\
962.7 \\
.8 \\
5.5 \\
- \\
5.5 \\
- \\
- \\
-\end{array}$ \\
\hline Primary Energy - Five Sectors C ......................... & $1,036.3$ & $2,161.3$ & $5,261.6$ & $5,125.0$ & $R_{5,422.7}$ & $R_{5,245.8}$ & $R_{5,353.4}$ & $5,813.2$ \\
\hline
\end{tabular}

a There are no direct fuel costs for hydroelectric, geothermal, wind, photovoltalc, or solar thermal energy.

beavy oll includes fuel oll nos. 4, 5, and 6, and residual fuel olls. Light oll includes fuel nos. 1 and 2, keroseno, and jet fuel.

- Blomass fuels are not included, except those consumed at electric utilltiles and those added to motor gasoline.

$R=$ Revised data.
-No consumption, Including cases where adjustments were made. See explanation of adjustments $\ln$ Section 6 of Appendix A.

$(s)=$ Value less than 0.05 million dollars.

Note: Totals may not equal sum of components due to independent rounding.

Sources: Data sources, estimation procedures, and assumptions are described in Appendlx A. 


\section{Energy Price and Expenditure Estimates by Source, Louisiana}

O 1970, 1975, 1980, 1985, and 1990-1993

\begin{tabular}{|c|c|c|c|c|c|c|c|c|}
\hline Energy Source & 1970 & 1975 & 1980 & 1985 & 1990 & 1991 & 1992 & 1893 \\
\hline & \multicolumn{8}{|c|}{ Prices In Dollars per Million Btu } \\
\hline 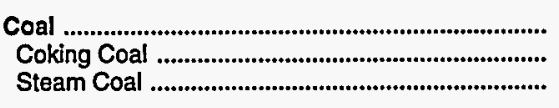 & $\begin{array}{l}- \\
-\end{array}$ & $\overline{-}$ & $\begin{array}{r}1.26 \\
1.26\end{array}$ & $\frac{2.14}{2.14}$ & $\begin{array}{r}1.68 \\
1 . \overline{6}\end{array}$ & $\begin{array}{r}1.65 \\
1.65\end{array}$ & $\begin{array}{r}1.54 \\
1.54\end{array}$ & $\frac{1.59}{1.59}$ \\
\hline Natural Gas & .27 & .75 & 1.61 & 3.09 & 2.11 & 1.89 & 2.09 & 2.50 \\
\hline 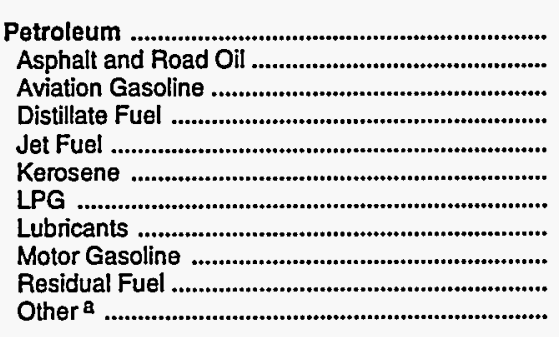 & $\begin{array}{r}1.48 \\
.66 \\
2.17 \\
.86 \\
.72 \\
.60 \\
1.12 \\
5.08 \\
2.86 \\
.45 \\
.70\end{array}$ & $\begin{array}{l}2.80 \\
1.78 \\
3.45 \\
2.34 \\
2.01 \\
2.02 \\
2.52 \\
7.48 \\
4.49 \\
1.62 \\
2.40\end{array}$ & $\begin{array}{r}5.98 \\
3.62 \\
9.02 \\
6.02 \\
6.34 \\
5.53 \\
5.30 \\
14.36 \\
9.89 \\
3.31 \\
6.28\end{array}$ & $\begin{array}{r}6.23 \\
4.17 \\
9.99 \\
5.01 \\
5.70 \\
3.57 \\
5.12 \\
17.61 \\
9.36 \\
3.60 \\
6.57\end{array}$ & $\begin{array}{r}R_{6.80} \\
2.91 \\
9.32 \\
7.61 \\
5.79 \\
7.60 \\
7.82 \\
R_{14.60} \\
9.47 \\
2.10 \\
R_{5.80}\end{array}$ & $\begin{array}{r}6.42 \\
3.08 \\
8.71 \\
7.20 \\
4.67 \\
6.95 \\
7.83 \\
R 16.79 \\
9.51 \\
2.84 \\
R 4.98\end{array}$ & $\begin{array}{r}R_{5.49} \\
2.17 \\
8.54 \\
6.99 \\
4.29 \\
7.30 \\
4.28 \\
R_{19.43} \\
9.23 \\
1.90 \\
R_{4.61}\end{array}$ & $\begin{array}{r}5.42 \\
2.30 \\
8.24 \\
6.88 \\
4.02 \\
6.29 \\
4.33 \\
18.94 \\
9.39 \\
1.88 \\
3.93\end{array}$ \\
\hline 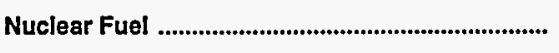 & - & - & - & .86 & .88 & .84 & .81 & .69 \\
\hline 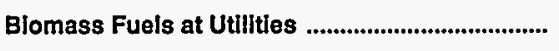 & - & - & - & - & - & - & - & - \\
\hline Primary Energy — Five Sectors ${ }^{b}$......................... & .65 & 1.60 & 3.70 & 4.48 & 4.02 & 3.80 & 3.50 & 3.61 \\
\hline $\begin{array}{l}\text { Electric Utility Fuel c } \\
\text { Electricity Purchased by End Users }\end{array}$ & $\begin{array}{r}.21 \\
4.69\end{array}$ & $\begin{array}{r}.73 \\
6.24\end{array}$ & $\begin{array}{r}2.19 \\
11.49\end{array}$ & $\begin{array}{r}2.46 \\
18.25\end{array}$ & $\begin{array}{r}1.49 \\
17.77\end{array}$ & $\begin{array}{r}1.40 \\
17.59\end{array}$ & $\begin{array}{r}1.52 \\
17.81\end{array}$ & $\begin{array}{r}1.65 \\
18.56\end{array}$ \\
\hline \multirow[t]{2}{*}{ 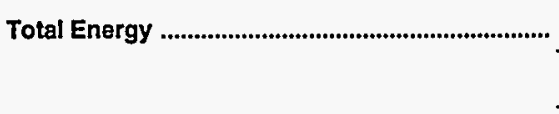 } & .94 & 2.03 & 4.50 & 6.33 & 6.00 & 5.70 & 5.33 & 5.55 \\
\hline & \multicolumn{8}{|c|}{ Expenditures in Millions of Dollars } \\
\hline 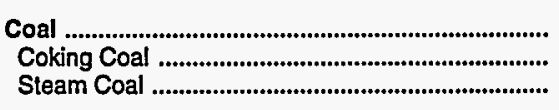 & $\begin{array}{l}- \\
-\end{array}$ & $\overline{-}$ & $\begin{array}{l}3.1 \\
\overline{3.1}\end{array}$ & $\begin{array}{c}340.0 \\
- \\
340.0\end{array}$ & $\begin{array}{c}351.2 \\
- \\
351.2\end{array}$ & $\begin{array}{c}353.8 \\
- \\
353.8\end{array}$ & $\begin{array}{c}344.8 \\
- \\
344.8\end{array}$ & $\begin{array}{c}354.0 \\
- \\
354.0\end{array}$ \\
\hline Natural Gas & 376.4 & $1,036.2$ & $2,396.3$ & $3,152.5$ & $2,466.5$ & $2,207.6$ & $2,439.5$ & $2,964.7$ \\
\hline $\begin{array}{l}\text { Petroleum } \\
\text { Asphalt and Road Oil } \\
\text { Aviation Gasoline } \\
\text { Distillate Fuel } \\
\text { Jet Fuel } \\
\text { Kerosene } \\
\text { LPG } \\
\text { Mubricants } \\
\text { Residual Fuer } \\
\text { Other }{ }^{\mathbf{a}}\end{array}$ & $\begin{array}{r}964.9 \\
9.7 \\
4.9 \\
59.1 \\
23.4 \\
8.5 \\
199.3 \\
49.0 \\
523.4 \\
31.1 \\
56.5\end{array}$ & $\begin{array}{r}2,705.4 \\
33.2 \\
5.1 \\
268.9 \\
67.9 \\
27.7 \\
481.4 \\
82.9 \\
1,018.8 \\
280.0 \\
439.5\end{array}$ & $\begin{array}{r}8,196.5 \\
46.8 \\
11.6 \\
752.1 \\
306.8 \\
179.2 \\
1,012.5 \\
174.1 \\
2,449.2 \\
1,265.9 \\
1,998.3\end{array}$ & $\begin{array}{r}6,761.1 \\
50.8 \\
8.6 \\
980.8 \\
410.5 \\
3.8 \\
1,293.9 \\
194.3 \\
2,424.2 \\
546.9 \\
847.3\end{array}$ & $\begin{array}{r}R_{7,816.0} \\
32.3 \\
5.1 \\
1,739.2 \\
845.1 \\
3.5 \\
1,343.2 \\
R_{181.2} \\
2,174.1 \\
302.9 \\
R_{1,189.4}\end{array}$ & $\begin{array}{r}R_{7,672.0} \\
30.6 \\
4.1 \\
1,458.1 \\
848.7 \\
3.4 \\
1,468.1 \\
R_{186.5} \\
2,148.7 \\
457.3 \\
R_{1,066.4}\end{array}$ & $\begin{array}{r}R_{6,594.0} \\
24.3 \\
3.8 \\
1,283.2 \\
653.1 \\
1.9 \\
840.6 \\
R_{220.0} \\
2,189.1 \\
R_{354.4} \\
A_{1,023.7}\end{array}$ & $\begin{array}{r}6,600.7 \\
28.4 \\
9.1 \\
1,408.3 \\
571.2 \\
2.2 \\
853.4 \\
218.4 \\
2,273.0 \\
328.1 \\
908.6\end{array}$ \\
\hline 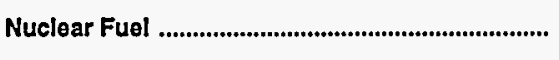 & - & - & - & 22.9 & 133.6 & 125.4 & 89.2 & 105.5 \\
\hline Biomass Fuels at Utillties ..................................... & - & - & - & - & - & - & - & - \\
\hline Primary Energy - Five Sectors ${ }^{b} \ldots \ldots \ldots \ldots . . . . . . . . . .$. & $1,341.4$ & $3,741.6$ & $10,595.9$ & $10,276.5$ & $R_{10,767.3}$ & $\mathrm{R}_{10,358.8}$ & $R_{9,467.4}$ & $10,024.8$ \\
\hline $\begin{array}{l}\text { Electric Utility Fuel }{ }^{c} \text {.................................................... } \\
\text { Electricity Purchased by End Users ...................... }\end{array}$ & $\begin{array}{r}-72.9 \\
435.9\end{array}$ & $\begin{array}{r}-303.4 \\
710.5\end{array}$ & $\begin{array}{r}-1,079.1 \\
1,899.6\end{array}$ & $\begin{array}{r}-1,168.2 \\
3,664.5\end{array}$ & $\begin{array}{r}-931.6 \\
3,739.3\end{array}$ & $\begin{array}{r}-869.3 \\
3,754.6\end{array}$ & $\begin{array}{r}-903.4 \\
3,823.6\end{array}$ & $\begin{array}{r}-1,058.1 \\
4,127.9\end{array}$ \\
\hline Total Energy .................................................................. & $1,704.4$ & $4,148.6$ & $11,416.4$ & $12,772.8$ & $R_{13,574.9}$ & $P_{13,244.1}$ & $R_{12,387.7}$ & $13,094.7$ \\
\hline
\end{tabular}

a Includes petroleum coke used at electric utlities.

b Biomass fuels are not included, except those consumed at electric utilities and those added to motor gasoline.

c There are no direct fuel costs for hydroelectric, geothermal, wind, photovoltaic, or solar thermal energy.

$R=$ Revised data.

-No consumption, including cases where adjustments were mado. Seo explanation of adjustments in Section 6 of Appendix A.

Note: Expenditure totals may not equal sum of components due to Independent rounding.

Sources: Data sources, estimation procedures, and assumptions are described in Appendlx A. 


\begin{tabular}{|c|c|c|c|c|c|c|c|c|}
\hline Sector and Energy Source & 1870 & 1975 & 1980 & 1985 & 1990 & 1991 & 1992 & 1993 \\
\hline 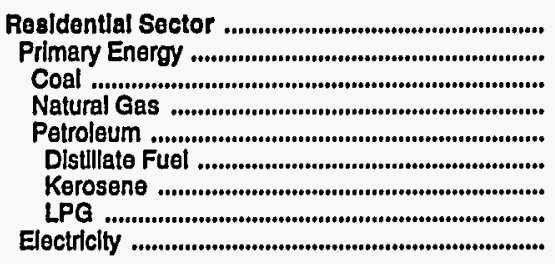 & $\begin{array}{r}2.29 \\
.90 \\
. \overline{.95} \\
.75 \\
.96 \\
1.60 \\
2.20 \\
6.58\end{array}$ & $\begin{array}{l}3.31 \\
1.55 \\
1.33 \\
4.36 \\
2.24 \\
3.40 \\
4.39 \\
7.96\end{array}$ & $\begin{array}{r}7.84 \\
3.56 \\
2.97 \\
3.28 \\
8.52 \\
6.65 \\
-\overline{8.54} \\
13.81\end{array}$ & $\begin{array}{r}13.04 \\
5.58 \\
5 . \overline{5} \\
7.47 \\
3.54 \\
4.24 \\
7.68 \\
20.27\end{array}$ & $\begin{array}{r}14.77 \\
6.12 \\
5 . \overline{5} \\
11.29 \\
6.46 \\
9.50 \\
11.43 \\
21.70\end{array}$ & $\begin{array}{r}14.56 \\
5.87 \\
2.81 \\
5.51 \\
12.75 \\
5.94 \\
8.75 \\
12.88 \\
21.68\end{array}$ & $\begin{array}{r}14.54 \\
5.76 \\
5.36 \\
11.69 \\
8.61 \\
12.68 \\
11.68 \\
22.02\end{array}$ & $\begin{array}{r}15.36 \\
6.13 \\
2.73 \\
5.88 \\
11.87 \\
7.73 \\
11.38 \\
11.88 \\
22.75\end{array}$ \\
\hline 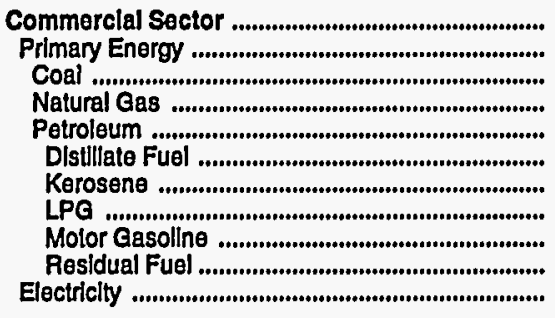 & $\begin{array}{r}1.62 \\
.48 \\
-37 \\
.37 \\
1.04 \\
.89 \\
.59 \\
1.06 \\
2.86 \\
.49 \\
5.07\end{array}$ & $\begin{array}{r}2.89 \\
1.25 \\
-\overline{.77} \\
2.19 \\
2.14 \\
2.01 \\
2.44 \\
4.49 \\
1.76 \\
6.99\end{array}$ & $\begin{array}{r}5.54 \\
3.40 \\
1.24 \\
2.60 \\
3.77 \\
6.36 \\
5.53 \\
5.22 \\
9.89 \\
3.55 \\
12.08\end{array}$ & $\begin{array}{r}12.08 \\
4.28 \\
- \\
5.09 \\
3.37 \\
2.85 \\
3.45 \\
5.08 \\
9.36 \\
4.12 \\
20.24\end{array}$ & $\begin{array}{r}14.77 \\
5.41 \\
- \\
5.04 \\
6.48 \\
5.74 \\
7.24 \\
7.76 \\
9.47 \\
2.62 \\
20.57\end{array}$ & $\begin{array}{r}14.46 \\
4.96 \\
1.73 \\
4.68 \\
5.91 \\
5.34 \\
6.61 \\
7.75 \\
9.51 \\
2.03 \\
20.30\end{array}$ & $\begin{array}{r}14.60 \\
4.86 \\
- \\
4.59 \\
6.28 \\
5.67 \\
5.94 \\
4.14 \\
9.23 \\
2.02 \\
20.73\end{array}$ & $\begin{array}{r}15.65 \\
5.10 \\
1.68 \\
5.15 \\
4.89 \\
4.74 \\
5.59 \\
4.23 \\
9.39 \\
1.92 \\
21.50\end{array}$ \\
\hline 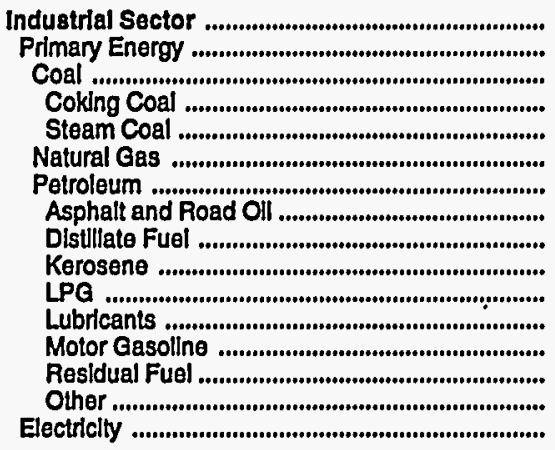 & $\begin{array}{r}.48 \\
.42 \\
- \\
- \\
.23 \\
.23 \\
.97 \\
.66 \\
.51 \\
.59 \\
1.06 \\
5.08 \\
2.86 \\
.49 \\
.70 \\
2.49\end{array}$ & $\begin{array}{r}1.40 \\
1.32 \\
- \\
- \\
.74 \\
2.41 \\
1.78 \\
1.81 \\
2.01 \\
2.44 \\
7.48 \\
4.49 \\
1.72 \\
2.40 \\
3.99\end{array}$ & $\begin{array}{r}3.30 \\
3.07 \\
1.24 \\
-\overline{1} \\
1.24 \\
1.24 \\
5.68 \\
3.62 \\
4.89 \\
5.53 \\
5.22 \\
14.36 \\
9.89 \\
3.68 \\
6.28 \\
9.02\end{array}$ & $\begin{array}{r}4.64 \\
3.96 \\
1.46 \\
1 . \overline{1} \\
1.46 \\
2.92 \\
5.33 \\
4.17 \\
2.96 \\
3.45 \\
5.08 \\
17.61 \\
9.36 \\
4.12 \\
6.57 \\
14.93\end{array}$ & $\begin{array}{r}4.15 \\
3.65 \\
1.56 \\
-\overline{1} \\
1.56 \\
1.92 \\
6.62 \\
2.91 \\
6.22 \\
7.24 \\
7.76 \\
\text { A } 14.60 \\
9.47 \\
2.62 \\
85.80 \\
12.27\end{array}$ & $\begin{array}{r}3.89 \\
3.37 \\
1.73 \\
- \\
1.73 \\
1.66 \\
6.24 \\
3.08 \\
5.68 \\
6.61 \\
7.75 \\
R 16.79 \\
9.51 \\
2.03 \\
R_{4.98} \\
12.15\end{array}$ & $\begin{array}{r}3.50 \\
2.92 \\
1.69 \\
-\overline{1.69} \\
1.85 \\
R 4.71 \\
2.17 \\
5.10 \\
5.94 \\
4.14 \\
R 19.43 \\
9.23 \\
2.02 \\
R 4.73 \\
12.38\end{array}$ & $\begin{array}{r}3.67 \\
3.06 \\
1.68 \\
- \\
1.68 \\
2.22 \\
4.51 \\
2.30 \\
4.81 \\
5.59 \\
4.23 \\
18.94 \\
9.39 \\
1.92 \\
4.23 \\
13.01\end{array}$ \\
\hline 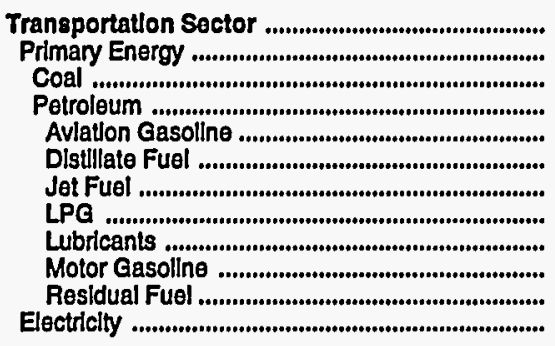 & $\begin{array}{r}1.97 \\
1.97 \\
1.97 \\
2.17 \\
1.09 \\
.72 \\
1.06 \\
5.08 \\
2.86 \\
.44 \\
5.07\end{array}$ & $\begin{array}{r}3.28 \\
3.28 \\
3.28 \\
3.45 \\
2.54 \\
2.01 \\
2.44 \\
7.48 \\
4.49 \\
1.54 \\
6.99\end{array}$ & $\begin{array}{r}6.87 \\
6.87 \\
- \\
6.87 \\
9.02 \\
6.84 \\
6.34 \\
5.22 \\
14.36 \\
9.89 \\
3.05 \\
12.08\end{array}$ & $\begin{array}{r}7.16 \\
7.16 \\
-\overline{7.16} \\
9.99 \\
6.38 \\
5.70 \\
5.08 \\
17.61 \\
9.36 \\
3.40 \\
20.24\end{array}$ & $\begin{array}{r}R_{6.92} \\
R_{6.92} \\
R_{6.92} \\
9.32 \\
8.48 \\
5.79 \\
7.76 \\
R_{14.60} \\
9.47 \\
2.07 \\
20.18\end{array}$ & $\begin{array}{r}R_{6.53} \\
R_{6.53} \\
R_{6.53} \\
8.71 \\
8.21 \\
4.67 \\
7.75 \\
R_{16.79} \\
9.51 \\
2.87 \\
18.68\end{array}$ & $\begin{array}{r}R_{6.06} \\
R_{6.06} \\
R_{6.06} \\
8.54 \\
8.14 \\
4.29 \\
4.14 \\
R_{19.43} \\
9.23 \\
1.90 \\
18.90\end{array}$ & $\begin{array}{r}6.23 \\
6.23 \\
- \\
6.23 \\
8.24 \\
8.13 \\
4.02 \\
4.23 \\
18.94 \\
9.39 \\
1.87 \\
20.85\end{array}$ \\
\hline 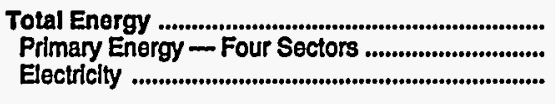 & $\begin{array}{r}.94 \\
.74 \\
4.69\end{array}$ & $\begin{array}{l}2.03 \\
1.78 \\
6.24\end{array}$ & $\begin{array}{r}4.50 \\
4.02 \\
11.49\end{array}$ & $\begin{array}{r}6.33 \\
5.01 \\
18.25\end{array}$ & $\begin{array}{r}6.00 \\
4.80 \\
17.77\end{array}$ & $\begin{array}{r}5.70 \\
4.50 \\
17.59\end{array}$ & $\begin{array}{r}5.33 \\
4.06 \\
17.81\end{array}$ & $\begin{array}{r}5.55 \\
4.19 \\
18.56\end{array}$ \\
\hline 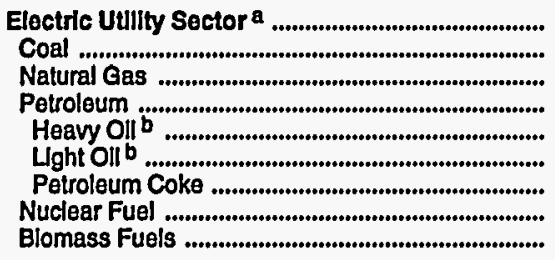 & $\begin{array}{l}21 \\
- \\
.21 \\
.57 \\
.55 \\
.60 \\
= \\
=\end{array}$ & $\begin{array}{r}.73 \\
-64 \\
1.76 \\
1.76 \\
1.92 \\
- \\
- \\
-\end{array}$ & $\begin{array}{r}2.19 \\
-\overrightarrow{01} \\
3.68 \\
3.58 \\
4.37 \\
- \\
- \\
-\end{array}$ & $\begin{array}{r}2.46 \\
2.19 \\
2.73 \\
5.08 \\
3.51 \\
5.84 \\
-\overline{6} \\
-\end{array}$ & $\begin{array}{r}1.49 \\
1.70 \\
1.66 \\
4.15 \\
2.47 \\
5.01 \\
\overline{88} \\
-\end{array}$ & $\begin{array}{r}1.40 \\
1.65 \\
1.53 \\
4.32 \\
3.30 \\
4.56 \\
- \\
.84 \\
-\end{array}$ & 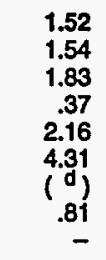 & 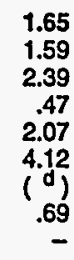 \\
\hline Primary Energy - Flve Sectors C ....................... & .65 & 1.60 & $\mathbf{3 . 7 0}$ & 4.48 & 4.02 & 3.80 & 3.50 & 3.61 \\
\hline
\end{tabular}

a There are no direct fuel costs for hydroelectric, geothermal, wind, photovoltalc, or solar thermal energy.

Heavy oll Includes fuel oll nos. 4, 5, and 6, and residual fuel oils. Light oll Includes fuel oll nos. 1 and 2, kerosene, and jet fuel.

- Blomass fuels are not included, except those consumed at electric utlltiles and those added to motor gasoline.

Utilities used petroleum coke at no charge.

$R=$ Revised data.

-No consumption, including cases where adjustments were made. See explanation of adjustments in Section 6 of Appendix A.

Sources: Data sources, estimation procedures, and assumptions are described in Appondlx A. 
L Energy Expenditure Estimates by Sector, Louisiana $1970,1975,1980,1985$, and 1990-1993

(Million Dollars)

\begin{tabular}{|c|c|c|c|c|c|c|c|c|}
\hline Sector and Energy Source & 1970 & 1975 & 1980 & 1985 & 1990 & 1991 & 1992 & 1993 \\
\hline $\begin{array}{l}\text { Residential Sector } \\
\text { Primary Energy } \\
\text { Coal . } \\
\text { Natural Gas . } \\
\text { Petroleum } \\
\text { Distillate Fuel . } \\
\text { Kerosene }\end{array}$ & $\begin{array}{r}299.1 \\
89.4 \\
-. \\
66.7 \\
22.8 \\
(s) \\
.2 \\
22.5 \\
209.6\end{array}$ & $\begin{array}{r}490.0 \\
166.2 \\
131 . \overline{6} \\
34.6 \\
.1 \\
.4 \\
34.0 \\
323.8\end{array}$ & $\begin{array}{r}1,077.9 \\
285.0 \\
.1 \\
248.7 \\
36.2 \\
.2 \\
-2 \\
36.0 \\
792.9\end{array}$ & $\begin{array}{r}1,767.3 \\
372.3 \\
-\overline{-} \\
344.3 \\
28.0 \\
.2 \\
.5 \\
27.3 \\
1,395.0\end{array}$ & $\begin{array}{r}1,945.2 \\
358.3 \\
- \\
325.2 \\
33.1 \\
.3 \\
.7 \\
32.1 \\
1,587.0\end{array}$ & $\begin{array}{r}1,950.1 \\
354.1 \\
(s) \\
315.0 \\
39.1 \\
.1 \\
.7 \\
38.4 \\
1,596.0\end{array}$ & $\begin{array}{r}1,946.6 \\
354.7 \\
309.2 \\
35.4 \\
(s) \\
.7 \\
44.8 \\
1,592.0\end{array}$ & $\begin{array}{r}2,116.8 \\
375.8 \\
(s) \\
344.7 \\
31.0 \\
(s) \\
.5 \\
30.5 \\
1,741.0\end{array}$ \\
\hline 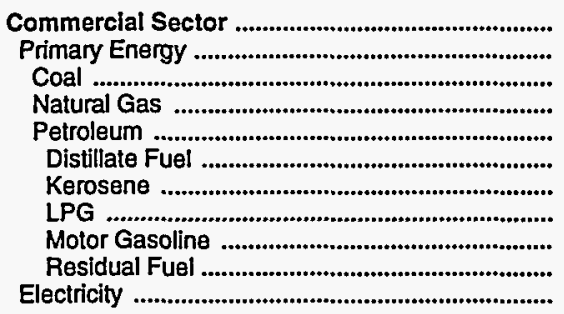 & $\begin{array}{r}187.4 \\
41.6 \\
- \\
26.6 \\
15.0 \\
4.3 \\
1.5 \\
1.9 \\
5.7 \\
1.6 \\
145.8\end{array}$ & $\begin{array}{r}318.5 \\
98.5 \\
-\overline{4} \\
40.5 \\
58.0 \\
18.2 \\
5.3 \\
3.3 \\
11.0 \\
20.2 \\
220.0\end{array}$ & $\begin{array}{r}981.0 \\
453.2 \\
.1 \\
107.7 \\
345.4 \\
14.8 \\
17.2 \\
3.9 \\
8.7 \\
300.8 \\
527.7\end{array}$ & $\begin{array}{r}1,395.4 \\
252.8 \\
159.7 \\
93.1 \\
62.2 \\
1.3 \\
3.2 \\
11.6 \\
14.9 \\
1,142.6\end{array}$ & $\begin{array}{r}1,348.5 \\
188.6 \\
-\overline{131.1} \\
57.5 \\
36.5 \\
.9 \\
3.8 \\
15.7 \\
.7 \\
1,159.9\end{array}$ & $\begin{array}{r}1,317.9 \\
172.0 \\
(s) \\
124.8 \\
47.2 \\
27.9 \\
.8 \\
4.1 \\
12.9 \\
1.5 \\
1,145.9\end{array}$ & $\begin{array}{r}1,334.2 \\
171.4 \\
- \\
136.3 \\
35.1 \\
20.0 \\
.3 \\
2.8 \\
11.9 \\
.1 \\
1,162.9\end{array}$ & $\begin{array}{r}1,401.6 \\
162.8 \\
(\mathrm{~s}) \\
134.1 \\
28.6 \\
23.9 \\
.8 \\
1.9 \\
2.0 \\
(\mathrm{~s}) \\
1,238.9\end{array}$ \\
\hline 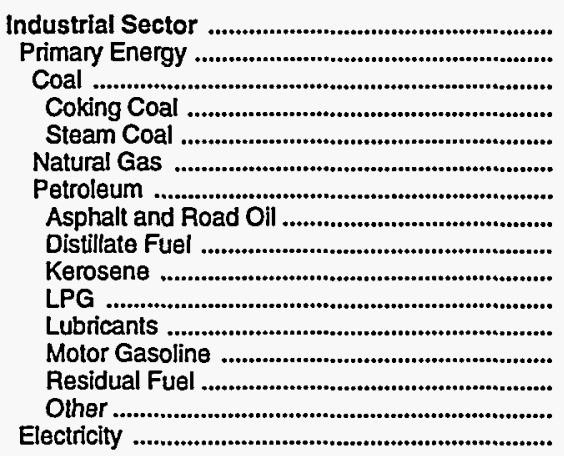 & $\begin{array}{r}589.6 \\
509.2 \\
- \\
- \\
- \\
210.8 \\
298.4 \\
9.7 \\
12.4 \\
6.8 \\
173.5 \\
32.4 \\
4.5 \\
2.5 \\
56.5 \\
80.5\end{array}$ & $\begin{array}{r}1,872.7 \\
1,706.1 \\
- \\
- \\
\overline{6} \\
624.6 \\
1,081.5 \\
33.2 \\
49.0 \\
22.0 \\
441.2 \\
59.0 \\
4.1 \\
33.6 \\
439.5 \\
166.6\end{array}$ & $\begin{array}{r}5,442.9 \\
4,864.0 \\
2.9 \\
\overline{2} \\
2.9 \\
1,150.2 \\
3,710.9 \\
46.8 \\
210.9 \\
161.9 \\
969.6 \\
111.3 \\
3.2 \\
208.8 \\
1,998.3 \\
578.8\end{array}$ & $\begin{array}{r}5,611.6 \\
4,484.9 \\
15.8 \\
- \\
15.8 \\
1,833.1 \\
2,635.9 \\
50.8 \\
164.5 \\
2.0 \\
1,261.4 \\
124.2 \\
23.9 \\
161.8 \\
847.3 \\
1,126.7\end{array}$ & $\begin{array}{r}R_{5,723.4} \\
R_{4,731.1} \\
24.8 \\
- \\
24.8 \\
1,544.3 \\
R_{3,161.9} \\
32.3 \\
487.0 \\
1.9 \\
1,305.2 \\
8_{115.9} \\
16.7 \\
13.6 \\
\mathrm{~A}_{1,189.4} \\
992.3\end{array}$ & $\begin{array}{r}R_{5,484.0} \\
R_{4,471.5} \\
17.8 \\
- \\
17.8 \\
1,362.2 \\
R_{3,091.5} \\
30.6 \\
424.2 \\
1.9 \\
1,423.6 \\
R_{119.3} \\
17.8 \\
7.7 \\
R_{1,066.4} \\
1,012.5\end{array}$ & $\begin{array}{r}R_{4,937.4} \\
R_{3,868.8} \\
18.7 \\
- \\
18.7 \\
1,507.9 \\
R_{2,342.1} \\
24.3 \\
338.1 \\
.9 \\
792.1 \\
R \quad 140.7 \\
16.7 \\
5.7 \\
R_{1,023.7} \\
1,068.6\end{array}$ & $\begin{array}{r}5,320.3 \\
4,172.4 \\
18.1 \\
- \\
18.1 \\
1,878.9 \\
2,275.3 \\
28.4 \\
342.6 \\
.9 \\
819.9 \\
139.6 \\
32.4 \\
2.9 \\
908.6 \\
1,147.9\end{array}$ \\
\hline 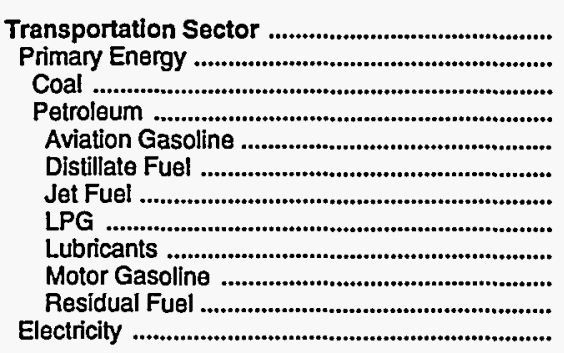 & $\begin{array}{r}628.3 \\
628.3 \\
- \\
628.3 \\
4.9 \\
42.1 \\
23.4 \\
1.4 \\
16.6 \\
513.2 \\
26.8 \\
\text { (s) }\end{array}$ & $\begin{array}{r}1,467.5 \\
1,467.4 \\
- \\
1,467.4 \\
5.1 \\
200.6 \\
67.9 \\
2.8 \\
23.9 \\
1,003.8 \\
163.2 \\
.1\end{array}$ & $\begin{array}{r}3,914.7 \\
3,914.6 \\
- \\
3,914.6 \\
11.6 \\
496.3 \\
306.8 \\
3.0 \\
62.8 \\
2,437.3 \\
596.8 \\
.1\end{array}$ & $\begin{array}{r}3,998.5 \\
3,998.4 \\
- \\
3,998.4 \\
8.6 \\
749.4 \\
410.5 \\
2.0 \\
70.0 \\
2,388.8 \\
368.9 \\
.2\end{array}$ & $\begin{array}{r}R_{4,557.8} \\
R_{4,557.6} \\
- \\
R_{4,557.6} \\
5.1 \\
1,210.7 \\
845.1 \\
2.1 \\
R_{65.3} \\
2,141.7 \\
287.5 \\
.2\end{array}$ & $\begin{array}{r}R_{4,492.1} \\
R_{4,491.9} \\
R_{4,491.9} \\
4.1 \\
1,004.0 \\
848.7 \\
2.1 \\
R_{67.2} \\
2,118.0 \\
447.7 \\
.2\end{array}$ & $\begin{array}{r}R_{4,169.4} \\
R_{4,169.2} \\
- \\
R_{4,169.2} \\
3.8 \\
923.3 \\
653.1 \\
1.0 \\
R_{79.3} \\
2,160.5 \\
\text { R }_{348.4} \\
.2\end{array}$ & $\begin{array}{r}4,256.1 \\
4,255.9 \\
-\overline{4,255.9} \\
9.1 \\
1,040.2 \\
571.2 \\
1.0 \\
78.7 \\
2,238.6 \\
317.0 \\
.2\end{array}$ \\
\hline $\begin{array}{l}\text { Total Energy . } \\
\text { Primary Energy - Four Sectors } \\
\text { Electricity . . }\end{array}$ & $\begin{array}{r}1,704.4 \\
1,268.5 \\
435.9\end{array}$ & $\begin{array}{r}4,148.6 \\
3,438.1 \\
710.5\end{array}$ & $\begin{array}{r}11,416.4 \\
9,516.8 \\
1,899.6\end{array}$ & $\begin{array}{r}12,772.8 \\
9,108.3 \\
3,664.5\end{array}$ & $\begin{array}{r}R_{13,574.9} \\
R_{9,635.6} \\
3,739.3\end{array}$ & $\begin{array}{r}\mathrm{R}_{13,244.1} \\
\mathrm{R}_{9,489.6} \\
3,754.6\end{array}$ & $\begin{array}{r}\mathrm{R}_{12,387.7} \\
\mathrm{R}_{8,564.0} \\
3,823.6\end{array}$ & $\begin{array}{r}13,094.7 \\
8,966.8 \\
4,127.9\end{array}$ \\
\hline 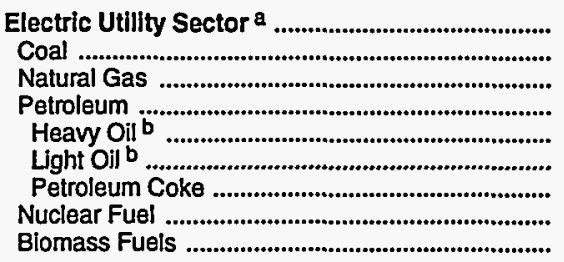 & $\begin{array}{r}72.9 \\
- \\
72.3 \\
.5 \\
.3 \\
.2 \\
- \\
- \\
-\end{array}$ & $\begin{array}{r}303.4 \\
239.5 \\
63.9 \\
62.9 \\
1.0 \\
- \\
- \\
-\end{array}$ & $\begin{array}{r}1,079.1 \\
- \\
889.7 \\
189.4 \\
159.5 \\
29.9 \\
= \\
-\end{array}$ & $\begin{array}{r}1,168.2 \\
324.2 \\
815.3 \\
5.8 \\
1.3 \\
4.5 \\
\overline{22.9} \\
-\end{array}$ & $\begin{array}{r}931.6 \\
326.3 \\
465.9 \\
5.8 \\
1.2 \\
4.7 \\
- \\
133.6 \\
-\end{array}$ & $\begin{array}{r}869.3 \\
336.0 \\
405.6 \\
2.3 \\
.3 \\
1.9 \\
- \\
125.4 \\
-\end{array}$ & $\begin{array}{r}903.4 \\
326.1 \\
486.0 \\
2.1 \\
.2 \\
1.9 \\
(d .9 \\
89.2 \\
-\end{array}$ & $\begin{array}{r}1,058.1 \\
335.8 \\
606.9 \\
9.9 \\
8.2 \\
1.7 \\
(d) \\
105.5 \\
-\end{array}$ \\
\hline Primary Energy — Five Sectors c ............................ & $1,341.4$ & $3,741.6$ & $10,595.9$ & $10,276.5$ & $A_{10,767.3}$ & $R_{10,358.8}$ & ${ }_{8,467.4}$ & $10,024.8$ \\
\hline
\end{tabular}

a There are no direct fuel costs for hydroelectric, geothermal, wind, photovoltaic, or solar themal energy.

Heavy oil includes fuel oil nos. 4, 5, and 6, and residual fuel oils. Light oil includes fuel nos. 1 and 2, kerosene, and jet fuel.

c Biomass fuels are not included, except those consumed at electric utilitities and those added to motor gasoline.

U Utilities used petroleum coke at no charge.

R=Revised data.

-No consumption, including cases where adjustments were made. See explanation of adjustments in Section 6 of Appendix A.

Note: Totals may not equal sum of components due to independent rounding.

Sources: Data sources, estimation procedures, and assumptions are described in Appendix A. 


\begin{tabular}{|c|c|c|c|c|c|c|c|c|}
\hline Energy Source & 1970 & 1975 & 1980 & 1985 & 1990 & 1991 & 1992 & 1993 \\
\hline & \multicolumn{8}{|c|}{ Prices in Dollars per Million Btu } \\
\hline 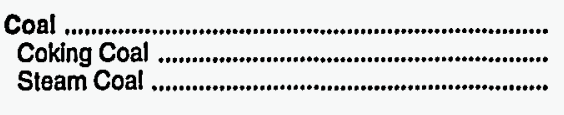 & 1.06 & $2 . \overline{-60}$ & $1 . \overline{89}$ & $\frac{2.58}{2.58}$ & $\begin{array}{r}2.72 \\
2.72\end{array}$ & $\begin{array}{r}2.67 \\
-\overline{2}\end{array}$ & $\begin{array}{r}2.67 \\
- \\
2.67\end{array}$ & $\begin{array}{r}2.36 \\
-\overline{36}\end{array}$ \\
\hline 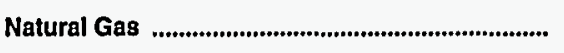 & 1.48 & 2.03 & 5.03 & 7.41 & 6.05 & 5.50 & 5.43 & 6.06 \\
\hline 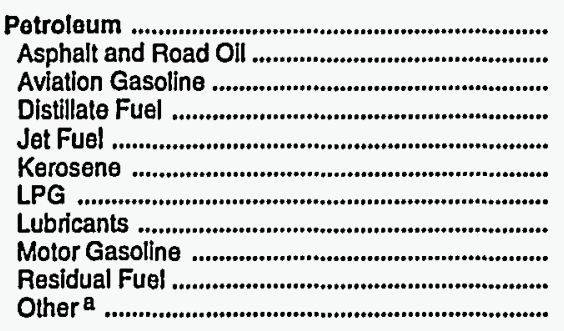 & $\begin{array}{r}1.46 \\
.73 \\
2.17 \\
1.37 \\
.75 \\
1.53 \\
2.37 \\
5.08 \\
3.02 \\
.38 \\
.89\end{array}$ & $\begin{array}{r}3.04 \\
1.99 \\
3.45 \\
2.78 \\
2.09 \\
3.10 \\
4.20 \\
7.48 \\
4.56 \\
1.79 \\
-\end{array}$ & $\begin{array}{r}7.00 \\
3.73 \\
9.02 \\
6.83 \\
6.51 \\
7.82 \\
7.86 \\
14.36 \\
9.69 \\
4.10 \\
-\end{array}$ & $\begin{array}{r}7.38 \\
5.21 \\
9.99 \\
8.00 \\
6.10 \\
8.78 \\
10.89 \\
17.61 \\
9.35 \\
4.37 \\
-\end{array}$ & $\begin{array}{r}\text { R } 7.00 \\
3.34 \\
9.32 \\
7.87 \\
5.92 \\
8.62 \\
13.04 \\
14.60 \\
9.74 \\
2.86 \\
-\end{array}$ & $\begin{array}{r}6.71 \\
3.05 \\
8.71 \\
7.56 \\
5.07 \\
8.29 \\
14.45 \\
16.79 \\
16.64 \\
9.64 \\
2.33 \\
\end{array}$ & $\begin{array}{r}\mathrm{A}_{6.57} \\
2.81 \\
8.54 \\
6.98 \\
5.20 \\
7.65 \\
\mathrm{~T} \\
\mathrm{r} \\
\mathrm{R} \\
19.49 \\
9.63 \\
2.42 \\
-\end{array}$ & $\begin{array}{r}6.54 \\
3.30 \\
8.24 \\
6.64 \\
4.43 \\
7.11 \\
12.59 \\
18.94 \\
9.59 \\
2.38 \\
-\end{array}$ \\
\hline 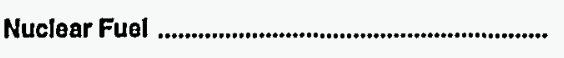 & - & .32 & .58 & .62 & .46 & .43 & .39 & .37 \\
\hline 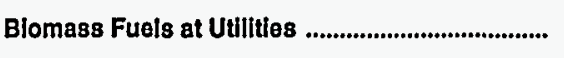 & - & - & - & - & - & - & - & - \\
\hline Primary Energy - Flve Sectors ${ }^{b} \ldots \ldots . . . . . . . . . . . . . . . .$. & 1.45 & 2.54 & 5.70 & 5.84 & 5.77 & 5.21 & 5.13 & 5.18 \\
\hline 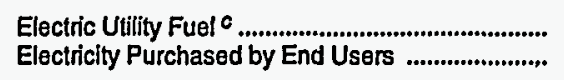 & $\begin{array}{r}.35 \\
5.92\end{array}$ & 9.71 & $\begin{array}{r}1.82 \\
16.30\end{array}$ & $\begin{array}{r}1.60 \\
20.16\end{array}$ & $\begin{array}{r}1.17 \\
22.42\end{array}$ & $\begin{array}{r}.73 \\
25.21\end{array}$ & $\begin{array}{r}.76 \\
26.52\end{array}$ & $\begin{array}{r}.60 \\
26.68\end{array}$ \\
\hline \multirow[t]{2}{*}{ Total Enorgy } & 1.95 & 3.77 & 8.44 & 9.49 & ${ }^{R} 9.50$ & 9.48 & ค9.29 & 9.39 \\
\hline & \multicolumn{8}{|c|}{ Expenditures in Millions of Dollars } \\
\hline 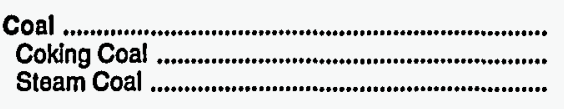 & $\frac{2.3}{2.3}$ & $\begin{array}{l}3.4 \\
3.4\end{array}$ & $\begin{array}{l}5.7 \\
5.7\end{array}$ & $\stackrel{13.2}{13.2}$ & $\begin{array}{l}18.0 \\
18.0\end{array}$ & $\begin{array}{l}25.0 \\
25.0\end{array}$ & $\begin{array}{l}57.3 \\
57.3\end{array}$ & $\begin{array}{l}26.5 \\
26.5\end{array}$ \\
\hline 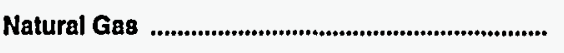 & 1.9 & 4.0 & 11.2 & 19.3 & 26.4 & 26.5 & 28.2 & 30.5 \\
\hline $\begin{array}{l}\text { Potroleum } \\
\text { Asphalt and Road Oil } \\
\text { Aviation Gasoline } \\
\text { Distillate Fuel } \\
\text { Jet Fuel } \\
\text { Kerosene } \\
\text { LPG }\end{array}$ & $\begin{array}{r}337.1 \\
3.4 \\
1.0 \\
94.2 \\
9.4 \\
15.5 \\
5.7 \\
5.2 \\
174.9 \\
27.5 \\
.4\end{array}$ & $\begin{array}{r}675.1 \\
9.2 \\
1.2 \\
186.5 \\
22.7 \\
18.2 \\
15.1 \\
7.6 \\
303.1 \\
111.7 \\
-\end{array}$ & $\begin{array}{r}1,388.1 \\
10.8 \\
3.7 \\
422.8 \\
66.7 \\
22.4 \\
25.2 \\
17.1 \\
598.7 \\
220.7 \\
-\end{array}$ & $\begin{array}{r}1,509.0 \\
75.6 \\
2.1 \\
446.4 \\
54.4 \\
51.8 \\
26.5 \\
19.1 \\
615.9 \\
217.2 \\
-\end{array}$ & $\begin{array}{r}R_{1,676.9} \\
14.3 \\
2.9 \\
549.8 \\
82.9 \\
32.1 \\
65.7 \\
R 17.8 \\
718.6 \\
192.7 \\
-\end{array}$ & $\begin{array}{r}R_{1,539.0} \\
20.0 \\
1.9 \\
456.3 \\
66.7 \\
34.9 \\
77.0 \\
R_{18.3} \\
714.7 \\
149.1 \\
-\end{array}$ & $\begin{array}{r}R_{1,476.9} \\
19.8 \\
1.8 \\
442.8 \\
54.8 \\
24.0 \\
50.9 \\
R_{21.6} \\
714.3 \\
146.8 \\
-\end{array}$ & $\begin{array}{r}1,542.4 \\
23.7 \\
1.5 \\
493.8 \\
36.6 \\
39.0 \\
62.1 \\
21.4 \\
724.5 \\
139.7 \\
-\end{array}$ \\
\hline 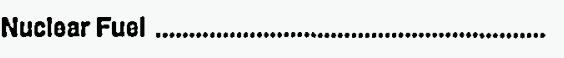 & - & 16.1 & 27.9 & 35.7 & 24.1 & 29.0 & 22.2 & 22.8 \\
\hline 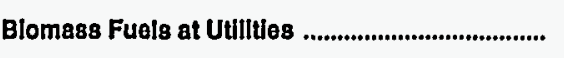 & - & - & - & - & - & - & - & - \\
\hline Primary Energy — Flve Sectors ${ }^{b}$ & 341.3 & 698.6 & $1,432.8$ & $1,577.2$ & $R_{1,745.5}$ & $P_{1,619.5}$ & $R_{1,584.6}$ & $1,622.3$ \\
\hline 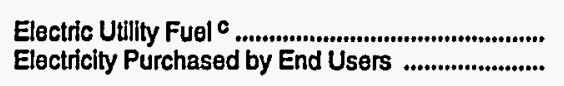 & $\begin{array}{l}-10.6 \\
102.3\end{array}$ & $\begin{array}{r}-48.1 \\
216.1\end{array}$ & $\begin{array}{r}-129.8 \\
455.3\end{array}$ & $\begin{array}{r}-127.6 \\
675.7\end{array}$ & $\begin{array}{r}-86.5 \\
882.0\end{array}$ & $\begin{array}{l}-59.3 \\
979.1\end{array}$ & $\begin{array}{r}-54.4 \\
1,038.9\end{array}$ & $\begin{array}{r}-41.7 \\
1,088.0\end{array}$ \\
\hline Total Energy ... & 433.1 & 866.7 & $1,758.3$ & $2,125.3$ & $\mathrm{R}_{2,541.0}$ & $R_{2,539.2}$ & $\mathrm{R}_{2,569.1}$ & $2,668.7$ \\
\hline
\end{tabular}

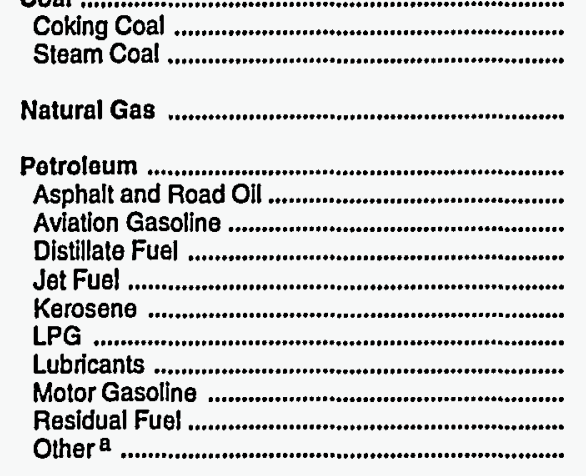

Nuclear Fuel

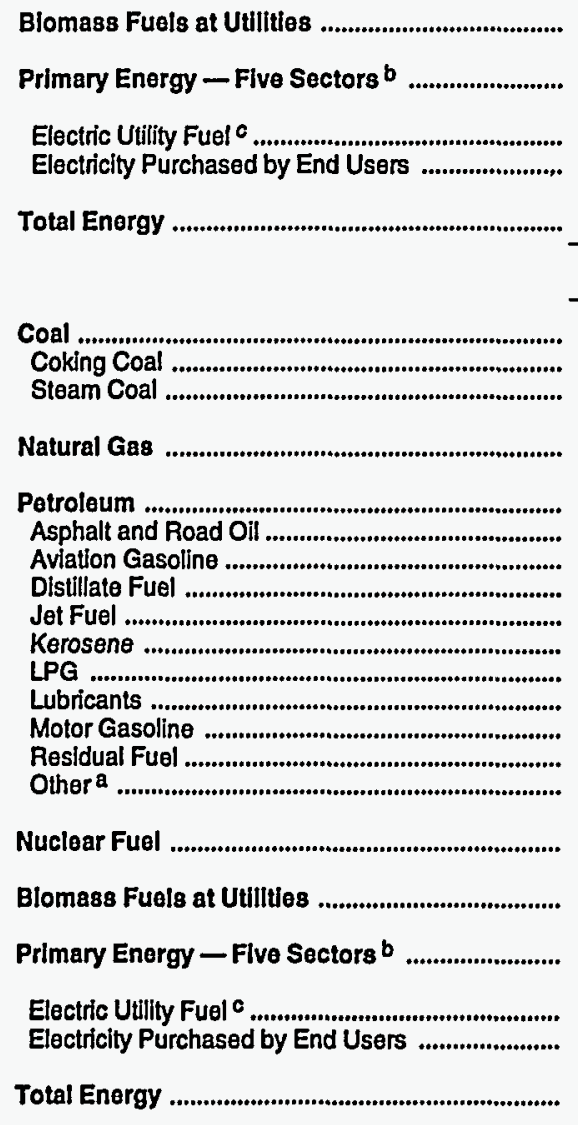

a Includes petroleum coke used at electric utilities.

b Biomass fuels are not included, except those consumed at electric utilities and those added to motor gasoline.

c There are no direct fuel costs for hydroelectric, geothermal, wind, photovoltaic, or solar thermal energy.

$R=$ Rovised data.
-No consumption, including cases where adjustments were made. See explanation of adjustments in Section 6 of Appendix A.

Note: Expenditure totals may not equal sum of components due to independent rounding.

Sources: Data sources, estimation procedures, and assumptions are described in Appendix A. 
(Dollars per Million Btu)

$\mathbf{N}$

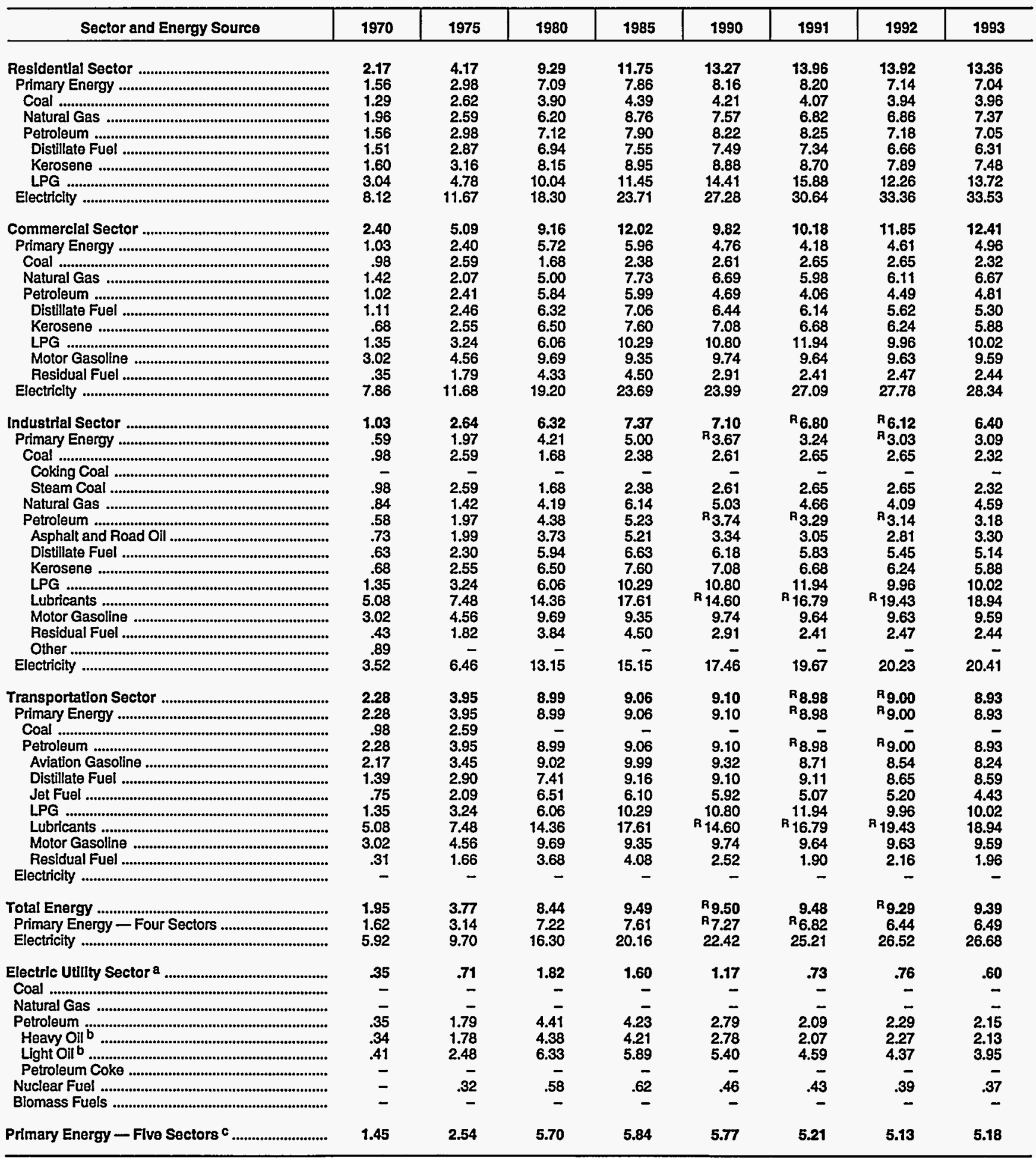

a There are no direct fuel costs for hydroelectric, geothermal, wind, photovoltalc, or solar thermal energy.

b Heavy oil includes fuel oll nos. 4, 5, and 6, and residual fuel oils. Light oil includes fuel oil nos. 1 and 2, kerosene, and jet fuel.

c Blomass fuels are not included, except those consumed at electric utilities and those added to motor gasoline.
$R=$ Revised data.

-No consumption, including cases where adjustments were made. See explanation of adjustments in Section 6 of Appendix A.

Sources: Data sources, estimation procedures, and assumptions are described in Appendix A. 
(Million Dollars)

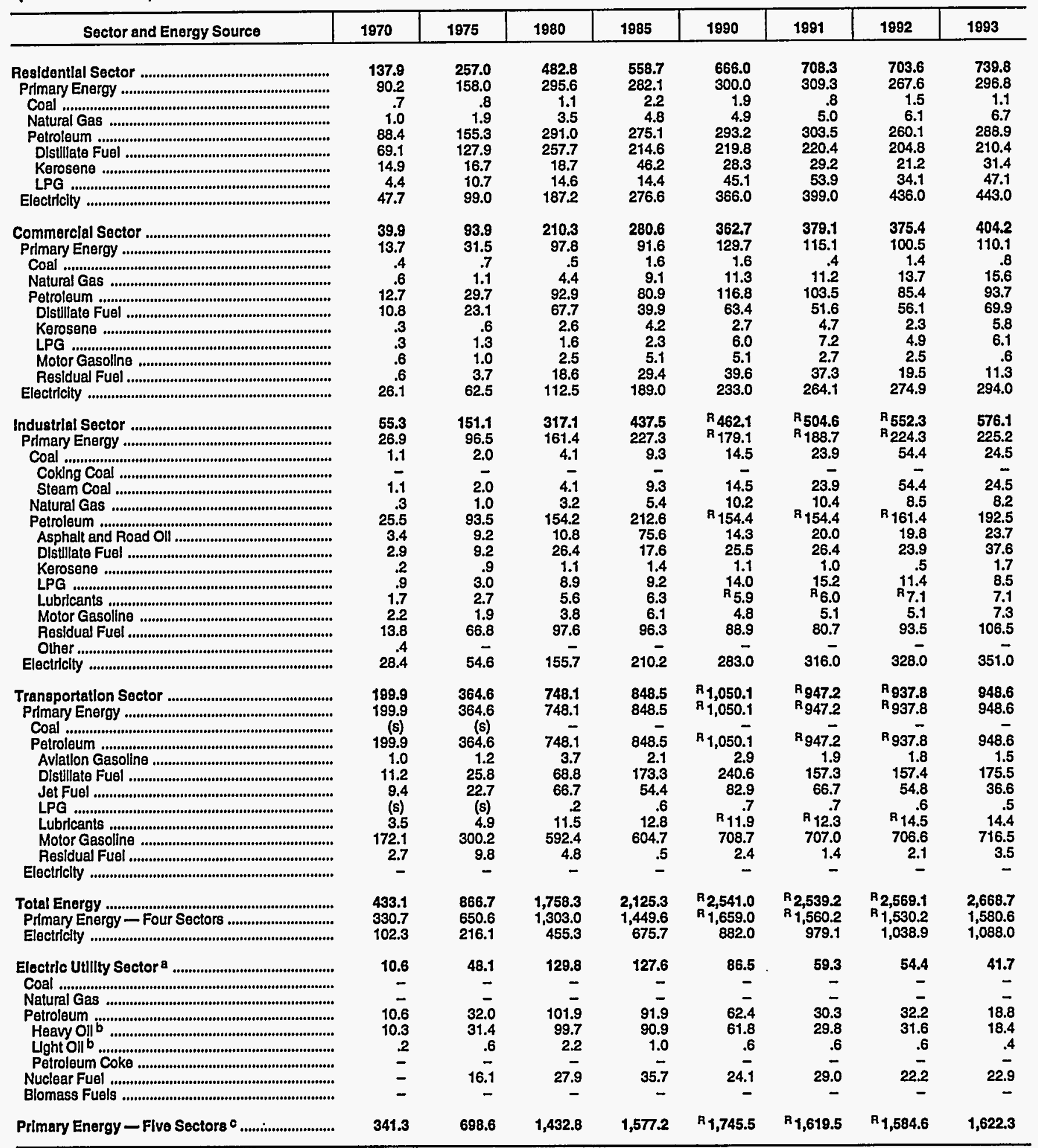

a There are no direct fuel costs for hydroelectric, geothermal, wind, photovoltalc, or solar thermal energy.

b Hoavy oll includes fuel oll nos. 4, 5, and 6, and residual fuel olls. Llght oll includes fuel nos. 1 and 2, kerosene, and jet fuel.

- Blomass fuels are not included, except those consumed at electric utllities and those added to motor gasoline.

$R=$ Revised data.
-No consumption, Including cases where adjustments were made. See explanation of adjustments in Section 6 of Appendix A.

(s) =Valuo less than 0.05 million dollars.

Note: Totals may not equal sum of components due to independent rounding.

Sources: Data sources, estimation procedures, and assumptions are described in Appendix A. 
M Energy Price and Expenditure Estimates by Source, Maryland

A 1970, 1975, 1980, 1985, and 1990-1993

\begin{tabular}{|c|c|c|c|c|c|c|c|c|}
\hline Energy Source & 1970 & 1975 & 1980 & 1985 & 1990 & 1991 & 1992 & 1993 \\
\hline & \multicolumn{8}{|c|}{ Prices in Dollars per Million Btu } \\
\hline $\begin{array}{l}\text { Coal } \\
\text { Coking Coal } \\
\text { Steam Coal }\end{array}$ & $\begin{array}{r}0.45 \\
.58 \\
.34\end{array}$ & $\begin{array}{l}1.69 \\
2.14 \\
1.28\end{array}$ & $\begin{array}{l}1.77 \\
2.38 \\
1.50\end{array}$ & $\begin{array}{l}1.75 \\
1.88 \\
1.71\end{array}$ & $\begin{array}{l}1.62 \\
1.71 \\
1.61\end{array}$ & $\begin{array}{l}1.62 \\
1.75 \\
1.61\end{array}$ & $\begin{array}{r}1.58 \\
1.58\end{array}$ & $\begin{array}{l}1.58 \\
1.73 \\
1.58\end{array}$ \\
\hline Natural Gas & 1.07 & 1.94 & 3.81 & 6.29 & 5.07 & 4.69 & 4.97 & 6.44 \\
\hline $\begin{array}{l}\text { Petroleum } \\
\text { Asphalt and Road Oil } \\
\text { Aviation Gasollne } \\
\text { Distillate Fuel } \\
\text { Jet Fuel } \\
\text { Kerosene } \\
\text { LPG }\end{array}$ & $\begin{array}{r}1.62 \\
.60 \\
2.17 \\
1.20 \\
.73 \\
1.46 \\
1.87 \\
5.08 \\
2.85 \\
.43 \\
1.53\end{array}$ & $\begin{array}{l}3.30 \\
1.89 \\
3.45 \\
2.61 \\
2.04 \\
3.24 \\
3.71 \\
7.48 \\
4.86 \\
1.87 \\
2.65\end{array}$ & $\begin{array}{r}7.66 \\
3.73 \\
9.02 \\
6.87 \\
6.46 \\
7.88 \\
6.81 \\
14.36 \\
9.93 \\
4.04 \\
8.91\end{array}$ & $\begin{array}{r}8.13 \\
4.95 \\
9.99 \\
7.83 \\
5.80 \\
8.63 \\
11.26 \\
17.61 \\
9.50 \\
4.06 \\
7.31\end{array}$ & $\begin{array}{r}R 7.92 \\
2.97 \\
9.32 \\
8.07 \\
5.47 \\
8.65 \\
11.58 \\
R_{14.60} \\
9.88 \\
3.03 \\
R_{6.22}\end{array}$ & $\begin{array}{r}\mathrm{R}_{7.73} \\
2.89 \\
8.71 \\
7.64 \\
4.78 \\
8.35 \\
12.52 \\
\mathrm{R}_{16.79} \\
9.56 \\
2.16 \\
\mathrm{R}_{6.35}\end{array}$ & 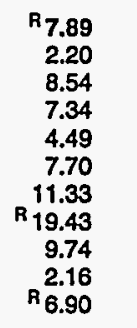 & $\begin{array}{r}7.53 \\
3.11 \\
8.24 \\
7.30 \\
4.16 \\
7.54 \\
11.51 \\
18.94 \\
9.39 \\
2.21 \\
6.66\end{array}$ \\
\hline 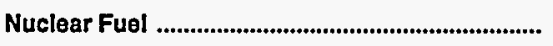 & - & .23 & .44 & .59 & .61 & .50 & .45 & .53 \\
\hline 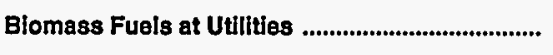 & - & - & - & .79 & - & - & - & - \\
\hline Primary Energy - Flve Sectors b ........................... & 1.17 & 2.62 & 4.92 & 5.39 & ${ }^{8} 5.46$ & $R_{4.93}$ & $R_{5.06}$ & 4.93 \\
\hline Electric Utility Fuel c & $\begin{array}{r}.40 \\
5.76\end{array}$ & $\begin{array}{r}1.36 \\
11.19\end{array}$ & $\begin{array}{r}1.66 \\
15.47\end{array}$ & $\begin{array}{r}1.65 \\
18.60\end{array}$ & $\begin{array}{r}1.88 \\
18.47\end{array}$ & $\begin{array}{r}1.46 \\
19.97\end{array}$ & $\begin{array}{r}1.35 \\
19.93\end{array}$ & $\begin{array}{r}1.38 \\
20.40\end{array}$ \\
\hline \multirow[t]{2}{*}{ 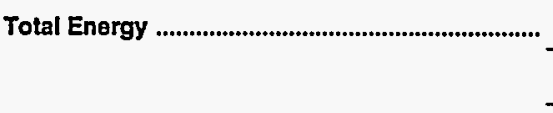 } & 1.79 & 4.02 & 7.70 & 9.10 & ${ }^{\mathrm{R}} 9.36$ & R 9.68 & ${ }^{\mathrm{R}} 9.92$ & 10.05 \\
\hline & \multicolumn{8}{|c|}{ Expenditures in Millions of Dollars } \\
\hline 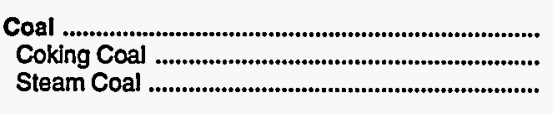 & $\begin{array}{r}139.6 \\
79.6 \\
60.0\end{array}$ & $\begin{array}{l}333.2 \\
200.6 \\
132.6\end{array}$ & $\begin{array}{l}416.8 \\
168.9 \\
247.9\end{array}$ & $\begin{array}{l}448.6 \\
107.4 \\
341.2\end{array}$ & $\begin{array}{r}463.9 \\
57.6 \\
406.3\end{array}$ & $\begin{array}{r}444.8 \\
46.6 \\
398.3\end{array}$ & $\begin{array}{l}390.0 \\
- \\
390.0\end{array}$ & $\begin{array}{r}412.6 \\
(s) \\
412.6\end{array}$ \\
\hline 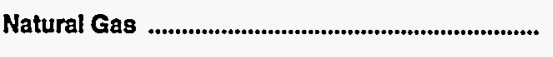 & 168.5 & 270.5 & 607.5 & 966.9 & 884.5 & 822.0 & 913.4 & 995.8 \\
\hline $\begin{array}{l}\text { Patroleum } \\
\text { Asphalt and Road OII } \\
\text { Aviatlon Gasoline } \\
\text { Distillate Fuel } \\
\text { Jet Fual } \\
\text { Kerosene } \\
\text { LPG }\end{array}$ & $\begin{array}{r}860.4 \\
11.1 \\
3.4 \\
138.3 \\
18.1 \\
19.3 \\
13.0 \\
19.2 \\
556.7 \\
58.7 \\
22.5\end{array}$ & $\begin{array}{r}1,942.2 \\
40.8 \\
3.6 \\
317.1 \\
34.6 \\
21.9 \\
33.0 \\
34.6 \\
1,115.0 \\
314.0 \\
27.6\end{array}$ & $\begin{array}{r}4,059.4 \\
65.3 \\
7.9 \\
872.6 \\
126.3 \\
52.2 \\
51.5 \\
63.1 \\
2,296.3 \\
415.8 \\
108.3\end{array}$ & $\begin{array}{r}3,877.0 \\
148.6 \\
3.8 \\
807.7 \\
125.7 \\
61.0 \\
73.3 \\
70.4 \\
2,275.7 \\
201.9 \\
108.9\end{array}$ & $\begin{array}{r}R_{3,962.6} \\
98.7 \\
3.5 \\
799.5 \\
110.9 \\
22.9 \\
82.4 \\
R_{65.7} \\
2,446.9 \\
188.4 \\
R_{143.8}\end{array}$ & $\begin{array}{r}{ }^{8} 3,811.9 \\
71.0 \\
3.3 \\
770.9 \\
87.8 \\
22.5 \\
91.3 \\
R_{67.6} \\
2,433.3 \\
127.0 \\
R_{137.1}\end{array}$ & $\begin{array}{r}R_{3,916.1} \\
51.2 \\
4.1 \\
784.4 \\
76.8 \\
16.5 \\
108.2 \\
\text { R } 79.7 \\
2,509.4 \\
106.3 \\
R_{179.6}\end{array}$ & $\begin{array}{r}3,951.3 \\
96.7 \\
4.2 \\
838.2 \\
69.9 \\
26.6 \\
102.9 \\
79.1 \\
2,445.5 \\
134.8 \\
153.3\end{array}$ \\
\hline 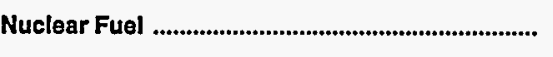 & - & 11.3 & 52.5 & 62.9 & 8.2 & 48.2 & 51.8 & 69.6 \\
\hline 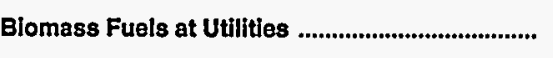 & - & - & - & .1 & - & - & - & - \\
\hline Primary Energy - Five Sectors ${ }^{b}$............................... & $1,168.5$ & $2,557.3$ & $5,136.2$ & $5,355.5$ & $\mathrm{R}_{5,319.2}$ & ${ }^{R_{5,127.0}}$ & $R_{5,271.3}$ & $5,429.2$ \\
\hline 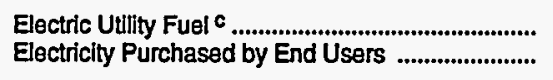 & $\begin{array}{r}-91.0 \\
442.4\end{array}$ & $\begin{array}{r}-352.5 \\
1,042.3\end{array}$ & $\begin{array}{r}-544.9 \\
1,825.5\end{array}$ & $\begin{array}{r}-536.2 \\
2,496.0\end{array}$ & $\begin{array}{r}-568.0 \\
3,122.0\end{array}$ & $\begin{array}{r}-558.0 \\
3,483.1\end{array}$ & $\begin{array}{r}-524.0 \\
3,468.0\end{array}$ & $\begin{array}{r}-594.9 \\
3,749.0\end{array}$ \\
\hline Cotal Energy & $1,519.9$ & $3,247.1$ & $6,416.7$ & 7,315.3 & $\mathrm{R}_{7,873.2}$ & ${ }^{R_{8,052.1}}$ & ${ }^{R} 8,215,3$ & $8,583.3$ \\
\hline
\end{tabular}

a Includes petroleum coke used at electric utilities.

b Biomass fuels are not included, except those consumed at electric utilities and those added to motor gasoline.

c There are no direct fuel costs for hydroelectric, geothermal, wind, photovoltalc, or solar thermal energy.

$R=$ Revised data.

-No consumption, including cases where adjustments were made. See

explanation of adjustments in Section 6 of Appendix A.

(s)=Value less than 0.05 million dollars.

Note: Expenditure totals may not equal sum of components due to independent rounding.

Sources: Data sources, estimation procedures, and assumptions are described in Appendlx A. 


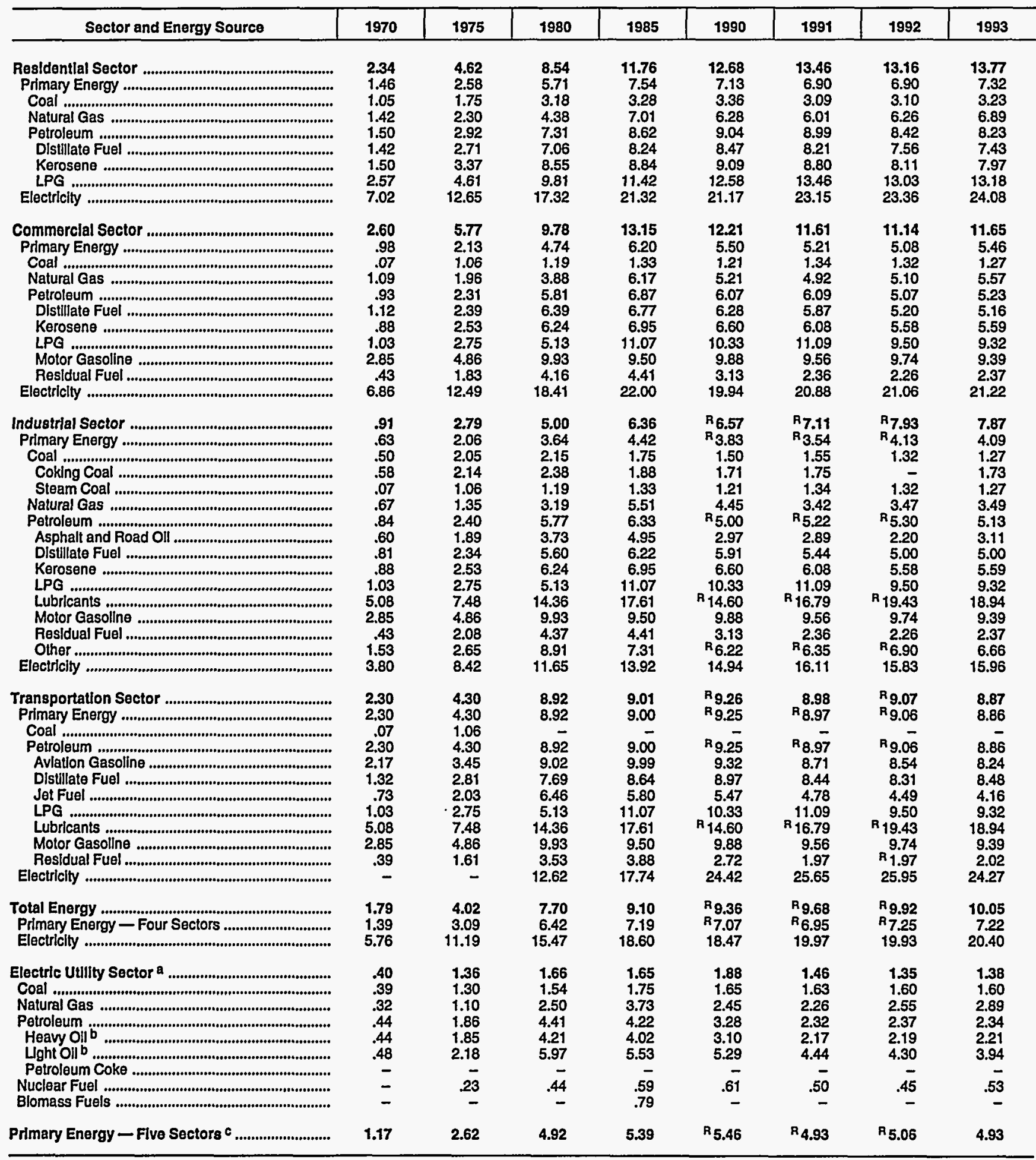

There are no direct fuel costs for hydroelectric, geothermal, wind, photovoltalc, or solar thermal energy.

Heavy oll includes fuel oil nos. 4, 5, and 6, and residual fuel olls. Light oll includes fuel oll nos. 1 and 2 , kerosene, and jet fuel.

c Blomass fuels are not Included, except those consumed at electric utilitites and those added to motor gasoline.
$R=$ Revised data.

- No consumption, including cases where adjustments were made. See explanation of adjustments in Section 6 of Appendix A.

Sources: Data sources, estimation procedures, and assumptions are describod in Appendix A. 
M Energy Expenditure Estimates by Sector, Maryland

A 1970, 1975, 1980, 1985, and 1990-1993

$\mathbf{R}$ (Million Dollars)

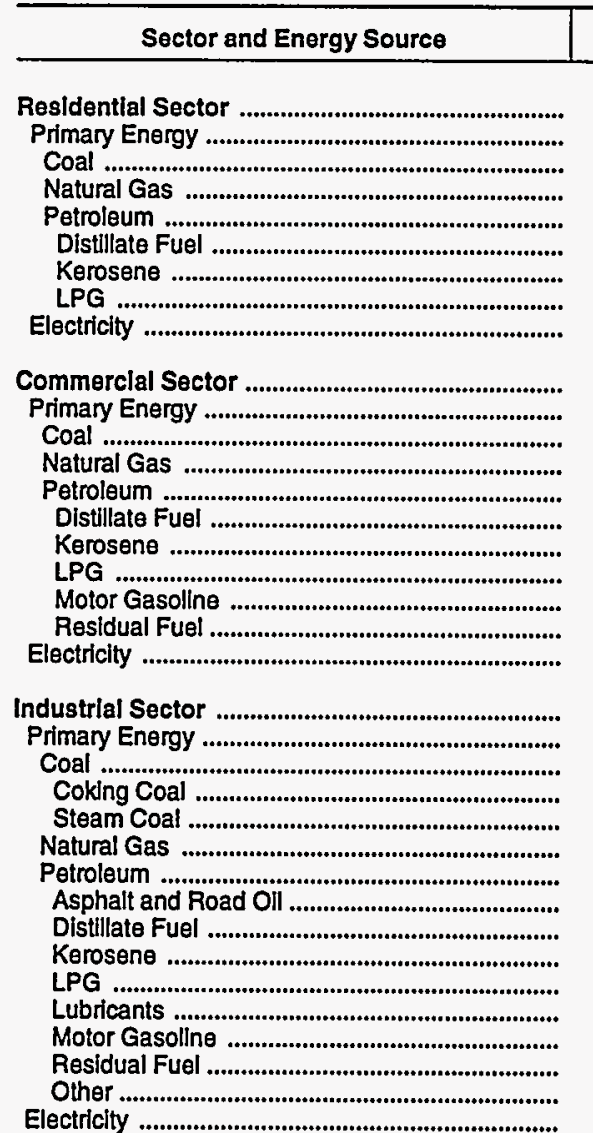

Transportation Sector

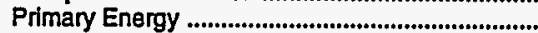
Coal

Petroleum ...................

Aviation Gasoling Distillate Fuel

Jet Fuel

Lubricants Motor Gasoline

Residual Fuel

Electricity

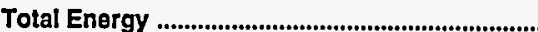

Primary Energy - Four Sectors ...............................

Electricity

Electric Utility Sector a .........................................

Coal

Natural Gas

Petroleum

Heavy Oil $b$

Uight OIl ${ }^{b}$

Nuclear Fuel ....
Blomass Fuels.

Primary Energy - Five Sectors $c$

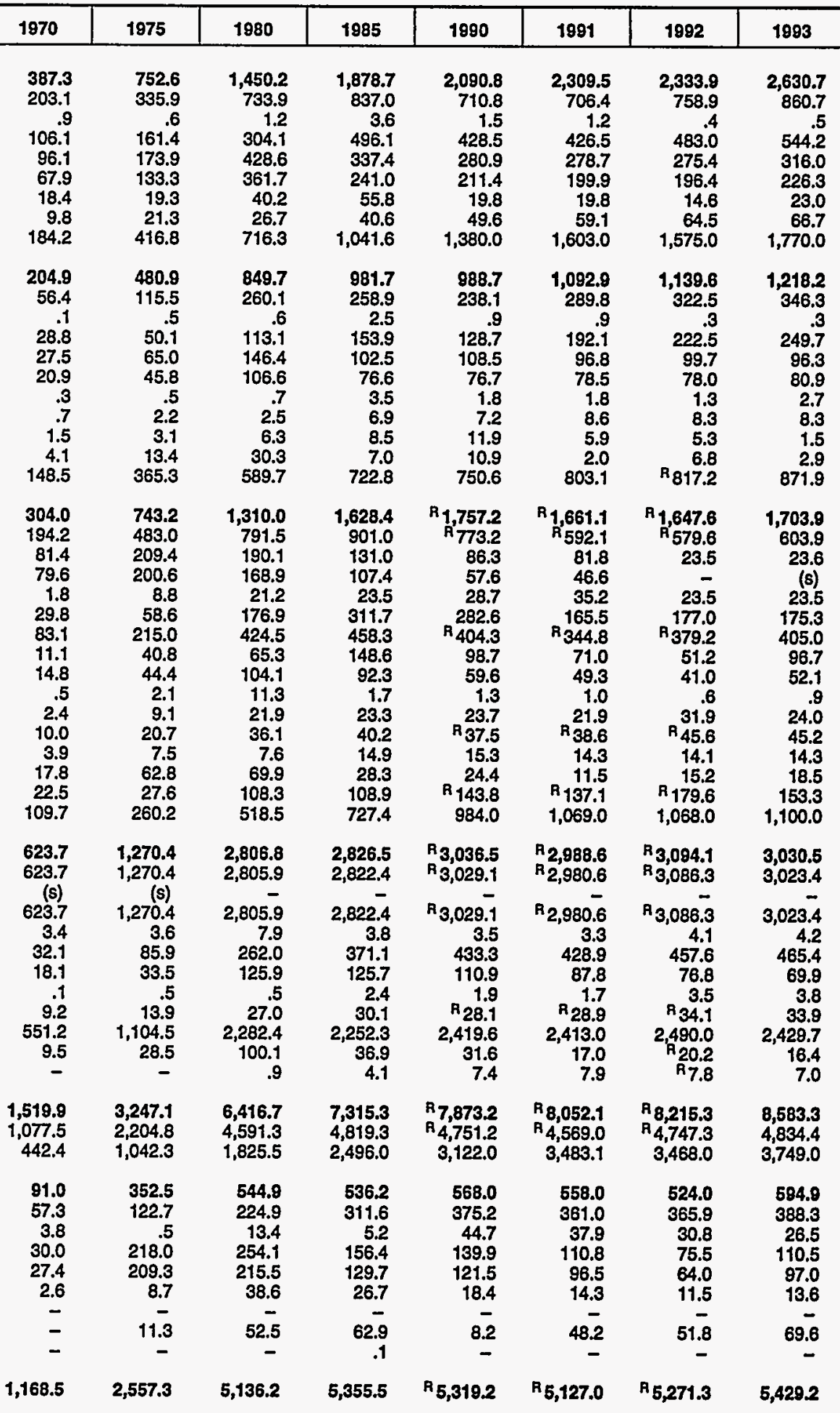

-No consumptlon, including cases where adjustments were made. See explanation of adjustments in Section 6 of Appendix A.

$(s)=$ Value less than 0.05 million dollars.

Note: Totals may not equal sum of components due to independent rounding.

Sources: Data sources, estlmation procedures, and assumptions are described in Appendix A.
There are no direct fuel costs for hydroelectric, geothermal, wind

b Heavy oll includes fuel oll nos.

oil includes fuel nos. 1 and 2 , kerosene, and jet fuel.

c Blomass fuels are not included, except those consumed at electric utilities and those added to motor gasoline.

$R=$ Revised data. 


\begin{tabular}{|c|c|c|c|c|c|c|c|c|}
\hline Energy Source & 1970 & 1975 & 1980 & 1985 & 1990 & 1991 & 1892 & 1993 \\
\hline & \multicolumn{8}{|c|}{ Prices in Dollars per Million Btu } \\
\hline 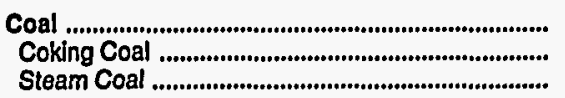 & $\overrightarrow{.55}$ & $\begin{array}{l}1.57 \\
1.57\end{array}$ & 2.03 & $\begin{array}{r}2.03 \\
2.03\end{array}$ & $\frac{1.77}{1.77}$ & $\frac{1.75}{1.75}$ & $\frac{1.74}{1.74}$ & $\begin{array}{r}1.71 \\
1.71\end{array}$ \\
\hline 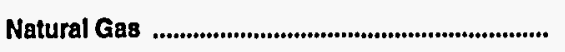 & 1.58 & 2.86 & 4.88 & 6.25 & 5.55 & 5.66 & 5.66 & 6.10 \\
\hline 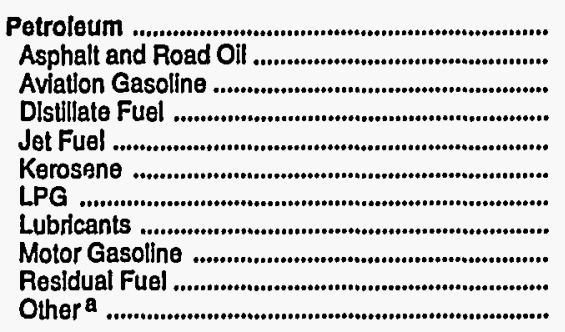 & $\begin{array}{r}1.24 \\
.63 \\
2.17 \\
1.34 \\
.75 \\
1.36 \\
2.31 \\
5.08 \\
2.86 \\
.39 \\
1.86\end{array}$ & $\begin{array}{l}2.95 \\
1.95 \\
3.45 \\
2.74 \\
2.10 \\
2.99 \\
3.74 \\
7.48 \\
4.73 \\
1.95 \\
3.28\end{array}$ & $\begin{array}{r}6.60 \\
3.73 \\
9.02 \\
6.87 \\
6.51 \\
7.06 \\
6.62 \\
14.36 \\
9.69 \\
3.84 \\
8.66\end{array}$ & $\begin{array}{r}7.28 \\
5.21 \\
9.99 \\
8.05 \\
6.04 \\
8.65 \\
11.77 \\
17.61 \\
9.18 \\
4.04 \\
9.25\end{array}$ & $\begin{array}{r}7.13 \\
3.36 \\
9.32 \\
7.99 \\
5.83 \\
8.41 \\
12.12 \\
14.60 \\
9.53 \\
2.88 \\
\text { R } \\
7.46\end{array}$ & $\begin{array}{r}6.97 \\
3.05 \\
8.71 \\
7.53 \\
5.00 \\
7.17 \\
7.77 \\
13.73 \\
R \quad 16.79 \\
10.01 \\
2.21 \\
R 6.91\end{array}$ & $\begin{array}{r}\mathrm{R}_{6.94} \\
2.79 \\
8.54 \\
6.93 \\
4.63 \\
7.03 \\
711.48 \\
\mathrm{R} \\
\mathrm{1} \\
\mathrm{9} .43 \\
9.83 \\
2.33 \\
\mathrm{R} 7.58\end{array}$ & $\begin{array}{r}7.04 \\
3.30 \\
8.24 \\
6.84 \\
4.36 \\
6.84 \\
11.49 \\
18.94 \\
9.73 \\
2.57 \\
6.79\end{array}$ \\
\hline 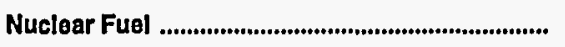 & .20 & .18 & .41 & .60 & .62 & .61 & .50 & .49 \\
\hline Blomass Fuels at Utilitles .............................................. & - & - & - & - & - & - & - & - \\
\hline Primary Energy — Flve Sectors b ............................ & 1.25 & 2.83 & 6.04 & 6.22 & $\mathrm{R}_{6.00}$ & R $_{5.92}$ & $\mathbf{R}_{5.88}$ & 6.10 \\
\hline Electric Utility Fuel c & $\begin{array}{r}.37 \\
7.29\end{array}$ & $\begin{array}{r}1.66 \\
13.93\end{array}$ & $\begin{array}{r}3.41 \\
21.13\end{array}$ & $\begin{array}{r}2.74 \\
24.34\end{array}$ & $\begin{array}{r}2.15 \\
25.93\end{array}$ & $\begin{array}{r}1.85 \\
27.94\end{array}$ & $\begin{array}{r}1.90 \\
28.31\end{array}$ & $\begin{array}{r}1.97 \\
29.24\end{array}$ \\
\hline \multirow[t]{2}{*}{ 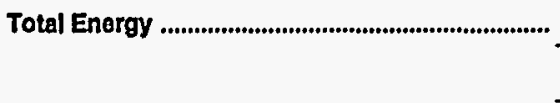 } & 1.90 & 4.15 & 8.90 & 10.09 & $\mathrm{R}_{10.53}$ & $R_{10.82}$ & 10.46 & 10.52 \\
\hline & \multicolumn{8}{|c|}{ Expenditures in Millions of Dollars } \\
\hline $\begin{array}{l}\text { Coal } \\
\text { Coking Coal } \\
\text { Steam Coal }\end{array}$ & $\begin{array}{l}11.7 \\
11.7\end{array}$ & $\begin{array}{c}38.5 \\
38.5\end{array}$ & $\frac{46.4}{46.4}$ & 223.8 & $\begin{array}{l}200.4 \\
-\overline{-} \\
200.4\end{array}$ & $\begin{array}{l}204.7 \\
204.7\end{array}$ & $\begin{array}{c}193.2 \\
19 \overline{3} .2\end{array}$ & $\begin{array}{r}168.5 \\
168.5\end{array}$ \\
\hline 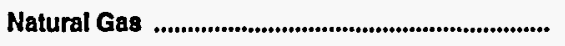 & 234.1 & 441.3 & 901.9 & $1,395.1$ & $1,479.7$ & $1,470.6$ & $1,720.9$ & $1,964.2$ \\
\hline $\begin{array}{l}\text { Petroleum } \\
\text { Asphalt and Road Oil } \\
\text { Avlatlon Gasoline } \\
\text { Distillate Fuel } \\
\text { Jet Fuel }\end{array}$ Kerosene & \begin{tabular}{r|}
$1,537.4$ \\
12.0 \\
3.0 \\
461.9 \\
33.3 \\
16.3 \\
15.9 \\
29.2 \\
743.8 \\
210.9 \\
11.2
\end{tabular} & $\begin{array}{r}3,325.6 \\
23.7 \\
4.0 \\
934.7 \\
95.0 \\
14.7 \\
32.2 \\
35.7 \\
1,357.3 \\
808.9 \\
19.6\end{array}$ & $\begin{array}{r}6,042.8 \\
30.5 \\
12.5 \\
1,504.7 \\
315.8 \\
27.9 \\
51.7 \\
73.2 \\
2,619.1 \\
1,306.9 \\
100.5\end{array}$ & $\begin{array}{r}5,682.3 \\
36.4 \\
6.8 \\
1,550.3 \\
238.4 \\
36.2 \\
72.9 \\
81.7 \\
2,643.9 \\
915.4 \\
100.5\end{array}$ & $\begin{array}{r}\mathrm{R}_{5,585.0} \\
29.9 \\
4.5 \\
1,567.8 \\
323.3 \\
14.7 \\
115.6 \\
\mathrm{R} 76.2 \\
2,789.2 \\
581.2 \\
\mathrm{R} 82.5\end{array}$ & $\begin{array}{r}\mathrm{R}_{5,315.5} \\
40.0 \\
2.0 \\
1,456.0 \\
264.2 \\
15.0 \\
95.2 \\
R_{78.4} \\
2,864.3 \\
424.7 \\
\mathrm{R}_{75.7}\end{array}$ & $\begin{array}{r}{ }^{R} 5,196.4 \\
29.0 \\
1.9 \\
1,419.2 \\
206.1 \\
16.9 \\
77.8 \\
R 92.6 \\
2,864.4 \\
401.8 \\
R_{86.7}\end{array}$ & $\begin{array}{r}5,215.9 \\
31.8 \\
3.5 \\
1,459.5 \\
190.6 \\
14.7 \\
87.1 \\
91.9 \\
2,864.8 \\
393.8 \\
78.1\end{array}$ \\
\hline 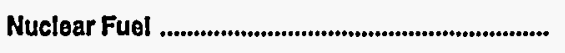 & 2.7. & 7.5 & 14.3 & 39.8 & 33.6 & 29.0 & 25.5 & 22.7 \\
\hline 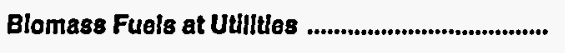 & - & - & - & - & - & - & - & - \\
\hline Primary Energy — Flue Sectors b ........................... & $1,785.8$ & $3,812.9$ & $7,005.4$ & $7,341.1$ & $\mathrm{R}_{7,298.6}$ & $\mathrm{~A}_{7,019.7}$ & $\mathrm{R}_{7,136.1}$ & $7,371.3$ \\
\hline 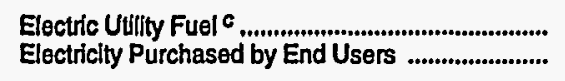 & $\begin{array}{r}-112.4 \\
612.9\end{array}$ & $\begin{array}{r}-524.9 \\
1,401.0\end{array}$ & $\begin{array}{r}-1,191.4 \\
2,398.4\end{array}$ & $\begin{array}{r}-1,011.3 \\
3,166.2\end{array}$ & $\begin{array}{r}-801.1 \\
4,021.1\end{array}$ & $\begin{array}{r}-661.1 \\
4,271.0\end{array}$ & $\begin{array}{r}-628.3 \\
4,347.0\end{array}$ & $\begin{array}{r}-562.5 \\
4,518.0\end{array}$ \\
\hline 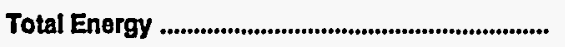 & $2,286.3$ & $4,689.0$ & $8,212.4$ & $9,496.0$ & $R_{10,518.6}$ & $R_{10,629.7}$ & $A_{10,854.7}$ & $11,326.8$ \\
\hline
\end{tabular}

a Includes petroleum coke used at electric utilities.

biomass fuels are not included, except those consumed at electric uttilites and those added to motor gasoline.

c There are no direct fuel costs for hydroelectric, goothermal, wind, photovoltalc, or solar themal energy.

-No consumption, including cases where adjustments were made. Se日 explanation of adjustments in Section 6 of Appendix A.

Note: Expenditure totals may not equal sum of components due to independent rounding.

Sources: Data sources, estimation procedures, and assumptions are $R=$ Revised data described in Appendix A. 
M Energy Price Estimates by Sector, Massachusetts

A $1970,1975,1980,1985$, and 1990-1993

$\mathbf{S}$ (Dollars per Million Btu)

S
$A$
$C$
$H$
$U$
$S$
$E$
$T$
$T$
$S$

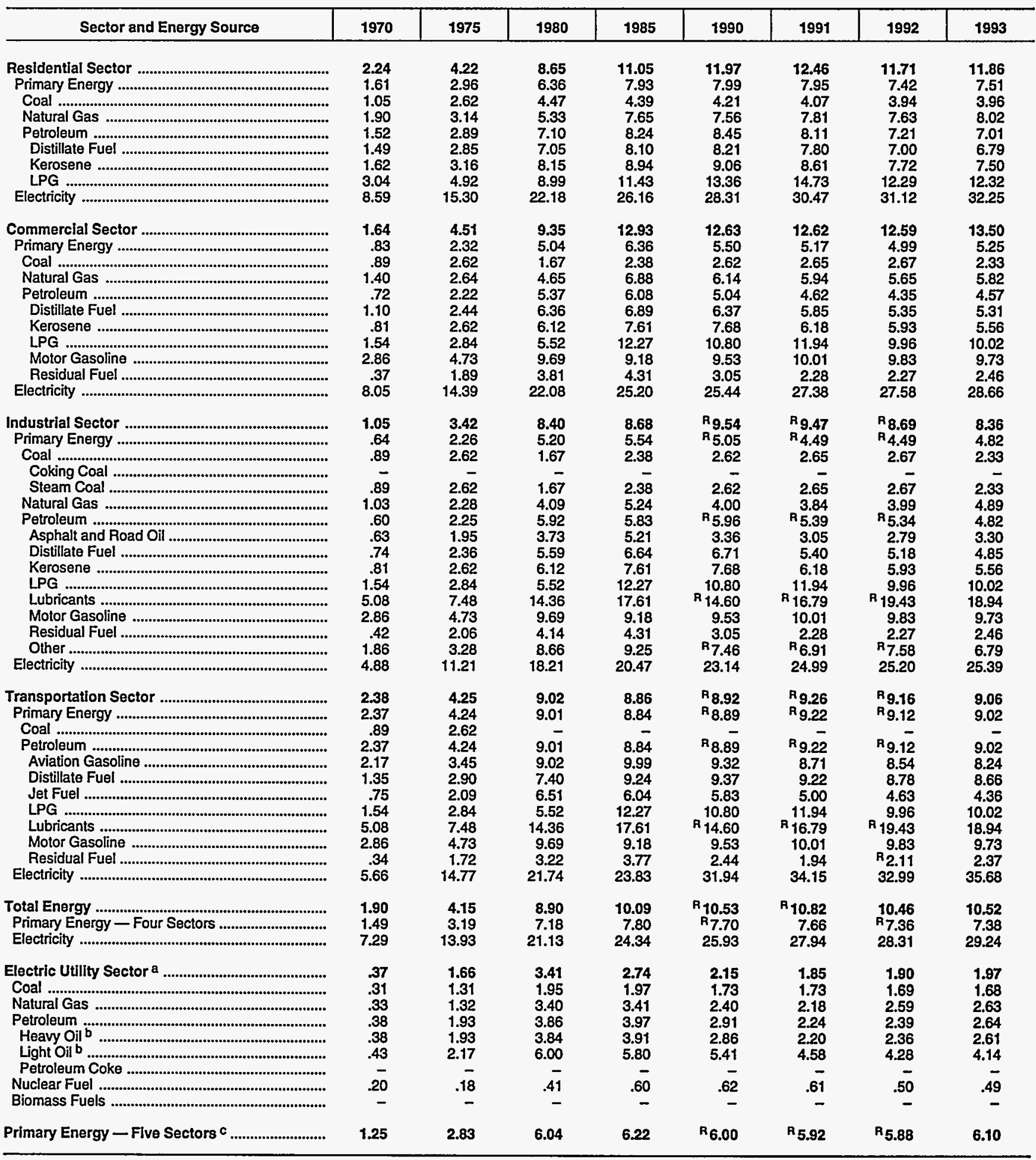

a There are no direct fuel costs for hydroelectric, geothermal, wind, photovoltaic, or solar thermal energy.

beavy oil includes fuel oil nos. 4, 5, and 6, and residual fuel oils. Light oil includes fuel oil nos. 1 and 2, kerosene, and jet fuel.

c Biomass fuels are not included, except those consumed at electric utilities and those added to motor gasoline.

R=Revised data.

$\rightarrow$ No consumption, including cases where adjustments were made. See explanation of adjustments in Section 6 of Appendix A.

Sources: Data sources, estimation procedures, and assumptions are described in Appendix A. 


\begin{tabular}{|c|c|c|c|c|c|c|c|c|}
\hline Sector and Energy Source & 1970 & 1975 & 1980 & 1985 & 1990 & 1991 & 1992 & 1993 \\
\hline 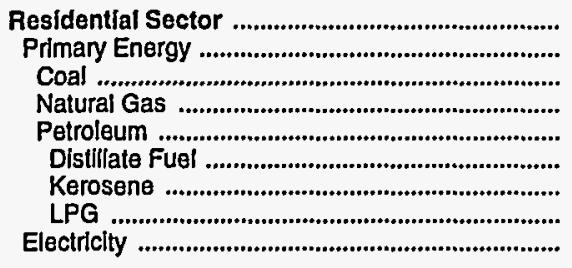 & $\begin{array}{r}793.8 \\
520.1 \\
2.6 \\
158.6 \\
358.9 \\
334.9 \\
13.2 \\
10.7 \\
273.7\end{array}$ & $\begin{array}{r}1,501.2 \\
945.4 \\
3.3 \\
284.4 \\
657.7 \\
628.7 \\
10.6 \\
18.4 \\
555.7\end{array}$ & $\begin{array}{r}2,362.9 \\
1,487.2 \\
5.2 \\
511.9 \\
970.1 \\
932.9 \\
14.9 \\
22.3 \\
875.7\end{array}$ & $\begin{array}{r}2,844.0 \\
1,692.1 \\
7.2 \\
765.7 \\
919.2 \\
847.8 \\
29.3 \\
42.1 \\
1,151.9\end{array}$ & $\begin{array}{r}3,243.9 \\
1,738.9 \\
3.1 \\
835.2 \\
900.6 \\
826.4 \\
8.4 \\
65.8 \\
1,505.0\end{array}$ & $\begin{array}{r}3,264.1 \\
1,665.1 \\
1.5 \\
835.0 \\
828.6 \\
755.8 \\
7.4 \\
65.4 \\
1,599.0\end{array}$ & $\begin{array}{r}3,434.4 \\
1,782.4 \\
2.4 \\
947.8 \\
832.2 \\
766.6 \\
11.4 \\
54.3 \\
1,652.0\end{array}$ & $\begin{array}{r}3,631.3 \\
1,894.3 \\
2.1 \\
1,009.8 \\
882.3 \\
812.0 \\
10.6 \\
59.7 \\
1,737.0\end{array}$ \\
\hline 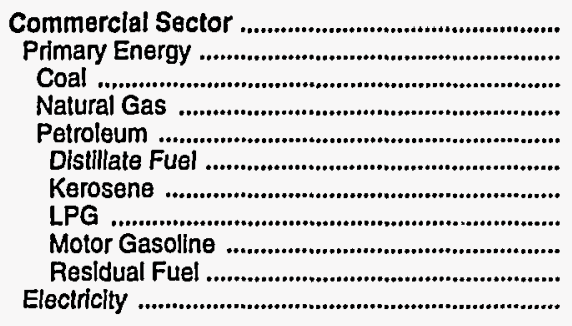 & $\begin{array}{r}390.4 \\
176.2 \\
1.7 \\
50.1 \\
124.4 \\
86.4 \\
.5 \\
1.0 \\
1.5 \\
35.0 \\
214.2\end{array}$ & $\begin{array}{r}964.2 \\
404.6 \\
2.6 \\
100.1 \\
301.8 \\
187.9 \\
.7 \\
1.9 \\
2.7 \\
108.6 \\
559.6\end{array}$ & $\begin{array}{r}1,645.4 \\
662.0 \\
2.0 \\
252.5 \\
407.5 \\
278.0 \\
1.0 \\
2.4 \\
9.7 \\
116.3 \\
983.4\end{array}$ & $\begin{array}{r}1,971.0 \\
631.5 \\
3.9 \\
291.5 \\
336.2 \\
228.9 \\
4.6 \\
8.0 \\
9.1 \\
85.6 \\
1,339.5\end{array}$ & $\begin{array}{r}2,357.0 \\
660.3 \\
2.2 \\
321.4 \\
336.6 \\
231.3 \\
5.5 \\
9.4 \\
3.4 \\
87.0 \\
1,696.7\end{array}$ & $\begin{array}{r}2,496.4 \\
679.7 \\
.8 \\
328.2 \\
350.8 \\
259.4 \\
7.0 \\
9.4 \\
9.6 \\
65.4 \\
1,816.7\end{array}$ & $\begin{array}{r}R_{2,502.7} \\
659.0 \\
2.2 \\
377.1 \\
279.7 \\
208.1 \\
2.4 \\
7.8 \\
8.5 \\
52.9 \\
R_{1,843.7}\end{array}$ & $\begin{array}{r}2,575.4 \\
647.5 \\
1.2 \\
395.2 \\
251.1 \\
196.1 \\
3.6 \\
8.6 \\
2.7 \\
40.1 \\
1,927.9\end{array}$ \\
\hline 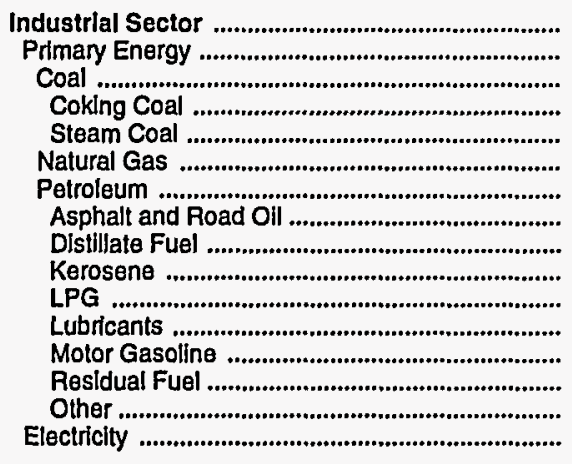 & $\begin{array}{r}277.7 \\
154.3 \\
3.2 \\
- \\
3.2 \\
23.5 \\
127.6 \\
12.0 \\
12.5 \\
2.5 \\
4.0 \\
15.6 \\
1.7 \\
68.1 \\
11.2 \\
123.4\end{array}$ & $\begin{array}{r}660.3 \\
380.0 \\
6.9 \\
- \\
6.9 \\
55.0 \\
318.1 \\
23.7 \\
36.5 \\
3.4 \\
11.6 \\
16.0 \\
2.0 \\
205.3 \\
19.6 \\
280.3\end{array}$ & $\begin{array}{r}989.3 \\
461.9 \\
4.0 \\
- \\
4.0 \\
120.2 \\
337.7 \\
30.5 \\
61.5 \\
12.0 \\
26.5 \\
32.9 \\
4.6 \\
69.3 \\
100.5 \\
527.3\end{array}$ & $\begin{array}{r}1,330.1 \\
669.7 \\
10.4 \\
10.4 \\
177.8 \\
481.4 \\
36.4 \\
40.4 \\
2.3 \\
19.8 \\
36.7 \\
17.7 \\
227.8 \\
100.5 \\
660.4\end{array}$ & $\begin{array}{r}R_{1,332.0} \\
R_{530.0} \\
4.8 \\
- \\
4.8 \\
183.5 \\
R_{341.8} \\
29.9 \\
85.0 \\
.8 \\
38.1 \\
R 34.2 \\
20.6 \\
50.6 \\
R 82.5 \\
802.0\end{array}$ & $\begin{array}{r}R_{1,303.2} \\
R_{468.2} \\
5.6 \\
- \\
5.6 \\
218.5 \\
R_{244.1} \\
40.0 \\
37.6 \\
.6 \\
17.4 \\
R_{35.2} \\
17.4 \\
20.2 \\
R 75.7 \\
835.0\end{array}$ & $\begin{array}{r}R_{1,412.7} \\
R_{581.7} \\
10.3 \\
- \\
10.3 \\
293.4 \\
R_{278.0} \\
29.0 \\
55.9 \\
3.1 \\
13.4 \\
R^{2} \\
11.5 \\
17.3 \\
31.0 \\
R_{86.7} \\
831.0\end{array}$ & $\begin{array}{r}1,591.1 \\
759.2 \\
6.7 \\
- \\
6.7 \\
480.9 \\
271.6 \\
31.8 \\
39.6 \\
.5 \\
16.6 \\
41.2 \\
8.9 \\
54.7 \\
78.1 \\
832.0\end{array}$ \\
\hline $\begin{array}{l}\text { Transportatlon Sector } \\
\text { Primary Energy } \\
\text { Coal } \\
\text { Petroleum } \\
\text { Avlation Gasoline } \\
\text { Distillate Fubl } \\
\text { Jet Fuel } \\
\text { LPG } \\
\text { Motor Gasoline } \\
\text { Residual Fuel } \\
\text { Electricity }\end{array}$ & $\begin{array}{r}824.4 \\
822.8 \\
(\mathrm{~s}) \\
82.8 \\
3.0 \\
25.2 \\
33.3 \\
.2 \\
13.6 \\
740.6 \\
7.0 \\
1.6\end{array}$ & $\begin{array}{r}1,563.4 \\
1,558.0 \\
(s) \\
1,558.0 \\
4.0 \\
75.8 \\
94.4 \\
.3 \\
19.6 \\
1,352.5 \\
11.3 \\
5.4\end{array}$ & $\begin{array}{r}3,214.8 \\
3,202.9 \\
- \\
3,202.9 \\
12.5 \\
211.1 \\
315.5 \\
.5 \\
40.4 \\
2,604.7 \\
18.2 \\
11.9\end{array}$ & $\begin{array}{r}3,350.9 \\
3,336.5 \\
- \\
3,336.5 \\
6.8 \\
405.4 \\
238.4 \\
3.1 \\
45.0 \\
2,617.1 \\
20.7 \\
14.4\end{array}$ & $\begin{array}{r}R_{3,585.7} \\
R_{3,568.3} \\
- \\
R_{3,568.3} \\
4.5 \\
409.8 \\
323.3 \\
2.3 \\
R_{42.0} \\
2,765.1 \\
21.2 \\
17.4\end{array}$ & $\begin{array}{r}R_{3,565.9} \\
R_{3,545.6} \\
- \\
R_{3,545.6} \\
2.0 \\
390.5 \\
264.2 \\
3.0 \\
R_{43.2} \\
2,837.3 \\
5.4 \\
20.3\end{array}$ & $\begin{array}{r}R_{3,504.9} \\
R_{3,484.6} \\
- \\
R_{3,484.6} \\
1.9 \\
378.7 \\
206.1 \\
2.3 \\
R_{51.0} \\
2,838.7 \\
5.8 \\
R_{20.3}\end{array}$ & $\begin{array}{r}3,528.9 \\
3,507.8 \\
- \\
3,507.8 \\
3.5 \\
402.4 \\
190.6 \\
2.2 \\
50.6 \\
2,853.2 \\
5.2 \\
21.1\end{array}$ \\
\hline 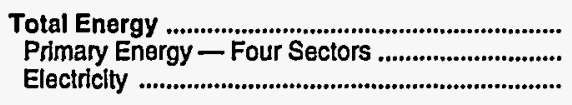 & $\begin{array}{r}2,286.3 \\
1,673.3 \\
612.9\end{array}$ & $\begin{array}{l}4,689.0 \\
3,288.0 \\
1,401.0\end{array}$ & $\begin{array}{l}8,212.4 \\
5,814.0 \\
2,398.4\end{array}$ & $\begin{array}{l}9,496.0 \\
6,329.8 \\
3,166.2\end{array}$ & $\begin{array}{r}R_{10,518.6} \\
R_{6,497.5} \\
4,021.1\end{array}$ & $\begin{array}{r}R_{10,629.7} \\
R_{6,358.7} \\
4,271.0\end{array}$ & $\begin{array}{r}R_{10,854.7} \\
R_{6,507.7} \\
4,347.0\end{array}$ & $\begin{array}{r}11,326.8 \\
6,808.8 \\
4,518.0\end{array}$ \\
\hline 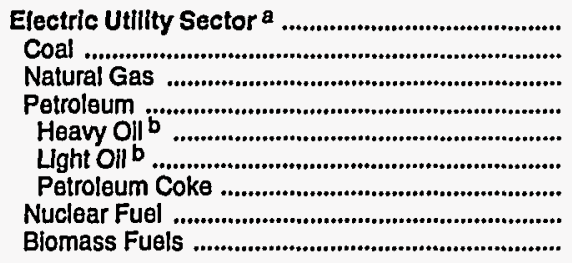 & $\begin{array}{r}112.4 \\
4.2 \\
1.9 \\
103.7 \\
100.8 \\
2.9 \\
-\overline{2.7} \\
-\end{array}$ & $\begin{array}{r}524.9 \\
25.6 \\
1.9 \\
490.0 \\
483.6 \\
6.3 \\
- \\
7.5 \\
-\end{array}$ & $\begin{array}{r}1,191.4 \\
35.2 \\
17.3 \\
1,124.6 \\
1,103.1 \\
21.5 \\
- \\
14.3 \\
-\end{array}$ & $\begin{array}{r}1,011.3 \\
202.4 \\
160.1 \\
609.0 \\
581.3 \\
27.8 \\
- \\
39.8 \\
-\end{array}$ & $\begin{array}{r}801.1 \\
190.3 \\
139.5 \\
437.7 \\
422.3 \\
15.4 \\
- \\
33.6 \\
-\end{array}$ & $\begin{array}{r}661.1 \\
196.7 \\
89.0 \\
346.4 \\
333.8 \\
12.6 \\
- \\
29.0 \\
-\end{array}$ & $\begin{array}{r}628.3 \\
178.3 \\
102.6 \\
321.9 \\
312.1 \\
9.8 \\
- \\
25.5 \\
-\end{array}$ & $\begin{array}{r}562.5 \\
158.5 \\
78.3 \\
303.1 \\
293.8 \\
9.3 \\
- \\
22.7 \\
-\end{array}$ \\
\hline Primary Energy - Five Sectors c ....................... & $1,785.8$ & $3,812.9$ & $7,005.4$ & $7,341.1$ & $R_{7,298.6}$ & $\mathrm{P}_{7,019.7}$ & $A_{7,136.1}$ & $7,371.3$ \\
\hline
\end{tabular}

a There are no direct fuel costs for hydroelectric, geothermal, wind, photovoltalc, or solar thermal energy.

b Heavy oll Includes fuel oil nos. 4, 5, and 6, and residual fuel oils. Light oll Includes fuel nos. 1 and 2 , kerosene, and jet fuel.

c Blomass fuels are not included, except those consumed at electric utllittes and those added to motor gasoline.

$R=$ Revised data.

-No consumption, including cases where adjustments were made. See explanation of adjustments in Section 6 of Appendix A.

$(s)=V a l u e$ less than 0.05 million dollars.

Note: Totals may not equal sum of components due to independent rounding.

Sources: Data sources, estimation procedures, and assumptions are described in Appendix A. 
M Energy Price and Expenditure Estimates by Source, Michigan 1970, 1975, 1980, 1985, and 1990-1993

$\mathbf{C}$
$\mathbf{H}$
$\mathbf{G}$
$\mathbf{A}$

\begin{tabular}{|c|c|c|c|c|c|c|c|c|}
\hline Energy Source & 1970 & 1975 & 1980 & 1985 & 1990 & 1991 & 1992 & 1993 \\
\hline & \multicolumn{8}{|c|}{ Prices in Dollars per Million Btu } \\
\hline Coal & $\begin{array}{r}0.44 \\
.55 \\
.42\end{array}$ & $\begin{array}{l}1.23 \\
2.07 \\
1.04\end{array}$ & $\begin{array}{l}1.71 \\
2.27 \\
1.62\end{array}$ & $\begin{array}{l}1.92 \\
2.08 \\
1.90\end{array}$ & $\begin{array}{l}1.63 \\
1.80 \\
1.63\end{array}$ & $\begin{array}{l}1.62 \\
1.72 \\
1.62\end{array}$ & $\frac{1.58}{1.58}$ & $\begin{array}{l}1.55 \\
1.68 \\
1.55\end{array}$ \\
\hline 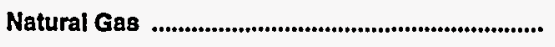 & .77 & 1.42 & 3.05 & 6.70 & 4.33 & 4.43 & 4.38 & 4.39 \\
\hline $\begin{array}{l}\text { Petroleum } \\
\text { Asphalt and Road OII } \\
\text { Aviation Gasoline } \\
\text { Distillate Fuel } \\
\text { Jet Fuel } \\
\text { Kerosene } \\
\text { LPG }\end{array}$ & $\begin{array}{r}2.00 \\
.84 \\
2.17 \\
1.09 \\
.74 \\
.89 \\
1.89 \\
5.08 \\
2.71 \\
.59 \\
1.25\end{array}$ & $\begin{array}{l}3.72 \\
2.12 \\
3.45 \\
2.49 \\
2.08 \\
2.60 \\
3.76 \\
7.48 \\
4.72 \\
1.96 \\
2.92\end{array}$ & $\begin{array}{r}8.48 \\
4.04 \\
9.02 \\
6.76 \\
6.38 \\
6.29 \\
6.62 \\
14.36 \\
10.09 \\
3.90 \\
7.80\end{array}$ & $\begin{array}{r}8.67 \\
5.00 \\
9.99 \\
7.71 \\
6.09 \\
8.52 \\
8.74 \\
17.61 \\
9.10 \\
4.45 \\
8.21\end{array}$ & $\begin{array}{r}{ }^{R} 8.24 \\
3.06 \\
9.32 \\
7.53 \\
5.65 \\
8.28 \\
10.26 \\
R_{14.60} \\
8.78 \\
3.00 \\
{ }^{8} 6.65\end{array}$ & \begin{tabular}{r|}
$R 7.89$ \\
2.87 \\
8.71 \\
6.98 \\
4.94 \\
7.60 \\
10.13 \\
$R \quad 16.79$ \\
8.37 \\
2.54 \\
$R 5.91$
\end{tabular} & $\begin{array}{r}\mathrm{R} 7.71 \\
2.25 \\
8.54 \\
6.84 \\
4.57 \\
7.20 \\
9.50 \\
\mathrm{R} \\
\mathbf{1 9 . 4 3} \\
8.16 \\
2.50 \\
\mathrm{R} 5.88\end{array}$ & $\begin{array}{r}7.7 \\
2.99 \\
8.24 \\
6.91 \\
4.26 \\
7.01 \\
9.62 \\
18.94 \\
8.35 \\
2.78 \\
5.40\end{array}$ \\
\hline 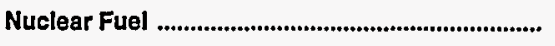 & .36 & .28 & .49 & .80 & .79 & .65 & .67 & .60 \\
\hline 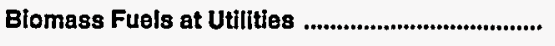 & - & - & - & - & - & - & - & - \\
\hline Primary Energy - Five Sectors ${ }^{b}$ & 1.12 & 2.23 & 4.39 & 5.18 & $\mathrm{R}_{4.50}$ & ${ }^{R} 4.37$ & ${ }^{A} 4.48$ & 4.40 \\
\hline $\begin{array}{l}\text { Electric Utility Fuel } \mathrm{c} \\
\text { Electricity Purchased by End Users }\end{array}$ & $\begin{array}{r}.38 \\
5.55\end{array}$ & $\begin{array}{l}1.02 \\
9.78\end{array}$ & $\begin{array}{r}1.58 \\
15.40\end{array}$ & $\begin{array}{r}1.72 \\
19.88\end{array}$ & $\begin{array}{r}1.41 \\
20.85\end{array}$ & $\begin{array}{r}1.32 \\
21.18\end{array}$ & $\begin{array}{r}1.36 \\
21.24\end{array}$ & $\begin{array}{r}1.25 \\
20.98\end{array}$ \\
\hline \multirow[t]{2}{*}{ 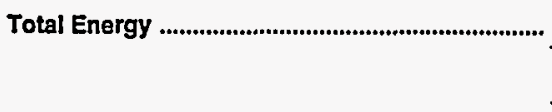 } & 1.70 & 3.33 & 6.61 & 8.48 & ${ }^{R_{8.08}}$ & ${ }^{n_{8.14}}$ & ${ }^{\mathrm{R}} 7.98$ & 8.00 \\
\hline & \multicolumn{8}{|c|}{ Expendltures in Millions of Dollars } \\
\hline 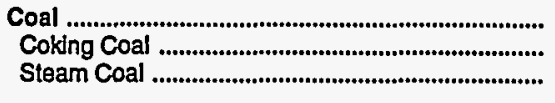 & $\begin{array}{r}363.6 \\
73.4 \\
290.3\end{array}$ & $\begin{array}{l}925.0 \\
290.3 \\
634.7\end{array}$ & $\begin{array}{r}1,299.2 \\
250.1 \\
1,049.1\end{array}$ & $\begin{array}{r}1,499.0 \\
149.7 \\
1,349.3\end{array}$ & $\begin{array}{r}1,283.4 \\
51.3 \\
1,232.1\end{array}$ & $\begin{array}{r}1,231.9 \\
26.3 \\
1,205.6\end{array}$ & $\begin{array}{l}1,108.6 \\
1,108.6\end{array}$ & $\begin{array}{r}1,101.0 \\
(s) \\
1,100.9\end{array}$ \\
\hline Natural Gas & 620.2 & $1,235.6$ & 2,596.2 & $3,954.1$ & $3,433.8$ & $3,538.2$ & $3,783.5$ & $3,882.2$ \\
\hline $\begin{array}{l}\text { Potroleum } \\
\text { Asphalt and Road Oil } \\
\text { Aviation Gasoline } \\
\text { Distillate Fuel } \\
\text { Jet Fuel } \\
\text { Kerosene } \\
\text { LPG }\end{array}$ & $\begin{array}{r}1,925.7 \\
21.5 \\
7.9 \\
240.6 \\
30.4 \\
15.7 \\
43.9 \\
97.3 \\
1,378.2 \\
33.7 \\
56.4\end{array}$ & \begin{tabular}{r|}
$4,020.3$ \\
54.6 \\
6.0 \\
610.6 \\
66.8 \\
19.9 \\
103.9 \\
124.9 \\
$2,686.4$ \\
217.0 \\
130.2
\end{tabular} & $\begin{array}{r}8,007.8 \\
94.0 \\
22.2 \\
1,087.9 \\
236.9 \\
44.0 \\
163.6 \\
285.1 \\
5,144.7 \\
315.1 \\
614.4\end{array}$ & $\begin{array}{r}7,044.2 \\
92.2 \\
10.1 \\
1,140.8 \\
223.6 \\
24.5 \\
440.4 \\
318.2 \\
4,465.3 \\
58.0 \\
273.2\end{array}$ & $\begin{array}{r}\text { R 7,176.2 } \\
80.1 \\
10.1 \\
1,022.2 \\
319.7 \\
12.7 \\
542.2 \\
\text { R } 296.8 \\
4,581.8 \\
44.2 \\
\text { R } 268.4\end{array}$ & $\begin{array}{r}{ }^{R} 6,887.5 \\
68.0 \\
9.0 \\
1,015.5 \\
283.9 \\
15.5 \\
578.5 \\
R_{305.4} \\
4,458.5 \\
23.2 \\
{ }_{234.0}\end{array}$ & $\begin{array}{r}{ }^{8} 6,877.2 \\
53.0 \\
7.8 \\
1,007.9 \\
260.5 \\
10.2 \\
567.8 \\
{ }^{8} 360.3 \\
4,343.7 \\
22.2 \\
{ }^{R} 243.7\end{array}$ & $\begin{array}{r}7,338.8 \\
88.4 \\
8.2 \\
1,154.9 \\
247.7 \\
18.0 \\
569.3 \\
357.6 \\
4,639.9 \\
30.5 \\
224.3\end{array}$ \\
\hline 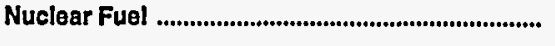 & 1.5 & 22.2 & 85.1 & 117.0 & 181.4 & 189.1 & 135.6 & 183.3 \\
\hline 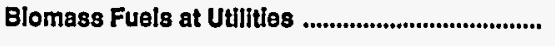 & - & - & - & - & - & - & - & - \\
\hline Primary Energy - Flve Sectors ${ }^{b}$............................ & $2,811.0$ & $6,203.2$ & $11,988.3$ & $12,614.4$ & $A_{12,074.8}$ & $R_{11,946.7}$ & $\mathrm{~A}_{11,804.8}$ & $12,505.3$ \\
\hline 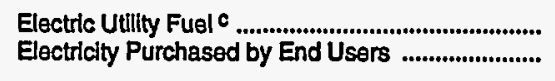 & $\begin{array}{r}-223.6 \\
1,041.7\end{array}$ & $\begin{array}{r}-733.0 \\
2,139.6\end{array}$ & $\begin{array}{r}-1,246.3 \\
3,647.5\end{array}$ & $\begin{array}{r}-1,312.2 \\
4,993.3\end{array}$ & $\begin{array}{r}-1,280.4 \\
5,786.9\end{array}$ & $\begin{array}{r}-1,281.2 \\
6,041.6\end{array}$ & $\begin{array}{r}-1,142.1 \\
6,008.9\end{array}$ & $\begin{array}{r}-1,180.3 \\
6,213.3\end{array}$ \\
\hline 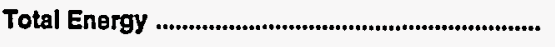 & $3,729.2$ & $7,609.8$ & $14,389.5$ & $16,295.4$ & $R_{16,591.2}$ & $R_{16,707.1}$ & ${ }^{R_{16,771.6}}$ & $17,538.3$ \\
\hline
\end{tabular}

a Inciudes petroleum coke used at electric utllities.

b Blomass fuels are not included, except those consumed at electric uttlities and those added to motor gasoline.

- There are no direct fuel costs for hydroelectric, geothermal, wind, photovoltalc, or solar thermal energy.

$R=$ Revised data.

-No consumption, including cases where adjustments were made. See

explanation of adjustments in Section 6 of Appendix A.

$(s)=$ Value less than 0.05 million dollars.

Note: Expenditure totals may not equal sum of components due to Independent rounding.

Sources: Data sources, estimation procodures, and assumptions ase described In Appendix A. 


\begin{tabular}{|c|c|c|c|c|c|c|c|c|}
\hline Sector and Energy Source & 1970 & 1975 & 1980 & 1985 & 1990 & 1991 & 1992 & 1993 \\
\hline 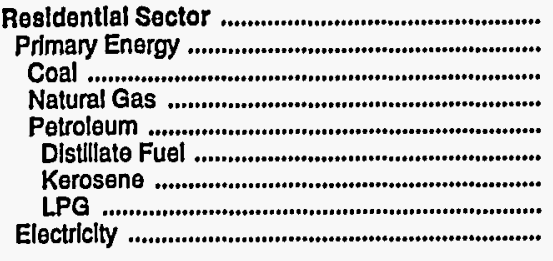 & $\begin{array}{l}1.74 \\
1.10 \\
1.43 \\
1.00 \\
1.35 \\
1.23 \\
1.56 \\
2.07 \\
6.99\end{array}$ & $\begin{array}{r}3.14 \\
1.93 \\
3.07 \\
1.58 \\
2.78 \\
2.51 \\
3.12 \\
4.29 \\
11.32\end{array}$ & $\begin{array}{r}5.56 \\
3.72 \\
3.70 \\
3.13 \\
7.25 \\
7.05 \\
8.43 \\
8.08 \\
16.76\end{array}$ & $\begin{array}{r}8.82 \\
6.41 \\
3.86 \\
6.14 \\
8.30 \\
7.66 \\
8.77 \\
9.63 \\
21.62\end{array}$ & $\begin{array}{r}8.53 \\
5.37 \\
3.39 \\
4.80 \\
9.43 \\
7.57 \\
8.66 \\
11.37 \\
22.94\end{array}$ & $\begin{array}{r}8.71 \\
5.36 \\
3.15 \\
4.88 \\
8.56 \\
7.06 \\
8.09 \\
10.11 \\
23.64\end{array}$ & $\begin{array}{r}8.40 \\
5.24 \\
3.18 \\
4.88 \\
7.89 \\
6.53 \\
7.48 \\
9.17 \\
23.77\end{array}$ & $\begin{array}{r}\mathbf{8 . 4 7} \\
\mathbf{5 . 2 6} \\
3.42 \\
4.87 \\
\mathbf{8 . 0 1} \\
6.54 \\
7.49 \\
9.27 \\
23.92\end{array}$ \\
\hline 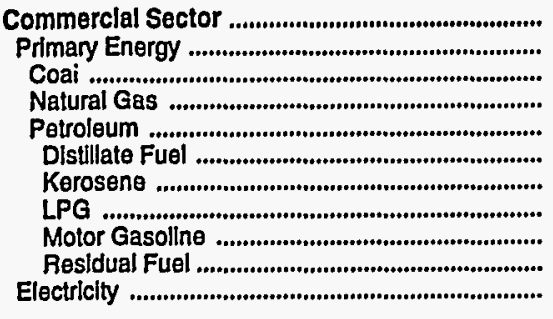 & $\begin{array}{r}2.11 \\
.88 \\
.53 \\
.83 \\
1.23 \\
1.05 \\
.74 \\
1.39 \\
2.71 \\
.64 \\
7.12\end{array}$ & $\begin{array}{r}3.40 \\
1.63 \\
1.49 \\
1.45 \\
2.69 \\
2.33 \\
2.44 \\
2.51 \\
4.72 \\
1.97 \\
11.41\end{array}$ & $\begin{array}{r}6.38 \\
3.53 \\
1.82 \\
3.13 \\
6.86 \\
6.53 \\
6.14 \\
5.16 \\
10.09 \\
3.97 \\
17.60\end{array}$ & $\begin{array}{r}10.12 \\
5.68 \\
1.99 \\
5.61 \\
6.84 \\
6.24 \\
7.21 \\
8.32 \\
9.10 \\
4.39 \\
23.36\end{array}$ & $\begin{array}{r}10.17 \\
4.62 \\
1.77 \\
4.43 \\
6.93 \\
5.35 \\
6.66 \\
9.36 \\
8.78 \\
3.15 \\
24.21\end{array}$ & $\begin{array}{r}10.21 \\
4.67 \\
1.80 \\
4.53 \\
6.63 \\
4.74 \\
5.90 \\
10.15 \\
8.37 \\
2.79 \\
24.16\end{array}$ & $\begin{array}{r}10.09 \\
4.62 \\
1.73 \\
4.48 \\
6.48 \\
4.54 \\
5.93 \\
9.77 \\
8.16 \\
2.66 \\
24.44\end{array}$ & $\begin{array}{r}10.98 \\
4.59 \\
1.71 \\
4.50 \\
6.49 \\
4.39 \\
5.25 \\
9.95 \\
8.35 \\
2.83 \\
23.63\end{array}$ \\
\hline 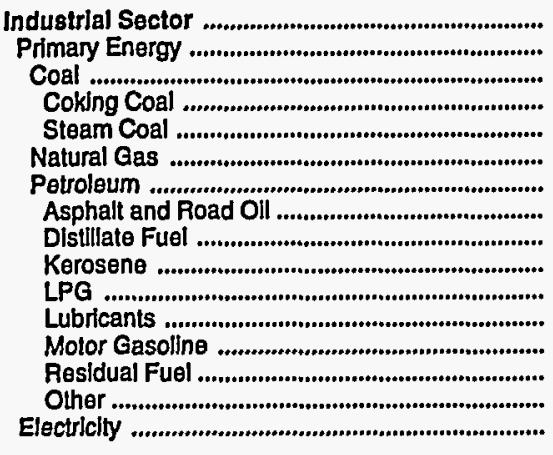 & $\begin{array}{r}1.01 \\
.71 \\
.54 \\
.55 \\
.53 \\
.53 \\
1.27 \\
.84 \\
.68 \\
.74 \\
1.39 \\
5.08 \\
2.71 \\
.54 \\
1.25 \\
3.74\end{array}$ & $\begin{array}{l}2.54 \\
1.81 \\
1.82 \\
2.07 \\
1.49 \\
1.22 \\
2.83 \\
2.12 \\
2.27 \\
2.44 \\
2.51 \\
7.48 \\
4.72 \\
1.98 \\
2.92 \\
7.83\end{array}$ & $\begin{array}{r}5.00 \\
3.68 \\
2.04 \\
2.27 \\
1.82 \\
2.87 \\
6.78 \\
4.04 \\
5.56 \\
6.14 \\
5.16 \\
14.36 \\
10.09 \\
3.23 \\
7.80 \\
13.18\end{array}$ & $\begin{array}{r}7.02 \\
4.75 \\
2.03 \\
2.08 \\
1.99 \\
4.95 \\
8.01 \\
5.00 \\
6.51 \\
7.21 \\
8.32 \\
17.61 \\
9.10 \\
4.39 \\
8.21 \\
16.75\end{array}$ & $\begin{array}{r}R_{6.44} \\
R_{4.09} \\
1.78 \\
1.80 \\
1.77 \\
3.72 \\
R_{6.91} \\
3.06 \\
6.01 \\
6.66 \\
9.36 \\
R_{14.60} \\
8.78 \\
3.15 \\
R_{6.65} \\
17.14\end{array}$ & $\begin{array}{r}{ }^{8} 6.77 \\
R_{4.30} \\
1.78 \\
1.72 \\
1.80 \\
3.85 \\
R_{6.93} \\
2.87 \\
5.32 \\
5.90 \\
10.15 \\
R_{16.79} \\
8.37 \\
2.79 \\
R_{5.91} \\
17.25\end{array}$ & $\begin{array}{r}R_{6.74} \\
R_{4.32} \\
1.73 \\
- \\
1.73 \\
3.78 \\
\text { R }_{6.95} \\
2.25 \\
5.35 \\
5.93 \\
9.77 \\
\text { R }_{19.43} \\
8.16 \\
2.66 \\
R_{5.88} \\
17.29\end{array}$ & $\begin{array}{r}6.07 \\
4.23 \\
1.71 \\
1.68 \\
1.71 \\
3.78 \\
6.59 \\
2.99 \\
4.74 \\
5.25 \\
9.95 \\
18.94 \\
8.35 \\
2.83 \\
5.40 \\
15.65\end{array}$ \\
\hline 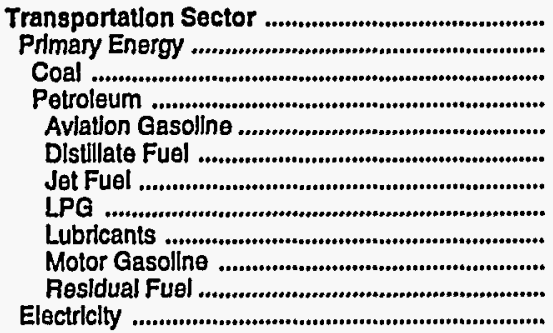 & $\begin{array}{r}2.50 \\
2.50 \\
.53 \\
2.50 \\
2.17 \\
1.27 \\
.74 \\
1.39 \\
5.08 \\
2.71 \\
.57 \\
-\end{array}$ & $\begin{array}{l}4.46 \\
4.46 \\
1.49 \\
4.46 \\
3.45 \\
2.77 \\
2.08 \\
2.51 \\
7.48 \\
4.72 \\
1.76 \\
-\end{array}$ & $\begin{array}{r}9.63 \\
9.63 \\
- \\
9.63 \\
9.02 \\
7.19 \\
6.38 \\
5.16 \\
14.36 \\
10.09 \\
3.49 \\
-\end{array}$ & $\begin{array}{r}8.96 \\
8.96 \\
- \\
8.96 \\
9.99 \\
8.55 \\
6.09 \\
8.32 \\
17.61 \\
9.10 \\
4.38 \\
-\end{array}$ & $\begin{array}{r}R_{8.52} \\
R_{8.52} \\
- \\
R_{8.52} \\
9.32 \\
8.24 \\
5.65 \\
9.36 \\
R_{14.60} \\
8.78 \\
2.42 \\
-\end{array}$ & $\begin{array}{r}R_{8.12} \\
R_{8.12} \\
R_{8.12} \\
8.71 \\
7.88 \\
4.94 \\
10.15 \\
R_{16.79} \\
8.37 \\
2.09 \\
26.87\end{array}$ & $\begin{array}{r}R_{7.94} \\
R_{7.94} \\
-\bar{R} 7.94 \\
8.54 \\
7.74 \\
4.57 \\
9.77 \\
R_{19.43} \\
8.16 \\
R_{2.14} \\
27.20\end{array}$ & $\begin{array}{r}8.06 \\
8.06 \\
-\overline{8} \\
8.06 \\
8.24 \\
7.78 \\
4.26 \\
9.95 \\
18.94 \\
8.35 \\
2.46 \\
26.72\end{array}$ \\
\hline 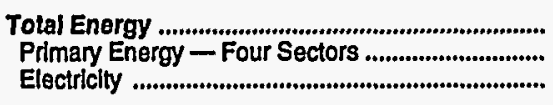 & $\begin{array}{l}1.70 \\
1.34 \\
5.55\end{array}$ & $\begin{array}{l}3.33 \\
2.65 \\
9.78\end{array}$ & $\begin{array}{r}6.61 \\
5.54 \\
15.40\end{array}$ & $\begin{array}{r}8.48 \\
6.77 \\
19.88\end{array}$ & $\begin{array}{l}R_{8.08} \\
R_{6.08} \\
20.85\end{array}$ & $\begin{array}{l}R_{8.14} \\
R_{6.04} \\
21.18\end{array}$ & $\begin{array}{l}R_{7.98} \\
R_{5.91} \\
21.24\end{array}$ & $\begin{array}{r}8.00 \\
5.97 \\
20.98\end{array}$ \\
\hline 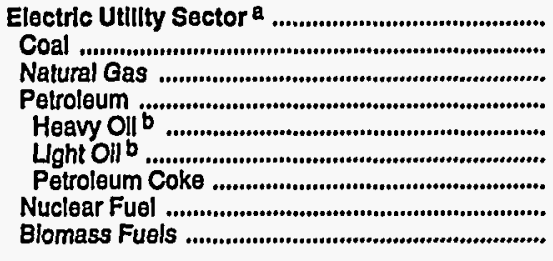 & $\begin{array}{l}.38 \\
.36 \\
.42 \\
.63 \\
.63 \\
.65 \\
-36 \\
-\end{array}$ & $\begin{array}{r}1.02 \\
.92 \\
1.28 \\
1.98 \\
1.97 \\
2.05 \\
. \overline{28} \\
-\end{array}$ & $\begin{array}{r}1.58 \\
1.56 \\
2.74 \\
4.24 \\
4.10 \\
6.07 \\
-49 \\
-\end{array}$ & $\begin{array}{r}1.72 \\
1.88 \\
4.43 \\
5.15 \\
4.64 \\
5.60 \\
-80 \\
-\end{array}$ & $\begin{array}{r}1.41 \\
1.60 \\
2.11 \\
3.26 \\
2.89 \\
4.60 \\
- \\
.79 \\
-\end{array}$ & $\begin{array}{r}1.32 \\
1.59 \\
1.96 \\
2.89 \\
2.44 \\
4.51 \\
- \\
.65 \\
-\end{array}$ & $\begin{array}{r}1.36 \\
1.56 \\
1.95 \\
2.92 \\
2.44 \\
4.39 \\
- \\
.57 \\
-\end{array}$ & $\begin{array}{r}1.25 \\
1.53 \\
2.42 \\
3.05 \\
2.77 \\
3.97 \\
- \\
.60 \\
-\end{array}$ \\
\hline Primary Energy - Flve Sectors c ........................ & 1.12 & 2.23 & 4.39 & 5.18 & $\mathrm{~A}_{4.50}$ & $R_{4.37}$ & $R_{4.48}$ & 4.40 \\
\hline
\end{tabular}

There are no drect fuel costs for hydroelectric, geothermal, wind, photovoltalc, or solar thermal energy.

b Heavy oll includes fuel oll nos. 4, 5, and 6 , and residual fuel olls. Light oil includes fuel oll nos. 1 and 2, kerosene, and jet fuel.

olomass fuels are not included, except those consumed at electric utllttes and those added to motor gasoline.
R=Revised data.

- No consumption, Including cases where adjustments were made. See explanation of adjustments in Section 6 of Appendix A.

Sources: Data sources, estimation procodures, and assumptions are described in Appendix A. 

1970, 1975, 1980, 1985, and 1990-1993

(Million Dollars)

\begin{tabular}{|c|c|c|c|c|c|c|c|c|}
\hline Sector and Energy Source & 1970 & 1975 & 1980 & 1985 & 1990 & 1991 & 1992 & 1993 \\
\hline $\begin{array}{l}\text { Residential Sector } \\
\text { Primary Energy } \\
\text { Coal } \\
\text { Natural Gas . } \\
\text { Petroleum } \\
\text { Distillate Fuel } \\
\text { Kerosene }\end{array}$ & $\begin{array}{r}939.0 \\
530.9 \\
10.3 \\
345.1 \\
175.5 \\
135.5 \\
4.8 \\
35.2 \\
408.1\end{array}$ & $\begin{array}{r}1,732.6 \\
925.9 \\
10.1 \\
542.8 \\
373.0 \\
284.4 \\
5.3 \\
83.3 \\
806.7\end{array}$ & $\begin{array}{r}3,000.8 \\
1,727.5 \\
9.8 \\
1,236.0 \\
481.8 \\
377.7 \\
4.0 \\
100.1 \\
1,273.3\end{array}$ & $\begin{array}{r}4,237.7 \\
2,592.6 \\
8.4 \\
2,143.5 \\
440.7 \\
266.0 \\
21.1 \\
153.6 \\
1,645.1\end{array}$ & $\begin{array}{r}4,097.2 \\
2,115.2 \\
7.9 \\
1,643.5 \\
463.8 \\
183.7 \\
10.7 \\
269.5 \\
1,982.0\end{array}$ & $\begin{array}{r}4,339.9 \\
2,181.9 \\
7.3 \\
1,709.6 \\
465.0 \\
187.5 \\
12.8 \\
264.7 \\
2,158.0\end{array}$ & $\begin{array}{r}4,312.5 \\
2,230.5 \\
5.3 \\
1,811.9 \\
413.3 \\
161.0 \\
8.7 \\
243.6 \\
2,082.0\end{array}$ & $\begin{array}{r}4,495.6 \\
2,310.6 \\
7.0 \\
1,863.8 \\
439.8 \\
158.0 \\
15.1 \\
266.7 \\
2,185.0\end{array}$ \\
\hline $\begin{array}{l}\text { Commerclal Sector } \\
\text { Primary Energy } \\
\text { Coal . } \\
\text { Natural Gas } \\
\text { Petroleum } \\
\text { Distillate Fuel } \\
\text { Kerosene } \\
\text { LPG } \\
\text { Motor Gasoline } \\
\text { Residual Fuel } \\
\text { Electricity }\end{array}$ & $\begin{array}{r}475.7 \\
159.3 \\
7.0 \\
111.4 \\
40.9 \\
21.4 \\
1.7 \\
4.2 \\
11.4 \\
2.2 \\
316.4\end{array}$ & $\begin{array}{r}935.8 \\
367.7 \\
9.0 \\
269.8 \\
88.9 \\
48.7 \\
3.1 \\
8.6 \\
23.7 \\
4.8 \\
568.1\end{array}$ & $\begin{array}{r}1,802.2 \\
795.3 \\
8.8 \\
606.7 \\
179.8 \\
118.8 \\
.5 \\
11.3 \\
43.6 \\
5.6 \\
1,006.9\end{array}$ & $\begin{array}{r}2,531.9 \\
1,063.7 \\
8.0 \\
905.1 \\
150.6 \\
85.7 \\
.5 \\
23.4 \\
33.4 \\
7.6 \\
1,468.2\end{array}$ & $\begin{array}{r}2,692.3 \\
876.3 \\
7.7 \\
738.2 \\
130.5 \\
53.9 \\
.7 \\
39.2 \\
35.3 \\
1.4 \\
1,816.0\end{array}$ & $\begin{array}{r}2,788.2 \\
912.6 \\
7.7 \\
778.1 \\
126.8 \\
53.5 \\
.6 \\
46.9 \\
25.8 \\
.1 \\
1,875.6\end{array}$ & $\begin{array}{r}R_{2,806.8} \\
930.0 \\
5.3 \\
808.2 \\
116.6 \\
46.7 \\
.2 \\
45.8 \\
23.7 \\
.2 \\
1,876.7\end{array}$ & $\begin{array}{r}3,377.6 \\
938.9 \\
6.5 \\
839.9 \\
92.4 \\
37.7 \\
.8 \\
50.5 \\
3.4 \\
.1 \\
2,438.7\end{array}$ \\
\hline 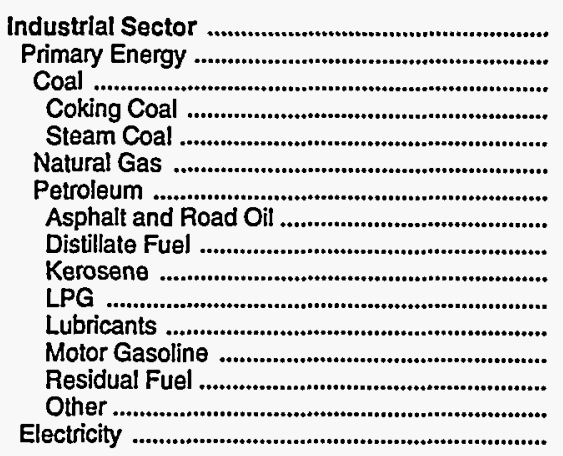 & $\begin{array}{r}858.9 \\
541.7 \\
172.7 \\
73.4 \\
99.3 \\
136.5 \\
232.5 \\
21.5 \\
33.3 \\
9.2 \\
4.3 \\
56.5 \\
39.2 \\
12.0 \\
56.4 \\
317.2\end{array}$ & $\begin{array}{r}2,043.9 \\
1,279.1 \\
449.1 \\
290.3 \\
158.8 \\
362.3 \\
467.7 \\
54.6 \\
115.9 \\
11.4 \\
11.2 \\
64.9 \\
46.9 \\
32.6 \\
130.2 \\
764.8\end{array}$ & $\begin{array}{r}3,733.4 \\
2,366.0 \\
448.3 \\
250.1 \\
198.2 \\
700.3 \\
1,217.4 \\
94.0 \\
155.7 \\
39.5 \\
49.7 \\
156.4 \\
51.3 \\
56.3 \\
614.4 \\
1,367.4\end{array}$ & $\begin{array}{r}4,154.6 \\
2,274.7 \\
344.4 \\
149.7 \\
194.6 \\
884.9 \\
1,045.4 \\
92.2 \\
160.5 \\
2.9 \\
254.6 \\
174.6 \\
56.9 \\
30.5 \\
273.2 \\
1,880.0\end{array}$ & $\begin{array}{r}R_{4,168.8} \\
R_{2,169.9} \\
209.7 \\
51.3 \\
158.4 \\
1,041.2 \\
R_{919.0} \\
80.1 \\
119.2 \\
1.3 \\
223.8 \\
\text { R }_{162.8} \\
44.8 \\
20.4 \\
\mathrm{R}_{266.4} \\
1,998.9\end{array}$ & $\begin{array}{r}R_{4,130.5} \\
R_{2,122.9} \\
164.9 \\
26.3 \\
138.7 \\
1,032.4 \\
R_{925.6} \\
66.0 \\
141.8 \\
2.1 \\
257.3 \\
R_{167.6} \\
48.8 \\
7.9 \\
R_{234.0} \\
2,007.6\end{array}$ & $\begin{array}{r}R_{4,283.0} \\
R_{2,233.2} \\
131.8 \\
-\overline{131.8} \\
1,143.2 \\
\text { R }_{958.1} \\
53.0 \\
144.2 \\
1.4 \\
269.5 \\
\text { R }_{197.7} \\
40.7 \\
7.9 \\
\text { R }_{243.7} \\
2,049.9\end{array}$ & $\begin{array}{r}3,817.5 \\
2,228.3 \\
133.9 \\
(s) \\
133.9 \\
1,161.1 \\
933.2 \\
88.4 \\
123.6 \\
2.1 \\
242.3 \\
196.2 \\
45.3 \\
10.9 \\
224.3 \\
1,589.2\end{array}$ \\
\hline 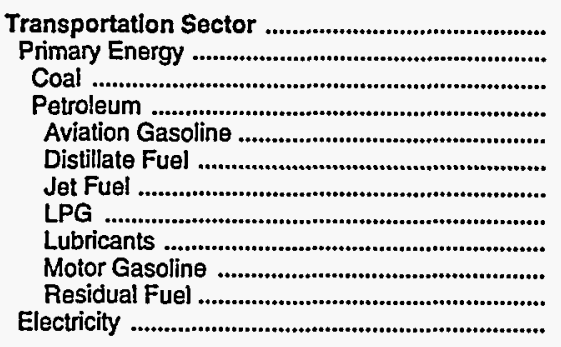 & $\begin{array}{r}1,455.6 \\
1,455.6 \\
.3 \\
1,455.3 \\
7.9 \\
46.9 \\
30.4 \\
.3 \\
40.8 \\
1,327.5 \\
1.5 \\
-\end{array}$ & $\begin{array}{r}2,897.5 \\
2,897.5 \\
.1 \\
2,897.4 \\
6.0 \\
144.2 \\
65.8 \\
.9 \\
60.0 \\
2,615.8 \\
4.7 \\
-\end{array}$ & $\begin{array}{r}5,853.1 \\
5,853.1 \\
- \\
5,853.1 \\
22.2 \\
408.1 \\
236.9 \\
2.4 \\
128.6 \\
5,049.8 \\
5.1 \\
-\end{array}$ & $\begin{array}{r}5,371.2 \\
5,371.2 \\
- \\
5,371.2 \\
10.1 \\
607.5 \\
223.6 \\
8.7 \\
143.6 \\
4,375.0 \\
2.7 \\
-\end{array}$ & $\begin{array}{r}R_{5,632.9} \\
R_{5,632.9} \\
R_{5,632.9} \\
10.1 \\
656.3 \\
319.7 \\
9.7 \\
R_{133.9} \\
4,501.7 \\
1.4 \\
-\end{array}$ & $\begin{array}{r}R_{5,448.5} \\
R_{5,448.1}- \\
- \\
R_{5,448.1} \\
9.0 \\
625.2 \\
283.9 \\
9.6 \\
R_{137.8} \\
4,381.9 \\
.7 \\
.4\end{array}$ & $\begin{array}{r}R_{5,369.3} \\
R_{5,369.0} \\
- \\
R_{5,369.0} \\
7.8 \\
648.5 \\
260.5 \\
8.9 \\
R_{162.6} \\
4,279.3 \\
1.3 \\
R .3\end{array}$ & $\begin{array}{r}5,847.5 \\
5,847.2 \\
5,847.2 \\
8.2 \\
827.7 \\
247.7 \\
9.8 \\
161.4 \\
4,591.2 \\
1.1 \\
.3\end{array}$ \\
\hline 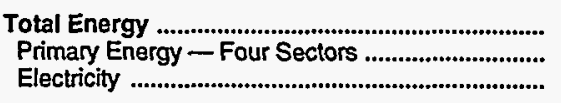 & $\begin{array}{l}3,729.2 \\
2,687.5 \\
1,041.7\end{array}$ & $\begin{array}{l}7,609.8 \\
5,470.2 \\
2,139.6\end{array}$ & $\begin{array}{r}14,389.5 \\
10,742.0 \\
3,647.5\end{array}$ & $\begin{array}{r}16,295.4 \\
11,302.2 \\
4,993.3\end{array}$ & $\begin{array}{r}R_{16,591.2} \\
R_{10,794.4} \\
5,796.9\end{array}$ & $\begin{array}{r}R_{16,707.1} \\
R_{10,665.5} \\
6,041.6\end{array}$ & $\begin{array}{r}R_{16,771.6} \\
R_{10,762.7} \\
6,008.9\end{array}$ & $\begin{array}{r}17,538.3 \\
11,325.0 \\
6,213.3\end{array}$ \\
\hline 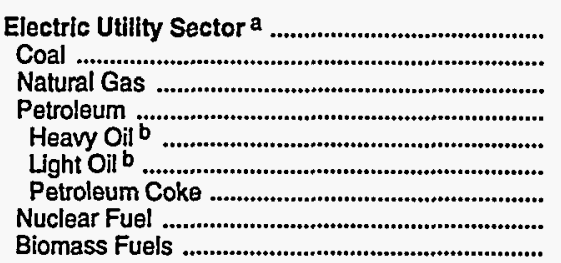 & $\begin{array}{r}223.6 \\
173.4 \\
27.2 \\
21.5 \\
17.9 \\
3.6 \\
- \\
1.5 \\
-\end{array}$ & $\begin{array}{r}733.0 \\
456.8 \\
60.7 \\
193.2 \\
174.9 \\
18.4 \\
- \\
22.2 \\
-\end{array}$ & $\begin{array}{r}1,246.3 \\
832.3 \\
53.2 \\
275.7 \\
248.2 \\
27.5 \\
- \\
85.1 \\
-\end{array}$ & $\begin{array}{r}1,312.2 \\
1,138.3 \\
20.6 \\
36.3 \\
15.2 \\
21.1 \\
-\overline{117.0} \\
-\end{array}$ & $\begin{array}{r}1,280.4 \\
1,058.2 \\
10.8 \\
30.0 \\
20.9 \\
9.1 \\
181.4 \\
-\end{array}$ & $\begin{array}{r}1,281.2 \\
1,052.0 \\
18.1 \\
22.0 \\
14.5 \\
7.5 \\
189.1 \\
-\end{array}$ & $\begin{array}{r}1,142.1 \\
966.2 \\
20.1 \\
20.2 \\
12.8 \\
7.5 \\
- \\
135.5 \\
-\end{array}$ & $\begin{array}{r}1,180.3 \\
953.5 \\
17.3 \\
26.1 \\
18.3 \\
7.9 \\
- \\
183.3 \\
-\end{array}$ \\
\hline 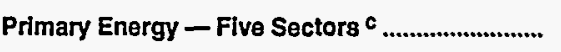 & $2,911.0$ & $6,203.2$ & $11,988.3$ & $12,614.4$ & $12,074.8$ & ${ }_{11,946.7}$ & $R_{11,904.8}$ & $12,505.3$ \\
\hline
\end{tabular}

a There are no direct fuel costs for hydroelectric, geothermal, wind, photovoltaic, or solar thermal energy.

Heavy oil includes fuel oil nos. 4,5 , and 6 , and residual fuel oils. Light oil includes fuel nos. 1 and 2 , kerosene, and jet fuel.

c Biomass fuels are not included, except those consumed at electric utilities and those added to motor gasoline.

$R=$ Revised data.
-No consumption, including cases where adjustments were made. See explanation of adjustments in Section 6 of Appendix A.

(s)=Value less than 0.05 million dollars.

Note: Totals may not equal sum of components due to independent rounding.

Sources: Data sources, estimation procedures, and assumptions are described in Appendix A. 
Energy Price and Expenditure Estimates by Source, Minnesota 1970, 1975, 1980, 1985, and 1990-1993

\begin{tabular}{|c|c|c|c|c|c|c|c|c|}
\hline Energy Source & 1970 & 1975 & 1980 & 1985 & 1990 & 1991 & 1992 & 1993 \\
\hline & \multicolumn{8}{|c|}{ Prices In Dollars per Million Btu } \\
\hline 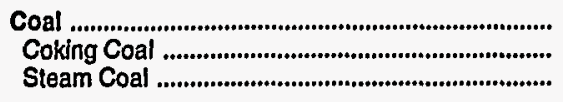 & $\begin{array}{r}0.41 \\
.53 \\
.40\end{array}$ & $\begin{array}{r}0.83 \\
1.80 \\
.69\end{array}$ & 1.12 & $\begin{array}{r}1.51 \\
1.51\end{array}$ & $\frac{1.32}{1.32}$ & $\frac{1.30}{1.30}$ & $\frac{1.24}{1.24}$ & $\frac{1.21}{1.21}$ \\
\hline 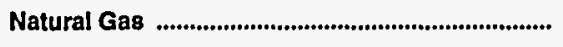 & .66 & 1.17 & 2.85 & 5.13 & 3.87 & 3.70 & 3.99 & 4.36 \\
\hline 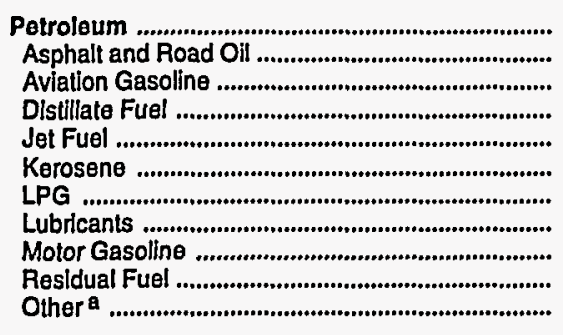 & $\begin{array}{r}2.01 \\
.68 \\
2.17 \\
1.08 \\
.75 \\
1.34 \\
1.80 \\
5.08 \\
2.97 \\
.59 \\
.74\end{array}$ & $\begin{array}{l}3.57 \\
2.19 \\
3.45 \\
2.51 \\
2.09 \\
2.78 \\
3.67 \\
7.48 \\
4.63 \\
1.80 \\
1.72\end{array}$ & $\begin{array}{r}7.88 \\
3.31 \\
9.02 \\
6.72 \\
6.47 \\
7.05 \\
5.82 \\
14.36 \\
9.55 \\
3.52 \\
3.82\end{array}$ & $\begin{array}{r}8.45 \\
4.99 \\
9.99 \\
7.55 \\
5.93 \\
8.30 \\
8.08 \\
17.61 \\
9.73 \\
4.05 \\
4.75\end{array}$ & $\begin{array}{r}R_{8.25} \\
3.31 \\
9.32 \\
7.89 \\
5.68 \\
8.12 \\
8.85 \\
R_{14.60} \\
9.56 \\
2.50 \\
2.32\end{array}$ & $\begin{array}{r}R_{7.88} \\
3.19 \\
8.71 \\
7.06 \\
4.87 \\
7.34 \\
7.89 \\
R \quad 16.79 \\
9.31 \\
2.11 \\
1.85\end{array}$ & $\begin{array}{r}R_{7.56} \\
2.71 \\
8.54 \\
R_{6.83} \\
4.64 \\
R_{6.69} \\
7.05 \\
R_{19.43} \\
9.11 \\
R_{1.79} \\
1.73\end{array}$ & $\begin{array}{r}7.82 \\
2.93 \\
8.24 \\
6.90 \\
4.33 \\
6.43 \\
8.54 \\
18.94 \\
9.51 \\
2.18 \\
1.66\end{array}$ \\
\hline 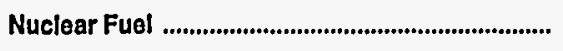 & - & .24 & .44 & .50 & .48 & .47 & .44 & .41 \\
\hline 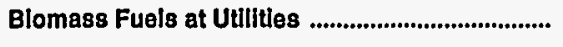 & .65 & .92 & 1.74 & - & .91 & .98 & .87 & .55 \\
\hline Primary Energy - Five Sectors b ....................... & 1.27 & 2.13 & 4.42 & 5.27 & 4.51 & 4.42 & $R_{4.45}$ & 4.59 \\
\hline 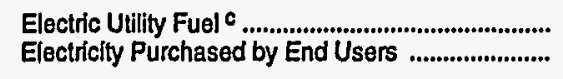 & 6.10 & $\begin{array}{r}.52 \\
8.64\end{array}$ & $\begin{array}{r}.91 \\
13.26\end{array}$ & $\begin{array}{r}1.09 \\
15.81\end{array}$ & $\begin{array}{r}1.03 \\
15.68\end{array}$ & $\begin{array}{r}1.02 \\
16.07\end{array}$ & $\begin{array}{r}.97 \\
16.24\end{array}$ & $\begin{array}{r}.93 \\
16.46\end{array}$ \\
\hline \multirow[t]{2}{*}{ 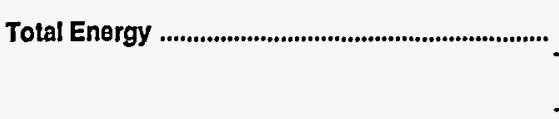 } & 1.86 & 3.18 & 6.91 & 8.42 & $\mathrm{R}_{8.11}$ & $R_{7.95}$ & $\mathrm{R}_{7.87}$ & 8.08 \\
\hline & \multicolumn{8}{|c|}{ Expenditures in Millions of Dollars } \\
\hline 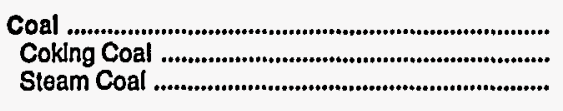 & $\begin{array}{r}74.1 \\
8.6 \\
65.5\end{array}$ & $\begin{array}{r}159.8 \\
45.4 \\
114.4\end{array}$ & $\begin{array}{c}270.7 \\
- \\
270.7\end{array}$ & $\begin{array}{c}341.4 \\
\overline{341.4}\end{array}$ & $\begin{array}{c}429.1 \\
-\overline{429.1}\end{array}$ & $\begin{array}{c}390.2 \\
390.2\end{array}$ & $\begin{array}{c}371.0 \\
- \\
371.0\end{array}$ & $\begin{array}{c}392.2 \\
- \\
392.2\end{array}$ \\
\hline Natural Gas & 220.6 & 381.4 & 785.0 & $1,283.0$ & $1,066.5$ & $1,110.6$ & $1,170.0$ & $1,354.6$ \\
\hline 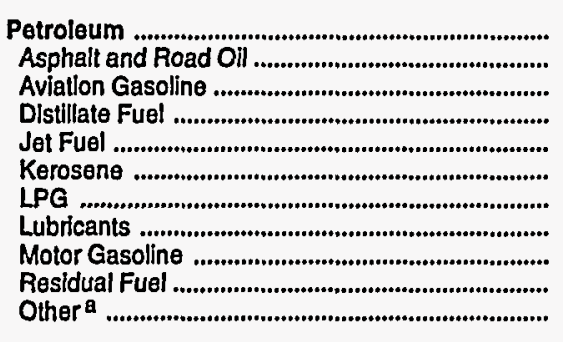 & $\begin{array}{r}988.1 \\
20.0 \\
3.0 \\
140.5 \\
14.7 \\
12.8 \\
60.1 \\
28.5 \\
688.9 \\
14.9 \\
4.6\end{array}$ & \begin{tabular}{r|}
$1,902.1$ \\
67.2 \\
3.7 \\
355.7 \\
66.5 \\
13.5 \\
124.8 \\
45.5 \\
$1,172.9$ \\
38.4 \\
13.7
\end{tabular} & $\begin{array}{r}3,784.8 \\
78.4 \\
8.8 \\
837.2 \\
188.3 \\
8.5 \\
163.0 \\
97.5 \\
2,319.4 \\
56.3 \\
27.5\end{array}$ & $\begin{array}{r}3,928.6 \\
165.1 \\
7.8 \\
852.5 \\
261.4 \\
8.6 \\
154.0 \\
108.8 \\
2,335.5 \\
15.8 \\
19.1\end{array}$ & $\begin{array}{r}R_{3,863.3} \\
132.5 \\
10.0 \\
849.2 \\
164.0 \\
1.9 \\
187.7 \\
R_{101.5} \\
2,385.6 \\
12.2 \\
18.5\end{array}$ & $\begin{array}{r}R_{3,842.0} \\
106.6 \\
8.3 \\
872.2 \\
137.3 \\
2.3 \\
185.8 \\
R 104.5 \\
2,398.2 \\
10.0 \\
17.0\end{array}$ & $\begin{array}{r}R_{3,870.1} \\
95.9 \\
5.8 \\
R_{859.7} \\
173.8 \\
2.0 \\
202.3 \\
R_{123.2} \\
2,379.8 \\
9.2 \\
18.3\end{array}$ & $\begin{array}{r}4,165.2 \\
93.2 \\
5.5 \\
845.8 \\
231.3 \\
2.2 \\
269.8 \\
122.3 \\
2,565.6 \\
11.6 \\
17.9\end{array}$ \\
\hline 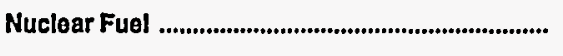 & - & 25.5 & 48.6 & 62.5 & 61.7 & 60.7 & 52.0 & 52.9 \\
\hline Blomass Fuels at Utilities ................................... & .1 & (8) & (8) & - & 3.7 & 4.1 & 3.6 & 2.4 \\
\hline Primary Energy - Flve Sectors ${ }^{b} \ldots . . . . . . . . . . . . . . . .$. & $1,282.9$ & $2,468.9$ & $4,889.2$ & $5,615.6$ & $R_{5,424.5}$ & $R_{5,407.5}$ & $\mathrm{R}_{\mathbf{5 , 4 6 6 . 6}}$ & $5,967.2$ \\
\hline $\begin{array}{l}\text { Electric Utility Fuel c ...................................................... } \\
\text { Electricity Purchased by End Users ..................... }\end{array}$ & $\begin{array}{r}-65.3 \\
427.5\end{array}$ & $\begin{array}{r}-144.2 \\
769.9\end{array}$ & $\begin{array}{r}-311.3 \\
1,481.2\end{array}$ & $\begin{array}{r}-355.5 \\
2,062.8\end{array}$ & $\begin{array}{r}-454.0 \\
2,491.2\end{array}$ & $\begin{array}{r}-439.2 \\
2,638.6\end{array}$ & $\begin{array}{r}-402.9 \\
2,592.8\end{array}$ & $\begin{array}{r}-409.7 \\
2,727.9\end{array}$ \\
\hline 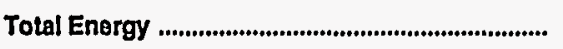 & $1,645.1$ & $3,094.6$ & $6,059.1$ & $7,322.9$ & $R_{7,461.7}$ & $R_{7,606.9}$ & ${ }^{R_{7,656.6}}$ & $8,285.5$ \\
\hline
\end{tabular}

a Includes petroleum coke used at electric utilities.

b Biomass fuels are not included, except those consumed at electric utilitles and those added to motor gasoline.

c There are no direct fuel costs for hydroelectric, geothermal, wind, photovoltalc, or solar themal energy.

$\mathrm{R}=$ Revised data.

-No consumption, Including cases where adjustments were made. See explanation of adjustments in Section 6 of Appendix A.

(s)=Value less than 0.05 million dollars.

Note: Expenditure totals may not equal sum of components due to independent rounding.

Sources: Data sources, estimation procedures, and assumptions are described in Appendix A. 
I 1970, 1975, 1980, 1985, and 1990-1993

N (Dollars per Million Btu)

\begin{tabular}{|c|c|c|c|c|c|c|c|c|}
\hline Sector and Energy Source & 1970 & 1975 & 1980 & 1985 & 1990 & 1991 & 1992 & 1993 \\
\hline 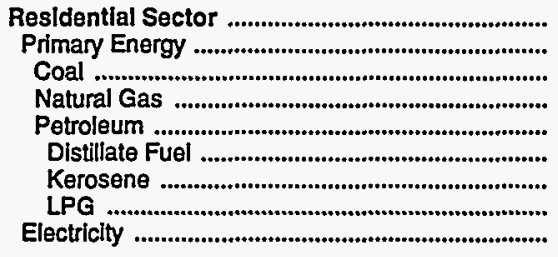 & $\begin{array}{l}2.17 \\
1.29 \\
1.55 \\
1.09 \\
1.54 \\
1.26 \\
1.52 \\
2.03 \\
7.30\end{array}$ & $\begin{array}{l}3.38 \\
2.15 \\
3.04 \\
1.57 \\
3.12 \\
2.55 \\
2.91 \\
4.22 \\
9.90\end{array}$ & $\begin{array}{r}6.92 \\
4.47 \\
4.32 \\
3.24 \\
7.25 \\
7.20 \\
8.02 \\
7.34 \\
16.06\end{array}$ & $\begin{array}{r}9.34 \\
6.22 \\
4.10 \\
5.78 \\
7.81 \\
7.79 \\
8.82 \\
7.79 \\
19.01\end{array}$ & $\begin{array}{r}9.25 \\
5.32 \\
3.46 \\
4.61 \\
7.97 \\
7.75 \\
8.77 \\
8.35 \\
19.94\end{array}$ & $\begin{array}{r}8.97 \\
5.07 \\
3.81 \\
4.47 \\
7.12 \\
6.96 \\
7.88 \\
7.42 \\
20.28\end{array}$ & $\begin{array}{r}9.20 \\
5.32 \\
3.47 \\
4.81 \\
R 7.11 \\
R 6.29 \\
R 7.12 \\
8.37 \\
20.55\end{array}$ & $\begin{array}{r}9.43 \\
5.67 \\
3.28 \\
5.25 \\
7.22 \\
6.36 \\
7.20 \\
8.24 \\
20.76\end{array}$ \\
\hline 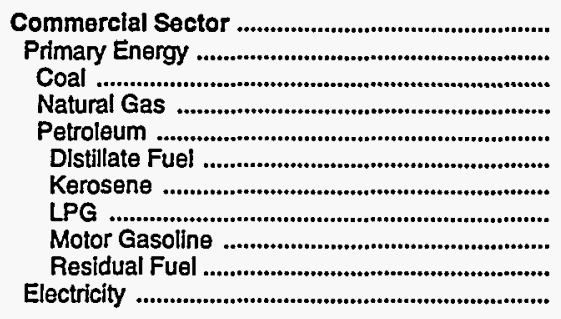 & $\begin{array}{r}1.42 \\
.76 \\
.47 \\
.69 \\
1.13 \\
1.05 \\
.89 \\
1.20 \\
2.97 \\
.58 \\
7.72\end{array}$ & $\begin{array}{r}2.56 \\
1.39 \\
.87 \\
1.16 \\
2.61 \\
2.34 \\
2.54 \\
2.61 \\
4.63 \\
1.97 \\
10.38\end{array}$ & $\begin{array}{r}5.37 \\
3.47 \\
1.77 \\
2.89 \\
6.73 \\
6.60 \\
-\overline{-} \\
4.87 \\
9.55 \\
4.48 \\
12.95\end{array}$ & $\begin{array}{r}7.84 \\
5.39 \\
2.04 \\
5.18 \\
6.62 \\
6.34 \\
6.81 \\
8.32 \\
9.73 \\
4.10 \\
17.53\end{array}$ & $\begin{array}{r}7.66 \\
4.57 \\
2.05 \\
3.96 \\
7.65 \\
5.75 \\
6.44 \\
9.36 \\
9.56 \\
2.50 \\
17.69\end{array}$ & $\begin{array}{l}7.33 \\
3.93 \\
1.87 \\
3.76 \\
5.52 \\
4.88 \\
5.74 \\
8.34 \\
9.31 \\
2.11 \\
18.01\end{array}$ & $\begin{array}{r}7.64 \\
4.13 \\
1.93 \\
4.06 \\
R \\
R .94 \\
\mathrm{R} 4.70 \\
\mathrm{R} 5.58 \\
5.97 \\
9.11 \\
\mathrm{R} 1.79 \\
18.16\end{array}$ & $\begin{array}{r}7.97 \\
4.58 \\
1.99 \\
4.47 \\
6.22 \\
4.99 \\
5.33 \\
8.84 \\
9.51 \\
2.18 \\
18.39\end{array}$ \\
\hline 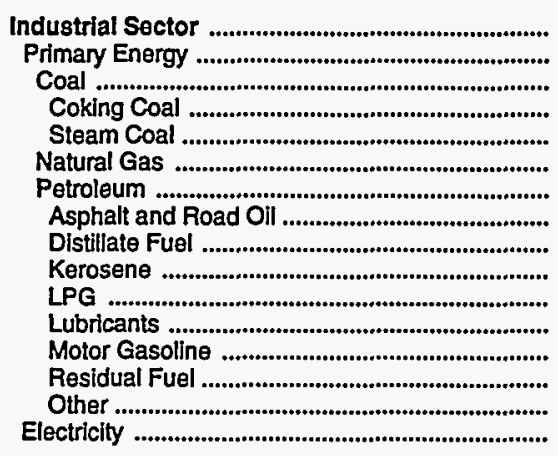 & $\begin{array}{r}1.11 \\
.78 \\
.49 \\
.53 \\
.47 \\
.42 \\
1.16 \\
.68 \\
.83 \\
.89 \\
1.20 \\
5.08 \\
2.97 \\
.55 \\
.82 \\
4.17\end{array}$ & $\begin{array}{r}2.33 \\
1.73 \\
1.33 \\
1.80 \\
.87 \\
.83 \\
2.62 \\
2.19 \\
2.39 \\
2.54 \\
2.61 \\
7.48 \\
4.63 \\
1.74 \\
1.77 \\
6.73\end{array}$ & $\begin{array}{r}5.08 \\
3.60 \\
1.77 \\
- \\
1.77 \\
2.51 \\
5.08 \\
3.31 \\
5.66 \\
5.92 \\
4.87 \\
14.36 \\
9.55 \\
2.97 \\
3.82 \\
11.22\end{array}$ & $\begin{array}{r}6.97 \\
5.00 \\
2.04 \\
2 . \overline{-} \\
4.04 \\
6.44 \\
4.99 \\
6.18 \\
6.81 \\
8.32 \\
17.61 \\
9.73 \\
4.10 \\
4.75 \\
12.65\end{array}$ & $\begin{array}{r}\mathrm{R}_{6.23} \\
\mathrm{R}_{3.92} \\
2.05 \\
- \\
2.05 \\
2.96 \\
\mathrm{R}_{5.30} \\
3.31 \\
5.85 \\
6.44 \\
9.36 \\
\mathrm{R}_{14.60} \\
9.56 \\
2.50 \\
4.22 \\
12.14\end{array}$ & 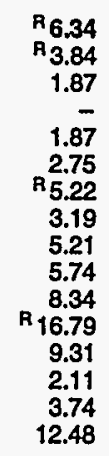 & $\begin{array}{r}R_{6.16} \\
R_{3.74} \\
1.93 \\
-\overline{1} \\
1.93 \\
3.02 \\
R_{4.72} \\
2.71 \\
R_{5.07} \\
R_{5.58} \\
5.97 \\
R_{19.43} \\
9.11 \\
R_{1.79} \\
3.34 \\
12.69\end{array}$ & $\begin{array}{r}6.36 \\
3.90 \\
1.99 \\
1.99 \\
3.17 \\
5.12 \\
2.93 \\
4.84 \\
5.33 \\
8.84 \\
18.94 \\
9.51 \\
2.18 \\
3.08 \\
12.88\end{array}$ \\
\hline 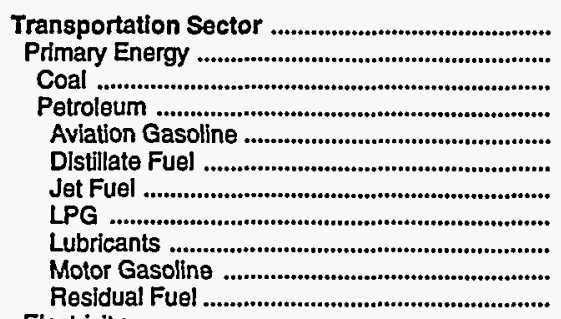 & $\begin{array}{r}2.64 \\
2.64 \\
.47 \\
2.64 \\
2.17 \\
1.24 \\
.75 \\
1.20 \\
5.08 \\
2.97 \\
.57\end{array}$ & $\begin{array}{r}4.13 \\
4.13 \\
.87 \\
4.13 \\
3.45 \\
2.67 \\
2.09 \\
2.61 \\
7.48 \\
4.63 \\
1.70\end{array}$ & $\begin{array}{r}8.88 \\
8.88 \\
8.88 \\
9.02 \\
7.16 \\
6.47 \\
4.87 \\
14.36 \\
9.55 \\
3.81\end{array}$ & $\begin{array}{r}9.16 \\
9.16 \\
9 . \overline{16} \\
9.99 \\
8.68 \\
5.93 \\
8.32 \\
17.61 \\
9.73 \\
3.91\end{array}$ & $\begin{array}{r}\text { R } 9.23 \\
R_{9.23} \\
R_{9.23} \\
9.32 \\
9.19 \\
5.68 \\
9.36 \\
R_{14.60} \\
9.56 \\
-\end{array}$ & $\begin{array}{r}\mathrm{R}_{8.86} \\
\mathrm{R}_{8.86} \\
\mathrm{R}_{8.86} \\
8.71 \\
8.28 \\
4.87 \\
8.34 \\
\mathrm{P}_{16.79} \\
9.31 \\
1.98\end{array}$ & $\begin{array}{r}R_{8.60} \\
{ }_{8.60} \\
{ }_{8} 8.60 \\
8.54 \\
8.12 \\
4.64 \\
5.97 \\
{ }_{19.43} \\
9.11 \\
R_{1.32}\end{array}$ & $\begin{array}{r}8.69 \\
8.69 \\
- \\
8.69 \\
8.24 \\
8.22 \\
4.33 \\
8.84 \\
18.94 \\
9.51 \\
1.51\end{array}$ \\
\hline 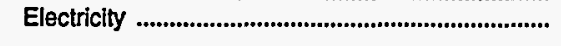 & - & - & . & - & - & - & - & (n) \\
\hline 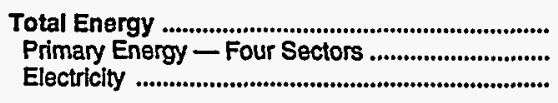 & $\begin{array}{l}1.86 \\
1.50 \\
6.10\end{array}$ & $\begin{array}{l}3.18 \\
2.63 \\
8.64\end{array}$ & $\begin{array}{r}6.91 \\
5.98 \\
13.26\end{array}$ & $\begin{array}{r}8.42 \\
7.12 \\
15.81\end{array}$ & $\begin{aligned} R_{8} 8.11 \\
R_{6.53} \\
15.68\end{aligned}$ & $\begin{array}{l}R_{7.95} \\
R_{6.27} \\
16.07\end{array}$ & $\begin{array}{l}R_{7.87} \\
R_{6.23} \\
16.24\end{array}$ & $\begin{array}{r}8.08 \\
6.47 \\
16.46\end{array}$ \\
\hline 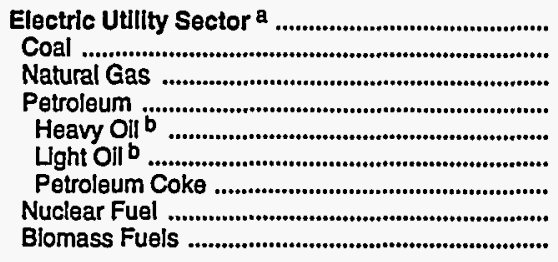 & $\begin{array}{l}.34 \\
.34 \\
.26 \\
.73 \\
.74 \\
.85 \\
.28 \\
.65\end{array}$ & $\begin{array}{r}.52 \\
.62 \\
.64 \\
2.03 \\
1.95 \\
2.26 \\
.54 \\
.24 \\
.92\end{array}$ & $\begin{array}{r}.91 \\
1.04 \\
1.99 \\
4.86 \\
4.46 \\
5.80 \\
. \overline{44} \\
1.74\end{array}$ & $\begin{array}{r}1.09 \\
1.43 \\
3.69 \\
5.96 \\
3.99 \\
5.97 \\
. \overline{-} \\
-\end{array}$ & $\begin{array}{r}1.03 \\
1.25 \\
1.92 \\
1.25 \\
1.86 \\
5.33 \\
.76 \\
.48 \\
.91\end{array}$ & $\begin{array}{l}1.02 \\
1.26 \\
1.70 \\
1.09 \\
2.16 \\
4.63 \\
.77 \\
.47 \\
.98\end{array}$ & $\begin{array}{r}.97 \\
1.19 \\
1.84 \\
.90 \\
1.47 \\
4.51 \\
.70 \\
.44 \\
.87\end{array}$ & $\begin{array}{r}.93 \\
1.13 \\
2.45 \\
.99 \\
1.65 \\
4.42 \\
.71 \\
.41 \\
.55\end{array}$ \\
\hline 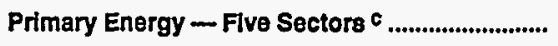 & 1.27 & 2.13 & 4.42 & 5.27 & 4.51 & 4.42 & $R_{4.45}$ & 4.59 \\
\hline
\end{tabular}

a There are no direct fuel costs for hydroelectric, geothermal, wind, photovoltalc, or solar thermal energy.

Heavy oil includes fuel oll nos. 4, 5, and 6, and residual fuel olls. Light oll includes fuel oll nos. 1 and 2, kerosene, and jet fuel.

c Blomass fuels are not included, except those consumed at electric

$R=$ Revised data.

-No consumption, Including cases where adjustments were made. See explanation of adjustments in Section 6 of Appendlx $A$.

Sources: Data sources, estimation procedures, and assumptions are utilities and those added to motor gasoline. 
(Million Dollars)

\begin{tabular}{|c|c|c|c|c|c|c|c|c|}
\hline Sector and Energy Source & 1970 & 1975 & 1980 & 1985 & 1990 & 1891 & 1992 & 1993 \\
\hline 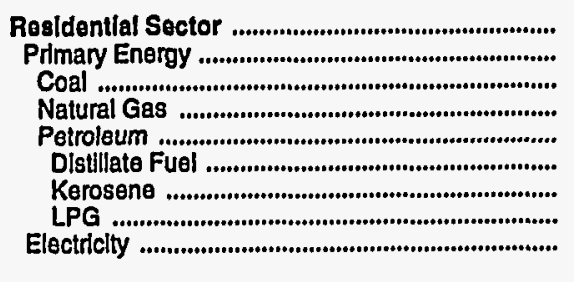 & $\begin{array}{r}455.3 \\
230.2 \\
6.5 \\
111.5 \\
112.2 \\
52.9 \\
10.3 \\
48.9 \\
225.0\end{array}$ & $\begin{array}{r}739.8 \\
395.8 \\
4.8 \\
179.5 \\
211.6 \\
107.6 \\
9.2 \\
94.8 \\
344.1\end{array}$ & $\begin{array}{r}1,315.8 \\
672.0 \\
4.5 \\
333.8 \\
333.7 \\
249.5 \\
5.2 \\
79.0 \\
643.8\end{array}$ & $\begin{array}{r}1,732.8 \\
872.5 \\
6.0 \\
618.7 \\
247.8 \\
173.6 \\
6.8 \\
67.4 \\
860.3\end{array}$ & $\begin{array}{r}1,745.8 \\
734.8 \\
3.9 \\
495.3 \\
235.7 \\
145.5 \\
1.5 \\
88.8 \\
1,011.0\end{array}$ & $\begin{array}{r}1,868.2 \\
785.2 \\
2.2 \\
529.5 \\
253.4 \\
166.2 \\
1.8 \\
85.4 \\
1,083.0\end{array}$ & $\begin{array}{r}R_{1,828.4} \\
R_{787.5} \\
.5 \\
551.9 \\
R_{235.0} \\
R_{125.5} \\
R_{1.5} \\
107.9 \\
1,041.0\end{array}$ & $\begin{array}{r}2,012.9 \\
907.9 \\
2.3 \\
655.3 \\
250.4 \\
118.9 \\
1.4 \\
130.0 \\
1,105.0\end{array}$ \\
\hline 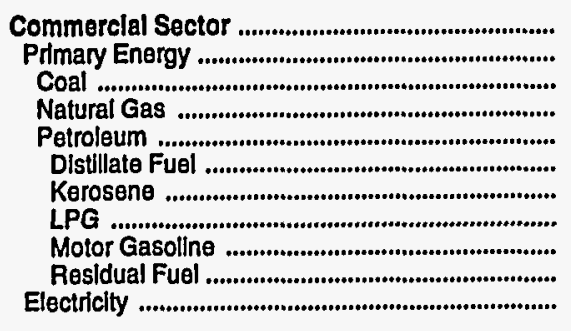 & $\begin{array}{r}163.0 \\
79.2 \\
3.7 \\
53.2 \\
22.3 \\
10.7 \\
1.3 \\
5.1 \\
3.7 \\
1.4 \\
83.7\end{array}$ & $\begin{array}{r}325.9 \\
154.3 \\
2.5 \\
104.2 \\
47.6 \\
24.1 \\
1.7 \\
10.3 \\
8.6 \\
2.8 \\
171.6\end{array}$ & $\begin{array}{r}522.6 \\
269.7 \\
3.4 \\
183.6 \\
82.7 \\
55.5 \\
- \\
9.3 \\
17.1 \\
.9 \\
252.8\end{array}$ & $\begin{array}{r}990.3 \\
543.4 \\
5.6 \\
400.2 \\
137.6 \\
101.1 \\
.9 \\
12.7 \\
17.1 \\
5.8 \\
446.9\end{array}$ & $\begin{array}{r}978.4 \\
446.4 \\
4.3 \\
310.5 \\
131.7 \\
31.5 \\
.2 \\
17.6 \\
78.3 \\
4.1 \\
532.0\end{array}$ & $\begin{array}{r}948.7 \\
385.7 \\
2.0 \\
327.2 \\
56.5 \\
25.9 \\
.1 \\
17.0 \\
9.7 \\
3.9 \\
563.0\end{array}$ & $\begin{array}{r}R_{938.8} \\
R_{380.8} \\
.5 \\
337.8 \\
R_{42.4} \\
R_{20.8} \\
2 \\
13.6 \\
5.6 \\
2.2 \\
558.0\end{array}$ & $\begin{array}{r}1,021.3 \\
442.3 \\
2.5 \\
391.6 \\
48.2 \\
19.0 \\
.3 \\
24.6 \\
2.4 \\
1.8 \\
579.0\end{array}$ \\
\hline 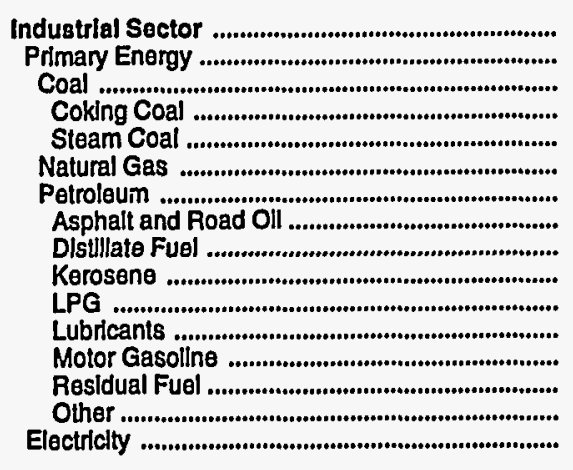 & $\begin{array}{r}323.8 \\
205.0 \\
20.8 \\
8.6 \\
12.2 \\
40.6 \\
143.6 \\
20.0 \\
37.5 \\
1.2 \\
5.6 \\
9.1 \\
56.3 \\
9.4 \\
4.4 \\
118.8\end{array}$ & $\begin{array}{r}725.0 \\
470.8 \\
67.6 \\
45.4 \\
22.2 \\
83.5 \\
319.7 \\
67.2 \\
111.0 \\
2.5 \\
18.8 \\
11.4 \\
76.1 \\
19.0 \\
13.5 \\
254.2\end{array}$ & $\begin{array}{r}1,356.2 \\
771.6 \\
31.9 \\
- \\
31.9 \\
251.7 \\
488.1 \\
78.4 \\
188.2 \\
3.3 \\
73.5 \\
28.2 \\
67.1 \\
22.0 \\
27.5 \\
584.6\end{array}$ & $\begin{array}{r}1,611.5 \\
855.9 \\
43.3 \\
- \\
43.3 \\
259.4 \\
553.2 \\
165.1 \\
172.5 \\
.9 \\
70.2 \\
31.4 \\
87.8 \\
6.2 \\
19.1 \\
755.7\end{array}$ & $\begin{array}{r}R_{1,729.0} \\
R_{780.7} \\
48.8 \\
- \\
48.8 \\
250.7 \\
R_{481.2} \\
132.5 \\
160.6 \\
.3 \\
79.5 \\
\text { R } 29.3 \\
55.8 \\
8.0 \\
15.2 \\
948.3\end{array}$ & $\begin{array}{r}R_{1,743.0} \\
R_{750.4} \\
28.5 \\
- \\
28.5 \\
243.8 \\
R_{478.2} \\
106.6 \\
170.2 \\
.3 \\
81.8 \\
R_{30.2} \\
70.5 \\
6.0 \\
12.5 \\
992.6\end{array}$ & $\begin{array}{r}R_{1,785.3} \\
R_{791.6} \\
37.8 \\
-. \\
37.8 \\
271.2 \\
R_{482.5} \\
95.9 \\
R_{182.6} \\
.3 \\
79.6 \\
R_{35.6} \\
67.8 \\
6.9 \\
13.8 \\
993.8\end{array}$ & $\begin{array}{r}1,878.7 \\
834.8 \\
49.5 \\
- \\
49.5 \\
298.1 \\
487.2 \\
93.2 \\
162.1 \\
.5 \\
112.0 \\
35.3 \\
61.0 \\
9.7 \\
13.3 \\
1,043.9\end{array}$ \\
\hline 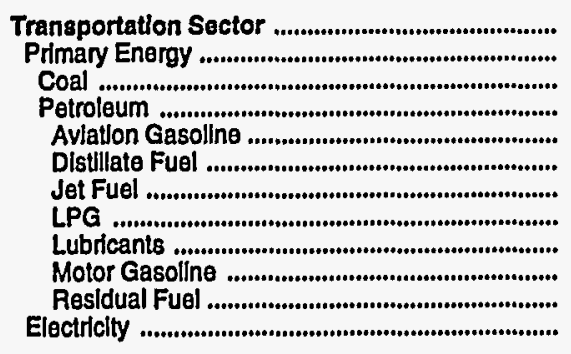 & $\begin{array}{r}703.1 \\
703.1 \\
(s) \\
703.1 \\
3.0 \\
36.6 \\
14.7 \\
.4 \\
19.3 \\
628.9 \\
1 \\
-\end{array}$ & $\begin{array}{r}1,303.8 \\
1,303.8 \\
(s) \\
1,303.7 \\
3.7 \\
104.1 \\
66.5 \\
.9 \\
34.1 \\
1,088.1 \\
6.2 \\
-\end{array}$ & $\begin{array}{r}2,864.5 \\
2,864.5 \\
- \\
2,864.5 \\
8.8 \\
338.4 \\
188.3 \\
1.2 \\
69.3 \\
2,235.3 \\
23.2 \\
-\end{array}$ & $\begin{array}{r}2,988.3 \\
2,988.3 \\
-\overrightarrow{3} \\
2,988.3 \\
7.8 \\
403.6 \\
261.4 \\
3.7 \\
77.4 \\
2,230.6 \\
3.8 \\
-\end{array}$ & $\begin{array}{r}R_{3,008.5} \\
R_{3,008.5} \\
- \\
R_{3,008.5} \\
10.0 \\
508.9 \\
164.0 \\
1.9 \\
A_{72.2} \\
2,251.5 \\
- \\
-\end{array}$ & $\begin{array}{r}A_{3,047.0} \\
A_{3,047.0} \\
- \\
A_{3,047.0} \\
8.3 \\
507.6 \\
137.3 \\
1.6 \\
A_{74.3} \\
2,318.0 \\
(s) \\
-\end{array}$ & $\begin{array}{r}A_{3,104.0} \\
A_{3,104.0} \\
- \\
A_{3,104.0} \\
5.8 \\
529.2 \\
173.8 \\
1.2 \\
R_{87.6} \\
2,306.4 \\
(8) \\
-\end{array}$ & $\begin{array}{r}3,372.5 \\
3,372.5 \\
- \\
3,372.5 \\
5.5 \\
543.6 \\
231.3 \\
3.2 \\
87.0 \\
2,502.1 \\
(\mathrm{~s}) \\
-\end{array}$ \\
\hline 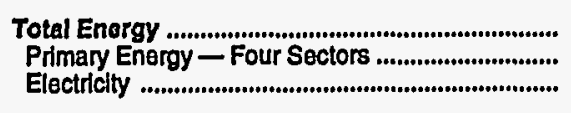 & $\begin{array}{r}1,645.1 \\
1,217.6 \\
427.5\end{array}$ & $\begin{array}{r}3,094.6 \\
2,324.7 \\
769.9\end{array}$ & $\begin{array}{l}6,059.1 \\
4,577.9 \\
1,481.2\end{array}$ & $\begin{array}{l}7,322.9 \\
5,260.1 \\
2,062.8\end{array}$ & $\begin{array}{r}R_{7,461.7} \\
R_{4,970.5} \\
2,491.2\end{array}$ & $\begin{array}{r}R_{7,608.9} \\
R_{4,968.3} \\
2,638.6\end{array}$ & $\begin{array}{r}R_{7,656.6} \\
R_{5,063.8} \\
2,592.8\end{array}$ & $\begin{array}{l}8,285.5 \\
5,557.6 \\
2,727.9\end{array}$ \\
\hline 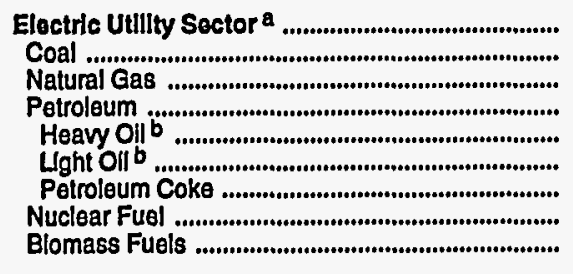 & $\begin{array}{r}65.3 \\
43.1 \\
15.3 \\
6.9 \\
3.9 \\
2.7 \\
.2 \\
-.1\end{array}$ & $\begin{array}{r}144.2 \\
84.9 \\
14.2 \\
19.5 \\
10.4 \\
8.9 \\
.2 \\
25.5 \\
(\mathrm{~s})\end{array}$ & $\begin{array}{r}311.3 \\
230.9 \\
16.0 \\
15.8 \\
10.1 \\
5.6 \\
- \\
48.6 \\
(s)\end{array}$ & $\begin{array}{r}355.5 \\
286.5 \\
4.7 \\
1.7 \\
(8) \\
1.7 \\
- \\
62.5 \\
-\end{array}$ & $\begin{array}{r}454.0 \\
372.2 \\
10.1 \\
6.2 \\
(8) \\
2.8 \\
3.3 \\
61.7 \\
3.7\end{array}$ & $\begin{array}{r}439.2 \\
357.4 \\
10.1 \\
6.9 \\
(8) \\
2.4 \\
4.4 \\
60.7 \\
4.1\end{array}$ & $\begin{array}{r}402.9 \\
332.1 \\
9.1 \\
6.1 \\
(\mathrm{~s}) \\
1.6 \\
4.5 \\
52.0 \\
3.6\end{array}$ & $\begin{array}{r}409.7 \\
337.8 \\
9.7 \\
6.9 \\
(8) \\
2.3 \\
4.6 \\
52.9 \\
2.4\end{array}$ \\
\hline Primary Energy - Five Sectors ${ }^{\circ} \ldots . . . \ldots . . . . . . . . . . . . . . . .$. & $1,282.9$ & $2,468.9$ & $4,889.2$ & $5,615.6$ & $R_{5,424.5}$ & $A_{5,407.5}$ & $\mathrm{~A}_{5,466.6}$ & $5,967.2$ \\
\hline
\end{tabular}

a There are no direct fuel costs for hydroelectric, geothermal, wind, photovoltalc, or solar thermal energy.

b Heavy oll includes fuel oll nos. 4, 5, and 6, and residual fuel oils. Light oll includes fuel nos. 1 and 2, kerosene, and jet fuel.

- Blomass fuels are not included, except those consumed at electric uttllites and those added to motor gasoline.

$R=$ Revised data.
-No consumption, Inciuding cases where adjustments were made. See explanation of adjustments in Section 6 of Appendix A.

(s)=Value less than 0.05 million dollars.

Note: Totals may not equal sum of components due to independent rounding.

Sources: Data sources, estimation procedures, and assumptions are described In Appendlx A. 
M Energy Price and Expenditure Estimates by Source, Mississippi 1970, 1975, 1980, 1985, and 1990-1993

\begin{tabular}{|c|c|c|c|c|c|c|c|c|}
\hline Energy Source & 1970 & 1975 & 1980 & 1985 & 1990 & 1991 & 1992 & 1993 \\
\hline & \multicolumn{8}{|c|}{ Prices in Dollars per Million Btu } \\
\hline Coal & 0.26 & 0.83 & 1.84 & 2.50 & 1.66 & 1.67 & 1.60 & 1.64 \\
\hline 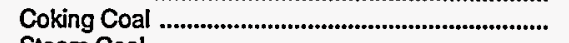 & - & - & - & - & - & - & - & - \\
\hline 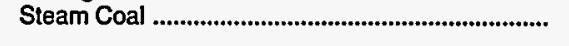 & .26 & .83 & 1.84 & 2.50 & 1.66 & 1.67 & 1.60 & 1.64 \\
\hline 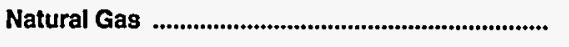 & .38 & .87 & 2.55 & 3.76 & 2.76 & 2.59 & 2.71 & 3.31 \\
\hline Petroleum & 2.12 & 3.10 & 7.04 & 7.59 & ${ }^{R} 7.23$ & $\mathrm{R}_{6.87}$ & $\mathrm{R}_{6.72}$ & 6.56 \\
\hline 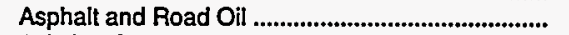 & .66 & 1.77 & 3.56 & 4.81 & 2.93 & 3.29 & 2.04 & 2.36 \\
\hline 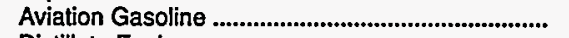 & 2.17 & 3.45 & 9.02 & 9.99 & 9.32 & 8.71 & 8.54 & 8.24 \\
\hline 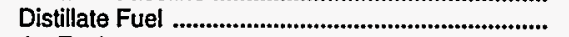 & 1.32 & 2.24 & 6.89 & 6.68 & 6.75 & 6.41 & 6.66 & 6.76 \\
\hline Jet Fuel & .73 & 2.03 & 6.39 & 5.84 & 5.16 & 4.59 & 4.33 & 3.96 \\
\hline 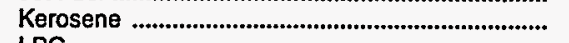 & .83 & 2.03 & 6.99 & 8.35 & 5.54 & 5.35 & 7.70 & 7.35 \\
\hline LPG & 1.83 & 3.42 & 6.31 & 7.40 & 6.38 & 7.21 & 5.99 & 7.54 \\
\hline 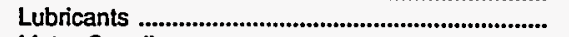 & 5.08 & 7.48 & 14.36 & 17.61 & $F_{14.60}$ & $R_{16.79}$ & $\mathrm{~F}_{19.43}$ & 18.94 \\
\hline 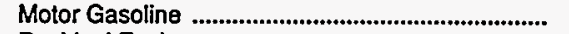 & 2.84 & 4.34 & 10.53 & 8.75 & 9.21 & 8.92 & 8.62 & 8.83 \\
\hline 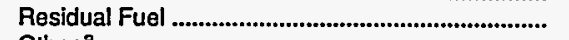 & .45 & 1.67 & 2.84 & 4.06 & 2.33 & 1.75 & $R_{1.70}$ & $1.69^{\circ}$ \\
\hline Other a & .50 & 1.48 & 3.39 & 4.93 & ${ }^{R} 5.16$ & $R_{4.29}$ & $R_{4.01}$ & 3.68 \\
\hline 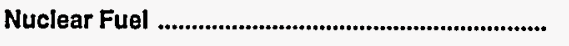 & - & - & - & 1.13 & 1.11 & .88 & .70 & .56 \\
\hline Biomass Fuels at Utilities & - & - & - & - & - & - & - & - \\
\hline Primary Energy - Five Sectors ${ }^{b}$ & 1.15 & 2.27 & 4.92 & 5.17 & $R_{4.58}$ & $R_{4.32}$ & 4.37 & 4.48 \\
\hline 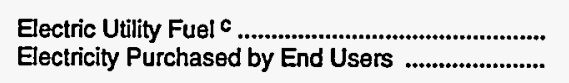 & $\begin{array}{r}.27 \\
4.44\end{array}$ & $\begin{array}{l}1.24 \\
7.58\end{array}$ & $\begin{array}{r}2.16 \\
13.69\end{array}$ & $\begin{array}{r}2.30 \\
17.05\end{array}$ & $\begin{array}{r}1.54 \\
18.05\end{array}$ & $\begin{array}{r}1.35 \\
17.79\end{array}$ & $\begin{array}{r}1.31 \\
17.84\end{array}$ & $\begin{array}{r}1.42 \\
18.28\end{array}$ \\
\hline \multirow[t]{2}{*}{ Total Energy } & 1.71 & 3.20 & 7.32 & 8.20 & ${ }^{R} 8.26$ & ${ }^{R} 7.93$ & $R_{7.83}$ & 8.29 \\
\hline & \multicolumn{8}{|c|}{ Expenditures in Millions of Dollars } \\
\hline Coal & 3.5 & 27.5 & 137.6 & 273.2 & 172.3 & 159.4 & 139.2 & 163.0 \\
\hline 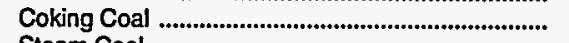 & - & - & - & - & - & - & - & - \\
\hline 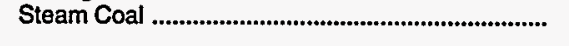 & 3.5 & 27.5 & 137.6 & 273.2 & 172.3 & 159.4 & 139.2 & 163.0 \\
\hline Natural Gas & 111.2 & 154.3 & - 553.4 & 710.7 & 557.5 & 524.1 & 529.3 & 589.5 \\
\hline Petroleum ............................. & 518.8 & $1,099.2$ & $2,475.0$ & $2,336.5$ & $R_{2,583.4}$ & $R_{2,531.6}$ & $\mathrm{R}_{2,540.4}$ & $2,583.5$ \\
\hline 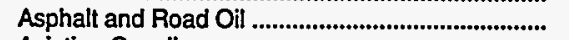 & 7.7 & 30.4 & 48.1 & 65.5 & 48.8 & 55.2 & 29.4 & 30.5 \\
\hline 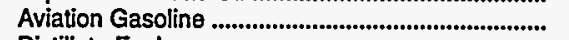 & 3.5 & 3.5 & 9.4 & 5.4 & 6.2 & 4.8 & 4.1 & 3.5 \\
\hline 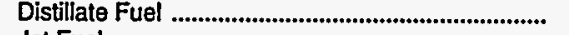 & 46.2 & 127.6 & 383.8 & 619.3 & 633.8 & 576.4 & 594.0 & 578.4 \\
\hline 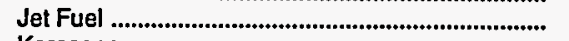 & 6.3 & 16.3 & 53.3 & 134.1 & 201.1 & 208.6 & 269.5 & 186.0 \\
\hline 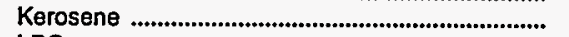 & 12.5 & 16.5 & 9.6 & 4.1 & 1.7 & 1.9 & 1.7 & 2.7 \\
\hline LPG & 59.4 & 102.3 & 125.9 & 124.4 & 163.8 & 158.8 & 134.5 & 120.4 \\
\hline 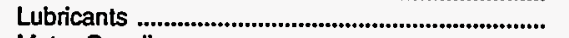 & 16.2 & 30.9 & 57.1 & 63.7 & ${ }^{R} 59.4$ & ${ }_{61.1}$ & R 72.1 & 71.6 \\
\hline 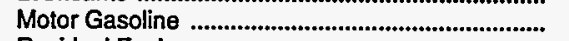 & 362.5 & 633.5 & $1,481.0$ & $1,267.2$ & $1,399.1$ & $1,396.1$ & $1,382.8$ & $1,480.4$ \\
\hline 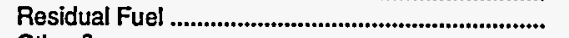 & 1.9 & 126.6 & 284.7 & 33.5 & 50.2 & 51.3 & A 35.6 & 95.2 \\
\hline Other ${ }^{a}$ & 2.7 & 11.6 & 22.3 & 19.2 & $R_{19.2}$ & $R_{17.4}$ & $R_{16.7}$ & 14.9 \\
\hline Nuclear Fuel & - & - & - & 53.1 & 87.9 & 86.7 & 61.3 & 47.0 \\
\hline 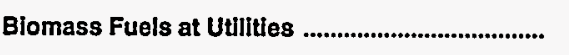 & - & - & - & - & - & - & - & - \\
\hline Primary Energy - Five Sectors ${ }^{b}$ & 633.5 & $1,281.1$ & $3,166.1$ & $3,373.5$ & $\mathrm{R}_{3,401.1}$ & $\mathrm{~A}_{3,301.9}$ & $R_{3,270.2}$ & $3,383.0$ \\
\hline $\begin{array}{l}\text { Electric Utility Fuel c } \\
\text { Electricity Purchased by End Users }\end{array}$ & $\begin{array}{r}-31.7 \\
225.9\end{array}$ & $\begin{array}{r}-154.7 \\
486.0\end{array}$ & $\begin{array}{r}-438.6 \\
1,075.9\end{array}$ & $\begin{array}{r}-476.1 \\
1,455.8\end{array}$ & $\begin{array}{r}-387.1 \\
1,914.5\end{array}$ & $\begin{array}{r}-346.5 \\
1,940.5\end{array}$ & $\begin{array}{r}-298.8 \\
R_{1,958.0}\end{array}$ & $\begin{array}{r}-359.5 \\
2,087.0\end{array}$ \\
\hline Total Energy & 827.7 & $1,612.4$ & $3,803.3$ & $4,353.2$ & $R_{4,928.5}$ & $R_{4,895.8}$ & $R_{4,929.4}$ & $5,110.5$ \\
\hline
\end{tabular}

a Includes petroleum coke used at electric utilities.

b Biomass fuels are not included, except those consumed at electric utilities and those added to motor gasoline.

$c$ There are no direct fuel costs for hydroelectric, geothermal, wind, photovoltaic, or solar thermal energy.

$R=$ Revised data.
-No consumption, including cases where adjustments were made. Seo explanation of adjustments in Section 6 of Appendix A.

Note: Expenditure totals may not equal sum of components due to independent rounding.

Sources: Data sources, estimation procedures, and assumptions are described in Appendix A. 


\begin{tabular}{|c|c|c|c|c|c|c|c|c|}
\hline Sector and Energy Source & 1970 & 1975 & 1980 & 1985 & 1990 & 1991 & 1992 & 1993 \\
\hline 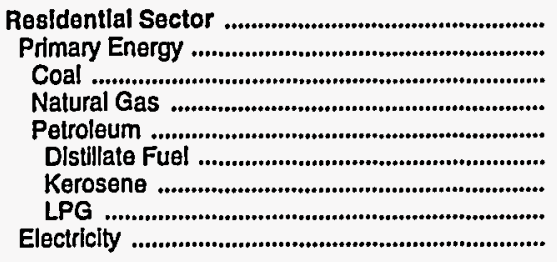 & $\begin{array}{r}2.39 \\
1.31 \\
.86 \\
2.14 \\
1.24 \\
2.06 \\
2.16 \\
5.06\end{array}$ & $\begin{array}{r}4.43 \\
2.34 \\
1.38 \\
3.98 \\
2.49 \\
3.79 \\
4.10 \\
8.06\end{array}$ & $\begin{array}{r}9.08 \\
4.45 \\
2.97 \\
3.36 \\
8.41 \\
6.89 \\
10.48 \\
8.35 \\
14.38\end{array}$ & $\begin{array}{r}12.18 \\
5.85 \\
2.74 \\
5.33 \\
7.78 \\
7.07 \\
10.96 \\
7.71 \\
18.12\end{array}$ & $\begin{array}{r}13.93 \\
6.17 \\
2.70 \\
5.16 \\
9.47 \\
4.59 \\
7.12 \\
9.50 \\
20.19\end{array}$ & $\begin{array}{r}14.03 \\
6.20 \\
2.81 \\
5.06 \\
10.61 \\
4.23 \\
6.55 \\
10.70 \\
20.16\end{array}$ & $\begin{array}{r}13.85 \\
5.56 \\
2.69 \\
4.70 \\
9.31 \\
7.66 \\
11.88 \\
9.28 \\
20.55\end{array}$ & $\begin{array}{r}14.26 \\
6.24 \\
2.73 \\
5.11 \\
10.30 \\
7.16 \\
11.10 \\
10.29 \\
20.87\end{array}$ \\
\hline 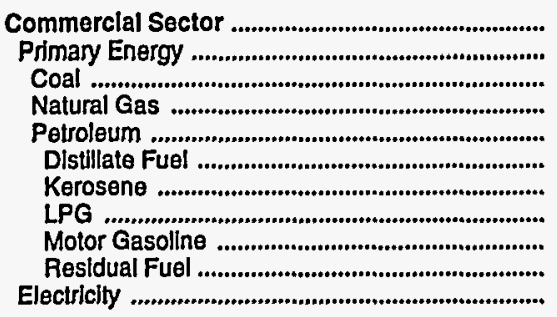 & $\begin{array}{r}1.96 \\
.70 \\
-57 \\
.59 \\
1.39 \\
.96 \\
-\overline{1.33} \\
2.84 \\
.49 \\
5.53\end{array}$ & $\begin{array}{r}3.34 \\
1.29 \\
. \overline{2} \\
2.17 \\
2.18 \\
2 . \overline{6} \\
4.34 \\
1.72 \\
8.59\end{array}$ & $\begin{array}{r}6.70 \\
3.17 \\
1.65 \\
2.97 \\
3.36 \\
6.27 \\
- \\
4.92 \\
10.53 \\
3.02 \\
15.87\end{array}$ & $\begin{array}{r}11.75 \\
5.37 \\
1.85 \\
4.95 \\
6.23 \\
5.74 \\
7.17 \\
7.19 \\
8.75 \\
4.33 \\
19.50\end{array}$ & $\begin{array}{r}13.17 \\
4.52 \\
1.74 \\
4.34 \\
5.09 \\
4.08 \\
5.08 \\
5.02 \\
9.21 \\
- \\
21.33\end{array}$ & $\begin{array}{r}13.11 \\
4.26 \\
1.74 \\
4.15 \\
4.65 \\
3.80 \\
4.64 \\
5.67 \\
8.92 \\
2.03 \\
21.24\end{array}$ & $\begin{array}{r}13.11 \\
4.28 \\
1.71 \\
3.92 \\
5.61 \\
5.04 \\
5.22 \\
4.71 \\
8.62 \\
2.08 \\
21.56\end{array}$ & $\begin{array}{r}13.58 \\
4.37 \\
1.64 \\
4.28 \\
4.88 \\
4.39 \\
5.12 \\
4.82 \\
8.83 \\
-\overline{14} \\
22.14\end{array}$ \\
\hline 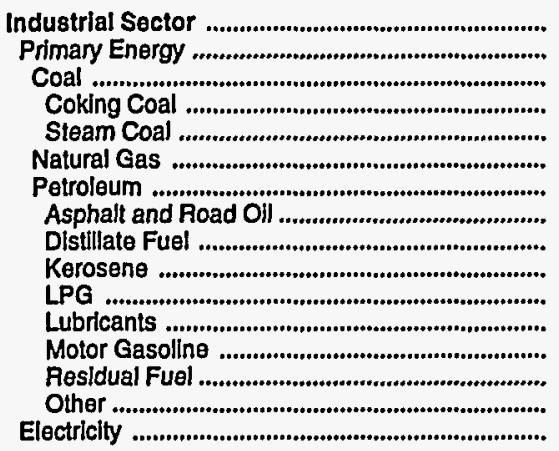 & $\begin{array}{r}.70 \\
.50 \\
.33 \\
- \\
.33 \\
.29 \\
.94 \\
.66 \\
.74 \\
.79 \\
1.33 \\
5.08 \\
2.84 \\
.40 \\
.50 \\
2.94\end{array}$ & $\begin{array}{r}1.94 \\
1.32 \\
1.11 \\
1 . \overline{11} \\
.71 \\
2.05 \\
1.77 \\
1.70 \\
1.86 \\
2.66 \\
7.48 \\
4.34 \\
1.77 \\
1.48 \\
6.39\end{array}$ & $\begin{array}{r}4.90 \\
3.61 \\
1.65 \\
- \\
1.65 \\
2.66 \\
4.61 \\
3.56 \\
5.55 \\
6.21 \\
4.92 \\
14.36 \\
10.53 \\
2.82 \\
3.39 \\
11.42\end{array}$ & $\begin{array}{r}6.13 \\
4.72 \\
1.85 \\
-\overline{1} \\
1.85 \\
3.68 \\
6.45 \\
4.81 \\
6.24 \\
7.17 \\
7.19 \\
17.61 \\
8.75 \\
4.33 \\
4.93 \\
13.94\end{array}$ & $\begin{array}{r}R_{5.27} \\
R_{3.43} \\
1.74 \\
-\overline{1.74} \\
2.49 \\
R_{4.64} \\
2.93 \\
4.42 \\
5.08 \\
5.02 \\
R_{14.60} \\
9.21 \\
3.02 \\
R_{5.16} \\
13.63\end{array}$ & $\begin{array}{r}A_{5} .21 \\
R_{3.29} \\
1.74 \\
-\overline{1.74} \\
2.28 \\
R_{4.77} \\
3.29 \\
4.04 \\
4.64 \\
5.67 \\
\text { A } 16.79 \\
8.92 \\
2.03 \\
R_{4.29} \\
13.16\end{array}$ & $\begin{array}{r}R_{5.25} \\
R_{3.26} \\
1.71 \\
-1.71 \\
2.40 \\
R_{4.62} \\
2.04 \\
4.54 \\
5.22 \\
4.71 \\
R_{19.43} \\
8.62 \\
2.08 \\
R_{4.01} \\
12.93\end{array}$ & $\begin{array}{r}5.78 \\
3.44 \\
1.64 \\
- \\
1.64 \\
2.92 \\
4.58 \\
2.36 \\
4.45 \\
5.12 \\
4.82 \\
18.94 \\
8.83 \\
2.02 \\
3.68 \\
13.45\end{array}$ \\
\hline 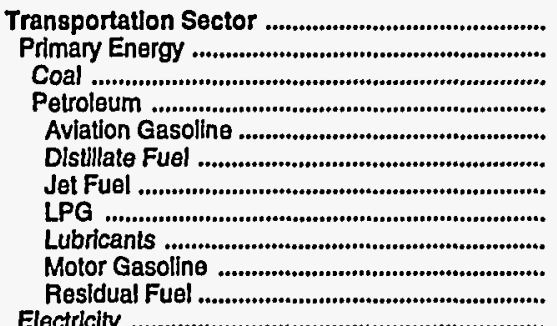 & $\begin{array}{r}2.64 \\
2.64 \\
.33 \\
2.64 \\
2.17 \\
2.02 \\
.73 \\
1.33 \\
5.08 \\
2.84 \\
.43\end{array}$ & $\begin{array}{l}3.91 \\
3.91 \\
1.11 \\
3.91 \\
3.45 \\
2.75 \\
2.03 \\
2.66 \\
7.48 \\
4.34 \\
1.49\end{array}$ & $\begin{array}{r}8.71 \\
8.71 \\
-\overrightarrow{8.71} \\
9.02 \\
7.67 \\
6.39 \\
4.92 \\
14.36 \\
10.53 \\
2.55\end{array}$ & $\begin{array}{r}7.97 \\
7.97 \\
- \\
7.97 \\
9.99 \\
7.05 \\
5.84 \\
7.19 \\
17.61 \\
8.75 \\
4.03\end{array}$ & $\begin{array}{r}R_{8.15} \\
R_{8.15} \\
- \\
R_{8.15} \\
9.32 \\
8.25 \\
5.16 \\
5.02 \\
R_{14.60} \\
9.21 \\
2.01\end{array}$ & $\begin{array}{r}R_{7.40} \\
R_{7.40} \\
- \\
R_{7.40} \\
8.71 \\
7.73 \\
4.59 \\
5.67 \\
R_{16.79} \\
8.92 \\
1.71\end{array}$ & $\begin{array}{r}R_{7.20} \\
R_{7.20} \\
R_{7.20} \\
8.54 \\
7.63 \\
4.33 \\
4.71 \\
\text { R }_{19.43} \\
8.62 \\
R_{1.65}\end{array}$ & $\begin{array}{r}7.38 \\
7.38 \\
- \\
7.38 \\
8.24 \\
7.66 \\
3.96 \\
4.82 \\
18.94 \\
8.83 \\
1.58\end{array}$ \\
\hline 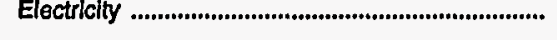 & - & - & - & - & - & - & - & - \\
\hline 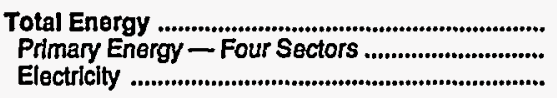 & $\begin{array}{l}1.71 \\
1.39 \\
4.44\end{array}$ & $\begin{array}{l}3.20 \\
2.56 \\
7.58\end{array}$ & $\begin{array}{r}7.32 \\
6.18 \\
13.69\end{array}$ & $\begin{array}{r}8.20 \\
6.50 \\
17.05\end{array}$ & $\begin{array}{l}A_{8.26} \\
R_{6.14} \\
18.05\end{array}$ & $\begin{array}{l}\text { A }_{7.93} \\
\text { A }_{5.81} \\
17.79\end{array}$ & $\begin{array}{l}R 7.83 \\
R 5.72 \\
17.84\end{array}$ & $\begin{array}{r}8.29 \\
6.02 \\
18.28\end{array}$ \\
\hline 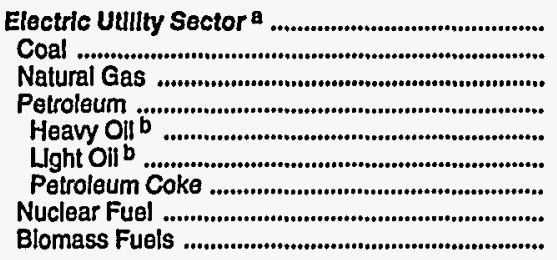 & $\begin{array}{l}.27 \\
.26 \\
.27 \\
.48 \\
.48 \\
.61 \\
- \\
- \\
-\end{array}$ & $\begin{array}{r}1.24 \\
.82 \\
.83 \\
1.70 \\
1.69 \\
2.08 \\
= \\
- \\
-\end{array}$ & $\begin{array}{r}2.16 \\
1.84 \\
2.11 \\
3.06 \\
3.03 \\
5.47 \\
- \\
- \\
-\end{array}$ & $\begin{array}{r}2.30 \\
2.54 \\
2.80 \\
4.78 \\
4.16 \\
5.97 \\
-\overline{13} \\
-\end{array}$ & $\begin{array}{r}1.54 \\
1.65 \\
1.76 \\
2.44 \\
2.35 \\
4.80 \\
-\overline{1} \\
1.11 \\
-\end{array}$ & $\begin{array}{r}1.35 \\
1.67 \\
1.57 \\
2.25 \\
1.94 \\
4.74 \\
-88 \\
-\end{array}$ & $\begin{array}{r}1.31 \\
1.60 \\
1.80 \\
1.96 \\
1.85 \\
4.68 \\
- \\
.70 \\
-\end{array}$ & $\begin{array}{r}1.42 \\
1.64 \\
2.42 \\
1.76 \\
1.75 \\
4.39 \\
- \\
.56 \\
-\end{array}$ \\
\hline Primary Energy - Five Sectors c ........................ & 1.15 & 2.27 & 4.92 & 5.17 & $R_{4.58}$ & $\mathrm{R}_{4.32}$ & 4.37 & 4.48 \\
\hline
\end{tabular}

a There are no direct fuel costs for hydroelectric, geothermal, wind, photovoltalc, or solar thermal energy.

b Heavy oil includes fuel oll nos. 4, 5, and 6, and residual fuel olls. Llght oll includes fuel oil nos. 1 and 2, kerosene, and let fuel.

c Biomass fuels are not included, except those consumed at electric utilitles and those added to motor gasoline.
R=Revised data.

- No consumption, including cases where adjustments were made. See explanation of adjustments in Section 6 of Appendix A.

Sources: Data sources, estimation procedures, and assumptions are described in Appendix A. 


\section{Energy Expenditure Estimates by Sector, Mississippi}

I 1970, 1975, 1980, 1985, and 1990-1993

$S$ (Million Dollars)

\begin{tabular}{|c|c|c|c|c|c|c|c|c|}
\hline Sector and Energy Source & 1970 & 1975 & 1980 & 1985 & 1990 & 1991 & 1992 & 1893 \\
\hline $\begin{array}{l}\text { Residentlal Sector } \\
\text { Primary Energy } \\
\text { Coal } \\
\text { Natural Gas } \\
\text { Petroleum } \\
\text { Distillate Fuel } \\
\text { Kerosene }\end{array}$ & $\begin{array}{r}194.5 \\
75.9 \\
- \\
32.4 \\
43.5 \\
.6 \\
.9 \\
42.0 \\
118.7\end{array}$ & $\begin{array}{r}334.1 \\
111.5 \\
-\overline{4} \\
41.6 \\
70.0 \\
2.8 \\
2.7 \\
64.4 \\
222.5\end{array}$ & $\begin{array}{r}661.9 \\
173.0 \\
(5) \\
102.6 \\
70.4 \\
.3 \\
2.6 \\
67.5 \\
488.9\end{array}$ & $\begin{array}{r}841.3 \\
195.3 \\
(s) \\
140.4 \\
54.9 \\
.1 \\
1.7 \\
53.2 \\
646.0\end{array}$ & $\begin{array}{r}1,053.3 \\
208.3 \\
(s) \\
133.5 \\
74.8 \\
(8) \\
.5 \\
74.3 \\
845.0\end{array}$ & $\begin{array}{r}1,068.1 \\
207.1 \\
(s) \\
134.2 \\
72.9 \\
(s) \\
.8 \\
72.0 \\
861.0\end{array}$ & $\begin{array}{r}1,061.8 \\
190.8 \\
(8) \\
131.1 \\
59.6 \\
.1 \\
1.0 \\
58.6 \\
871.0\end{array}$ & $\begin{array}{r}1,171.5 \\
231.6 \\
(8) \\
148.3 \\
23.3 \\
.1 \\
1.5 \\
81.6 \\
940.0\end{array}$ \\
\hline $\begin{array}{l}\text { Commercial Sector } \\
\text { Primary Energy } \\
\text { Coal } \\
\text { Natural Gas } \\
\text { Petroleum } \\
\text { Distillate Fuel } \\
\text { Kerosene }\end{array}$ & $\begin{array}{r}77.5 \\
20.5 \\
13.9 \\
6.7 \\
.6 \\
\overline{4} \\
1.6 \\
.1 \\
57.0\end{array}$ & $\begin{array}{r}161.8 \\
45.1 \\
22 . \overline{6} \\
22.5 \\
3.0 \\
7 . \overline{7} \\
2.4 \\
9.7 \\
116.7\end{array}$ & $\begin{array}{r}420.3 \\
143.5 \\
(s) \\
64.1 \\
79.4 \\
.9 \\
- \\
7.0 \\
6.8 \\
64.7 \\
276.8\end{array}$ & $\begin{array}{r}544.5 \\
136.6 \\
(s) \\
84.1 \\
52.5 \\
35.7 \\
1.6 \\
8.8 \\
6.2 \\
.3 \\
407.9\end{array}$ & $\begin{array}{r}646.7 \\
107.7 \\
(s) \\
78.6 \\
29.1 \\
14.0 \\
.2 \\
6.9 \\
8.0 \\
539.0\end{array}$ & $\begin{array}{r}642.1 \\
100.1 \\
(s) \\
75.9 \\
24.1 \\
13.4 \\
.2 \\
6.7 \\
3.8 \\
(s) \\
542.0\end{array}$ & $\begin{array}{r}641.4 \\
102.4 \\
(8) \\
74.1 \\
28.3 \\
15.0 \\
.3 \\
5.2 \\
7.8 \\
(s) \\
539.0\end{array}$ & $\begin{array}{r}654.7 \\
101.7 \\
(8) \\
84.1 \\
17.6 \\
8.4 \\
.2 \\
6.8 \\
2.3 \\
553.0\end{array}$ \\
\hline 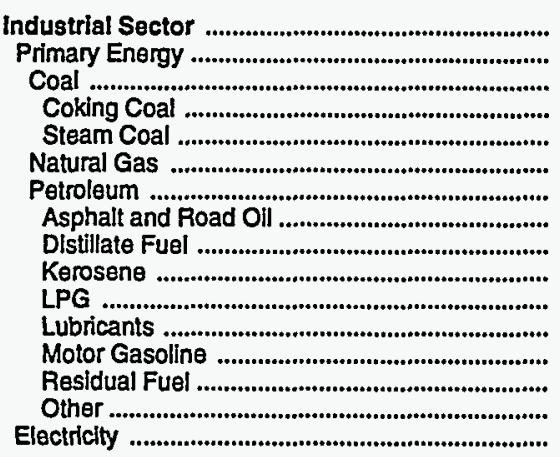 & $\begin{array}{r}146.6 \\
96.4 \\
.4 \\
- \\
.4 \\
37.6 \\
58.4 \\
7.7 \\
13.3 \\
11.6 \\
10.5 \\
7.5 \\
4.6 \\
.5 \\
2.7 \\
50.2\end{array}$ & $\begin{array}{r}365.8 \\
219.1 \\
.6 \\
- \\
.6 \\
63.2 \\
155.3 \\
30.4 \\
43.4 \\
13.8 \\
25.9 \\
17.0 \\
5.0 \\
8.3 \\
11.6 \\
146.7\end{array}$ & $\begin{array}{r}803.2 \\
492.9 \\
2.0 \\
\overline{2} \\
2.0 \\
182.6 \\
308.3 \\
48.1 \\
111.3 \\
7.0 \\
48.6 \\
29.7 \\
4.1 \\
37.3 \\
22.3 \\
310.2\end{array}$ & $\begin{array}{r}1,151.1 \\
749.2 \\
10.7 \\
- \\
10.7 \\
330.6 \\
407.8 \\
65.5 \\
196.0 \\
.8 \\
56.4 \\
33.1 \\
34.5 \\
2.2 \\
19.2 \\
401.9\end{array}$ & $\begin{array}{r}R_{1}, 134.7 \\
R_{604.2} \\
10.9 \\
-\overline{1} \\
10.9 \\
226.3 \\
R_{366.9} \\
48.8 \\
145.9 \\
1.0 \\
80.2 \\
R_{30.9} \\
27.8 \\
13.1 \\
R_{19.2} \\
530.5\end{array}$ & $\begin{array}{r}R_{1}, 090.4 \\
R_{552.9} \\
9.8 \\
- \\
9.8 \\
213.4 \\
R_{329.7} \\
55.2 \\
113.6 \\
.9 \\
77.8 \\
R_{31.8} \\
31.4 \\
1.6 \\
R_{17.4} \\
537.5\end{array}$ & $\begin{array}{r}\mathrm{A}_{1,079.7} \\
\mathrm{R}_{531.7} \\
9.9 \\
-\overline{9} \\
9.9 \\
223.9 \\
\mathrm{R}_{297.9} \\
29.4 \\
114.7 \\
.5 \\
69.1 \\
\mathrm{~A}_{37.5} \\
28.9 \\
1.1 \\
R_{16.7} \\
\mathrm{R}_{548.0}\end{array}$ & $\begin{array}{r}1,094.4 \\
500.4 \\
10.4 \\
-\overline{1} \\
10.4 \\
258.6 \\
231.4 \\
30.5 \\
97.3 \\
1.0 \\
30.2 \\
37.2 \\
17.8 \\
2.5 \\
14.9 \\
594.0\end{array}$ \\
\hline 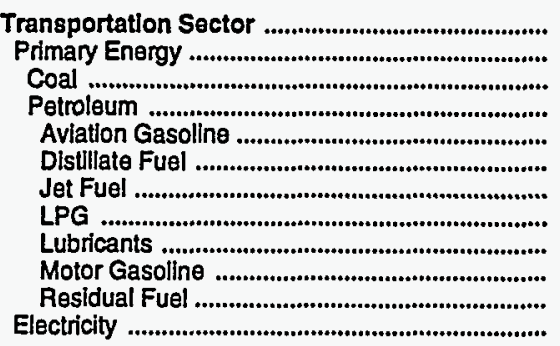 & $\begin{array}{r}409.1 \\
409.1 \\
(\mathrm{~s}) \\
409.1 \\
3.5 \\
31.6 \\
6.3 \\
2.4 \\
8.7 \\
356.5 \\
(\mathrm{~s}) \\
-\end{array}$ & $\begin{array}{r}750.7 \\
750.7 \\
(s) \\
750.7 \\
3.5 \\
75.1 \\
16.3 \\
4.6 \\
13.9 \\
626.2 \\
11.1 \\
-\end{array}$ & $\begin{array}{r}1,918.0 \\
1,918.0 \\
1,918.0 \\
9.4 \\
269.0 \\
53.3 \\
2.7 \\
27.4 \\
1,470.2 \\
86.0 \\
-\end{array}$ & $\begin{array}{r}1,816.3 \\
1,816.3 \\
- \\
1,816.3 \\
5.4 \\
385.5 \\
134.1 \\
6.0 \\
30.6 \\
1,226.6 \\
28.1 \\
-\end{array}$ & $\begin{array}{r}R_{2,093.8} \\
R_{2,093.8} \\
R_{2,093.8} \\
6.2 \\
472.5 \\
201.1 \\
2.4 \\
R_{28.5} \\
1,363.4 \\
19.7 \\
-\end{array}$ & $\begin{array}{r}R_{2,085.3} \\
R_{2,095.3} \\
-R_{2,095.3} \\
4.8 \\
447.1 \\
208.6 \\
2.2 \\
R_{29.4} \\
1,360.9 \\
42.3 \\
-\end{array}$ & $\begin{array}{r}\mathrm{R}_{2,146.6} \\
\mathrm{R}_{2,146.6} \\
\mathrm{R}_{2,146.6} \\
4.1 \\
463.4 \\
269.5 \\
1.6 \\
\mathrm{R}_{34.6} \\
1,346.1 \\
\mathrm{R}_{27.2} \\
-\end{array}$ & $\begin{array}{r}2,189.8 \\
2,189.8 \\
-\overline{-189.8} \\
3.5 \\
471.6 \\
186.0 \\
1.8 \\
34.4 \\
1,460.3 \\
32.3 \\
-\end{array}$ \\
\hline 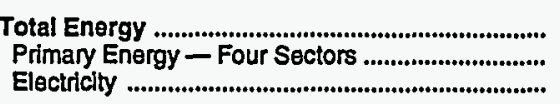 & $\begin{array}{l}827.7 \\
601.9 \\
225.9\end{array}$ & $\begin{array}{r}1,612.4 \\
1,126.4 \\
486.0\end{array}$ & $\begin{array}{l}3,803.3 \\
2,727.5 \\
1,075.9\end{array}$ & $\begin{array}{l}4,353.2 \\
2,897.4 \\
1,455.8\end{array}$ & $\begin{array}{r}R_{4,928.5} \\
R_{3,014.0} \\
1,914.5\end{array}$ & $\begin{array}{rl}R_{4} & 4,895.8 \\
R_{2,955.4} \\
1,940.5\end{array}$ & $\begin{array}{l}R_{4,929.4} \\
R_{2,971.4} \\
R_{1,958.0}\end{array}$ & $\begin{array}{l}5,110.5 \\
3,023.5 \\
2,087.0\end{array}$ \\
\hline 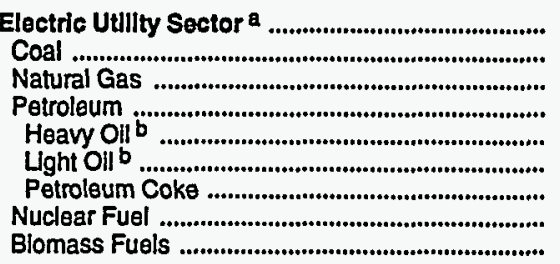 & $\begin{array}{r}31.7 \\
3.1 \\
27.3 \\
1.3 \\
1.2 \\
\text { (s) } \\
- \\
=\end{array}$ & $\begin{array}{r}154.7 \\
26.9 \\
26.9 \\
100.8 \\
97.6 \\
3.2 \\
= \\
=\end{array}$ & $\begin{array}{r}438.6 \\
135.5 \\
204.2 \\
98.9 \\
96.7 \\
2.2 \\
= \\
=\end{array}$ & $\begin{array}{r}476.1 \\
262.4 \\
155.6 \\
4.9 \\
2.8 \\
2.1 \\
-.1 \\
53.1\end{array}$ & $\begin{array}{r}387.1 \\
161.3 \\
119.1 \\
18.8 \\
17.4 \\
1.4 \\
-\overline{87.9} \\
-\end{array}$ & $\begin{array}{r}346.5 \\
149.6 \\
100.6 \\
9.5 \\
7.4 \\
2.2 \\
- \\
86.7 \\
-\end{array}$ & $\begin{array}{r}298.8 \\
129.3 \\
100.2 \\
8.0 \\
7.2 \\
.8 \\
- \\
61.3 \\
-\end{array}$ & $\begin{array}{r}359.5 \\
152.6 \\
88.5 \\
61.3 \\
60.4 \\
.9 \\
47.0 \\
-\end{array}$ \\
\hline 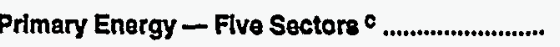 & 633.5 & $1,281.1$ & $3,166.1$ & $3,373.5$ & $A_{3,401.1}$ & $R_{3,301.0}$ & ค $_{3,270,2}$ & $3,383.0$ \\
\hline
\end{tabular}

a There are no direct fuel costs for hydroelectric, geothermal, wind, photovoltalc, or solar thermal energy.

$b$ Heavy oil includes fuel oil nos. 4, 5, and 6 , and residual tuel olls. Light oll includes fuel nos. 1 and 2, kerosene, and jet fuel.

c Blomass fuels are not included, except those consumed at electric utilitites and those added to motor gasoline.

$R=$ Revised data.

-No consumption, Including cases where adjustments were made. Seo explanation of adjustments in Section 6 of Appendix A.

(s)=Value less than 0.05 million collars.

Note: Totals may not equal sum of components due to independent rounding.

Sources: Data sources, estlmation procedures, and assumptions are described in Appendix A. 


\begin{tabular}{|c|c|c|c|c|c|c|c|c|}
\hline Energy Source & 1970 & 1975 & 1980 & 1985 & 1990 & 1991 & 1992 & 1993 \\
\hline & \multicolumn{8}{|c|}{ Prices in Dollars per Million Btu } \\
\hline 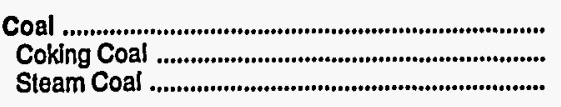 & $\begin{array}{r}0.29 \\
.38 \\
.28\end{array}$ & $\begin{array}{r}0.62 \\
1.60 \\
.60\end{array}$ & $\begin{array}{l}1.22 \\
1.81 \\
1.21\end{array}$ & $\begin{array}{l}1.51 \\
1.93 \\
1.51\end{array}$ & 1.35 & 1.34 & $1 . \overline{34}$ & 1.25 \\
\hline 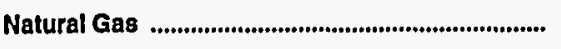 & .64 & 1.16 & 2.95 & 4.94 & 4.69 & 4.52 & 4.60 & 4.88 \\
\hline 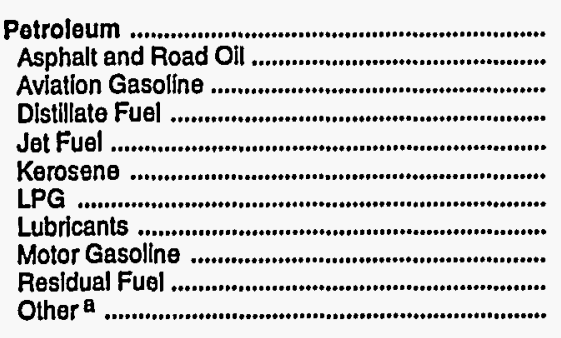 & $\begin{array}{r}1.97 \\
.71 \\
2.17 \\
1.05 \\
.75 \\
.89 \\
1.79 \\
5.08 \\
2.73 \\
.56 \\
1.44\end{array}$ & $\begin{array}{l}3.66 \\
2.05 \\
3.45 \\
2.52 \\
2.09 \\
2.45 \\
3.06 \\
7.48 \\
4.55 \\
1.78 \\
2.76\end{array}$ & $\begin{array}{r}8.04 \\
3.59 \\
9.02 \\
6.61 \\
6.47 \\
6.43 \\
6.32 \\
14.36 \\
9.33 \\
3.33 \\
7.23\end{array}$ & $\begin{array}{r}7.88 \\
4.77 \\
9.99 \\
6.70 \\
5.90 \\
7.78 \\
8.12 \\
17.61 \\
8.56 \\
4.09 \\
7.85\end{array}$ & $\begin{array}{r}R_{7.80} \\
3.11 \\
9.32 \\
7.49 \\
5.68 \\
8.34 \\
8.91 \\
R_{14.60} \\
8.61 \\
2.54 \\
R_{5.87}\end{array}$ & $\begin{array}{r}R_{7.43} \\
3.22 \\
8.71 \\
6.94 \\
4.81 \\
7.42 \\
7.96 \\
R_{16.79} \\
8.30 \\
2.15 \\
R_{5.50}\end{array}$ & $\begin{array}{r}R_{7.23} \\
2.46 \\
8.54 \\
6.69 \\
4.57 \\
5.86 \\
7.33 \\
R_{19.43} \\
8.11 \\
2.22 \\
R_{5.54}\end{array}$ & $\begin{array}{r}7.14 \\
2.86 \\
8.24 \\
6.64 \\
4.24 \\
6.15 \\
8.00 \\
18.94 \\
8.18 \\
2.23 \\
4.66\end{array}$ \\
\hline Nuclear Fuel ................................................................ & - & - & - & .82 & .74 & .71 & .58 & .56 \\
\hline 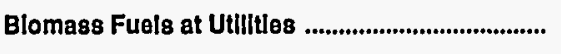 & - & - & - & - & - & - & - & .52 \\
\hline Prlmary Energy — Flve Sectors b ....................... & 1.16 & 2.08 & 4.38 & 4.58 & 4.52 & $\mathrm{R}_{4.31}$ & $R_{4.35}$ & 4.49 \\
\hline 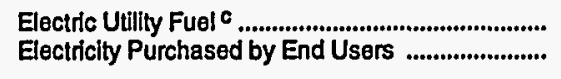 &. .26 & 8.64 & $\begin{array}{r}1.25 \\
13.91\end{array}$ & $\begin{array}{r}1.41 \\
17.16\end{array}$ & $\begin{array}{r}1.27 \\
18.94\end{array}$ & $\begin{array}{r}1.24 \\
18.95\end{array}$ & $\begin{array}{r}1.23 \\
18.79\end{array}$ & $\begin{array}{r}1.14 \\
18.55\end{array}$ \\
\hline \multirow[t]{2}{*}{ Total Energy } & 1.84 & 3.32 & 7.20 & 8.30 & $R_{8.84}$ & $\mathrm{R}_{8.67}$ & $R_{8.46}$ & 8.47 \\
\hline & \multicolumn{8}{|c|}{ Expenditures in Millions of Dollars } \\
\hline 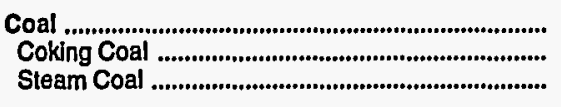 & $\begin{array}{r}80.3 \\
3.1 \\
77.2\end{array}$ & $\begin{array}{r}266.8 \\
11.9 \\
254.9\end{array}$ & $\begin{array}{r}647.4 \\
9.6 \\
637.7\end{array}$ & $\begin{array}{r}800.8 \\
12.0 \\
788.8\end{array}$ & $\begin{array}{c}728.0 \\
- \\
728.0\end{array}$ & $\begin{array}{c}717.7 \\
- \\
717.7\end{array}$ & $\begin{array}{c}699.3 \\
- \\
699.3\end{array}$ & $\begin{array}{c}582.1 \\
- \\
582.1\end{array}$ \\
\hline 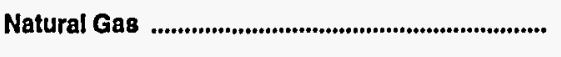 & 265.4 & 423.0 & 928.2 & $1,284.0$ & $1,107.8$ & $1,157.2$ & $1,099.3$ & $1,322.7$ \\
\hline 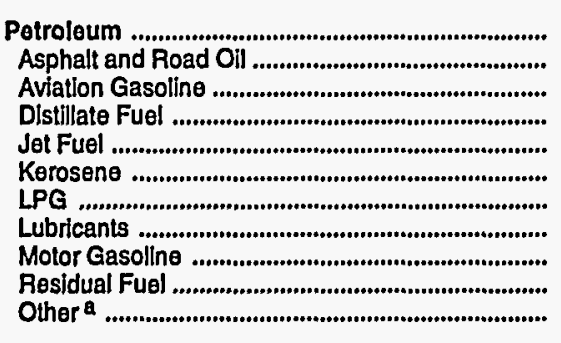 & $\begin{array}{r}1,127.1 \\
26.8 \\
2.0 \\
99.1 \\
34.1 \\
3.2 \\
79.5 \\
35.5 \\
803.2 \\
11.4 \\
32.4\end{array}$ & $\begin{array}{r}2,208.7 \\
73.3 \\
3.2 \\
261.8 \\
98.2 \\
3.9 \\
147.5 \\
58.3 \\
1,490.4 \\
21.7 \\
50.4\end{array}$ & $\begin{array}{r}4,698.9 \\
95.3 \\
7.4 \\
708.2 \\
229.5 \\
11.5 \\
211.7 \\
139.6 \\
2,889.0 \\
23.2 \\
383.7\end{array}$ & $\begin{array}{r}4,438.7 \\
135.9 \\
6.8 \\
765.2 \\
196.6 \\
6.6 \\
163.3 \\
155.8 \\
2,699.9 \\
18.8 \\
289.7\end{array}$ & $\begin{array}{r}R_{4,762.4} \\
92.2 \\
5.9 \\
905.3 \\
213.8 \\
2.1 \\
222.1 \\
R_{145.3} \\
2,879.3 \\
10.0 \\
R_{286.4}\end{array}$ & $\begin{array}{r}R_{4,573.5} \\
86.7 \\
5.1 \\
820.7 \\
204.2 \\
2.7 \\
248.2 \\
R_{149.5} \\
2,786.1 \\
7.4 \\
R_{262.8}\end{array}$ & $\begin{array}{r}R_{4,628.6} \\
62.6 \\
4.9 \\
R_{874.9} \\
194.6 \\
1.4 \\
225.0 \\
R_{176.4} \\
2,780.7 \\
9.3 \\
R_{298.6}\end{array}$ & $\begin{array}{r}4,750.4 \\
77.0 \\
3.9 \\
880.9 \\
216.8 \\
1.9 \\
276.6 \\
175.1 \\
2,840.4 \\
15.1 \\
262.8\end{array}$ \\
\hline Nuclear Fuel ................................................................. & - & - & - & 71.3 & 62.9 & 76.5 & 49.7 & 50.1 \\
\hline Blomass Fuels at Utllitles .................................... & - & - & - & - & - & - & - & (8) \\
\hline Primary Energy - Five Sectors b ...................... & $1,472.7$ & $2,898.5$ & $6,274.5$ & $6,594.8$ & $R_{6,661.1}$ & $R_{6,524.9}$ & $R_{6,476.9}$ & $6,705.3$ \\
\hline $\begin{array}{l}\text { Electric Utility Fuel c .................................................. } \\
\text { Electricity Purchased by End Users ................... }\end{array}$ & $\begin{array}{r}-76.3 \\
542.4\end{array}$ & $\begin{array}{r}-234.0 \\
974.3\end{array}$ & $\begin{array}{r}-639.6 \\
2,022.4\end{array}$ & $\begin{array}{r}-811.7 \\
2,712.0\end{array}$ & $\begin{array}{r}-754.7 \\
3,485.0\end{array}$ & $\begin{array}{r}-774.2 \\
3,654.0\end{array}$ & $\begin{array}{r}-715.7 \\
3,489.0\end{array}$ & $\begin{array}{r}-609.6 \\
3,710.0\end{array}$ \\
\hline 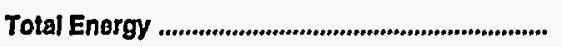 & $1,938.9$ & $3,638.8$ & $7,657.4$ & $8,495.2$ & $R_{9,391.4}$ & $\mathrm{R}_{\mathbf{9 , 4 0 4 . 7}}$ & $R_{9,250,2}$ & $9,805.8$ \\
\hline
\end{tabular}

\footnotetext{
a Includes petroleum coke used at electric utilities.

b Blomass fuels are not included, except those consumed at electric utllitles and those added to motor gasoline.

c There are no direct fuel costs for hydroelectric, geothermal, wind, photovoltalc, or solar thermal energy.

$\mathbf{R}=$ Revised data.

-No consumption, Including cases where adjustments were made. See
}

explanation of adjustments in Section 6 of Appendix A.

(s) $=$ Value less than 0.05 million dollars.

Note: Expenditure totals may not equal sum of components due to independent rounding.

Sources: Data sources, estimation procedures, and assumptions are described in Appendix A. 
M Energy Price Estimates by Sector, Missouri

$1970,1975,1980,1985$, and 1990-1993

$\mathbf{S}$ (Dollars per Million Btu)

\begin{tabular}{|c|c|c|c|c|c|c|c|c|}
\hline Sector and Energy Source & 1970 & 1975 & 1980 & 1985 & 1990 & 1991 & 1992 & 1993 \\
\hline 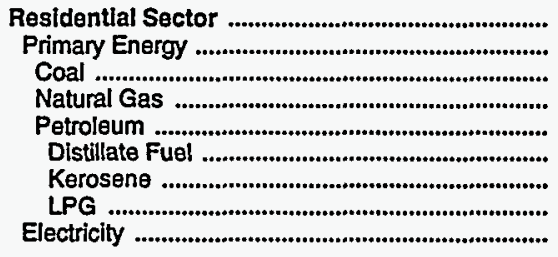 & $\begin{array}{r}2.09 \\
1.13 \\
.86 \\
.96 \\
1.81 \\
1.19 \\
1.43 \\
1.95 \\
7.86\end{array}$ & $\begin{array}{r}3.39 \\
1.85 \\
1.72 \\
1.48 \\
3.14 \\
2.62 \\
2.88 \\
3.26 \\
10.06\end{array}$ & $\begin{array}{r}6.88 \\
3.80 \\
1.70 \\
3.23 \\
7.01 \\
6.85 \\
7.95 \\
7.06 \\
15.21\end{array}$ & $\begin{array}{r}9.66 \\
5.60 \\
1.73 \\
5.40 \\
7.33 \\
6.70 \\
8.45 \\
7.53 \\
19.27\end{array}$ & $\begin{array}{r}11.22 \\
5.63 \\
1.56 \\
5.15 \\
9.33 \\
7.27 \\
9.16 \\
9.61 \\
21.56\end{array}$ & $\begin{array}{r}11.24 \\
5.55 \\
1.53 \\
5.10 \\
8.34 \\
6.75 \\
8.51 \\
8.54 \\
21.66\end{array}$ & $\begin{array}{r}10.98 \\
5.40 \\
1.33 \\
5.10 \\
7.31 \\
\text { R } 5.36 \\
\mathrm{R} 6.76 \\
7.52 \\
21.80\end{array}$ & $\begin{array}{r}10.90 \\
5.55 \\
1.72 \\
5.35 \\
7.05 \\
5.46 \\
6.88 \\
7.23 \\
21.29\end{array}$ \\
\hline 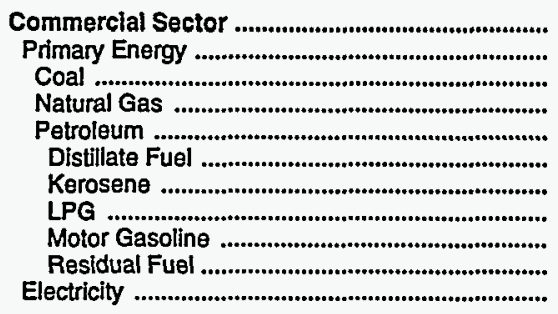 & $\begin{array}{r}1.66 \\
.69 \\
.49 \\
.62 \\
.93 \\
1.03 \\
.82 \\
1.27 \\
2.73 \\
.57 \\
7.00\end{array}$ & $\begin{array}{l}2.87 \\
1.36 \\
1.17 \\
1.14 \\
2.38 \\
2.45 \\
2.40 \\
2.49 \\
4.55 \\
1.77 \\
9.46\end{array}$ & $\begin{array}{r}6.87 \\
3.32 \\
1.58 \\
2.88 \\
5.74 \\
6.49 \\
6.10 \\
5.42 \\
9.33 \\
3.47 \\
14.33\end{array}$ & $\begin{array}{r}10.26 \\
5.06 \\
1.56 \\
4.88 \\
6.54 \\
5.75 \\
6.61 \\
9.12 \\
8.56 \\
4.11 \\
17.94\end{array}$ & $\begin{array}{r}11.38 \\
4.56 \\
1.31 \\
4.48 \\
6.44 \\
5.47 \\
6.86 \\
7.82 \\
8.61 \\
2.60 \\
18.98\end{array}$ & $\begin{array}{r}11.15 \\
4.48 \\
1.34 \\
4.46 \\
5.66 \\
4.79 \\
5.96 \\
6.94 \\
8.30 \\
2.21 \\
18.79\end{array}$ & $\begin{array}{r}11.20 \\
4.44 \\
1.35 \\
4.46 \\
5.23 \\
4.06 \\
R 5.03 \\
6.98 \\
8.11 \\
2.28 \\
18.80\end{array}$ & $\begin{array}{r}11.00 \\
4.75 \\
1.36 \\
4.74 \\
5.96 \\
3.99 \\
4.65 \\
9.21 \\
8.18 \\
2.26 \\
18.45\end{array}$ \\
\hline 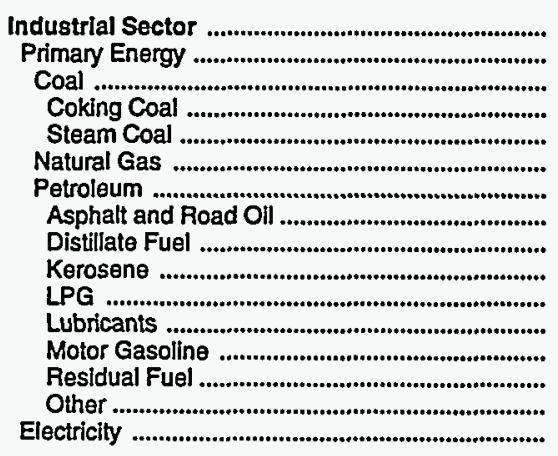 & $\begin{array}{r}1.12 \\
.77 \\
.47 \\
.38 \\
.49 \\
.40 \\
1.19 \\
.71 \\
.77 \\
.82 \\
1.27 \\
5.08 \\
2.73 \\
.53 \\
1.44 \\
4.01\end{array}$ & $\begin{array}{r}2.40 \\
1.75 \\
1.24 \\
1.60 \\
1.17 \\
.80 \\
2.69 \\
2.05 \\
2.25 \\
2.40 \\
2.49 \\
7.48 \\
4.55 \\
1.82 \\
2.77 \\
6.46\end{array}$ & $\begin{array}{r}5.40 \\
4.53 \\
1.61 \\
1.81 \\
1.58 \\
2.61 \\
6.39 \\
3.59 \\
5.83 \\
6.10 \\
5.42 \\
14.36 \\
9.33 \\
3.09 \\
7.30 \\
11.21\end{array}$ & $\begin{array}{r}6.39 \\
5.04 \\
1.62 \\
1.93 \\
1.56 \\
4.14 \\
6.93 \\
4.77 \\
6.00 \\
6.61 \\
9.12 \\
17.61 \\
8.56 \\
4.11 \\
7.85 \\
13.14\end{array}$ & $\begin{array}{r}R_{6.36} \\
R_{4.56} \\
1.31 \\
- \\
1.31 \\
4.14 \\
\mathrm{R}_{5.63} \\
3.11 \\
6.23 \\
6.86 \\
7.82 \\
\mathrm{R}_{14.60} \\
8.61 \\
2.60 \\
\mathrm{R}_{5.87} \\
14.50\end{array}$ & $\begin{array}{r}R_{6.27} \\
R_{4.42} \\
1.34 \\
\overline{1} \\
1.34 \\
4.04 \\
\mathrm{R}_{5.42} \\
3.22 \\
5.42 \\
5.96 \\
6.94 \\
\mathrm{R}_{16.79} \\
8.30 \\
2.21 \\
\mathrm{R}_{5.50} \\
14.37\end{array}$ & $\begin{array}{r}R_{6.11} \\
R_{4.31} \\
1.35 \\
-\overline{1} \\
1.35 \\
3.86 \\
R_{5.21} \\
2.46 \\
R_{4.57} \\
R_{5.03} \\
6.98 \\
R_{19.43} \\
8.11 \\
2.28 \\
R 5.54 \\
14.02\end{array}$ & $\begin{array}{r}6.16 \\
4.46 \\
1.36 \\
1.36 \\
4.23 \\
5.29 \\
2.86 \\
4.22 \\
4.65 \\
9.21 \\
18.94 \\
8.18 \\
2.26 \\
5.10 \\
13.82\end{array}$ \\
\hline $\begin{array}{l}\text { Transportation Sector } \\
\text { Primary Energy } \\
\text { Coal } \\
\text { Petroleum } \\
\text { Aviation Gasoline } \\
\text { Distillate Fuel } \\
\text { Jet Fuel } \\
\text { LPG }\end{array}$ & $\begin{array}{r}2.32 \\
2.32 \\
.49 \\
2.32 \\
2.17 \\
1.24 \\
.75 \\
1.27 \\
5.08 \\
2.73 \\
.55 \\
-\end{array}$ & $\begin{array}{l}4.07 \\
4.07 \\
1.17 \\
4.07 \\
3.45 \\
2.72 \\
2.09 \\
2.49 \\
7.48 \\
4.55 \\
1.73 \\
-\end{array}$ & $\begin{array}{r}8.76 \\
8.76 \\
- \\
8.76 \\
9.02 \\
6.97 \\
6.47 \\
5.42 \\
14.36 \\
9.33 \\
3.38 \\
-\end{array}$ & $\begin{array}{r}8.19 \\
8.19 \\
8.19 \\
9.99 \\
7.04 \\
5.90 \\
9.12 \\
17.61 \\
8.56 \\
3.88\end{array}$ & $\begin{array}{r}R_{8.30} \\
R_{8.30} \\
- \\
R_{8.30} \\
9.32 \\
7.87 \\
5.68 \\
7.82 \\
R_{14.60} \\
8.61 \\
1.65\end{array}$ & $\begin{array}{r}R 7.91 \\
\text { R } 7.91 \\
- \\
R 7.91 \\
8.71 \\
7.42 \\
4.81 \\
6.94 \\
\text { R } 16.79 \\
8.30 \\
-\end{array}$ & $\begin{array}{r}R 7.76 \\
R 7.76 \\
- \\
R 7.76 \\
8.54 \\
7.31 \\
4.57 \\
6.98 \\
R 19.43 \\
8.11 \\
1.31 \\
-\end{array}$ & $\begin{array}{r}7.70 \\
7.70 \\
- \\
7.70 \\
8.24 \\
7.26 \\
4.24 \\
9.21 \\
18.94 \\
8.18 \\
1.61 \\
-\end{array}$ \\
\hline 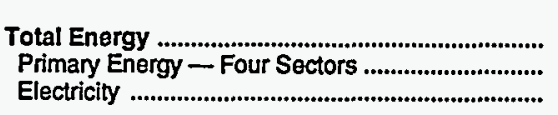 & $\begin{array}{l}1.84 \\
1.44 \\
6.17\end{array}$ & $\begin{array}{l}3.32 \\
2.71 \\
8.64\end{array}$ & $\begin{array}{r}7.20 \\
6.13 \\
13.91\end{array}$ & $\begin{array}{r}8.30 \\
6.68 \\
17.16\end{array}$ & $\begin{array}{c}R_{8.84} \\
R_{6.72} \\
18.94\end{array}$ & $\begin{array}{l}{ }^{R_{8.67}} \\
{ }^{R_{6.45}} \\
18.95\end{array}$ & $\begin{array}{l}R_{8.46} \\
R_{6.35} \\
18.79\end{array}$ & $\begin{array}{r}8.47 \\
6.37 \\
18.55\end{array}$ \\
\hline 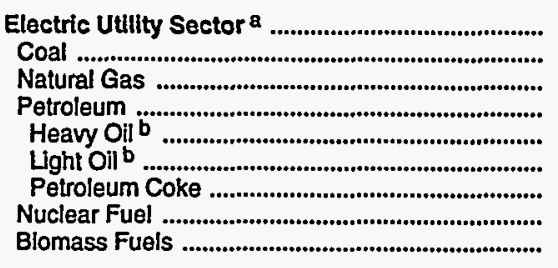 & $\begin{array}{l}.26 \\
.25 \\
.26 \\
.62 \\
.55 \\
.69 \\
- \\
= \\
-\end{array}$ & $\begin{array}{r}.57 \\
.54 \\
.59 \\
2.05 \\
1.74 \\
2.26 \\
.65 \\
-\end{array}$ & $\begin{array}{r}1.25 \\
1.19 \\
2.22 \\
5.07 \\
3.45 \\
6.02 \\
.67 \\
- \\
-\end{array}$ & $\begin{array}{r}1.41 \\
1.50 \\
3.31 \\
5.60 \\
3.99 \\
5.76 \\
1.38 \\
.82 \\
-\end{array}$ & $\begin{array}{r}1.27 \\
1.35 \\
1.72 \\
4.99 \\
1.80 \\
5.11 \\
. \overline{-74} \\
-\end{array}$ & $\begin{array}{r}1.24 \\
1.34 \\
1.49 \\
4.23 \\
1.33 \\
4.78 \\
.71 \\
-\end{array}$ & $\begin{array}{r}1.23 \\
1.34 \\
1.87 \\
3.92 \\
1.45 \\
4.26 \\
-58 \\
-5\end{array}$ & $\begin{array}{r}1.14 \\
1.24 \\
2.32 \\
1.58 \\
1.64 \\
4.01 \\
.64 \\
.56 \\
.52\end{array}$ \\
\hline Primary Energy — Five Sectors ${ }^{c} \ldots . . . . . . . . . . . . . . . . . . . . . . .$. & 1.16 & 2.08 & 4.38 & 4.58 & 4.52 & $R_{4.31}$ & $R_{4.35}$ & 4.49 \\
\hline
\end{tabular}

a There are no direct fuel costs for hydroelectric, geothermal, wind, photovoltalc, or solar thermal energy.

Heavy oil includes fuel oil nos. 4, 5, and 6, and residual fuel oils. Light oil includes fuel oil nos. 1 and 2, kerosene, and jet fuel.

c Biomass fuels are not included, except those consumed at electric

R=Revised data

-No consumption, including cases where adjustments were made. See explanation of adjustments in Section 6 of Appendix A.

Sources: Data sources, estimation procedures, and assumptions are utilitites and those added to motor gasoline.

described in Appendix A. 


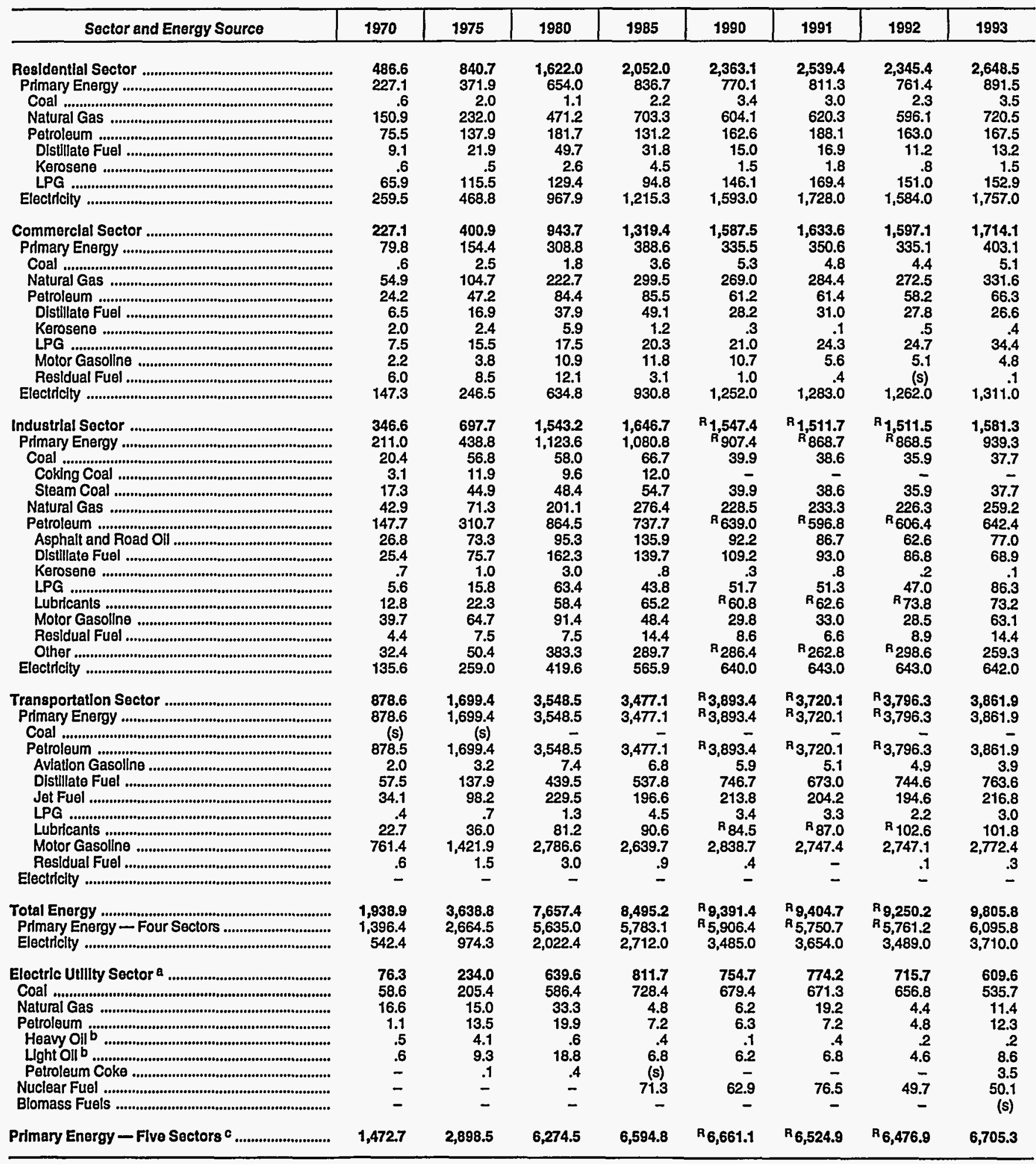

There are no direct fuel costs for hydroelectric, geothermal, wind, photovoltalc, or solar themal energy.

Heavy oll includes fuel oil nos. 4, 5, and 6, and residual fuel oils. Light oll Includes fuel nos. 1 and 2, kerosene, and jet fuel.

c Blomass fuels are not Included, except those consumed at electric utllitles and those added to motor gasoline.

$\mathrm{R}=$ Revised data.
-No consumption, including cases where adjustments were made. See explanation of adjustments in Section 6 of Appendix A.

(s) =Value less than 0.05 million dollars.

Note: Totals may not equal sum of components due to independent rounding.

Sources: Data sources, estimation procedures, and assumptions are described in Appendix A. 
M Energy Price and Expenditure Estimates by Source, Montana 1970, 1975, 1980, 1985, and 1990-1993

\begin{tabular}{|c|c|c|c|c|c|c|c|c|}
\hline Energy Source & 1970 & 1975 & 1980 & 1985 & 1990 & 1991 & 1992 & 1893 \\
\hline & \multicolumn{8}{|c|}{ Prices in Dollars per Million Btu } \\
\hline $\begin{array}{l}\text { Coal } \\
\text { Coking Coal } \\
\text { Steam Coal }\end{array}$ & $\begin{array}{r}0.22 \\
.22\end{array}$ & $\frac{0.33}{.33}$ & $\frac{0.53}{.53}$ & $\begin{array}{r}0.75 \\
.75\end{array}$ & $\frac{0.70}{.70}$ & $\begin{array}{l}R_{0.70} \\
R_{.70}\end{array}$ & $\begin{array}{r}R_{0.73} \\
R_{.73}\end{array}$ & $\frac{0.73}{.73}$ \\
\hline Natural Gas & .57 & 1.07 & 3.14 & 4.84 & 4.16 & 4.06 & 4.42 & 4.21 \\
\hline $\begin{array}{l}\text { Petroleum } \\
\text { Asphalt and Road Oil } \\
\text { Aviation Gasoline } \\
\text { Distillate Fuel full. } \\
\text { Jet Fuel } \\
\text { Kerosene } \\
\text { LPG }\end{array}$ & $\begin{array}{r}1.89 \\
.59 \\
2.17 \\
1.14 \\
.76 \\
.94 \\
2.10 \\
5.08 \\
2.89 \\
.39 \\
.52\end{array}$ & $\begin{array}{l}3.41 \\
1.84 \\
3.45 \\
2.58 \\
2.12 \\
2.63 \\
3.62 \\
7.48 \\
4.78 \\
1.99 \\
1.40\end{array}$ & $\begin{array}{r}7.13 \\
3.67 \\
9.02 \\
6.59 \\
6.59 \\
-\overline{6} \\
6.49 \\
14.36 \\
9.99 \\
3.28 \\
2.38\end{array}$ & $\begin{array}{r}7.37 \\
4.83 \\
9.99 \\
6.39 \\
6.64 \\
11.07 \\
7.60 \\
17.61 \\
9.16 \\
3.03 \\
2.17\end{array}$ & $\begin{array}{r}\mathrm{R}_{7.84} \\
2.64 \\
9.32 \\
7.58 \\
6.26 \\
6.62 \\
9.11 \\
\mathrm{R} 14.60 \\
9.56 \\
3.03 \\
2.02\end{array}$ & $\begin{array}{r}\mathrm{R} 7.66 \\
3.29 \\
8.71 \\
7.34 \\
5.47 \\
7.18 \\
9.87 \\
\mathrm{R} \\
16.79 \\
9.07 \\
2.36 \\
1.72\end{array}$ & $\begin{array}{r}\mathrm{R} 7.64 \\
2.86 \\
8.54 \\
7.52 \\
5.46 \\
7.04 \\
8.72 \\
\mathrm{R} \\
19.43 \\
9.39 \\
1.70 \\
1.29\end{array}$ & $\begin{array}{r}7.77 \\
2.80 \\
8.24 \\
7.59 \\
5.39 \\
8.39 \\
8.43 \\
18.94 \\
9.83 \\
2.32 \\
1.06\end{array}$ \\
\hline 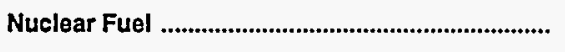 & - & - & - & - & - & - & - & - \\
\hline 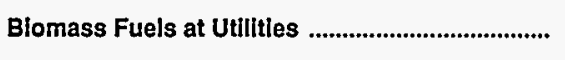 & .65 & .92 & 1.74 & .79 & (d) & (d) & (d) & (d) \\
\hline Primary Energy — Five Sectors ${ }^{b}$............................ & 1.26 & 2.41 & 4.79 & 4.69 & 3.82 & 3.63 & 3.63 & 4.06 \\
\hline 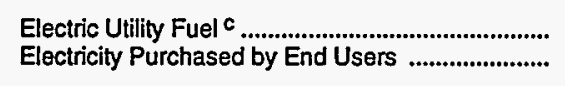 & $\begin{array}{r}.23 \\
2.88\end{array}$ & $\begin{array}{r}.34 \\
4.05\end{array}$ & $\begin{array}{r}.72 \\
5.80\end{array}$ & $\begin{array}{r}.72 \\
10.72\end{array}$ & $\begin{array}{r}.68 \\
11.67\end{array}$ & $\begin{array}{r}.68 \\
12.22\end{array}$ & $\begin{array}{r}.71 \\
12.39\end{array}$ & $\begin{array}{r}.70 \\
12.87\end{array}$ \\
\hline \multirow[t]{2}{*}{ 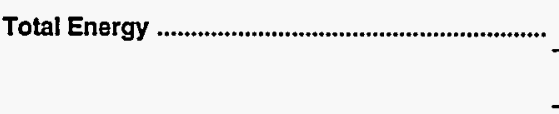 } & 1.56 & 2.78 & 6.02 & 7.48 & 7.81 & $R_{7.75}$ & $\mathrm{R}_{7.86}$ & 7.82 \\
\hline & \multicolumn{8}{|c|}{ Expenditures in Millions of Dollars } \\
\hline 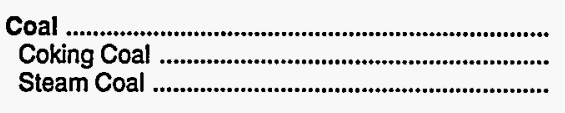 & $\begin{array}{l}2.6 \\
\overline{2.6}\end{array}$ & $\begin{array}{l}6.2 \\
-6.2\end{array}$ & $\begin{array}{l}31.8 \\
\overline{31.8}\end{array}$ & $\begin{array}{l}74.0 \\
74.0\end{array}$ & $\begin{array}{l}\mathrm{R}_{115.6} \\
\mathrm{R}_{115.6}\end{array}$ & $\begin{array}{l}R_{126.3} \\
R_{126.3}\end{array}$ & $\begin{array}{l}R_{138.5} \\
R_{138.5}\end{array}$ & $\begin{array}{l}115.0 \\
\overline{-} \\
115.0\end{array}$ \\
\hline 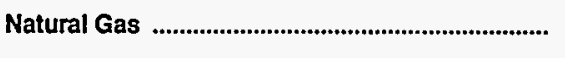 & 45.1 & 78.2 & 166.0 & 204.7 & 162.9 & 169.1 & 179.8 & 187.8 \\
\hline $\begin{array}{l}\text { Petroleum } \\
\text { Asphalt and Road Oil } \\
\text { Aviation Gasoline } \\
\text { Distillate Fuel } \\
\text { Jet Fuel } \\
\text { Kerosene } \\
\text { LPG }\end{array}$ & $\begin{array}{r}204.2 \\
5.3 \\
.5 \\
31.9 \\
2.7 \\
2.0 \\
9.9 \\
6.2 \\
140.7 \\
.7 \\
4.4\end{array}$ & $\begin{array}{r}461.4 \\
11.3 \\
1.4 \\
114.2 \\
9.7 \\
1.8 \\
17.1 \\
9.4 \\
266.6 \\
17.6 \\
12.3\end{array}$ & $\begin{array}{r}1,050.4 \\
24.9 \\
7.3 \\
288.2 \\
34.1 \\
- \\
41.9 \\
21.5 \\
546.8 \\
68.3 \\
17.4\end{array}$ & $\begin{array}{r}1,062.3 \\
46.9 \\
4.6 \\
421.3 \\
25.2 \\
.6 \\
39.8 \\
24.0 \\
490.2 \\
2.4 \\
7.1\end{array}$ & $\begin{array}{r}\text { R } 992.5 \\
26.0 \\
5.2 \\
327.6 \\
24.8 \\
.3 \\
55.5 \\
R 22.4 \\
515.5 \\
.2 \\
14.8\end{array}$ & $\begin{array}{r}R 972.4 \\
29.5 \\
4.8 \\
355.6 \\
19.0 \\
.1 \\
36.7 \\
R_{23.1} \\
493.3 \\
(s) \\
10.3\end{array}$ & $\begin{array}{r}\text { R } 992.7 \\
24.8 \\
3.3 \\
338.1 \\
26.3 \\
(s) \\
31.1 \\
R_{27.2} \\
529.3 \\
(s) \\
12.5\end{array}$ & $\begin{array}{r}1,089.5 \\
31.7 \\
2.6 \\
353.8 \\
27.1 \\
.4 \\
65.7 \\
27.0 \\
567.9 \\
4.8 \\
8.3\end{array}$ \\
\hline 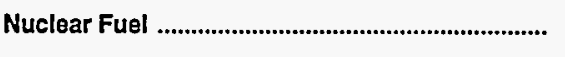 & - & - & - & - & - & - & - & - \\
\hline 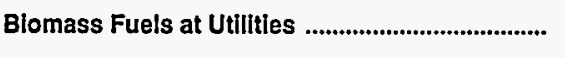 & .5 & .1 & .3 & .5 & $\left({ }^{d}\right)$ & (d) & $\left({ }^{d}\right)$ & $(d)$ \\
\hline 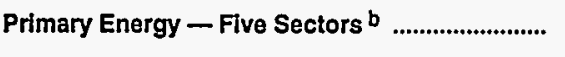 & 252.4 & 545.9 & $1,248.6$ & $1,341.5$ & $R_{1,271.1}$ & $R_{1,267.9}$ & $R_{1,310.9}$ & $1,402.4$ \\
\hline 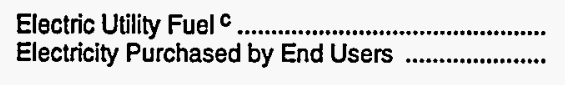 & $\begin{array}{r}-3.4 \\
84.1\end{array}$ & $\begin{array}{r}-6.4 \\
119.8\end{array}$ & $\begin{array}{r}-44.3 \\
207.7\end{array}$ & $\begin{array}{r}-69.2 \\
488.6\end{array}$ & $\begin{array}{r}-110.6 \\
510.4\end{array}$ & $\begin{array}{r}-119.3 \\
545.8\end{array}$ & $\begin{array}{r}-132.7 \\
542.0\end{array}$ & $\begin{array}{r}-106.7 \\
555.3\end{array}$ \\
\hline Total Energy & 333.1 & 659.3 & $1,412.0$ & $1,760.9$ & $R_{1,670.8}$ & $R_{1,694.4}$ & $R_{1,720.3}$ & $1,850.9$ \\
\hline
\end{tabular}

a Includes petroleum coke used at electric utilities.

b Biomass fuels are not included, except those consumed at electric utilities and those added to motor gasoline.

c There are no direct fuel costs for hydroelectric, geothermal, wind, photovoltaic, or solar thermal energy.

Utilities used biomass fuels at no charge or received a fee for accepting

R=Revised data.

-No consumption, including cases where adjustments were made. See explanation of adjustments in Section 6 of Appendix A.

Note: Expenditure totals may not equal sum of components due to independent rounding.

Sources: Data sources, estimation procedures, and assumptions are them.

described in Appendix A. 
(Dollars per Million Btu)

\begin{tabular}{|c|c|c|c|c|c|c|c|c|}
\hline Sector and Energy Source & 1970 & 1975 & 1980 & 1985 & 1990 & 1991 & 1992 & 1993 \\
\hline 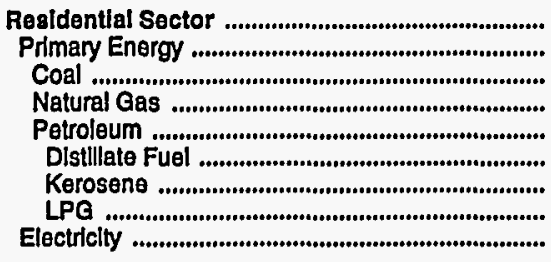 & $\begin{array}{r}1.87 \\
1.06 \\
.80 \\
.88 \\
2.03 \\
1.28 \\
2.35 \\
6.57\end{array}$ & $\begin{array}{r}2.73 \\
1.74 \\
1.06 \\
1.27 \\
3.37 \\
2.84 \\
-\overline{3.88} \\
7.02\end{array}$ & $\begin{array}{r}5.36 \\
3.91 \\
1.35 \\
3.02 \\
7.08 \\
6.92 \\
7 . \overline{-} \\
9.04\end{array}$ & $\begin{array}{r}8.27 \\
5.40 \\
.98 \\
4.82 \\
8.10 \\
7.92 \\
11.40 \\
8.18 \\
13.77\end{array}$ & $\begin{array}{r}R_{8.92} \\
R_{5.30} \\
R_{1.32} \\
4.47 \\
8.69 \\
6.42 \\
9.23 \\
9.99 \\
15.97\end{array}$ & $\begin{array}{r}R_{g .03} \\
R_{5.15} \\
R_{1.16} \\
4.40 \\
8.48 \\
6.11 \\
8.79 \\
10.42 \\
16.86\end{array}$ & $\begin{array}{r}9.38 \\
R 5.17 \\
R 1.26 \\
4.70 \\
7.67 \\
5.99 \\
8.61 \\
8.65 \\
17.12\end{array}$ & $\begin{array}{r}9.08 \\
5.11 \\
1.27 \\
4.84 \\
6.79 \\
6.02 \\
8.66 \\
7.36 \\
16.94\end{array}$ \\
\hline 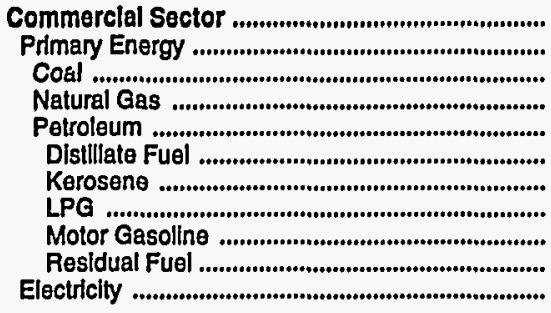 & $\begin{array}{r}1.51 \\
.78 \\
.48 \\
.60 \\
1.64 \\
1.06 \\
.94 \\
1.49 \\
2.89 \\
.34 \\
5.74\end{array}$ & $\begin{array}{r}2.39 \\
1.49 \\
.79 \\
1.07 \\
2.89 \\
2.49 \\
2.63 \\
2.77 \\
4.78 \\
2.03 \\
6.39\end{array}$ & $\begin{array}{r}5.12 \\
3.76 \\
2.04 \\
3.12 \\
6.87 \\
6.45 \\
5 . \overline{5} \\
9.99 \\
4.42 \\
8.50\end{array}$ & $\begin{array}{r}8.25 \\
5.39 \\
1.66 \\
5.10 \\
6.09 \\
6.25 \\
6.85 \\
7.19 \\
9.16 \\
3.03 \\
12.49\end{array}$ & $\begin{array}{r}8.41 \\
R 4.69 \\
R 1.54 \\
4.52 \\
6.93 \\
5.13 \\
6.20 \\
8.30 \\
9.56 \\
3.03 \\
13.49\end{array}$ & $\begin{array}{r}8.57 \\
4.39 \\
R \quad 1.59 \\
4.23 \\
6.16 \\
4.46 \\
6.58 \\
8.68 \\
9.07 \\
2.36 \\
14.36\end{array}$ & $\begin{array}{r}9.28 \\
R_{4.58} \\
R \quad 1.52 \\
4.36 \\
6.58 \\
5.01 \\
6.18 \\
8.82 \\
9.39 \\
1.70 \\
14.85\end{array}$ & $\begin{array}{r}9.00 \\
4.72 \\
1.51 \\
4.59 \\
6.20 \\
5.31 \\
6.33 \\
8.79 \\
9.83 \\
2.32 \\
14.68\end{array}$ \\
\hline 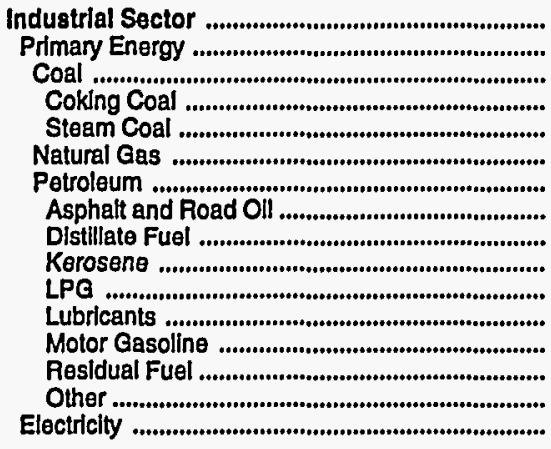 & $\begin{array}{r}.80 \\
.64 \\
.48 \\
-\overline{.48} \\
.33 \\
.95 \\
.59 \\
.87 \\
.94 \\
1.49 \\
5.08 \\
2.89 \\
.45 \\
.52 \\
1.33\end{array}$ & $\begin{array}{r}1.79 \\
1.75 \\
.79 \\
-79 \\
.79 \\
.93 \\
2.32 \\
1.84 \\
2.44 \\
2.63 \\
2.77 \\
7.48 \\
4.78 \\
1.99 \\
1.40 \\
1.96\end{array}$ & $\begin{array}{r}3.73 \\
3.91 \\
2.04 \\
-\overline{2} \\
2.04 \\
3.11 \\
4.23 \\
3.67 \\
5.19 \\
- \\
5.85 \\
14.36 \\
9.99 \\
3.28 \\
2.38 \\
3.05\end{array}$ & $\begin{array}{r}5.91 \\
5.49 \\
1.66 \\
- \\
1.66 \\
4.71 \\
5.89 \\
4.83 \\
6.02 \\
6.85 \\
7.19 \\
17.61 \\
9.16 \\
3.03 \\
2.17 \\
7.35\end{array}$ & $\begin{array}{r}R_{5.40} \\
R_{4.18} \\
R_{1.54} \\
- \\
R_{1.54} \\
3.19 \\
R_{4.68} \\
2.64 \\
5.45 \\
6.20 \\
8.30 \\
R_{14.60} \\
9.56 \\
3.03 \\
2.02 \\
8.39\end{array}$ & $\begin{array}{r}R_{5.54} \\
R_{4.35} \\
R_{1.59} \\
- \\
R_{1.59} \\
3.13 \\
R_{4.99} \\
3.29 \\
5.79 \\
6.58 \\
8.68 \\
R_{16.79} \\
9.07 \\
2.36 \\
1.72 \\
8.54\end{array}$ & $\begin{array}{r}R_{5} .26 \\
R_{4.00} \\
R_{1} .52 \\
R_{1.52} \\
4.10 \\
R_{4.28} \\
2.86 \\
5.44 \\
6.18 \\
8.82 \\
R_{19.43} \\
9.39 \\
1.70 \\
1.29 \\
8.50\end{array}$ & $\begin{array}{r}5.16 \\
4.00 \\
1.51 \\
1.51 \\
1.51 \\
2.71 \\
4.71 \\
2.80 \\
5.57 \\
6.33 \\
8.79 \\
18.94 \\
9.83 \\
2.32 \\
1.06 \\
9.09\end{array}$ \\
\hline 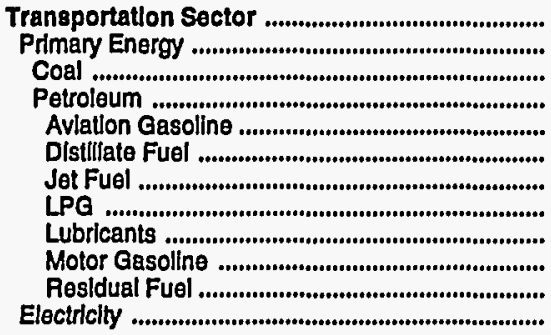 & $\begin{array}{r}2.34 \\
2.34 \\
.48 \\
2.34 \\
2.17 \\
1.24 \\
.76 \\
1.49 \\
5.08 \\
2.89 \\
.34 \\
-\end{array}$ & $\begin{array}{r}4.02 \\
4.02 \\
.79 \\
4.02 \\
3.45 \\
2.65 \\
2.12 \\
2.77 \\
7.48 \\
4.78 \\
2.01 \\
-\end{array}$ & $\begin{array}{r}8.92 \\
8.92 \\
- \\
8.92 \\
9.02 \\
7.15 \\
6.59 \\
5.85 \\
14.36 \\
9.99 \\
- \\
-\end{array}$ & $\begin{array}{r}8.42 \\
8.42 \\
8 . \overline{2} \\
8.42 \\
9.99 \\
6.80 \\
6.64 \\
7.19 \\
17.61 \\
9.16 \\
4.01 \\
-\end{array}$ & $\begin{array}{r}R_{9.35} \\
R_{9.35} \\
-\overline{A_{9.35}} \\
9.32 \\
9.18 \\
6.26 \\
8.30 \\
R_{14.60} \\
9.56 \\
- \\
-\end{array}$ & $\begin{array}{r}R_{8.97} \\
R_{8.97} \\
\text { R } 8.97 \\
8.71 \\
8.94 \\
5.47 \\
8.68 \\
R 16.79 \\
9.07 \\
- \\
-\end{array}$ & $\begin{array}{r}R_{9.13} \\
R_{9.13} \\
R_{9.13} \\
8.54 \\
8.85 \\
5.46 \\
8.82 \\
R_{19.43} \\
9.39 \\
- \\
-\end{array}$ & $\begin{array}{r}9.42 \\
9.42 \\
- \\
9.42 \\
8.24 \\
8.96 \\
5.39 \\
8.79 \\
18.94 \\
9.83 \\
- \\
-\end{array}$ \\
\hline 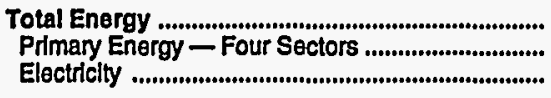 & $\begin{array}{l}1.56 \\
1.35 \\
2.88\end{array}$ & $\begin{array}{l}2.78 \\
2.59 \\
4.05\end{array}$ & $\begin{array}{l}6.02 \\
6.06 \\
5.80\end{array}$ & $\begin{array}{r}7.48 \\
6.70 \\
10.72\end{array}$ & $\begin{array}{r}7.81 \\
R_{6.82} \\
11.67\end{array}$ & $\begin{array}{r}R_{7.75} \\
R_{6.60} \\
12.22\end{array}$ & $\begin{array}{r}R_{7.86} \\
R_{6.73} \\
12.39\end{array}$ & $\begin{array}{r}7.82 \\
6.69 \\
12.87\end{array}$ \\
\hline 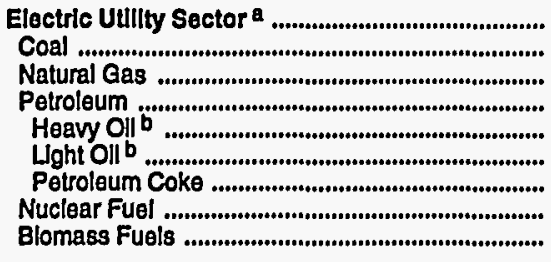 & $\begin{array}{l}23 \\
.19 \\
.27 \\
.33 \\
.33 \\
.36 \\
- \\
.65\end{array}$ & $\begin{array}{r}.34 \\
.30 \\
.38 \\
2.00 \\
1.99 \\
2.30 \\
- \\
.92\end{array}$ & $\begin{array}{r}.72 \\
.44 \\
3.87 \\
5.01 \\
-\overline{01} \\
- \\
-\overline{-} \\
1.74\end{array}$ & $\begin{array}{r}.72 \\
.71 \\
.59 \\
6.11 \\
-\overline{11} \\
6.11 \\
- \\
.79\end{array}$ & $\begin{array}{r}.68 \\
.67 \\
1.45 \\
5.43 \\
\overline{5} .43 \\
\overline{-} \\
(\bar{d})\end{array}$ & $\begin{array}{r}.68 \\
.67 \\
3.94 \\
4.72 \\
\overline{-} \\
4.72 \\
\overline{-} \\
\overline{0})\end{array}$ & $\begin{array}{r}.71 \\
.71 \\
3.42 \\
5.09 \\
-\overline{9} \\
5.09 \\
- \\
\overrightarrow{(d)}\end{array}$ & $\begin{array}{r}.70 \\
.69 \\
2.68 \\
5.26 \\
\overline{-} \\
5.26 \\
\overline{-} \\
(\bar{d})\end{array}$ \\
\hline Primary Energy - Flve Sectore $c$........................... & 1.26 & 2.41 & 4.79 & 4.69 & 3.82 & 3.63 & 3.63 & 4.06 \\
\hline
\end{tabular}

a There are no direct fuel costs for hydroelectric, geothermal, wind, photovoltalc, or solar thermal energy.

b Heavy oll includes fuel oll nos. 4, 5, and 6, and residual fuel oils. Light oll Includes fuel oll nos. 1 and 2, kerosene, and jet fuel.

- Blomass luels are not Included, except those consumed at electric uttlities and those added to motor gasoline.

d utilities used blomass fuels at no charge or recelved a fee for accepting

them.

R=Revised data.

No consumption, including cases where adjustments were made. See explanation of adjustments in Section 6 of Appendlx A.

Sources: Data sources, estimation procedures, and assumptions are described in Appendix A. 
M Energy Expenditure Estimates by Sector, Montana

O 1970, 1975, 1980, 1985, and 1990-1993

$\mathrm{N}$ (Million Dollars)

\begin{tabular}{|c|c|c|c|c|c|c|c|c|}
\hline Sector and Energy Source & 1970 & 1975 & 1980 & 1985 & 1990 & 1991 & 1992 & 1993 \\
\hline $\begin{array}{l}\text { Residentlal Sector } \\
\text { Primary Energy } \\
\text { Coal } \\
\text { Natural Gas } \\
\text { Petroleum } \\
\text { Distillate Fuel } \\
\text { Kerosene } \\
\text { EPG } \\
\text { Electricity }\end{array}$ & $\begin{array}{r}66.7 \\
32.3 \\
.1 \\
22.5 \\
9.7 \\
1.9 \\
- \\
7.9 \\
34.4\end{array}$ & $\begin{array}{r}106.4 \\
55.1 \\
.1 \\
31.2 \\
23.8 \\
9.7 \\
- \\
14.0 \\
51.3\end{array}$ & $\begin{array}{r}187.8 \\
98.0 \\
.1 \\
58.9 \\
38.9 \\
17.0 \\
- \\
21.9 \\
89.9\end{array}$ & $\begin{array}{r}297.4 \\
127.6 \\
(s) \\
93.2 \\
34.3 \\
15.9 \\
.6 \\
17.8 \\
169.8\end{array}$ & $\begin{array}{r}R_{301.1} \\
R_{118.1} \\
R .5 \\
77.3 \\
40.3 \\
10.8 \\
.1 \\
29.4 \\
183.0\end{array}$ & $\begin{array}{r}R 321.7 \\
R_{122.7} \\
R .3 \\
83.2 \\
39.2 \\
12.7 \\
(s) \\
26.5 \\
199.0\end{array}$ & $\begin{array}{r}R_{298.6} \\
\text { R } 106.5 \\
\text { R.2 } \\
80.0 \\
26.3 \\
7.6 \\
(\mathrm{~s}) \\
18.7 \\
192.0\end{array}$ & $\begin{array}{r}332.5 \\
124.5 \\
.1 \\
100.2 \\
24.2 \\
9.4 \\
.3 \\
14.5 \\
208.0\end{array}$ \\
\hline $\begin{array}{l}\text { Commercial Sector } \\
\text { Primary Energy } \\
\text { Coal ... } \\
\text { Natural Gas } \\
\text { Petroleum } \\
\text { Distillate Fuel } \\
\text { Kerosene } \\
\text { MPG } \\
\text { Metor Gasoline } \\
\text { Electricity }\end{array}$ & $\begin{array}{r}41.3 \\
18.0 \\
.1 \\
11.5 \\
6.5 \\
1.7 \\
.5 \\
.9 \\
3.3 \\
(s) \\
23.3\end{array}$ & $\begin{array}{r}73.1 \\
37.2 \\
.1 \\
20.4 \\
16.7 \\
9.7 \\
.8 \\
1.8 \\
4.4 \\
(s) \\
35.9\end{array}$ & $\begin{array}{r}127.1 \\
66.4 \\
.4 \\
44.9 \\
21.2 \\
13.0 \\
- \\
3.1 \\
4.8 \\
.2 \\
60.7\end{array}$ & $\begin{array}{r}296.5 \\
115.7 \\
.2 \\
75.5 \\
40.0 \\
31.4 \\
(s) \\
2.8 \\
3.5 \\
2.4 \\
180.8\end{array}$ & $\begin{array}{r}R_{219.8} \\
R_{70.8} \\
R_{1.1} \\
56.4 \\
13.3 \\
4.6 \\
(5) \\
4.3 \\
4.2 \\
.2 \\
149.0\end{array}$ & $\begin{array}{r}R_{231.9} \\
R_{69.0} \\
R .8 \\
55.9 \\
12.2 \\
5.3 \\
(\mathrm{~s}) \\
3.9 \\
3.0 \\
(\mathrm{~s}) \\
163.0\end{array}$ & $\begin{array}{r}R_{235.0} \\
A_{63.0} \\
.4 \\
51.6 \\
11.1 \\
4.9 \\
(s) \\
3.4 \\
2.7 \\
(s) \\
172.0\end{array}$ & $\begin{array}{r}249.8 \\
74.8 \\
.2 \\
64.8 \\
9.8 \\
6.0 \\
(s) \\
3.1 \\
.6 \\
.1 \\
175.0\end{array}$ \\
\hline 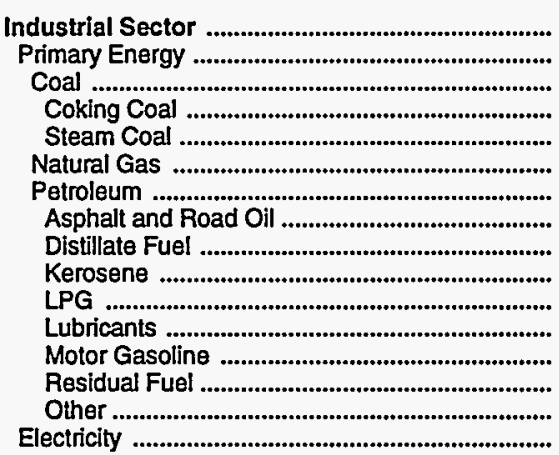 & $\begin{array}{r}67.2 \\
40.7 \\
.3 \\
- \\
.3 \\
10.4 \\
30.0 \\
5.3 \\
6.5 \\
1.5 \\
.9 \\
1.4 \\
9.6 \\
.4 \\
4.4 \\
26.5\end{array}$ & $\begin{array}{r}156.8 \\
124.1 \\
.8 \\
- \\
.8 \\
26.1 \\
97.2 \\
11.3 \\
35.5 \\
1.0 \\
.8 \\
2.1 \\
19.4 \\
14.9 \\
12.3 \\
32.6\end{array}$ & $\begin{array}{r}329.7 \\
272.6 \\
6.0 \\
- \\
6.0 \\
45.2 \\
221.4 \\
24.9 \\
58.2 \\
- \\
15.9 \\
4.4 \\
32.5 \\
68.1 \\
17.4 \\
57.1\end{array}$ & $\begin{array}{r}493.1 \\
355.2 \\
6.8 \\
- \\
6.8 \\
35.7 \\
312.7 \\
46.9 \\
203.3 \\
(\mathrm{~s}) \\
17.9 \\
5.0 \\
32.6 \\
(\mathrm{~s}) \\
7.1 \\
138.0\end{array}$ & $\begin{array}{r}A_{396.4} \\
R_{218.0} \\
R_{6.2} \\
- \\
R_{6.2} \\
28.4 \\
R_{183.4} \\
26.0 \\
87.2 \\
.2 \\
19.8 \\
R_{4.6} \\
30.7 \\
(s) \\
14.8 \\
178.4\end{array}$ & $\begin{array}{r}R_{419.4} \\
R_{235.5} \\
R_{8.2} \\
- \\
R_{8.2} \\
28.7 \\
A_{198.5} \\
29.5 \\
120.0 \\
.1 \\
4.8 \\
R_{4.8} \\
29.1 \\
(5) \\
10.3 \\
183.9\end{array}$ & $\begin{array}{r}R_{393.5} \\
R_{215.5} \\
R_{7.2} \\
- \\
R_{7.2} \\
47.3 \\
R_{161.0} \\
24.8 \\
82.0 \\
(s) \\
7.8 \\
R_{5.6} \\
28.2 \\
(s) \\
12.5 \\
177.9\end{array}$ & $\begin{array}{r}429.7 \\
257.4 \\
10.3 \\
- \\
10.3 \\
32.0 \\
215.1 \\
31.7 \\
88.7 \\
(\mathrm{~s}) \\
46.8 \\
5.6 \\
29.3 \\
4.7 \\
8.3 \\
172.3\end{array}$ \\
\hline $\begin{array}{l}\text { Transportation Sector } \\
\text { Primary Energy } \\
\text { Coal } \\
\text { Petroleum } \\
\text { Aviation Gasoline } \\
\text { Distillate Fuel } \\
\text { Jet Fual } \\
\text { LuG } \\
\text { Motor Gasoline }\end{array}$ & $\begin{array}{r}157.9 \\
157.9 \\
(\mathrm{~s}) \\
157.9 \\
.5 \\
21.9 \\
2.7 \\
.2 \\
4.7 \\
127.7 \\
3\end{array}$ & $\begin{array}{r}323.1 \\
323.1 \\
(\mathrm{~s}) \\
323.1 \\
1.4 \\
59.2 \\
9.7 \\
.5 \\
7.3 \\
242.9 \\
2.0\end{array}$ & $\begin{array}{r}767.3 \\
767.3 \\
- \\
767.3 \\
7.3 \\
198.3 \\
34.1 \\
1.0 \\
17.1 \\
509.5\end{array}$ & $\begin{array}{r}673.8 \\
673.8 \\
- \\
673.8 \\
4.6 \\
169.4 \\
25.2 \\
1.3 \\
19.1 \\
454.2\end{array}$ & $\begin{array}{r}R_{753.6} \\
R_{753.6} \\
- \\
R_{753.6} \\
5.2 \\
223.1 \\
24.8 \\
2.0 \\
R_{17.8} \\
480.6\end{array}$ & $\begin{array}{r}R_{721.3} \\
R_{721.3} \\
- \\
R_{721.3} \\
4.8 \\
216.6 \\
19.0 \\
1.5 \\
R_{18.3} \\
461.2\end{array}$ & $\begin{array}{r}\text { R } 793.2 \\
\text { R } 793.2 \\
- \\
R_{793.2} \\
3.3 \\
242.6 \\
26.3 \\
1.1 \\
\text { R } 21.6 \\
498.4\end{array}$ & $\begin{array}{r}838.9 \\
838.9 \\
- \\
838.9 \\
2.6 \\
248.3 \\
27.1 \\
1.4 \\
21.4 \\
538.1\end{array}$ \\
\hline Electricity & 13 & $\begin{array}{c}2.0 \\
-\end{array}$ & - & (5) & $\overline{-}$ & $\overline{-}$ & $\overline{-}$ & $\overline{-}$ \\
\hline 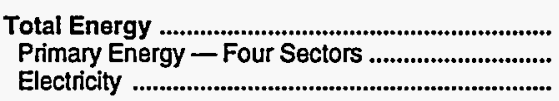 & $\begin{array}{r}333.1 \\
249.0 \\
84.1\end{array}$ & $\begin{array}{l}659.3 \\
539.5 \\
119.8\end{array}$ & $\begin{array}{r}1,412.0 \\
1,204.3 \\
207.7\end{array}$ & $\begin{array}{r}1,760.9 \\
1,272.3 \\
488.6\end{array}$ & $\begin{array}{r}R_{1,670.8} \\
R_{1,160.4} \\
510.4\end{array}$ & $\begin{array}{r}R_{1,694.4} \\
R_{1,148.6} \\
545.8\end{array}$ & $\begin{array}{r}R_{1,720.3} \\
R_{1,178.3} \\
542.0\end{array}$ & $\begin{array}{r}1,850.9 \\
1,295.7 \\
555.3\end{array}$ \\
\hline $\begin{array}{l}\text { Electric Utility Sector } \\
\text { Coal } \\
\text { Natural Gas } \\
\text { Petroleum } \\
\text { Heavy Oil } \mathrm{b} \\
\text { Light Oil } \mathrm{b} \\
\text { Petroleum } \mathrm{Coke} \\
\text { Nuclear Fuel } \\
\text { Biomass Fuels }\end{array}$ & $\begin{array}{r}3.4 \\
2.2 \\
.7 \\
.1 \\
.1 \\
(s) \\
- \\
.5\end{array}$ & $\begin{array}{r}6.4 \\
5.2 \\
.5 \\
.7 \\
.7 \\
(s) \\
- \\
.1\end{array}$ & $\begin{array}{r}44.3 \\
25.3 \\
17.0 \\
1.7 \\
\overline{1} \\
1.7 \\
- \\
\overline{-}\end{array}$ & $\begin{array}{r}69.2 \\
67.1 \\
.3 \\
1.4 \\
- \\
1.4 \\
- \\
- \\
.5\end{array}$ & $\begin{array}{r}110.6 \\
107.9 \\
.7 \\
2.0 \\
- \\
2.0 \\
- \\
- \\
\left({ }^{d}\right)\end{array}$ & $\begin{array}{r}119.3 \\
116.9 \\
1.3 \\
1.1 \\
\overline{1.1} \\
- \\
- \\
(\mathrm{d})\end{array}$ & $\begin{array}{r}132.7 \\
130.8 \\
.9 \\
1.0 \\
\overline{1.0} \\
- \\
\overline{(d)}\end{array}$ & $\begin{array}{r}106.7 \\
104.4 \\
.8 \\
1.5 \\
\overline{1.5} \\
- \\
\overline{(d)}\end{array}$ \\
\hline Primary Energy — Five Sectors c ......................... & 252.4 & 545.9 & $1,248.6$ & $1,341.5$ & $\mathrm{P}_{1,271.1}$ & $R_{1,267.9}$ & $R_{1,310.9}$ & $1,402.4$ \\
\hline
\end{tabular}

a There are no direct fuel costs for hydroelectric, geothermal, wind, photovoltaic, or solar thermal energy.

Heavy oil includes fuel oil nos. 4, 5, and 6 , and residual fuel oils. Light oil includes fuel nos. 1 and 2, kerosene, and jet fuel.

$c$ Biomass fuels are not included, except those consumed at electric utilities and those added to motor gasoline.

d Utilities used biomass fuels at no charge or recelved a fee for accepting

R=Revised data.

- No consumption, including cases where adjustments were made. See explanation of adjustments in Section 6 of Appendix A.

$(s)=$ Value less than 0.05 million dollars.

Note: Totals may not equal sum of components due to independent rounding.

Sources: Data sources, estimation procedures, and assumptions are described in Appendix A. them. 


\begin{tabular}{|c|c|c|c|c|c|c|c|c|}
\hline Energy Source & 1970 & 1975 & 1980 & 1985 & 1990 & 1991 & 1992 & 1993 \\
\hline & \multicolumn{8}{|c|}{ Prices in Dollars per Million Btu } \\
\hline 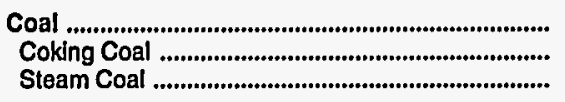 & $\frac{0.32}{.32}$ & $\begin{array}{r}0.86 \\
.86\end{array}$ & $\frac{1.27}{1.27}$ & $1 . \overline{17}$ & $\frac{0.78}{.78}$ & $\begin{array}{r}0.78 \\
.78\end{array}$ & $\begin{array}{r}0.78 \\
. \overline{78}\end{array}$ & $\begin{array}{r}0.79 \\
.79\end{array}$ \\
\hline Natural Gas & .50 & .90 & 2.40 & 4.43 & 3.93 & 3.93 & 4.10 & 4.25 \\
\hline $\begin{array}{l}\text { Petroleum } \\
\text { Asphalt and Road Oil } \\
\text { Aviation Gasoline } \\
\text { Distillate Fuel . } \\
\text { Jet Fuel }\end{array}$ & $\begin{array}{r}2.12 \\
.71 \\
2.17 \\
.95 \\
.75 \\
1.18 \\
1.56 \\
5.08 \\
3.03 \\
.48 \\
.51\end{array}$ & $\begin{array}{l}3.74 \\
2.07 \\
3.45 \\
2.38 \\
2.09 \\
2.63 \\
3.07 \\
7.48 \\
4.76 \\
1.74 \\
3.42\end{array}$ & $\begin{array}{r}8.27 \\
3.89 \\
9.02 \\
6.24 \\
6.47 \\
5.56 \\
5.70 \\
14.36 \\
10.06 \\
3.21 \\
7.71\end{array}$ & $\begin{array}{r}8.34 \\
5.04 \\
9.99 \\
6.86 \\
6.19 \\
8.86 \\
7.00 \\
17.61 \\
9.67 \\
4.28 \\
13.38\end{array}$ & $\begin{array}{r}\mathrm{R}_{8.34} \\
3.15 \\
9.32 \\
7.60 \\
6.03 \\
6.56 \\
8.83 \\
\mathrm{R}_{14.60} \\
9.49 \\
2.22 \\
\mathrm{R}_{8.13}\end{array}$ & $\begin{array}{r}R 8.05 \\
3.18 \\
8.71 \\
7.12 \\
5.01 \\
6.20 \\
7.79 \\
\mathrm{R}_{16.79} \\
9.43 \\
2.02 \\
\mathrm{R} 7.96\end{array}$ & $\begin{array}{r}\mathrm{R} 7.85 \\
2.62 \\
8.54 \\
6.87 \\
4.64 \\
6.00 \\
7.31 \\
\mathrm{R} 19.43 \\
9.09 \\
1.97 \\
\mathrm{R} 10.06\end{array}$ & $\begin{array}{r}7.88 \\
2.92 \\
8.24 \\
6.78 \\
4.33 \\
5.74 \\
6.53 \\
18.94 \\
9.33 \\
2.27 \\
8.42\end{array}$ \\
\hline 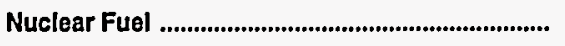 & - & .17 & .44 & .65 & .61 & .62 & .57 & .64 \\
\hline 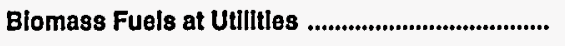 & - & - & - & - & - & - & .99 & 1.09 \\
\hline Primary Energy — Flve Sectors b ........................... & 1.21 & 1.96 & 4.18 & 4.85 & $R_{4.28}$ & $R_{4.06}$ & $R_{4.06}$ & 4.09 \\
\hline 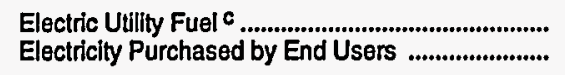 & $\begin{array}{r}.30 \\
5.12\end{array}$ & $\begin{array}{r}.50 \\
6.89\end{array}$ & $\begin{array}{r}1.00 \\
11.76\end{array}$ & $\begin{array}{r}1.01 \\
15.70\end{array}$ & $\begin{array}{r}.73 \\
16.34\end{array}$ & $\begin{array}{r}.72 \\
16.65\end{array}$ & $\begin{array}{r}.69 \\
16.18\end{array}$ & $\begin{array}{r}.74 \\
16.24\end{array}$ \\
\hline \multirow[t]{2}{*}{ Total Energy } & 1.72 & 2.90 & 6.52 & 8.09 & ${ }^{\mathrm{R}} 8.38$ & ${ }^{\mathrm{R}} 8.13$ & ${ }^{R_{8.11}}$ & 8.04 \\
\hline & \multicolumn{8}{|c|}{ Expenditures In Milllons of Dollars } \\
\hline 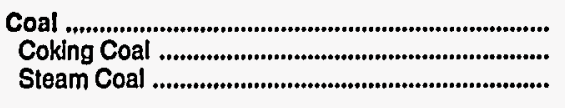 & $\begin{array}{l}9.6 \\
\overline{9.6}\end{array}$ & $\begin{array}{l}28.4 \\
28.4\end{array}$ & $\begin{aligned} 119.4 \\
-\overline{119.4}\end{aligned}$ & $\begin{array}{l}135.5 \\
- \\
135.5\end{array}$ & $\begin{array}{r}110.2 \\
110.2\end{array}$ & $\begin{array}{r}118.4 \\
\overline{-} \\
118.4\end{array}$ & $\begin{array}{c}110.2 \\
- \\
110.2\end{array}$ & $\begin{aligned} & 131.0- \\
& 131.0\end{aligned}$ \\
\hline Natural Gas & 104.1 & 184.3 & 354.1 & 523.7 & 415.4 & 438.4 & 418.8 & 512.1 \\
\hline 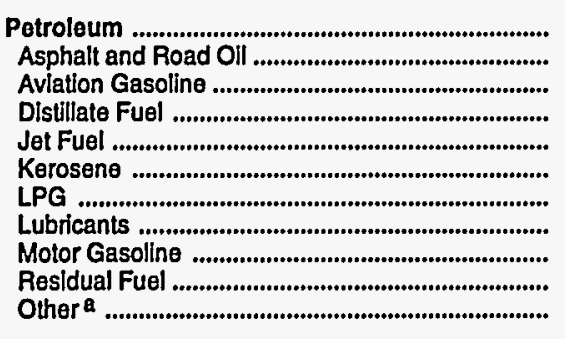 & $\begin{array}{r}404.8 \\
5.3 \\
2.2 \\
41.4 \\
7.3 \\
3.9 \\
33.2 \\
14.8 \\
294.4 \\
2.3 \\
.1\end{array}$ & $\begin{array}{r}775.1 \\
10.3 \\
2.5 \\
117.9 \\
19.3 \\
8.2 \\
65.4 \\
22.3 \\
516.3 \\
11.2 \\
1.6\end{array}$ & $\begin{array}{r}1,564.1 \\
18.6 \\
9.7 \\
332.7 \\
56.2 \\
2.0 \\
94.2 \\
33.9 \\
1,008.9 \\
4.3 \\
3.7\end{array}$ & $\begin{array}{r}1,576.8 \\
15.8 \\
4.9 \\
494.9 \\
45.9 \\
3.7 \\
65.3 \\
37.8 \\
901.2 \\
1.7 \\
5.6\end{array}$ & $\begin{array}{r}\text { P }_{1,697.7} \\
29.0 \\
3.9 \\
551.7 \\
50.0 \\
1.5 \\
93.2 \\
\text { R } 35.3 \\
914.9 \\
3.6 \\
R_{14.6}\end{array}$ & $\begin{array}{r}R_{1,631.9} \\
29.9 \\
3.7 \\
539.9 \\
33.1 \\
.6 \\
89.2 \\
R_{36.3} \\
881.7 \\
2.5 \\
14.9\end{array}$ & $\begin{array}{r}8_{1,621.6} \\
15.6 \\
3.5 \\
R_{563.5} \\
30.7 \\
.7 \\
85.5 \\
R_{42.8} \\
857.8 \\
2.3 \\
R_{19.2}\end{array}$ & $\begin{array}{r}1,618.9 \\
15.5 \\
3.0 \\
555.0 \\
27.8 \\
.8 \\
70.3 \\
42.5 \\
883.8 \\
4.0 \\
16.4\end{array}$ \\
\hline 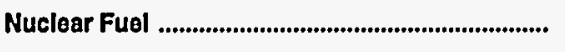 & - & 11.0 & 27.7 & 29.3 & 49.3 & 53.4 & 53.6 & 46.5 \\
\hline 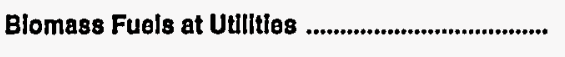 & - & - & - & - & - & - & .1 & .1 \\
\hline 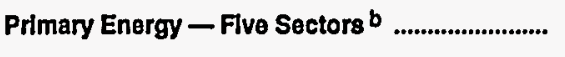 & 518.6 & 998.7 & $2,065.3$ & $2,265.3$ & $R_{2,272.5}$ & $R_{2,242.1}$ & $R_{2,204.3}$ & $2,308.6$ \\
\hline 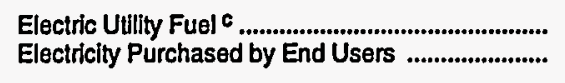 & $\begin{array}{r}.22 .3 \\
170.3\end{array}$ & $\begin{array}{r}-68.1 \\
271.2\end{array}$ & $\begin{array}{r}-164.7 \\
550.6\end{array}$ & $\begin{array}{r}-158.7 \\
841.2\end{array}$ & $\begin{array}{r}-161.1 \\
996.0\end{array}$ & $\begin{array}{r}-169.5 \\
1,019.0\end{array}$ & $\begin{array}{r}-159.2 \\
982.0\end{array}$ & $\begin{array}{r}-172.8 \\
1,039.0\end{array}$ \\
\hline 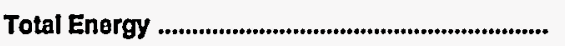 & 666.6 & $1,201.8$ & $2,451.2$ & $2,947.8$ & $\mathrm{R}_{\mathbf{3 , 1 0 7 . 5}}$ & ${ }^{A_{3,091.6}}$ & $R_{3,027.1}$ & $3,174.9$ \\
\hline
\end{tabular}

a Includes petroleum coke used at electric utilities.

b Biomass fuels are not included, except those consumed at electric utilities and those added to motor gasoline.

c There are no direct fuel costs for hydroelectric, geothermal, wind, photovoltalc, or solar thermal energy.

$R=$ Revised data.
-No consumption, including cases where adjustments were made. See explanation of adjustments in Section 6 of Appendix A.

Note: Expenditure totals may not equal sum of components due to independent rounding.

Sources: Data sources, estimation procedures, and assumptions are described in Appendix A. 
N Energy Price Estimates by Sector, Nebraska

E 1970,1975, 1980, 1985, and 1990-1993

B (Dollars per Million Btu)

\begin{tabular}{|c|c|c|c|c|c|c|c|c|}
\hline Sector and Energy Source & 1970 & 1975 & 1980 & 1985 & 1990 & 1991 & 1992 & 1893 \\
\hline 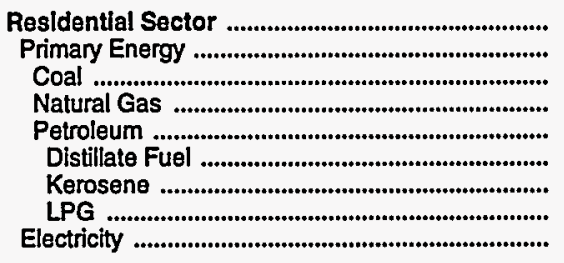 & $\begin{array}{r}1.84 \\
1.04 \\
1.08 \\
.84 \\
1.69 \\
1.19 \\
1.39 \\
1.78 \\
6.21\end{array}$ & $\begin{array}{l}2.95 \\
1.74 \\
2.16 \\
1.29 \\
3.39 \\
2.62 \\
2.74 \\
3.57 \\
8.13\end{array}$ & $\begin{array}{r}5.83 \\
3.32 \\
3.60 \\
2.78 \\
6.84 \\
6.85 \\
7.55 \\
6.82 \\
13.22\end{array}$ & $\begin{array}{r}8.83 \\
5.37 \\
2.76 \\
5.10 \\
7.49 \\
7.92 \\
9.74 \\
7.12 \\
17.30\end{array}$ & $\begin{array}{r}9.45 \\
4.96 \\
2.42 \\
4.67 \\
7.57 \\
6.74 \\
8.28 \\
7.79 \\
18.23\end{array}$ & $\begin{array}{r}9.19 \\
4.94 \\
2.36 \\
4.71 \\
6.79 \\
6.25 \\
7.69 \\
6.92 \\
17.86\end{array}$ & $\begin{array}{r}9.44 \\
5.10 \\
2.39 \\
4.92 \\
6.48 \\
R 5.51 \\
6.78 \\
6.65 \\
18.36\end{array}$ & $\begin{array}{r}9.41 \\
5.21 \\
2.44 \\
5.09 \\
6.31 \\
5.65 \\
6.95 \\
6.45 \\
18.33\end{array}$ \\
\hline 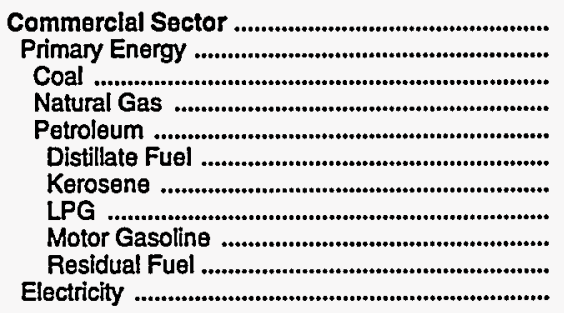 & $\begin{array}{r}1.36 \\
.59 \\
.16 \\
.52 \\
1.09 \\
1.03 \\
.79 \\
1.09 \\
3.03 \\
.50 \\
4.87\end{array}$ & $\begin{array}{r}2.36 \\
1.17 \\
.81 \\
1.00 \\
2.60 \\
2.45 \\
2.39 \\
2.46 \\
4.76 \\
1.75 \\
6.96\end{array}$ & $\begin{array}{r}5.01 \\
2.62 \\
1.69 \\
2.33 \\
6.81 \\
6.49 \\
5.17 \\
5.19 \\
10.06 \\
3.22 \\
12.86\end{array}$ & $\begin{array}{r}8.34 \\
4.68 \\
2.46 \\
4.29 \\
7.21 \\
6.79 \\
7.81 \\
6.92 \\
9.67 \\
16.78\end{array}$ & $\begin{array}{l}8.87 \\
4.17 \\
1.48 \\
3.92 \\
7.01 \\
5.07 \\
6.36 \\
9.36 \\
9.49 \\
2.22 \\
17.22\end{array}$ & $\begin{array}{r}8.57 \\
4.08 \\
1.54 \\
3.93 \\
6.51 \\
4.44 \\
5.53 \\
8.34 \\
9.43 \\
2.03 \\
16.82\end{array}$ & $\begin{array}{r}9.03 \\
4.20 \\
1.57 \\
4.07 \\
5.67 \\
4.18 \\
R 5.17 \\
7.73 \\
9.09 \\
1.97 \\
17.12\end{array}$ & $\begin{array}{r}9.29 \\
4.41 \\
1.55 \\
4.38 \\
4.92 \\
4.12 \\
4.81 \\
6.58 \\
9.33 \\
2.27 \\
17.29\end{array}$ \\
\hline $\begin{array}{l}\text { Industrial Sector } \\
\text { Primary Energy } \\
\text { Coal } \\
\text { Coking Coal } \\
\text { Steam Coal } \\
\text { Natural Gas } \\
\text { Petroleum } \\
\text { Asphalt and Road Oil } \\
\text { Distillate Fuel }\end{array}$ & $\begin{array}{r}.88 \\
.69 \\
.16 \\
.16 \\
.32 \\
1.26 \\
.71 \\
.73 \\
.79 \\
1.09 \\
5.08 \\
3.03 \\
.40 \\
.51 \\
3.42\end{array}$ & $\begin{array}{r}1.77 \\
1.48 \\
.81 \\
.81 \\
.69 \\
.69 \\
2.93 \\
2.07 \\
2.25 \\
2.39 \\
2.46 \\
7.48 \\
4.76 \\
1.74 \\
3.42 \\
4.96\end{array}$ & $\begin{array}{r}4.50 \\
3.87 \\
1.69 \\
1.69 \\
2.21 \\
5.88 \\
3.89 \\
4.94 \\
5.17 \\
5.19 \\
14.36 \\
10.06 \\
3.13 \\
7.71 \\
8.71\end{array}$ & $\begin{array}{r}6.42 \\
5.59 \\
2.46 \\
2.46 \\
3.67 \\
7.47 \\
5.04 \\
7.09 \\
7.81 \\
6.92 \\
17.61 \\
9.67 \\
4.28 \\
13.38 \\
11.47\end{array}$ & $\begin{array}{r}6.11 \\
R 4.86 \\
1.48 \\
1 . \overline{-} \\
1.08 \\
3.02 \\
6.14 \\
3.15 \\
5.78 \\
6.36 \\
9.36 \\
\mathrm{R} \\
14.60 \\
9.49 \\
2.22 \\
8.13 \\
12.31\end{array}$ & $\begin{array}{r}\mathrm{R}_{5.73} \\
4.45 \\
1.54 \\
1.54 \\
2.80 \\
5.60 \\
3.18 \\
5.02 \\
5.53 \\
8.34 \\
\mathrm{R} \\
16.79 \\
9.43 \\
2.03 \\
\mathrm{R} 7.96 \\
12.19\end{array}$ & $\begin{array}{r}R_{5.64} \\
R_{4.37} \\
1.57 \\
-\overline{1.57} \\
2.98 \\
R_{5.46} \\
2.62 \\
4.70 \\
\mathrm{R}_{5.17} \\
7.73 \\
\mathrm{R} 19.43 \\
9.09 \\
1.97 \\
\mathrm{R} 10.06 \\
11.90\end{array}$ & $\begin{array}{r}5.22 \\
4.00 \\
1.55 \\
1.55 \\
3.17 \\
5.02 \\
2.92 \\
4.37 \\
4.81 \\
6.58 \\
18.94 \\
9.33 \\
2.27 \\
8.42 \\
11.81\end{array}$ \\
\hline 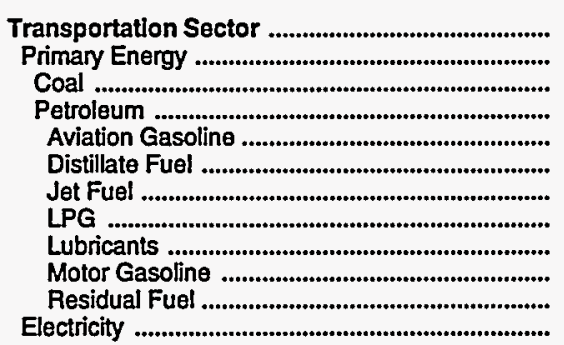 & $\begin{array}{r}2.51 \\
2.51 \\
.16 \\
2.51 \\
2.17 \\
1.14 \\
.75 \\
1.09 \\
5.08 \\
3.03 \\
.50 \\
-\end{array}$ & $\begin{array}{r}4.15 \\
4.15 \\
.81 \\
4.15 \\
3.45 \\
2.50 \\
2.09 \\
2.46 \\
7.48 \\
4.76 \\
1.74 \\
-\end{array}$ & $\begin{array}{r}9.20 \\
9.20 \\
- \\
9.20 \\
9.02 \\
7.06 \\
6.47 \\
5.19 \\
14.36 \\
10.06 \\
- \\
-\end{array}$ & $\begin{array}{r}8.70 \\
8.70 \\
- \\
8.70 \\
9.99 \\
6.68 \\
6.19 \\
6.92 \\
17.61 \\
9.67 \\
- \\
-\end{array}$ & $\begin{array}{r}\text { R9.12 } \\
{ }^{R} 9.12 \\
{ }^{R} 9.12 \\
9.32 \\
8.66 \\
6.03 \\
9.36 \\
\text { R } 14.60 \\
9.49 \\
- \\
-\end{array}$ & $\begin{array}{r}R_{9.00} \\
R_{9.00} \\
R_{9.00} \\
8.71 \\
8.44 \\
5.01 \\
8.34 \\
R_{16.79} \\
9.43 \\
-\end{array}$ & $\begin{array}{r}\mathrm{R}_{8.72} \\
\mathrm{R}_{8.72} \\
\mathrm{R}_{8.72} \\
8.54 \\
8.20 \\
4.64 \\
7.73 \\
\mathrm{R}_{19.43} \\
9.09 \\
- \\
-\end{array}$ & $\begin{array}{r}8.89 \\
8.89 \\
- \\
8.89 \\
8.24 \\
8.29 \\
4.33 \\
6.58 \\
18.94 \\
9.33 \\
\overline{-}\end{array}$ \\
\hline 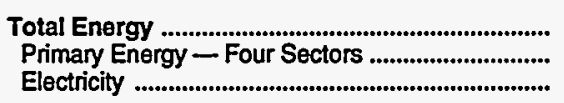 & $\begin{array}{l}1.72 \\
1.40 \\
5.12\end{array}$ & $\begin{array}{l}2.90 \\
2.48 \\
6.89\end{array}$ & $\begin{array}{r}6.52 \\
5.77 \\
11.76\end{array}$ & $\begin{array}{r}8.09 \\
6.78 \\
15.70\end{array}$ & $\begin{array}{l}{ }^{R} 8.38 \\
{ }^{8} 6.81 \\
16.34\end{array}$ & $\begin{array}{l}R_{8.13} \\
R_{6.54} \\
16.05\end{array}$ & $\begin{array}{l}R_{8.11} \\
R_{6.54} \\
16.18\end{array}$ & $\begin{array}{r}8.04 \\
6.46 \\
16.24\end{array}$ \\
\hline 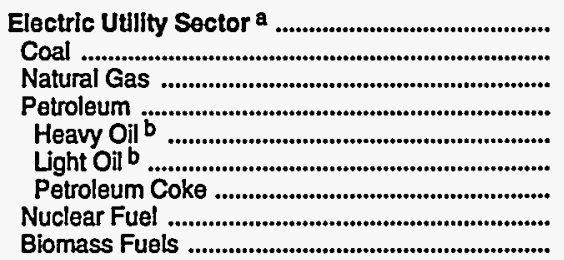 & $\begin{array}{l}.30 \\
.35 \\
.27 \\
.54 \\
.49 \\
.63 \\
- \\
- \\
-\end{array}$ & $\begin{array}{r}.50 \\
.87 \\
.63 \\
1.77 \\
1.73 \\
1.85 \\
.17 \\
-\end{array}$ & $\begin{array}{r}1.00 \\
1.24 \\
1.82 \\
4.14 \\
3.21 \\
6.19 \\
.44 \\
-\end{array}$ & $\begin{array}{r}1.01 \\
1.11 \\
3.58 \\
5.89 \\
\overline{-19} \\
. \overline{65} \\
-\end{array}$ & $\begin{array}{r}.73 \\
.75 \\
2.01 \\
6.89 \\
1.86 \\
7.03 \\
.61 \\
-\end{array}$ & $\begin{array}{r}.72 \\
.75 \\
1.97 \\
4.20 \\
1.41 \\
4.57 \\
.62 \\
-\end{array}$ & $\begin{array}{r}.69 \\
.75 \\
2.38 \\
4.65 \\
4.65 \\
. \overline{5} \\
.99\end{array}$ & $\begin{array}{r}.74 \\
.76 \\
2.73 \\
4.20 \\
4 . \overline{0} \\
. \overline{6} \\
1.09\end{array}$ \\
\hline Primary Energy — Flve Sectors c ............................ & 1.21 & 1.96 & 4.18 & 4.85 & $R_{4.28}$ & $\mathrm{P}_{4.06}$ & $\mathrm{P}_{4.06}$ & 4.09 \\
\hline
\end{tabular}

a There are no direct fuel costs for hydroelectric, geothermal, wind, photovoltaic, or solar thermal energy.

beavy oil includes fuel oil nos. 4,5 , and 6 , and residual fuel oils. Light oil includes fuel oil nos. 1 and 2, kerosene, and jet fuel.

c Biomass fuels are not included, except those consumed at electric utilities and those added to motor gasoline.

$R=$ Revised data.

- No consumption, including cases where adjustments were made. See explanation of adjustments in Section 6 of Appendix A.

Sources: Data sources, estimation procedures, and assumptions are described in Appendix $A$. 


\begin{tabular}{|c|c|c|c|c|c|c|c|c|}
\hline Sector and Energy Source & 1970 & 1975 & 1980 & 1985 & 1890 & 1991 & 1992 & 1993 \\
\hline 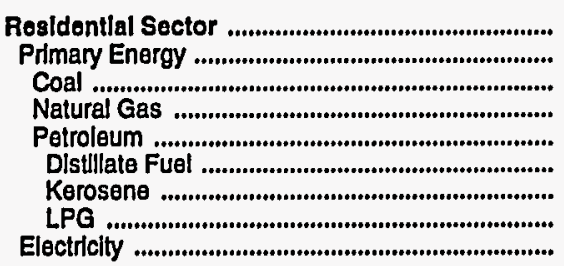 & $\begin{array}{r}167.4 \\
80.3 \\
.3 \\
49.6 \\
30.4 \\
1.4 \\
3.0 \\
26.1 \\
87.0\end{array}$ & $\begin{array}{r}249.4 \\
119.2 \\
.1 \\
68.9 \\
50.1 \\
2.6 \\
5.8 \\
41.7 \\
130.3\end{array}$ & $\begin{array}{r}433.0 \\
184.0 \\
.4 \\
133.5 \\
50.0 \\
14.4 \\
.4 \\
35.2 \\
249.1\end{array}$ & $\begin{array}{r}643.2 \\
277.7 \\
.3 \\
233.9 \\
43.5 \\
15.7 \\
2.2 \\
25.6 \\
365.5\end{array}$ & $\begin{array}{r}648.4 \\
225.4 \\
.1 \\
190.9 \\
34.4 \\
6.6 \\
.2 \\
27.6 \\
423.0\end{array}$ & $\begin{array}{r}680.7 \\
245.7 \\
.3 \\
207.3 \\
38.1 \\
7.2 \\
.2 \\
30.7 \\
435.0\end{array}$ & $\begin{array}{r}645.9 \\
234.8 \\
.1 \\
199.6 \\
35.1 \\
4.7 \\
.4 \\
30.0 \\
411.0\end{array}$ & $\begin{array}{r}724.6 \\
272.6 \\
.1 \\
239.3 \\
33.2 \\
5.5 \\
.4 \\
27.2 \\
452.0\end{array}$ \\
\hline 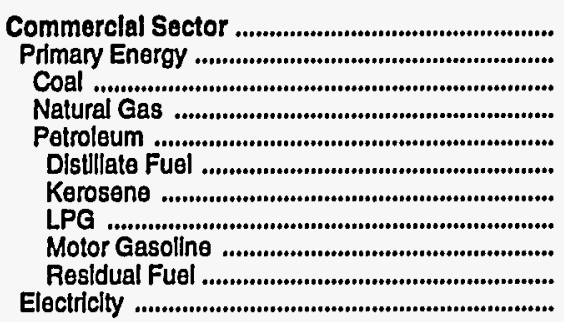 & $\begin{array}{r}89.9 \\
31.7 \\
.1 \\
24.7 \\
6.8 \\
1.2 \\
.3 \\
2.8 \\
1.7 \\
.8 \\
58.3\end{array}$ & $\begin{array}{r}143.1 \\
56.2 \\
.1 \\
42.9 \\
13.3 \\
2.5 \\
1.0 \\
5.1 \\
3.0 \\
1.7 \\
86.9\end{array}$ & $\begin{array}{r}298.5 \\
120.0 \\
.4 \\
99.1 \\
20.5 \\
6.8 \\
.6 \\
4.7 \\
7.9 \\
.5 \\
178.5\end{array}$ & $\begin{array}{r}538.2 \\
211.0 \\
.4 \\
166.0 \\
44.6 \\
31.7 \\
.5 \\
4.4 \\
8.0 \\
327.2\end{array}$ & $\begin{array}{r}541.9 \\
162.9 \\
.1 \\
140.8 \\
22.0 \\
7.3 \\
.8 \\
5.9 \\
7.7 \\
.3 \\
379.0\end{array}$ & $\begin{array}{r}561.8 \\
172.8 \\
.2 \\
155.9 \\
16.7 \\
4.7 \\
.1 \\
6.5 \\
5.0 \\
.3 \\
389.0\end{array}$ & $\begin{array}{r}533.4 \\
155.4 \\
.1 \\
137.6 \\
17.7 \\
6.6 \\
(s) \\
6.2 \\
4.4 \\
.5 \\
378.0\end{array}$ & $\begin{array}{r}549.1 \\
162.1 \\
.1 \\
148.3 \\
13.7 \\
7.3 \\
.1 \\
4.9 \\
1.0 \\
.3 \\
387.0\end{array}$ \\
\hline $\begin{array}{l}\text { Industrlal Sector } \\
\text { Primary Energy } \\
\text { Coal } \\
\text { Coking Coal } \\
\text { Steam Coal }\end{array}$ & $\begin{array}{r}92.3 \\
67.4 \\
.8 \\
.8 \\
17.0 \\
49.6 \\
5.3 \\
14.0 \\
.6 \\
3.4 \\
4.9 \\
21.0 \\
.3 \\
.1\end{array}$ & $\begin{array}{r}230.9 \\
177.0 \\
4.8 \\
4.8 \\
49.8 \\
49.2 \\
122.9 \\
10.3 \\
42.3 \\
1.5 \\
16.5 \\
8.8 \\
41.1 \\
.8 \\
1.6\end{array}$ & $\begin{array}{r}486.6 \\
363.7 \\
8.7 \\
\overline{8.7} \\
101.1 \\
253.9 \\
18.6 \\
98.1 \\
.9 \\
51.0 \\
3.6 \\
77.7 \\
.3 \\
3.7 \\
123.0\end{array}$ & $\begin{array}{r}589.9 \\
441.4 \\
11.9 \\
11.9 \\
119.4 \\
110.0 \\
310.0 \\
15.8 \\
177.4 \\
1.0 \\
33.9 \\
4.0 \\
70.7 \\
1.7 \\
5.6 \\
148.5\end{array}$ & $\begin{array}{r}A_{572.4} \\
A_{378.4} \\
6.6 \\
-\overline{6.6} \\
76.5 \\
76.5 \\
\mathrm{R}_{295.3} \\
29.0 \\
139.3 \\
.5 \\
57.7 \\
R_{3.7} \\
47.1 \\
3.3 \\
R_{14.6} \\
194.0\end{array}$ & $\begin{array}{r}\mathrm{R}_{556.5} \\
\mathrm{R}_{361.6} \\
9.4 \\
-\overline{9.4} \\
68.3 \\
\mathrm{R}_{283.8} \\
29.9 \\
136.1 \\
.3 \\
50.0 \\
\mathrm{R}_{3.8} \\
46.6 \\
2.2 \\
14.9 \\
195.0\end{array}$ & $\begin{array}{r}R_{543.0} \\
R_{350.0} \\
9.4 \\
-\overline{9.4} \\
97.2 \\
77.2 \\
R_{263.3} \\
15.6 \\
R_{134.5} \\
.2 \\
48.0 \\
R_{4.5} \\
39.4 \\
1.8 \\
R_{19.2} \\
193.0\end{array}$ & $\begin{array}{r}566.8 \\
366.8 \\
10.6 \\
10.6 \\
119.5 \\
236.7 \\
15.5 \\
125.3 \\
.3 \\
37.0 \\
4.5 \\
34.1 \\
3.7 \\
16.4 \\
200.0\end{array}$ \\
\hline 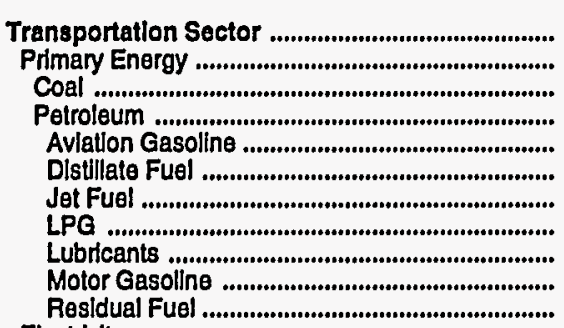 & $\begin{array}{r}317.0 \\
317.0 \\
((s) \\
317.0 \\
2.2 \\
24.4 \\
7.3 \\
.9 \\
9.8 \\
271.7 \\
.7\end{array}$ & $\begin{array}{r}578.3 \\
578.3 \\
(s) \\
578.3 \\
2.5 \\
67.2 \\
19.3 \\
2.1 \\
13.6 \\
472.2 \\
1.5\end{array}$ & $\begin{array}{r}1,233.0 \\
1,233.0 \\
- \\
1,233.0 \\
9.7 \\
210.2 \\
56.2 \\
3.3 \\
30.3 \\
923.3 \\
-\end{array}$ & $\begin{array}{r}1,176.5 \\
1,176.5 \\
- \\
1,176.5 \\
4.9 \\
268.1 \\
45.9 \\
1.4 \\
33.8 \\
822.4 \\
-\end{array}$ & $\begin{array}{r}R_{1,344.7} \\
R_{1,344.7} \\
R_{1,344.7} \\
3.9 \\
397.2 \\
50.0 \\
2.1 \\
R_{31.5} \\
860.1 \\
-\end{array}$ & $\begin{array}{r}\mathrm{R}_{1,292.6} \\
\mathrm{R}_{1,292.6} \\
\mathrm{R}_{1,292.6} \\
3.7 \\
391.2 \\
33.1 \\
1.9 \\
1.9 \\
32.5 \\
830.2 \\
-\end{array}$ & $\begin{array}{r}R_{1} 1,304.9 \\
R_{1,304.9} \\
R_{1,304.9} \\
3.5 \\
417.1 \\
30.7 \\
1.3 \\
R_{38.3} \\
814.0 \\
-\end{array}$ & $\begin{array}{r}1,334.4 \\
1,334.4 \\
- \\
1,334.4 \\
3.0 \\
415.8 \\
27.8 \\
1.1 \\
38.0 \\
848.6 \\
-\end{array}$ \\
\hline Electricity & - & - & - & - & - & - & - & - \\
\hline 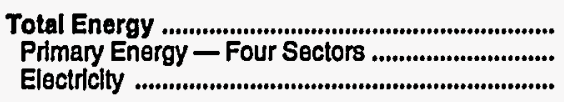 & $\begin{array}{l}666.6 \\
496.3 \\
170.3\end{array}$ & $\begin{array}{r}1,201.8 \\
930.6 \\
271.2\end{array}$ & $\begin{array}{r}2,451.2 \\
1,900.6 \\
550.6\end{array}$ & $\begin{array}{r}2,847.8 \\
2,106.6 \\
841.2\end{array}$ & $\begin{array}{r}R_{3,107.5} \\
R_{2,111.5} \\
996.0\end{array}$ & $\begin{array}{r}R_{3,091.6} \\
R_{2,072.6} \\
1,019.0\end{array}$ & $\begin{array}{r}A_{3,027.1} \\
{ }^{2}, 045.1 \\
982.0\end{array}$ & $\begin{array}{l}3,174.8 \\
2,135.8 \\
1,039.0\end{array}$ \\
\hline 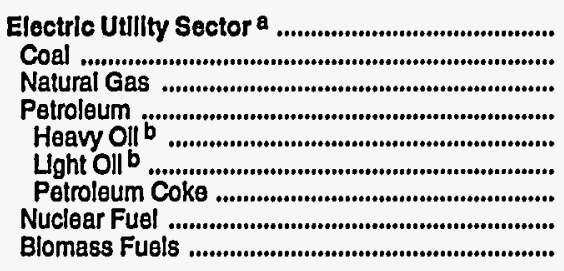 & $\begin{array}{r}22.3 \\
8.5 \\
12.8 \\
1.0 \\
.6 \\
.5 \\
= \\
=\end{array}$ & $\begin{array}{r}68.1 \\
23.4 \\
23.3 \\
10.5 \\
7.2 \\
3.3 \\
\overline{-1} \\
11.0 \\
-\end{array}$ & $\begin{array}{r}164.7 \\
109.8 \\
20.5 \\
6.7 \\
3.6 \\
3.1 \\
-\overline{2} \\
27.7 \\
-\end{array}$ & $\begin{array}{r}158.7 \\
122.9 \\
4.4 \\
2.1 \\
2 . \overline{2} \\
29.3 \\
-\end{array}$ & $\begin{array}{r}161.1 \\
103.4 \\
7.2 \\
1.3 \\
(8) \\
1.3 \\
\overline{-} \\
49.3 \\
-\end{array}$ & $\begin{array}{r}169.5 \\
108.5 \\
6.8 \\
.8 \\
(8) \\
.7 \\
53.4 \\
-\end{array}$ & $\begin{array}{r}159.2 \\
100.6 \\
4.3 \\
.7 \\
\overline{.7} \\
53.6 \\
.1\end{array}$ & $\begin{array}{r}172.8 \\
120.2 \\
5.0 \\
1.0 \\
1.0 \\
46.5 \\
.1\end{array}$ \\
\hline Primary Energy - Fivo Sectors c ............................. & 518.6 & 998.7 & $2,065.3$ & $2,265.3$ & $R_{2,272.5}$ & $R_{2,242.1}$ & $R_{2,204.3}$ & $2,308.6$ \\
\hline
\end{tabular}

a There are no direct fuel costs for hydroelectric, geothermal, wind, photovoltalc, or solar thermal energy.

b Heavy oll includes fuel oll nos. 4, 5, and 6, and residual fuel olls. Lught oll Includes fuel nos. 1 and 2, kerosene, and jet fuel.

- Blomass fuels are not Included, except those consumed at electric utllities and those added to motor gasoline.

$R=$ Revised data.
-No consumption, including cases where adjustments were made. See explanation of adjustments in Section 6 of Appendix A.

(s)=Value less than 0.05 million dollars.

Note: Totals may not equal sum of components due to Independent rounding.

Sources: Data sources, estimation procedures, and assumptions are described in Appendix A. 
N Energy Price and Expenditure Estimates by Source, Nevada

E 1970, 1975, 1980, 1985, and 1990-1993

\begin{tabular}{|c|c|c|c|c|c|c|c|c|}
\hline Energy Source & 1970 & 1975 & 1980 & 1985 & 1990 & 1991 & 1992 & 1993 \\
\hline & \multicolumn{8}{|c|}{ Prices in Dollars per Mlllion Btu } \\
\hline 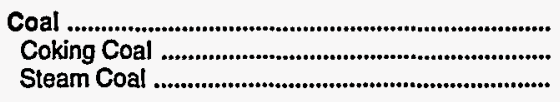 & $\begin{array}{r}0.37 \\
-37\end{array}$ & $\begin{array}{r}0.35 \\
.35\end{array}$ & 1.06 & $\begin{array}{c}1.62 \\
1.62\end{array}$ & $\begin{array}{r}1.49 \\
1.49\end{array}$ & $\begin{array}{r}1.41 \\
1.41\end{array}$ & $\frac{1.46}{1.46}$ & $\begin{array}{r}1.47 \\
1.47\end{array}$ \\
\hline Natural Gas & .61 & 1.31 & 3.10 & 5.44 & 3.68 & 3.72 & 3.65 & 4.05 \\
\hline 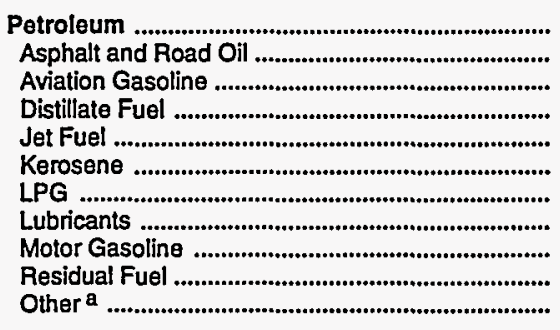 & $\begin{array}{r}1.95 \\
.59 \\
2.17 \\
1.29 \\
.76 \\
.77 \\
2.91 \\
5.08 \\
3.07 \\
.58 \\
.43\end{array}$ & $\begin{array}{r}3.40 \\
1.83 \\
3.45 \\
2.75 \\
2.12 \\
2.42 \\
4.03 \\
7.48 \\
4.74 \\
1.98 \\
-\end{array}$ & $\begin{array}{r}7.67 \\
3.69 \\
9.02 \\
6.97 \\
6.59 \\
-\overline{7} \\
7.07 \\
14.36 \\
9.96 \\
3.58 \\
4.04\end{array}$ & $\begin{array}{r}7.77 \\
4.78 \\
9.99 \\
7.32 \\
6.22 \\
14.41 \\
11.46 \\
17.61 \\
8.77 \\
4.45 \\
3.39\end{array}$ & $\begin{array}{r}R_{7.88} \\
2.60 \\
9.32 \\
7.59 \\
6.26 \\
10.02 \\
11.36 \\
R_{14.60} \\
9.10 \\
2.93 \\
-\end{array}$ & $\begin{array}{r}R_{7.46} \\
3.27 \\
8.71 \\
7.33 \\
5.00 \\
9.48 \\
12.20 \\
R_{16.79} \\
8.83 \\
3.69 \\
-\end{array}$ & $\begin{array}{r}R_{7.68} \\
2.91 \\
8.54 \\
7.30 \\
4.70 \\
10.27 \\
12.75 \\
R_{19.43} \\
9.30 \\
3.15 \\
-\end{array}$ & $\begin{array}{r}7.68 \\
2.84 \\
8.24 \\
7.37 \\
4.69 \\
11.86 \\
11.98 \\
18.94 \\
9.47 \\
3.38 \\
-\end{array}$ \\
\hline 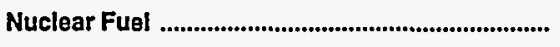 & - & - & - & - & - & - & - & - \\
\hline Blomass Fuels at Utilities ...................................... & - & - & - & - & - & - & - & - \\
\hline Primany Energy - Five Sectors b ..................... & 1.32 & 1.82 & 4.69 & 4.89 & $R_{4.57}$ & 4.27 & 4.39 & 4.54 \\
\hline $\begin{array}{l}\text { Electric Utility Fuel c ................................................. } \\
\text { Electricity Purchased by End Users .................... }\end{array}$ & $\begin{array}{r}.36 \\
3.89\end{array}$ & $\begin{array}{r}.59 \\
6.86\end{array}$ & $\begin{array}{r}1.68 \\
13.18\end{array}$ & $\begin{array}{r}1.79 \\
16.75\end{array}$ & $\begin{array}{r}1.59 \\
15.77\end{array}$ & $\begin{array}{r}1.48 \\
16.47\end{array}$ & $\begin{array}{r}1.55 \\
16.69\end{array}$ & $\begin{array}{r}1.60 \\
17.28\end{array}$ \\
\hline \multirow[t]{2}{*}{ Total Energy .......................................................... } & 1.96 & 3.52 & 8.18 & 9.03 & ${ }^{\text {A } 9.00}$ & 8.78 & $R_{9.08}$ & 8.92 \\
\hline & \multicolumn{8}{|c|}{ Expendltures in Millions of Dollars } \\
\hline 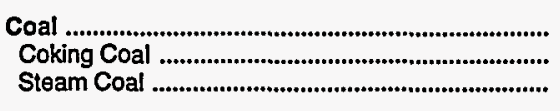 & $\begin{array}{l}6.4 \\
6.4\end{array}$ & $\begin{array}{c}35.8 \\
- \\
35.8\end{array}$ & $\begin{array}{c}99.1 \\
- \\
99.1\end{array}$ & $\begin{array}{c}204.2 \\
- \\
204.2\end{array}$ & $\begin{array}{c}\mathrm{A}_{247.5} \\
\mathrm{R}_{247.5}\end{array}$ & $\begin{array}{c}R_{254.1} \\
- \\
R_{254.1}\end{array}$ & $\begin{array}{c}R_{261.9} \\
R_{261.9}\end{array}$ & $\begin{array}{c}253.1 \\
- \\
253.1\end{array}$ \\
\hline 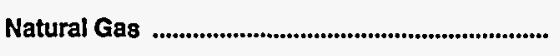 & 34.5 & 85.5 & 191.5 & 222.8 & 243.0 & 245.1 & 252.7 & 345.6 \\
\hline $\begin{array}{l}\text { Petroleum } \\
\text { Asphalt and Road Oil } \\
\text { Aviation Gasoline } \\
\text { Distillate Fuel } \\
\text { Jet Fuel } \\
\text { Kerosene } \\
\text { LPG } \\
\text { Lubricants } \\
\text { Retor Gasoline } \\
\text { Other }{ }^{\mathrm{a}}\end{array}$ & $\begin{array}{r}176.6 \\
2.4 \\
2.0 \\
21.2 \\
19.2 \\
.1 \\
9.2 \\
3.2 \\
118.7 \\
.5 \\
(s)\end{array}$ & $\begin{array}{r}393.4 \\
10.2 \\
3.4 \\
41.1 \\
69.2 \\
.4 \\
7.4 \\
5.4 \\
239.7 \\
16.7 \\
-\end{array}$ & $\begin{array}{r}1,125.9 \\
15.0 \\
9.4 \\
160.9 \\
266.2 \\
- \\
22.9 \\
9.4 \\
587.0 \\
55.0 \\
.1\end{array}$ & $\begin{array}{r}1,055.0 \\
26.8 \\
5.3 \\
230.4 \\
197.0 \\
4.3 \\
40.6 \\
10.5 \\
535.6 \\
4.4 \\
.1\end{array}$ & $\begin{array}{r}R_{1,350.4} \\
18.7 \\
5.2 \\
325.2 \\
212.9 \\
1.1 \\
56.9 \\
\mathbf{P}_{9.8} \\
710.2 \\
8.4 \\
-\end{array}$ & $\begin{array}{r}R_{1,292.7} \\
23.3 \\
4.9 \\
302.6 \\
182.5 \\
1.2 \\
45.9 \\
R_{10.1} \\
712.1 \\
10.2 \\
-\end{array}$ & $\begin{array}{r}R_{1,344.6} \\
16.2 \\
4.5 \\
311.9 \\
161.8 \\
1.4 \\
41.6 \\
R_{11.9} \\
783.8 \\
11.4 \\
-\end{array}$ & $\begin{array}{r}1,389.4 \\
21.6 \\
4.7 \\
326.3 \\
171.1 \\
1.0 \\
36.0 \\
11.8 \\
807.0 \\
9.8 \\
-\end{array}$ \\
\hline 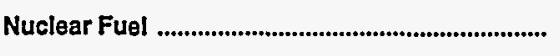 & - & - & - & - & - & - & - & - \\
\hline Blomass Fuels at Utilitles .................................... & - & - & - & - & - & - & - & - \\
\hline Primary Energy - Flve Sectors b ...................... & 217.5 & $\mathbf{5 1 4 . 7}$ & $1,416.5$ & $1,481.9$ & $R_{1,840.9}$ & $R_{1,791.8}$ & $R_{1,859,2}$ & $1,988.0$ \\
\hline Electric Utility Fuel c .................................................. & $\begin{array}{r}-15.1 \\
75.7\end{array}$ & $\begin{array}{l}-79.8 \\
179.0\end{array}$ & $\begin{array}{r}-226.1 \\
468.2\end{array}$ & $\begin{array}{r}-238.1 \\
634.3\end{array}$ & $\begin{array}{r}-301.8 \\
879.9\end{array}$ & $\begin{array}{r}-296.7 \\
914.1\end{array}$ & $\begin{array}{r}-314.8 \\
987.2\end{array}$ & $\begin{array}{r}-308.1 \\
1,066.2\end{array}$ \\
\hline Total Energy & 278.1 & 613.9 & $1,658.6$ & $1,878.1$ & $R_{2,419.0}$ & $R_{2,409.3}$ & $R_{\mathbf{2 , 5 3 1 . 6}}$ & $2,746.1$ \\
\hline
\end{tabular}

a Includes petroleum coke used at electric utilities.

b Biomass fuels are not included, except those consumed at electric utilities and those added to motor gasoline.

c There are no direct fuel costs for hydroelectric, geothermal, wind, photovoltaic, or solar thermal energy.

$\mathbf{R}=$ Revised data.

-No consumption, including cases where adjustments were made. Seo

explanation of adjustments in Section 6 of Appendix A.

(s) =Value less than 0.05 million dollars.

Note: Expenditure totals may not equal sum of components due to independent rounding.

Sources: Data sources, estimation procedures, and assumptions are described in Appendix A. 


\begin{tabular}{|c|c|c|c|c|c|c|c|c|}
\hline Sector and Energy Source & 1970 & 1975 & 1980 & 1985 & 1990 & 1991 & 1992 & 1993 \\
\hline 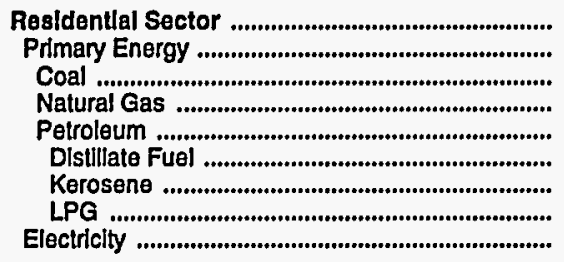 & $\begin{array}{r}2.70 \\
1.76 \\
1.31 \\
1.39 \\
2.51 \\
1.27 \\
3 . \overline{5} \\
4.46\end{array}$ & $\begin{array}{l}4.30 \\
2.18 \\
1.55 \\
1.83 \\
3.72 \\
2.82 \\
4 . \overline{0} \\
7.54\end{array}$ & $\begin{array}{r}8.74 \\
4.58 \\
5.13 \\
3.87 \\
8.31 \\
6.92 \\
-\overline{9} \\
9.28 \\
14.21\end{array}$ & $\begin{array}{r}12.59 \\
7.61 \\
4.54 \\
6.63 \\
10.68 \\
7.55 \\
15.04 \\
12.40 \\
18.83\end{array}$ & $\begin{array}{r}11.26 \\
6.61 \\
5.03 \\
5.49 \\
11.10 \\
6.76 \\
13.48 \\
13.10 \\
16.72\end{array}$ & $\begin{array}{r}11.32 \\
6.39 \\
4.07 \\
5.41 \\
11.21 \\
6.58 \\
13.11 \\
13.42 \\
17.28\end{array}$ & $\begin{array}{r}12.06 \\
6.46 \\
4.14 \\
5.40 \\
11.98 \\
R 7.74 \\
15.41 \\
14.23 \\
18.12\end{array}$ & $\begin{array}{r}12.20 \\
6.25 \\
3.87 \\
5.49 \\
11.08 \\
6.96 \\
13.88 \\
12.92 \\
19.08\end{array}$ \\
\hline $\begin{array}{l}\text { Commerclal Sector } \\
\text { Primary Energy } \\
\text { Coal .... } \\
\text { Natural Gas } \\
\text { Petroleum } \\
\text { Distlllate Fuel } \\
\text { Kerosene } \\
\text { LPG } \\
\text { Motor Gasoline } \\
\text { Restdual Fuel } \\
\text { Electricity }\end{array}$ & $\begin{array}{r}2.15 \\
.77 \\
.52 \\
.70 \\
1.34 \\
1.12 \\
.77 \\
1.16 \\
3.07 \\
.62 \\
4.74\end{array}$ & $\begin{array}{r}3.88 \\
1.59 \\
.82 \\
1.45 \\
2.99 \\
2.62 \\
2.42 \\
2.45 \\
4.74 \\
2.00 \\
8.01\end{array}$ & $\begin{array}{r}7.73 \\
4.29 \\
1.36 \\
3.68 \\
6.78 \\
6.60 \\
- \\
4.99 \\
9.96 \\
3.53 \\
15.39\end{array}$ & $\begin{array}{r}11.26 \\
6.17 \\
1.58 \\
5.77 \\
8.02 \\
7.76 \\
9.06 \\
9.63 \\
8.77 \\
4.80 \\
18.24\end{array}$ & $\begin{array}{r}10.51 \\
4.77 \\
R \mathbf{1 . 5 6} \\
4.25 \\
7.50 \\
6.79 \\
7.19 \\
9.03 \\
9.10 \\
2.85 \\
17.39\end{array}$ & $\begin{array}{r}10.44 \\
4.58 \\
\mathrm{R} 1.56 \\
4.19 \\
7.26 \\
6.38 \\
6.46 \\
9.29 \\
8.83 \\
2.54 \\
17.88\end{array}$ & $\begin{array}{r}10.77 \\
4.60 \\
\mathrm{R} 1.53 \\
4.19 \\
7.37 \\
6.54 \\
6.65 \\
9.26 \\
9.30 \\
2.44 \\
17.85\end{array}$ & $\begin{array}{r}10.60 \\
4.59 \\
1.53 \\
4.25 \\
6.15 \\
5.74 \\
6.23 \\
9.21 \\
9.47 \\
- \\
18.38\end{array}$ \\
\hline 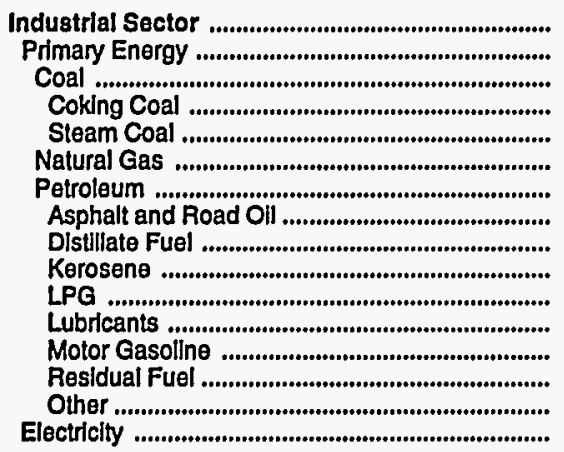 & $\begin{array}{r}1.02 \\
.75 \\
.52 \\
- \\
.52 \\
.52 \\
1.04 \\
.59 \\
.96 \\
.77 \\
1.16 \\
5.08 \\
3.07 \\
.49 \\
.43 \\
2.14\end{array}$ & $\begin{array}{r}2.18 \\
1.61 \\
.82 \\
\overline{.82} \\
1.06 \\
2.25 \\
1.83 \\
2.25 \\
2.42 \\
2.45 \\
7.48 \\
4.74 \\
1.83 \\
4 . \overline{23}\end{array}$ & $\begin{array}{r}7.20 \\
3.67 \\
1.36 \\
- \\
1.36 \\
2.83 \\
5.10 \\
3.69 \\
5.56 \\
-\overline{4} \\
4.99 \\
14.36 \\
9.96 \\
3.75 \\
4.04 \\
11.63\end{array}$ & $\begin{array}{r}8.06 \\
5.72 \\
1.58 \\
- \\
1.58 \\
4.05 \\
6.97 \\
4.78 \\
7.97 \\
9.06 \\
9.63 \\
17.61 \\
8.77 \\
4.80 \\
3.39 \\
12.91\end{array}$ & $\begin{array}{r}R_{7.99} \\
R_{4.95} \\
R_{1.56} \\
- \\
R_{1.56} \\
3.98 \\
5.67 \\
2.60 \\
6.32 \\
7.19 \\
9.03 \\
R_{14.60} \\
9.10 \\
2.85 \\
-\overline{13.76}\end{array}$ & $\begin{array}{r}R_{8.04} \\
R_{4.59} \\
R_{1.56} \\
- \\
R_{1.56} \\
4.06 \\
R_{5.24} \\
3.27 \\
5.67 \\
6.46 \\
9.29 \\
R_{16.79} \\
8.83 \\
2.54 \\
-\overline{14.53}\end{array}$ & $\begin{array}{r}R_{8.23} \\
R_{4.65} \\
R_{1.53} \\
- \\
R_{1.53} \\
3.93 \\
R_{5.41} \\
2.91 \\
5.84 \\
6.65 \\
9.26 \\
R_{19.43} \\
9.30 \\
2.44 \\
-\overline{14.43}\end{array}$ & $\begin{array}{r}7.46 \\
4.24 \\
1.53 \\
-\overline{1} \\
1.53 \\
4.15 \\
4.84 \\
2.84 \\
5.48 \\
6.23 \\
9.21 \\
18.94 \\
9.47 \\
2.36 \\
-\overline{14} \\
14.77\end{array}$ \\
\hline 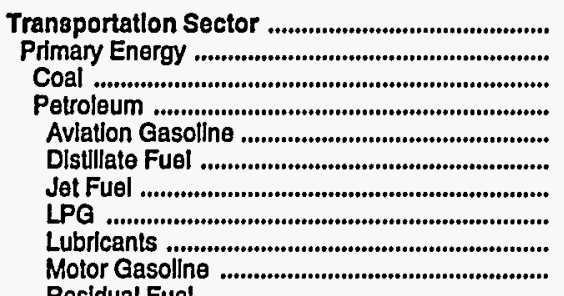 & $\begin{array}{r}2.08 \\
2.08 \\
.52 \\
2.08 \\
2.17 \\
1.50 \\
.76 \\
1.16 \\
5.08 \\
3.07\end{array}$ & $\begin{array}{r}3.66 \\
3.66 \\
.82 \\
3.66 \\
3.45 \\
3.01 \\
2.12 \\
2.45 \\
7.48 \\
4.74\end{array}$ & $\begin{array}{r}8.44 \\
8.44 \\
-\overline{8.44} \\
9.02 \\
7.36 \\
6.59 \\
4.99 \\
14.36 \\
9.96\end{array}$ & $\begin{array}{r}7.79 \\
7.79 \\
- \\
7.79 \\
9.99 \\
6.97 \\
6.22 \\
9.63 \\
17.61 \\
8.77\end{array}$ & $\begin{array}{r}R_{8.37} \\
R_{8.37} \\
- \\
R_{8.37} \\
9.32 \\
8.97 \\
6.26 \\
9.03 \\
R_{14.60} \\
9.10\end{array}$ & $\begin{array}{r}7.85 \\
7.85 \\
- \\
7.85 \\
8.71 \\
8.89 \\
5.00 \\
9.29 \\
\text { A } 16.79 \\
8.83\end{array}$ & $\begin{array}{r}\mathrm{A}_{8.09} \\
\mathrm{R}_{\mathbf{8 . 0 9}} \\
\mathrm{A}_{\mathbf{8 . 0 9}} \\
\mathbf{8 . 5 4} \\
8.53 \\
4.70 \\
9.26 \\
\mathrm{R}_{19.43} \\
9.30\end{array}$ & $\begin{array}{r}8.19 \\
8.19 \\
- \\
8.19 \\
8.24 \\
8.80 \\
4.69 \\
9.21 \\
18.94 \\
9.47\end{array}$ \\
\hline 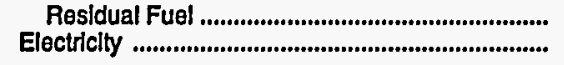 & .60 & $\begin{array}{r}2.36 \\
-\end{array}$ & - & - & $\overline{-}$ & $\overline{-}$ & $\overline{-}$ & $\overline{-}$ \\
\hline 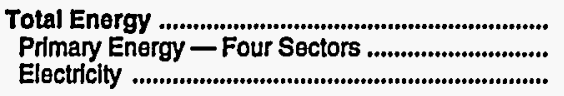 & $\begin{array}{l}1.96 \\
1.65 \\
3.89\end{array}$ & $\begin{array}{l}3.52 \\
2.93 \\
6.86\end{array}$ & $\begin{array}{r}8.18 \\
7.12 \\
13.18\end{array}$ & $\begin{array}{r}9.03 \\
7.31 \\
16.75\end{array}$ & $\begin{array}{r}R_{9.00} \\
7.22 \\
15.77\end{array}$ & $\begin{array}{r}8.78 \\
6.83 \\
16.47\end{array}$ & $\begin{array}{r}R_{9.08} \\
R_{7.03} \\
16.69\end{array}$ & $\begin{array}{r}8.92 \\
6.83 \\
17.28\end{array}$ \\
\hline 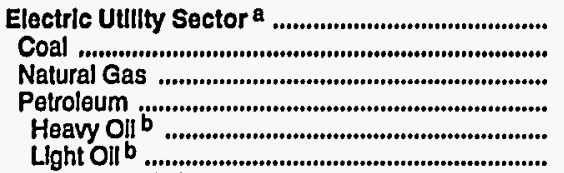 & $\begin{array}{l}.36 \\
.31 \\
.38 \\
.62 \\
.61 \\
.70\end{array}$ & $\begin{array}{r}.59 \\
.34 \\
1.09 \\
2.00 \\
1.98 \\
2.47\end{array}$ & $\begin{array}{l}1.68 \\
1.05 \\
2.59 \\
3.60 \\
3.58 \\
5.58\end{array}$ & $\begin{array}{l}1.79 \\
1.62 \\
4.07 \\
4.91 \\
3.71 \\
6.12\end{array}$ & $\begin{array}{l}1.59 \\
1.49 \\
1.96 \\
3.50 \\
2.93 \\
6.47\end{array}$ & $\begin{array}{l}1.48 \\
1.41 \\
1.73 \\
4.05 \\
3.86 \\
5.20\end{array}$ & $\begin{array}{l}1.55 \\
1.46 \\
1.87 \\
3.41 \\
3.23 \\
4.90\end{array}$ & $\begin{array}{l}1.60 \\
1.47 \\
2.38 \\
3.68 \\
3.55 \\
5.08\end{array}$ \\
\hline 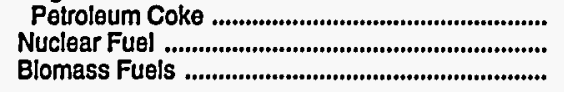 & $\begin{array}{l}- \\
-\end{array}$ & $\begin{array}{l}- \\
-\end{array}$ & $\begin{array}{l}- \\
-\end{array}$ & $\overline{-}$ & $\overrightarrow{-}$ & $\begin{array}{l}- \\
-\end{array}$ & $\begin{array}{l}- \\
-\end{array}$ & $\begin{array}{l}- \\
-\end{array}$ \\
\hline Primary Energy — Flve Sectors c ........................ & 1.32 & 1.82 & 4.69 & 4.89 & $R_{4.57}$ & 4.27 & 4.39 & 4.54 \\
\hline
\end{tabular}

There are no direct fuel costs for hydroelectric, geothermal, wind, photovoltalc, or solar thermal energy.

beavy oil includes fuel oll nos. 4, 5, and 6, and residual fuel olls. Light oll Includes fuel oll nos. 1 and 2, kerosene, and jet fuel.

- Blomass fuels are not included, except those consumed at electric utllitles and those added to motor gasolline.
$R=$ Revised data.

- No consumption, Including cases where adjustments were made. See explanation of adjustments in Section 6 of Appendix $A$.

Sources: Data sources, estimation procedures, and assumptions are described in Appendix A. 
N Energy Expenditure Estimates by Sector, Nevada

E $1970,1975,1980,1985$, and 1990-1993

V (Million Dollars)

\begin{tabular}{|c|c|c|c|c|c|c|c|c|}
\hline Sector and Energy Source & 1970 & 1975 & 1980 & 1985 & 1990 & 1991 & 1992 & 1893 \\
\hline $\begin{array}{l}\text { Residential Sector } \\
\text { Primary Energy } \\
\text { Coal } \\
\text { Natural Gas }\end{array}$ & $\begin{array}{r}52.6 \\
22.4 \\
.7 \\
10.9 \\
10.7 \\
2.4 \\
\overline{8} \\
8.3 \\
30.3\end{array}$ & $\begin{array}{r}104.0 \\
31.9 \\
.1 \\
21.6 \\
10.1 \\
4.4 \\
\overrightarrow{5.8} \\
72.1\end{array}$ & $\begin{array}{r}255.1 \\
75.9 \\
.2 \\
53.6 \\
22.1 \\
7.5 \\
-\overline{1} \\
14.6 \\
179.2\end{array}$ & $\begin{array}{r}389.4 \\
134.3 \\
.1 \\
88.7 \\
45.6 \\
12.5 \\
4.0 \\
29.1 \\
265.1\end{array}$ & $\begin{array}{r}462.1 \\
146.1 \\
.2 \\
97.1 \\
48.9 \\
9.4 \\
.6 \\
38.8 \\
316.0\end{array}$ & $\begin{array}{r}493.2 \\
152.2 \\
.1 \\
107.3 \\
44.8 \\
8.5 \\
.8 \\
35.6 \\
341.0\end{array}$ & $\begin{array}{r}519.9 \\
144.9 \\
(8) \\
101.6 \\
43.2 \\
9.8 \\
.8 \\
32.6 \\
375.0\end{array}$ & $\begin{array}{r}563.9 \\
154.9 \\
.1 \\
117.7 \\
37.1 \\
7.3 \\
.8 \\
29 \\
29.0 \\
409.0\end{array}$ \\
\hline 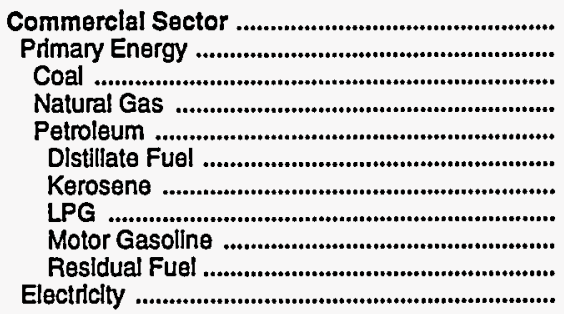 & $\begin{array}{r}43.7 \\
10.3 \\
.5 \\
7.3 \\
2.5 \\
1.0 \\
(s) \\
.5 \\
.8 \\
.1 \\
33.4\end{array}$ & $\begin{array}{r}106.6 \\
28.1 \\
.1 \\
23.2 \\
4.8 \\
2.0 \\
.2 \\
.5 \\
1.7 \\
.4 \\
78.6\end{array}$ & $\begin{array}{r}151.1 \\
57.9 \\
.1 \\
39.6 \\
18.3 \\
13.6 \\
\overline{1.4} \\
3.2 \\
.2 \\
93.2\end{array}$ & $\begin{array}{r}310.4 \\
98.4 \\
(\mathrm{~s}) \\
74.9 \\
23.4 \\
14.6 \\
.2 \\
4.0 \\
3.8 \\
.8 \\
212.0\end{array}$ & $\begin{array}{r}358.8 \\
88.8 \\
.1 \\
66.0 \\
22.7 \\
13.8 \\
.1 \\
4.7 \\
4.0 \\
(s) \\
270.0\end{array}$ & $\begin{array}{r}377.7 \\
92.7 \\
(s) \\
73.6 \\
19.0 \\
10.9 \\
.1 \\
4.3 \\
3.6 \\
(s) \\
285.0\end{array}$ & $\begin{array}{r}387.4 \\
88.4 \\
(s) \\
69.8 \\
18.6 \\
11.3 \\
.2 \\
3.7 \\
3.4 \\
(8) \\
299.0\end{array}$ & $\begin{array}{r}418.0 \\
102.1 \\
(s) \\
77.3 \\
24.7 \\
20.4 \\
.1 \\
3.7 \\
.6 \\
316.0\end{array}$ \\
\hline 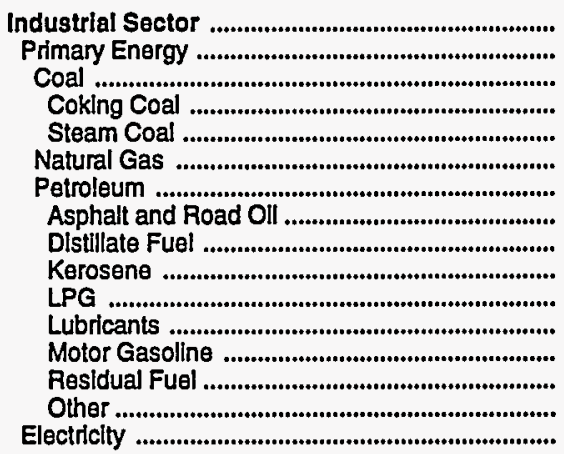 & $\begin{array}{r}29.7 \\
17.7 \\
.9 \\
-.9 \\
.9 \\
5.8 \\
11.0 \\
2.4 \\
4.7 \\
(s) \\
.4 \\
.7 \\
2.7 \\
.1 \\
\text { (s) } \\
12.0\end{array}$ & $\begin{array}{r}66.4 \\
38.1 \\
1.5 \\
\overline{1.5} \\
11.4 \\
25.2 \\
10.2 \\
9.3 \\
.2 \\
1.0 \\
1.2 \\
2.9 \\
.5 \\
28.3\end{array}$ & $\begin{array}{r}273.4 \\
77.6 \\
4.6 \\
- \\
4.6 \\
21.9 \\
51.1 \\
15.0 \\
21.1 \\
\overline{6.9} \\
2.2 \\
5.8 \\
(8) \\
195.8\end{array}$ & $\begin{array}{r}300.9 \\
143.7 \\
4.1 \\
-\overline{4.1} \\
24.2 \\
115.4 \\
26.8 \\
71.1 \\
(8) \\
6.5 \\
2.4 \\
6.0 \\
2.5 \\
1 \\
157.2\end{array}$ & $\begin{array}{r}R_{495.0} \\
R_{201.0} \\
R_{6.1} \\
R_{6.1} \\
30.8 \\
R_{164.1} \\
18.7 \\
120.0 \\
.3 \\
14.6 \\
R_{2.3} \\
8.1 \\
.1 \\
-\overline{2} \\
294.0\end{array}$ & $\begin{array}{r}R_{459.1} \\
R_{171.0} \\
R_{7.1} \\
R_{7.1} \\
25.5 \\
R_{138.4} \\
23.3 \\
98.0 \\
.3 \\
5.3 \\
R_{2.3} \\
8.3 \\
.9 \\
288.1\end{array}$ & $\begin{array}{r}R_{488.2} \\
R_{175.1} \\
R_{6.1} \\
- \\
R_{6.1} \\
34.7 \\
R_{134.2} \\
16.2 \\
101.2 \\
.4 \\
4.4 \\
R_{2.8} \\
8.4 \\
.8 \\
313.2\end{array}$ & $\begin{array}{r}563.4 \\
222.1 \\
7.0 \\
-\overline{0} \\
7.0 \\
98.5 \\
116.7 \\
21.6 \\
81.8 \\
(8) \\
2.5 \\
2.7 \\
7.0 \\
1.0 \\
-\overline{3} \\
341.3\end{array}$ \\
\hline 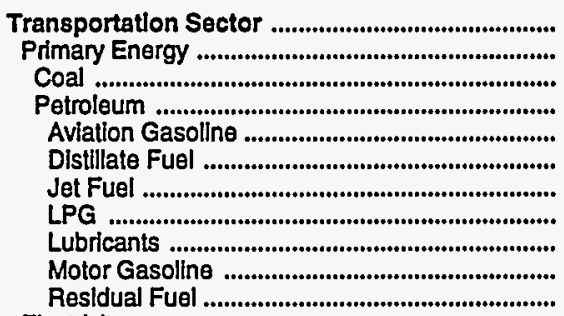 & $\begin{array}{r}152.1 \\
152.1 \\
(s) \\
152.1 \\
2.0 \\
13.0 \\
19.2 \\
(s) \\
2.6 \\
115.3 \\
\text { (s) }\end{array}$ & $\begin{array}{r}336.9 \\
336.9 \\
(s) \\
336.9 \\
3.4 \\
24.7 \\
69.2 \\
.1 \\
4.2 \\
235.2 \\
.1\end{array}$ & $\begin{array}{r}978.9 \\
978.9 \\
- \\
978.9 \\
9.4 \\
118.0 \\
266.2 \\
.1 \\
7.3 \\
578.0 \\
-\end{array}$ & $\begin{array}{r}867.5 \\
867.5 \\
867.5 \\
5.3 \\
130.3 \\
197.0 \\
1.1 \\
8.1 \\
525.7 \\
-\end{array}$ & $\begin{array}{r}R_{1}, 103.2 \\
R_{1,103.2} \\
- \\
R_{1,103.2} \\
5.2 \\
178.7 \\
212.9 \\
.7 \\
R_{7.6} \\
698.1 \\
-\end{array}$ & $\begin{array}{r}R_{1}, 079.2 \\
R_{1,079.2} \\
R_{1,079.2} \\
4.9 \\
183.1 \\
182.5 \\
.7 \\
R_{7.8} \\
700.2 \\
-\end{array}$ & $\begin{array}{r}R_{1,136.1} \\
R_{1,136.1} \\
- \\
R_{1,136.1} \\
4.5 \\
187.7 \\
161.8 \\
.8 \\
R_{9.2} \\
772.0 \\
-\end{array}$ & $\begin{array}{r}1,200.8 \\
1,200.8 \\
- \\
1,200.8 \\
4.7 \\
215.6 \\
171.1 \\
.9 \\
9.1 \\
799.4 \\
-\end{array}$ \\
\hline 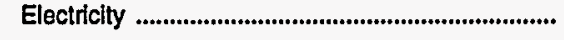 & . & - & - & - & - & - & - & - \\
\hline 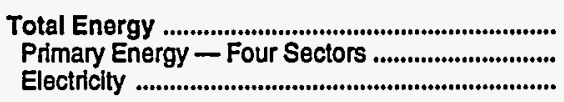 & $\begin{array}{r}278.1 \\
202.5 \\
75.7\end{array}$ & $\begin{array}{l}613.9 \\
434.9 \\
179.0\end{array}$ & $\begin{array}{r}1,658.6 \\
1,190.4 \\
468.2\end{array}$ & $\begin{array}{r}1,878.1 \\
1,243.9 \\
634.3\end{array}$ & $\begin{array}{r}R_{2}, 419.0 \\
R_{1,539.1} \\
879.9\end{array}$ & $\begin{array}{r}A_{2}, 409.3 \\
A_{1,495.2} \\
914.1\end{array}$ & $\begin{array}{r}\mathrm{R}_{2,531.6} \\
\mathrm{R}_{1,544.4} \\
887.2\end{array}$ & $\begin{array}{l}2,746.1 \\
1,679.9 \\
1,066.2\end{array}$ \\
\hline 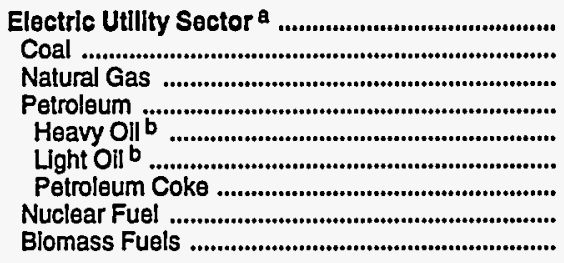 & $\begin{array}{r}15.1 \\
4.3 \\
10.5 \\
.4 \\
.3 \\
.1 \\
= \\
=\end{array}$ & $\begin{array}{r}79.8 \\
34.1 \\
29.3 \\
16.5 \\
15.7 \\
.8 \\
= \\
=\end{array}$ & $\begin{array}{r}226.1 \\
94.2 \\
76.4 \\
55.5 \\
54.8 \\
.7 \\
- \\
- \\
-\end{array}$ & $\begin{array}{r}238.1 \\
199.9 \\
35.0 \\
3.1 \\
1.2 \\
1.9 \\
= \\
-\end{array}$ & $\begin{array}{r}301.8 \\
241.1 \\
49.1 \\
11.6 \\
8.2 \\
3.4 \\
= \\
=\end{array}$ & $\begin{array}{r}296.7 \\
246.8 \\
38.6 \\
11.3 \\
9.2 \\
2.0 \\
- \\
-\end{array}$ & $\begin{array}{r}314.8 \\
255.7 \\
46.6 \\
12.4 \\
10.5 \\
1.9 \\
- \\
= \\
-\end{array}$ & $\begin{array}{r}308.1 \\
246.0 \\
52.1 \\
10.0 \\
8.8 \\
1.2 \\
= \\
=\end{array}$ \\
\hline Primary Energy - Flve Sectors ${ }^{\circ} \ldots \ldots . . . . . . . . . . . . . . . . . . .$. & 217.5 & 514.7 & $1,416.5$ & $1,481.9$ & $R_{1,840.9}$ & $A_{1,791.8}$ & $R_{1,859.2}$ & $1,988.0$ \\
\hline
\end{tabular}

a There are no direct fuel costs for hydroelectric, geothermal, wind, photovoltaic, or solar thermal energy.

beavy oil Includes fuel oil nos. 4, 5, and 6, and residual fuel oils. Light oil includes fuel nos. 1 and 2, kerosene, and jet fuel.

c Blomass fuels are not included, except those consumed at electric utilities and those added to motor gasoline.

$\mathrm{R}=$ Revised data.
-No consumption, including cases where adjustments were made. See explanation of adjustments in Section 6 of Append IXA.

$(s)=$ Value less than 0.05 million dollars.

Note: Totals may not equal sum of components due to independent rounding.

Sources: Data sources, estimation procedures, and assumptions are described in Appendix A. 


\begin{tabular}{|c|c|c|c|c|c|c|c|c|}
\hline Energy Source & 1970 & 1975 & 1980 & 1985 & 1990 & 1991 & 1992 & 1993 \\
\hline & \multicolumn{8}{|c|}{ Prices in Dollars per Million Btu } \\
\hline 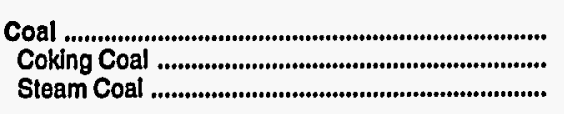 & $\frac{0.37}{.37}$ & $\overline{1.22}$ & $\frac{1.60}{1.60}$ & $2 . \overline{03}$ & $\frac{1.82}{1.82}$ & $\frac{1.81}{1.81}$ & $\frac{1.73}{1.73}$ & $\begin{array}{r}1.66 \\
1.66\end{array}$ \\
\hline Natural Gas & 1.65 & 2.24 & 4.27 & 6.44 & 6.38 & 6.12 & 6.23 & 6.56 \\
\hline $\begin{array}{l}\text { Potroleum } \\
\text { Asphalt and Road Oil } \\
\text { Aviation Gasoline } \\
\text { Distlllate Fuel } \\
\text { Jet Fuel }\end{array}$ & $\begin{array}{r}1.60 \\
.70 \\
2.17 \\
1.38 \\
.75 \\
1.50 \\
2.06 \\
5.08 \\
2.92 \\
.42 \\
1.76\end{array}$ & $\begin{array}{l}3.24 \\
2.05 \\
3.45 \\
2.80 \\
2.10 \\
3.09 \\
3.53 \\
7.48 \\
4.54 \\
1.85 \\
4.01\end{array}$ & $\begin{array}{r}7.29 \\
4.00 \\
9.02 \\
6.97 \\
6.51 \\
8.05 \\
6.89 \\
14.36 \\
10.11 \\
3.82 \\
8.04\end{array}$ & $\begin{array}{r}7.78 \\
5.20 \\
9.99 \\
7.51 \\
6.53 \\
8.71 \\
11.42 \\
17.61 \\
9.26 \\
3.81 \\
10.67\end{array}$ & $\begin{array}{r}7.24 \\
3.34 \\
9.32 \\
7.30 \\
6.40 \\
8.56 \\
11.55 \\
\mathrm{R} 14.60 \\
9.66 \\
2.43 \\
10.72\end{array}$ & $\begin{array}{r}7.32 \\
3.05 \\
8.71 \\
6.69 \\
5.36 \\
7.55 \\
12.82 \\
\mathrm{R} 16.79 \\
9.55 \\
1.90 \\
\mathrm{R} 11.27\end{array}$ & $\begin{array}{r}7.20 \\
2.78 \\
8.54 \\
6.30 \\
4.86 \\
7.07 \\
11.54 \\
R_{19.43} \\
9.50 \\
1.93 \\
18 \\
R_{16.00}\end{array}$ & $\begin{array}{r}7.18 \\
3.30 \\
8.24 \\
6.16 \\
4.58 \\
6.89 \\
11.37 \\
18.94 \\
9.42 \\
1.91 \\
12.87\end{array}$ \\
\hline Nuclear Fuel .................................................................... & - & - & - & - & 1.03 & .84 & .95 & .57 \\
\hline Blomass Fuels at Utiltiles ............................................. & - & - & - & - & - & - & - & - \\
\hline Primary Energy - Flve Sectors b .......................... & 1.41 & 2.88 & 6.16 & 6.41 & $\mathrm{R}_{5.38}$ & 4.74 & 4.57 & 4.30 \\
\hline 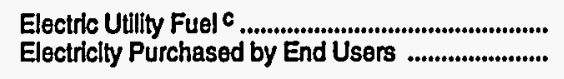 & $\begin{array}{r}.36 \\
6.74\end{array}$ & $\begin{array}{r}1.43 \\
12.68\end{array}$ & $\begin{array}{r}2.68 \\
19.55\end{array}$ & $\begin{array}{r}2.46 \\
23.28\end{array}$ & $\begin{array}{r}1.58 \\
26.67\end{array}$ & $\begin{array}{r}1.22 \\
26.76\end{array}$ & $\begin{array}{r}1.24 \\
29.23\end{array}$ & $\begin{array}{r}.95 \\
31.81\end{array}$ \\
\hline \multirow[t]{2}{*}{ 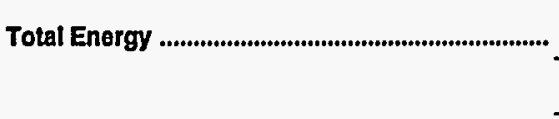 } & 2.20 & 4.38 & 9.59 & 10.68 & 11.28 & ${ }^{\mathrm{R}} 11.12$ & 11.31 & 11.58 \\
\hline & \multicolumn{8}{|c|}{ Expenditures in Mlllions of Dollars } \\
\hline 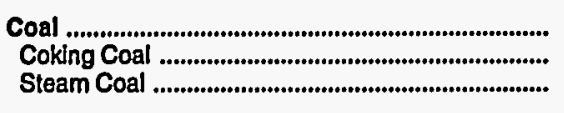 & $\begin{array}{c}10.1 \\
\overline{10.1}\end{array}$ & $\begin{array}{l}31.9 \\
\overline{31.9}\end{array}$ & $\begin{array}{l}46.9 \\
\overline{46.9}\end{array}$ & $\begin{array}{l}80.5 \\
\overline{0} 0.5\end{array}$ & $\begin{array}{l}57.2 \\
57.2\end{array}$ & $\begin{array}{l}62.7 \\
- \\
62.7\end{array}$ & $\begin{array}{c}60.1 \\
- \\
60.1\end{array}$ & $\begin{array}{l}62.1 \\
- \\
62.1\end{array}$ \\
\hline 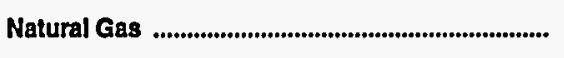 & 11.2 & 17.2 & 41.0 & 69.7 & 92.2 & 86.9 & 105.4 & 109.8 \\
\hline 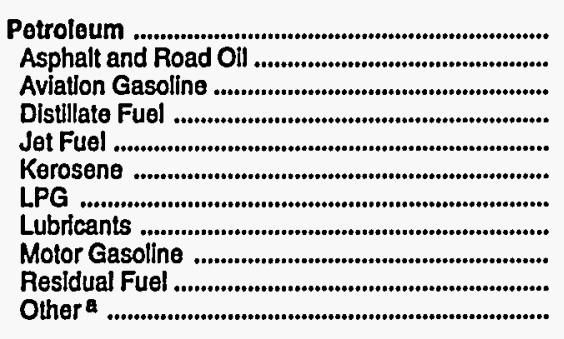 & $\begin{array}{r}225.2 \\
2.5 \\
.4 \\
61.9 \\
4.2 \\
6.6 \\
6.5 \\
2.2 \\
124.4 \\
14.7 \\
1.6\end{array}$ & $\begin{array}{r}441.9 \\
5.9 \\
.6 \\
116.9 \\
10.3 \\
8.1 \\
18.7 \\
3.2 \\
223.4 \\
53.2 \\
1.7\end{array}$ & $\begin{array}{r}971.1 \\
6.7 \\
1.8 \\
236.1 \\
27.3 \\
15.5 \\
31.3 \\
7.3 \\
498.1 \\
135.5 \\
11.6\end{array}$ & $\begin{array}{r}989.6 \\
29.5 \\
1.2 \\
229.4 \\
18.4 \\
44.5 \\
65.3 \\
8.1 \\
502.8 \\
82.4 \\
8.1\end{array}$ & $\begin{array}{r}R_{1,112.1} \\
26.6 \\
1.0 \\
269.2 \\
22.7 \\
12.9 \\
88.9 \\
87.6 \\
594.2 \\
80.3 \\
8.8\end{array}$ & $\begin{array}{r}R_{1,040.5} \\
13.3 \\
1.1 \\
R_{247.7} \\
13.9 \\
13.8 \\
76.5 \\
R_{7.8} \\
608.8 \\
48.0 \\
9.6\end{array}$ & $\begin{array}{r}R_{1,027.4} \\
14.6 \\
.8 \\
242.8 \\
10.2 \\
11.7 \\
73.7 \\
R_{9.2} \\
604.4 \\
45.8 \\
14.2\end{array}$ & $\begin{array}{r}1,052.4 \\
7.0 \\
1.8 \\
241.2 \\
9.9 \\
15.4 \\
88.7 \\
9.1 \\
618.2 \\
49.2 \\
11.9\end{array}$ \\
\hline 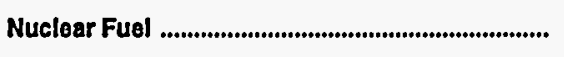 & - & - & - & - & 45.0 & 61.4 & 80.0 & 55.5 \\
\hline Blomass Fuels at Utilities ............................................ & - & - & - & - & - & - & - & - \\
\hline Primary Energy — Five Sectors b .......................... & 246.4 & 491.0 & $1,059.1$ & $1,139.8$ & $\mathrm{~A}_{1,306.5}$ & $R_{1,251.5}$ & $R_{1,272.9}$ & $1,279.8$ \\
\hline 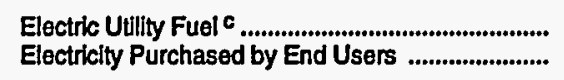 & $\begin{array}{r}-15.6 \\
83.5\end{array}$ & $\begin{array}{r}-58.2 \\
207.7\end{array}$ & $\begin{array}{r}-150.9 \\
394.5\end{array}$ & $\begin{array}{r}-131.5 \\
588.4\end{array}$ & $\begin{array}{r}-156.9 \\
817.1\end{array}$ & $\begin{array}{r}-149.3 \\
800.0\end{array}$ & $\begin{array}{r}-163.0 \\
893.0\end{array}$ & $\begin{array}{r}-139.4 \\
951.0\end{array}$ \\
\hline 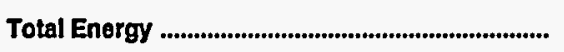 & 314.3 & 640.5 & $1,302.6$ & $1,596.7$ & $R_{1,966.7}$ & $R_{1,902.2}$ & $R_{2,003.0}$ & $2,091.4$ \\
\hline
\end{tabular}

\footnotetext{
a Includes petroleum coke used at electric utilities.

b Blomass fuels are not included, except those consumed at electric utilitiles and those added to motor gasoline.

c There are no direct fuel costs for hydroelectric, geothermal, wind, photovoltalc, or solar thermal energy.

$R=$ Revised data.

$\rightarrow$ No consumption, including cases where adjustments were made. See explanation of adjustments in Section 6 of Appendix A.

Note: Expenditure totals may not equal sum of components due to independent rounding.

Sources: Data sources, estimation procedures, and assumptions are described in Appendix A.
} 
N Energy Price Estimates by Sector, New Hampshire

E $1970,1975,1980,1985$, and 1990-1993

W (Dollars per Million Btu)

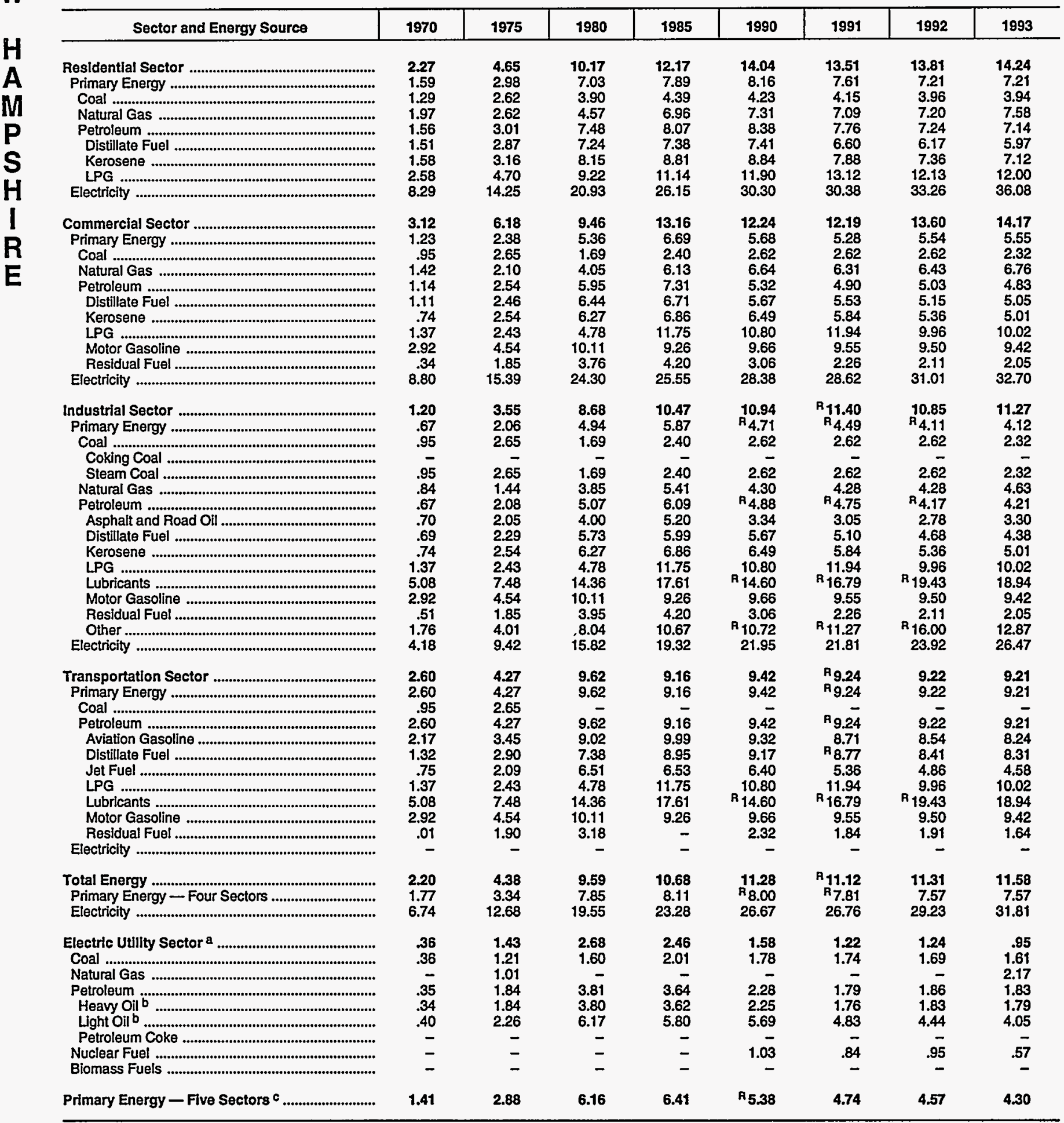

\footnotetext{
a There are no direct fuel costs for hydroelectric, geothermal, wind, photovoltaic, or solar thermal energy.

beavy oil includes fuel oll nos. 4,5 , and 6 , and residual fuel olls. Light oil includes fuel oil nos. 1 and 2, kerosene, and jet fuel.

$c$ Biomass fuels are not included, except those consumed at electric utilities and those added to motor gasoline.
}

$R=$ Revised data.

- No consumption, Including cases where adjustments were made. See explanation of adjustments in Section 6 of Appendlx A.

Sources: Data sources, estimation procedures, and assumptions are described in Appendix A. 


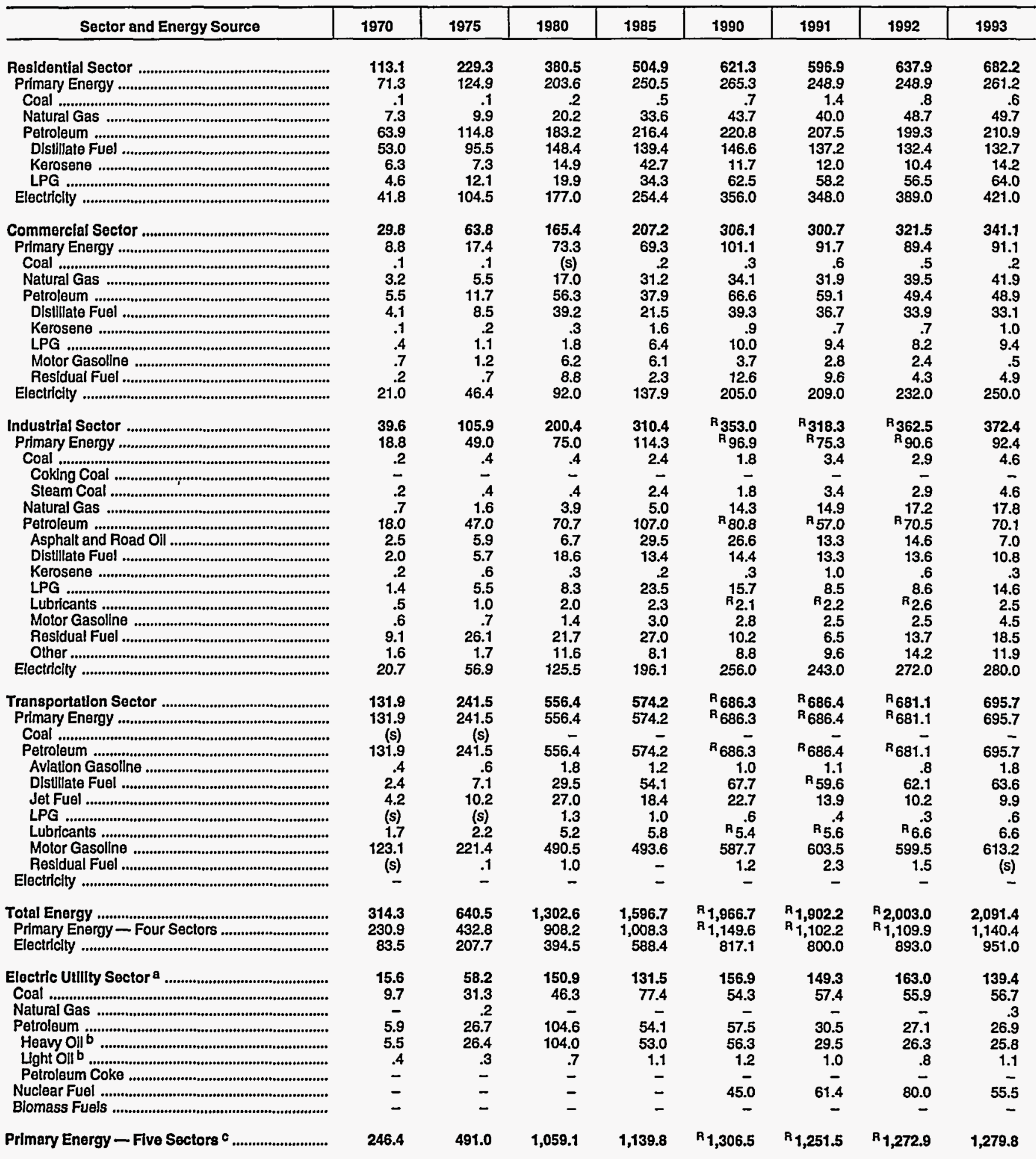

a There are no direct fuel costs for hydroelectric, geothermal, wind, photovoltalc, or solar thermal energy.

b Heavy oll Includes fuel oll nos. 4, 5, and 6, and residual fuel olls. Light oll includes fue! nos. 1 and 2, kerosene, and jet fuel.

c Blomass luels are not included, except those consumed at electric utlititles and those added to motor gasoline.

$R=$ Revised data. $\rightarrow$ No consumption, including cases where adjustments were made. See explanation of adjustments in Section 6 of Appendix A.

(s) $=$ Value less than 0.05 million dollars.

Note: Totals may not equal sum of components due to independent rounding.

Sources: Data sources, estimation procedures, and assumptions are described in Appendix A. 
N Energy Price and Expenditure Estimates by Source, New Jersey

E 1970, 1975, 1980, 1985, and 1990-1993

$\mathbf{W}$

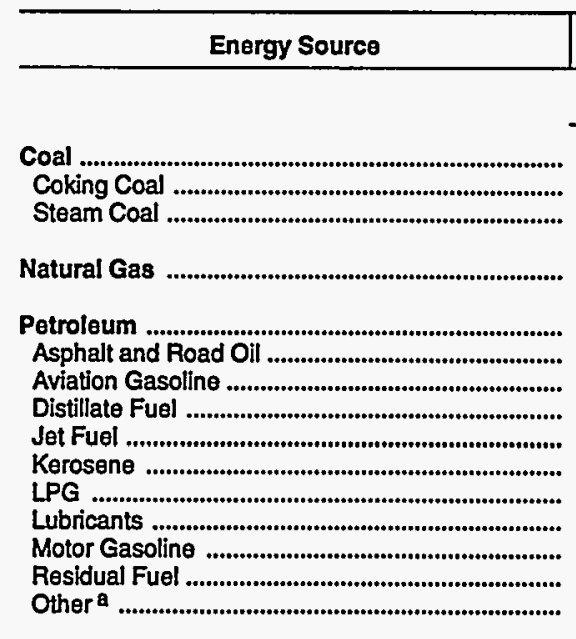

Nuclear Fuel

\begin{tabular}{c|c|c|c|c|c}
1970 & 1975 & 1980 & 1985 & 1990 & \\
\multicolumn{6}{c}{ Prices in Dollars per Million Btu } \\
\hline
\end{tabular}

Coal.

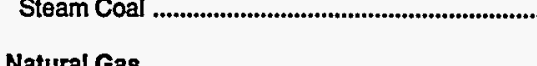

Petroloum

Asphalt and Road Oil

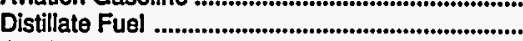

Jet Fuel .

LPG

Lubricants

Motor Gasoline.

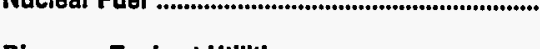

Blomass Fuels at Utilities

Primary Energy - Five Sectors b

$\begin{array}{rr}0.45 & 1.58 \\ .58 & 1.5 \\ .44 & 1.58 \\ 1.28 & 2.29 \\ 1.42 & 3.27 \\ .68 & 1.89 \\ 2.17 & 3.45 \\ 1.29 & 2.73 \\ .72 & 2.03 \\ 1.18 & 2.86 \\ 1.63 & 3.5 \\ 5.08 & 7.48 \\ 2.99 & 4.79 \\ .45 & 2.08 \\ 1.10 & 2.70 \\ & \end{array}$

$\begin{array}{lll}.20 & .18 & .34\end{array}$

$1 . \overline{8} \quad 1 . \overline{1}$

1.92

1.79

1.77

1991

1992

1993

172

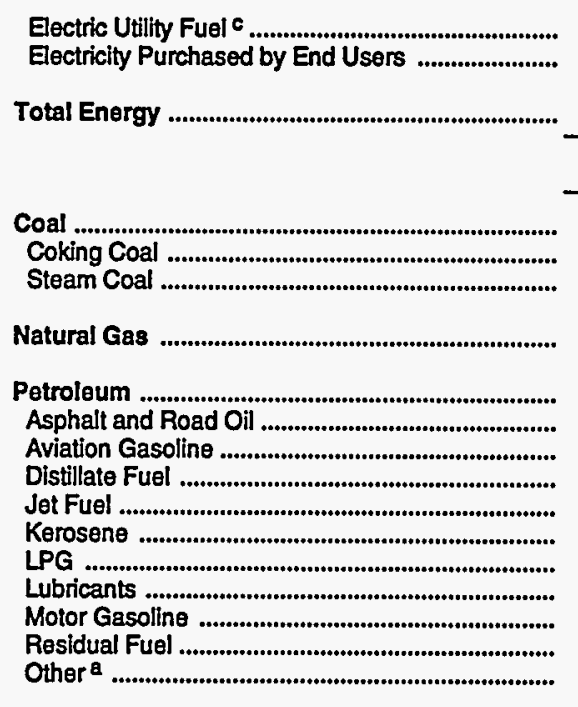

Nuclear Fual

4.15

6.18

1.79

$1 . \overline{77}$

1.72

1.75

2.29

4.15
7.17

3.65

9.02

6.75

6.26

6.35

5.72
14.36

14.36
9.94

4.53

7.07

7.48

4.89

9.99

7.92

5.76
8.91

8.91
11.86
17.61

17.61
8.95

4.35

7.86

5.05

4.91

$1 . \overline{7}$

$1 . \overline{75}$

R 7.23

2.86

9.32

7.85

5.60

8.15
10.98

10.98
$R \quad 14.60$

9.03

3.25

${ }^{H_{6}} 6.84$

2.70

8.71

8.71
7.38
4.79

4.79

8.09

10.26
16.79

16.79
8.91

2.63
$\mathrm{~A}_{5.45}$

5.02

5.07

R 6.01

.61

R 6.71

2.26

8.54

6.83

4.44

6.55

10.90

$R_{19.43}$

9.04
2.66

2.66
$R_{5.29}$

6.44

.71

.61

.56

2.92

8.24

6.85

4.15

6.63

11.93

18.94

9.09

2.51

4.75

.58

$-$

1.31

$-$

$-$

$-$

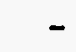

-

-

$\begin{array}{rrrrrrr}.42 & 1.71 & 2.67 & 1.90 & 1.20 & 1.12 & 1.04 \\ 6.24 & 13.61 & 21.26 & 28.18 & 26.63 & 27.76 & 27.91\end{array}$

28.18

26.63

27.76

27.91

1.01
29.31

$\begin{array}{lll}9.16 & \text { P }_{8.78} & 8.87\end{array}$

Expenditures in Millions of Dollars

\begin{tabular}{|c|c|c|c|c|c|c|c|}
\hline 1.95 & 4.26 & 8.34 & 9.62 & 9.25 & 9.16 & ${ }^{\mathrm{P}} 8.78$ & 8.87 \\
\hline \multicolumn{8}{|c|}{ Expenditures in Mlllions of Dollars } \\
\hline $\begin{array}{r}55.6 \\
5.3 \\
50.4\end{array}$ & $\begin{array}{c}95.9 \\
\overline{95.9}\end{array}$ & $\begin{array}{c}124.5 \\
- \\
124.5\end{array}$ & $\begin{array}{c}198.1 \\
- \\
198.1\end{array}$ & $\begin{array}{c}144.4 \\
\overrightarrow{-} \\
144.4\end{array}$ & $\begin{array}{c}109.3 \\
- \\
109.3\end{array}$ & $\begin{array}{c}108.0 \\
- \\
108.0\end{array}$ & $\begin{array}{c}109.6 \\
109.6\end{array}$ \\
\hline 413.8 & 556.5 & $1,434.3$ & $2,371.8$ & $2,184.4$ & $2,295.8$ & $2,757.9$ & $2,842.4$ \\
\hline $\begin{array}{r}2,001.4 \\
26.3 \\
1.7 \\
468.7 \\
26.9 \\
12.2 \\
40.9 \\
60.2 \\
1,040.8 \\
215.4 \\
108.3\end{array}$ & $\begin{array}{r}4,088.2 \\
62.9 \\
1.6 \\
947.8 \\
71.4 \\
19.6 \\
95.0 \\
79.0 \\
1,951.3 \\
575.0 \\
284.7\end{array}$ & $\begin{array}{r}8,945.0 \\
105.7 \\
3.8 \\
2,072.7 \\
308.7 \\
61.0 \\
134.3 \\
206.5 \\
3,797.7 \\
1,419.1 \\
835.5\end{array}$ & $\begin{array}{r}8,834.9 \\
153.6 \\
9.3 \\
1,861.2 \\
1,430.6 \\
70.9 \\
300.8 \\
230.4 \\
3,546.4 \\
644.1 \\
587.5\end{array}$ & $\begin{array}{r}R_{8,197.0} \\
68.0 \\
5.6 \\
1,595.2 \\
1,470.6 \\
33.7 \\
161.3 \\
R_{214.9} \\
3,694.1 \\
303.1 \\
R_{650.4}\end{array}$ & $\begin{array}{r}R_{7,713.9} \\
56.1 \\
4.4 \\
1,427.9 \\
1,183.7 \\
28.2 \\
218.9 \\
R_{221.2} \\
3,728.0 \\
278.4 \\
R_{566.8}\end{array}$ & $\begin{array}{r}R_{7,558.0} \\
50.6 \\
5.3 \\
1,336.4 \\
1,160.8 \\
30.5 \\
256.1 \\
R_{261.0} \\
3_{, 641.0} \\
R_{255.2} \\
R_{561.1}\end{array}$ & $\begin{array}{r}7,155.3 \\
160.8 \\
5.0 \\
1,359.7 \\
1,131.5 \\
19.5 \\
155.5 \\
259.0 \\
3,363.9 \\
194.0 \\
506.4\end{array}$ \\
\hline 7.6 & 6.1 & 27.9 & 135.8 & 155.8 & 162.9 & 129.6 & 153.4 \\
\hline
\end{tabular}

Blomass Fuels at Utllitles

Primary Energy - Five Sectors b

$2,478.5$

$10,531.7$

$11,540.6$

$R_{10,681.6}$
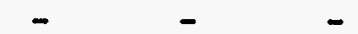

Electric Utility Fuelc

Eectricity Purchased by End Users

$-182.1$

451.6

$-881.5$

$-730.2$

$-478$.

$R_{10,281}$

98

$10,553.4$

$10,260.7$

799.5

$1,966.1$

$\mathbf{3 , 5 3 8 . 5}$

$5,148.3$

$5,688.7$

$\begin{array}{rr}-454.0 & -354.9\end{array}$

$-376.5$

$3,096.0$

$6,261.3$

$13,188.7$

$\begin{array}{llll}15,958.8 & R_{15,892.3} & R_{15,916.3} & R_{16,173.5}\end{array}$

$16,408.0$

a Includes petroleum coke used at electric utilities.

b Biomass fuels are not included, except those consumed at electric utilities and those added to motor gasoline.

c There are no direct fuel costs for hydroelectric, geothermal, wind, photovoltaic, or solar thermal energy.

$R=$ Revised data.

-No consumption, including cases where adjustments were made. See explanation of adjustments in Section 6 of Appendix A.

Note: Expenditure totals may not equal sum of components due to independent rounding.

Sources: Data sources, estimation procedures, and assumptions are described in Appendix A. 


\begin{tabular}{|c|c|c|c|c|c|c|c|c|}
\hline Sector and Energy Source & 1970 & 1975 & 1980 & 1985 & 1990 & 1991 & 1992 & 1993 \\
\hline 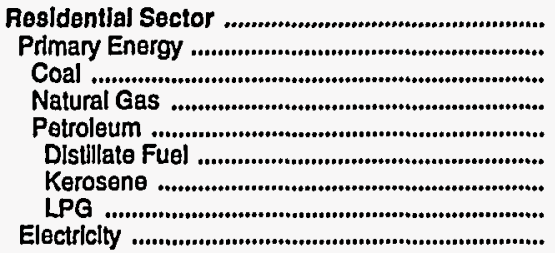 & $\begin{array}{l}2.28 \\
1.62 \\
1.13 \\
1.84 \\
1.46 \\
1.43 \\
1.72 \\
3.00 \\
7.83\end{array}$ & $\begin{array}{r}4.50 \\
2.75 \\
2.09 \\
2.61 \\
2.86 \\
2.81 \\
3.51 \\
4.94 \\
15.77\end{array}$ & $\begin{array}{r}8.97 \\
6.02 \\
3.17 \\
4.90 \\
7.14 \\
7.06 \\
9.27 \\
9.83 \\
24.08\end{array}$ & $\begin{array}{r}12.09 \\
7.70 \\
3.07 \\
7.33 \\
8.27 \\
8.09 \\
10.06 \\
10.95 \\
32.24\end{array}$ & $\begin{array}{r}12.20 \\
7.09 \\
3.14 \\
6.44 \\
8.69 \\
8.39 \\
10.42 \\
14.08 \\
30.35\end{array}$ & $\begin{array}{r}12.66 \\
7.10 \\
2.90 \\
6.57 \\
8.48 \\
8.02 \\
9.97 \\
15.07 \\
31.69\end{array}$ & $\begin{array}{r}12.05 \\
7.00 \\
2.68 \\
6.77 \\
7.69 \\
7.21 \\
8.96 \\
13.82 \\
31.85\end{array}$ & $\begin{array}{r}12.62 \\
6.95 \\
2.89 \\
6.75 \\
7.53 \\
7.06 \\
8.78 \\
13.51 \\
33.43\end{array}$ \\
\hline 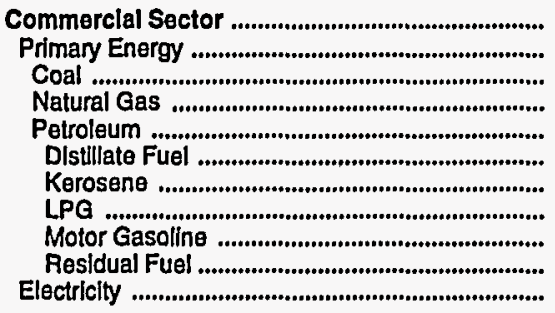 & $\begin{array}{r}2.01 \\
.99 \\
.23 \\
1.38 \\
.83 \\
1.14 \\
.79 \\
1.43 \\
2.99 \\
.45 \\
7.62\end{array}$ & $\begin{array}{r}5.19 \\
2.34 \\
1.27 \\
2.26 \\
2.39 \\
2.48 \\
2.50 \\
3.38 \\
4.79 \\
2.04 \\
14.97\end{array}$ & $\begin{array}{r}9.22 \\
5.14 \\
1.49 \\
4.45 \\
5.51 \\
6.47 \\
5.81 \\
5.15 \\
9.94 \\
4.66 \\
22.49\end{array}$ & $\begin{array}{r}13.90 \\
6.37 \\
1.74 \\
6.49 \\
6.29 \\
6.94 \\
6.82 \\
12.00 \\
8.95 \\
4.56 \\
29.02\end{array}$ & $\begin{array}{r}12.77 \\
5.43 \\
1.60 \\
5.07 \\
6.21 \\
6.50 \\
6.60 \\
10.09 \\
9.03 \\
3.47 \\
26.50\end{array}$ & $\begin{array}{r}13.00 \\
5.26 \\
1.58 \\
5.08 \\
5.67 \\
6.09 \\
5.93 \\
9.15 \\
8.91 \\
2.64 \\
27.43\end{array}$ & $\begin{array}{r}12.86 \\
5.33 \\
1.57 \\
5.40 \\
5.15 \\
5.28 \\
5.35 \\
10.15 \\
9.04 \\
2.56 \\
\text { A }_{27.65}\end{array}$ & $\begin{array}{r}13.56 \\
5.24 \\
1.50 \\
5.40 \\
4.79 \\
5.37 \\
5.01 \\
10.95 \\
9.09 \\
2.71 \\
28.84\end{array}$ \\
\hline 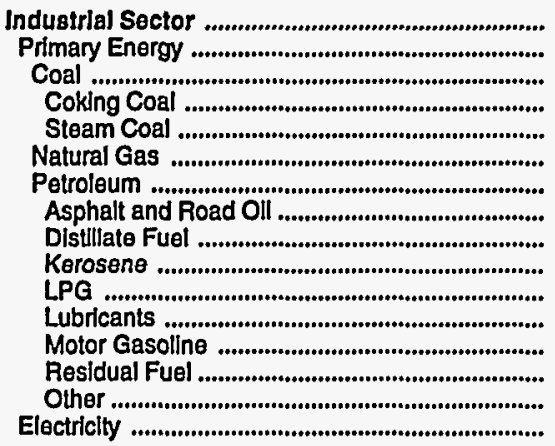 & $\begin{array}{r}1.18 \\
.86 \\
.40 \\
.58 \\
.23 \\
.68 \\
.93 \\
.68 \\
.76 \\
.79 \\
1.43 \\
5.08 \\
2.99 \\
.48 \\
1.10 \\
3.89\end{array}$ & $\begin{array}{r}3.43 \\
2.47 \\
1.27 \\
1.27 \\
1.65 \\
2.61 \\
1.89 \\
2.36 \\
2.50 \\
3.38 \\
7.48 \\
4.79 \\
2.15 \\
2.70 \\
10.03\end{array}$ & $\begin{array}{r}6.99 \\
5.59 \\
1.49 \\
1 . \overline{ } \\
1.49 \\
3.63 \\
5.97 \\
3.65 \\
5.48 \\
5.81 \\
5.15 \\
14.36 \\
9.94 \\
4.69 \\
7.07 \\
16.96\end{array}$ & $\begin{array}{r}9.36 \\
6.82 \\
1.74 \\
- \\
1.74 \\
5.39 \\
7.68 \\
4.89 \\
6.16 \\
6.82 \\
12.00 \\
17.61 \\
8.95 \\
4.56 \\
7.86 \\
22.54\end{array}$ & $\begin{array}{r}R_{7.70} \\
R_{5.29} \\
1.60 \\
- \\
1.60 \\
3.85 \\
R_{6.08} \\
2.86 \\
5.96 \\
6.60 \\
10.09 \\
R_{14.60} \\
9.03 \\
3.47 \\
R_{6.01} \\
21.57\end{array}$ & $\begin{array}{r}R_{7.58} \\
R 4.98 \\
1.58 \\
- \\
1.58 \\
3.56 \\
R \quad 5.86 \\
2.70 \\
5.36 \\
5.93 \\
9.15 \\
R 16.79 \\
8.91 \\
2.64 \\
R 5.45 \\
22.46\end{array}$ & $\begin{array}{r}R_{6.76} \\
R_{4.62} \\
1.57 \\
- \\
1.57 \\
3.34 \\
R_{5.89} \\
2.26 \\
4.84 \\
5.35 \\
10.15 \\
R_{19.43} \\
9.04 \\
2.56 \\
R 5.29 \\
22.59\end{array}$ & $\begin{array}{r}6.40 \\
4.30 \\
1.50 \\
- \\
1.50 \\
3.57 \\
5.04 \\
2.92 \\
4.52 \\
5.01 \\
10.95 \\
18.94 \\
9.09 \\
2.71 \\
4.75 \\
23.69\end{array}$ \\
\hline 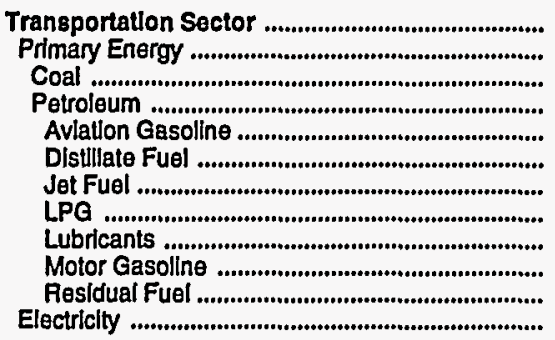 & $\begin{array}{r}2.39 \\
2.39 \\
.23 \\
2.39 \\
2.17 \\
1.57 \\
.72 \\
1.43 \\
5.08 \\
2.99 \\
.41 \\
4.62\end{array}$ & $\begin{array}{r}4.32 \\
4.32 \\
1.27 \\
4.32 \\
3.45 \\
3.21 \\
2.01 \\
3.38 \\
7.48 \\
4.79 \\
1.81 \\
11.14\end{array}$ & $\begin{array}{r}8.60 \\
8.60 \\
- \\
8.60 \\
9.02 \\
7.34 \\
6.27 \\
5.15 \\
14.36 \\
9.94 \\
3.94 \\
14.91\end{array}$ & $\begin{array}{r}7.54 \\
7.53 \\
- \\
7.53 \\
9.99 \\
8.51 \\
5.76 \\
12.00 \\
17.61 \\
8.95 \\
4.18 \\
21.28\end{array}$ & $\begin{array}{r}R_{7.55} \\
7.53 \\
-5 \\
7.53 \\
9.32 \\
8.64 \\
5.60 \\
10.09 \\
R 14.60 \\
9.03 \\
2.99 \\
47.11\end{array}$ & $\begin{array}{r}7.10 \\
\text { R } 7.09 \\
- \\
\text { R } 7.09 \\
8.71 \\
8.00 \\
4.79 \\
9.15 \\
\text { R } 16.79 \\
8.91 \\
2.55 \\
47.74\end{array}$ & $\begin{array}{r}R_{6.99} \\
R_{6.97} \\
- \\
R_{6.97} \\
8.54 \\
7.59 \\
4.44 \\
10.15 \\
R_{19.43} \\
9.04 \\
2.63 \\
48.95\end{array}$ & $\begin{array}{r}6.90 \\
6.88 \\
- \\
6.88 \\
8.24 \\
7.66 \\
4.15 \\
10.95 \\
18.94 \\
9.09 \\
2.35 \\
51.44\end{array}$ \\
\hline 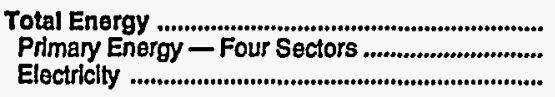 & $\begin{array}{l}1.95 \\
1.57 \\
6.24\end{array}$ & $\begin{array}{r}4.26 \\
3.24 \\
13.61\end{array}$ & $\begin{array}{r}8.34 \\
6.82 \\
21.26\end{array}$ & $\begin{array}{r}9.62 \\
7.32 \\
28.18\end{array}$ & $\begin{array}{r}9.25 \\
6.78 \\
26.63\end{array}$ & $\begin{array}{r}9.16 \\
R_{6.48} \\
27.76\end{array}$ & $\begin{array}{l}R_{8.78} \\
R_{6.27} \\
27.91\end{array}$ & $\begin{array}{r}8.87 \\
6.08 \\
29.31\end{array}$ \\
\hline 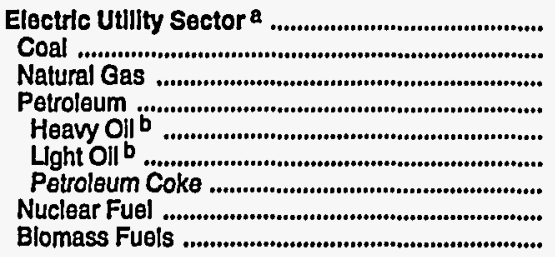 & $\begin{array}{l}.42 \\
.45 \\
.39 \\
.45 \\
.45 \\
.45 \\
.20 \\
.\end{array}$ & $\begin{array}{r}1.71 \\
1.59 \\
.95 \\
2.12 \\
2.12 \\
2.14 \\
\overline{18} \\
-\end{array}$ & $\begin{array}{r}2.67 \\
1.80 \\
3.01 \\
4.98 \\
4.79 \\
5.93 \\
- \\
.34 \\
-\end{array}$ & $\begin{array}{r}1.90 \\
1.92 \\
3.97 \\
4.62 \\
4.41 \\
6.24 \\
- \\
.71 \\
-\end{array}$ & $\begin{array}{r}1.20 \\
1.80 \\
2.17 \\
3.87 \\
3.56 \\
5.45 \\
- \\
.61 \\
-\end{array}$ & $\begin{array}{r}1.12 \\
1.78 \\
1.96 \\
3.26 \\
2.96 \\
4.81 \\
- \\
.61 \\
-\end{array}$ & $\begin{array}{r}1.04 \\
1.73 \\
2.11 \\
3.23 \\
3.02 \\
4.51 \\
- \\
.56 \\
-\end{array}$ & $\begin{array}{r}1.01 \\
1.77 \\
2.30 \\
2.89 \\
2.65 \\
4.04 \\
- \\
.58 \\
-\end{array}$ \\
\hline Primary Energy - Flve Sectors c ........................ & 1.31 & 2.99 & 6.04 & 6.20 & $\mathrm{R}_{5.62}$ & 5.34 & $R_{5.36}$ & 5.13 \\
\hline
\end{tabular}

a There are no direct fuel costs for hydroelectric, geothermal, wind, pholovoltalc, or solar thermal energy.

b Heavy oll includes fuel oll nos. 4, 5, and 6, and residual fuel oils. Light oll includes fuel oll nos. 1 and 2, kerosene, and jet fuel.

- Blomass fuels are not Included, except those consumed at electric utllities and those added to motor gasollne.
$R=$ Revised data.

- No consumption, Including cases where adjustments were made. See explanation of adjustments in Section 6 of Appendix A

Sources: Data sources, estimation procedures, and assumptions are described in Appendix A. 


\section{N Energy Expenditure Estimates by Sector, New Jersey}

E $1970,1975,1980,1985$, and 1990-1993

W (Million Dollars)

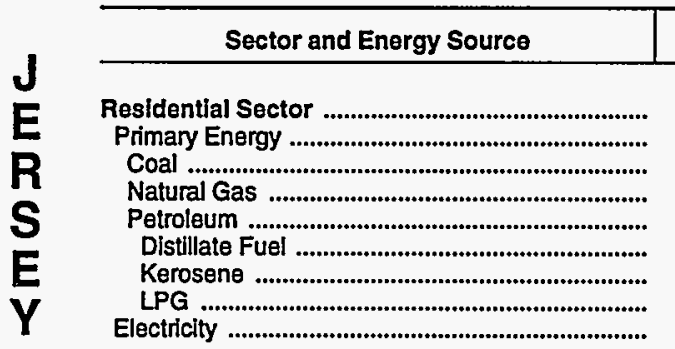

Commerclal Sector

Primary Energy

Coal .

Natural Gas

Petroleum

Distillate Fue

Kerosene

LPG

Motor Gasoline

Residual Fuel

Electricity

Industrial Sector

Primary Energy

Coal

Coking Coal

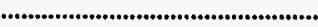

Natural Gas

Petroleum

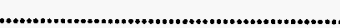

Asphait and Road Oil

Distillate Fuel

Kerosene

LPG

Lubricants

Residual Fuel

Other ...

Electricity

Transportation Sector

Primary Energy

Coal

etroleum

Aviation Gasoline

Distillate Fuel

Jet Fuel

LPG

Lubricants

Motor Gasoline

Residual Fuel

Electricity

Total Energy ...

Primary Energy - Four Sectors .............................

Electricity

Electric Utility Sector a

Coal .

Natural Gas

Petroleum

Heavy Oil b

Light Oll $b$.

Petroleum Coke

Nuclear Fuel ...

Biomass Fuels

Primary Energy - Five Sectors $c$

1970

1975

882.8
558.7
2.4
264.7
291.6
274.6
7.5
9.5
324.1

$1,657.8$

1980

$3,062.8$

2.2
348.4

527.3

501.0

8.6
17.7

17.7
780.0

478.8

$1,085.2$

197.9

79.3

79.3
118.2

118.2
74.0

74.0
1.3

9.6

9.6
32.5

280.9

560.5

560.5
366.5

7.5

5.3

2.2
51.4

51.4
307.6

26.3

38.6

3.4
30.0

30.0

42.5

6.3
52.1

52.1
108.3

194.0

378.0

.9
124.2

252.9

149.4

149.4
2.4
2.1

15.9
83.0

707.2

1,277.1

799.8

2.0

2.0

75.5

722.4

62.9

109.5

8.7

73.9

51.6

5.9

125.3

284.7

477.3

$1,173.9$

$1,173.4$

$1,173.4$

1.7
78.3

78.3

26.9

17.7
.6

$1,024.9$

23.3
.5

2,241.2

2,239.5

(s)

2,239.5

1.6
165.5

166.5

64.9

1.2

27.5
1.929 .5

$1,929.5$

48.3
1.7

$3,096.0 \quad 6,261.3$

$6,261.3$
$4,295.2$

$1,966.1$

$1,721.3$

2.4
691.2

$1,027.7$

985.9

13.8

28.0
$1,341.5$

$2,259.8$

964.5

964.5
.8

278.0

685.8

345.2

1.3
2.6

15.5

321.1

$1,295.3$

$3,002.0$

$2,101.8$

1.2

1.2

217.5

$1,883.2$

105.7

230.9
45.9

102.9

144.4

7.7

410.2

835.5

900.1

4,864.2

$4,862.5$

$4,862 . \overline{5}$

3.8
438.1

438.1

284.6

62.1
3.774 .5

,774.5

298.7
1.6

$13,188.7$

$9,650.2$

$3,538.5$

$10,810.5$
$5,148.3$

\section{5}

451.6

182.1
45.4

18.4

110.6

107.5

90.8

8.4

346.2

318.4

120.2

247.6

485.8

389.1

96.7

27.9

7.6

6.1

$4,746.7$

$10,531.7$

1985

4.4
$1,130.9$

939.9

852.0

51.7

36.2
$1,889.6$

$2,984.5$

914.0

1.9
553.5

358.6

227.9

3.0

7.0

89.7

2,070.5

$3,038.6$

$1,856.8$

15.0

15.0

433.0

$1,408.9$

153.6

89.3

16.2
252.8

161.2

21.7

126.5

587.5

$1,181.8$

$5,970.8$

$5,964.4$

$5,964.4$

9.3
667.6

$1,430.6$

4.8
69.3

69.3
3.493 .6

289.

\section{2}

176.8

254.5

163.1

138.7

24.4

1990

$\begin{array}{ll}3,964.8 & 3,881.6 \\ 2,075.2 & 1,758.6\end{array}$

.6
$1,133.0$
625.0

561.7

17.4

45.9
$2,123.0$

.

$3,404.3$
943.7

601.2

342.4

262.0

6.7

5.8

35.6

32.3
$2,460.6$

$R_{2,619.1}$

$R_{1,530.4}$

11.1

11.

11.1
343.6
$R_{1,175.7}$

68.0
100.3

9.6

106.9

$R_{150.3}$

21.7

68.5
650.4

$1,088.7$

$R_{5,987.3}$

$\mathrm{R}_{5,970.9}$

A $5,970.9^{-}$

5.6
651.7

651.7

$1,470.6$

2.8
$R_{64.6}$

$3,636.8$

138.9

16.4 


\begin{tabular}{|c|c|c|c|c|c|c|c|c|}
\hline Energy Source & 1970 & 1975 & 1980 & 1985 & 1990 & 1991 & 1992 & 1993 \\
\hline & \multicolumn{8}{|c|}{ Prices in Dollars per Million Btu } \\
\hline $\begin{array}{l}\text { Coal } \\
\text { Coking Coal } \\
\text { Steam Coal }\end{array}$ & $\frac{0.14}{.14}$ & $\frac{0.23}{. \overline{3}}$ & $\frac{0.56}{.56}$ & $\begin{array}{l}1.09 \\
1.09\end{array}$ & $\frac{1.32}{1.32}$ & $\frac{1.38}{-}$ & $\frac{1.32}{1.32}$ & $\frac{1.37}{-}$ \\
\hline 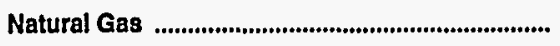 & .39 & .75 & 2.66 & 4.60 & 3.84 & 3.62 & 3.67 & 3.91 \\
\hline $\begin{array}{l}\text { Petroleum } \\
\text { Asphalt and Road Oil } \\
\text { Avlation Gasoline } \\
\text { Distillate Fuel } \\
\text { Jet Fuel } \\
\text { Kerosene } \\
\text { LPG }\end{array}$ & $\begin{array}{r}1.92 \\
.58 \\
2.17 \\
1.07 \\
.76 \\
1.03 \\
1.35 \\
5.08 \\
2.94 \\
.34 \\
.50\end{array}$ & $\begin{array}{l}3.43 \\
1.84 \\
3.45 \\
2.42 \\
2.12 \\
2.26 \\
3.17 \\
7.48 \\
4.72 \\
1.66 \\
1.37\end{array}$ & $\begin{array}{r}7.80 \\
3.67 \\
9.02 \\
6.80 \\
6.59 \\
6.91 \\
5.84 \\
14.36 \\
9.58 \\
3.80 \\
2.65\end{array}$ & $\begin{array}{r}7.85 \\
4.79 \\
9.99 \\
6.53 \\
6.24 \\
7.31 \\
8.21 \\
17.61 \\
9.14 \\
3.98 \\
2.59\end{array}$ & $\begin{array}{r}{ }^{R} 8.02 \\
2.76 \\
9.32 \\
7.27 \\
6.01 \\
6.05 \\
8.02 \\
R_{14.60} \\
9.23 \\
2.75 \\
R_{4.27}\end{array}$ & $\begin{array}{r}R_{7.50} \\
3.45 \\
8.71 \\
6.89 \\
4.84 \\
5.58 \\
6.08 \\
R_{16.79} \\
9.10 \\
2.27 \\
R 3.50\end{array}$ & $\begin{array}{r}R 7.39 \\
2.99 \\
8.54 \\
7.32 \\
4.57 \\
5.23 \\
4.45 \\
\mathrm{R}_{19.43} \\
9.33 \\
2.09 \\
\mathrm{R}_{3.12}\end{array}$ & $\begin{array}{r}7.91 \\
3.12 \\
8.24 \\
7.60 \\
4.50 \\
5.53 \\
5.25 \\
18.94 \\
10.13 \\
1.96 \\
2.84\end{array}$ \\
\hline 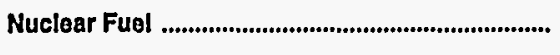 & - & - & - & - & - & - & - & - \\
\hline 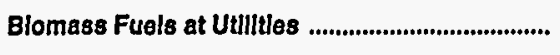 & - & - & - & - & - & - & - & - \\
\hline Primany Energy - Five Sectors ${ }^{b}$............................ & .85 & 1.63 & 3.71 & 3.99 & 4.15 & 4.25 & $R_{4.06}$ & 4.29 \\
\hline $\begin{array}{l}\text { Electric Utility Fuel }{ }^{c} \\
\text { Electricily Purchased by End Users }\end{array}$ & $\begin{array}{r}.20 \\
5.62\end{array}$ & $\begin{array}{r}.45 \\
7.99\end{array}$ & $\begin{array}{r}1.02 \\
15.52\end{array}$ & $\begin{array}{r}1.33 \\
21.20\end{array}$ & $\begin{array}{r}1.37 \\
20.99\end{array}$ & $\begin{array}{r}1.42 \\
21.09\end{array}$ & $\begin{array}{r}1.38 \\
21.03\end{array}$ & $\begin{array}{r}1.45 \\
21.40\end{array}$ \\
\hline \multirow[t]{2}{*}{ 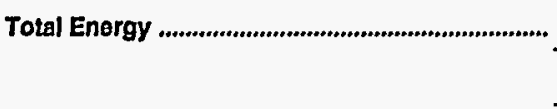 } & 1.46 & 2.88 & 7.08 & 9.26 & ${ }^{R} 9.18$ & $\mathrm{R}_{8.73}$ & $\mathrm{R}_{8.62}$ & 9.12 \\
\hline & \multicolumn{8}{|c|}{ Expenditures in Millions of Dollars } \\
\hline $\begin{array}{l}\text { Coal } \\
\text { Coking Coal } \\
\text { Steam Coal }\end{array}$ & $\overline{14.3}$ & $\begin{array}{l}30.0 \\
30.0\end{array}$ & $\begin{array}{r}114.2 \\
114.2\end{array}$ & $\begin{array}{l}293.7 \\
293.7\end{array}$ & $\begin{array}{c}363.4 \\
-\overline{3} \\
363.4\end{array}$ & $\begin{array}{l}322.0 \\
-\overline{2}\end{array}$ & $\begin{array}{r}353.8 \\
353.8\end{array}$ & $\begin{aligned} 369.6 \\
369.6\end{aligned}$ \\
\hline 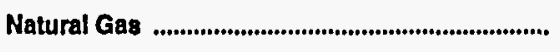 & 80.7 & 134.8 & 394.1 & 350.8 & 348.8 & 352.7 & 341.2 & 387.8 \\
\hline $\begin{array}{l}\text { Potroleum } \\
\text { Asphalt and Road Oil } \\
\text { Aviation Gasoline } \\
\text { Distillate Fuel } \\
\text { Jet Fuel }\end{array}$ & $\begin{array}{r}292.3 \\
4.6 \\
1.2 \\
33.6 \\
12.9 \\
5.8 \\
22.0 \\
8.3 \\
202.9 \\
.4 \\
.5\end{array}$ & $\begin{array}{r}653.3 \\
19.9 \\
1.4 \\
94.7 \\
30.9 \\
8.4 \\
41.3 \\
14.4 \\
409.2 \\
31.0 \\
2.0\end{array}$ & $\begin{array}{r}1,507.8 \\
27.7 \\
7.6 \\
315.6 \\
96.0 \\
52.5 \\
98.6 \\
28.9 \\
850.8 \\
23.5 \\
6.6\end{array}$ & $\begin{array}{r}1,483.4 \\
47.7 \\
4.8 \\
323.9 \\
97.7 \\
7.9 \\
88.8 \\
32.2 \\
859.3 \\
19.0 \\
2.0\end{array}$ & $\begin{array}{r}\text { R }_{1,680.5} \\
26.6 \\
4.0 \\
386.4 \\
96.2 \\
1.9 \\
230.3 \\
R_{30.1} \\
898.7 \\
2.0 \\
R_{4.2}\end{array}$ & $\begin{array}{r}\text { R }_{1,692.9} \\
34.9 \\
4.1 \\
378.4 \\
65.2 \\
2.0 \\
257.4 \\
830.9 \\
914.9 \\
1.1 \\
\text { R }_{3.9}\end{array}$ & $\begin{array}{r}R_{1,700.8} \\
37.2 \\
4.0 \\
425.7 \\
71.3 \\
.7 \\
168.4 \\
R_{36.5} \\
952.7 \\
.8 \\
3.6\end{array}$ & $\begin{array}{r}1,808.5 \\
50.4 \\
2.9 \\
364.6 \\
82.5 \\
.5 \\
181.6 \\
36.2 \\
1,084.8 \\
1.7 \\
3.1\end{array}$ \\
\hline Nuclear Fuel ................................................................... & - & - & - & - & - & - & - & - \\
\hline 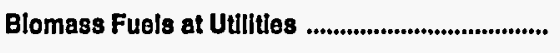 & - & - & - & - & - & - & - & - \\
\hline Primary Energy - Five Sectors b ............................. & 387.3 & 818.1 & $2,016.1$ & $2,127.9$ & $R_{2,392.7}$ & $\mathrm{R}_{2,367,5}$ & $R_{2,395.8}$ & $2,566.0$ \\
\hline 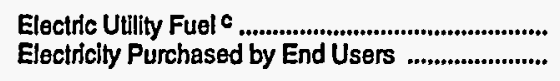 & $\begin{array}{r}32.0 \\
106.6\end{array}$ & $\begin{array}{r}-95.4 \\
179.5\end{array}$ & $\begin{array}{r}-268.0 \\
460.2\end{array}$ & $\begin{array}{r}-392.6 \\
836.0\end{array}$ & $\begin{array}{r}-414.2 \\
963.6\end{array}$ & $\begin{array}{r}-371.1 \\
987.1\end{array}$ & $\begin{array}{r}-398.9 \\
1,009.6\end{array}$ & $\begin{array}{r}-431.5 \\
1,057.9\end{array}$ \\
\hline 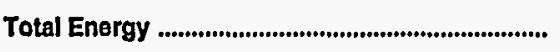 & 461.8 & 902.3 & $2,208.2$ & $2,571.3$ & $R_{2,942.1}$ & $\mathrm{~F}_{2,983.6}$ & $\mathrm{R}_{3,006.5}$ & $3,192.4$ \\
\hline
\end{tabular}

\footnotetext{
Includes petroleum coke used at electric utilities.

b Blomass fuels are not included, except those consumed at electric utilities and those added to motor gasoline.

c There are no direct fuel costs for hydroelectric, geothermal, wind, photovoltalc, or solar thermal energy.

R=Revised data.
}

- No consumption, including cases where adjustments were made. See explanation of adjustments in Section 6 of Appendix A.

Note: Expenditure totals may not equal sum of components due to independent rounding.

Sources: Data sources, estimation procedures, and assumptions are described in Appendix A. 
N Energy Price Estimates by Sector, New Mexico

E 1970, 1975, 1980, 1985, and 1990-1993

W (Dollars per Million Btu)

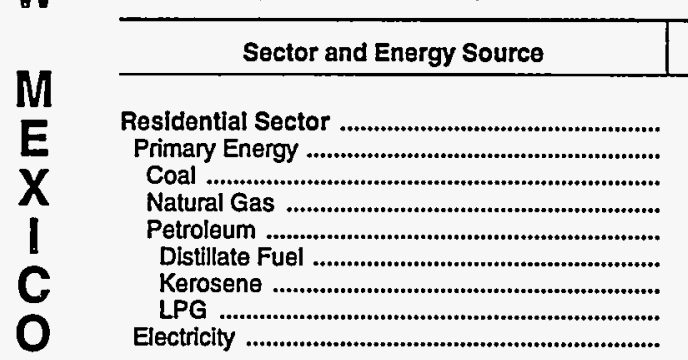

Commercial Sector

Primary Energy

Coal

Petroleum

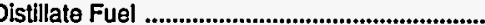

Kerosene

LPG

Motor Gasolin日

Residual Fuel ...............................................

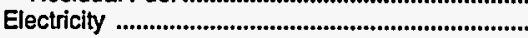

Industrial Sector

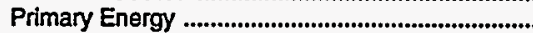

Coal

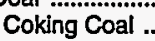

- ln

Natural Gas

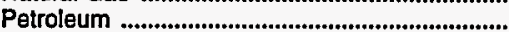

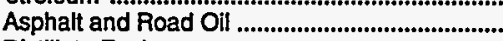

Kerosene

LPG

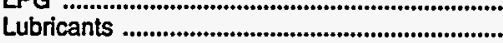

Motor Gasoline

Residual Fuel

Other

Electricity

Transportation Sector

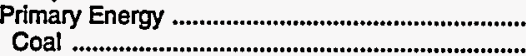

Petroleum ....................................................................

Aviation Gasoline

Distillate Fuel ...........................................................

Jet Fuel

LPG ..........

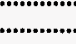

Motor Gasolin

$$
\text { Electricity }
$$

Total Energy ...

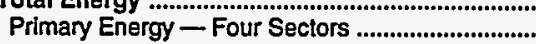

Electricity

Electric Utility Sector a

Coal

Natural Gas

Petroleum .

Heav Oil b

Light Oil b

Petroleum Coke

Blomass Fuels

Primary Energy - Flve Sectors ${ }^{c}$...
Natural Gas

Distillate Fuel ......................................................

Residual Fue

\begin{tabular}{l|l|l}
\hline 1970 & 1975 & 1980 \\
\hline
\end{tabular}

1970
1.78
1.00
.90
.86
1.60
.98
1.49
1.61
8.1

$1.78 \quad 3.06$

$1.00 \quad 1.64$

$\begin{array}{ll}.90 & . \overline{4}\end{array}$

$\begin{array}{ll}1.60 & 4.12\end{array}$

$\begin{array}{ll}.98 & 2.82\end{array}$

$.49 \quad 3.05$

$\begin{array}{rr}1.61 & 4.16 \\ 8.15 & 10.47\end{array}$

1.37

.50
.56

$\begin{array}{r}.56 \\ .44 \\ \hline\end{array}$

1.34

1.01

1.01
1.12

2.94

$5 . \overline{8}$

\subsection{4}

.94

.74

3.03

2.62

2.22

4.72

-

7.90

1.52

.65

.49
.56

.56

56
25

.25
1.01

.58

.95
1.01

1.01
1.12

5.08

2.94

.41
.50

.50
3.44

\subsection{8}

2.28
.56

.56
2.28

2.28
2.17

2.17
1.15

.76
1.12

1.12

5.08
2.94

2.94
.38

.

1.46

1.46
1.20

5.62

20

.14

.30

.23

.23
.27
-

-

.85

1.27

-

.

.58
2.13
1.84

1.84

2.05
2.22

2.22
2.60

7.48

4.72

1.60

1.37
5.54

2.62
2.12

2.60

7.48

7.48
4.72

4.72
-

\subsection{8}

2.49

7.99

.45
.23

.23
.69

1.70
1.89

1.89

1.63

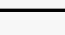

\subsection{6}

3.78

3.78
3.17

3.17
7.29

6.79

7.95

7.19

18.89

6.79

3.50

.88
2.79

6.85

6.57

6.80

5.36

9.58

$15 . \overline{5}$

\subsection{5}

4.06

.88

.88

2.46

5.34
3.67

6.42

6.80

5.36

14.36

9.58

3.82

2.65
12.11

$4.03 \quad 8.69$

\begin{tabular}{ll}
4.03 & 8.69 \\
4.03 & 8.69 \\
\hline
\end{tabular}

$4 . \overline{0} \quad 8 . \overline{69}$

$\begin{array}{ll}4.03 & 8.69 \\ 3.45 & 9.02\end{array}$

6.97
6.59

6.59
5.36

5.36
14.36

$\begin{array}{r}9.58 \\ \hline\end{array}$

6.20

15.52

1.02

.56
2.47

2.47
5.21

5.21
3.70

6.53

-

$3.71 \quad 3.99$

$\mathbf{R}=$ Revised data

a There are no direct fuel costs for hydroelectric, geothermal, wind, photovoltaic, or solar thermal energy.

b Heavy oil includes fuel oil nos. 4, 5, and 6, and residual fuel olls. Light oil includes fuel oll nos. 1 and 2 , kerosene, and jet fuel.

c Blomass fuels are not included, except those consumed at electric utilities and those added to motor gasoline.

-No consumption, including cases where adjustments were made. Seo explanation of adjustments in Section 6 of Appendix A.

Sources: Data sources, estimation procedures, and assumptions are described in Appendix A. 


\begin{tabular}{|c|c|c|c|c|c|c|c|c|}
\hline Sector and Energy Source & 1970 & 1975 & 1980 & 1985 & 1990 & 1991 & 1992 & 1993 \\
\hline 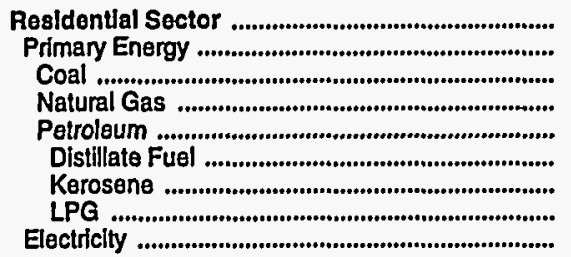 & $\begin{array}{r}82.0 \\
41.0 \\
(s) \\
28.6 \\
12.4 \\
(s) \\
.2 \\
12.2 \\
41.0\end{array}$ & $\begin{array}{r}127.1 \\
57.2 \\
- \\
37.0 \\
20.2 \\
.1 \\
.5 \\
19.6 \\
69.9\end{array}$ & $\begin{array}{r}292.1 \\
134.1 \\
.8 \\
95.0 \\
38.3 \\
.4 \\
6.0 \\
31.9 \\
158.1\end{array}$ & $\begin{array}{r}470.7 \\
201.3 \\
.2 \\
133.4 \\
67.8 \\
.8 \\
2.0 \\
64.9 \\
269.4\end{array}$ & $\begin{array}{r}536.6 \\
217.7 \\
.1 \\
159.6 \\
58.0 \\
.5 \\
.2 \\
57.4 \\
319.0\end{array}$ & $\begin{array}{r}545.4 \\
212.4 \\
.1 \\
160.7 \\
51.6 \\
.3 \\
.3 \\
51.0 \\
333.0\end{array}$ & $\begin{array}{r}535.4 \\
191.4 \\
.2 \\
149.3 \\
41.9 \\
.5 \\
.2 \\
41.3 \\
344.0\end{array}$ & $\begin{array}{r}560.1 \\
203.1 \\
.2 \\
173.9 \\
29.1 \\
.2 \\
.2 \\
28.7 \\
357.0\end{array}$ \\
\hline 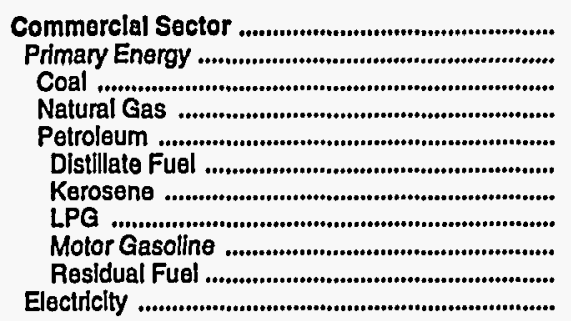 & $\begin{array}{r}62.7 \\
19.0 \\
(s) \\
15.7 \\
3.2 \\
.6 \\
(s) \\
1.5 \\
1.1 \\
43.7\end{array}$ & $\begin{array}{r}99.4 \\
25.4 \\
18.2 \\
7.2 \\
2.7 \\
.1 \\
2.2 \\
2.3 \\
\overline{2} \\
74.0\end{array}$ & $\begin{array}{r}296.3 \\
112.4 \\
.5 \\
71.7 \\
40.1 \\
5.1 \\
25.4 \\
4.2 \\
5.5 \\
-\overline{1} \\
184.0\end{array}$ & $\begin{array}{r}488.9 \\
129.7 \\
.1 \\
97.2 \\
32.4 \\
14.8 \\
2.4 \\
9.7 \\
5.4 \\
.1 \\
359.2\end{array}$ & $\begin{array}{r}580.3 \\
137.3 \\
.1 \\
105.0 \\
32.3 \\
17.3 \\
.5 \\
8.4 \\
6.1 \\
\overline{1} \\
443.0\end{array}$ & $\begin{array}{r}578.4 \\
126.5 \\
.1 \\
103.7 \\
22.6 \\
11.9 \\
.6 \\
4.7 \\
5.4 \\
\overline{4} \\
452.0\end{array}$ & $\begin{array}{r}569.8 \\
107.8 \\
.2 \\
93.7 \\
13.9 \\
6.1 \\
.2 \\
2.6 \\
4.9 \\
-\overline{2} \\
462.0\end{array}$ & $\begin{array}{r}620.2 \\
132.1 \\
.2 \\
120.3 \\
11.7 \\
8.1 \\
.2 \\
2.5 \\
.9 \\
- \\
488.0\end{array}$ \\
\hline 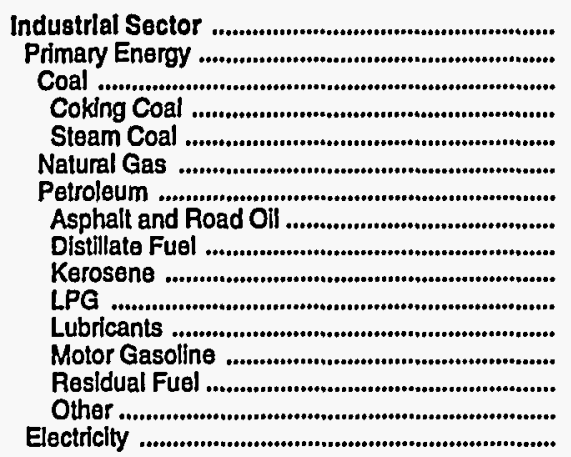 & $\begin{array}{r}76.8 \\
54.9 \\
.1 \\
- \\
.1 \\
18.7 \\
36.0 \\
4.6 \\
11.7 \\
5.5 \\
7.3 \\
3.2 \\
3.0 \\
.3 \\
.5 \\
21.9\end{array}$ & $\begin{array}{r}165.0 \\
129.4 \\
- \\
- \\
-\overline{3} .8 \\
96.5 \\
19.9 \\
27.5 \\
7.8 \\
17.5 \\
5.4 \\
3.6 \\
12.8 \\
2.0 \\
35.6\end{array}$ & $\begin{array}{r}436.1 \\
318.0 \\
.2 \\
- \\
.2 \\
84.5 \\
233.4 \\
27.7 \\
82.1 \\
21.1 \\
61.9 \\
10.3 \\
4.2 \\
19.4 \\
6.6 \\
118.1\end{array}$ & $\begin{array}{r}473.3 \\
265.9 \\
2.5 \\
- \\
2.5 \\
21.1 \\
242.3 \\
47.7 \\
130.5 \\
3.5 \\
11.7 \\
11.5 \\
17.3 \\
18.0 \\
2.0 \\
207.5\end{array}$ & $\begin{array}{r}R_{524.1} \\
R_{322.5} \\
1.1 \\
-\overline{1.1} \\
34.1 \\
R_{287.3} \\
26.6 \\
65.9 \\
1.2 \\
161.3 \\
R_{10.7} \\
15.9 \\
1.4 \\
R_{4.2} \\
201.6\end{array}$ & 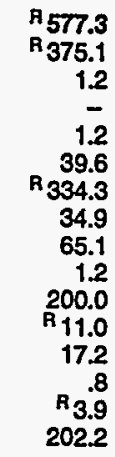 & $\begin{array}{r}R_{500.0} \\
R_{296.4} \\
1.4 \\
- \\
1.4 \\
55.5 \\
R_{241.5} \\
37.2 \\
47.5 \\
.3 \\
123.1 \\
R_{13.0} \\
16.1 \\
.7 \\
3.6 \\
203.6\end{array}$ & $\begin{array}{r}532.5 \\
319.7 \\
1.7 \\
-\overline{1.7} \\
31.8 \\
286.1 \\
50.4 \\
39.1 \\
.2 \\
148.7 \\
12.9 \\
29.9 \\
1.7 \\
3.1 \\
212.8\end{array}$ \\
\hline 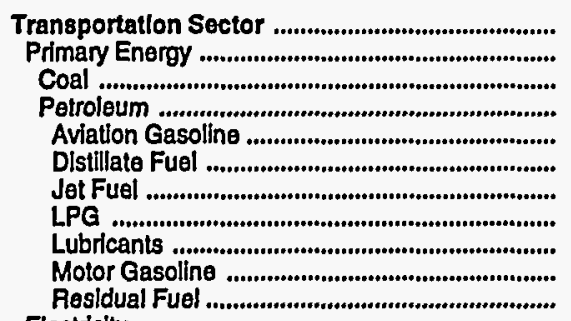 & $\begin{array}{r}240.4 \\
240.4 \\
(s) \\
240.4 \\
1.2 \\
21.2 \\
12.9 \\
1.0 \\
5.1 \\
198.9 \\
\text { (s) }\end{array}$ & $\begin{array}{r}510.8 \\
510.8 \\
510.8 \\
1.4 \\
64.0 \\
30.9 \\
2.0 \\
9.0 \\
403.4 \\
-\end{array}$ & $\begin{array}{r}1,183.6 \\
1,183.6 \\
1,183.6 \\
7.6 \\
219.7 \\
96.0 \\
.6 \\
18.6 \\
841.2 \\
\end{array}$ & $\begin{array}{r}1,138.4 \\
1,138.4 \\
- \\
1,138.4 \\
4.8 \\
176.1 \\
97.7 \\
2.5 \\
20.7 \\
836.6 \\
-\end{array}$ & $\begin{array}{r}R_{1,301.0} \\
R_{1,301.0} \\
R_{1,301.0} \\
4.0 \\
301.4 \\
96.2 \\
3.3 \\
R_{19.3} \\
876.7 \\
-\end{array}$ & $\begin{array}{r}R_{1,282.4} \\
R_{1,282.4} \\
R_{1,282.4} \\
4.1 \\
299.3 \\
65.2 \\
1.6 \\
R_{19.9} \\
892.3 \\
-\end{array}$ & $\begin{array}{r}R_{1,401.3} \\
R_{1,401.3} \\
R_{1,401.3} \\
4.0 \\
369.5 \\
71.3 \\
1.4 \\
R_{23.5} \\
931.6 \\
-\end{array}$ & $\begin{array}{r}1,479.6 \\
1,479.6 \\
- \\
1,479.6 \\
2.9 \\
315.2 \\
82.5 \\
1.7 \\
23.3 \\
1,054.0 \\
-\end{array}$ \\
\hline 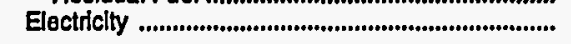 & & - & - & - & - & - & - & - \\
\hline 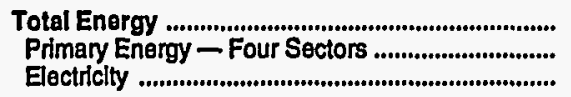 & $\begin{array}{l}461.8 \\
355.2 \\
106.6\end{array}$ & $\begin{array}{l}902.3 \\
722.7 \\
179.5\end{array}$ & $\begin{array}{r}2,208.2 \\
1,748.0 \\
460.2\end{array}$ & $\begin{array}{r}2,571.3 \\
1,735.3 \\
836.0\end{array}$ & $\begin{array}{r}P_{2,942.1} \\
P_{1,978.5} \\
963.6\end{array}$ & $\begin{array}{r}R_{2}, 983.6 \\
A_{1,996.4} \\
987.1\end{array}$ & $\begin{array}{r}R_{3,006.5} \\
R_{1,996.9} \\
1,009.6\end{array}$ & $\begin{array}{l}3,192.4 \\
2,134.5 \\
1,057.9\end{array}$ \\
\hline 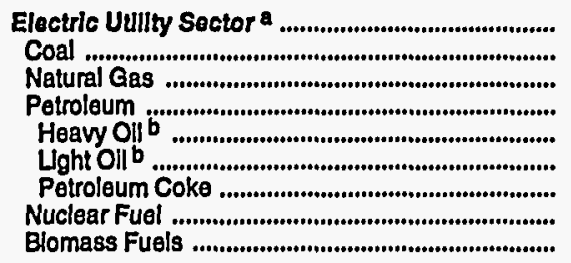 & $\begin{array}{r}32.0 \\
14.2 \\
17.7 \\
.1 \\
.1 \\
\text { (s) }\end{array}$ & $\begin{array}{r}95.4 \\
30.0 \\
46.8 \\
18.6 \\
18.2 \\
.4\end{array}$ & $\begin{array}{r}268.0 \\
112.8 \\
142.9 \\
12.3 \\
4.1 \\
8.2 \\
= \\
=\end{array}$ & $\begin{array}{r}392.6 \\
290.9 \\
99.1 \\
2.6 \\
.9 \\
1.6 \\
- \\
- \\
-\end{array}$ & $\begin{array}{r}414.2 \\
362.1 \\
50.2 \\
2.0 \\
.6 \\
1.3 \\
= \\
-\end{array}$ & $\begin{array}{r}371.1 \\
320.5 \\
48.6 \\
2.0 \\
.3 \\
1.8 \\
- \\
- \\
-\end{array}$ & $\begin{array}{r}398.9 \\
352.1 \\
44.7 \\
2.2 \\
(s) \\
2.1 \\
- \\
-\end{array}$ & $\begin{array}{r}431.5 \\
367.6 \\
61.8 \\
2.1 \\
(s) \\
2.1 \\
= \\
-\end{array}$ \\
\hline Primary Energy - Five Sectors ${ }^{\circ}$............................ & 387.3 & B18.1 & $2,016.1$ & $2,127.9$ & $R_{2,392.7}$ & $R_{2,367.5}$ & $R_{2,395.8}$ & $2,566.0$ \\
\hline
\end{tabular}

There are no direct fuel costs for hydroelectric, geothermal, wind, photovoltalc, or solar thermal energy.

beavy oil Includes fuel oil nos. 4, 5, and 6, and residual fuel oils. Light oil Includes fuel nos. 1 and 2, kerosene, and jet fuel.

- Blomass fuels are not included, except those consumed at electric utilltles and those added to motor gasoline.

R=Revised data.
-No consumption, including cases where adjustments were made. See explanation of adjustments in Section 6 of Appendix A.

$(s)=$ Value less than 0.05 million dollars.

Note: Totals may not equal sum of components due to independent rounding.

Sources: Data sources, estimation procedures, and assumptions are described in Appendix A. 


\section{N Energy Price and Expenditure Estimates by Source, New York \\ E $1970,1975,1980,1985$, and 1990-1993}

\begin{tabular}{|c|c|c|c|c|c|c|c|c|}
\hline Energy Source & 1970 & 1975 & 1980 & 1985 & 1990 & 1991 & 1992 & 1993 \\
\hline & \multicolumn{8}{|c|}{ Prices in Dollars per Million Btu } \\
\hline 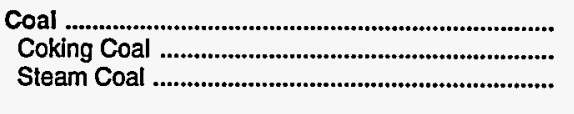 & $\begin{array}{r}0.51 \\
.58 \\
.49\end{array}$ & $\begin{array}{l}1.53 \\
2.14 \\
1.27\end{array}$ & $\begin{array}{l}1.78 \\
2.38 \\
1.56\end{array}$ & $\begin{array}{l}1.81 \\
1.88 \\
1.80\end{array}$ & $\begin{array}{l}1.66 \\
1.71 \\
1.66\end{array}$ & $\begin{array}{l}1.65 \\
1.72 \\
1.64\end{array}$ & $\begin{array}{l}1.56 \\
1.73 \\
1.54\end{array}$ & $\begin{array}{l}1.56 \\
1.73 \\
1.54\end{array}$ \\
\hline Natural Gas & 1.07 & 2.16 & 4.10 & 5.94 & 5.25 & 5.18 & 5.48 & 6.00 \\
\hline $\begin{array}{l}\text { Petroleum } \\
\text { Asphalt and Road Oil } \\
\text { Aviation Gasoline } \\
\text { Distillate Fuel } \\
\text { Jet Fuel } \\
\text { Kerosene } \\
\text { LPG }\end{array}$ & $\begin{array}{r}1.36 \\
.70 \\
2.17 \\
1.24 \\
.72 \\
1.39 \\
2.24 \\
5.08 \\
2.92 \\
.43 \\
1.17\end{array}$ & $\begin{array}{l}2.96 \\
1.84 \\
3.45 \\
2.66 \\
2.02 \\
3.06 \\
3.99 \\
7.48 \\
4.80 \\
1.93 \\
2.67\end{array}$ & $\begin{array}{r}6.93 \\
3.67 \\
9.02 \\
6.78 \\
6.27 \\
7.78 \\
7.43 \\
14.36 \\
10.26 \\
4.10 \\
7.05\end{array}$ & $\begin{array}{r}7.39 \\
4.81 \\
9.99 \\
7.92 \\
6.51 \\
8.31 \\
11.40 \\
17.61 \\
8.79 \\
4.38 \\
6.57\end{array}$ & $\begin{array}{r}{ }^{R} 7.10 \\
2.96 \\
9.32 \\
8.13 \\
6.03 \\
8.82 \\
12.76 \\
\mathrm{R}_{14.60} \\
8.83 \\
3.64 \\
\mathrm{R}_{6.06}\end{array}$ & $\begin{array}{r}R 7.12 \\
2.76 \\
8.71 \\
7.98 \\
5.18 \\
8.58 \\
13.54 \\
\text { R } \\
16.79 \\
9.44 \\
2.73 \\
R 4.49\end{array}$ & $\begin{array}{r}R 7.14 \\
2.30 \\
8.54 \\
7.52 \\
4.84 \\
7.60 \\
12.96 \\
R 19.43 \\
9.28 \\
2.71 \\
R 4.09\end{array}$ & $\begin{array}{r}7.15 \\
2.81 \\
8.24 \\
7.38 \\
4.47 \\
7.23 \\
12.14 \\
18.94 \\
9.30 \\
2.69 \\
3.88\end{array}$ \\
\hline 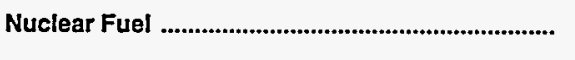 & .20 & .31 & .56 & .67 & .65 & .55 & .45 & .57 \\
\hline 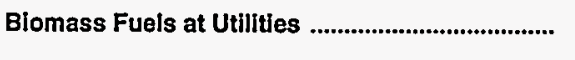 & - & - & 1.74 & - & - & - & - & 1.40 \\
\hline Primary Energy - Flve Sectors ${ }^{b}$ & 1.17 & 2.60 & 5.45 & 5.87 & ${ }^{R} 5.51$ & ${ }^{R} 5.37$ & 5.47 & 5.65 \\
\hline 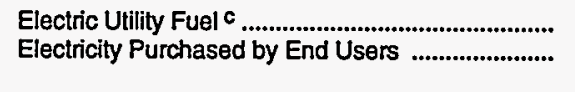 & $\begin{array}{r}.42 \\
6.72\end{array}$ & $\begin{array}{r}1.53 \\
14.04\end{array}$ & $\begin{array}{r}2.68 \\
19.64\end{array}$ & $\begin{array}{r}2.54 \\
26.97\end{array}$ & $\begin{array}{r}2.20 \\
27.51\end{array}$ & $\begin{array}{r}1.74 \\
28.68\end{array}$ & $\begin{array}{r}1.65 \\
29.86\end{array}$ & $\begin{array}{r}1.62 \\
31.43\end{array}$ \\
\hline \multirow[t]{2}{*}{ 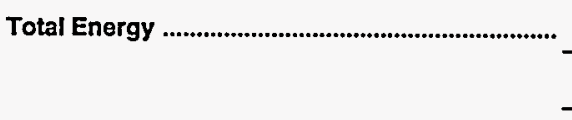 } & 1.81 & 4.11 & 8.08 & 10.38 & 10.64 & 10.84 & $R_{10.80}$ & 11.05 \\
\hline & \multicolumn{8}{|c|}{ Expenditures in Millions of Dollars } \\
\hline 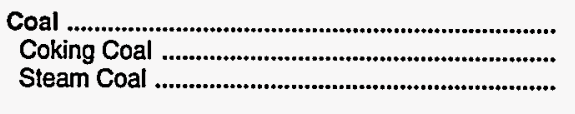 & $\begin{array}{r}308.4 \\
96.4 \\
212.0\end{array}$ & $\begin{array}{l}477.1 \\
197.8 \\
279.3\end{array}$ & $\begin{array}{l}558.6 \\
197.6 \\
361.0\end{array}$ & $\begin{array}{r}546.0 \\
58.5 \\
487.6\end{array}$ & $\begin{array}{r}574.9 \\
62.2 \\
512.7\end{array}$ & $\begin{array}{r}566.9 \\
56.6 \\
510.4\end{array}$ & $\begin{array}{r}525.4 \\
53.4 \\
472.0\end{array}$ & $\begin{array}{r}479.4 \\
57.7 \\
421.7\end{array}$ \\
\hline 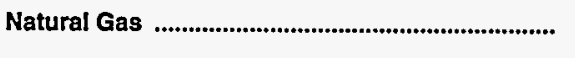 & 771.3 & $1,255.2$ & $3,087.1$ & $4,637.2$ & $4,614.1$ & $4,630.4$ & $5,365.3$ & $5,789.5$ \\
\hline $\begin{array}{l}\text { Petroleum } \\
\text { Asphalt and Road Oil } \\
\text { Aviation Gasoline } \\
\text { Distillate Fuel } \\
\text { Jet Fuel } \\
\text { Kerosene } \\
\text { LPG }\end{array}$ & $\begin{array}{r}3,611.9 \\
26.0 \\
2.7 \\
803.3 \\
155.5 \\
55.3 \\
37.2 \\
67.8 \\
2,005.9 \\
409.7 \\
48.5\end{array}$ & $\begin{array}{r}7,618.7 \\
69.9 \\
4.8 \\
1,626.9 \\
441.7 \\
90.3 \\
70.3 \\
88.4 \\
3,368.0 \\
1,740.1 \\
118.2\end{array}$ & $\begin{array}{r}14,901.7 \\
121.3 \\
14.6 \\
2,862.3 \\
1,275.3 \\
101.8 \\
139.0 \\
182.1 \\
6,865.7 \\
2,964.1 \\
375.5\end{array}$ & $\begin{array}{r}12,246.0 \\
230.1 \\
11.1 \\
2,861.6 \\
139.0 \\
250.5 \\
202.3 \\
203.2 \\
6,299.5 \\
1,827.8 \\
220.7\end{array}$ & $\begin{array}{r}R_{12,395.9} \\
108.4 \\
3.6 \\
3,141.0 \\
183.5 \\
114.2 \\
256.9 \\
R_{189.6} \\
6,419.2 \\
1,756.9 \\
\text { R }_{222.4}\end{array}$ & $\begin{array}{r}R_{11,840.8} \\
117.0 \\
2.9 \\
2,861.6 \\
153.0 \\
128.8 \\
352.7 \\
R_{195.1} \\
6,644.3 \\
1,164.1 \\
R_{221.3}\end{array}$ & $\begin{array}{r}\mathrm{A}_{11,179.0} \\
105.4 \\
3.2 \\
2,877.9 \\
144.6 \\
80.2 \\
332.4 \\
\mathrm{R}_{230.2} \\
6,294.2 \\
\mathrm{R}_{877.4} \\
\mathrm{R}_{233.4}\end{array}$ & $\begin{array}{r}11,335.5 \\
150.3 \\
2.5 \\
3,012.4 \\
128.2 \\
99.3 \\
268.7 \\
228.5 \\
6,431.0 \\
812.7 \\
201.9\end{array}$ \\
\hline 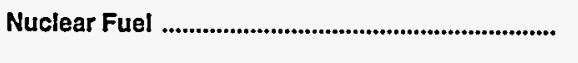 & 9.2 & 44.9 & 118.3 & 175.2 & 164.7 & 169.4 & 117.2 & 163.8 \\
\hline 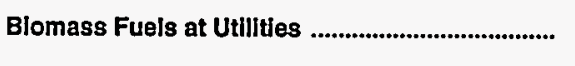 & - & - & 2 & - & - & - & - & .2 \\
\hline Primary Energy - Five Sectors ${ }^{b}$ & $4,700.7$ & $9,395.9$ & $18,666.0$ & $17,604.4$ & $R_{17,749.6}$ & $R_{17,207.5}$ & $R_{17,186.9}$ & $17,768.4$ \\
\hline 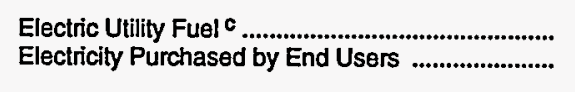 & $\begin{array}{r}-335.3 \\
2,006.4\end{array}$ & $\begin{array}{r}-1,326.8 \\
4,580.0\end{array}$ & $\begin{array}{r}-2,427.3 \\
7,044.4\end{array}$ & $\begin{array}{r}-2,320.5 \\
10,369.9\end{array}$ & $\begin{array}{r}-2,383.6 \\
12,087.5\end{array}$ & $\begin{array}{r}-1,848.0 \\
12,665.0\end{array}$ & $\begin{array}{r}-1,509.3 \\
R+13,091.0\end{array}$ & $\begin{array}{l}-1,361.7 \\
13,961.1\end{array}$ \\
\hline 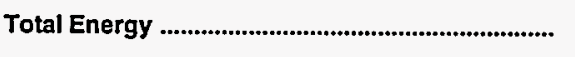 & $6,371.7$ & $12,649.1$ & $23,283.2$ & $25,653.8$ & ${ }^{R} 27,453.5$ & $R_{28,024.5}$ & ${ }^{R} 28,768.5$ & $30,367.8$ \\
\hline
\end{tabular}

\footnotetext{
a Includes petroleum coke used at electric utilities.

b Biomass fuels are not included, except those consumed at electric utilities and those added to motor gasoline.

c There are no direct fuel costs for hydroelectric, geothermal, wind, photovoltaic, or solar thermal energy.

$R=$ Revised data.
}

- No consumption, including cases where adjustments were made. See explanation of adjustments in Section 6 of Appendix A.

Note: Expenditure totals may not equal sum of components due to independent rounding.

Sources: Data sources, estimation procedures, and assumptions are described in Appendix A. 


\begin{tabular}{|c|c|c|c|c|c|c|c|c|}
\hline Sector and Energy Source & 1970 & 1975 & 1980 & 1985 & 1990 & 1991 & 1992 & 1993 \\
\hline 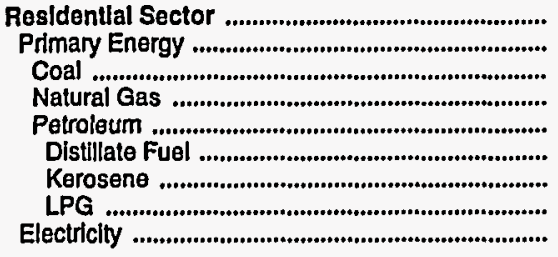 & $\begin{array}{l}2.19 \\
1.43 \\
1.43 \\
1.37 \\
1.48 \\
1.43 \\
1.56 \\
2.70 \\
8.83\end{array}$ & $\begin{array}{r}4.40 \\
2.70 \\
2.78 \\
2.50 \\
2.89 \\
2.81 \\
3.28 \\
4.48 \\
16.44\end{array}$ & $\begin{array}{r}8.42 \\
5.80 \\
3.26 \\
4.85 \\
7.22 \\
7.08 \\
8.49 \\
9.12 \\
23.08\end{array}$ & $\begin{array}{r}11.98 \\
7.91 \\
3.61 \\
7.54 \\
8.58 \\
8.35 \\
9.30 \\
11.12 \\
31.84\end{array}$ & $\begin{array}{r}12.88 \\
7.75 \\
3.59 \\
7.19 \\
8.92 \\
8.44 \\
9.40 \\
13.64 \\
33.54\end{array}$ & $\begin{array}{r}13.29 \\
7.77 \\
3.44 \\
7.16 \\
9.06 \\
8.35 \\
9.30 \\
14.59 \\
35.09\end{array}$ & $\begin{array}{r}13.00 \\
7.67 \\
3.21 \\
7.37 \\
8.38 \\
7.71 \\
8.59 \\
14.43 \\
36.43\end{array}$ & $\begin{array}{r}13.69 \\
7.93 \\
3.25 \\
7.92 \\
8.02 \\
7.51 \\
8.36 \\
13.35 \\
38.61\end{array}$ \\
\hline 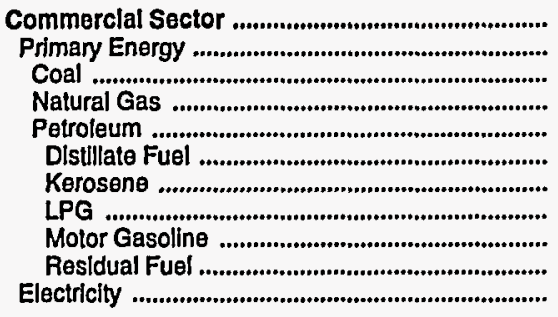 & $\begin{array}{r}1.99 \\
.80 \\
.48 \\
1.17 \\
.67 \\
1.14 \\
.73 \\
1.45 \\
2.92 \\
.42 \\
7.80\end{array}$ & $\begin{array}{r}5.42 \\
2.11 \\
1.36 \\
1.97 \\
2.19 \\
2.48 \\
2.51 \\
3.09 \\
4.80 \\
1.90 \\
16.57\end{array}$ & $\begin{array}{r}9.27 \\
4.70 \\
1.67 \\
4.17 \\
5.09 \\
6.48 \\
5.68 \\
5.79 \\
10.26 \\
4.18 \\
23.21\end{array}$ & $\begin{array}{r}13.68 \\
5.84 \\
1.91 \\
5.95 \\
5.86 \\
7.03 \\
6.79 \\
11.95 \\
8.79 \\
4.64 \\
30.86\end{array}$ & $\begin{array}{r}13.10 \\
5.26 \\
1.76 \\
5.43 \\
5.15 \\
6.70 \\
6.86 \\
10.33 \\
8.83 \\
3.75 \\
29.44\end{array}$ & $\begin{array}{r}13.22 \\
4.91 \\
1.74 \\
5.33 \\
4.52 \\
6.42 \\
5.83 \\
11.09 \\
9.44 \\
2.83 \\
30.31\end{array}$ & $\begin{array}{r}13.36 \\
5.00 \\
1.74 \\
5.59 \\
4.37 \\
5.76 \\
5.57 \\
9.50 \\
9.28 \\
2.89 \\
31.48\end{array}$ & $\begin{array}{r}13.78 \\
5.12 \\
1.67 \\
5.99 \\
4.20 \\
5.60 \\
5.17 \\
9.32 \\
9.30 \\
2.88 \\
32.87\end{array}$ \\
\hline 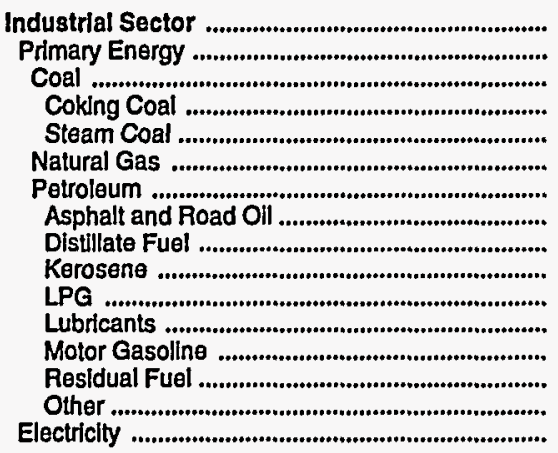 & $\begin{array}{r}.97 \\
.69 \\
.53 \\
.58 \\
.48 \\
.68 \\
.81 \\
.70 \\
.70 \\
.73 \\
1.45 \\
5.08 \\
2.92 \\
.49 \\
1.17 \\
3.51\end{array}$ & $\begin{array}{l}2.85 \\
2.06 \\
1.82 \\
2.14 \\
1.36 \\
1.47 \\
2.35 \\
1.84 \\
2.36 \\
2.51 \\
3.09 \\
7.48 \\
4.80 \\
2.01 \\
2.67 \\
7.97\end{array}$ & $\begin{array}{r}5.39 \\
3.98 \\
2.08 \\
2.38 \\
1.67 \\
3.43 \\
5.33 \\
3.67 \\
5.36 \\
5.68 \\
5.79 \\
14.36 \\
10.26 \\
3.78 \\
7.05 \\
12.11\end{array}$ & $\begin{array}{r}7.01 \\
4.77 \\
1.90 \\
1.88 \\
1.91 \\
5.13 \\
6.17 \\
4.81 \\
6.13 \\
6.79 \\
11.95 \\
17.61 \\
8.79 \\
4.64 \\
6.57 \\
15.34\end{array}$ & $\begin{array}{r}R_{7.45} \\
R_{4.24} \\
1.74 \\
1.71 \\
1.76 \\
4.72 \\
R_{5.41} \\
2.96 \\
6.20 \\
6.86 \\
10.33 \\
R_{14.60} \\
8.83 \\
3.75 \\
R_{6.06} \\
16.94\end{array}$ & $\begin{array}{r}R_{7.30} \\
R_{4.00} \\
1.73 \\
1.72 \\
1.74 \\
4.60 \\
R_{4.80} \\
2.76 \\
5.27 \\
5.83 \\
11.09 \\
R_{16.79} \\
9.44 \\
2.83 \\
R_{4.49} \\
18.07\end{array}$ & $\begin{array}{r}R_{7.34} \\
R_{4.07} \\
1.74 \\
1.73 \\
1.74 \\
4.79 \\
R_{4.44} \\
2.30 \\
5.03 \\
5.57 \\
9.50 \\
R_{19.43} \\
9.28 \\
2.89 \\
R_{4.09} \\
19.05\end{array}$ & $\begin{array}{r}7.21 \\
4.12 \\
1.70 \\
1.73 \\
1.67 \\
5.03 \\
4.32 \\
2.81 \\
4.67 \\
5.17 \\
9.32 \\
18.94 \\
9.30 \\
2.88 \\
3.88 \\
19.53\end{array}$ \\
\hline 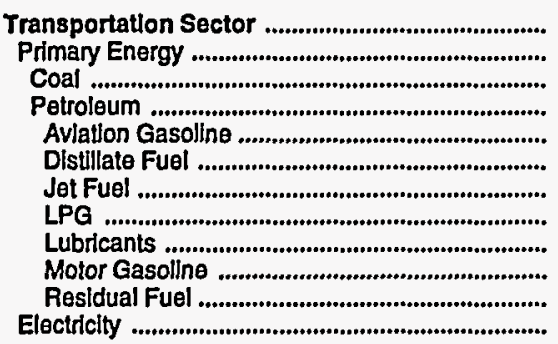 & $\begin{array}{r}2.14 \\
2.12 \\
.48 \\
2.12 \\
2.17 \\
1.44 \\
.72 \\
1.45 \\
5.08 \\
2.92 \\
.37 \\
4.82\end{array}$ & $\begin{array}{r}4.02 \\
3.95 \\
1.36 \\
3.95 \\
3.45 \\
2.84 \\
2.01 \\
3.09 \\
7.48 \\
4.80 \\
1.67 \\
13.66\end{array}$ & $\begin{array}{r}8.86 \\
8.82 \\
- \\
8.82 \\
9.02 \\
7.45 \\
6.27 \\
5.79 \\
14.36 \\
10.26 \\
3.53 \\
15.02\end{array}$ & $\begin{array}{r}8.84 \\
8.74 \\
- \\
8.74 \\
9.99 \\
8.48 \\
6.51 \\
11.95 \\
17.61 \\
8.79 \\
4.08 \\
19.65\end{array}$ & $\begin{array}{r}{ }_{8} 8.88 \\
R_{8.75} \\
- \\
R_{8.75} \\
9.32 \\
8.99 \\
6.03 \\
10.33 \\
R_{14.60} \\
8.83 \\
3.13 \\
23.32\end{array}$ & $\begin{array}{r}{ }^{R_{9.22}} \\
R_{9.10} \\
- \\
R_{9.10} \\
8.71 \\
9.06 \\
5.18 \\
11.09 \\
R_{16.79} \\
9.44 \\
2.38 \\
23.13\end{array}$ & $\begin{array}{r}R_{9.10} \\
R_{8.95} \\
- \\
R_{8.95} \\
8.54 \\
8.89 \\
4.84 \\
9.50 \\
A_{19.43} \\
9.28 \\
R_{2.33} \\
25.53\end{array}$ & $\begin{array}{r}9.15 \\
9.00 \\
- \\
9.00 \\
8.24 \\
9.04 \\
4.47 \\
9.32 \\
18.94 \\
9.30 \\
2.29 \\
26.78\end{array}$ \\
\hline 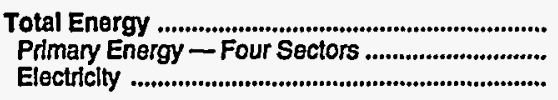 & $\begin{array}{l}1.81 \\
1.36 \\
6.72\end{array}$ & $\begin{array}{r}4.11 \\
2.93 \\
14.04\end{array}$ & $\begin{array}{r}8.08 \\
6.44 \\
19.64\end{array}$ & $\begin{array}{r}10.38 \\
7.32 \\
26.97\end{array}$ & $\begin{array}{l}10.64 \\
R 7.18 \\
27.51\end{array}$ & $\begin{array}{r}10.84 \\
R 7.17 \\
28.68\end{array}$ & $\begin{array}{r}R_{10.80} \\
7.04 \\
29.86\end{array}$ & $\begin{array}{r}11.05 \\
7.12 \\
31.43\end{array}$ \\
\hline 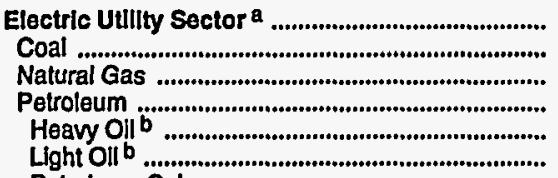 & $\begin{array}{l}.42 \\
.47 \\
.38 \\
.42 \\
.42 \\
.44\end{array}$ & $\begin{array}{r}1.53 \\
1.18 \\
.88 \\
1.95 \\
1.94 \\
2.16\end{array}$ & $\begin{array}{l}2.68 \\
1.47 \\
2.67 \\
4.26 \\
4.25 \\
5.63\end{array}$ & $\begin{array}{l}2.54 \\
1.72 \\
3.48 \\
4.29 \\
4.26 \\
6.11\end{array}$ & $\begin{array}{l}2.20 \\
1.61 \\
2.38 \\
3.65 \\
3.60 \\
6.34\end{array}$ & $\begin{array}{l}1.74 \\
1.59 \\
2.23 \\
2.76 \\
2.72 \\
5.30\end{array}$ & $\begin{array}{l}1.65 \\
1.49 \\
2.41 \\
2.66 \\
2.63 \\
4.96\end{array}$ & $\begin{array}{l}1.62 \\
1.50 \\
2.65 \\
2.61 \\
2.56 \\
4.69\end{array}$ \\
\hline $\begin{array}{l}\text { Petroleum Coke } \\
\text { Nuclear Fuel } \\
\text { Blomass Fuels }\end{array}$ &.$\overline{20}$ &.$\overline{31}$ &.$\overline{56}$ &.$\overline{67}$ &.$\overline{65}$ &.$\overline{5}$ &.$\overline{45}$ & $\begin{array}{r}.57 \\
1.40\end{array}$ \\
\hline Primary Energy - Flve Sectors c ........................ & 1.17 & 2.60 & 5.45 & 5.87 & $\mathrm{~A}_{5.51}$ & $R_{5.37}$ & 5.47 & 5.65 \\
\hline
\end{tabular}

There are no direct fuel costs for hydroelectric, geothermal, wind, photovoltaic, or solar thermal energy.

$b$ Heavy oll includes fuel oil nos. 4, 5, and 6, and residual fuel oils. Light oll includes fuel oll nos. 1 and 2, kerosene, and jet fuel.

c Blomass fuels are not included, except those consumed at electric utllitles and those added to motor gasoline.
R=Revised data

No consumption, including cases where adjustments were made. See explanation of adjustments in Section 6 of Appendix A.

Sources: Data sources, estimation procedures, and assumptions are described in Appendix A. 
N Energy Expenditure Estimates by Sector, New York

E 1970, 1975, 1980, 1985, and 1990-1993

W (Million Dollars)

\begin{tabular}{|c|c|c|c|c|c|c|c|c|}
\hline Sector and Energy Source & 1970 & 1975 & 1980 & 1985 & 1990 & 1991 & 1992 & 1983 \\
\hline 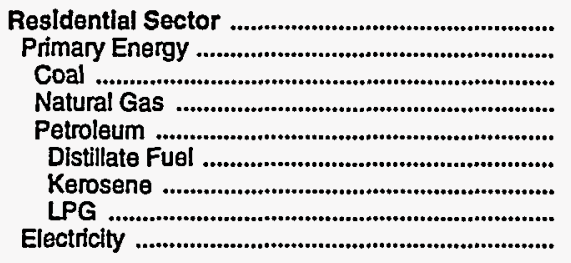 & $\begin{array}{r}1,844.5 \\
1,076.5 \\
12.8 \\
484.5 \\
579.2 \\
501.4 \\
49.4 \\
28.3 \\
768.0\end{array}$ & $\begin{array}{r}3,490.4 \\
1,879.9 \\
14.2 \\
830.2 \\
1,035.4 \\
914.6 \\
69.6 \\
51.2 \\
1,610.5\end{array}$ & $\begin{array}{r}5,798.8 \\
3,390.0 \\
13.7 \\
1,654.8 \\
1,721.5 \\
1,554.5 \\
82.9 \\
84.1 \\
2,408.8\end{array}$ & $\begin{array}{r}7,860.2 \\
4,301.5 \\
17.7 \\
2,478.1 \\
1,805.7 \\
1,506.7 \\
169.7 \\
129.3 \\
3,558.6\end{array}$ & $\begin{array}{r}8,526.0 \\
4,111.9 \\
11.5 \\
2,501.1 \\
1,599.3 \\
1,303.6 \\
94.1 \\
201.6 \\
4,414.0\end{array}$ & $\begin{array}{r}8,785.5 \\
4,095.5 \\
11.2 \\
2,490.9 \\
1,593.4 \\
1,216.4 \\
110.6 \\
266.3 \\
4,690.1\end{array}$ & $\begin{array}{r}9,271.5 \\
4,458.5 \\
10.2 \\
2,870.5 \\
1,577.8 \\
1,257.2 \\
61.0 \\
259.7 \\
4,813.0\end{array}$ & $\begin{array}{r}9,932.9 \\
4,676.8 \\
9.5 \\
3,131.4 \\
1,536.0 \\
1,255.2 \\
74.2 \\
206.6 \\
5,256.1\end{array}$ \\
\hline 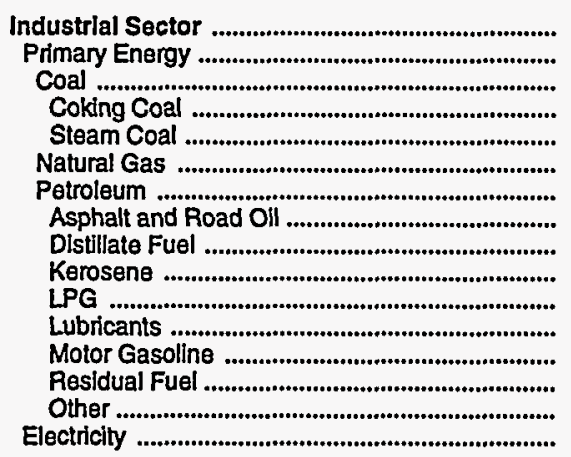 & $\begin{array}{r}803.2 \\
581.2 \\
164.5 \\
96.4 \\
68.1 \\
80.0 \\
336.7 \\
26.0 \\
68.8 \\
3.3 \\
5.6 \\
30.9 \\
50.3 \\
103.2 \\
48.5 \\
322.1\end{array}$ & $\begin{array}{r}1,961.1 \\
1,226.5 \\
283.3 \\
197.8 \\
85.5 \\
156.0 \\
787.2 \\
69.9 \\
216.9 \\
14.8 \\
11.4 \\
45.3 \\
34.1 \\
276.6 \\
118.2 \\
734.6\end{array}$ & $\begin{array}{r}3,374.0 \\
2,056.0 \\
304.2 \\
197.6 \\
106.6 \\
398.4 \\
1,353.4 \\
121.3 \\
289.8 \\
13.4 \\
43.8 \\
89.5 \\
82.7 \\
337.3 \\
375.5 \\
1,318.1\end{array}$ & $\begin{array}{r}3,238.1 \\
1,737.7 \\
180.4 \\
58.5 \\
121.9 \\
526.2 \\
1,031.1 \\
230.1 \\
172.1 \\
47.6 \\
42.2 \\
99.8 \\
56.5 \\
162.0 \\
220.7 \\
1,500.4\end{array}$ & $\begin{array}{r}R_{3,160.1} \\
R_{1,344.6} \\
143.0 \\
62.2 \\
80.8 \\
473.7 \\
R_{727.9} \\
108.4 \\
123.0 \\
9.7 \\
22.7 \\
R 93.1 \\
52.8 \\
95.7 \\
R_{222.4} \\
1,815.5\end{array}$ & $\begin{array}{r}R_{3,301.6} \\
A_{1,383.7} \\
142.4 \\
56.6 \\
85.9 \\
561.4 \\
R_{679.8} \\
117.0 \\
93.4 \\
11.1 \\
44.3 \\
R_{95.8} \\
54.4 \\
42.4 \\
R_{221.3} \\
1,918.0\end{array}$ & $\begin{array}{r}R_{3,565.9} \\
R_{1,548.9} \\
124.0 \\
53.4 \\
70.7 \\
727.3 \\
R_{697.6} \\
105.4 \\
91.3 \\
6.4 \\
37.6 \\
R_{113.1} \\
54.1 \\
56.2 \\
R_{233.4} \\
R_{2,017.1}\end{array}$ & $\begin{array}{r}3,703.5 \\
1,691.5 \\
129.2 \\
57.7 \\
71.5 \\
829.3 \\
732.9 \\
150.3 \\
110.1 \\
7.1 \\
32.3 \\
112.2 \\
48.1 \\
70.9 \\
201.9 \\
2,012.0\end{array}$ \\
\hline 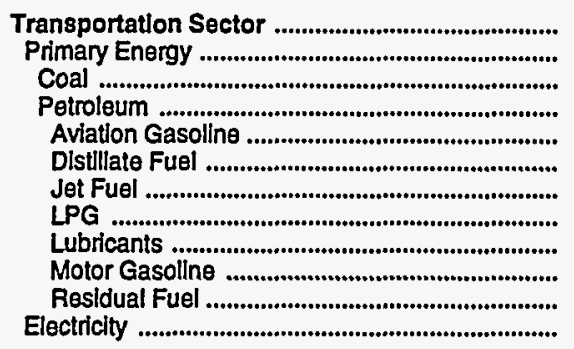 & $\begin{array}{r}2,299.1 \\
2,267.8 \\
.2 \\
2,267.6 \\
2.7 \\
89.5 \\
155.5 \\
.6 \\
36.9 \\
1,939.4 \\
43.0 \\
31.3\end{array}$ & $\begin{array}{r}4,140.7 \\
4,043.8 \\
(s) \\
4,043.7 \\
4.8 \\
173.7 \\
423.1 \\
1.4 \\
43.1 \\
3,304.6 \\
93.0 \\
96.9\end{array}$ & $\begin{array}{r}8,915.6 \\
8,809.9 \\
8,809.9 \\
14.6 \\
447.5 \\
1,274.5 \\
1.7 \\
92.7 \\
6,727.2 \\
251.7 \\
105.6\end{array}$ & $\begin{array}{r}7,256.8 \\
7,106.5 \\
- \\
7,106.5 \\
11.1 \\
669.3 \\
139.0 \\
6.3 \\
103.4 \\
6,154.6 \\
22.7 \\
150.3\end{array}$ & $\begin{array}{r}\text { R } 7,991.4 \\
\text { R } 7,798.2 \\
- \\
\text { R } 7,798.2 \\
3.6 \\
1,170.8 \\
183.5 \\
5.7 \\
R 96.5 \\
6,311.0 \\
27.1 \\
193.2\end{array}$ & $\begin{array}{r}R_{8,106.3} \\
R_{7,922.6} \\
- \\
\text { R }_{7,922.6} \\
2.9 \\
1,047.4 \\
153.0 \\
6.3 \\
R 99.3 \\
6,554.4 \\
59.3 \\
183.6\end{array}$ & $\begin{array}{r}R_{7,778.5} \\
R_{7,582.5} \\
- \\
R_{7,582.5} \\
3.2 \\
1,051.1 \\
144.6 \\
5.0 \\
R_{117.1} \\
R_{1} 206.9 \\
R_{54.6} \\
R_{196.1}\end{array}$ & $\begin{array}{r}8,007.6 \\
7,809.5 \\
- \\
7,809.5 \\
2.5 \\
1,138.2 \\
128.2 \\
4.3 \\
116.2 \\
6,373.3 \\
46.9 \\
198.1\end{array}$ \\
\hline 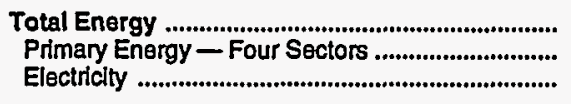 & $\begin{array}{l}6,371.7 \\
4,365.4 \\
2,006.4\end{array}$ & $\begin{array}{r}12,649.1 \\
8,069.1 \\
4,580.0\end{array}$ & $\begin{array}{r}23,283.2 \\
16,238.8 \\
7,044.4\end{array}$ & $\begin{array}{l}25,653.8 \\
15,283.9 \\
10,369.9\end{array}$ & $\begin{array}{r}R_{27,453.5} \\
R_{15,366.0} \\
12,087.5\end{array}$ & $\begin{array}{r}R_{28,024.5} \\
R_{15,359.5} \\
12,665.0\end{array}$ & $\begin{array}{l}R_{28,768.5} \\
R_{15,677.6} \\
R_{13,091.0}\end{array}$ & $\begin{array}{l}30,367.8 \\
16,406.6 \\
13,961.1\end{array}$ \\
\hline 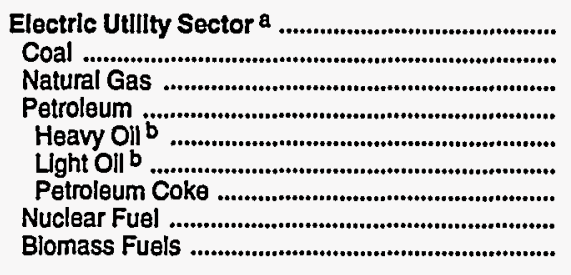 & $\begin{array}{r}335.3 \\
127.6 \\
40.9 \\
157.7 \\
149.6 \\
8.1 \\
-\overline{9} \\
-\end{array}$ & $\begin{array}{r}1,326.8 \\
173.3 \\
12.2 \\
1,096.4 \\
1,029.8 \\
66.6 \\
\overline{-} \\
44.9 \\
-\end{array}$ & $\begin{array}{r}2,427.3 \\
233.8 \\
343.4 \\
1,731.5 \\
1,706.9 \\
24.5 \\
- \\
118.3 \\
.2\end{array}$ & $\begin{array}{r}2,320.5 \\
337.5 \\
622.1 \\
1,185.7 \\
1,156.5 \\
29.2 \\
- \\
175.2 \\
-\end{array}$ & $\begin{array}{r}2,383.6 \\
414.1 \\
549.3 \\
1,255.5 \\
1,218.0 \\
37.5 \\
. .5 \\
164.7 \\
-\end{array}$ & $\begin{array}{r}1,848.0 \\
406.8 \\
486.2 \\
785.7 \\
758.4 \\
27.3 \\
169.4 \\
-\end{array}$ & $\begin{array}{r}1,509.3 \\
384.8 \\
518.6 \\
488.7 \\
476.7 \\
12.1 \\
117.2 \\
-\end{array}$ & $\begin{array}{r}1,361.7 \\
336.1 \\
469.0 \\
392.6 \\
377.1 \\
15.5 \\
163.8 \\
.2\end{array}$ \\
\hline Primary Energy — Flve Sectors ' & $4,700.7$ & $9,395.9$ & $18,666.0$ & $17,604.4$ & $R_{17,749.6}$ & R $_{17,207.5}$ & $\beta_{17,186.9}$ & $17,768.4$ \\
\hline
\end{tabular}

a There are no direct fuel costs for hydroelectric, geothermal, wind, photovoltalc, or solar thermal energy.

beavy oil includes fuel oil nos. 4, 5, and 6, and residual fuel olls. Light oil Includes fuel nos. 1 and 2 , kerosene, and jet fuel.

c Blomass fuels are not included, except those consumed at electric utilities and those added to motor gasoline.

$R=$ Revised data.

-No consumption, including cases where adjustments were made. See explanation of adjustments in section 6 of Appendix A.

(s) =Value less than 0.05 million dollars.

Note: Totals may not equal sum of components due to Independent rounding.

Sources: Data sources, estimation procedures, and assumptions are described in Appendix A. 


\begin{tabular}{|c|c|c|c|c|c|c|c|c|}
\hline Energy Source & 1970 & 1975 & 1980 & 1985 & 1990 & 1991 & 1992 & 1993 \\
\hline & \multicolumn{8}{|c|}{ Prices In Dollars per Mtilion Btu } \\
\hline 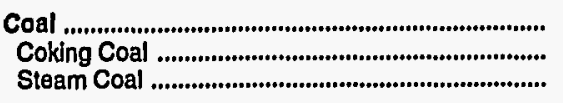 & $\begin{array}{r}0.43 \\
-43\end{array}$ & $\frac{1.12}{1.12}$ & $\frac{1.58}{1.58}$ & $\begin{array}{r}1.97 \\
1.97\end{array}$ & $\begin{array}{r}1.79 \\
1.79\end{array}$ & $\begin{array}{r}1.77 \\
1.77\end{array}$ & $\begin{array}{r}1.73 \\
1.73\end{array}$ & $\begin{array}{r}1.71 \\
1.71\end{array}$ \\
\hline Natural Gas & .69 & 1.57 & 3.55 & 5.29 & 4.19 & 4.09 & 4.29 & 4.79 \\
\hline 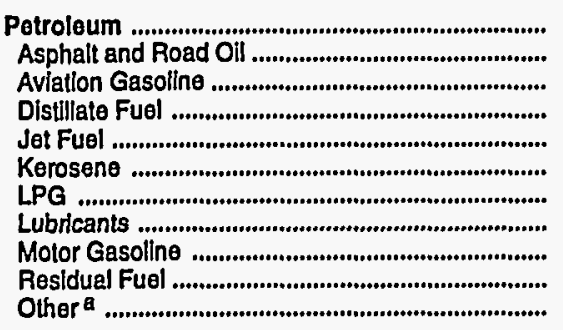 & $\begin{array}{r}1.94 \\
.67 \\
2.17 \\
1.13 \\
.73 \\
1.32 \\
1.89 \\
5.08 \\
2.82 \\
.46 \\
1.10\end{array}$ & $\begin{array}{l}3.68 \\
1.80 \\
3.45 \\
2.74 \\
2.03 \\
2.86 \\
3.24 \\
7.48 \\
4.55 \\
1.90 \\
2.54\end{array}$ & $\begin{array}{r}8.15 \\
3.57 \\
9.02 \\
6.80 \\
6.46 \\
7.67 \\
6.11 \\
14.36 \\
9.91 \\
3.72 \\
7.44\end{array}$ & $\begin{array}{r}8.21 \\
4.94 \\
9.99 \\
7.52 \\
5.77 \\
8.57 \\
9.72 \\
17.61 \\
9.03 \\
4.45 \\
7.23\end{array}$ & $\begin{array}{r}R_{8.42} \\
2.95 \\
9.32 \\
8.05 \\
5.65 \\
8.38 \\
10.31 \\
R_{14.60} \\
9.44 \\
3.11 \\
R_{6.33}\end{array}$ & $\begin{array}{r}\mathbf{A}_{8.14} \\
3.08 \\
8.71 \\
7.56 \\
4.79 \\
7.85 \\
11.09 \\
R_{16.79} \\
9.23 \\
2.37 \\
\mathbf{R}_{5.06}\end{array}$ & $\begin{array}{r}R_{7.74} \\
2.32 \\
8.54 \\
7.15 \\
4.48 \\
7.43 \\
10.16 \\
\mathrm{~A}_{19.43} \\
8.96 \\
2.46 \\
R_{4.82}\end{array}$ & $\begin{array}{r}7.67 \\
2.88 \\
8.24 \\
7.10 \\
4.19 \\
7.47 \\
9.56 \\
18.94 \\
8.93 \\
2.35 \\
4.49\end{array}$ \\
\hline 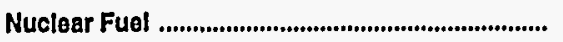 & - & .29 & .36 & .54 & .54 & .52 & .51 & .48 \\
\hline Blomass Fuels at Utilltles .................................... & - & - & - & - & - & - & - & - \\
\hline Prlmary Energy - Flve Sectors b ....................... & 1.20 & 2.47 & 4.63 & 4.78 & $\mathrm{~F}_{4.64}$ & $\mathrm{R}_{4.40}$ & 4.36 & 4.35 \\
\hline 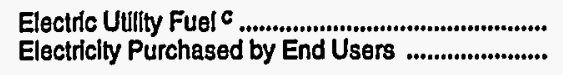 & $\begin{array}{r}.41 \\
4.17\end{array}$ & $\begin{array}{l}1.05 \\
7.92\end{array}$ & $\begin{array}{r}1.48 \\
11.72\end{array}$ & $\begin{array}{r}1.56 \\
17.46\end{array}$ & $\begin{array}{r}1.33 \\
18.74\end{array}$ & $\begin{array}{r}1.27 \\
18.96\end{array}$ & $\begin{array}{r}1.35 \\
19.33\end{array}$ & $\begin{array}{r}1.34 \\
19.44\end{array}$ \\
\hline \multirow[t]{2}{*}{ Total Energy ....................................................... } & 2.02 & 4.11 & 8.02 & 9.55 & 10.01 & 9.97 & $R_{9.76}$ & 9.92 \\
\hline & \multicolumn{8}{|c|}{ Expenditures in Millions of Dollars } \\
\hline 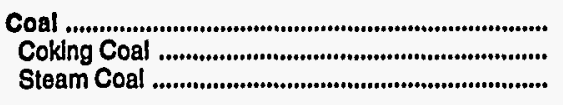 & $\frac{210.3}{210.3}$ & $\begin{array}{c}533.2 \\
\overline{533.2}\end{array}$ & $\begin{array}{c}985.6 \\
\overline{9} \\
985.6\end{array}$ & $\begin{array}{c}1,084.5 \\
- \\
1,084.5\end{array}$ & $\begin{array}{c}945.7 \\
- \\
945.7\end{array}$ & $\begin{array}{c}925.6 \\
- \\
925.6\end{array}$ & $\begin{array}{c}1,038.0 \\
- \\
1,038.0\end{array}$ & $\begin{array}{c}1,096.2 \\
- \\
1,096.2\end{array}$ \\
\hline 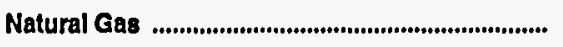 & 102.8 & 178.1 & 529.3 & 705.5 & 656.5 & 675.7 & 768.8 & 891.0 \\
\hline 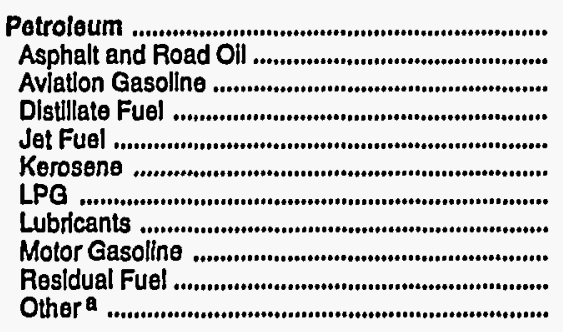 & $\begin{array}{r}1,201.9 \\
16.1 \\
1.7 \\
149.3 \\
18.7 \\
86.6 \\
39.1 \\
26.2 \\
835.7 \\
19.7 \\
8.8\end{array}$ & $\begin{array}{r}2,354.1 \\
36.4 \\
3.8 \\
339.0 \\
42.3 \\
94.7 \\
77.6 \\
42.8 \\
1,599.1 \\
92.9 \\
25.5\end{array}$ & $\begin{array}{r}5,423.4 \\
73.3 \\
9.8 \\
955.5 \\
185.3 \\
141.7 \\
179.1 \\
105.0 \\
3,448.9 \\
211.1 \\
113.6\end{array}$ & $\begin{array}{r}5,663.5 \\
113.2 \\
8.8 \\
1,087.3 \\
213.6 \\
231.9 \\
264.4 \\
117.2 \\
3,362.0 \\
174.3 \\
90.8\end{array}$ & $\begin{array}{r}R_{6,033.3} \\
82.4 \\
10.0 \\
1,175.0 \\
174.2 \\
77.2 \\
321.7 \\
R_{109.3} \\
3,823.8 \\
101.2 \\
R_{158.4}\end{array}$ & $\begin{array}{r}A_{5,843.8} \\
78.2 \\
7.5 \\
1,054.3 \\
116.3 \\
86.2 \\
413.1 \\
R_{112.5} \\
3,734.7 \\
91.1 \\
R_{150.0}\end{array}$ & $\begin{array}{r}R_{5,796.5} \\
65.4 \\
6.6 \\
1,072.1 \\
116.4 \\
85.4 \\
408.4 \\
R_{132.7} \\
3,635.6 \\
116.5 \\
R_{157.5}\end{array}$ & $\begin{array}{r}6,011.3 \\
88.9 \\
4.9 \\
1,094.6 \\
114.2 \\
88.8 \\
409.2 \\
131.7 \\
3,820.8 \\
119.3 \\
138.9\end{array}$ \\
\hline 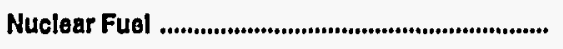 & $\boldsymbol{~} \boldsymbol{-}$ & 4.4 & 22.9 & 111.8 & 150.4 & 169.8 & 122.8 & 123.0 \\
\hline Blomass Fuele at Utilitles ...................................... & - & - & $\rightarrow$ & - & - & - & - & - \\
\hline Primary Energy - Five Sectors b ...................... & $1,515.0$ & $3,069.8$ & $6,961.2$ & $7,565.3$ & R $7,786.0$ & ค 7,614.9 & $\mathrm{R}_{7,726.0}$ & $8,121.4$ \\
\hline 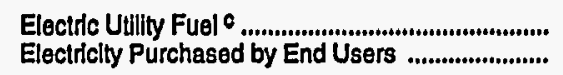 & $\begin{array}{r}-190.7 \\
576.2\end{array}$ & $\begin{array}{r}-473.6 \\
1,393.1\end{array}$ & $\begin{array}{r}-967.2 \\
2,553.8\end{array}$ & $\begin{array}{r}-1,097.2 \\
4,305.3\end{array}$ & $\begin{array}{r}-973.5 \\
5,715.3\end{array}$ & $\begin{array}{r}.992 .1 \\
5,973.0\end{array}$ & $\begin{array}{r}-1,043.7 \\
6,213.0\end{array}$ & $\begin{array}{r}-1,118.3 \\
6,618.0\end{array}$ \\
\hline 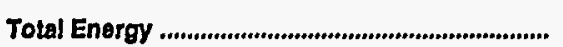 & $1,900.4$ & $3,989.4$ & $8,547.8$ & $10,773.4$ & $R_{12,527.9}$ & $R_{12,595.8}$ & $A_{12,895.4}$ & $13,621.1$ \\
\hline
\end{tabular}

a Includes petroleum coke used at electric uillities.

b Blomass fuels are not Included, except those consumed at electric utllities and those added to motor gasoline.

- There are no direct fuel costs for hydroelectric, geothermal, wind, photovoltaic, or solar themal energy.

- No consumption, including cases where adjustments were made. See explanation of adjustments in Section 6 of Appendix A.

Note: Expenditure totals may not equal sum of components due to independent rounding.

Sources: Data sources, estimation procedures, and assumptions are $R=$ Revised data. 


\section{N Energy Price Estimates by Sector, North Carolina \\ O 1970, 1975, 1980, 1985, and 1990-1993 \\ R (Dollars per Million Btu)}

\begin{tabular}{|c|c|c|c|c|c|c|c|c|}
\hline Sector and Energy Source & 1970 & 1975 & 1980 & 1985 & 1990 & 1991 & 1992 & 1993 \\
\hline 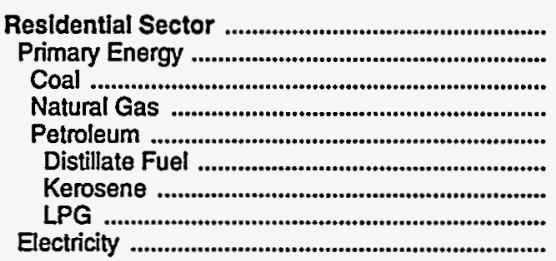 & $\begin{array}{l}2.41 \\
1.40 \\
1.14 \\
1.27 \\
1.44 \\
1.31 \\
1.40 \\
2.28 \\
5.45\end{array}$ & $\begin{array}{l}5.15 \\
2.69 \\
2.06 \\
1.99 \\
2.97 \\
2.71 \\
2.96 \\
4.32 \\
9.31\end{array}$ & $\begin{array}{r}9.62 \\
6.15 \\
2.70 \\
4.06 \\
7.30 \\
6.95 \\
7.96 \\
7.67 \\
13.91\end{array}$ & $\begin{array}{r}14.09 \\
7.86 \\
2.75 \\
6.38 \\
8.69 \\
8.02 \\
8.73 \\
10.27 \\
20.48\end{array}$ & $\begin{array}{r}16.56 \\
7.68 \\
2.78 \\
5.98 \\
9.22 \\
7.95 \\
8.65 \\
11.22 \\
22.98\end{array}$ & $\begin{array}{r}16.72 \\
7.70 \\
2.58 \\
6.05 \\
9.21 \\
7.48 \\
8.14 \\
11.67 \\
23.29\end{array}$ & $\begin{array}{r}16.44 \\
7.39 \\
2.65 \\
6.38 \\
8.44 \\
7.06 \\
7.68 \\
10.29 \\
23.78\end{array}$ & $\begin{array}{r}16.72 \\
7.67 \\
2.79 \\
6.75 \\
8.72 \\
7.07 \\
7.70 \\
11.03 \\
23.96\end{array}$ \\
\hline 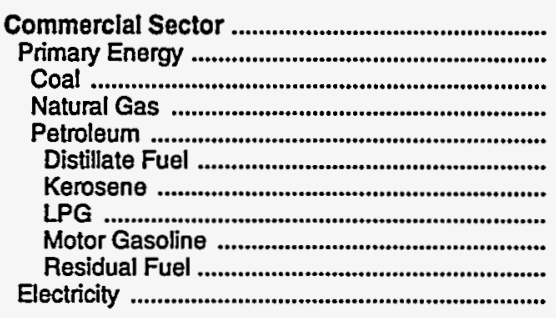 & $\begin{array}{r}2.51 \\
.98 \\
.53 \\
.94 \\
1.23 \\
1.02 \\
.77 \\
1.41 \\
2.82 \\
.67 \\
4.60\end{array}$ & $\begin{array}{l}5.16 \\
2.01 \\
1.53 \\
1.71 \\
2.66 \\
2.34 \\
2.37 \\
2.67 \\
4.55 \\
1.79 \\
8.46\end{array}$ & $\begin{array}{r}8.50 \\
4.73 \\
1.71 \\
3.67 \\
6.58 \\
6.33 \\
6.12 \\
5.24 \\
9.91 \\
3.80 \\
12.28\end{array}$ & $\begin{array}{r}12.78 \\
6.15 \\
1.90 \\
5.65 \\
7.24 \\
6.90 \\
7.73 \\
9.33 \\
9.03 \\
4.46 \\
18.18\end{array}$ & $\begin{array}{r}13.66 \\
5.28 \\
1.80 \\
4.49 \\
7.00 \\
6.04 \\
6.66 \\
9.41 \\
9.44 \\
3.16 \\
18.94\end{array}$ & $\begin{array}{r}13.80 \\
4.93 \\
1.71 \\
4.39 \\
6.37 \\
4.92 \\
5.96 \\
10.59 \\
9.23 \\
2.42 \\
19.11\end{array}$ & $\begin{array}{r}13.90 \\
4.81 \\
1.73 \\
4.63 \\
5.88 \\
4.10 \\
5.02 \\
10.04 \\
8.96 \\
2.49 \\
19.50\end{array}$ & $\begin{array}{r}14.00 \\
5.11 \\
1.73 \\
5.32 \\
5.36 \\
4.82 \\
5.41 \\
8.27 \\
8.93 \\
2.36 \\
19.41\end{array}$ \\
\hline $\begin{array}{l}\text { Industrial Sector } \\
\text { Primary Energy } \\
\text { Coal } \\
\text { Coking Coal } \\
\text { Steam Coal } \\
\text { Natural Gas } \\
\text { Petroleum } \\
\text { Asphalt and Road Oil } \\
\text { Distillate Fuel } \\
\text { Kerosene } \\
\text { LPG }\end{array}$ & $\begin{array}{r}1.06 \\
.68 \\
.53 \\
- \\
.53 \\
.50 \\
.86 \\
.67 \\
.71 \\
.77 \\
1.41 \\
5.08 \\
2.82 \\
.45 \\
1.10 \\
2.76\end{array}$ & $\begin{array}{l}2.99 \\
1.91 \\
1.53 \\
- \\
1.53 \\
1.34 \\
2.31 \\
1.80 \\
2.19 \\
2.37 \\
2.67 \\
7.48 \\
4.55 \\
1.92 \\
2.54 \\
6.36\end{array}$ & $\begin{array}{r}5.34 \\
4.03 \\
1.71 \\
- \\
1.71 \\
3.32 \\
5.03 \\
3.57 \\
5.49 \\
6.12 \\
5.24 \\
14.36 \\
9.91 \\
3.72 \\
7.44 \\
9.28\end{array}$ & $\begin{array}{r}7.26 \\
4.89 \\
1.90 \\
- \\
1.90 \\
4.75 \\
6.44 \\
4.94 \\
6.92 \\
7.73 \\
9.33 \\
17.61 \\
9.03 \\
4.46 \\
7.23 \\
13.83\end{array}$ & $\begin{array}{r}\mathrm{R}_{6.61} \\
\mathrm{R}_{3.82} \\
1.80 \\
- \\
1.80 \\
3.36 \\
\mathrm{R}_{5.42} \\
2.95 \\
5.96 \\
6.66 \\
9.41 \\
\mathrm{R}_{14.60} \\
9.44 \\
3.16 \\
\mathrm{R} \\
13.33 \\
13.99\end{array}$ & 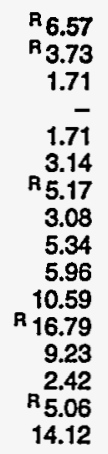 & $\begin{array}{r}R_{6.41} \\
R_{3.56} \\
1.73 \\
- \\
1.73 \\
3.23 \\
\mathrm{P}_{4.66} \\
2.32 \\
4.50 \\
5.02 \\
10.04 \\
\mathrm{R}_{19.43} \\
8.96 \\
2.49 \\
\mathrm{R}_{4.82} \\
14.44\end{array}$ & $\begin{array}{r}6.53 \\
3.66 \\
1.73 \\
- \\
1.73 \\
3.61 \\
4.47 \\
2.88 \\
4.84 \\
5.41 \\
8.27 \\
18.94 \\
8.93 \\
2.36 \\
4.49 \\
14.36\end{array}$ \\
\hline $\begin{array}{l}\text { Transportation Sector } \\
\text { Primary Energy } \\
\text { Coal } \\
\text { Petroleum } \\
\text { Aviation Gasoline } \\
\text { Distillate Fuel } \\
\text { Jet Fuel } \\
\text { LPG } \\
\text { Lubricants } \\
\text { Motor Gasoline } \\
\text { Residual Fuel }\end{array}$ & $\begin{array}{r}2.52 \\
2.52 \\
.53 \\
2.52 \\
2.17 \\
1.30 \\
.73 \\
1.41 \\
5.08 \\
2.82 \\
.27 \\
-\end{array}$ & $\begin{array}{l}4.27 \\
4.27 \\
1.53 \\
4.27 \\
3.45 \\
3.12 \\
2.03 \\
2.67 \\
7.48 \\
4.55 \\
1.56 \\
-\end{array}$ & $\begin{array}{r}9.35 \\
9.35 \\
- \\
9.35 \\
9.02 \\
7.34 \\
6.46 \\
5.24 \\
14.36 \\
9.91 \\
3.43 \\
-\end{array}$ & \begin{tabular}{r|}
8.62 \\
8.62 \\
8.62 \\
9.99 \\
7.66 \\
5.77 \\
9.33 \\
17.61 \\
9.03 \\
3.78 \\
\end{tabular} & $\begin{array}{r}\text { R9.10 } \\
{ }^{R} 9.10 \\
\text { R9.10 } \\
9.32 \\
8.75 \\
5.65 \\
9.41 \\
\text { R } 14.60 \\
9.44 \\
2.65\end{array}$ & $\begin{array}{r}\text { R } 8.86 \\
\text { R } 8.86 \\
\text { R } 8.86 \\
8.71 \\
8.37 \\
4.79 \\
10.59 \\
\text { R } 16.79 \\
9.23 \\
2.00\end{array}$ & $\begin{array}{r}\mathrm{A}_{8.59} \\
\mathrm{R}_{8.59} \\
\mathrm{P}_{8.59} \\
8.54 \\
8.01 \\
4.48 \\
10.04 \\
\mathrm{R}_{19.43} \\
8.96 \\
2.19 \\
\end{array}$ & $\begin{array}{r}8.53 \\
8.53 \\
8.53 \\
8.24 \\
7.82 \\
4.19 \\
8.27 \\
18.94 \\
8.93 \\
2.04 \\
-\end{array}$ \\
\hline $\begin{array}{l}\text { Total Energy } \\
\text { Primary Energy - Four Sectors } \\
\text { Electricity }\end{array}$ & $\begin{array}{l}2.02 \\
1.65 \\
4.17\end{array}$ & $\begin{array}{l}4.11 \\
3.27 \\
7.92\end{array}$ & $\begin{array}{r}8.02 \\
7.07 \\
11.72\end{array}$ & $\begin{array}{r}9.55 \\
7.34 \\
17.46\end{array}$ & $\begin{array}{r}10.01 \\
\text { R } 7.20 \\
18.74\end{array}$ & $\begin{array}{r}9.97 \\
86.99 \\
18.96\end{array}$ & $\begin{array}{l}\text { R } 9.76 \\
\text { R }_{6.68} \\
19.33\end{array}$ & $\begin{array}{r}9.92 \\
6.78 \\
19.44\end{array}$ \\
\hline 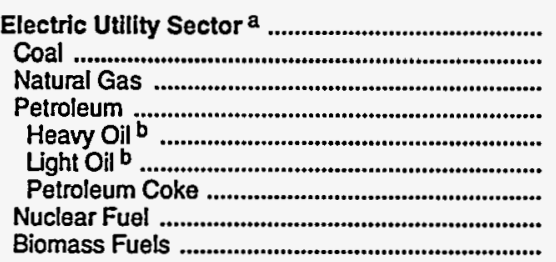 & $\begin{array}{l}.41 \\
.41 \\
.37 \\
.79 \\
.69 \\
.83 \\
- \\
- \\
-\end{array}$ & $\begin{array}{r}1.05 \\
1.07 \\
1.41 \\
1.89 \\
1.78 \\
2.22 \\
-29 \\
-\end{array}$ & $\begin{array}{r}1.48 \\
1.57 \\
3.15 \\
5.82 \\
3.82 \\
5.82 \\
. \overline{-36} \\
-\end{array}$ & $\begin{array}{r}1.56 \\
1.98 \\
4.78 \\
5.68 \\
- \\
5.68 \\
- \\
.54 \\
-\end{array}$ & $\begin{array}{r}1.33 \\
1.78 \\
3.10 \\
5.12 \\
\overline{5} \\
5.12 \\
.54 \\
-\end{array}$ & $\begin{array}{r}1.27 \\
1.78 \\
2.68 \\
4.74 \\
\overline{4} \\
4.74 \\
.52 \\
-\end{array}$ & $\begin{array}{r}1.35 \\
1.73 \\
2.86 \\
4.41 \\
-\overline{4} \\
-\overline{.51} \\
-\end{array}$ & $\begin{array}{r}1.34 \\
1.70 \\
3.52 \\
4.05 \\
\overline{4} \\
4.05 \\
. \overline{8}\end{array}$ \\
\hline Primary Energy — Five Sectors c ............................. & 1.20 & 2.47 & 4.63 & 4.78 & $R_{4.64}$ & $R_{4.40}$ & 4.36 & 4.35 \\
\hline
\end{tabular}

a There are no direct fuel costs for hydroelectric, geothermal, wind, photovoltaic, or solar thermal energy.

beavy oil includes fuel oil nos. 4, 5, and 6, and residual fuel oils. Light oil includes fuel oil nos. 1 and 2 , kerosene, and jet fuel.

c Biomass fuels are not included, except those consumed at electric utilities and those added to motor gasoline.

R=Revised data.

-No consumption, including cases where adjustments were made. See explanation of adjustments in Section 6 of Appendix A.

Sources: Data sources, estimation procedures, and assumptions are described in Appendix A. 


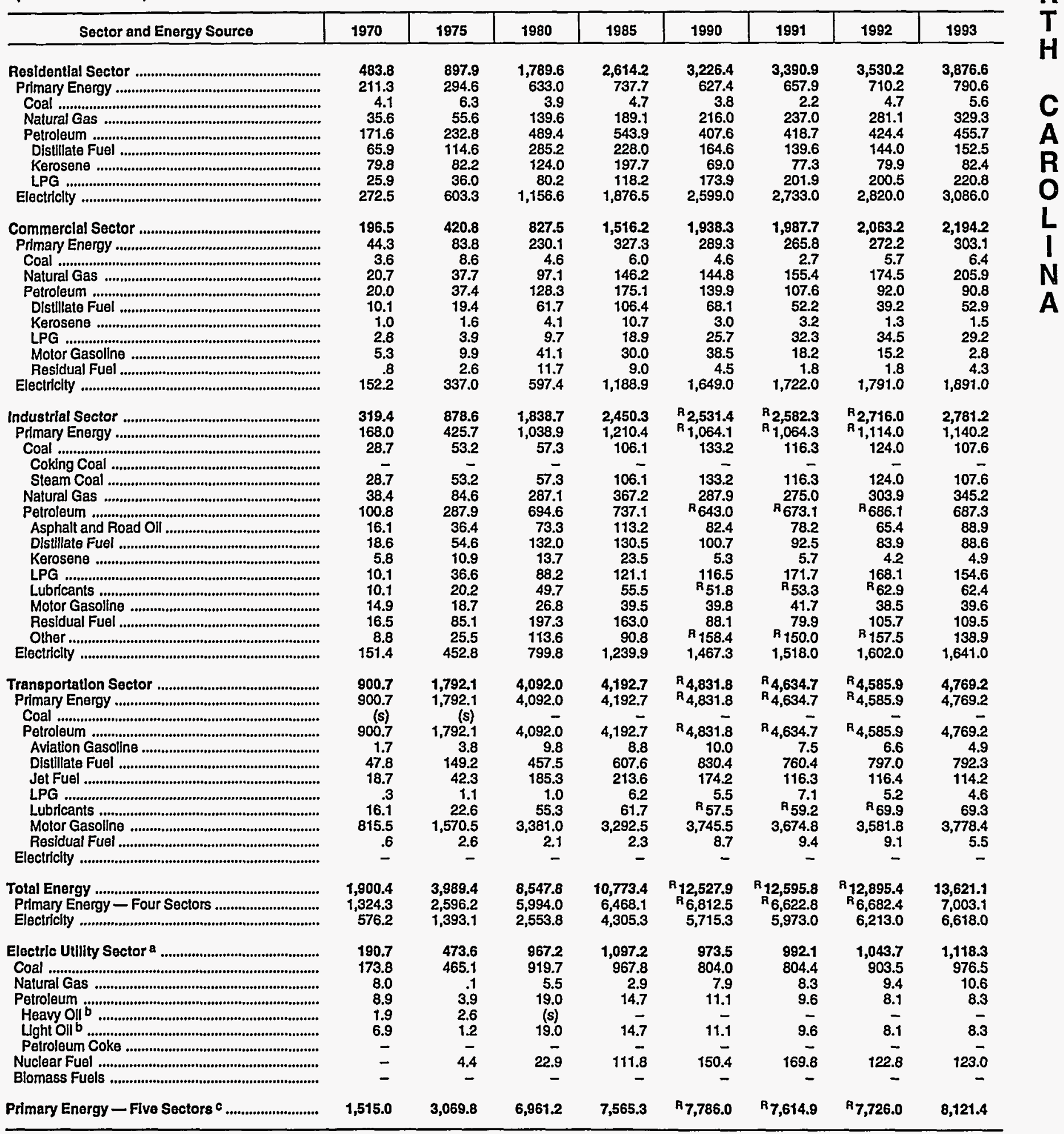

a There are no direct fuel costs for hydroelectric, geothermal, wind, photovoltalc, or solar thermal energy.

beavy oil includes fuel oil nos. 4, 5, and 6, and residual fuel oils. Light oll Includes fuel nos. 1 and 2, kerosene, and jet fuel.

- Blomass fuels are not Included, except those consumed at electric utilitiles and those added to motor gasoline.

$\mathrm{R}=$ Revised data.

$\rightarrow$ No consumption, including cases where adjustments were made. See explanation of adjustments in Section 6 of Appendix A.

$(\mathrm{s})=$ Value less than 0.05 million dollars.

Note: Totals may not equal sum of components due to independent rounding.

Sources: Data sources, estimation procedures, and assumptions are described in Appendix A. 
N Energy Price and Expenditure Estimates by Source, North Dakota

O 1970, 1975, 1980, 1985, and 1990-1993

\begin{tabular}{|c|}
\hline Energy Source \\
\hline 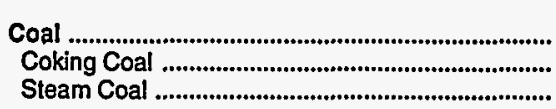 \\
\hline 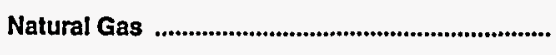 \\
\hline $\begin{array}{l}\text { Petroleum } \\
\text { Asphalt and Road Oil } \\
\text { Aviation Gasoline } \\
\text { Distillate Fuel } \\
\text { Jet Fuel }\end{array}$ \\
\hline
\end{tabular}

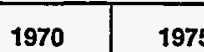

1980

1985

1990

1991

1992

1993

Prices in Dollars per Million Btu

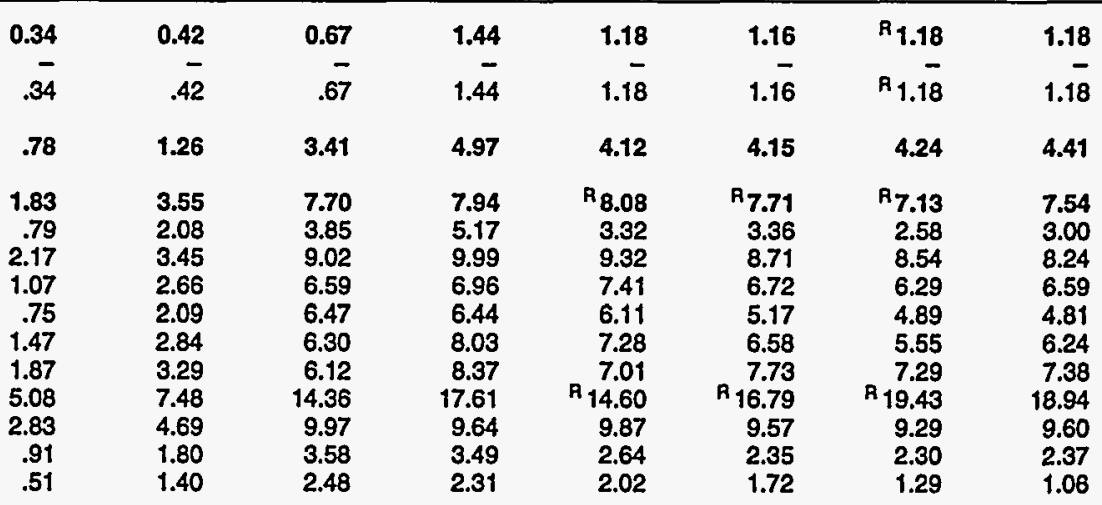

Nuclear Fuel

Biomass Fuels at Utilities

Primary Energy - Five Sectors b .

Electric Utility Fuel $\mathrm{c}$

Electricity Purchased by End Users

Total Energy

$(-. .$.

2.02

1.29

1.08

\begin{tabular}{|c|c|c|c|c|c|c|c|c|}
\hline \multirow[t]{2}{*}{ 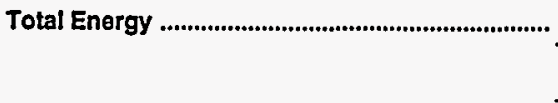 } & 1.97 & 3.47 & 7.28 & 7.08 & ${ }^{R} 6.61$ & ${ }^{R} 6.48$ & 6.12 & 6.37 \\
\hline & \multicolumn{8}{|c|}{ Expenditures in Millions of Dollars } \\
\hline $\begin{array}{l}\text { Coal } \\
\text { Coking Coal } \\
\text { Steam Coal }\end{array}$ & $\frac{19.5}{19.5}$ & $\begin{array}{l}28.8 \\
- \\
28.8\end{array}$ & $\frac{110.2}{110.2}$ & $\begin{array}{l}436.0 \\
- \\
436.0\end{array}$ & $4 \overline{-}$ & $\begin{array}{l}\mathrm{R}_{439.9} \\
\overline{-} \\
\mathrm{R}_{439.9}\end{array}$ & $\begin{array}{l}R_{472.6} \\
\overline{-} \\
R_{472.6}\end{array}$ & $\begin{array}{l}470.9 \\
470.9\end{array}$ \\
\hline 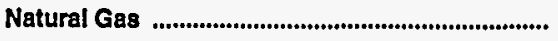 & 14.9 & 31.1 & 77.6 & 118.4 & 98.9 & 110.1 & 109.8 & 125.2 \\
\hline $\begin{array}{l}\text { Patroleum } \\
\text { Asphalt and Road Oil } \\
\text { Aviation Gasoline } \\
\text { Distillate Fuel } \\
\text { Jet Fuel }\end{array}$ & $\begin{array}{r}201.2 \\
7.3 \\
1.0 \\
30.9 \\
8.3 \\
2.0 \\
12.1 \\
5.1 \\
130.2 \\
3.2 \\
.8\end{array}$ & $\begin{array}{r}393.6 \\
14.6 \\
1.5 \\
68.8 \\
20.9 \\
1.1 \\
19.3 \\
7.2 \\
247.6 \\
10.0 \\
2.6\end{array}$ & $\begin{array}{r}938.4 \\
19.2 \\
2.9 \\
312.6 \\
59.7 \\
.5 \\
.5 \\
29.0 \\
15.5 \\
480.1 \\
13.6 \\
5.3\end{array}$ & $\begin{array}{r}887.8 \\
35.9 \\
.2 \\
303.8 \\
58.3 \\
.7 \\
16.3 \\
17.2 \\
446.7 \\
6.2 \\
2.3\end{array}$ & $\begin{array}{r}\text { R } 827.9 \\
17.9 \\
1.3 \\
291.9 \\
29.0 \\
39 \\
.2 \\
35.5 \\
\text { R } 16.1 \\
420.1 \\
4.0 \\
1.8\end{array}$ & $\begin{array}{r}{ }^{8} 827.7 \\
17.3 \\
1.2 \\
290.1 \\
27.0 \\
.4 \\
.4 \\
55.9 \\
R 16.6 \\
414.9 \\
2.8 \\
1.4\end{array}$ & $\begin{array}{r}R_{793.2} \\
25.1 \\
1.2 \\
257.6 \\
37.1 \\
.2 \\
46.5 \\
R 19.5 \\
401.9 \\
2.7 \\
1.4\end{array}$ & $\begin{array}{r}827.1 \\
18.2 \\
2.6 \\
285.3 \\
32.6 \\
.4 \\
35.9 \\
19.4 \\
427.7 \\
3.9 \\
1.2\end{array}$ \\
\hline 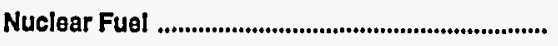 & - & - & - & - & - & - & - & - \\
\hline 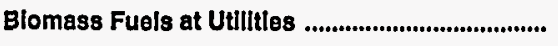 & - & - & - & - & - & - & - & - \\
\hline Primary Energy — Five Sectors b ........................... & 235.6 & 453.5 & $1,126.3$ & $1,442.2$ & $R_{1,370.7}$ & $R_{1,377.7}$ & $R_{1,375.7}$ & $1,423.2$ \\
\hline $\begin{array}{l}\text { Electric Utility Fuel c } \\
\text { Electricity Purchased by End Users }\end{array}$ & $\begin{array}{r}-12.3 \\
67.3\end{array}$ & $\begin{array}{r}-15.8 \\
108.0\end{array}$ & $\begin{array}{r}-87.9 \\
210.2\end{array}$ & $\begin{array}{r}-203.4 \\
407.5\end{array}$ & $\begin{array}{r}-198.3 \\
400.8\end{array}$ & $\begin{array}{r}-209.5 \\
415.8\end{array}$ & $\begin{array}{r}-220.9 \\
411.7\end{array}$ & $\begin{array}{r}.220 .3 \\
430.6\end{array}$ \\
\hline 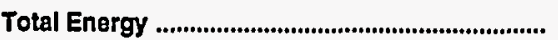 & 290.6 & 545.7 & $1,248.6$ & $1,646.2$ & $R_{1,573.2}$ & $\mathrm{~A}_{\mathbf{1 , 5 8 4 . 0}}$ & $R_{1,566.5}$ & $1,633.6$ \\
\hline
\end{tabular}

a Includes petroleum coke used at electric utilities.

b Biomass fuels are not included, except those consumed at electric utilities and those added to motor gasoline.

c There are no direct fuel costs for hydroelectric, geothermal, wind, photovoltaic, or solar thermal energy.

$R=$ Revised data.

- No consumption, including cases where adjustments were made. See explanation of adjustments in Section 6 of Appendix $A$.

Note: Expenditure totals may not equal sum of components due to independent roúnding.

Sources: Data sources, estimation procedures, and assumptions are described in Appendix A. 
(Dollars per Million Btu)

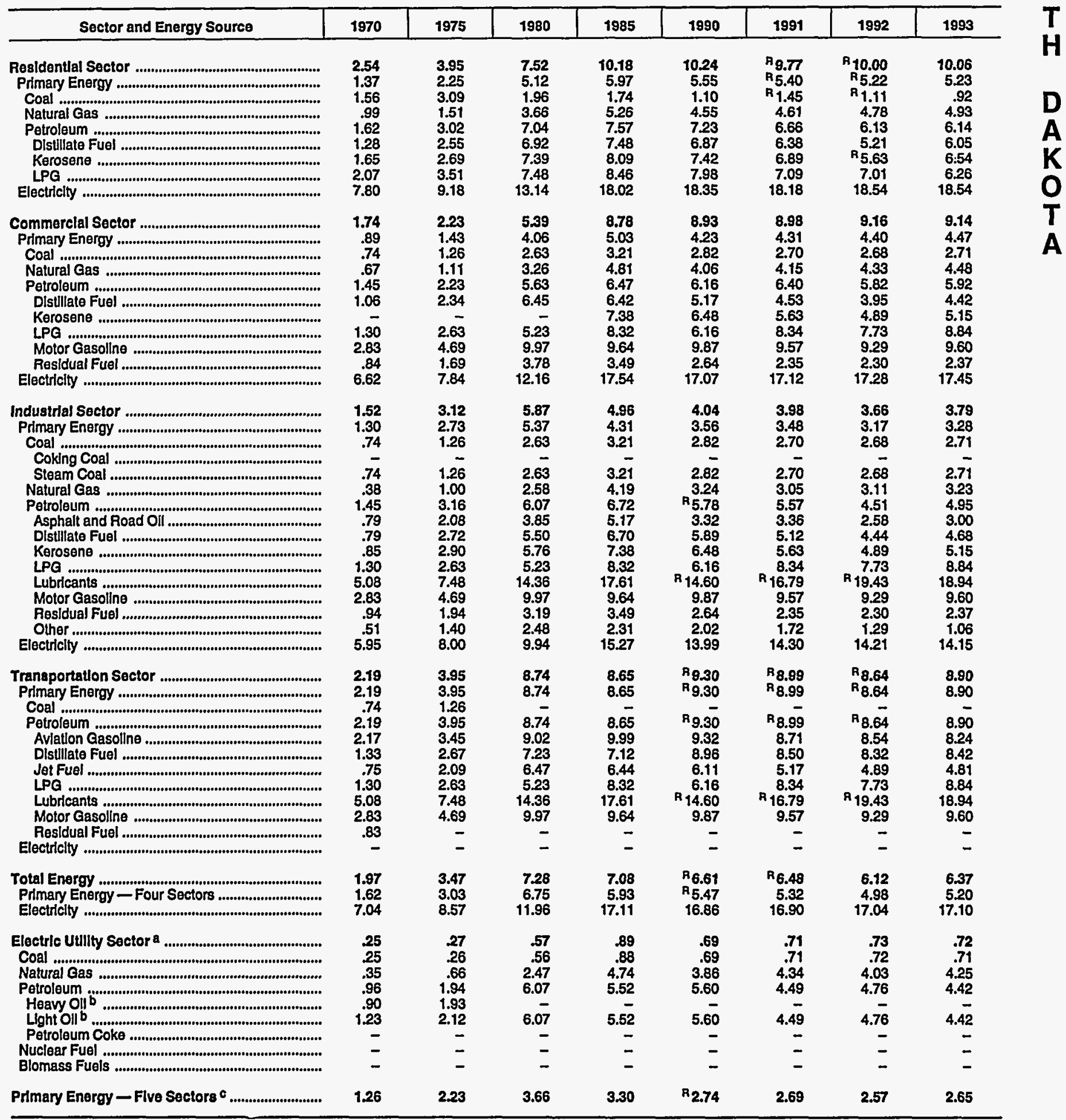

There are no direct fuel costs for hydroelectric, geothermal, wind, photovoltalc, or solar thermal energy.

b Heavy oll includes fuel oll nos, 4, 5, and 6, and residual fuel olls. Light oll includes fuel oil nos. 1 and 2 , kerosene, and jet fuel.

- Blomass fuels are not included, except those consumed at electric utilitles and those added to motor gasoline.
$R=$ Revised data.

$\rightarrow$ No consumption, Including cases where adjustments were made. See explanation of adjustments in Section 6 of Appendix A.

Sources: Data sources, estimation procedures, and assumptions are described in Appendix A. 
N Energy Expenditure Estimates by Sector, North Dakota

O 1970, 1975, 1980, 1985, and 1990-1993

$\mathbf{R}$ (Million Dollars)

\begin{tabular}{|c|c|c|c|c|c|c|c|c|}
\hline Sector and Energy Source & 1970 & 1975 & 1980 & 1985 & 1990 & 1991 & 1992 & 1993 \\
\hline 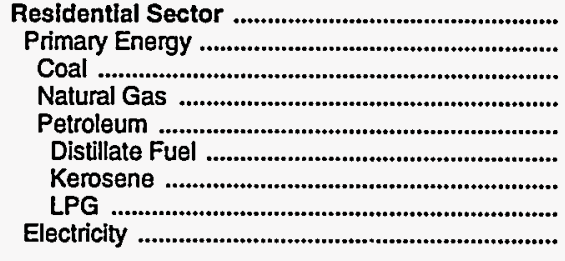 & $\begin{array}{r}66.8 \\
29.6 \\
1.2 \\
8.4 \\
20.0 \\
8.2 \\
1.8 \\
10.0 \\
37.2\end{array}$ & $\begin{array}{r}104.4 \\
44.9 \\
2.3 \\
15.4 \\
27.3 \\
11.5 \\
.3 \\
15.4 \\
59.5\end{array}$ & $\begin{array}{r}210.1 \\
100.0 \\
1.3 \\
37.1 \\
61.6 \\
47.3 \\
.2 \\
14.0 \\
110.1\end{array}$ & $\begin{array}{r}299.1 \\
114.0 \\
1.6 \\
57.9 \\
54.5 \\
48.8 \\
.6 \\
5.2 \\
185.1\end{array}$ & $\begin{array}{r}281.8 \\
96.8 \\
.7 \\
43.2 \\
52.9 \\
33.8 \\
.2 \\
18.9 \\
185.0\end{array}$ & $\begin{array}{r}R_{301.6} \\
R_{109.6} \\
R_{1.0} \\
49.8 \\
58.8 \\
33.5 \\
.3 \\
25.0 \\
192.0\end{array}$ & $\begin{array}{r}\mathrm{R}_{2} 287.3 \\
\mathrm{R}_{96.3} \\
\mathrm{R} .7 \\
48.5 \\
\mathrm{R}_{47.1} \\
19.5 \\
.2 \\
27.5 \\
191.0\end{array}$ & $\begin{array}{r}303.7 \\
100.7 \\
.7 \\
56.0 \\
44.0 \\
26.5 \\
.3 \\
17.2 \\
203.0\end{array}$ \\
\hline 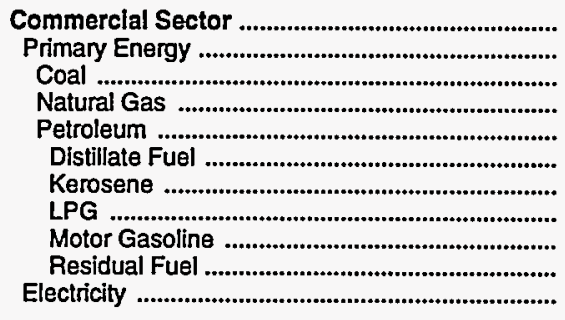 & $\begin{array}{r}27.9 \\
12.2 \\
1.0 \\
5.8 \\
5.4 \\
1.5 \\
-\overline{1.1} \\
2.2 \\
.5 \\
15.7\end{array}$ & $\begin{array}{r}49.0 \\
27.5 \\
1.7 \\
13.7 \\
12.0 \\
2.4 \\
- \\
2.0 \\
2.3 \\
5.2 \\
21.5\end{array}$ & $\begin{array}{r}127.8 \\
80.3 \\
3.2 \\
37.8 \\
39.2 \\
24.1 \\
1.7 \\
3.8 \\
9.5 \\
47.5\end{array}$ & $\begin{array}{r}202.2 \\
81.0 \\
5.4 \\
51.7 \\
23.9 \\
18.1 \\
(\mathrm{~s}) \\
.9 \\
3.5 \\
1.4 \\
121.2\end{array}$ & $\begin{array}{r}191.4 \\
57.4 \\
3.4 \\
42.9 \\
11.1 \\
4.5 \\
(s) \\
2.6 \\
3.6 \\
.4 \\
133.9\end{array}$ & $\begin{array}{r}201.6 \\
61.6 \\
3.3 \\
46.6 \\
11.7 \\
4.2 \\
(s) \\
5.2 \\
2.2 \\
.1 \\
140.0\end{array}$ & $\begin{array}{r}192.1 \\
58.1 \\
3.1 \\
44.1 \\
10.9 \\
3.6 \\
(s) \\
5.3 \\
1.8 \\
.2 \\
134.0\end{array}$ & $\begin{array}{r}200.9 \\
62.9 \\
3.6 \\
50.6 \\
8.8 \\
3.7 \\
(5) \\
4.3 \\
.5 \\
.2 \\
138.0\end{array}$ \\
\hline 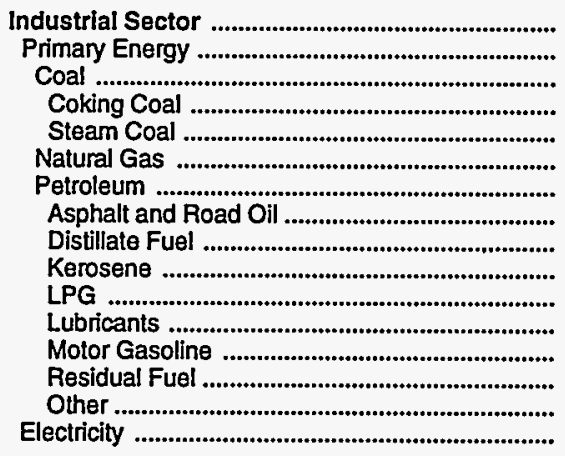 & $\begin{array}{r}77.3 \\
63.0 \\
5.4 \\
- \\
5.4 \\
.7 \\
56.9 \\
7.3 \\
10.0 \\
.3 \\
1.0 \\
.9 \\
34.4 \\
2.3 \\
.8\end{array}$ & $\begin{array}{r}143.2 \\
116.2 \\
9.4 \\
- \\
9.4 \\
1.9 \\
105.0 \\
14.6 \\
25.6 \\
.8 \\
1.8 \\
1.0 \\
54.1 \\
4.6 \\
2.6 \\
27.0\end{array}$ & $\begin{array}{r}279.1 \\
226.5 \\
20.2 \\
-\overline{2} \\
20.2 \\
2.6 \\
203.7 \\
19.2 \\
78.8 \\
.3 \\
13.0 \\
2.3 \\
80.7 \\
4.1 \\
5.3 \\
52.6\end{array}$ & $\begin{array}{r}556.5 \\
455.3 \\
228.0 \\
228.0 \\
228.0 \\
8.7 \\
218.6 \\
35.9 \\
108.4 \\
.1 \\
9.9 \\
2.5 \\
54.7 \\
4.8 \\
2.3 \\
101.1\end{array}$ & $\begin{array}{r}R_{507.6} \\
R_{425.8} \\
243.2 \\
- \\
243.2 \\
12.9 \\
R_{169.7} \\
17.9 \\
89.0 \\
(\mathrm{~s}) \\
13.7 \\
R_{2.4} \\
41.2 \\
3.7 \\
1.8 \\
81.9\end{array}$ & $\begin{array}{r}R_{505.2} \\
R_{421.5} \\
228.0 \\
-\overline{-} \\
228.0 \\
13.7 \\
R_{179.8} \\
17.3 \\
91.2 \\
.1 \\
25.3 \\
R_{2.4} \\
39.4 \\
2.7 \\
1.4 \\
83.8\end{array}$ & $\begin{array}{r}{ }^{8} 509.7 \\
\mathrm{R}_{423.0} \\
249.6 \\
- \\
249.6 \\
17.2 \\
\mathrm{R}_{156.1} \\
25.1 \\
76.0 \\
(\mathrm{~s}) \\
13.2 \\
\mathrm{P}_{2.9} \\
35.1 \\
2.5 \\
1.4 \\
86.7\end{array}$ & $\begin{array}{r}510.3 \\
420.8 \\
248.2 \\
248.2 \\
18.5 \\
154.0 \\
18.2 \\
80.3 \\
(\mathrm{~s}) \\
13.9 \\
2.8 \\
34.0 \\
3.6 \\
1.2 \\
89.6\end{array}$ \\
\hline 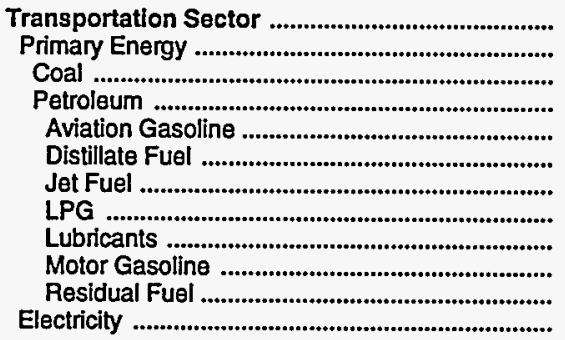 & $\begin{array}{r}118.6 \\
118.6 \\
(s) \\
118.6 \\
1.0 \\
11.1 \\
8.3 \\
(s) \\
4.2 \\
93.6 \\
.2 \\
-\end{array}$ & $\begin{array}{r}249.1 \\
249.1 \\
(s) \\
249.1 \\
1.5 \\
29.2 \\
20.9 \\
(s) \\
6.2 \\
191.2 \\
-\end{array}$ & $\begin{array}{r}631.5 \\
631.5 \\
-. \\
631.5 \\
2.9 \\
159.9 \\
59.7 \\
.2 \\
13.2 \\
395.6 \\
-\end{array}$ & $\begin{array}{r}588.4 \\
588.4 \\
- \\
588.4 \\
.2 \\
126.3 \\
58.3 \\
.3 \\
14.7 \\
388.6 \\
-\end{array}$ & $\begin{array}{r}R_{592.4} \\
R_{592.4} \\
- \\
R_{592.4} \\
1.3 \\
162.7 \\
39.0 \\
.3 \\
\text { R } 13.7 \\
375.3 \\
= \\
-\end{array}$ & $\begin{array}{r}R_{575.5} \\
\mathrm{R}_{575.5} \\
- \\
\mathrm{R}_{575.5} \\
1.2 \\
159.4 \\
27.0 \\
.5 \\
\mathrm{R} 14.1 \\
373.3 \\
- \\
-\end{array}$ & $\begin{array}{r}\mathrm{R}_{577.4} \\
\mathrm{R}_{577.4} \\
- \\
\mathrm{R}_{577.4} \\
1.2 \\
156.9 \\
37.1 \\
.5 \\
\mathrm{R}_{16.7} \\
365.0 \\
-\end{array}$ & $\begin{array}{r}618.6 \\
618.6 \\
- \\
618.6 \\
2.6 \\
173.1 \\
32.6 \\
.6 \\
16.5 \\
393.2 \\
- \\
-\end{array}$ \\
\hline 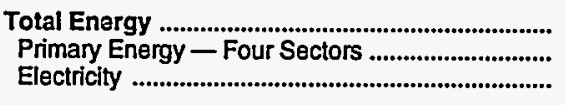 & $\begin{array}{r}290.6 \\
223.3 \\
67.3\end{array}$ & $\begin{array}{l}545.7 \\
437.7 \\
108.0\end{array}$ & $\begin{array}{r}1,248.6 \\
1,038.3 \\
210.2\end{array}$ & $\begin{array}{r}1,646.2 \\
1,238.7 \\
407.5\end{array}$ & $\begin{array}{r}R_{1}, 573.2 \\
R_{1}, 172.4 \\
400.8\end{array}$ & $\begin{array}{r}R_{1}, 584.0 \\
R_{1,168.2} \\
415.8\end{array}$ & $\begin{array}{r}R_{1,566.5} \\
1,154.7 \\
411.7\end{array}$ & $\begin{array}{r}1,633.6 \\
1,203.0 \\
430.6\end{array}$ \\
\hline 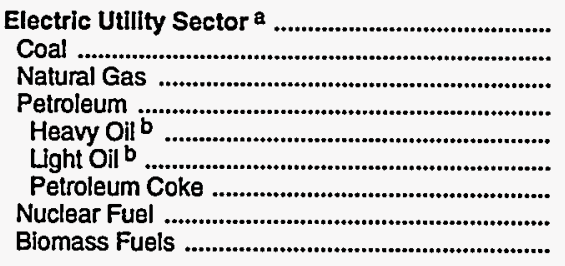 & $\begin{array}{r}12.3 \\
12.0 \\
.1 \\
.2 \\
.1 \\
(s) \\
= \\
=\end{array}$ & $\begin{array}{r}15.8 \\
15.4 \\
.1 \\
.2 \\
.2 \\
\text { (s) } \\
- \\
=\end{array}$ & $\begin{array}{r}87.9 \\
85.5 \\
(\mathrm{~s}) \\
2.4 \\
\overrightarrow{2.4} \\
- \\
-\end{array}$ & $\begin{array}{r}203.4 \\
201.1 \\
(\mathrm{~s}) \\
2.4 \\
-\overrightarrow{2.4} \\
- \\
- \\
-\end{array}$ & $\begin{array}{r}198.3 \\
196.5 \\
(s) \\
1.8 \\
\overline{1.8} \\
= \\
-\end{array}$ & $\begin{array}{r}209.5 \\
207.7 \\
(s) \\
1.8 \\
- \\
1.8 \\
- \\
- \\
-\end{array}$ & $\begin{array}{r}220.9 \\
219.3 \\
(s) \\
1.6 \\
\overline{1.6} \\
= \\
= \\
-\end{array}$ & $\begin{array}{r}220.3 \\
218.5 \\
(s) \\
1.8 \\
-\overline{1.8} \\
= \\
=\end{array}$ \\
\hline Primary Energy — Five Sectors ${ }^{c} \ldots \ldots . . . . . . . . . . . . . . . . . . .$. & 235.6 & 453.5 & $1,126.3$ & $1,442.2$ & $\mathrm{~F}_{1,370.7}$ & $\mathrm{~A}_{\mathbf{1}, \mathbf{3 7 7 . 7}}$ & $\mathrm{R}_{1,375.7}$ & 1,423.2 \\
\hline
\end{tabular}

a There are no direct fuel costs for hydroelectric, geothermal, wind, photovoltaic, or solar thermal energy.

b Heavy oil includes fuel oil nos. 4, 5, and 6, and residual fuel oils. Light oil includes fuel nos. 1 and 2, kerosene, and jet fuel.

c Biomass tuels are not included, except those consumed at electric utilities and those added to motor gasoline.

$R=$ Revised data.

-No consumption, including cases where adjustments were made. Se日 explanation of adjustments in Section 6 of Appendix A.

(s) =Value less than 0.05 million dollars.

Note: Totals may not equal sum of components due to independent rounding.

Sources: Data sources, estimation procedures, and assumptions are described in Appendix A. 
Energy Price and Expenditure Estimates by Source, Ohio $1970,1975,1980,1985$, and 1990-1993

\begin{tabular}{|c|c|c|c|c|c|c|c|c|}
\hline Energy Source & 1970 & 1975 & 1980 & 1985 & 1990 & 1991 & 1992 & 1993 \\
\hline & \multicolumn{8}{|c|}{ Prices in Dollars per Million Btu } \\
\hline 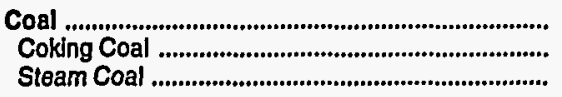 & $\begin{array}{r}0.35 \\
.42 \\
.33\end{array}$ & $\begin{array}{l}1.14 \\
1.57 \\
1.03\end{array}$ & $\begin{array}{l}1.56 \\
2.00 \\
1.47\end{array}$ & $\begin{array}{l}1.72 \\
2.05 \\
1.68\end{array}$ & $\begin{array}{l}1.54 \\
1.80 \\
1.51\end{array}$ & $\begin{array}{l}1.49 \\
1.72 \\
1.48\end{array}$ & $\begin{array}{l}1.46 \\
1.74 \\
1.44\end{array}$ & $\begin{array}{l}1.43 \\
1.68 \\
1.42\end{array}$ \\
\hline 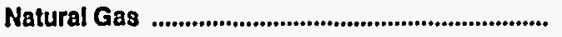 & .74 & 1.30 & 3.27 & 5.32 & 4.53 & 4.53 & 4.55 & 5.03 \\
\hline 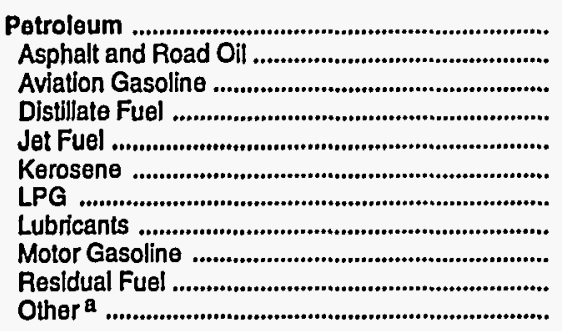 & $\begin{array}{r}2.17 \\
.74 \\
2.17 \\
1.13 \\
.74 \\
1.11 \\
1.73 \\
5.08 \\
2.93 \\
.61 \\
1.14\end{array}$ & $\begin{array}{l}3.79 \\
2.03 \\
3.45 \\
2.53 \\
2.09 \\
2.72 \\
3.73 \\
7.48 \\
4.73 \\
2.14 \\
2.36\end{array}$ & $\begin{array}{r}7.70 \\
3.66 \\
9.02 \\
6.44 \\
6.38 \\
6.87 \\
5.48 \\
14.36 \\
9.45 \\
3.34 \\
6.93\end{array}$ & $\begin{array}{r}8.63 \\
4.43 \\
9.99 \\
7.66 \\
6.04 \\
7.76 \\
9.51 \\
17.61 \\
9.15 \\
4.21 \\
7.57\end{array}$ & $\begin{array}{r}R_{8.36} \\
3.13 \\
9.32 \\
7.79 \\
5.73 \\
7.72 \\
10.42 \\
R_{14.60} \\
9.35 \\
2.60 \\
R_{6.17}\end{array}$ & $\begin{array}{r}R_{8.20} \\
2.96 \\
8.71 \\
7.39 \\
4.95 \\
7.05 \\
10.04 \\
R_{16.79} \\
9.23 \\
2.35 \\
R_{5.62}\end{array}$ & $\begin{array}{r}R_{7.91} \\
2.33 \\
8.54 \\
7.12 \\
4.64 \\
6.73 \\
9.39 \\
R_{19.43} \\
8.98 \\
2.27 \\
R_{5.38}\end{array}$ & $\begin{array}{r}8.06 \\
2.91 \\
8.24 \\
7.17 \\
4.30 \\
6.38 \\
9.60 \\
18.94 \\
9.07 \\
2.42 \\
5.04\end{array}$ \\
\hline 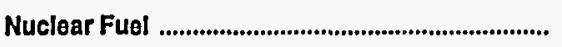 & - & - & .28 & 1.09 & 1.24 & 1.14 & 1.12 & 1.07 \\
\hline 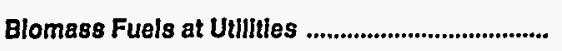 & .65 & .92 & 1.74 & .79 & $(d)$ & (d) & $(d)$ & $\left({ }^{d}\right)$ \\
\hline Primary Energy - Five Sectors ${ }^{b}$...................... & .97 & 2.00 & 4.08 & 4.81 & 4.34 & $R_{4.22}$ & $R_{4.16}$ & 4.38 \\
\hline 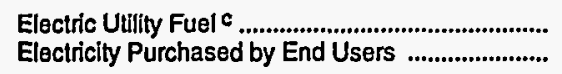 & $\begin{array}{r}.30 \\
4.68\end{array}$ & $\begin{array}{r}.98 \\
7.94\end{array}$ & $\begin{array}{r}1.50 \\
12.97\end{array}$ & $\begin{array}{r}1.70 \\
16.90\end{array}$ & $\begin{array}{r}1.50 \\
17.33\end{array}$ & $\begin{array}{r}1.45 \\
17.98\end{array}$ & $\begin{array}{r}1.40 \\
17.84\end{array}$ & $\begin{array}{r}1.39 \\
18.30\end{array}$ \\
\hline \multirow[t]{2}{*}{ 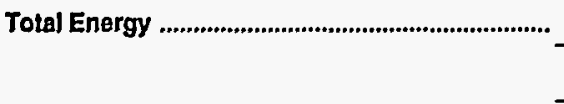 } & 1.50 & 3.05 & 6.23 & 8.25 & $R_{8.24}$ & ${ }^{A_{8.39}}$ & $R_{8.23}$ & 8.58 \\
\hline & \multicolumn{8}{|c|}{ Expenditures In Millions of Dollars } \\
\hline 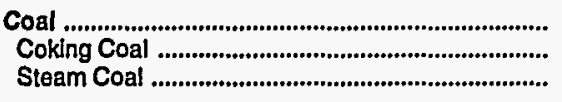 & $\begin{array}{l}556.1 \\
146.6 \\
409.5\end{array}$ & $\begin{array}{r}1,847.6 \\
519.3 \\
1,328.3\end{array}$ & $\begin{array}{r}2,390.1 \\
549.5 \\
1,840.7\end{array}$ & $\begin{array}{r}2,383.9 \\
287.8 \\
2,096.1\end{array}$ & $\begin{array}{r}2,194.3 \\
239.0 \\
1,955.3\end{array}$ & $\begin{array}{r}2,109.9 \\
170.7 \\
1,939.3\end{array}$ & $\begin{array}{r}2,073.3 \\
175.2 \\
1,898.0\end{array}$ & $\begin{array}{r}2,051.3 \\
130.3 \\
1,921.0\end{array}$ \\
\hline 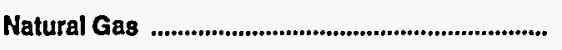 & 769.2 & $1,243.3$ & $2,887.6$ & $3,944.8$ & $3,390.6$ & $3,509.2$ & $3,705.9$ & $4,221.5$ \\
\hline 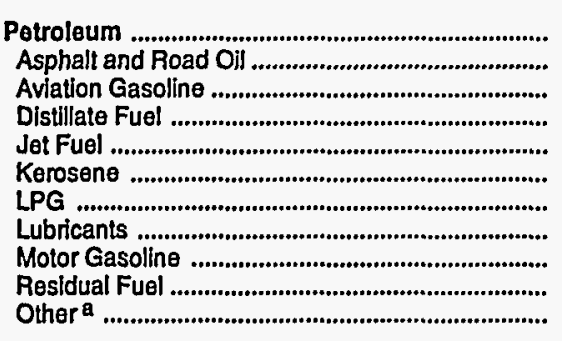 & $\begin{array}{r}2,223.4 \\
44.3 \\
7.8 \\
224.5 \\
24.4 \\
40.7 \\
56.5 \\
111.9 \\
1,637.3 \\
17.6 \\
58.5\end{array}$ & $\begin{array}{r}4,355.9 \\
118.0 \\
8.5 \\
621.6 \\
70.7 \\
55.5 \\
127.6 \\
163.8 \\
2,949.3 \\
117.1 \\
123.6\end{array}$ & $\begin{array}{r}9,908.7 \\
178.1 \\
21.5 \\
1,828.0 \\
259.2 \\
95.5 \\
883.2 \\
332.7 \\
5,623.4 \\
122.1 \\
565.1\end{array}$ & $\begin{array}{r}9,047.6 \\
186.3 \\
16.6 \\
1,605.0 \\
245.3 \\
75.2 \\
934.9 \\
371.3 \\
5,224.6 \\
33.6 \\
354.7\end{array}$ & $\begin{array}{r}{ }^{R} 8,774.5 \\
205.0 \\
11.2 \\
1,663.1 \\
343.5 \\
39.4 \\
405.0 \\
R_{346.3} \\
5,394.3 \\
20.5 \\
{ }^{2} 346.2\end{array}$ & $\begin{array}{r}R_{8,443.8} \\
176.5 \\
9.4 \\
1,534.9 \\
291.0 \\
38.8 \\
397.8 \\
R_{356.4} \\
5,326.0 \\
13.3 \\
R_{299.7}\end{array}$ & $\begin{array}{r}R_{8,442.8} \\
153.2 \\
9.7 \\
1,589.0 \\
279.0 \\
35.6 \\
490.4 \\
R_{420.5} \\
5,127.9 \\
15.4 \\
R_{322.1}\end{array}$ & $\begin{array}{r}8,831.9 \\
148.4 \\
8.6 \\
1,655.0 \\
259.1 \\
49.0 \\
507.7 \\
417.3 \\
5,468.7 \\
21.4 \\
296.7\end{array}$ \\
\hline Nuclear Fuel & - & - & 6.4 & 23.0 & 141.3 & 182.1 & 176.6 & 114.2 \\
\hline 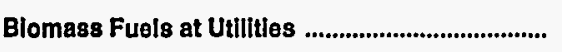 & (s) & (s) & (s) & 2.2 & $\left({ }^{d}\right)$ & $\left({ }^{d}\right)$ & (d) & (d) \\
\hline Primary Energy - Five Sectors b ........................ & $3,548.7$ & $7,446.8$ & $15,193.0$ & $15,401.3$ & $R_{14,500.7}$ & $R_{14,245.0}$ & $R_{14,398.6}$ & $15,219.0$ \\
\hline 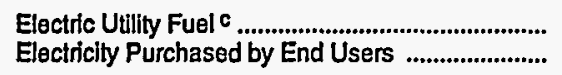 & $\begin{array}{r}-245.5 \\
1,344.2\end{array}$ & $\begin{array}{r}-1,046.9 \\
2,773.5\end{array}$ & $\begin{array}{r}-1,729.9 \\
4,904.7\end{array}$ & $\begin{array}{r}-1,919.7 \\
7,080.8\end{array}$ & $\begin{array}{r}-1,920.0 \\
8,321.6\end{array}$ & $\begin{array}{r}-1,960.3 \\
8,827.3\end{array}$ & $\begin{array}{r}-1,927.6 \\
8,717.8\end{array}$ & $\begin{array}{r}-1,887.6 \\
9,163.4\end{array}$ \\
\hline 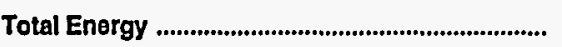 & $4,647.4$ & $9,173.4$ & $18,367.8$ & $20,562.4$ & $R_{20,902.3}$ & $R_{21,112.0}$ & $R_{21,188.9}$ & $22,494.7$ \\
\hline
\end{tabular}

a Includes petroleum coke used at electric utilities.

b Biomass fuels are not included, except those consumed at electric utilities and those added to motor gasoline.

$c$ There are no direct fuel costs for hydroelectric, geothermal, wind, photovoltaic, or solar thermal energy.

Utilities used blomass fuels at no charge or received a fee for accepting them.

$\mathbf{R}=$ Revised data.

-No consumption, including cases where adjustments were made. Seo explanation of adjustments in Section 6 of Appendix A.

$(s)=$ Value less than 0.05 million dollars.

Note: Expenditure totals may not equal sum of components due to independent rounding.

Sources: Data sources, estimation procedures, and assumptions are described in Appendix A. 
I (Dollars per Million Btu)

\begin{tabular}{|c|c|c|c|c|c|c|c|c|}
\hline Sector and Energy Source & 1970 & 1975 & 1980 & 1985 & 1990 & 1991 & 1992 & 1993 \\
\hline 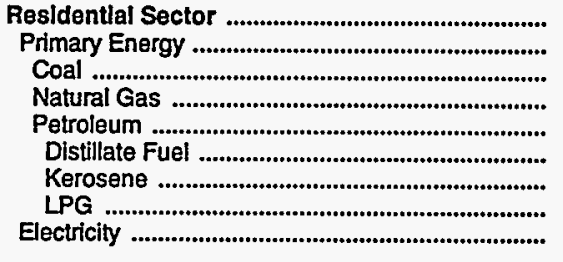 & $\begin{array}{r}1.69 \\
.98 \\
1.05 \\
.88 \\
1.54 \\
1.41 \\
1.42 \\
2.14 \\
6.99\end{array}$ & $\begin{array}{r}3.12 \\
1.74 \\
2.62 \\
1.47 \\
2.96 \\
2.51 \\
2.90 \\
4.53 \\
10.93\end{array}$ & $\begin{array}{r}6.37 \\
3.92 \\
3.07 \\
3.49 \\
6.94 \\
6.63 \\
8.07 \\
7.66 \\
16.29\end{array}$ & $\begin{array}{r}9.77 \\
6.02 \\
3.00 \\
5.79 \\
8.36 \\
7.55 \\
8.43 \\
10.09 \\
22.49\end{array}$ & $\begin{array}{r}10.21 \\
5.52 \\
2.80 \\
5.09 \\
9.16 \\
7.43 \\
8.30 \\
12.05 \\
23.58\end{array}$ & $\begin{array}{r}10.34 \\
5.41 \\
2.64 \\
5.06 \\
8.34 \\
6.89 \\
7.70 \\
10.71 \\
23.91\end{array}$ & $\begin{array}{r}9.99 \\
5.30 \\
2.55 \\
5.02 \\
7.78 \\
6.33 \\
7.07 \\
10.72 \\
24.15\end{array}$ & $\begin{array}{r}10.49 \\
5.72 \\
2.65 \\
5.50 \\
7.69 \\
6.36 \\
7.10 \\
9.89 \\
24.51\end{array}$ \\
\hline 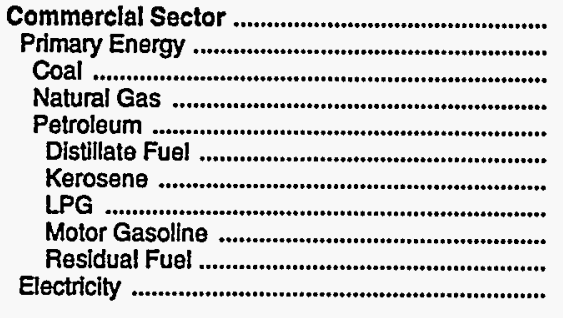 & $\begin{array}{r}1.87 \\
.76 \\
.40 \\
.75 \\
1.26 \\
1.20 \\
.84 \\
1.39 \\
2.93 \\
.69 \\
6.33\end{array}$ & $\begin{array}{r}3.55 \\
1.51 \\
1.31 \\
1.31 \\
2.74 \\
2.33 \\
2.48 \\
2.83 \\
4.73 \\
2.20 \\
10.10\end{array}$ & $\begin{array}{r}7.07 \\
3.76 \\
1.34 \\
3.26 \\
7.13 \\
6.28 \\
6.01 \\
5.34 \\
9.45 \\
3.58 \\
15.71\end{array}$ & $\begin{array}{r}10.76 \\
5.24 \\
1.49 \\
5.34 \\
6.98 \\
6.10 \\
6.93 \\
9.43 \\
9.15 \\
4.18 \\
20.91\end{array}$ & $\begin{array}{r}11.28 \\
4.61 \\
1.44 \\
4.50 \\
7.19 \\
5.49 \\
6.41 \\
9.36 \\
9.35 \\
2.54 \\
21.30\end{array}$ & $\begin{array}{r}11.53 \\
4.65 \\
1.41 \\
4.56 \\
6.74 \\
4.85 \\
5.57 \\
9.59 \\
9.23 \\
2.30 \\
21.56\end{array}$ & $\begin{array}{r}11.25 \\
4.55 \\
1.44 \\
4.56 \\
6.17 \\
4.66 \\
5.52 \\
8.88 \\
8.98 \\
2.23 \\
21.72\end{array}$ & $\begin{array}{r}11.68 \\
4.97 \\
1.42 \\
5.05 \\
6.29 \\
4.62 \\
5.21 \\
9.47 \\
9.07 \\
2.42 \\
21.78\end{array}$ \\
\hline 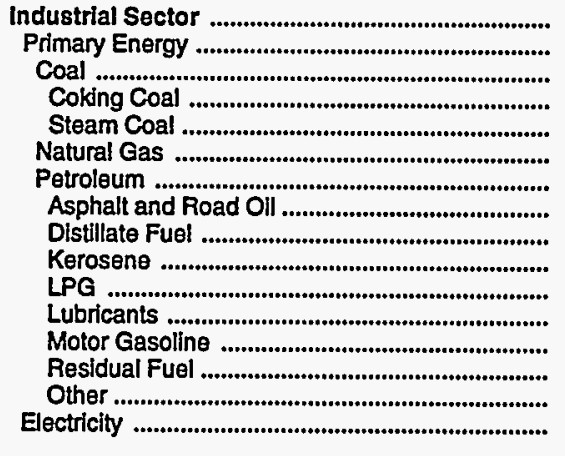 & $\begin{array}{r}.83 \\
.60 \\
.41 \\
.42 \\
.40 \\
.57 \\
1.21 \\
.74 \\
.77 \\
.84 \\
1.39 \\
5.08 \\
2.93 \\
.55 \\
1.14 \\
2.90\end{array}$ & $\begin{array}{l}2.16 \\
1.60 \\
1.47 \\
1.57 \\
1.31 \\
1.08 \\
2.59 \\
2.03 \\
2.31 \\
2.48 \\
2.83 \\
7.48 \\
4.73 \\
2.17 \\
2.36 \\
5.61\end{array}$ & $\begin{array}{r}4.43 \\
3.56 \\
1.79 \\
2.00 \\
1.34 \\
3.01 \\
5.73 \\
3.68 \\
5.45 \\
6.01 \\
5.34 \\
14.36 \\
9.45 \\
3.31 \\
6.93 \\
9.73\end{array}$ & $\begin{array}{r}6.19 \\
4.69 \\
1.78 \\
2.05 \\
1.49 \\
4.66 \\
7.94 \\
4.43 \\
6.25 \\
6.93 \\
9.43 \\
17.61 \\
9.15 \\
4.18 \\
7.57 \\
11.75\end{array}$ & $\begin{array}{r}R_{5} .68 \\
R_{3.71} \\
1.63 \\
1.80 \\
1.44 \\
3.92 \\
R_{6.01} \\
3.13 \\
5.78 \\
6.41 \\
9.36 \\
R_{14.60} \\
9.35 \\
2.54 \\
R_{6.17} \\
11.81\end{array}$ & $\begin{array}{r}\mathbf{A}_{5.85} \\
\mathbf{A}_{\mathbf{3} .71} \\
1.55 \\
1.72 \\
1.41 \\
3.92 \\
\mathrm{R}_{\mathbf{5 . 9 1}} \\
2.96 \\
\mathbf{5 . 0 3} \\
\mathbf{5 . 5 7} \\
9.59 \\
\mathrm{R}_{16.79} \\
9.23 \\
2.30 \\
\mathrm{R}_{\mathbf{5} .62} \\
\mathbf{1 2 . 3 1}\end{array}$ & $\begin{array}{r}A_{5} .95 \\
R_{3.97} \\
1.59 \\
1.74 \\
1.44 \\
4.01 \\
R_{5.94} \\
2.33 \\
4.98 \\
5.52 \\
8.88 \\
R_{19.43} \\
8.98 \\
2.23 \\
R_{5} .38 \\
12.14\end{array}$ & $\begin{array}{r}6.26 \\
4.19 \\
1.53 \\
1.68 \\
1.42 \\
4.47 \\
6.06 \\
2.91 \\
4.70 \\
5.21 \\
9.47 \\
18.94 \\
9.07 \\
2.42 \\
5.04 \\
12.45\end{array}$ \\
\hline 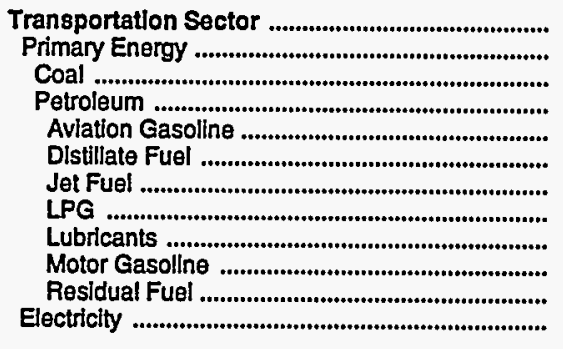 & $\begin{array}{r}2.66 \\
2.66 \\
.40 \\
2.66 \\
2.17 \\
1.26 \\
.74 \\
1.39 \\
5.08 \\
2.93 \\
.64 \\
4.05\end{array}$ & $\begin{array}{l}4.39 \\
4.38 \\
1.31 \\
4.39 \\
3.45 \\
2.76 \\
2.08 \\
2.83 \\
7.48 \\
4.73 \\
1.61 \\
7.63\end{array}$ & $\begin{array}{r}8.87 \\
8.87 \\
- \\
8.87 \\
9.02 \\
6.95 \\
6.38 \\
5.34 \\
14.36 \\
9.45 \\
3.02 \\
13.51\end{array}$ & $\begin{array}{r}8.92 \\
8.92 \\
8 . \overline{2} \\
8.92 \\
9.99 \\
8.28 \\
6.04 \\
9.43 \\
17.61 \\
9.15 \\
22 . \overline{10}\end{array}$ & $\begin{array}{r}{ }^{A} 8.96 \\
R_{8.96} \\
R_{8.96} \\
9.32 \\
8.44 \\
5.73 \\
9.36 \\
R_{14.60} \\
9.35 \\
2.70 \\
17.97\end{array}$ & $\begin{array}{r}R_{8.80} \\
R_{8.80} \\
R_{8.80} \\
8.71 \\
8.22 \\
4.95 \\
9.59 \\
R_{16.79} \\
9.23 \\
2.26 \\
17.84\end{array}$ & $\begin{array}{r}R_{8.57} \\
R_{8.56} \\
-\overline{8.56} \\
8.54 \\
8.02 \\
4.64 \\
8.88 \\
R_{19.43} \\
8.98 \\
R_{2.41} \\
18.26\end{array}$ & $\begin{array}{r}8.64 \\
8.64 \\
-\overline{8.64} \\
8.24 \\
8.10 \\
4.30 \\
9.47 \\
18.94 \\
9.07 \\
2.42 \\
18.27\end{array}$ \\
\hline 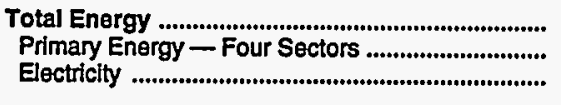 & $\begin{array}{l}1.50 \\
1.17 \\
4.68\end{array}$ & $\begin{array}{l}3.05 \\
2.41 \\
7.94\end{array}$ & $\begin{array}{r}6.23 \\
5.24 \\
12.97\end{array}$ & $\begin{array}{r}8.25 \\
6.51 \\
16.90\end{array}$ & $\begin{array}{l}{ }_{8} 8.24 \\
R_{6.12} \\
17.33\end{array}$ & $\begin{array}{r}A_{8.39} \\
R_{6.07} \\
17.98\end{array}$ & $\begin{array}{r}R_{8.23} \\
R_{5.97} \\
17.84\end{array}$ & $\begin{array}{r}8.58 \\
6.29 \\
18.30\end{array}$ \\
\hline 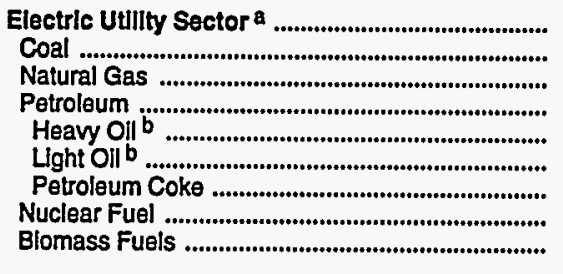 & $\begin{array}{r}.30 \\
.29 \\
.39 \\
.72 \\
.69 \\
.75 \\
- \\
.65\end{array}$ & $\begin{array}{r}.98 \\
.95 \\
1.19 \\
2.29 \\
2.18 \\
2.35 \\
- \\
-\overline{9}\end{array}$ & $\begin{array}{r}1.50 \\
1.48 \\
2.90 \\
5.11 \\
3.58 \\
5.72 \\
-\overline{8} \\
.28 \\
1.74\end{array}$ & $\begin{array}{r}1.70 \\
1.69 \\
5.09 \\
5.71 \\
4.43 \\
6.09 \\
-09 \\
1.09 \\
.79\end{array}$ & $\begin{array}{l}1.50 \\
1.52 \\
2.55 \\
4.84 \\
3.12 \\
5.40 \\
-\overline{2} \\
1.24 \\
(0)\end{array}$ & $\begin{array}{l}1.45 \\
1.48 \\
2.18 \\
4.35 \\
2.58 \\
4.91 \\
-\overline{14} \\
\left({ }^{\circ}\right)\end{array}$ & $\begin{array}{l}1.40 \\
1.44 \\
2.24 \\
4.27 \\
2.72 \\
4.51 \\
-\overline{12} \\
(10)\end{array}$ & $\begin{array}{l}1.39 \\
1.41 \\
2.86 \\
4.02 \\
2.70 \\
4.07 \\
-\overline{1.07} \\
\left({ }^{\circ}\right)\end{array}$ \\
\hline Primary Energy - Flve Sectors c .......................... & .97 & 2.00 & 4.08 & 4.81 & 4.34 & $R_{4,22}$ & $R_{4.16}$ & 4.38 \\
\hline
\end{tabular}

a There are no direct fuel costs for hydroelectric, geothermal, wind, photovoltalc, or solar thermal energy.

beavy oll includes fuel oll nos. 4, 5, and 6, and residual fuel olls. Light oil includes fuel oil nos. 1 and 2, kerosene, and jet fuel.

c Biomass fuels are not included, except those consumed at electric utilities and those added to motor gasoline.

d Utilities used blomass fuels at no charge or recelved a fee for accepting them.

$R=$ Revised data.

-No consumption, including cases where adjustments were made. See explanation of adjustments in Section 6 of Appendix A.

Sources: Data sources, estimation procedures, and assumptions are describod in Appendix A. 


\begin{tabular}{|c|c|c|c|c|c|c|c|c|}
\hline Soctor and Energy Source & 1970 & 1975 & 1980 & 1985 & 1890 & 1991 & 1992 & 1993 \\
\hline 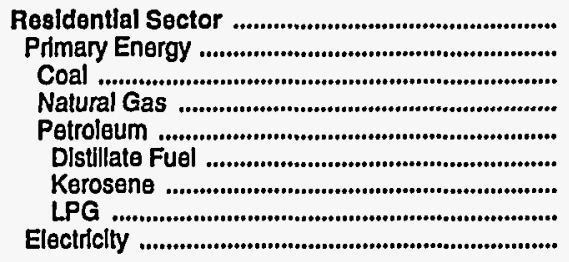 & $\begin{array}{r}1,090.9 \\
559.8 \\
13.8 \\
414.0 \\
132.0 \\
76.5 \\
24.1 \\
31.5 \\
531.1\end{array}$ & $\begin{array}{r}1,980.1 \\
940.4 \\
23.3 \\
643.4 \\
273.6 \\
157.8 \\
33.8 \\
82.0 \\
1,039.7\end{array}$ & $\begin{array}{r}3,675.4 \\
1,815.5 \\
14.0 \\
1,396.3 \\
405.3 \\
286.8 \\
46.5 \\
72.0 \\
1,859.9\end{array}$ & $\begin{array}{r}4,967.8 \\
2,363.4 \\
21.7 \\
1,978.7 \\
363.0 \\
196.7 \\
45.0 \\
121.3 \\
2,604.3\end{array}$ & $\begin{array}{r}5,085.2 \\
2,036.1 \\
15.5 \\
1,631.0 \\
389.6 \\
176.5 \\
29.4 \\
183.7 \\
3,049.0\end{array}$ & $\begin{array}{r}5,420.9 \\
2,080.9 \\
11.0 \\
1,698.7 \\
371.2 \\
169.4 \\
29.5 \\
172.3 \\
3,340.0\end{array}$ & $\begin{array}{r}5,365.2 \\
2,140.2 \\
13.0 \\
1,771.3 \\
355.9 \\
171.9 \\
29.2 \\
154.9 \\
3,225.0\end{array}$ & $\begin{array}{r}5,911.0 \\
2,403.0 \\
13.2 \\
2,022.0 \\
367.8 \\
165.7 \\
33.8 \\
168.3 \\
3,508.0\end{array}$ \\
\hline 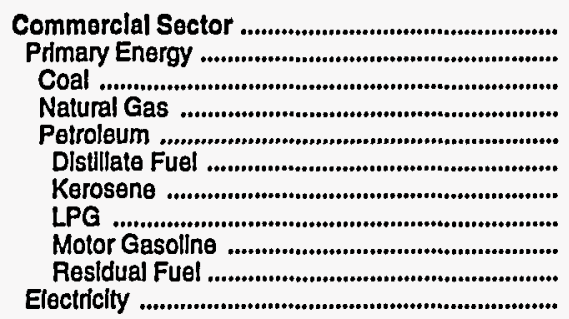 & $\begin{array}{r}545.7 \\
176.6 \\
9.5 \\
140.0 \\
27.1 \\
13.0 \\
.7 \\
3.6 \\
6.2 \\
3.6 \\
369.2\end{array}$ & $\begin{array}{r}1,023.2 \\
332.5 \\
21.5 \\
227.6 \\
83.5 \\
29.0 \\
1.5 \\
9.0 \\
23.7 \\
20.1 \\
690.7\end{array}$ & $\begin{array}{r}2,031.3 \\
781.1 \\
11.2 \\
551.1 \\
218.8 \\
94.8 \\
4.4 \\
8.9 \\
102.2 \\
8.5 \\
1,250.2\end{array}$ & $\begin{array}{r}3,041.6 \\
959.5 \\
19.7 \\
799.0 \\
140.9 \\
72.4 \\
17.3 \\
20.0 \\
29.0 \\
2.2 \\
2,082.1\end{array}$ & $\begin{array}{r}3,357.3 \\
823.7 \\
14.8 \\
671.9 \\
136.9 \\
52.8 \\
6.9 \\
25.2 \\
51.7 \\
.4 \\
2,533.6\end{array}$ & $\begin{array}{r}3,559.3 \\
850.7 \\
10.8 \\
715.9 \\
123.9 \\
45.6 \\
5.7 \\
27.2 \\
44.8 \\
.6 \\
2,708.6\end{array}$ & $\begin{array}{r}\text { R }_{3,554.4} \\
875.1 \\
13.3 \\
758.5 \\
103.2 \\
45.7 \\
2.1 \\
22.6 \\
31.7 \\
1.0 \\
\text { R }_{2,679.3}\end{array}$ & $\begin{array}{r}3,769.0 \\
963.6 \\
13.0 \\
859.8 \\
90.8 \\
37.2 \\
5.9 \\
28.4 \\
18.7 \\
.4 \\
2,805.4\end{array}$ \\
\hline 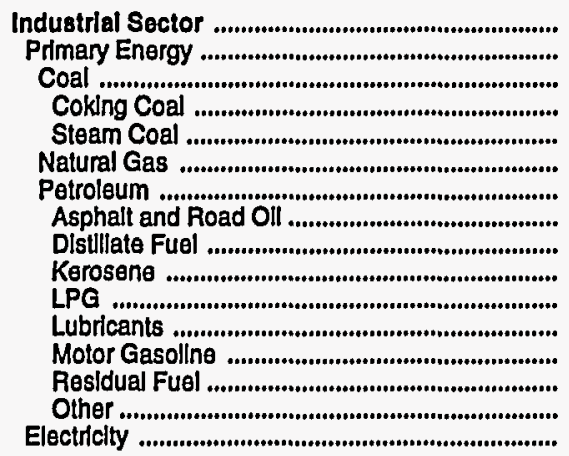 & $\begin{array}{r}1,252.9 \\
809.5 \\
301.9 \\
146.6 \\
155.3 \\
208.6 \\
301.1 \\
44.3 \\
50.5 \\
15.9 \\
20.7 \\
73.7 \\
29.7 \\
7.9 \\
58.5 \\
443.4\end{array}$ & $\begin{array}{r}2,870.3 \\
1,828.4 \\
815.2 \\
519.3 \\
296.0 \\
366.0 \\
647.2 \\
118.0 \\
149.7 \\
20.2 \\
34.7 \\
90.2 \\
37.7 \\
73.0 \\
123.6 \\
1,042.0\end{array}$ & $\begin{array}{r}5,786.1 \\
3,993.6 \\
723.6 \\
549.5 \\
174.1 \\
926.5 \\
2,343.4 \\
178.1 \\
396.8 \\
44.5 \\
797.9 \\
208.6 \\
57.3 \\
95.1 \\
565.1 \\
1,792.6\end{array}$ & $\begin{array}{r}5,917.6 \\
3,526.4 \\
473.5 \\
287.8 \\
185.7 \\
1,163.5 \\
1,889.4 \\
186.3 \\
243.0 \\
12.9 \\
780.7 \\
232.8 \\
51.6 \\
27.5 \\
354.7 \\
2,391.2\end{array}$ & $\begin{array}{r}R_{5,419.7} \\
R_{2,683.1} \\
405.4 \\
239.0 \\
166.4 \\
1,084.4 \\
R_{1,193.3} \\
205.0 \\
173.1 \\
3.2 \\
183.8 \\
R_{217.1} \\
47.5 \\
17.4 \\
R_{346.2} \\
2,736.6\end{array}$ & $\begin{array}{r}R_{5,301.7} \\
R_{2,525.4} \\
336.4 \\
170.7 \\
165.7 \\
1,087.4 \\
R_{1,101.6} \\
176.5 \\
153.7 \\
3.6 \\
188.2 \\
R_{223.5} \\
46.7 \\
9.9 \\
R_{299.7} \\
2,776.2\end{array}$ & $\begin{array}{r}R_{5,672.9} \\
R_{2,862.1} \\
315.1 \\
175.2 \\
139.9 \\
1,169.3 \\
R_{1,377.7} \\
153.2 \\
185.3 \\
4.3 \\
304.8 \\
R_{263.6} \\
131.8 \\
12.5 \\
R_{322.1} \\
2,810.8\end{array}$ & $\begin{array}{r}5,722.8 \\
2,875.4 \\
273.0 \\
130.3 \\
142.6 \\
1,331.7 \\
1,270.7 \\
148.4 \\
178.3 \\
9.2 \\
302.5 \\
261.6 \\
53.5 \\
20.4 \\
296.7 \\
2,847.4\end{array}$ \\
\hline 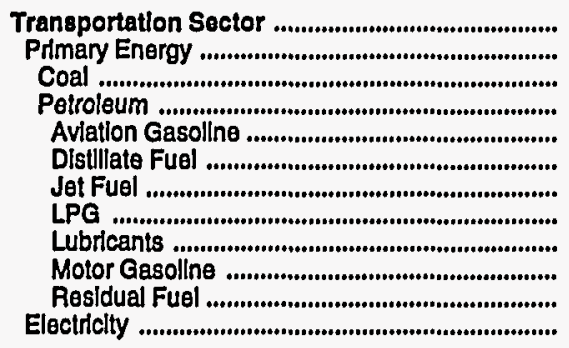 & $\begin{array}{r}1,757.9 \\
1,757.3 \\
.4 \\
1,756.8 \\
7.8 \\
81.2 \\
24.4 \\
.7 \\
.7 \\
38.3 \\
1,601.5 \\
3.1 \\
.6\end{array}$ & $\begin{array}{r}3,299.8 \\
3,298.6 \\
.1 \\
3,298.5 \\
8.5 \\
251.5 \\
69.2 \\
1.9 \\
73.6 \\
2,887.8 \\
6.0 \\
1.2\end{array}$ & $\begin{array}{r}6,874.8 \\
6,872.9 \\
- \\
6,872.9 \\
21.5 \\
994.9 \\
259.2 \\
4.4 \\
124.1 \\
5,463.9 \\
4.8 \\
2.0\end{array}$ & $\begin{array}{r}6,635.5 \\
6,632.3 \\
6,632 . \overline{3} \\
16.6 \\
1,074.9 \\
245.3 \\
12.9 \\
138.5 \\
5,144.0 \\
\overline{3} \\
3.2\end{array}$ & $\begin{array}{r}R_{7,040.2} \\
R_{7,037.8} \\
R_{7,037.8} \\
11.2 \\
1,246.5 \\
343.5 \\
12.2 \\
R 129.2 \\
5,295.1 \\
.1 \\
2.4\end{array}$ & $\begin{array}{r}R_{6,830.1} \\
R_{6,827.7} \\
- \\
R_{6,827.7} \\
9.4 \\
1,149.6 \\
291.0 \\
10.1 \\
R_{133.0} \\
5,234.4 \\
.1 \\
2.4\end{array}$ & $\begin{array}{r}R_{6,596.4} \\
R_{6,593.7} \\
- \\
R_{6,593.7} \\
9.7 \\
1,174.9 \\
279.0 \\
8.1 \\
A_{156.9} \\
4,964.3 \\
.8 \\
R_{2.7}\end{array}$ & $\begin{array}{r}7,092.0 \\
7,089.4 \\
- \\
7,089.4 \\
8.6 \\
1,260.9 \\
259.1 \\
8.4 \\
155.7 \\
5,396.4 \\
.2 \\
2.6\end{array}$ \\
\hline 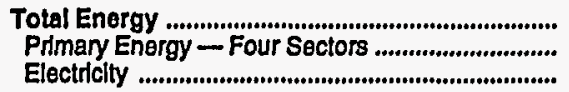 & $\begin{array}{l}4,647.4 \\
3,303.2 \\
1,344.2\end{array}$ & $\begin{array}{l}9,173.4 \\
6,399.9 \\
2,773.5\end{array}$ & $\begin{array}{r}18,367.8 \\
13,463.1 \\
4,904.7\end{array}$ & $\begin{array}{r}20,562.4 \\
13,481.6 \\
7,080.8\end{array}$ & $\begin{array}{r}R_{20,902.3} \\
R_{12,580.7} \\
8,321.6\end{array}$ & $\begin{array}{r}\text { P }_{21,112.0} \\
\text { F }_{12,284.7} \\
8,827.3\end{array}$ & $\begin{array}{r}\mathrm{F}_{21,188.9} \\
\mathrm{~F}_{12,471.1} \\
8,717.8\end{array}$ & $\begin{array}{r}22,494.7 \\
13,331.3 \\
9,163.4\end{array}$ \\
\hline 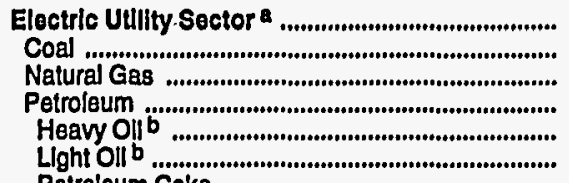 & $\begin{array}{r}245.5 \\
230.5 \\
8.6 \\
6.4 \\
3.0 \\
3.4\end{array}$ & $\begin{array}{r}1,046.9 \\
987.4 \\
6.3 \\
53.2 \\
18.0 \\
35.2\end{array}$ & $\begin{array}{r}1,729.9 \\
1,641.4 \\
13.7 \\
68.3 \\
13.6 \\
54.7\end{array}$ & $\begin{array}{r}1,919.7 \\
1,869.0 \\
3.6 \\
22.0 \\
3.9 \\
18.0\end{array}$ & $\begin{array}{r}1,920.0 \\
1,758.7 \\
3.2 \\
16.9 \\
2.7 \\
14.2\end{array}$ & $\begin{array}{r}1,960.3 \\
1,751.8 \\
7.1 \\
19.4 \\
2.7 \\
16.7\end{array}$ & $\begin{array}{r}1,927.6 \\
1,731.8 \\
6.8 \\
12.3 \\
1.1 \\
11.2\end{array}$ & $\begin{array}{r}1,887.6 \\
1,752.1 \\
8.1 \\
13.3 \\
.3 \\
12.9\end{array}$ \\
\hline 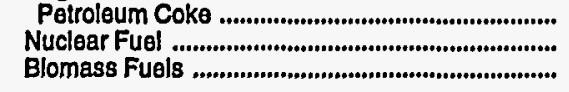 & $\overline{-}$ & $\overline{-}$ & $\begin{array}{l}\overline{6.4} \\
(\mathrm{~s})\end{array}$ & $\begin{array}{r}23 . \overline{0} \\
2.2\end{array}$ & $(\bar{d}) \overline{3}$ & (d) & $\left(\begin{array}{c}176.6 \\
\text { d) }\end{array}\right.$ & $(114 . \bar{d}$ \\
\hline Primary Energy - Flve Sectors c ............................ & $3,548.7$ & $7,446.8$ & $15,193.0$ & $15,401.3$ & $R_{14,500.7}$ & $R_{14,245.0}$ & $R_{14,398.6}$ & $15,219.0$ \\
\hline
\end{tabular}

a There are no direct fuel costs for hydroelectric, geothemal, wind, photovoltalc, or solar thermal energy.

Heavy oll Includes fuel oll nos. 4, 5, and 6, and residual fuel olls. Light oll Includes fuel nos. 1 and 2, kerosene, and let fuel.

c Blomass fuels are not Included, except those consumed at electric utilitiles and those added to motor gasoline.

Utllites used blomass fuels at no charge or received a fee for accepting

R=Revised data.

- No consumptlon, including cases where adjustments were made. See explanation of adjustments in Section 6 of Appendix A.

(s)=Value less than 0.05 million dollars.

Note: Totals may not equal sum of components due to Independent rounding.

Sources: Data sources, estimation procedures, and assumptions are described in Appendlx A. 
O Energy Price and Expenditure Estimates by Source, Oklahoma $1970,1975,1980,1985$, and 1990-1993

\begin{tabular}{|c|c|c|c|c|c|c|c|c|}
\hline Energy Source & 1970 & 1975 & 1980 & 1985 & 1990 & 1991 & 1992 & 1993 \\
\hline & \multicolumn{8}{|c|}{ Prices in Dollars per Million Btu } \\
\hline 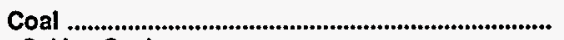 & 0.57 & 0.96 & 1.25 & 1.69 & 1.40 & 1.32 & 1.24 & 1.25 \\
\hline 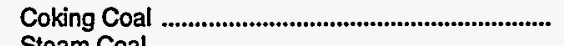 & & $\overline{0}$ & $\overline{25}$ & $\overline{1}$ & $\overline{1}$ & $\overline{130}$ & $\overline{1}$ & $\overline{125}$ \\
\hline 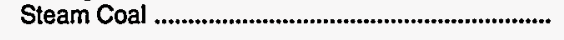 & .57 & .96 & 1.25 & 1.69 & 1.40 & 1.32 & 1.24 & 1.25 \\
\hline Natural Gas & .35 & .75 & 1.96 & 3.41 & 2.80 & 2.78 & 3.02 & 3.17 \\
\hline $\begin{array}{l}\text { Petroleum } \\
\text { Asphalt and Road Oil } \\
\text { Aviation Gasoline } \\
\text { Distillate Fuel } \\
\text { Jet Fuel }\end{array}$ & $\begin{array}{r}1.97 \\
.68 \\
2.17 \\
.90 \\
.72 \\
.66 \\
1.42 \\
5.08 \\
2.82 \\
.50 \\
.58\end{array}$ & $\begin{array}{l}3.52 \\
1.86 \\
3.45 \\
2.36 \\
2.01 \\
2.41 \\
2.91 \\
7.48 \\
4.52 \\
1.58 \\
1.45\end{array}$ & $\begin{array}{r}7.97 \\
3.60 \\
9.02 \\
6.77 \\
6.34 \\
6.52 \\
6.03 \\
14.36 \\
9.79 \\
3.23 \\
2.90\end{array}$ & $\begin{array}{r}7.43 \\
4.20 \\
9.99 \\
5.54 \\
5.87 \\
4.07 \\
7.21 \\
17.61 \\
8.76 \\
3.41 \\
3.36\end{array}$ & $\begin{array}{r}\text { R } 7.86 \\
3.14 \\
9.32 \\
7.49 \\
5.93 \\
7.46 \\
6.50 \\
\text { R } 14.60 \\
9.00 \\
2.46 \\
\text { R } 3.30\end{array}$ & $\begin{array}{r}\text { R } 7.41 \\
3.40 \\
8.71 \\
6.93 \\
4.73 \\
6.75 \\
6.46 \\
16.79 \\
8.72 \\
1.77 \\
R 2.92\end{array}$ & $\begin{array}{r}\mathrm{R} 7.01 \\
2.47 \\
8.54 \\
6.69 \\
4.42 \\
6.50 \\
5.38 \\
\mathrm{R}_{19.43} \\
8.37 \\
2.29 \\
\mathrm{R}_{2.40}\end{array}$ & $\begin{array}{r}7.20 \\
2.74 \\
8.24 \\
6.75 \\
4.12 \\
6.06 \\
7.17 \\
18.94 \\
8.47 \\
2.42 \\
2.06\end{array}$ \\
\hline 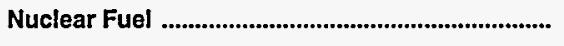 & - & - & - & - & - & - & - & - \\
\hline Biomass Fuels at Utilities & - & - & - & - & - & - & - & - \\
\hline Primary Energy －Five Sectors ${ }^{b}$ & 1.03 & 1.90 & 4.04 & 4.56 & F $_{4.21}$ & $P_{4.03}$ & $\mathrm{~F}_{4.03}$ & 4.06 \\
\hline 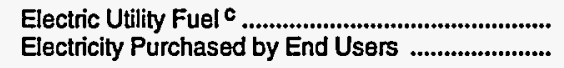 & $\begin{array}{r}.19 \\
5.76\end{array}$ & $\begin{array}{r}.61 \\
6.64\end{array}$ & $\begin{array}{r}1.63 \\
11.80\end{array}$ & $\begin{array}{r}2.30 \\
17.23\end{array}$ & $\begin{array}{r}2.05 \\
16.08\end{array}$ & $\begin{array}{r}1.92 \\
16.99\end{array}$ & $\begin{array}{r}1.88 \\
17.04\end{array}$ & $\begin{array}{r}1.88 \\
17.51\end{array}$ \\
\hline \multirow[t]{2}{*}{ 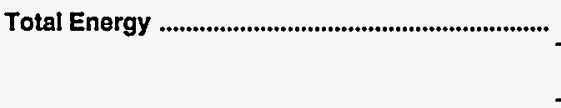 } & 1.83 & 3.06 & 6.43 & 7.58 & R7.39 & ${ }^{\text {B } 7.25}$ & ${ }^{\mathrm{R}} 7.15$ & 7.31 \\
\hline & \multicolumn{8}{|c|}{ Expenditures in Millions of Dollars } \\
\hline 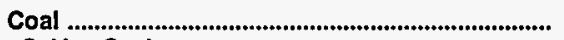 & 0.1 & 0.5 & 132.5 & 400.2 & 388.1 & 384.4 & 381.7 & 414.5 \\
\hline 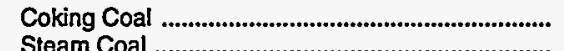 & - & - & - & - & - & - & - & - \\
\hline 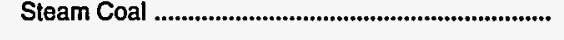 & .1 & .5 & 132.5 & 400.2 & 388.1 & 384.4 & 381.7 & 414.5 \\
\hline 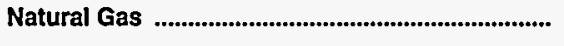 & 152.7 & 392.2 & $1,209.5$ & $1,633.3$ & $1,307.5$ & $1,262.6$ & $1,281.6$ & $1,429.6$ \\
\hline Petroleum . & 636.9 & $1,329.1$ & $3,202.7$ & $3,206.5$ & $R_{3,090.6}$ & $\mathrm{R}_{2,972.2}$ & $\mathrm{R}_{3,024.5}$ & $3,053.1$ \\
\hline 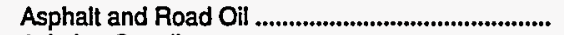 & 20.7 & 69.9 & 115.1 & 111.7 & 73.1 & 77.5 & 47.9 & 67.7 \\
\hline 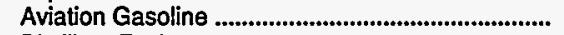 & 4.9 & 5.4 & 14.9 & 11.0 & 6.9 & 4.9 & 5.3 & 4.3 \\
\hline 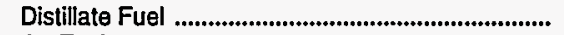 & 28.7 & 128.1 & 478.2 & 592.0 & 669.9 & 572.5 & 634.4 & 643.7 \\
\hline 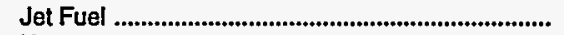 & 17.2 & 43.2 & 170.5 & 190.6 & 259.8 & 279.1 & 321.7 & 207.9 \\
\hline 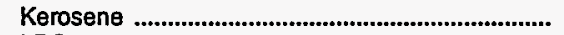 & 4.1 & 4.5 & 12.6 & 2.6 & 1.6 & 1.2 & 1.1 & .9 \\
\hline LPG & 50.3 & 99.1 & 196.2 & 204.8 & 75.6 & 111.7 & & 142.5 \\
\hline 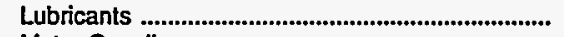 & 19.2 & 36.8 & 118.1 & 131.8 & $R_{122.9}$ & $\mathrm{~A}_{126.5}$ & $R_{149.3}$ & 148.2 \\
\hline 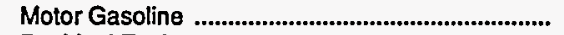 & 481.9 & 913.4 & $2,038.2$ & $1,940.7$ & $1,851.0$ & $1,778.1$ & $1,754,2$ & $1,814.7$ \\
\hline $\begin{array}{l}\text { Residual Fuel } \\
\text { Other }\end{array}$ & 2.2 & 5.7 & 13.1 & 2.4 & $\begin{array}{r}7.6 \\
\text { R2. }\end{array}$ & $\begin{array}{r}2.0 \\
R_{187}\end{array}$ & 5.9 & 6.9 \\
\hline (n) & & 20.1 & 40 & 0.0 & & 80.0 & . 2 & 10.0 \\
\hline 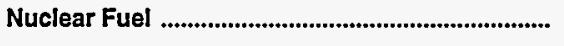 & - & - & - & - & - & - & - & - \\
\hline 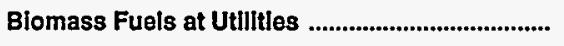 & - & - & - & - & - & - & - & - \\
\hline Primary Energy — Five Sectors ${ }^{b}$............................. & 789.7 & $1,721.8$ & $4,544.7$ & $5,240.0$ & $R_{4,786.2}$ & $\mathrm{~A}_{4,619.2}$ & $R_{4,687.8}$ & $4,897.2$ \\
\hline 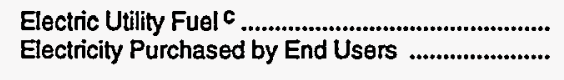 & $\begin{array}{r}-46.8 \\
311.7\end{array}$ & $\begin{array}{r}-190.0 \\
509.6\end{array}$ & $\begin{array}{r}-727.3 \\
1,211.3\end{array}$ & $\begin{array}{r}-988.5 \\
2,141.2\end{array}$ & $\begin{array}{r}.904 .7 \\
2,316.2\end{array}$ & $\begin{array}{r}-862.2 \\
2,266.6\end{array}$ & $R_{2,207.6}^{-835.3}$ & $\begin{array}{r}-873.1 \\
2,403.3\end{array}$ \\
\hline 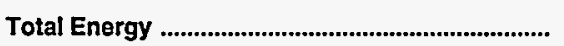 & $1,054.5$ & $2,041.4$ & $5,028.7$ & $6,392.8$ & ${ }^{R_{6,197.7}}$ & $R_{6,023.6}$ & $R_{6,060.1}$ & $6,427.4$ \\
\hline
\end{tabular}

a Includes petroleum coke used at electric utilities.

b Biomass fuels are not included, except those consumed at electric utilities and those added to motor gasoline.

c There are no direct fuel costs for hydroelectric, geothermal, wind, photovoltaic, or solar thermal energy.

$\mathrm{R}=$ Revised data.
-No consumption, including cases where adjustments were made. Seo explanation of adjustments in Section 6 of Appendix A.

Note: Expenditure totals may not equal sum of components due to independent rounding.

Sources: Data sources, estimation procedures, and assumptions are described in Appendix A. 


\begin{tabular}{|c|c|c|c|c|c|c|c|c|}
\hline Sector and Energy Source & 1970 & 1975 & 1980 & 1985 & 1990 & 1991 & 1992 & 1993 \\
\hline 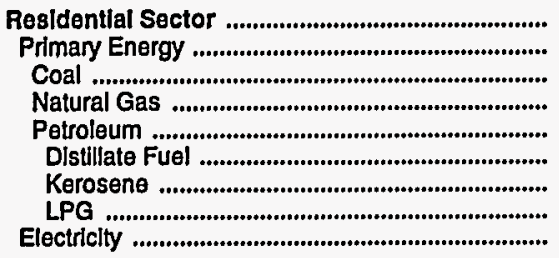 & $\begin{array}{r}2.24 \\
.99 \\
.90 \\
.81 \\
1.60 \\
.89 \\
1.41 \\
1.60 \\
7.42\end{array}$ & $\begin{array}{l}3.19 \\
1.62 \\
1.58 \\
1.22 \\
3.12 \\
2.22 \\
2.88 \\
3.13 \\
8.22\end{array}$ & $\begin{array}{r}6.40 \\
2.84 \\
2.54 \\
2.46 \\
7.29 \\
6.60 \\
7.95 \\
7.29 \\
13.50\end{array}$ & $\begin{array}{r}10.09 \\
4.76 \\
2.83 \\
4.49 \\
7.47 \\
3.73 \\
4.57 \\
7.78 \\
19.37\end{array}$ & $\begin{array}{r}11.38 \\
4.94 \\
2.41 \\
4.71 \\
8.28 \\
7.37 \\
9.02 \\
8.27 \\
19.29\end{array}$ & $\begin{array}{r}11.31 \\
4.84 \\
2.36 \\
4.66 \\
7.36 \\
6.84 \\
8.38 \\
7.35 \\
20.62\end{array}$ & $\begin{array}{r}11.51 \\
5.04 \\
2.43 \\
4.85 \\
8.09 \\
6.25 \\
7.65 \\
8.11 \\
21.01\end{array}$ & $\begin{array}{r}11.26 \\
5.06 \\
2.16 \\
4.84 \\
8.80 \\
6.28 \\
7.69 \\
8.82 \\
20.94\end{array}$ \\
\hline 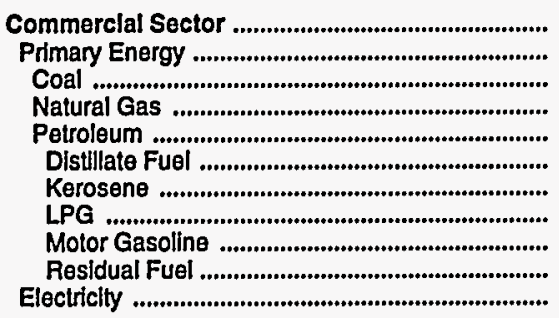 & $\begin{array}{r}1.68 \\
.61 \\
.45 \\
.51 \\
1.18 \\
.82 \\
.62 \\
1.12 \\
2.82 \\
.47 \\
5.50\end{array}$ & $\begin{array}{r}2.96 \\
1.24 \\
.94 \\
.94 \\
2.58 \\
2.12 \\
2.37 \\
2.56 \\
4.52 \\
1.46 \\
6.73\end{array}$ & $\begin{array}{r}6.12 \\
2.74 \\
1.39 \\
2.30 \\
7.20 \\
6.31 \\
6.42 \\
5.72 \\
9.79 \\
3.42 \\
11.91\end{array}$ & $\begin{array}{r}10.56 \\
4.46 \\
1.79 \\
4.32 \\
5.24 \\
3.20 \\
3.89 \\
7.01 \\
8.76 \\
-\overline{2} \\
18.02\end{array}$ & $\begin{array}{r}10.57 \\
4.20 \\
1.33 \\
3.84 \\
6.29 \\
5.48 \\
6.89 \\
5.33 \\
9.00 \\
2.38 \\
16.65\end{array}$ & $\begin{array}{r}10.65 \\
4.07 \\
1.32 \\
3.86 \\
5.61 \\
4.79 \\
5.99 \\
6.10 \\
8.72 \\
1.66 \\
17.59\end{array}$ & $\begin{array}{r}11.15 \\
4.25 \\
1.39 \\
4.14 \\
5.26 \\
4.59 \\
5.90 \\
4.47 \\
8.37 \\
2.29 \\
17.68\end{array}$ & $\begin{array}{r}11.18 \\
4.39 \\
1.41 \\
4.33 \\
5.35 \\
4.46 \\
5.46 \\
6.67 \\
8.47 \\
18.04\end{array}$ \\
\hline 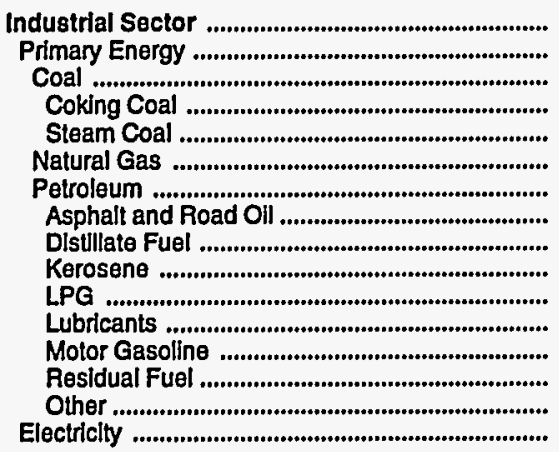 & $\begin{array}{r}.76 \\
.53 \\
- \\
- \\
- \\
.25 \\
.82 \\
.68 \\
.54 \\
.62 \\
1.12 \\
5.08 \\
2.82 \\
.53 \\
.58 \\
3.13\end{array}$ & $\begin{array}{r}1.70 \\
1.39 \\
.94 \\
\overline{-} \\
.94 \\
.72 \\
2.07 \\
1.86 \\
2.09 \\
2.37 \\
2.56 \\
7.48 \\
4.52 \\
1.65 \\
1.45 \\
4.29\end{array}$ & $\begin{array}{r}3.86 \\
3.23 \\
1.39 \\
- \\
1.39 \\
2.11 \\
4.92 \\
3.60 \\
5.68 \\
6.42 \\
5.72 \\
14.36 \\
9.79 \\
3.22 \\
2.90 \\
9.31\end{array}$ & $\begin{array}{r}4.90 \\
3.80 \\
1.79 \\
- \\
1.79 \\
3.23 \\
5.00 \\
4.20 \\
3.34 \\
3.89 \\
7.01 \\
17.61 \\
8.76 \\
3.39 \\
3.36 \\
13.33\end{array}$ & $\begin{array}{r}R_{3.59} \\
R_{2} .53 \\
1.33 \\
- \\
1.33 \\
1.71 \\
R_{5.16} \\
3.14 \\
5.92 \\
6.89 \\
5.33 \\
R_{14.60} \\
9.00 \\
2.38 \\
R_{3.30} \\
10.64\end{array}$ & $\begin{array}{r}\mathrm{A}_{3.74} \\
\mathrm{R}_{2.61} \\
1.32 \\
\overline{1} \\
1.32 \\
1.67 \\
\text { R }_{5.28} \\
3.40 \\
5.15 \\
5.99 \\
6.10 \\
\text { R } 16.79 \\
8.72 \\
1.66 \\
\text { R }_{2.92} \\
11.30\end{array}$ & $\begin{array}{r}\mathrm{R}_{3.85} \\
\mathrm{R}_{2.73} .39 \\
1.39 \\
\overline{1.39} \\
1.98 \\
\mathrm{R}_{4.77} \\
2.47 \\
5.07 \\
5.90 \\
4.47 \\
\mathrm{R}_{19.43} \\
8.37 \\
2.29 \\
\mathrm{R}_{2.40} \\
11.32\end{array}$ & $\begin{array}{r}4.02 \\
2.87 \\
1.41 \\
-71 \\
1.41 \\
2.15 \\
4.96 \\
2.74 \\
4.69 \\
5.46 \\
6.67 \\
18.94 \\
8.47 \\
2.42 \\
2.06 \\
12.13\end{array}$ \\
\hline $\begin{array}{l}\text { Transportatlon Sector } \\
\text { Primary Energy } \\
\text { Coal } \\
\text { Petroleum } \\
\text { Avlatlon Gasoline } \\
\text { Distillate Fuel } \\
\text { Jet Fuel } \\
\text { LPG } \\
\text { Lubricants } \\
\text { Motor Gasoline } \\
\text { Residual Fuel }\end{array}$ & $\begin{array}{r}2.44 \\
2.44 \\
2 . \overline{4} \\
2.17 \\
1.11 \\
.72 \\
1.12 \\
5.08 \\
2.82 \\
.46\end{array}$ & $\begin{array}{r}4.11 \\
4.11 \\
.94 \\
4.11 \\
3.45 \\
2.61 \\
2.01 \\
.2 .56 \\
7.48 \\
4.52 \\
1.79\end{array}$ & $\begin{array}{r}9.12 \\
9.12 \\
9.12 \\
9.02 \\
7.30 \\
6.34 \\
5.72 \\
14.36 \\
9.79 \\
-\end{array}$ & $\begin{array}{r}8.27 \\
8.27 \\
-\overline{8.27} \\
9.99 \\
7.15 \\
5.87 \\
7.01 \\
17.61 \\
8.76 \\
-\end{array}$ & $\begin{array}{r}R_{8.44} \\
R_{8.44} \\
R_{8.44} \\
9.32 \\
8.00 \\
5.93 \\
5.33 \\
R_{14.60} \\
9.00 \\
-\end{array}$ & $\begin{array}{r}R_{7.88} \\
R_{7.88} \\
-R_{7.88} \\
8.71 \\
7.59 \\
4.73 \\
6.10 \\
R_{16.79} \\
8.72 \\
-\end{array}$ & $\begin{array}{r}R_{7.49} \\
R_{7.49} \\
R_{7.49} \\
8.54 \\
7.34 \\
4.42 \\
4.47 \\
R_{19.43} \\
8.37 \\
-\end{array}$ & $\begin{array}{r}7.70 \\
7.70 \\
- \\
7.70 \\
8.24 \\
7.31 \\
4.12 \\
6.67 \\
18.94 \\
8.47 \\
-\end{array}$ \\
\hline 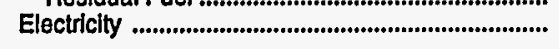 & - & - & - & - & - & - & - & - \\
\hline 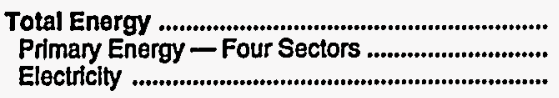 & $\begin{array}{l}1.83 \\
1.42 \\
5.76\end{array}$ & $\begin{array}{l}3.06 \\
2.59 \\
6.64\end{array}$ & $\begin{array}{r}6.43 \\
5.62 \\
11.80\end{array}$ & $\begin{array}{r}7.58 \\
5.91 \\
17.23\end{array}$ & $\begin{array}{r}R_{7.39} \\
A_{5.58} \\
16.08\end{array}$ & $\begin{array}{r}R_{7.25} \\
R_{5.39} \\
16.99\end{array}$ & $\begin{array}{l}R_{7.15} \\
R_{5.37} \\
17.04\end{array}$ & $\begin{array}{r}7.31 \\
5.42 \\
17.51\end{array}$ \\
\hline 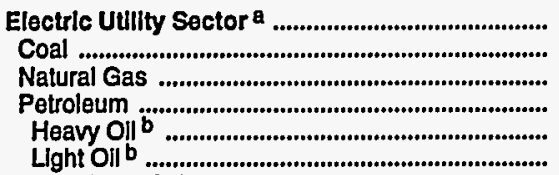 & $\begin{array}{l}.19 \\
.39 \\
.19 \\
.50 \\
.46 \\
.56\end{array}$ & $\begin{array}{r}.61 \\
.43 \\
.61 \\
1.75 \\
1.45 \\
1.92\end{array}$ & $\begin{array}{l}1.63 \\
1.23 \\
1.74 \\
5.29 \\
3.44 \\
5.30\end{array}$ & $\begin{array}{l}2.30 \\
1.68 \\
2.95 \\
5.34 \\
3.73 \\
5.54\end{array}$ & $\begin{array}{l}2.05 \\
1.40 \\
3.01 \\
4.34 \\
3.02 \\
7.28\end{array}$ & $\begin{array}{l}1.92 \\
1.32 \\
2.87 \\
3.84 \\
3.33 \\
4.09\end{array}$ & $\begin{array}{l}1.88 \\
1.23 \\
3.08 \\
3.54 \\
2.16 \\
4.36\end{array}$ & $\begin{array}{l}1.88 \\
1.24 \\
3.11 \\
3.16 \\
2.07 \\
3.50\end{array}$ \\
\hline Petroleum Coke ....................................................... & - & $\overline{-}$ & - & $\overline{-}$ & - & - & $\overline{-}$ & $\overline{-}$ \\
\hline 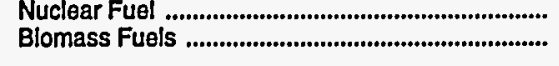 & $\overline{-}$ & $\bar{z}$ & $\overline{-}$ & $\overline{-}$ & $\overline{-}$ & $\overline{-}$ & $\overrightarrow{-}$ & $\overline{-}$ \\
\hline Primary Energy - Flve Sectors c ....................... & 1.03 & 1.90 & 4.04 & 4.56 & $\mathrm{R}_{4.21}$ & $R_{4.03}$ & $R_{4.03}$ & 4.06 \\
\hline
\end{tabular}

a There are no direct fuel costs for hydroelectric, geothermal, wind, photovoltalc, or solar thermal energy.

b Heavy oll includes fuel oil nos. 4, 5, and 6, and residual fuel oils. Light oll includes fuel oll nos. 1 and 2 , kerosene, and jet fuel.

c Blomass fuels are not included, except those consumed at electric

R=Revised data.

-No consumption, Including cases where adjustments were made. See explanation of adjustments in Section 6 of Appendix A.

Sources: Data sources, estimation procedures, and assumptions are described in Appendix A. utllitles and those added to motor gasoline. 


\begin{tabular}{|c|c|c|c|c|c|c|c|c|}
\hline Sector and Energy Source & 1970 & 1975 & 1980 & 1985 & 1990 & 1991 & 1992 & 1993 \\
\hline $\begin{array}{l}\text { Residentlal Sector } \\
\text { Primary Energy . } \\
\text { Coal } \\
\text { Natural Gas } \\
\text { Petroleum } \\
\text { Distillate Fuel } \\
\text { Kerosene } \\
\text { LPG }\end{array}$ & $\begin{array}{r}285.3 \\
100.7 \\
(s) \\
65.1 \\
35.5 \\
(s) \\
.4 \\
35.1 \\
184.6\end{array}$ & $\begin{array}{r}422.0 \\
163.3 \\
(s) \\
97.3 \\
66.0 \\
.2 \\
.4 \\
65.4 \\
258.7\end{array}$ & $\begin{array}{r}804.6 \\
237.8 \\
.6 \\
188.5 \\
48.6 \\
.6 \\
.9 \\
47.1 \\
566.8\end{array}$ & \begin{tabular}{r|}
$1,359.4$ \\
407.8 \\
.1 \\
348.3 \\
59.4 \\
1.8 \\
.8 \\
56.8 \\
951.6
\end{tabular} & $\begin{array}{r}1,477.7 \\
353.7 \\
(s) \\
315.0 \\
38.7 \\
(s) \\
.5 \\
38.2 \\
1,124.0\end{array}$ & $\begin{array}{r}1,441.6 \\
363.6 \\
(s) \\
326.6 \\
36.9 \\
(s) \\
.5 \\
36.5 \\
1,078.0\end{array}$ & $\begin{array}{r}\text { R }_{1,381.7} \\
359.6 \\
(\mathrm{~s}) \\
326.4 \\
33.2 \\
.1 \\
.5 \\
32.6 \\
1,022.0\end{array}$ & $\begin{array}{r}1,564.3 \\
428.3 \\
(s) \\
387.1 \\
41.2 \\
(s) \\
.3 \\
40.9 \\
1,136.0\end{array}$ \\
\hline $\begin{array}{l}\text { Commerclal Sector } \\
\text { Primary Energy } \\
\text { Coal } \\
\text { Natural Gas . } \\
\text { Petroleum } \\
\text { Distillate Fuel } \\
\text { Kerosene } \\
\text { LPG } \\
\text { Motor Gasoline } \\
\text { Residual Fuel } \\
\text { Electricity }\end{array}$ & $\begin{array}{r}115.4 \\
32.5 \\
(\mathrm{~s}) \\
22.9 \\
9.6 \\
.5 \\
.8 \\
4.3 \\
3.4 \\
.6 \\
82.9\end{array}$ & $\begin{array}{r}219.6 \\
63.1 \\
(s) \\
39.1 \\
24.0 \\
5.0 \\
1.4 \\
9.4 \\
6.3 \\
1.8 \\
156.5\end{array}$ & $\begin{array}{r}509.6 \\
143.8 \\
.6 \\
108.4 \\
34.8 \\
11.6 \\
.5 \\
6.5 \\
15.5 \\
.6 \\
365.8\end{array}$ & $\begin{array}{r}937.9 \\
218.0 \\
.1 \\
179.8 \\
38.2 \\
13.1 \\
.4 \\
9.0 \\
15.6 \\
719.9\end{array}$ & $\begin{array}{r}962.7 \\
186.7 \\
(\mathrm{~s}) \\
145.9 \\
40.8 \\
17.2 \\
.5 \\
4.3 \\
17.6 \\
1.2 \\
776.0\end{array}$ & $\begin{array}{r}845.4 \\
185.4 \\
(s) \\
154.8 \\
30.6 \\
13.5 \\
.3 \\
5.3 \\
10.6 \\
.8 \\
760.0\end{array}$ & $\begin{array}{r}919.6 \\
170.6 \\
(s) \\
149.0 \\
21.5 \\
10.0 \\
.1 \\
3.2 \\
7.6 \\
.6 \\
749.1\end{array}$ & $\begin{array}{r}892.4 \\
196.4 \\
(8) \\
180.7 \\
15.7 \\
8.4 \\
.2 \\
5.5 \\
1.6 \\
- \\
796.0\end{array}$ \\
\hline 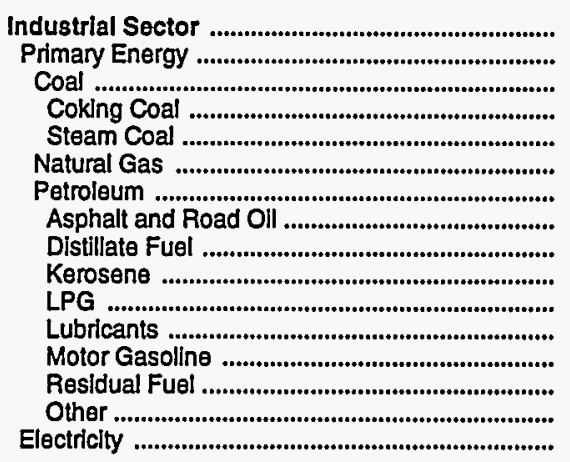 & $\begin{array}{r}122.6 \\
78.3 \\
- \\
- \\
\overline{18.1} \\
60.2 \\
20.7 \\
6.3 \\
2.9 \\
8.7 \\
5.1 \\
7.6 \\
1.2 \\
7.7 \\
44.2\end{array}$ & $\begin{array}{r}352.2 \\
257.7 \\
.4 \\
-.4 \\
.4 \\
66.6 \\
190.6 \\
69.9 \\
49.3 \\
2.7 \\
19.7 \\
12.4 \\
10.4 \\
3.2 \\
23.1 \\
94.5\end{array}$ & $\begin{array}{r}1,110.5 \\
831.9 \\
7.8 \\
- \\
7.8 \\
310.6 \\
513.5 \\
115.1 \\
122.5 \\
11.1 \\
137.7 \\
50.4 \\
18.4 \\
12.5 \\
45.7 \\
278.6\end{array}$ & $\begin{array}{r}1,495.1 \\
1,025.4 \\
32.6 \\
- \\
32.6 \\
486.9 \\
505.8 \\
111.7 \\
134.9 \\
1.4 \\
135.5 \\
56.3 \\
44.9 \\
2.2 \\
18.9 \\
469.7\end{array}$ & $\begin{array}{r}R_{1,079.1} \\
R_{662.9} \\
16.8 \\
- \\
16.8 \\
315.5 \\
R_{330.5} \\
73.1 \\
106.6 \\
.6 \\
31.1 \\
R_{52.5} \\
39.2 \\
5.3 \\
R_{22.2} \\
416.1\end{array}$ & $\begin{array}{r}\mathrm{A}_{1}, 088.7 \\
\mathrm{R}_{660.1} \\
21.3 \\
-\overline{2} \\
21.3 \\
282.9 \\
\mathrm{P}_{355.9} \\
77.5 \\
95.8 \\
.4 \\
67.5 \\
\mathrm{R}_{54.0} \\
41.0 \\
1.0 \\
\mathrm{R}_{18.7} \\
428.6\end{array}$ & $\begin{array}{r}\mathrm{R}_{1,134.8} \\
\mathrm{R}_{698.4} \\
23.1 \\
- \\
23.1 \\
330.0 \\
\mathrm{R}_{345.3} \\
47.9 \\
123.9 \\
.6 \\
49.3 \\
\mathrm{R}_{63.7} \\
36.5 \\
5.1 \\
18.2 \\
436.5\end{array}$ & $\begin{array}{r}1,254.4 \\
783.0 \\
37.9 \\
-\overline{3} \\
37.9 \\
365.8 \\
379.4 \\
67.7 \\
85.4 \\
.4 \\
93.9 \\
63.3 \\
45.6 \\
6.9 \\
16.3 \\
471.3\end{array}$ \\
\hline 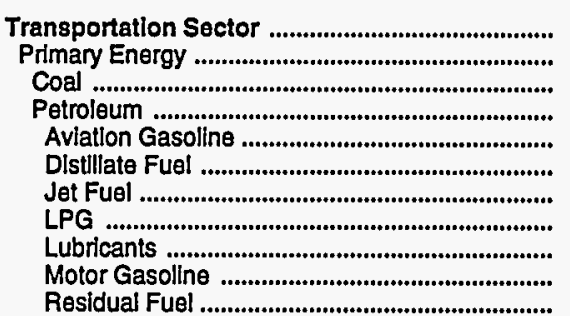 & $\begin{array}{r}531.2 \\
531.2 \\
- \\
531.2 \\
4.9 \\
21.7 \\
17.2 \\
2.2 \\
14.1 \\
470.9 \\
.2\end{array}$ & $\begin{array}{r}1,047.7 \\
1,047.7 \\
(s) \\
1,047.7 \\
5.4 \\
73.0 \\
43.2 \\
4.5 \\
24.4 \\
896.7 \\
.5\end{array}$ & $\begin{array}{r}2,604.0 \\
2,604.0 \\
2,- \\
2,604.0 \\
14.9 \\
341.7 \\
170.5 \\
4.9 \\
67.7 \\
2,004.2 \\
-\end{array}$ & $\begin{array}{r}2,600.4 \\
2,600.4 \\
- \\
2,600.4 \\
11.0 \\
439.7 \\
190.6 \\
3.4 \\
75.5 \\
1,880.2 \\
-\end{array}$ & $\begin{array}{r}\mathrm{R}_{2,678.2} \\
\mathrm{R}_{2,678.2} \\
- \\
\mathrm{R}_{2,678.2} \\
6.9 \\
545.0 \\
259.8 \\
1.9 \\
\mathrm{R} 70.5 \\
1,794.2 \\
-\end{array}$ & $\begin{array}{r}R_{2,547.9} \\
R_{2,547.9} \\
- \\
R_{2,547.9} \\
4.9 \\
462.5 \\
279.1 \\
2.4 \\
R_{72.5} \\
1,726.5 \\
-\end{array}$ & $\begin{array}{r}\mathrm{R}_{2,623.9} \\
\mathrm{R}_{2,623.9} \\
- \\
\mathrm{R}_{2,623.9} \\
5.3 \\
499.9 \\
321.7 \\
1.3 \\
\mathrm{R} 85.5 \\
1,710.1 \\
-\end{array}$ & $\begin{array}{r}2,616.4 \\
2,616.4 \\
- \\
2,616.4 \\
4.3 \\
549.5 \\
207.9 \\
2.3 \\
84.9 \\
1,767.5 \\
\end{array}$ \\
\hline Electriclty & 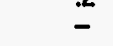 & 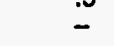 & - & - & - & - & 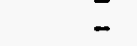 & $\overline{-}$ \\
\hline 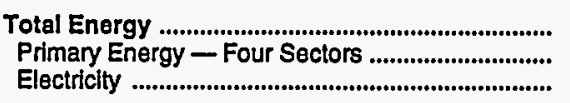 & $\begin{array}{r}1,054.5 \\
742.8 \\
311.7\end{array}$ & $\begin{array}{r}2,041.4 \\
1,531.8 \\
509.6\end{array}$ & $\begin{array}{l}5,028.7 \\
3,817.4 \\
1,211.3\end{array}$ & $\begin{array}{l}6,392.8 \\
4,251.6 \\
2,141.2\end{array}$ & $\begin{array}{r}R_{6,197.7} \\
R_{3,881.5} \\
2,316.2\end{array}$ & $\begin{array}{r}\mathrm{A}_{6,023.6} \\
\mathrm{~A}_{3,757.0} \\
2,266.6\end{array}$ & $\begin{array}{l}R_{6,060.1} \\
R_{3,852.5} \\
R_{2,207.6}\end{array}$ & $\begin{array}{l}6,427.4 \\
4,024.1 \\
2,403.3\end{array}$ \\
\hline $\begin{array}{l}\text { Electric Utllty Sector a } \\
\text { Coal } \\
\text { Natural Gas } \\
\text { Petroleum } \\
\text { Heavy Oll } \\
\text { Light Oll } \\
\text { Petroleum Coke } \\
\text { Nuclear Fuel } \\
\text { Blomass Fuels }\end{array}$ & $\begin{array}{r}46.8 \\
(\mathrm{~s}) \\
46.5 \\
.4 \\
.2 \\
.2 \\
- \\
-\end{array}$ & $\begin{array}{r}190.0 \\
(5) \\
189.1 \\
.9 \\
.3 \\
.6 \\
= \\
=\end{array}$ & $\begin{array}{r}727.3 \\
123.5 \\
602.0 \\
1.8 \\
(s) \\
1.8 \\
- \\
= \\
-\end{array}$ & $\begin{array}{r}988.5 \\
367.4 \\
618.3 \\
2.7 \\
.2 \\
2.5 \\
= \\
=\end{array}$ & $\begin{array}{r}904.7 \\
371.3 \\
531.1 \\
2.3 \\
1.1 \\
1.2 \\
- \\
- \\
-\end{array}$ & $\begin{array}{r}862.2 \\
363.1 \\
498.2 \\
.9 \\
.3 \\
.6 \\
= \\
-\end{array}$ & $\begin{array}{r}835.3 \\
358.6 \\
476.1 \\
.6 \\
.1 \\
.5 \\
= \\
= \\
-\end{array}$ & $\begin{array}{r}873.1 \\
376.5 \\
498.1 \\
.5 \\
.1 \\
.4 \\
= \\
-\end{array}$ \\
\hline Primary Energy — Five Sectors c .............................. & 789.7 & $1,721.8$ & 4,544.7 & $5,240.0$ & $R_{4,786.2}$ & $R_{4,619.2}$ & $R_{4,687.8}$ & $4,897.2$ \\
\hline
\end{tabular}

a There are no direct fuel costs for hydroelectric, geothermal, wind, photovoltalc, or solar thermal energy.

b Heavy oil includes fuel oil nos. 4, 5, and 6, and residual fuel olls. Light oll includes fuel nos. 1 and 2, kerosene, and jet fuel.

c Blomass fuels are not included, except those consumed at electric utilittes and those added to motor gasoline.

$R=$ Revised data.
-No consumption, including cases where adjustments were made. See explanation of adjustments in Section 6 of Appendlx A.

(s)=Value less than 0.05 million dollars.

Note: Totals may not equal sum of components due to Independent rounding.

Sources: Data sources, estimation procedures, and assumptlons are described in Appendix A. 


\begin{tabular}{|c|c|c|c|c|c|c|c|c|}
\hline Energy Source & 1970 & 1975 & 1980 & 1985 & 1890 & 1991 & 1992 & 1993 \\
\hline & \multicolumn{8}{|c|}{ Prices in Dollars per Million Btu } \\
\hline 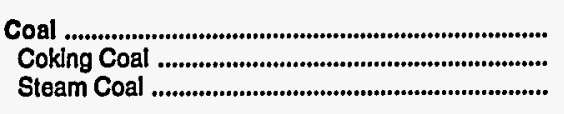 & $\frac{0.57}{.57}$ & $1 . \overline{0}$ & $\frac{1.72}{1.72}$ & $\begin{array}{r}2.15 \\
2.15\end{array}$ & $\frac{1.22}{1.22}$ & $\frac{1.17}{1.17}$ & $\begin{array}{r}1.18 \\
1 . \overline{18}\end{array}$ & $\frac{1.20}{1.20}$ \\
\hline 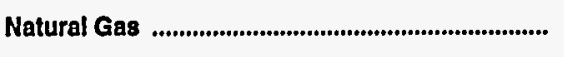 & .81 & 1.44 & 4.69 & 5.60 & 4.28 & 4.01 & 3.85 & 4.14 \\
\hline 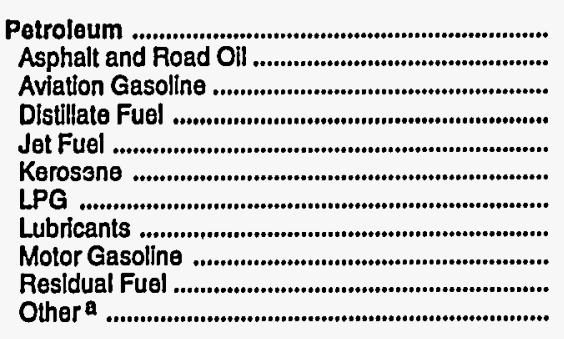 & $\begin{array}{r}1.88 \\
.58 \\
2.17 \\
1.21 \\
.73 \\
1.48 \\
2.21 \\
5.08 \\
2.83 \\
.51 \\
.78\end{array}$ & $\begin{array}{l}3.49 \\
1.78 \\
3.45 \\
2.62 \\
2.04 \\
2.84 \\
4.17 \\
7.48 \\
4.45 \\
2.06 \\
1.61\end{array}$ & $\begin{array}{r}7.86 \\
3.60 \\
9.02 \\
6.62 \\
6.21 \\
7.61 \\
7.09 \\
14.36 \\
9.75 \\
3.92 \\
4.04\end{array}$ & $\begin{array}{r}7.96 \\
4.42 \\
9.99 \\
7.98 \\
6.16 \\
9.85 \\
9.18 \\
17.61 \\
8.87 \\
4.70 \\
6.43\end{array}$ & $\begin{array}{r}\text { A } 7.78 \\
3.15 \\
9.32 \\
7.72 \\
5.93 \\
8.35 \\
10.37 \\
\text { R } 14.60 \\
9.45 \\
3.50 \\
2.50\end{array}$ & $\begin{array}{r}\mathbf{R} .44 \\
3.28 \\
8.71 \\
7.78 \\
5.01 \\
8.21 \\
10.84 \\
\mathrm{R} \\
16.79 \\
9.14 \\
2.56 \\
2.19\end{array}$ & $\begin{array}{r}R_{7.58} \\
2.81 \\
8.54 \\
7.80 \\
4.67 \\
7.57 \\
10.35 \\
R_{19.43} \\
10.03 \\
R_{1.89} \\
1.72\end{array}$ & $\begin{array}{r}8.02 \\
2.95 \\
8.24 \\
7.99 \\
4.62 \\
7.52 \\
10.43 \\
18.94 \\
10.24 \\
2.04 \\
1.61\end{array}$ \\
\hline 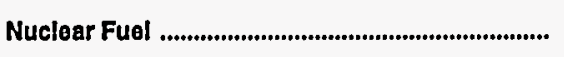 & - & .20 & .36 & .54 & .44 & .43 & .53 & - \\
\hline 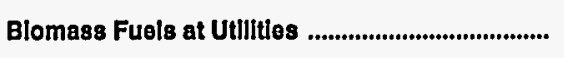 & .65 & .92 & 1.74 & - & .85 & 1.63 & 1.61 & 2.48 \\
\hline Primary Energy - Five Sectors b ......................... & 1.63 & 2.97 & 6.28 & 6.29 & $R_{6.04}$ & $R_{6.08}$ & $R_{5.76}$ & 6.55 \\
\hline $\begin{array}{l}\text { Electric Utillty Fuel }{ }^{\circ} \\
\text { Electriclity Purchased by End Users }\end{array}$ & $\begin{array}{r}.48 \\
2.90\end{array}$ & $\begin{array}{l}2.04 \\
4.13\end{array}$ & $\begin{array}{r}.59 \\
7.59\end{array}$ & $\begin{array}{r}.67 \\
13.08\end{array}$ & $\begin{array}{r}.78 \\
12.25\end{array}$ & $\begin{array}{r}1.01 \\
12.45\end{array}$ & $\begin{array}{r}.95 \\
12.64\end{array}$ & $\begin{array}{r}1.51 \\
12.97\end{array}$ \\
\hline \multirow[t]{2}{*}{ 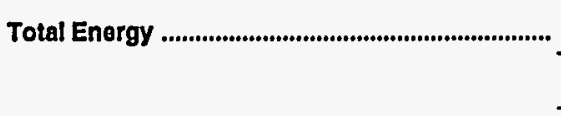 } & 1.87 & 3.22 & 7.34 & 8.77 & $R_{8.33}$ & $\mathbf{P}_{8.08}$ & $R_{8.16}$ & 8.51 \\
\hline & \multicolumn{8}{|c|}{ Expendltures in Millions of Dollars } \\
\hline $\begin{array}{l}\text { Coal } \\
\text { Coking Coal } \\
\text { Steam Coal }\end{array}$ & $\begin{array}{l}1.7 \\
\overline{1.7}\end{array}$ & $\begin{array}{l}2.8 \\
2.8\end{array}$ & $\frac{20.8}{20.8}$ & $\begin{array}{l}21.6 \\
\overline{21.6}\end{array}$ & $\begin{array}{c}18.1 \\
\overline{19.1}\end{array}$ & $\begin{array}{c}38.5 \\
38.5\end{array}$ & $\frac{48.1}{4 \overrightarrow{8} .1}$ & $\begin{array}{r}44.4 \\
\overrightarrow{44.4}\end{array}$ \\
\hline Natural Gas ............................................................................ & 68.7 & 139.9 & 320.8 & 432.9 & 438.3 & 472.4 & 459.7 & 560.2 \\
\hline 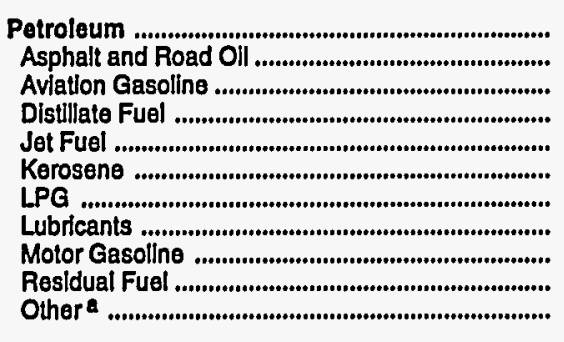 & $\begin{array}{r}541.1 \\
8.3 \\
3.3 \\
89.2 \\
8.6 \\
1.8 \\
10.2 \\
23.7 \\
371.2 \\
18.5 \\
6.3\end{array}$ & $\begin{array}{r}1,039.9 \\
38.0 \\
3.0 \\
199.4 \\
24.0 \\
3.6 \\
10.0 \\
30.8 \\
675.3 \\
45.4 \\
10.4\end{array}$ & $\begin{array}{r}2,584.6 \\
59.2 \\
11.8 \\
643.9 \\
86.5 \\
4.8 \\
31.7 \\
65.4 \\
1,562.9 \\
100.0 \\
18.1\end{array}$ & $\begin{array}{r}2,507.2 \\
83.3 \\
7.1 \\
715.0 \\
74.3 \\
3.8 \\
44.7 \\
73.0 \\
1,353.9 \\
142.9 \\
9.2\end{array}$ & $\begin{array}{r}R_{2,766.1} \\
63.3 \\
5.7 \\
767.2 \\
111.3 \\
1.2 \\
52.1 \\
568.1 \\
1,566.3 \\
98.9 \\
32.1\end{array}$ & $\begin{array}{r}R_{2,701.3} \\
57.9 \\
5.5 \\
731.8 \\
105.8 \\
1.0 \\
61.1 \\
R 70.1 \\
1,541.7 \\
101.7 \\
24.6\end{array}$ & $\begin{array}{r}R_{2,794.9} \\
61.4 \\
5.6 \\
697.8 \\
105.9 \\
1.3 \\
53.7 \\
582.7 \\
1,681.6 \\
778.2 \\
26.8\end{array}$ & $\begin{array}{r}2,865.2 \\
65.2 \\
4.6 \\
657.3 \\
112.7 \\
1.8 \\
58.7 \\
82.1 \\
1,803.3 \\
59.6 \\
20.1\end{array}$ \\
\hline 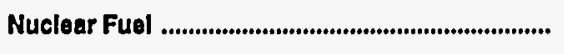 & - & (8) & 21.4 & 40.6 & 28.6 & 6.8 & 26.0 & - \\
\hline Blomass Fuels at Utiltiles ........................................ & .3 & (8) & 2.9 & - & (8) & (8) & .1 & .3 \\
\hline Prlmary Energy — Flve Sectore b ............................ & 611.8 & $1,182.6$ & $2,950.6$ & $3,002.3$ & $R_{3,252,1}$ & $\mathrm{~A}_{3,218.9}$ & $R_{3,328.8}$ & $3,470.1$ \\
\hline 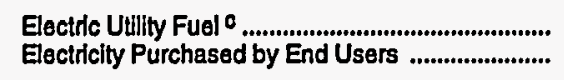 & $\begin{array}{r}-.8 \\
248.3\end{array}$ & $\begin{array}{r}-\cdot 4 \\
458.4\end{array}$ & $\begin{array}{r}-41.1 \\
950.4\end{array}$ & $\begin{array}{r}-54.5 \\
1,573.1\end{array}$ & $\begin{array}{r}-67.9 \\
1,797.0\end{array}$ & $\begin{array}{r}-58.1 \\
1,855.0\end{array}$ & $\begin{array}{r}-96.9 \\
1,850.1\end{array}$ & $\begin{array}{r}-77.4 \\
1,973.0\end{array}$ \\
\hline 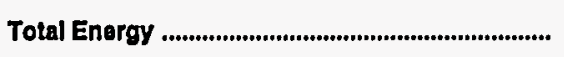 & 859.3 & $1,640.6$ & $3,860.0$ & $4,520.9$ & $R_{4,981.2}$ & $\mathrm{~A}_{5,015.8}$ & $R_{5,082.0}$ & $6,365.7$ \\
\hline
\end{tabular}

a Includes potroleum coke used at electric utilities.

b Biomass fuels are not Included, except those consumed at electric utilities and those added to motor gasoline.

- There are no direct fuel costs for hydroelectric, geothermal, wind, photovoltalc, or solar themal energy.

R=Revised data.

-No consumption, Including cases where adjustments were made. See

explanation of adjustments in Section 6 of Appendix $A$.

(s)=Value less than 0.05 million dollars.

Note: Expenditure totais may not equal sum of components due to independent, rounding.

Sources: Data sources, estimation procedures, and assumptions are described in Appendix A. 
O Energy Price Estimates by Sector, Oregon

R 1970, 1975, 1980, 1985, and 1990-1993

E (Dollars per Million Btu)

$\mathbf{G}$

\begin{tabular}{|c|c|c|c|c|c|c|c|c|}
\hline Sector and Energy Source & 1970 & 1975 & 1980 & 1985 & 1990 & 1991 & 1992 & 1993 \\
\hline $\begin{array}{l}\text { Resldentlal Sector } \\
\text { Primary Energy } \\
\text { Coal } \\
\text { Natural Gas } \\
\text { Petroleum } \\
\text { Distillate Fuel } \\
\text { Kerosene } \\
\text { EPG }\end{array}$ & $\begin{array}{r}2.47 \\
1.53 \\
.95 \\
1.45 \\
1.63 \\
1.41 \\
2.79 \\
2.66 \\
3.65\end{array}$ & $\begin{array}{l}3.78 \\
2.42 \\
1.14 \\
2.11 \\
3.03 \\
2.80 \\
3.82 \\
5.27 \\
5.27\end{array}$ & $\begin{array}{l}8.04 \\
6.20 \\
4.26 \\
5.36 \\
7.35 \\
7.02 \\
9.80 \\
9.00 \\
9.37\end{array}$ & $\begin{array}{r}10.78 \\
6.94 \\
3.67 \\
6.73 \\
7.24 \\
7.00 \\
9.85 \\
8.73 \\
13.72\end{array}$ & $\begin{array}{r}10.95 \\
6.69 \\
3.77 \\
6.13 \\
7.82 \\
6.99 \\
9.84 \\
13.92 \\
13.85\end{array}$ & $\begin{array}{r}11.01 \\
6.52 \\
5.21 \\
5.95 \\
8.01 \\
6.73 \\
9.47 \\
14.26 \\
14.11\end{array}$ & $\begin{array}{r}11.38 \\
6.37 \\
3.76 \\
5.94 \\
7.66 \\
6.33 \\
8.91 \\
12.88 \\
14.46\end{array}$ & $\begin{array}{r}11.40 \\
6.56 \\
3.77 \\
6.17 \\
8.10 \\
6.62 \\
9.31 \\
13.14 \\
14.71\end{array}$ \\
\hline 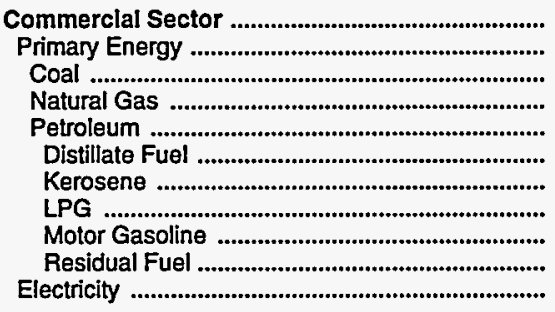 & $\begin{array}{r}2.29 \\
1.16 \\
.53 \\
1.22 \\
1.14 \\
1.22 \\
.93 \\
1.12 \\
2.83 \\
.79 \\
3.90\end{array}$ & $\begin{array}{l}3.67 \\
2.21 \\
1.04 \\
1.79 \\
2.68 \\
2.60 \\
2.58 \\
2.76 \\
4.45 \\
2.45 \\
5.20\end{array}$ & $\begin{array}{l}7.29 \\
5.66 \\
2.24 \\
4.88 \\
6.39 \\
6.71 \\
6.54 \\
5.40 \\
9.75 \\
4.90 \\
8.86\end{array}$ & $\begin{array}{r}10.91 \\
6.24 \\
2.48 \\
6.06 \\
6.58 \\
6.42 \\
9.86 \\
9.45 \\
8.87 \\
4.12 \\
14.96\end{array}$ & $\begin{array}{r}10.15 \\
5.17 \\
2.54 \\
4.74 \\
5.96 \\
5.91 \\
6.74 \\
9.03 \\
9.45 \\
3.03 \\
14.05\end{array}$ & $\begin{array}{r}10.23 \\
4.79 \\
2.65 \\
4.61 \\
5.29 \\
5.28 \\
6.05 \\
9.29 \\
9.14 \\
2.36 \\
14.29\end{array}$ & $\begin{array}{r}10.59 \\
4.80 \\
2.45 \\
4.56 \\
5.49 \\
5.46 \\
5.91 \\
9.26 \\
10.03 \\
2.30 \\
14.30\end{array}$ & $\begin{array}{r}10.59 \\
4.87 \\
2.33 \\
4.84 \\
5.04 \\
5.32 \\
6.07 \\
9.21 \\
10.24 \\
2.20 \\
14.48\end{array}$ \\
\hline 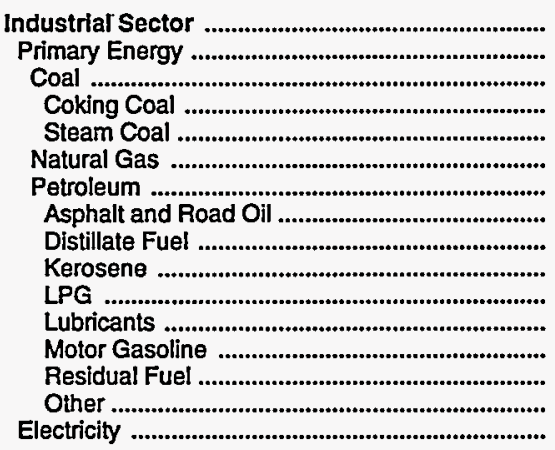 & $\begin{array}{r}.78 \\
.67 \\
.53 \\
- \\
.53 \\
.46 \\
.83 \\
.58 \\
.80 \\
.93 \\
1.12 \\
5.08 \\
2.83 \\
.33 \\
.78 \\
1.26\end{array}$ & $\begin{array}{r}1.73 \\
1.59 \\
1.04 \\
- \\
1.04 \\
.92 \\
2.16 \\
1.78 \\
2.29 \\
2.58 \\
2.76 \\
7.48 \\
4.45 \\
1.85 \\
1.61 \\
2.13\end{array}$ & $\begin{array}{r}4.56 \\
4.53 \\
2.24 \\
- \\
2.24 \\
4.21 \\
4.84 \\
3.60 \\
5.62 \\
6.54 \\
5.40 \\
14.36 \\
9.75 \\
3.39 \\
4.04 \\
4.65\end{array}$ & $\begin{array}{r}6.93 \\
5.58 \\
2.48 \\
- \\
2.48 \\
4.65 \\
6.42 \\
4.42 \\
8.72 \\
9.86 \\
9.45 \\
17.61 \\
8.87 \\
4.12 \\
6.43 \\
10.32\end{array}$ & $\begin{array}{r}R_{5.72} \\
R_{4.01} \\
2.54 \\
- \\
2.54 \\
3.39 \\
R_{4.58} \\
3.15 \\
5.96 \\
6.74 \\
9.03 \\
R_{14.60} \\
9.45 \\
3.03 \\
2.50 \\
9.27\end{array}$ & $\begin{array}{r}\mathbf{R}_{5.59} \\
\mathbf{R}_{3.86} \\
2.65 \\
- \\
2.65 \\
3.31 \\
\mathrm{R}_{4.51} \\
3.28 \\
5.35 \\
6.05 \\
9.29 \\
\mathrm{R}_{16.79} \\
9.14 \\
2.36 \\
2.19 \\
9.23\end{array}$ & $\begin{array}{r}R_{5.27} \\
R_{3.52} \\
2.45 \\
- \\
2.45 \\
3.24 \\
\text { P }_{3.86} \\
2.81 \\
5.22 \\
5.91 \\
9.26 \\
R_{19.43} \\
10.03 \\
2.30 \\
1.72 \\
9.42\end{array}$ & $\begin{array}{r}5.46 \\
3.71 \\
2.33 \\
-\overline{2} \\
2.33 \\
3.34 \\
4.15 \\
2.95 \\
5.36 \\
6.07 \\
9.21 \\
18.94 \\
10.24 \\
2.20 \\
1.61 \\
9.74\end{array}$ \\
\hline 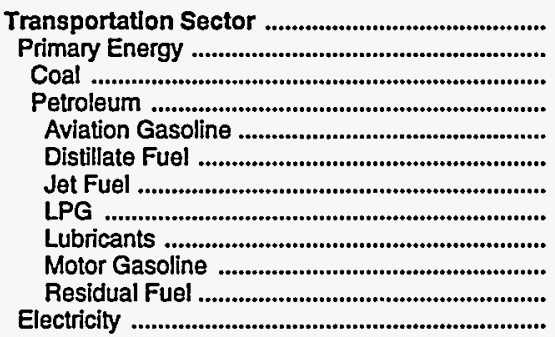 & $\begin{array}{r}2.41 \\
2.41 \\
.53 \\
2.41 \\
2.17 \\
1.34 \\
.73 \\
1.12 \\
5.08 \\
2.83 \\
.71 \\
-\end{array}$ & $\begin{array}{r}3.98 \\
3.98 \\
1.04 \\
3.98 \\
3.45 \\
2.69 \\
2.04 \\
2.76 \\
7.48 \\
4.45 \\
2.21 \\
-\end{array}$ & $\begin{array}{r}8.81 \\
8.81 \\
- \\
8.81 \\
9.02 \\
6.96 \\
6.21 \\
5.40 \\
14.36 \\
9.75 \\
4.14 \\
-\end{array}$ & $\begin{array}{r}8.40 \\
8.40 \\
- \\
8.40 \\
9.99 \\
8.27 \\
6.16 \\
9.45 \\
17.61 \\
8.87 \\
5.02 \\
-\end{array}$ & $\begin{array}{r}R_{8.55} \\
R_{8.55} \\
- \\
R_{8.55} \\
9.32 \\
8.54 \\
5.93 \\
9.03 \\
R_{14.60} \\
9.45 \\
3.59 \\
13.90\end{array}$ & $\begin{array}{r}R_{7.99} \\
R_{7.99} \\
- \\
R_{7.99} \\
8.71 \\
8.63 \\
5.01 \\
9.29 \\
R_{16.79} \\
9.14 \\
2.58 \\
15.56\end{array}$ & $\begin{array}{r}A_{8.38} \\
R_{8.38} \\
- \\
R_{8.38} \\
8.54 \\
8.63 \\
4.67 \\
9.26 \\
R_{19.43} \\
10.03 \\
R_{1.84} \\
14.54\end{array}$ & $\begin{array}{r}8.89 \\
8.89 \\
- \\
8.89 \\
8.24 \\
8.93 \\
4.62 \\
9.21 \\
18.94 \\
10.24 \\
2.00 \\
15.45\end{array}$ \\
\hline 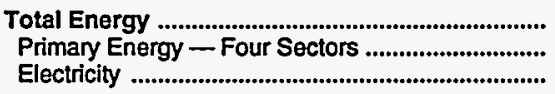 & $\begin{array}{l}1.87 \\
1.63 \\
2.90\end{array}$ & $\begin{array}{l}3.22 \\
2.97 \\
4.13\end{array}$ & $\begin{array}{l}7.34 \\
7.26 \\
7.59\end{array}$ & $\begin{array}{r}8.77 \\
7.46 \\
13.08\end{array}$ & $\begin{array}{l}R_{8.33} \\
R_{7.05} \\
12.25\end{array}$ & $\begin{array}{r}A_{8.08} \\
{ }^{R_{6.70}} \\
12.45\end{array}$ & $\begin{array}{l}R_{8.16} \\
R_{6.79} \\
12.64\end{array}$ & $\begin{array}{r}8.51 \\
7.09 \\
12.97\end{array}$ \\
\hline $\begin{array}{l}\text { Electric Utllity Sector }{ }^{\mathrm{a}} \\
\text { Coal } \\
\text { Natural Gas } \\
\text { Petroleum } \\
\text { Heavy Oil }{ }^{b} \\
\text { Light Oil } \mathrm{b} \text {. } \\
\text { Petroleum Coke } \\
\text { Nuclear Fuel } \\
\text { Biomass Fuels }\end{array}$ & $\begin{array}{r}.48 \\
.37 \\
.80 \\
.80 \\
.83 \\
\overline{-} \\
.65\end{array}$ & $\begin{array}{r}2.04 \\
1 . \overline{2} \\
2.31 \\
\overline{-31} \\
. \overline{0} \\
.92\end{array}$ & $\begin{array}{r}.59 \\
1.41 \\
4.29 \\
6.53 \\
- \\
6.53 \\
\overline{36} \\
.36 \\
1.74\end{array}$ & $\begin{array}{r}.67 \\
2.00 \\
- \\
5.67 \\
- \\
5.67 \\
\overline{-} \\
.54 \\
-\end{array}$ & $\begin{array}{r}.78 \\
1.08 \\
3.03 \\
3.47 \\
-\overline{7} \\
3.47 \\
\overline{.44} \\
.85\end{array}$ & $\begin{array}{r}1.01 \\
1.08 \\
1.57 \\
4.75 \\
- \\
4.75 \\
-\overline{43} \\
1.63\end{array}$ & $\begin{array}{r}.95 \\
1.10 \\
1.94 \\
4.49 \\
-\overline{4} \\
4.49 \\
-\overline{53} \\
1.61\end{array}$ & $\begin{array}{r}1.51 \\
1.12 \\
2.25 \\
3.83 \\
-\overline{3} \\
3.83 \\
- \\
\overline{-} \\
2.48\end{array}$ \\
\hline Primary Energy - Flve Sectors ${ }^{c}$ & 1.63 & 2.97 & 6.28 & 6.29 & ${ }^{R_{6.04}}$ & ${ }^{R_{6.08}}$ & $R_{5.76}$ & 6.55 \\
\hline
\end{tabular}

a There are no direct fuel costs for hydroelectric, geothermal, wind, photovoltaic, or solar thermal energy.

Heavy oil includes fuel oll nos. 4, 5, and 6, and residual fuel olls. Light oil includes fuel oil nos. 1 and 2, kerosene, and jet fuel.

c Biomass fuels are not included, except those consumed at electric

$R=$ Revised data.

- No consumption, including cases where adjustments were made. Se日 explanation of adjustments in Section 6 of Appendix A.

Sources: Data sources, estimation procedures, and assumptions are described in Appendix A. utilities and those added to motor gasoline. 


\begin{tabular}{|c|c|c|c|c|c|c|c|c|}
\hline Sector and Energy Source & 1970 & 1975 & 1980 & 1985 & 1990 & 1991 & 1992 & 1993 \\
\hline $\begin{array}{l}\text { Residential Sector } \\
\text { Primary Energy } \\
\text { Coal } \\
\text { Natural Gas } \\
\text { Petroleum . } \\
\text { Distlllate Fuel } \\
\text { Kerosene } \\
\text { LPG }\end{array}$ & $\begin{array}{r}188.1 \\
65.4 \\
.2 \\
29.8 \\
35.3 \\
25.6 \\
1.0 \\
8.7 \\
122.8\end{array}$ & $\begin{array}{r}327.8 \\
110.3 \\
.1 \\
63.1 \\
47.1 \\
39.0 \\
1.0 \\
7.1 \\
217.4\end{array}$ & $\begin{array}{r}640.2 \\
207.3 \\
.6 \\
103.1 \\
103.5 \\
82.5 \\
2.1 \\
19.0 \\
432.9\end{array}$ & $\begin{array}{r}944.3 \\
264.2 \\
.1 \\
148.8 \\
115.4 \\
96.8 \\
2.3 \\
16.3 \\
680.0\end{array}$ & $\begin{array}{r}966.2 \\
239.3 \\
.1 \\
146.6 \\
92.6 \\
72.7 \\
.7 \\
19.2 \\
727.0\end{array}$ & $\begin{array}{r}1,013.6 \\
245.6 \\
(s) \\
161.4 \\
84.2 \\
58.3 \\
.7 \\
25.2 \\
768.0\end{array}$ & $\begin{array}{r}953.0 \\
203.0 \\
(s) \\
142.6 \\
60.4 \\
39.4 \\
.9 \\
20.2 \\
750.0\end{array}$ & $\begin{array}{r}1,093.1 \\
255.0 \\
.1 \\
191.2 \\
63.8 \\
39.9 \\
1.0 \\
22.9 \\
838.0\end{array}$ \\
\hline 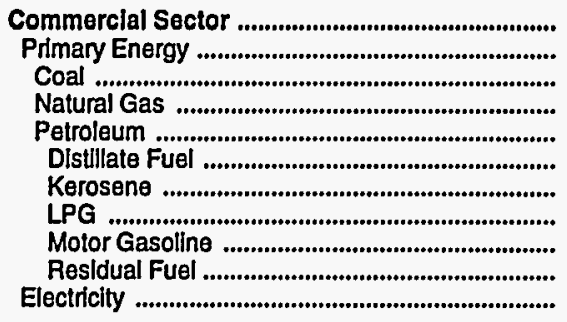 & $\begin{array}{r}126.1 \\
37.4 \\
.3 \\
14.5 \\
22.6 \\
11.5 \\
.2 \\
.6 \\
3.7 \\
6.6 \\
88.7\end{array}$ & $\begin{array}{r}225.8 \\
69.7 \\
.2 \\
29.6 \\
39.8 \\
18.8 \\
.5 \\
.7 \\
5.1 \\
14.8 \\
156.1\end{array}$ & $\begin{array}{r}509.3 \\
193.4 \\
.6 \\
77.5 \\
115.3 \\
70.0 \\
1.4 \\
2.0 \\
14.9 \\
27.0 \\
316.0\end{array}$ & $\begin{array}{r}718.7 \\
191.1 \\
.1 \\
118.9 \\
72.0 \\
51.8 \\
1.4 \\
3.1 \\
10.8 \\
4.9 \\
527.6\end{array}$ & $\begin{array}{r}746.3 \\
166.6 \\
.1 \\
99.2 \\
67.4 \\
46.0 \\
.3 \\
2.2 \\
13.4 \\
5.5 \\
579.7\end{array}$ & $\begin{array}{r}756.4 \\
151.9 \\
(\mathrm{~s}) \\
106.1 \\
45.8 \\
30.6 \\
.1 \\
2.9 \\
8.4 \\
3.8 \\
604.5\end{array}$ & $\begin{array}{r}745.6 \\
132.0 \\
(\mathrm{~s}) \\
92.6 \\
39.3 \\
24.4 \\
.2 \\
2.6 \\
8.7 \\
3.5 \\
613.6\end{array}$ & $\begin{array}{r}781.2 \\
145.6 \\
.1 \\
121.2 \\
24.3 \\
17.0 \\
.4 \\
2.8 \\
1.7 \\
2.4 \\
635.6\end{array}$ \\
\hline 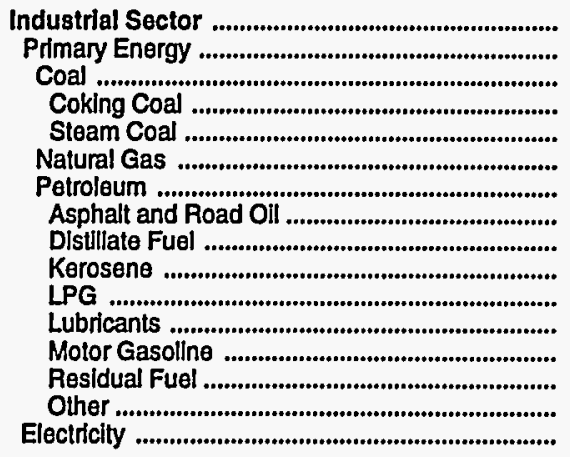 & $\begin{array}{r}119.1 \\
82.2 \\
1.2 \\
-\overline{1.2} \\
23.9 \\
57.1 \\
8.3 \\
14.8 \\
.6 \\
.8 \\
8.7 \\
10.7 \\
7.0 \\
6.3 \\
36.8\end{array}$ & $\begin{array}{r}268.3 \\
183.5 \\
2.5 \\
- \\
2.5 \\
47.2 \\
133.9 \\
38.0 \\
35.1 \\
2.1 \\
2.1 \\
8.6 \\
13.1 \\
24.5 \\
10.4 \\
84.8\end{array}$ & $\begin{array}{r}650.4 \\
448.7 \\
8.5 \\
- \\
8.5 \\
138.8 \\
301.5 \\
59.2 \\
128.4 \\
1.4 \\
9.5 \\
19.3 \\
21.4 \\
44.2 \\
18.1 \\
201.6\end{array}$ & $\begin{array}{r}862.3 \\
496.9 \\
7.5 \\
-\overline{5} \\
7.5 \\
165.2 \\
324.2 \\
83.3 \\
128.5 \\
.1 \\
18.9 \\
21.5 \\
22.4 \\
40.3 \\
9.2 \\
365.4\end{array}$ & $\begin{array}{r}\mathrm{R} 932.0 \\
\mathrm{R}_{442.0} \\
3.6 \\
- \\
3.6 \\
169.6 \\
\mathrm{R}_{268.7} \\
63.3 \\
98.7 \\
.2 \\
24.7 \\
\mathrm{R}_{20.1} \\
21.0 \\
8.6 \\
32.1 \\
490.0\end{array}$ & $\begin{array}{r}\mathrm{R}_{905.7} \\
\mathrm{R}_{423.7} \\
5.0 \\
- \\
5.0 \\
187.7 \\
\mathrm{R}_{231.0} \\
57.9 \\
71.4 \\
.1 \\
27.7 \\
\mathrm{R}_{20.7} \\
23.5 \\
5.2 \\
24.6 \\
482.0\end{array}$ & $\begin{array}{r}R 917.0 \\
R 431.0 \\
5.7 \\
- \\
5.7 \\
196.6 \\
R 228.7 \\
61.4 \\
69.1 \\
.3 \\
26.0 \\
R 24.4 \\
13.4 \\
7.3 \\
26.8 \\
486.0\end{array}$ & $\begin{array}{r}962.9 \\
463.9 \\
5.2 \\
- \\
5.2 \\
210.9 \\
247.8 \\
65.2 \\
76.0 \\
.4 \\
28.2 \\
24.2 \\
24.3 \\
9.4 \\
20.1 \\
499.0\end{array}$ \\
\hline 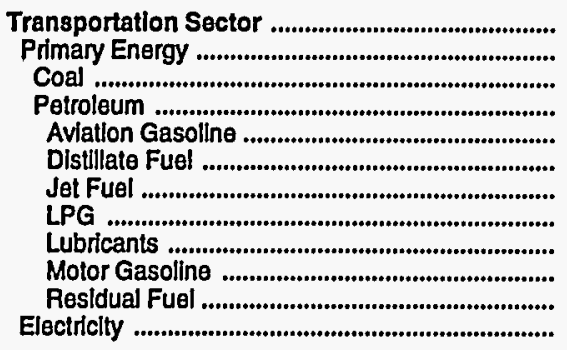 & $\begin{array}{r}426.0 \\
426.0 \\
(s) \\
426.0 \\
3.3 \\
37.4 \\
8.6 \\
.1 \\
15.0 \\
356.7 \\
4.8 \\
-\end{array}$ & $\begin{array}{r}818.7 \\
818.7 \\
(s) \\
818.7 \\
3.0 \\
106.2 \\
24.0 \\
.1 \\
22.3 \\
657.1 \\
6.1 \\
-\end{array}$ & $\begin{array}{r}2,060.1 \\
2,060.1 \\
- \\
2,060.1 \\
11.8 \\
358.9 \\
86.5 \\
1.3 \\
46.1 \\
1,526.7 \\
28.8 \\
-\end{array}$ & $\begin{array}{r}1,995.5 \\
1,995.5 \\
- \\
1,995.5 \\
7.1 \\
437.8 \\
74.3 \\
6.5 \\
51.5 \\
1,320.7 \\
97.6 \\
-\end{array}$ & $\begin{array}{r}R_{2,336.6} \\
R_{2,336.3} \\
- \\
R_{2,336.3} \\
5.7 \\
548.7 \\
111.3 \\
6.0 \\
R_{48.0} \\
1,531.8 \\
84.8 \\
.4\end{array}$ & $\begin{array}{r}\mathrm{R}_{2,340.1} \\
\mathrm{R}_{2,339.7} \\
- \\
\mathrm{R}_{2,339.7} \\
5.5 \\
570.9 \\
105.8 \\
5.3 \\
\mathrm{R}_{49.4} \\
1,509.9 \\
92.8 \\
.5\end{array}$ & $\begin{array}{r}\mathrm{R}_{2,466.4} \\
\mathrm{R}_{2,466.0} \\
- \\
\mathrm{R}_{2,466.0} \\
5.6 \\
564.4 \\
105.9 \\
4.9 \\
\mathrm{R}_{58.3} \\
1,659.5 \\
\mathrm{R}_{67.3} \\
.4\end{array}$ & $\begin{array}{r}2,528.5 \\
2,528.1 \\
- \\
2,528.1 \\
4.6 \\
523.1 \\
112.7 \\
4.7 \\
57.9 \\
1,777.3 \\
47.8 \\
.4\end{array}$ \\
\hline $\begin{array}{l}\text { Tolal Energy } \\
\text { Primary Energy - Four Sectors } \\
\text { Electricity }\end{array}$ & $\begin{array}{l}859.3 \\
611.0 \\
248.3\end{array}$ & $\begin{array}{r}1,640.6 \\
1,182.2 \\
458.4\end{array}$ & $\begin{array}{r}3,860.0 \\
2,909.5 \\
950.4\end{array}$ & $\begin{array}{l}4,520.9 \\
2,947.8 \\
1,573.1\end{array}$ & $\begin{array}{r}R_{4,981.2} \\
R_{3,184.2} \\
1,797.0\end{array}$ & $\begin{array}{r}\mathrm{P}_{5,015.8} \\
\mathrm{R}_{3,160.8} \\
1,855.0\end{array}$ & $\begin{array}{r}R_{5,082.0} \\
R_{3,232.0} \\
1,850.1\end{array}$ & $\begin{array}{l}5,365.7 \\
3,392.7 \\
1,973.0\end{array}$ \\
\hline 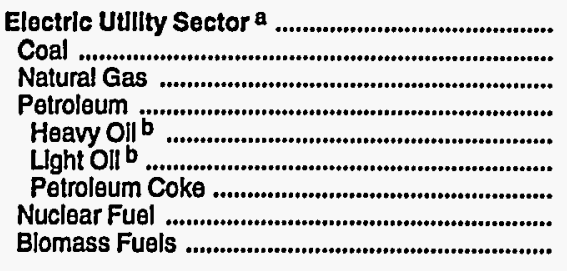 & $\begin{array}{l}.8 \\
.4 \\
.1 \\
.1 \\
(s) \\
- \\
.3\end{array}$ & $\begin{array}{l}.4 \\
\overline{(s)} \\
.4 \\
\overline{.4} \\
\overline{(s)} \\
\text { (s) }\end{array}$ & $\begin{array}{r}41.1 \\
11.2 \\
1.4 \\
4.2 \\
\overline{4.2} \\
\overline{21.4} \\
2.9\end{array}$ & $\begin{array}{r}54.5 \\
13.9 \\
\overline{.1} \\
\overline{.} \\
\overline{.} \\
40.6 \\
-\end{array}$ & $\begin{array}{r}67.9 \\
15.3 \\
22.9 \\
1.1 \\
\overline{1.1} \\
\overline{28.6} \\
(\mathrm{~s})\end{array}$ & $\begin{array}{r}58.1 \\
33.5 \\
17.2 \\
.6 \\
\overline{.6} \\
\overline{6.8} \\
(\mathrm{~s})\end{array}$ & $\begin{array}{r}96.9 \\
42.3 \\
27.9 \\
.5 \\
\\
.5 \\
- \\
26.0 \\
.1\end{array}$ & $\begin{array}{r}77.4 \\
39.1 \\
36.8 \\
1.2 \\
\overline{1.2} \\
\overline{-} \\
\overline{.3}\end{array}$ \\
\hline Primary Energy - Flve Sectors c ............................. & 611.8 & $1,182.6$ & $2,950.6$ & $3,002.3$ & ${ }^{R}, 252.1$ & ${ }^{R} 3,218.9$ & $R_{3,328.8}$ & $3,470.1$ \\
\hline
\end{tabular}

a There are no direct fuel costs for hydroelectric, geothermal, wind, photovoltalc, or solar thermal energy.

b Heavy oll Includes fuel oll nos, 4, 5, and 6, and residual fuel oils. Light oll Includes fuel nos. 1 and 2, kerosene, and jet fuel.

c Blomass fuels are not included, except those consumed at electric utlitiles and those added to motor gasoline.

R=Rovised data.
-No consumption, including cases where adjustments were made. See explanation of adjustments in Section 6 of Appendix A.

$(s)=$ Value less than 0.05 million dollars.

Note: Totals may not equal sum of components due to independent rounding.

Sources: Data sources, estimation procedures, and assumptions are described in Appendix A. 
P Energy Price and Expenditure Estimates by Source, Pennsylvania

E $1970,1975,1980,1985$, and 1990-1993

\begin{tabular}{|c|c|c|c|c|c|c|c|c|}
\hline Energy Source & 1970 & 1975 & 1980 & 1985 & 1990 & 1991 & 1992 & 1993 \\
\hline & \multicolumn{8}{|c|}{ Prices in Dollars per Million Btu } \\
\hline 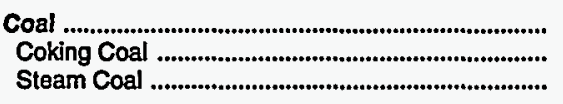 & $\begin{array}{r}0.39 \\
.44 \\
.35\end{array}$ & $\begin{array}{l}1.21 \\
1.52 \\
1.03\end{array}$ & $\begin{array}{l}1.59 \\
2.20 \\
1.35\end{array}$ & $\begin{array}{l}1.64 \\
1.88 \\
1.58\end{array}$ & $\begin{array}{l}1.57 \\
1.71 \\
1.54\end{array}$ & $\begin{array}{l}1.59 \\
1.75 \\
1.55\end{array}$ & $\begin{array}{l}1.54 \\
1.73 \\
1.50\end{array}$ & $\begin{array}{l}1.50 \\
1.73 \\
1.45\end{array}$ \\
\hline Natural Gas & .87 & 1.53 & 3.37 & 5.74 & 5.34 & 5.38 & 5.22 & 5.33 \\
\hline $\begin{array}{l}\text { Petroleum } \\
\text { Asphalt and Road Oil } \\
\text { Aviation Gasoline } \\
\text { Distillate Fuel } \\
\text { Jet Fuel }\end{array}$ & $\begin{array}{r}1.71 \\
.78 \\
2.17 \\
1.17 \\
.72 \\
1.39 \\
1.82 \\
5.08 \\
2.92 \\
.47 \\
1.15\end{array}$ & $\begin{array}{l}3.45 \\
1.97 \\
3.45 \\
2.65 \\
2.01 \\
2.88 \\
3.39 \\
7.48 \\
4.72 \\
2.02 \\
2.35\end{array}$ & $\begin{array}{r}7.67 \\
3.72 \\
9.02 \\
6.70 \\
6.27 \\
7.75 \\
6.16 \\
14.36 \\
9.71 \\
4.30 \\
6.54\end{array}$ & $\begin{array}{r}8.06 \\
4.95 \\
9.99 \\
7.73 \\
5.84 \\
8.37 \\
9.75 \\
17.61 \\
9.01 \\
4.38 \\
7.15\end{array}$ & 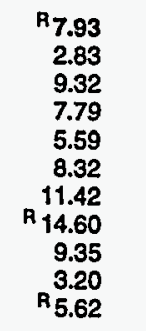 & $\begin{array}{r}R_{7.99} \\
2.76 \\
8.71 \\
7.61 \\
4.81 \\
8.08 \\
12.18 \\
\mathrm{P}_{16.79} \\
9.44 \\
2.31 \\
\mathbf{R}_{5.77}\end{array}$ & $\begin{array}{r}\mathrm{R}_{7.85} \\
2.31 \\
8.54 \\
7.20 \\
4.49 \\
7.29 \\
\mathrm{P}_{10.31}^{10.43} \\
\mathrm{P}_{2.33} \\
\mathrm{P}_{2.33} \\
\mathrm{P}_{6.33}\end{array}$ & $\begin{array}{r}7.63 \\
3.15 \\
8.24 \\
7.06 \\
4.17 \\
6.99 \\
10.60 \\
18.94 \\
9.27 \\
2.35 \\
5.63\end{array}$ \\
\hline 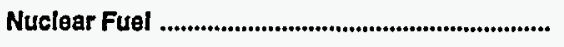 & .21 & .25 & .42 & .92 & .83 & .77 & .68 & .66 \\
\hline 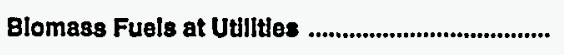 & - & - & - & - & - & - & - & - \\
\hline Primary Energy — Five Sectors ${ }^{b}$ & .97 & 2.02 & 4.04 & 4.45 & $R_{4.03}$ & $\mathrm{R}_{4.05}$ & R 3.93 & 3.94 \\
\hline 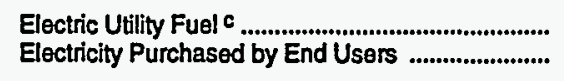 & $\begin{array}{r}.34 \\
5.23\end{array}$ & $\begin{array}{r}.93 \\
10.37\end{array}$ & $\begin{array}{r}1.55 \\
15.17\end{array}$ & $\begin{array}{r}1.61 \\
21.24\end{array}$ & $\begin{array}{r}1.32 \\
22.46\end{array}$ & $\begin{array}{r}1.28 \\
23.51\end{array}$ & $\begin{array}{r}1.19 \\
23.61\end{array}$ & $\begin{array}{r}1.18 \\
23.26\end{array}$ \\
\hline \multirow[t]{2}{*}{ 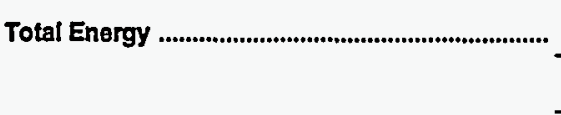 } & 1.47 & 3.26 & 6.41 & 8.48 & ${ }^{A_{8}} 8.59$ & ${ }^{\mathrm{A}} 8.95$ & ${ }^{A_{8}} 8.67$ & 8.63 \\
\hline & \multicolumn{8}{|c|}{ Expenditures in Mlllions of Dollars } \\
\hline $\begin{array}{l}\text { Coal } \\
\text { Coking Coal } \\
\text { Steam Coal }\end{array}$ & $\begin{array}{l}656.6 \\
317.5 \\
339.1\end{array}$ & $\begin{array}{r}1,991.7 \\
913.7 \\
1,078.1\end{array}$ & $\begin{array}{l}2,594.6 \\
1,005.0 \\
1,589.6\end{array}$ & $\begin{array}{r}2,307.2 \\
492.9 \\
1,814.3\end{array}$ & $\begin{array}{r}2,244.7 \\
480.0 \\
1,764.7\end{array}$ & $\begin{array}{r}2,163.0 \\
412.9 \\
1,750.1\end{array}$ & $\begin{array}{r}2,169.0 \\
458.8 \\
1,710.2\end{array}$ & $\begin{array}{r}2,116.3 \\
479.5 \\
1,636.8\end{array}$ \\
\hline 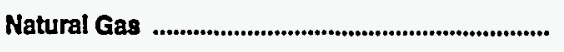 & 653.4 & 964.8 & 2,489.5 & $3,444.8$ & $3,291.5$ & $3,285.9$ & $3,404.8$ & $3,540.2$ \\
\hline 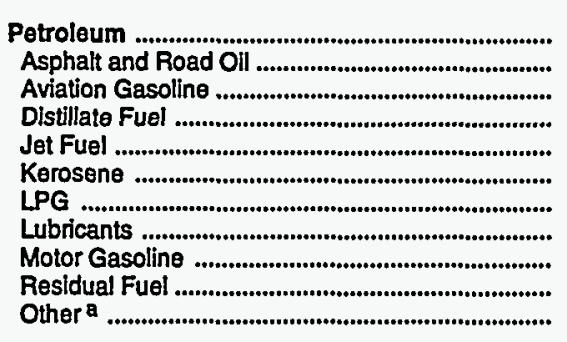 & $\begin{array}{r}2,447.5 \\
34.0 \\
7.3 \\
429.1 \\
36.9 \\
33.6 \\
32.7 \\
118.5 \\
1,559.6 \\
157.4 \\
38.6\end{array}$ & $\begin{array}{r}4,720.6 \\
74.2 \\
7.4 \\
1,039.9 \\
97.3 \\
55.5 \\
76.5 \\
152.0 \\
2,695.2 \\
441.3 \\
81.4\end{array}$ & $\begin{array}{r}10,484.8 \\
127.1 \\
15.3 \\
2,665.1 \\
360.1 \\
121.5 \\
163.8 \\
354.3 \\
5,507.0 \\
798.1 \\
372.4\end{array}$ & $\begin{array}{r}9,331.7 \\
161.3 \\
10.5 \\
2,418.9 \\
334.6 \\
168.9 \\
262.3 \\
395.4 \\
4,825.9 \\
483.8 \\
270.1\end{array}$ & $\begin{array}{r}R_{9,469.4} \\
140.3 \\
6.8 \\
2,444.5 \\
380.7 \\
76.0 \\
251.3 \\
R_{368.8} \\
5,246.8 \\
338.3 \\
\text { म }_{213.8}\end{array}$ & $\begin{array}{r}R_{9,275.6} \\
113.6 \\
5.1 \\
2,346.4 \\
309.5 \\
81.7 \\
327.9 \\
R 379.6 \\
5,310.9 \\
214.3 \\
R 186.9\end{array}$ & $\begin{array}{r}\mathbf{R}_{9,230.5} \\
92.4 \\
7.0 \\
2,304.5 \\
277.8 \\
75.6 \\
338.5 \\
R_{447.9} \\
5,267.8 \\
R_{205.6} \\
\mathrm{R}_{213.5}\end{array}$ & $\begin{array}{r}9,471.9 \\
127.2 \\
6.2 \\
2,516.8 \\
278.1 \\
81.5 \\
215.6 \\
444.5 \\
5,353.8 \\
257.0 \\
191.2\end{array}$ \\
\hline Nuclear Fuel ................................................................. & 1.1 & 44.3 & 55.4 & 262.2 & 511.5 & 474.0 & 436.0 & $\$ 16.7$ \\
\hline Blomass Fuels at Utilities ............................................. & - & - & $\sim$ & - & - & - & - & - \\
\hline Primary Energy — Five Sectore b .......................... & $\mathbf{3 , 7 5 8 . 7}$ & $7,721.4$ & $15,624.2$ & $15,345.9$ & $A_{15,517.1}$ & $\mathrm{R}_{15,198.7}$ & $A_{15,240,3}$ & $15,545.1$ \\
\hline 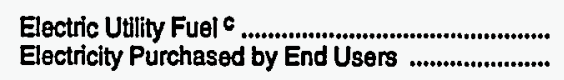 & $\begin{array}{r}-296.5 \\
1,330.2\end{array}$ & $\begin{array}{r}-1,047.7 \\
3,060.5\end{array}$ & $\begin{array}{r}-1,997.2 \\
5,096.8\end{array}$ & $\begin{array}{r}-2,233.1 \\
7,203.2\end{array}$ & $\begin{array}{r}-2,215.1 \\
8,736.6\end{array}$ & $\begin{array}{r}-2,122.4 \\
9,235.0\end{array}$ & $\begin{array}{r}-1,992.9 \\
9,274.4\end{array}$ & $\begin{array}{r}-1,991.2 \\
0,429.7\end{array}$ \\
\hline 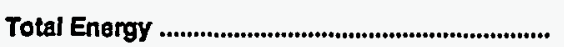 & $4,792,4$ & $8,734.3$ & $18,723.8$ & $20,315.9$ & $R_{22,038.6}$ & $R_{22,311.3}$ & $\mathrm{R}_{22,521.3}$ & $22,983.6$ \\
\hline
\end{tabular}

a Includes petroleum coke used at electric utilities.

b Biomass fuels are not included, except those consumed at electric utilities and those added to motor gasoline.

c There are no direct fuel costs for hydroelectric, geothermal, wind, photovoltaic, or solar themal energy.

$\mathrm{R}=$ Rovised data.

-No consumption, including cases where adjustments were made. See explanation of adjustments in Section 6 of Appendix A.

Note: Expenditure totals may not equal sum of components due to independent rounding.

Sources: Data sources, estimation procedures, and assumptions are described in Appendix A. 


\begin{tabular}{|c|c|c|c|c|c|c|c|c|}
\hline Sector and Ënergy Source & 1970 & 1975 & 1980 & 1985 & 1990 & 1991 & 1992 & 1993 \\
\hline 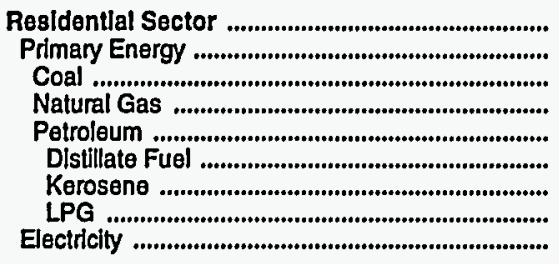 & $\begin{array}{l}1.98 \\
1.26 \\
1.03 \\
1.20 \\
1.41 \\
1.35 \\
1.57 \\
2.47 \\
7.15\end{array}$ & $\begin{array}{r}3.97 \\
2.32 \\
2.57 \\
1.89 \\
2.89 \\
2.81 \\
3.12 \\
4.42 \\
12.80\end{array}$ & $\begin{array}{r}7.17 \\
4.92 \\
2.70 \\
3.73 \\
7.10 \\
6.95 \\
8.05 \\
9.00 \\
17.42\end{array}$ & $\begin{array}{r}10.75 \\
6.95 \\
2.83 \\
6.50 \\
8.12 \\
7.82 \\
8.68 \\
11.63 \\
25.05\end{array}$ & $\begin{array}{r}11.94 \\
6.80 \\
2.96 \\
6.37 \\
8.30 \\
7.84 \\
8.70 \\
12.94 \\
27.02\end{array}$ & $\begin{array}{r}12.34 \\
6.88 \\
2.64 \\
6.53 \\
8.23 \\
7.62 \\
8.46 \\
13.85 \\
28.09\end{array}$ & $\begin{array}{r}11.77 \\
6.48 \\
2.69 \\
6.37 \\
7.31 \\
6.79 \\
7.54 \\
11.87 \\
28.33\end{array}$ & $\begin{array}{r}11.83 \\
6.60 \\
2.79 \\
6.60 \\
7.03 \\
6.60 \\
7: 32 \\
11.86 \\
27.99\end{array}$ \\
\hline 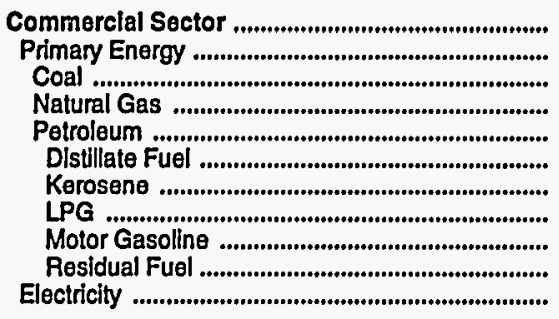 & $\begin{array}{r}1.90 \\
.90 \\
.32 \\
.93 \\
1.12 \\
1.09 \\
.74 \\
1.40 \\
2.92 \\
.47 \\
6.71\end{array}$ & $\begin{array}{r}4.48 \\
1.94 \\
1.25 \\
1.67 \\
2.56 \\
2.48 \\
2.52 \\
2.84 \\
4.72 \\
2.02 \\
11.88\end{array}$ & $\begin{array}{r}7.57 \\
3.95 \\
1.33 \\
3.49 \\
6.08 \\
6.39 \\
6.01 \\
5.36 \\
9.71 \\
4.43 \\
16.63\end{array}$ & $\begin{array}{r}11.38 \\
5.76 \\
1.61 \\
5.99 \\
6.56 \\
6.78 \\
7.14 \\
8.92 \\
9.01 \\
4.70 \\
23.28\end{array}$ & $\begin{array}{r}12.06 \\
5.54 \\
1.47 \\
5.79 \\
6.23 \\
6.11 \\
6.39 \\
10.33 \\
9.35 \\
3.46 \\
24.01\end{array}$ & $\begin{array}{r}12.45 \\
5.46 \\
1.43 \\
5.80 \\
6.13 \\
5.98 \\
6.04 \\
11.09 \\
9.44 \\
2.51 \\
24.65\end{array}$ & $\begin{array}{r}12.17 \\
5.19 \\
1.42 \\
5.67 \\
5.51 \\
5.57 \\
5.66 \\
9.50 \\
9.33 \\
2.51 \\
\text { R }_{25.11}\end{array}$ & $\begin{array}{r}12.35 \\
5.29 \\
1.36 \\
5.78 \\
5.12 \\
5.36 \\
5.63 \\
9.32 \\
9.27 \\
2.54 \\
24.59\end{array}$ \\
\hline 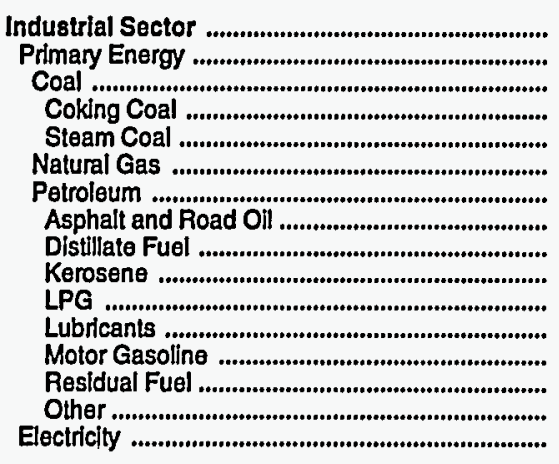 & $\begin{array}{l}.78 \\
.55 \\
.41 \\
.44 \\
.32 \\
.57 \\
.98 \\
.78 \\
.70 \\
.74 \\
1.40 \\
5.08 \\
2.92 \\
.50 \\
1.15 \\
3.55\end{array}$ & $\begin{array}{l}2.24 \\
1.62 \\
1.47 \\
1.52 \\
1.25 \\
1.07 \\
2.55 \\
1.97 \\
2.38 \\
2.52 \\
2.84 \\
7.48 \\
4.72 \\
2.07 \\
2.35 \\
7.99\end{array}$ & $\begin{array}{r}4.30 \\
3.13 \\
2.03 \\
2.20 \\
1.33 \\
3.00 \\
6.03 \\
3.72 \\
5.67 \\
6.01 \\
5.36 \\
14.36 \\
9.71 \\
4.07 \\
6.74 \\
12.87\end{array}$ & $\begin{array}{r}6.08 \\
3.96 \\
1.81 \\
1.88 \\
1.61 \\
4.77 \\
7.78 \\
4.95 \\
6.45 \\
7.14 \\
8.92 \\
17.61 \\
9.01 \\
4.70 \\
7.98 \\
17.07\end{array}$ & $\begin{array}{r}R_{5.62} \\
R_{3.33} \\
1.65 \\
1.71 \\
1.47 \\
4.02 \\
R_{5.92} \\
2.83 \\
5.78 \\
6.39 \\
10.33 \\
R_{14.60} \\
9.35 \\
3.46 \\
R_{6.51} \\
17.51\end{array}$ & $\begin{array}{r}R_{5.96} \\
R_{3.38} \\
1.65 \\
1.75 \\
1.43 \\
3.88 \\
R_{6.39} \\
2.76 \\
5.46 \\
6.04 \\
11.09 \\
R_{16.79} \\
9.44 \\
2.51 \\
R_{6.87} \\
18.43\end{array}$ & $\begin{array}{r}R_{5.73} \\
R_{3.31} \\
1.65 \\
1.73 \\
1.42 \\
3.62 \\
R_{6.54} \\
2.31 \\
5.11 \\
5.66 \\
9.50 \\
R_{19.43} \\
9.33 \\
2.51 \\
R_{7.57} \\
18.21\end{array}$ & $\begin{array}{r}5.53 \\
3.20 \\
1.63 \\
1.73 \\
1.36 \\
3.71 \\
6.28 \\
3.15 \\
5.09 \\
5.63 \\
9.32 \\
18.94 \\
9.27 \\
2.54 \\
6.63 \\
17.69\end{array}$ \\
\hline 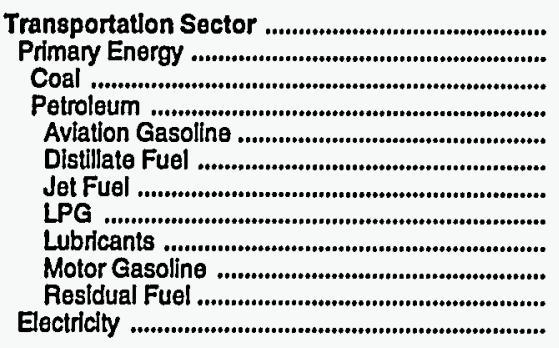 & $\begin{array}{r}2.48 \\
2.48 \\
.32 \\
2.48 \\
2.17 \\
1.35 \\
.72 \\
1.40 \\
5.08 \\
2.92 \\
.42 \\
3.66\end{array}$ & $\begin{array}{l}4.16 \\
4.15 \\
1.25 \\
4.15 \\
3.45 \\
2.64 \\
2.01 \\
2.84 \\
7.48 \\
4.72 \\
1.80 \\
8.41\end{array}$ & $\begin{array}{r}8.86 \\
8.85 \\
-\overline{-} \\
8.85 \\
9.02 \\
7.05 \\
6.27 \\
5.36 \\
14.36 \\
9.71 \\
3.76 \\
15.14\end{array}$ & $\begin{array}{r}8.67 \\
8.65 \\
- \\
8.65 \\
9.99 \\
8.35 \\
5.84 \\
8.92 \\
17.61 \\
9.01 \\
4.14 \\
21.08\end{array}$ & $\begin{array}{r}R_{8.73} \\
R_{8.70} \\
- \\
R_{8.70} \\
9.32 \\
8.79 \\
5.59 \\
10.33 \\
R_{14.60} \\
9.35 \\
2.82 \\
31.66\end{array}$ & $\begin{array}{r}{ }^{R_{8.69}} \\
R_{8.66} \\
R_{8.66} \\
8.71 \\
8.56 \\
4.81 \\
11.09 \\
R_{16.79} \\
9.44 \\
2.10 \\
32.74\end{array}$ & $\begin{array}{r}R_{8.54} \\
R_{8.51} \\
R_{8.51} \\
8.54 \\
8.43 \\
4.49 \\
9.50 \\
R_{19.43} \\
9.33 \\
2.18 \\
33.43\end{array}$ & $\begin{array}{r}8.48 \\
8.45 \\
- \\
8.45 \\
8.24 \\
8.32 \\
4.17 \\
9.32 \\
18.94 \\
9.27 \\
2.15 \\
33.84\end{array}$ \\
\hline 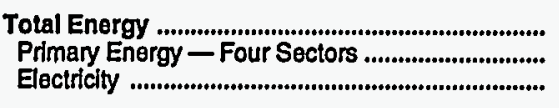 & $\begin{array}{l}1.47 \\
1.15 \\
5.23\end{array}$ & $\begin{array}{r}3.26 \\
2.48 \\
10.37\end{array}$ & $\begin{array}{r}6.41 \\
5.27 \\
15.17\end{array}$ & $\begin{array}{r}8.48 \\
6.37 \\
21.24\end{array}$ & $\begin{array}{l}{ }_{8} 8.59 \\
{ }^{8} 6.11 \\
22.46\end{array}$ & $\begin{array}{l}R_{8.95} \\
A_{6.23} \\
23.51\end{array}$ & $\begin{array}{r}\mathrm{A}_{8.67} \\
\mathrm{R}_{6.01} \\
23.61\end{array}$ & $\begin{array}{r}8.63 \\
6.00 \\
23.26\end{array}$ \\
\hline 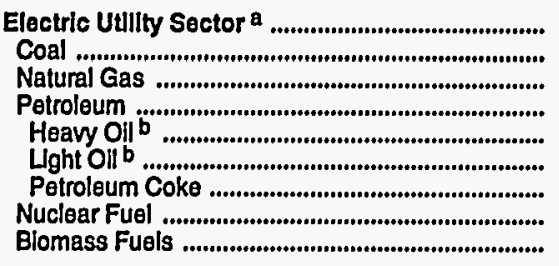 & $\begin{array}{l}.34 \\
.31 \\
.41 \\
.47 \\
.47 \\
.49 \\
.21 \\
.\end{array}$ & $\begin{array}{r}.93 \\
.96 \\
1.47 \\
2.12 \\
2.07 \\
2.27 \\
.25 \\
-\end{array}$ & $\begin{array}{r}1.55 \\
1.33 \\
3.60 \\
4.60 \\
4.52 \\
5.85 \\
.72 \\
.42 \\
-\end{array}$ & $\begin{array}{r}1.61 \\
1.56 \\
5.08 \\
4.30 \\
4.32 \\
5.85 \\
1.27 \\
.92 \\
-\end{array}$ & $\begin{array}{r}1.32 \\
1.52 \\
2.95 \\
3.32 \\
3.31 \\
5.48 \\
.90 \\
.83 \\
-\end{array}$ & $\begin{array}{r}1.28 \\
1.55 \\
2.95 \\
2.48 \\
2.40 \\
4.76 \\
.84 \\
.77 \\
-\end{array}$ & $\begin{array}{r}1.19 \\
1.48 \\
2.97 \\
2.36 \\
2.45 \\
4.31 \\
.78 \\
.68 \\
-\end{array}$ & $\begin{array}{r}1.18 \\
1.44 \\
2.58 \\
2.36 \\
2.40 \\
4.05 \\
.58 \\
.66 \\
-\end{array}$ \\
\hline Prlmary Energy - Flve Sectors c ...................... & .97 & 2.02 & 4.04 & 4.45 & $R_{4.03}$ & $\mathrm{R}_{4.05}$ & $\mathrm{~A}_{3.93}$ & 3.94 \\
\hline
\end{tabular}

a There are no direct fuel costs for hydroelectric, geothermal, wind, photovoltaic, or solar thermal energy.

Heavy oll includes fuel oll nos. 4, 5, and 6, and residual fuel oils. Light oll includes fuel oll nos. 1 and 2, kerosene, and jet fuel.

c Biomass fuels are not included, except those consumed at electric utilitiles and those added to motor gasoline.

R=Revised data.

-No consumption, including cases where adjustments were made. See explanation of adjustments in Section 6 of Appendix A.

Sources: Data sources, estimation procedures, and assumptions are described in Appendix A. 
P Energy Expenditure Estimates by Sector, Pennsylvania

E 1970, 1975, 1980, 1985, and 1990-1993

N (Million Dollars)

\begin{tabular}{|c|c|c|c|c|c|c|c|c|}
\hline Sector and Energy Source & 1970 & 1975 & 1980 & 1985 & 1990 & 1991 & 1992 & 1993 \\
\hline $\begin{array}{l}\text { Residentlal Sector } \\
\text { Primary Energy } \\
\text { Coal } \\
\text { Natural Gas }\end{array}$ & $\begin{array}{r}1,269.9 \\
708.4 \\
48.4 \\
367.4 \\
292.7 \\
245.1 \\
29.9 \\
17.6 \\
561.5\end{array}$ & $\begin{array}{r}2,383.3 \\
1,174.8 \\
59.9 \\
527.3 \\
587.6 \\
517.2 \\
35.8 \\
34.7 \\
1,208.5\end{array}$ & $\begin{array}{r}4,325.2 \\
2,437.1 \\
51.3 \\
1,098.2 \\
1,287.5 \\
1,127.1 \\
107.8 \\
52.6 \\
1,888.1\end{array}$ & $\begin{array}{r}5,704.2 \\
2,910.7 \\
42.7 \\
1,644.9 \\
1,223.1 \\
986.4 \\
140.4 \\
96.3 \\
2,793.4\end{array}$ & $\begin{array}{r}6,121.4 \\
2,602.4 \\
52.2 \\
1,586.5 \\
963.6 \\
776.8 \\
68.0 \\
118.8 \\
3,519.0\end{array}$ & $\begin{array}{r}6,478.3 \\
2,683.3 \\
47.0 \\
1,640.8 \\
995.4 \\
776.0 \\
72.3 \\
147.2 \\
3,795.0\end{array}$ & $\begin{array}{r}6,504.2 \\
2,711.2 \\
52.5 \\
1,759.1 \\
899.6 \\
698.2 \\
67.7 \\
133.7 \\
3,793.0\end{array}$ & $\begin{array}{r}6,836.9 \\
2,877.8 \\
44.2 \\
1,839.9 \\
993.7 \\
803.5 \\
68.7 \\
121.5 \\
3,959.0\end{array}$ \\
\hline 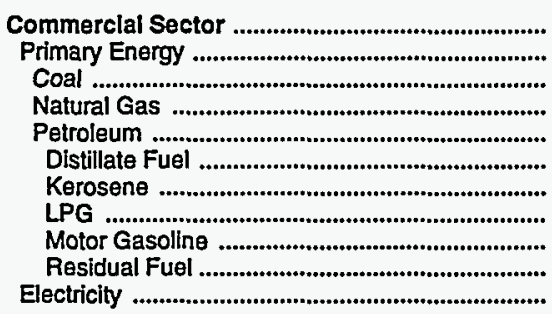 & $\begin{array}{r}507.0 \\
198.6 \\
12.3 \\
95.9 \\
90.4 \\
34.4 \\
1.2 \\
1.8 \\
37.6 \\
15.4 \\
308.4\end{array}$ & $\begin{array}{r}1,111.1 \\
356.8 \\
23.3 \\
169.1 \\
164.3 \\
79.4 \\
2.5 \\
3.9 \\
32.5 \\
46.0 \\
754.2\end{array}$ & $\begin{array}{r}1,969.1 \\
734.4 \\
23.0 \\
422.8 \\
288.6 \\
218.2 \\
6.6 \\
5.5 \\
16.0 \\
42.4 \\
1,234.7\end{array}$ & $\begin{array}{r}2,979.5 \\
1,024.2 \\
24.3 \\
714.6 \\
285.3 \\
194.8 \\
14.5 \\
13.0 \\
21.2 \\
41.8 \\
1,955.3\end{array}$ & $\begin{array}{r}3,527.0 \\
1,049.1 \\
22.4 \\
754.1 \\
272.7 \\
198.8 \\
5.4 \\
16.7 \\
34.2 \\
17.5 \\
2,477.9\end{array}$ & $\begin{array}{r}3,694.9 \\
1,031.2 \\
25.2 \\
753.3 \\
252.7 \\
190.0 \\
4.5 \\
20.8 \\
27.5 \\
10.0 \\
2,663.7\end{array}$ & $\begin{array}{r}\text { R }_{3,775.9} \\
1,045.9 \\
29.7 \\
788.1 \\
228.1 \\
175.6 \\
3.3 \\
18.9 \\
16.4 \\
14.0 \\
\text { ค }_{2,730.0}\end{array}$ & $\begin{array}{r}3,834.3 \\
1,041.4 \\
20.2 \\
789.4 \\
231.8 \\
187.3 \\
5.5 \\
16.8 \\
4.2 \\
17.9 \\
2,792.9\end{array}$ \\
\hline $\begin{array}{l}\text { Industrial Sector } \\
\text { Primary Energy } \\
\text { Coal } \\
\text { Coking Coal } \\
\text { Steam Coal } \\
\text { Natural Gas . } \\
\text { Petroleum } \\
\text { Asphalt and Road Oil } \\
\text { Distillate Fuel } \\
\text { Kerosene } \\
\text { LPG }\end{array}$ & $\begin{array}{r}1,309.6 \\
851.1 \\
381.8 \\
317.5 \\
64.3 \\
186.2 \\
283.1 \\
34.0 \\
38.9 \\
2.5 \\
12.6 \\
77.6 \\
18.1 \\
60.9 \\
38.6 \\
458.4\end{array}$ & $\begin{array}{r}3,123.8 \\
2,031.6 \\
1,085.7 \\
913.7 \\
172.0 \\
266.6 \\
679.3 \\
74.2 \\
144.8 \\
17.1 \\
36.2 \\
102.3 \\
27.2 \\
196.0 \\
81.4 \\
1,092.1\end{array}$ & $\begin{array}{r}5,468.1 \\
3,503.3 \\
1,155.8 \\
1,005.0 \\
150.8 \\
957.9 \\
1,389.6 \\
127.1 \\
358.4 \\
7.1 \\
102.8 \\
240.0 \\
29.9 \\
153.1 \\
371.0 \\
1,964.8\end{array}$ & $\begin{array}{r}5,350.4 \\
2,920.1 \\
647.4 \\
492.9 \\
154.6 \\
1,077.2 \\
1,195.4 \\
161.3 \\
212.3 \\
14.0 \\
145.0 \\
267.9 \\
60.4 \\
70.5 \\
264.1 \\
2,430.3\end{array}$ & $\begin{array}{r}R_{5}, 364.9 \\
R_{2,662.4} \\
629.4 \\
480.0 \\
149.3 \\
943.7 \\
R_{1}, 089.3 \\
140.3 \\
210.8 \\
4.6 \\
109.9 \\
R_{249.9} \\
57.6 \\
107.9 \\
R 208.3 \\
2,702.6\end{array}$ & $\begin{array}{r}R_{5}, 171.8 \\
R_{2,433.7} \\
556.5 \\
412.9 \\
143.6 \\
885.5 \\
R_{991.7} \\
113.6 \\
168.5 \\
4.9 \\
152.4 \\
R_{257.2} \\
62.2 \\
51.1 \\
R 181.8 \\
2,738.1\end{array}$ & $\begin{array}{r}\mathrm{A}_{5,260.9} \\
\mathrm{R}_{2,544.5} \\
605.7 \\
458.8 \\
146.9 \\
848.2 \\
\mathrm{R}_{1,090.7} \\
92.4 \\
184.0 \\
4.5 \\
179.4 \\
\mathrm{R}_{303.4} \\
65.8 \\
52.4 \\
\mathrm{R}_{208.7} \\
2,716.3\end{array}$ & $\begin{array}{r}5,132.6 \\
2,488.9 \\
624.7 \\
479.5 \\
145.1 \\
888.9 \\
975.3 \\
127.2 \\
179.6 \\
7.3 \\
70.8 \\
301.1 \\
46.7 \\
54.7 \\
187.9 \\
2,643.7\end{array}$ \\
\hline 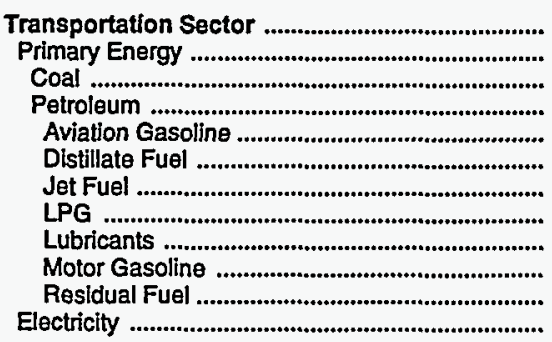 & $\begin{array}{r}1,705.9 \\
1,704.0 \\
.4 \\
1,703.6 \\
7.3 \\
99.5 \\
36.9 \\
.7 \\
40.9 \\
1,503.8 \\
14.6 \\
1.9\end{array}$ & $\begin{array}{r}3,116.1 \\
3,110.5 \\
.1 \\
3,110.4 \\
7.4 \\
254.4 \\
96.2 \\
1.7 \\
49.7 \\
2,635.5 \\
65.5 \\
5.6\end{array}$ & $\begin{array}{r}6,961.5 \\
6,952.3 \\
- \\
6,952.3 \\
15.3 \\
885.1 \\
360.1 \\
2.9 \\
114.3 \\
5,461.1 \\
113.4 \\
9.2\end{array}$ & $\begin{array}{r}6,281.9 \\
6,257.7 \\
- \\
6,257.7 \\
10.5 \\
977.0 \\
334.6 \\
8.0 \\
127.5 \\
4,744.3 \\
55.7 \\
24.1\end{array}$ & $\begin{array}{r}R_{7,025.3} \\
R_{6,988.1} \\
- \\
\text { R }_{6,988.1} \\
6.8 \\
1,220.2 \\
380.7 \\
5.9 \\
R_{119.0} \\
5,155.0 \\
100.5 \\
37.2\end{array}$ & $\begin{array}{r}{ }^{R} 6,966.3 \\
R_{6,928.0} \\
- \\
R_{6,928.0} \\
5.1 \\
1,186.8 \\
309.5 \\
7.5 \\
R_{122.4} \\
5,221.2 \\
75.4 \\
38.2\end{array}$ & $\begin{array}{r}\mathrm{R}_{6,980.8} \\
\mathrm{R}_{6,945.8} \\
- \\
\mathrm{R}_{6,945.8} \\
7.0 \\
1,228.7 \\
277.8 \\
6.5 \\
R_{144.5} \\
5,185.6 \\
R_{95.8} \\
R_{35.0}\end{array}$ & $\begin{array}{r}7,180.0 \\
7,145.9 \\
-\overline{7,145.9} \\
6.2 \\
1,326.4 \\
278.1 \\
6.6 \\
143.4 \\
5,302.9 \\
82.4 \\
34.1\end{array}$ \\
\hline 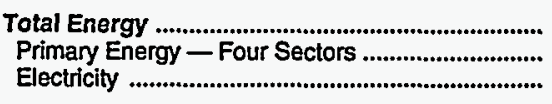 & $\begin{array}{l}4,792.4 \\
3,462.2 \\
1,330.2\end{array}$ & $\begin{array}{l}9,734.3 \\
6,673.8 \\
3,060.5\end{array}$ & $\begin{array}{r}18,723.9 \\
13,627.1 \\
5,096.8\end{array}$ & $\begin{array}{r}20,315.9 \\
13,112.8 \\
7,203.2\end{array}$ & $\begin{array}{r}R_{22,038.6} \\
R_{13,302.0} \\
8,736.6\end{array}$ & $\begin{array}{r}R_{22,311.3} \\
R_{13,076.3} \\
9,235.0\end{array}$ & $\begin{array}{r}R_{22,521.8} \\
R_{13,247.4} \\
9,274.4\end{array}$ & $\begin{array}{r}22,983.6 \\
13,553.9 \\
9,429.7\end{array}$ \\
\hline 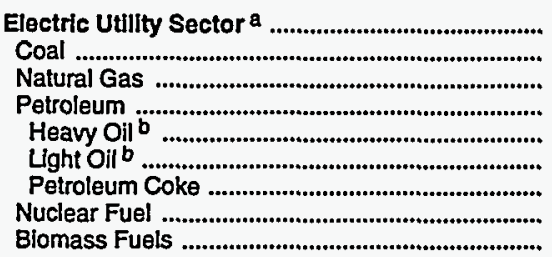 & $\begin{array}{r}296.5 \\
213.6 \\
4.0 \\
77.8 \\
66.6 \\
11.3 \\
-\overline{1.1} \\
-\end{array}$ & $\begin{array}{r}1,047.7 \\
822.7 \\
1.8 \\
178.9 \\
133.8 \\
45.2 \\
- \\
44.3 \\
-\end{array}$ & $\begin{array}{r}1,997.2 \\
1,364.4 \\
10.5 \\
566.8 \\
489.2 \\
76.2 \\
1.4 \\
55.4 \\
-\end{array}$ & $\begin{array}{r}2,233.1 \\
1,592.7 \\
8.0 \\
370.2 \\
315.7 \\
48.5 \\
6.0 \\
262.2 \\
-\end{array}$ & $\begin{array}{r}2,215.1 \\
1,540.7 \\
7.2 \\
155.8 \\
112.5 \\
37.8 \\
5.4 \\
511.5 \\
-\end{array}$ & $\begin{array}{r}2,122.4 \\
1,534.3 \\
6.2 \\
108.0 \\
77.8 \\
25.1 \\
5.0 \\
474.0 \\
-\end{array}$ & $\begin{array}{r}1,992.9 \\
1,481.1 \\
9.5 \\
66.3 \\
43.5 \\
18.0 \\
4.8 \\
436.0\end{array}$ & $\begin{array}{r}1,991.2 \\
1,427.3 \\
22.0 \\
125.2 \\
102.0 \\
19.9 \\
3.2 \\
416.7\end{array}$ \\
\hline Primary Energy — Five Sectors ${ }^{c} \ldots \ldots . . . . . . . . . . . . . . . . . .$. & $3,758.7$ & $\mathbf{7 , 7 2 1 . 4}$ & $15,624.2$ & $15,345.9$ & $\mathrm{R}_{15, \mathbf{5 1 7 . 1}}$ & $R_{15,198.7}$ & $R_{15,240.3}$ & $15,545.1$ \\
\hline
\end{tabular}

a There are no direct fuel costs for hydroelectric, geothermal, wind, photovoltaic, or solar thermal energy.

beavy oil includes tuel oil nos. 4, 5, and 6, and residual fuel oils. Light oil includes fuel nos. 1 and 2, kerosene, and jet fuel.

c Biomass fuels are not included, except those consumed at electric utilities and those added to motor gasoline.

$R=$ Revised data.

- No consumption, including cases where adjustments were made. Seo explanation of adjustments in Section 6 of Appendlx A.

Note: Totals may not equal sum of components due to independent rounding.

Sources: Data sources, estimation procedures, and assumptions are described in Appendix A. 


\begin{tabular}{|c|c|c|c|c|c|c|c|c|}
\hline Energy Source & 1970 & 1975 & 1980 & 1985 & 1890 & 1991 & 1992 & 1993 \\
\hline & \multicolumn{8}{|c|}{ Prices in Dollars per Million Btu } \\
\hline 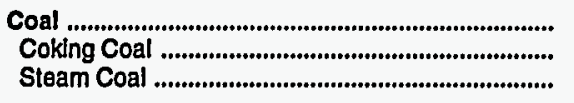 & $\overline{0.94}$ & $2 . \overline{2.64}$ & $\frac{2.37}{2.37}$ & 3.03 & $\frac{3.56}{3.56}$ & 3.51 & $\begin{array}{r}3.43 \\
3.43\end{array}$ & $\begin{array}{l}3.31 \\
3.31\end{array}$ \\
\hline Natural Gas & 1.38 & 2.74 & 5.09 & 6.66 & 5.83 & 5.94 & 5.52 & 5.97 \\
\hline 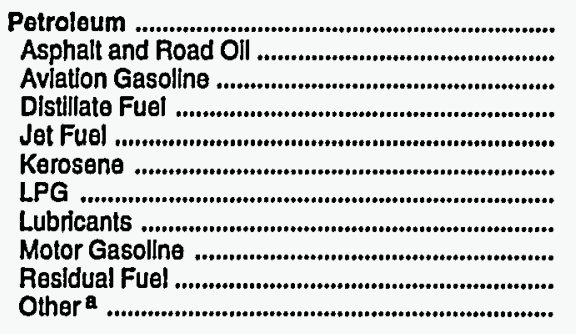 & $\begin{array}{r}1.41 \\
.66 \\
2.17 \\
1.35 \\
.75 \\
1.50 \\
1.77 \\
5.08 \\
2.90 \\
.43 \\
1.86\end{array}$ & $\begin{array}{l}3.17 \\
1.86 \\
3.45 \\
2.76 \\
2.09 \\
2.98 \\
3.50 \\
7.48 \\
4.50 \\
1.92 \\
4.18\end{array}$ & $\begin{array}{r}7.59 \\
3.58 \\
9.02 \\
7.06 \\
6.51 \\
7.44 \\
6.57 \\
14.36 \\
9.72 \\
4.03 \\
8.20\end{array}$ & $\begin{array}{r}7.64 \\
5.18 \\
9.99 \\
8.08 \\
6.10 \\
8.91 \\
11.70 \\
17.61 \\
9.13 \\
4.66 \\
7.37\end{array}$ & $\begin{array}{r}R 8.21 \\
3.34 \\
9.32 \\
8.53 \\
6.03 \\
8.90 \\
12.46 \\
R 14.60 \\
10.03 \\
3.40 \\
9.71\end{array}$ & $\begin{array}{r}R 8.51 \\
3.05 \\
8.71 \\
7.85 \\
5.23 \\
7.85 \\
13.91 \\
16.79 \\
10.09 \\
2.86 \\
9.71\end{array}$ & $\begin{array}{r}R 7.73 \\
2.78 \\
8.54 \\
7.23 \\
4.79 \\
7.19 \\
12.21 \\
\text { R } 19.43 \\
9.83 \\
2.57 \\
9.71\end{array}$ & $\begin{array}{r}7.96 \\
3.30 \\
8.24 \\
7.16 \\
4.49 \\
7.24 \\
12.23 \\
18.94 \\
9.83 \\
2.84 \\
9.71\end{array}$ \\
\hline 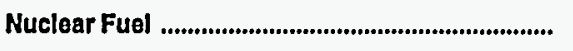 & - & - & - & - & - & - & - & - \\
\hline Blomass Fuels at Utilties ............................................ & - & - & - & - & - & - & - & - \\
\hline Primary Enorgy — Five Sectors b ........................... & 1.40 & 3.11 & 7.06 & 7.43 & ${ }^{\mathrm{R}} 7.57$ & ${ }^{R} 7.54$ & ${ }^{R} 6.76$ & 7.08 \\
\hline $\begin{array}{l}\text { Electric Utillty Fuel } \mathrm{c} \\
\text { Electricity Purchased by End Users }\end{array}$ & $\begin{array}{r}.43 \\
6.85\end{array}$ & $\begin{array}{r}1.84 \\
13.78\end{array}$ & $\begin{array}{r}3.91 \\
20.67\end{array}$ & $\begin{array}{r}3.82 \\
24.73\end{array}$ & $\begin{array}{r}2.60 \\
26.85\end{array}$ & $\begin{array}{r}2.23 \\
29.75\end{array}$ & $\begin{array}{r}2.16 \\
30.12\end{array}$ & $\begin{array}{r}2.92 \\
30.52\end{array}$ \\
\hline \multirow[t]{2}{*}{ 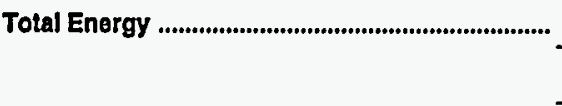 } & 1.91 & 4.16 & 9.06 & 8.67 & ${ }^{R_{10.62}}$ & $\mathrm{R}_{10.53}$ & 9.34 & 9.75 \\
\hline & \multicolumn{8}{|c|}{ Expenditures In Millions of Dollars } \\
\hline 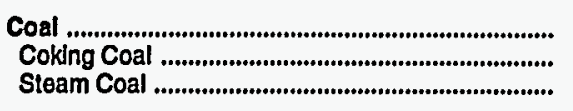 & $\begin{array}{c}0.2 \\
-.2\end{array}$ & $\begin{array}{l}0.4 \\
-.4\end{array}$ & $\begin{array}{l}0.4 \\
. .4\end{array}$ & $\begin{array}{l}0.6 \\
- \\
.6\end{array}$ & $\begin{array}{l}0.5 \\
- \\
.5\end{array}$ & $\begin{array}{l}0.4 \\
-4\end{array}$ & $\begin{array}{l}0.4 \\
-.4\end{array}$ & $\begin{array}{l}0.2 \\
- \\
.2\end{array}$ \\
\hline Natural Gas & 35.2 & 64.3 & 142.7 & 204.6 & 213.7 & 330.8 & 435.4 & 462.7 \\
\hline $\begin{array}{l}\text { Petrolaum } \\
\text { Asphalt and Road Oll } \\
\text { Avlatton Gasoline } \\
\text { Distillate Fuel } \\
\text { Jet Fuel } \\
\text { Kerosene } \\
\text { LPG }\end{array}$ & $\begin{array}{r}234.1 \\
4.1 \\
1.6 \\
67.9 \\
.6 \\
3.7 \\
2.5 \\
3.9 \\
122.0 \\
25.7 \\
2.2\end{array}$ & $\begin{array}{r}434.3 \\
16.5 \\
5.0 \\
128.5 \\
3.2 \\
2.2 \\
6.5 \\
4.4 \\
211.9 \\
52.9 \\
3.4\end{array}$ & $\begin{array}{r}794.4 \\
24.7 \\
12.2 \\
207.0 \\
12.8 \\
3.5 \\
7.1 \\
11.5 \\
429.7 \\
63.9 \\
22.0\end{array}$ & $\begin{array}{r}856.3 \\
102.3 \\
1.5 \\
209.6 \\
17.1 \\
6.8 \\
21.1 \\
12.8 \\
415.5 \\
65.5 \\
4.1\end{array}$ & $\begin{array}{r}\mathrm{R} 825.2 \\
36.3 \\
2.0 \\
230.4 \\
26.4 \\
2.7 \\
22.6 \\
\mathrm{R} 11.9 \\
459.0 \\
30.8 \\
3.0\end{array}$ & $\begin{array}{r}\mathrm{R} 781.8 \\
9.3 \\
1.3 \\
231.7 \\
19.4 \\
2.3 \\
23.4 \\
23.4 \\
\mathrm{R} \\
459.3 \\
19.8 \\
2.4\end{array}$ & $\begin{array}{r}\mathrm{R} 778.6 \\
27.7 \\
1.3 \\
223.4 \\
15.1 \\
2.1 \\
20.2 \\
20.2 \\
\mathrm{R}_{14.5} \\
452.0 \\
19.4 \\
2.9\end{array}$ & $\begin{array}{r}783.6 \\
17.9 \\
.3 \\
228.0 \\
13.4 \\
2.1 \\
22.6 \\
14.4 \\
458.4 \\
23.6 \\
2.9\end{array}$ \\
\hline 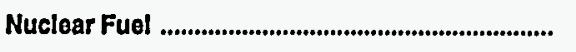 & - & - & - & - & - & - & - & - \\
\hline Blomass Fuels at Utilttes ........................................... & - & - & - & - & - & - & - & - \\
\hline 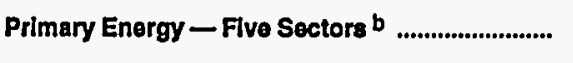 & 269.6 & 498.9 & 937.5 & $1,061.5$ & $R_{1,039.3}$ & $R_{1,113.0}$ & $R_{1,214.4}$ & $1,246.5$ \\
\hline 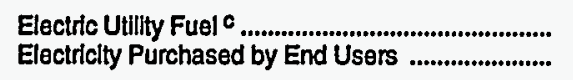 & $\begin{array}{r}-9.3 \\
90.7\end{array}$ & $\begin{aligned}-18.1 \\
209.3\end{aligned}$ & $\begin{array}{r}-47.5 \\
361.9\end{array}$ & $\begin{array}{r}-27.4 \\
458.2\end{array}$ & $\begin{array}{l}-20.6 \\
588.1\end{array}$ & $\begin{array}{r}-5.7 \\
650.0\end{array}$ & $\begin{array}{r}-3.4 \\
657.0\end{array}$ & $\begin{array}{r}-2.5 \\
681.9\end{array}$ \\
\hline 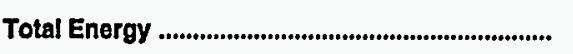 & 350.9 & 690.1 & $1,252.0$ & $1,492.3$ & $R_{1,606.9}$ & $R_{1,757.2}$ & $R_{1,867.9}$ & $1,926.0$ \\
\hline
\end{tabular}

\footnotetext{
ancludes petroleum coke used at electric utllities.

b Blomass fuels are not included, except those consumed at electric uttlities and those added to motor gasoline.

c There are no direct fuel costs for hydroelectric, geothermal, wind, photovoltalc, or solar thermal energy.

$R=$ Rovised data.
}

-No consumption, including cases where adjustments were made. See explanation of adjustments in Section 6 of Appendix A.

Note: Expenditure totals may not equal sum of components due to independent rounding.

Sources: Data sources, estimation procedures, and assumptions are described in Appendix A. 
R Energy Price Estimates by Sector, Rhode Island

H 1970, 1975, 1980, 1985, and 1990-1993

O (Dollars per Million Btu)

\begin{tabular}{|c|c|c|c|c|c|c|c|c|}
\hline Sector and Energy Source & 1970 & 1975 & 1980 & 1985 & 1990 & 1991 & 1992 & 1993 \\
\hline $\begin{array}{l}\text { ResIdentlal Sector } \\
\text { Primary Energy } \\
\text { Coal ... } \\
\text { Natural Gas } \\
\text { Petroleum } \\
\text { Distillate Fuel } \\
\text { Kerosene } \\
\text { LPG } \\
\text { Electricity }\end{array}$ & $\begin{array}{r}2.19 \\
1.59 \\
.98 \\
1.79 \\
1.52 \\
1.49 \\
1.70 \\
2.55 \\
8.44\end{array}$ & $\begin{array}{r}4.33 \\
2.94 \\
2.62 \\
3.04 \\
2.90 \\
2.85 \\
3.16 \\
5.49 \\
15.43\end{array}$ & $\begin{array}{r}9.08 \\
6.60 \\
4.47 \\
5.58 \\
7.33 \\
7.29 \\
8.15 \\
8.57 \\
22.64\end{array}$ & $\begin{array}{r}10.90 \\
8.03 \\
4.39 \\
7.62 \\
8.33 \\
8.15 \\
8.93 \\
11.44 \\
26.77\end{array}$ & $\begin{array}{r}11.84 \\
7.82 \\
4.21 \\
7.03 \\
8.73 \\
8.38 \\
9.18 \\
13.81 \\
28.86\end{array}$ & $\begin{array}{r}12.38 \\
7.78 \\
4.07 \\
7.42 \\
8.17 \\
7.71 \\
8.44 \\
15.22 \\
32.17\end{array}$ & $\begin{array}{r}11.64 \\
7.45 \\
3.94 \\
7.54 \\
7.37 \\
7.04 \\
7.71 \\
13.80 \\
32.74\end{array}$ & $\begin{array}{r}11.93 \\
7.60 \\
3.96 \\
7.94 \\
7.27 \\
6.89 \\
7.55 \\
13.58 \\
33.42\end{array}$ \\
\hline $\begin{array}{l}\text { Commerclal Sector } \\
\text { Primary Energy } \\
\text { Coal ... } \\
\text { Natural Gas } \\
\text { Petroleum } \\
\text { Distillate Fuel } \\
\text { Kerosene } \\
\text { LPG } \\
\text { Motor Gasoline } \\
\text { Residual Fuel } \\
\text { Electricity }\end{array}$ & $\begin{array}{r}2.08 \\
1.00 \\
.90 \\
1.44 \\
.85 \\
1.10 \\
.78 \\
1.20 \\
2.90 \\
.44 \\
7.02\end{array}$ & $\begin{array}{r}5.23 \\
2.39 \\
2.65 \\
2.71 \\
2.28 \\
2.44 \\
2.59 \\
2.66 \\
4.50 \\
1.81 \\
13.84\end{array}$ & $\begin{array}{r}10.70 \\
5.44 \\
1.67 \\
5.00 \\
6.05 \\
6.46 \\
5.29 \\
9.72 \\
3.96 \\
20.45\end{array}$ & $\begin{array}{r}12.55 \\
6.36 \\
2.38 \\
6.45 \\
6.28 \\
7.45 \\
8.34 \\
12.03 \\
9.13 \\
4.96 \\
24.56\end{array}$ & $\begin{array}{r}13.12 \\
5.79 \\
2.62 \\
6.04 \\
5.57 \\
7.25 \\
8.26 \\
10.80 \\
10.03 \\
3.35 \\
26.28\end{array}$ & $\begin{array}{r}13.63 \\
5.47 \\
2.65 \\
5.87 \\
5.09 \\
6.39 \\
6.57 \\
11.94 \\
10.09 \\
2.92 \\
28.97\end{array}$ & $\begin{array}{r}14.06 \\
5.56 \\
2.67 \\
6.21 \\
4.74 \\
6.14 \\
5.87 \\
9.96 \\
9.83 \\
2.71 \\
29.41\end{array}$ & $\begin{array}{r}14.19 \\
5.78 \\
2.33 \\
6.90 \\
4.47 \\
5.87 \\
6.07 \\
10.02 \\
9.83 \\
2.82 \\
30.08\end{array}$ \\
\hline 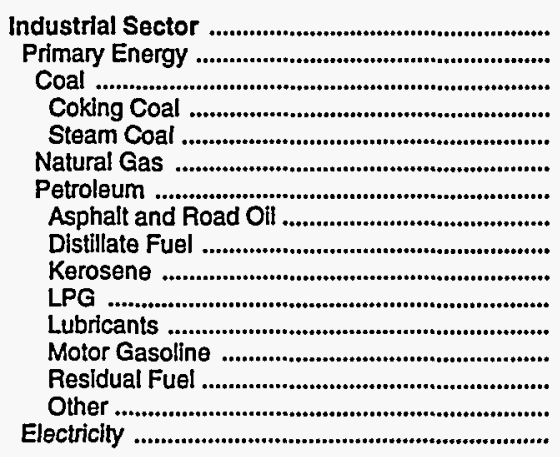 & $\begin{array}{r}1.06 \\
.66 \\
.90 \\
-\overline{0} \\
.90 \\
.85 \\
.62 \\
.66 \\
.71 \\
.78 \\
1.20 \\
5.08 \\
2.90 \\
.42 \\
1.86 \\
4.83\end{array}$ & $\begin{array}{r}3.20 \\
2.15 \\
2.65 \\
-\overline{2.65} \\
2.10 \\
2.17 \\
1.86 \\
2.34 \\
2.59 \\
2.66 \\
7.48 \\
4.50 \\
2.05 \\
4.18 \\
11.36\end{array}$ & $\begin{array}{r}7.27 \\
4.91 \\
1.67 \\
- \\
1.67 \\
4.45 \\
5.07 \\
3.58 \\
5.65 \\
6.18 \\
5.29 \\
14.36 \\
9.72 \\
4.24 \\
8.20 \\
18.39\end{array}$ & $\begin{array}{r}7.48 \\
5.59 \\
2.38 \\
- \\
2.38 \\
5.70 \\
5.58 \\
5.18 \\
7.28 \\
8.34 \\
12.03 \\
17.61 \\
9.13 \\
4.96 \\
7.37 \\
21.93\end{array}$ & $\begin{array}{r}R_{8.13} \\
R_{4.56} \\
R_{2.62} \\
- \\
R_{2.62} \\
5.18 \\
R_{4.39} \\
3.34 \\
7.21 \\
8.26 \\
10.80 \\
R_{14.60} \\
10.03 \\
3.35 \\
9.71 \\
24.46\end{array}$ & $\begin{array}{r}R_{7.70} \\
R_{5.17} \\
- \\
- \\
- \\
5.25 \\
R_{4.90} \\
3.05 \\
5.74 \\
6.57 \\
11.94 \\
R 16.79 \\
10.09 \\
2.92 \\
9.71 \\
27.09\end{array}$ & $\begin{array}{r}R_{5.90} \\
R_{4.39} \\
- \\
- \\
- \\
4.58 \\
R_{3.80} \\
2.78 \\
5.12 \\
5.87 \\
9.96 \\
R_{19.43} \\
9.83 \\
2.71 \\
9.71 \\
26.96\end{array}$ & $\begin{array}{r}6.48 \\
4.86 \\
- \\
- \\
- \\
4.97 \\
4.44 \\
3.30 \\
5.30 \\
6.07 \\
10.02 \\
18.94 \\
9.83 \\
2.82 \\
9.71 \\
26.44\end{array}$ \\
\hline 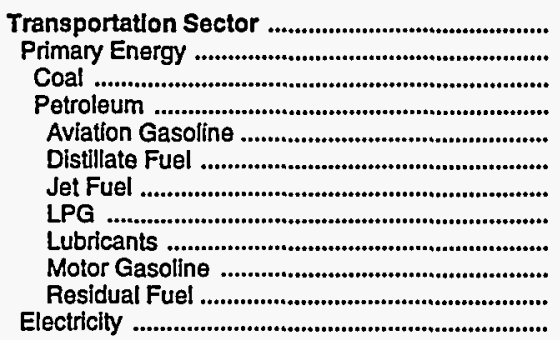 & $\begin{array}{r}2.17 \\
2.17 \\
- \\
2.17 \\
2.17 \\
1.36 \\
.75 \\
1.20 \\
5.08 \\
2.90 \\
.41 \\
-\end{array}$ & $\begin{array}{r}4.19 \\
4.19 \\
2.65 \\
4.19 \\
3.45 \\
2.90 \\
2.09 \\
2.66 \\
7.48 \\
4.50 \\
1.71 \\
-\end{array}$ & $\begin{array}{r}9.40 \\
9.40 \\
-\overline{9} \\
9.40 \\
9.02 \\
7.41 \\
6.51 \\
5.29 \\
14.36 \\
9.72 \\
3.34 \\
-\end{array}$ & $\begin{array}{r}9.03 \\
9.03 \\
- \\
9.03 \\
9.99 \\
8.89 \\
6.10 \\
12.03 \\
17.61 \\
9.13 \\
- \\
-\end{array}$ & $\begin{array}{r}R_{9.72} \\
R_{9.72} \\
- \\
R_{9.72} \\
9.32 \\
9.93 \\
6.03 \\
10.80 \\
R_{14.60} \\
10.03 \\
3.42 \\
-\end{array}$ & $\begin{array}{r}R_{9.71} \\
R_{9.71} \\
{ }^{8} 9.71 \\
8.71 \\
9.39 \\
5.23 \\
11.94 \\
\text { R } 16.79 \\
10.09 \\
2.16 \\
-\end{array}$ & $\begin{array}{r}R_{9.45} \\
R_{9.45} \\
- \\
R_{9.45} \\
8.54 \\
8.91 \\
4.79 \\
9.96 \\
R_{19.43} \\
9.83 \\
1.86 \\
-\end{array}$ & $\begin{array}{r}9.49 \\
9.49 \\
- \\
9.49 \\
8.24 \\
9.00 \\
4.49 \\
10.02 \\
18.94 \\
9.83 \\
2.96 \\
-\end{array}$ \\
\hline 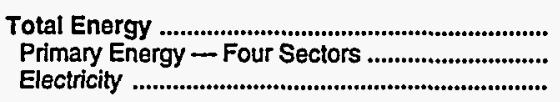 & $\begin{array}{l}1.91 \\
1.53 \\
6.85\end{array}$ & $\begin{array}{r}4.16 \\
3.19 \\
13.78\end{array}$ & $\begin{array}{r}9.06 \\
7.37 \\
20.67\end{array}$ & $\begin{array}{r}9.67 \\
7.62 \\
24.73\end{array}$ & $\begin{array}{r}R_{10.62} \\
7.87 \\
26.85\end{array}$ & $\begin{array}{r}R_{10.53} \\
7.63 \\
29.75\end{array}$ & $\begin{array}{r}9.34 \\
R_{6.80} \\
30.12\end{array}$ & $\begin{array}{r}9.75 \\
7.10 \\
30.52\end{array}$ \\
\hline 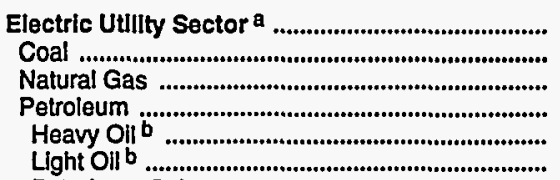 & $\begin{array}{r}.43 \\
-39 \\
.44 \\
.44 \\
.48\end{array}$ & $\begin{array}{l}1.84 \\
1.15 \\
1.84 \\
1.84 \\
2.00\end{array}$ & $\begin{array}{l}3.91 \\
-\overline{3} \\
4.00 \\
3.97 \\
6.03\end{array}$ & $\begin{array}{l}3.82 \\
3.37 \\
4.08 \\
4.03 \\
5.83\end{array}$ & $\begin{array}{l}2.60 \\
2.17 \\
3.68 \\
3.59 \\
5.53\end{array}$ & $\begin{array}{l}2.23 \\
1.98 \\
2.70 \\
2.41 \\
4.70\end{array}$ & $\begin{array}{l}2.16 \\
2.13 \\
2.17 \\
1.95 \\
4.43\end{array}$ & $\begin{array}{l}2.92 \\
2.39 \\
3.41 \\
3.20 \\
4.11\end{array}$ \\
\hline 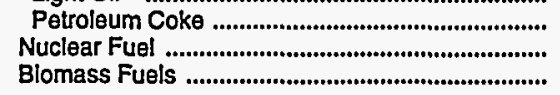 & $\begin{array}{l}- \\
-\end{array}$ & $\overline{-}$ & $\overline{-}$ & $\overline{-}$ & $\overline{-}$ & $\begin{array}{l}- \\
-\end{array}$ & $\overline{-}$ & - \\
\hline Primary Energy - Flve Sectors $c \ldots \ldots \ldots \ldots \ldots . . . . . . . . .$. & 1.40 & 3.11 & 7.06 & 7.43 & $\mathrm{P}_{7.57}$ & $\mathrm{R}_{7.54}$ & $\mathbf{R}_{6.76}$ & 7.08 \\
\hline
\end{tabular}

a There are no direct fuel costs for hydroelectric, geothermal, wind, photovoltaic, or solar thermal energy.

beavy oil includes fuel oil nos. 4,5 , and 6 , and residual fuel oils. Light oll includes fuel oil nos. 1 and 2 , kerosene, and jet fuel.

c Biomass fuels are not included, except those consumed at electric utilities and those added to motor gasoline.

R=Revised data.

- No consumption, including cases where adjustments were made. Se日 explanation of adjustments in Sectlon 6 of Appendix A.

Sources: Data sources, estimation procedures, and assumptions are described in Appendix A. 


\begin{tabular}{|c|c|c|c|c|c|c|c|c|}
\hline Sector and Energy Source & 1970 & 1975 & 1980 & 1985 & 1990 & 1991 & 1992 & 1993 \\
\hline 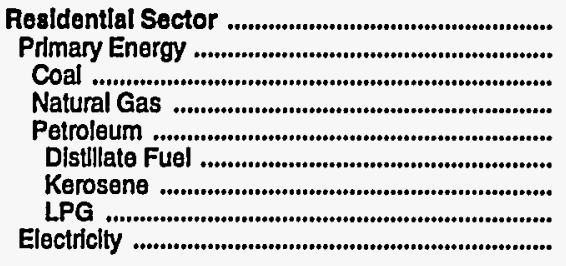 & $\begin{array}{r}117.5 \\
77.5 \\
.1 \\
21.9 \\
55.5 \\
50.7 \\
3.2 \\
1.5 \\
40.0\end{array}$ & $\begin{array}{r}223.2 \\
134.5 \\
.1 \\
40.2 \\
94.1 \\
89.6 \\
1.6 \\
3.0 \\
88.7\end{array}$ & $\begin{array}{r}367.9 \\
225.8 \\
.2 \\
79.5 \\
146.1 \\
140.0 \\
2.5 \\
3.6 \\
142.1\end{array}$ & $\begin{array}{r}478.8 \\
298.8 \\
.3 \\
.3 \\
118.0 \\
180.5 \\
162.4 \\
6.6 \\
11.5 \\
180.0\end{array}$ & $\begin{array}{r}502.8 \\
268.8 \\
.3 \\
128.0 \\
140.5 \\
124.6 \\
2.0 \\
13.9 \\
234.0\end{array}$ & $\begin{array}{r}530.5 \\
270.5 \\
.2 \\
132.5 \\
137.7 \\
120.7 \\
1.7 \\
15.4 \\
260.0\end{array}$ & \begin{tabular}{r|}
566.9 \\
302.9 \\
.3 \\
153.6 \\
149.0 \\
134.0 \\
1.6 \\
13.4 \\
264.0
\end{tabular} & $\begin{array}{r}585.2 \\
310.3 \\
.2 \\
161.1 \\
149.0 \\
131.6 \\
1.7 \\
15.6 \\
275.0\end{array}$ \\
\hline 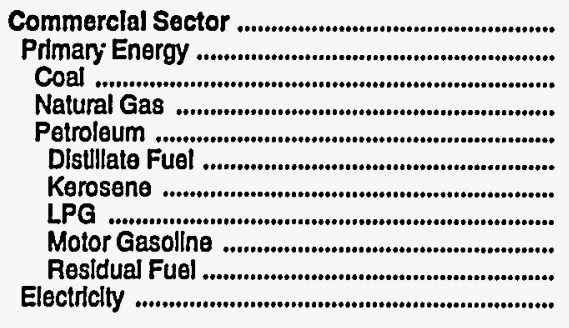 & $\begin{array}{r}51.1 \\
20.3 \\
.1 \\
7.5 \\
12.8 \\
9.4 \\
(s) \\
.1 \\
.6 \\
2.7 \\
30.8\end{array}$ & $\begin{array}{r}113.5 \\
39.1 \\
.1 \\
11.6 \\
27.3 \\
19.3 \\
(8) \\
.3 \\
1.0 \\
6.9 \\
74.4\end{array}$ & $\begin{array}{r}197.1 \\
65.1 \\
(s) \\
34.5 \\
30.6 \\
23.2 \\
-\overline{.4} \\
2.5 \\
4.5 \\
132.0\end{array}$ & $\begin{array}{r}271.9 \\
90.9 \\
.1 \\
50.6 \\
40.2 \\
19.2 \\
.2 \\
2.1 \\
1.5 \\
17.2 \\
181.0\end{array}$ & $\begin{array}{r}336.4 \\
95.4 \\
.1 \\
50.1 \\
45.2 \\
28.4 \\
.1 \\
1.9 \\
2.0 \\
12.7 \\
241.0\end{array}$ & $\begin{array}{r}357.8 \\
93.8 \\
.1 \\
49.9 \\
43.8 \\
28.9 \\
(s) \\
2.1 \\
1.9 \\
10.8 \\
264.0\end{array}$ & $\begin{array}{r}359.4 \\
91.5 \\
.1 \\
57.4 \\
33.9 \\
21.6 \\
.1 \\
1.7 \\
1.7 \\
8.9 \\
267.9\end{array}$ & $\begin{array}{r}380.3 \\
101.3 \\
.1 \\
65.4 \\
35.9 \\
21.9 \\
.1 \\
2.0 \\
.5 \\
11.4 \\
279.0\end{array}$ \\
\hline 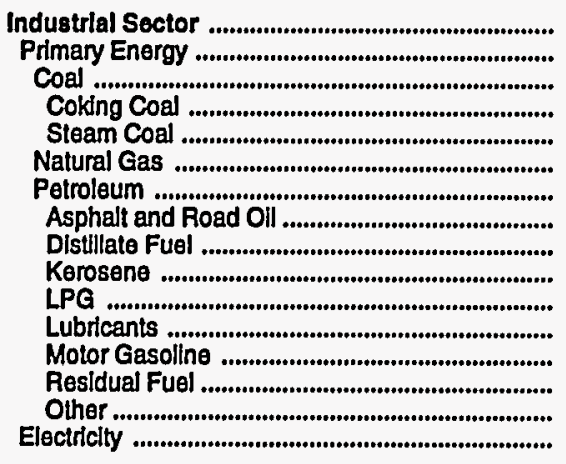 & $\begin{array}{r}45.0 \\
25.1 \\
(s) \\
-\bar{s} \\
5.0 \\
20.1 \\
4.1 \\
2.8 \\
.4 \\
.7 \\
1.5 \\
(s) \\
8.3 \\
2.2 \\
19.9\end{array}$ & $\begin{array}{r}114.7 \\
68.5 \\
.1 \\
- \\
.1 \\
12.4 \\
56.0 \\
16.5 \\
6.0 \\
.6 \\
2.9 \\
1.8 \\
.1 \\
24.7 \\
3.4 \\
46.2\end{array}$ & $\begin{array}{r}198.3 \\
110.5 \\
.2 \\
- \\
.2 \\
23.1 \\
87.2 \\
24.7 \\
13.6 \\
1.1 \\
2.9 \\
5.4 \\
.1 \\
17.4 \\
22.0 \\
87.8\end{array}$ & $\begin{array}{r}285.7 \\
188.5 \\
.2 \\
- \\
.2 \\
27.2 \\
161.0 \\
102.3 \\
10.5 \\
(5) \\
6.5 \\
6.0 \\
1.3 \\
30.3 \\
4.1 \\
97.3\end{array}$ & $\begin{array}{r}\mathrm{R}_{209.3} \\
\mathrm{R}_{96.3} \\
(\mathrm{~s}) \\
\overline{(\mathrm{s})} \\
23.3 \\
\mathrm{R} 73.0 \\
36.3 \\
9.9 \\
.7 \\
6.1 \\
\mathrm{R} 5.6 \\
1.8 \\
9.7 \\
3.0 \\
113.0\end{array}$ & 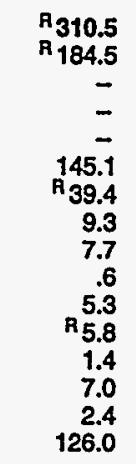 & $\begin{array}{r}R 408.3 \\
R_{283.3} \\
- \\
= \\
\\
223.3 \\
{ }^{2} 60.0 \\
27.7 \\
8.4 \\
.4 \\
4.6 \\
R_{6.8} \\
1.4 \\
7.8 \\
2.9 \\
125.0\end{array}$ & $\begin{array}{r}417.8 \\
289.9 \\
- \\
- \\
235.2 \\
54.6 \\
17.9 \\
8.9 \\
.3 \\
4.7 \\
6.8 \\
2.5 \\
10.7 \\
2.9 \\
128.0\end{array}$ \\
\hline 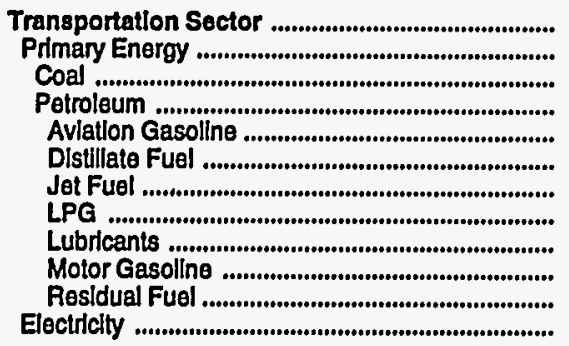 & $\begin{array}{r}137.4 \\
137.4 \\
- \\
137.4 \\
1.6 \\
4.8 \\
.6 \\
.1 \\
2.4 \\
121.4 \\
6.5 \\
-\end{array}$ & $\begin{array}{r}238.7 \\
238.7 \\
(s) \\
238.7 \\
5.0 \\
13.3 \\
3.2 \\
.3 \\
2.6 \\
210.8 \\
3.5 \\
-\end{array}$ & $\begin{array}{r}488.7 \\
488.7 \\
- \\
488.7 \\
12.2 \\
29.2 \\
12.8 \\
.2 \\
6.1 \\
427.1 \\
1.2 \\
-\end{array}$ & $\begin{array}{r}455.9 \\
455.9 \\
- \\
455.9 \\
1.5 \\
16.9 \\
17.1 \\
1.0 \\
6.8 \\
412.7 \\
- \\
-\end{array}$ & $\begin{array}{r}R_{558.3} \\
R_{558.3} \\
R_{558.3} \\
2.0 \\
66.9 \\
26.4 \\
.8 \\
R_{6.3} \\
455.2 \\
.7 \\
-\end{array}$ & $\begin{array}{r}R_{558.5} \\
R_{558.5} \\
- \\
R_{558.5} \\
1.3 \\
74.0 \\
19.4 \\
.6 \\
R_{6.5} \\
456.5 \\
.1 \\
-\end{array}$ & $\begin{array}{r}R_{533.2} \\
R_{533.2} \\
- \\
R_{533.2} \\
1.3 \\
59.0 \\
15.1 \\
.5 \\
7.7 \\
449.0 \\
.7 \\
-\end{array}$ & $\begin{array}{r}542.6 \\
542.6 \\
542.6 \\
.3 \\
65.2 \\
13.4 \\
.3 \\
7.6 \\
455.4 \\
.4 \\
-\end{array}$ \\
\hline 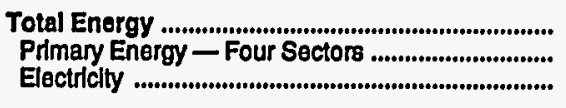 & $\begin{array}{r}350.9 \\
260.3 \\
90.7\end{array}$ & $\begin{array}{l}690.1 \\
480.8 \\
209.3\end{array}$ & $\begin{array}{r}1,252.0 \\
890.1 \\
361.9\end{array}$ & $\begin{array}{r}1,492.3 \\
1,034.1 \\
458.2\end{array}$ & $\begin{array}{r}R_{1,606.9} \\
R_{1,018.8} \\
588.1\end{array}$ & $\begin{array}{r}R_{1}, 757.2 \\
R_{1,107.2} \\
650.0\end{array}$ & $\begin{array}{r}R_{1}, 867.9 \\
R_{1,210.9} \\
657.0\end{array}$ & $\begin{array}{r}1,926.0 \\
1,244.0 \\
681.9\end{array}$ \\
\hline 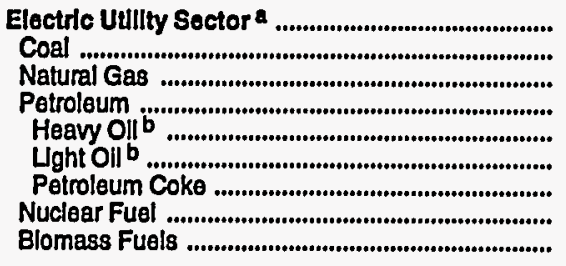 & $\begin{array}{r}8.3 \\
.9 \\
8.4 \\
8.2 \\
.2 \\
= \\
-\end{array}$ & $\begin{array}{r}18.1 \\
\overline{(s)} \\
18.1 \\
17.8 \\
.3 \\
- \\
- \\
-\end{array}$ & $\begin{array}{r}47.5 \\
5.7 \\
41.8 \\
40.8 \\
1.0 \\
= \\
-\end{array}$ & $\begin{array}{r}27.4 \\
\overline{8.8} \\
18.6 \\
17.9 \\
.7 \\
\overline{-} \\
-\end{array}$ & $\begin{array}{r}20.6 \\
12.3 \\
8.3 \\
7.7 \\
.6 \\
= \\
=\end{array}$ & $\begin{array}{r}5.7 \\
- \\
3.3 \\
2.4 \\
1.9 \\
.5 \\
= \\
-\end{array}$ & $\begin{array}{l}3.4 \\
\overline{1.0} \\
2.4 \\
2.0 \\
.4 \\
\overline{-} \\
=\end{array}$ & $\begin{array}{r}2.5 \\
1.0 \\
1.5 \\
1.1 \\
.4 \\
= \\
=\end{array}$ \\
\hline Primary Energy - Flve Sectors ${ }^{\circ}$............................ & 269.6 & 488.9 & 937.5 & $1,061.5$ & $R_{1,039.3}$ & $R_{1,113.0}$ & $n_{1,214.4}$ & $1,246.5$ \\
\hline
\end{tabular}

a There are no direct fuel costs for hydroelectric, geothermal, wind, photovoltalc, or solar thermal energy.

beavy oll inciudes fuel oll nos. 4, 5, and 6, and residual fuel olls. Light oll includes fuel nos. 1 and 2, kerosene, and jet fuel.

- Blomass fuels are not Included, except those consumed at electric uttilttles and those added to motor gasoline.

$\mathrm{P}=$ Revised data.
-No consumption, Including cases where adjustments were made. See explanation of adjustments in Section 6 of Appendlx A.

$(s)=$ Value less than 0.05 million dollars.

Note: Totals may not equal sum of components due to Independent rounding.

Sources: Data sources, estimation procedures, and assumptions are described in Appendix A. 


\section{S Energy Price and Expenditure Estimates by Source, South Carolina}

$1970,1975,1980,1985$, and 1990-1993

\begin{tabular}{|c|c|c|c|c|c|c|c|c|}
\hline Enargy Source & 1970 & 1975 & 1980 & 1985 & 1990 & 1991 & 1992 & 1993 \\
\hline & \multicolumn{8}{|c|}{ Prices In Dollars per Million Btu } \\
\hline 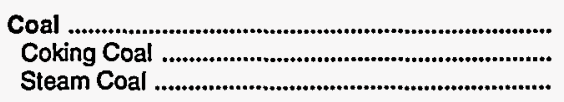 & $\begin{array}{r}0.47 \\
.47\end{array}$ & $\begin{array}{r}1.25 \\
1.25\end{array}$ & $\begin{array}{r}1.60 \\
1.60\end{array}$ & $\frac{1.88}{1.86}$ & $\begin{array}{r}1.73 \\
1.73\end{array}$ & $\frac{1.64}{1.64}$ & $\begin{array}{r}1.57 \\
- \\
1.57\end{array}$ & $\begin{array}{r}1.60 \\
1.60\end{array}$ \\
\hline 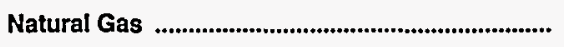 & .57 & 1.16 & 3.07 & 5.06 & 4.01 & 3.66 & 3.96 & 4.19 \\
\hline $\begin{array}{l}\text { Petroleum } \\
\text { Asphalt and Road Oil } \\
\text { Aviation Gasoline . } \\
\text { Distillate Fuel } \\
\text { Jet Fuel } \\
\text { Kerosene } \\
\text { LPG } \\
\text { Motor Gasoline } \\
\text { Residual Fuel } \\
\text { Other }{ }^{\mathrm{a}}\end{array}$ & $\begin{array}{r}1.89 \\
.67 \\
2.17 \\
1.03 \\
.73 \\
1.43 \\
2.04 \\
5.08 \\
2.75 \\
.42 \\
1.58\end{array}$ & $\begin{array}{l}3.43 \\
1.78 \\
3.45 \\
2.68 \\
2.03 \\
3.02 \\
3.56 \\
7.48 \\
4.35 \\
1.40 \\
3.34\end{array}$ & $\begin{array}{r}8.07 \\
3.58 \\
9.02 \\
6.84 \\
6.46 \\
7.92 \\
5.77 \\
14.36 \\
10.18 \\
3.43 \\
6.70\end{array}$ & $\begin{array}{r}7.98 \\
4.99 \\
9.99 \\
7.06 \\
6.11 \\
8.01 \\
9.87 \\
17.61 \\
8.84 \\
4.36 \\
6.03\end{array}$ & $\begin{array}{r}R_{7.84} \\
2.90 \\
9.32 \\
7.68 \\
6.07 \\
8.30 \\
10.35 \\
R_{14.60} \\
8.80 \\
3.11 \\
R_{4.69}\end{array}$ & $\begin{array}{r}R_{7.55} \\
3.06 \\
8.71 \\
7.21 \\
5.12 \\
7.75 \\
10.82 \\
R_{16.79} \\
8.56 \\
2.42 \\
R_{4.32}\end{array}$ & $\begin{array}{r}R_{7.16} \\
2.27 \\
8.54 \\
6.62 \\
4.82 \\
6.33 \\
9.35 \\
R_{19.43} \\
8.25 \\
2.50 \\
R_{3.97}\end{array}$ & $\begin{array}{r}7.04 \\
2.93 \\
8.24 \\
6.51 \\
4.49 \\
6.43 \\
9.20 \\
18.94 \\
8.20 \\
2.25 \\
3.81\end{array}$ \\
\hline 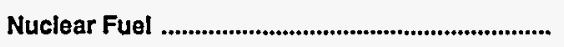 & .19 & .19 & .44 & .62 & .53 & .52 & .50 & .50 \\
\hline Blomass Fuels at Utilities & - & - & - & - & - & - & - & - \\
\hline Primary Energy - Five Sectors ${ }^{b} \ldots . . . . . . . . . . . . . . . . .$. & 1.20 & 1.86 & 4.09 & 3.80 & 3.45 & 3.31 & 3.13 & 3.11 \\
\hline $\begin{array}{l}\text { Electric Utility Fuel c ................................................... } \\
\text { Electricity Purchased by End Users .................... }\end{array}$ & $\begin{array}{r}.42 \\
3.98\end{array}$ & $\begin{array}{r}.56 \\
7.72\end{array}$ & $\begin{array}{r}1.14 \\
11.11\end{array}$ & $\begin{array}{r}1.11 \\
15.99\end{array}$ & $\begin{array}{r}.94 \\
16.39\end{array}$ & $\begin{array}{r}.90 \\
16.50\end{array}$ & $\begin{array}{r}.84 \\
16.21\end{array}$ & $\begin{array}{r}.88 \\
16.53\end{array}$ \\
\hline \multirow[t]{2}{*}{ Total Energy } & 1.83 & 3.79 & 7.25 & 8.85 & 8.89 & $R_{8.77}$ & 8.51 & 8.57 \\
\hline & \multicolumn{8}{|c|}{ Expenditures In Millions of Dollars } \\
\hline 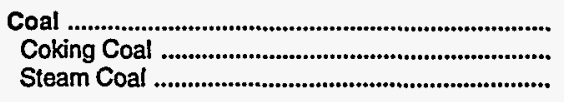 & $\frac{65.3}{65.3}$ & $\begin{array}{c}174.7 \\
174.7\end{array}$ & $\begin{array}{c}392.2 \\
- \\
392.2\end{array}$ & $\begin{array}{c}493.6 \\
-\overline{493.6}\end{array}$ & $\begin{array}{c}499.0 \\
- \\
499.0\end{array}$ & $\begin{array}{c}478.2 \\
- \\
478.2\end{array}$ & $\begin{array}{c}451.3 \\
- \\
451.3\end{array}$ & $\begin{array}{c}528.2 \\
- \\
528.2\end{array}$ \\
\hline Natural Gas & 91.4 & 143.3 & 441.2 & 495.3 & 525.9 & 492.8 & 549.8 & 598.9 \\
\hline 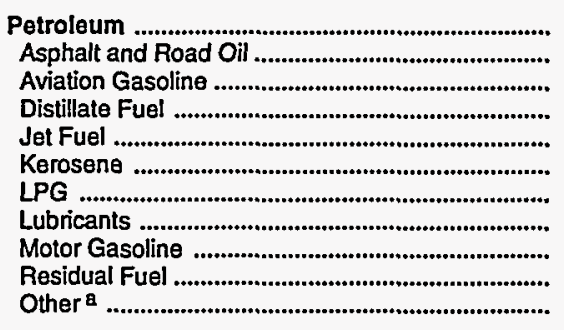 & $\begin{array}{r}569.7 \\
9.9 \\
2.5 \\
56.7 \\
12.4 \\
19.3 \\
22.6 \\
11.9 \\
415.8 \\
14.2 \\
4.4\end{array}$ & $\begin{array}{r}1,166.8 \\
28.9 \\
2.5 \\
130.7 \\
29.5 \\
17.5 \\
42.3 \\
20.9 \\
809.3 \\
67.5 \\
17.5\end{array}$ & $\begin{array}{r}2,937.1 \\
36.4 \\
6.8 \\
424.9 \\
107.1 \\
60.7 \\
67.4 \\
47.3 \\
1,899.0 \\
155.3 \\
132.2\end{array}$ & $\begin{array}{r}2,808.2 \\
45.3 \\
6.9 \\
482.8 \\
105.3 \\
67.4 \\
112.4 \\
52.8 \\
1,751.7 \\
80.0 \\
103.7\end{array}$ & $\begin{array}{r}R_{3,144.9} \\
38.1 \\
4.8 \\
650.4 \\
97.4 \\
31.0 \\
109.3 \\
R_{49.2} \\
1,987.6 \\
47.9 \\
R_{129.2}\end{array}$ & $\begin{array}{r}R_{3,079.0} \\
39.4 \\
7.9 \\
642.3 \\
95.8 \\
37.4 \\
141.0 \\
R_{50.7} \\
1,912.1 \\
37.0 \\
R_{115.6}\end{array}$ & $\begin{array}{r}R_{2,869.0} \\
31.1 \\
9.7 \\
529.5 \\
68.0 \\
18.8 \\
121.8 \\
R_{59.8} \\
1,869.9 \\
37.7 \\
R_{122.7}\end{array}$ & $\begin{array}{r}2,924.8 \\
45.8 \\
7.0 \\
517.4 \\
49.7 \\
27.7 \\
121.5 \\
59.3 \\
1,940.1 \\
53.9 \\
102.3\end{array}$ \\
\hline 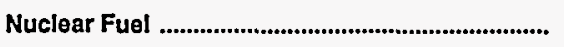 & (8) & 40.6 & 83.4 & 214.5 & 242.8 & 240.5 & 241.2 & 247.1 \\
\hline 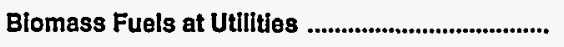 & - & - & - & - & - & - & - & - \\
\hline Primary Energy - Five Sectors b .......................... & 726.5 & $1,525.4$ & $3,854.0$ & $4,011.6$ & $A_{4,412.6}$ & $R_{4,290.5}$ & $A_{4,111.3}$ & $4,299.0$ \\
\hline 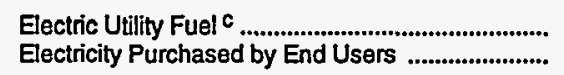 & $\begin{array}{r}-65.0 \\
294.7\end{array}$ & $\begin{array}{r}-205.4 \\
782.8\end{array}$ & $\begin{array}{r}-467.6 \\
1,412.5\end{array}$ & $\begin{array}{r}-601.3 \\
2,523.7\end{array}$ & $\begin{array}{r}-656.9 \\
3,113.0\end{array}$ & $\begin{array}{r}-640.7 \\
3,212.0\end{array}$ & $\begin{array}{r}-603.7 \\
3,230.0\end{array}$ & $\begin{array}{r}-675.1 \\
3,471.0\end{array}$ \\
\hline 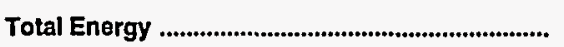 & 956.2 & $2,102.7$ & $4,798.9$ & $5,934.0$ & ${ }^{R_{6,868.6}}$ & ${ }^{R_{6,861.7}}$ & $P_{6,737.6}$ & $7,095.0$ \\
\hline
\end{tabular}

a Includes petroleum coke used at electric utilities.

b Biomass fuels are not included, except those consumed at electric

explanation of adjustments in Section 6 of Appendix A.

(s) =Value less than 0.05 million dollars.

utilities and those added to motor gasoline.
$c$ There are no direct fuel costs tor hydroelectric, geothemal, wind,

Note: Expenditure totals may not equal sum of components due to independent rounding.

Sources: Data sources, estimation procedures, and assumptions are R=Revised data.

- No consumption, including cases where adjustments were made. See described in Appendix A. 
Energy Price Estimates by Sector, South Carolina

1970, 1975, 1980, 1985, and 1990-1993

(Dollars per Million Btu)

\begin{tabular}{|c|c|c|c|c|c|c|c|c|}
\hline Sector and Energy Source & 1970 & 1975 & 1980 & 1985 & 1990 & 1991 & 1992 & 1993 \\
\hline 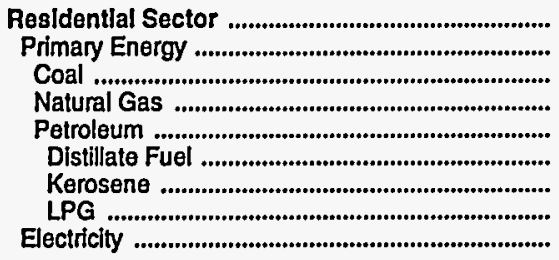 & $\begin{array}{l}2.82 \\
1.51 \\
1.20 \\
1.32 \\
1.64 \\
1.30 \\
1.58 \\
2.46 \\
5.64\end{array}$ & $\begin{array}{l}5.78 \\
2.71 \\
2.47 \\
2.08 \\
3.29 \\
2.69 \\
3.16 \\
4.28 \\
9.60\end{array}$ & $\begin{array}{r}9.73 \\
5.76 \\
3.19 \\
4.06 \\
7.50 \\
6.95 \\
8.27 \\
7.47 \\
13.69\end{array}$ & $\begin{array}{r}14.90 \\
7.44 \\
3.48 \\
6.44 \\
8.38 \\
7.19 \\
8.25 \\
9.72 \\
20.54\end{array}$ & $\begin{array}{r}16.32 \\
7.87 \\
3.34 \\
6.97 \\
9.01 \\
7.57 \\
8.69 \\
10.57 \\
20.95\end{array}$ & $\begin{array}{r}16.21 \\
7.76 \\
3.15 \\
6.80 \\
8.95 \\
7.06 \\
8.10 \\
11.00 \\
21.17\end{array}$ & $\begin{array}{r}15.95 \\
7.12 \\
3.11 \\
6.84 \\
7.64 \\
5.87 \\
6.73 \\
8.87 \\
21.06\end{array}$ & $\begin{array}{r}16.07 \\
7.04 \\
3.26 \\
6.94 \\
7.43 \\
5.90 \\
6.77 \\
8.70 \\
21.48\end{array}$ \\
\hline 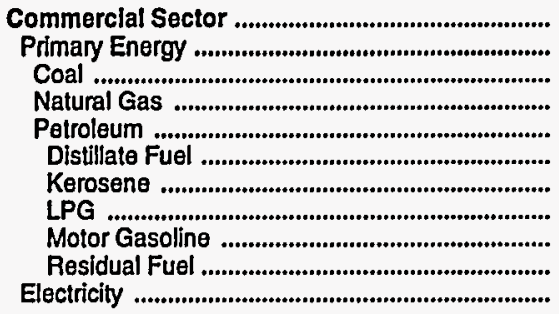 & $\begin{array}{r}2.35 \\
.93 \\
.50 \\
.86 \\
1.28 \\
1.01 \\
.63 \\
1.39 \\
2.75 \\
.46 \\
4.85\end{array}$ & $\begin{array}{l}4.84 \\
1.58 \\
1.53 \\
1.22 \\
2.58 \\
2.32 \\
2.29 \\
2.68 \\
4.35 \\
1.15 \\
8.55\end{array}$ & $\begin{array}{r}7.65 \\
3.57 \\
1.70 \\
3.11 \\
6.70 \\
6.33 \\
5.15 \\
4.24 \\
10.18 \\
3.41 \\
12.07\end{array}$ & $\begin{array}{r}12.85 \\
5.92 \\
1.77 \\
5.60 \\
7.07 \\
6.18 \\
6.93 \\
10.09 \\
8.84 \\
4.50 \\
18.01\end{array}$ & $\begin{array}{r}13.93 \\
6.11 \\
1.74 \\
5.74 \\
7.13 \\
5.75 \\
6.35 \\
10.05 \\
8.80 \\
3.25 \\
17.92\end{array}$ & $\begin{array}{r}14.02 \\
5.61 \\
1.71 \\
5.41 \\
6.52 \\
4.64 \\
5.62 \\
10.60 \\
8.56 \\
2.59 \\
18.15\end{array}$ & $\begin{array}{r}13.62 \\
5.36 \\
1.72 \\
5.50 \\
5.24 \\
3.41 \\
4.18 \\
10.04 \\
8.25 \\
2.61 \\
18.00\end{array}$ & $\begin{array}{r}13.60 \\
5.30 \\
1.72 \\
5.66 \\
5.27 \\
4.02 \\
4.51 \\
9.91 \\
8.20 \\
2.30 \\
18.14\end{array}$ \\
\hline 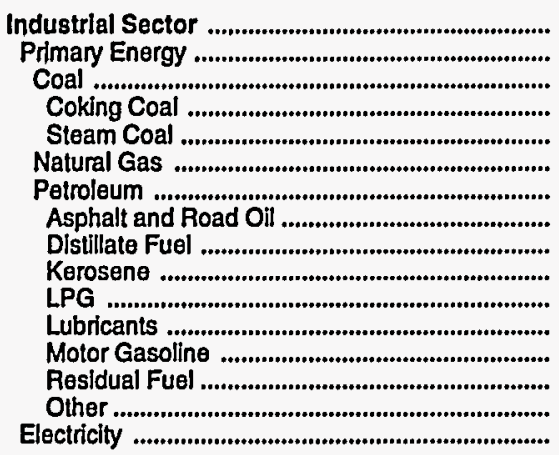 & $\begin{array}{r}.87 \\
.57 \\
.50 \\
- \\
.50 \\
.45 \\
.83 \\
.67 \\
.58 \\
.63 \\
1.39 \\
5.08 \\
2.75 \\
.40 \\
1.58 \\
2.41\end{array}$ & $\begin{array}{r}2.48 \\
1.56 \\
1.53 \\
-\overline{1.53} \\
1.00 \\
2.27 \\
1.78 \\
2.12 \\
2.29 \\
2.68 \\
7.48 \\
4.35 \\
1.82 \\
3.34 \\
5.80\end{array}$ & $\begin{array}{r}4.40 \\
3.35 \\
1.70 \\
- \\
1.70 \\
2.89 \\
4.88 \\
3.58 \\
4.62 \\
5.15 \\
4.24 \\
14.36 \\
10.18 \\
3.53 \\
6.70 \\
8.56\end{array}$ & $\begin{array}{r}6.40 \\
4.17 \\
1.77 \\
-\overline{1} \\
1.77 \\
4.57 \\
6.24 \\
4.99 \\
6.21 \\
6.93 \\
10.09 \\
17.61 \\
8.84 \\
4.50 \\
6.03 \\
12.02\end{array}$ & $\begin{array}{r}5.86 \\
R_{3.43} \\
1.74 \\
- \\
1.74 \\
3.26 \\
R_{4.96} \\
2.90 \\
5.68 \\
6.35 \\
10.05 \\
R_{14.60} \\
8.80 \\
3.25 \\
R_{4.69} \\
12.24\end{array}$ & $\begin{array}{r}R_{5.80} \\
R_{3.23} \\
1.71 \\
- \\
1.71 \\
2.87 \\
R_{4.84} \\
3.06 \\
5.03 \\
5.62 \\
10.60 \\
16.79 \\
8.56 \\
2.59 \\
R_{4.32} \\
12.20\end{array}$ & $\begin{array}{r}R_{5.59} \\
R_{3.13} \\
1.72 \\
-\overline{1.72} \\
3.05 \\
R_{4.26} \\
2.27 \\
3.74 \\
4.18 \\
10.04 \\
19.43 \\
8.25 \\
2.61 \\
R_{3.97} \\
11.82\end{array}$ & $\begin{array}{r}5.56 \\
3.12 \\
1.72 \\
-\overline{1} \\
1.72 \\
3.26 \\
4.01 \\
2.93 \\
4.04 \\
4.51 \\
9.91 \\
18.94 \\
8.20 \\
2.30 \\
3.81 \\
11.89\end{array}$ \\
\hline 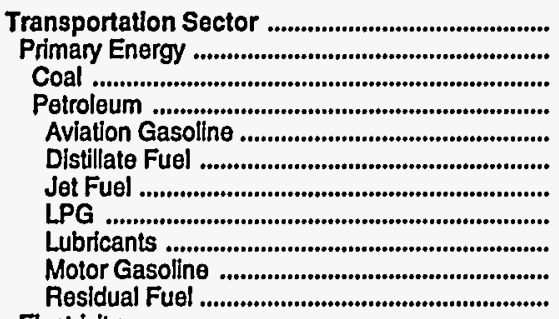 & $\begin{array}{r}2.34 \\
2.34 \\
.50 \\
2.34 \\
2.17 \\
1.32 \\
.73 \\
1.39 \\
5.08 \\
2.75 \\
.41\end{array}$ & $\begin{array}{l}4.04 \\
4.04 \\
1.53 \\
4.04 \\
3.45 \\
3.01 \\
2.03 \\
2.68 \\
7.48 \\
4.35 \\
1.52\end{array}$ & $\begin{array}{r}9.42 \\
9.42 \\
- \\
9.42 \\
9.02 \\
7.63 \\
6.46 \\
4.24 \\
14.36 \\
10.18 \\
2.90\end{array}$ & $\begin{array}{r}8.39 \\
8.39 \\
- \\
8.39 \\
9.99 \\
7.36 \\
6.11 \\
10.09 \\
17.61 \\
8.84 \\
3.82\end{array}$ & $\begin{array}{r}8.49 \\
8.49 \\
- \\
8.49 \\
9.32 \\
8.17 \\
6.07 \\
10.05 \\
\times 14.60 \\
8.80 \\
2.58\end{array}$ & $\begin{array}{r}R_{8.12} \\
R_{8.12} \\
- \\
R_{8.12} \\
8.71 \\
7.77 \\
5.12 \\
10.60 \\
R_{16.79} \\
8.56 \\
2.06\end{array}$ & $\begin{array}{r}R_{7.91} \\
R_{7.91} \\
- \\
R_{7.91} \\
8.54 \\
7.39 \\
4.82 \\
10.04 \\
\text { R }_{19.43} \\
8.25 \\
R_{2.14}\end{array}$ & $\begin{array}{r}7.84 \\
7.84 \\
- \\
7.84 \\
8.24 \\
7.17 \\
4.49 \\
9.91 \\
18.94 \\
8.20 \\
2.00\end{array}$ \\
\hline 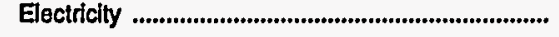 & - & - & - & - & - & - & - & - \\
\hline 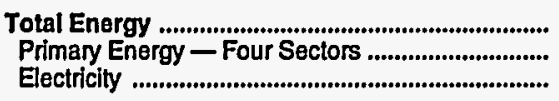 & $\begin{array}{l}1.83 \\
1.47 \\
3.98\end{array}$ & $\begin{array}{l}3.79 \\
2.91 \\
7.72\end{array}$ & $\begin{array}{r}7.25 \\
6.34 \\
11.11\end{array}$ & $\begin{array}{r}8.85 \\
6.65 \\
15.99\end{array}$ & $\begin{array}{r}8.89 \\
R_{6.45} \\
16.39\end{array}$ & $\begin{array}{l}R_{8.77} \\
R_{6.21} \\
16.50\end{array}$ & $\begin{array}{r}8.51 \\
5.92 \\
16.21\end{array}$ & $\begin{array}{r}8.57 \\
5.86 \\
16.53\end{array}$ \\
\hline 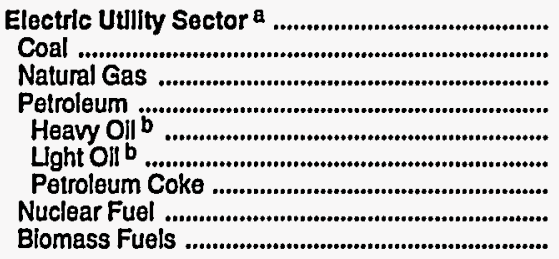 & $\begin{array}{r}.42 \\
.43 \\
.37 \\
.52 \\
.46 \\
.70 \\
\overline{1} \\
.19 \\
-\end{array}$ & $\begin{array}{r}.56 \\
1.14 \\
.71 \\
1.17 \\
1.14 \\
2.41 \\
. \overline{19} \\
-\end{array}$ & $\begin{array}{r}1.14 \\
1.56 \\
2.41 \\
3.91 \\
3.44 \\
5.78 \\
- \\
.44 \\
-\end{array}$ & $\begin{array}{r}1.11 \\
1.91 \\
4.54 \\
5.72 \\
3.94 \\
5.73 \\
- \\
.62 \\
-\end{array}$ & $\begin{array}{r}.94 \\
1.72 \\
1.72 \\
6.00 \\
3.02 \\
6.22 \\
- \\
.53 \\
-\end{array}$ & $\begin{array}{r}.90 \\
1.63 \\
1.49 \\
4.54 \\
2.22 \\
4.75 \\
- \\
.52 \\
-\end{array}$ & $\begin{array}{r}.84 \\
1.53 \\
1.69 \\
4.39 \\
2.38 \\
4.62 \\
- \\
.50 \\
-\end{array}$ & $\begin{array}{r}.88 \\
1.57 \\
2.91 \\
3.60 \\
2.19 \\
4.26 \\
- \\
.50 \\
-\end{array}$ \\
\hline Primary Energy — Flve Sectors c ....................... & 1.20 & 1.86 & 4.09 & 3.80 & 3.45 & $\mathbf{3 . 3 1}$ & 3.13 & 3.11 \\
\hline
\end{tabular}

a There are no direct fuel costs for hydroelectric, geothermal, wind, photovoltaic, of solar thermal energy.

b Heavy oil includes fuel oil nos. 4,5 , and 6 , and residual fuel oils. Light oil includes fuel oll nos. 1 and 2, kerosene, and jet fuel.

c Blomass fuels are not included, except those consumed at electric utilities and those added to motor gasoline.

$R=$ Revised data.

-No consumption, including cases where adjustments were made. See explanation of adjustments in Section 6 of Appendix A.

Sources: Data sources, estimation procedures, and assumptions are described in Appendix A. 
S Energy Expenditure Estimates by Sector, South Carolina $1970,1975,1980,1985$, and 1990-1993

(Million Dollars)

\begin{tabular}{|c|c|c|c|c|c|c|c|c|}
\hline Sector and Energy Source & 1970 & 1975 & 1980 & 1985 & 1990 & 1991 & 1992 & 1993 \\
\hline 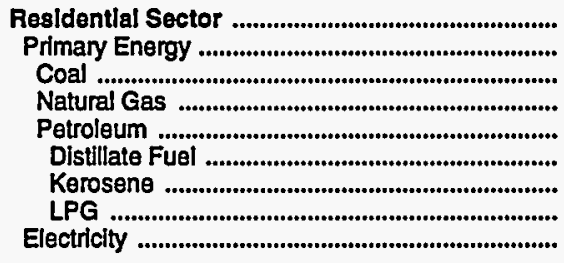 & $\begin{array}{r}222.0 \\
80.7 \\
2.5 \\
25.6 \\
52.6 \\
18.2 \\
18.0 \\
16.5 \\
141.3\end{array}$ & $\begin{array}{r}435.8 \\
113.5 \\
4.9 \\
38.8 \\
69.8 \\
26.6 \\
15.4 \\
27.9 \\
322.3\end{array}$ & $\begin{array}{r}833.8 \\
246.2 \\
5.4 \\
79.1 \\
161.7 \\
64.0 \\
56.3 \\
41.5 \\
587.6\end{array}$ & $\begin{array}{r}1,308.2 \\
280.8 \\
2.0 \\
108.7 \\
170.0 \\
48.3 \\
56.6 \\
65.1 \\
1,027.5\end{array}$ & $\begin{array}{r}1,573.1 \\
268.2 \\
.2 \\
131.9 \\
136.1 \\
44.5 \\
27.1 \\
64.5 \\
1,305.0\end{array}$ & $\begin{array}{r}1,641.4 \\
290.4 \\
.6 \\
136.9 \\
152.9 \\
41.1 \\
33.6 \\
78.3 \\
1,351.0\end{array}$ & $\begin{array}{r}1,627.7 \\
266.7 \\
.8 \\
157.4 \\
108.4 \\
23.6 \\
16.8 \\
68.0 \\
1,361.0\end{array}$ & $\begin{array}{r}1,813.7 \\
297.7 \\
3.3 \\
173.8 \\
120.6 \\
28.6 \\
24.8 \\
67.2 \\
1,516.0\end{array}$ \\
\hline 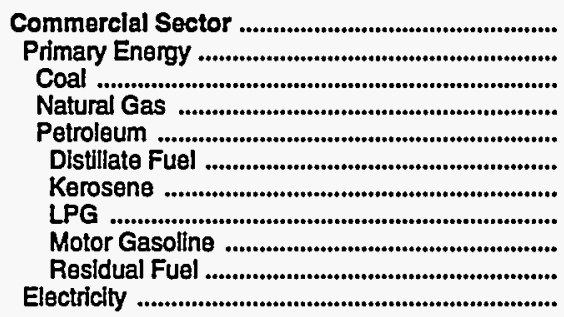 & $\begin{array}{r}93.5 \\
23.4 \\
1.9 \\
12.3 \\
9.2 \\
4.2 \\
.2 \\
1.6 \\
3.0 \\
.2 \\
70.1\end{array}$ & $\begin{array}{r}251.5 \\
43.7 \\
5.7 \\
21.5 \\
16.5 \\
6.8 \\
.3 \\
3.1 \\
5.1 \\
1.2 \\
207.8\end{array}$ & $\begin{array}{r}473.4 \\
115.0 \\
5.3 \\
73.5 \\
36.2 \\
17.8 \\
.7 \\
4.1 \\
12.8 \\
.7 \\
358.4\end{array}$ & $\begin{array}{r}747.7 \\
146.9 \\
1.9 \\
88.0 \\
57.1 \\
30.3 \\
1.9 \\
11.9 \\
10.7 \\
2.3 \\
600.8\end{array}$ & $\begin{array}{r}910.7 \\
134.7 \\
.2 \\
90.8 \\
43.7 \\
20.3 \\
.4 \\
10.8 \\
11.8 \\
.4 \\
776.0\end{array}$ & $\begin{array}{r}927.0 \\
122.0 \\
.6 \\
87.8 \\
33.6 \\
14.1 \\
.4 \\
13.3 \\
5.4 \\
.4 \\
805.0\end{array}$ & $\begin{array}{r}935.5 \\
127.5 \\
.9 \\
94.0 \\
32.6 \\
13.3 \\
.3 \\
13.6 \\
4.5 \\
.9 \\
808.0\end{array}$ & $\begin{array}{r}1,003.0 \\
138.0 \\
2.9 \\
99.5 \\
35.6 \\
19.9 \\
.5 \\
13.5 \\
1.3 \\
.4 \\
865.0\end{array}$ \\
\hline 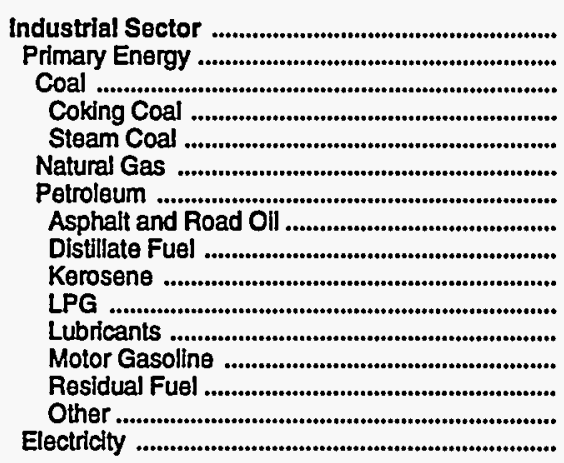 & $\begin{array}{r}183.5 \\
100.2 \\
22.0 \\
- \\
22.0 \\
36.4 \\
41.8 \\
9.9 \\
8.9 \\
1.1 \\
4.1 \\
4.6 \\
4.8 \\
4.0 \\
4.4 \\
83.3\end{array}$ & $\begin{array}{r}498.9 \\
246.3 \\
43.2 \\
- \\
43.2 \\
72.3 \\
130.8 \\
28.9 \\
25.2 \\
1.9 \\
10.6 \\
11.3 \\
4.8 \\
30.7 \\
17.5 \\
252.6\end{array}$ & $\begin{array}{r}1,184.6 \\
718.1 \\
74.9 \\
- \\
74.9 \\
275.2 \\
367.9 \\
36.4 \\
50.4 \\
3.7 \\
21.3 \\
24.6 \\
5.1 \\
94.2 \\
132.2 \\
466.5\end{array}$ & $\begin{array}{r}1,675.8 \\
780.4 \\
111.3 \\
-\overline{111.3} \\
296.3 \\
372.8 \\
45.3 \\
61.4 \\
8.8 \\
30.3 \\
27.4 \\
32.6 \\
63.1 \\
103.7 \\
895.4\end{array}$ & $\begin{array}{r}R_{1,787.2} \\
R_{755.2} \\
101.2 \\
- \\
101.2 \\
290.9 \\
R_{363.2} \\
38.1 \\
64.5 \\
3.5 \\
30.9 \\
R_{25.6} \\
32.3 \\
39.1 \\
R_{129.2} \\
1,032.0\end{array}$ & $\begin{array}{r}R_{1,753.1} \\
R_{697.1} \\
95.5 \\
- \\
95.5 \\
253.1 \\
R_{348.5} \\
39.4 \\
61.6 \\
3.5 \\
45.7 \\
R_{26.3} \\
30.2 \\
26.1 \\
R_{115.6} \\
1,056.0\end{array}$ & $\begin{array}{r}R_{1,773.2} \\
R_{712.3} \\
94.2 \\
-\overline{9} \\
94.2 \\
295.2 \\
R_{322.8} \\
31.1 \\
38.7 \\
1.6 \\
37.1 \\
\text { R } 31.1 \\
31.0 \\
29.4 \\
R_{122.7} \\
1,061.0\end{array}$ & $\begin{array}{r}1,831.3 \\
741.3 \\
103.8 \\
- \\
103.8 \\
320.1 \\
317.3 \\
45.8 \\
36.8 \\
2.4 \\
37.8 \\
30.8 \\
16.7 \\
44.7 \\
102.3 \\
1,090.0\end{array}$ \\
\hline 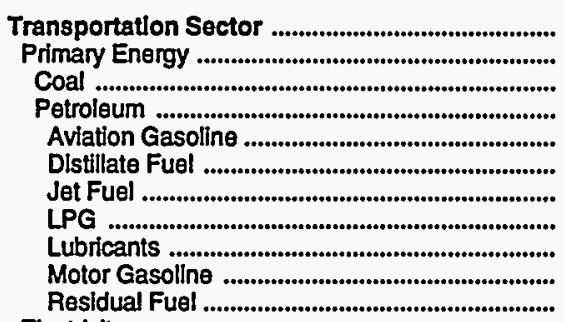 & $\begin{array}{r}457.0 \\
457.0 \\
(s) \\
457.0 \\
2.5 \\
22.3 \\
12.4 \\
.3 \\
7.3 \\
408.0 \\
4.1\end{array}$ & $\begin{array}{r}916.4 \\
916.4 \\
(\mathrm{~s}) \\
916.4 \\
2.5 \\
70.5 \\
29.5 \\
.8 \\
9.7 \\
799.5 \\
4.0\end{array}$ & $\begin{array}{r}2,307.1 \\
2,307.1 \\
- \\
2,307.1 \\
6.8 \\
273.6 \\
107.1 \\
.5 \\
22.7 \\
1,881.0 \\
15.4\end{array}$ & $\begin{array}{r}2,202.3 \\
2,202.3 \\
-\overline{2} \\
2,202.3 \\
6.9 \\
336.7 \\
105.3 \\
5.1 \\
25.4 \\
1,708.4 \\
14.6\end{array}$ & $\begin{array}{r}R_{2,597.5} \\
R_{2,597.5} \\
R_{2,597.5} \\
4.8 \\
516.8 \\
97.4 \\
3.2 \\
R_{23.6} \\
1,943.5 \\
8.3\end{array}$ & $\begin{array}{r}R_{2,540.3} \\
R_{2,540.3} \\
R_{2,540.3} \\
7.9 \\
521.8 \\
95.8 \\
3.7 \\
R_{24.3} \\
1,876.5 \\
10.3\end{array}$ & $\begin{array}{r}R_{2,401.2} \\
R_{2,401.2} \\
R_{2,401.2} \\
9.7 \\
450.0 \\
68.0 \\
3.2 \\
R_{28.7} \\
1,834.4 \\
7.2\end{array}$ & $\begin{array}{r}2,447.0 \\
2,447.0 \\
2, \\
2,447.0 \\
7.0 \\
428.7 \\
49.7 \\
2.9 \\
28.5 \\
1,922.1 \\
8.0\end{array}$ \\
\hline 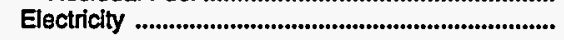 & - & - & - & - & - & - & - & - \\
\hline 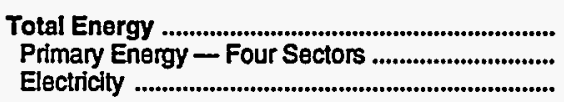 & $\begin{array}{l}956.2 \\
661.4 \\
294.7\end{array}$ & $\begin{array}{r}2,102.7 \\
1,319.9 \\
782.8\end{array}$ & $\begin{array}{l}4,798.9 \\
3,386.4 \\
1,412.5\end{array}$ & $\begin{array}{l}5,934.0 \\
3,410.3 \\
2,523.7\end{array}$ & $\begin{array}{r}{ }_{R}^{R} 6,868.6 \\
R_{3,755.6} \\
3,113.0\end{array}$ & $\begin{array}{r}R_{6,861.7} \\
R_{3,649.8} \\
3,212.0\end{array}$ & $\begin{array}{r}{ }_{1}^{R_{6,737.6}} \\
{ }_{3,507.6} \\
3,230.0\end{array}$ & $\begin{array}{l}7,095.0 \\
3,624.0 \\
3,471.0\end{array}$ \\
\hline 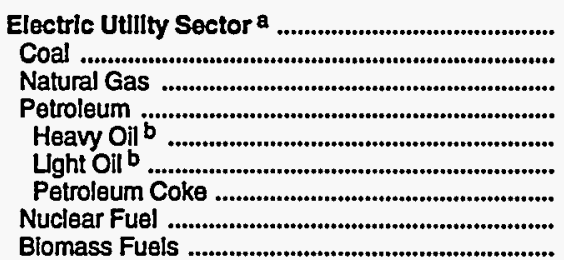 & $\begin{array}{r}65.0 \\
39.0 \\
17.1 \\
9.0 \\
5.9 \\
3.1 \\
- \\
\text { (s) }\end{array}$ & $\begin{array}{r}205.4 \\
120.9 \\
10.7 \\
33.3 \\
31.6 \\
1.7 \\
- \\
40.6\end{array}$ & $\begin{array}{r}467.6 \\
306.6 \\
13.4 \\
64.1 \\
45.0 \\
19.1 \\
- \\
83.4 \\
-\end{array}$ & $\begin{array}{r}601.3 \\
378.4 \\
2.3 \\
6.1 \\
(s) \\
6.1 \\
- \\
214.5 \\
-\end{array}$ & $\begin{array}{r}656.9 \\
397.5 \\
12.3 \\
4.4 \\
.2 \\
4.3 \\
- \\
242.8 \\
-\end{array}$ & $\begin{array}{r}640.7 \\
381.4 \\
15.0 \\
3.8 \\
.2 \\
3.7 \\
- \\
240.5 \\
-\end{array}$ & $\begin{array}{r}603.7 \\
355.3 \\
3.1 \\
4.1 \\
.2 \\
3.9 \\
-\overline{2} \\
241.2 \\
-\end{array}$ & $\begin{array}{r}675.1 \\
418.2 \\
5.5 \\
4.3 \\
.8 \\
3.5 \\
- \\
247.1 \\
-\end{array}$ \\
\hline Primary Energy - Flve Sectors c ............................ & 726.5 & $1,525.4$ & $3,854.0$ & $4,011.6$ & $R_{4,412.6}$ & $R_{4,290.5}$ & $9_{4,111.3}$ & $4,299.0$ \\
\hline
\end{tabular}

a There are no direct fuel costs for hydroslectric, geothermal, wind, photovoltaic, or solar themal energy.

b Heavy oll includes fuel oil nos. 4,5, and 6, and residual fuel olls. Light oll includes fuel nos. 1 and 2, kerosene, and jet fuel.

c Biomass fuels are not included, except those consumed at electric utilities and those added to motor gasoline.

$R=$ Revised data.

-No consumption, Including cases where adjustments were made. See explanation of adjustments in Section 6 of Appendix A.

(s)=Value less than 0.05 million dollars.

Note: Totals may not equal sum of components due to independent rounding.

Sources: Data sources, estimation procedures, and assumptions are described in Appendix A. 
Energy Price and Expenditure Estimates by Source, South Dakota 1970, 1975, 1980, 1985, and 1990-1993

\begin{tabular}{|c|c|c|c|c|c|c|c|c|}
\hline Energy Source & 1970 & 1975 & 1980 & 1985 & 1990 & 1991 & 1992 & 1993 \\
\hline & \multicolumn{8}{|c|}{ Prlces In Dollars per Million Btu } \\
\hline 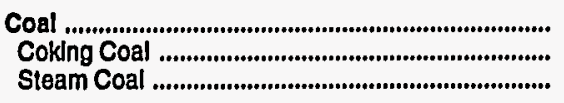 & $\frac{0.41}{.41}$ & $\begin{array}{r}0.53 \\
.53\end{array}$ & $\begin{array}{r}0.85 \\
.85\end{array}$ & $\begin{array}{r}1.37 \\
1.37\end{array}$ & 1.22 & $\frac{1.22}{1.22}$ & $\frac{1.21}{1.21}$ & $\frac{1.20}{1.20}$ \\
\hline 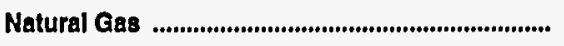 & .69 & 1.04 & 2.83 & 5.01 & 4.41 & 4.21 & 4.44 & 4.60 \\
\hline 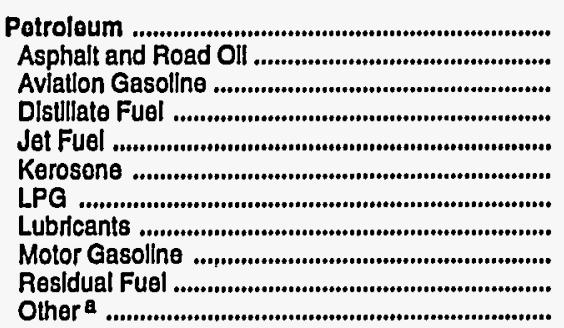 & $\begin{array}{r}2.05 \\
.76 \\
2.17 \\
.97 \\
.75 \\
1.47 \\
1.61 \\
5.08 \\
2.97 \\
.70 \\
-\end{array}$ & $\begin{array}{r}3.77 \\
2.09 \\
3.45 \\
2.60 \\
2.09 \\
2.84 \\
3.05 \\
7.48 \\
4.70 \\
2.15 \\
-\end{array}$ & $\begin{array}{r}6.19 \\
3.82 \\
9.02 \\
6.53 \\
6.47 \\
7.20 \\
5.50 \\
14.36 \\
10.14 \\
3.28 \\
-\end{array}$ & $\begin{array}{r}8.15 \\
5.06 \\
9.99 \\
6.98 \\
6.29 \\
9.03 \\
7.89 \\
17.61 \\
9.26 \\
4.43 \\
-\end{array}$ & $\begin{array}{r}R_{7.93} \\
3.24 \\
9.32 \\
6.52 \\
6.21 \\
6.03 \\
8.35 \\
R_{14.60} \\
9.40 \\
2.61 \\
-\end{array}$ & $\begin{array}{r}R_{7.47} \\
3.24 \\
8.71 \\
6.04 \\
5.36 \\
5.57 \\
7.19 \\
R_{16.79} \\
8.92 \\
2.33 \\
-\end{array}$ & $\begin{array}{r}A_{7.40} \\
2.71 \\
8.54 \\
6.56 \\
5.16 \\
6.17 \\
7.81 \\
R_{19.43} \\
8.65 \\
2.28 \\
-\end{array}$ & $\begin{array}{r}7.58 \\
3.06 \\
8.24 \\
6.28 \\
4.90 \\
6.73 \\
7.98 \\
18.94 \\
9.03 \\
2.41 \\
-\end{array}$ \\
\hline Nuclear Fuel ............................................................... & - & - & - & - & - & - & - & - \\
\hline Blomas8 Fuele at Utilitles .................................. & - & - & - & - & - & - & - & - \\
\hline Prlmary Energy - Flve Sectors b ..................... & 1.65 & 2.72 & 6.74 & 6.13 & $R_{6.05}$ & $\mathrm{P}_{\mathbf{5 . 5 2}}$ & 5.66 & 5.80 \\
\hline $\begin{array}{l}\text { Electric Utillty Fuel }{ }^{0} \text {................................................. } \\
\text { Electrlcity Purchased by End Users ..................... }\end{array}$ & $\begin{array}{r}.41 \\
7.38\end{array}$ & 8.21 & $\begin{array}{r}.83 \\
12.95\end{array}$ & $\begin{array}{r}1.22 \\
17.38\end{array}$ & $\begin{array}{r}1.19 \\
17.95\end{array}$ & $\begin{array}{r}1.16 \\
17.93\end{array}$ & $\begin{array}{r}1.15 \\
18.23\end{array}$ & $\begin{array}{r}1.13 \\
18.17\end{array}$ \\
\hline \multirow[t]{2}{*}{ 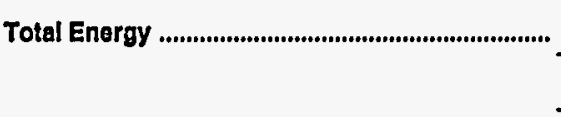 } & 2.13 & 3.64 & 7.77 & 8.63 & $R_{8.61}$ & 8.30 & $\mathbf{R}_{8.31}$ & 8.37 \\
\hline & \multicolumn{8}{|c|}{ Expenditures in Millions of Dollars } \\
\hline 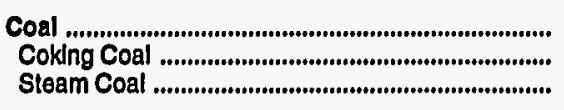 & $\begin{array}{l}2.3 \\
\overline{2.3}\end{array}$ & $\begin{array}{c}12.9 \\
12.9\end{array}$ & $\begin{array}{c}30.9 \\
- \\
30.9\end{array}$ & $\begin{array}{c}47.3 \\
- \\
47.3\end{array}$ & $\begin{array}{c}39.8 \\
- \\
39.8\end{array}$ & $\begin{array}{c}44.0 \\
\sim \\
44.0\end{array}$ & $\begin{array}{c}40.6 \\
- \\
40.6\end{array}$ & $\begin{array}{c}41.2 \\
- \\
41.2\end{array}$ \\
\hline Natural Gas .................................................................... & 25.2 & 33.7 & 67.7 & 125.9 & 111.0 & 108.4 & 110.2 & 131.8 \\
\hline 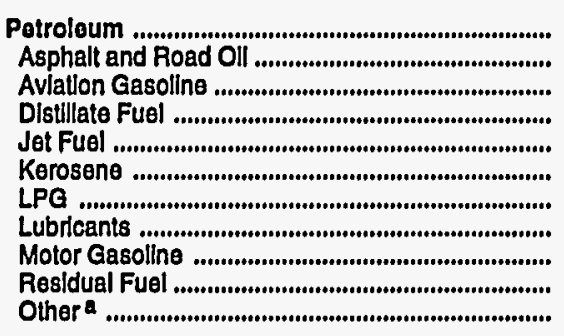 & $\begin{array}{r}212.9 \\
4.5 \\
1.1 \\
24.8 \\
4.7 \\
.1 \\
16.5 \\
5.1 \\
154.6 \\
1.4 \\
-\end{array}$ & $\begin{array}{r}389.2 \\
11.9 \\
1.3 \\
58.2 \\
11.9 \\
.1 \\
33.2 \\
7.3 \\
262.4 \\
2.9 \\
-\end{array}$ & $\begin{array}{r}833.6 \\
16.1 \\
4.4 \\
182.6 \\
46.0 \\
.6 \\
51.2 \\
13.9 \\
516.3 \\
2.5 \\
-\end{array}$ & $\begin{array}{r}775.7 \\
28.3 \\
4.4 \\
203.4 \\
34.6 \\
2.1 \\
35.3 \\
15.5 \\
451.2 \\
1.0 \\
-\end{array}$ & $\begin{array}{r}R 836.9 \\
17.0 \\
4.4 \\
210.0 \\
36.8 \\
.3 \\
111.7 \\
R 14.5 \\
441.3 \\
1.0 \\
-\end{array}$ & $\begin{array}{r}R_{726.2} \\
16.5 \\
2.7 \\
206.2 \\
10.8 \\
.2 \\
46.6 \\
R 14.9 \\
427.3 \\
1.0 \\
-\end{array}$ & $\begin{array}{r}R_{767.0} \\
15.9 \\
2.7 \\
213.7 \\
35.4 \\
.3 \\
54.6 \\
R 17.6 \\
424.8 \\
2.1 \\
-\end{array}$ & $\begin{array}{r}822.5 \\
13.1 \\
2.2 \\
227.8 \\
31.6 \\
.3 \\
74.6 \\
17.4 \\
453.8 \\
1.8 \\
-\end{array}$ \\
\hline Nuclear Fuel .............................................................. & - & - & - & - & - & - & - & - \\
\hline Blomas8 Fuele at Utllities ........................................ & - & - & - & - & - & - & - & - \\
\hline Prlmary Energy - Flve Sectors b ...................... & 240.5 & 435.9 & 932.1 & 849.0 & $R_{987.7}$ & $R_{879.6}$ & $R_{917.9}$ & 895.5 \\
\hline 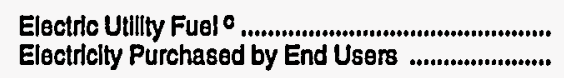 & $\begin{array}{r}-4.7 \\
70.6\end{array}$ & $\begin{array}{r}-16.0 \\
113.6\end{array}$ & $\begin{array}{l}-28.7 \\
224.7\end{array}$ & $\begin{array}{r}-36.2 \\
335.0\end{array}$ & $\begin{array}{r}34.4 \\
388.0\end{array}$ & $\begin{array}{r}-36.4 \\
409.0\end{array}$ & $\begin{array}{r}-33.5 \\
404.0\end{array}$ & $\begin{array}{r}32.7 \\
428.0\end{array}$ \\
\hline Total Enor & 306.4 & 533.5 & $1,128.1$ & $1,247.7$ & $A_{1,341.3}$ & $A_{1,252.2}$ & $R_{1,288.4}$ & $1,390,9$ \\
\hline
\end{tabular}

\footnotetext{
a Includes petroleum coke used at electric utillities.

b Blomass fuels are not Included, except those consumed at electric utllitles and those added to motor gasoline.

c There are no direct fuel costs.for hydroelectric, geothemal, wind, photovoltalc, or solar thermal energy.

$R=$ Revised data.
}

-No consumption, Including cases where adjustments were made. See explanation of adjustments in Section 6 of Appendix A.

Note: Expenditure totals may not equal sum of components due to Independent rounding.

Sources: Data sources, estimation procodures, and assumptlons are described in Appendix A. 
S Energy Price Estimates by Sector, South Dakota

O 1970, 1975, 1980, 1985, and 1990-1993

(Dollars per Million Btu)

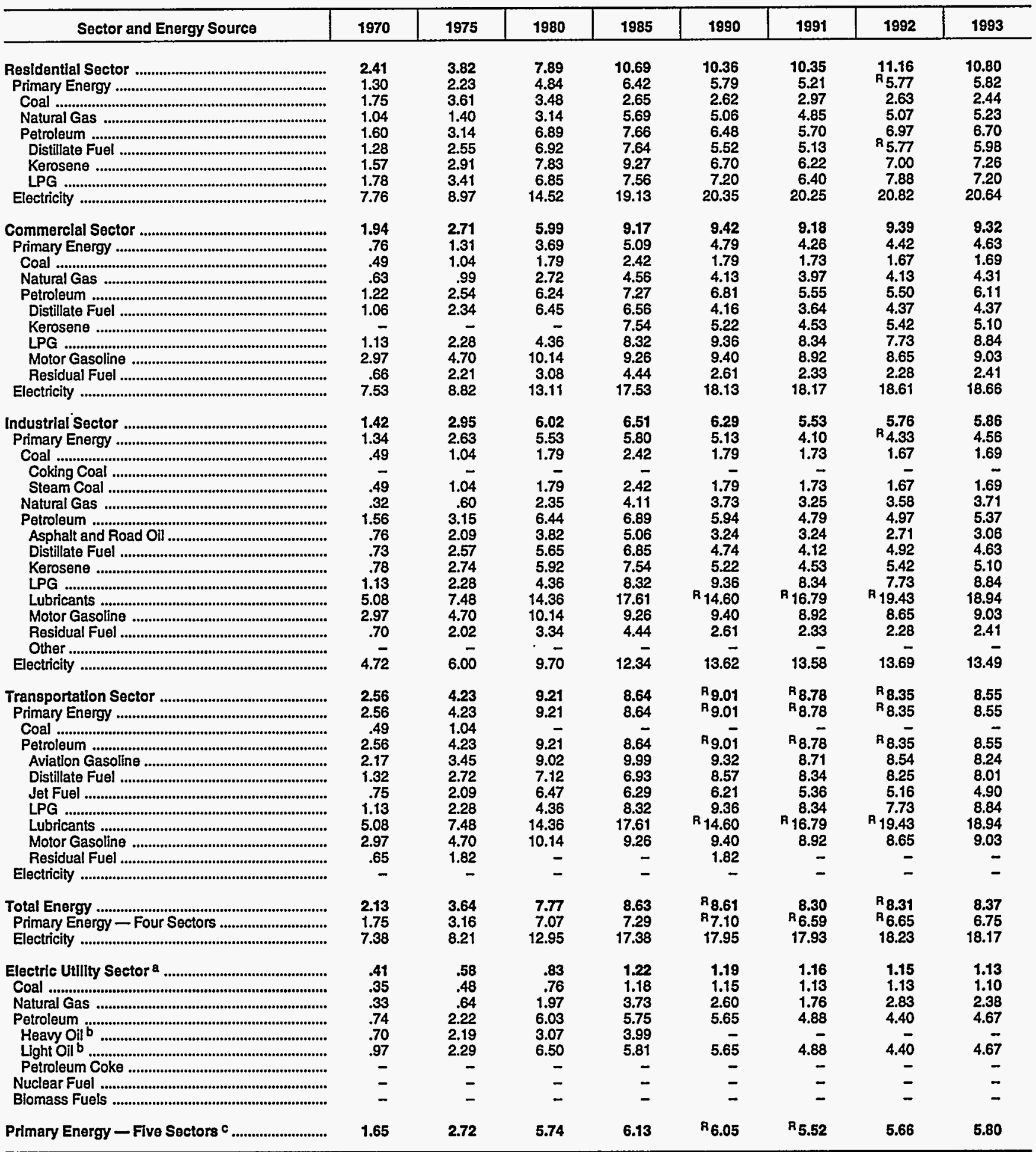

a There are no direct fuel costs for hydroelectric, geothermal, wind, photovoltaic, or solar thermal energy.

Heavy oil includes fuel oil nos. 4, 5, and 6, and residual fuel oils. Light oil includes fuel oil nos. 1 and 2 , kerosene, and jet fuel.

c Biomass fuels are not included, except those consumed at electric utillities and those added to motor gasoline.

$R=$ Revised data.

-No consumption, including cases where adjustments were made. Seo explanation of adjustments in Section 6 of Appendix A.

Sources: Data sources, estimation procedures, and assumptions are described in Appendix A. 


\begin{tabular}{|c|c|c|c|c|c|c|c|c|}
\hline Sector and Energy Source & 1970 & 1975 & 1980 & 1985 & 1990 & 1991 & 1992 & 1993 \\
\hline 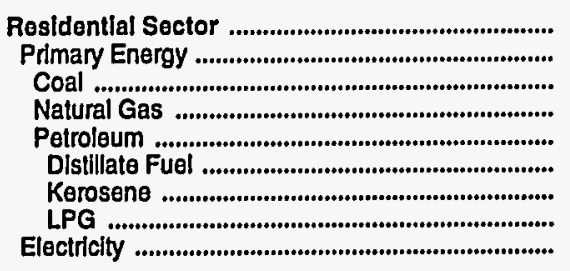 & $\begin{array}{r}76.0 \\
34.0 \\
.4 \\
14.3 \\
19.4 \\
5.7 \\
.1 \\
13.5 \\
42.0\end{array}$ & $\begin{array}{r}114.4 \\
51.1 \\
.5 \\
16.7 \\
33.9 \\
8.5 \\
(s) \\
25.3 \\
63.3\end{array}$ & $\begin{array}{r}223.8 \\
93.9 \\
.4 \\
33.1 \\
60.5 \\
30.7 \\
.4 \\
29.3 \\
129.9\end{array}$ & $\begin{array}{r}300.4 \\
119.7 \\
.3 \\
65.3 \\
54.1 \\
33.1 \\
1.8 \\
19.1 \\
180.7\end{array}$ & $\begin{array}{r}322.8 \\
123.8 \\
.1 \\
52.4 \\
71.3 \\
25.9 \\
.2 \\
45.2 \\
199.0\end{array}$ & $\begin{array}{r}314.2 \\
104.2 \\
.1 \\
55.4 \\
48.7 \\
24.0 \\
.2 \\
24.5 \\
210.0\end{array}$ & $\begin{array}{r}302.4 \\
100.4 \\
(\mathrm{~s}) \\
55.6 \\
44.8 \\
15.9 \\
.1 \\
28.7 \\
202.0\end{array}$ & $\begin{array}{r}340.9 \\
121.9 \\
(s) \\
65.9 \\
56.0 \\
20.6 \\
.2 \\
35.2 \\
219.0\end{array}$ \\
\hline 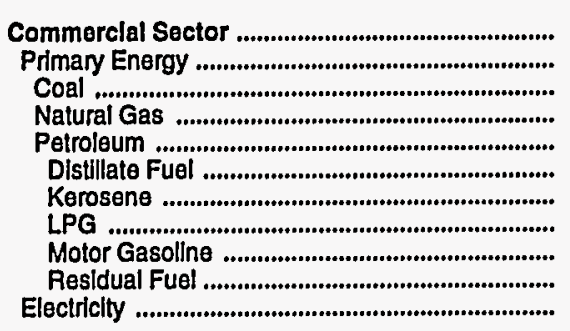 & $\begin{array}{r}35.7 \\
11.6 \\
.2 \\
7.2 \\
4.2 \\
1.9 \\
- \\
1.5 \\
.8 \\
.1 \\
24.1\end{array}$ & $\begin{array}{r}49.4 \\
19.5 \\
.3 \\
11.4 \\
7.8 \\
3.1 \\
. \\
3.0 \\
1.4 \\
.3 \\
29.9\end{array}$ & $\begin{array}{r}95.3 \\
44.4 \\
.4 \\
23.1 \\
20.9 \\
13.7 \\
\overline{3.3} \\
3.5 \\
.4 \\
51.0\end{array}$ & $\begin{array}{r}177.6 \\
66.2 \\
.5 \\
46.0 \\
19.7 \\
10.6 \\
(\mathrm{~s}) \\
3.7 \\
4.8 \\
.5 \\
111.5\end{array}$ & $\begin{array}{r}167.6 \\
55.6 \\
.1 \\
35.9 \\
19.6 \\
5.0 \\
(s) \\
10.4 \\
3.8 \\
.4 \\
112.0\end{array}$ & $\begin{array}{r}170.1 \\
51.1 \\
.1 \\
38.3 \\
12.8 \\
4.1 \\
(s) \\
5.6 \\
2.5 \\
.5 \\
119.0\end{array}$ & $\begin{array}{r}171.5 \\
52.5 \\
(\mathrm{~s}) \\
38.2 \\
14.2 \\
\mathrm{R} 6.2 \\
(\mathrm{~s}) \\
5.0 \\
2.5 \\
.5 \\
119.0\end{array}$ & $\begin{array}{r}185.3 \\
61.3 \\
(\mathrm{~s}) \\
46.8 \\
14.5 \\
6.3 \\
(\mathrm{~s}) \\
7.6 \\
.5 \\
124.0\end{array}$ \\
\hline 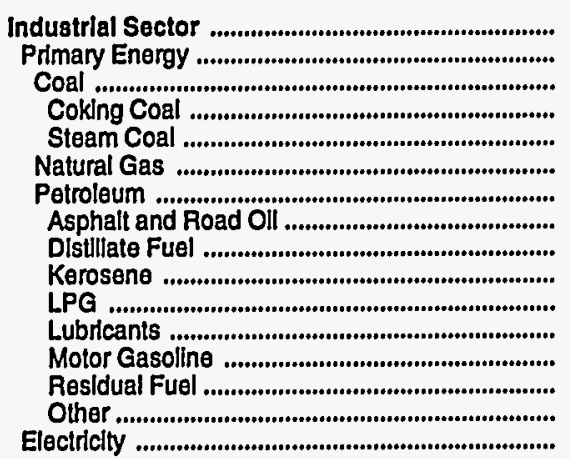 & $\begin{array}{r}57.5 \\
53.0 \\
(\mathrm{~s}) \\
\overline{(\mathrm{s})} \\
2.2 \\
50.7 \\
4.5 \\
9.9 \\
(\mathrm{~s}) \\
1.3 \\
.4 \\
34.5 \\
.2 \\
\overline{4} \\
4.5\end{array}$ & $\begin{array}{r}107.6 \\
87.3 \\
1.2 \\
\overline{1.2} \\
3.5 \\
32.6 \\
11.9 \\
24.5 \\
(5) \\
4.5 \\
.9 \\
40.1 \\
.7 \\
20.4\end{array}$ & $\begin{array}{r}227.6 \\
183.9 \\
4.4 \\
4.4 \\
11.0 \\
168.5 \\
16.1 \\
54.0 \\
.2 \\
17.5 \\
.3 \\
78.5 \\
2.0 \\
-\overline{2} \\
43.8\end{array}$ & $\begin{array}{r}210.4 \\
167.6 \\
11.7 \\
-\overline{7} \\
11.7 \\
14.6 \\
141.3 \\
28.3 \\
66.6 \\
.2 \\
11.7 \\
.3 \\
33.8 \\
.4 \\
-\overline{4} \\
42.9\end{array}$ & $\begin{array}{r}259.7 \\
182.7 \\
6.9 \\
- \\
6.9 \\
22.0 \\
153.8 \\
17.0 \\
56.5 \\
.1 \\
55.4 \\
.3 \\
24.0 \\
.6 \\
- \\
77.0\end{array}$ & $\begin{array}{r}216.3 \\
136.3 \\
8.7 \\
- \\
8.7 \\
15.4 \\
112.2 \\
16.5 \\
56.1 \\
.1 \\
16.0 \\
.3 \\
22.7 \\
.5 \\
-\overline{1} \\
80.0\end{array}$ & $\begin{array}{r}227.4 \\
144.4 \\
7.7 \\
-\overline{7.7} \\
76.3 \\
120.4 \\
15.9 \\
62.5 \\
.1 \\
20.4 \\
.4 \\
19.5 \\
1.6 \\
-\overline{1} \\
83.0\end{array}$ & $\begin{array}{r}253.3 \\
168.3 \\
9.8 \\
- \\
9.8 \\
18.7 \\
139.8 \\
13.1 \\
68.0 \\
(5) \\
31.0 \\
4 \\
25.6 \\
1.8 \\
- \\
85.0\end{array}$ \\
\hline 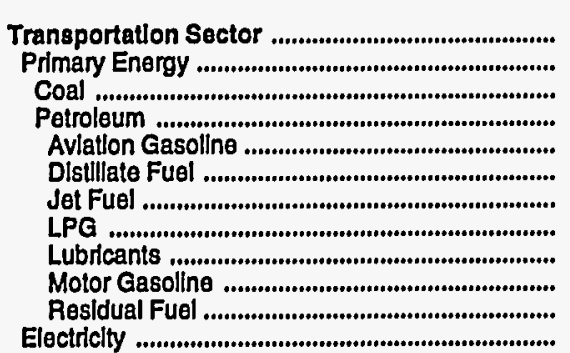 & $\begin{array}{r}137.2 \\
137.2 \\
(s) \\
137.2 \\
1.1 \\
7.1 \\
4.7 \\
.2 \\
4.7 \\
119.3 \\
(s) \\
-1\end{array}$ & $\begin{array}{r}262.1 \\
262.1 \\
(s) \\
262.1 \\
1.3 \\
21.1 \\
11.9 \\
.5 \\
6.3 \\
220.8 \\
(s) \\
-\end{array}$ & $\begin{array}{r}581.3 \\
581.3 \\
- \\
581.3 \\
4.4 \\
82.0 \\
46.0 \\
1.1 \\
13.6 \\
434.3 \\
- \\
-\end{array}$ & $\begin{array}{r}559.3 \\
559.3 \\
-\overline{3} \\
559.3 \\
4.4 \\
91.8 \\
34.6 \\
.7 \\
15.2 \\
412.7 \\
- \\
-\end{array}$ & $\begin{array}{r}R_{591.1} \\
R_{591.1} \\
- \\
{ }^{R} 591.1 \\
4.4 \\
121.5 \\
36.8 \\
.8 \\
R_{14.1} \\
413.5 \\
\text { (s) }\end{array}$ & $\begin{array}{r}R_{551.5} \\
R_{551.5} \\
R_{551.5} \\
2.7 \\
121.0 \\
10.8 \\
.4 \\
R_{14.6} \\
402.1 \\
- \\
-\end{array}$ & $\begin{array}{r}R_{587.1} \\
R_{587.1} \\
- \\
R_{587.1} \\
2.7 \\
128.5 \\
35.4 \\
.5 \\
R_{17.2} \\
402.8 \\
- \\
-\end{array}$ & $\begin{array}{r}611.3 \\
611.3 \\
. \\
611.3 \\
2.2 \\
132.0 \\
31.6 \\
.8 \\
17.0 \\
427.7 \\
- \\
-\end{array}$ \\
\hline 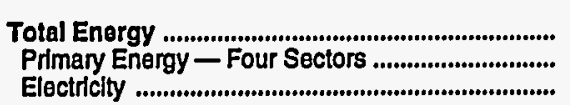 & $\begin{array}{r}306.4 \\
235.8 \\
70.6\end{array}$ & $\begin{array}{l}533.5 \\
419.9 \\
113.6\end{array}$ & $\begin{array}{r}1,128.1 \\
903.5 \\
224.7\end{array}$ & $\begin{array}{r}1,247.7 \\
912.7 \\
335.0\end{array}$ & $\begin{array}{r}\mathrm{R}_{1,341.3} \\
\mathrm{H}_{953.3} \\
388.0\end{array}$ & $\begin{array}{r}R_{1,252.2} \\
R_{843.2} \\
409.0\end{array}$ & $\begin{array}{r}\mathrm{R}_{1,288.4} \\
\mathrm{R}_{884.4} \\
404.0\end{array}$ & $\begin{array}{r}1,390.9 \\
962.8 \\
428.0\end{array}$ \\
\hline 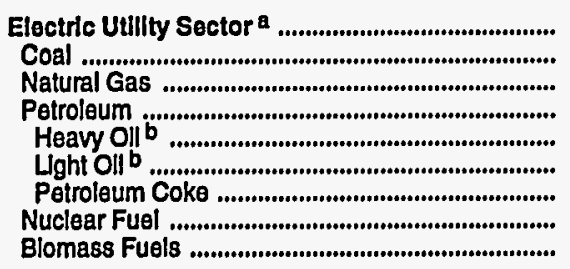 & $\begin{array}{l}4.7 \\
1.8 \\
1.5 \\
1.5 \\
1.2 \\
.3 \\
= \\
=\end{array}$ & $\begin{array}{r}16.0 \\
11.0 \\
2.1 \\
2.9 \\
2.0 \\
.9 \\
= \\
-\end{array}$ & $\begin{array}{r}28.7 \\
25.8 \\
.5 \\
2.4 \\
.2 \\
2.2 \\
= \\
= \\
-\end{array}$ & $\begin{array}{r}36.2 \\
34.8 \\
.1 \\
1.3 \\
(s) \\
1.3 \\
- \\
- \\
-\end{array}$ & $\begin{array}{r}34.4 \\
32.8 \\
.6 \\
1.1 \\
\overline{1.1} \\
= \\
=\end{array}$ & $\begin{array}{r}36.4 \\
35.1 \\
.3 \\
1.0 \\
\overline{1.0} \\
\overline{-} \\
\overline{-}\end{array}$ & $\begin{array}{r}33.5 \\
32.8 \\
.1 \\
.5 \\
-5 \\
. \\
= \\
-\end{array}$ & $\begin{array}{r}32.7 \\
31.4 \\
.4 \\
.9 \\
- \\
.9 \\
- \\
- \\
-\end{array}$ \\
\hline PrImary Energy — Flve Sectore ${ }^{\circ}$............................. & 240.5 & 435.8 & 832.1 & 949.0 & R 987.7 & ค 879.6 & R 917.9 & 995.5 \\
\hline
\end{tabular}

There are no direct fuel costs for hydroelectric, geothermal, wind, photovoltalc, or solar thermal energy.

Heavy oll Includes fuel oll nos. 4, 5, and 6, and residual fuel olls. Light oll includes fuel nos. 1 and 2, kerosene, and lat fuel.

- Blomass fuels are not Included, except those consumed at electric ullitites and those added to motor gasoline.

$R=$ Revised data.
-No consumption, including cases where adjustments were made. See explanation of adjustments in Section 6 of Appendix A.

(s)=Value less than 0.05 million dollars.

Note: Totals may not equal sum of components due to Independent rounding.

Sources: Data sources, estimation procedures, and assumptions are described in Appendix A. 

$1970,1975,1980,1985$, and 1990-1993

\begin{tabular}{|c|c|c|c|c|c|c|c|c|}
\hline Energy Source & 1970 & 1975 & 1980 & 1985 & 1990 & 1891 & 1992 & 1993 \\
\hline & \multicolumn{8}{|c|}{ Prices In Dollare per Million Btu } \\
\hline $\begin{array}{l}\text { Coal } \\
\text { Coking Coal } \\
\text { Steam Coal }\end{array}$ & $\begin{array}{r}0.26 \\
.38 \\
.25\end{array}$ & $\begin{array}{r}0.91 \\
1.60 \\
.91\end{array}$ & $\begin{array}{l}1.54 \\
1.81 \\
1.54\end{array}$ & $\begin{array}{l}1.55 \\
1.93 \\
1.55\end{array}$ & $\begin{array}{l}1.35 \\
1.83 \\
1.35\end{array}$ & $\begin{array}{l}1.28 \\
1.80 \\
1.28\end{array}$ & $\overline{1.30}$ & $\frac{1.28}{1.28}$ \\
\hline Natural Gas & .54 & .93 & 2.69 & 4.46 & 3.98 & 3.91 & 4.14 & 4.49 \\
\hline $\begin{array}{l}\text { Petroloum } \\
\text { Asphalt and Road Oll } \\
\text { Avlation Gasoline } \\
\text { Distillate Fuel } \\
\text { Jet Fuel } \\
\text { Kerosene } \\
\text { LPG } \\
\text { Lubricants } \\
\text { Motor Gasoline } \\
\text { Residual Fuel } \\
\text { Other }{ }^{a}\end{array}$ & $\begin{array}{r}2.06 \\
.67 \\
2.17 \\
1.11 \\
.73 \\
1.19 \\
1.92 \\
5.08 \\
2.84 \\
.36 \\
.81\end{array}$ & $\begin{array}{l}3.76 \\
1.79 \\
3.45 \\
2.68 \\
2.03 \\
2.93 \\
3.44 \\
7.48 \\
4.58 \\
1.78 \\
2.24\end{array}$ & $\begin{array}{r}8.37 \\
3.56 \\
9.02 \\
6.80 \\
6.39 \\
7.21 \\
6.42 \\
14.36 \\
9.89 \\
3.36 \\
6.24\end{array}$ & $\begin{array}{r}7.89 \\
4.76 \\
9.99 \\
6.44 \\
5.83 \\
6.99 \\
8.97 \\
17.61 \\
8.85 \\
4.80 \\
6.79\end{array}$ & $\begin{array}{r}\mathrm{R} 8.23 \\
2.94 \\
9.32 \\
7.89 \\
5.58 \\
6.93 \\
10.88 \\
114.60 \\
9.40 \\
3.14 \\
\mathrm{R} \\
\mathrm{F} .59\end{array}$ & $\begin{array}{r}\mathrm{R} 7.86 \\
3.30 \\
8.71 \\
7.34 \\
4.82 \\
6.38 \\
9.70 \\
\mathbf{R} \\
16.79 \\
9.00 \\
2.34 \\
\mathrm{R} \\
\mathrm{A.87}\end{array}$ & $\begin{array}{r}R 7.62 \\
2.03 \\
8.54 \\
7.08 \\
4.56 \\
R_{5.51} \\
8.47 \\
R_{19.43} \\
8.92 \\
R_{2.51} \\
R_{4.44}\end{array}$ & $\begin{array}{r}7.64 \\
2.37 \\
8.24 \\
7.04 \\
4.19 \\
5.46 \\
9.32 \\
18.94 \\
9.03 \\
2.67 \\
4.04\end{array}$ \\
\hline 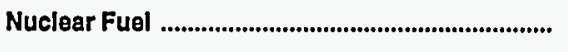 & - & - & .38 & .78 & .84 & .85 & .94 & .95 \\
\hline 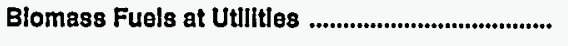 & - & - & - & - & - & - & - & - \\
\hline 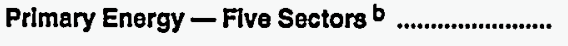 & 1.02 & 2.13 & 4.43 & 4.26 & 4.22 & $\mathbf{R}_{3.89}$ & ${ }^{A} 4.02$ & 4.21 \\
\hline 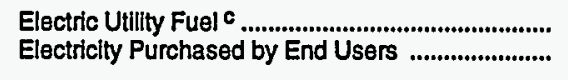 & .23 & $\begin{array}{r}.89 \\
5.83\end{array}$ & $\begin{array}{r}1.57 \\
10.67\end{array}$ & $\begin{array}{r}1.41 \\
14.65\end{array}$ & $\begin{array}{r}1.24 \\
15.59\end{array}$ & $\begin{array}{r}1.15 \\
15.29\end{array}$ & $\begin{array}{r}1.20 \\
15.28\end{array}$ & $\begin{array}{r}1.28 \\
15.33\end{array}$ \\
\hline \multirow[t]{2}{*}{ 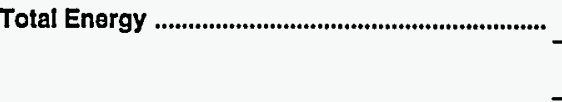 } & 1.71 & 3.54 & 7.30 & 8.16 & $\mathrm{R}_{8.56}$ & $R_{8.31}$ & ${ }^{A_{8.17}}$ & 8.22 \\
\hline & \multicolumn{8}{|c|}{ Expenditures in Millions of Dollare } \\
\hline 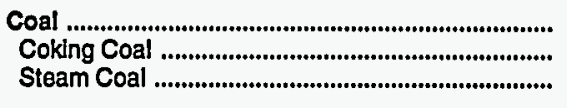 & $\begin{array}{r}103.1 \\
2.5 \\
100.7\end{array}$ & $\begin{array}{r}431.0 \\
8.9 \\
422.1\end{array}$ & $\begin{array}{r}888.2 \\
5.0 \\
883.2\end{array}$ & $\begin{array}{r}829.3 \\
8.0 \\
821.3\end{array}$ & $\begin{array}{r}812.8 \\
3.3 \\
809.6\end{array}$ & $\begin{array}{r}725.0 \\
.9 \\
724.2\end{array}$ & $\begin{array}{l}765.0 \\
765.0\end{array}$ & $\begin{aligned} 880.2 \\
880.2\end{aligned}$ \\
\hline 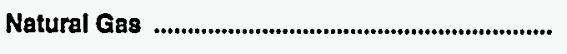 & 123.6 & 186.1 & 670.8 & 813.4 & 804.8 & 828.0 & 938.5 & $1,067.8$ \\
\hline $\begin{array}{l}\text { Petroleum } \\
\text { Asphalt and Road OIl } \\
\text { Aviation Gasoline } \\
\text { Distlllate Fuel } \\
\text { Jet Fuel } \\
\text { Kerosene } \\
\text { LPG } \\
\text { Lubricants } \\
\text { Motor Gasoline } \\
\text { Resldual Fuel } \\
\text { Other }{ }^{\mathrm{a}}\end{array}$ & $\begin{array}{r}831.6 \\
16.2 \\
1.3 \\
70.8 \\
13.6 \\
27.9 \\
23.0 \\
25.4 \\
625.1 \\
1.1 \\
27.2\end{array}$ & \begin{tabular}{r|}
$1,870.9$ \\
44.8 \\
1.2 \\
272.8 \\
45.1 \\
38.0 \\
48.8 \\
60.3 \\
$1,292.7$ \\
4.3 \\
62.9
\end{tabular} & $\begin{array}{r}4,365.1 \\
79.8 \\
13.2 \\
759.3 \\
149.8 \\
62.7 \\
65.3 \\
108.0 \\
2,853.4 \\
28.2 \\
245.4\end{array}$ & $\begin{array}{r}4,235.0 \\
139.3 \\
7.8 \\
835.9 \\
160.1 \\
43.9 \\
73.1 \\
120.6 \\
2,698.2 \\
9.6 \\
146.6\end{array}$ & $\begin{array}{r}R_{4,665.1} \\
113.0 \\
8.2 \\
1,097.0 \\
131.7 \\
17.2 \\
113.2 \\
R_{112.5} \\
2,846.3 \\
4.6 \\
R_{221.4}\end{array}$ & $\begin{array}{r}R_{4,278.4} \\
117.3 \\
6.4 \\
966.9 \\
82.9 \\
12.4 \\
111.6 \\
R_{115.7} \\
2,655.2 \\
4.0 \\
R_{186.0}\end{array}$ & $\begin{array}{r}P_{4,431.6} \\
71.2 \\
14.8 \\
P_{991.2} \\
115.3 \\
13.8 \\
145.5 \\
R 136.6 \\
2,746.7 \\
4.5 \\
R_{192.0}\end{array}$ & $\begin{array}{r}4,580.0 \\
77.3 \\
16.4 \\
983.3 \\
155.5 \\
12.7 \\
118.6 \\
135.5 \\
2,904.3 \\
5.9 \\
170.4\end{array}$ \\
\hline 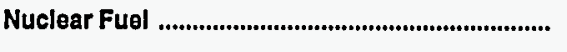 & - & - & 2.1 & 81.1 & 125.9 & 151.1 & 166.4 & 33.6 \\
\hline 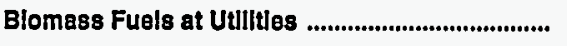 & - & - & - & - & - & - & - & - \\
\hline Primary Energy - Five Sectors ${ }^{b} \ldots \ldots \ldots \ldots \ldots \ldots . . . . . . . . .$. & $1,058.3$ & $2,487.9$ & $5,826.4$ & $6,058.7$ & ${ }^{R_{6,408.7}}$ & $A_{5,984.4}$ & ${ }^{R} 6,291.6$ & $6,561.6$ \\
\hline 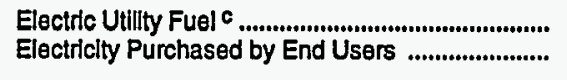 & $\begin{array}{r}-80.9 \\
504.6\end{array}$ & $\begin{array}{r}-376.4 \\
1,357.0\end{array}$ & $\begin{array}{r}-804.8 \\
2,656.5\end{array}$ & $\begin{array}{r}-846.9 \\
3,409.7\end{array}$ & $\begin{array}{r}-803.6 \\
4,054.9\end{array}$ & $\begin{array}{r}-745.1 \\
4,041.4\end{array}$ & $\begin{array}{r}-791.8 \\
R_{4,040.0}\end{array}$ & $\begin{array}{r}-784.6 \\
4,115.3\end{array}$ \\
\hline 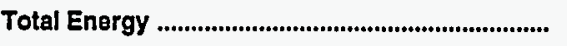 & $1,482.0$ & $3,468.5$ & 7,678.1 & $8,621.5$ & ${ }^{R_{9,660.0}}$ & $\mathrm{R}_{9,280.7}$ & ${ }^{A} 9,539.7$ & $9,892.3$ \\
\hline
\end{tabular}

a Includes petroleum coke used at electric utillities.

b Blomass fuels are not included, except those consumed at electric utilitiles and those added to motor gasoline.

c There are no direct fuel costs for hydroelectric, geothermal, wind, photovoltaic, or solar thermal energy.

$R=$ Revised data.
-No consumption, including cases where adjustments were made. See explanation of adjustments in Section 6 of Appendix $A$.

Note: Expenditure totals may not equal sum of components due to Independent rounding.

Sources: Data sources, estimation procedures, and assumptions are described in Appendlx A. 
(Dollars per Million Btu)

\begin{tabular}{|c|c|c|c|c|c|c|c|c|}
\hline Sector and Energy Source & 1970 & 1975 & 1980 & 1985 & 1990 & 1991 & 1992 & 1993 \\
\hline 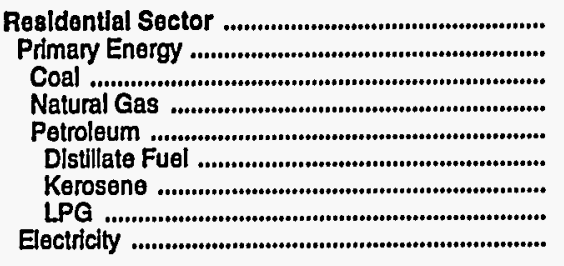 & $\begin{array}{r}2.15 \\
1.15 \\
.74 \\
.89 \\
1.82 \\
1.24 \\
1.62 \\
2.16 \\
3.34\end{array}$ & $\begin{array}{l}4.46 \\
1.93 \\
1.75 \\
1.25 \\
3.56 \\
2.49 \\
3.38 \\
3.84 \\
6.62\end{array}$ & $\begin{array}{r}7.79 \\
3.73 \\
1.97 \\
2.85 \\
7.95 \\
6.89 \\
9.09 \\
7.65 \\
10.43\end{array}$ & $\begin{array}{r}10.97 \\
5.44 \\
1.85 \\
4.96 \\
7.95 \\
6.59 \\
7.09 \\
9.23 \\
14.28\end{array}$ & $\begin{array}{r}12.53 \\
5.67 \\
1.77 \\
4.94 \\
10.19 \\
6.59 \\
7.09 \\
11.90 \\
16.68\end{array}$ & $\begin{array}{r}12.36 \\
5.63 \\
1.75 \\
5.02 \\
9.28 \\
6.12 \\
6.58 \\
10.57 \\
16.54\end{array}$ & $\begin{array}{r}12.35 \\
5.73 \\
1.61 \\
5.33 \\
8.15 \\
5.21 \\
5.61 \\
9.41 \\
16.71\end{array}$ & $\begin{array}{r}12.32 \\
5.90 \\
1.79 \\
5.50 \\
8.51 \\
5.30 \\
5.70 \\
9.61 \\
16.87\end{array}$ \\
\hline 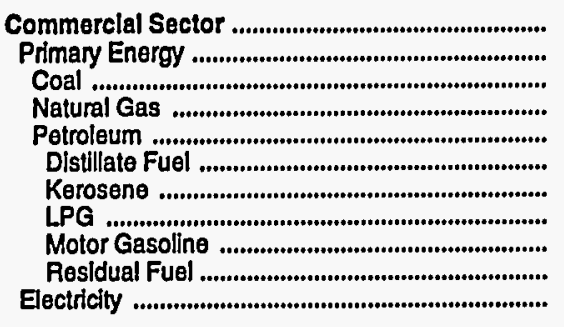 & $\begin{array}{r}1.87 \\
.75 \\
.35 \\
.70 \\
1.46 \\
1.06 \\
.78 \\
1.27 \\
2.84 \\
.42 \\
4.97\end{array}$ & $\begin{array}{l}3.48 \\
1.38 \\
1.17 \\
1.09 \\
2.88 \\
2.29 \\
2.32 \\
2.39 \\
4.58 \\
1.77 \\
8.27\end{array}$ & $\begin{array}{r}7.97 \\
3.57 \\
1.39 \\
2.95 \\
7.05 \\
6.49 \\
6.16 \\
4.96 \\
9.89 \\
3.44 \\
13.29\end{array}$ & $\begin{array}{r}8.95 \\
5.04 \\
1.60 \\
4.75 \\
6.04 \\
5.66 \\
6.78 \\
8.66 \\
8.85 \\
4.80 \\
17.05\end{array}$ & $\begin{array}{r}10.62 \\
4.76 \\
1.40 \\
4.64 \\
6.98 \\
4.96 \\
6.49 \\
9.36 \\
9.40 \\
3.16 \\
18.02\end{array}$ & $\begin{array}{r}10.51 \\
4.69 \\
1.42 \\
4.61 \\
6.45 \\
4.34 \\
5.64 \\
8.34 \\
9.00 \\
2.48 \\
17.99\end{array}$ & $\begin{array}{r}9.11 \\
4.84 \\
1.41 \\
4.91 \\
5.36 \\
3.95 \\
5.10 \\
7.73 \\
8.92 \\
R_{2} .67 \\
19.35\end{array}$ & $\begin{array}{r}8.78 \\
5.02 \\
1.41 \\
5.09 \\
5.34 \\
3.87 \\
4.71 \\
8.84 \\
9.03 \\
2.72 \\
20.14\end{array}$ \\
\hline 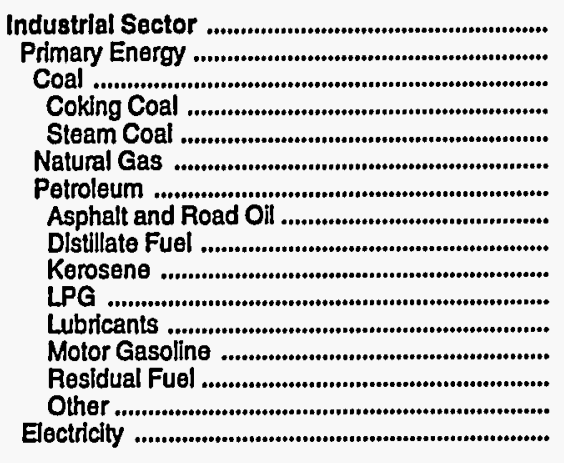 & $\begin{array}{r}.93 \\
.54 \\
.35 \\
.38 \\
.35 \\
.38 \\
.86 \\
.67 \\
.72 \\
.78 \\
1.27 \\
5.08 \\
2.84 \\
.36 \\
.81 \\
2.05\end{array}$ & $\begin{array}{l}2.56 \\
1.38 \\
1.22 \\
1.60 \\
1.17 \\
.73 \\
2.28 \\
1.79 \\
2.11 \\
2.32 \\
2.39 \\
7.48 \\
4.58 \\
1.89 \\
2.24 \\
4.87\end{array}$ & $\begin{array}{r}5.10 \\
3.36 \\
1.41 \\
1.81 \\
1.39 \\
2.54 \\
5.51 \\
3.56 \\
5.50 \\
6.16 \\
4.96 \\
14.36 \\
9.89 \\
3.36 \\
6.24 \\
9.71\end{array}$ & $\begin{array}{r}6.81 \\
3.87 \\
1.61 \\
1.93 \\
1.60 \\
4.11 \\
6.37 \\
4.76 \\
5.91 \\
6.78 \\
8.66 \\
17.61 \\
8.85 \\
4.80 \\
6.79 \\
14.22\end{array}$ & $\begin{array}{r}6.18 \\
R_{3.33} \\
1.41 \\
1.83 \\
1.40 \\
3.29 \\
R_{5.11} \\
2.94 \\
5.65 \\
6.49 \\
9.36 \\
R 14.60 \\
9.40 \\
3.16 \\
R 5.59 \\
13.74\end{array}$ & $\begin{array}{r}5.96 \\
R 3.19 \\
1.42 \\
1.80 \\
1.42 \\
3.12 \\
R 4.88 \\
3.30 \\
4.91 \\
5.64 \\
8.34 \\
R_{16.79} \\
9.00 \\
2.48 \\
R 4.87 \\
13.21\end{array}$ & $\begin{array}{r}R_{6.22} \\
R_{3.18} \\
1.41 \\
- \\
1.41 \\
3.34 \\
R_{4.45} \\
2.03 \\
4.44 \\
5.10 \\
7.73 \\
R_{19.43} \\
8.92 \\
R_{2.67} \\
R_{4.44} \\
R_{13.50}\end{array}$ & $\begin{array}{r}6.37 \\
3.22 \\
1.41 \\
-\overline{1.41} \\
3.76 \\
4.28 \\
2.37 \\
4.10 \\
4.71 \\
8.84 \\
18.94 \\
9.03 \\
2.72 \\
4.04 \\
13.54\end{array}$ \\
\hline 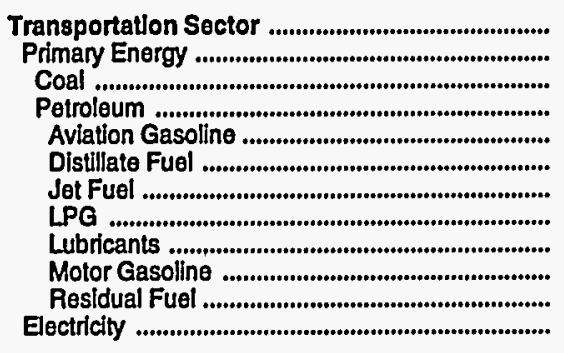 & $\begin{array}{r}2.49 \\
2.49 \\
.35 \\
2.49 \\
2.17 \\
1.28 \\
.73 \\
1.27 \\
5.08 \\
2.84 \\
.42 \\
4.97\end{array}$ & $\begin{array}{l}4.19 \\
4.19 \\
1.17 \\
4.19 \\
3.45 \\
3.02 \\
2.03 \\
2.39 \\
7.48 \\
4.58 \\
1.67 \\
8.27\end{array}$ & $\begin{array}{r}9.20 \\
9.20 \\
9 . \\
9.20 \\
9.02 \\
7.25 \\
6.39 \\
4.96 \\
14.36 \\
9.89 \\
3.45 \\
13.29\end{array}$ & $\begin{array}{r}8.29 \\
8.29 \\
- \\
8.29 \\
9.99 \\
6.73 \\
5.83 \\
8.66 \\
17.61 \\
8.85 \\
= \\
17.05\end{array}$ & $\begin{array}{r}\text { R }_{8.97} \\
\text { R }_{8.97} \\
- \\
R_{8.97} \\
9.32 \\
8.36 \\
5.58 \\
9.36 \\
\text { R }_{14.60} \\
9.40 \\
2.22 \\
20.12\end{array}$ & $\begin{array}{r}R_{8.57} \\
R_{8.57} \\
- \\
R_{8.57} \\
8.71 \\
7.84 \\
4.82 \\
8.34 \\
R_{16.79} \\
9.00 \\
1.71 \\
20.09\end{array}$ & $\begin{array}{r}R_{8.48} \\
R_{8.48} \\
-\overline{8} \\
R_{8.48} \\
8.54 \\
7.82 \\
4.56 \\
7.73 \\
R_{19.43} \\
8.92 \\
R_{1.65} \\
21.62\end{array}$ & $\begin{array}{r}8.44 \\
8.44 \\
-\overline{8.44} \\
8.24 \\
7.80 \\
4.19 \\
8.84 \\
18.94 \\
9.03 \\
1.58 \\
23.08\end{array}$ \\
\hline 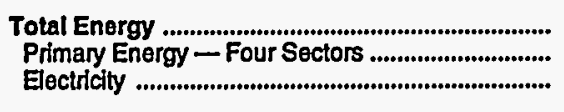 & $\begin{array}{l}1.71 \\
1.42 \\
2.85\end{array}$ & $\begin{array}{l}3.54 \\
2.82 \\
5.83\end{array}$ & $\begin{array}{r}7.30 \\
6.25 \\
10.67\end{array}$ & $\begin{array}{r}8.16 \\
6.32 \\
14.65\end{array}$ & $\begin{array}{r}R_{8.56} \\
R_{6.45} \\
15.59\end{array}$ & $\begin{array}{r}A_{8.31} \\
{ }^{A_{6.14}} \\
15.29\end{array}$ & $\begin{array}{r}R_{8.17} \\
R_{6.09} \\
15.28\end{array}$ & $\begin{array}{r}8.22 \\
6.18 \\
15.33\end{array}$ \\
\hline $\begin{array}{l}\text { Electrlc Utulty Sector a } \\
\text { Coal } \\
\text { Natural Gas } \\
\text { Petroleum }\end{array}$ & $\begin{array}{l}.23 \\
.23 \\
.25 \\
-\end{array}$ & $\begin{array}{r}.89 \\
.87 \\
2 . \overline{19}\end{array}$ & $\begin{array}{l}1.57 \\
1.56 \\
2.33 \\
6.39\end{array}$ & $\begin{array}{r}1.41 \\
1.54 \\
- \\
5.85\end{array}$ & $\begin{array}{l}1.24 \\
1.34 \\
2.72 \\
5.61\end{array}$ & $\begin{array}{l}1.15 \\
1.25 \\
2.44 \\
4.98\end{array}$ & $\begin{array}{l}1.20 \\
1.27 \\
2.42 \\
4.80\end{array}$ & $\begin{array}{l}1.26 \\
1.26 \\
2.69 \\
4.31\end{array}$ \\
\hline 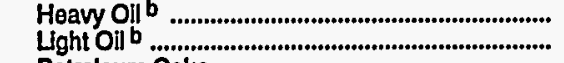 & $\overline{-}$ & $2 . \overline{19}$ & $6 . \overline{39}$ & $5 . \overline{85}$ & $5 . \overline{61}$ & $4 . \overline{8}$ & $4 . \overline{80}$ & $4 . \overline{31}$ \\
\hline 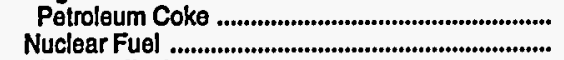 & - & $\overline{-}$ &.$\overline{38}$ & $\overline{.78}$ &.$\overline{84}$ &. $\overrightarrow{85}$ &.$\overline{94}$ &.$\overline{95}$ \\
\hline Blomass Fuls ........................................................ & - & - & - & - & - & - & - & - \\
\hline Primary Energy - Five Sectors c .......................... & 1.02 & 2.13 & 4.43 & 4.26 & 4.22 & $\mathrm{R}_{3.99}$ & $R_{4.02}$ & 4.21 \\
\hline
\end{tabular}

a There are no direct fuel costs for hydroelectric, geothermal, wind, photovoltalc, or solar thermal energy.

beavy oil includes fuel oil nos. 4, 5, and 6, and residual fuel oils. Light oil includes fuel oil nos. 1 and 2, kerosene, and jet fuel.

Blomass fuels are not included, except those consumed at electric utilities and those added to motor gasoline.
$R=$ Revised data.

-No consumption, including cases where adjustments were made. See explanation of adjustments in Section 6 of Appendix A.

Sources: Data sources, estimation procedures, and assumptions are described in Appendix A. 


\section{T Energy Expenditure Estimates by Sector, Tennessee}

E 1970, 1975, 1980, 1985, and 1990-1993

N (Million Dollars)

\begin{tabular}{|c|c|c|c|c|c|c|c|c|}
\hline Sector and Energy Source & 1970 & 1975 & 1980 & 1985 & 1990 & 1991 & 1992 & 1993 \\
\hline 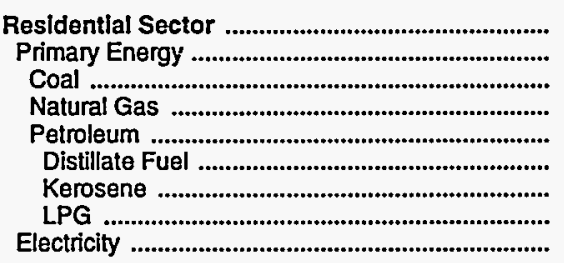 & $\begin{array}{r}288.7 \\
84.5 \\
3.3 \\
42.5 \\
38.7 \\
1.2 \\
18.6 \\
18.9 \\
204.2\end{array}$ & $\begin{array}{r}650.3 \\
129.7 \\
4.7 \\
56.8 \\
68.1 \\
3.4 \\
25.3 \\
39.5 \\
520.6\end{array}$ & $\begin{array}{r}1,149.1 \\
216.5 \\
3.9 \\
129.8 \\
82.8 \\
12.4 \\
28.3 \\
42.2 \\
932.6\end{array}$ & $\begin{array}{r}1,529.1 \\
284.5 \\
2.7 \\
202.0 \\
79.8 \\
9.9 \\
29.7 \\
40.2 \\
1,244.6\end{array}$ & $\begin{array}{r}1,973.3 \\
336.3 \\
3.4 \\
236.8 \\
96.1 \\
9.1 \\
9.1 \\
13.0 \\
74.0 \\
1,637.0\end{array}$ & $\begin{array}{r}2,023.4 \\
352.4 \\
2.8 \\
256.2 \\
93.5 \\
9.6 \\
10.0 \\
74.0 \\
1,671.0\end{array}$ & $\begin{array}{r}2,062.2 \\
380.2 \\
2.2 \\
287.2 \\
90.8 \\
7.9 \\
11.5 \\
71.5 \\
1,682.0\end{array}$ & $\begin{array}{r}2,167.7 \\
429.7 \\
1.8 \\
335.2 \\
92.6 \\
6.3 \\
10.0 \\
76.3 \\
1,738.0\end{array}$ \\
\hline 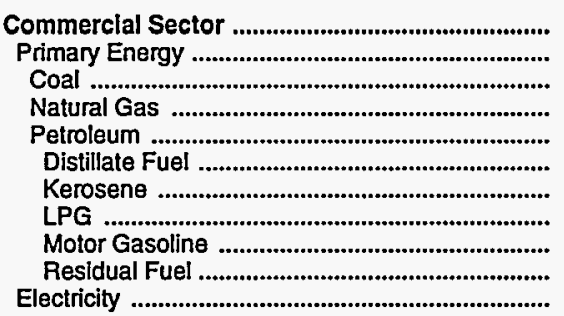 & $\begin{array}{r}153.4 \\
45.6 \\
2.9 \\
30.4 \\
12.2 \\
2.6 \\
1.8 \\
2.0 \\
5.9 \\
(s) \\
107.8\end{array}$ & $\begin{array}{r}289.4 \\
79.4 \\
5.8 \\
47.9 \\
25.7 \\
7.9 \\
3.4 \\
4.3 \\
10.1 \\
(s) \\
210.0\end{array}$ & $\begin{array}{r}853.7 \\
209.2 \\
5.1 \\
132.1 \\
72.0 \\
38.4 \\
3.6 \\
4.8 \\
24.2 \\
1.0 \\
644.5\end{array}$ & $\begin{array}{r}924.1 \\
350.9 \\
4.3 \\
213.2 \\
133.4 \\
101.7 \\
6.4 \\
6.7 \\
15.6 \\
2.9 \\
573.3\end{array}$ & $\begin{array}{r}1,072.5 \\
268.5 \\
4.8 \\
209.1 \\
54.6 \\
18.4 \\
2.5 \\
10.3 \\
22.7 \\
.7 \\
804.0\end{array}$ & $\begin{array}{r}1,074.1 \\
269.2 \\
3.9 \\
218.7 \\
46.5 \\
15.2 \\
1.0 \\
10.3 \\
19.8 \\
.3 \\
805.0\end{array}$ & $\begin{array}{r}780.3 \\
292.4 \\
3.5 \\
235.5 \\
\text { R } 53.5 \\
R_{23.9} \\
2.0 \\
10.4 \\
16.2 \\
1.0 \\
487.9\end{array}$ & $\begin{array}{r}734.3 \\
315.3 \\
2.5 \\
267.5 \\
45.3 \\
21.1 \\
1.6 \\
12.4 \\
9.6 \\
6.6 \\
419.0\end{array}$ \\
\hline $\begin{array}{l}\text { Industrial Sector } \\
\text { Primary Energy } \\
\text { Coal } \\
\text { Coking Coal } \\
\text { Steam Coal } \\
\text { Natural Gas } \\
\text { Petroleum } \\
\text { Asphalt and Road Oil } \\
\text { Distillate Fuel } \\
\text { Kerosene } \\
\text { LPG }\end{array}$ & $\begin{array}{r}340.0 \\
147.4 \\
20.3 \\
2.5 \\
17.8 \\
46.3 \\
80.8 \\
16.2 \\
13.3 \\
7.5 \\
1.7 \\
10.3 \\
3.5 \\
1.1 \\
27.2 \\
192.6\end{array}$ & $\begin{array}{r}975.9 \\
349.5 \\
60.8 \\
8.9 \\
52.0 \\
81.4 \\
207.3 \\
44.8 \\
57.6 \\
9.4 \\
3.9 \\
23.7 \\
2.8 \\
2.3 \\
62.9 \\
626.3\end{array}$ & $\begin{array}{r}2,067.7 \\
988.3 \\
94.4 \\
5.0 \\
89.4 \\
306.4 \\
587.5 \\
79.8 \\
136.3 \\
30.8 \\
17.1 \\
49.2 \\
1.9 \\
27.0 \\
245.4 \\
1,079.4\end{array}$ & $\begin{array}{r}2,680.1 \\
1,088.3 \\
164.6 \\
8.0 \\
156.6 \\
398.1 \\
525.6 \\
139.3 \\
119.5 \\
7.8 \\
21.0 \\
54.9 \\
29.9 \\
6.6 \\
146.6 \\
1,591.8\end{array}$ & $\begin{array}{r}R_{2}, 647.9 \\
R_{1,034.0} \\
136.1 \\
3.3 \\
132.8 \\
357.4 \\
R_{540.5} \\
113.0 \\
96.2 \\
1.7 \\
24.6 \\
R_{51.2} \\
28.6 \\
3.9 \\
R_{21.4} 221.4 \\
1,613.9\end{array}$ & $\begin{array}{r}\mathrm{R}_{2,550.1} \\
\mathrm{~A}_{984.7} \\
132.9 \\
.9 \\
132.0 \\
354.4 \\
\mathrm{R}_{497.4} \\
117.3 \\
77.2 \\
1.4 \\
23.3 \\
\mathrm{R}_{52.7} \\
26.3 \\
3.2 \\
31.2 \\
\mathrm{R}_{196.0} \\
1,565.4\end{array}$ & $\begin{array}{r}R_{2,926.7} \\
R_{1,056.6} \\
130.9 \\
- \\
130.9 \\
415.1 \\
R_{510.6} \\
71.2 \\
94.6 \\
.4 \\
60.3 \\
R_{62.2} \\
26.9 \\
R_{3.1} \\
R_{192.0} \\
R_{1,870.1}\end{array}$ & $\begin{array}{r}3,014.6 \\
1,056.3 \\
139.5 \\
- \\
139.5 \\
460.8 \\
456.0 \\
77.3 \\
80.7 \\
1.0 \\
25.3 \\
61.7 \\
34.4 \\
5.2 \\
170.4 \\
1,958.3\end{array}$ \\
\hline 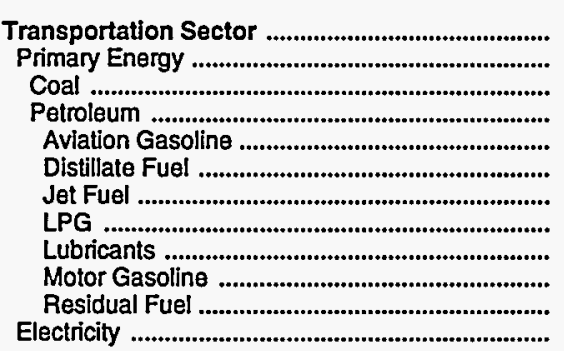 & $\begin{array}{r}699.9 \\
699.9 \\
(\mathrm{~s}) \\
699.8 \\
1.3 \\
53.6 \\
13.6 \\
.5 \\
15.1 \\
615.7 \\
(\mathrm{~s}) \\
(\mathrm{s})\end{array}$ & $\begin{array}{r}1,553.0 \\
1,553.0 \\
(s) \\
1,553.0 \\
1.2 \\
187.1 \\
45.1 \\
1.1 \\
36.6 \\
1,279.8 \\
2.0 \\
\text { (s) }\end{array}$ & $\begin{array}{r}3,607.6 \\
3,607.6 \\
- \\
3,607.6 \\
13.2 \\
557.1 \\
149.8 \\
1.1 \\
58.9 \\
2,827.3 \\
.1 \\
(s)\end{array}$ & $\begin{array}{r}3,488.2 \\
3,488.2 \\
- \\
3,488.2 \\
7.8 \\
596.7 \\
160.1 \\
5.2 \\
65.7 \\
2,652.7 \\
\overline{(s)}\end{array}$ & $\begin{array}{r}\mathrm{R}_{3,966.3} \\
\mathrm{R}_{3,966.3} \\
\mathrm{R}_{3,966.3} \\
8.2 \\
965.8 \\
131.7 \\
4.3 \\
\mathrm{R}_{61.3} \\
2,794.9 \\
.1 \\
\text { (s) }\end{array}$ & $\begin{array}{r}R_{3,633.1} \\
\mathrm{R}_{3,633.1} \\
- \\
\mathrm{R}_{3,633.1} \\
6.4 \\
857.0 \\
92.9 \\
4.1 \\
\mathrm{R}_{63.1} \\
2,609.1 \\
.5 \\
\text { (s) }\end{array}$ & $\begin{array}{r}R_{3,770.5} \\
R_{3,770.5} \\
- \\
R_{3,770.5} \\
14.8 \\
858.6 \\
115.3 \\
3.4 \\
R_{74.4} \\
2,703.6 \\
.5 \\
(\mathrm{~s})\end{array}$ & $\begin{array}{r}3,975.7 \\
3,975.7 \\
- \\
3,975.7 \\
16.4 \\
864.8 \\
155.5 \\
4.7 \\
73.8 \\
2,860.3 \\
.2 \\
(s)\end{array}$ \\
\hline 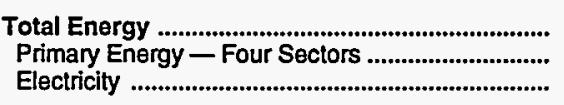 & $\begin{array}{r}1,482.0 \\
977.4 \\
504.6\end{array}$ & $\begin{array}{l}3,468.5 \\
2,111.6 \\
1,357.0\end{array}$ & $\begin{array}{l}7,678.1 \\
5,021.6 \\
2,656.5\end{array}$ & $\begin{array}{l}8,621.5 \\
5,211.8 \\
3,409.7\end{array}$ & $\begin{array}{r}\text { F }_{9,660.0} \\
\text { F }_{5,605.1} \\
4,054.9\end{array}$ & $\begin{array}{r}\text { R }_{9,280.7} \\
\mathrm{R}_{5,239.3} \\
4,041.4\end{array}$ & $\begin{array}{l}R_{9,539.7} \\
R_{5,499.6} \\
R_{4,040.0}\end{array}$ & $\begin{array}{l}9,892.3 \\
5,777.0 \\
4,115.3\end{array}$ \\
\hline 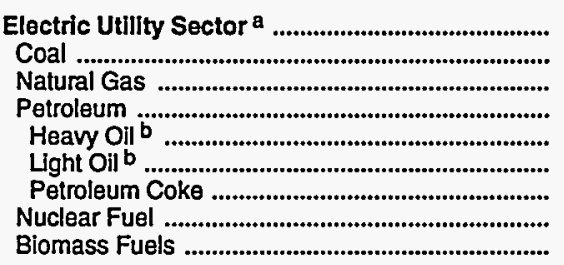 & $\begin{array}{r}80.9 \\
76.5 \\
4.4 \\
= \\
= \\
= \\
= \\
-\end{array}$ & $\begin{array}{r}376.4 \\
359.6 \\
\overline{-} \\
16.7 \\
\overline{-} \\
16.7 \\
- \\
=\end{array}$ & $\begin{array}{r}804.8 \\
784.9 \\
2.6 \\
15.1 \\
\overline{15.1} \\
\overline{2.1} \\
-\end{array}$ & $\begin{array}{r}846.9 \\
757.7 \\
\overline{8.1} \\
\overline{8.1} \\
\overline{-1.1} \\
-\end{array}$ & $\begin{array}{r}803.6 \\
668.5 \\
1.6 \\
7.6 \\
\overline{7.6} \\
\overline{-} \\
125.9 \\
-\end{array}$ & $\begin{array}{r}745.1 \\
585.5 \\
.5 \\
7.9 \\
\overline{7.9} \\
\overline{-} \\
151.1 \\
-\end{array}$ & $\begin{array}{r}791.8 \\
628.5 \\
.7 \\
6.3 \\
\overline{6.3} \\
\overline{-} \\
156.4 \\
-\end{array}$ & $\begin{array}{r}784.6 \\
736.4 \\
4.3 \\
10.4 \\
\overline{10.4} \\
3 . \overline{6} \\
-\end{array}$ \\
\hline Primary Energy — Five Sectors c ............................... & $1,058.3$ & $2,487.9$ & $5,826.4$ & $6,058.7$ & ${ }_{6,408.7}$ & $R_{5,984.4}$ & $\mathrm{R}_{6,291.5}$ & $6,561.6$ \\
\hline
\end{tabular}

a There are no direct fuel costs for hydroelectric, geothermal, wind, photovoltaic, or solar thermal energy.

b Heavy oil includes fuel oil nos. 4, 5, and 6, and residual fuel oils. Light oil includes fuel nos. 1 and 2, kerosene, and jet fuel.

c Biomass fuels are not included, except those consumed at electric utilities and those added to motor gasoline.

$\mathrm{R}=$ Revised data.

-No consumption, including cases where adjustments were made. See explanation of adjustments in Section 6 of Appendix A.

(s)=Value less than 0.05 million dollars.

Note: Totals may not equal sum of components due to independent rounding.

Sources: Data sources, estimation procedures, and assumptions are described in Appendix A. 


\begin{tabular}{|c|c|c|c|c|c|c|c|c|}
\hline Energy Source & 1970 & 1975 & 1980 & 1985 & 1990 & 1991 & 1992 & 1993 \\
\hline & \multicolumn{8}{|c|}{ Prices In Dollars per Million Btu } \\
\hline 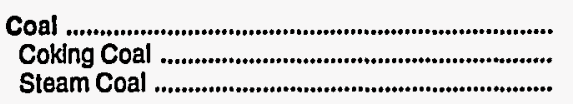 & $\begin{array}{r}0.39 \\
.38 \\
.86\end{array}$ & $\begin{array}{r}0.61 \\
1.60 \\
.46\end{array}$ & $\begin{array}{l}1.22 \\
1.81 \\
1.19\end{array}$ & $\begin{array}{l}1.60 \\
1.93 \\
1.59\end{array}$ & $1 . \overline{44}$ & $\begin{array}{r}1.49 \\
1.49\end{array}$ & $\begin{array}{r}1.48 \\
1.48\end{array}$ & $\frac{1.42}{1.42}$ \\
\hline 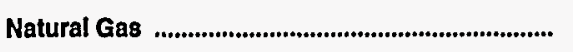 & .29 & .89 & 2.17 & 3.38 & 2.47 & 2.30 & 2.47 & 2.79 \\
\hline 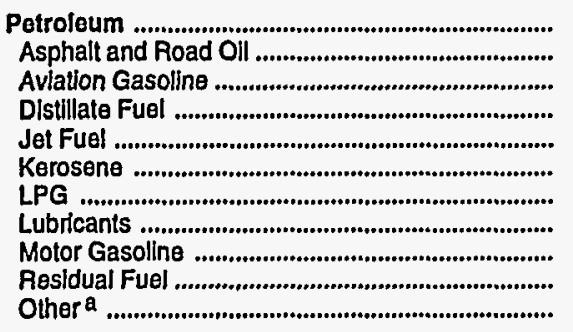 & $\begin{array}{r}1.55 \\
.74 \\
2.17 \\
.94 \\
.72 \\
.76 \\
1.07 \\
5.08 \\
2.66 \\
.42 \\
.79\end{array}$ & $\begin{array}{l}3.03 \\
1.77 \\
3.45 \\
2.35 \\
2.01 \\
2.24 \\
2.49 \\
7.48 \\
4.36 \\
1.61 \\
2.49\end{array}$ & $\begin{array}{r}6.63 \\
3.79 \\
9.02 \\
6.73 \\
6.34 \\
6.91 \\
5.29 \\
14.36 \\
9.26 \\
2.49 \\
6.59\end{array}$ & $\begin{array}{r}6.53 \\
4.20 \\
9.99 \\
6.43 \\
5.67 \\
7.57 \\
4.42 \\
17.61 \\
8.79 \\
4.00 \\
7.00\end{array}$ & $\begin{array}{r}6.22 \\
2.94 \\
9.32 \\
6.74 \\
5.41 \\
4.69 \\
4.13 \\
\mathrm{R}_{14.60} \\
9.16 \\
2.93 \\
\mathrm{R}_{5.89}\end{array}$ & $\begin{array}{r}{ }^{8} 5.98 \\
3.33 \\
8.71 \\
6.58 \\
4.64 \\
4.56 \\
4.21 \\
\mathrm{R}_{16.79} \\
9.09 \\
2.97 \\
\mathrm{R} 5.08\end{array}$ & $\begin{array}{r}\mathrm{R}_{5.80} \\
2.33 \\
8.54 \\
6.92 \\
4.32 \\
5.23 \\
3.97 \\
\mathrm{R}_{19.43} \\
9.05 \\
\mathrm{R}_{1.90} \\
\mathrm{R}_{4.88}\end{array}$ & $\begin{array}{r}5.79 \\
3.14 \\
8.24 \\
6.99 \\
4.01 \\
5.57 \\
3.81 \\
18.94 \\
9.16 \\
1.88 \\
4.37\end{array}$ \\
\hline 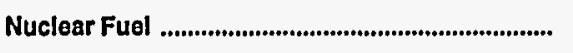 & - & - & - & - & .56 & .49 & .52 & .69 \\
\hline 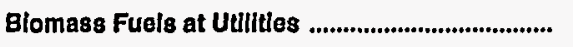 & .65 & .92 & 1.74 & .79 & .35 & .35 & .35 & .35 \\
\hline Primary Energy - Five Sectors b ............................. & .85 & 1.93 & 4.22 & 4.61 & 4.02 & $\mathrm{R}_{3.84}$ & $R_{3.84}$ & 3.92 \\
\hline 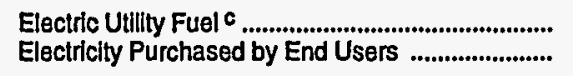 & $\begin{array}{r}.25 \\
4.52\end{array}$ & $\begin{array}{r}.73 \\
6.81\end{array}$ & $\begin{array}{r}1.65 \\
12.69\end{array}$ & $\begin{array}{r}2.44 \\
18.58\end{array}$ & $\begin{array}{r}1.67 \\
17.09\end{array}$ & $\begin{array}{r}1.61 \\
17.91\end{array}$ & $\begin{array}{r}1.67 \\
18.21\end{array}$ & $\begin{array}{r}1.81 \\
18.93\end{array}$ \\
\hline \multirow[t]{2}{*}{ 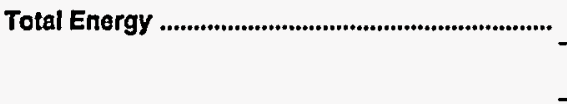 } & 1.28 & 2.80 & 6.00 & 7.04 & 6.43 & 6.30 & 6.28 & 6.46 \\
\hline & \multicolumn{8}{|c|}{ Expenditures in Millions of Dollars } \\
\hline 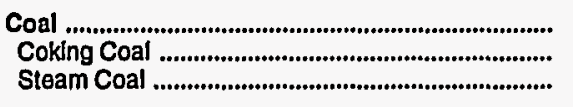 & $\begin{array}{r}11.9 \\
11.6 \\
.2\end{array}$ & $\begin{array}{r}120.2 \\
41.0 \\
79.2\end{array}$ & $\begin{array}{r}892.5 \\
47.9 \\
844.6\end{array}$ & $\begin{array}{r}1,832.9 \\
20.9 \\
1,812.0\end{array}$ & $\begin{array}{c}1,918.8 \\
1,918.8\end{array}$ & $\begin{array}{c}1,981.4 \\
\overline{1} \\
1,981.4\end{array}$ & $\begin{array}{l}1,961.2 \\
1,961.2\end{array}$ & $\begin{array}{l}2,008.2 \\
2,008.2\end{array}$ \\
\hline Natural Gas & 804.9 & $2,361.3$ & $6,838.0$ & $9,815.8$ & $7,311.4$ & $6,953.6$ & $7,289.1$ & $8,847.6$ \\
\hline 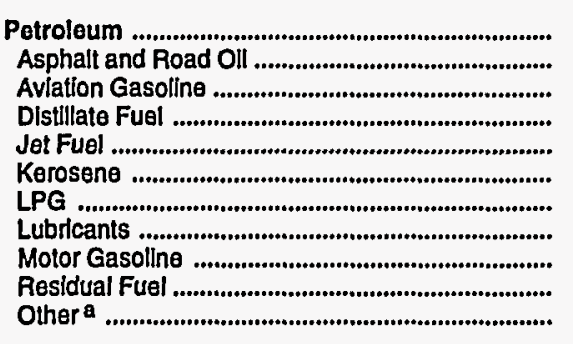 & $\begin{array}{r}3,376.3 \\
58.0 \\
22.0 \\
176.6 \\
97.4 \\
32.5 \\
611.9 \\
129.6 \\
1,976.0 \\
36.0 \\
236.4\end{array}$ & $\begin{array}{r}8,323.8 \\
95.7 \\
22.8 \\
735.9 \\
306.2 \\
91.3 \\
1,452.4 \\
196.2 \\
4,020.6 \\
383.7 \\
1,019.0\end{array}$ & \begin{tabular}{r|}
$23,998.1$ \\
274.5 \\
57.5 \\
$2,823.7$ \\
$1,098.5$ \\
601.2 \\
$3,670.4$ \\
465.0 \\
$8,805.7$ \\
969.9 \\
$5,231.6$
\end{tabular} & $\begin{array}{r}24,077.8 \\
329.2 \\
66.4 \\
3,522.2 \\
2,383.1 \\
33.3 \\
4,077.4 \\
519.0 \\
9,479.5 \\
710.5 \\
2,957.1\end{array}$ & $\begin{array}{r}\mathbf{R}_{24,464.3} \\
273.0 \\
39.4 \\
3,230.1 \\
2,931.6 \\
5.5 \\
4,376.3 \\
\mathrm{R}_{484.1} \\
9,831.0 \\
505.9 \\
\mathbf{R}_{2,807.3}\end{array}$ & $\begin{array}{r}\text { R } 23,763.4 \\
206.8 \\
28.8 \\
3,244.5 \\
2,377.9 \\
2.4 \\
4,873.8 \\
R_{498.2} \\
9,485.4 \\
524.9 \\
\mathrm{R}_{2,520.5}\end{array}$ & $\begin{array}{r}R_{23,787.1} \\
182.3 \\
33.8 \\
3,637.3 \\
2,198.6 \\
5.1 \\
4,783.0 \\
R_{587.8} \\
9,543.5 \\
R_{364.0} \\
R_{2,451.7}\end{array}$ & $\begin{array}{r}23,576.0 \\
264.9 \\
28.8 \\
3,736.8 \\
1,975.4 \\
4.8 \\
4,556.3 \\
583.3 \\
9,981.5 \\
263.4 \\
2,180.9\end{array}$ \\
\hline 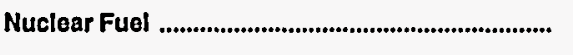 & - & - & - & - & 94.9 & 103.3 & 135.9 & 91.7 \\
\hline 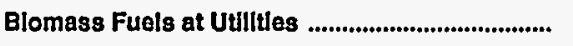 & .7 & .9 & 1.4 & 2.5 & 1.0 & 1.0 & 1.0 & 1.1 \\
\hline Primary Energy - Fivo Sectors ${ }^{b}$............................... & $4,193.8$ & $10,806.1$ & $31,730.0$ & $35,729.0$ & ${ }^{P_{33,810.3}}$ & $\mathrm{H}_{32,802.7}$ & $R_{33,174,4}$ & $34,524.6$ \\
\hline 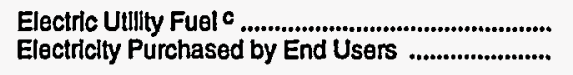 & $\begin{array}{r}-267.6 \\
1,421.0\end{array}$ & $\begin{array}{r}-1,099.3 \\
2,895.0\end{array}$ & $\begin{array}{r}-3,576.1 \\
7,434.5\end{array}$ & $\begin{array}{r}-5,652.8 \\
13,119.7\end{array}$ & $\begin{array}{r}-4,165.2 \\
13,431.1\end{array}$ & $\begin{array}{r}-4,063.1 \\
14,270.8\end{array}$ & $\begin{array}{r}-4,210.8 \\
14,457.3\end{array}$ & $\begin{array}{r}-4,678.8 \\
15,644.3\end{array}$ \\
\hline . & $5,347.2$ & $12,601.8$ & $35,588.4$ & $43,195.9$ & ค $_{43,076.2}$ & $R_{43,010.4}$ & $R_{43,420.9}$ & $45,490.2$ \\
\hline
\end{tabular}

a Includes petroleum coke used at electric utilities.

b Blomass fuels are not included, except those consumed at electric utillties and those added to motor gasoline.

$c$ There are no direct fuel costs for hydroelectric, geothermal, wind, photovoltalc, or solar thermal energy.

$R=$ Revised data.
-No consumption, Including cases where adjustments were made. See explanation of adjustments in Section 6 of Appendix A.

Note: Expenditure totals may not equal sum of components due to Independent rounding.

Sources: Data sources, estimation procedures, and assumptions are described in Appendix A. 
T Energy Price Estimates by Sector, Texas 1970, 1975, 1980, 1985, and 1990-1993

X (Dollars per Million Btu)

\begin{tabular}{|c|c|c|c|c|c|c|c|c|}
\hline Sector and Energy Source & $1970^{\circ}$ & 1975 & 1980 & 1985 & 1990 & 1991 & 1992 & 1993 \\
\hline $\begin{array}{l}\text { Residentlal Sector } \\
\text { Primary Energy } \\
\text { Coal } \\
\text { Natural Gas } \\
\text { Petroleum } \\
\text { Distillate Fuel } \\
\text { Kerosene }\end{array}$ & $\begin{array}{r}2.48 \\
1.06 \\
.90 \\
.90 \\
1.70 \\
.98 \\
1.29 \\
1.71 \\
6.31\end{array}$ & $\begin{array}{l}4.08 \\
1.79 \\
1 . \overline{8} \\
3.45 \\
2.24 \\
3.01 \\
3.50 \\
8.74\end{array}$ & $\begin{array}{r}8.55 \\
3.69 \\
2.54 \\
3.31 \\
7.43 \\
6.51 \\
8.35 \\
7.39 \\
14.92\end{array}$ & $\begin{array}{r}13.88 \\
5.87 \\
2.83 \\
5.55 \\
8.52 \\
6.99 \\
8.40 \\
8.53 \\
21.99\end{array}$ & $\begin{array}{r}14.13 \\
5.99 \\
2.41 \\
5.55 \\
10.36 \\
4.32 \\
5.20 \\
10.39 \\
21.11\end{array}$ & $\begin{array}{r}14.64 \\
5.86 \\
2.36 \\
5.49 \\
11.61 \\
3.98 \\
4.78 \\
11.71 \\
22.17\end{array}$ & $\begin{array}{r}14.90 \\
5.75 \\
2.43 \\
5.51 \\
10.03 \\
5.46 \\
6.57 \\
10.07 \\
22.69\end{array}$ & $\begin{array}{r}15.46 \\
5.96 \\
2.16 \\
5.74 \\
9.75 \\
5.78 \\
6.94 \\
9.80 \\
23.45\end{array}$ \\
\hline 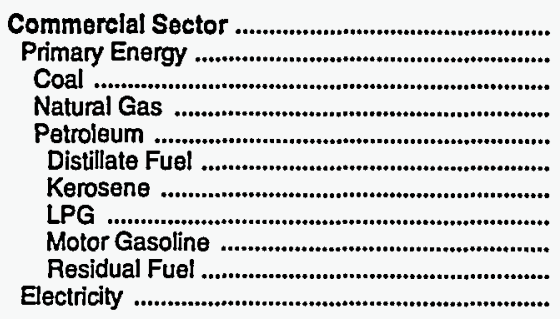 & $\begin{array}{r}1.91 \\
.56 \\
.86 \\
.44 \\
1.01 \\
.90 \\
.76 \\
1.00 \\
2.66 \\
.48 \\
5.19\end{array}$ & $\begin{array}{r}3.92 \\
1.41 \\
1 . \overline{-} \\
2.37 \\
2.14 \\
2.23 \\
2.42 \\
4.36 \\
1.87 \\
7.59\end{array}$ & $\begin{array}{r}7.77 \\
3.89 \\
.89 \\
2.90 \\
6.24 \\
6.23 \\
6.89 \\
5.22 \\
9.26 \\
2.53 \\
14.12\end{array}$ & $\begin{array}{r}12.20 \\
5.23 \\
1.60 \\
4.70 \\
6.38 \\
6.15 \\
7.43 \\
4.30 \\
8.79 \\
3.87 \\
20.06\end{array}$ & $\begin{array}{r}11.58 \\
4.25 \\
1.14 \\
3.97 \\
5.64 \\
3.84 \\
4.85 \\
4.00 \\
9.16 \\
2.60 \\
18.12\end{array}$ & $\begin{array}{r}12.03 \\
4.03 \\
1.23 \\
3.86 \\
5.13 \\
3.57 \\
4.43 \\
4.11 \\
9.09 \\
1.95 \\
19.11\end{array}$ & $\begin{array}{r}12.23 \\
4.12 \\
1.27 \\
3.90 \\
5.62 \\
4.42 \\
5.03 \\
3.91 \\
9.05 \\
2.07 \\
19.56\end{array}$ & $\begin{array}{r}12.17 \\
3.84 \\
1.16 \\
3.80 \\
4.33 \\
4.13 \\
5.23 \\
3.74 \\
9.16 \\
20 . \overline{-}\end{array}$ \\
\hline 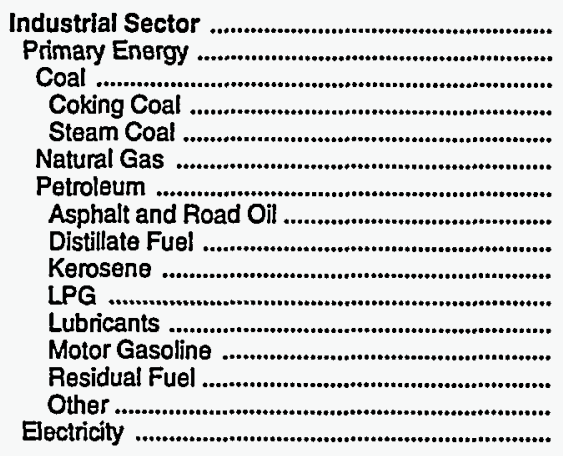 & $\begin{array}{r}.63 \\
.53 \\
.38 \\
.38 \\
.86 \\
.20 \\
.96 \\
.74 \\
.66 \\
.76 \\
1.00 \\
5.08 \\
2.66 \\
.37 \\
.79 \\
2.51\end{array}$ & $\begin{array}{l}1.95 \\
1.73 \\
1.20 \\
1.60 \\
1.01 \\
.92 \\
2.41 \\
1.77 \\
2.02 \\
2.23 \\
2.42 \\
7.48 \\
4.36 \\
1.51 \\
2.49 \\
4.70\end{array}$ & $\begin{array}{r}4.74 \\
4.34 \\
1.28 \\
1.81 \\
.89 \\
2.24 \\
5.92 \\
3.79 \\
6.09 \\
6.89 \\
5.22 \\
14.36 \\
9.26 \\
3.69 \\
6.59 \\
9.99\end{array}$ & $\begin{array}{r}5.08 \\
4.31 \\
1.64 \\
1.93 \\
1.60 \\
3.07 \\
5.42 \\
4.20 \\
6.39 \\
7.43 \\
4.30 \\
17.61 \\
8.79 \\
3.87 \\
7.00 \\
14.15\end{array}$ & $\begin{array}{r}4.04 \\
3.43 \\
1.14 \\
-\overline{14} \\
1.14 \\
2.09 \\
4.64 \\
2.94 \\
4.17 \\
4.85 \\
4.00 \\
\mathrm{R} 14.60 \\
9.16 \\
2.60 \\
\mathrm{R} 5.89 \\
11.82\end{array}$ & $\begin{array}{r}R_{3.89} \\
3.26 \\
1.23 \\
-\overline{1.23} \\
1.86 \\
4.51 \\
3.33 \\
3.81 \\
4.43 \\
4.11 \\
16.79 \\
9.09 \\
1.95 \\
R 5.08 \\
12.16\end{array}$ & $\begin{array}{r}3.91 \\
R_{3.28} \\
1.27 \\
- \\
1.27 \\
2.02 \\
4.33 \\
2.33 \\
4.32 \\
5.03 \\
3.91 \\
\text { R } 19.43 \\
9.05 \\
2.07 \\
R_{4.88} \\
12.31\end{array}$ & $\begin{array}{r}3.97 \\
3.33 \\
1.16 \\
- \\
1.16 \\
2.46 \\
4.12 \\
3.14 \\
4.49 \\
5.23 \\
3.74 \\
18.94 \\
9.16 \\
1.94 \\
4.38 \\
12.67\end{array}$ \\
\hline 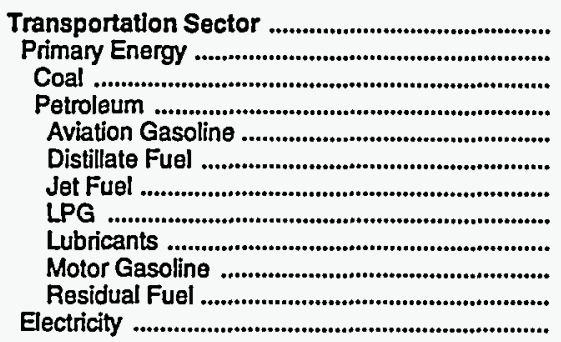 & $\begin{array}{r}2.07 \\
2.07 \\
.86 \\
2.07 \\
2.17 \\
1.05 \\
.72 \\
1.00 \\
5.08 \\
2.66 \\
.42 \\
-\end{array}$ & $\begin{array}{l}3.54 \\
3.54 \\
1.01 \\
3.54 \\
3.45 \\
2.49 \\
2.01 \\
2.42 \\
7.48 \\
4.36 \\
1.63 \\
-\end{array}$ & $\begin{array}{r}7.42 \\
7.42 \\
- \\
7.42 \\
9.02 \\
7.09 \\
6.34 \\
5.22 \\
14.36 \\
9.26 \\
2.15 \\
-\end{array}$ & $\begin{array}{r}7.44 \\
7.44 \\
- \\
7.44 \\
9.99 \\
6.50 \\
5.67 \\
4.30 \\
17.61 \\
8.79 \\
4.03 \\
-\end{array}$ & $\begin{array}{r}R 7.57 \\
R 7.57 \\
- \\
R 7.57 \\
9.32 \\
8.20 \\
5.41 \\
4.00 \\
R \quad 14.60 \\
9.16 \\
2.94 \\
-\end{array}$ & $\begin{array}{r}\text { R7.30 } \\
\text { R7.30 } \\
-\overline{-} \\
\text { R7.30 } \\
8.71 \\
7.84 \\
4.64 \\
4.11 \\
\text { R } 16.79 \\
9.09 \\
3.00 \\
-\end{array}$ & $\begin{array}{r}7.13 \\
7.13 \\
- \\
7.13 \\
8.54 \\
7.99 \\
4.32 \\
3.91 \\
R 19.43 \\
9.05 \\
1.90 \\
-\end{array}$ & $\begin{array}{r}7.33 \\
7.33 \\
- \\
7.33 \\
8.24 \\
7.94 \\
4.01 \\
3.74 \\
18.94 \\
9.16 \\
1.87 \\
-\end{array}$ \\
\hline 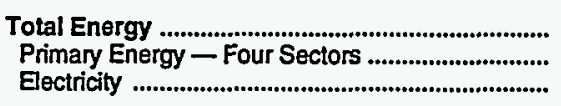 & $\begin{array}{l}1.28 \\
1.02 \\
4.52\end{array}$ & $\begin{array}{l}2.80 \\
2.38 \\
6.81\end{array}$ & $\begin{array}{r}6.00 \\
5.27 \\
12.69\end{array}$ & $\begin{array}{r}7.04 \\
5.54 \\
18.58\end{array}$ & $\begin{array}{r}6.43 \\
5.01 \\
17.09\end{array}$ & $\begin{array}{r}6.30 \\
4.77 \\
17.91\end{array}$ & $\begin{array}{r}6.28 \\
4.73 \\
18.21\end{array}$ & $\begin{array}{r}6.46 \\
4.80 \\
18.93\end{array}$ \\
\hline 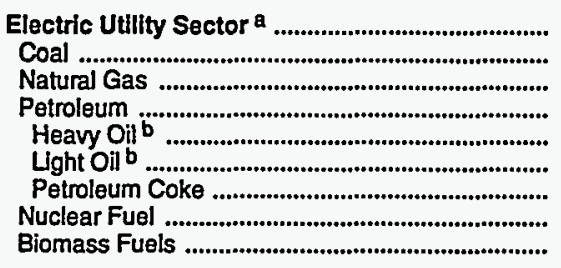 & $\begin{array}{l}.25 \\
. \overline{24} \\
.51 \\
.47 \\
.63 \\
\overline{-} \\
.65\end{array}$ & $\begin{array}{r}.73 \\
.23 \\
.76 \\
1.89 \\
1.89 \\
2.03 \\
= \\
.92\end{array}$ & $\begin{array}{l}1.65 \\
1.21 \\
1.84 \\
3.35 \\
2.59 \\
3.83 \\
- \\
1.74\end{array}$ & $\begin{array}{r}2.44 \\
1.59 \\
3.15 \\
4.90 \\
4.36 \\
5.57 \\
- \\
.79\end{array}$ & $\begin{array}{r}1.67 \\
1.45 \\
2.10 \\
5.14 \\
3.50 \\
5.78 \\
.56 \\
.35\end{array}$ & $\begin{array}{r}1.61 \\
1.50 \\
1.97 \\
4.56 \\
3.47 \\
4.91 \\
\overline{.49} \\
.35\end{array}$ & $\begin{array}{l}1.67 \\
1.49 \\
2.20 \\
3.35 \\
2.16 \\
4.11 \\
-52 \\
.55\end{array}$ & $\begin{array}{r}1.81 \\
1.44 \\
2.41 \\
2.09 \\
2.07 \\
3.85 \\
.84 \\
.69 \\
.35\end{array}$ \\
\hline Primary Energy — Flve Sectors ${ }^{c}$.............................. & .85 & 1.93 & 4.22 & 4.61 & 4.02 & $\mathrm{R}_{3.84}$ & R3.84 & 3.92 \\
\hline
\end{tabular}

There are no direct fuel costs for hydroelectric, geothermal, wind, photovoltaic, or solar thermal energy.

b Heavy oil includes fuel oil nos. 4, 5, and 6, and residual fuel oils. Light oil includes fuel oil nos. 1 and 2 , kerosene, and jet fuel.

c Biomass fuels are not included, except those consumed at electric utilities and those added to motor gasoline.
$R=$ Revised data.

-No consumption, including cases where adjustments were made. See explanation of adjustments in Section 6 of Appendix A.

Sources: Data sources, estimation procedures, and assumptions are described in Appendix $A$. 


\begin{tabular}{|c|c|c|c|c|c|c|c|c|}
\hline Sector and Energy Source & 1970 & 1875 & 1980 & 1985 & 1990 & 1991 & 1992 & 1993 \\
\hline 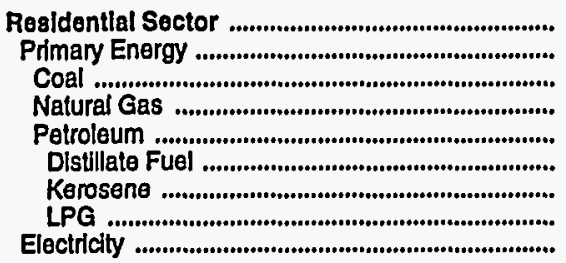 & $\begin{array}{r}1,015.6 \\
314.3 \\
(s) \\
213.8 \\
100.5 \\
.8 \\
.2 \\
.2 \\
99.5 \\
701.2\end{array}$ & $\begin{array}{r}1,726.1 \\
506.5 \\
-\overline{3} \\
353.8 \\
152.7 \\
3.5 \\
.7 \\
148.5 \\
1,219.6\end{array}$ & $\begin{array}{r}3,852.3 \\
942.0 \\
(s) \\
765.9 \\
176.1 \\
.3 \\
9.4 \\
166.4 \\
2,910.3\end{array}$ & $\begin{array}{r}6,839.0 \\
1,457.1 \\
.2 \\
1,226.8 \\
230.2 \\
1.6 \\
5.4 \\
223.3 \\
5,381.8\end{array}$ & $\begin{array}{r}7,386.7 \\
1,449.7 \\
.2 \\
1,217.6 \\
231.9 \\
.1 \\
.8 \\
231.1 \\
5,947.0\end{array}$ & $\begin{array}{r}7,802.0 \\
1,441.0 \\
.2 \\
1,268.8 \\
172.0 \\
.1 \\
.9 \\
171.0 \\
17\end{array}$ & $\begin{array}{r}7,710.8 \\
1,367.8 \\
.2 \\
1,240.9 \\
126.8 \\
.1 \\
.8 \\
.8 \\
125.9 \\
6,343.0\end{array}$ & $\begin{array}{r}8,518.1 \\
1,501.1 \\
.1 \\
1,369.9 \\
131.1 \\
.1 \\
1.2 \\
129.8 \\
7,017.0\end{array}$ \\
\hline 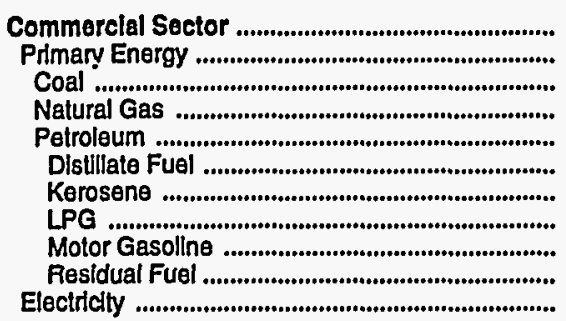 & $\begin{array}{r}511.7 \\
106.4 \\
(s) \\
66.3 \\
40.1 \\
4.4 \\
15.6 \\
10.3 \\
9.7 \\
.2 \\
405.2\end{array}$ & $\begin{array}{r}1,115.4 \\
238.3 \\
122.6 \\
115.7 \\
20.8 \\
53.1 \\
18.1 \\
15.7 \\
7.9 \\
877.2\end{array}$ & $\begin{array}{r}3,079.0 \\
956.5 \\
(\mathrm{~s}) \\
504.3 \\
452.2 \\
103.1 \\
126.9 \\
20.7 \\
160.5 \\
40.9 \\
2,122.5\end{array}$ & $\begin{array}{r}5,327.5 \\
1,211.5 \\
.2 \\
741.3 \\
470.0 \\
343.3 \\
10.6 \\
19.9 \\
90.2 \\
6.1 \\
4,116.0\end{array}$ & $\begin{array}{r}5,291.4 \\
914.3 \\
.2 \\
713.5 \\
200.6 \\
73.3 \\
.7 \\
15.7 \\
109.8 \\
1.2 \\
4,377.0\end{array}$ & $\begin{array}{r}5,582.4 \\
878.4 \\
.2 \\
725.7 \\
152.4 \\
61.4 \\
.3 \\
10.6 \\
77.5 \\
2.7 \\
4,704.0\end{array}$ & $\begin{array}{r}5,724.9 \\
915.0 \\
.2 \\
755.3 \\
159.4 \\
79.9 \\
1.9 \\
8.6 \\
68.8 \\
.2 \\
4,810.0\end{array}$ & $\begin{array}{r}6,165.6 \\
955.6 \\
.1 \\
882.0 \\
73.5 \\
56.3 \\
.7 \\
8.7 \\
7.7 \\
- \\
5,210.0\end{array}$ \\
\hline 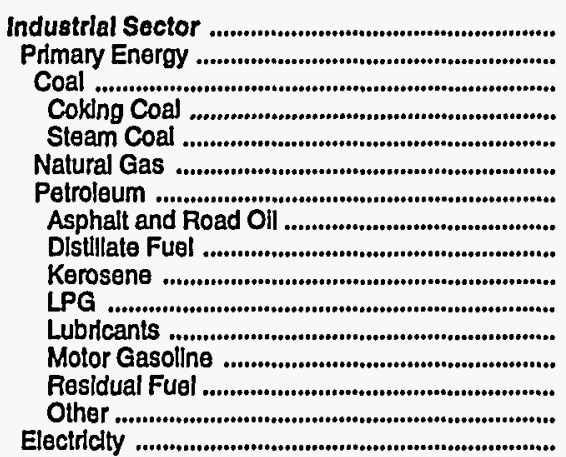 & $\begin{array}{r}1,514.4 \\
1,199.9 \\
11.8 \\
11.6 \\
.2 \\
258.3 \\
929.8 \\
58.0 \\
33.9 \\
16.7 \\
481.0 \\
79.6 \\
19.7 \\
4.5 \\
236.4 \\
314.5\end{array}$ & $\begin{array}{r}4,527.0 \\
3,728.8 \\
93.3 \\
41.0 \\
52.3 \\
834.9 \\
2,800.6 \\
95.7 \\
168.1 \\
37.5 \\
1,241.2 \\
117.3 \\
22.8 \\
99.0 \\
1,019.0 \\
798.2\end{array}$ & $\begin{array}{r}16,088.6 \\
13,687.0 \\
80.9 \\
47.9 \\
32.9 \\
2,840.6 \\
10,765.5 \\
274.5 \\
701.9 \\
464.9 \\
3,470.9 \\
298.7 \\
22.9 \\
300.1 \\
5,231.6 \\
2,401.7\end{array}$ & $\begin{array}{r}16,529.5 \\
12,907.7 \\
138.5 \\
20.9 \\
117.6 \\
3,940.8 \\
8,828.4 \\
329.2 \\
1,016.2 \\
17.4 \\
3,824.9 \\
333.4 \\
217.1 \\
133.2 \\
2,957.1 \\
3,621.9\end{array}$ & $\begin{array}{r}R_{14,733.8} \\
R_{11,626.8} \\
69.9 \\
- \\
69.9 \\
3,188.7 \\
R_{8,368.3} \\
273.0 \\
627.6 \\
4.1 \\
4,122.5 \\
R_{311.0} \\
207.5 \\
15.2 \\
R_{2,807.3} \\
3,107.1\end{array}$ & $\begin{array}{r}\mathrm{R}_{14,674.9} \\
\mathrm{R}_{11,469.1} \\
77.8 \\
-\overline{77.8} \\
2,915.7 \\
\mathrm{R}_{8,475.6} \\
206.8 \\
512.4 \\
1.2 \\
4,687.0 \\
\mathrm{R}_{320.1} \\
220.4 \\
7.2 \\
\text { म }_{2,520.5} \\
3,205.7\end{array}$ & $\begin{array}{r}R_{14,942.3} \\
R_{11,638.0} \\
76.9 \\
- \\
76.9 \\
3,112.5 \\
R_{8,448.5} \\
182.3 \\
579.3 \\
2.3 \\
4,644.1 \\
R_{377.6} \\
206.3 \\
4.7 \\
R_{2,451.7} \\
3,304.4\end{array}$ & $\begin{array}{r}15,452.4 \\
12,035.1 \\
82.0 \\
- \\
82.0 \\
3,947.0 \\
8,006.2 \\
264.9 \\
583.2 \\
2.9 \\
4,413.1 \\
374.7 \\
165.4 \\
22.7 \\
2,179.2 \\
3,417.3\end{array}$ \\
\hline 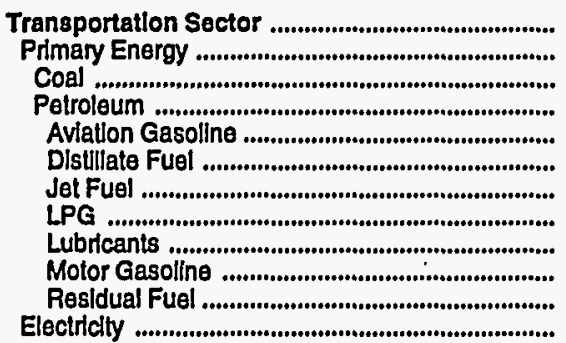 & $\begin{array}{r}2,305.6 \\
2,305.6 \\
(s) \\
2,305.5 \\
22.0 \\
137.5 \\
97.4 \\
21.1 \\
50.0 \\
1,846.6 \\
30.9 \\
-\end{array}$ & $\begin{array}{r}5,233.3 \\
5,233.3 \\
(s) \\
5,233.3 \\
22.8 \\
542.6 \\
306.2 \\
44.6 \\
78.9 \\
3,982.0 \\
256.2 \\
-\end{array}$ & $\begin{array}{r}12,568.4 \\
12,568.4 \\
- \\
12,568.4 \\
57.5 \\
1,993.2 \\
1,098.5 \\
12.4 \\
166.3 \\
8,622.3 \\
618.1 \\
-\end{array}$ & $\begin{array}{r}14,499.9 \\
14,499.9 \\
- \\
14,499.9 \\
66.4 \\
2,136.1 \\
2,383.1 \\
9.4 \\
185.6 \\
9,172.2 \\
547.0 \\
-\end{array}$ & $\begin{array}{r}R_{15,654.3} \\
A_{15,654.3} \\
- \\
R_{15,654.3} \\
39.4 \\
2,505.6 \\
2,931.6 \\
7.0 \\
R_{173.1} \\
9,513.7 \\
484.0 \\
-\end{array}$ & $\begin{array}{r}R_{14,951.1} \\
R_{14,951.1} \\
- \\
R_{14,951.1} \\
28.8 \\
2,660.7 \\
2,377.9 \\
5.1 \\
R_{178.1} \\
9,187.6 \\
512.8 \\
-\end{array}$ & $\begin{array}{r}R_{15,042.9} \\
R_{15,042.9} \\
- \\
R_{15,042.9} \\
33.8 \\
2,970.9 \\
2,198.6 \\
4.4 \\
R_{210.2} \\
9,268.4 \\
R_{356.7} \\
-\end{array}$ & $\begin{array}{r}15,354.1 \\
15,354.1 \\
- \\
15,354.1 \\
28.8 \\
3,091.8 \\
1,975.4 \\
4.7 \\
208.6 \\
9,808.4 \\
236.4 \\
-\end{array}$ \\
\hline 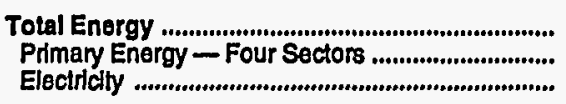 & $\begin{array}{l}5,347.2 \\
3,926.2 \\
1,421.0\end{array}$ & $\begin{array}{r}12,601.8 \\
9,706.8 \\
2,895.0\end{array}$ & $\begin{array}{r}35,588.4 \\
28,153.9 \\
7,434.5\end{array}$ & $\begin{array}{l}43,195.9 \\
30,076.2 \\
13,119.7\end{array}$ & $\begin{array}{r}R_{43,076.2} \\
R_{29,645.2} \\
13,431.1\end{array}$ & $\begin{aligned} R_{43,010.4} \\
R_{28,739.6} \\
14,270.8\end{aligned}$ & $\begin{array}{r}R_{43,420.9} \\
R_{28,963.6} \\
14,457.3\end{array}$ & $\begin{array}{l}45,490.2 \\
29,845.9 \\
15,644.3\end{array}$ \\
\hline 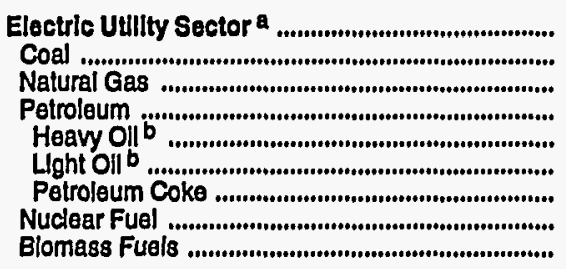 & $\begin{array}{r}267.6 \\
266 . \overline{5} \\
.5 \\
.3 \\
.2 \\
\overline{-} \\
\overline{.7}\end{array}$ & $\begin{array}{r}1,099.3 \\
26.9 \\
1,050.0 \\
21.5 \\
20.6 \\
.9 \\
\overline{-} \\
.9\end{array}$ & $\begin{array}{r}3,576.1 \\
811.7 \\
2,727.1 \\
35.9 \\
10.7 \\
25.1 \\
- \\
1.4\end{array}$ & $\begin{array}{r}5,652.8 \\
1,694.0 \\
3,907.0 \\
49.3 \\
24.2 \\
25.1 \\
- \\
\overline{2.5}\end{array}$ & $\begin{array}{r}4,165.2 \\
1,848.5 \\
2,191.6 \\
29.2 \\
5.6 \\
23.6 \\
94.9 \\
1.0\end{array}$ & $\begin{array}{r}4,063.1 \\
1,903.1 \\
2,043.5 \\
12.2 \\
2.3 \\
10.0 \\
-5 \\
103.3 \\
1.0\end{array}$ & $\begin{array}{r}4,210.8 \\
1,884.0 \\
2,180.4 \\
9.5 \\
2.4 \\
7.1 \\
-\overline{135.9} \\
1.0\end{array}$ & $\begin{array}{r}4,678.8 \\
1,926.1 \\
2,648.6 \\
11.2 \\
4.3 \\
5.4 \\
1.6 \\
91.7 \\
1.1\end{array}$ \\
\hline 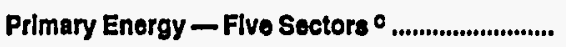 & $4,193.8$ & $10,806.1$ & $31,730.0$ & $35,729.0$ & $\mathrm{~A}_{33,810.3}$ & $A_{32,802.7}$ & $R_{33,174,4}$ & $34,524.6$ \\
\hline
\end{tabular}

a There are no direct fual costs for hydroelectric, geothermal, wind, photovoltalc, or solar thermal energy.

Heavy oll Includes fuel oil nos. 4, 5, and 6 , and residual fuel olls. Light oll includes fuel nos. 1 and 2, kerosene, and Jet fuel.

Blomass fuels are not included, except those consumed at electric utilities and those added to motor gasollne.

$R=$ Revlsed data.
-No consumption, including cases where adjustments were made. See explanation of adjustments in Section 6 of Appendix A.

(s) =Value less than 0.05 million dollars.

Note: Totals may not equal sum of components due to independent rounding.

Sources: Data sources, estimation procedures, and assumptlons are described in Appendix A. 
U Energy Price and Expenditure Estimates by Source, Utah

T 1970, 1975, 1980, 1985, and 1990-1993

A

\begin{tabular}{|c|c|c|c|c|c|c|c|c|}
\hline Energy Source & 1970 & 1975 & 1980 & 1985 & 1990 & 1991 & 1992 & 1993 \\
\hline & \multicolumn{8}{|c|}{ Prices in Dollars per Million Btu } \\
\hline 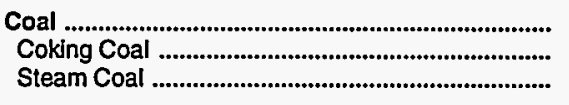 & $\begin{array}{r}0.38 \\
.43 \\
.28\end{array}$ & $\begin{array}{r}0.92 \\
1.38 \\
.55\end{array}$ & $\begin{array}{l}1.35 \\
1.97 \\
1.16\end{array}$ & $\begin{array}{l}1.48 \\
1.93 \\
1.39\end{array}$ & $\begin{array}{l}1.24 \\
1.84 \\
1.18\end{array}$ & $\begin{array}{l}1.28 \\
1.99 \\
1.21\end{array}$ & $\begin{array}{l}1.28 \\
2.00 \\
1.21\end{array}$ & $\begin{array}{l}1.24 \\
1.95 \\
1.19\end{array}$ \\
\hline Natural Gas & .57 & 1.07 & 2.33 & 4.01 & 4.17 & 4.21 & 4.18 & 3.99 \\
\hline $\begin{array}{l}\text { Petroleum } \\
\text { Asphalt and Road Oil 10 } \\
\text { Aviation Gasoline } \\
\text { Distillate Fuel } \\
\text { Jet Fuel } \\
\text { Kerosene } \\
\text { LPG }\end{array}$ & $\begin{array}{r}1.67 \\
.57 \\
2.17 \\
1.09 \\
.76 \\
.76 \\
2.12 \\
5.08 \\
2.75 \\
.39 \\
.51\end{array}$ & $\begin{array}{l}3.28 \\
1.79 \\
3.45 \\
2.61 \\
2.12 \\
2.42 \\
4.37 \\
7.48 \\
4.52 \\
1.71 \\
1.41\end{array}$ & $\begin{array}{r}7.48 \\
3.65 \\
9.02 \\
6.54 \\
6.59 \\
5.82 \\
5.68 \\
14.36 \\
9.80 \\
3.70 \\
2.85\end{array}$ & $\begin{array}{r}7.91 \\
4.85 \\
9.99 \\
6.69 \\
6.25 \\
9.95 \\
8.69 \\
17.61 \\
9.09 \\
3.86 \\
3.68\end{array}$ & $\begin{array}{r}R_{7.86} \\
2.70 \\
9.32 \\
8.00 \\
5.75 \\
10.07 \\
8.66 \\
R_{14.60} \\
9.09 \\
2.67 \\
2.02\end{array}$ & $\begin{array}{r}R 7.34 \\
3.25 \\
8.71 \\
7.60 \\
5.13 \\
9.76 \\
9.21 \\
R 16.79 \\
8.81 \\
2.31 \\
1.72\end{array}$ & $\begin{array}{r}\text { म } 7.59 \\
2.96 \\
8.54 \\
7.60 \\
4.96 \\
11.94 \\
9.44 \\
9.44 \\
19.43 \\
8.95 \\
1.78 \\
1.29\end{array}$ & $\begin{array}{r}7.43 \\
2.91 \\
8.24 \\
6.81 \\
4.91 \\
6.16 \\
9.13 \\
18.94 \\
9.02 \\
1.96 \\
1.06\end{array}$ \\
\hline Nuclear Fuel & - & - & - & - & - & - & - & - \\
\hline 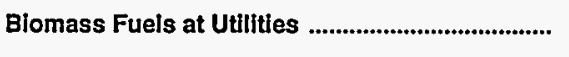 & - & - & - & - & - & - & - & - \\
\hline 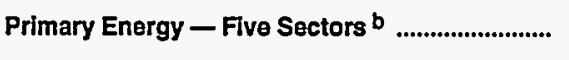 & 1.02 & 2.04 & 4.05 & 4.28 & 3.53 & $\mathrm{R}_{3.61}$ & 3.58 & 3.51 \\
\hline 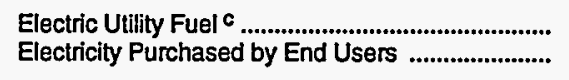 & $\underset{5.31}{.25}$ & $\begin{array}{r}.51 \\
7.06\end{array}$ & $\begin{array}{r}1.20 \\
13.11\end{array}$ & $\begin{array}{r}1.39 \\
19.05\end{array}$ & $\begin{array}{r}1.18 \\
16.09\end{array}$ & $\begin{array}{r}1.21 \\
16.08\end{array}$ & $\begin{array}{r}1.22 \\
15.61\end{array}$ & $\begin{array}{r}1.21 \\
15.69\end{array}$ \\
\hline \multirow[t]{2}{*}{ 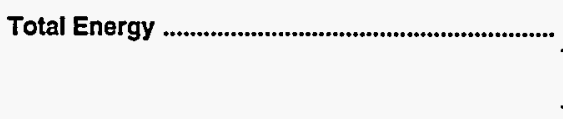 } & 1.31 & 2.59 & 5.77 & 7.24 & ${ }^{R} 7.13$ & 6.94 & ${ }^{\mathrm{R}} 7.16$ & 6.93 \\
\hline & \multicolumn{8}{|c|}{ Expenditures in Millions of Dollars } \\
\hline $\begin{array}{l}\text { Coal } \\
\text { Coking Coal } \\
\text { Steam Coal }\end{array}$ & $\begin{array}{r}30.1 \\
22.7 \\
7.4\end{array}$ & $\begin{array}{r}107.0 \\
71.7 \\
35.3\end{array}$ & $\begin{array}{r}227.1 \\
77.9 \\
149.2\end{array}$ & $\begin{array}{r}295.0 \\
64.8 \\
230.2\end{array}$ & $\begin{array}{r}455.0 \\
60.8 \\
394.2\end{array}$ & $\begin{array}{r}441.1 \\
63.5 \\
377.6\end{array}$ & $\begin{array}{r}463.2 \\
59.8 \\
403.4\end{array}$ & $\begin{array}{r}458.5 \\
52.6 \\
406.0\end{array}$ \\
\hline Natural Gas & 61.5 & 113.6 & 255.6 & 439.9 & 419.4 & 512.8 & 474.7 & 512.6 \\
\hline $\begin{array}{l}\text { Petroleum } \\
\text { Asphalt and Road Oil } \\
\text { Aviation Gasoline } \\
\text { Distillate Fuel } \\
\text { Jet Fuel } \\
\text { Kerosene } \\
\text { LPG }\end{array}$ & $\begin{array}{r}252.9 \\
6.0 \\
1.9 \\
32.4 \\
7.6 \\
1.1 \\
6.7 \\
7.9 \\
177.5 \\
10.3 \\
1.5\end{array}$ & $\begin{array}{r}612.1 \\
14.5 \\
2.8 \\
137.5 \\
22.4 \\
2.0 \\
15.4 \\
10.5 \\
357.3 \\
43.5 \\
6.2\end{array}$ & $\begin{array}{r}1,399.3 \\
35.8 \\
6.3 \\
319.7 \\
96.4 \\
3.4 \\
23.8 \\
26.1 \\
799.6 \\
74.8 \\
13.4\end{array}$ & $\begin{array}{r}1,281.0 \\
50.8 \\
4.7 \\
231.5 \\
133.0 \\
1.8 \\
43.4 \\
29.1 \\
775.3 \\
1.7 \\
9.7\end{array}$ & $\begin{array}{r}R_{1,404.7} \\
24.7 \\
5.0 \\
342.1 \\
171.0 \\
.8 \\
32.4 \\
R_{27.1} \\
794.2 \\
2.1 \\
5.4\end{array}$ & $\begin{array}{r}R_{1,434.7} \\
61.9 \\
5.2 \\
344.7 \\
170.3 \\
.9 \\
23.9 \\
\mathrm{R}_{27.9} \\
795.4 \\
.3 \\
4.0\end{array}$ & $\begin{array}{r}R_{1,453.6} \\
32.1 \\
5.7 \\
356.7 \\
156.3 \\
.3 \\
22.7 \\
R_{33.0} \\
842.4 \\
.2 \\
4.1\end{array}$ & $\begin{array}{r}1,463.1 \\
33.4 \\
4.7 \\
317.1 \\
152.6 \\
.3 \\
25.2 \\
32.7 \\
892.1 \\
2.0 \\
2.8\end{array}$ \\
\hline Nuclear Fuel & - & - & - & - & - & - & - & - \\
\hline 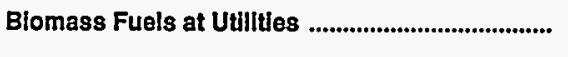 & - & - & - & - & - & - & - & - \\
\hline Primary Energy - Five Sectors ${ }^{b}$ & 344.5 & 832.7 & $1,882.0$ & $2,015.9$ & $\mathrm{R}_{2,279.2}$ & $\mathrm{R}_{2,388.6}$ & $\mathrm{R}_{2,391.5}$ & $2,434.2$ \\
\hline 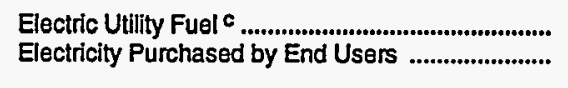 & $\begin{array}{r}-6.4 \\
92.0\end{array}$ & $\begin{array}{r}-26.2 \\
186.9\end{array}$ & $\begin{array}{r}-141.2 \\
469.3\end{array}$ & $\begin{array}{r}-208.0 \\
830.7\end{array}$ & $\begin{array}{r}-370.7 \\
830.9\end{array}$ & $\begin{array}{l}-362.7 \\
857.3\end{array}$ & $\begin{array}{r}-395.5 \\
868.1\end{array}$ & $\begin{array}{r}-399.0 \\
886.7\end{array}$ \\
\hline 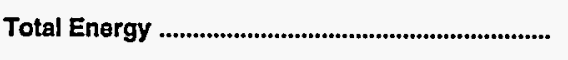 & 430.2 & 993.4 & $2,210.1$ & $2,638.6$ & $\mathrm{~A}_{2,739.3}$ & $R_{2,883.2}$ & $\mathrm{R}_{2,864.0}$ & $2,922.0$ \\
\hline
\end{tabular}

a Includes petroleum coke used at electric utilities.

b Blomass fuels are not included, except those consumed at electric utilities and those added to motor gasoline.

c There are no direct fuel costs for hydroelectric, geothermal, wind, photovoltalc, or solar thermal energy.

$R=$ Revised data.

-No consumption, including cases where adjustments were made. Se日 explanation of adjustments in Section 6 of Appendix A.

Note: Expenditure totals may not equal sum of components dus to Independent rounding.

Sources: Data sources, estimation procedures, and assumptions are described in Appendix A. 
(Dollars per Million Btu)

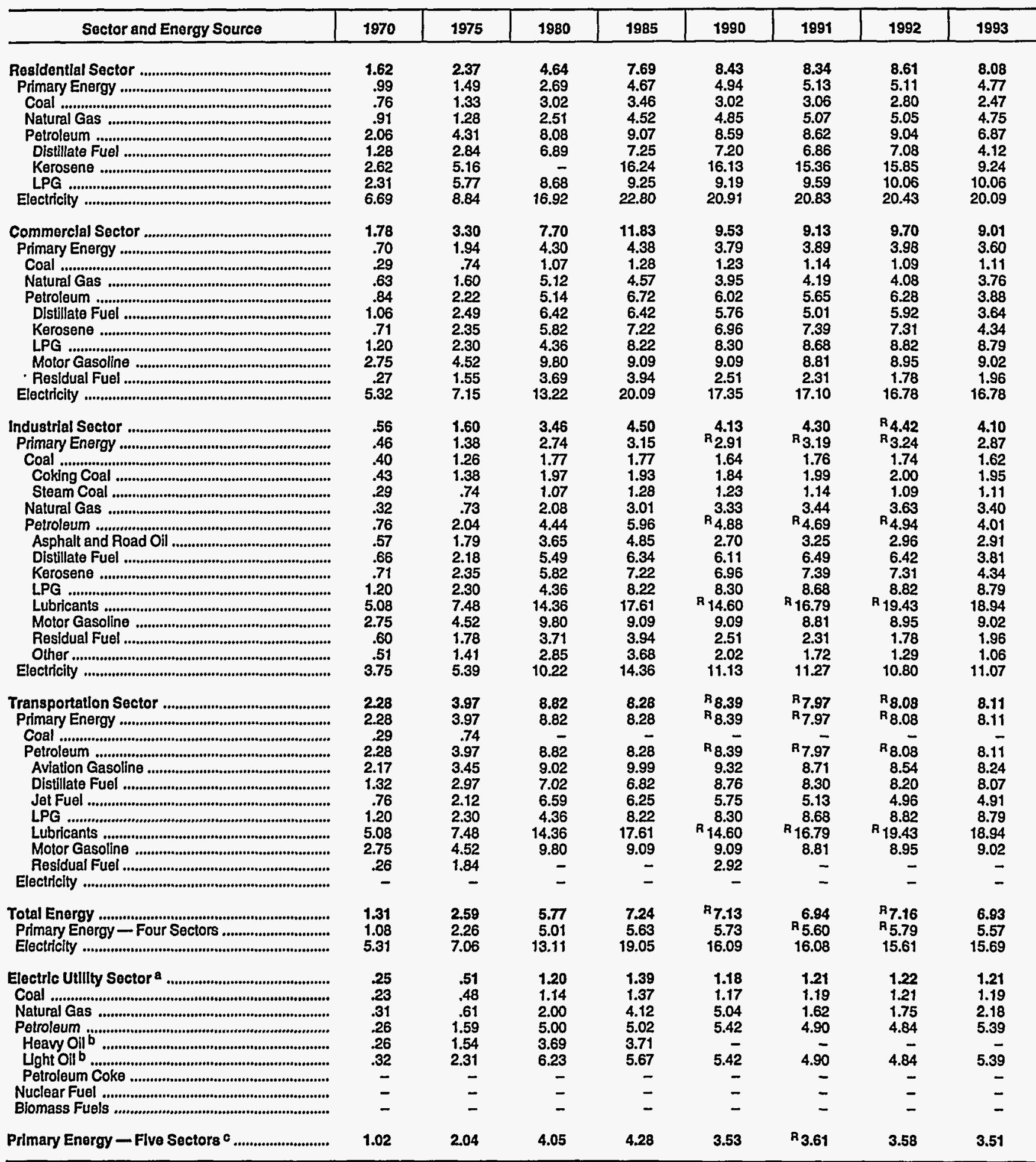

a There are no direct fuel costs for hydroelectric, geothermal, wind, photovoltalc, or solar thermal energy.

beavy oil includes fuel oil nos. 4, 5, and 6, and residual fuel oils. Light oll includes fuel oil nos. 1 and 2, kerosene, and jet fuel.

- Biomass fuels are not included, except those consumed at electric utilities and those added to motor gasoline.
$R=$ Revised data.

-No consumption, including cases where adjustments were made. See explanation of adjustments in Section 6 of Appendix A.

Sources: Data sources, estimation procedures, and assumptions are described in Appendix A. 
U Energy Expenditure Estimates by Sector, Utah

T 1970, 1975, 1980, 1985, and 1990-1993

A (Million Dollars)

H

\begin{tabular}{|c|c|c|c|c|c|c|c|c|}
\hline Sector and Energy Source & 1970 & 1975 & 1980 & 1985 & 1890 & 1991 & 1992 & 1993 \\
\hline $\begin{array}{l}\text { Residentlal Sector } \\
\text { Primary Energy } \\
\text { Coal } \\
\text { Natural Gas } \\
\text { Petroleum } \\
\text { Distillate Fuel } \\
\text { Kerosene } \\
\text { LPG }\end{array}$ & $\begin{array}{r}84.4 \\
45.9 \\
.7 \\
37.9 \\
7.2 \\
1.1 \\
.1 \\
6.0 \\
38.5\end{array}$ & $\begin{array}{r}167.5 \\
92.3 \\
1.5 \\
72.8 \\
18.1 \\
5.9 \\
.1 \\
.1 \\
12.1 \\
75.2\end{array}$ & $\begin{array}{r}359.4 \\
179.4 \\
5.8 \\
158.0 \\
15.6 \\
4.5 \\
11 . \overline{1} \\
179.9\end{array}$ & $\begin{array}{r}627.6 \\
317.5 \\
7.2 \\
285.3 \\
25.0 \\
3.1 \\
.9 \\
.9 \\
21.0 \\
310.1\end{array}$ & $\begin{array}{r}559.1 \\
256.1 \\
6.5 \\
229.3 \\
20.3 \\
5.7 \\
.4 \\
.4 \\
14.1 \\
303.0\end{array}$ & $\begin{array}{r}620.9 \\
303.9 \\
7.6 \\
275.1 \\
21.3 \\
6.4 \\
.4 \\
14.4 \\
317.0\end{array}$ & $\begin{array}{r}579.4 \\
265.4 \\
5.0 \\
243.2 \\
17.1 \\
4.8 \\
.2 \\
12.2 \\
314.0\end{array}$ & $\begin{array}{r}603.1 \\
279.1 \\
2.4 \\
265.6 \\
11.1 \\
3.6 \\
.2 \\
7.3 \\
324.0\end{array}$ \\
\hline 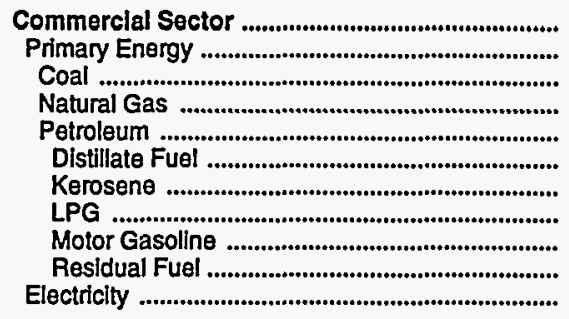 & $\begin{array}{r}49.0 \\
14.8 \\
.5 \\
6.0 \\
8.2 \\
3.2 \\
.2 \\
.6 \\
2.9 \\
1.4 \\
34.3\end{array}$ & $\begin{array}{r}106.9 \\
46.4 \\
1.5 \\
9.2 \\
35.8 \\
18.8 \\
.4 \\
.9 \\
5.0 \\
10.7 \\
60.5\end{array}$ & $\begin{array}{r}216.4 \\
74.7 \\
3.8 \\
1.8 \\
69.0 \\
38.4 \\
1.1 \\
1.0 \\
4.1 \\
24.4 \\
141.7\end{array}$ & $\begin{array}{r}391.3 \\
76.3 \\
4.9 \\
41.7 \\
29.6 \\
20.2 \\
.8 \\
.8 \\
3.3 \\
4.2 \\
1.1 \\
315.0\end{array}$ & $\begin{array}{r}413.9 \\
94.9 \\
4.9 \\
69.8 \\
20.3 \\
12.1 \\
.2 \\
2.3 \\
4.6 \\
4.6 \\
1.2 \\
319.0\end{array}$ & $\begin{array}{r}437.5 \\
112.5 \\
5.2 \\
86.8 \\
20.5 \\
13.7 \\
.4 \\
2.3 \\
3.8 \\
.3 \\
325.0\end{array}$ & $\begin{array}{r}433.5 \\
98.5 \\
3.7 \\
73.0 \\
21.8 \\
16.2 \\
(s) \\
1.9 \\
3.4 \\
.2 \\
335.0\end{array}$ & $\begin{array}{r}443.5 \\
104.5 \\
2.0 \\
91.8 \\
10.6 \\
7.8 \\
.1 \\
1.1 \\
1.0 \\
.7 \\
339.0\end{array}$ \\
\hline 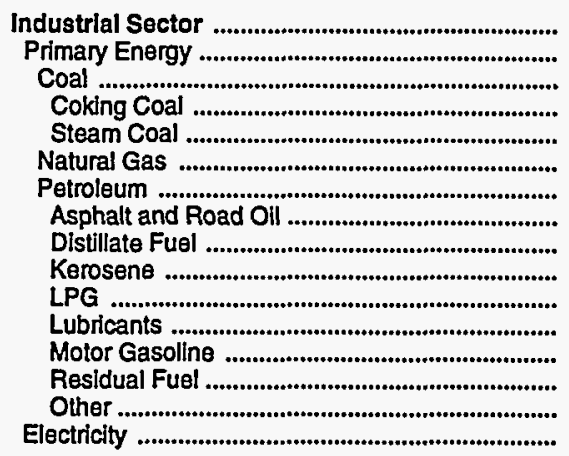 & $\begin{array}{r}89.2 \\
70.0 \\
26.4 \\
22.7 \\
3.6 \\
16.5 \\
27.1 \\
6.0 \\
6.0 \\
.8 \\
.1 \\
2.9 \\
3.8 \\
6.0 \\
1.5 \\
19.2\end{array}$ & $\begin{array}{r}268.0 \\
216.8 \\
81.2 \\
71.7 \\
9.5 \\
29.9 \\
105.7 \\
14.5 \\
40.9 \\
1.5 \\
2.3 \\
3.3 \\
6.3 \\
30.5 \\
6.2 \\
51.2\end{array}$ & $\begin{array}{r}524.1 \\
376.4 \\
89.9 \\
77.9 \\
12.0 \\
86.0 \\
200.6 \\
35.8 \\
70.9 \\
2.2 \\
11.5 \\
9.2 \\
8.5 \\
49.1 \\
13.4 \\
147.7\end{array}$ & $\begin{array}{r}534.8 \\
329.1 \\
78.3 \\
64.8 \\
13.5 \\
111.8 \\
139.0 \\
50.8 \\
40.8 \\
.1 \\
16.8 \\
10.3 \\
10.5 \\
(s) \\
9.7 \\
205.7\end{array}$ & $\begin{array}{r}R 522.1 \\
R 313.2 \\
80.1 \\
60.8 \\
19.3 \\
115.8 \\
115.8 \\
R^{117.3} \\
24.7 \\
53.6 \\
.2 \\
14.4 \\
R 9.6 \\
9.4 \\
\text { (s) } \\
5.4 \\
208.9\end{array}$ & $\begin{array}{r}R_{5} 597.3 \\
R_{382.0} \\
77.0 \\
63.5 \\
13.4 \\
141.9 \\
R_{163.1} \\
61.9 \\
71.6 \\
.1 \\
5.8 \\
R 9.9 \\
9.7 \\
(5) \\
4.0 \\
215.3\end{array}$ & 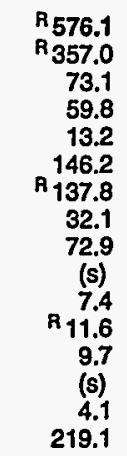 & $\begin{array}{r}552.6 \\
328.9 \\
71.4 \\
52.6 \\
18.9 \\
140.5 \\
116.9 \\
33.4 \\
40.6 \\
.1 \\
15.4 \\
11.6 \\
11.7 \\
1.4 \\
2.8 \\
223.7\end{array}$ \\
\hline 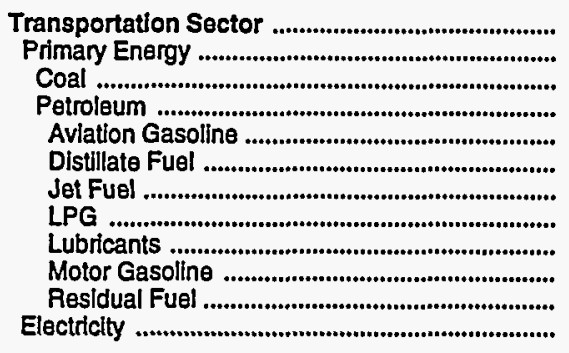 & $\begin{array}{r}207.5 \\
207.5 \\
(\mathrm{~s}) \\
207.5 \\
1.9 \\
22.1 \\
7.6 \\
(\mathrm{~s}) \\
5.0 \\
170.9 \\
(\mathrm{~s}) \\
-\end{array}$ & $\begin{array}{r}451.0 \\
451.0 \\
(\mathrm{~s}) \\
451.0 \\
2.8 \\
71.7 \\
22.4 \\
.1 \\
7.2 \\
346.0 \\
.8 \\
-\end{array}$ & $\begin{array}{r}1,110.3 \\
1,110.3 \\
-. \\
1,110.3 \\
6.3 \\
203.5 \\
96.4 \\
.2 \\
16.9 \\
787.0 \\
= \\
-\end{array}$ & $\begin{array}{r}1,085.0 \\
1,085.0 \\
- \\
1,085.0 \\
4.7 \\
165.5 \\
133.0 \\
2.2 \\
18.8 \\
760.7 \\
- \\
-\end{array}$ & $\begin{array}{r}R_{1,244.2} \\
R_{1,244.2} \\
R_{1,244.2} \\
5.0 \\
268.1 \\
171.0 \\
1.5 \\
R_{17.6} \\
780.2 \\
.9 \\
-\end{array}$ & $\begin{array}{r}R_{1,227.5} \\
R_{1,227.5} \\
- \\
R_{1,227.5} \\
5.2 \\
250.7 \\
170.3 \\
1.4 \\
R_{18.1} \\
781.9 \\
- \\
-\end{array}$ & $\begin{array}{r}R_{1,275.1} \\
R_{1,275.1} \\
- \\
R_{1,275.1} \\
5.7 \\
261.1 \\
156.3 \\
1.3 \\
R_{21.3} \\
829.3 \\
= \\
-\end{array}$ & $\begin{array}{r}1,322.8 \\
1,322.8 \\
- \\
1,322.8 \\
4.7 \\
263.5 \\
152.6 \\
1.3 \\
21.2 \\
879.5 \\
- \\
-\end{array}$ \\
\hline 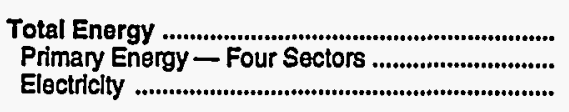 & $\begin{array}{r}430.2 \\
338.2 \\
92.0\end{array}$ & $\begin{array}{l}993.4 \\
806.5 \\
186.9\end{array}$ & $\begin{array}{r}2,210.1 \\
1,740.8 \\
469.3\end{array}$ & $\begin{array}{r}2,638.6 \\
1,807.9 \\
830.7\end{array}$ & $\begin{array}{r}R_{2,739.3} \\
\mathrm{R}_{1,908.5} \\
830.9\end{array}$ & $\begin{array}{r}R_{2,883.2} \\
R_{2,025.9} \\
857.3\end{array}$ & $\begin{array}{r}A_{2}, 864.0 \\
{ }_{1}, 995.9 \\
868.1\end{array}$ & $\begin{array}{r}2,822.0 \\
2,035.2 \\
886.7\end{array}$ \\
\hline 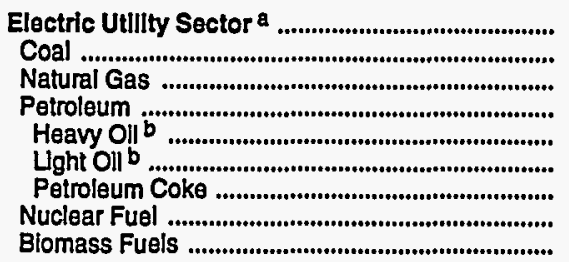 & $\begin{array}{l}6.4 \\
2.5 \\
1.0 \\
2.9 \\
2.8 \\
(8) \\
= \\
=\end{array}$ & $\begin{array}{r}26.2 \\
22.8 \\
1.8 \\
1.6 \\
1.5 \\
.1 \\
= \\
=\end{array}$ & $\begin{array}{r}141.2 \\
127.6 \\
9.8 \\
3.8 \\
1.4 \\
2.4 \\
= \\
=\end{array}$ & $\begin{array}{r}208.0 \\
204.6 \\
1.0 \\
2.4 \\
.6 \\
1.8 \\
= \\
=\end{array}$ & $\begin{array}{r}370.7 \\
363.5 \\
4.6 \\
2.6 \\
\overline{2.6} \\
- \\
-\end{array}$ & $\begin{array}{r}362.7 \\
351.4 \\
8.9 \\
2.3 \\
\overline{2.3} \\
- \\
-\end{array}$ & $\begin{array}{r}395.5 \\
381.5 \\
12.3 \\
1.7 \\
\overline{1.7} \\
\overline{-} \\
-\end{array}$ & $\begin{array}{r}399.0 \\
382.7 \\
14.6 \\
1.7 \\
\overline{1.7} \\
= \\
=\end{array}$ \\
\hline Primary Energy - Flve Sectors ${ }^{\circ}$................................... & 344.5 & 832.7 & $1,882.0$ & $2,015.9$ & $A_{2,279.2}$ & $\mathrm{~A}_{2,388.6}$ & A $2,391.5$ & $2,434.2$ \\
\hline
\end{tabular}

a There are no direct fuel costs for hydroelectric, geothermal, wind, photovoltalc, or solar thermal energy.

b Heavy oil includes fuel oll nos. 4, 5, and 6, and residual fusl olls. Light oll Includes fuel nos. 1 and 2 , kerosene, and jet fuel.

- Blomass fuels are not included, except those consumed at electric utllities and those added to motor gasoline.

$R=$ Revised data.

-No consumption, Including cases where adjustments were made. See explanation of adjustments in Section 6 of Appendlx $A$.

(s) =Value less than 0.05 million dollars.

Note: Totals may not equal sum of components due to Independent rounding.

Sources: Data sources, estimation procodures, and assumptlons are described In Appendlx A. 


\begin{tabular}{|c|c|c|c|c|c|c|c|c|}
\hline Energy Source & 1970 & 1975 & 1880 & 1985 & 1990 & 1881 & 1992 & 1993 \\
\hline & \multicolumn{8}{|c|}{ Prices In Dollars per Milllon Btu } \\
\hline 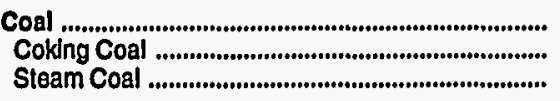 & $\begin{array}{r}0.72 \\
.72\end{array}$ & $2 . \overline{35}$ & $2 . \overline{4}$ & $2.8 \overline{-}$ & $\begin{array}{r}3.73 \\
3.73\end{array}$ & $\begin{array}{r}3.07 \\
3.07\end{array}$ & 2.91 & $\begin{array}{r}3.62 \\
3.62\end{array}$ \\
\hline Natural Gas ................................................................. & 1.41 & 1.87 & 5.62 & 5.59 & 4.65 & 4.51 & 5.03 & 4.99 \\
\hline 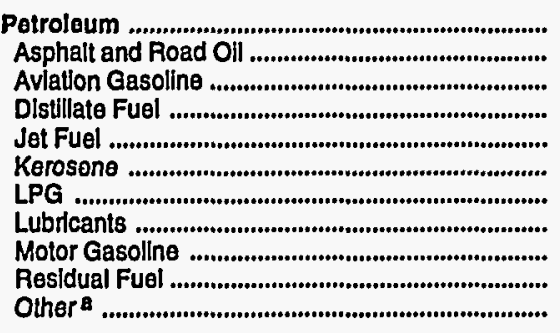 & $\begin{array}{r}1.97 \\
.68 \\
2.17 \\
1.37 \\
.75 \\
1.53 \\
2.15 \\
5.08 \\
3.09 \\
.66 \\
4.14\end{array}$ & $\begin{array}{l}3.64 \\
1.91 \\
3.45 \\
2.77 \\
2.22 \\
3.03 \\
4.12 \\
7.48 \\
4.69 \\
1.92 \\
4.95\end{array}$ & $\begin{array}{r}8.42 \\
3.66 \\
9.02 \\
7.01 \\
6.55 \\
7.82 \\
7.56 \\
14.36 \\
10.12 \\
4.05 \\
12.01\end{array}$ & $\begin{array}{r}8.88 \\
5.20 \\
9.99 \\
8.13 \\
6.10 \\
8.83 \\
11.71 \\
17.61 \\
9.53 \\
4.54 \\
13.38\end{array}$ & $\begin{array}{r}R_{9.24} \\
3.34 \\
9.32 \\
8.15 \\
6.60 \\
8.72 \\
13.14 \\
R_{14.60} \\
9.66 \\
3.32 \\
14.39\end{array}$ & $\begin{array}{r}\boldsymbol{R}_{8.81} \\
3.05 \\
8.71 \\
7.66 \\
5.07 \\
8.40 \\
14.29 \\
R_{16.79} \\
9.48 \\
2.51 \\
15.96\end{array}$ & $\begin{array}{r}R_{8.50} \\
2.79 \\
8.54 \\
7.11 \\
4.72 \\
7.62 \\
11.75 \\
R_{19.43} \\
9.39 \\
2.53 \\
23.81\end{array}$ & $\begin{array}{r}8.39 \\
3.30 \\
8.24 \\
7.04 \\
5.16 \\
7.35 \\
11.62 \\
18.94 \\
9.35 \\
2.62 \\
18.55\end{array}$ \\
\hline Nuclear Fuel ............................................................ & - & .31 & .58 & .64 & .57 & .56 & .53 & .54 \\
\hline Blomas8 Fuels at Utilities ....................................... & - & - & 1.74 & .79 & 2.82 & 2.60 & 2.74 & 2.60 \\
\hline Primary Energy - Five Sectors b ........................ & 1.92 & 2.41 & $\mathbf{5 . 6 7}$ & 5.93 & 6.00 & $\mathbf{R}_{5.65}$ & $R_{5.75}$ & 5.90 \\
\hline 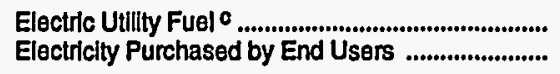 & $\begin{array}{r}.72 \\
6.05\end{array}$ & $\begin{array}{r}.36 \\
10.33\end{array}$ & $\begin{array}{r}.69 \\
14.33\end{array}$ & $\begin{array}{r}.72 \\
20.81\end{array}$ & $\begin{array}{r}.66 \\
24.24\end{array}$ & $\begin{array}{r}.65 \\
25.30\end{array}$ & $\begin{array}{r}.62 \\
25.86\end{array}$ & $\begin{array}{r}.60 \\
26.47\end{array}$ \\
\hline \multirow[t]{2}{*}{ 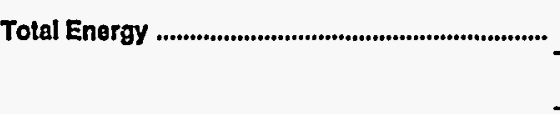 } & 2.40 & 4.40 & 929 & 10.53 & 11.60 & $R_{11.20}$ & $\mathrm{R}_{11.11}$ & 11.12 \\
\hline & \multicolumn{8}{|c|}{ Expenditures in Millions of Dollars } \\
\hline 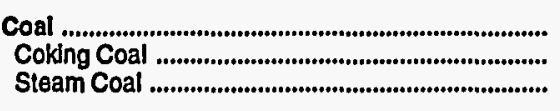 & $\begin{array}{l}1.5 \\
1.5\end{array}$ & $\begin{array}{l}1.7 \\
1.7\end{array}$ & $\begin{array}{l}1.3 \\
\overline{1.3}\end{array}$ & $\begin{array}{l}5.6 \\
\overline{5.6}\end{array}$ & $\begin{array}{l}0.8 \\
- \\
.8\end{array}$ & $\begin{array}{l}0.9 \\
- \\
.9\end{array}$ & $\begin{array}{l}1.5 \\
\overline{1.5}\end{array}$ & $\begin{array}{c}0.5 \\
- \\
.5\end{array}$ \\
\hline 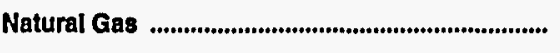 & 3.8 & 7.5 & 22.2 & 27.7 & 31.0 & 31.5 & 38.1 & 36.0 \\
\hline 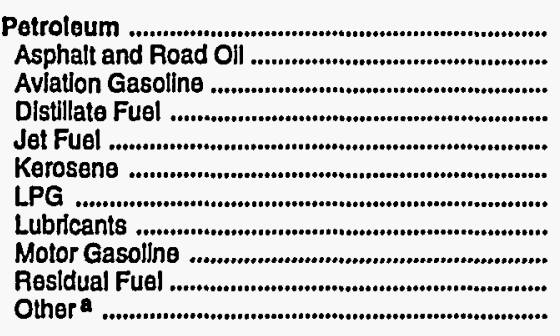 & $\begin{array}{r}145.6 \\
1.2 \\
.2 \\
45.7 \\
.5 \\
4.4 \\
4.4 \\
2.0 \\
82.5 \\
3.7 \\
1.0\end{array}$ & \begin{tabular}{r|}
250.8 \\
.4 \\
.2 \\
75.0 \\
2.2 \\
5.4 \\
12.8 \\
2.5 \\
140.2 \\
9.6 \\
2.5
\end{tabular} & $\begin{array}{r}518.8 \\
1.0 \\
1.1 \\
167.3 \\
5.6 \\
12.5 \\
18.5 \\
5.9 \\
288.9 \\
12.0 \\
5.9\end{array}$ & $\begin{array}{r}586.5 \\
11.4 \\
1.1 \\
198.5 \\
6.7 \\
28.9 \\
33.4 \\
6.5 \\
290.9 \\
3.5 \\
5.6\end{array}$ & $\begin{array}{r}{ }^{*} 633.4 \\
.6 \\
.7 \\
192.0 \\
6.6 \\
11.0 \\
66.8 \\
R_{6.1} \\
337.8 \\
5.0 \\
6.8\end{array}$ & $\begin{array}{r}R_{659.0} \\
10.7 \\
.7 \\
190.1 \\
4.6 \\
13.1 \\
84.4 \\
R_{6.3} \\
337.2 \\
4.2 \\
7.9\end{array}$ & $\begin{array}{r}R_{671.9} \\
6.2 \\
.6 \\
206.9 \\
3.0 \\
9.9 \\
81.5 \\
17.4 \\
339.3 \\
4.5 \\
12.6\end{array}$ & $\begin{array}{r}678.7 \\
.7 \\
.5 \\
219.6 \\
3.5 \\
11.5 \\
68.8 \\
7.3 \\
348.3 \\
7.9 \\
10.5\end{array}$ \\
\hline Nuclear Fuel ................................................................ & - & 12.0 & 18.7 & 20.8 & 22.1 & 24.9 & 212 & 19.5 \\
\hline Blomass Fuels at Utilities ...................................... & - & - & .9 & 2.3 & 27 & 29 & 2.6 & 1.7 \\
\hline Primary Enorgy - Five Sectors b ....................... & 150.9 & 272.0 & 561.9 & 6429 & ${ }^{n_{690.1}}$ & $R_{719.3}$ & R735.3 & 736.4 \\
\hline 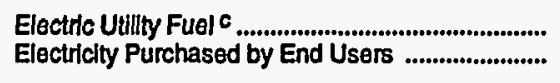 & $\begin{array}{r}-2.2 \\
53.9\end{array}$ & $\begin{array}{r}-14.5 \\
105.6\end{array}$ & $\begin{array}{r}-23.4 \\
193.1\end{array}$ & $\begin{array}{r}-26.1 \\
285.1\end{array}$ & $\begin{array}{r}-26.8 \\
390.0\end{array}$ & $\begin{array}{r}-30.1 \\
406.1\end{array}$ & $\begin{array}{r}-25.7 \\
436.0\end{array}$ & $\begin{array}{r}22.3 \\
453.0\end{array}$ \\
\hline 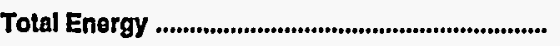 & 202.6 & 363.0 & 731.7 & 901.9 & $R_{1,053.3}$ & $R_{1,095.2}$ & $R_{1,145.6}$ & $1,167.1$ \\
\hline
\end{tabular}

a Includes petroleum coke used at electric utilities.

b Blomass fuels are not included, except those consumed at electric utllitles and those added to motor gasoline.

c There are no direct fuel costs for hydroelectric, geothermal, wind, photovoltalc, or solar thermal energy.

$R=$ Revised data.
-No consumption, including cases where adjustments were made. See explanation of adjustments in Section 6 of Appendix A.

Note: Expenditure totals may not equal sum of components due to independent rounding.

Sources: Data sources, estimation procedures, and assumptlons are described in Appendix A. 
V Energy Price Estimates by Sector, Vermont

E 1970, 1975, 1980, 1985, and 1990-1993

$\mathbf{R}$ (Dollars per Million Btu)

\begin{tabular}{|c|c|c|c|c|c|c|c|c|}
\hline Sector and Energy Source & 1970 & 1975 & 1980 & 1985 & 1990 & 1991 & 1992 & 1993 \\
\hline 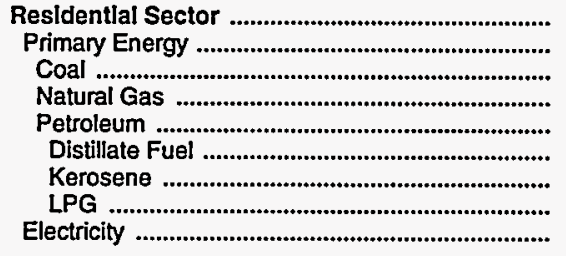 & $\begin{array}{l}2.24 \\
1.58 \\
1.37 \\
1.97 \\
1.57 \\
1.51 \\
1.63 \\
2.55 \\
6.68\end{array}$ & $\begin{array}{r}4.52 \\
3.04 \\
2.62 \\
2.62 \\
3.07 \\
2.87 \\
3.16 \\
4.72 \\
11.47\end{array}$ & $\begin{array}{r}9.65 \\
7.43 \\
4.42 \\
6.30 \\
7.56 \\
7.32 \\
8.15 \\
9.28 \\
15.76\end{array}$ & $\begin{array}{r}11.08 \\
8.41 \\
4.91 \\
6.33 \\
8.67 \\
8.08 \\
8.98 \\
11.79 \\
21.20\end{array}$ & $\begin{array}{r}13.58 \\
9.05 \\
4.73 \\
5.87 \\
9.49 \\
8.02 \\
8.92 \\
13.76 \\
27.22\end{array}$ & $\begin{array}{r}13.62 \\
9.22 \\
4.59 \\
6.31 \\
9.61 \\
7.71 \\
8.57 \\
15.17 \\
27.94\end{array}$ & $\begin{array}{r}12.85 \\
8.26 \\
4.46 \\
6.73 \\
8.48 \\
6.98 \\
7.76 \\
12.37 \\
27.99\end{array}$ & $\begin{array}{r}12.76 \\
7.86 \\
4.48 \\
6.20 \\
8.09 \\
6.84 \\
7.61 \\
12.20 \\
28.85\end{array}$ \\
\hline 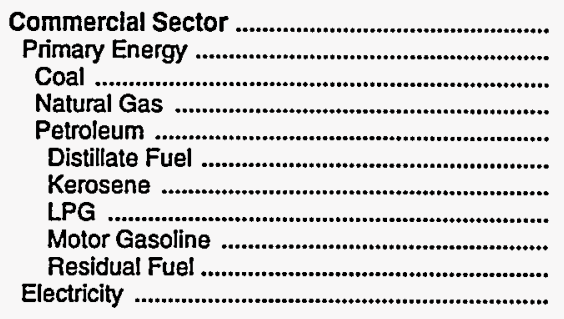 & $\begin{array}{r}2.18 \\
1.06 \\
.87 \\
1.43 \\
1.04 \\
1.11 \\
.92 \\
1.37 \\
3.09 \\
.79 \\
6.78\end{array}$ & $\begin{array}{r}4.51 \\
2.33 \\
2.60 \\
2.10 \\
2.35 \\
2.46 \\
2.65 \\
2.92 \\
4.69 \\
1.91 \\
11.34\end{array}$ & $\begin{array}{r}9.00 \\
5.90 \\
1.65 \\
6.22 \\
5.93 \\
6.48 \\
6.39 \\
5.59 \\
10.12 \\
4.09 \\
15.56\end{array}$ & $\begin{array}{r}12.72 \\
6.80 \\
2.38 \\
5.76 \\
7.93 \\
7.56 \\
7.67 \\
11.46 \\
9.53 \\
4.54 \\
24.02\end{array}$ & $\begin{array}{r}14.40 \\
6.54 \\
2.62 \\
5.12 \\
7.16 \\
7.09 \\
7.41 \\
10.80 \\
9.66 \\
3.33 \\
25.16\end{array}$ & $\begin{array}{r}14.27 \\
6.31 \\
2.57 \\
5.30 \\
6.69 \\
6.47 \\
6.79 \\
11.94 \\
9.48 \\
2.51 \\
26.42\end{array}$ & $\begin{array}{r}14.00 \\
5.98 \\
2.58 \\
5.69 \\
6.12 \\
5.76 \\
6.18 \\
9.96 \\
9.39 \\
2.54 \\
27.37\end{array}$ & $\begin{array}{r}14.02 \\
5.59 \\
2.32 \\
5.26 \\
5.74 \\
5.74 \\
5.89 \\
10.02 \\
9.35 \\
2.62 \\
27.60\end{array}$ \\
\hline 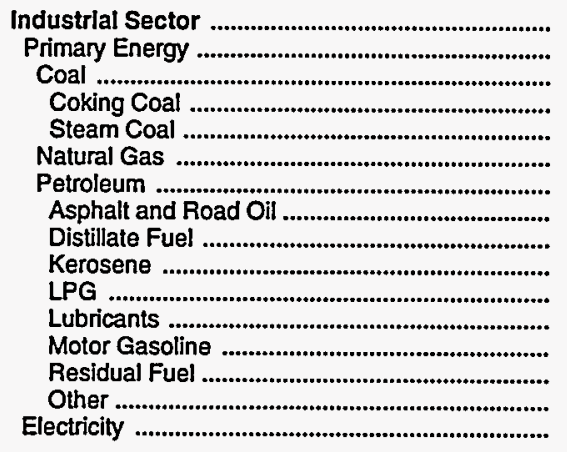 & $\begin{array}{r}1.71 \\
.96 \\
.87 \\
- \\
.87 \\
.85 \\
.97 \\
.68 \\
.84 \\
.92 \\
1.37 \\
5.08 \\
3.09 \\
.53 \\
4.14 \\
4.52\end{array}$ & $\begin{array}{r}3.74 \\
2.41 \\
2.60 \\
- \\
2.60 \\
1.44 \\
2.63 \\
1.91 \\
2.38 \\
2.65 \\
2.92 \\
7.48 \\
4.69 \\
1.93 \\
4.95 \\
7.61\end{array}$ & $\begin{array}{r}7.69 \\
5.73 \\
1.65 \\
- \\
1.65 \\
4.94 \\
5.96 \\
3.66 \\
5.84 \\
6.39 \\
5.59 \\
14.36 \\
10.12 \\
4.01 \\
12.01 \\
11.37\end{array}$ & $\begin{array}{r}10.87 \\
6.50 \\
2.38 \\
2.38 \\
2.38 \\
4.91 \\
7.01 \\
5.20 \\
6.70 \\
7.67 \\
11.46 \\
17.61 \\
9.53 \\
4.54 \\
13.38 \\
18.40\end{array}$ & $\begin{array}{r}R_{11.59} \\
R_{6.32} \\
2.62 \\
- \\
2.62 \\
3.56 \\
R_{7.36} \\
3.34 \\
6.47 \\
7.41 \\
10.80 \\
R_{14.60} \\
9.66 \\
3.33 \\
14.39 \\
19.31\end{array}$ & $\begin{array}{r}10.06 \\
R_{5.38} \\
2.57 \\
- \\
2.57 \\
3.03 \\
R_{5.88} \\
3.05 \\
5.93 \\
6.79 \\
11.94 \\
R_{16.79} \\
9.48 \\
2.51 \\
15.96 \\
20.66\end{array}$ & $\begin{array}{r}R_{10.66} \\
R_{5.65} \\
2.58 \\
- \\
2.58 \\
3.29 \\
R_{6.34} \\
2.79 \\
5.40 \\
6.18 \\
9.96 \\
\mathrm{R}_{19.43} \\
9.39 \\
2.54 \\
23.81 \\
21.37\end{array}$ & $\begin{array}{r}11.45 \\
5.79 \\
- \\
- \\
- \\
3.58 \\
6.44 \\
3.30 \\
5.14 \\
5.89 \\
10.02 \\
18.94 \\
9.35 \\
2.62 \\
18.55 \\
21.91\end{array}$ \\
\hline 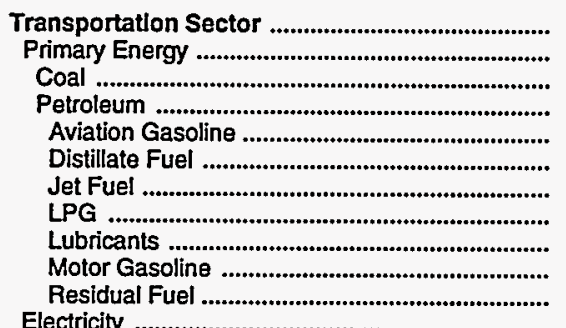 & $\begin{array}{r}2.94 \\
2.94 \\
.87 \\
2.94 \\
2.17 \\
1.43 \\
.75 \\
1.37 \\
5.08 \\
3.09 \\
.76\end{array}$ & $\begin{array}{l}4.49 \\
4.49 \\
2.60 \\
4.49 \\
3.45 \\
2.90 \\
2.09 \\
2.92 \\
7.48 \\
4.69 \\
1.84\end{array}$ & $\begin{array}{r}9.72 \\
9.72 \\
- \\
9.72 \\
9.02 \\
7.41 \\
6.51 \\
5.59 \\
14.36 \\
10.12 \\
-\end{array}$ & $\begin{array}{r}9.46 \\
9.46 \\
- \\
9.46 \\
9.99 \\
9.30 \\
6.10 \\
11.46 \\
17.61 \\
9.53 \\
-\end{array}$ & $\begin{array}{r}R_{9.62} \\
R_{9.62} \\
- \\
R_{9.62} \\
9.32 \\
9.66 \\
6.60 \\
10.80 \\
R_{14.60} \\
9.66 \\
2.76\end{array}$ & $\begin{array}{r}R_{9.39} \\
R_{9.39} \\
- \\
R_{9.39} \\
8.71 \\
9.14 \\
5.07 \\
11.94 \\
R_{16.79} \\
9.48 \\
2.16\end{array}$ & $\begin{array}{r}R_{9.25} \\
R_{9.25} \\
\bar{~} \\
R_{9.25} \\
8.54 \\
8.67 \\
4.72 \\
9.96 \\
R_{19.43} \\
9.39 \\
2.24\end{array}$ & $\begin{array}{r}9.16 \\
9.16 \\
- \\
9.16 \\
8.24 \\
8.46 \\
5.16 \\
10.02 \\
18.94 \\
9.35 \\
-\end{array}$ \\
\hline 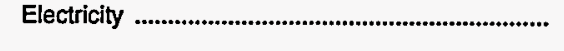 & - & - & - & - & - & - & - & - \\
\hline 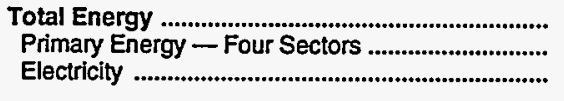 & $\begin{array}{l}2.40 \\
1.97 \\
6.05\end{array}$ & $\begin{array}{r}4.40 \\
3.56 \\
10.33\end{array}$ & $\begin{array}{r}9.29 \\
8.25 \\
14.33\end{array}$ & $\begin{array}{r}10.53 \\
8.57 \\
20.81\end{array}$ & $\begin{array}{r}11.60 \\
8.88 \\
24.24\end{array}$ & $\begin{array}{r}R_{11.29} \\
8.51 \\
25.30\end{array}$ & $\begin{array}{r}11.11 \\
8.22 \\
25.86\end{array}$ & $\begin{array}{r}11.12 \\
8.13 \\
26.47\end{array}$ \\
\hline 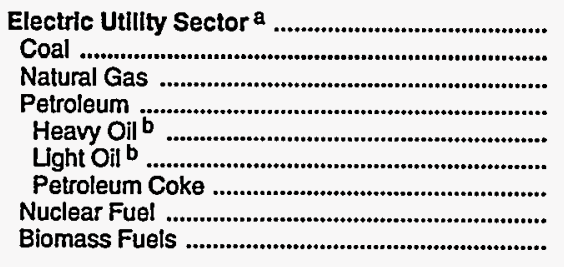 & $\begin{array}{r}.72 \\
.49 \\
. \overline{.91} \\
.83 \\
.92 \\
- \\
- \\
-\end{array}$ & $\begin{array}{r}.36 \\
2.05 \\
1.17 \\
2.41 \\
1.95 \\
2.42 \\
-31 \\
-\end{array}$ & $\begin{array}{r}.69 \\
1.73 \\
4.50 \\
6.28 \\
-\overline{6} \\
6.28 \\
- \\
.58 \\
1.74\end{array}$ & $\begin{array}{r}.72 \\
2.03 \\
4.84 \\
5.83 \\
-\overline{8} \\
5.83 \\
\overline{64} \\
.79\end{array}$ & $\begin{array}{r}.66 \\
-\overline{42} \\
2.42 \\
5.53 \\
-\overline{53} \\
\overline{-57} \\
.57 \\
2.82\end{array}$ & $\begin{array}{r}.65 \\
-\overline{74} \\
1.7 .70 \\
- \\
4.70 \\
- \\
.56 \\
2.60\end{array}$ & $\begin{array}{r}.62 \\
-\overline{02} \\
2.02 \\
4.43 \\
-\overline{43} \\
4.43 \\
. \overline{3} \\
2.74\end{array}$ & $\begin{array}{r}.60 \\
-\overline{0} \\
2.02 \\
4.85 \\
\overline{-} \\
4.85 \\
\overline{-} \\
.54 \\
2.60\end{array}$ \\
\hline Primary Energy — Flve Sectors $c \ldots . . . . . . . . . . . . . . . . . .$. & 1.92 & 2.41 & 5.67 & 5.93 & 6.00 & $\mathrm{R}_{5.65}$ & $R_{5.75}$ & 5.90 \\
\hline
\end{tabular}

a There are no direct fuel costs for hydroelectric, geothermal, wind, photovoltaic, or solar thermal energy.

beavy oil includes fuel oil nos. 4, 5, and 6, and residual fuel oils. Light oil includes fuel oil nos. 1 and 2, kerosene, and jet fuel.

c Biomass fuels are not included, except those consumed at electric utifities and those added to motor gasoline.

R=Revised data.

-No consumption, Including cases where adjustments were made. See explanation of adjustments in Section 6 of Appendix A.

Sources: Data sources, estimation procedures, and assumptions are described in Appendix A. 


\begin{tabular}{|c|c|c|c|c|c|c|c|c|}
\hline Sector and Energy Source & 1970 & 1975 & 1980 & 1985 & 1990 & 1991 & 1992 & 1993 \\
\hline 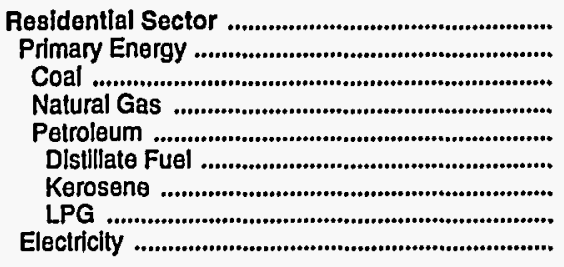 & $\begin{array}{r}71.8 \\
44.1 \\
.6 \\
2.1 \\
41.4 \\
34.0 \\
4.0 \\
3.4 \\
27.7\end{array}$ & $\begin{array}{r}125.1 \\
69.3 \\
.5 \\
3.0 \\
65.8 \\
51.9 \\
4.2 \\
9.7 \\
55.8\end{array}$ & $\begin{array}{r}219.8 \\
124.1 \\
.7 \\
8.1 \\
115.3 \\
92.5 \\
10.6 \\
12.1 \\
95.8\end{array}$ & $\begin{array}{r}278.9 \\
167.7 \\
2.3 \\
9.1 \\
156.3 \\
104.5 \\
26.2 \\
25.5 \\
111.2\end{array}$ & $\begin{array}{r}336.2 \\
168.2 \\
.5 \\
12.4 \\
155.3 \\
90.2 \\
9.8 \\
55.3 \\
168.0\end{array}$ & $\begin{array}{r}352.8 \\
182.7 \\
.3 \\
13.7 \\
168.6 \\
91.5 \\
12.0 \\
65.1 \\
170.0\end{array}$ & $\begin{array}{r}363.4 \\
179.4 \\
.4 \\
16.9 \\
162.1 \\
89.1 \\
9.2 \\
63.8 \\
184.0\end{array}$ & $\begin{array}{r}367.7 \\
173.7 \\
.4 \\
15.7 \\
157.6 \\
94.5 \\
10.1 \\
53.0 \\
194.0\end{array}$ \\
\hline 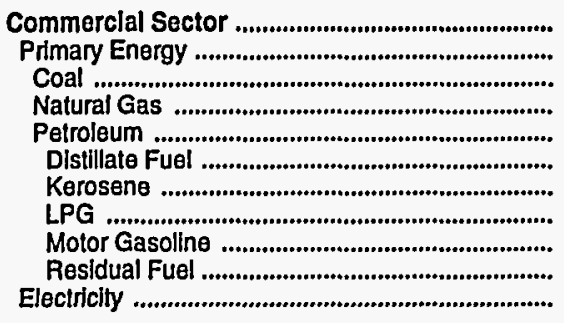 & $\begin{array}{r}23.2 \\
9.1 \\
.2 \\
.8 \\
8.1 \\
5.1 \\
.1 \\
.3 \\
.4 \\
2.1 \\
14.1\end{array}$ & $\begin{array}{r}45.0 \\
17.6 \\
.4 \\
1.6 \\
15.6 \\
9.1 \\
.2 \\
1.1 \\
.7 \\
4.5 \\
27.4\end{array}$ & $\begin{array}{r}88.3 \\
39.3 \\
.2 \\
5.1 \\
34.1 \\
23.4 \\
1.6 \\
1.3 \\
1.7 \\
6.1 \\
49.0\end{array}$ & $\begin{array}{r}121.1 \\
42.5 \\
1.6 \\
9.0 \\
31.9 \\
23.3 \\
1.6 \\
4.4 \\
2.0 \\
.7 \\
78.6\end{array}$ & $\begin{array}{r}177.6 \\
46.5 \\
.2 \\
10.3 \\
36.0 \\
23.2 \\
.5 \\
7.7 \\
2.1 \\
2.5 \\
131.0\end{array}$ & $\begin{array}{r}188.4 \\
50.4 \\
.1 \\
10.8 \\
39.4 \\
26.4 \\
.6 \\
9.0 \\
1.4 \\
2.1 \\
138.0\end{array}$ & $\begin{array}{r}200.6 \\
53.6 \\
.2 \\
13.1 \\
40.3 \\
27.4 \\
.5 \\
9.1 \\
1.7 \\
1.7 \\
147.0\end{array}$ & $\begin{array}{r}201.5 \\
49.6 \\
.1 \\
12.5 \\
36.9 \\
24.9 \\
1.1 \\
7.7 \\
.3 \\
2.9 \\
152.0\end{array}$ \\
\hline 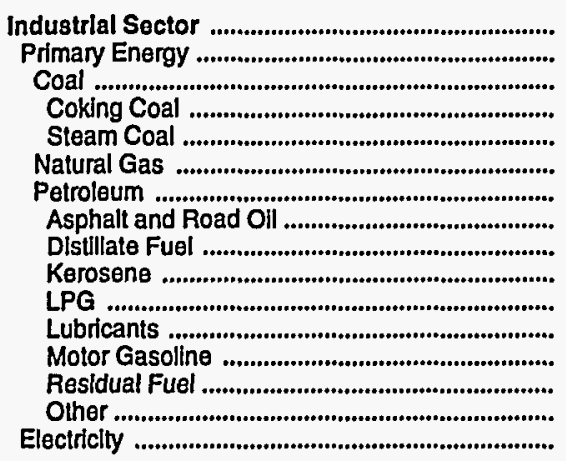 & $\begin{array}{r}21.7 \\
9.5 \\
.1 \\
- \\
.1 \\
.9 \\
8.5 \\
1.2 \\
2.3 \\
.2 \\
.6 \\
.5 \\
1.1 \\
1.5 \\
1.0 \\
12.1\end{array}$ & $\begin{array}{r}43.0 \\
20.7 \\
.1 \\
. \\
.1 \\
2.2 \\
18.3 \\
.4 \\
5.1 \\
1.0 \\
1.9 \\
.5 \\
1.9 \\
5.1 \\
2.5 \\
22.3\end{array}$ & $\begin{array}{r}94.0 \\
45.6 \\
.1 \\
- \\
.1 \\
7.9 \\
37.6 \\
1.0 \\
17.1 \\
.3 \\
5.0 \\
1.3 \\
1.0 \\
5.9 \\
5.9 \\
48.4\end{array}$ & $\begin{array}{r}153.4 \\
58.0 \\
.3 \\
. \\
.3 \\
9.1 \\
48.6 \\
11.4 \\
17.5 \\
1.1 \\
2.9 \\
1.5 \\
5.8 \\
2.8 \\
5.6 \\
95.3\end{array}$ & $\begin{array}{r}R_{134.6} \\
R_{43.6} \\
.1 \\
-.1 \\
.6 .6 \\
R_{37.0} \\
.6 \\
17.6 \\
.7 \\
3.3 \\
R 1.4 \\
4.1 \\
2.4 \\
6.8 \\
91.0\end{array}$ & $\begin{array}{r}R_{155.6} \\
R_{57.6} \\
.5 \\
- \\
.5 \\
5.1 \\
R_{52.0} \\
10.7 \\
15.4 \\
.4 \\
9.7 \\
P_{1.4} \\
4.4 \\
2.1 \\
7.9 \\
98.0\end{array}$ & $\begin{array}{r}R_{164.3} \\
R_{59.3} \\
.9 \\
- \\
.9 \\
6.4 \\
R_{51.9} \\
6.2 \\
16.0 \\
.2 \\
8.2 \\
R_{1.7} \\
4.4 \\
2.7 \\
12.6 \\
105.0\end{array}$ & $\begin{array}{r}159.3 \\
52.3 \\
- \\
- \\
- \\
7.3 \\
45.0 \\
.7 \\
15.3 \\
.3 \\
7.8 \\
1.7 \\
3.7 \\
5.0 \\
10.5 \\
107.0\end{array}$ \\
\hline 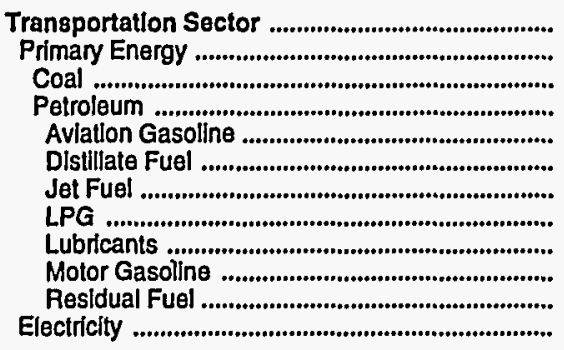 & $\begin{array}{r}86.0 \\
86.0 \\
(s) \\
86.0 \\
.2 \\
2.9 \\
.5 \\
(s) \\
1.5 \\
81.0 \\
(s) \\
-\end{array}$ & $\begin{array}{r}149.9 \\
149.9 \\
(\mathrm{~s}) \\
149.9 \\
.2 \\
8.5 \\
1.5 \\
(5) \\
2.1 \\
137.6 \\
(\mathrm{~s}) \\
-\end{array}$ & $\begin{array}{r}329.5 \\
329.5 \\
- \\
329.5 \\
1.1 \\
32.7 \\
4.9 \\
(8) \\
4.5 \\
286.2 \\
- \\
-\end{array}$ & $\begin{array}{r}348.5 \\
348.5 \\
- \\
348.5 \\
1.1 \\
52.0 \\
6.7 \\
.6 \\
5.1 \\
283.1 \\
- \\
-\end{array}$ & $\begin{array}{r}A_{404.9} \\
R_{404.9} \\
- \\
R_{404.9} \\
.7 \\
60.7 \\
6.6 \\
.4 \\
R_{4.7} \\
331.7 \\
.1 \\
-\end{array}$ & $\begin{array}{r}R_{398.4} \\
R_{398.4} \\
- \\
R_{398.4} \\
.7 \\
56.4 \\
4.6 \\
.5 \\
R_{4.9} \\
331.5 \\
(\mathrm{~s}) \\
-\end{array}$ & $\begin{array}{r}R_{417.3} \\
R_{417.3} \\
- \\
R_{417.3} \\
.6 \\
74.3 \\
3.0 \\
.4 \\
R_{5.7} \\
333.2 \\
.1 \\
-\end{array}$ & $\begin{array}{r}438.6 \\
438.6 \\
- \\
438.6 \\
.5 \\
84.4 \\
3.5 \\
.3 \\
5.7 \\
344.3 \\
- \\
-\end{array}$ \\
\hline 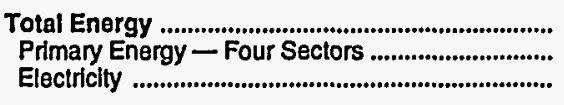 & $\begin{array}{r}202.6 \\
148.7 \\
53.9\end{array}$ & $\begin{array}{l}363.0 \\
257.5 \\
105.6\end{array}$ & $\begin{array}{l}731.7 \\
538.5 \\
193.1\end{array}$ & $\begin{array}{l}901.9 \\
616.7 \\
285.1\end{array}$ & $\begin{array}{r}R_{1,053.3} \\
\mathbf{3} 663.3 \\
390.0\end{array}$ & $\begin{array}{r}R_{1,095.2} \\
R_{689.2} \\
406.1\end{array}$ & $\begin{array}{r}R_{1,145.6} \\
R_{709.6} \\
436.0\end{array}$ & $\begin{array}{r}1,167.1 \\
714.2 \\
453.0\end{array}$ \\
\hline 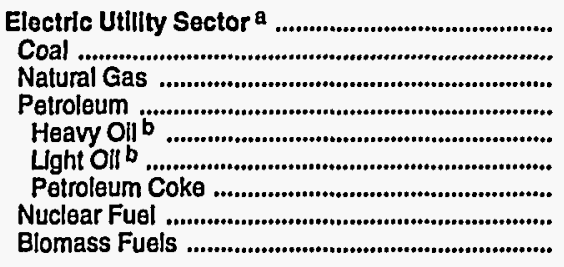 & $\begin{array}{l}2.2 \\
.7 \\
\overline{1.6} \\
.1 \\
1.4 \\
= \\
=\end{array}$ & $\begin{array}{r}14.5 \\
.7 \\
.7 \\
1.2 \\
(s) \\
1.2 \\
- \\
12.0 \\
-\end{array}$ & $\begin{array}{r}23.4 \\
.4 \\
1.1 \\
2.3 \\
-\overline{2} \\
2.3 \\
-\overline{7} \\
18.7 \\
.9\end{array}$ & $\begin{array}{r}26.1 \\
1.4 \\
.5 \\
1.1 \\
- \\
1.1 \\
-\overline{2} \\
20.8 \\
2.3\end{array}$ & $\begin{array}{r}26.8 \\
\overline{-} \\
1.7 \\
.2 \\
\overline{.} \\
.2 \\
\overline{2} \\
22.1 \\
2.7\end{array}$ & $\begin{array}{r}30.1 \\
\overline{1.9} \\
.4 \\
\overline{.4} \\
\overline{-} \\
24.9 \\
2.9\end{array}$ & $\begin{array}{r}25.7 \\
- \\
1.6 \\
.2 \\
\overline{2} \\
.2 \\
\overline{2} \\
21.2 \\
2.6\end{array}$ & $\begin{array}{r}22.3 \\
- \\
.5 \\
.5 \\
- \\
.5 \\
\overline{-} \\
19.5 \\
1.7\end{array}$ \\
\hline Primary Energy - Five Sectors $c$........................ & 150.9 & 272.0 & 561.9 & 642.9 & $R_{690.1}$ & ${ }^{R} 719.3$ & ${ }^{A} 735.3$ & 736.4 \\
\hline
\end{tabular}

a There are no direct fuel costs for hydroelectric, geothermal, wind, photovoltalc, or solar thermal energy.

b Heavy oll includes fuel oll nos. 4, 5, and 6, and residual fuel oils. Light oll includes fuel nos. 1 and 2, kerosene, and jet fuel.

c Blomass fuels are not included, except those consumed at electric utilities and those added to motor gasoline.

$R=$ Revised data.
-No consumption, including cases where adjustments were made. See explanation of adjustments in Section 6 of Appendix A.

$(s)=$ Value less than 0.05 million dollars.

Note: Totals may not equal sum of components due to independent rounding.

Sources: Data sources, estimation procedures, and assumptions are described in Appendix A. 


\section{Energy Price and Expenditure Estimates by Source, Virginia \\ I 1970, 1975, 1980, 1985, and 1990-1993}

\begin{tabular}{|c|c|c|c|c|c|c|c|c|}
\hline Energy Source & 1970 & 1975 & 1980 & 1985 & 1890 & 1991 & 1992 & 1983 \\
\hline & \multicolumn{8}{|c|}{ Prices In Dollars por Million Btu } \\
\hline 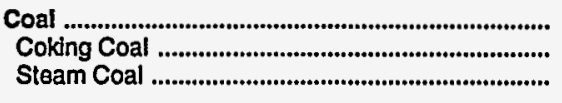 & $\begin{array}{r}0.41 \\
.40 \\
.41\end{array}$ & $1 . \overline{30}$ & $\begin{array}{l}1.72 \\
1.86 \\
1.71\end{array}$ & $\begin{array}{l}1.80 \\
1.93 \\
1.78\end{array}$ & $\begin{array}{l}1.60 \\
1.80 \\
1.59\end{array}$ & $\begin{array}{l}1.57 \\
1.72 \\
1.56\end{array}$ & $\begin{array}{l}1.54 \\
1.74 \\
1.53\end{array}$ & $\begin{array}{l}1.54 \\
1.68 \\
1.52\end{array}$ \\
\hline 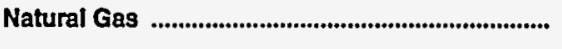 & .96 & 1.71 & 3.62 & 5.68 & 4.67 & 4.76 & 4.77 & 5.14 \\
\hline $\begin{array}{l}\text { Petroleum } \\
\text { Asphalt and Road Oil } \\
\text { Avlation Gasoline } \\
\text { Distillate Fuel } \\
\text { Jet Fuel } \\
\text { Kerosene } \\
\text { LPG }\end{array}$ & $\begin{array}{r}1.49 \\
.68 \\
2.17 \\
1.14 \\
.73 \\
1.36 \\
1.95 \\
5.08 \\
2.85 \\
.31 \\
.75\end{array}$ & $\begin{array}{l}3.16 \\
1.82 \\
3.45 \\
2.60 \\
2.03 \\
2.93 \\
3.63 \\
7.48 \\
4.77 \\
1.80 \\
2.66\end{array}$ & $\begin{array}{r}7.47 \\
3.60 \\
9.02 \\
6.84 \\
6.46 \\
7.59 \\
6.33 \\
14.36 \\
9.97 \\
3.75 \\
7.25\end{array}$ & $\begin{array}{r}8.12 \\
4.93 \\
9.99 \\
7.77 \\
5.79 \\
8.15 \\
9.87 \\
17.61 \\
9.33 \\
4.26 \\
8.23\end{array}$ & $\begin{array}{r}R 7.97 \\
2.99 \\
9.32 \\
7.85 \\
5.53 \\
8.33 \\
11.25 \\
R \quad 14.60 \\
9.46 \\
3.24 \\
R 6.52\end{array}$ & 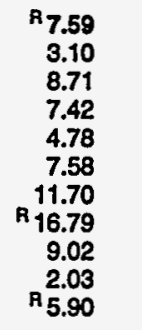 & 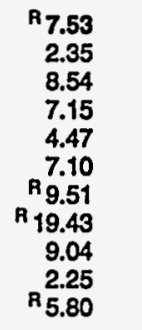 & $\begin{array}{r}7.50 \\
2.96 \\
8.24 \\
6.96 \\
4.16 \\
6.79 \\
9.91 \\
18.94 \\
9.15 \\
2.02 \\
5.35\end{array}$ \\
\hline 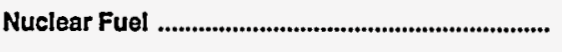 & - & .28 & .74 & .55 & .47 & .53 & .43 & .43 \\
\hline 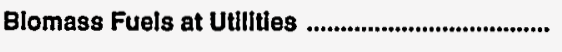 & - & - & - & - & - & - & - & - \\
\hline Primary Energy - Flve Sectors ${ }^{b}$ & 1.17 & 2.52 & 5.31 & 5.14 & $R_{4.91}$ & 4.63 & 4.63 & 4.73 \\
\hline 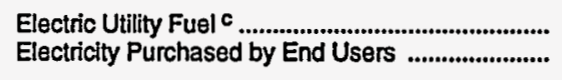 & $\begin{array}{r}.35 \\
4.91\end{array}$ & $\begin{array}{l}1.24 \\
9.63\end{array}$ & $\begin{array}{r}2.00 \\
15.77\end{array}$ & $\begin{array}{r}1.18 \\
17.06\end{array}$ & $\begin{array}{r}1.06 \\
17.70\end{array}$ & $\begin{array}{r}1.05 \\
17.89\end{array}$ & $\begin{array}{r}1.01 \\
18.44\end{array}$ & $\begin{array}{r}1.07 \\
18.30\end{array}$ \\
\hline \multirow[t]{2}{*}{ Total Energy } & 1.80 & 3.97 & 8.02 & 8.79 & ${ }^{R} 8.86$ & 8.77 & $\mathrm{R}_{8.88}$ & 9.10 \\
\hline & \multicolumn{8}{|c|}{ Expenditures in Millions of Dollare } \\
\hline $\begin{array}{l}\text { Coal } \\
\text { Coking Coal } \\
\text { Steam Coal }\end{array}$ & $\begin{array}{r}113.6 \\
.3 \\
113.3\end{array}$ & $\begin{array}{l}220.7 \\
220.7\end{array}$ & $\begin{array}{r}398.1 \\
33.0 \\
365.1\end{array}$ & $\begin{array}{r}531.3 \\
45.7 \\
485.5\end{array}$ & $\begin{array}{r}532.6 \\
42.7 \\
489.9\end{array}$ & $\begin{array}{r}557.8 \\
44.9 \\
512.9\end{array}$ & $\begin{array}{r}629.1 \\
45.4 \\
483.7\end{array}$ & $\begin{array}{r}532.8 \\
43.4 \\
489.4\end{array}$ \\
\hline 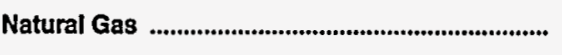 & 126.6 & 205.0 & 548.0 & 783.7 & 830.8 & 818.0 & 941.8 & $1,115.5$ \\
\hline $\begin{array}{l}\text { Petroleum } \\
\text { Asphalt and Road Oil } \\
\text { Aviation Gasoline } \\
\text { Distillate Fuel } \\
\text { Jet Fuel } \\
\text { Kerosene } \\
\text { LPG }\end{array}$ & $\begin{array}{r}1,105.6 \\
10.2 \\
3.9 \\
163.6 \\
44.9 \\
38.9 \\
17.8 \\
22.2 \\
727.8 \\
65.0 \\
11.3\end{array}$ & $\begin{array}{r}2,590.2 \\
28.1 \\
4.4 \\
344.3 \\
131.9 \\
37.7 \\
41.2 \\
33.3 \\
1,484.6 \\
462.4 \\
22.4\end{array}$ & $\begin{array}{r}5,723.8 \\
62.6 \\
9.9 \\
980.1 \\
444.2 \\
73.9 \\
70.2 \\
82.9 \\
3,092.9 \\
575.1 \\
332.0\end{array}$ & $\begin{array}{r}5,516.5 \\
132.0 \\
6.6 \\
1,139.9 \\
357.1 \\
186.2 \\
138.4 \\
92.5 \\
3,086.1 \\
221.1 \\
156.4\end{array}$ & $\begin{array}{r}\text { R }_{5,909.4} \\
93.3 \\
3.3 \\
1,276.8 \\
489.8 \\
64.9 \\
162.0 \\
R_{86.3} \\
3,475.0 \\
152.2 \\
R_{105.8}\end{array}$ & $\begin{array}{r}R_{5,448.6} \\
76.9 \\
5.1 \\
1,158.0 \\
318.7 \\
67.1 \\
193.6 \\
R_{88.8} \\
3,340.2 \\
108.8 \\
\text { P }_{91.3}\end{array}$ & $\begin{array}{r}\mathrm{A}_{\mathbf{5}, 381.1} \\
58.5 \\
4.4 \\
1,099.6 \\
294.4 \\
59.0 \\
\mathrm{R}_{161.5} \\
\mathrm{R}_{104.8} \\
3,400.3 \\
\mathrm{R}_{105.5} \\
\mathrm{R}_{93.1}\end{array}$ & $\begin{array}{r}5,572.0 \\
72.7 \\
4.4 \\
1,142.1 \\
279.6 \\
66.8 \\
169.0 \\
104.0 \\
3,546.1 \\
101.2 \\
86.1\end{array}$ \\
\hline 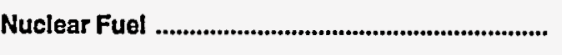 & - & 27.7 & 92.8 & 131.5 & 119.6 & 135.2 & 108.2 & 103.7 \\
\hline 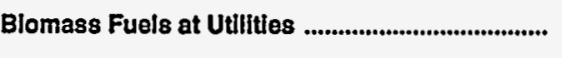 & - & - & - & - & - & - & - & - \\
\hline Primary Energy - Five Sectors b ............................. & $1,345.8$ & $3,043.6$ & $6,762.6$ & $6,962.9$ & ${ }^{\text {R7,392.4 }}$ & $R_{6,958.6}$ & $A_{6,960.2}$ & $7,324.0$ \\
\hline 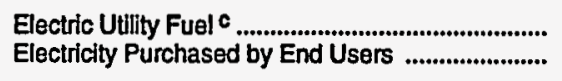 & $\begin{array}{r}-101.4 \\
494.4\end{array}$ & $\begin{array}{r}-455.1 \\
1,280.5\end{array}$ & $\begin{array}{r}-726.4 \\
2,581.5\end{array}$ & $\begin{array}{r}-514.6 \\
3,343.0\end{array}$ & $\begin{array}{r}-508.9 \\
4,374.4\end{array}$ & $\begin{array}{r}-532.0 \\
4,558.0\end{array}$ & $\begin{array}{r}-500.1 \\
4,781.6\end{array}$ & $\begin{array}{r}-561.6 \\
5,053.4\end{array}$ \\
\hline 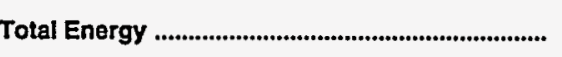 & $1,738.8$ & $3,869.0$ & $8,617.8$ & $9,791.3$ & $P_{11,257.8}$ & ${ }^{R_{10,985.5}}$ & $R_{11,241.7}$ & $11,815.7$ \\
\hline
\end{tabular}

a Includes petroleum coke used at electric utilities.

b Biomass fuels are not included, except those consumed at electric utilities and those added to motor gasoline.

c There are no direct fuel costs for hydroelectric, geothermal, wind, photovoltaic, or solar thermal energy.

$R=$ Revised data.

-No consumption, including cases where adjustments were made. See explanation of adjustments in Section 6 of Appendix A.

Note: Expenditure totals may not equal sum of components due to independent rounding.

Sources: Data sources, estimation procedures, and assumptions are described in Appendix A. 


\begin{tabular}{|c|c|c|c|c|c|c|c|c|}
\hline Sector and Energy Source & 1970 & 1975 & 1980 & 1985 & 1990 & 1991 & 1992 & 1993 \\
\hline 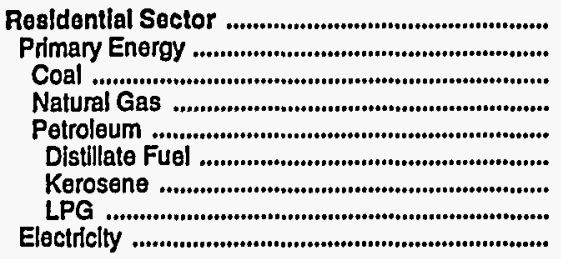 & $\begin{array}{l}2.45 \\
1.45 \\
1.34 \\
1.45 \\
1.45 \\
1.37 \\
1.44 \\
2.27 \\
6.11\end{array}$ & $\begin{array}{r}5.19 \\
2.60 \\
2.73 \\
2.20 \\
2.88 \\
2.69 \\
2.99 \\
4.43 \\
11.05\end{array}$ & $\begin{array}{r}10.23 \\
5.75 \\
3.85 \\
4.20 \\
7.32 \\
7.10 \\
7.96 \\
8.07 \\
17.80\end{array}$ & $\begin{array}{r}12.45 \\
7.51 \\
3.92 \\
6.76 \\
8.34 \\
7.89 \\
8.30 \\
10.48 \\
19.49\end{array}$ & $\begin{array}{r}14.29 \\
7.60 \\
3.48 \\
6.48 \\
9.15 \\
8.25 \\
8.68 \\
13.03 \\
21.24\end{array}$ & $\begin{array}{r}14.50 \\
7.45 \\
3.35 \\
6.53 \\
8.79 \\
7.54 \\
7.93 \\
13.55 \\
21.51\end{array}$ & $\begin{array}{r}14.31 \\
6.90 \\
3.32 \\
6.44 \\
7.72 \\
6.93 \\
7.28 \\
10.59 \\
22.37\end{array}$ & $\begin{array}{r}14.53 \\
7.28 \\
3.37 \\
7.19 \\
7.65 \\
6.66 \\
7.01 \\
11.58 \\
22.19\end{array}$ \\
\hline 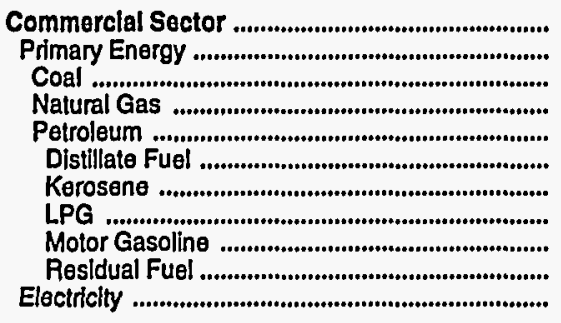 & $\begin{array}{r}2.53 \\
.94 \\
.42 \\
.94 \\
1.18 \\
1.08 \\
.65 \\
1.48 \\
2.85 \\
.32 \\
4.84\end{array}$ & $\begin{array}{l}5.50 \\
1.93 \\
1.47 \\
1.69 \\
2.60 \\
2.37 \\
2.36 \\
2.79 \\
4.77 \\
1.85 \\
9.49\end{array}$ & $\begin{array}{r}10.06 \\
4.30 \\
1.64 \\
3.71 \\
6.32 \\
6.46 \\
5.94 \\
4.60 \\
9.97 \\
3.91 \\
15.79\end{array}$ & $\begin{array}{r}12.09 \\
5.81 \\
1.69 \\
5.76 \\
6.72 \\
6.54 \\
6.81 \\
9.35 \\
9.33 \\
4.29 \\
17.35\end{array}$ & $\begin{array}{r}12.21 \\
5.10 \\
1.64 \\
4.73 \\
6.56 \\
6.11 \\
6.45 \\
9.21 \\
9.46 \\
3.31 \\
17.14\end{array}$ & $\begin{array}{r}12.32 \\
4.91 \\
1.61 \\
4.66 \\
6.02 \\
5.38 \\
5.64 \\
9.80 \\
9.02 \\
2.32 \\
17.16\end{array}$ & $\begin{array}{r}12.36 \\
4.82 \\
1.63 \\
4.79 \\
5.53 \\
4.99 \\
5.79 \\
8.34 \\
9.04 \\
2.41 \\
17.73\end{array}$ & $\begin{array}{r}12.26 \\
5.06 \\
1.65 \\
5.36 \\
5.11 \\
4.81 \\
5.49 \\
8.20 \\
9.15 \\
2.22 \\
17.53\end{array}$ \\
\hline 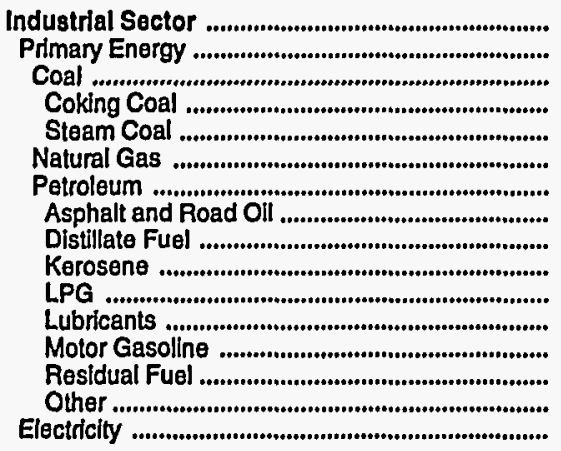 & $\begin{array}{r}.81 \\
.57 \\
.42 \\
.40 \\
.42 \\
.49 \\
.79 \\
.68 \\
.60 \\
.65 \\
1.48 \\
5.08 \\
2.85 \\
.34 \\
.96 \\
3.08\end{array}$ & $\begin{array}{r}2.50 \\
1.74 \\
1.47 \\
1 . \overline{-} \\
1.08 \\
2.18 \\
1.82 \\
2.19 \\
2.36 \\
2.79 \\
7.48 \\
4.77 \\
1.81 \\
2.66 \\
7.37\end{array}$ & $\begin{array}{r}4.83 \\
3.77 \\
1.69 \\
1.86 \\
1.64 \\
2.99 \\
5.58 \\
3.60 \\
5.33 \\
5.94 \\
4.60 \\
14.36 \\
9.97 \\
3.58 \\
7.25 \\
12.19\end{array}$ & $\begin{array}{r}5.38 \\
4.10 \\
1.74 \\
1.93 \\
1.69 \\
4.60 \\
6.44 \\
4.93 \\
6.10 \\
6.81 \\
9.35 \\
17.61 \\
9.33 \\
4.29 \\
8.23 \\
12.47\end{array}$ & $\begin{array}{r}R_{4.78} \\
3.28 \\
1.67 \\
1.80 \\
1.64 \\
3.52 \\
A_{5.14} \\
2.99 \\
5.78 \\
6.45 \\
9.21 \\
R_{14.60} \\
9.46 \\
3.31 \\
R_{6.52} \\
12.51\end{array}$ & $\begin{array}{r}R_{4.62} \\
R_{3.11} \\
1.63 \\
1.72 \\
1.61 \\
3.66 \\
R_{5.12} \\
3.10 \\
5.04 \\
5.64 \\
9.80 \\
R_{16.79} \\
9.02 \\
2.32 \\
R_{5.90} \\
12.40\end{array}$ & $\begin{array}{r}R_{4.74} \\
R_{3.11} \\
1.65 \\
1.74 \\
1.63 \\
3.58 \\
R_{4.77} \\
2.35 \\
5.18 \\
5.79 \\
8.34 \\
R_{19.43} \\
9.04 \\
2.41 \\
R_{5.80} \\
12.54\end{array}$ & $\begin{array}{r}4.96 \\
3.30 \\
1.66 \\
1.68 \\
1.65 \\
3.71 \\
4.82 \\
2.96 \\
4.92 \\
5.49 \\
8.20 \\
18.94 \\
9.15 \\
2.22 \\
5.35 \\
12.29\end{array}$ \\
\hline 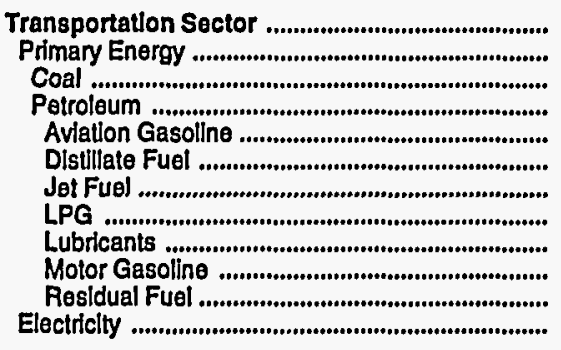 & $\begin{array}{r}1.95 \\
1.95 \\
.42 \\
1.95 \\
2.17 \\
1.25 \\
.73 \\
1.48 \\
5.08 \\
2.85 \\
.30 \\
-\end{array}$ & $\begin{array}{r}3.91 \\
3.91 \\
1.47 \\
3.91 \\
3.45 \\
2.72 \\
2.03 \\
2.79 \\
7.48 \\
4.77 \\
1.61 \\
-\end{array}$ & $\begin{array}{r}8.73 \\
8.72 \\
-\overline{-} \\
8.72 \\
9.02 \\
7.27 \\
6.46 \\
4.60 \\
14.36 \\
9.97 \\
3.32 \\
14.65\end{array}$ & $\begin{array}{r}8.55 \\
8.55 \\
- \\
8.55 \\
9.99 \\
8.34 \\
5.79 \\
9.35 \\
17.61 \\
9.33 \\
4.18 \\
17.33\end{array}$ & $\begin{array}{r}R_{8.47} \\
8.46 \\
-\overline{8.46} \\
9.32 \\
8.40 \\
5.53 \\
9.21 \\
R_{14.60} \\
9.46 \\
3.03 \\
15.55\end{array}$ & $\begin{array}{r}R_{8.09} \\
8.08 \\
-\overline{8} \\
8.08 \\
8.71 \\
8.10 \\
4.78 \\
9.80 \\
R_{16.79} \\
9.02 \\
1.82 \\
15.68\end{array}$ & $\begin{array}{r}R_{8.11} \\
R_{8.11} \\
R_{8.11} \\
8.54 \\
7.79 \\
4.47 \\
8.34 \\
R_{19.43} \\
9.04 \\
2.05 \\
16.33\end{array}$ & $\begin{array}{r}8.17 \\
8.16 \\
-\overline{8} \\
8.16 \\
8.24 \\
7.72 \\
4.16 \\
8.20 \\
18.94 \\
9.15 \\
1.83 \\
16.32\end{array}$ \\
\hline 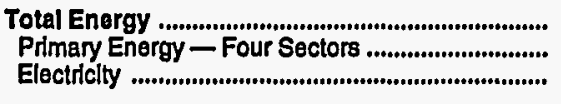 & $\begin{array}{l}1.80 \\
1.44 \\
4.91\end{array}$ & $\begin{array}{l}3.97 \\
3.08 \\
9.63\end{array}$ & $\begin{array}{r}8.02 \\
6.62 \\
15.77\end{array}$ & $\begin{array}{r}8.79 \\
7.02 \\
17.06\end{array}$ & $\begin{array}{r}R_{8.86} \\
6.72 \\
17.70\end{array}$ & $\begin{array}{r}8.77 \\
\mathrm{R}_{6.45} \\
17.89\end{array}$ & $\begin{array}{r}R_{8.88} \\
R_{6.42} \\
18.44\end{array}$ & $\begin{array}{r}9.10 \\
6.61 \\
18.30\end{array}$ \\
\hline 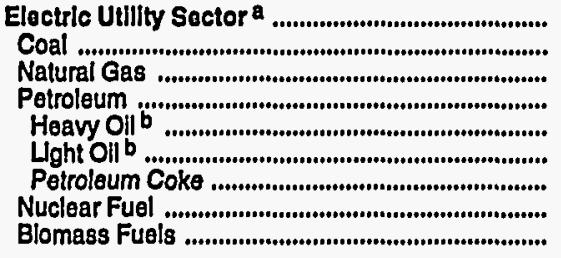 & $\begin{array}{l}.35 \\
.38 \\
.29 \\
.32 \\
.31 \\
.35 \\
.35 \\
- \\
-\end{array}$ & $\begin{array}{r}1.24 \\
1.14 \\
.99 \\
1.85 \\
1.84 \\
2.18 \\
-\overline{28} \\
-\end{array}$ & $\begin{array}{r}2.00 \\
1.71 \\
2.89 \\
4.03 \\
3.94 \\
5.86 \\
-\overline{14} \\
-\end{array}$ & $\begin{array}{r}1.18 \\
1.80 \\
3.44 \\
4.60 \\
4.37 \\
5.57 \\
- \\
.55 \\
-\end{array}$ & $\begin{array}{r}1.08 \\
1.55 \\
2.58 \\
4.14 \\
3.60 \\
5.83 \\
\overline{-4} \\
-\end{array}$ & $\begin{array}{r}1.05 \\
1.52 \\
1.82 \\
2.36 \\
2.13 \\
4.64 \\
- \\
.53 \\
-\end{array}$ & $\begin{array}{r}1.01 \\
1.47 \\
2.37 \\
2.59 \\
2.33 \\
4.71 \\
\overrightarrow{.43} \\
-\end{array}$ & $\begin{array}{r}1.07 \\
1.47 \\
2.79 \\
2.16 \\
2.03 \\
4.11 \\
- \\
.43 \\
-\end{array}$ \\
\hline Prlmary Energy - Flve Sectors 0 ......................... & 1.17 & 2.52 & 5.31 & 6.14 & $R_{4.91}$ & 4.63 & 4.63 & 4.73 \\
\hline
\end{tabular}

a There are no direct fuel costs for hydroelectric, geothermal, wind, pholovoltalc, or solar thermal energy.

beavy oll includes fuel oil nos. 4, 5, and 6, and residual fuel olls. Light oll includes fuel oil nos. 1 and 2 , kerosene, and jet fuel.

- Blomass fuels are not Included, except those consumed at electric utilities and those added to motor gasoline.
R=Revised data.

-No consumption, Including cases where adjustments were made. See explanation of adjustments in Section 6 of Appendix $A$.

Sources: Data sources, estimation procedures, and assumptions are described in Appendix A. 

1970, 1975, 1980, 1985, and 1990-1993

$\mathbf{R}$ (Million Dollars)

\begin{tabular}{|c|c|c|c|c|c|c|c|c|}
\hline Sector and Energy Source & 1970 & 1975 & 1980 & 1985 & 1990 & 1991 & 1992 & 1993 \\
\hline $\begin{array}{l}\text { Residential Sector } \\
\text { Primary Energy } \\
\text { Coal .at } \\
\text { Natural Gas } \\
\text { Petroleum } \\
\text { Distillate Fuel } \\
\text { Kerosene } \\
\text { LPG }\end{array}$ & $\begin{array}{r}446.8 \\
206.2 \\
5.3 \\
73.8 \\
127.1 \\
77.7 \\
37.1 \\
12.3 \\
240.5\end{array}$ & $\begin{array}{r}918.4 \\
319.8 \\
7.3 \\
109.5 \\
203.0 \\
142.4 \\
34.9 \\
25.7 \\
598.6\end{array}$ & $\begin{array}{r}1,851.9 \\
653.6 \\
6.4 \\
233.9 \\
413.3 \\
305.3 \\
63.4 \\
44.7 \\
1,198.3\end{array}$ & $\begin{array}{r}2,326.7 \\
826.1 \\
9.3 \\
342.4 \\
474.4 \\
236.3 \\
170.0 \\
68.1 \\
1,500.6\end{array}$ & $\begin{array}{r}2,796.4 \\
757.4 \\
7.2 \\
347.2 \\
403.0 \\
245.6 \\
57.1 \\
100.3 \\
2,039.0\end{array}$ & $\begin{array}{r}2,920.6 \\
747.6 \\
4.1 \\
368.6 \\
374.9 \\
201.9 \\
59.5 \\
113.6 \\
2,173.0\end{array}$ & $\begin{array}{r}3,035.5 \\
762.5 \\
5.7 \\
417.7 \\
339.7 \\
192.9 \\
53.0 \\
93.2 \\
2,273.0\end{array}$ & $\begin{array}{r}3,311.4 \\
852.4 \\
9.2 \\
491.7 \\
351.5 \\
192.5 \\
59.2 \\
99.9 \\
2,459.0\end{array}$ \\
\hline $\begin{array}{l}\text { Commercial Sector } \\
\text { Primary Energy } \\
\text { Coal } \\
\text { Natural Gas } \\
\text { Petroleum } \\
\text { Distillate Fuel } \\
\text { Kerosene } \\
\text { LPG } \\
\text { Motor Gasoline } \\
\text { Residual Fuel }\end{array}$ & $\begin{array}{r}228.6 \\
50.2 \\
3.1 \\
28.9 \\
18.2 \\
13.1 \\
.3 \\
1.4 \\
3.1 \\
.2 \\
178.4\end{array}$ & $\begin{array}{r}557.1 \\
103.5 \\
7.2 \\
55.5 \\
40.8 \\
26.8 \\
.6 \\
.6 \\
2.9 \\
7.8 \\
2.9 \\
453.5\end{array}$ & $\begin{array}{r}1,161.9 \\
247.7 \\
5.0 \\
144.9 \\
97.8 \\
61.5 \\
1.5 \\
4.5 \\
19.4 \\
10.9 \\
914.2\end{array}$ & $\begin{array}{r}1,630.4 \\
357.6 \\
7.4 \\
203.3 \\
146.9 \\
93.7 \\
8.3 \\
10.7 \\
22.4 \\
11.9 \\
1,272.8\end{array}$ & $\begin{array}{r}1,981.8 \\
338.8 \\
6.3 \\
202.3 \\
130.2 \\
84.4 \\
5.1 \\
12.5 \\
23.6 \\
4.6 \\
1,643.0\end{array}$ & $\begin{array}{r}2,042.2 \\
321.3 \\
3.6 \\
213.8 \\
103.9 \\
66.8 \\
4.7 \\
14.5 \\
16.1 \\
1.7 \\
1,721.0\end{array}$ & $\begin{array}{r}R_{2,157.5} \\
349.8 \\
5.1 \\
252.3 \\
92.5 \\
56.8 \\
4.2 \\
13.0 \\
15.1 \\
3.4 \\
R_{1,807.6}\end{array}$ & $\begin{array}{r}2,277.8 \\
398.0 \\
8.3 \\
296.1 \\
93.6 \\
67.8 \\
5.0 \\
12.5 \\
5.8 \\
2.5 \\
1,879.8\end{array}$ \\
\hline $\begin{array}{l}\text { Industrial Sector } \\
\text { Primary Energy } \\
\text { Coal . } \\
\text { Coking Coal } \\
\text { Steam Coal } \\
\text { Natural Gas } \\
\text { Petroleum } \\
\text { Asphalt and Road Oil } \\
\text { Distillate Fuel } \\
\text { Kerosene } \\
\text { LPG } \\
\text { Lubricants } \\
\text { Motor Gasoline } \\
\text { Residual Fuel } \\
\text { Other }\end{array}$ & $\begin{array}{r}207.8 \\
132.2 \\
42.1 \\
.3 \\
41.8 \\
22.5 \\
67.6 \\
10.2 \\
15.3 \\
1.5 \\
3.8 \\
8.9 \\
9.8 \\
8.6 \\
9.6 \\
75.5\end{array}$ & $\begin{array}{r}577.1 \\
348.7 \\
97.0 \\
- \\
97.0 \\
39.4 \\
212.4 \\
28.1 \\
36.8 \\
2.2 \\
12.0 \\
13.9 \\
11.5 \\
85.4 \\
22.4 \\
228.3\end{array}$ & $\begin{array}{r}1,474.8 \\
1,007.3 \\
148.4 \\
33.0 \\
115.4 \\
161.9 \\
697.0 \\
62.6 \\
111.0 \\
9.0 \\
20.3 \\
36.8 \\
14.6 \\
110.9 \\
332.0 \\
467.5\end{array}$ & $\begin{array}{r}1,599.0 \\
1,032.6 \\
183.7 \\
45.7 \\
138.0 \\
232.5 \\
616.4 \\
132.0 \\
105.7 \\
8.0 \\
56.1 \\
41.0 \\
33.6 \\
83.5 \\
156.4 \\
566.4 \\
6.4\end{array}$ & $\begin{array}{r}\mathrm{R}_{1}, 623.3 \\
\mathrm{~A}_{934.9} \\
195.8 \\
42.7 \\
153.1 \\
263.7 \\
\mathrm{R}_{475.3} \\
93.3 \\
102.0 \\
2.7 \\
47.1 \\
\mathrm{R}_{38.3} \\
34.8 \\
51.3 \\
\mathrm{R}_{105.8} \\
688.4\end{array}$ & $\begin{array}{r}\mathbf{R}_{1,510.6} \\
\mathrm{R}_{850.7} \\
217.1 \\
44.9 \\
172.2 \\
217.6 \\
\mathrm{R}_{416.0} \\
76.9 \\
85.4 \\
2.9 \\
61.9 \\
\text { R }_{39.4} \\
31.8 \\
26.3 \\
\mathrm{R}_{91.3} \\
659.9\end{array}$ & $\begin{array}{r}\mathrm{R}_{1}, \mathbf{5 2 6 . 6} \\
\mathrm{R}_{830.0} \\
191.0 \\
45.4 \\
145.6 \\
244.6 \\
\mathrm{R}_{394.4} \\
58.5 \\
75.2 \\
1.8 \\
\mathrm{R}_{52.2} \\
\mathrm{R}_{46.5} \\
31.8 \\
35.2 \\
\mathrm{~A}_{93.1} \\
696.6\end{array}$ & $\begin{array}{r}1,547.7 \\
837.4 \\
160.4 \\
43.4 \\
117.0 \\
270.6 \\
406.4 \\
72.7 \\
84.2 \\
2.7 \\
53.4 \\
46.1 \\
30.5 \\
30.6 \\
86.1 \\
710.4\end{array}$ \\
\hline 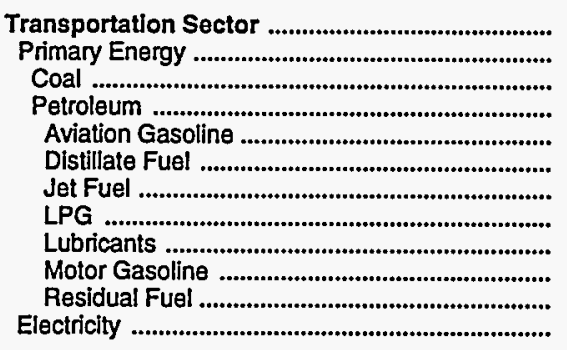 & $\begin{array}{r}855.7 \\
855.7 \\
.1 \\
855.7 \\
3.9 \\
56.0 \\
44.9 \\
.3 \\
13.3 \\
714.9 \\
22.4 \\
-\end{array}$ & $\begin{array}{r}1,816.5 \\
1,816.5 \\
(s) \\
1,816.5 \\
4.4 \\
130.4 \\
131.9 \\
.6 \\
19.4 \\
1,465.4 \\
64.4 \\
-\end{array}$ & $\begin{array}{r}4,129.1 \\
4,127.6 \\
- \\
4,127.6 \\
9.9 \\
475.3 \\
444.2 \\
.8 \\
46.1 \\
3,058.9 \\
92.3 \\
1.5\end{array}$ & $\begin{array}{r}4,235.2 \\
4,232.0 \\
- \\
4,232.0 \\
6.6 \\
693.2 \\
357.1 \\
3.4 \\
51.5 \\
3,030.1 \\
89.9 \\
3.2\end{array}$ & $\begin{array}{r}R_{4,856.4} \\
R_{4,852.4} \\
- \\
R_{4,852.4} \\
3.3 \\
828.5 \\
489.8 \\
2.1 \\
R_{48.0} \\
3,416.5 \\
64.1 \\
4.0\end{array}$ & $\begin{array}{r}R_{4,512.0} \\
R_{4,508.0} \\
- \\
R_{4,508.0} \\
5.1 \\
795.7 \\
318.7 \\
3.6 \\
R_{49.4} \\
3,292.2 \\
43.2 \\
4.0\end{array}$ & $\begin{array}{r}R_{4,522.2} \\
R_{4,517.9} \\
- \\
R_{4,517.9} \\
4.4 \\
767.3 \\
294.4 \\
3.1 \\
R_{58.3} \\
3,353.5 \\
R_{37.0} \\
\text { R } 4.3\end{array}$ & $\begin{array}{r}4,678.8 \\
4,674.6 \\
- \\
4,674.6 \\
4.4 \\
792.2 \\
279.6 \\
3.2 \\
57.9 \\
3,509.8 \\
27.5 \\
4.2\end{array}$ \\
\hline 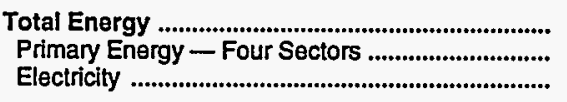 & $\begin{array}{r}1,738.8 \\
1,244.4 \\
494.4\end{array}$ & $\begin{array}{l}3,869.0 \\
2,588.5 \\
1,280.5\end{array}$ & $\begin{array}{l}8,617.8 \\
6,036.3 \\
2,581.5\end{array}$ & $\begin{array}{l}9,791.3 \\
6,448.3 \\
3,343.0\end{array}$ & $\begin{array}{r}R_{11,257.8} \\
R_{6,883.4} \\
4,374.4\end{array}$ & $\begin{array}{r}R_{10,985.5} \\
R_{6,427.5} \\
4,558.0\end{array}$ & $\begin{array}{r}R_{11,241.7} \\
R_{6,460.1} \\
4,781.6\end{array}$ & $\begin{array}{r}11,815.7 \\
6,762.4 \\
5,053.4\end{array}$ \\
\hline $\begin{array}{l}\text { Electric Utillty Sector }{ }^{\mathrm{a}} \\
\text { Coal } \\
\text { Natural Gas } \\
\text { Petroleum } \\
\text { Heavy Oil }{ }^{\mathrm{b}} \\
\text { Light Oil } \mathrm{b} \\
\text { Petroleum Coke } \\
\text { Nuclear Fuel } \\
\text { Biomass Fuels }\end{array}$ & $\begin{array}{r}101.4 \\
63.1 \\
1.3 \\
37.0 \\
33.8 \\
1.5 \\
1.8 \\
= \\
-\end{array}$ & $\begin{array}{r}455.1 \\
109.3 \\
.5 \\
317.6 \\
309.7 \\
7.9 \\
- \\
27.7 \\
-\end{array}$ & $\begin{array}{r}726.4 \\
238.2 \\
7.3 \\
388.1 \\
361.0 \\
27.1 \\
-\overline{-} \\
92.8 \\
-\end{array}$ & $\begin{array}{r}514.6 \\
330.9 \\
5.5 \\
46.7 \\
35.7 \\
11.0 \\
-.5 \\
131.5\end{array}$ & $\begin{array}{r}508.9 \\
323.2 \\
17.6 \\
48.5 \\
32.2 \\
16.4 \\
- \\
119.6 \\
-\end{array}$ & $\begin{array}{r}532.0 \\
333.0 \\
18.0 \\
45.8 \\
37.6 \\
8.2 \\
-\overline{135.2} \\
-\end{array}$ & $\begin{array}{r}500.1 \\
327.4 \\
27.2 \\
37.3 \\
29.9 \\
7.4 \\
- \\
108.2 \\
-\end{array}$ & $\begin{array}{r}561.6 \\
355.0 \\
57.1 \\
45.9 \\
40.6 \\
5.3 \\
\overline{-} \overline{103.7} \\
-\end{array}$ \\
\hline Primary Energy — Flve Sectors c ............................. & $1,345.8$ & $3,043.6$ & $6,762.6$ & $6,962.9$ & $\mathrm{R}_{7,392.4}$ & $\mathrm{R}_{6,959.6}$ & $\mathrm{P}_{6,960.2}$ & $7,324.0$ \\
\hline
\end{tabular}

a There are no direct fuel costs for hydroelectric, geothermal, wind, photovoltaic, or solar thermal energy.

beavy oil includes fuel oil nos. 4, 5, and 6, and residual fuel oils. Light oil includes fuel nos. 1 and 2 , kerosene, and jet fuel.

c Biomass fuels are not included, except those consumed at electric utilities and those added to motor gasoline.

$R=$ Revised data.

-No consumption, including cases where adjustments were made. See explanation of adjustments in Section 6 of Appendix A.

$(s)=$ Value less than 0.05 million dollars.

Note: Totals may not equal sum of components due to independent rounding.

Sources: Data sources, estimation procedures, and assumptions are described in Appendix A. 


\begin{tabular}{|c|c|c|c|c|c|c|c|c|}
\hline Energy Source & 1970 & 1975 & 1980 & 1985 & 1990 & 1991 & 1992 & 1993 \\
\hline & \multicolumn{8}{|c|}{ Prices in Dollars per Million Btu } \\
\hline 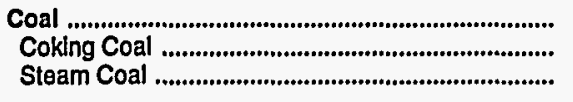 & .5 .54 & $\frac{0.61}{.61}$ & 1.15 & $\frac{1.74}{1.74}$ & $\frac{1.65}{1.65}$ & $\frac{1.65}{1.65}$ & $\frac{1.45}{1.45}$ & $\frac{1.45}{1.45}$ \\
\hline 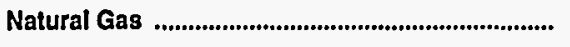 & .71 & 1.60 & 4.48 & 5.23 & 3.60 & 3.55 & 3.70 & 3.97 \\
\hline 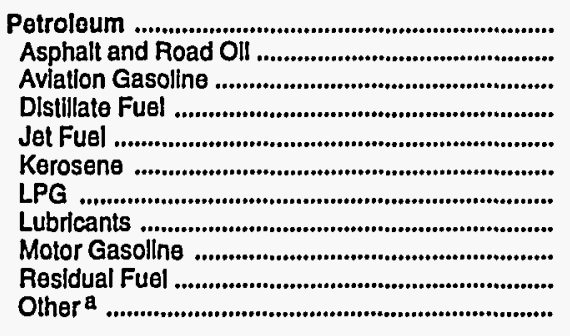 & $\begin{array}{r}1.71 \\
.66 \\
2.17 \\
1.18 \\
.73 \\
1.62 \\
2.50 \\
5.08 \\
2.92 \\
.32 \\
.60\end{array}$ & $\begin{array}{l}3.24 \\
1.77 \\
3.45 \\
2.55 \\
2.04 \\
3.07 \\
4.46 \\
7.48 \\
4.62 \\
1.93 \\
1.50\end{array}$ & $\begin{array}{r}7.11 \\
3.70 \\
9.02 \\
6.68 \\
6.21 \\
8.53 \\
6.78 \\
14.36 \\
9.92 \\
3.24 \\
2.97\end{array}$ & $\begin{array}{r}7.64 \\
4.17 \\
9.99 \\
7.80 \\
6.03 \\
7.22 \\
9.11 \\
17.61 \\
9.31 \\
4.53 \\
3.43\end{array}$ & $\begin{array}{r}R_{7.07} \\
3.18 \\
9.32 \\
7.98 \\
5.68 \\
8.97 \\
10.14 \\
R 14.60 \\
9.45 \\
2.70 \\
2.28\end{array}$ & $\begin{array}{r}R_{7.17} \\
3.40 \\
8.71 \\
7.78 \\
4.76 \\
8.58 \\
10.74 \\
R_{16.79} \\
9.14 \\
5.18 \\
2.09\end{array}$ & $\begin{array}{r}R_{6.28} \\
2.85 \\
8.54 \\
7.75 \\
4.56 \\
8.00 \\
10.58 \\
R_{19.43} \\
9.47 \\
R_{1.85} \\
1.56\end{array}$ & $\begin{array}{r}6.73 \\
3.30 \\
8.24 \\
8.12 \\
4.54 \\
8.65 \\
10.24 \\
18.94 \\
9.42 \\
2.00 \\
1.37\end{array}$ \\
\hline 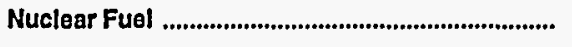 & .18 & 24 & .43 & .71 & .47 & .45 & .38 & .46 \\
\hline Blomass Fuels at Utilitles ........................................ & .65 & - & - & .79 & .61 & .76 & ค. .78 & .80 \\
\hline Primary Energy — Flve Sectors ${ }^{b} \ldots . . . . . . . . . . . . . . . . .$. & 1.42 & 2.51 & 5.83 & 5.92 & $\mathbf{R}_{5.65}$ & $R_{5.74}$ & $R_{5.08}$ & 5.26 \\
\hline $\begin{array}{l}\text { Electric Utllity Fuel }{ }^{c} \text {................................................ } \\
\text { Electriclty Purchased by End Users ..................... }\end{array}$ & $\begin{array}{r}.18 \\
2.02\end{array}$ & $\begin{array}{r}.46 \\
2.77\end{array}$ & $\begin{array}{r}.91 \\
4.16\end{array}$ & $\begin{array}{l}1.17 \\
9.18\end{array}$ & $\begin{array}{r}1.09 \\
10.03\end{array}$ & $\begin{array}{l}1.16 \\
9.96\end{array}$ & $\begin{array}{r}1.07 \\
10.13\end{array}$ & $\begin{array}{r}1.04 \\
10.77\end{array}$ \\
\hline \multirow[t]{2}{*}{ 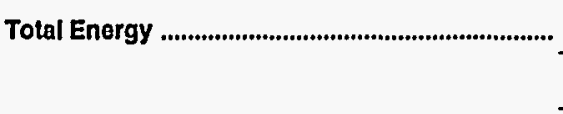 } & 1.59 & 2.82 & 5.98 & 7.67 & $\mathrm{R}_{7.36}$ & 7.37 & ${ }^{R} 6.87$ & 7.30 \\
\hline & \multicolumn{8}{|c|}{ Expenditures In Millions of Dollars } \\
\hline $\begin{array}{l}\text { Coal } \\
\text { Coking Coal } \\
\text { Steam Coal }\end{array}$ & $\begin{array}{l}3.2 \\
\overrightarrow{3.2}\end{array}$ & $\frac{46.9}{46.9}$ & $\begin{array}{c}104.2 \\
- \\
104.2\end{array}$ & $\begin{array}{c}163.4 \\
- \\
163.4\end{array}$ & $\begin{array}{c}141.3 \\
\overline{-} \\
141.3\end{array}$ & $\begin{array}{c}147.0 \\
- \\
147.0\end{array}$ & $\begin{array}{c}153.6 \\
- \\
153.6\end{array}$ & $\begin{array}{c}141.8 \\
- \\
141.8\end{array}$ \\
\hline 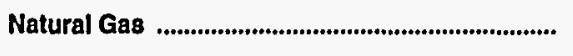 & 97.2 & 242.3 & 530.5 & 686.4 & 554.3 & 590.0 & 610.5 & 771.1 \\
\hline 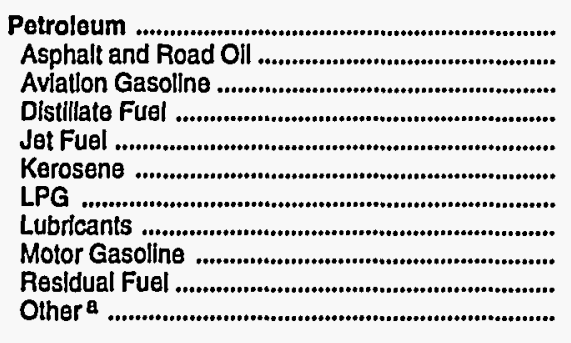 & $\begin{array}{r}812.2 \\
10.2 \\
3.8 \\
123.0 \\
43.3 \\
2.2 \\
15.3 \\
20.5 \\
553.3 \\
17.9 \\
22.7\end{array}$ & $\begin{array}{r}1,631.0 \\
34.1 \\
4.8 \\
248.4 \\
160.7 \\
6.0 \\
11.5 \\
28.2 \\
994.2 \\
82.8 \\
60.3\end{array}$ & $\begin{array}{r}3,938.8 \\
50.3 \\
16.2 \\
715.7 \\
419.5 \\
5.8 \\
33.4 \\
61.2 \\
2,222.4 \\
327.7 \\
86.5\end{array}$ & $\begin{array}{r}4,205.7 \\
56.4 \\
10.2 \\
923.8 \\
522.2 \\
49.6 \\
69.3 \\
68.3 \\
2,151.5 \\
314.2 \\
40.2\end{array}$ & $\begin{array}{r}R_{4,923.1} \\
52.4 \\
14.7 \\
1,012.1 \\
716.0 \\
3.8 \\
71.9 \\
R 63.7 \\
2,639.3 \\
269.6 \\
79.6\end{array}$ & $\begin{array}{r}R_{4,909.0} \\
67.0 \\
11.8 \\
902.9 \\
572.7 \\
3.4 \\
79.3 \\
P_{65.6} \\
2,604.1 \\
552.2 \\
50.1\end{array}$ & $\begin{array}{r}R_{4,767.0} \\
57.2 \\
12.5 \\
832.0 \\
619.4 \\
2.1 \\
75.2 \\
R_{77.4} \\
2,747.7 \\
R_{269.4} \\
74.1\end{array}$ & $\begin{array}{r}4,612.4 \\
64.4 \\
8.2 \\
730.5 \\
570.3 \\
3.1 \\
71.4 \\
76.8 \\
2,838.3 \\
196.8 \\
52.6\end{array}$ \\
\hline 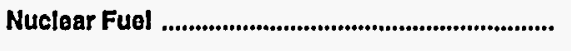 & 5.2 & 8.7 & 9.6 & 61.4 & 29.1 & 20.4 & 23.2 & 35.2 \\
\hline Blomass Fuels at UtIlitles ................................... & (8) & - & - & 2.3 & 2.1 & 2.2 & $R_{2.9}$ & 3.2 \\
\hline Primary Energy - Flve Sectors ${ }^{b} \ldots \ldots \ldots \ldots \ldots \ldots \ldots$ & 917.9 & $1,928.9$ & $4,583.0$ & $5,119.2$ & $R_{5,649.9}$ & $\mathrm{~A}_{5,668.6}$ & $R_{5,557.2}$ & $5,563.7$ \\
\hline 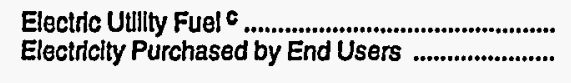 & $\begin{array}{r}-5.2 \\
316.8\end{array}$ & $\begin{array}{r}-46.5 \\
523.9\end{array}$ & $\begin{array}{r}-95.7 \\
953.4\end{array}$ & $\begin{array}{r}-203.6 \\
2,331.7\end{array}$ & $\begin{array}{r}-157.2 \\
3,033.7\end{array}$ & $\begin{array}{r}-152.5 \\
3,069.4\end{array}$ & $\begin{array}{r}R-182.5 \\
3,014.8\end{array}$ & $\begin{array}{r}-184.3 \\
3,248.9\end{array}$ \\
\hline 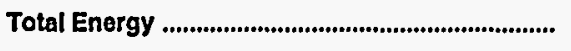 & $1,229.4$ & $2,406.2$ & $5,440.7$ & $7,247.3$ & $\mathrm{~A}_{\mathbf{8 , 5 2 6 . 5}}$ & ${ }^{\mathrm{P}_{8,585.5}}$ & $\mathrm{R}_{8,389.4}$ & $8,628.3$ \\
\hline
\end{tabular}

a Includes petroleum coke used at electric utilities.

b Blomass fuels are not included, except those consumed at electric utilitles and those added to motor gasoline.

c There are no direct fuel costs for hydroelectric, geothermal, wind, photovoltalc, or solar thermal energy.

$R=$ Revised data.

-No consumption, including cases where adjustments were made. See

explanation of adjustments in Section 6 of Appendix $A$.

$(s)=$ Value less than 0.05 million dollars.

Note: Expenditure totals may not equal sum of components due to independent rounding.

Sources: Data sources, estimation procedures, and assumptions are described in Appendix A. 
A $1970,1975,1980,1985$, and 1990-1993

S (Dollars per Million Btu)

\begin{tabular}{|c|c|c|c|c|c|c|c|c|}
\hline Sector and Energy Source & 1970 & 1975 & 1980 & 1985 & 1990 & 1991 & 1992 & 1993 \\
\hline 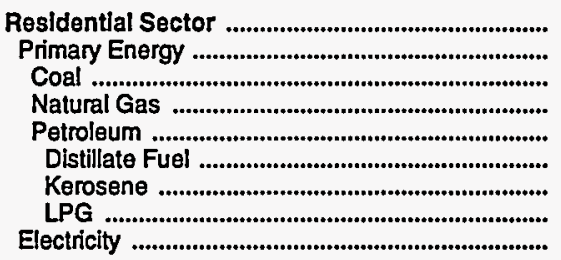 & $\begin{array}{l}2.12 \\
1.47 \\
.95 \\
1.33 \\
1.57 \\
1.40 \\
2.47 \\
3.05 \\
3.12\end{array}$ & $\begin{array}{l}3.23 \\
2.54 \\
1.14 \\
2.18 \\
2.97 \\
2.80 \\
3.61 \\
5.73 \\
3.94\end{array}$ & $\begin{array}{l}5.73 \\
5.99 \\
4.26 \\
5.05 \\
7.40 \\
7.27 \\
9.80 \\
8.12 \\
5.56\end{array}$ & $\begin{array}{r}9.53 \\
6.82 \\
3.67 \\
6.35 \\
7.88 \\
7.76 \\
9.92 \\
8.46 \\
11.14\end{array}$ & $\begin{array}{r}10.22 \\
6.02 \\
3.77 \\
4.87 \\
8.45 \\
7.90 \\
10.10 \\
12.32 \\
12.88\end{array}$ & $\begin{array}{r}9.99 \\
5.67 \\
5.21 \\
4.54 \\
8.70 \\
7.80 \\
9.97 \\
12.62 \\
12.79\end{array}$ & $\begin{array}{r}10.27 \\
5.67 \\
3.76 \\
4.84 \\
8.40 \\
7.24 \\
9.25 \\
12.21 \\
13.07\end{array}$ & $\begin{array}{r}10.41 \\
5.69 \\
3.77 \\
5.05 \\
8.70 \\
7.67 \\
9.80 \\
11.37 \\
13.47\end{array}$ \\
\hline 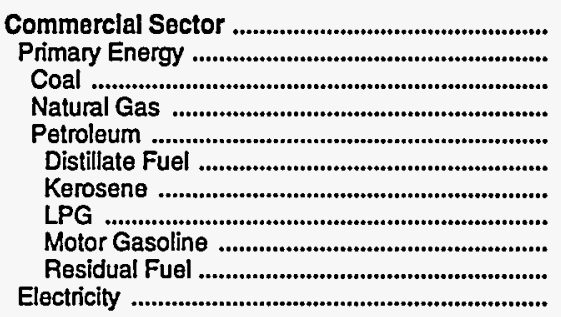 & $\begin{array}{r}1.90 \\
1.12 \\
.52 \\
1.05 \\
1.21 \\
1.21 \\
.84 \\
1.19 \\
2.92 \\
.33 \\
3.21\end{array}$ & $\begin{array}{r}2.94 \\
2.06 \\
.90 \\
1.75 \\
2.87 \\
2.60 \\
2.31 \\
2.67 \\
4.62 \\
2.45 \\
4.10\end{array}$ & $\begin{array}{l}5.35 \\
5.03 \\
2.28 \\
4.59 \\
6.76 \\
6.90 \\
7.04 \\
5.60 \\
9.92 \\
3.61 \\
5.67\end{array}$ & $\begin{array}{r}7.94 \\
5.62 \\
2.30 \\
5.24 \\
6.38 \\
6.53 \\
7.02 \\
9.34 \\
9.31 \\
4.05 \\
10.57\end{array}$ & $\begin{array}{r}8.63 \\
4.65 \\
2.45 \\
4.02 \\
6.52 \\
6.17 \\
6.80 \\
9.03 \\
9.45 \\
2.84 \\
11.63\end{array}$ & $\begin{array}{r}8.57 \\
4.29 \\
2.73 \\
3.94 \\
5.72 \\
5.38 \\
5.93 \\
9.29 \\
9.14 \\
2.30 \\
11.77\end{array}$ & $\begin{array}{r}9.21 \\
4.38 \\
2.72 \\
4.18 \\
5.91 \\
5.27 \\
5.91 \\
9.26 \\
9.47 \\
2.26 \\
12.15\end{array}$ & $\begin{array}{r}9.45 \\
4.47 \\
2.63 \\
4.37 \\
5.92 \\
5.57 \\
6.07 \\
9.21 \\
9.42 \\
2.08 \\
12.77\end{array}$ \\
\hline $\begin{array}{l}\text { Industrial Sector } \\
\text { Primary Energy } \\
\text { Coal } \\
\text { Coking Coal } \\
\text { Steam Coal }\end{array}$ & $\begin{array}{r}.67 \\
.55 \\
.52 \\
- \\
.52 \\
.38 \\
.67 \\
.66 \\
.73 \\
.84 \\
1.19 \\
5.08 \\
2.92 \\
.33 \\
.60 \\
.97\end{array}$ & $\begin{array}{r}1.52 \\
1.58 \\
.90 \\
. \overline{0} \\
1.29 \\
1.85 \\
1.77 \\
2.05 \\
2.31 \\
2.67 \\
7.48 \\
4.62 \\
1.78 \\
1.50 \\
1.37\end{array}$ & $\begin{array}{r}3.40 \\
4.08 \\
2.28 \\
-\overline{2.28} \\
4.09 \\
4.19 \\
3.70 \\
6.06 \\
7.04 \\
5.60 \\
14.36 \\
9.92 \\
3.36 \\
2.97 \\
2.26\end{array}$ & $\begin{array}{r}5.40 \\
4.88 \\
2.30 \\
-\overline{2} \\
2.30 \\
4.58 \\
5.22 \\
4.17 \\
6.20 \\
7.02 \\
9.34 \\
17.61 \\
9.31 \\
4.05 \\
3.43 \\
6.23\end{array}$ & $\begin{array}{r}5.00 \\
R_{3.48} \\
2.45 \\
-\overline{2.45} \\
2.64 \\
R_{4.18} \\
3.18 \\
6.01 \\
6.80 \\
9.03 \\
R_{14.60} \\
9.45 \\
2.84 \\
2.28 \\
7.00\end{array}$ & $\begin{array}{r}4.91 \\
\mathrm{R}_{3.43} \\
2.73 \\
-\overline{2} \\
2.73 \\
2.71 \\
\mathrm{R}_{4.14} \\
3.40 \\
5.24 \\
5.93 \\
9.29 \\
\mathrm{P} 16.79 \\
9.14 \\
2.30 \\
2.09 \\
6.72\end{array}$ & $\begin{array}{r}4.51 \\
\mathrm{P}_{3.10} \\
2.72 \\
- \\
2.72 \\
2.82 \\
\mathrm{P}_{3.32} \\
2.85 \\
5.23 \\
5.91 \\
5.91 \\
9.26 \\
\mathrm{R}_{19.43} \\
9.47 \\
2.26 \\
1.56 \\
6.56\end{array}$ & $\begin{array}{r}4.74 \\
3.20 \\
2.63 \\
- \\
2.63 \\
3.11 \\
3.32 \\
3.30 \\
5.37 \\
6.07 \\
9.21 \\
18.94 \\
9.42 \\
2.08 \\
1.37 \\
7.02\end{array}$ \\
\hline 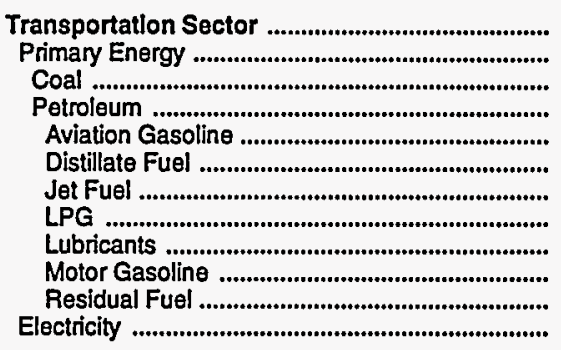 & $\begin{array}{r}2.23 \\
2.23 \\
.52 \\
2.23 \\
2.17 \\
1.32 \\
.73 \\
1.19 \\
5.08 \\
2.92 \\
.30 \\
2.16\end{array}$ & $\begin{array}{l}3.73 \\
3.73 \\
.90 \\
3.73 \\
3.45 \\
2.65 \\
2.04 \\
2.67 \\
7.48 \\
4.62 \\
2.14 \\
3.20\end{array}$ & $\begin{array}{r}7.86 \\
7.86 \\
- \\
7.86 \\
9.02 \\
6.72 \\
6.21 \\
5.60 \\
14.36 \\
9.92 \\
3.15 \\
4.26\end{array}$ & $\begin{array}{r}8.24 \\
8.24 \\
- \\
8.24 \\
9.99 \\
8.77 \\
6.03 \\
9.34 \\
17.61 \\
9.31 \\
5.02 \\
8.28\end{array}$ & $\begin{array}{r}\text { A7.51 } \\
\text { R7.51 } \\
\text { R7.51 } \\
9.32 \\
9.04 \\
5.68 \\
9.03 \\
R_{14.60} \\
9.45 \\
2.69 \\
9.15\end{array}$ & $\begin{array}{r}R_{7.58} \\
R_{7.58} \\
R_{7.58} \\
8.71 \\
8.94 \\
4.76 \\
9.29 \\
R_{16.79} \\
9.14 \\
5.36 \\
9.38\end{array}$ & $\begin{array}{r}\mathrm{R}_{6.70} \\
\mathrm{R}_{6.70} \\
- \\
\mathrm{R}_{6.70} \\
8.54 \\
8.68 \\
4.56 \\
9.26 \\
\mathrm{R}_{19.43} \\
9.47 \\
\mathrm{R}_{1.84} \\
9.81\end{array}$ & $\begin{array}{r}7.18 \\
7.18 \\
7.18 \\
7.24 \\
9.06 \\
4.54 \\
9.21 \\
18.94 \\
9.42 \\
2.00 \\
10.37\end{array}$ \\
\hline 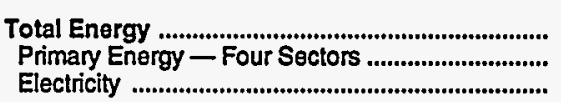 & $\begin{array}{l}1.59 \\
1.48 \\
2.02\end{array}$ & $\begin{array}{l}2.82 \\
2.83 \\
2.77\end{array}$ & $\begin{array}{l}5.98 \\
6.59 \\
4.16\end{array}$ & $\begin{array}{l}7.67 \\
7.11 \\
9.18\end{array}$ & $\begin{array}{r}R_{7.36} \\
6.41 \\
10.03\end{array}$ & $\begin{array}{r}7.37 \\
R_{6.44} \\
9.96\end{array}$ & $\begin{array}{l}A_{6.87} \\
A_{5.82} \\
10.13\end{array}$ & $\begin{array}{r}7.30 \\
6.11 \\
10.77\end{array}$ \\
\hline 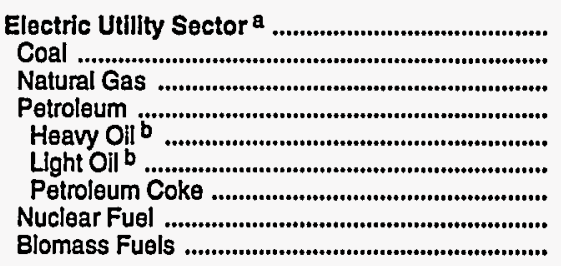 & $\begin{array}{l}.18 \\
\overline{-} \\
.33 \\
.32 \\
.37 \\
. \overline{18} \\
.65\end{array}$ & $\begin{array}{r}.46 \\
.57 \\
2 . \overline{9} \\
2.50 \\
2.41 \\
. \overline{4} \\
-\end{array}$ & $\begin{array}{r}.91 \\
.96 \\
3.43 \\
3.93 \\
3.58 \\
6.40 \\
. \overline{3} \\
-\end{array}$ & $\begin{array}{r}1.17 \\
1.65 \\
4.54 \\
5.72 \\
5 . \overline{2} \\
. \overline{71} \\
.79\end{array}$ & $\begin{array}{r}1.09 \\
1.58 \\
3.03 \\
5.09 \\
3.05 \\
5.15 \\
. \overline{.7} \\
.61\end{array}$ & $\begin{array}{r}1.16 \\
1.55 \\
3.83 \\
5.74 \\
6.19 \\
5.72 \\
. \overline{.45} \\
.76\end{array}$ & $\begin{array}{r}1.07 \\
1.37 \\
3.16 \\
R_{4.47} \\
\mathrm{R}_{2.18} \\
4.68 \\
-.38 \\
\mathrm{R} \\
.78\end{array}$ & $\begin{array}{l}1.04 \\
1.36 \\
3.76 \\
4.66 \\
2.31 \\
4.69 \\
.46 \\
.80\end{array}$ \\
\hline Primary Energy - Flve Sectors ${ }^{\circ}$............................. & 1.42 & 2.51 & 5.83 & 5.92 & $R_{5.65}$ & $\mathrm{R}_{5.74}$ & $A_{5.08}$ & 5.26 \\
\hline
\end{tabular}

a There are no direct fuel costs for hydroelectric, geothermal, wind, photovoltaic, or solar thermal energy.

beavy oil includes fuel oil nos. 4, 5, and 6, and residual fuel olls. Light oil includes fuel oil nos. 1 and 2, kerosene, and jet fuel.

$c$ Biomass fuels are not included, except those consumed at electric utilities and those added to motor gasoline.
R=Revlsed data.

- No consumption, Including cases where adjustments were made. See explanation of adjustments in Section 6 of Appendix A.

Sources: Data sources, estimation procedures, and assumptlons are described in Appendix A. 


\begin{tabular}{|c|c|c|c|c|c|c|c|c|}
\hline Sector and Energy Source & 1970 & 1975 & 1980 & 1985 & 1990 & 1991 & 1992 & 1993 \\
\hline 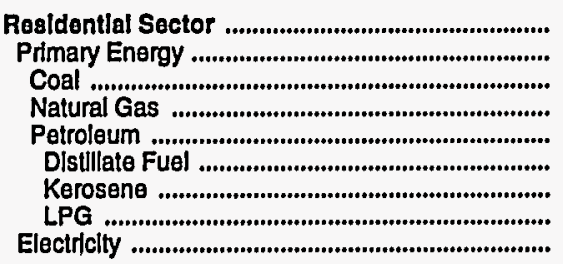 & $\begin{array}{r}280.8 \\
117.3 \\
.3 \\
44.8 \\
72.2 \\
57.4 \\
1.6 \\
13.2 \\
163.5\end{array}$ & $\begin{array}{r}427.3 \\
169.4 \\
.2 \\
78.1 \\
91.1 \\
78.3 \\
4.2 \\
8.6 \\
258.0\end{array}$ & $\begin{array}{r}794.5 \\
330.6 \\
5.5 \\
158.0 \\
167.2 \\
144.9 \\
3.6 \\
18.7 \\
463.8\end{array}$ & $\begin{array}{r}1,447.8 \\
386.0 \\
6.5 \\
217.8 \\
161.7 \\
140.0 \\
4.8 \\
16.8 \\
1,061.8\end{array}$ & $\begin{array}{r}1,640.6 \\
374.6 \\
1.9 \\
202.5 \\
170.1 \\
138.0 \\
2.8 \\
29.3 \\
1,266.0\end{array}$ & $\begin{array}{r}1,679.6 \\
375.6 \\
3.3 \\
216.3 \\
156.1 \\
112.8 \\
2.6 \\
40.7 \\
1,304.0\end{array}$ & $\begin{array}{r}1,603.4 \\
335.5 \\
2.7 \\
215.2 \\
117.5 \\
77.1 \\
1.5 \\
38.9 \\
1,268.0\end{array}$ & $\begin{array}{r}1,811.9 \\
389.9 \\
3.4 \\
278.5 \\
108.0 \\
67.8 \\
2.4 \\
37.8 \\
1,422.0\end{array}$ \\
\hline 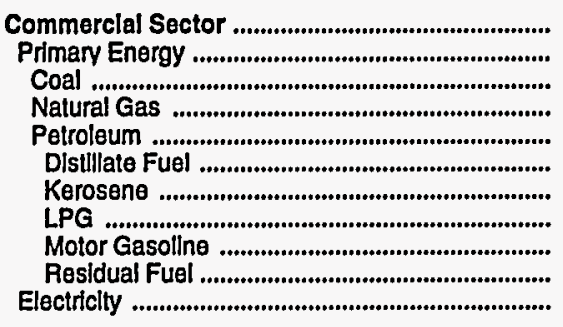 & $\begin{array}{r}116.7 \\
43.0 \\
.3 \\
20.4 \\
22.3 \\
15.7 \\
.1 \\
.9 \\
4.7 \\
1.0 \\
73.6\end{array}$ & $\begin{array}{r}242.3 \\
97.0 \\
.2 \\
58.2 \\
38.6 \\
23.0 \\
.3 \\
.7 \\
9.1 \\
5.5 \\
145.3\end{array}$ & $\begin{array}{r}502.6 \\
234.8 \\
5.4 \\
148.7 \\
80.7 \\
43.1 \\
.7 \\
2.3 \\
24.9 \\
9.7 \\
267.8\end{array}$ & $\begin{array}{r}1,095.0 \\
411.2 \\
7.6 \\
193.3 \\
210.4 \\
162.4 \\
8.2 \\
3.3 \\
17.4 \\
19.0 \\
683.8\end{array}$ & $\begin{array}{r}1,110.5 \\
256.9 \\
2.3 \\
160.3 \\
94.3 \\
75.2 \\
.6 \\
3.8 \\
13.9 \\
1.0 \\
853.6\end{array}$ & $\begin{array}{r}1,122.2 \\
239.7 \\
3.2 \\
169.7 \\
66.8 \\
50.4 \\
.6 \\
5.3 \\
9.1 \\
1.5 \\
882.5\end{array}$ & $\begin{array}{r}1,139.7 \\
205.3 \\
3.6 \\
163.7 \\
38.0 \\
25.0 \\
.4 \\
5.2 \\
6.5 \\
.8 \\
{ }_{1934.4}\end{array}$ & $\begin{array}{r}1,233.8 \\
233.3 \\
4.4 \\
198.1 \\
30.9 \\
21.9 \\
.5 \\
5.4 \\
2.4 \\
.8 \\
1,000.4\end{array}$ \\
\hline 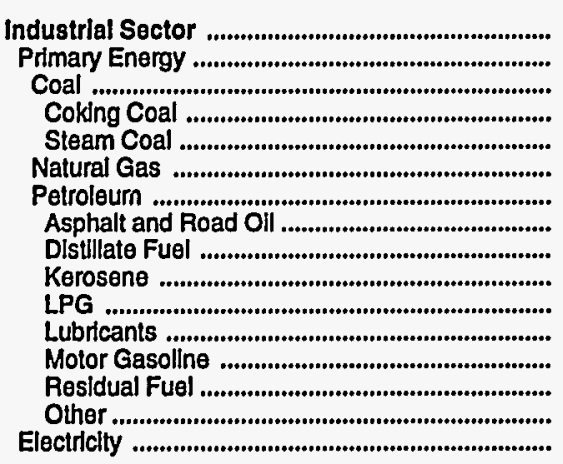 & $\begin{array}{r}198.1 \\
118.4 \\
2.7 \\
-\overline{2.7} \\
32.0 \\
83.7 \\
10.2 \\
19.6 \\
.5 \\
1.1 \\
8.2 \\
8.4 \\
13.1 \\
22.7 \\
79.7\end{array}$ & $\begin{array}{r}446.2 \\
325.6 \\
9.8 \\
-\overline{9} \\
9.8 \\
106.0 \\
209.8 \\
34.1 \\
44.8 \\
1.5 \\
1.8 \\
8.7 \\
10.6 \\
47.9 \\
60.3 \\
120.6\end{array}$ & $\begin{array}{r}903.4 \\
681.7 \\
16.2 \\
-5 \\
16.2 \\
220.5 \\
445.0 \\
50.3 \\
150.7 \\
1.5 \\
10.5 \\
17.6 \\
14.5 \\
113.3 \\
86.5 \\
221.7\end{array}$ & $\begin{array}{r}1,316.9 \\
731.1 \\
10.3 \\
- \\
10.3 \\
274.9 \\
445.8 \\
56.4 \\
99.4 \\
36.6 \\
38.0 \\
19.6 \\
33.8 \\
121.8 \\
40.2 \\
585.8\end{array}$ & $\begin{array}{r}R_{1,509.6} \\
R_{595.9} \\
12.7 \\
- \\
12.7 \\
190.8 \\
R_{392.3} \\
52.4 \\
155.2 \\
.4 \\
29.2 \\
R 18.3 \\
32.5 \\
24.7 \\
79.6 \\
913.7\end{array}$ & $\begin{array}{r}R_{1,430.9} \\
R_{548.5} \\
11.7 \\
- \\
11.7 \\
203.4 \\
R_{333.4} \\
67.0 \\
121.0 \\
.2 \\
25.1 \\
R_{18.8} \\
38.1 \\
13.1 \\
50.1 \\
882.3\end{array}$ & $\begin{array}{r}A_{1,365.1} \\
A_{553.3} \\
9.2 \\
- \\
9.2 \\
213.7 \\
R_{330.5} \\
57.2 \\
102.8 \\
.2 \\
24.1 \\
R_{22.2} \\
40.1 \\
9.7 \\
74.1 \\
811.8\end{array}$ & $\begin{array}{r}1,386.4 \\
560.6 \\
9.2 \\
- \\
9.2 \\
275.2 \\
276.2 \\
64.4 \\
82.5 \\
.2 \\
21.1 \\
22.0 \\
26.0 \\
7.3 \\
52.6 \\
825.9\end{array}$ \\
\hline 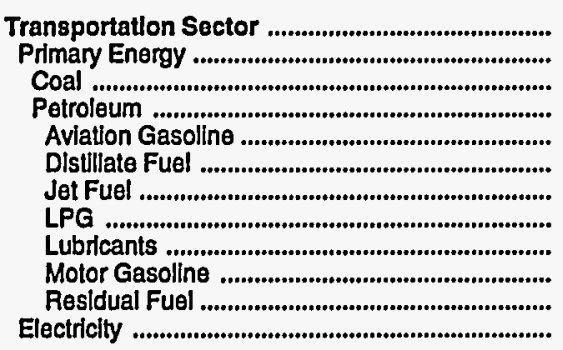 & $\begin{array}{r}633.9 \\
633.9 \\
(s) \\
633.9 \\
3.8 \\
30.3 \\
43.3 \\
.2 \\
12.3 \\
540.2 \\
3.8 \\
(5)\end{array}$ & $\begin{array}{r}1,290.3 \\
1,290.3 \\
(s) \\
1,290.3 \\
4.8 \\
102.2 \\
160.7 \\
.4 \\
19.4 \\
974.5 \\
28.3 \\
(s)\end{array}$ & $\begin{array}{r}3,240.3 \\
3,240.3 \\
- \\
3,240.3 \\
16.2 \\
375.8 \\
419.5 \\
1.9 \\
43.6 \\
2,183.1 \\
200.3 \\
(s)\end{array}$ & $\begin{array}{r}3,387.6 \\
3,387.3 \\
- \\
3,387.3 \\
10.2 \\
521.4 \\
522.2 \\
11.1 \\
48.7 \\
2,100.2 \\
173.4 \\
.4\end{array}$ & $\begin{array}{r}R_{4,265.8} \\
R_{4,265.4} \\
- \\
R_{4,265.4} \\
14.7 \\
642.8 \\
716.0 \\
9.6 \\
R_{45.4} \\
2,592.9 \\
243.9 \\
.4\end{array}$ & $\begin{array}{r}A_{4,352.8} \\
R_{4,352.2} \\
- \\
R_{4,352.2} \\
11.8 \\
618.2 \\
572.7 \\
8.2 \\
R_{46.8} \\
2,556.9 \\
537.6 \\
.5\end{array}$ & $\begin{array}{r}R_{4,281.2} \\
R_{4,280.6} \\
-\overline{-} \\
R_{4,280.6} \\
12.5 \\
626.7 \\
619.4 \\
7.0 \\
R_{55.2} \\
2,701.1 \\
R_{258.9} \\
\text { R.6 }\end{array}$ & $\begin{array}{r}4,196.1 \\
4,195.6 \\
- \\
4,195.6 \\
8.2 \\
556.6 \\
570.3 \\
7.1 \\
54.7 \\
2,809.9 \\
188.8 \\
.6\end{array}$ \\
\hline 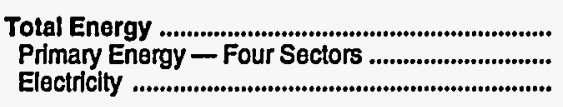 & $\begin{array}{r}1,229.4 \\
912.6 \\
316.8\end{array}$ & $\begin{array}{r}2,406.2 \\
1,882.3 \\
523.9\end{array}$ & $\begin{array}{r}5,440.7 \\
4,487.4 \\
953.4\end{array}$ & $\begin{array}{l}7,247.3 \\
4,915.6 \\
2,331.7\end{array}$ & $\begin{array}{r}R_{8,526.5} \\
R_{5,492.8} \\
3,033.7\end{array}$ & $\begin{array}{r}\mathrm{A}_{8,585.5} \\
\mathrm{~A}_{5,516.1} \\
\mathbf{3 , 0 6 9 . 4}\end{array}$ & $\begin{array}{r}R_{8,389.4} \\
R_{5,374.7} \\
3,014.8\end{array}$ & $\begin{array}{l}8,628.3 \\
5,379.4 \\
3,248.9\end{array}$ \\
\hline 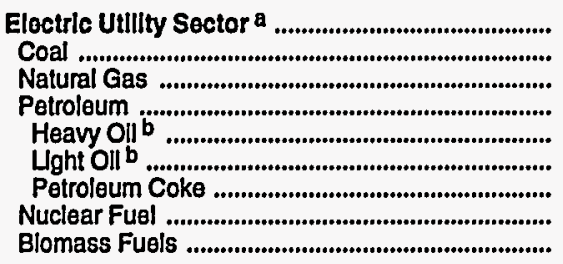 & $\begin{array}{l}5.2 \\
- \\
-\overline{2} \\
(s) \\
(s) \\
\overline{(s)} \\
\text { (s) }\end{array}$ & $\begin{array}{r}46.5 \\
36.7 \\
\overline{1.2} \\
1.1 \\
.1 \\
\overline{8.7} \\
-\end{array}$ & $\begin{array}{r}95.7 \\
77.1 \\
3.3 \\
5.7 \\
4.5 \\
1.1 \\
- \\
9.6 \\
-\end{array}$ & $\begin{array}{r}203.6 \\
139.0 \\
.4 \\
.6 \\
- \\
.6 \\
- \\
61.4 \\
2.3\end{array}$ & $\begin{array}{r}157.2 \\
124.4 \\
.6 \\
.9 \\
(s) \\
.9 \\
- \\
29.1 \\
2.1\end{array}$ & $\begin{array}{r}152.5 \\
128.9 \\
.6 \\
.5 \\
(s) \\
.5 \\
- \\
20.4 \\
2.2\end{array}$ & $\begin{array}{r}R_{182.5} \\
138.2 \\
17.8 \\
A^{3} .3 \\
(\mathrm{~s}) \\
.3 \\
-\overline{3} \\
23.2 \\
\text { R }_{2.9}\end{array}$ & $\begin{array}{r}184.3 \\
124.8 \\
19.3 \\
1.7 \\
(\mathrm{~s}) \\
1.7 \\
-\overline{35.2} \\
3.2\end{array}$ \\
\hline Primary Energy — Five Sectors c ....................... & 917.9 & $1,928.9$ & $4,583.0$ & 5,1192 & $R_{5,649.9}$ & $\mathrm{~A}_{5,668.6}$ & $\mathrm{~A}_{5,557.2}$ & $5,563.7$ \\
\hline
\end{tabular}

a There are no direct fuel costs for hydroelectric, geothermal, wind, photovoltalc, or solar thermal energy.

Heavy oll includes fuel oil nos. 4, 5, and 6, and residual fuel oils. Light oll includes fuel nos. 1 and 2, kerosene, and jet fuel.

c Blomass fuels are not included, except those consumed at electric utllities and those added to motor gasoline.

$R=$ Revised data.

- No consumption, including cases where adjustments were made. See explanation of adjustments in Section 6 of Appendix A.

(s) =Value less than 0.05 million dollars.

Note: Totals may not equal sum of components due to independent rounding.

Sources: Data sources, estimation procedures, and assumptions are described in Appendix A. 


\section{W Energy Price and Expenditure Estimates by Source, West Virginia \\ E 1970, 1975, 1980, 1985, and 1990-1993}

\begin{tabular}{|c|c|c|c|c|c|c|c|c|}
\hline Energy Source & 1970 & 1975 & 1980 & 1985 & 1990 & 1991 & 1992 & 1993 \\
\hline & \multicolumn{8}{|c|}{ Prices in Dollars per Million Btu } \\
\hline Coal & 0.31 & 1.02 & 1.46 & 1.61 & 1.47 & 1.51 & 1.47 & 1.43 \\
\hline 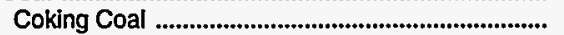 & .40 & 1.51 & 1.86 & 1.93 & 1.80 & 1.72 & 1.74 & 1.68 \\
\hline 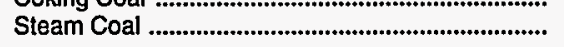 & .28 & .94 & 1.41 & 1.59 & 1.45 & 1.50 & 1.46 & 1.41 \\
\hline Natural Gas & .62 & 1.16 & 3.18 & 5.28 & 4.40 & 4.59 & 4.41 & 4.33 \\
\hline 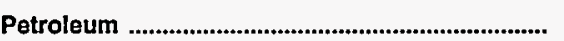 & 1.77 & 3.62 & 8.06 & 8.38 & 8.03 & ${ }^{R} 7.68$ & $\mathrm{R}_{7.54}$ & 7.47 \\
\hline 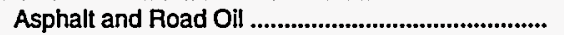 & .68 & 1.81 & 3.58 & 4.91 & 3.07 & 3.11 & 2.35 & 2.90 \\
\hline 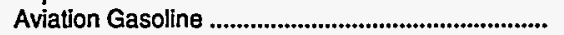 & 2.17 & 3.45 & 9.02 & 9.99 & 9.32 & 8.71 & - & 8.24 \\
\hline Distillate Fuel ......................................................... & 1.40 & 3.36 & 7.24 & 8.01 & 8.04 & 7.16 & 7.38 & 7.16 \\
\hline 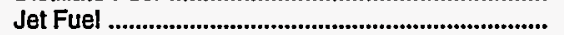 & .73 & 2.05 & 6.46 & 6.87 & 6.41 & 5.58 & 5.31 & 4.19 \\
\hline Kerosene ....................................................................... & 1.49 & 2.83 & 8.19 & 8.10 & 8.59 & 7.62 & 7.28 & 7.18 \\
\hline LPG & 1.67 & 3.25 & 6.16 & 9.02 & 10.79 & 11.12 & $\AA_{10.43}$ & 10.23 \\
\hline 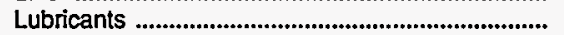 & 5.08 & 7.48 & 14.36 & 17.61 & $R_{14.60}$ & $R_{16.79}$ & R 19.43 & 18.94 \\
\hline 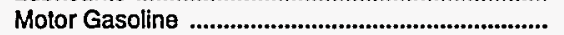 & 2.86 & 4.61 & 9.96 & 9.19 & 9.96 & 9.92 & 9.49 & 9.87 \\
\hline 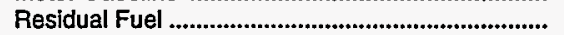 & .58 & 1.89 & 3.33 & 4.01 & 2.68 & 1.92 & 2.18 & 2.41 \\
\hline Other a & .87 & 2.87 & 7.19 & 7.37 & R 5.87 & ${ }^{\mathrm{R}} 5.18$ & $R_{4.93}$ & 4.39 \\
\hline Nuclear Fuel & - & - & - & - & - & - & - & - \\
\hline 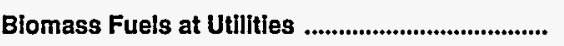 & .65 & - & - & - & - & - & - & - \\
\hline Primary Energy — Five Sectors b ............................ & .68 & 1.58 & 3.16 & 3.22 & 3.13 & 3.17 & $\mathrm{R}_{3.10}$ & 3.08 \\
\hline 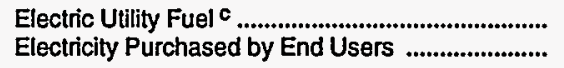 & $\begin{aligned} .26 \\
3.96\end{aligned}$ & $\begin{array}{r}.88 \\
8.30\end{array}$ & $\begin{array}{r}1.43 \\
10.58\end{array}$ & $\begin{array}{r}1.62 \\
14.19\end{array}$ & $\begin{array}{r}1.48 \\
13.90\end{array}$ & $\begin{array}{r}1.53 \\
14.22\end{array}$ & $\begin{array}{r}1.48 \\
14.84\end{array}$ & $\begin{array}{r}1.43 \\
15.34\end{array}$ \\
\hline \multirow[t]{2}{*}{ 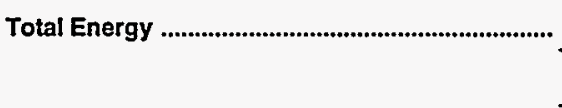 } & 1.13 & 2.79 & 5.78 & 7.34 & 6.71 & 6.84 & ${ }^{\mathrm{R}} 6.88$ & 6.84 \\
\hline & \multicolumn{8}{|c|}{ Expenditures in Millions of Dollars } \\
\hline$\ldots$ & 187.2 & 833.9 & $1,253.8$ & $1,399.0$ & $1,286.3$ & $1,208.8$ & $1,183.9$ & $1,144.8$ \\
\hline 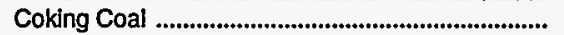 & 55.3 & 178.3 & 190.2 & 72.4 & 93.1 & 86.8 & 75.4 & 79.1 \\
\hline 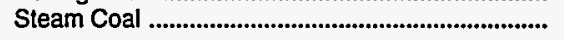 & 131.9 & 655.6 & $1,063.6$ & $1,326.6$ & $1,193.2$ & $1,122.0$ & $1,108.5$ & $1,065.7$ \\
\hline Natural Gas & 108.3 & 171.0 & 415.1 & 510.6 & 471.2 & 454.7 & 478.3 & 500.7 \\
\hline Petroleum .. & 383.5 & 927.6 & $2,380.6$ & $1,950.0$ & $R_{2,144.0}$ & $\mathrm{P}_{2,013.3}$ & $R_{1,981.9}$ & $2,010.1$ \\
\hline 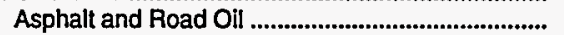 & 3.9 & 11.3 & 17.0 & 14.0 & 14.8 & 10.9 & 8.6 & 8.2 \\
\hline Aviation Gasoline ............................................................. & .9 & 1.0 & 3.0 & 1.9 & 1.7 & 1.5 & - & 1.1 \\
\hline Distillate Fuel ........................................................... & 31.9 & 114.2 & 441.1 & 451.7 & 456.3 & 400.2 & 405.4 & 447.8 \\
\hline 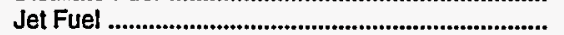 & 1.2 & & 12.9 & 9.0 & 9.8 & 7.3 & 8.0 & 6.0 \\
\hline Kerosene ……………………………………………. & 2.7 & 5.2 & 23.0 & 32.0 & 14.4 & 13.0 & 13.9 & 17.3 \\
\hline LPG & 7.8 & 18.1 & 77.7 & 36.9 & 59.8 & 71.3 & ${ }^{R} 63.0$ & 64.7 \\
\hline 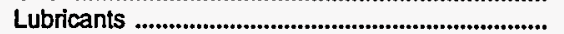 & 21.1 & 31.1 & 58.4 & 65.2 & ${ }^{R} 60.8$ & $R_{62.6}$ & $R_{73.8}$ & 73.2 \\
\hline 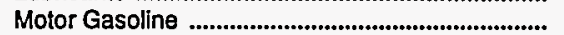 & 237.6 & 467.7 & $1,014.2$ & 894.0 & $1,021.8$ & $1,007.4$ & 989.9 & $1,017.3$ \\
\hline 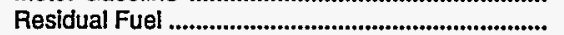 & 7.5 & 26.2 & 24.8 & 22.2 & 18.7 & 9.5 & 6.4 & 6.3 \\
\hline Other ${ }^{a}$ & 69.0 & 250.0 & 708.4 & 423.1 & $R_{485.9}$ & $R_{429.5}$ & $R_{412.9}$ & 368.1 \\
\hline Nuclear Fuel & - & - & - & - & - & - & - & - \\
\hline 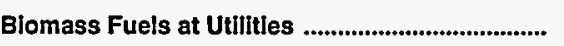 & (8) & - & - & - & - & - & - & - \\
\hline Primary Energy — Five Sectors $b$ & 679.1 & $1,932.5$ & $4,049.5$ & $3,859.5$ & ${ }^{P_{3,901.5}}$ & $\mathrm{R}_{3, \mathbf{6 7 6 . 8}}$ & ${ }^{R} 3,644.1$ & $3,655.6$ \\
\hline 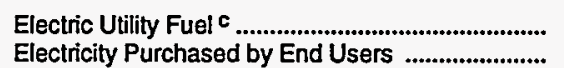 & $\begin{array}{r}-89.9 \\
204.3\end{array}$ & $\begin{array}{r}-531.0 \\
477.3\end{array}$ & $\begin{array}{r}-997.7 \\
748.8\end{array}$ & $\begin{array}{r}-1,261.8 \\
1,000.4\end{array}$ & $\begin{array}{r}-1,108.0 \\
1,088.8\end{array}$ & $\begin{array}{r}-1,056.7 \\
1,133.0\end{array}$ & $\begin{array}{r}-1,043.6 \\
1,192.8\end{array}$ & $\begin{array}{r}-994.2 \\
1,265.7\end{array}$ \\
\hline 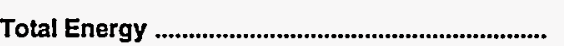 & 793.5 & $1,878.7$ & $3,800.5$ & 3,598.1 & $\mathrm{R}_{3,882.3}$ & $\mathrm{R}_{3,753.1}$ & $\mathrm{~F}_{3,793.3}$ & $3,927.1$ \\
\hline
\end{tabular}

a Includes petroleum coke used at electric utilities.

b Biomass fuels are not included, except those consumed at electric utilities and those added to motor gasoline.

c There are no direct fuel costs for hydroelectric, geothermal, wind, photovoltaic, or solar thermal energy.

$\mathrm{R}=$ Revised data.

$\rightarrow$ No consumption, including cases where adjustments were made. See

explanation of adjustments in Section 6 of Appendix A.

$(s)=V a l u e$ less than 0.05 million dollars.

Note: Expenditure totals may not equal sum of components due to independent rounding.

Sources: Data sources, estimation procedures, and assumptions are described in Appendix A. 


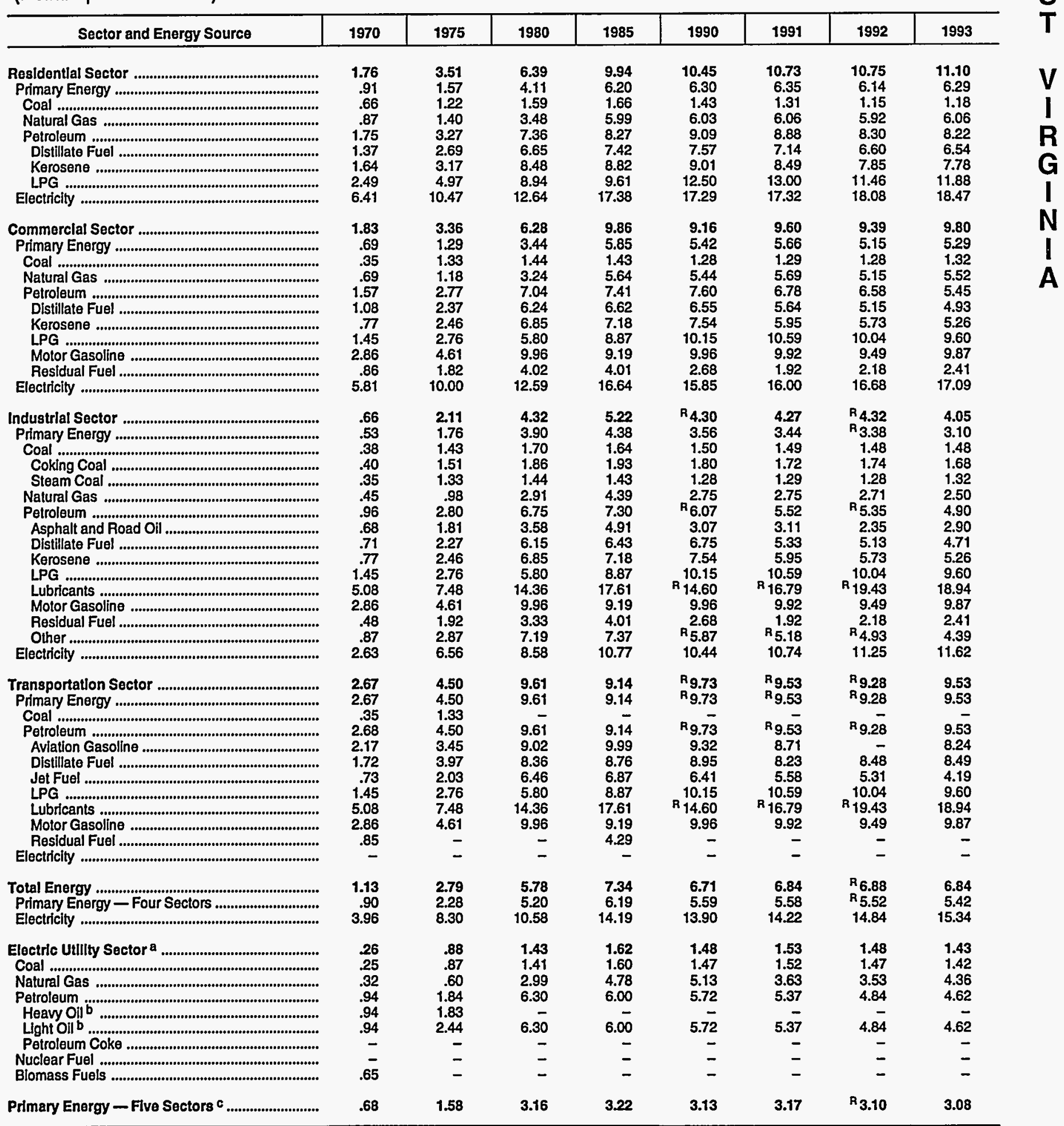

a There are no direct fuel costs for hydroelectric, geothermal, wind, photovoltalc, or solar thermal energy.

beavy oll includes fuel oll nos. 4, 5, and 6, and residual fuel oils. Light oll includes fuel oil nos. 1 and 2, kerosene, and jet fuel.

c Blomass fuels are not included, except those consumed at electric utilitles and those added to motor gasoline.
$R=$ Revised data.

-No consumption, including cases where adjustments were made. See explanation of adjustments in Section 6 of Appendix A.

Sources: Data sources, estimation procedures, and assumptions are described in Appendix A. 
W Energy Expenditure Estimates by Sector, West Virginia

E 1970, 1975, 1980, 1985, and 1990-1993

$\mathbf{S}$ (Million Dollars)

\begin{tabular}{|c|c|c|c|c|c|c|c|c|}
\hline Sector and Energy Source & 1970 & 1975 & 1980 & 1985 & 1980 & 1891 & 1992 & 1893 \\
\hline 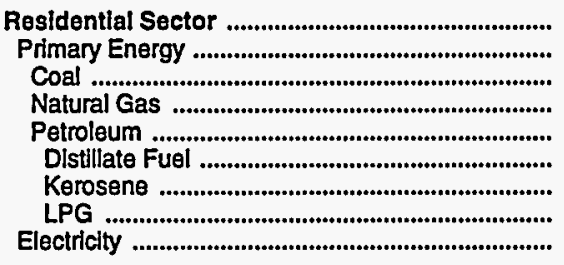 & $\begin{array}{r}135.3 \\
59.7 \\
1.1 \\
51.7 \\
7.0 \\
2.0 \\
2.5 \\
2.5 \\
75.6\end{array}$ & $\begin{array}{r}273.1 \\
95.2 \\
2.4 \\
74.5 \\
18.3 \\
9.1 \\
3.1 \\
6.1 \\
177.9\end{array}$ & $\begin{array}{r}538.5 \\
253.6 \\
2.1 \\
173.6 \\
77.9 \\
45.3 \\
19.6 \\
13.0 \\
284.9\end{array}$ & $\begin{array}{r}681.3 \\
283.2 \\
1.2 \\
234.7 \\
47.3 \\
20.0 \\
19.5 \\
7.8 \\
398.1\end{array}$ & $\begin{array}{r}714.8 \\
267.8 \\
2.2 \\
210.6 \\
54.9 \\
25.3 \\
10.7 \\
18.9 \\
447.0\end{array}$ & $\begin{array}{r}742.3 \\
263.3 \\
1.1 \\
211.8 \\
50.3 \\
22.3 \\
9.5 \\
18.5 \\
479.0\end{array}$ & $\begin{array}{r}773.2 \\
271.2 \\
.9 \\
222.7 \\
47.6 \\
17.8 \\
10.9 \\
18.9 \\
502.0\end{array}$ & $\begin{array}{r}831.8 \\
284.8 \\
1.1 \\
227.1 \\
56.6 \\
21.6 \\
14.3 \\
20.7 \\
547.0\end{array}$ \\
\hline $\begin{array}{l}\text { Commerclal Sector } \\
\text { Primary Energy } \\
\text { Coal . } \\
\text { Natural Gas } \\
\text { Petroleum } \\
\text { Distillate Fuel } \\
\text { Kerosene } \\
\text { LPG } \\
\text { Motor Gasoline } \\
\text { Residual Fuel }\end{array}$ & $\begin{array}{r}62.5 \\
18.1 \\
1.0 \\
15.3 \\
1.8 \\
.6 \\
.1 \\
.3 \\
.8 \\
(\mathrm{~s}) \\
44.4\end{array}$ & $\begin{array}{r}137.8 \\
40.3 \\
4.9 \\
30.2 \\
5.2 \\
2.9 \\
.1 \\
.6 \\
1.4 \\
.1 \\
97.5\end{array}$ & $\begin{array}{r}252.4 \\
95.2 \\
3.5 \\
73.4 \\
18.3 \\
9.5 \\
1.4 \\
1.5 \\
5.7 \\
.1 \\
157.1\end{array}$ & $\begin{array}{r}403.6 \\
150.2 \\
1.8 \\
103.7 \\
44.7 \\
23.3 \\
5.2 \\
1.3 \\
14.8 \\
.1 \\
253.4\end{array}$ & $\begin{array}{r}443.3 \\
168.3 \\
3.7 \\
124.7 \\
39.9 \\
16.9 \\
2.0 \\
2.7 \\
17.2 \\
1.1 \\
275.0\end{array}$ & $\begin{array}{r}456.7 \\
166.7 \\
2.0 \\
128.6 \\
36.1 \\
17.0 \\
2.2 \\
2.7 \\
13.7 \\
.6 \\
290.0\end{array}$ & $\begin{array}{r}463.8 \\
160.8 \\
1.7 \\
133.8 \\
25.3 \\
9.6 \\
1.0 \\
2.9 \\
10.9 \\
.8 \\
303.0\end{array}$ & $\begin{array}{r}488.1 \\
163.1 \\
2.1 \\
143.1 \\
17.9 \\
12.5 \\
1.1 \\
3.0 \\
1.0 \\
.3 \\
325.0\end{array}$ \\
\hline $\begin{array}{l}\text { Industrial Sector } \\
\text { Primary Energy } \\
\text { Coal } \\
\text { Coking Coal } \\
\text { Steam Coal }\end{array}$ & $\begin{array}{r}327.8 \\
243.5 \\
97.9 \\
55.3 \\
42.6 \\
41.2 \\
104.5 \\
3.9 \\
4.5 \\
.2 \\
5.0 \\
15.4 \\
1.7 \\
4.8 \\
69.0 \\
84.3\end{array}$ & $\begin{array}{r}805.7 \\
703.8 \\
304.0 \\
178.3 \\
125.7 \\
66.1 \\
333.7 \\
11.3 \\
19.1 \\
2.0 \\
11.2 \\
20.3 \\
1.9 \\
17.9 \\
250.0 \\
201.9\end{array}$ & $\begin{array}{r}1,731.5 \\
1,424.8 \\
275.7 \\
190.2 \\
85.6 \\
167.9 \\
981.2 \\
17.0 \\
125.3 \\
2.0 \\
62.9 \\
36.6 \\
4.3 \\
24.7 \\
708.4 \\
306.7\end{array}$ & $\begin{array}{r}1,283.3 \\
934.4 \\
147.6 \\
72.4 \\
75.2 \\
171.6 \\
615.1 \\
14.0 \\
69.7 \\
7.2 \\
27.2 \\
40.9 \\
11.1 \\
22.1 \\
423.1 \\
348.9\end{array}$ & $\begin{array}{r}R_{1,400.2} \\
R_{1,033.4} \\
185.3 \\
93.1 \\
92.2 \\
135.2 \\
R_{712.9} \\
14.8 \\
104.3 \\
1.7 \\
37.5 \\
R_{38.1} \\
13.0 \\
17.6 \\
R_{485.9} \\
366.8\end{array}$ & $\begin{array}{r}R_{1,270.1} \\
R_{906.1} \\
160.1 \\
86.8 \\
73.4 \\
113.8 \\
R_{632.1} \\
10.9 \\
79.2 \\
1.3 \\
49.5 \\
R_{39.2} \\
13.5 \\
8.9 \\
R_{429.5} \\
364.0\end{array}$ & $\begin{array}{r}R_{1,248.6} \\
R_{860.9} \\
147.0 \\
75.4 \\
71.6 \\
121.0 \\
\text { P }_{592.8} \\
8.6 \\
64.6 \\
1.9 \\
R_{40.4} \\
\text { P }_{46.3} \\
12.4 \\
5.7 \\
\text { R }_{412.9} \\
387.8\end{array}$ & $\begin{array}{r}1,234.3 \\
840.6 \\
157.6 \\
79.1 \\
78.4 \\
129.9 \\
553.1 \\
8.2 \\
74.3 \\
1.9 \\
40.3 \\
45.9 \\
8.3 \\
6.0 \\
368.1 \\
393.7\end{array}$ \\
\hline 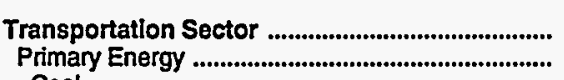 & $\begin{array}{l}267.9 \\
267.9\end{array}$ & 562.1 & $\begin{array}{l}1,278.1 \\
1,278.1\end{array}$ & $\begin{array}{l}1,229.9 \\
1,229.9\end{array}$ & $\begin{array}{l}A_{1,324.1} \\
A_{1,324.1}\end{array}$ & $\begin{array}{l}F_{1,284.1} \\
F_{1,284.1}\end{array}$ & $\begin{array}{l}R_{1,307.6} \\
R_{1,307.6}\end{array}$ & $\begin{array}{l}1,372.8 \\
1,372.8\end{array}$ \\
\hline Coal & 267.7 & $\begin{aligned}(s) \\
562.1\end{aligned}$ & $1,278.1$ & $1,229.9$ & $\mathrm{R}_{1,324.1}$ & $\mathrm{R}_{1,284.1}$ & $R_{1,307.6}$ & $1,37 \overline{2.8}$ \\
\hline 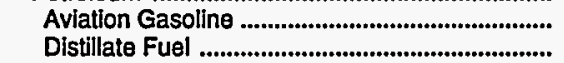 & $\begin{array}{r}.9 \\
24.8\end{array}$ & $\begin{array}{r}1.0 \\
83.0\end{array}$ & $\begin{array}{r}3.0 \\
236.1\end{array}$ & $\begin{array}{r}1.9 \\
325.9\end{array}$ & $\begin{array}{r}1.7 \\
297.6\end{array}$ & $\begin{array}{r}1.5 \\
271.0\end{array}$ & $304 . \overline{7}$ & $\begin{array}{r}1.1 \\
329.8\end{array}$ \\
\hline 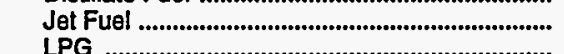 & 1.2 & 2.7 & 12.8 & 9.0 & 9.8 & 7.3 & 8.0 & 6.0 \\
\hline 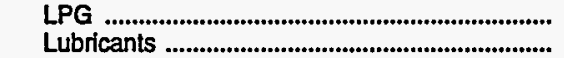 & & $\begin{array}{r}.1 \\
10.9\end{array}$ & $\begin{array}{r}.3 \\
21.8\end{array}$ & 24.3 & $\mathrm{R}_{22.7}^{.7}$ & $R_{23.3}^{.7}$ & $R_{27.5} .8$ & $\begin{array}{r}.7 \\
27.3\end{array}$ \\
\hline 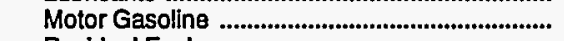 & 235.1 & 464.3 & $1,004.2$ & 868.1 & 991.6 & 980.3 & 966.6 & $1,008.0$ \\
\hline 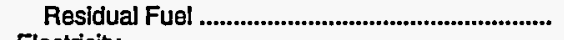 & (s) & - & - & (s) & - & - & - & - \\
\hline 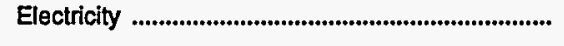 & 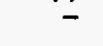 & - & - & $\because$ & - & - & - & - \\
\hline 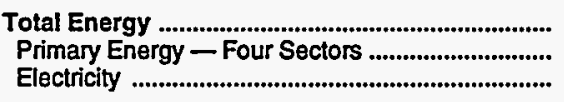 & $\begin{array}{l}793.5 \\
589.2 \\
204.3\end{array}$ & $\begin{array}{r}1,878.7 \\
1,401.5 \\
477.3\end{array}$ & $\begin{array}{r}3,800.5 \\
3,051.8 \\
748.8\end{array}$ & $\begin{array}{l}3,598.1 \\
2,597.7 \\
1,000.4\end{array}$ & $\begin{array}{r}R_{3,882.3} \\
R_{2,793.5} \\
1,088.8\end{array}$ & $\begin{array}{r}R_{3,753.1} \\
R_{2,620.1} \\
1,133.0\end{array}$ & $\begin{array}{r}R_{3,793.3} \\
R_{2,600.5} \\
1,192.8\end{array}$ & $\begin{array}{l}3,927.1 \\
2,661.4 \\
1,265.7\end{array}$ \\
\hline $\begin{array}{l}\text { Eloctric Utility Sector }{ }^{a} \\
\text { Coal }\end{array}$ & $\begin{array}{l}89.9 \\
87.1\end{array}$ & $\begin{array}{l}531.0 \\
522.5\end{array}$ & $\begin{array}{l}997.7 \\
972.5\end{array}$ & $\begin{array}{l}1,261.8 \\
1,248.3\end{array}$ & $\begin{array}{l}1,108.0 \\
1,095.1\end{array}$ & $\begin{array}{l}1,056.7 \\
1,045.6\end{array}$ & $\begin{array}{l}1,043.6 \\
1,034.2\end{array}$ & $\begin{array}{l}994.2 \\
984.0\end{array}$ \\
\hline Natural Gas & .2 & .1 & 2 & .6 & .7 & .5 & .7 & .6 \\
\hline Petroleum & 2.6 & 8.3 & 25.1 & 12.9 & 12.3 & 10.6 & 8.7 & 9.6 \\
\hline $\begin{array}{l}\text { Heavy Oild } \\
\text { Light Oil }\end{array}$ & $\begin{array}{l}2.5 \\
(\mathrm{~s})\end{array}$ & $\begin{array}{r}8.2 \\
.2\end{array}$ & $25 . \overline{1}$ & $12 \overline{9}$ & $12 . \overline{3}$ & $10 . \overline{6}$ & $8 . \overline{7}$ & $9 . \overline{6}$ \\
\hline 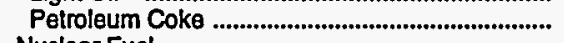 & - & - & - & - & - & - & - & - \\
\hline 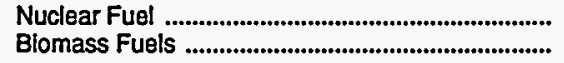 & $\overrightarrow{(s)}$ & $\overline{-}$ & $\overline{-}$ & $\overline{-}$ & $\overline{-}$ & - & $\overline{-}$ & $\overline{-}$ \\
\hline Primary Energy — Five Sectors c ........................... & 679.1 & $1,932.5$ & $4,049.5$ & $3,859.5$ & $\mathrm{~A}_{3,801.5}$ & $R_{3,676.8}$ & $R_{3,644.1}$ & $3,655.6$ \\
\hline
\end{tabular}

a There are no direct fuel costs for hydroelectric, geothermal, wind, photovoltalc, or solar thermal energy.

beavy oil includes fuel oil nos. 4, 5, and 6, and residual fuel oils. Light oil includes fuel nos. 1 and 2, kerosene, and jet fuel.

c Biomass fuels are not inciuded, except those consumed at electric utilities and those added to motor gasoline.

$\mathrm{R}=$ Revised data.

-No consumption, including cases where adjustments were made. See explanation of adjustments in Section 6 of Appendix A.

(s) $=$ Value less than 0.05 million dollars.

Note: Totals may not equal sum of components due to independent rounding.

Sources: Data sources, estimation procedures, and assumptions are described in Appendix A. 


\begin{tabular}{|c|c|c|c|c|c|c|c|c|}
\hline Energy Source & 1970 & 1975 & 1980 & 1985 & 1990 & 1991 & 1992 & 1993 \\
\hline & \multicolumn{8}{|c|}{ Prices in Dollars per Million Btu } \\
\hline 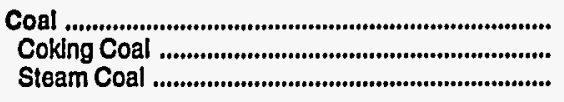 & $\begin{array}{r}0.51 \\
.53 \\
.51\end{array}$ & $\begin{array}{l}1.05 \\
1.80 \\
1.03\end{array}$ & $\begin{array}{l}1.44 \\
2.27 \\
1.43\end{array}$ & $\begin{array}{l}1.76 \\
2.08 \\
1.76\end{array}$ & $\frac{1.41}{1 . \overline{41}}$ & $1 . \overline{41}$ & $1 . \overline{38}$ & $\begin{array}{r}1.27 \\
1.27\end{array}$ \\
\hline 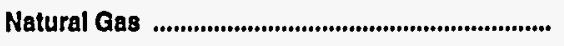 & .79 & 1.30 & 3.43 & 5.37 & 4.56 & 4.40 & 4.60 & 4.92 \\
\hline $\begin{array}{l}\text { Potroloum } \\
\text { Asphalt and Road Oll } \\
\text { Avlatlon Gasollne } \\
\text { Dlstillate Fuel } \\
\text { Jet Fuel }\end{array}$ & $\begin{array}{r}1.89 \\
.76 \\
2.17 \\
1.07 \\
.74 \\
1.17 \\
1.88 \\
5.08 \\
2.65 \\
.57 \\
1.65\end{array}$ & $\begin{array}{l}3.65 \\
2.05 \\
3.45 \\
2.47 \\
2.08 \\
2.71 \\
3.60 \\
7.48 \\
4.54 \\
1.79 \\
3.21\end{array}$ & $\begin{array}{r}8.13 \\
3.85 \\
9.02 \\
6.59 \\
6.38 \\
7.05 \\
6.51 \\
14.36 \\
9.43 \\
3.48 \\
8.73\end{array}$ & $\begin{array}{r}8.68 \\
4.87 \\
9.99 \\
7.66 \\
6.19 \\
8.20 \\
8.55 \\
17.61 \\
9.33 \\
4.59 \\
8.73\end{array}$ & $\begin{aligned} & 8.52 \\
& 3.13 \\
& 9.32 \\
& 7.81 \\
& 5.99 \\
& 7.68 \\
& 9.78 \\
& 14.60 \\
& 9.38 \\
& 9.41 \\
& 27.76\end{aligned}$ & $\begin{array}{r}R 8.23 \\
3.10 \\
8.71 \\
7.21 \\
5.26 \\
7.11 \\
8.42 \\
R_{16.79} \\
9.18 \\
2.35 \\
R 7.35\end{array}$ & $\begin{array}{r}R 8.03 \\
2.59 \\
8.54 \\
6.99 \\
4.64 \\
6.48 \\
7.97 \\
R 19.43 \\
8.94 \\
2.23 \\
R 7.99\end{array}$ & $\begin{array}{r}8.03 \\
3.19 \\
8.24 \\
6.98 \\
4.26 \\
6.45 \\
8.63 \\
18.94 \\
8.97 \\
2.50 \\
6.89\end{array}$ \\
\hline 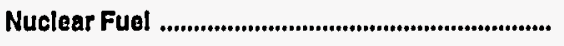 & .15 & .32 & .47 & .68 & .48 & .45 & .40 & .40 \\
\hline Blomass Fuels at Utilitles ........................................... & .65 & - & 1.74 & .79 & .68 & .73 & ค. .76 & .69 \\
\hline Primary Energy - Flve Sectors b ............................ & 1.16 & 2.12 & 4.42 & 5.01 & $\mathrm{P}_{4.64}$ & $\mathrm{R}_{4.50}$ & 4.47 & 4.56 \\
\hline $\begin{array}{l}\text { Electric Uttllty Fuel }{ }^{c} \\
\text { Electirelty Purchased by End Users }\end{array}$ & $\begin{array}{r}.39 \\
6.02\end{array}$ & $\begin{array}{r}.71 \\
8.88\end{array}$ & $\begin{array}{r}1.25 \\
13.34\end{array}$ & $\begin{array}{r}1.42 \\
16.87\end{array}$ & $\begin{array}{r}1.15 \\
15.77\end{array}$ & $\begin{array}{r}1.15 \\
16.01\end{array}$ & $\begin{array}{r}R_{1.11} \\
16.12\end{array}$ & $\begin{array}{r}1.02 \\
16.24\end{array}$ \\
\hline \multirow[t]{2}{*}{ 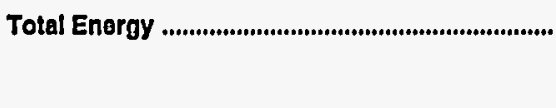 } & 1.75 & 3.28 & 6.85 & 8.61 & 8.22 & ${ }^{R_{8.08}}$ & 8.04 & 8.18 \\
\hline & \multicolumn{8}{|c|}{ Expendilures in Mlilions of Dollars } \\
\hline 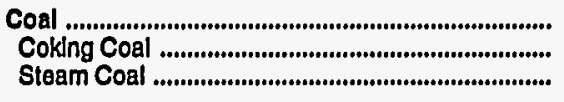 & $\begin{array}{r}198.2 \\
5.0 \\
191.2\end{array}$ & $\begin{array}{r}285.6 \\
12.0 \\
273.6\end{array}$ & $\begin{array}{r}472.2 \\
12.3 \\
459.9\end{array}$ & $\begin{array}{r}636.0 \\
.1 \\
635.9\end{array}$ & $\begin{array}{c}560.2 \\
\overline{560.2}\end{array}$ & $\begin{array}{l}573.5 \\
- \\
573.5\end{array}$ & $\begin{array}{c}550.5 \\
- \\
550.5\end{array}$ & $\begin{array}{l}513.5 \\
\overline{513.5}\end{array}$ \\
\hline Natural Gas & 267.1 & 474.2 & $1,184.8$ & $1,634.5$ & $1,371.6$ & $1,420.9$ & $1,494.7$ & $1,680.8$ \\
\hline $\begin{array}{l}\text { Petroloum } \\
\text { Asphalt and Road Oll } \\
\text { Avlation Gasollne } \\
\text { Dlstlllate Fuel } \\
\text { Jet Fuel }\end{array}$ & $\begin{array}{r}952.8 \\
23.6 \\
3.6 \\
161.6 \\
6.7 \\
19.9 \\
54.3 \\
30.6 \\
633.6 \\
8.8 \\
10.0\end{array}$ & $\begin{array}{r}1,898.3 \\
41.1 \\
3.0 \\
382.3 \\
26.0 \\
15.0 \\
112.5 \\
41.9 \\
1,230.6 \\
19.3 \\
24.5\end{array}$ & \begin{tabular}{r|}
$3,832.2$ \\
77.1 \\
5.6 \\
863.2 \\
86.1 \\
8.9 \\
143.2 \\
88.8 \\
$2,457.8$ \\
27.6 \\
73.8
\end{tabular} & $\begin{array}{r}3,776.2 \\
54.6 \\
5.1 \\
1,007.8 \\
57.8 \\
10.9 \\
164.3 \\
99.1 \\
2,280.9 \\
9.3 \\
86.4\end{array}$ & $\begin{array}{r}R_{3,978.7} \\
76.4 \\
5.7 \\
1,048.6 \\
47.9 \\
2.1 \\
233.6 \\
R^{2} 92.4 \\
2,390.6 \\
13.2 \\
{ }^{8} 68.3\end{array}$ & $\begin{array}{r}\text { P }_{3,909.1} \\
68.5 \\
4.6 \\
966.2 \\
39.8 \\
2.0 \\
255.9 \\
R^{R} 95.1 \\
2,405.7 \\
8.6 \\
R 62.7\end{array}$ & $\begin{array}{r}\text { R }_{3,813.1} \\
53.3 \\
5.2 \\
926.8 \\
44.9 \\
1.9 \\
223.5 \\
\text { R } 112.2 \\
2,361.7 \\
8.7 \\
\text { R } 74.9\end{array}$ & $\begin{array}{r}4,010.0 \\
68.9 \\
5.0 \\
995.2 \\
45.9 \\
2.8 \\
265.8 \\
111.3 \\
2,433.5 \\
13.8 \\
67.7\end{array}$ \\
\hline 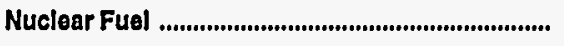 & .3 . & 36.6 & 50.3 & 69.2 & 57.9 & 53.4 & 47.5 & 48.5 \\
\hline Blomass Fuels at Uttlitles ............................................ & .1 & - & 1.1 & .7 & 1.2 & 1.2 & $R_{1.2}$ & 1.6 \\
\hline Primary Energy - Flve Sectors b .............................. & $1,416.4$ & $2,692.7$ & $5,540.7$ & $6,116.6$ & $R_{5,969.7}$ & ${ }^{R_{5}, 958.1}$ & ${ }^{R_{5}, 906.9}$ & $6,254.3$ \\
\hline 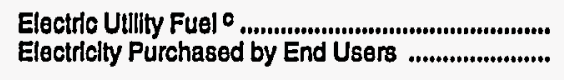 & $\begin{array}{r}-109.2 \\
501.0\end{array}$ & $\begin{array}{r}-245.2 \\
932.2\end{array}$ & $\begin{array}{r}-494.9 \\
1,669.5\end{array}$ & $\begin{array}{r}-612.9 \\
2,601.0\end{array}$ & $\begin{array}{r}-544.4 \\
2,620.3\end{array}$ & $\begin{array}{r}-557.3 \\
2,760.5\end{array}$ & $\begin{array}{r}-530.0 \\
2,772.0\end{array}$ & $\begin{array}{r}-498.7 \\
2,913.7\end{array}$ \\
\hline 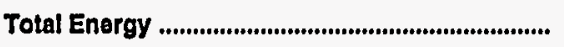 & $1,808.2$ & $3,379.7$ & $6,715.3$ & $8,104.7$ & $R_{8,045.6}$ & $R_{8,161.2}$ & ${ }^{R_{8,148.9}}$ & $8,669.3$ \\
\hline
\end{tabular}

a Includes petroleum coke used at electric utlittes.

b Blomass fuels are not included, except those consumed at electric uttllites and those added to motor gasoline.

- There are no direct fuel costs for hydroelectric, geothermal, wind, photovoltalc, or solar thermal energy.

$R=$ Revised data.

- No consumptlon, Including cases where adjustments were made. See explanation of adjustments in Section 6 of Appendix A.

Note: Expenditure totals may not equal sum of components due to independent rounding.

Sources: Data sources, estimation procedures, and assumptions are described in Appendix A. 
W Energy Price Estimates by Sector, Wisconsin

I 1970, 1975, 1980, 1985, and 1990-1993

S (Dollars per Million Btu)

\begin{tabular}{|c|c|c|c|c|c|c|c|c|}
\hline Sector and Energy Source & 1970 & 1975 & 1980 & 1985 & 1990 & 1991 & 1992 & 1993 \\
\hline 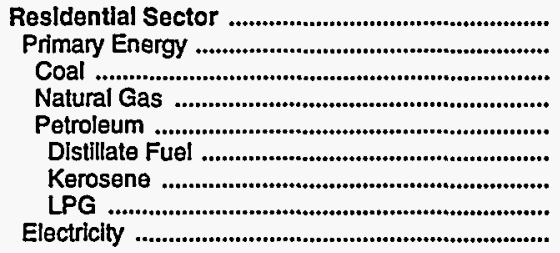 & $\begin{array}{l}2.06 \\
1.33 \\
1.63 \\
1.22 \\
1.42 \\
1.21 \\
1.47 \\
2.07 \\
6.75\end{array}$ & $\begin{array}{r}3.48 \\
2.24 \\
3.10 \\
1.71 \\
2.94 \\
2.57 \\
2.97 \\
4.15 \\
10.04\end{array}$ & $\begin{array}{r}6.85 \\
4.78 \\
3.92 \\
3.81 \\
6.82 \\
6.60 \\
8.11 \\
7.69 \\
15.04\end{array}$ & $\begin{array}{r}10.03 \\
6.80 \\
4.26 \\
6.41 \\
7.75 \\
7.44 \\
8.45 \\
8.72 \\
19.73\end{array}$ & $\begin{array}{r}9.80 \\
6.37 \\
3.37 \\
5.71 \\
8.17 \\
7.13 \\
8.09 \\
10.03 \\
19.44\end{array}$ & $\begin{array}{r}9.59 \\
6.14 \\
3.43 \\
5.57 \\
7.60 \\
6.78 \\
7.69 \\
8.91 \\
19.73\end{array}$ & $\begin{array}{r}9.64 \\
6.11 \\
3.41 \\
5.82 \\
6.90 \\
6.19 \\
7.03 \\
7.99 \\
20.25\end{array}$ & $\begin{array}{r}9.98 \\
6.52 \\
3.35 \\
6.27 \\
7.18 \\
6.23 \\
7.07 \\
8.59 \\
20.62\end{array}$ \\
\hline 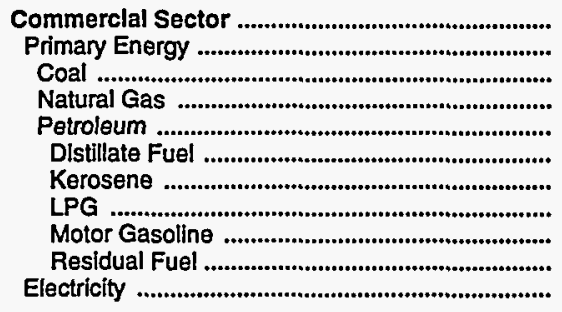 & $\begin{array}{r}2.05 \\
.84 \\
.66 \\
.82 \\
1.08 \\
1.04 \\
.83 \\
1.35 \\
2.65 \\
.59 \\
7.28\end{array}$ & $\begin{array}{r}3.55 \\
1.50 \\
1.51 \\
1.29 \\
2.43 \\
2.39 \\
2.41 \\
2.63 \\
4.54 \\
1.66 \\
10.13\end{array}$ & $\begin{array}{r}6.92 \\
3.80 \\
1.47 \\
3.43 \\
6.21 \\
6.30 \\
5.72 \\
5.33 \\
9.43 \\
4.31 \\
15.25\end{array}$ & $\begin{array}{r}9.51 \\
5.50 \\
2.11 \\
5.14 \\
6.73 \\
6.43 \\
6.98 \\
8.32 \\
9.33 \\
4.50 \\
18.90\end{array}$ & $\begin{array}{r}9.29 \\
5.04 \\
1.80 \\
4.72 \\
6.36 \\
5.65 \\
7.06 \\
9.36 \\
9.38 \\
2.41 \\
17.03\end{array}$ & $\begin{array}{r}9.09 \\
4.77 \\
1.78 \\
4.59 \\
5.57 \\
4.88 \\
6.17 \\
7.61 \\
9.18 \\
2.35 \\
17.17\end{array}$ & $\begin{array}{r}9.34 \\
4.90 \\
1.74 \\
4.77 \\
5.56 \\
4.85 \\
5.76 \\
7.93 \\
8.94 \\
2.23 \\
17.46\end{array}$ & $\begin{array}{r}9.45 \\
5.16 \\
1.70 \\
5.10 \\
5.61 \\
4.72 \\
5.45 \\
8.70 \\
8.97 \\
2.50 \\
17.56\end{array}$ \\
\hline 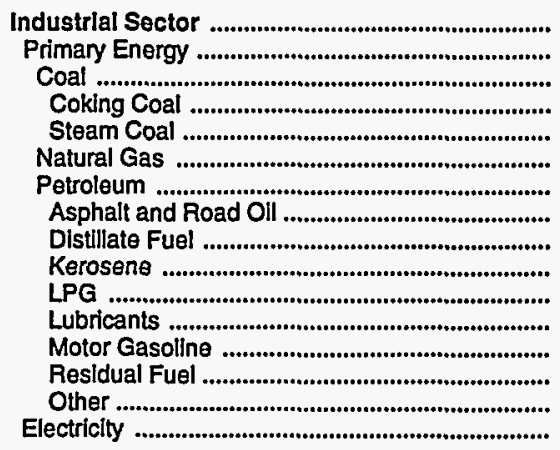 & $\begin{array}{r}1.00 \\
.76 \\
.65 \\
.53 \\
.66 \\
.54 \\
1.14 \\
.76 \\
.76 \\
.83 \\
1.35 \\
5.08 \\
2.65 \\
.57 \\
2.05 \\
4.23\end{array}$ & $\begin{array}{l}2.18 \\
1.65 \\
1.55 \\
1.80 \\
1.51 \\
1.03 \\
2.70 \\
2.05 \\
2.23 \\
2.41 \\
2.63 \\
7.48 \\
4.54 \\
2.06 \\
3.29 \\
6.63\end{array}$ & $\begin{array}{r}4.56 \\
3.61 \\
1.55 \\
2.27 \\
1.47 \\
3.12 \\
5.95 \\
3.85 \\
5.18 \\
5.72 \\
5.33 \\
14.36 \\
9.43 \\
3.31 \\
8.78 \\
10.10\end{array}$ & $\begin{array}{r}6.35 \\
4.69 \\
2.11 \\
2.08 \\
2.11 \\
4.44 \\
7.57 \\
4.87 \\
6.30 \\
6.98 \\
8.32 \\
17.61 \\
9.33 \\
4.50 \\
8.84 \\
12.64\end{array}$ & $\begin{array}{r}R_{5.53} \\
R_{3.84} \\
1.80 \\
- \\
1.80 \\
3.37 \\
R_{5.97} \\
3.13 \\
6.37 \\
7.06 \\
9.36 \\
R_{14.60} \\
9.38 \\
2.41 \\
R 7.76 \\
11.69\end{array}$ & $\begin{array}{r}R_{5.42} \\
R_{3.69} \\
1.78 \\
- \\
1.78 \\
3.14 \\
R_{5.81} \\
3.10 \\
5.56 \\
6.17 \\
7.61 \\
{ }^{\prime} 16.79 \\
9.18 \\
2.35 \\
\text { R } 7.35 \\
11.81\end{array}$ & $\begin{array}{r}\text { R5.51 } \\
\text { R } 3.76 \\
1.74 \\
- \\
1.74 \\
3.35 \\
\text { R } 5.77 \\
2.59 \\
5.19 \\
5.76 \\
7.93 \\
\text { R } 19.43 \\
8.94 \\
2.23 \\
\text { R } 8.19 \\
11.72\end{array}$ & $\begin{array}{r}5.59 \\
3.85 \\
1.70 \\
- \\
1.70 \\
3.48 \\
5.67 \\
3.19 \\
4.92 \\
5.45 \\
8.70 \\
18.94 \\
8.97 \\
2.50 \\
7.37 \\
11.68\end{array}$ \\
\hline 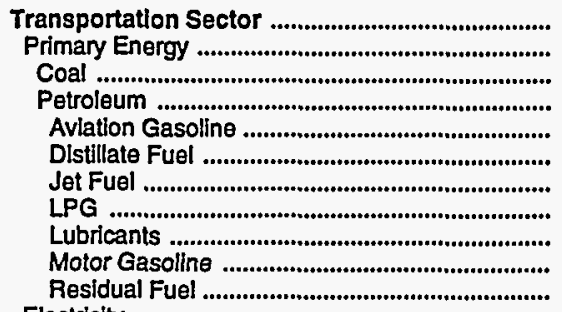 & $\begin{array}{r}2.49 \\
2.49 \\
.66 \\
2.49 \\
2.17 \\
1.33 \\
.74 \\
1.35 \\
5.08 \\
2.65 \\
.55\end{array}$ & $\begin{array}{l}4.24 \\
4.24 \\
1.51 \\
4.24 \\
3.45 \\
2.62 \\
2.08 \\
2.63 \\
7.48 \\
4.54 \\
1.44\end{array}$ & $\begin{array}{r}8.99 \\
8.99 \\
-\overline{8.99} \\
9.02 \\
7.28 \\
6.38 \\
5.33 \\
14.36 \\
9.43 \\
3.80\end{array}$ & $\begin{array}{r}9.18 \\
9.18 \\
- \\
9.18 \\
9.99 \\
8.69 \\
6.19 \\
8.32 \\
17.61 \\
9.33 \\
4.71\end{array}$ & $\begin{array}{r}R_{9.22} \\
R_{9.22} \\
R_{9.22} \\
9.32 \\
8.79 \\
5.99 \\
9.36 \\
R_{14.60} \\
9.38 \\
2.80\end{array}$ & $\begin{array}{r}R_{9.00} \\
R_{9.00} \\
R_{9.00} \\
8.71 \\
8.40 \\
5.26 \\
7.61 \\
R_{16.79} \\
9.18 \\
2.29\end{array}$ & $\begin{array}{r}{ }^{P_{8.75}} \\
\mathrm{P}_{8.75} \\
\mathrm{P}_{8.75} \\
8.54 \\
8.21 \\
4.64 \\
7.93 \\
\mathrm{~A}_{19.43} \\
8.94 \\
\mathrm{P}_{2.41}\end{array}$ & $\begin{array}{r}8.78 \\
8.78 \\
8.78 \\
8.24 \\
8.34 \\
4.26 \\
8.70 \\
18.94 \\
8.97 \\
2.42\end{array}$ \\
\hline Electricity & - & - & - & - & - & - & - & - \\
\hline 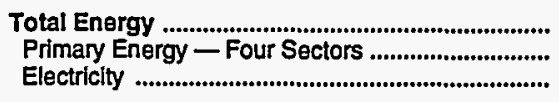 & $\begin{array}{l}1.75 \\
1.38 \\
6.02\end{array}$ & $\begin{array}{l}3.28 \\
2.65 \\
8.88\end{array}$ & $\begin{array}{r}6.85 \\
5.90 \\
13.34\end{array}$ & $\begin{array}{r}8.61 \\
6.99 \\
16.87\end{array}$ & $\begin{array}{r}8.22 \\
{ }^{8} 6.68 \\
15.77\end{array}$ & $\begin{array}{l}R_{8.06} \\
R_{6.43} \\
16.01\end{array}$ & $\begin{array}{r}8.04 \\
{ }_{6}^{6.39} \\
16.12\end{array}$ & $\begin{array}{r}8.18 \\
6.54 \\
16.24\end{array}$ \\
\hline 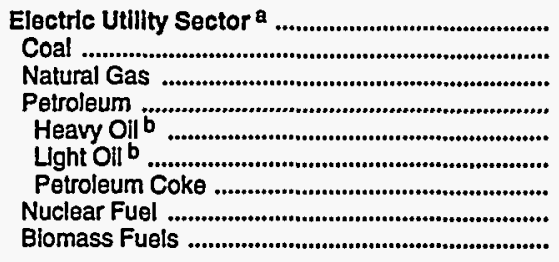 & $\begin{array}{l}.39 \\
.39 \\
.42 \\
.54 \\
.56 \\
.67 \\
.36 \\
.15 \\
.65\end{array}$ & $\begin{array}{r}.71 \\
.86 \\
.82 \\
1.93 \\
1.65 \\
2.30 \\
.72 \\
.32 \\
-\end{array}$ & $\begin{array}{r}1.25 \\
1.42 \\
2.94 \\
5.35 \\
4.28 \\
5.58 \\
1.17 \\
.47 \\
1.74\end{array}$ & $\begin{array}{r}1.42 \\
1.71 \\
4.11 \\
5.12 \\
-\overline{8} \\
5.48 \\
1.38 \\
.58 \\
.79\end{array}$ & $\begin{array}{r}1.15 \\
1.36 \\
2.93 \\
5.26 \\
-\overline{6} \\
5.26 \\
\overline{8} \\
.68\end{array}$ & $\begin{array}{r}1.15 \\
1.36 \\
2.70 \\
4.46 \\
\overline{46} \\
4.46 \\
\overline{.45} \\
.73\end{array}$ & $\begin{array}{r}R_{1.11} \\
1.33 \\
2.40 \\
3.31 \\
- \\
4.64 \\
.83 \\
.40 \\
\text { R..76 }\end{array}$ & $\begin{array}{r}1.02 \\
1.21 \\
2.63 \\
2.26 \\
-0 \\
4.09 \\
.28 \\
.40 \\
.69\end{array}$ \\
\hline Primary Energy - Flve Sectors c ......................... & 1.16 & 2.12 & 4.42 & 5.01 & $R_{4.64}$ & $\mathrm{P}_{\mathbf{4 . 5 0}}$ & 4.47 & 4.56 \\
\hline
\end{tabular}

a There are no direct fuel costs for hydroelectric, geothermal, wind, photovoltalc, or solar thermal energy

b Heavy oil includes fuel oil nos. 4, 5, and 6, and residual fuel oils. Uight oll includes fuel oil nos. 1 and 2, kerosene, and jet fuel.

c Blomass fuels are not included, except those consumed at electric

R=Revised data

- No consumption, including cases where adjustments were made. See explanation of adjustments in Section 6 of Appendix A.

Sources: Data sources, estimation procedures, and assumptions are utilitios and those added to motor gasoline. 


\begin{tabular}{|c|c|c|c|c|c|c|c|c|}
\hline Sector and Energy Source & 1970 & 1975 & 1980 & 1985 & 1990 & 1991 & 1992 & 1993 \\
\hline 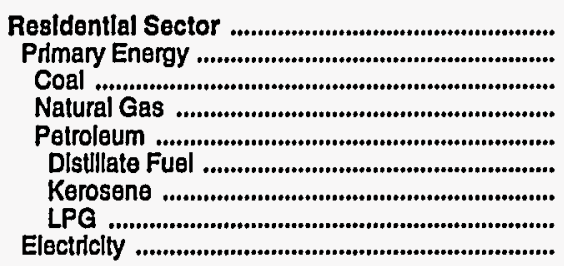 & $\begin{array}{r}512.5 \\
286.3 \\
15.5 \\
131.2 \\
139.5 \\
82.3 \\
13.4 \\
43.9 \\
226.2\end{array}$ & $\begin{array}{r}882.0 \\
478.4 \\
11.9 \\
209.5 \\
257.0 \\
164.8 \\
8.9 \\
83.2 \\
403.6\end{array}$ & $\begin{array}{r}1,575.9 \\
878.3 \\
1.7 \\
473.2 \\
403.4 \\
313.4 \\
5.7 \\
84.3 \\
697.6\end{array}$ & \begin{tabular}{r|}
$2,233.8$ \\
$1,136.1$ \\
1.0 \\
751.6 \\
383.5 \\
278.4 \\
9.3 \\
95.7 \\
$1,097.7$
\end{tabular} & $\begin{array}{r}2,087.9 \\
1,000.9 \\
.2 \\
654.6 \\
346.0 \\
192.5 \\
1.3 \\
152.2 \\
1,087.0\end{array}$ & $\begin{array}{r}2,236.9 \\
1,068.8 \\
.3 \\
696.1 \\
372.4 \\
202.4 \\
1.3 \\
168.7 \\
1,168.0\end{array}$ & $\begin{array}{r}2,188.5 \\
1,040.5 \\
.1 \\
724.4 \\
316.0 \\
171.5 \\
1.2 \\
143.4 \\
1,148.0\end{array}$ & $\begin{array}{r}2,409.1 \\
1,187.0 \\
1.1 \\
825.0 \\
360.9 \\
186.3 \\
1.9 \\
172.8 \\
1,222.0\end{array}$ \\
\hline 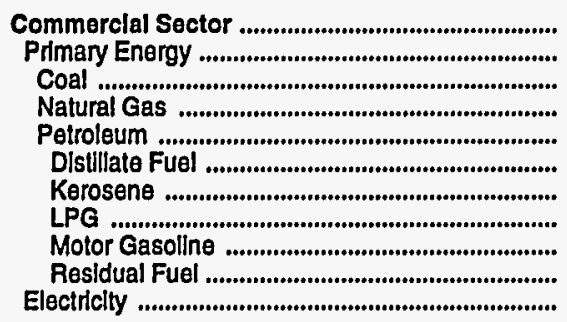 & $\begin{array}{r}229.6 \\
76.1 \\
11.7 \\
45.5 \\
18.8 \\
11.5 \\
.6 \\
5.0 \\
.8 \\
.9 \\
153.5\end{array}$ & $\begin{array}{r}425.5 \\
137.1 \\
10.7 \\
88.6 \\
37.8 \\
24.9 \\
.6 \\
9.3 \\
1.2 \\
1.8 \\
288.4\end{array}$ & $\begin{array}{r}868.0 \\
346.5 \\
1.2 \\
266.9 \\
78.5 \\
61.8 \\
1.8 \\
10.3 \\
3.8 \\
.8 \\
521.5\end{array}$ & \begin{tabular}{r|}
$1,311.2$ \\
531.6 \\
.9 \\
378.3 \\
152.5 \\
118.8 \\
.7 \\
16.1 \\
13.9 \\
3.0 \\
779.6
\end{tabular} & $\begin{array}{r}1,199.0 \\
420.0 \\
.1 \\
315.2 \\
104.7 \\
60.3 \\
.3 \\
25.1 \\
15.7 \\
3.3 \\
779.0\end{array}$ & $\begin{array}{r}1,246.8 \\
426.8 \\
.3 \\
330.5 \\
96.0 \\
55.7 \\
.3 \\
25.4 \\
11.9 \\
2.6 \\
820.0\end{array}$ & $\begin{array}{r}1,255.7 \\
425.7 \\
.1 \\
343.2 \\
82.5 \\
43.9 \\
.3 \\
25.1 \\
9.9 \\
3.2 \\
830.0\end{array}$ & $\begin{array}{r}1,339.2 \\
478.1 \\
1.0 \\
397.9 \\
79.2 \\
42.6 \\
.3 \\
30.9 \\
2.4 \\
3.1 \\
861.0\end{array}$ \\
\hline 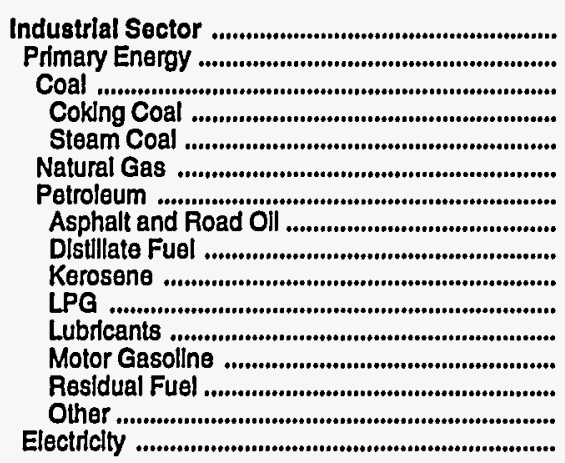 & $\begin{array}{r}407.6 \\
286.3 \\
78.0 \\
5.0 \\
73.0 \\
77.3 \\
131.0 \\
23.6 \\
35.1 \\
5.9 \\
5.0 \\
13.6 \\
34.4 \\
3.9 \\
9.5 \\
121.3\end{array}$ & $\begin{array}{r}744.3 \\
504.1 \\
84.6 \\
12.0 \\
72.6 \\
159.5 \\
260.0 \\
41.1 \\
92.9 \\
5.5 \\
19.1 \\
19.3 \\
48.4 \\
9.3 \\
24.4 \\
240.2\end{array}$ & $\begin{array}{r}1,390.1 \\
939.7 \\
84.6 \\
12.3 \\
72.3 \\
404.2 \\
450.9 \\
77.1 \\
108.3 \\
1.3 \\
47.0 \\
43.2 \\
80.9 \\
19.4 \\
73.7 \\
450.4\end{array}$ & $\begin{array}{r}1,735.0 \\
1,011.4 \\
104.7 \\
.1 \\
104.6 \\
499.2 \\
407.5 \\
54.6 \\
112.5 \\
.8 \\
47.0 \\
48.3 \\
55.7 \\
2.2 \\
86.2 \\
723.7\end{array}$ & $\begin{array}{r}R_{1,658.1} \\
\text { R }_{903.7} \\
85.0 \\
- \\
85.0 \\
394.8 \\
\mathrm{R}_{423.9} \\
76.4 \\
133.4 \\
.4 \\
52.3 \\
\mathrm{R}_{45.0} \\
38.2 \\
9.8 \\
\mathrm{R}_{68.3} \\
754.3\end{array}$ & $\begin{array}{r}R_{1,663.4} \\
R_{890.9} \\
81.3 \\
-\overline{8} \\
81.3 \\
386.9 \\
R_{422.7} \\
68.5 \\
132.8 \\
.3 \\
57.9 \\
\mathrm{R}_{46.3} \\
48.1 \\
6.0 \\
{ }^{R} 62.7 \\
772.5\end{array}$ & $\begin{array}{r}\text { R }_{1,697.1} \\
\text { R }_{903.0} \\
77.6 \\
- \\
77.6 \\
420.9 \\
\text { R }_{404.6} \\
53.3 \\
126.3 \\
.4 \\
51.5 \\
\text { R } 54.7 \\
38.3 \\
5.4 \\
\text { R } 74.7 \\
794.0\end{array}$ & $\begin{array}{r}1,789.2 \\
958.6 \\
73.9 \\
- \\
73.9 \\
449.7 \\
434.9 \\
68.9 \\
136.7 \\
.6 \\
57.5 \\
54.2 \\
38.9 \\
10.6 \\
67.6 \\
830.7\end{array}$ \\
\hline 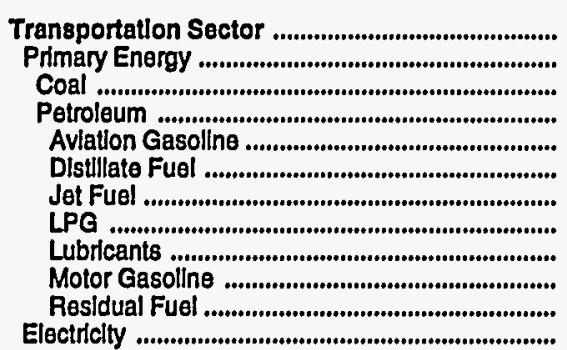 & $\begin{array}{r}658.5 \\
658.5 \\
.1 \\
658.4 \\
3.6 \\
32.3 \\
6.7 \\
.4 \\
17.0 \\
598.4 \\
(5) \\
\end{array}$ & $\begin{array}{r}1,328.0 \\
1,328.0 \\
(s) \\
1,328.0 \\
3.0 \\
92.4 \\
25.5 \\
.9 \\
22.6 \\
1,181.0 \\
2.6 \\
-\end{array}$ & $\begin{array}{r}2,881.3 \\
2,881.3 \\
-3 \\
2,881.3 \\
5.6 \\
363.6 \\
86.1 \\
1.6 \\
45.5 \\
2,373.2 \\
5.6\end{array}$ & $\begin{array}{r}2,824.7 \\
2,824.7 \\
2,824.7 \\
5.1 \\
490.1 \\
57.8 \\
5.5 \\
50.8 \\
2,211.3 \\
4.1\end{array}$ & $\begin{array}{r}R_{3,100.6} \\
R_{3,100.6} \\
R_{3,100.6} \\
5.7 \\
658.9 \\
47.9 \\
4.0 \\
R_{47.4} \\
2,336.7 \\
(s)\end{array}$ & $\begin{array}{r}R_{3,014.1} \\
R_{3,014.1} \\
- \\
R_{3,014.1} \\
4.6 \\
571.4 \\
39.8 \\
3.8 \\
R_{48.8} \\
2,345.7 \\
(s) \\
-\end{array}$ & $\begin{array}{r}R_{3,007.6} \\
R_{3,007.6} \\
-R_{3,007.6} \\
5.2 \\
583.0 \\
44.9 \\
3.5 \\
R^{2} 57.5 \\
2,313.4 \\
.1 \\
-\end{array}$ & $\begin{array}{r}3,131.8 \\
3,131.8 \\
- \\
3,131.8 \\
5.0 \\
626.7 \\
45.9 \\
4.7 \\
57.1 \\
2,392.3 \\
.2 \\
-\end{array}$ \\
\hline 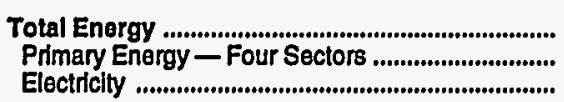 & $\begin{array}{r}1,808.2 \\
1,307.2 \\
501.0\end{array}$ & $\begin{array}{r}3,379.7 \\
2,447.5 \\
932.2\end{array}$ & $\begin{array}{l}6,715.3 \\
5,045.8 \\
1,669.5\end{array}$ & $\begin{array}{l}8,104.7 \\
5,503.7 \\
2,601.0\end{array}$ & $\begin{array}{r}R_{8,045.6} \\
R_{5,425.2} \\
2,620.3\end{array}$ & $\begin{array}{r}R_{8,161.2} \\
R_{5,400.7} \\
2,760.5\end{array}$ & $\begin{array}{r}R_{8,148.9} \\
R_{5,376.9} \\
2,772.0\end{array}$ & $\begin{array}{l}8,669.3 \\
5,755.5 \\
2,913.7\end{array}$ \\
\hline 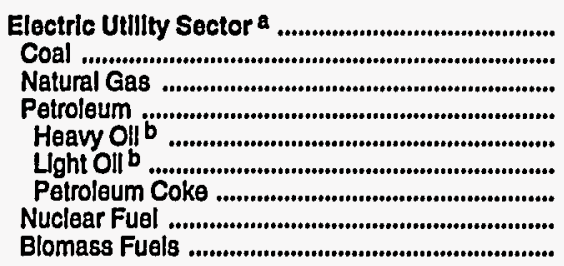 & $\begin{array}{r}109.2 \\
90.8 \\
13.1 \\
5.0 \\
4.0 \\
.5 \\
.5 \\
.3 \\
.1\end{array}$ & $\begin{array}{r}245.2 \\
178.3 \\
16.7 \\
13.6 \\
5.7 \\
7.7 \\
.2 \\
36.6 \\
-\end{array}$ & $\begin{array}{r}494.9 \\
384.7 \\
40.6 \\
18.1 \\
1.8 \\
16.2 \\
.1 \\
50.3 \\
1.1\end{array}$ & $\begin{array}{r}612.9 \\
529.4 \\
5.4 \\
8.2 \\
\overline{8.0} \\
.2 \\
69.2 \\
.7\end{array}$ & $\begin{array}{r}544.4 \\
474.9 \\
7.0 \\
3.5 \\
\overline{3.5} \\
\overline{-} \\
57.9 \\
1.2\end{array}$ & $\begin{array}{r}557.3 \\
491.6 \\
7.4 \\
3.8 \\
-\overline{3} \\
3.8 \\
53.4 \\
1.2\end{array}$ & $\begin{array}{r}\text { R } 530.0 \\
472.7 \\
6.3 \\
2.4 \\
\overline{-} \\
2.2 \\
.2 \\
47.5 \\
R_{1.2}\end{array}$ & $\begin{array}{r}498.7 \\
437.5 \\
8.2 \\
3.1 \\
-\overline{2.9} \\
.2 \\
48.5 \\
1.6\end{array}$ \\
\hline Prlmary Energy — Flve Sactors c c........................... & $1,416.4$ & $2,692.7$ & $5,540.7$ & $6,116.6$ & $\mathrm{P}_{5,969.7}$ & $\mathrm{R}_{5,958.1}$ & $R_{5,906,9}$ & $6,254.3$ \\
\hline
\end{tabular}

There are no direct fuel costs for hydroelectric, geothermal, wind, photovoltalc, or solar thermal energy.

b Heavy oll Includes fuel oil nos. 4, 5, and 6, and residual fuel olls. Light oll includes fuel nos. 1 and 2, kerosene, and jet fuel.

- Blomass tuels are not included, except those consumed at electric utilities and those added to motor gasoline.

$\mathrm{R}=$ Revised data.
-No consumption, Including cases where adjustments were made. See explanation of adjustments in Section 6 of Appendix A.

(s) =Value less than 0.05 million dollars.

Note: Totals may not equal sum of components due to Independent rounding.

Sources: Data sources, estimation procedures, and assumptions are described in Appendlx A. 
W Energy Price and Expenditure Estimates by Source, Wyoming

Y 1970, 1975, 1980, 1985, and 1990-1993

$\mathbf{O}$
$\mathbf{M}$
$\mathbf{I}$
$\mathbf{N}$
$\mathbf{G}$

\begin{tabular}{|c|c|c|c|c|c|c|c|c|}
\hline Energy Source & 1970 & 1975 & 1980 & 1985 & 1990 & 1991 & 1992 & 1993 \\
\hline & \multicolumn{8}{|c|}{ Prices in Dollars per Million Btu } \\
\hline 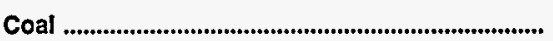 & 0.16 & 0.31 & 0.70 & 1.00 & 0.86 & 0.86 & 0.79 & 0.83 \\
\hline 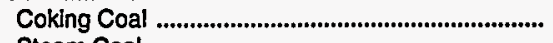 & - & - & $\overline{-}$ & - & - & $\overline{-}$ & $-\overline{0}$ & $\overline{0}$ \\
\hline 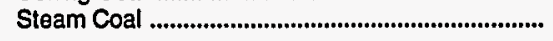 & .16 & .31 & .70 & 1.00 & .86 & .86 & .79 & .83 \\
\hline 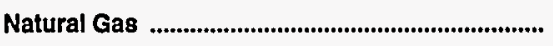 & .38 & .71 & 2.45 & 4.28 & 3.57 & 3.48 & 3.17 & 3.70 \\
\hline $\begin{array}{l}\text { Petroleum } \\
\text { Asphalt and Road Oil } \\
\text { Aviation Gasoline } \\
\text { Distlllate Fuel } \\
\text { Jet Fuel } \\
\text { Kerosene } \\
\text { LPG }\end{array}$ & $\begin{array}{r}1.73 \\
.58 \\
2.17 \\
1.11 \\
.76 \\
.96 \\
1.64 \\
5.08 \\
2.93 \\
.55 \\
.51\end{array}$ & $\begin{array}{l}3.28 \\
1.80 \\
3.45 \\
2.51 \\
2.12 \\
2.47 \\
3.46 \\
7.48 \\
4.77 \\
1.71 \\
1.39\end{array}$ & $\begin{array}{r}7.16 \\
3.66 \\
9.02 \\
6.44 \\
6.59 \\
5.76 \\
5.77 \\
14.36 \\
10.28 \\
3.56 \\
2.53\end{array}$ & $\begin{array}{r}7.67 \\
4.82 \\
9.99 \\
7.32 \\
6.53 \\
10.22 \\
8.13 \\
17.61 \\
8.87 \\
3.14 \\
2.37\end{array}$ & $\begin{array}{r}{ }^{A} 7.60 \\
2.71 \\
9.32 \\
7.56 \\
6.45 \\
6.66 \\
8.10 \\
\text { R } 14.60 \\
8.66 \\
2.46 \\
2.02\end{array}$ & $\begin{array}{r}\mathrm{R}_{7.43} \\
3.43 \\
8.71 \\
7.10 \\
6.05 \\
6.86 \\
9.68 \\
\mathrm{R} \\
\mathrm{16.79} \\
8.40 \\
2.26 \\
1.72\end{array}$ & $\begin{array}{r}{ }^{R} 7.30 \\
3.07 \\
8.54 \\
6.76 \\
5.88 \\
5.14 \\
7.19 \\
\mathrm{R}_{19.43} \\
8.73 \\
1.75 \\
1.29\end{array}$ & $\begin{array}{r}7.38 \\
2.93 \\
8.24 \\
7.06 \\
5.71 \\
6.43 \\
6.71 \\
18.94 \\
8.68 \\
2.05 \\
1.06\end{array}$ \\
\hline 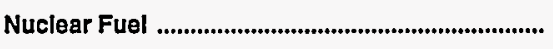 & - & - & - & - & - & - & - & - \\
\hline 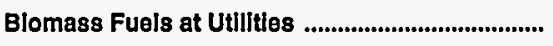 & - & - & - & - & - & - & - & - \\
\hline Primary Energy - Five Sectors ${ }^{b}$ & .84 & 1.51 & 3.00 & 2.54 & 2.26 & $R_{2.22}$ & 2.09 & 2.30 \\
\hline 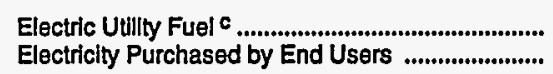 & .14 & $\begin{array}{r}.26 \\
4.63\end{array}$ & $\begin{array}{r}.59 \\
7.45\end{array}$ & $\begin{array}{r}.93 \\
12.54\end{array}$ & $\begin{array}{r}.84 \\
12.37\end{array}$ & $\begin{array}{r}.84 \\
12.53\end{array}$ & $\begin{array}{r}.76 \\
12.56\end{array}$ & $\begin{array}{r}.81 \\
12.55\end{array}$ \\
\hline \multirow[t]{2}{*}{ 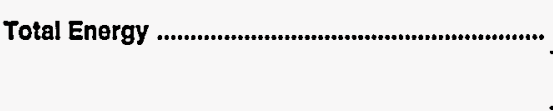 } & 1.31 & 2.53 & 5.79 & 6.87 & ${ }^{R_{6.42}}$ & 6.23 & $\mathrm{R}_{5.86}$ & 6.11 \\
\hline & \multicolumn{8}{|c|}{ Expenditures in Millions of Dollare } \\
\hline $\begin{array}{l}\text { Coal } \\
\text { Coking Coal } \\
\text { Steam Coal }\end{array}$ & $\frac{10.2}{10.2}$ & $\begin{array}{l}39.8 \\
39.8\end{array}$ & $\begin{array}{l}187.2 \\
18 \overline{7.2}\end{array}$ & $\begin{array}{l}406.6 \\
406.6\end{array}$ & $\begin{array}{l}395.9 \\
395.9\end{array}$ & $\begin{array}{l}387.8 \\
\overline{3} \overline{7} .8\end{array}$ & $\begin{array}{l}389.9 \\
-\overline{-} \\
389.9\end{array}$ & $\begin{array}{l}386.2 \\
38 \overline{8.2}\end{array}$ \\
\hline 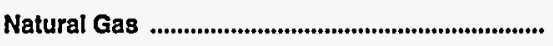 & 28.4 & 36.4 & 91.6 & 176.5 & 162.9 & 178.9 & 230.6 & 280.6 \\
\hline $\begin{array}{l}\text { Patroleum } \\
\text { Asphalt and Road Oil } \\
\text { Avlation Gasollne } \\
\text { Distlllate Fuel } \\
\text { Jet Fuel } \\
\text { Kerosenen } \\
\text { LPG }\end{array}$ & $\begin{array}{r}152.0 \\
4.2 \\
2.8 \\
32.7 \\
.5 \\
1.6 \\
10.6 \\
3.5 \\
90.8 \\
2.7 \\
2.1\end{array}$ & $\begin{array}{r}359.5 \\
7.2 \\
3.8 \\
111.2 \\
1.5 \\
2.4 \\
21.6 \\
7.0 \\
184.4 \\
13.6 \\
6.9\end{array}$ & $\begin{array}{r}1,097.6 \\
28.2 \\
4.9 \\
498.4 \\
6.0 \\
2.0 \\
22.7 \\
42.7 \\
18.1 \\
458.9 \\
24.0 \\
16.4\end{array}$ & $\begin{array}{r}825.5 \\
53.6 \\
2.6 \\
326.9 \\
5.6 \\
1.2 \\
51.1 \\
20.2 \\
357.2 \\
1.4 \\
5.8\end{array}$ & $\begin{array}{r}{ }^{R} 828.8 \\
17.1 \\
1.7 \\
422.8 \\
5.1 \\
.2 \\
.2 \\
35.5 \\
818.9 \\
321.4 \\
(8) \\
6.1\end{array}$ & $\begin{array}{r}R 775.1 \\
23.1 \\
1.2 \\
364.6 \\
4.1 \\
.4 \\
40.7 \\
40.7 \\
19.4 \\
317.9 \\
(8) \\
3.6\end{array}$ & $\begin{array}{r}R 784.3 \\
15.7 \\
1.1 \\
365.7 \\
5.0 \\
.2 \\
29.1 \\
29.1 \\
R_{22.9} \\
340.8 \\
(8) \\
3.8\end{array}$ & $\begin{array}{r}847.7 \\
14.7 \\
.8 \\
414.2 \\
4.5 \\
.7 \\
41.7 \\
22.7 \\
345.0 \\
.4 \\
3.0\end{array}$ \\
\hline Nuclear Fuel & - & - & - & - & - & - & - & - \\
\hline 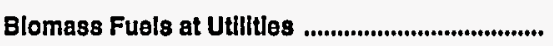 & - & - & - & - & - & - & - & - \\
\hline Primary Energy — Five Sectors ${ }^{b}$ & 180.6 & 435.7 & $1,376.3$ & 1,408.6 & $A_{1,387,6}$ & $R_{1,342,8}$ & $R_{1,404.7}$ & $1,514.5$ \\
\hline $\begin{array}{l}\text { Electric Utility Fuel }{ }^{c} \\
\text { Electricly Purchased by End Users }\end{array}$ & $\begin{array}{r}-8.9 \\
46.9\end{array}$ & $\begin{array}{r}-30.3 \\
70.0\end{array}$ & $\begin{array}{r}-140.7 \\
176.1\end{array}$ & $\begin{array}{r}-346.3 \\
427.3\end{array}$ & $\begin{array}{l}349.8 \\
481.6\end{array}$ & $\begin{array}{r}-340.2 \\
487.6\end{array}$ & $\begin{array}{r}-340.1 \\
487.6\end{array}$ & $\begin{array}{r}341.4 \\
493.7\end{array}$ \\
\hline 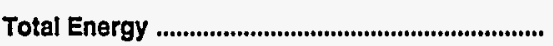 & 228.5 & 475.4 & $1,411.7$ & $1,489.7$ & $8_{1,519.4}$ & $R_{1,490.3}$ & $R_{1,552.2}$ & $1,666.7$ \\
\hline
\end{tabular}

\footnotetext{
a Includes petroleum coke used at electric utilities.

b Blomass fuels are not Included, except those consumed at electric utilitites and those added to motor gasoline.

c There are no direct fuel costs for hydroelectric, geothermal, wind, photovoltalc, or solar thermal energy.

$R=$ Revised data.

$\rightarrow$ No consumption, Including cases where adjustments were made. See
}

explanation of adjustments in Section 6 of Appendlx A.

(s) $=$ Value less than 0.05 million dollars.

Note: Expenditure totals may not equal sum of components due to Independent rounding.

Sources: Data sources, estimation procedures, and assumptions are described in Appendix A. 


\begin{tabular}{|c|c|c|c|c|c|c|c|c|}
\hline Sector and Energy Source & 1970 & 1975 & 1980 & 1985 & 1990 & 1991 & 1992 & 1993 \\
\hline 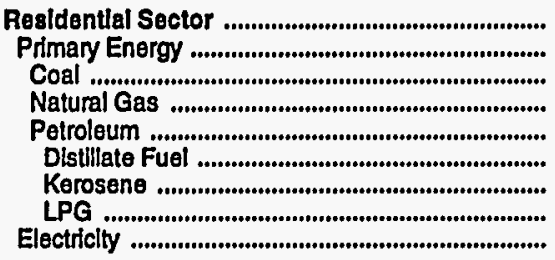 & $\begin{array}{r}1.45 \\
.89 \\
.66 \\
.67 \\
1.93 \\
1.28 \\
1.70 \\
1.96 \\
7.52\end{array}$ & $\begin{array}{r}2.78 \\
1.83 \\
.99 \\
1.09 \\
4.13 \\
2.84 \\
3.17 \\
4.20 \\
7.58\end{array}$ & $\begin{array}{r}5.58 \\
3.42 \\
.87 \\
2.66 \\
7.23 \\
6.94 \\
-\overline{7} \\
7.25 \\
11.66\end{array}$ & $\begin{array}{r}8.12 \\
5.18 \\
2.29 \\
4.92 \\
7.97 \\
10.07 \\
12.67 \\
7.51 \\
16.60\end{array}$ & $\begin{array}{r}8.43 \\
4.96 \\
1.32 \\
4.40 \\
10.39 \\
6.35 \\
7.98 \\
10.72 \\
17.55\end{array}$ & $\begin{array}{r}8.56 \\
5.17 \\
1.16 \\
4.47 \\
10.19 \\
6.05 \\
7.60 \\
11.18 \\
17.56\end{array}$ & $\begin{array}{r}8.62 \\
4.79 \\
1.26 \\
4.46 \\
7.57 \\
4.79 \\
6.02 \\
8.10 \\
17.79\end{array}$ & $\begin{array}{r}8.29 \\
4.66 \\
1.27 \\
4.52 \\
7.74 \\
6.02 \\
7.58 \\
8.06 \\
17.53\end{array}$ \\
\hline 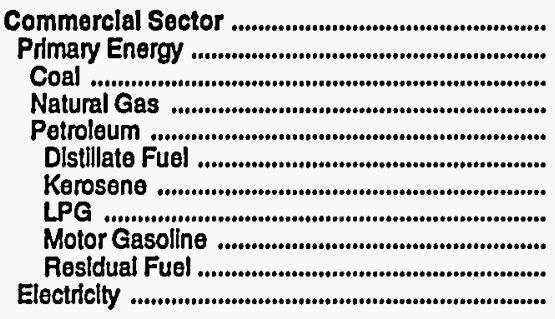 & $\begin{array}{r}1.10 \\
.55 \\
.42 \\
.43 \\
1.32 \\
1.06 \\
.86 \\
1.22 \\
2.93 \\
.55 \\
5.28\end{array}$ & $\begin{array}{r}1.86 \\
1.08 \\
.90 \\
.72 \\
2.77 \\
2.49 \\
2.42 \\
2.48 \\
4.77 \\
2.03 \\
5.48\end{array}$ & $\begin{array}{r}5.94 \\
3.95 \\
1.72 \\
2.50 \\
6.70 \\
6.47 \\
5.76 \\
5.07 \\
10.28 \\
3.59 \\
11.21\end{array}$ & $\begin{array}{r}8.84 \\
5.27 \\
1.89 \\
4.83 \\
7.52 \\
7.95 \\
8.71 \\
8.38 \\
8.87 \\
3.14 \\
15.38\end{array}$ & $\begin{array}{r}8.37 \\
3.97 \\
1.12 \\
4.07 \\
5.98 \\
5.08 \\
6.13 \\
6.34 \\
8.66 \\
2.46 \\
15.54\end{array}$ & $\begin{array}{r}8.24 \\
3.92 \\
1.14 \\
4.07 \\
5.84 \\
4.41 \\
6.51 \\
8.10 \\
8.40 \\
2.26 \\
15.50\end{array}$ & $\begin{array}{r}8.71 \\
3.95 \\
1.13 \\
4.03 \\
5.35 \\
4.01 \\
4.95 \\
6.43 \\
8.73 \\
- \\
15.26\end{array}$ & $\begin{array}{r}8.00 \\
3.73 \\
1.10 \\
4.01 \\
5.56 \\
5.31 \\
6.33 \\
6.23 \\
8.68 \\
15.02\end{array}$ \\
\hline 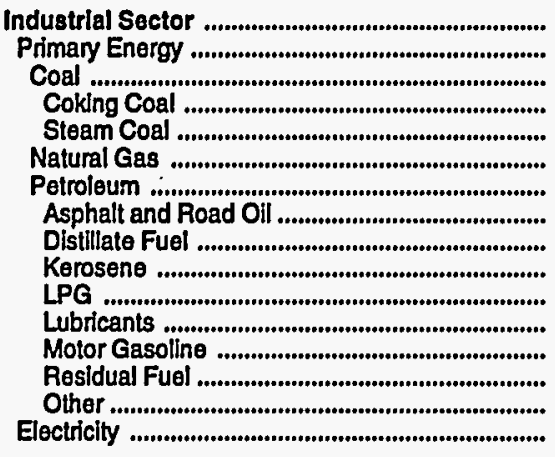 & $\begin{array}{r}.75 \\
.54 \\
.42 \\
.4 \\
.42 \\
.24 \\
.95 \\
.58 \\
.80 \\
.86 \\
1.22 \\
5.08 \\
2.93 \\
.55 \\
.51 \\
3.23\end{array}$ & $\begin{array}{r}1.66 \\
1.46 \\
.90 \\
. \overline{0} \\
.55 \\
2.27 \\
1.80 \\
2.30 \\
2.42 \\
2.48 \\
7.48 \\
4.77 \\
1.65 \\
1.39 \\
3.44\end{array}$ & $\begin{array}{r}3.79 \\
3.62 \\
1.72 \\
-\overline{1} \\
1.72 \\
2.32 \\
4.91 \\
3.66 \\
5.44 \\
5.76 \\
5.07 \\
14.36 \\
10.28 \\
3.55 \\
2.53 \\
5.12\end{array}$ & $\begin{array}{r}5.34 \\
4.23 \\
1.89 \\
-\overline{1} \\
1.89 \\
3.38 \\
6.70 \\
4.82 \\
7.65 \\
8.71 \\
8.38 \\
17.61 \\
8.87 \\
3.14 \\
2.37 \\
10.15\end{array}$ & $\begin{array}{r}R_{4.30} \\
2.69 \\
1.12 \\
-\overline{1.12} \\
2.94 \\
R_{4.85} \\
2.71 \\
5.39 \\
6.13 \\
6.34 \\
R_{14.60} \\
8.66 \\
2.46 \\
2.02 \\
10.16\end{array}$ & $\begin{array}{r}4.31 \\
R_{2.87} \\
1.14 \\
-\overline{14} \\
2.86 \\
R 5.38 \\
3.43 \\
5.72 \\
6.51 \\
8.10 \\
R_{16.79} \\
8.40 \\
2.26 \\
1.72 \\
10.24\end{array}$ & $\begin{array}{r}3.82 \\
2.57 \\
1.13 \\
\overline{1} \\
1.13 \\
2.75 \\
R 4.50 \\
3.07 \\
4.35 \\
4.95 \\
6.43 \\
R 19.43 \\
8.73 \\
1.75 \\
1.29 \\
10.32\end{array}$ & $\begin{array}{r}4.27 \\
3.10 \\
1.10 \\
1.10 \\
3.43 \\
5.14 \\
2.93 \\
5.57 \\
6.33 \\
6.23 \\
18.94 \\
8.68 \\
2.05 \\
1.06 \\
10.27\end{array}$ \\
\hline 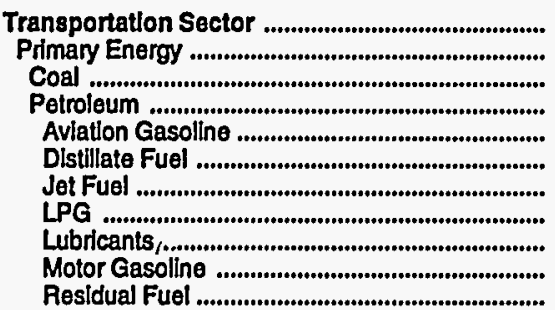 & $\begin{array}{r}2.19 \\
2.19 \\
.42 \\
2.19 \\
2.17 \\
1.31 \\
.76 \\
1.22 \\
5.08 \\
2.93 \\
.54\end{array}$ & $\begin{array}{r}3.95 \\
3.95 \\
.90 \\
3.95 \\
3.45 \\
2.70 \\
2.12 \\
2.48 \\
7.48 \\
4.77 \\
-\end{array}$ & $\begin{array}{r}8.94 \\
8.94 \\
-\overline{8} \\
8.94 \\
9.02 \\
7.39 \\
6.59 \\
5.07 \\
14.36 \\
10.28 \\
-\end{array}$ & $\begin{array}{r}8.25 \\
8.25 \\
8.25 \\
9.99 \\
7.05 \\
6.53 \\
8.38 \\
17.61 \\
8.87 \\
4.01\end{array}$ & $\begin{array}{r}\mathrm{A}_{8.56} \\
\mathrm{R}_{8.56} \\
-\overline{8} \\
\mathrm{R}_{8.56} \\
9.32 \\
8.38 \\
6.45 \\
6.34 \\
\mathrm{R}_{14.60} \\
8.66 \\
-\end{array}$ & $\begin{array}{r}R_{8.25} \\
R_{8.25} \\
- \\
R_{8.25} \\
8.71 \\
7.92 \\
6.05 \\
8.10 \\
R_{16.79} \\
8.40 \\
-\end{array}$ & $\begin{array}{r}{ }^{R_{8.45}} \\
{ }_{8} 8.45 \\
-\overline{8} \\
{ }_{8.45} \\
8.54 \\
7.97 \\
5.88 \\
6.43 \\
A_{19.43} \\
8.73 \\
-\end{array}$ & $\begin{array}{r}8.28 \\
8.28 \\
- \\
8.28 \\
8.24 \\
7.74 \\
5.71 \\
6.23 \\
18.94 \\
8.68 \\
-\end{array}$ \\
\hline 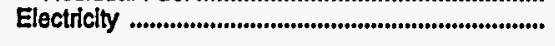 & - & - & - & - & - & - & - & - \\
\hline 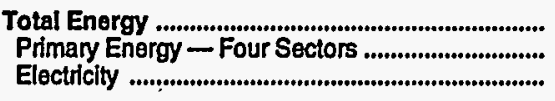 & $\begin{array}{l}1.31 \\
1.11 \\
4.53\end{array}$ & $\begin{array}{l}2.53 \\
2.35 \\
4.63\end{array}$ & $\begin{array}{l}5.79 \\
5.61 \\
7.45\end{array}$ & $\begin{array}{r}6.87 \\
5.82 \\
12.54\end{array}$ & $\begin{array}{r}R_{6.42} \\
R_{5.25} \\
12.37\end{array}$ & $\begin{array}{r}6.23 \\
\mathbf{5 . 0 1} \\
12.53\end{array}$ & $\begin{array}{r}R_{5.86} \\
R_{4.71} \\
12.56\end{array}$ & $\begin{array}{r}6.11 \\
5.03 \\
12.55\end{array}$ \\
\hline 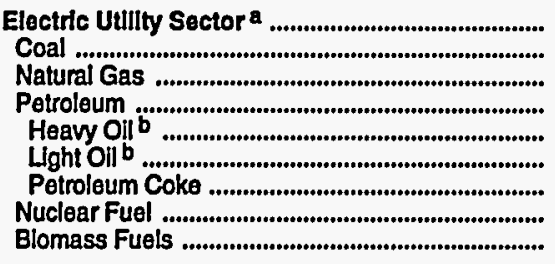 & $\begin{array}{l}.14 \\
.14 \\
.22 \\
.67 \\
.58 \\
.76 \\
- \\
- \\
-\end{array}$ & $\begin{array}{r}.26 \\
.25 \\
.94 \\
2.01 \\
1.99 \\
2.44 \\
- \\
- \\
-\end{array}$ & $\begin{array}{r}.59 \\
.57 \\
4.61 \\
6.98 \\
-\overline{8} \\
6.98 \\
- \\
- \\
-\end{array}$ & $\begin{array}{r}.93 \\
.92 \\
4.33 \\
6.00 \\
\overline{0} \\
6.00 \\
- \\
- \\
-\end{array}$ & $\begin{array}{r}.84 \\
.84 \\
3.15 \\
5.27 \\
- \\
5.27 \\
- \\
- \\
-\end{array}$ & $\begin{array}{r}.84 \\
.83 \\
3.34 \\
4.94 \\
-\overline{94} \\
- \\
- \\
-\end{array}$ & $\begin{array}{r}.76 \\
.76 \\
3.20 \\
4.79 \\
- \\
4.79 \\
- \\
- \\
-\end{array}$ & $\begin{array}{r}.81 \\
.80 \\
3.30 \\
4.73 \\
- \\
4.73 \\
- \\
- \\
-\end{array}$ \\
\hline Primary Energy - Five Sectora c ......................... & .84 & 1.51 & 3.00 & 2.54 & 2.26 & $R_{2.22}$ & 2.09 & 2.30 \\
\hline
\end{tabular}

There are no direct fuel costs for hydroelectric, geothemal, wind, photovoltaic, or solar themal energy.

6 Heavy oil includes fuel oil nos. 4, 5, and 6, and residual fuel oils. Light oll includes fuel oil nos. 1 and 2, kerosene, and jet fuel.

c Biomass fuels are not Included, except those consumed at electric utilittes and those added to motor gasoline.
$R=$ Revised data.

-No consumption, including cases where adjustments were made. See explanation of adjustments in Section 6 of Appendix A.

Sounces: Data sources, estimation procedures, and assumptions are described in Appendix A. 
W Energy Expenditure Estimates by Sector, Wyoming

Y 1970, 1975, 1980, 1985, and 1990-1993

O (Million Dollars)

\begin{tabular}{c} 
Sector and Energy Source \\
\cline { 2 - 4 }
\end{tabular}

\begin{tabular}{l|l}
1970 & 1975 \\
\hline
\end{tabular}

1975

1980

1985

1990

1991

1991

1992

51.3

102.2

195.4

179.7

194.4

175.8

Commercial Sector

Primary Energy

Coal

Natural Gas

Petroleum

Distillate Fuel

Kerosene

LPG

Motor Gasoline

Residual Fuel

Electricity

Industrial Sector

Primary Energy

Coal

Coking Coal

Steam Coal

Natural Gas

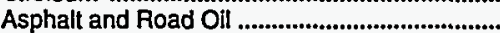

Distillate Fuel

Kerosene

Lubricants

Motor Gasoline

Residual Fuel

Other

Transportation Sector

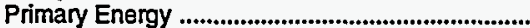

Coal

Petroleum .

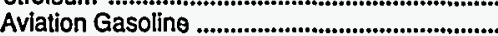

Distillate Fuel

Jet Fuel

LPG

Lubricants

Motor Gasoline

Residual Fuel

Electricity

Total Energy

Primary Energy — Four Sectors ..............................

Electricity ......................................................................

Electric Utility Sector

Coal

Natural Gas

Petroleum

Heavy Oll

Light Oil b

Petroleum Coke

Nuclear Fuel .

Biomass Fuels

Primary Energy - Five Sectors $c$

$\begin{array}{rr}35.7 & \\ 20.2 & \\ .1 & \\ 12.3 & \\ 7.9 & \\ .1 & \\ .4 & \\ 7.4 & 15.5\end{array}$

28.3

12.3

15.6

.4

15.0

23.0

$21.2 \quad 27.9$

$9.4 \quad 13.4$

.1

6.1

3.2

46.1
.6

27.5
18.1

18.1
.9

17.1

56.1

92.6
1.5

74.2

17.0

3.0
.6

.6
13.4

102.8

$84.0 \quad 198.3$

$40.4 \quad 76.5$

2.1

13.2

25.1

16.1

2.1

5.5

43.5

2.3
46.4

46.4
27.8

20.4

.3

2.6

3.1
1.4

121.8

$59.2 \quad 154.8$

$39.7 \quad 122.4$

$1.7 \quad 10.6$

$1.7 \quad 10.6$

$9.5 \quad 16.7$

$28.5 \quad 95.0$

4.2

8.9

.8

2.1

8.5

.9

2.1

19.6

112.4

112.4

(s)
112.3

112.3
2.8

2.8
23.4

.5

.4

81.0

1.6
-

490.1

413.6

566.2

363.5

$49.6 \quad 62.2$

$49 . \overline{6}$

50.0

314.0

28.2

198.0

1.3

22.0

5.0

19.7

23.4

16.4

6.9

32.5

76.5

241.4

735.4

241.4

(s)
241.4

3.8

62.4

1.5

1.1
4.9

167.8

-

228.5

475.4
405.4

735.4

$735 . \overline{4}$

4.9

276.4

6.0

1.4

13.1

433.7

62.2

55.3
246.1

53.6

122.6

.3
33.6

5.6

24.7

(s)

5.8
202.7

529.6

529.6

529.6

2.6
176.1

176.1
5.6

1.4
14.6

329.4

(s)

76.7
1.2
55.6

55.6
19.9

.9
.1

18.9

103.0

174.4

51.4

1.9
37.7

11.8

6.4

(s)

2.0

3.4

(s)
123.0

$R_{\mathbf{5 0 3 . 9}}$

म 248.3
4.93

46.3

46.3

69.3

R 132.7

17.1

71.3

.1
14.0

$\mathrm{R}_{5.2}$

18.9

(s)

6.1
255.6

$R_{661.4}$

$R_{661.4}$

A $661.4^{-}$

1.7

341.2

5

R 13.7

299.1
-

$1,411.7$
$1,235.6$
176.1

$1,489.7$
$1,062.3$

$R_{1,519.4}$

$1,037.8$
481.6

ค $1,002.6$

$1,002.6$
487.6

$A_{1,552,2}$

R $1,064.6$

$1,064.6$
487.6

$1,666.7$

8

8.9
8.3
.5

30.3

140.7

134.9

134.9
.9
5.0

3463

349.8

340.2

336.4

.3
3.5

340.1

337.0

.3
2.8

2.8

2.8

-

$1,404.7$

$1,514.5$

a There are no direct fuel costs for hydroelectric, geothermal, wind,

photovoltaic, or solar thermal energy.
$b$ Heavy oil includes fuel oil nos. 4,5 , and 6 , and residual fuel oils. Light oil includes fuel nos. 1 and 2 , kerosene, and jet fuel.

c Biomass fuels are not included, except those consumed at electric utilities and those added to motor gasoline.

$R=R e v i s e d$ data.

-No consumption, inciuding cases where adjustments were made. See explanation of adjustments in Section 6 of Appendix A.

(s)=Value less than 0.05 million dollars.

Note: Totals may not equal sum of components due to independent rounding.

Sources: Data sources, estimation procedures, and assumptions are described in Appendix A. 
Appendix A

\section{Documentation}




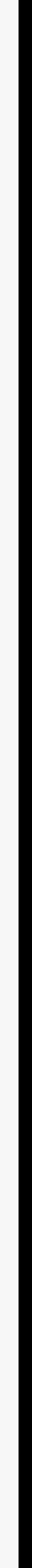




\section{Documentation}

\section{Section 1. Overview}

This appendix documents data sources and procedures used to develop the estimates in the State Energy Price and Expenditure Data System (SEPEDS) that are presented in the "Statistical Tables" of this report. Information is provided for each of the four major energy sources: coal, natural gas, petroleum, and electricity. The last section describes adjustments for consumption of process fuel and intermediate products. Many of the price estimates used in SEPEDS were developed by the Battelle Pacific Northwest Laboratories under contract to EIA.

\section{Price Estimation Methodologies}

Price data in the State Energy Price and Expenditure Report are expressed in dollars per million Btu. If the source data are in physical units, they are divided by the appropriate conversion factors to create the Btu prices. Estimated prices are used only when specific State-level prices are not available for a given energy source and sector. In some cases, prices for energy consumed in one sector in a State are assigned to another sector in the same State. Specific examples are: industrial steam coal prices are assigned to the commercial and transportation sectors' steam coal use; industrial kerosene prices are assigned to commercial kerosene uses; industrial liquefied petroleum gases (LPG) prices are assigned to LPG used by the commercial and transportation sectors; industrial lubricants prices are assigned to transportation lubricants uses; and transportation motor gasoline prices are assigned to commercial and industrial use of motor gasoline.

In addition, there are a few cases where State-level prices could not be identified for any economic sector for a given energy source for some or all years. In these instances, a national-level price is used for all States for a given year. The procedures for estimating these national-level prices are presented in the body of the documentation under each energy source as appropriate. The cases where a nationallevel price is assigned to all States in all years are: transportation use of aviation gasoline; industrial and transportation use of lubricants; and the components of other petroleum products used in the industrial sector.

Finally, within a given energy source and sector where price data are usually available, there are some cases of missing prices. Two general approaches are used to assign or estimate prices in cases where consumption occurs but no price is directly available from the data sources. The first approach is to assign an adjacent State price or the simple average of adjacent States' prices. When this approach is not feasible, the consumption-weighted price from the Census division or region or the Petroleum Administration for Defense district or subdistrict in which the State is located is assigned.

Three State groupings used in the report-U.S. Census regions and divisions, Federal regions, and Petroleum Administration for Defense districts-are shown in Figures A1, A2, and A3, respectively, on the following pages. Throughout the documentation, the term "State" includes the District of Columbia. States are often designated by the two-letter postal code abbreviations shown in the map legends.

\section{Expenditures}

Energy consumption estimates used to calculate expenditures in SEPEDS are taken from the Energy Information Administration (EIA) State Energy Data Report 1993, Consumption Estimates, DOE/EIA0214(93), published in July 1995. The data system from which the consumption report is developed is called the State Energy Data System (SEDS). Full documentation of SEDS data sources and the methods used to estimate energy consumption are described in the consumption report.

Note: Throughout this report, the term "State" includes the District of Columbia. 
D To calculate energy expenditures, SEDS consumption is adjusted to remove quantities of process fuel and intermediate products used in the industrial and transportation sectors that are not purchased directly by end users. Energy expenditures, in million dollars, are calculated by multiplying SEPEDS prices for each fuel in dollars per million Btu times the SEDS adjusted consumption for the fuel in billion Btu.

\section{End-Use Sector, State, and National Consump- tion-Weighted Average Prices}

Aggregated prices shown in this report are the consumption-weighted averages of the various energy sources included in the aggregation. The SEPEDS calculation is performed by summing the expenditures for the energy sources and dividing by the sum of the corresponding consumption values. For example, the average price for residential petroleum is the sum of residential expenditures for distillate fuel oil, kerosene, and liquefied petroleum gas divided by the sum of residential consumption of those fuels.

Primary Energy and Electricity. Within each enduse sector, the sum of all fuels used directly as energy, i.e., coal, natural gas, and petroleum products, is called primary energy. Electricity is considered a secondary energy source because it is generated from the primary energy sources consumed by electric utilities. Within each sector, the primary energy price is calculated by summing the expenditures for coal (the sum of coking coal and steam coal), natural gas, and petroleum (the sum of all petroleum products used by the sector) and dividing by the sum of all the consumption for those fuels. The average price of all energy consumed by each sector is the sum of the expenditures for primary energy and electricity divided by the sum of the consumption of the primary energy sources and electricity.

State and National Totals. The price for "Primary Energy - Four Sectors" is the sum of the residential, commercial, industrial, and transportation sectors' expenditures for primary energy fuels divided by the sum of the end-use sectors' consumption of the primary energy sources. The total electricity price is the sum of electricity expenditures for the four sectors divided by the four sectors' electricity consumption. The "Total Energy" average price, then, is the sum of the four sectors' primary energy and electricity expenditures divided by the corresponding consumption values.

Electric Utility Sector. Electric utilities comprise a fifth energy consuming sector, although they are not considered an end-use sector. The average price paid by electric utilities for primary energy sources is the sum of their expenditures for coal, natural gas, petroleum products, nuclear fuel, and biomass fuels divided by the quantities consumed.

Primary Energy - Five Sectors. The average price for the "Primary Energy - Five Sectors" shown in the SEPER tables is the sum of the four end-use sectors' and the electric utility sector's expenditures for coal, natural gas, petroleum, and other fuels divided by the sum of five sectors' consumption of those fuels. The distinction between this average price and the "Total Energy" average price is that this price includes all fossil and other fuels consumed, including those used to generate electricity. The "Total Energy" price is the average price of fuels consumed directly as energy by the four end-use sectors and the much higher price of the four sectors' electricity consumption. 


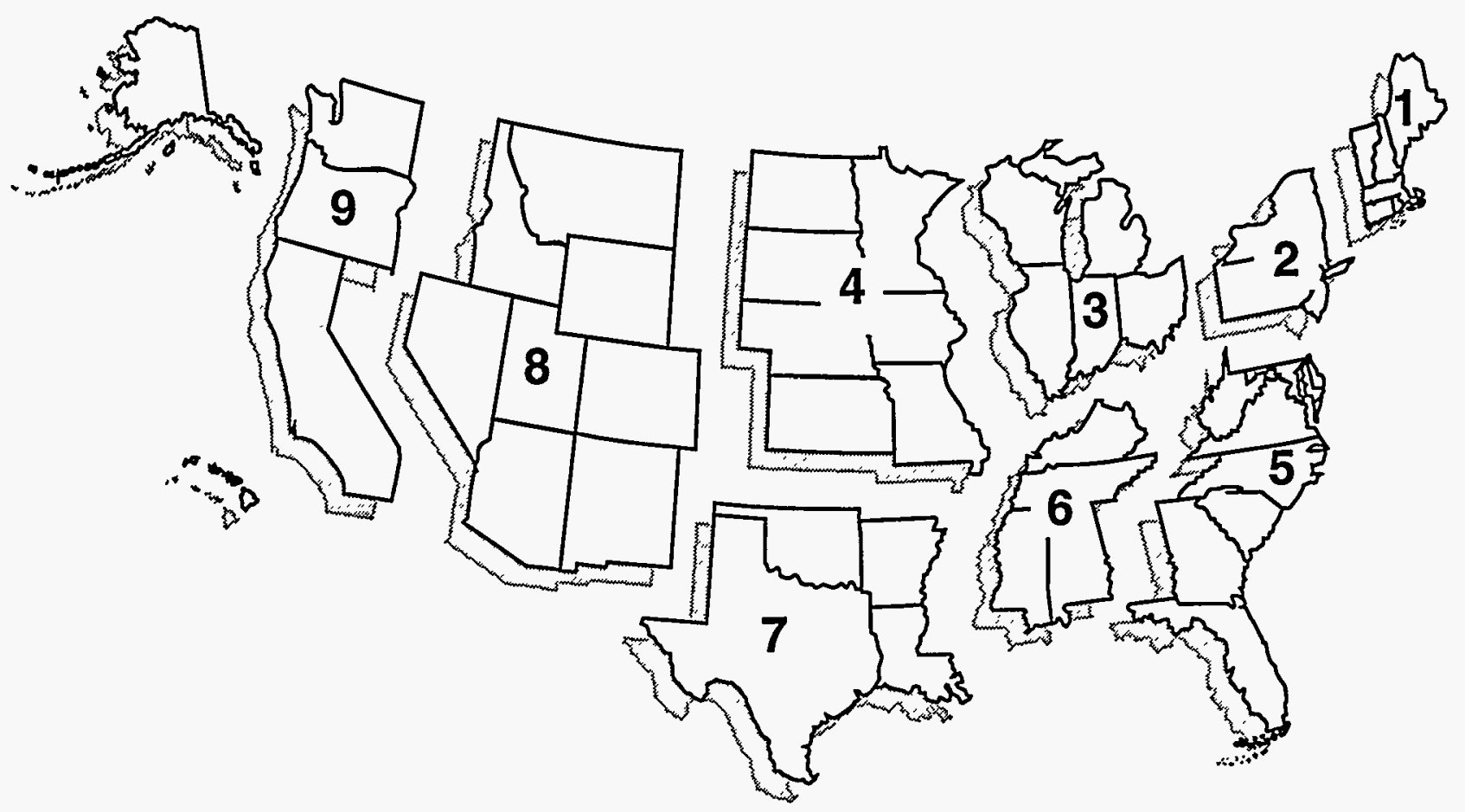

\section{Region 1}

Northeast

Division 1

(New England)

Connecticut (CT)

Maine (ME)

Massachusetts (MA)

New Hampshire (NH)

Rhode Island (RI)

Vermont (VT)

Division 2

(Middle Atlantic)

New Jersey (NJ)

New York (NY)

Pennsylvania (PA)

\section{Region 2}

Midwest

Division 3

(East North Central)

Illinois (IL)

Indiana (IN)

Michigan (MI)

Ohio (OH)

Wisconsin (WI)

Division 4

(West North Central)

Iowa (IA)

Kansas (KS)

Minnesota (MN)

Missouri (MO)

Nebraska (NE)

North Dakota (ND)

South Dakota (SD)
Region 3

South

Division 5

(South Atlantic)

Delaware (DE)

District of Columbia (DC)

Florida (FL)

Georgia (GA)

Maryland (MD)

North Carolina (NC)

South Carolina (SC)

Virginia (VA)

West Virginia (WV)

\section{Division 6}

(East South Central)

Alabama (AL)

Kentucky (KY)

Mississippi (MS)

Tennessee (TN)
Region 4

West

\section{Division 8}

(Mountain)

Arizona (AZ)

Colorado (CO)

Idaho (ID)

Montana (MT)

Nevada (NV)

New Mexico (NM)

Utah (UT)

Wyoming (WY)

\section{Division 9}

(Pacific)

Alaska (AK)

California (CA)

Hawaii (HI)

Oregon (OR)

Washington (WA)

\section{Division 7}

(West South Central)

Arkansas (AR)

Louisiana (LA)

Oklahoma (OK)

Texas (TX) 


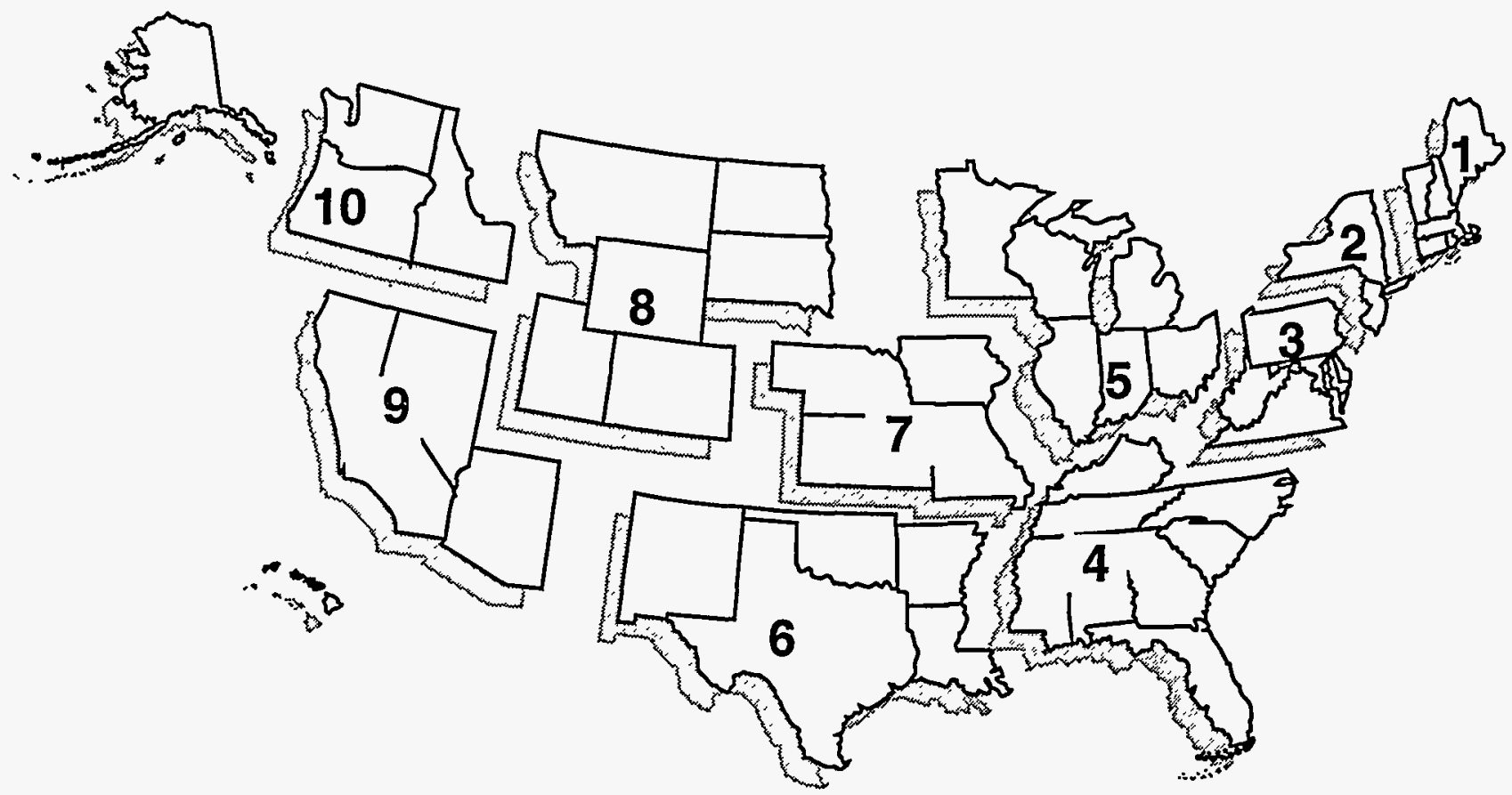

Region 1

New England

Connecticut (CT)

Maine (ME)

Massachusetts (MA)

New Hampshire (NH)

Rhode Island (RD)

Vermont (VT)

\section{Region 2}

New York/New Jersey

New Jersey (NJ)

New York (NY)

\section{Region 3}

Mid Atlantic

Delaware (DE)

District of Columbia (DC)

Maryland (MD)

Pennsylvania (PA)

Virginia (VA)

West Virginia (WV)
Region 4

South Atlantic

Alabama (AL)

Florida (FL)

Georgia (GA)

Kentucky (KY)

Mississippi (MS)

North Carolina (NC)

South Carolina (SC)

Tennessee (TN)

\section{Region 5}

Midwest

Illinois (II)

Indiana (IN)

Michigan (MI)

Minnesota (MN)

Ohio (OH)

Wisconsin (WI)
Region 6

Southwest

Arkansas (AR)

Louisiana (LA)

New Mexico (NM)

Oklahoma (OK)

Texas (TX)

Region 7

Central

Iowa (IA)

Kansas (KS)

Missouri (MS)

Nebraska (NE)
Region 8

North Central

Colorado (CO)

Montana (MT)

North Dakota (ND)

South Dakota (SD)

Utah (UT)

Wyoming (WY)

Region 9

West

Arizona

California (CA)

Hawaii (HI)

Nevada (NV)

Region 10

Northwest

Alaska (AK)

Idaho (ID)

Oregon (OR)

Washington (WA) 

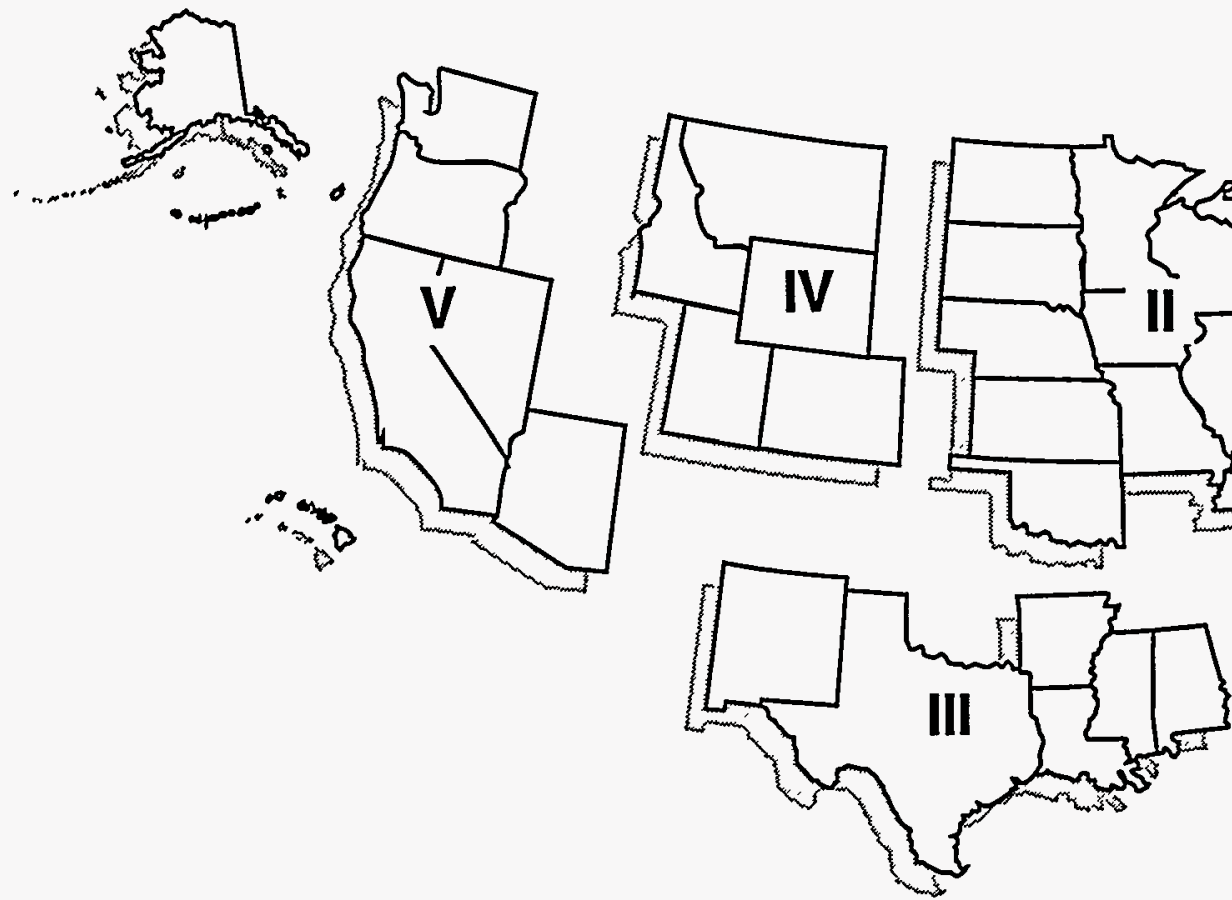

District IA

Connecticut (CT)

Maine (ME)

Massachusetts (MA)

New Hampshire (NH)

Rhode Island (RI)

Vermont (VT)

District IB

Delaware (DE)

District of Columbia (DC)

Maryland (MD)

New Jersey (NJ)

New York (NY)

Pennsylvania (PA)
District II

Illinois (IL)

Indiana (IN)

Iowa (IA)

Kansas (KS)

Kentucky (KY)

Michigan (MI)

Minnesota (MN)

Missouri (MO)

Nebraska (NE)

North Dakota (ND)

Ohio (OH)

Oklahoma (OK)

South Dakota (SD)

Tennessee (TN)

Wisconsin (WI)
District III

Alabama (AL)

Arkansas (AR)

Louisiana (LA)

Mississippi (MS)

New Mexico (NM)

Texas (TX)

\section{District IV}

Colorado (CO)

Idaho (ID)

Montana (MT)

Utah (UT)

Wyoming (WY)
District V

Alaska (AK)

Arizona (AZ)

California (CA)

Hawaii (HI)

Nevada (NV)

Oregon (OR)

Washington (WA)

\section{District IC}

Florida (FL)

Georgia (GA)

North Carolina (NC)

South Carolina (SC)

Virginia (VA)

West Virginia (WV) 



\section{Section 2. Coal}

Coal prices are developed for the following categories: coking coal; steam coal (all noncoking coal); and coal coke, imports and exports.

Coking coal, used in the industrial sector only, is a high-quality bituminous coal that is used to make coal coke. Steam coal, which may be used by all sectors, includes anthracite, bituminous coal, subbituminous coal, and lignite. In the industrial sector, coal consumption is the sum of coking coal and steam coal. The industrial coal price is the quantity-weighted average price of these two components.

Imports and exports of coal coke are confined to the national level and are accounted for in the industrial sector. Coal coke imports and exports are reported separately, and their prices and expenditures are not merged with other coal prices and expenditures.

\section{Coking Coal}

Coking coal is generally more expensive than steam coal; therefore, it is identified separately in developing the price estimates. Coking coal prices are those paid at coke plants for coal received and probably include taxes, although it is not clear from the source data collection forms. Analysts familiar with the survey believe that respondents would report the total cost of the coal (including cost, insurance, freight, and taxes) because that value is typically carried as the total expense in their bookkeeping systems.

\section{Physical Unit Prices: All Years}

Source publications contain physical unit prices for States or for groups of States. Individual State prices are used directly for their respective States. Group prices are assigned to each State within the group. Wherever individual State prices or State group prices are unavailable, prices are assigned from adjacent or nearby States or from States with similar coal use patterns as shown in Table A1.
Table A1. Coking Coal State Group Price and Adjacent State Price Assignments

\begin{tabular}{|c|c|c|}
\hline State & Years & State Prices Assigned \\
\hline $\mathrm{CA}$ & 1970-1982 & CA, CO, UT \\
\hline CO & 1970-1982 & $\mathrm{CA}, \mathrm{CO}, \mathrm{UT}$ \\
\hline IL & $1986-1993$ & IN \\
\hline$K Y$ & $\begin{array}{l}1970-1987 \\
1988-1993\end{array}$ & $\mathrm{KY}, \mathrm{MO}, \mathrm{TN}, \mathrm{TX}$ \\
\hline \multirow[t]{2}{*}{ MD } & 1970,1971 & $\mathrm{MD}, \mathrm{NJ}, \mathrm{NY}$ \\
\hline & $1983-1991,1993$ & PA \\
\hline \multirow[t]{3}{*}{ MI } & 1979 & MI, MN, WI \\
\hline & 1980-1985, 1987 & MI, WI \\
\hline & 1988-1991, 1993 & $\mathrm{OH}$ \\
\hline \multirow[t]{2}{*}{$M N$} & $1970-1978$ & MN, WI \\
\hline & 1979 & $M I, M N, W I$ \\
\hline MO & $\begin{array}{l}1970-1987 \\
1988\end{array}$ & $\begin{array}{l}\mathrm{KY}, \mathrm{MO}, \mathrm{TN}, \mathrm{TX} \\
\mathrm{AL}\end{array}$ \\
\hline NJ & 1970,1971 & $\mathrm{MD}, \mathrm{NJ}, \mathrm{NY}$ \\
\hline \multirow[t]{3}{*}{ NY } & 1970,1971 & $M D, N J, N Y$ \\
\hline & $1972-1982$ & MD, NY \\
\hline & 1983-1993 & PA \\
\hline \multirow[t]{2}{*}{ TN } & $1970-1987$ & KY, MO, TN, TX \\
\hline & 1988-1991 & AL \\
\hline$T X$ & $1970-1987$ & KY, MO, TN, TX \\
\hline \multirow[t]{3}{*}{ UT } & $1970-1982$ & $\mathrm{CA}, \mathrm{CO}, \mathrm{UT}$ \\
\hline & 1983-1986 & $T X$ \\
\hline & $1988-1993$ & IN \\
\hline \multirow[t]{4}{*}{ VA } & $1970,1971,1976,1977$ & WV \\
\hline & 1978-1982 & VA, WV \\
\hline & $1983-1986$ & KY \\
\hline & $1987-1993$ & $\mathrm{OH}$ \\
\hline \multirow[t]{3}{*}{ WI } & $1970-1978$ & MN, WI \\
\hline & 1979 & MI, MN, WI \\
\hline & 1980-1985, 1987 & MI, WI \\
\hline \multirow[t]{3}{*}{ wv } & $1978-1982$ & VA, WV \\
\hline & $1983-1986$ & KY \\
\hline & $1987-1993$ & $\mathrm{OH}$ \\
\hline
\end{tabular}

\section{Btu Prices: All Years}

Btu prices for States are calculated from the physical unit prices and the constant conversion factor for coking coal. U.S. Btu prices are calculated as the average of the State Btu prices, weighted by consumption data from the State Energy Data System (SEDS). 
C Data Sources

A Prices

1981 forward: Energy Information Administration, Quarterly Coal Report, October- December issue, Table A3 (1981-1991) and Table 39 (1992, 1993).

E
E
C
R
I
C
U
I
I
$T$
$Y$

1977-1980: Energy Information Administration, Coke and Coal Chemicals, Table 19 (1977), Table 15 (1978), and Table 7 (1979, 1980).

1970-1976: Bureau of Mines, U.S. Department of the Interior, Minerals Yearbook, "Coke and Coal Chemicals" chapter, Table 22.

Consumption

1970 forward: Energy Information Administration, State Energy Data System, coking coal consumption.

\section{Conversion Factor: All Years}

26.80 million Btu per short ton.

\section{Steam Coal}

Steam coal is used in all sectors. Price data are generally available in the electric utility, residential, and industrial sectors. However, no price data are directly available in the transportation and commercial sectors. The industrial sector steam coal prices are assigned as price estimates in these two sectors. Data sources and computational procedures for estimating coal prices are discussed by sector. Estimates of the amount of steam coal consumed by sector are taken from SEDS and are adjusted for process fuel consumption in the industrial sector. (See the "Consumption Adjustments for Calculating Expenditures" section on page 249.)

\section{Electric Utility Sector}

\section{Btu Prices: 1973 Forward}

State Btu prices, including all taxes, are taken from Cost and Quality of Fuels for Electric Utility Plants (CEQ) for 1973 forward and are converted from cents to dollars per million Btu. Where individual State prices are unavailable, quantity-weighted
Census division prices are assigned as shown in Table A2.

\section{Btu Prices: 1970 Through 1972}

Btu prices for States are taken from the Edison Electric Institute Statistical Yearbook and are converted from cents to dollars. DE, DC, and MD are each assigned the combined price for the three States. The steam coal electric utility sector AK price for 1971 is estimated as discussed below.

\section{Alaska Prices: All Years}

Since $C \mathcal{E} Q$ does not publish prices for $A K$, Btu prices for AK are taken from the Statistical Yearbook. For the years 1970, 1972, 1974, 1976, 1977, and 1979 forward, prices are taken directly from the Statistical Yearbook. Prices for 1971, 1973, 1975, and 1978 are estimated from the Statistical Yearbook prices for the United States and the average ratio of AK-to-U.S. prices for the years when AK prices are available. The 1971 and 1973 estimated prices are based on the average ratio for 1970 and 1972, while the 1975 and 1978 estimated prices are based on the average ratio for 1974, 1976, 1977, and 1979.

\section{U.S. Prices: All Years}

U.S. Btu prices are calculated as the average of the State Btu prices, weighted by consumption data from SEDS.

\section{Data Sources}

Prices

1973 forward: Energy Information Administration, Cost and Quality of Fuels for Electric Utility Plants,

Table A2. Electric Utility Sector Price Assignments, 1973 Forward

\begin{tabular}{lll}
\hline State & \multicolumn{1}{c}{ Years } & $\begin{array}{c}\text { State/Census Division } \\
\text { Prices Assigned }\end{array}$ \\
\hline CT & $1975-1979$ & New England \\
DC & 1976 & MD, VA \\
OK & 1973,1974 & West South Central \\
& 1975 & CO, KS, MO, NM, TX \\
OR & 1983,1989 & Pacific Contiguous \\
RI & 1974 & MA \\
VT & $1980,1983-1986$ & New England \\
& & \\
\hline
\end{tabular}


Table 3 (1973-1979), Table 51 (1980-1982), Table 50 (1983, 1984), Table 40 (1985-1989), Table 7 (1990, 1991), and Table 2 (1992, 1993).

1970 forward: Edison Electric Institute, Statistical Yearbook of the Electric Utility Industry, table titled "Analysis of Fuel for Electric Generation: Total Electric Utility Industry" (1970-1988), Table 29 (1989 forward).

\section{Consumption}

1970 forward: Energy Information Administration, State Energy Data System, electric utility sector coal consumption.

\section{Conversion Factors: All Years}

Btu prices are taken directly from the data sources; no explicit conversion factors are used.

\section{Residential Sector}

Residential sector steam coal prices are the average delivered prices for coal purchased by residential customers and include taxes.

\section{Prices: 1979 Forward}

Residential steam coal Btu prices for 1979 forward are not available. The residential steam coal prices calculated for 1974 through 1978 from Gas Househeating Survey (GHS) and the average Btu spot prices from the Cost and Quality of Fuels for Electric Utility Plants (CEQ) for 1974 through 1978 are used to estimate a regression equation. Electric utility coal spot prices from CEQ for 1979 forward are converted from cents per million Btu to dollars per million Btu and are used in the regression equation to estimate residential steam coal prices for 1979 forward. AK residential coal prices are estimated by using a different methodology, described on page 185.

Some States have GHS residential prices during the 1974 through 1978 period to use in the regression analysis, but are missing prices in the 1979 forward data used to calculate prices. For these missing data, $C E Q$ prices are assigned from other States for use in the regression as shown in Table A3. C\&Q prices for ND result in a negative price when used in the regression; therefore $\mathrm{MN}$ spot prices are assigned to ND for use in the regression.
Table A3. Residential Sector Coal Spot Price Assignments from $C \& Q, 1979$ Forward

\begin{tabular}{clccc}
\hline State & \multicolumn{1}{c}{ Years } & $\begin{array}{c}\text { State Prices } \\
\text { Assigned }\end{array}$ & A \\
\cline { 1 - 2 } CO & 1979,1981 & KS & \\
CT & 1975 & NY & \\
& $1976-1979$ & NH & \\
& $1980-1987,1993$ & MA & \\
DC & $1976-1993$ & MD & R \\
ID & $1974,1979-1982$ & NV & E \\
& $1975-1977$ & SD & S \\
& 1978 & ND & I \\
& $1983-1993$ & CO & D \\
MA & 1975 & VT & E \\
& $1976-1979$ & NH & N \\
ME & $1974,1975,1981,1983$ & VT & T \\
& $1976-1980,1982$ & NH & I \\
& $1984-1993$ & MA & A \\
MT & $1974,1975,1978$ & ND & L
\end{tabular}

Price estimates for 1974 through 1978 for some States are not available because there was no consumption. To calculate prices for 1979 forward, these States are assigned the final prices from selected States as shown in Table A4.

In addition, several States are assigned the simple average of the final prices of adjacent States as shown in Table A4. 


\begin{tabular}{|c|c|c|}
\hline State & Years & $\begin{array}{l}\text { State and Averaged } \\
\text { Final Prices Assigned }\end{array}$ \\
\hline \multirow[t]{3}{*}{$A R$} & $\begin{array}{l}\text { 1980, 1982, 1984, 1985, } \\
1987-1993\end{array}$ & $A L$ \\
\hline & $\begin{array}{l}1981 \\
1983\end{array}$ & MO, OK, TN, TX \\
\hline & $1982,1984,1985$ & CA, NM, NV, UT \\
\hline & $1987,1988,1990-1993$ & UT \\
\hline \multirow[t]{2}{*}{$\mathrm{CA}$} & $1979-1985$ & NV \\
\hline & $1987-1993$ & WA \\
\hline FL & $1980-1993$ & $\mathrm{GA}$ \\
\hline LA & $\begin{array}{l}1980,1982,1984,1986 \\
1988,1991,1993\end{array}$ & AL \\
\hline \multirow[t]{2}{*}{ MS } & $\begin{array}{l}1979,1980,1983,1984 \\
1986-1993\end{array}$ & $A L$ \\
\hline & 1985 & $A L, A R, T N$ \\
\hline MT & 1986-1993 & WY \\
\hline NM & 1979-1993 & $\mathrm{CO}$ \\
\hline OK & $1979-1993$ & $\mathrm{co}$ \\
\hline \multirow[t]{2}{*}{ OR } & $1979,1980,1982-1993$ & WA \\
\hline & 1981 & CA, ID, NV, WA \\
\hline TX & $1980-1982,1985-1993$ & co \\
\hline
\end{tabular}

\section{Prices: 1971 Through 1978}

For 1971 through 1978, Btu steam coal prices are calculated by using data from GHS. The price for a State is equal to the simple average of the city/utility price observations for that State. For 1971 and 1972, GHS reports physical unit prices rather than Btu prices (as published for 1973 through 1978) and, therefore, the State-level conversion factors for this sector from SEDS are used to convert to Btu prices for those years. AK residential coal prices are estimated by using a different methodology described on page 189.

A simple average of price observations in $\mathrm{CT}, \mathrm{MA}$, $\mathrm{ME}, \mathrm{NH}, \mathrm{RI}$, and VT is assigned to each of these States. To impute other missing prices in the 1971 through 1978 period, States are assigned simple averages of adjacent State prices or are directly assigned the single price of an adjacent or nearby State as listed in Table A5.

\section{Prices: 1970}

Since State-level coal price data for 1970 are not available from either GHS or CEQ, the 1970 residential sector coal prices are calculated by using the 1971 through 1978 data from the Statistical Yearbook for
Table A5. Residential Sector Spot Coal Price Assignments, 1971-1978

\begin{tabular}{lll}
\hline State & \multicolumn{1}{c}{ Years } & State Assigned or \\
Averaged Prices
\end{tabular}

the 39 States with some reported coal use from 1971 through 1983 and regression analysis.

For estimating the 1970 prices, States missing Statistical Yearbook data are assigned prices as follows: ID/1970 through 1978 from MT; MA/1976 through 1978 from CT; ME/1970 through 1978 from NH; RI/1973, 1975 through 1978 from CT; and WA/1970 through 1972 from OR. DC, DE, and MD are all assigned the combined Statistical Yearbook price for those States. Wherever individual State prices are unavailable, prices are assigned from an adjacent or nearby State as follows: CA from NV; NM from CO; OK from CO; OR from WA; and TX from CO. AK 
residential coal prices are estimated by using a different methodology, described below.

\section{Alaska Prices: All Years}

AK's Btu prices for 1981 forward are estimated from the WA State prices during that period. The average ratio of AK-to-WA prices during 1970 through 1977 is applied to the WA price each year that AK has consumption to estimate the $\mathrm{AK}$ price for the year.

AK physical unit prices for 1970 through 1977 are estimated by using the ratio of AK-to-U.S. electric utility sector prices.

\section{U.S. Prices: All Years}

U.S. Btu prices are calculated as the average of the State Btu prices, weighted by consumption data from SEDS.

\section{Data Sources}

\section{Prices}

1974 forward: Energy Information Administration, Cost and Quality of Fuels for Electric Plants, average spot coal prices, Table 2 (1974-1979), Table 44 (19801982), Table 49 (1983, 1984), Table 39 (1985-1989), Table 8 (1990, 1991), and Table $3(1992,1993)$.

1971-1978: American Gas Association, Gas Househeating Survey, table titled "Competitive Fuel Prices."

1970-1978: Edison Electric Institute, Statistical Yearbook of the Electric Utility Industry, Table 43S.

\section{Consumption}

1970 forward: Energy Information Administration, State Energy Data System, residential sector coal consumption.

Conversion Factors: 1971, 1972

Energy Information Administration, State Energy Data Report 1993, Consumption Estimates, Table D8.

\section{Commercial Sector}

Commercial sector prices are assigned from industrial steam coal prices. States without Btu industrial steam coal prices were assigned the prices from
Table A6. Commercial Sector Final Price Assignments 1980 Forward

\begin{tabular}{|c|c|c|}
\hline State & Years & $\begin{array}{c}\text { State } \\
\text { Prices Assigned }\end{array}$ \\
\hline CT & 1980 & MA \\
\hline$D C$ & $1980-1993$ & $\mathrm{MD}$ \\
\hline OK & 1970 & $\mathrm{KS}$ \\
\hline RI & $1982,1983,1991-1993$ & MA \\
\hline VT & 1993 & $\mathrm{NH}$ \\
\hline \multicolumn{3}{|c|}{$\begin{array}{l}\text { adjacent States, as shown in Table A6. U.S. Btu } \\
\text { prices are calculated as the average of the State Btu } \\
\text { prices, weighted by consumption data from SEDS. }\end{array}$} \\
\hline
\end{tabular}

\section{Industrial Sector}

For 1980 forward, prices are based on the average price of coal receipts at manufacturing plants. For 1970 through 1979, prices are based on the average cost of coal sold to manufacturing firms.

\section{Physical Unit Prices: 1980 Forward}

For 1984 forward, State prices are taken from the Quarterly Coal Report (QCR). The annual price is estimated as the average of the quarterly prices weighted by the quarterly receipts. For 1980 through 1983, prices are taken directly from Form EIA-3 data. Published prices in average cost per ton are used directly when available, while missing prices are estimated by using simple averages or price assignments from adjacent States. Although it is not clear from the data collection forms, it is assumed that the prices include taxes. Analysts familiar with the survey believe that respondents would report the total cost of the coal (including cost, insurance, freight, and taxes) because that value is typically carried as the total expense in their bookkeeping systems.

Missing State data are estimated by using simple averages of the published data for adjacent States. In a few cases, only a single adjacent State or Census division price is available for the estimation. The adjacent State and Census division price assignments used for estimations are shown in Table A7.

\section{Physical Unit Prices: 1971, 1974 Through 1979}

For 1971 and 1974 through 1979, available cost and quantity of bituminous coal, lignite, and anthracite from Annual Survey of Manufactures (ASM) or Census 
Table A7. Industrial Sector Steam Coal Price Estimates for 1980 Forward

A
L
I
D
S
T
I
A
L

\begin{tabular}{|c|c|c|}
\hline State & Years & $\begin{array}{l}\text { Prices Used } \\
\text { in the Estimation }\end{array}$ \\
\hline$A Z$ & $\begin{array}{l}1980 \\
1981,1984,1985\end{array}$ & $\begin{array}{l}\text { CA, UT } \\
\text { CA, CO, UT }\end{array}$ \\
\hline $\mathrm{CO}$ & $1980^{\circ}$ & KS, UT \\
\hline CT & $1981-1993$ & New England \\
\hline$D C$ & 1980,1981 & MD \\
\hline DE & $1980-1993$ & MD \\
\hline $\mathrm{FL}$ & 1980 & $A L, G A$ \\
\hline $\mathrm{HI}$ & 1982-1993 & $\mathrm{CA}$ \\
\hline LA & $1980-1985,1989-1993$ & $A R, T X$ \\
\hline MA & $1980-1983$ & NY \\
\hline & 1984-1993 & New England \\
\hline $\mathrm{ME}$ & $1980-1983$ & NY \\
\hline & $1984-1993$ & New England \\
\hline MS & $1980-1993$ & $A L, A R, T N$ \\
\hline MT & $\begin{array}{l}1983,1986-1993 \\
1984,1985\end{array}$ & $\begin{array}{l}\text { ID, WY } \\
\text { ID }\end{array}$ \\
\hline ND & $\begin{array}{l}1980-1982 \\
1983-1993\end{array}$ & $\begin{array}{l}\text { MN, MT } \\
\text { MN }\end{array}$ \\
\hline $\mathrm{NE}$ & $\begin{array}{l}1980 \\
1982,1983,1986-1993\end{array}$ & $\begin{array}{l}\text { IA, KS, MO } \\
\text { CO, IA, KS, MO, WY }\end{array}$ \\
\hline $\mathrm{NH}$ & $\begin{array}{l}1980-1983 \\
1984-1993\end{array}$ & $\begin{array}{l}\text { NY } \\
\text { New England }\end{array}$ \\
\hline NJ & $1980-1993$ & NY, PA \\
\hline NM & $\begin{array}{l}1980 \\
1981,1984-1988 \\
1982,1983 \\
1989-1993\end{array}$ & $\begin{array}{l}\text { TX, UT } \\
\text { CO, OK, TX } \\
A Z, C O, O K, T X \\
A Z, C O, T X\end{array}$ \\
\hline NV & $\begin{array}{l}1980,1981,1984,1985 \\
1983,1986-1993\end{array}$ & $\begin{array}{l}\mathrm{CA}, \mathrm{ID}, \mathrm{UT} \\
\mathrm{AZ}, \mathrm{CA}, \mathrm{ID}, \mathrm{UT}\end{array}$ \\
\hline OK & $1980,1989-1993$ & $\mathrm{AR}, \mathrm{KS}, \mathrm{MO}, \mathrm{TX}$ \\
\hline OR & $\begin{array}{l}1980,1981,1983-1993 \\
1982\end{array}$ & $\begin{array}{l}C A, I D, W A \\
C A, I D, N V, W A\end{array}$ \\
\hline $\mathrm{Rl}$ & $\begin{array}{l}1980,1981 \\
1984-1989,1992,1993\end{array}$ & $\begin{array}{l}\text { NY } \\
\text { New England }\end{array}$ \\
\hline$S D$ & $\begin{array}{l}1980 \\
1981 \\
1982 \\
1983,1986-1993 \\
1984,1985\end{array}$ & $\begin{array}{l}\text { IA, MN, MT } \\
\text { IA, MN, MT, NE } \\
\text { IA, MN, MT, WY } \\
\text { IA, MN,WY } \\
\text { IA, MN, NE }\end{array}$ \\
\hline VT & $\begin{array}{l}1980-1983 \\
1984-1993\end{array}$ & $\begin{array}{l}\text { NY } \\
\text { New England }\end{array}$ \\
\hline WV & 1980 & $\mathrm{KY}, \mathrm{MD}, \mathrm{OH}, \mathrm{PA}, \mathrm{VA}$ \\
\hline$W Y$ & $\begin{array}{l}1980 \\
1981 \\
1984,1985\end{array}$ & $\begin{array}{l}\text { ID, MT, UT } \\
\text { CO, ID, MT, NE, UT } \\
\text { CO, ID, NE, UT }\end{array}$ \\
\hline
\end{tabular}

of Manufactures (CM) are used to calculate prices as average cost per unit of sales for covered States. (States with undisclosed data are not considered covered.) Although it is not clear from the data sources, the prices probably include taxes as explained for prices in the 1980 forward period.
For States with industrial steam coal use and for which $A S M$ or CM data are not available in 1971 and 1974 through 1979, adjacent State simple averages of available $A S M / C M$ data are used to impute prices. The assigned prices from adjacent States are shown in Table A8.

\section{Physical Unit Prices: 1970, 1972, 1973}

Steam coal industrial sector prices for 1970,1972, and 1973 (years for which no $A S M / C M$ prices are available) are estimated by using regression techniques. Values for the independent variable are steam coal electric utility sector physical unit prices and values for the dependent variable are the steam coal industrial physical unit prices (from $A S M$ or estimated as described above) for 1971, and 1974 through 1977. A few States are assigned electric utility prices for the dependent variable in the regression, as shown in Table A10.

Wherever individual State prices remain unavailable after the estimation using the above regression techniques, prices are assigned from adjacent or nearby States, as shown in Table A9.

\section{Physical Unit Prices: Alaska, All Years}

Steam coal industrial sector prices are estimated for Alaska by assumtng that the ratio of the Alaska price to the WA price in the industrial sector is the same as the ratio of the AK and WA prices in the electric utility sector for 1993. In 1970 through 1992, the ratio of the AK price to the U.S. price in the electric utility sector is used in the estimation.

\section{Btu Prices: All Years}

Btu prices for States are calculated from the physical unit prices and the conversion factors, which vary by State and by year. U.S. Btu prices are calculated as the average of the State Btu prices, weighted by consumption data from SEDS, adjusted for process fuel and coking coal consumption.

\section{Data Sources}

Prices

1984 forward: Energy Information Administration, Quarterly Coal Report, Tables 16 and 17 (1984 1st quarter through 1985 2nd quarter and 1986, 1987, and 1988 1st quarters); Tables 18 and 19 (1985 3rd and 4th quarters, 1986, 1987, and 1988 2nd through 
Table A8. Industrial Sector Steam Coal Price Estimates for 1971 and 1974-1979

\begin{tabular}{|c|c|c|}
\hline State & Years & $\begin{array}{l}\text { State Prices Used } \\
\text { in the Estimation }\end{array}$ \\
\hline AR & $\begin{array}{l}1971,1972,1974,1975 \\
1979\end{array}$ & $\begin{array}{l}\text { MO, TN } \\
\text { MO, TN, TX }\end{array}$ \\
\hline$A Z$ & $\begin{array}{l}1971 \\
1974-1978\end{array}$ & $\begin{array}{l}\text { CA, NV, UT } \\
\text { CA, UT }\end{array}$ \\
\hline co & $\begin{array}{l}1974-1978 \\
1979\end{array}$ & $\begin{array}{l}\mathrm{KS}, \mathrm{NE}, \mathrm{UT} \\
\mathrm{UT}\end{array}$ \\
\hline CT & $\begin{array}{l}1974-1978 \\
1979\end{array}$ & $\begin{array}{l}\text { MA, NY } \\
\text { NY }\end{array}$ \\
\hline$D C$ & $1971,1974-1979$ & MD, VA \\
\hline $\mathrm{DE}$ & $1971,1974-1979$ & $\mathrm{MD}, \mathrm{NJ}, \mathrm{PA}$ \\
\hline FL & 1979 & $A L, G A$ \\
\hline ID & 1974 & OR, UT \\
\hline & $\begin{array}{l}1975-1978 \\
1979\end{array}$ & $\begin{array}{l}\text { UT } \\
\text { UT, WA }\end{array}$ \\
\hline KS & 1979 & MO \\
\hline LA & $\begin{array}{l}1978 \\
1979\end{array}$ & $\begin{array}{l}\text { AR } \\
\text { TX }\end{array}$ \\
\hline MA & 1979 & NY \\
\hline ME & $\begin{array}{l}1975-1978 \\
1979\end{array}$ & $\begin{array}{l}\text { MA } \\
\text { NY }\end{array}$ \\
\hline MS & $\begin{array}{l}1971,1974,1975,1979 \\
1976-1978\end{array}$ & $\begin{array}{l}\text { AL, TN } \\
A L, A R, T N\end{array}$ \\
\hline MT & $\begin{array}{l}1974-1978 \\
1979\end{array}$ & $\begin{array}{l}\text { MN, NE, UT } \\
M N, U T\end{array}$ \\
\hline ND & $1974-1979$ & MN \\
\hline NE & 1979 & $I A, M O$ \\
\hline NH & $1971,1974-1979$ & MA \\
\hline NM & $\begin{array}{l}1971 \\
1974,1976-1978 \\
1979\end{array}$ & $\begin{array}{l}\text { CO, OK, TX, UT } \\
\text { KS, UT } \\
\text { UT }\end{array}$ \\
\hline NV & $\begin{array}{l}1974 \\
1975-1979\end{array}$ & $\begin{array}{l}\text { CA, OR, UT } \\
\text { CA, UT }\end{array}$ \\
\hline OK & $\begin{array}{l}1974,1975 \\
1976-1978 \\
1979\end{array}$ & $\begin{array}{l}\text { KS, MO } \\
\text { AR, KS, MO } \\
\text { MO, TX }\end{array}$ \\
\hline OR & $\begin{array}{l}1975-1978 \\
1979\end{array}$ & $\begin{array}{l}C A \\
C A, W A\end{array}$ \\
\hline RI & $\begin{array}{l}1971,1974-1978 \\
1979\end{array}$ & $\begin{array}{l}\text { MA } \\
\text { NY }\end{array}$ \\
\hline SD & $\begin{array}{l}1971,1974 \\
1975-1978 \\
1979\end{array}$ & $\begin{array}{l}\text { IA } \\
\text { IA, MN, NE } \\
\text { IA, MN }\end{array}$ \\
\hline$T X$ & $\begin{array}{l}1974,1975 \\
1976-1978\end{array}$ & $\begin{array}{l}\text { KS } \\
\text { AR, KS }\end{array}$ \\
\hline VT & $\begin{array}{l}1971,1974-1978 \\
1979\end{array}$ & $\begin{array}{l}\text { MA } \\
\text { NY }\end{array}$ \\
\hline WA & $\begin{array}{l}1974 \\
1975-1978\end{array}$ & $\begin{array}{l}\text { CA, OR } \\
\text { CA }\end{array}$ \\
\hline WY & $\begin{array}{l}1974-1978 \\
1979\end{array}$ & $\begin{array}{l}\text { NE, UT } \\
\text { UT }\end{array}$ \\
\hline
\end{tabular}

Table A9. Industrial Sector Final Price Assignments for 1970,1972 and 1973

\begin{tabular}{lll}
\hline State & \multicolumn{1}{c}{ Years } & \multicolumn{1}{c}{$\begin{array}{c}\text { State } \\
\text { Prices Assigned }\end{array}$} \\
\hline AR & 1972 & MO, TN \\
NH & $1970,1972,1973$ & MA \\
RI & $1970,1972,1973$ & MA \\
SD & $1970,1972,1973$ & IA \\
VT & $1970,1972,1973$ & MA \\
\hline
\end{tabular}

4th quarters, 1989-1991 1st through 4th quarters); and Tables 40 and 41 (1992 and 1993 1st through 4th quarters).

1980-1983: Energy Information Administration, "Quarterly Coal Consumption Report: Manufacturing Plants" (Form EIA-3). Only published data are used from Table 25 (1980), Table 11 (1981, 1982), and Table 2 (1983).

1971, 1974-1979: Bureau of the Census, U.S. Department of Commerce, Annual Survey of Manufactures and Census of Manufactures, Table 4 (1971) and Table 3 (1974-1979).

1970, 1972, 1973: Steam coal electric utility physical unit prices.

Consumption

1970 forward: Energy Information Administration, State Energy Data System, industrial sector coal consumption.

Table A10. Industrial Sector Price Assignments Used in the Regression Equation for 1971, and 1974-1979

\begin{tabular}{lll}
\hline State & Years & $\begin{array}{c}\text { State } \\
\text { Prices Assigned }\end{array}$ \\
\hline AR & $1973-1977$ & MO \\
CA & $1970-1977$ & NV \\
CT & $1975-1977$ & NY \\
DC & 1976,1977 & MD \\
ID & $1970-1977$ & MT \\
MA & 1976,1977 & NH \\
ME & $1970-1977$ & NH \\
OK & $1973-1975$ & KS \\
OR & $1973-1977$ & WA \\
TX & 1970 & NM \\
WA & $1970-1972$ & OR \\
\hline
\end{tabular}


Energy Information Administration, State Energy Data Report 1993, Consumption Estimates, Tables D10 and D11.

C The conversion factor for CT/1990 is assigned from $\mathrm{CT} / 1989$; the $\mathrm{NH} / 1976$ conversion factor is assigned from NH/1975; the RI/1977 conversion factor is assigned from RI/1976; and the VT/1986 conversion factor is assigned from VT/1985.

\section{Transportation Sector}

Transportation sector steam coal prices are assigned from industrial sector steam coal prices. U.S. Btu prices are calculated as the average of the State Btu prices, weighted by SEDS consumption data.

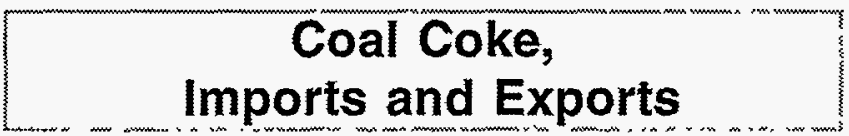

Imports and exports of coal coke are components of total U.S. energy consumption and are accounted for in the industrial sector. Prices and values of imports and exports are developed only for the United States; no attempt is made to estimate State-level prices or expenditures. Prices are f.a.s. (free alongside ship) values and do not include taxes. The quantities of U.S. coal coke imports and exports are taken from SEDS.

\section{Physical Unit Prices: All Years}

For 1980 forward, the Coke Plant Report, the Quarterly Coal Report, and Bureau of the Census computer tapes provide physical unit coal coke import and export prices in dollars per short ton. For 1970 through 1979, Coke and Coal Chemicals, International Coal, and the Minerals Yearbook provide coal coke import and export physical unit quantities and values in short tons and dollars, respectively. Values are equivalent to expenditures.

\section{Btu Prices: All Years}

For 1980 forward, Btu prices are computed by dividing the physical unit prices by the conversion factor. For 1970 through 1979, physical unit prices are computed by dividing the import and export values by their respective quantities, and Btu prices are computed by dividing the physical unit prices by the conversion factor.

\section{Data Sources}

Prices

1989 forward: Bureau of the Census, U.S. Department of Commerce, electronic data from "Monthly Report IM 145" and "Monthly Report EM 545."

1981-1988: Energy Information Administration, Quarterly Coal Report, October-December issues, Tables A11 and A13 (1981-1985) and Tables A10 and A12 (1986-1988).

1980: Energy Information Administration, Coke Plant Report, Tables 7 and 8.

1978-1979: Energy Information Administration, Coke and Coal Chemicals 1979, Tables 5 and 6.

1977: National Coal Association, International Coal 1980, tables titled "U.S. Imports of Solid Fuels and Customs Value" and "U.S. Exports of Coke and Value."

1976: Energy Information Administration, Coke and Coal Chemicals, Tables 19 and 20.

1970-1975: Bureau of Mines, U.S. Department of the Interior, Minerals Yearbook, "Coke and Coal Chemicals" chapter, Tables 19 and 20.

\section{Consumption}

1970 forward: Energy Information Administration, State Energy Data System, U.S. imports and exports of coal coke.

\section{Conversion Factor: All Years}

24.8 million Btu per short ton. 


\section{Section 3. Natural Gas}

Natural gas prices are developed for the residential, commercial, industrial, and electric utility sectors. Natural gas used for transportation is consumed solely in pipeline operations and is discussed in Section 6, "Consumption Adjustments for Calculating Expenditures," on page 249. Reported natural gas prices are retail prices for sales of natural gas to ultimate users.

In general, taxes are included in the prices. However, taxes collected by a utility from an end user and turned over to a Government authority frequently are not included in the revenues reported in the Natural Gas Annual or Natural Gas Production and Consumption and, therefore, are not included in the prices. Taxes paid by the utility (rather than the end user) are considered operating costs and are passed on to the end user as part of the rate. Therefore, Federal, State, business, and property taxes are typically included in the prices, while sales and other point-ofpurchase taxes typically are not.

Estimates of the amount of natural gas consumed by the residential, commercial, industrial, and electric utility sectors are taken from the State Energy Data System (SEDS). Estimates for the industrial sector are adjusted to remove estimated refinery consumption and lease and plant use of natural gas in each State. (See the discussion in Section 6, "Consumption Adjustments for Calculating Expenditures," on page 249.)

\section{Residential, Commercial, and Industrial Sectors}

\section{Prices: 1987 Forward}

All natural gas physical unit prices by State for the residential, commercial, and industrial sectors are taken from the Natural Gas Annual (NGA).

\section{Prices: 1970 Through 1986}

All natural gas physical unit prices for the residential, commercial, and industrial sectors are calculated from value and quantity of sales data from the Natural Gas Annual or its predecessor report, Natural Gas Production and Consumption. State prices are calculated directly from the data sources as average revenue per unit of sales by natural gas utilities. Prices for each of the three sectors are calculated by dividing the value of natural gas, reported in thousands of dollars, by the quantity of natural gas sold, as reported in million cubic feet.

For 1970 through 1979, both the value and quantity of sales data from the NGA are reported as composites for $\mathrm{MD}$ and $\mathrm{DC}$ and for $\mathrm{ME}, \mathrm{NH}$, and VT. In each case, the combined prices are assigned to each of the States in the composite.

\section{Btu Prices: All Years}

State Btu prices for all years are calculated by using the physical unit price series and the State-level nonelectric utility conversion factors. U.S. Btu prices are calculated as the average of the State Btu prices, weighted by consumption data from SEDS, adjusted for process fuel consumption in the industrial sector.

\section{Data Sources}

\section{Prices}

1980 forward: Energy Information Administration, Natural Gas Annual, Volume 1, Table 11 (1980), Table 14 (1981-1985), Table 15 (1986), Table 19 (1987), Table 22 (1988-1992), and Tables 25 (residential 1993) and 26 (commercial and industrial 1993).

1970-1979: Bureau of Mines, U.S. Department of the Interior, Natural Gas Production and Consumption, Table $6(1970,1979)$ and Table 7 (1971-1978). 
Consumption

1970 forward: Energy Information Administration, State Energy Data System, residential, commercial, and industrial natural gas consumption.

\section{Conversion Factors: All Years}

Energy Information Administration, factors published rounded in State Energy Data Report 1993, Consumption Estimates, Tables D4 and D5.

\section{Electric Utility Sector}

\section{Prices: 1973, 1974, 1983 Forward}

Prices for States are reported by Cost and Quality of Fuels for Electric Utility Plants (CEQ) for gas consumed at steam-electric plants only. Btu prices are taken from $C \mathcal{E} Q$, converted from cents to dollars per million Btu.

Where individual State prices are unavailable from CEQ, they are developed from the NGA. Physical unit prices prior to 1987 are calculated by dividing the value of natural gas, reported in thousands of dollars, by the quantity of natural gas sold, reported in million cubic feet. For 1987 forward, physical unit prices are taken directly from the NGA. Table A11 lists the States and years for which NGA data are used. Btu prices are calculated from the physical unit prices by using State-level electric utility conversion factors.

Prices are not available from either $C \mathcal{E} Q$ or $N G A$ for CT/1973; ID/1974, 1987; NH/1983; and OR/1983, $1984,1986,1989,1990$. In these cases, quantityweighted Census division prices from $C \mathcal{E} Q$ are assigned. In addition, prices for VT/1986 and WA/1986, 1987, 1990 use quantity-weighted Census division prices from $C \& Q$ for more consistent prices than those available from the NGA.

\section{Prices: 1980 Through 1982}

State Btu and physical unit prices for 1980 through 1982 are taken from $C E Q$ for all reporting plants. Physical unit prices are taken directly from the data source, while Btu prices are converted from cents to dollars per million Btu.
Table A11. Natural Gas Electric Utility Sector Prices from NGA, 1973 Forward

\begin{tabular}{ll}
\hline State & \multicolumn{1}{c}{ Years } \\
\hline AK & $1973-1990$ \\
CT & $1974-1976$ \\
ID & $1983-1986$ \\
MD & $1973,1974,1983-1985$ \\
NC & $1983-1990$ \\
ND & $1973,1974,1976-1986$ \\
NH & $1973,1974,1977,1987-1989$ \\
PA & 1973 \\
RI & 1976,1980 \\
SC & 1977 \\
SD & $1983-1990$ \\
TN & $1976,1980,1981,1983,1988-1993$ \\
UT & 1988,1989 \\
VT & $1983-1985,1989,1990$ \\
WA & $1978,1983-1985,1988,1989$ \\
WY & 1973,1975 \\
\end{tabular}

Note: NGA includes predecessor publications.

\section{Prices: 1975 Through 1979}

State prices are reported separately by $C \mathcal{E} Q$ for gas consumed at steam-electric plants and gas consumed at combustion turbine and internal combustion units. Weighted-average Btu prices are calculated by using the two $C \mathcal{E} Q$ prices and the respective gas deliveries for steam-electric and combustion use. The $\mathrm{NH} / 1977$ price is not available from CEQ. A combined price is computed from value and quantity of sales data from the $N G A$ for $\mathrm{ME}, \mathrm{NH}$, and VT and assigned to $\mathrm{NH} / 1977$.

\section{Prices: 1970 Through 1972}

State prices for 1970 through 1972 are taken from Natural Gas Production and Consumption and are calculated similarly to the way prices for the residential, commercial, and industrial sectors are calculated. Prices, as average revenue per unit of sales, are computed from value and quantity of sales data from the source reports. A combined price is reported for $\mathrm{NH}$ and VT for 1971 and 1972, and each of these States is assigned the combined price. State Btu prices are calculated from the physical unit prices by using the State-level electric utility conversion factors.

\section{U.S. Prices: All Years}

U.S. Btu prices are calculated as the average of the State Btu prices, weighted by consumption data from SEDS. 


\section{Data Sources}

Prices

1973 forward: Energy Information Administration, Cost and Quality of Fuels for Electric Utility Plants, tables numbered as follows:

\begin{tabular}{llc} 
Years & Price Data & Volume Data \\
\hline 1973,1974 & Table 10 & Table 9 \\
$1975-1979$ & Table 10, 16 & Table 9, 15 \\
$1980-1982$ & Table 48 & - \\
1983,1984 & Table 53 & - \\
$1985-1987$ & Table 43 & - \\
1988,1989 & Table 44 & - \\
1990,1991 & Table 19 & - \\
1992,1993 & Table 12 & - \\
\hline
\end{tabular}

1993: Energy Information Administration, Natural Gas Annual 1993, Table 26.

1980-1992: Energy Information Administration, Natural Gas Annual 1992, Volume 2, Table 23.
1976-1979: Energy Information Administration, Energy Data Reports, Natural Gas Production and Consumption, Table 7 (1976-1978) and Table 6 (1979).

1970-1975: Bureau of Mines, U.S. Department of the Interior, Natural Gas Production and Consumption, Table 6 (1970) and Table 7 (1971-1975).

\section{Consumption}

1970 forward: Energy Information Administration, State Energy Data System, electric utility natural gas consumption.

\section{Conversion Factors}

Btu prices that are calculated directly from Cost and Quality of Fuels for Electric Utility Plants (CEQ) require no conversion factors. When Natural Gas Annual (NGA) data are used to develop prices that are missing from $C \mathcal{E} Q$, conversion factors are used from the following source:

1970 forward: Energy Information Administration, State Energy Data Report 1993, Consumption Estimates, Tables D2 and D3. 


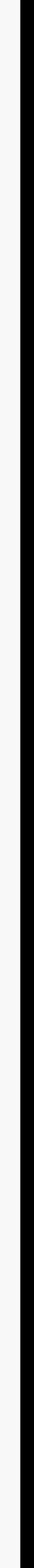




\section{Asphalt and Road Oil}

The State Energy Data System (SEDS) assumes that all asphalt and road oil consumption occurs in the industrial sector. Asphalt and road oil are used primarily for paving (79 percent of consumption in 1970 and 87 percent in 1993), with the remaining products used for roofing and sealing. Taxes are not included in the prices because most street and highway paving is done under contract to State, county, and other public authorities who are typically exempted from paying taxes.

\section{Physical Unit Prices: All Years}

Asphalt prices in physical units are developed from monthly reports in the Engineering News-Record, a construction industry weekly magazine published by McGraw-Hill, Inc. The source data consist of monthly reports from correspondents in 20 U.S. cities with price quotes for tank cars, drums, or both, for the three major types of asphalt products: asphalt cement (AC-20), asphalt emulsion (rapid set and slow set), and asphalt cutback.

For 1986 forward, the tank car price is used. However, for 1986 and 1987, the drum price is used if a tank car price is not available. For 1970 through 1985, when both tank car and drum prices are available, a simple average of the two prices is used. When only one price is available, that price is used.

Asphalt prices are developed by calculating a simple average annual price from the monthly prices for each city for the three products. City prices are assigned to States. CA, OH (1970 through 1985, 1992 forward), and PA have prices from two cities; in these cases, simple averages of the two city prices are used. No States have prices from more than two cities. States with no prices are assigned a Census division simple average price. State average asphalt prices are calculated as the quantity-weighted average prices of the three products for each State. If there is no Census division price, the simple average of the prices for the other Census divisions within that Census region is used. Quantity data for 1970 through 1980 are taken from the Bureau of Mines and EIA reports on sales of asphalt. Quantity data for 1981 forward are taken from the Report on Sales of Asphalt in the U.S., published by the Asphalt Institute. Non-paving asphalts are assumed to have the price of paving asphalt cement.

For 1970 through 1982, asphalt and road oil are estimated as separate data series. Asphalt prices are estimated as discussed above. Road oil prices are assumed to equal asphalt emulsion prices because specific prices are not available from any source.

\section{Btu Prices: All Years}

Asphalt prices in dollars per ton are converted to dollars per gallon by dividing by 235 gallons per ton for asphalt cement, 241 gallons per ton for emulsion, or 248.6 gallons per ton for cutback. These prices are then multiplied by 42 gallons per barrel and divided by 6.636 million Btu per barrel to get dollars per million Btu. Road oil unit prices of dollars per ton are converted to dollars per million Btu by using the constant conversion factors of 5.5 barrels per ton and 6.636 million Btu per barrel. The average price of all asphalt and road oil is the consumption-weighted average of the individual product prices.

U.S. Btu prices are calculated as the average of the State Btu prices, weighted by consumption data from SEDS.

\section{Data Sources}

Prices

1970 forward: McGraw-Hill, Inc., Engineering NewsRecord.

Quantities for Calculating Weighted Average Prices

1981 forward: Asphalt Institute, Asphalt Usage, United States and Canada. 
A 1978-1980: Energy Information Administration, Energy Data Reports, Sales of Asphalt, Table 2.

1977: Energy Information Administration, Energy Data Reports, Asphalt Sales, Annual, Table 2.

1971-1976: Bureau of Mines, U.S. Department of the Interior, Mineral Industry Survey, Asphalt Sales, Annual, Table 2.

1970: Bureau of Mines, U.S. Department of the Interior, Mineral Industry Survey, Asphalt Shipments, Annual, Table 2.

Consumption

1970 forward: Energy Information Administration, State Energy Data System, industrial sector, asphalt and road oil consumption.

\section{Conversion Factors: All Years}

Conversion factors used are: 235 gallons per ton of asphalt cement; 241 gallons per ton of emulsion; 248.6 gallons per ton of cutback; 42 gallons per barrel; 5.5 barrels per ton of road oil; 6.636 million Btu per barrel.

\section{Aviation Gasoline}

Aviation gasoline prices are developed for the transportation sector. Estimates of the amount of aviation gasoline consumed by the transportation sector are taken from SEDS. Aviation gasoline prices are national averages, excluding taxes, developed from several sources, depending on the years. In all cases, physical unit prices are developed and then converted to Btu prices. Federal and State excise taxes, as well as State and local sales taxes, are not included.

\section{Physical Unit Prices: 1976 Forward}

Aviation gasoline prices for 1978 forward are assumed to be the national average refiners sales prices to end users published in the EIA's Annual Energy Review. The 1976 and 1977 prices are assumed to be the national average retail prices published in the EIA's Monthly Energy Review.

\section{Physical Unit Prices: 1970 Through 1975}

For 1970 through 1975, aviation gasoline prices are not available. Prices are derived by dividing the national motor gasoline prices for those years by the 1976 national motor gasoline price and applying those percent changes to the 1976 national aviation gasoline price.

\section{Btu Prices: All Years}

Aviation gasoline Btu prices are calculated by converting the physical unit prices from cents per gallon to dollars per barrel (42 gallons per barrel) and then to dollars per million Btu (5.048 million Btu per barrel).

\section{Data Sources}

Prices

1979 forward: Energy Information Administration, Annual Energy Review 1994, Table 5.20, row titled "Sales Prices to End Users: Aviation Gasoline."

1978: Energy Information Administration, Annual Energy Review 1993, Table 5.21, row titled "Sales Prices to End Users: Aviation Gasoline."

1976, 1977: Energy Information Administration, Monthly Energy Review, April 1984, page 106, column titled "Aviation Gasoline, Retail."

1970-1975: Energy Information Administration, Annual Energy Review 1989, Table 70, column titled "Motor Gasoline, Leaded Regular, Nominal."

\section{Consumption}

1970 forward: Energy Information Administration, State Energy Data System, transportation sector, aviation gasoline consumption.

\section{Conversion Factor: All Years}

5.048 million Btu per barrel.

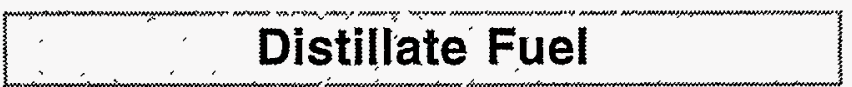

Distillate fuel prices are developed for all sectors. Distillate fuel in the transportation sector is assumed to be diesel fuel. Estimates of the amount of 
distillate fuel consumed in each sector are taken from the State Energy Data System (SEDS). Estimated consumption for the industrial sector is adjusted to remove the estimated refinery consumption of distillate fuel in each State. (See the discussion in Section 6, "Consumption Adjustments for Calculating Expenditures," on page 249.)

\section{Residential Sector}

Residential distillate prices are developed by using a variety of data sources and several estimation methods, depending on the years involved. In all cases, physical unit prices for States are developed first, then Btu prices are calculated by using the physical unit prices and the conversion factor. The prices contained in this series are the retail prices paid by consumers for residential heating oil, including taxes.

\section{Physical Unit Prices: 1983 Through 1990, 1992, and 1993}

For 1983 through 1990, 1992, and 1993 physical unit distillate prices in cents per gallon (excluding taxes) are generally available for 24 States from the Petroleum Marketing Annual (PMA). State-level prices for the States without $P M A$ prices are estimated by using price data from the American Gas Association (AGA), SEDS consumption data, and PMA Petroleum Administration for Defense (PAD) district prices. The estimation procedures are described below and include the addition of State general sales taxes.

1. State prices in cents per gallon are generally available from the PMA for the following 24 States: AK, CT, DC, DE, ID, IL, IN, MA, MD, ME, MI, MN, NH, NJ, NY, OH, OR, PA, RI, VA, VT, WA, WI, and WV. Prices for these States are converted from cents to dollars per gallon, and State general sales taxes from the Bureau of the Census publication State Government Tax Collections (SGTC) are added. The 1993 tax data are published in the Census publication State Tax Review (STR).

2. For the States that do not have prices in the $P M A$, prices are estimated by using AGA fuel oil prices, SEDS consumption data, and PMA PAD district prices for Districts II, III, IV, and V and Subdistrict IC (all the States in PAD Subdistricts IA and IB have published prices). The following steps are used to estimate the prices: a. Distillate prices from the PMA for PAD Districts II, III, IV, and V and Subdistrict IC are converted from cents per gallon to dollars per gallon.

b. The AGA lists fuel oil prices by company for the principal city served in dollars per million Btu, including State sales taxes. A simple average of the city-level prices is used to derive a State-level price for each of the States without PMA prices. These AGA State averages are converted from dollars per million Btu to dollars per gallon by using the AGA conversion factor of 7.194 gallons per million Btu. State general sales taxes from SGTC(STR) are subtracted to give State averages comparable to the PMA prices.

c. The AGA State prices derived in step $2 \mathrm{~b}$. are combined into PAD district averages by using SEDS consumption to weight each State's values. This procedure gives AGA consumption-weighted average prices for PAD Districts II, III, IV, and V and Subdistrict IC that are comparable to the volumeweighted PAD district prices published in the $P M A$. The AGA PAD district averages are calculated by using only the available States; if a State does not appear in the survey, it is not included in the PAD calculation.

d. Adjustment factors, ratios of the PMA PAD district price divided by the AGA derived PAD district price, are calculated for PAD Districts II, III, IV, and V, and Subdistrict IC.

e. Prices for the States not published in the PMA are calculated by multiplying the AGA State prices derived in step $2 \mathrm{~b}$. by the appropriate PAD district adjustment factor from step $2 \mathrm{~d}$. and adding State general sales taxes from the SGTC(STR).

f. States that do not have prices in either the $P M A$ or the AGA are assigned a PMA PAD district price, and State general sales taxes from the SGTC (STR) are added. The States with assigned PAD prices are as shown in Table A12.

\section{Physical Unit Prices: 1991}

Physical unit distillate prices in cents per gallon (excluding taxes) are available for 24 States from the PMA. Because prices are not available from AGA for 1991, State-level prices for the remaining 27 States 
Table A12. Distillate Residential Sector Price Assignments, 1983-1990, 1992, and 1993

\begin{tabular}{lll}
\hline State & \multicolumn{1}{c}{ Years } & Prices Assigned \\
\hline AR & 1988,1993 & PAD District III \\
AZ & 1992,1993 & PAD District V \\
CA & $1984,1992,1993$ & PAD District V \\
FL & 1993 & PAD District IC \\
HI & $1983-1990,1992,1993$ & PAD District V \\
IL & 1986 & PAD District II \\
KS & 1986,1989 & PAD District II \\
LA & 1986 & PAD District III \\
MS & $1983,1985,1986$ & PAD District III \\
NM & $1984-1990,1992,1993$ & PAD District III \\
OK & $1986,1989,1990,1992$, & PAD District II \\
& 1993 & \\
SD & 1986 & PAD District II \\
TX & 1992,1993 & PAD District III \\
UT & 1985 & PAD District IV \\
\end{tabular}

are estimated by using physical unit prices derived for 1990 in SEPEDS (described below) and the 1991 PMA Petroleum Administration for Defense (PAD) district prices. The estimation procedures are described below and include the addition of State general sales taxes.

1. State prices in cents per gallon are available from the PMA for the following 24 States: AK, CT, $\mathrm{DC}, \mathrm{DE}, \mathrm{ID}, \mathrm{IL}, \mathbb{I N}, \mathrm{MA}, \mathrm{MD}, \mathrm{ME}, \mathrm{MI}, \mathrm{MN}, \mathrm{NH}$, NJ, NY, OH, OR, PA, RI, VA, VT, WA, WI, and WV. Prices for these States are converted from cents to dollars per gallon, and State general sales taxes from the Bureau of the Census' State Government Tax Collections (SGTC) are added.

2. For the remaining 27 States that do not have prices in the $P M A$, prices are estimated by using the 1990 SEPEDS physical unit prices and PMA PAD district prices for 1990 and 1991. The following steps are used to estimate the prices:

a. For 1990, the Subdistrict IC price is withheld in the $P M A$ and the average of the VA and WV prices is used as the Subdistrict IC price.

b. The 1990 State prices derived from AGA and $P M A$, as described below, are adjusted by the percentage change in the 1990 and 1991 prices for each State's PMA PAD district or subdistrict. c. The State general sales taxes from SGTC are added.

\section{Physical Unit Prices: 1978 Through 1982}

Procedures for the 1978 through 1982 period are similar to those for 1983 forward except for changes in data sources. Annual physical unit prices are taken either directly from the Monthly Energy Review $(M E R)$ or are calculated from monthly regional price data, also from the MER. These data were collected on Form EIA-9A (formerly EIA Form 9 and FEA Form P112-M-1) and include taxes. Price data from Platt's Oil Price Handbook and Oilmanac (Platt's) and SEDS consumption data for 1978 through 1982 are used to compute State prices when only regional data are available. These calculations are described step-by-step below.

1. Annual State physical unit prices are generally available from the MER for the same 24 States covered by the PMA in 1983 and forward. These 24 States compose all of Federal Regions 1, 2, 3, 5 , and 10 (see Figure A2 on page 178). Prices for these States exclude taxes and are converted to dollars per gallon.

2. Of the States without MER prices, the 22 in Federal Regions 4, 7, 8, and 9 have annual prices estimated from the monthly Federal regional prices published in the MER. No regional prices are available for Federal Region 6 for the 1978 through 1982 period, and some monthly prices are missing in regions 7, 8, and 9 in 1980, 1981, and 1982.

a. Missing monthly prices for Federal regions are estimated with assigned prices as follows: the Region 9 November 1980 price is assigned to December 1980; an average of the Region 7 July and October 1982 prices is assigned to August and September 1982; an average of Region 8 June and September 1982 prices is assigned to July and August 1982; and an average of Region 3 August and October 1982 prices is assigned to the September 1982. Imputation of missing Region 6 prices for 1978 through 1982 and missing Region 9 prices for 1981 and 1982 is discussed later.

b. The simple average of monthly State-level normal heating degree-day data is averaged for all the States within each of the 10 Federal regions and used to estimate average Federal region heating degree-days. AK, DC, and HI 
are assigned the monthly heating degree-days from $M N, M D$, and $F L$, respectively.

c. Weighted average annual physical unit distillate prices for the residential sector are calculated for Federal Regions 4, 7, 8, and 9 (except for Region 9 in 1981 and 1982) by using the regional normal heating degree-days and the monthly regional prices from the MER.

d, In 1981, only March and May prices are available for Federal Region 9. To estimate the average annual price for this region, the relationship between the U.S. annual heating oil price (from the MER) and the U.S. March and May prices is expressed as a ratio and used with the Region 9 March and May prices to estimate the 1981 annual Region 9 price.

e. City-level prices from Platt's are assigned to States as shown in Table A13. The assigned State-level Platt's prices for States are consumption-weighted into Federal regions by using residential sector consumption data from SEDS.

f. Adjustment factors, ratios of the regional MER distillate prices to the regional Platt'sbased distillate prices, are calculated for Federal Regions 4, 7, 8, and 9 (except for 1982).

g. Since there are no monthly regional distillate prices from the MER for Federal Region 6 for 1978 through 1982 and Federal Region 9 for 1982, the adjustment factors for these regions are based on the adjustment factors for previous time periods. The Region 6 adjustment factor for each of the years in the 1978 through 1982 period is equal to 1.1313 , which is the average of the adjustment factor for the West South Central Census Division for 1976 and 1977. The Region 9 adjustment factor for 1982 is equal to 1.1995 , which is the average adjustment factor for Region 9 from 1978 through 1981.

h. The residential sector distillate State prices for the 27 States in Federal Regions 4, 6, 7, 8, and 9 are calculated by multiplying the regional adjustment factors, for each year and the State-level assigned Platt's prices.
For the years 1975 through 1977, no State-level data are available, and regional data from Form EIA-9A are available only at the Census division level, except for Federal region prices for November and December of 1977. Using a methodology similar to that described above for the allocation of regional data to States, adjustment factors are calculated at the regional level and applied to Platt's price data assigned to States. The resulting prices implicitly include average regional taxes but do not reflect individual State differences.

1. Monthly regional price data for 1975 and $\mathbf{1 9 7 6}$ are reported in the MER only for Census divisions. In 1977, however, monthly price data are reported for Census divisions for January through October and for Federal regions for November and December. The Federal region prices for November and December are assigned to their respective States and reaggregated into Census divisions in order to create a consistent set of monthly Census division prices for 1977. Annual residential sector distillate consumption data from SEDS are used to do the reaggregation.

2. The Census division monthly price data from the MER for 1975, 1976, and the first 10 months of 1977 are used with the estimated Census division price data for November and December 1977 to estimate State-level prices.

a. Missing monthly prices in the East South Central Division for June and November 1975 and the Mountain Division for March and July 1975 are estimated by using an average of the prices for the month preceding and the month following the missing month. Missing November and December West South Central Division prices in 1977 are estimated with the assignment of the October price to both months. No monthly price data are available for the West South Central Division in 1975; step $2 f$. below discusses how the calculations are handled for this division.

b. The monthly State-level normal heating degree-day data are averaged for the States within each Census division to estimate regional monthly heating degree-days. AK, $\mathrm{DC}$, and $\mathrm{HI}$ are assigned the monthly heating degree-days from MN, MD, and FL, respectively. 


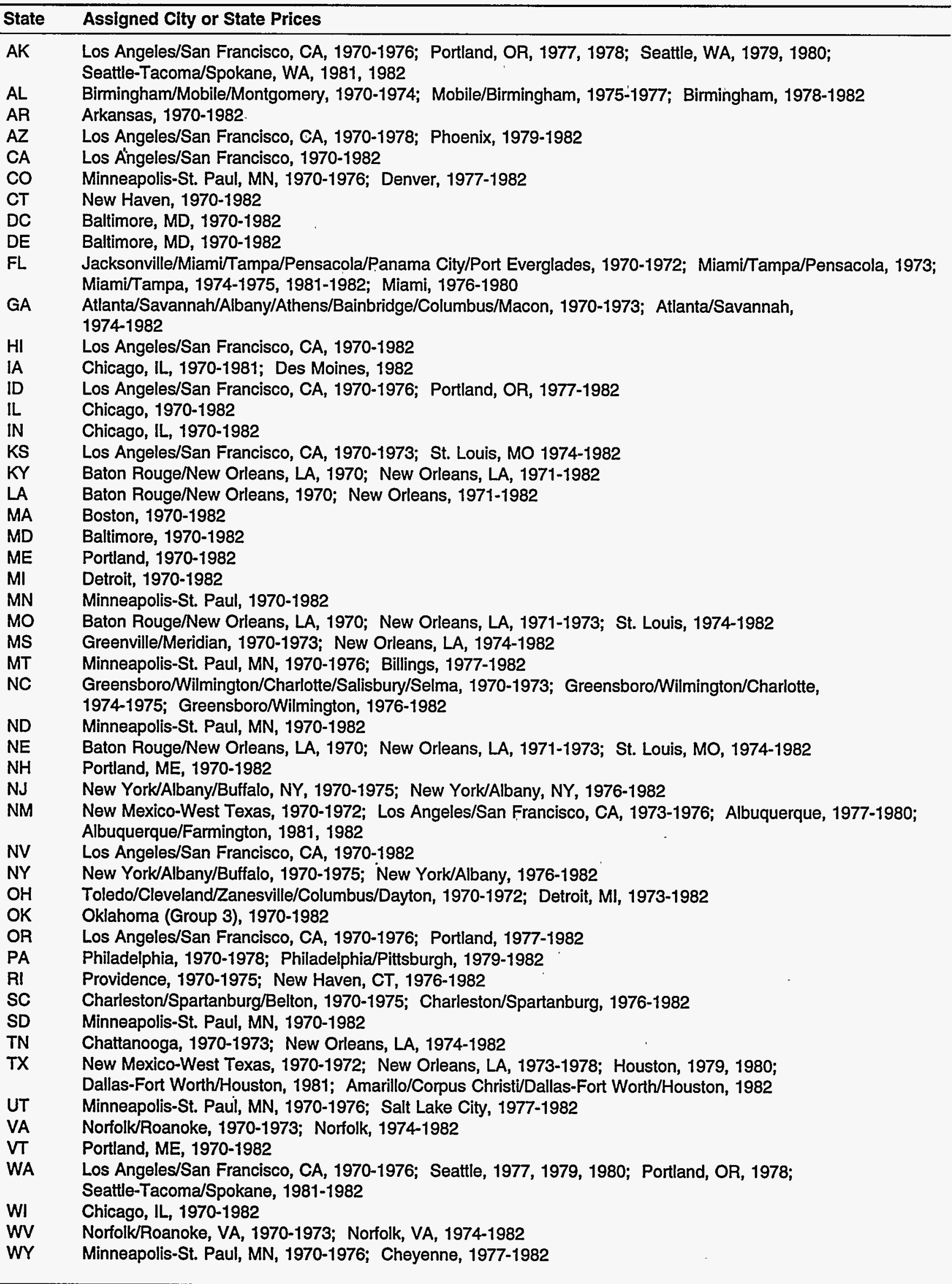


c. Weighted average annual distillate prices for Census divisions are calculated by using the monthly Census division price data from the $M E R$ and the normal heating degree-days estimated for Census divisions.

d. City-level No. 2 fuel oil refinery and terminal prices from Platt's for 1975 through 1977 are assigned to States as shown in Table A13. The assigned Platt's prices for States are consumption-weighted into Census divisions by using residential sector consumption data from SEDS.

e. Adjustment factors are calculated as the ratios of the $M E R$ distillate Census division prices to the Platt's distillate Census division prices.

f. Since there are no 1975 MER price data for the West South Central Division from which to calculate an adjustment factor, the 1975 adjustment factor for this region is assumed to be equal to the simple average of the West South Central adjustment factors for 1976 and 1977 (i.e., 1.1313).

g. The residential sector distillate State prices for all States are calculated by multiplying the regional adjustment factors for each year by the State-level assigned Platt's prices.

\section{Physical Unit Prices: 1970 Through 1974}

There are no regional or State-level distillate price data directly available for the 1970 through 1974 period. To estimate State prices, regional average prices are first derived from the relationship between U.S. prices and Federal region prices for 1975 through 1980. State prices are then estimated from the regional prices by using a methodology similar to that described for 1978 through 1982. The resulting prices implicitly include average regional taxes but do not reflect individual State differences.

1. The first step in the estimation of residential distillate prices for the 1970 through 1974 time period is to develop an equation that uses U.S. prices to estimate prices for Federal regions. Regression techniques are used for this purpose. U.S. prices for 1975 through 1980 from the Annual Energy Review ( $A E R$ ) are used as the independent variable for developing the equation; annual Federal region prices are used as the dependent variable. Federal region prices for 1978 through 1980 are calculated above, but MER prices for 1975 through 1977 are for Census divisions. To convert these annual Census division prices into Federal region prices, the estimated State prices for 1975 through 1977 are aggregated into Federal regions by using SEDS consumption data.

2. Regression techniques are applied to the pooled Federal region price data (dependent variable) and the U.S. prices from the $A E R$ (independent variable) for 1975 through 1980. U.S. prices for 1970 through 1974 are input to estimate annual Federal region prices for 1970 through 1974.

3. City-level prices from Platt's for 1970 through 1974 are assigned to States as shown in Table A13. The assigned State-level Platt's prices are consumption-weighted into Federal regions by using residential sector distillate consumption data from SEDS.

4. Adjustment factors, ratios of the regional $M E R$ distillate Federal region prices to the Platt'sbased distillate Federal region prices, are calculated.

5. The residential sector distillate prices for all States are calculated by multiplying the regional adjustment factors for each year by the Statelevel assigned Platt's prices.

\section{Btu Prices: All Years}

Btu prices for States are calculated by converting the physical unit prices from dollars per gallon to dollars per barrel (42 gallons per barrel) and then to dollars per million Btu (5.825 million Btu per barrel). U.S. Btu prices are calculated as the average of the State Btu prices, weighted by consumption data from SEDS.

\section{Data Sources}

\section{Prices}

1986 forward: Energy Information Administration, Petroleum Marketing Annual, Table 37 (1986-1988) and Table 39 (1989 forward), column titled "To Residential Consumers."

1983-1990, 1992, 1993: American Gas Association, Residential Natural Gas Market Survey (1989, 1990, 1992, 1993), Gas Househeating Survey (1983-1988), Appendix 2, "Competitive Fuel Prices," column titled "Fuel Oil." 
1993: Bureau of the Census, U.S. Department of Commerce, State Tax Review, Volume 54, No. 31, map titled "State Gasoline, Sales and Cigarette Tax Rates as of July 1, 1993."

1983-1992: Bureau of the Census, U.S. Department of Commerce, State Government Tax Collections, table titled "State Government Excises on General Sales, Motor Fuel, and Cigarettes, Beginning and End of Fiscal Year."

1983-1985: Energy Information Administration, $\mathrm{Pe}$ troleum Marketing Annual 1985, Volume 1, Table 26.

1970-1982: McGraw-Hill, Inc., Platt's Oil Price Handbook and Oilmanac, refinery and terminal prices for No. 2 fuel oil, average of highs and lows.

1975-1982: National Oceanic and Atmospheric Administration, U.S. Department of Commerce, State, Regional, and National Monthly and Seasonal Heating Degree-Days Weighted by Population (1980 Census), Historical Climatology Series 5-1, table titled "195180 State Pop. Wgt'd Heating Degree-Days."

1975-1982: Energy Information Administration, Monthly Energy Review, table titled "Residential Heating Oil Prices by Region," February 1978, page 67 (1975, 1976); April 1980, page 83 (1977, 1978); July 1982, page 87 (1979-1982).

1970-1982: Energy Information Administration, Annual Energy Review 1988, Table 67, "Motor Gasoline and Residential Heating Oil Prices, 1949-1988."

\section{Consumption}

1970 forward: Energy Information Administration, State Energy Data System, residential sector distillate consumption.

\section{Conversion Factor: All years}

5.825 million Btu per barrel

\section{Commercial Sector}

Commercial sector distillate prices are estimated by using several different data sources and estimation methodologies, depending on the years involved. For 1983 forward, retail prices paid by commercial/institutional establishments (excluding taxes) from Petroleum Marketing Annual (PMA) are supplemented with prices derived from the American Gas Association (AGA) Residential Gas Market Survey (except for 1991, when AGA data are not available). State general sales taxes from the Bureau of the Census are added. For 1970 through 1982, commercial distillate prices are based on refinery and terminal (wholesale) prices from Platt's and markups from Energy Prices: 1960-73 that include taxes. For both time periods, physical unit prices are calculated from the data sources, and Btu prices are computed by using the physical unit prices and the conversion factor.

\section{Physical Unit Prices: 1983 Through 1990, 1992, and 1993}

For 1983 through 1990, 1992, and 1993 physical unit distillate prices in cents per gallon (excluding taxes) are available for approximately 24 States from the $P M A$. State-level prices for the remaining States are estimated by using price data from the American Gas Association (AGA), SEDS consumption data and PMA Petroleum Administration for Defense (PAD) district prices. The estimation procedures are described below.

1. State prices in cents per gallon are generally available from the $P M A$ for the following 24 States: AK, CT, DC, DE, ID, IL, IN, MA, MD, ME, MI, MN, NH, NJ, NY, OH, OR, PA, RI, VA, VT, WA, WI, and WV. Prices for these States are converted from cents to dollars per gallon, and State general sales taxes from the Bureau of the Census' State Government Tax Collections (SGTC) are added. The 1993 tax data are published in the Census publication State Tax Review (STR).

2: For the States that do not have prices in the $P M A$, prices are estimated by using AGA fuel oil prices, SEDS consumption data, and PMA PAD district prices for Districts II, III, IV, and V and Subdistrict IC (all the States in PAD Subdistricts IA and IB have published prices). For 1990, the Subdistrict IC price is withheld in the PMA and the average of the VA and WV prices is used as the Subdistrict IC price. The following steps are used to estimate the prices:

a. Distillate prices from the PMA for PAD Districts II, III, IV, and V and Subdistrict IC are converted from cents per gallon to dollars per gallon.

b. The AGA lists fuel oil prices by company for the principal city served in dollars per million Btu, including State sales taxes. A simple average of the city-level prices is used to derive a State-level price for each of the 27 States without PMA prices. These AGA State 
averages are converted from dollars per million Btu to dollars per gallon by using the AGA conversion factor of 7.194 gallons per million Btu. State general sales taxes from SGTC (STR) are subtracted to give State averages comparable to the $P M A$ prices.

c. The AGA State prices derived in step $2 b$. are combined into PAD district averages by using SEDS consumption to weight each State's values. This procedure gives AGA consumptionweighted average prices for PAD Districts II, III, IV, and V and Subdistrict IC that are comparable to the volume-weighted PAD district prices published in the PMA. The AGA PAD district averages are calculated by using only the available States; if a State does not appear in the survey, it is not included in the PAD district calculation.

d. Adjustment factors, ratios of the PMA PAD district price divided by the AGA-derived PAD district price, are calculated for PAD Districts II, III, IV, and V, and Subdistrict IC.

e. Prices for the 27 States not published in the PMA are calculated by multiplying the AGA State prices derived in step $2 \mathrm{~b}$. by the appropriate PAD district adjustment factor from step $2 \mathrm{~d}$. and adding State general sales taxes from the $S G T C(S T R)$.

f. States that do not have prices in either the $P M A$ or the AGA are assigned a PMA PAD district price, and State general sales taxes from the SGTC (STR) are added. The States with assigned prices are as shown in Table A14.

\section{Physical Unit Prices: 1991}

Physical unit distillate prices in cents per gallon (excluding taxes) are available for 24 States from the $P M A$. Because prices are not available from AGA for 1991, State-level prices for the remaining 27 States are estimated by using physical unit prices derived for 1990 in SEPEDS (described below) and the 1991 PMA Petroleum Administration for Defense (PAD) district prices. The estimation procedures are described below.

1. State prices in cents per gallon are available from the PMA for the following 24 States: AK, CT, $\mathrm{DC}, \mathrm{DE}, \mathrm{ID}, \mathrm{IL}, \mathrm{IN}, \mathrm{MA}, \mathrm{MD}, \mathrm{ME}, \mathrm{MI}, \mathrm{MN}, \mathrm{NH}$, NJ, NY, OH, OR, PA, RI, VA, VT, WA, WI, and WV. Prices for these States are converted from
Table A14. Distillate Commercial Sector

Price Assignments, 1983-1990, 1992, and 1993

\begin{tabular}{lll}
\hline State & \multicolumn{1}{c}{ Years } & Prices Assigned \\
\hline AR & 1988,1993 & PAD District III \\
AZ & 1992,1993 & PAD District V \\
CA & $1984,1992,1993$ & PAD District V \\
FL & 1993 & PAD District IC \\
HI & $1983-1990,1992,1993$ & PAD District V \\
KS & 1986,1989 & PAD District II \\
LA & 1986 & PAD District III \\
MO & 1986 & PAD District II \\
MS & $1983,1985,1986$ & PAD District III \\
NM & $1984-1990,1992,1993$ & PAD District III \\
OK & $1986,1989,1990,1992$, & PAD District II \\
& 1993 & \\
SD & 1986 & PAD District II \\
TX & 1992,1993 & PAD District III \\
UT & 1985 & PAD District IV \\
\end{tabular}

cents to dollars per gallon, and State general sales taxes from the Bureau of the Census' State Government Tax Collections (SGTC) are added.

2. For the remaining 27 States that do not have prices in the $P M A$, prices are estimated by using the 1990 SEPEDS physical unit and PMA PAD district prices for 1990 and 1991. The following steps are used to estimate the prices:

a. For 1990, the Subdistrict IC price is withheld in the PMA and the average of the VA and WV prices is used as the Subdistrict IC price. For 1991, the PAD District III price is withheld in the PMA. The ratio of the PAD District II commercial consumers price to the PAD District II industrial price is applied to the PAD District III industrial price to estimate the withheld commercial price.

b. The 1990 State prices derived from AGA and $P M A$, as described below, are adjusted by the percentage change in the 1990 and 1991 prices for each State's PMA PAD district or subdistrict.

c. The State general sales taxes from SGTC are added.

\section{Physical Unit Prices: 1970 Through 1982}

Commercial sector distillate physical unit prices for 1970 through 1982 are calculated by using Platt's 
prices assigned to States and commercial sector markups estimated from Energy Prices: 1960-73. The resulting estimates implicitly include State-specific taxes.

1. The first step is to compute the markups. Energy Prices contains single price estimates for small commercial users and two price estimates for large commercial users for 10 cities: Boston, MA; Albany, NY; New York, NY; Charlotte, NC; Washington, DC; Chicago, IL; Detroit MI; Minneapolis/St. Paul, MN; St. Louis, $\mathrm{MO}$; and Seattle, WA. First, a simple average of the two large commercial prices is calculated for each city except for Albany and New York. In this case, all four large commercial prices are averaged together, since cities are assigned to their respective States.

2. For the nine States covered by the Energy Prices data (noted in step 1), the markup of the reported prices from Energy Prices over the assigned Platt's prices (Table A13 on page 198) and the markup of the residential prices calculated above for 1970 through 1972 over the Platt's prices is calculated.

3. At this point, residential and commercial sector retail markups have been computed for nine States for each of the years 1970 through 1972. The next step is to calculate the average retail markup for the 3-year period for each sector. A simple average of the markup ratios is calculated.

4. The average commercial and residential sector retail markups for the nine available States are assigned as shown in Table A15.

5. To translate the average commercial and residential markups for 1970 through 1972 into the estimated commercial sector retail markups to be used for 1970 through 1982, the relationship between these two markups is used, with the residential markups calculated for all States for each year. The calculation of the residential markups follows the same procedure used in step 2. above.

6. The commercial sector adjustment factors for each State for each of the years 1970 through 1982 are multiplied by the corresponding Platt's prices for 1970 through 1982 to calculate the final commercial sector physical unit prices.
Table A15. Distillate Fuel Commercial Sector Average Retail Markup Price Assignments, 1970-1972

\begin{tabular}{|c|c|}
\hline State & City Price Assignments \\
\hline AK & Seattle, WA \\
\hline $\mathrm{AL}$ & Charlotte, NC \\
\hline AR & St. Louis, MO \\
\hline$A Z$ & Seattle, WA \\
\hline CA & Seattle, WA \\
\hline $\mathrm{CO}$ & Minneapolis-St. Paul, MN \\
\hline CT & Boston, MA \\
\hline $\mathrm{DC}$ & Washington, DC \\
\hline$D E$ & Washington, DC \\
\hline $\mathrm{FL}$ & Charlotte, NC \\
\hline GA & Charlotte, NC \\
\hline $\mathrm{HI}$ & Seattle, WA \\
\hline IA & St. Louis, MO \\
\hline ID & Seattle, WA \\
\hline IL & Chicago, IL \\
\hline IN & Chicago, IL \\
\hline KS & St. Louis, MO \\
\hline $\mathrm{KY}$ & Chicago, IL \\
\hline LA & St. Louis, MO \\
\hline MA & Boston, MA \\
\hline MD & Washington, DC \\
\hline ME & Boston, MA \\
\hline MI & Detroit, MI \\
\hline MN & Minneapolis-St. Paul, MN \\
\hline MO & St. Louis, MO \\
\hline MS & Charlotte, NC \\
\hline MT & Minneapolis-St. Paul, MN \\
\hline NC & Charlotte, NC \\
\hline ND & Minneapolis-St. Paul, MN \\
\hline NE & St. Louis, MO \\
\hline NH & Boston, MA \\
\hline $\mathrm{NJ}$ & Albany and New York, NY \\
\hline NM & Seattle, WA \\
\hline NV & Seattle, WA \\
\hline NY & Albany and New York, NY \\
\hline $\mathrm{OH}$ & Detroit, MI \\
\hline OK & St. Louis, MO \\
\hline OR & Seattle, WA \\
\hline PA & Albany and New York, NY \\
\hline RI & Boston, MA \\
\hline SC & Charlotte, NC \\
\hline SD & Minneapolis-St. Paul, MN \\
\hline TN & Chicago, IL \\
\hline$T X$ & St. Louis, MO \\
\hline UT & Minneapolis-St. Paul, MN \\
\hline VA & Washington, DC \\
\hline VT & Boston, MA \\
\hline WA & Seattle, WA \\
\hline WI & Chicago, IL \\
\hline WV & Washington, DC \\
\hline WY & Minneapolis-St. Paul, MN \\
\hline
\end{tabular}


Btu prices for States are calculated by converting the physical unit prices from cents to dollars per gallon, then to dollars per barrel ( 42 gallons per barrel) and, finally, to dollars per million Btu (5.825 million Btu per barrel). U.S. prices are calculated as the average of the State Btu prices, weighted by consumption data from SEDS.

\section{Data Sources}

\section{Prices}

1986 forward: Energy Information Administration, Petroleum Marketing Annual, Table 37 (1986-1988) and Table 39 (1989 forward), column titled "To Commercial/Institutional Consumers."

1983-1990, 1992, 1993: American Gas Association, Residential Natural Gas Market Survey (1989, 1990, 1992, 1993), Gas Househeating Survey (1983-1988), Appendix 2, "Competitive Fuel Prices," column titled "Fuel Oil."

1993: Bureau of the Census, U.S. Department of Commerce, State Tax Review, Volume 54, No. 31, map titled "State Gasoline, Sales and Cigarette Tax Rates as of July 1,1993."

1983-1992: Bureau of the Census, U.S. Department of Commerce, State Government Tax Collections, table titled "State Government Excises on General Sales, Motor Fuel, and Cigarettes: Beginning and End of Fiscal Year."

1983-1985: Energy Information Administration, $\mathrm{Pe}$ troleum Marketing Annual 1985, Volume 1, Table 26.

1970-1982: McGraw-Hill, Inc., Platt's Oil Price Handbook and Oilmanac, refinery and terminal prices for No. 2 fuel oil, average of highs and lows.

1970-1982: Foster Associates, Inc., 1974, Energy Prices 1960-73, Tables 4-c and 5-b.

\section{Consumption}

1970 forward: Energy Information Administration, State Energy Data System, commercial sector distillate consumption.

\section{Conversion Factor: All Years}

5.825 million Btu per barrel
The electric utility price for distillate fuel is the average delivered cost of No. 2 fuel oil receipts at electric utilities (See Light Oil (Utilities) on page 214). For 1973 forward, these prices are taken from Cost and Quality of Fuels for Electric Utility Plants; for 1970 through 1972, prices from the Statistical Yearbook of the Electric Utility Industry are used with regression analysis. Btu prices are developed directly from the data sources and include all applicable taxes.

\section{Prices: 1973 Forward}

Btu prices for the years 1973 forward are based on the Btu prices reported in Cost and Quality of Fuels (CEQ). For 1973, 1974, and 1980 forward, Btu prices are taken directly from the data source. Btu prices in cents per million Btu ppublished in C\&Q are converted to dollars per million Btu. For 1975 through 1979, consumption-weighted average Btu prices are calculated from prices and consumption reported separately for steam-electric plants and for combustion turbine and internal combustion units. Because 1973 was the first year $C \& Q$ was published, there are many States with missing data. The estimation of the missing 1973 State prices follows the same procedure as prices for 1970 through 1972 and is discussed under that section.

Wherever individual State prices are unavailable, quantity-weighted Census division prices from $C \& Q$ are assigned as shown in Table A16.

U.S. Btu prices for all years are calculated as the average of the State Btu prices, weighted by consumption data from SEDS.

\section{Prices: 1970 Through 1972}

Btu prices for 1970 through 1972 (and AK and HI for 1973) are estimated by using data from Statistical Yearbook of the Electric Utility Industry. U.S. prices are then computed by using the State-level prices and the electric utility distillate consumption data from SEDS.

1. Regression techniques are used to arrive at the equation for estimating electric utility sector distillate prices for the 1970 through 1972 (or 1973) period. AL is treated as the reference State. The regression equation uses Statistical Yearbook State-level prices for 1974 through 1980 as the independent variable and the State-level prices calculated above for 1974 through 1980 as the

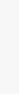




\begin{tabular}{|c|c|c|}
\hline & $\begin{array}{l}\text { Census Division } \\
\text { from } C \& Q, 1973 F\end{array}$ & $\begin{array}{l}\text { ce Assignments } \\
\text { ward }\end{array}$ \\
\hline State & Years & Census Division \\
\hline $\mathrm{CA}$ & $\begin{array}{l}\text { 1983-1985, 1987, 1988, } \\
1990-1992\end{array}$ & Pacific \\
\hline CT & 1973 & New England \\
\hline DC & 1973 & South Atlantic \\
\hline $\mathrm{DE}$ & 1973 & South Atlantic \\
\hline ID & $\begin{array}{l}1973,1974,1976,1980- \\
1993\end{array}$ & Mountain \\
\hline MD & 1973 & South Atlantic \\
\hline ME & 1973,1974 & New England \\
\hline MT & $1973-1975,1977,1983$ & Mountain \\
\hline $\mathrm{NH}$ & 1973,1974 & New England \\
\hline NJ & 1973,1974 & Mid-Atlantic \\
\hline OR & 1987,1988 & Pacific \\
\hline RI & $1976-1993$ & New England \\
\hline$S D$ & $1973,1974,1992$ & W. North Central \\
\hline TN & 1973 & E. South Central \\
\hline VT & $\begin{array}{l}1973,1974,1978,1983- \\
1992\end{array}$ & New England \\
\hline WA & $1973-1977$ & Pacific \\
\hline WV & 1973 & South Atlantic \\
\hline WY & 1973 & Mountain \\
\hline
\end{tabular}

dependent variable. Substituting Btu prices for 1970 through 1972 (or 1973) from the Statistical Yearbook into the regression equation yields the estimated electric utility sector State-level distillate prices.

2. Wherever individual State prices are unavailable, quantity-weighted Census division prices are assigned as follows: ID in 1970 through 1972; TN in 1970; and WA in 1970 and 1971.

3. U.S. Btu prices are calculated as the average of the State Btu prices, weighted by consumption data from SEDS.

\section{Alaska and Hawaii Prices}

The CEQ does not have prices for AK in 1973 forward or HI in 1973 through 1982 and 1992 forward. Prices are estimated by calculating the the ratio of the reported $C \& Q$ U.S. price and the Statistical Yearbook U.S. price and multiplying the ratio by the Statistical Yearbook State price for each year. AK prices for 1971, 1975, and 1978, for which Statistical Yearbook data are missing, are estimated from the average ratio of AK prices to U.S. prices in adjacent years (e.g., 1975 is calculated as the average of the
1974 and 1976 prices). Prices for 1970 through 1973 are calculated as described in the "Prices: 1970-1972" section.

\section{Data Sources}

Prices

1973 forward: Energy Information Administration, Cost and Quality of Fuels for Electric Utility Plants; Table 6 (1973, 1974); Tables 5, 6, 12, 13 (1975-1979); Table 45 (1980-1982); Table 51 (1983, 1984); Table 41 (1985-1989); Table 14 (1990, 1991); and Table 8 (1992, 1993).

1970 forward: Edison Electric Institute, Statistical Yearbook of the Electric Utility Industry, table titled, "Analysis of Fuel for Electric Generation-Total Electric Utility Industry," (1970-1988); and table titled, "Fossil Fuels Used for Electric Generation Total Electric Utility Industry," (1990 forward).

\section{Consumption}

1970 forward: Energy Information Administration, State Energy Data System, electric utility sector distillate consumption.

\section{Conversion Factors}

Btu prices are calculated directly from data sources; no explicit conversion factors are needed for any years for the electric utility sector.

\section{Industrial Sector}

The industrial sector distillate prices are developed by using a variety of data sources and several estimation methods, depending on the years involved. For 1983 forward, prices of No. 2 fuel oil (excluding taxes) from the Petroleum Marketing Annual (PMA) are supplemented with prices derived from the American Gas Association (AGA) Residential Gas Market Survey (except for 1991, when AGA data are not available). State general sales taxes from the $\mathrm{Bu}-$ reau of the Census are added. For 1970 through 1982 , prices are the average cost of distillate to manufacturing firms and implicitly include taxes that reflect individual State differences. 
Physical Unit Prices: 1983 Through 1990, 1992, and 1993

For 1983 through 1990, 1992, and 1993 physical unit distillate prices in cents per gallon (excluding taxes) are generally available for 24 States from the PMA. State-level prices for the remaining States are estimated by using price data from the American Gas Association (AGA), SEDS consumption data, and PMA Petroleum Administration for Defense (PAD) district prices. The estimation procedures are described below.

1. State prices in cents per gallon are generally available from the $P M A$ for the following 24 States: AK, CT, DC, DE, ID, IL, IN, MA, MD, ME, MI, MN, NH, NJ, NY, OH, OR, PA, RI, VA, VT, WA, WI, and WV. Prices for these States are converted from cents to dollars per gallon, and State general sales taxes from the Bureau of the Census' State Government Tax Collections (SGTC) are added. The 1993 tax data are published in the Census publication State Tax Review (STR).

2. For the States that do not have prices in the $P M A$, prices are estimated by using AGA fuel oil prices, SEDS consumption data, and PMA PAD district prices for Districts II, III, IV, and V and Subdistrict IC (all the States in PAD Subdistricts IA and IB have published prices). The following steps are used to estimate the prices:

a. Distillate prices from the PMA for PAD Districts II, III, IV, and V and Subdistrict IC are converted from cents per gallon to dollars per gallon.

b. The AGA lists fuel oil prices by company for the principal city served in dollars per million Btu, including State sales taxes. A simple average of the city-level prices is used to derive a State-level price for each of the 27 States without PMA prices. These AGA State averages are converted from dollars per million Btu to dollars per gallon by using the AGA conversion factor of 7.194 gallons per million Btu. State general sales taxes from SGTC (STR) are subtracted to give State averages comparable to the PMA prices.

c. The AGA State prices derived in step $2 \mathrm{~b}$. are combined into PAD district averages using SEDS consumption to weight each State's values. This procedure gives AGA consumption-weighted average prices for PAD Districts II, III, IV, and V and Subdistrict
IC that are comparable to the volumeweighted PAD district prices published in the $P M A$. The AGA PAD district averages are calculated by using only the available States; if a State does not appear in the survey, it is not included in the PAD calculation.

d. Adjustment factors, ratios of the PMA PAD district price divided by the AGA derived PAD district price, are calculated for PAD Districts II, III, IV, and V, and Subdistrict IC.

e. Prices for the 27 States not published in the $P M A$ are calculated by multiplying the AGA State prices derived in step $2 \mathrm{~b}$. by the appropriate PAD district adjustment factor from step $2 \mathrm{~d}$. and adding State general sales taxes from the SGTC (STR).

f. States that do not have prices in either the $P M A$ or the AGA are assigned a PMA PAD district price, and State general sales taxes from the SGTC (STR) are added. The States with assigned prices are shown in Table A17.

\section{Physical Unit Prices: 1991}

Physical unit distillate prices in cents per gallon (excluding taxes) are available for 21 States from the $P M A$. Because prices are not available from AGA for 1991, State-level prices for the remaining 30 States are estimated by using physical unit prices derived

Table A17. Distillate Industrial Sector PADD Price Assignments, 1983-1990, 1992, and 1993

\begin{tabular}{lll}
\hline State & \multicolumn{1}{c}{ Years } & Prices Assigned \\
\hline AK & 1988 & PAD District V \\
AR & 1988,1993 & PAD District III \\
AZ & 1992,1993 & PAD District V \\
CA & $1984,1992,1993$ & PAD District V \\
FL & 1993 & PAD District IC \\
HI & $1983-1990,1992,1993$ & PAD District V \\
KS & 1986,1989 & PAD District II \\
LA & 1986 & PAD District III \\
MS & $1983,1985,1986$ & PAD District III \\
NM & $1984-1990,1992,1993$ & PAD District III \\
OK & $1986,1989,1990$, & PAD District II \\
& 1992,1993 & \\
SD & 1986 & PAD District II \\
TX & 1992,1993 & PAD District III \\
UT & 1985 & PAD District IV \\
WY & 1991 & PAD District IV
\end{tabular}


for 1990 in SEPEDS (described below) and the 1991 PMA Petroleum Administration for Defense (PAD) district prices. The estimation procedures are as follows:

1. State prices in cents per gallon are available from the PMA for the following 21 States: $A K, C T$, $\mathrm{DE}, \mathrm{IL}, \mathrm{IN}, \mathrm{MA}, \mathrm{MD}, \mathrm{ME}, \mathrm{MI}, \mathrm{MN}, \mathrm{NH}, \mathrm{NJ}, \mathrm{NY}$, $\mathrm{OH}, \mathrm{PA}, \mathrm{RI}, \mathrm{VA}, \mathrm{VT}, \mathrm{WA}, \mathrm{WI}$, and WV. Prices for these States are converted from cents to dollars per gallon, and State general sales taxes from the Bureau of the Census' State Government Tax Collections (SGTC) are added.

2. For the remaining 30 States that do not have prices in the $P M A$, prices are estimated by using the 1990 SEPEDS physical unit and PMA PAD district prices for 1990 and 1991. The following steps are used to estimate the prices:

a. The 1990 State prices derived from AGA and $P M A$, as described below, are adjusted by the percentage change in the 1990 and 1991 prices for each State's PMA PAD district or subdistrict.

b. The State general sales taxes from SGTC are added.

\section{Physical Unit Prices: 1982}

In 1984, the Bureau of the Census announced that State-level fuel cost and quantity information would no longer be published in either the Annual Survey of Manufactures (ASM) or Census of Manufactures (CM). In addition, the $P M A$, the source for 1983 forward industrial sector distillate price data, did not contain 1982 prices. Because of this lack of price data, the 1982 industrial sector distillate prices are estimated, based on the relationship of industrial sector prices to electric utility sector prices for 1978 through 1981. The 1983 prices are not used in the estimation because they exclude taxes, while the 1978 through 1981 prices include taxes.

1. In order to calculate the average ratios of industrial-to-electric utility distillate prices, electric utility price assignments are made for: $\mathrm{AK}$ in 1978 through 1982 from WA; ID in 1979 through 1982 from MT; RI in 1978 through 1982 from CT; and VT in 1978 from ME.

2. The average 1978 through 1981 ratios of industrial-to-electric utility sector distillate prices are calculated for each State.
3. Prices for 1982 are estimated by multiplying the average ratios by the electric utility data for 1982.

\section{Physical Unit Prices: 1971, 1974 Through 1981}

For the years 1971 and 1974 through 1981, industrial sector distillate prices are calculated directly from cost and quantity data from the Annual Survey of Manufactures (ASM) or Census of Manufactures (CM) for all States where data are available. Taxes are included in the prices. There are no missing prices for 1971. Six States are missing some $A S M$ cost and quantity data for the 1974 through 1981 period. Cost and quantity data for these States are estimated as the simple average of the cost and quantity data for their adjacent States. The States, the years for which data are estimated, and the adjacent States used to make the estimation are shown in Table A18.

\section{Physical Unit Prices: 1970, 1972, 1973}

Since $A S M$ and $C M$ data are not available for these years, the prices must be estimated. Physical unit prices are based on the ratio of 1971 CM prices to the 1971-assigned Platt's prices (Table A13 on page 198). The resulting ratios for each State are used with the Platt's assigned prices for 1970, 1972, and 1973 to impute prices.

1. The first step is to calculate State-level ratios between prices calculated from the 1971 CM cost and quantity data and the 1971 assigned Platt's prices. There are no missing States in either of these two sets of prices.

2. State-level physical unit prices for 1970,1972 , and 1973 are estimated by multiplying the 1971 ratio by the assigned State-level Platt's prices for each respective year.

Table A18. Distillate Industrial Sector Price Assignments, 1974-1981

\begin{tabular}{lcl}
\hline State & Years & \multicolumn{1}{c}{ State Prices Used } \\
\hline HI & $1979-1981$ & CA \\
ND & $1979-1981$ & MN, MT, SD \\
NM & $1974-1979$ & AZ, CO, TX \\
NV & $1974-1981$ & AZ, CA, ID, OR, UT \\
OK & $1974-1978$ & AR, CO, KS, MO, TX \\
WY & $1974-1981$ & CO, ID, MT, NE, SD, UT \\
\hline
\end{tabular}


Btu prices for States are calculated by converting the physical unit prices from cents to dollars per gallon, then to dollars per barrel (42 gallons per barrel) and, finally, to dollars per million Btu (5.825 million Btu per barrel). U.S. Btu prices are calculated as the average of the State Btu prices, weighted by consumption data from SEDS, adjusted for process fuel consumption.

\section{Data Sources}

Prices

1986 forward: Energy Information Administration, Petroleum Marketing Annual, Table 37 (1986-1988) and Table 39 (1989 forward), column titled, "To Industrial Consumers."

1983-1990, 1992, 1993: American Gas Association, Residential Natural Gas Market Survey (1989, 1990, 1992, 1993), Gas Househeating Survey (1983-1988), Appendix 2, "Competitive Fuel Prices," column titled, "Fuel Oil."

1993: Bureau of the Census, U.S. Department of Commerce, State Tax Review, Volume 54, No. 31, map titled "State Gasoline, Sales and Cigarette Tax Rates as of July 1, 1993."

1983-1992: Bureau of the Census, U.S. Department of Commerce, State Government Tax Collections, table titled, "State Government Excises on General Sales, Motor Fuel, and Cigarettes: Beginning and End of Fiscal Year."

1983-1985: Energy Information Administration, $\mathrm{Pe}$ troleum Marketing Annual 1985, Volume 1, Table 26.

1970-1982: McGraw-Hill, Inc., Platt's Oil Price Handbook and Oilmanac, refinery and terminal prices for No. 2 fuel oil, average of highs and lows.

1971, 1977, and 1981: Bureau of the Census, U.S. Department of Commerce, Census of Manufactures, Table 4 (1971) and Table 3 (1977, 1981).

1974-1976 and 1978-1980: Bureau of the Census, U.S. Department of Commerce, Annual Survey of Manufactures, Table 3.
1970 forward: Energy Information Administration, State Energy Data System, industrial sector distillate consumption.

\section{Conversion Factor: All Years}

5.825 million Btu per barrel

\section{Transportation Sector}

Consumption of distillate fuel in the transportation sector includes distillate fuel used for vessel bunkering and for military and railroad use, plus on-highway diesel fuel use. Since on-highway diesel fuel use accounts for the largest portion of this sector-increasing from 55 percent in 1970 to 81 percent in 1993-prices and expenditures are calculated by using diesel prices. State physical unit prices for 1986 forward are taken from EIA's Petroleum Marketing Annual (PMA). Physical unit prices for earlier years are calculated by using PMA prices and consumption data from the U.S. Department of Transportation Highway Statistics to weight monthly or quarterly prices from the U.S. Department of Agriculture Agricultural Prices into annual prices. Btu prices for all years are calculated using the physical unit prices and the distillate conversion factor.

\section{Physical Unit Prices: 1986 Forward}

Diesel fuel physical unit prices for 1986 forward are based on the annual State-level price data available from the PMA for approximately 24 States and monthly tax rate information from Highway Statistics. Generally, the PMA provides prices in cents per gal-

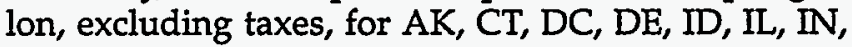
MA, MD, ME, MI, MN, NH, NJ, NY, OH, OR, PA, RI, VA, VT, WA, WI, and WV. State and Federal excise taxes on diesel fuel are added to PMA prices to derive final physical unit prices, which are converted to dollars per gallon. In cases where the tax rate is not constant through the year, an annual average tax is calculated based on the number of months each rate was in effect. State and local sales and other general taxes are not included.

For the remaining States for which no prices are published, the PMA PAD district prices for diesel fuel and motor gasoline and State motor gasoline prices are used. The State diesel fuel price is estimated as the ratio of the PAD district diesel fuel price to the PAD district motor gasoline price times the State 
motor gasoline price. The use of the ratio assumes that the relationship between the motor gasoline State and PAD district prices is similar to that of the diesel fuel State and PAD district prices. Motor gasoline prices to end users at all refiners' company outlets are used. When a State has no price available in that data series, the motor gasoline price to end users by all types of sellers through company outlets is used. States with no published diesel fuel or motor gasoline prices are assigned the motor gasoline price of an adjacent State. For 1991 forward, DC is assigned the MD price. State and Federal excise taxes are added as described above.

\section{Physical Unit Prices: 1983 Through 1985}

Diesel fuel physical unit prices for 1983 through 1985 are based on the annual State-level price data available from the PMA and monthly State and Federal tax rate information from Highway Statistics for 24 States. The prices for the remaining 27 States are calculated by using Agricultural Prices as outlined in the 1977 through 1982 methodology.

For the 24 States of AK, CT, DC, DE, ID, IL, IN, MA, MD, ME, MI, MN, NH, NJ, NY, OH, OR, PA, RI, VA, VT, WA, WI, and WV, the PMA provides physical unit prices, excluding taxes. In 1983 through 1985, the DC price is missing, and the MD price is assigned. In 1983, RI has no price and the PAD District IA average is assigned. A simple average of monthly State and Federal excise taxes is calculated as a combined average tax and added to the $P M A$ price for a final physical unit price. State and local sales and other general taxes are not included.

\section{Physical Unit Prices: 1977 Through 1982}

Monthly prices from Agricultural Prices and monthly special fuels consumption data from Highway Statistics are collected for the States. MD prices are assigned to DC. Prices include State and local per gallon taxes. Federal taxes and State and local sales and other general taxes are not included.

The volume-weighted annual diesel physical unit prices for States and the United States are calculated by using the monthly Agricultural Prices price data, weighted by the monthly Highway Statistics consumption data. AK/1977 through 1982 prices are estimated based on the assumption that the ratio of AK-to-U.S. diesel fuel price is the same as the ratio of the AK-to-U.S. motor gasoline price each year.

\section{Physical Unit Prices: 1970 Through 1976}

Quarterly prices from Agricultural Prices and monthly special fuels consumption data from Highway Statistics are collected for the States. Prices include State and local per gallon taxes. Federal taxes and State and local sales taxes and other general taxes are not included.

1. Prices for 1970 through 1972 are reported in cents per gallon and must be converted to dollars per gallon. Prices for 1973 through 1976 are already reported in dollars per gallon.

2. For 1971 through 1973, State-level prices are not available for CT, MA, ME, NH, RI, and VT. Each is assigned the New England price for the 3 years.

3. The third quarter $D E$ price is assigned to the missing fourth quarter DE price in 1972.

4. The combined MD/DE prices reported in 1973 are assigned to each of the States.

5. For 1970 through $1976, \mathrm{MD}$ (or $\mathrm{MD} / \mathrm{DE}$ ) prices are assigned to DC.

The monthly special fuels consumption for 1970 through 1976 are converted into quarterly consumption by summing the months for each quarter.

The consumption-weighted annual diesel physical unit prices for the States are calculated by using the quarterly weights and quarterly prices. For 1970 through 1972, the quarterly prices from Agriculture Prices are converted from cents per gallon to dollars per gallon. For 1973 forward, the prices are already in dollars per gallon in the source. AK/1970 through 1976 prices are estimated based on the assumption that the ratio of AK-to-U.S. diesel fuel price is the same as the ratio of AK-to-U.S. motor gasoline price each year.

\section{Btu Prices: All Years}

Btu prices for States are calculated by converting the physical unit prices from cents per gallon to dollars per barrel (42 gallons per barrel) and then to dollars per million Btu (5.825 million Btu per barrel). U.S. Btu prices are calculated as the average of the State Btu prices, weighted by consumption from SEDS. 


\section{Prices}

1986 forward: Energy Information Administration, Petroleum Marketing Annual, Table 36 (1986-1988) and Table 38 (1989 forward), column titled "Sales to End Users, Through Company-Operated Retail Outlets," for diesel fuel prices; Table 29 (1986-1988) and Table 30 (1989 forward), column titled "All Refiners, Sales to End Users, Through Company Outlets," for motor gasoline prices; and Table 28 (1986-1988) and Table 29 (1989 forward), column titled "Motor Gasoline Average, Through Company Outlets," for additional motor gasoline prices.

1983-1985: Energy Information Administration, Petroleum Marketing Annual 1985, Volume 1, Table 25, column titled "Sales to End Users, Sales Through Company-Operated Retail Outlets."

1970-1985: Crop Reporting Board, U.S. Department of Agriculture, Agriculture Prices, tables generally titled "Motor Supplies: Average Price Paid by Farmers for Motor Fuel" for 1970-1979, and "Diesel Fuel: Average Price Paid by States" for 1980-1985.

1970 forward: Federal Highway Administration, U.S. Department of Transportation, Highway Statistics, Table MF-121T for State tax rates, Table FE-101 (also EIA, Petroleum Marketing Annual, Table EN1) for Federal tax rates, and Table MF-25 for special fuels consumption data. Table MF-25 is not included in the 1976 volume but is publicly available directly from the Federal Highway Administration.

\section{Consumption}

1970 forward: Energy Information Administration, State Energy Data System, transportation sector distillate consumption.

\section{Conversion Factor: All Years}

5.825 million Btu per barrel

\section{Heavy Oil (Utilities)}

For all years, the price of heavy oil consumed at electric utilities is the average cost of No. 6 fuel oil (residual fuel oil) as reported in Cost and Quality of Fuels for Electric Utility Plants. (See Residual Fuel Electric Utility Sector on page 230.)
Jet fuel prices are estimated for all years for the transportation sector and for 1972 through 1982 for the electric utility sector. After 1982, electric utility use of jet fuel is reported as light oil. (See Light Oil (Utilities) on page 214).

\section{Transportation Sector}

Consumption estimates from SEDS are developed for two types of jet fuel: kerosene-type (used primarily by commercial aircraft and accounting for 92 percent of all jet fuel in 1993) and naphtha-type (used primarily by military aircraft and accounting for the remaining 8 percent in 1993). Prices are developed for kerosene-type jet fuel and are used as the price for both types of jet fuel. Taxes are not included in the prices.

\section{Physical Unit Prices: 1985 Forward}

Transportation sector jet fuel prices for 1985 forward are based on data from EIA's Petroleum Marketing Annual (PMA). Annual prices to end users are available for all States in most cases. Prices are converted to dollars per gallon. States without prices are assigned adjacent State or PAD subdistrict prices as shown in Table A19.

\section{Physical Unit Prices: 1983, 1984}

Prices for 1983 and 1984 are based on data from EIA's Petroleum Marketing Monthly (PMM). Monthly jet fuel prices in cents per gallon (excluding taxes) are available from the PMM for the PAD districts and subdistricts for January 1983 through August 1984. The monthly prices for each of the PAD districts or

Table A19. Jet Fuel Transportation Sector Price Assignments, 1985 Forward

\begin{tabular}{lll}
\hline State & \multicolumn{1}{c}{ Years } & \multicolumn{1}{c}{$\begin{array}{c}\text { PAD District or State } \\
\text { Prices Assigned }\end{array}$} \\
\hline DC & $1985-1988,1990,1993$ & MD \\
DE & 1987 & PAD Subdistrict IB \\
ME & $1985,1990,1991,1993$ & PAD Subdistrict IA \\
NH & 1987 & PAD Subdistrict IA \\
RI & $1985-1988$ & PAD Subdistrict IA \\
VT & $1985-1988,1991,1992$ & PAD Subdistrict IA \\
WV & 1993 & PAD Subdistrict IC \\
\hline
\end{tabular}


J subdistricts (IA, IB, IC, II, III, IV, and V) are assigned E to each State within a district whenever the State monthly price is unavailable. State-level data are available from September 1984 through December 1984. Annual prices are the simple averages of the monthly prices and are assigned directly to their respective States. These prices are converted to dollars per gallon. Missing 1984 prices are assigned values as shown in Table A20.

\section{Physical Unit Prices: 1976 Through 1982}

State-level jet fuel prices for 1976 through 1982 are calculated from the Producer Prices and Price Indexes (PPI) monthly indices for Census divisions and the jet fuel base prices by State for July 1975. The monthly price for each Census division is equal to the PPI monthly index times the jet fuel base price for July 1975 for that Census division. Census division monthly prices are assigned to each State within the Census division, and annual jet fuel prices are computed as simple averages of the monthly State prices.

\section{Physical Unit Prices: 1970 Through 1975}

Jet fuel physical unit State-level prices for the 1970 through 1975 period are based on U.S. annual wholesale prices from the PPI and the relationship of these prices to wholesale kerosene prices reported in Platt's. The U.S. prices are converted to Census divi-

Table A20. Jet Fuel Transportation Sector Price Assignments, 1983 and 1984

\begin{tabular}{lll}
\hline State & \multicolumn{1}{c}{ Months } & $\begin{array}{l}\text { PAD District or State } \\
\text { Prices Assigned }\end{array}$ \\
\hline CT & September & PAD Subdistrict IA \\
DC & September-December & MD \\
ID & September & PAD District IV \\
KS & November, December & PAD District II \\
ME & September-December & PAD Subdistrict IA \\
MS & October & PAD District III \\
ND & December & PAD District II \\
NE & October & PAD District II \\
NH & September, November, & PAD Subdistrict IA \\
& December & \\
NM & October-December & PAD District III \\
OR & November & PAD District V \\
RI & September-December & PAD Subdistrict IA \\
VT & September-December & PAD Subdistrict IA \\
WV & September & PAD Subdistrict IC \\
& & \\
\hline
\end{tabular}

sion prices, which are then assigned directly to States.

Preliminary U.S. jet fuel prices from the PPI for 1973 through 1980 are calculated by using the annual jet fuel price indices, the jet fuel U.S. base price for July 1975 ( 0.276 dollars per gallon) and the U.S. index for July 1975 (235.8). The index for 1973 is assumed to be equal to a simple average of the 11 available monthly indices.

The calculated preliminary U.S. jet fuel prices from the PPI are used as the dependent variable in a regression equation for 1973 through 1980 where the wholesale kerosene prices from Platt's are the independent variable. The regression equation is used to estimate U.S. annual jet fuel prices for 1970 through 1972.

Jet fuel prices for Census divisions are estimated by using the preliminary U.S. prices derived above for 1970 through 1975 (calculated directly from the PPI data for 1973 through 1975 and estimated for 1970 through 1972). These prices are used as inputs to a regression equation which establishes a linear relationship between preliminary U.S. prices and Census division prices for the years 1970 through 1975. Census division prices are assigned to each State within the Census division.

\section{Btu Prices: All Years}

Btu prices for States are calculated from the physical unit prices and the Btu conversion factor. U.S. Btu prices are calculated as the average of the State Btu prices, weighted by consumption data from SEDS.

\section{Data Sources}

\section{Prices}

1985 forward: Energy Information Administration, Petroleum Marketing Annual, Table 21, column titled "Kerosene-Type Jet Fuel" (1985); and Table 33 (19861988) and Table 35 (1989 forward), column titled "Kerosene-Type Jet Fuel, Sales to End Users."

1983, 1984: Energy Information Administration, Petroleum Marketing Monthly, Table 14 (January 1984), Table 19 (February-September 1984), and Table 25 (October 1984-January 1985).

1973-1982: Bureau of Labor Statistics, U.S. Department of Labor, Producer Prices and Price Indexes, 
Supplement, table titled "Producer price indexes for refined petroleum products by region."

1970-1975: McGraw Hill, Inc., Platt's Oil Price Handbook and Oilmanac, 57th Edition, page 480.

Consumption

1970 forward: Energy Information Administration, State Energy Data System, transportation sector jet fuel consumption.

\section{Conversion Factor: All Years}

5.670 million Btu per barrel

\section{Electric Utility Sector}

Jet fuel electric utility consumption estimates are taken from SEDS for 1972 through 1982 only. For 1970 and 1971, no parallel series is available, and for the years after 1982, the series is a part of light oil. All applicable taxes are included in the prices.

\section{Btu Prices: 1975 Through 1982}

For the States that consumed kerosene-type jet fuel at electric utilities during these years, the Btu prices are taken directly. from Cost and Quality of Fuels for Electric Utility Plants (CEQ).

\section{Btu Prices: 1972 Through 1974}

Because C\&Q prices are not available for 1972 through 1974, prices are estimated from $C E Q$ prices for 1975 and 1976 and Agricultural Prices data for 1972 through 1976.

1. Simple annual averages of Agricultural Prices quarterly values are calculated for 1972 through 1976. New England Census Division prices are assigned to $\mathrm{CT}, \mathrm{MA}, \mathrm{ME}, \mathrm{NH}, \mathrm{RI}$, and VT.

2. The average annual prices based on Agricultural Prices values for 1975 and 1976 are used as the independent variables in a regression where the dependent variables are State-level prices based on CEQ prices for 1975 and 1976.

3. State-level price estimates for 1972 through 1974 are derived from the results of the regression analysis and the Agricultural Prices values for 1972 through 1974.

\section{U.S. Btu Prices: All Years}

U.S. Btu prices are calculated as the average of the State Btu prices, weighted by consumption data from SEDS.

\section{Data Sources}

Prices

1975-1982: Energy Information Administration, Cost and Quality of Fuels for Electric Utility Plants, Tables 6 and 13 (1975), Table 13 (1976-1979), and Table 47 (1980-1982).

1972-1976: Crop Reporting Board, U.S. Department of Agriculture, Agriculture Prices, table titled "Household Supplies: Average Prices Paid by Farmers for Lawn Mowers and Petroleum Products."

\section{Consumption}

1972-1982: Energy Information Administration, State Energy Data System, electric utility sector kerosenetype jet fuel consumption.

\section{Conversion Factors: All Years}

Because Btu prices are available directly from the data sources, no conversion factors are used.

\section{Kerosene}

Kerosene prices are developed for the residential and industrial sectors, and the industrial sector prices are assigned to the commercial sector. Estimates of the amount of kerosene consumed by the residential, commercial, and industrial sectors are taken from the State Energy Data System (SEDS).

\section{Residential Sector}

\section{Physical Unit Prices: 1985 Forward}

In July 1985, the Bureau of Labor Statistics ceased publication of the Producer Prices and Price Indexes (PPI) data series that is used to estimate State Energy Price and Expenditure Data System residential kerosene prices for 1970 through 1984. The method used to estimate residential kerosene prices for 1985 
forward is based on the observation that average U.S. kerosene and distillate fuel prices from 1980 through 1984 were highly correlated. Residential kerosene prices for 1985 forward are estimated by applying the percentage changes in residential distillate fuel prices for each year to the residential kerosene prices for the previous year in each State. Taxes are included in the distillate fuel oil prices and are, therefore, reflected in the kerosene price estimates.

\section{Physical Unit Prices: 1977 Through 1984}

R

Because Agricultural Prices kerosene price data are not available after 1976, monthly Census division $P P I$ prices and price indices are used as the basis for the residential kerosene series from 1977 through 1984. To maintain consistency in the series, the PPI prices are multiplied by an adjustment factor that accounts for the relationship between $P P I$ and Agricultural Prices data for quarters in which the two series overlap. In the description of computational procedures below, the adjustment factor is derived first, the PPI prices for 1977 through 1984 are estimated, and the final kerosene physical unit and Btu prices for States are calculated. The final residential sector kerosene prices approximate the average prices paid by farmers. Taxes are included in the source data from Agricultural Prices and are, therefore, reflected in the final price estimates.

The first step is to compute the adjustment factor relating PPI and Agricultural Prices data.

1. Monthly $P P I$ prices for the 18 months covered from July 1975 through December 1976 are calculated from the July 1975 base prices and monthly indices for Census divisions.

2. The calculated Census division monthly prices are assigned to each State within the respective Census division.

3. Volume-weighted quarterly PPI- based prices for States are calculated by using the monthly volume weights developed from Retail Sales and Inventories sales data for "other distillate fuel oil."

4. The adjustment factor relating $P P I$ and Agricultural Prices data is calculated as the simple average of the ratios of the quarterly kerosene price by State from Agricultural Prices to the calculated quarterly PPI-based kerosene prices by State.

The next step is the calculation of monthly Statelevel prices from $P P I$ kerosene Census division data for 1977 through 1984.

1. Monthly Census division PPI prices are calculated by using the July 1975 base prices and the monthly price indices for 1977 through 1984. The missing monthly indices for February, June, July, and October 1980 for the East South Central Division are assumed to be equal to the index for the preceding month.

2. Census division monthly prices are assigned to each State within their respective Census divisions.

The next step is the calculation of annual physical unit State prices.

1. Annual PPI-based physical unit prices for States are computed from the monthly PPI prices and the monthly consumption weights.

2. Final residential kerosene prices for States are estimated as the product of the annual PPI-based State price and the adjustment factor calculated above.

\section{Physical Unit Prices: 1970 Through 1976}

Physical unit prices for States are calculated from quarterly price data in Agricultural Prices and consumption weights derived from Retail Sales and Inventories of Fuel Oil. Taxes are included in the source data.

The quarterly physical unit price data from Agricultural Prices for 1970 through 1976 are published in several different forms. The first step in the calculation of prices for these years is to organize the published Agricultural Prices data into a consistent form.

1. For 1971 through 1973 , no quarterly prices are available for CT, MA, ME, NH, RI, and VT. Each of these States is assigned the quarterly prices reported for the New England Census Division.

2. For 1973 , combined $\mathrm{MD} / \mathrm{DE}$ quarterly prices are reported instead of separate State prices. For this year, the combined prices are assigned to both States.

3. No prices are reported for $A K, D C / 1970$ through 1976. Quarterly weighted Census division 
prices are assigned to $A K$, and $M D$ prices are assigned to DC for all 7 years.

In order to weight the quarterly prices from Agricultural Prices into annual State prices, monthly quantity weights are calculated from Retail Sales and Inventories of Fuel Oil. This assumes that the "other distillate oil" consumption data by PAD districts is kerosene.

1. Monthly weights are computed by using simple averaging of all available "other distillate oil" sales data for each month for each PAD district. Since data are available from November 1978 to March 1981, some months have averages based on three data points while others are based on one or two data points. For example, the average weight for March is the simple average of the 1979, 1980, and 1981 March volumes published in Retail Sales and Inventories of Fuel Oil.

2. Each month's share of average annual sales is calculated by PAD district from the average monthly sales figures. These shares, which become the monthly weights, are then assigned to each State within its respective district.

Final State annual kerosene physical unit prices are calculated as the weighted average of the Agricultural Prices quarterly prices. The monthly weights (shares) are converted to quarterly weights by summing the shares for months within a particular quarter. These same weights are used with the Statelevel price data for each year from 1970 to 1976.

\section{Alaska Btu Prices: 1970 Through 1979, 1983, 1984}

Kerosene residential prices for $\mathrm{AK}$ are estimated on the basis of the assumption that the ratio of AK-toU.S. kerosene residential prices is the same as the ratio of AK-to-U.S. distillate fuel residential prices.

\section{Btu Prices: All Years}

Btu prices for States are calculated from the physical unit prices and the conversion factor. U.S. Btu prices are calculated as the average of the State Btu prices, weighted by consumption data from SEDS.

\section{Data Sources}

Prices

1985 forward: Residential sector distillate fuel price estimates (in physical units) from the State Energy Price and Expenditure Data System.

1975-1984: Bureau of Labor Statistics, U.S. Department of Labor, Producer Prices and Price Indexes, Supplement, table titled "Producer price indexes for refined petroleum products by region."

1978-1981: Energy Information Administration, Retail Sales and Inventories of Fuel Oil, Table 2.

1970-1976: Crop Reporting Board, U.S. Department of Agriculture, Agricultural Prices, table titled "Household Supplies: Average Price Paid by Farmers for Lawn Mowers and Petroleum Products."

\section{Consumption}

1970 forward: Energy Information Administration, State Energy Data System, residential sector kerosene consumption.

\section{Conversion Factor: All Years}

5.670 million Btu per barrel

\section{Commercial Sector}

State prices from the industrial sector are assigned to the commercial sector. Although there is no industrial consumption of kerosene in DC in 1989, 1990, 1992 , and 1993, an industrial price is calculated to be assigned to the commercial sector consumption.

\section{Industrial Sector}

The industrial sector kerosene prices are based on wholesale price and price index data and on the industrial sector distillate prices. The procedures vary slightly for 1970 through 1974, 1975 through 1984, and 1985 forward. In 1970 through 1984, physical unit prices are calculated first; then Btu prices are computed by using the physical unit prices and the conversion factor. Prices approximate an average kerosene price for the manufacturing sector. Taxes are included in the distillate fuel oil prices and are, therefore, reflected in the kerosene price estimates. 
Physical Unit Prices: 1985 Forward

The discontinuation of the published PPI data from the Bureau of Labor Statistics affected the estimation of industrial sector kerosene prices. To estimate prices for 1985 forward, the percentage change in industrial sector distillate fuel prices for each year is applied to the industrial sector kerosene prices for the previous year on a State-by-State basis.

\section{Physical Unit Prices: 1975 Through 1984}

Physical unit industrial kerosene prices for 1975 through 1984 are estimated from PPI base prices and indices for kerosene and No. 2 distillate oil, and from the industrial sector distillate prices in physical units. The ratio of PPI kerosene prices to PPI distillate prices is used as an adjustment factor to estimate kerosene prices.

Annual wholesale prices are calculated from PPI annual indices for kerosene and No. 2 distillate fuel oil and their respective July 1975 base prices for Census divisions. Annual average distillate price indices for 1976 are estimated as the simple average of monthly indices. Census division prices for both kerosene and fuel oil No. 2 are assigned to each State within the respective Census divisions. The industrial sector physical unit kerosene prices for States are computed by using the distillate industrial physical unit prices and the ratio of $P P I$ kerosene prices to $P P I$ fuel oil No. 2 prices.

\section{Physical Unit Prices: 1970 Through 1974}

Physical unit State-level prices for 1970 through 1974 are estimated from the distillate industrial prices and the average ratio of kerosene to distillate prices from PPI for 1975 through 1978. The average annual wholesale price ratio between kerosene and fuel oil No. 2 (distillate) is calculated from PPI-based data for the years 1975 through 1978. State-level kerosene industrial physical unit prices are calculated as the product of the ratios and the industrial sector distillate prices for 1970 through 1974.

\section{Btu Prices: All Years}

Btu prices for States are computed by converting the physical unit prices in dollars per gallon to dollars per barrel (42 gallons per barrel) and then to dollars per million Btu (5.670 million Btu per barrel). U.S. Btu prices are calculated as the average of the State
Btu prices, weighted by consumption data from SEDS.

\section{Data Sources}

Prices

1970 forward: Industrial sector distillate fuel price estimates (in physical units) from the State Energy Price and Expenditure Data System.

1975-1985: Bureau of Labor Statistics, U.S. Department of Labor, Producer Prices and Price Indexes, Supplement, table titled "Producer price indexes for refined petroleum products by region."

\section{Consumption}

1970 forward: Energy Information Administration, State Energy Data System, industrial sector kerosene consumption.

\section{Conversion Factor: All Years}

5.670 million Btu per barrel

\section{Light Oil (Utilities)}

Beginning with 1980, the price of light oil consumed at electric utilities is the average delivered cost of No. 2 fuel oil as reported in Cost and Quality of Fuels for Electric Utility Plants. For earlier years, the price is the consumption-weighted average of the kerosene-type jet fuel price and No. 2 fuel oil. (See also Distillate Fuel Electric Utility Sector on page 203 and Jet Fuel Electric Utility Sector on page 211.)

\section{Liquefied Petroleum Gases}

Liquefied petroleum gases (LPG) prices are developed for the residential and the industrial sectors. State prices for the commercial and transportation sectors are assigned from the industrial sector. Estimates of the amount of LPG consumed by sector are taken from the State Energy Data System (SEDS) and are adjusted for process fuel and intermediate product consumption in the industrial sector. (See the discussion under "Consumption Adjustments for Calculating Expenditures" on page 249.) 


\section{Residential Sector}

Residential sector LPG prices are estimated as the average prices of propane delivered to residential consumers in areas where natural gas is available as a competing fuel. Taxes are included in the prices. For 1973 forward, Btu prices are reported in the data source. For 1970 through 1972, physical unit prices are calculated first and Btu prices are calculated by using the physical unit prices and Btu conversion factors. Prices for AK and HI in all years are estimated by a different methodology described in a separate section on page 216.

\section{Btu Prices: 1973 Through 1990, 1992 Forward}

Propane prices by city are reported by the American Gas Association (AGA) directly in dollars per million $\mathrm{Btu}$, including taxes. The simple average of available city prices is used as the State annual average. Prices that fall outside a reasonable range are omitted from consideration for Central Hudson Gas and Electric for NY in1979 through 1981; Arkansas Louisiana Gas for AR in 1989; Public Service Electric \& Gas for NJ in 1989; Northwestern Public Service for SD in 1989; City of Long Beach for CA in 1989 and 1990; Orange \& Rockland Utilities for NY in 1989 and 1990; Pike County Light \& Power for PA in 1989 and 1990; Commonwealth Gas Co., and Fitchburg Gas \& Electric for MA in 1993; Providence Gas Co. for RI in 1993; and South Jersey Gas Co. for NJ in 1993.

To estimate missing prices (other than $\mathrm{AK}$ and $\mathrm{HI}$, which are described in a separate section that follows), simple averages of adjacent States' prices are used as shown in Table A21. Estimated data for one State are not used to estimate prices for another State.

\section{Btu Prices: 1991}

Propane prices from the American Gas Association (AGA) are not available for 1991. Propane prices from the EIA Petroleum Marketing Annual (PMA) are used to calculate the percentage change in propane prices between 1990 and 1991 for each PAD district or subdistrict. These percentages are applied to the 1990 State residential LPG prices from the State Energy Price and Expenditure Data System (SEPEDS) to estimate 1991 prices for the contiguous 48 States and the District of Columbia. Prices for LPG in AK and HI are developed by using the methodology described on page 216.
Prices for PAD Subdistricts IA and IB and PAD District $V$ are not available for 1990 in the $P M A$, and prices for PAD Subdistrict IA and PAD District $V$ for 1991 are not available. To estimate the missing PAD district prices, a ratio of the end-users price to the resale price for propane published for an adjacent district is calculated and applied to the known resale price for the PAD districts and subdistricts without an end-users price. For 1990, the PAD District I endusers-to-resale ratio is multiplied by the PAD Subdistricts IA and IB resale prices to estimate an end-users price for those Subdistricts. For 1991, the PAD Subdistrict IB end-users-to-resale ratio is multiplied by the PAD Subdistrict IA resale prices to estimate an end-users price. For both years, the U.S. end-users-to-resale price ratio is applied to the PAD District $V$ resale price to estimate a PAD District $V$ end-users price.

\section{Physical Unit Prices: 1971, 1972}

Physical unit residential LPG prices are based on the city-level propane prices reported by AGA in cents per gallon. Prices for missing States are estimated. The AGA prices are the average delivered prices for propane purchased by residential consumers as of December 31.

1. City-level propane prices from AGA are assigned to their respective States. The AL/1971 price for

Table A21. LPG Residential Sector Price Assignments, 1973 Forward

\begin{tabular}{lll}
\hline State & \multicolumn{1}{c}{ Years } & $\begin{array}{l}\text { State Prices Used } \\
\text { in the Estimation }\end{array}$ \\
\hline AR & 1977 & MO, MS, OK, TN, TX \\
CT & 1990 & MA, NY, RI \\
DC & $1973-1983,1990$ & MD \\
DE & 1976,1984 & MD, NJ, PA \\
ID & 1977 & MT, NV, OR, UT, WA, WY \\
LA & 1977 & MS, TX \\
ME & $1973-1977,1985$, & MA, NH, VT \\
& 1986,1992 & \\
MO & 1986 & IA, IL, KS \\
ND & 1973 & MN, MT, SD \\
NM & 1987,1988 & AZ, CO, UT \\
NV & 1973,1975 & AZ, CA, ID, OR, UT, WY \\
OR & 1976 & CA, ID, NV, WA \\
SD & 1986 & MN, MT, ND \\
UT & $1974,1978,1985$, & AZ, CO, ID, NV, WY \\
& 1993 & \\
VT & 1979 & MA, NH, NY \\
WV & 1992 & KY, MD, OH, PA, VA \\
& &
\end{tabular}


the Phoenix City Utilities System is omitted because it falls outside a reasonable range.

2. Physical unit prices for a State are calculated directly from the available city/utility price observations reported by AGA. Final physical unit prices are equal to the simple average of the price observations for each State.

3. MD prices are assigned for missing $D C$ prices. $\mathrm{AK}$ and $\mathrm{HI}$ prices are discussed in a separate section that follows.

\section{Physical Unit Prices: 1970}

A Since AGA did not publish LPG prices prior to 1971, the residential sector LPG prices for 1970 are estimated. To maintain continuity with the AGA prices for 1971 forward, prices for 1970 are estimated by using simple regression analysis. The relationship between AGA data for 1971 and 1972 and corresponding Agricultural Prices data is the basis for the estimation.

1. Before regression analysis can be applied, Agricultural Prices data for 1970 through 1972 are prepared for 49 States (no AK or HI prices are available). These prices include taxes. Development of $\mathrm{AK}$ and $\mathrm{HI}$ prices are described in a separate section on page 216.

a. State-level prices for small purchases, representing residential end users, for 1970 through 1972 are published by Agricultural Prices in cents per pound. When price per pound data are not available, price per gallon data, representing larger volume purchases, are used. These prices per gallon are multiplied by 0.543 , the average ratio of price per pound to price per gallon for the United States for 1970 through 1972, to create uniform input data in price per pound.

b. For 1971 and 1972, the price reported for the New England Region is assigned to CT, MA, $\mathrm{ME}, \mathrm{NH}, \mathrm{RI}$, and VT.

c. Data in cents per pound are converted to dollars per gallon by multiplying by the propane conversion factor of 4.2 pounds per gallon, from the Petroleum Products Handbook and dividing by 100 .

d. Missing prices use adjacent States' average prices as shown in Table A22.
2. The physical unit AGA prices and Agricultural Prices data for 1971 through 1972 (excluding AK and $\mathrm{HI}$ ) are used with simple regression analysis to estimate final physical unit LPG residential prices.

\section{Alaska and Hawaii Prices: All Years}

Prices cannot be estimated for $\mathrm{AK}$ and $\mathrm{HI}$ by using adjacent State price assignments. Missing prices for these two States are estimated by computing ratios of the AK or HI prices to the simple average U.S. prices calculated from the AGA data for years when AK or $\mathrm{HI}$ prices are available and applying these ratios to the U.S. simple average prices in years when prices need to be estimated.

1. AGA prices for $A K$ are available in 1972 and 1980. The 1972 AK-to-US ratio is used to estimate prices for 1970, 1971, and 1973 through 1979. The 1980 AK-to-US price ratio is used to estimate prices for 1981 forward.

2. AGA prices for $\mathrm{HI}$ are available in 1971,1977 through 1979, and 1989. The $1971 \mathrm{HI}$-to-US AGA is used to estimate prices for 1970 and 1972 through 1974. The average ratio of the HI-to-US prices for 1977 through 1979 is used to estimate prices for 1975, 1976, and 1980 through 1984. The 1989 HI-to-US ratio is used to estimate prices for 1985 through 1988 and 1990 forward.

\section{Btu Prices: All Years}

For 1973 forward, Btu prices for States are taken directly from the data sources. For 1970 through 1972, Btu prices for States are calculated by converting the physical unit prices by using the factors cited in Table A27 on page 220. U.S. Btu prices are calculated as the average of the State Btu prices, weighted by consumption data from SEDS.

Table A22. LPG Residential Agricultural Prices Assigned to Estimate 1970 Prices

\begin{tabular}{lll}
\hline State & Years & State Prices Used \\
\hline DC & $1970-1972$ & MD \\
NV & 1970,1971 & AZ, CA, ID, UT \\
OR & $1971-1972$ & CA, ID \\
UT & 1972 & AZ, CO, ID, NV, WY \\
WA & $1970-1972$ & CA, ID \\
\hline
\end{tabular}


Prices

1971-1990, 1992, 1993: American Gas Association (AGA), Gas Househeating Survey (1971-1988), Residential Gas Market Survey (1989 and 1990), and Residential Natural Gas Market Survey (1992 and 1993), Appendix 2, "Competitive Fuel Prices."

1991: Energy Information Administration, State Energy Price and Expenditure Data System, 1990 residential sector LPG prices.

1991: Energy Information Administration, Petroleum Marketing Annual, Table 35 (1990 and 1991), columns titled "Propane (Consumer Grade)."

1970-1972: Crop Reporting Board, U.S. Department of Agriculture, Agricultural Prices, table titled "Average Price Paid by Farmers for Lawn Mowers and Petroleum Products, Specified Dates, by State," column titled "L.P. Gas."

\section{Consumption}

1970 forward: Energy Information Administration, State Energy Data System, residential sector LPG consumption.

\section{Conversion Factors}

1970-1972: Energy Information Administration, State Energy Data Report 1993, Consumption Estimates, Table D1, as shown in Table A27 on page 220.

1970-1972: 4.2 pounds per gallon from Guthrie, Virgil, ed., 1960. Petroleum Products Handbook. John Wiley and Sons, Inc., New York, New York: pages 35.

Conversion factors are not necessary for other years because Btu prices are available directly from the data sources.

\section{Commercial Sector}

State prices from the industrial sector are assigned to the commercial sector.
The industrial sector LPG prices are estimated from average LPG prices to manufacturing firms, average LPG prices to farmers, or refiner and gas plant operator sales to end users, depending on the data sources for the different years. Prices for 1985 forward are taken from the Petroleum Marketing Annual (PMA). Prices for 1978 through 1981 are taken from the Annual Survey of Manufactures (ASM) or the Census of Manufactures (CM) and prices for 1970 through 1977 and 1982 through 1984 are derived from Agricultural Prices and scaled to the $A S M / C M$ prices by using the ratio of $A S M / C M$ to Agricultural Prices LPG prices for the years 1978 through 1981, when both price series were available. Taxes are included in the 1970 through 1984 estimates but are not included in the prices for 1985 forward.

\section{Physical Unit Prices: 1985 Forward}

Industrial sector LPG physical unit State prices for 1985 forward are estimated by using physical unit annual prices in the Petroleum Marketing Annual (PMA) for consumer grade propane sales to end-users. Where prices are not available, the PAD district price is assigned to the State as shown in Table A23. One exception is AR for 1992 and 1993. Because the neighboring States in PADD III are LPG producers, the PADD III price is uncharacteristically lower than previously reported prices for AR. Therefore, the 3 monthly prices available for AR in 1992 are averaged to derive an annual price. In 1993, the MO price is assigned to $A R$.

When a PAD district price is not available, a consumption-weighted average price is calculated by using available prices for States within the district and the SEDS industrial sector LPG consumption for those States. A PAD District V price for 1985 is calculated as a consumption-weighted average of $\mathrm{AK}$, CA, OR, and WA prices; a 1986 PAD Subdistrict IA price uses the average of $\mathrm{CT}$ and $\mathrm{NH}$ prices; and PAD Subdistrict IA prices for 1987 through 1988 use the average of $C T$ and $M A$ prices.

When a PAD district price is not available and there are no State data within the PAD district to develop a consumption-weighted average, a different methodology is used. The source table also contains resale prices. To estimate the missing sales to endusers PAD district price, a ratio of the end-users price to the resale price for an adjacent PAD district or subdistrict is calculated and applied to the known resale price for the PAD district or subdistrict that does not 
$L$ have an end-users price. PAD prices used in the es-

P timations are shown in Table A24.

$\mathbf{G}$

Physical Unit Prices: 1982 Through 1984, 1970 I
D
U
S
$\mathbf{T}$
$\mathbf{R}$
$\mathbf{I}$
$\mathbf{A}$
$\mathbf{L}$

Industrial sector LPG physical unit prices for 1982

Table A23. LPG Industrial Sector PAD District Price Assignments, 1985 Forward

\begin{tabular}{|c|c|c|}
\hline State & Years & PAD Assignments \\
\hline AK & 1986-1988, 1990-1993 & District V \\
\hline AL & $1985-1988$ & District III \\
\hline$A Z$ & $1985-1993$ & District V \\
\hline $\mathrm{CA}$ & $1990-1993$ & District V \\
\hline co & 1991 & District IV \\
\hline CT & $1990-1993$ & Subdistrict IA \\
\hline DC & $1985-1993$ & Subdistrict IB \\
\hline DE & $1986-1993$ & Subdistrict IB \\
\hline $\mathrm{FL}$ & $1990-1993$ & Subdistrict IC \\
\hline GA & $1985,1990-1993$ & Subdistrict IC \\
\hline $\mathrm{HI}$ & $1985-1993$ & District V \\
\hline IA & $1986,1991-1993$ & District II \\
\hline ID & $1986,1990-1993$ & District IV \\
\hline IN & $1990^{\circ}$ & District II \\
\hline KS & 1986-1989, 1992 & District II \\
\hline MA & $1986,1990-1993$ & Subdistrict IA \\
\hline$M D$ & $1988,1990-1993$ & Subdistrict IB \\
\hline ME & $1986-1993$ & Subdistrict IA \\
\hline MI & $1985-1988,1990$ & District II \\
\hline MN & $\begin{array}{l}1985,1986,1988-1991, \\
1993\end{array}$ & District II \\
\hline MS & $1990-1993$ & District III \\
\hline MT & $1990-1993$ & District IV \\
\hline NC & 1991,1992 & Subdistrict IC \\
\hline ND & $1985,1986,1991-1993$ & District II \\
\hline NE & 1986-1992 & District II \\
\hline NH & $1987-1993$ & Subdistrict IA \\
\hline NM & 1993 & District III \\
\hline NV & $1985-1988,1990-1993$ & District V \\
\hline NY & 1990-1993 & Subdistrict IB \\
\hline $\mathrm{OH}$ & 1990 & District II \\
\hline OK & 1986,1987 & District II \\
\hline OR & $1986,1990-1993$ & District $V$ \\
\hline PA & $1990-1993$ & Subdistrict IB \\
\hline RI & $1986-1993$ & Subdistrict IA \\
\hline sc & 1992 & Subdistrict IC \\
\hline SD & $1985-1993$ & District II \\
\hline TN & $1990-1993$ & District II \\
\hline UT & $1986-1988,1990-1993$ & District IV \\
\hline VT & $1986-1993$ & Subdistrict IA \\
\hline WA & 1986-1993 & District V \\
\hline WI & $1985,1986,1990$ & District II \\
\hline WV & $1989-1993$ & Subdistrict IC \\
\hline WY & 1987,1988 & District IV \\
\hline
\end{tabular}

Table A24. LPG Industrial Sector, PAD District Price Estimates, 1990 Forward

\begin{tabular}{lll}
\hline Year & $\begin{array}{c}\text { Missing PAD } \\
\text { Prices }\end{array}$ & \multicolumn{1}{c}{$\begin{array}{c}\text { Prices Used in } \\
\text { Estimation }\end{array}$} \\
\hline 1990 & Subdistrict IA & PAD District I \\
& Subdistrict IB & PAD District I \\
& District V & U.S. \\
1991 & Subdistrict IA & PAD Subdistrict IB \\
& District V & U.S. \\
1992 & Subdistrict IA & PAD Subdistrict IC \\
& Subdistrict IB & PAD Subdistrict IC \\
1993 & Subdistrict IA & PAD Subdistrict IC \\
& Subdistrict IB & PAD Subdistrict IC \\
\hline
\end{tabular}

through 1984 and 1970 through 1977 are estimated on the basis of the relationship between State-level LPG prices from Agricultural Prices and the prices calculated from Annual Survey of Manufactures ( $A S M$ ) or Census of Manufactures (CM) for 1978 through 1981.

1. Before the adjustment factor relating Agricultural Prices and $A S M / C M$ data is computed, monthly Agricultural Prices data are converted into annual prices and missing data are estimated.

a. Annual LPG prices are calculated as simple averages of the monthly prices from Agricultural Prices for the years 1977 through 1984. The only States missing data are WV in 1977 through 1981 and AK, DC, and HI in 1977 through 1984. WV is assigned the simple average of the $\mathrm{KY}, \mathrm{MD}, \mathrm{OH}, \mathrm{PA}$, and VA prices. $A K, D C$, and $\mathrm{HI}$ prices are discussed below.

b. The average ratio of $A S M / C M$-based final prices for 1978 through 1981 and the 1978 through 1981 Agricultural Prices annual prices is calculated for 48 States (excluding AK, DC, and $\mathrm{HI}$ ) as the simple average of the ratio over the 4 years. This average ratio is used as an adjustment factor.

2. Final industrial sector LPG prices for 1982 through 1984 and 1970 through 1977 are estimated by using the State-level adjustment factors and annual average LPG prices from $A g$ ricultural Prices for these years.

a. Annual average LPG prices are calculated for 1982 through 1984 and 1970 through 1977 as the simple average of the monthly prices. 
b. Agricultural Prices published annual average prices in dollars per gallon for all States in 1975 and 1976. For DE in 1970 through 1974, MD in 1970 through 1974, VA in 1970 through 1974 , and WV in 1970 through 1972, only prices for small volume purchases in cents per pound were published. These are converted to cents per gallon by multiplying by 1.96 , the average ratio of cents per gallon to cents per pound for the United States for 1970 through 1974.

c. For 1970 through 1972, Agricultural Prices are converted from cents per gallon to dollars per gallon.

d. For 1971 through 1973, the New England price per gallon reported by Agricultural Prices is assigned to $\mathrm{CT}, \mathrm{MA}, \mathrm{ME}, \mathrm{NH}, \mathrm{RI}$, and VT.

e. MD prices are assigned to DC in 1970 through 1972, 1974 through 1977, and 1982 through 1984. The combined MD/DE price in 1973 is assigned to $\mathrm{MD}, \mathrm{DE}$, and $\mathrm{DC}$.

f. Excluding AK and HI, States missing Agricultural Prices LPG prices are assigned the simple average price of adjacent States. The States with missing data and the adjacent State assignments are shown in Table A25.

g. Industrial sector LPG physical unit prices for 1970 through 1977 and 1982 through 1984 for all States (except AK, DC, and HI) are calculated by using the estimated annual

Table A25. LPG Industrial Sector Price Assignments, 1970-1976

\begin{tabular}{lll}
\hline State & Years & $\begin{array}{l}\text { State Prices Used } \\
\text { in the Estimation }\end{array}$ \\
\hline CT & 1974 & NY \\
MA & 1974 & NY \\
ME & 1974 & NY \\
NH & 1974 & NY \\
NV & $1970-1971$ & AZ, CA, ID, UT \\
& $1973-1974$ & AZ, CA, ID \\
OR & $1970-1974$ & CA, ID \\
RI & 1974 & NY \\
& $1975-1976$ & CT, MA, NY \\
UT & 1972 & AZ, CO, ID, NV, WY \\
& $1973-1974$ & AZ, CO, ID, WY \\
VT & 1974 & NY \\
WA & $1970-1974$ & CA, ID \\
\hline
\end{tabular}

Agricultural Prices data for the respective year and the State-level average ratios as adjustment factors.

3. AK prices for 1970 through 1977 and 1982 through 1984 and HI prices for 1970 through 1977 and 1982 through 1984 are estimated by using the relationship between $A S M / C M$ based prices for these States and the U.S. price reported by Agricultural Prices (1979 through 1981 for AK and 1978 through 1981 for $\mathrm{HI})$. The average ratio for the available years for the two States is calculated and used with the Agricultural Prices U.S. prices for the years to be estimated.

\section{Physical Unit Prices: 1978 Through 1981}

For 1978 through 1981, the industrial sector LPG prices are either calculated directly from cost and quantity data from the $A S M$ or the $C M$ or are estimated by using the relationship of $A S M / C M$ data to LPG price data from Agricultural Prices.

1. For 1978 through 1981, industrial sector physical unit prices for LPG are calculated as the average cost per unit from cost and quantity data published in $A S M / C M$. Since sales are reported in pounds, the prices are converted to dollars per gallon. The conversion factor of 4.5 pounds per gallon is from $A S M / C M$.

2. The AK price for 1978 is the consumptionweighted average Census division price. In addition, four States have prices estimated as the simple average of the prices of adjacent States, and $\mathrm{DC}$ is assigned the MD price as shown in Table A26.

\section{Btu Prices: All Years}

Btu prices for States and the United States are calculated from the physical unit prices and the conversion factors shown in Table A27. U.S. Btu

Table A26. LPG Industrial Sector Price Assignments, 1978-1981

\begin{tabular}{lll}
\hline State & \multicolumn{1}{c}{ Years } & \multicolumn{1}{c}{ State Prices Used } \\
\hline AR & 1978 & LA, MO, MS, OK, TX \\
DC & $1978-1981$ & MD \\
LA & 1980 & AR, MS, TX \\
NM & $1979-1981$ & AZ, CO, OK, TX \\
WY & $1978-1981$ & CO, ID, MT, ND, NE, SD, UT \\
\hline
\end{tabular}


L prices are calculated as the average of the State Btu U prices, weighted by consumption data from SEDS, B adjusted for process fuel and intermediate product $\mathbf{R}$ consumption.

\section{Data Sources}

Prices

1985 forward: Energy Information Administration, Petroleum Marketing Annual, Table 21 (1985), Table 33 (1986-1988), and Table 35 (1989 forward), columns titled "Propane (Consumer Grade)", "Sales to End Users" and "Sales for Resale."

1970-1984: Crop Reporting Board, U.S. Department of Agriculture, Agricultural Prices, tables titled "Average Price Paid by Farmers for Lawn Mowers and Petroleum Products, Specified Dates, by State," column titled "L.P. Gas," (1970-1976); "Household Supplies: Average Price Paid by Farmers" (19771979); "L.P. Gas: Average Price Paid by States" (1980); and "L.P. Gas: Average Price Paid by Months by States" (1981-1984).

1981: Bureau of the Census, U.S. Department of Commerce, 1982 Census of Manufactures, Fuels and Electric Energy Consumed, Part 2, States and Standard Metropolitan Statistical Areas by Major Industry Groups, Table 3, State-level quantity and cost of liquefied petroleum gases.

1978-1980: Bureau of the Census, U.S. Department of Commerce, Annual Survey of Manufactures, Fuels and Electric Energy Consumed, States by Industry Group and

Table A27. LPG Btu Conversion Factors 1970 Forward (Million Btu per Barrel)

\begin{tabular}{cccc}
\hline Year & $\begin{array}{c}\text { Conversion } \\
\text { Factor }\end{array}$ & Year & $\begin{array}{c}\text { Conversion } \\
\text { Factor }\end{array}$ \\
\hline 1970 & 3.779 & 1982 & 3.615 \\
1971 & 3.772 & 1983 & 3.614 \\
1972 & 3.760 & 1984 & 3.599 \\
1973 & 3.746 & 1985 & 3.603 \\
1974 & 3.730 & 1986 & 3.640 \\
1975 & 3.715 & 1987 & 3.659 \\
1976 & 3.711 & 1988 & 3.652 \\
1977 & 3.677 & 1989 & 3.683 \\
1978 & 3.669 & 1990 & 3.625 \\
1979 & 3.680 & 1991 & 3.614 \\
1980 & 3.674 & 1992 & 3.624 \\
1981 & 3.643 & 1993 & 3.606 \\
& & & \\
\hline
\end{tabular}

Standard Metropolitan Statistical Areas by Major Industry Group, Table 3, State-level quantity and cost of liquefied petroleum gases.

\section{Consumption}

1970 forward: Energy Information Administration, State Energy Data System, industrial sector LPG consumption.

\section{Conversion Factors}

1970 forward: Energy Information Administration, State Energy Data Report 1993, Consumption Estimates, Table D1, as shown in Table A27.

1978-1981: 4.5 pounds per gallon from Annual Survey of Manufactures, Appendix C.

\section{Transportation Sector}

State prices from the industrial sector are assigned to the transportation sector.

\section{Lubricants}

Lubricant prices are developed for the industrial sector and assigned to the transportation sector. State-level prices are not available for either sector; national-level prices are assigned to all States and do not include end-user taxes paid at the time of sale. Estimates of lubricant consumption by the industrial and transportation sectors are taken from the State Energy Data System (SEDS).

\section{Physical Unit Prices: All Years}

Prices of lubricants are estimated from U.S. Department of Commerce, Bureau of the Census, data for three product categories:

1. Lubricating oils made in refineries (SIC 29117.21) and not made in refineries (SIC 29920.21).

2. Lubricating greases made in refineries (SIC 29117.31) and not made in refineries (SIC 29920.31).

3. Lubricating oils and greases, not specifically known (n.s.k.), made in refineries (SIC 29117.00) and not made in refineries (SIC 29920.00 for 
establishments with 10 employees or more and SIC 29920.02 for establishments with fewer than 10 employees).

For the years where Census of Manufactures (CM) data are available (1967, 1972, 1977, and 1982), total shipments are calculated by adding the shipments for the three product categories. Shipments for the third product category are withheld and estimated by dividing their value of shipments sum by the weighted average cost of the product categories 29920.21 and 29920.31 .

Total shipments in each year for which $C M$ data are available is divided by the estimated SEDS total lubricants consumption (in physical units) for that year to establish a shipments-to-consumption ratio. Ratios for the years not covered by the CM (i.e., 1968 through 1971, 1973 through 1976, and 1978 through 1981) are estimated by linear interpolation, and the 1982 value is used for 1983 forward. Total shipments for the years not covered by the $C M$ are estimated by multiplying SEDS consumption data by the appropriate shipment-to-consumption ratio.

Estimated shipment prices are calculated by dividing the value of shipments shown in the CM or the $A n$ nual Survey of Manufactures by the estimated shipments for each product category. The shipment prices are used to represent wholesale prices.

End-user prices in dollars per barrel are estimated by multiplying the shipment prices by trade ratio factors that represent the wholesale-to-retail markup. The trade ratio factors are developed from Bureau of Economic Analysis (BEA) data for 1972 and 1977. For 1972, the sum of data called "purchasers value" for the three product categories is divided by the sum of the "producers value" for the three categories to derive a trade ratio. A similar calculation is made for 1977, but the terms "purchase value" and "basic value" are used in the source data.

The 1972 ratio is used for 1970 through 1972 and the 1977 ratio is used for 1977 forward. The values for 1973 through 1976 are estimated by linear interpolation by using the 1972 and 1977 values. The trade ratios for 1982 and later years are not used because the range of petroleum products included in the ratios was expanded by BEA and the ratios no longer represent the specific mark-up for lubricants.
Btu prices are obtained by dividing the prices in dollars per barrel by the conversion factor 6.065 million Btu per barrel.

\section{Data Sources}

Prices

1970, 1971, 1973 through 1976, 1978 through 1981, and 1983 through 1993: Bureau of the Census, U.S. Department of Commerce, Annual Survey of Manufactures; Lubricating Oils and Greases (Industries 29117 and 29920).

1972, 1977, and 1982: Bureau of the Census, U.S. Department of Commerce, Census of Manufactures, Petroleum Refining; Lubricating Oils and Greases (Industries 29117 and 29920).

1972 and 1977: Bureau of Economic Analysis, U.S. Department of Commerce, Input-Output Table Work Tapes for SIC Codes 29117 and 29920).

\section{Consumption}

1970 forward: Energy Information Administration, State Energy Data System, lubricants consumption.

\section{Conversion Factor: All Years}

6.065 million Btu per barrel

\section{Motor Gasoline}

Motor gasoline prices are developed for the transportation sector, and the transportation sector prices are assigned to the commercial and industrial sectors. Motor gasoline consumed in privately-owned vehicles is accounted for in the transportation sector. Estimates of motor gasoline consumed by the transportation, commercial, and industrial sectors used in calculating expenditures are taken from SEDS. Prices in this series are retail prices (usually service station prices), including taxes.

\section{Physical Unit Prices: 1983 Forward}

Motor gasoline physical unit prices for 1983 forward are based on annual State-level prices or assigned PAD District prices from the Petroleum Marketing 
Annual (PMA) except for prices for certain States and years as noted in Table A28 that are derived from the Bureau of Labor Statistics' Consumer Prices: Energy (CPI).

State and Federal motor gasoline taxes are added to the prices from the PMA. Monthly State tax information and annual Federal tax information are taken from Highway Statistics. The monthly State taxes are averaged to create an average annual tax for each State which is combined with the Federal tax to adjust the PMA price. Due to the lack of uniformity in application, State and local general sales taxes are not included.

The PMA average sales price (excluding taxes) of finished motor gasoline to end users through company outlets is used, under the assumption that this price most closely approximates retail motor gasoline prices. Finished motor gasoline includes leaded and unleaded motor gasoline and gasohol.

In the States and years (shown in Table A28) where prices are derived from the CPI, monthly CPI city prices are weighted by monthly consumption from Highway Statistics. All taxes are included in the CPI data.

Motor gasoline prices for $\mathrm{HI}$ are not collected or published in the CPI after December 1986. The following method is used to derive HI prices for 1987 through 1990, when PMA prices for HI became available. The monthly HI CPI prices are used to calculate annual averages for 1983 through 1986 . The annual averages are divided by the PMA PAD District $\mathrm{V}$ price (with $\mathrm{HI}$ State and Federal taxes added)

Table A28. Motor Gasoline Price Assignments, 1983 Forward

\begin{tabular}{lll}
\hline State & \multicolumn{1}{c}{ Years } & \multicolumn{1}{c}{ Source } \\
\hline AK & $1983-1986$ & CPI \\
CT & $1989-1993$ & PMA, PAD Subdistrict IA \\
DC & $1983-1993$ & CPI \\
DE & $1991-1993$ & PMA, PAD Subdistrict IB \\
HI & $1983-1986$ & $C P I$ \\
& $1987-1990$ & See text \\
ID & 1993 & $P M A$, PAD District IV \\
MD & $1985-1993$ & $C P I$ (Baltimore) \\
ME & $1985-1988,1990-1993$ & PMA, PAD Subdistrict IA \\
MT & $1991-1993$ & PMA, PAD Subdistrict IV \\
SD & $1987,1991,1992$ & PMA, PAD District II \\
VT & $1989-1993$ & $P M A$, PAD Subdistrict IA \\
WY & 1985 & PMA, PAD District IV \\
& & \\
\hline
\end{tabular}

for each year to develop annual ratios of the two prices. The four ratios for 1983 through 1986 are simple averaged to give one ratio that is multiplied by the PMA PAD District V prices for the 1987 through 1990 to estimate HI prices for those years. State and Federal taxes are added to the estimates.

\section{Physical Unit Prices: 1982}

Monthly physical unit motor gasoline prices for 1982 are taken from the Platt's Oil Price Handbook and Oilmanac (Platt's) table, "AAA 'Fuel Gauge' Report," the CPI, or both. Table A29 summarizes price data availability by source. The Platt's prices are reported for both leaded and unleaded motor gasoline and for both full-service and self-service for all States except $\mathrm{AK}$ and HI. All available Platt's prices for 1982 are used in the calculation of motor gasoline prices. The continuity of these prices with prices published by Platt's in previous years suggests that taxes are included.

The available CPI monthly physical unit motor gasoline prices for 1982 are for all types of motor gasoline and cover 25 States as shown in Table A30. The CPI prices are assigned to any State that has a county included in the Standard Metropolitan Statistical Area (SMSA) definitions used by the Bureau of Labor Statistics. These "all types" prices cover leaded regular, unleaded regular, and leaded premium, including taxes. All the available CPI prices for 1982 are also used in the calculation of motor gasoline prices. Complete monthly data exist for the 25 States covered by the CPI. The CPI Detailed Report of April 1986 explicitly states that Federal, State, and local taxes are included.

To combine the product-specific Platt's prices with the "all types" prices published in the CPI, the Platt's prices are weighted into "all types" prices by using annual U.S. data from the Monthly Energy Review $(M E R)$ to calculate shares for leaded and unleaded motor gasoline (no breakdowns for regular and premium are possible because of data limitations).

Motor gasoline price data reported by Platt's for 1982 cover the following months: February, April, June, August, November, and December. The missing 6 months are assigned prices as follows: January is assigned the February price, and the other missing months are assigned the average price of the preceding and succeeding months. A missing February price for $M O$ is assumed to be equal to the April price, and a missing price for $O R$ is assumed to be equal to the average of the April and August prices. 


\begin{tabular}{|c|c|c|c|c|}
\hline Years & Source & Grades Covered & $\begin{array}{c}\text { Composite } \\
\text { Price }\end{array}$ & $\begin{array}{l}\text { Missing States } \\
\text { All Sources }\end{array}$ \\
\hline 1982 & $\begin{array}{l}\text { Platt's } \\
C P I\end{array}$ & $\begin{array}{l}\text { leaded } \\
\text { unleaded } \\
\text { leaded regular } \\
\text { leaded premium } \\
\text { unleaded regular }\end{array}$ & $\begin{array}{l}\text { no } \\
\text { no } \\
\text { yes } \\
\text { yes } \\
\text { yes }\end{array}$ & none \\
\hline 1979-1981 & $\begin{array}{l}\text { Platt's } \\
\text { CPI }\end{array}$ & $\begin{array}{l}\text { leaded regular } \\
\text { leaded premium } \\
\text { unleaded regular } \\
\text { unleaded premium } \\
\text { leaded regular } \\
\text { leaded premium } \\
\text { unleaded regular }\end{array}$ & $\begin{array}{l}\text { no } \\
\text { no } \\
\text { no } \\
\text { no } \\
\text { yes } \\
\text { yes } \\
\text { yes }\end{array}$ & $\begin{array}{l}\text { AR, DE, ME, MS, MT, ND, NH, } \\
\text { OK, RI, SC, SD, VT, WV, WY }\end{array}$ \\
\hline 1978 & $\begin{array}{l}\text { Platt's } \\
\text { CPI }\end{array}$ & $\begin{array}{l}\text { leaded regular } \\
\text { leaded regular } \\
\text { leaded premium } \\
\text { unleaded regular }\end{array}$ & $\begin{array}{l}\text { no } \\
\text { yes } \\
\text { yes } \\
\text { yes }\end{array}$ & none \\
\hline 1976,1977 & $\begin{array}{l}\text { Platt's } \\
\text { CPI }\end{array}$ & $\begin{array}{l}\text { leaded regular } \\
\text { leaded regular } \\
\text { leaded premium } \\
\text { unleaded regular }\end{array}$ & $\begin{array}{l}\text { no } \\
\text { no } \\
\text { no } \\
\text { no }\end{array}$ & AK \\
\hline 1974,1975 & $\begin{array}{l}\text { Platt's } \\
\text { CPI }\end{array}$ & $\begin{array}{l}\text { leaded regular } \\
\text { leaded regular } \\
\text { leaded premium }\end{array}$ & $\begin{array}{l}\text { no } \\
\text { no } \\
\text { no }\end{array}$ & AK \\
\hline $1970-1973$ & Platt's & leaded regular & no & AK, HI \\
\hline
\end{tabular}

For States with data from Platt's only, prices by product type (leaded and unleaded) are first calculated as the simple average of full-service and self-service prices for that product for each month and State. The resulting prices are then weighted into monthly composite prices by using U.S. leaded and unleaded shares of motor gasoline product supplied from the $M E R$. The following 26 States have data only from Platt's: AL, AR, AZ, CT, DE, IA, ID, LA, ME, MS, MT, NC, ND, NE, NH, NM, NV, OK, RI, SC, SD, TN, UT, VT, WV, and WY.

Platt's reports two prices for each motor gasoline product for each year: one full-service price and one self-service price. These two prices are combined by using a simple average into a single product price for each State for each month.

The unleaded U.S. share of total motor gasoline consumption is reported in the MER as 52.1 percent in 1982. Assuming that the remaining motor gasoline consumption is leaded, the leaded portion of total consumption is 47.9 percent. These shares are used for all States and months to calculate the composite prices from the leaded and unleaded prices.

For AK and HI, the only States with data only from the CPI, the "all types" monthly prices reported are used directly as monthly composite prices.

For States with price data from both Platt's and the CPI, the Platt's data are first combined into product type prices and weighted with the MER shares. The resulting combined prices for all motor gasoline types are averaged together with the combined CPI city prices assigned to the respective month and State. The following 23 States have monthly composite prices computed in this way: $\mathrm{CA}, \mathrm{CO}$, $\mathrm{DC}, \mathrm{FL}, \mathrm{GA}, \mathrm{IL}, \mathrm{IN}, \mathrm{KS}, \mathrm{KY}, \mathrm{MA}, \mathrm{MD}, \mathrm{MI}, \mathrm{MN}, \mathrm{MO}$, NJ, NY, OH, OR, PA, TX, VA, WA, and WI.

1. Leaded and unleaded gasoline prices are calculated as simple averages of full-service and self-service prices from Platt's and are then 

from Consumer Prices: Energy, 1978-1982

\begin{tabular}{ll}
\hline State & City Price Assignments \\
\hline AK & Anchorage \\
CA & $\begin{array}{l}\text { Los Angeles-Long Beach-Anaheim, San Diego, } \\
\end{array}$ \\
CO & San Francisco, Oakland \\
DC & Washer-Boulder \\
FL & Miami \\
GA & Atlanta \\
HI & Honolulu \\
IL & Chicago-Northwestern Indiana, St. Louis \\
IN & Chicago-Northwestern Indiana, Cincinnati \\
KS & Kansas City \\
KY & Cincinnati \\
MA & Boston \\
MD & Baltimore, Washington \\
MI & Detroit \\
MN & Minneapolis-St. Paul \\
MO & St. Louis, Kansas City \\
NJ & New York-Northeastern NJ, Philadelphia \\
NY & New York-Northeastern NJ, Buffalo \\
OH & Cincinnati, Cleveland \\
OR & Portland \\
PA & Philadelphia, Northeastern PA, Pittsburgh \\
TX & Dallas-Ft. Worth, Houston \\
VA & Washington \\
WA & Seattle-Everett, Portland \\
WI & Milwaukee, Minneapolis-St. Paul \\
& \\
\hline
\end{tabular}

Note: All types of motor gasoline are included.

weighted into a composite price by use of $M E R$ shares of leaded and unleaded motor gasoline consumption.

2. Monthly "all types" motor gasoline prices covering leaded regular, leaded premium, and unleaded regular are taken directly from the CPI. If there is more than one CPI price observation for a month and State, the CPI prices are simple averages.

3. Using a simple average, the composite Platt's prices are combined with the "all types" CPI prices for that State. The resulting prices are the monthly composite prices for 1982 .

Annual physical unit prices for all States are calculated from the monthly motor gasoline prices calculated above and weighted by the monthly motor gasoline consumption volumes for States from Highway Statistics.
Physical Unit Prices: 1979 Through 1981

For 1979 through 1981, Platt's monthly motor gasoline prices are taken from a table titled "Platt's/Lundberg Summary." Prices are available for cities by product-type, by grade, and by type of service (full service, self service). Four products and grades of motor gasoline are covered: leaded regular, unleaded regular, leaded premium, and unleaded premium. These data cover 37 States and taxes are included. The CPI reports "all types" prices, including taxes, for the cities listed in Table A30. Platt's city price assignments to States for 1979 through 1981 are shown in Table A31.

The computation of monthly composite prices for 1979 through 1981 varies, depending on the available data sources for each State. Monthly composite prices are estimated for the 14 States which do not have reported price data from either data source. If both Platt's and the CPI report prices for a city, the CPI price is used.

1. For States with city price observations only from Platt's, prices for leaded and unleaded motor gasoline are combined by use of simple averaging, regardless of the type of service, and are converted to dollars per gallon. The leaded and unleaded prices are then weighted together into a monthly composite price. The following 12 States have prices only from Platt's for 1979 through 1981: AL, AZ, CT, IA, ID, LA, NC, NE, NM, NV, TN, and UT.

a. The Platt's prices for 1981 end in September of that year; monthly prices by grade and service type for October, November, and December are assumed to be equal to the corresponding September prices.

b. Leaded and unleaded prices are calculated for each State by simple averaging of all prices available for each product (leaded, unleaded), regardless of service type or grade of motor gasoline (regular, premium). All city prices for each State are averaged together.

c. Leaded and unleaded shares of total motor gasoline consumption for the United States are calculated from the MER for each year 1979 through 1981 . The monthly product type prices are weighted in to composite prices by using the national leaded and unleaded shares as weights. 
Table A31. Motor Gasoline Price Assignments from Platt's, 1979-1981

\begin{tabular}{ll}
\hline State & City Price Assignments \\
\hline AL & Birmingham \\
AZ & Phoenix, Tucson \\
CA & Bakersfield, Fresno, Los Angeles, Sacramento, \\
& San Diego, San Francisco, Stockton \\
CO & Denver \\
CT & New' Haven \\
DC & Washington \\
FL & Miami, Tampa-St. Petersburg \\
GA & Atlanta \\
IA & Des Moines \\
ID & Boise \\
IL & Chicago \\
IN & Indianapolis \\
KY & Louisville \\
LA & New Orleans \\
MA & Boston \\
MD & Baltimore \\
MI & Detroit \\
MN & Minneapolis \\
MO & Kansas City, St. Louis \\
NC & Charlotte \\
NE & Omaha \\
NJ & Newark \\
NM & Albuquerque \\
NV & Las Vegas, Reno \\
NY & Long Island, Rochester \\
OH & Cincinnati \\
OR & Portland \\
PA & Philadelphia, Pittsburgh \\
TN & Memphis \\
TX & El Paso, Houston \\
UT & Salt Lake City \\
VA & Norfolk \\
WA & Seattle, Spokane \\
WI & Milwaukee \\
& \\
\hline &
\end{tabular}

2. For States with city price observations only from the CPI, the monthly "all types" prices are used directly for States with only one price observation per month. For States with multiple observations, monthly prices are combined by simple averaging. States with $C P I$ data only are: $A K, C O, D C, G A, H I, I L, K S, M A, M D, M I, M N$, $\mathrm{MO}, \mathrm{NJ}, \mathrm{OH}, \mathrm{OR}, \mathrm{PA}$, and WI.

3. For the eight States with price observations from both Platt's and the CPI (CA, FL, IN, KY, NY, TX, VA, and WA), monthly composite prices for 1979 through 1981 are calculated by using three steps:

a. The Platt's prices are combined into single "all types" prices as described above by using leaded and unleaded grades of motor gasoline shares as weights.

b. The CPI prices are combined by State.

c. Using simple averaging, the composite Platt's price for each State is combined with the "all types" CPI price for that State. The resulting prices are the monthly composite prices for 1979 through 1981.

4. Fourteen States are not covered by price data from either Platt's or the CPI in1979 through 1981. These States are AR, DE, ME, MS, MT, ND, NH, OK, RI, SC, SD, VT, WV, and WY. Monthly composite prices for these States are estimated by using the monthly State-level composite prices for 1982 and Census region monthly prices from the CPI for 1979 through 1982.

a. The ratio between the 1982 State prices and the 1982 CPI Census region prices corresponding to each State is calculated for use as an adjustment factor in 1979, 1980, and 1981.

b. The monthly price for each of the 14 missing States is assumed to be the product of the 1982 Census region adjustment factor for that State times the monthly motor gasoline price for that Census region from the CPI.

Annual physical unit prices for all States are calculated from the monthly motor gasoline prices calculated above and weighted by the monthly motor gasoline consumption volumes for States from Highway Statistics.

\section{Physical Unit Prices: 1978}

The Platt's monthly leaded regular motor gasoline prices cover all States except AK and HI. The Platt's city assignments to States are shown in Table A32. In 1978, the CPI motor gasoline coverage was expanded from 21 States to 25 States (28 SMSAs) and an "all types" price was published that covers leaded regular, leaded premium, and unleaded regular. The CPI SMSA assignments to States for 1978 through 1982 are shown in Table A30 on page 224. Both the $C P I$ and the Platt's prices include taxes.

Since both sources report a single price for each city or SMSA, product weights are not needed to compute monthly composite prices. Instead, city price observations are assigned to States as shown in Table A30 and Table A32. Price observations are combined by using simple averaging by State and month. If 
Table A32. Motor Gasoline Price Assignments from Platt's, 1970-1978

State City Price Assignments

AL Birmingham

AR Little Rock

AZ Phoenix

CA Los Angeles, San Francisco

CO Denver

CT Hartiord

DC Washington

DE Wilmington

FL Miami

GA Atlanta

IA Des Moines

ID Boise

IL Chicago

IN Indianapolis

KS Wichita

KY Louisville

LA New Orleans

MA Boston

MD Baltimore

ME Portland

MI Detroit

MN Twin Cities

MO St. Louis

MS Jackson

MT Great Falls

NC Charlotte

ND Fargo

NE Omaha

NH Manchester

NJ Newark

NM Albuquerque

NV Reno

NY Buffalo, New York

$\mathrm{OH}$ Cincinnati, Cleveland

OK Tulsa

OR Portland

PA Philadelphia

RI Providence

SC Charleston

SD Huron

TN Memphis

TX Dallas, El Paso, Houston

UT Salt Lake City

VA Norfolk

VT Burlington

WA Seattle, Spokane

WI Milwaukee

WV Charleston

WY Cheyenne

both Platt's and the CPI cover a city/SMSA, the CPI price is used. Platt's prices are converted to dollars per gallon; the CPI prices are already expressed in dollars. All States are covered by the data sources, so no imputation is required for 1978. The following 26 States have prices only from Platt's: $\mathrm{AL}, \mathrm{AR}, \mathrm{AZ}, \mathrm{CT}$, DE, IA, ID, LA, ME, MS, MT, NC, ND, NE, NH, NM, NV, OK, RI, SC, SD, TN, UT, VT, WV, and WY. The following 19 States are covered only by the CPI: AK, CA, CO, DC, FL, GA, HI, IL, MA, MD, MI, MN, MO, NJ, NY, OH, OR, PA, and WI. Six States have price data from both sources: IN, KS, KY, TX, VA, and WA.

Annual physical unit prices for all States are calculated from the monthly motor gasoline prices calculated above and weighted by the monthly motor gasoline consumption volumes for States from Highway Statistics.

\section{Physical Unit Prices: 1976, 1977}

The calculation of monthly composite State prices for 1976 and 1977 depends upon the source of data. Different procedures are used for States with only Platt's data, States with only CPI data, and States with both Platt's and CPI data. If both data sources cover a city, only the CPI price is used for that city. City price assignments to States are given in Table A32 for Platt's and in Table A33 for the CPI. Prices from both sources include taxes. AK is the only State for which prices need to be estimated.

For States with data from Platt's only, the monthly prices reported in Platt's are either used directly or combined by simple averaging if there is more than one price observation for a State in a given month. The reported prices in cents per gallon are converted to dollars per gallon. Prices for the following 29 States are calculated by using this procedure and cover only leaded regular motor gasoline: $A L, A R$, $\mathrm{AZ}, \mathrm{CO}, \mathrm{CT}, \mathrm{DE}, \mathrm{FL}, \mathrm{IA}, \mathrm{ID}, \mathrm{LA}, \mathrm{ME}, \mathrm{MS}, \mathrm{MT}, \mathrm{NC}$, ND, NE, NH, NM, NV, OK, OR, RI, SC, SD, TN, UT, $\mathrm{VT}, \mathrm{WV}$, and WY.

If State-level motor gasoline prices for 1976 and 1977 are available only from the CPI, monthly composite prices are calculated as weighted averages of leaded and unleaded prices. Prices for 15 States are calculated by using data only from the CPI: CA, DC, GA, $\mathrm{HI}, \mathrm{IL}, \mathrm{MA}, \mathrm{MD}, \mathrm{MI}, \mathrm{MN}, \mathrm{MO}, \mathrm{NJ}, \mathrm{NY}, \mathrm{OH}, \mathrm{PA}$, and WI.

1. The weights used in this process are nationallevel shares of leaded and unleaded motor gasoline product supplied. For 1977, the leaded and unleaded share of 0.725 and 0.275 , respectively, are taken from the MER. For 1976, MER data for 1977 through 1984 are used to estimate 
Table A33. Motor Gasoline Price Assignments from Consumer Prices: Energy 1974-1977

\begin{tabular}{ll}
\hline State & City Price Assignments \\
\hline CA & Los Angeles-Long Beach, San Diego, \\
DC & San Francisco-Oakland \\
GA & Atlanta \\
HI & Honolulu \\
IL & Chicago, St. Louis \\
IN & Cincinnati, Chicago \\
KS & Kansas City \\
KY & Cincinnatl \\
MA & Boston \\
MD & Baltimore, Washington \\
MI & Detroit \\
MN & Minneapolis-St. Paul \\
MO & St. Louis, Kansas City \\
NJ & New York-Northeastern NJ, Philadephia \\
NY & Buffalo, New York-Northeastern NJ \\
OH & Cincinnati, Cleveland \\
PA & Philadelphia, Pittsburgh \\
TX & Dallas, Houston \\
VA & Washington \\
WA & Seattle \\
WI & Milwaukee, Minneapolis-St. Paul \\
\hline
\end{tabular}

Note: Prices are avallable separately for leaded regular, leaded premium, and unleaded regular $(1976,1977)$; "all types" prices are not available.

the unleaded share by using simple regression. The unleaded percentages for 1977 through 1984 are converted to shares and used to estimate leaded and unleaded shares of motor gasoline. The resulting 1976 leaded share is 0.744 and the unleaded share is 0.256 .

2. The next step is to calculate monthly composite leaded and unleaded prices for each State. If more than one $C P I$ price observation is available for a particular grade of motor gasoline (leaded or unleaded) for a State in a given month, the CPI observations are combined by grade by using simple averaging. Regular and premium prices are averaged for an estimate of State-level leaded prices.

3. Final monthly composite prices for 1976 and 1977 are calculated by using the leaded and unleaded composite prices calculated above and the $M E R$-based leaded and unleaded shares as volume weights.

For States with price data from both Platt's and the $C P I$, all price observations are averaged together by product type. If both sources report prices for a city, the CPI price is used. Once composite leaded and unleaded prices have been calculated separately for each State, the leaded and unleaded consumption shares are used to weight the product-type prices into the final monthly composite motor gasoline prices. Six States are calculated with data from both Platt's and the CPI: IN, KS, KY, TX, VA, and WA.

1. Monthly leaded composite prices are calculated by combining Platt's prices with the CPI prices for leaded regular and premium motor gasoline by month, since the Platt's prices cover only regular leaded fuel. If both data sources cover a city, the CPI prices are used.

2. Since the CPI is the only source of unleaded gasoline price data for 1976 through 1977, monthly unleaded composite prices are calculated from CPI data only.

3. Final monthly composite prices for the six States with price data from both Platt's and the CPI are calculated by using annual U.S. leaded and unleaded shares and leaded and unleaded monthly composite prices.

Prices for 1976 and 1977 for AK, the only State not covered by price data from either data source, are estimated based on the average relationship between the State and the national average price for years in which data are available. The national average price used for these estimations is a simple average of the prices of the 49 States for which data are available in all years (i.e., excluding $\mathrm{AK}$ and $\mathrm{HI}$ for all years). Annual prices for AK are estimated on the basis of the average AK-to-U.S. price relationship for 1978 and 1979.

Annual physical unit prices (excluding AK) are calculated from the monthly motor gasoline prices calculated above and weighted by the monthly motor gasoline consumption volumes for States from Highway Statistics.

\section{Physical Unit Prices: 1974, 1975}

The Platt's price data for 1974 through 1975 cover only leaded regular motor gasoline. Beginning in 1974, motor gasoline price data are also available from the CPI for selected SMSAs. An SMSA price is assigned to each State with counties included in the definition of that SMSA; for the years 1974 through 1977, prices for 23 SMSAs cover 21 States. The State assignments of SMSA prices for 1974 through 1977 are given in Table A33 on page 227. For 1974 and 
1975, CPI prices are reported separately for leaded regular and leaded premium motor gasoline. According to the CPI Detailed Report, April 1986, these prices include taxes; the Platt's prices also include taxes. AK is the only State not covered by either of these two data sources; prices for $\mathrm{AK}$ are imputed for 1974 and 1975.

The Platt's regular leaded prices and the CPI regular and premium leaded motor gasoline prices, including taxes, are assigned to their respective States as shown in Table A32 and Table A33. If both sources cover a city, the CPI price is used. The following 29 States are covered only by Platt's: $A L, A R, A Z, C O$, CT, DE, FL, IA, ID, LA, ME, MS, MT, NC, ND, NE, NH, NM, NV, OK, OR, RI, SC, SD, TN, UT, VT, WV, and WY. The following 15 States are covered only by CPI: CA, DC, GA, HI, IL, MA, MD, MI, MN, MO, NJ, NY, OH, PA, and WI. The following six States have both Platt's and CPI data for a particular city: IN, KS, $\mathrm{KY}, \mathrm{TX}, \mathrm{VA}$, and WA.

All price observations assigned to a State, regardless of grade or data source, are added together and divided by the number of observations. As part of this calculation, Platt's prices are converted from cents per gallon to dollars per gallon.

Neither Platt's nor the CPI reports price data for AK. The methodology of the estimation of annual AK prices is the same as used in 1976 and 1977.

Annual physical unit prices for the remaining 50 States (excluding $A K$ ) are calculated from the monthly motor gasoline prices calculated above and weighted by the monthly motor gasoline consiumption volumes for States from Highway Statistics.

\section{Physical Unit Prices: 1970 Through 1973}

Monthly motor gasoline physical unit prices for 1970 through 1973 are available only from Platt's, where city prices covering 49 States are reported in a table titled "Service Station Prices: Gasoline (Including Taxes)." These prices, as shown in Table A29, are for leaded regular gasoline only and include taxes.

Monthly average city prices from Platt's are assigned to the State in which the city is located. Platt's city price assignments to States are given in Table A32.

Monthly composite prices for 1970 through 1973 are equal to the reported monthly Platt's prices or, if more than one city is available for a given State in a certain month, are a simple average of the assigned city prices. The reported prices are converted from cents to dollars per gallon.

Platt's does not report data for either AK or HI for 1970 through 1973. The methodology of the estimation of $\mathrm{AK}$ and $\mathrm{HI}$ prices is the same as that used for 1976 and 1977.

Annual physical unit prices (excluding $\mathrm{AK}$ and $\mathrm{HI}$ ) are calculated from the monthly motor gasoline prices weighted by the monthly motor gasoline consumption volumes for States from Highway Statistics.

\section{Btu Prices: All Years}

Btu prices for States are computed by converting the physical unit prices in dollars per gallon to dollars per barrel (42 gallons per barrel) and then to dollars per million Btu (5.253 million Btu per barrel). U.S. Btu prices are calculated as the average of the State Btu prices, weighted by consumption data from SEDS.

\section{Data Sources}

\section{Prices}

1986 forward: Energy Information Administration, Petroleum Marketing Annual, Table 29 (1986-1988) and Table 30 (1989 forward), column titled "All Refiners, Sales to End Users, Through Company Outlets."

1983-1985: Energy Information Administration, $\mathrm{Pe}$ troleum Marketing Annual, 1985, Volume 1, Table 16, column titled "All Refiners and Gas Plant Operators, Sales to End-users, Through Company Outlets."

1974 forward: Bureau of Labor Statistics, U.S. Department of Labor, Consumer Prices: Energy, computer printouts of monthly gasoline prices.

1970-1982: McGraw-Hill, Inc., Platt's Oil Price Handbook and Oilmanac;: table titled "AAA 'Fuel-gauge' Report" (1982); table titled "Platt's/Lundberg Summary," (1979-1981); and table titled "Service Station Prices: Gasoline (Including Taxes)," (1970-1978).

1974-1982: Bureau of Labor Statistics, CPI Detailed Report, April 1986, Technical Notes, page 110.

1982: Energy Information Administration, Form EIA-25 "Prime Supplier Monthly Report" computer tape, unpublished data. 
1976-1984: Energy Information Administration, Monthly Energy Review, January 1985, table titled "Petroleum: Finished Motor Gasoline Supply and Disposition.".

Taxes

1983 forward (State Taxes): Federal Highway Administration, U.S. Department of Transportation, Highway Statistics, Tables MF-26 and MF-121T.

1983 through 1990 (Federal Taxes): Energy Information Administration, Petroleum Marketing Annual, 1990 , Table EN1.

1991 forward (Federal Taxes): Energy Information Administration, Petroleum Marketing Annual, Table EN1.

\section{Consumption}

1970 forward: Energy Information Administration, State Energy Data System, transportation sector, motor gasoline consumption.

\section{Conversion Factor: All Years}

5.253 million Btu per barrel

\section{Petroleum Coke (Utilities)}

Petroleum coke is consumed by the electric utility and industrial sectors. The portion of petroleum coke consumed by the industrial sector ( 95 percent in 1993) is included in the category "other petroleum products," which is discussed on page 240 . The remaining petroleum coke is used for electricity generation in 12 States over the period 1970 forward. A maximum of seven States reported consumption in any single year. Estimates of the annual consumption of petroleum coke by electric utilities are taken from SEDS. Estimates of the deliveries of petroleum coke, used in the price calculation described below, are taken from the Cost and Quality of Fuels for Electric Utility Plants (CEQ). The CEQ prices are the delivered costs of the fuels and include all taxes, transportation, and other charges paid by the utilities.
Btu Prices: 1972 Forward

The principal data source for petroleum coke used by electric utilities is an EIA public use computer tape covering the period 1972 forward. From 1972 through 1982, steam plants with a maximum capacity of 25 megawatts were included. For 1983 and subsequent years, the reporting threshold was raised to 50 megawatts capacity. In addition to the computer tape, the data are also published, but not for the full period. From 1978 through 1982, C\&Q was published monthly and annually; data for calculating petroleum coke prices are in only the monthly reports. For 1983 and subsequent years, CEQ was published annually and includes petroleum coke prices for individual States and for the Nation.

The computer tape, Cost and Quality of Fuels for Electric Utility Plants (Form 423) Historic Files, shows quantity in short tons, estimated Btu per pound, and price in dollars per million Btu. The data are presented by plant, by State, and by month. The Btu price is calculated as the annual sum of the unit prices, weighted by the total Btu in each reported delivery, divided by the annual sum of the Btu delivered to all electric utility plants within the State.

Some States have electric utility petroleum coke consumption in SEDS but no deliveries or price data in the $C \mathcal{E} Q$. Those States are assigned prices from neighboring States as shown in Table A34. The petroleum coke consumed in LA in 1992 and 1993 was obtained with no cost to the electric utility. The SEDS consumption is included with a zero price in calculating the electricity utility sector average price for LA and the US. The high DE prices prior to 1981 are actual reported prices.

Table A34. Petroleum Coke Electric Utility Price Assignments, 1972 Forward

\begin{tabular}{llc}
\hline State & Years & $\begin{array}{c}\text { State Prices } \\
\text { Assigned }\end{array}$ \\
\hline DE & $1981-1991$ & PA \\
KS & 1975 & MO \\
MO & 1983,1985 & MN \\
NY & 1974 & PA \\
WI & 1985 & MN
\end{tabular}


For the years 1970 and 1971, prices are estimated by using the gross domestic product implicit price deflator. The deflator for 1970 or 1971 is divided by the 1972 deflator and the quotient is multiplied by the 1972 price for each State to develop the price estimates for 1970 and 1971. The deflators are 35.1 in 1970, 37.1 in 1971, and 38.8 in 1972.

Although SEDS has a consumption estimate for NJ in F 1971, there are no NJ price data for any year in the U Form 423 Historic Files. Form 423 data for PA in E 1972 are used to estimate a PA price for 1971, which L is assigned to NJ. The Form 423 PA prices for 1972 and 1971 do not appear in SEPEDS since SEDS has no petroleum coke consumption in PA for those years.

\section{U.S. Btu Prices: All Years}

U.S. Btu prices are calculated as the average of the State Btu prices, weighted by consumption data from SEDS.

\section{Data Sources}

Prices

1972 forward: Energy Information Administration, electronic data from "Cost and Quality of Fuels for Electric Utility Plants (Form 423) Historic Files," as published in the following reports:

- 1983 forward: Energy Information Administration, Cost and Quality of Fuels for Electric Utility Plants, Table $20(1983,1984)$, Table 12 (19851989), Table 40 (1990, 1991), and Table 28 (1992, 1993).

- 1978-1982: Energy Information Administration, Cost and Quality of Fuels for Electric Utility Plants, table titled "Wood Chips, Refuse, and Petroleum Coke Used as Fuel by Steam-Electric Units."

1970-1972: Energy Information Administration, Annual Energy Review 1992, Appendix C. Gross Domestic Product and Implicit Price Deflator.

\section{Consumption}

1970 forward: Energy Information Administration, State Energy Data System, electric utility petroleum coke consumption.
No conversion factors are required; Btu prices are calculated directly from data sources.

\section{Residual Fuel}

Residual fuel prices are developed for the electric utility, industrial, commercial, and transportation sectors. Estimates of the amount of residual fuel consumed by sector are taken from SEDS and are adjusted for process fuel consumption in the industrial sector. (See the "Consumption Adjustments for Calculating Expenditures" section on page 249.)

\section{Electric Utility Sector}

The electric utility price for residual fuel (heavy oil) is the average delivered cost of No. 6 fuel oil receipts at electric utilities. For 1973 forward, Btu prices are developed directly from the data sources. For 1970 through 1972, prices are estimated by using simple regression analysis. All taxes, transportation, and other charges paid by utilities are included in the prices for all years.

\section{Btu Prices: 1973 Forward}

Electric utility sector residual fuel prices for 1973 forward are taken from Cost and Quality of Fuels for Electric Utility Plants (CEQ). For 1973 through 1979, Btu prices are calculated as the weighted average of contract and spot prices for No. 6 fuel oil. Table A35 lists the States and years for which consumption is indicated by SEDS but no price is shown in C\&Q. For these States, the Census division price, as shown in $C \mathcal{E} Q$, is assigned as the State price.

For 1983 forward, CEQ reports prices for steam electric plants of 50 megawatt capacity or greater. For 1980 through 1982, C\&Q prices cover all reporting plants of 25 megawatt capacity or greater.

Prices for AK in 1973 forward and for $\mathrm{HI}$ in 1973 through 1982 are developed by using the ratio of the CEQ U.S. price and the Statistical Yearbook U.S. price multiplied by the Statistical Yearbook State price. In 1973,1975 , and 1978, there was no AK price in the Statistical Yearbook. AK prices for 1973, 1975, and 1978 are based on the ratio of U.S. to AK prices for 1974, the average ratio for 1976 and 1977, and the average ratio for 1979 and 1980, respectively. 
Table A35. Residual Fuel Electric Utility

Census Division Price

Assignments, 1970 Forward

\begin{tabular}{|c|c|c|}
\hline State & Years of Asșigned Prices & Census Division \\
\hline AL & $1975-1979$ & New England \\
\hline AR & 1987, 1992, 1993 & West South Central \\
\hline$A Z$ & $1984,1985,1991-1993$ & Mountain \\
\hline co & $1982,1987,1989-1992$ & Mountain \\
\hline $\mathrm{DC}$ & $1982-1993$ & South Atlantic \\
\hline GA & 1991 & South Atlantic \\
\hline IA & $1970-1985$ & West North Central \\
\hline IN & $1970-1979$ & East North Central \\
\hline KS & $\begin{array}{l}\text { 1980, 1981, 1985-1987, } \\
1989-1992\end{array}$ & West North Central \\
\hline KY & $1970-1979$ & East South Central \\
\hline MN & $\begin{array}{l}1984,1985,1987-1990 \\
1992,1993\end{array}$ & West North Central \\
\hline MT & $1970-1979$ & Mountain \\
\hline NC & $\begin{array}{l}1976,1977,1979,1980 \\
1982,1984\end{array}$ & South Atlantic \\
\hline ND & .1970-1979 & West North Central \\
\hline NE & $1981-1983,1990,1991$ & West North Central \\
\hline NM & $1979-1982,1989-1993$ & Mountain \\
\hline NV & 1983,1985 & Mountain \\
\hline $\mathrm{OH}$ & 1992,1993 & East North Central \\
\hline OK & $\begin{array}{l}1977,1978,1980,1982- \\
1987,1989,1991-1993\end{array}$ & West South Central \\
\hline OR & $1970,1973,1974$ & Pacific \\
\hline sc & $1983,1985-1993$ & South Atlantic \\
\hline SD & $1981-1988$ & West North Central \\
\hline TN & 1979 & East South Central \\
\hline$T X$ & 1992,1993 & West South Central \\
\hline UT & $1982,1983,1986$ & Mountain \\
\hline VT & $1970-1979$ & New England \\
\hline WA & $\begin{array}{l}1970,1971,1975-1978 \\
1981-1983,1986-1988 \\
1992,1993\end{array}$ & Pacific \\
\hline WV & $1970-1977,1979$ & South Atlantic \\
\hline WY & $1970-1979$ & Mountain \\
\hline
\end{tabular}

\section{Btu Prices: 1970 Through 1972}

State-level Btu prices for 1970 through 1972 are estimated by using regression techniques and price data from the Statistical Yearbook. The regression equations use Statistical Yearbook State-level prices for 1973 through 1980 as the independent variable and the State-level prices calculated above (including the estimations for $\mathrm{AK}$ and $\mathrm{HI}$ ) as the dependent variable. Pacific regional price averages are assigned for the missing WA prices in 1970 and 1971. The average of 1970 and 1972 AK Statistical Yearbook prices is substituted for the missing 1971 AK price, and a similar calculation is made for the 1973, 1975, and 1978 prices.

\section{U.S. Btu Prices: All Years}

U.S. Btu prices are calculated as the average of the State Btu prices, weighted by consumption data from SEDS.

\section{Data Sources}

Prices

1973 forward: Energy Information Administration, Cost and Quality of Fuels for Electric Utility Plants, Table 6 (1973-1979), Table 45 (1980-1982), Table 51 (1983, 1984), Table 41 (1985-1989), Table 14 (1990, 1991), and Table 8 (1992, 1993).

1970 forward: Edison Electric Institute, Statistical Yearbook of the Electric Utility Industry, Table 43 (19701979), Table 26 (1980-1983), Table 28 (1984-1986), and Table 29 (1987 forward).

Consumption

1970 forward: Energy Information Administration, State Energy Data System, electric utility sector heavy oil consumption.

\section{Conversion Factors: All Years}

Because Btu prices are available directly from the data sources, no conversion factors are used.

\section{Industrial Sector}

The industrial sector residual fuel prices for 1984 forward are developed from refiner/reseller prices of residual fuel as published in the Petroleum Marketing Annual. Residual fuel prices for 1970 through 1983 are calculated or estimated by using average costs of residual fuel to manufacturing firms published in two Bureau of the Census reports and Platt's Oil Price Handbook and Oilmanac. Price data in these sources are available for the years 1971 and 1974 through 1981; prices for 1970, 1972, 1973, 1982, and 1983 are estimated. Prices for all years include taxes.

\section{Physical Unit Prices: 1984 Forward}

Residual fuel industrial sector physical unit prices are calculated by using refiner/reseller prices to end users from the Petroleum Marketing Annual (PMA). The States that do not have PMA prices are assigned their PAD district or subdistrict price as shown in 
Table A36, with the exception of AK. AK industrial residual fuel price for 1984 forward is based on the WA industrial residual fuel price and the ratio of the AK-to-WA industrial distillate fuel prices for each year. Tax data from Bureau of the Census publications are added to develop final sector prices.

\section{Physical Unit Prices: 1982, 1983}

After 1981, the U.S. Department of Commerce's Annual Survey of Manufactures and the Census of Manufactures ( $A S M / C M$ ) ceased publication of fuelspecific State-level residual fuel data from which prices can be calculated. Prices for 1982 and 1983 are estimated from the average relationship between the ASM/CM-based prices generated for 1978 through 1981 and the assigned Platt's No. 6 fuel oil prices for 1978 through 1981 (Table A37). These average ratios are calculated at the State-level for all States except AK, which shows no industrial sector residual fuel use reported in SEDS for 1982 and 1983. Physical unit residual fuel industrial prices for 1982 and 1983 are calculated by using the assigned Platt's prices for 1982 and 1983 (Table A37) and the State-level average ratios. The resulting estimates implicitly include taxes that reflect individual State differences.

Physical Unit Prices: 1971, 1974 Through 1981

For the years 1971 and 1974 through 1981, industrial sector residual prices are calculated directly from

Table A36. Residual Fuel Industrial Sector Price Assignments, 1984 Forward

\begin{tabular}{|c|c|c|}
\hline State & Years & $\begin{array}{l}\text { PAD District Prices } \\
\text { Assigned }\end{array}$ \\
\hline AR & 1985 & PAD District III \\
\hline$A Z$ & 1984-1991, 1993 & PAD District V \\
\hline $\mathrm{CO}$ & $1986,1988,1991-1993$ & PAD District IV \\
\hline ID & $1985,1986,1989-1992$ & PAD District IV \\
\hline MS & 1988, 1991, 1992 & PAD District III \\
\hline MT & 1992 & PAD District IV \\
\hline ND & 1988-1992 & PAD District II \\
\hline NM & $\begin{array}{l}1984-1986,1990,1991, \\
1993\end{array}$ & PAD District III \\
\hline NV & $1986,1988,1991-1993$ & PAD District V \\
\hline OK & 1992,1993 & PAD District II \\
\hline OR & 1989 & PAD District V \\
\hline Sc & 1993 & PAD Subdistrict IC \\
\hline SD & $1990-1993$ & PAD District \|I \\
\hline UT & 1989-1992 & PAD District IV \\
\hline WV & 1984 & PAD Subdistrict IC \\
\hline WY & 1989-1991, 1993 & PAD District IV \\
\hline
\end{tabular}

cost and quantity data reported by the $A S M / C M$. For all States with available cost and quantity data, prices are equal to the average cost of residual fuel to manufacturers. Taxes are included in the published cost data. Missing data for these years are assigned from the average prices of adjacent States as shown in Table A38.

\section{Physical Unit Prices: 1970, 1972, 1973}

Since $A S M / C M$ data are not available for 1970,1972 , or 1973, prices for these years must be estimated. Physical unit prices are based on the ratio of the 1971 $C M$ prices to the 1971 assigned No. 6 fuel oil prices from Platt's Oil Price Handbook and Oilmanac (Table A37). The estimated $1971 \mathrm{CM}$ prices for NM and WY are used in the calculations. The resulting ratios for each State are used with the Platt's assigned prices for 1970, 1972, and 1973 to estimate prices. The final estimates implicitly include State-specific taxes.

\section{Btu Prices: All Years}

Btu prices for States are calculated from the physical unit prices and the conversion factor. U.S. Btu prices are calculated as the average of the State Btu prices, weighted by consumption data from SEDS, which are adjusted for process fuel consumption.

\section{Data Sources}

\section{Prices}

1984 forward: Energy Information Administration, Petroleum Marketing Annual, Table A4 (1984-1990), Table A3 (1991-1993).

1984 forward: Industrial sector distillate fuel price estimates from the SEPER data system (AK and WA only).

1970-1983: McGraw-Hill, Inc., Platt's Oil Price Handbook and Oilmanac, refinery and terminal prices for No. 6 fuel oil, average of highs and lows.

1971, 1977, 1981: Bureau of the Census, U.S. Department of Commerce, Census of Manufactures, Fuels and Electric Energy Consumed, Part 2, Table 3. (Dates shown on the report covers are, respectively, 1972, 1977, and 1982.)

1974-1976 and 1978-1980: Bureau of the Census, U.S. Department of Commerce, Annual Survey of 


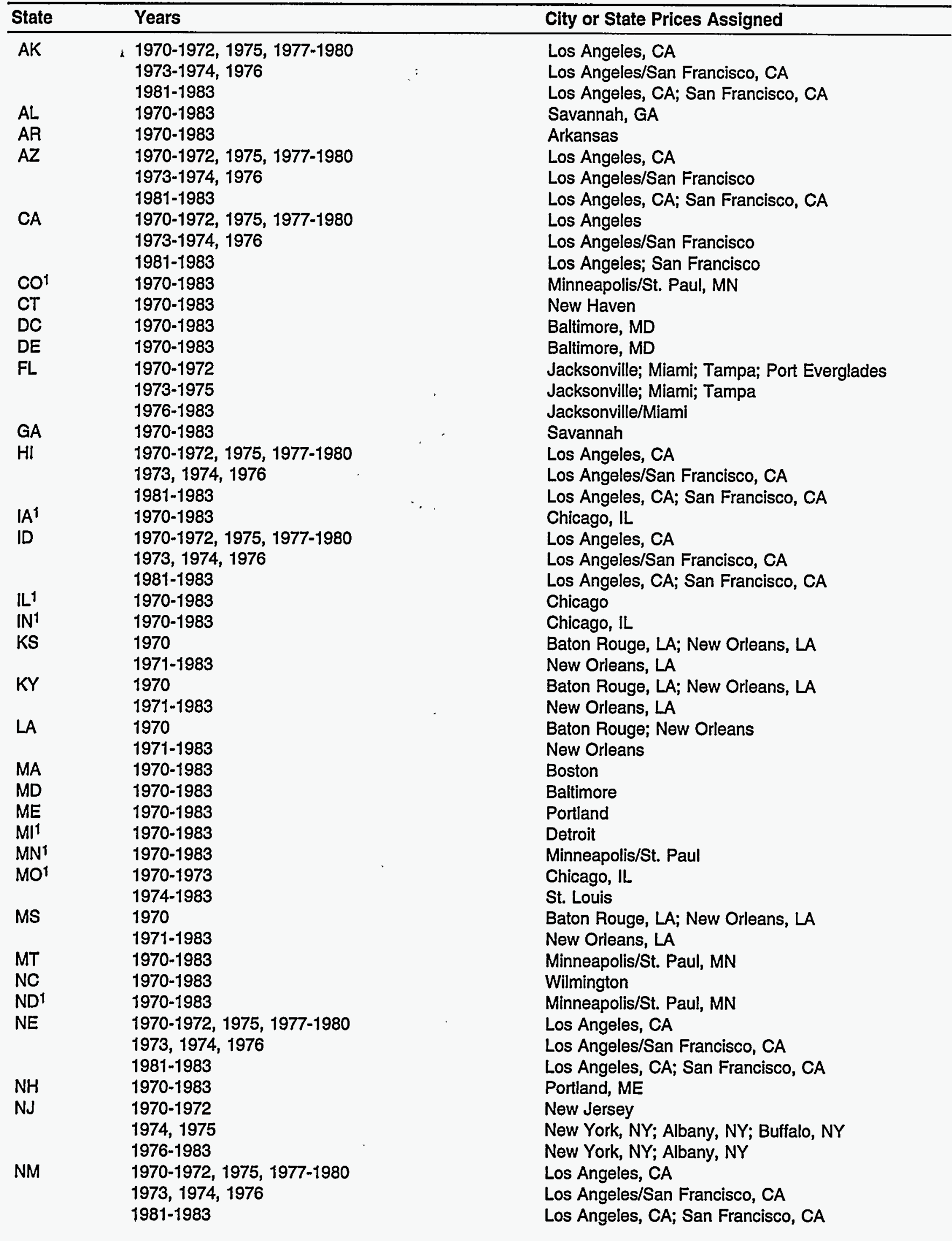

See footnotes at end of table. 
R

RI

1970-1983

1970-1975

1976-1983

SC 1970-1983

$S^{1} \quad 1970-1983$

TN 1970

N
1971-1983

1970-1972

1973-1983

1970-1983

1970-1983

1970-1983

1970-1972, 1975, 1978, 1979

$1973,1974,1976$

1980-1983

$\begin{array}{ll}\text { WI } & 1970-1983 \\ \text { WV } & 1970-1983\end{array}$

$W^{1} \quad 1970-1983$

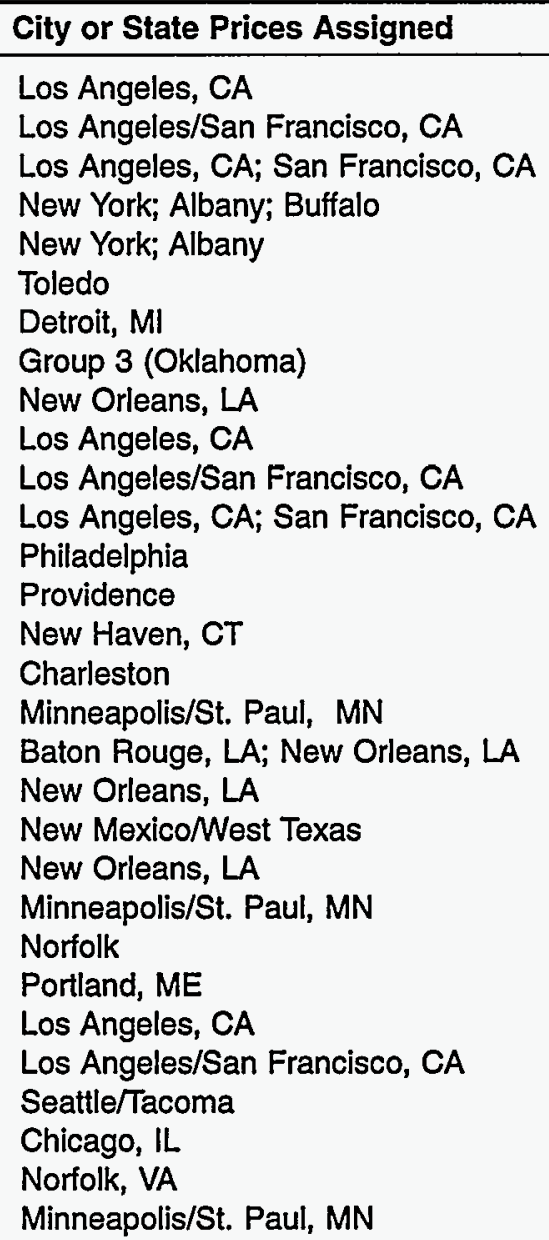

\footnotetext{
${ }_{2}^{1}$ Raw data from Platt's must be converted from cents per gallon to dollars per barrel.

${ }^{2}$ As shown in Platt's.
}

factures, Fuels and Electric Energy Consumed, States by Industry Group, Table 3.

Taxes

1993: Bureau of the Census, U.S. Department of Commerce, State Tax Review, Volume 54, No. 31, map titled "State Gasoline, Sales, and Cigarette Tax Rates as of July $1,1993, "$ sales tax rates.

1987-1992: Bureau of the Census, U.S. Department of Commerce, State Government Tax Collections, Table 8, column titled "Percentage rate, September 1."

1984-1986: Bureau of the Census, U.S. Department of Commerce, Statistical Abstract of the United States, table titled "State Government Tax Collections and Excise Taxes," column titled "Excise Taxes, General sales and gross receipts."
Table A38. Residual Fuel Industrial Sector Price Assignments, 1971, 1974-1981

\begin{tabular}{lll}
\hline State & \multicolumn{1}{c}{ Years } & State Prices Used \\
\hline AK & 1980,1981 & HI, WA \\
DC & $1979-1981$ & MD, VA \\
MT & $1974-1979$ & ID, ND, SD \\
ND & 1980 & MN, MT, SD \\
NM & $1971,1974-1981$ & AZ, CO, TX \\
NV & $1974-1978$ & AZ, CA, ID, OR, UT \\
OK & $1974-1978,1980$ & AR, CO, KS, MO, TX \\
SD & 1981 & IA, MN, MT, ND, NE \\
WY & $1971,1974-1981$ & CO, NE, UT \\
& & \\
\hline
\end{tabular}




\section{Consumption}

1970 forward: Energy Information Administration, State Energy Data System, industrial sector residual consumption.

\section{Conversion Factor: All Years}

6.287 million Btu per barrel

\section{Commercial Sector}

For 1984 forward, State-level commercial sector residual fuel prices are developed from refiner/reseller prices of residual fuel to end users published in the PMA. For 1970 through 1983, commercial sector residual fuel prices are estimated for all States from national-level residual fuel prices and the State-level electric utility sector residual fuel prices. State and Federal taxes are included in the final prices for all years.

\section{Physical Unit Prices: 1984 Forward}

Commercial sector residual fuel physical unit prices are calculated by using refiner/reseller prices to end users from the PMA. The States that do not have $P M A$ prices are assigned their PAD district or subdistrict price (Table A39), with the exception of AK. The AK commercial residual fuel prices for 1984 forward are based on the WA commercial residual fuel price and the ratio of the AK-to-WA commercial distillate

\section{Table A39. Residual Fuel Commercial Sector Price Assignments, 1984 Forward}

\begin{tabular}{lll}
\hline State & \multicolumn{1}{c}{ Years } & \multicolumn{1}{c}{$\begin{array}{c}\text { PAD District Prices } \\
\text { Assigned }\end{array}$} \\
\hline AZ & $1984,1985,1988,1991$ & PAD District V \\
CO & $1986,1992,1993$ & PAD District IV \\
ID & $1985,1986,1989-1992$ & PAD District IV \\
MS & $1988,1991,1992$ & PAD District III \\
MT & 1992 & PAD District IV \\
ND & $1988,1989-1992$ & PAD District II \\
NM & 1984,1985 & PAD District III \\
NV & $1986,1988,1991,1992$ & PAD District V \\
OK & 1992 & PAD District II \\
OR & 1989 & PAD District V \\
SC & 1993 & PAD Subdistrict IC \\
SD & $1990-1993$ & PAD District II \\
UT & $1989-1992$ & PAD District IV \\
WV & 1984 & PAD Subdistrict IC \\
WY & $1989-1991$ & PAD District IV \\
& & \\
\hline
\end{tabular}

fuel prices for each year. Tax data from Bureau of the Census publications are added to develop final sector prices.

\section{Physical Unit Prices: 1976 Through 1983}

The commercial sector residual fuel physical unit prices for 1976 through 1983 are estimated from the electric utility residual fuel prices and the U.S. average retail residual fuel prices (with taxes added) for each year. The resulting price estimates implicitly include taxes that reflect individual State differences.

1. The first step in the estimation of the commercial residual fuel physical unit State prices is to convert the State-level tax rates reported in the Bureau of the Census publications into the volume-weighted average U.S. sales tax rate by using commercial residual consumption data from SEDS.

2. A preliminary U.S. residual fuel oil price, including taxes, is computed by using the average U.S. tax rate estimated above and the annual average U.S. residual fuel price to end users (average retail price excluding taxes) from the Monthly Energy Review (MER).

3. Commercial sector physical unit residual fuel prices for States are computed by using the electric utility sector residual fuel prices. To do this calculation, the ratio of the State-level and U.S. prices in the commercial sector is assumed to be the same as the ratio of State and U.S. prices in the electric utility sector. Some States are missing electric utility prices for 1976 through 1983; these are estimated by using adjacent States' average prices (Table A40).

\section{Physical Unit Prices: 1970 Through 1975}

Since no national or State-level retail residual prices are available from published data sources, commercial sector residual prices for 1970 through 1975 are estimated. The estimation method is based on the assumption that the average ratio of State-to-U.S. prices is the same in the commercial and electric utility sectors. The average ratio for 1976 through 1979 of the MER U.S. tax-adjusted prices to the electric utility sector U.S. prices is calculated and used as an adjustment factor with State-level electric utility sector prices for 1970 through 1975. The resulting price estimates implicitly include taxes that reflect individual State differences. 


\begin{tabular}{|c|c|c|}
\hline & $\begin{array}{l}\text { Residual Fuel } \\
\text { Price Assignn }\end{array}$ & ents, 1970-1983 \\
\hline State & Years & $\begin{array}{l}\text { State Prices Used } \\
\text { in the Estimation }\end{array}$ \\
\hline AL & $\begin{array}{l}1970-1974,1980 \\
1982,1983\end{array}$ & $\mathrm{FL}, \mathrm{GA}, \mathrm{MS}$ \\
\hline ID & $\begin{array}{l}1980,1981,1983 \\
1982\end{array}$ & $\begin{array}{l}\mathrm{CA}, \mathrm{CO} \\
\mathrm{CA}\end{array}$ \\
\hline IN & $1980-1983$ & IL, MI, OH \\
\hline KY & $1980-1983$ & $\mathrm{IL}, \mathrm{MO}, \mathrm{OH}, \mathrm{VA}$ \\
\hline MT & $\begin{array}{l}1980,1983 \\
1982\end{array}$ & $\begin{array}{l}\mathrm{CO}, \mathrm{MN} \\
\mathrm{MN}\end{array}$ \\
\hline NC & 1981,1983 & GA, VA \\
\hline \multirow[t]{2}{*}{ ND } & 1980,1983 & $M N, S D$ \\
\hline & 1981,1982 & MN \\
\hline OR & $1975-1983$ & $\mathrm{CA}$ \\
\hline TN & $1970-1978,1980-1983$ & $A R, G A, M O, M S, V A$ \\
\hline VT & $1980-1983$ & $\mathrm{ME}, \mathrm{NH}, \mathrm{NY}$ \\
\hline WI & 1982,1983 & IL, MI, MN \\
\hline WV & $1980-1983$ & $\mathrm{MD}, \mathrm{OH} ; \mathrm{PA}, \mathrm{VA}$ \\
\hline \multirow[t]{3}{*}{ WY } & 1980 & $\mathrm{CO}, \mathrm{NE}, \mathrm{SD}, \mathrm{UT}$ \\
\hline & 1981,1983 & $\mathrm{CO}$ \\
\hline & 1982 & MN \\
\hline
\end{tabular}

1. The average ratio of the $M E R$ tax-adjusted U.S. prices and the electric utility sector U.S. prices is calculated for 1976 through 1979.

2. State-level commercial sector residual fuel prices are calculated by using the electric utility sector physical unit price series for 1970 through 1975 and the average ratio computed above. Price assignments for States missing electric utility sector data are shown in Table A40.

\section{Btu Prices: All Years}

Btu prices for States are calculated from the physical unit prices and the conversion factor. U.S. Btu prices are calculated as the average of the State Btu prices, weighted by consumption data from SEDS.

\section{Data Sources}

Prices

1984 forward: Energy Information Administration, Petroleum Marketing Annual, Table A4 (1984-1990), Table A3 (1991-1993).
1984 forward: Commercial sector distillate fuel price estimates from the SEPER data system (AK and WA only).

1978-1983: Energy Information Administration, Monthly Energy Review, December 1988, table titled "Refiner Sales Prices of Residual Fuel Oil," column titled "Average Sales to End Users."

1976, 1977: Energy Information Administration, Monthly Energy Review, December 1983, table titled "Average No. 6 Residual Fuel Oil Prices," column titled "Average, Retail."

1970-1983: Electric utility sector residual fuel price estimates (in physical units) from the SEPER data system.

Taxes

1993: Bureau of the Census, U.S. Department of Commerce, State Tax Review, Volume 54, No. 31, map titled "State Gasoline, Sales, and Cigarette Tax Rates as of July 1,1993," sales tax rates.

1987-1992: Bureau of the Census, U.S. Department of Commerce, State Government Tax Collections, Table 8, column titled "Percentage rate, September 1."

1976-1986: Bureau of the Census, U.S. Department of Commerce, Statistical Abstract of the United States, table titled "State Government Tax Collections and Excise Taxes," column titled "Excise Taxes, General sales and gross receipts."

\section{Consumption}

1970 forward: Energy Information Administration, State Energy Data System, commercial sector residual fuel consumption.

\section{Conversion Factor: All Years}

6.287 million Btu per barrel

\section{Transportation Sector}

Residual fuel is consumed in the transportation sector for vessel bunkering, military use, and railroads. In 1970, vessels consumed 74 percent of the transportation use of residual fuel, and the military and railroads accounted for 24 percent and 2 percent, respectively. By 1993, vessel use had grown to 99 percent, military use had dropped to 1 percent, and the railroads' share was insignificant. Prices are 
developed for vessel bunkering, and electric utility prices are assigned to the military and railroad uses. Tax adjustments are made as described below. The transportation sector average price for each State and year is the consumption-weighted average of the prices of the three uses.

\section{Physical Unit Prices: All Years}

Vessel Bunkering. Physical unit prices are calculated from actual or estimated U.S. average bunker $C$ prices and electric utility State and U.S. residual fuel prices for each year. The ratio of U.S. bunker C price to U.S. residual fuel electric utility price is multiplied by the State residual fuel electric utility price to obtain the State bunker $C$ price. Taxes are calculated as described for the commercial sector (1976 through 1983) and added, so that final State vessel bunkering price estimates implicitly include State-specific taxes. Other procedures are described separately by groups of years:

1. For 1982 forward, national average prices for residual fuel with sulfur content greater than 1 percent are taken from the Annual Energy Review and used as proxies for bunker $C$ prices.

2. For 1975 through 1981 , national average bunker $C$ prices are available from the Monthly Petroleum Product Price Report (MPPPR). Annual average U.S. prices for 1975 and 1976 are calculated as the simple average of the monthly prices for each respective year because annual average prices are not shown in the MPPPR.

3. For 1970 through 1974 , no U.S. bunker C prices are available. To estimate State-level prices for these years, the average ratio of published bunker $C$ prices and electric utility sector prices for 1975 through 1979 is calculated and multiplied by the State-level electric utility prices for 1970 through 1974.

Missing State prices are assigned adjacent States' average prices as shown in Table A41.

Military and Railroad Use. For all years, electric utility sector residual fuel prices are assigned to military and railroad uses. The electric utility prices include taxes. Since the military does not pay State taxes, their prices are adjusted to remove taxes. Estimation of the tax adjustment is described under the commercial sector.

In some cases, States have no reported consumption of residual fuel oil in the electric utility sector and,
Table A41. Residual Fuel Transportation Sector Price Assignments, 1970-1986

\begin{tabular}{|c|c|c|}
\hline State & Years & $\begin{array}{l}\text { State Prices Used } \\
\text { in the Estimation }\end{array}$ \\
\hline$A L$ & $1970-1974,1980-1986$ & FL, GA, MS \\
\hline $\mathrm{CO}$ & 1986 & KS, NM, UT \\
\hline CT & 1978 & $\mathrm{NH}, \mathrm{VT}$ \\
\hline \multirow[t]{2}{*}{ DC } & 1975 & MD \\
\hline & 1978 & PA \\
\hline GA & 1978 & KY, MS \\
\hline ID & 1970,1979 & $\mathrm{CA}, \mathrm{CO}$ \\
\hline IL & 1975 & $|A, I N, W|$ \\
\hline $\mathbb{N}$ & $1980-1986$ & IL, MI, OH \\
\hline $\mathrm{KS}$ & 1975 & MO, NE \\
\hline KY & $1980-1984$ & $\mathrm{IL}, \mathrm{MO}, \mathrm{OH}, \mathrm{VA}$ \\
\hline MD & 1978 & DE, PA \\
\hline ME & 1975 & $V T$ \\
\hline MN & 1986 & IL, MI \\
\hline MT & $1983-1985$ & $\mathrm{CO}, \mathrm{MN}, \mathrm{SD}$ \\
\hline \multirow[t]{3}{*}{ NC } & 1975 & GA \\
\hline & 1978 & KY \\
\hline & $1981,1983,1985,1986$ & GA, VA \\
\hline \multirow[t]{2}{*}{ ND } & $1982-1984$ & $M N, S D$ \\
\hline & 1986 & $S D$ \\
\hline $\mathrm{NH}$ & 1975 & $\mathrm{VT}$ \\
\hline NM & 1983,1984 & $\mathrm{CO}$ \\
\hline NV & 1975,1978 & $\mathrm{CA}$ \\
\hline $\mathrm{OH}$ & 1975 & IN, MI \\
\hline OK & 1975 & MO, TX \\
\hline \multirow[t]{2}{*}{ OR } & 1972 & $C A, W A$ \\
\hline & $1975-1986$ & $\mathrm{CA}$ \\
\hline \multirow[t]{2}{*}{$\mathrm{SC}$} & 1975,1984 & GA \\
\hline & 1978 & $\mathrm{AL}, \mathrm{FL}$ \\
\hline SD & 1975,1978 & MN, ND \\
\hline
\end{tabular}

TN $1970,1971,1973,1974$,

$1976,1977,1980-1982$

1975

1978

UT 1984

1975

VA 1975

1978

WA 1984,1985

WI 1978, 1982-1985

1986

WV 1985

WY $1981,1982,1985$

therefore, no final price in that sector. Since the electric utilities sector prices are used as a base in the calculation of prices in the other sectors, intermediate prices are calculated for OR in 1971 and for all States that need prices for 1987 forward. 
Average Prices. Transportation sector prices are the average of bunker fuel, military, and railroad prices, weighted by each category's share of total transportation consumption from SEDS.

\section{Btu Prices: All Years}

Btu prices for States are calculated from the physical unit prices and the residual fuel conversion factor. U.S. Btu prices are calculated as the average of the State Btu prices, weighted by consumption data from SEDS.

\section{Data Sources}

Prices

1982 forward: Energy Information Administration, Annual Energy Review 1994, Table 5.20, row titled "Sales Prices to End Users, Residual Fuel Oil, Greater Than 1 Percent Sulfur Content."

1976-1981: Energy Information Administration, Monthly Petroleum Product Price Report, Table 3.

1975: Federal Energy Administration, Monthly Petroleum Product Price Report, Table 3.

1970-1986: Electric utility sector residual fuel price estimates (in physical units) from SEPEDS.

\section{Taxes}

1993: Bureau of the Census, U.S. Department of Commerce, State Tax Review, Volume 54, No. 31, map titled "State Gasoline, Sales, and Cigarette Tax Rates as of July 1,1993," sales tax rates.

1987-1992: Bureau of the Census, U.S. Department of Commerce, State Government Tax Collections, Table 8, column titled "Percentage rate, September 1."

1976-1986: Bureau of the Census, U.S. Department of Commerce, Statistical Abstract of the United States, table titled "State Government Tax Collections and Excise Taxes," column titled "Excise Taxes, General sales and gross receipts."

\section{Consumption}

1970 forward: Energy Information Administration, State Energy Data System, transportation sector residual fuel consumption, including the subcategories for vessel bunkering, military, and railroad uses.

\section{Conversion Factor: All Years}

6.287 million Btu per barrel.

\section{Other Petroleum}

Sixteen separate products are included in the category called "other petroleum." Of the 16 products, prices are developed for the 7 noted with asterisks (*) below and described in the following paragraphs. All of these products are assigned to the industrial sector.

1. Aviation gasoline blending components

2. Crude oil

3. Miscellaneous products (*)

4. Motor gasoline blending components

5. Natural gasoline, including isopentane (19701983)

6. Pentanes plus (1984 forward)

7. Petrochemical feedstocks, naphtha $\left(^{*}\right.$

8. Petrochemical feedstocks, other oils $\left(^{*}\right)$

9. Petrochemical feedstocks, still gas (1970-1985) $\left(^{*}\right)$

10. Petroleum coke $\left(^{*}\right)$

11. Plant condensate (1970-1983)

12. Special naphthas $\left(^{*}\right)$

13. Still gas

14. Unfinished oils

15. Unfractionated stream (1970-1983)

16. Waxes $(*)$

\section{Physical Unit Prices: All Years}

Only national-level prices are developed for the seven other petroleum products because State-level price information is not available, and taxes are not included in any of the estimates. Consumption for the other nine products are completely removed as process fuel or intermediate products. (See the "Consumption Adjustments for Calculating Expenditures" section on page 249.)

Starting in 1984, three products-natural gasoline, plant condensate, and unfractionated stream-are dropped, and pentanes plus is added in the Energy Information Administration (EIA) reporting system that is the basis of the consumption estimates. Natural gasoline (including isopentane) and plant condensate are reported together as the new product, pentanes plus. Unfractionated stream is dropped because its components are reported separately as liquefied petroleum gases. 
The products in this category vary from inexpensive (absorption oils similar to kerosene) to very expensive (hydraulic fluids). The price estimates are based on the evidence presented in the Bureau of Mines Minerals Yearbooks of the 1970's indicating that the greater part of the miscellaneous product line consists of finished petrochemicals, especially the aromatic hydrocarbons: benzene, toluene, and the xylenes.

Price estimates for 1972, 1977, 1982, 1987, and 1992 are taken from Census of Manufactures (CM) data on quantity and value of "aromatics" and "other finished petroleum products" shipped by petroleum refining industries, i.e., Standard Industrial Code (SIC) 2911. The ratio of miscellaneous-products-tocrude-oil price for these 5 years varies widely. The following ratios, shown rounded, are used to estimate miscellaneous products prices for the years indicated:

$\begin{array}{ll}\text { 1970 - 1974: } & 1.91 \text { times the crude oil price } \\ \text { 1975 - 1979: } & 2.42 \text { times the crude oil price } \\ 1980 \text { - 1984: } & 1.56 \text { times the crude oil price. } \\ 1985 \text { - 1989: } & 1.99 \text { times the crude oil price. } \\ 1990 \text { - forward: } & 1.86 \text { times the crude oil price }\end{array}$

Quantity data for 1992 are published in pounds and are converted to barrels by use of the conversion factors of 7.282 pounds per gallon and 42 gallons per barrel.

\section{Price Data Sources}

1970 Forward: Energy Information Administration, Annual Energy Review 1994, Table 5.19, column titled "Composite, Nominal."

1972, 1977, 1982, 1987, 1992: Bureau of the Census, U.S. Department of Commerce, Census of Manufactures, data for Standard Industrial Code (SIC) 2911 on "Quantity and Value of Shipments by All Producers" as shown in Table 6a from MC77-I-29A, Product Codes 2911054, 2911056 (1972 and 1977); Table 6a-1 from MC87-I-29A, Product Codes 2911D55 and 2911D57 (1982 and 1987); and Table 6a-1 from MC92I-29A, Product Codes 2911D 55 and 2911D 57 (1992).

\section{Physical Unit Conversion Factors}

1992: Gas Processors Suppliers Association in cooperation with the Gas Processors Association, Engineering Data Book, 9th Edition, 1972, 4th Revision, printed 1979, pages 16-2 and 16-3, lines 42-47.
Naphthas for petrochemical feedstock use are those oils with boiling points less than $401^{\circ} \mathrm{F}$. Consumer prices for 1978 through 1980 are derived from the special Annual Survey of Manufactures (ASM) series on "Hydrocarbon, Coal, and Coke Materials Consumed," by using data for industries in SIC 2869 (industrial organic chemicals) and SIC 2821 (plastics materials, synthetic resins, and nonvulcanizable elastomers). A price estimate for 1982 is obtained from the CM, based on data for SIC 2869 only. Since the ratio of petrochemical-naphtha-to-crude-oil price is reasonably constant in $1978,1979,1980$, and 1982, the simple average of the four ratios, 1.23 , is used to estimate prices for petrochemical feedstocks, naphthas, for all other years.

\section{Price Data Sources}

1970-1977, 1981, 1983 forward: Energy Information Administration, Annual Energy Review 1994, Table 5.19, column titled "Composite, Nominal."

1982: Bureau of the Census, U.S. Department of Commerce, 1982 Census of Manufactures, M82-I-28F3(P), page 6, SIC 2869.

1980: Bureau of the Census, U.S. Department of Commerce, 1980 Annual Survey of Manufactures, M80(AS)-4.3, page 9, SIC 2821.

1978, 1979: Bureau of the Census, U.S. Department of Commerce, 1979 Annual Survey of Manufactures, M79(AS)-4.3, page 8, SIC 2821 and 2869.

\section{Petrochemical Feedstocks, Other Oils}

Petrochemical feedstocks referred to as "other oils" or "gas oils" are those oils with boiling points equal to or greater than $401^{\circ} \mathrm{F}$. Consumer prices for 3 years are obtained from the data on gas oils presented in the special $A S M$ series on hydrocarbons consumed by using data for industries in SIC 2865 (cyclic crudes and intermediates). The other-oils-tocrude-oil price ratio is quite stable, and the average ratio for the 3-year period, 1.607, is used to estimate prices for petrochemical feedstocks, other oils, for all other years.

\section{Price Data Sources}

1970-1977, 1981 forward: Energy Information Administration, Annual Energy Review 1994, Table 5.19, column titled "Composite, Nominal." 
1979, 1980: Bureau of the Census, U.S. Department of Commerce, 1980 Annual Survey of Manufactures, M80(AS)-4.3, page 9, SIC 2865.

1978: Bureau of the Census, U.S. Department of Commerce, 1979 Annual Survey of Manufactures, M79(AS)-4.3, page 8, SIC 2865.

\section{Petrochemical Feedstocks, Still Gas (1970 Through 1985)}

The source data for still gas is a mixture of consumer prices and producer prices for industries in SIC 2869 and SIC 2911 (petroleum refining). The still-gas-tocrude-oil price ratio is somewhat variable because still gas is a highly variable gaseous mixture. Value and quantity are available for 1972, 1977 through 1980, and 1982. In imputing prices for years when data from the CM or $A S M$ are not available, the average still-gas-to-crude-oil price ratio, 0.759 , is used. After 1985, EIA data series no longer report feedstock and refinery use of still gas separately and all SEDS industrial consumption is removed from SEPER. (See the "Consumption Adjustments for Calculating Expenditures" section on page 249.)

\section{Price Data Sources}

1970, 1971, 1981, 1983-1985: Energy Information Administration, Annual Energy Review 1994, Table 5.19, "Composite, Nominal."

1982: Bureau of the Census, U.S. Department of Commerce, 1987 Census of Manufactures, MC87-I-29A, Table 6a, SIC 2911.

1979, 1980: Bureau of the Census, U.S. Department of Commerce, 1980 Annual Survey of Manufactures, M80(AS)-4.3, page 9, SIC 2869.

1978: Bureau of the Census, U.S. Department of Commerce, 1979 Annual Survey of Manufactures, M79(AS)-4.3, page 28, SIC 2869.

1972, 1977: Bureau of the Census, U.S. Department of Commerce, 1977 Census of Manufactures, MC77-129A, page 29A-20, SIC 2911.

\section{Petroleum Coke}

Petroleum coke is consumed by the industrial and electric utility sectors. The portion of petroleum coke consumed by the electric utility sector (about 5 percent in 1993) is described in the Petroleum Coke (Utilities) section on page 229. The remaining petro- leum coke is marketed to industrial consumers in two forms, calcined and uncalcined. Calcined coke is about three times as expensive as uncalcined. A quantity-weighted U.S. average price is calculated by using U.S. Department of Commerce exports data and is assigned to all States with industrial petroleum coke consumption. The weighted average price is calculated by dividing the sum of the values of calcined and uncalcined petroleum coke exports by the sum of the two quantities exported. The physical unit conversion factor for petroleum coke is 5 barrels per short ton.

\section{Price Data Sources}

1989 forward: Bureau of the Census, U.S. Department of Commerce, December issues of EM-545, Foreign and Domestic Exports, for Petroleum Coke, Not Calcined, Commodity 2713110000 and Petroleum Coke, Calcined, Commodity 2713120000.

1988: Bureau of the Census, U.S. Department of Commerce, December issue of EM-522, U.S. Exports, Schedule B, Community by Country, Petroleum Coke, Except Calcined, Commodity 5213150, and Petroleum Coke, Calcined, Commodity 5175120.

1987: Bureau of the Census, U.S. Department of Commerce, December issue of EM-622, U.S. Exports, Schedule B, Commodity by Country, Petroleum Coke, Except Calcined, Commodity 5213150, and Petroleum Coke, Calcined, Commodity 5175120.

1986: Bureau of the Census, U.S. Department of Commerce, December issue of EM-546, U.S. Exports, Schedule B, Commodity by Country, Petroleum Coke, Except Calcined, Commodity 5213150, and Petroleum Coke, Calcined, Commodity 5175120.

1978-1985: Bureau of the Census, U.S. Department of Commerce, FT-446, U.S. Exports, Schedule B, Commodity by Country, Petroleum Coke, Except Calcined, Commodity 5213150, and Petroleum Coke, Calcined, Commodity 5175120 .

1970-1977: Bureau of the Census, U.S. Department of Commerce, December issues of FT-410, U.S. Exports, Schedule B, Commodity by Country, Petroleum Coke, Except Calcined, Commodity 3329420, and Petroleum Coke, Calcined, Commodity 3329410.

\section{Special Naphthas}

Prices for special naphthas are developed as the simple averages of the city prices for "varnish makers and painters naphtha" and two types of "solvent 
naphtha" that are published in the Chemical Marketing Reporter. For 1984 forward, the prices are averaged from the first issue of each month; for 1974, 1979, and 1980, when petroleum prices were increasing rapidly, prices are averaged from 10 randomly-selected issues; and for all other years, prices are averaged from at least 5 randomlyselected issues.

\section{Price Data Source}

1970 forward: Schnell Publishing Co., Inc., Chemical Marketing Reporter, selected monthly issues.

\section{Waxes}

Waxes data include fully refined crystalline wax, other refined crystalline wax, and microcrystalline wax. Price estimates for 1970 through 1973 and 1986 forward are obtained by dividing the value of exports by the quantity exported. For 1974 through 1985, prices are estimated by applying price indices to a representative base price. Producer prices for 1967 for the three waxes are available from data in the 1967 Census of Manufactures. A weighted-average price for 1967 of $\$ 15.75$ per barrel is obtained by summing the values of shipments of the three waxes and dividing the sum by the total quantity shipped. An annual composite price index for these three waxes is listed in the Bureau of Labor Statistics publication Producer Prices and Producer Price Indexes for April 1974 through June 1985. Price estimates for 1975 through 1984 are derived by multiplying the published price indices by the estimated 1967 base price. The indices for 1974 and 1985 are estimated as the simple average of monthly price indices that are available for that year. The physical unit conversion factors for wax are 280 pounds per barrel and 1 pounds equals 0.45359237 kilograms.

\section{Price Data Sources}

1989 forward: Bureau of the Census, U.S. Department of Commerce, December issues of Report No. EM-545, titled Foreign and Domestic Exports for Paraffin Wax Less Than 0.75 Percent Oil (Commodity 2712200000) and Other Mineral Waxes NESOI (Commodity 2712900000 ).

1987, 1988: Bureau of the Census, U.S. Department of Commerce, December issues of Report No. EM546 (1987) and EM-522 (1988), titled U.S. Exports, Schedule B, Commodity by Country for Paraffin Wax and Other Petroleum Waxes Unblended incl Microcrystalline Wax (Commodity 4925200).
1986: Bureau of the Census, U.S. Department of Commerce, December issue of EM-546, U.S. Exports, Schedule B, Commodity by Country for Paraffin Wax, Crystalline, Fully Refined (Commodity 4925210), Paraffin Wax, Crystalline, Except Fully Refined (Commodity 4925220), and Petroleum Waxes, NSPF incl Microcrystalline Wax (Commodity 4925240).

1974-1985: Bureau of Labor Statistics, U.S. Department of Labor, Producer Prices and Producer Price Indexes, Annual Supplement, Commodity Code 0577.

1974-1985: Bureau of the Census, U.S. Department of Commerce, Census of Manufactures, 1967, page 29 A15, quantity and value of shipments of waxes in 1967.

1970-1973: Bureau of the Census, U.S. Department of Commerce, December issues of FT-410, U.S. Exports, Schedule B, Commodity by Country for Paraffin Wax, Crystalline, Fully Refined (Commodity 3326220), Paraffin Wax, Crystalline, Except Fully Refined (Commodity 3326230), and Microcrystalline Wax (Commodity 3326210).

\section{Btu Prices: All Years}

Btu prices for the seven petroleum products are calculated by converting physical unit prices from dollars per barrel to dollars per million Btu by using the conversion factors shown in Table A42. The U.S. average price that is developed for each product is assigned to the industrial sector of States in years where there is consumption. The State-level and U.S. "other petroleum" average price are the average of the seven petroleum products, weighted by SEDS consumption data. The variable State average prices reflect the different mix of products consumed.

Table A42. Other Petroleum Products Btu Conversion Factors

\begin{tabular}{lc}
\hline Petroleum Product & $\begin{array}{c}\text { Million Btu } \\
\text { per barrel }\end{array}$ \\
\hline Miscellaneous Products & 5.796 \\
Petrochemical Feedstocks & \\
Naphtha & 5.248 \\
Other Oils & 5.825 \\
Still Gas & 6.000 \\
Petroleum Coke & 6.024 \\
Special Naphthas & 5.248 \\
Waxes & 5.537 \\
\hline
\end{tabular}


- Table A43 shows national-level estimated prices and T expenditures by the other petroleum product compoH nents for the years 1970, 1975, 1980, 1985, and 1990 H forward.

$\mathbf{R}$

$\mathbf{P}$

Table A43. Other Petroleum Price and Expenditure Estimates for the Industrial Sector, United States, 1970, 1975, 1980, 1985, and 1990-1993

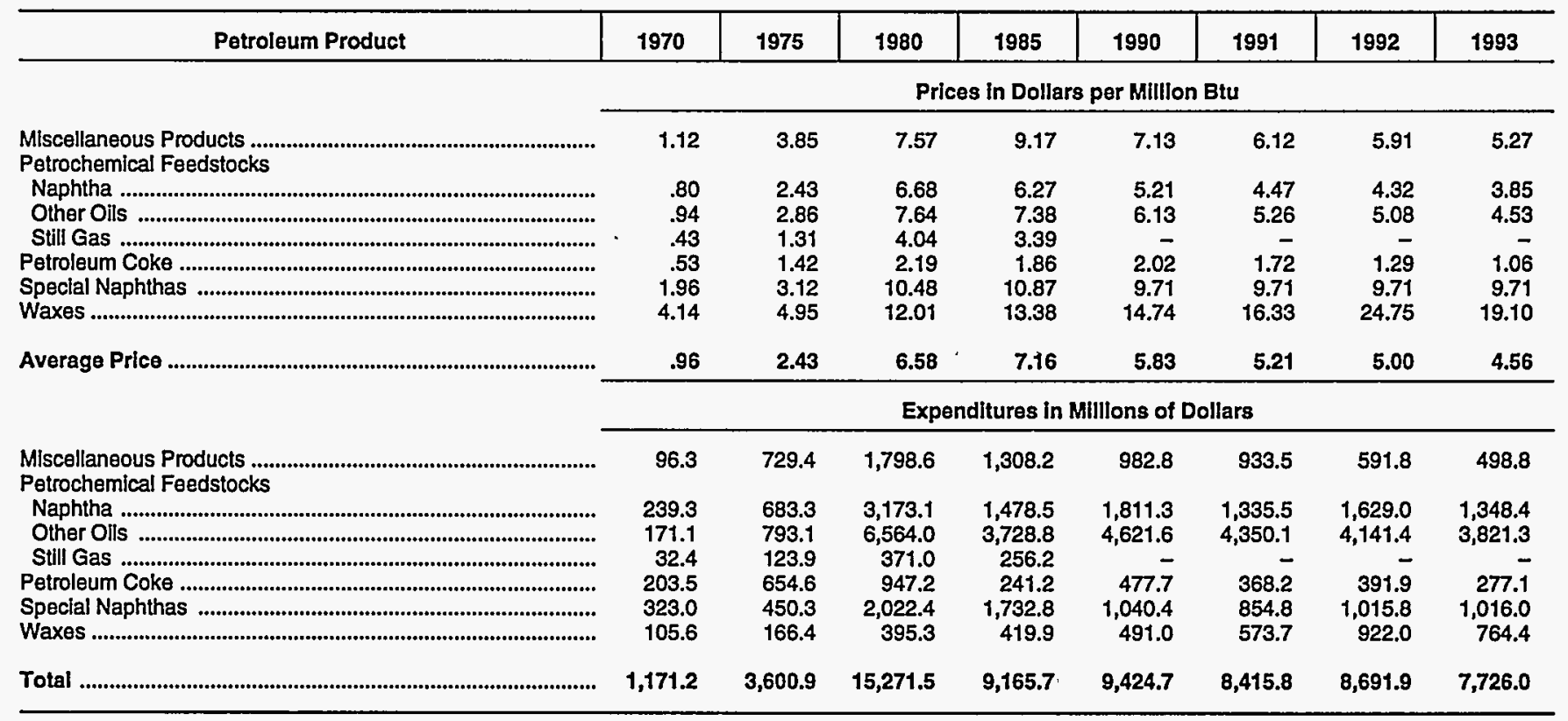

-Consumption data for thls series are not avallable after 1985 . See text.

Note: Expenditure totals may not equal sum of components due to independent rounding.

Source: State Energy Price and Expenditure Data System 1993. 


\section{Section 5. Electricity}

Electricity prices are retail prices for sales to ultimate users. Prices are developed for the residential, commercial, industrial, and transportation sectors. In general, taxes are included in the prices. However, taxes collected by a utility from an end user and turned over to a government authority are generally not included in the revenues reported in the Electric Power Annual or the Statistical Yearbook and are not included in the prices. Taxes paid by the utility (rather than the end user) are considered operating costs and are passed on to the end user as part of the rate. Therefore, Federal, State, business, and property taxes are typically included in the prices, while sales and other point-of-purchase taxes usually are not.

Consumption is based on sales by electric utilities to ultimate users. Electricity consumption data by State for the residential, commercial, industrial, and transportation sectors are obtained from the State Energy Data System (SEDS). Consumption of electricity in the industrial sector is adjusted for estimated refinery use in each State. (See the discussion in Section 6 "Consumption Adjustments for Calculating Expenditures," on page 249.)

\section{Conversion Factor: All Years}

\section{3,412 Btu per kilowatthour}

\section{Physical Unit Prices: 1987 Forward}

For 1987 forward, physical unit prices for States are calculated for all four sectors as the average revenue per unit of sales for all electric utilities reported in a State. Revenues and sales data from the Electric Power Annual are used to calculate physical unit prices. Data for the residential and industrial sectors are drawn from their respective columns. Data for the transportation sector are taken from the column titled "Other" that includes sales to railroads and railways. SEDS consumption is used to determine the portion of the data in the column titled "Other" that is not sold to the transportation sector. These data are added to the column titled "Commercial" to arrive at the revenues and sales data for the commercial sector.

For 1987 forward, prices are based on preliminary revenues and sales data in the given year and are replaced with revised data in the following year. There are no missing prices in any of the four sectors.

\section{Physical Unit Prices: 1970 Through 1986}

For 1970 through 1986, preliminary physical unit prices for States are calculated for all four sectors as the average revenue per unit of sales for all electric utilities reported in a State. The calculation of physical prices uses the revenues and sales data from the Statistical Yearbook for each year in the series. Data for the residential sector and industrial sector are drawn from their respective columns. The commercial sector is the sum of the columns titled "Commercial," "Street and Highway Lighting," "Other Public Authorities," and "Interdepartmental." The transportation sector is the column titled "Railroads and Railways."

For 1980 through 1986, prices are based on preliminary revenues and sales data in the given year and are replaced with revised data in the following year. The only exception to this rule is the revenues data for AR in 1981; preliminary data are used in this case because of an apparent error in the revised data.

For 1970 through 1981, MD prices are assigned to DC. There are no other missing prices for the residential, commercial, and industrial sectors.

In the transportation sector, numerous price assignments are made due to the lack of sector-specific price data. Generally, electricity usage in the transportation sector is small; the sector's electricity use ranged from 0.1 percent to 0.2 percent of total U.S. electricity consumption in 1970 through 1986 . From 1970 through 1986, only 15 States used measurable amounts of electricity in the transportation sector (CA, DC, FL, GA, IL, LA, MA, MD, NJ, NY, OH, PA, 
TN, VA, and WA). A few individual State prices are unavailable and are assigned the commercial sector prices: LA/1970 through 1986, and TN/1970 through 1986. (Prices are available for LA/1970, 1972, 1973, but those prices are replaced by commercial sector prices to maintain a consistent series for the State.)

In addition, MA/1985, 1986 transportation prices are estimated by multiplying the MA/1985, 1986 commercial prices by the average of the ratios of the commercial-to-transportation sector prices for 1980 through 1984. Similarly, the VA/1977 transportation price is estimated by multiplying the VA/1977 commercial price by the average of the ratios of the commercial-to-transportation sectors prices for 1978 through 1982.

$N$ In order to reconcile national-level electricity prices U based on the Statistical Yearbook with the EIA national-level electricity prices published in the Annual Energy Review (AER), yearly adjustment factors are calculated for the residential, commercial, and industrial sectors as follows: a preliminary U.S. price for each sector is calculated as the average of the State prices, weighted by SEDS consumption. These preliminary U.S. prices are divided by the national-level electricity prices published in the $A E R$, and the quotient is used as an adjustment factor. The preliminary State prices are multiplied by the adjustment factor to produce the final physical unit State prices in those sectors. Since no transportation sector prices are published in the $A E R$, no adjustments are made to that sector and the final physical unit prices are derived solely from the Statistical Yearbook sales and revenue data. The annual adjustment factors for the residential, commercial, and industrial sectors are shown in Table A44.

\section{Btu Prices: All Years}

Btu prices for States are calculated by dividing the physical unit prices by the conversion factor. U.S. Btu prices are calculated as the average of the State Btu prices, weighted by consumption data from SEDS, adjusted for process fuel consumption in the industrial sector.

\section{Data Sources}

Prices

1987 forward: Energy Information Administration, Electric Power Annual 1988, Tables 19 and 21 (1987 data); Electric Power Annual, Tables 27 and 29 (19881990) and Tables 26 and 28 (1991 forward).
Table A44. Annual Electricity Price Adjustment Factors

\begin{tabular}{cccc}
\hline Year & Residential & Commercial & Industrial \\
\hline 1970 & 1.05121 & 1.05712 & 1.06832 \\
1971 & 1.05632 & 1.05926 & 1.05504 \\
1972 & 1.05271 & 1.05514 & 1.05765 \\
1973 & 1.06626 & 1.06188 & 1.05991 \\
1974 & 1.09572 & 1.08098 & 1.08732 \\
1975 & 1.09257 & 1.08098 & 1.08732 \\
1976 & 1.07753 & 1.07755 & 1.06891 \\
1977 & 1.06746 & 1.07675 & 1.06820 \\
1978 & 1.06654 & 1.08273 & 1.06861 \\
1979 & 1.06986 & 1.08349 & 1.06441 \\
1980 & 1.04457 & 1.06109 & 1.06781 \\
1981 & 1.05821 & 1.06943 & 1.06523 \\
1982 & 1.06654 & 1.06351 & 1.05597 \\
1983 & 1.05421 & 1.05301 & 1.05537 \\
1984 & 0.99693 & 1.01924 & 0.99015 \\
1985 & 1.00010 & 1.02008 & 0.98355 \\
1986 & 0.99854 & 1.01518 & 0.98618 \\
& & & \\
\hline
\end{tabular}

Source: ElA calculations based on data from the Annual Energy Review and the Statistical Yearbook of the Electric Utility Industry.

1970-1986: Edison Electric Institute, Statistical Yearbook of the Electric Utility Industry, tables titled "Revenues: Total Electric Utility Industry" and "Energy Sales: Total Electric Utility Industry."

1970-1986: Energy Information Administration, Annual Energy Review 1989, Table 95, "Retail Prices of Electricity Sold by Electric Utilities, 1960-1989."

\section{Consumption}

1970 forward: Energy Information Administration, State Energy Data System, electricity consumption by end-use sector.

\section{Nuclear Fuel for Generation of Electricity}

Nuclear fuel prices are developed for the electric utility sector. State-level data on the amount of electricity generated from nuclear power plants are taken from SEDS. Utilities operating nuclear plants report fuel costs per kilowatthour to the U.S. Department of Energy annually. These data are collected on a plant-by-plant basis and include all taxes, transportation, and handling costs paid by the utilities. The 
State where the nuclear power plant is located is assigned the reported nuclear fuel cost. For States with two or more nuclear power plants, the average fuel cost, weighted by generation, is attributed to the State.

\section{Physical Unit and Btu Prices: All Years}

State-level nuclear fuel prices are estimated in two steps: (1) the total cost of fuels consumed at all nuclear power plants in a State is divided by their total generation of electricity, and (2) the cost per output created in step 1 is divided by an annual U.S. average thermal conversion factor to create the price in dollars per million Btu. Occasionally, the fuel costs at nuclear power plants include small amounts of non-nuclear fuels, which are necessary to continue essential plant operations during refueling or maintenance of the reactor.

A price is estimated by another method for States where generation and fuel cost data are not available for years in which the State Energy Data Report (SEDR) shows nuclear electric power generation. The ratio of the change in the national nuclear fuel price is used to estimate nuclear fuel prices for missing years within a State. The ratio of national prices used in the estimation is the ratio before missing prices are assigned. The States and years for which nuclear prices are estimated are shown in Table A45.

Nuclear electricity generation levels are negative for CO in 1985, for TN in 1986 and 1987, and for OR in 1993, indicating that the nuclear power plants used

Table A45. Nuclear Electricity Fuel Price Estimates, 1970 Through 1989

\begin{tabular}{ll}
\hline \multicolumn{1}{c}{ State } & \multicolumn{1}{c}{ Years } \\
\hline Alabama & $1973,1974,1976$ \\
Arizona & 1985 \\
Arkansas & 1980 \\
Colorado & $1977,1978,1982-1984,1986-1989$ \\
Georgia & 1974 \\
Maine & 1972 \\
Mississippi & 1984 \\
Missouri & 1984,1985 \\
North Carolina & 1982 \\
Ohio & 1986 \\
Oregon & 1975 \\
South Carolina & 1970,1985 \\
Tennessee & 1980 \\
Washington & $1970-1987$ \\
Wisconsin & 1970 \\
\end{tabular}

more energy than they supplied. In these cases the fuel prices and expenditures are set to zero. In 1986, there is generation in $\mathrm{CO}$ but price data are not available. The price for $\mathrm{CO}$ in 1986 is estimated by using the ratio of the 1984-to-1986 U.S. average prices and applying that ratio to the $1984 \mathrm{CO}$ price.

For MO in 1985, a large credit resulting from litigation is assigned to fuel costs, creating an artificially low price. The $1986 \mathrm{MO}$ uranium price, which is in the range of the prices of other nuclear fuel plants, is used to estimate the 1985 price by applying the ratio of the 1986-to-1985 national prices.

The 1985 Historical Plant Costs and Annual Production Expenses for Selected Electric Plants has a footnote for the Duke Power Catawba plant in SC stating that the reported production expenses represent only 12.5 percent of the actual production expenses. The production expenses used in the calculation for the Catawba plant are adjusted accordingly.

Prices for GA in 1978, NC in 1982, and OH in 1986 are assigned because the prices calculated by using reported data are significantly different from prices for other years for these States.

There are no prices available for WA in 1970 through 1987 and national prices are assigned for those years.

\section{Data Sources}

\section{Prices}

1992, 1993: Prices are developed by the Energy Information Administration, Office of Coal, Nuclear, and Alternate Fuels, from data compiled by the Utility Data Institute/McGraw-Hill. The data are collected on Federal Energy Regulatory Commission, FERC Form 1, "Annual Report of Major Electric Utilities, Licensees, and Others" and Energy Information Administration, Form EIA-412, "Annual Report of Public Electric Utilities."

1988-1991: Energy Information Administration, Electric Plant Cost and Power Production Expenses, Table 16 (1988-1990) and Table 14 (1991).

1982-1987: Energy Information Administration, Historical Plant Costs and Annual Production Expenses for Selected Electric Plants, Table 18 (1982-1984) and Table 20 (1985-1987).

1979-1981: Energy Information Administration, Thermal Electric Plant Construction Cost and Annual 
Production Expenses, pages 267-279 (1979), Table 11 (1980 and 1981).

1975-1978: Energy Information Administration, Steam Electric Plant Construction Cost and Annual Production Expenses, "Section II-Nuclear Plants."

1970-1974: Federal Power Commission, Steam Electric Plant Construction Costs and Annual Production Expenses, data sheets for Nuclear Plants (1970-1973), and "Section II-Nuclear Plants" (1974).

Consumption

1970 forward: Energy Information Administration, State Energy Data System, electricity generated by nuclear power.

\section{Conversion Factors}

1970 forward: Energy Information Administration, State Energy Data Report 1993, Consumption Estimates, Table D1, as shown in Table A46.

\section{Biomass Fuels for Generation of Electricity}

Biomass fuels prices are developed for the electric utility sector; data for biomass fuels consumed by other sectors are not available, except for ethanol added to motor gasoline. State-level data on the

Table A46. Nuclear Steam-Electric Thermal Conversion Factors

\begin{tabular}{cccc}
\hline Year & $\begin{array}{c}\text { Conversion } \\
\text { Factor }\end{array}$ & Year & $\begin{array}{c}\text { Conversion } \\
\text { Factor }\end{array}$ \\
\hline 1970 & 10.977 & 1982 & 11.073 \\
1971 & 10.837 & 1983 & 10.905 \\
1972 & 10.792 & 1984 & 10.843 \\
1973 & 10.903 & 1985 & 10.813 \\
1974 & 11.161 & 1986 & 10.799 \\
1975 & 11.013 & 1987 & 10.776 \\
1976 & 11.047 & 1988 & 10.743 \\
1977 & 10.769 & 1989 & 10.724 \\
1978 & 10.941 & 1990 & 10.680 \\
1979 & 10.879 & 1991 & 10.740 \\
1980 & 10.908 & 1992 & 10.678 \\
1981 & 11.030 & 1993 & 10.682 \\
& & & \\
\hline
\end{tabular}

amount of electricity generated at electric utilities from biomass fuels are taken from SEDS and are collected on Form EIA-759 (formerly, FPC-4). All electric utilities are required to report on EIA-759 but no price data are collected. State and national biomass fuels prices are developed for the electric utility sector from data reported on other EIA and Federal Energy Regulatory Commission (FERC) forms and from telephone surveys. Taxes are included in the prices for all years.

\section{Prices: All Years}

1989 Forward. State-level prices for biomass fuels used at electric utilities, in dollars per million Btu, are calculated from data obtained from FERC Form 1 and Form EIA-412 and by follow-up telephone surveys of the electric utilities that are not required to submit those forms. For States with more than one utility using biomass fuels, a consumption-weighted average price is calculated. There are anomalies that are unique to biomass fuels used at utilities. In some cases, there is no charge for the fuel. In other cases of municipal and industrial waste, the electric utilities charge a "tipping fee" for accepting the waste. That is, instead of paying for the fuel, the electric utilities are paid to take the fuel. For States where all utilities pay nothing for the fuel or charge a fee for receiving it (see Table A47), a price of zero is assigned. Although the corresponding consumption is included in calculating the average price for fuels consumed by electric utilities in the State and the United States, the expenditure included is zero. The State and U.S. average electric utility fuel prices. are, therefore, slightly lower because some of the fuel did not cost anything.

1983 Through 1988. A U.S. average price in dollars per million Btu is calculated and assigned to all States. The national price is a consumptionweighted average price based on data obtained from FERC Form 1 and Form EIA-412 and by follow up

\section{Table A47. Biomass Fuels Used at Electric Utilities at No Cost or For a Fee, 1989 Forward}

\begin{tabular}{lc}
\hline State & Years \\
\hline California & $1989-1993$ \\
Connecticut & $1989-1993$ \\
Hawaii & 1989,1990 \\
Montana & $1989-1993$ \\
Ohio & $1989-1993$ \\
\hline
\end{tabular}


telephone surveys of the electric utilities that report use of biomass fuels for generating electricity.

Prices are erratic for biomass fuels used at utilities. In addition to the anomalies of no charge for the fuel and the "tipping fee" mentioned above, handling refuse-derived fuel is more labor intensive than handling conventional fossil fuels. The labor expenses are included in the plant's operating costs, not the fuel costs. Biomass fuels prices are also erratic because the demand is relatively small and the pricing mechanism, even for a single facility, may change from year to year. A price or quantity change by a single major user affects the national price more significantly than for any other fuel.

1978 Through 1982. National average prices are derived from data collected on FPC Form 423 and published monthly by EIA in Cost and Quality of Fuels for Electric Utility Plants (CEQ). For these years, fossil-fueled plants with a combined capacity of 25 megawatts or greater were required to report on FPC Form 423. Annual prices of biomass fuels sold to electric utilities are developed as quantity-weighted monthly prices for those plants where wood chips and refuse were used as fuel. Beginning in 1983, the reporting threshold was raised to 50 megawatts, and very few plants reported use of biomass fuels on the Form 423 in 1983 and subsequent years.

A detailed review of data in $C \& Q$ showed that some entries were in error by factors of 10,100 , or 1,000 . Accordingly, the following corrections were made. For 1982, the February, March, and April quantities for the Florida Power Corporation were divided by 1,000 to make them 80,40 , and 60 short tons, respectively. The March, April, and May costs for Northern States Power were multiplied by 100 to make them $\$ 0.70$ per million Btu. For the 5 months from November 1979 through March 1980, the reported quantities of wood delivered to Burlington Electric Co. were divided by 10 , to place them in the range of 7,980 to 9,390 short tons. For the 8 months from June 1978 through January 1979, seed corn delivered to the Logansport Indiana Electric Department were included in the biomass fuel. For February 1978, the reported quantity of wood delivered to the United Power Associates was divided by 1,000 to make it 90 short tons.
1970 Through 1977. The annual prices for wood chips are derived by deflating the 1978 price by using the gross domestic product implicit price deflator based on 1987 dollars. The deflators are shown in Table A48.

Table A48. Price Deflators Used for Biomass Fuels Prices, 1970-1977

\begin{tabular}{lccc}
\hline Years & Deflator & Years & Deflator \\
\hline 1970 & 35.1 & 1975 & 49.2 \\
1971 & 37.1 & 1976 & 52.3 \\
1972 & 38.8 & 1977 & 55.9 \\
1973 & 41.3 & 1978 & 60.3 \\
1974 & 44.9 & & \\
\hline
\end{tabular}

\section{Data Sources}

Prices

1983 forward: Energy Information Administration, data reported on FERC Form 1, "Annual Report of Major Electric Utilities, Licensees and Others" and Form EIA-412, "Annual Report of Public Electric Utilities" and follow-up telephone surveys of the electric utilities that report use of biomass fuels for generating electricity.

1978-1982: Energy Information Administration, Cost and Quality of Fuels for Electric Utility Plants, table titled "Wood Chips, Refuse, and Petroleum Coke Used as Fuel by Steam-Electric Plants."

1970-1978: Energy Information Administration, Annual Energy Review 1991, Appendix C, Gross Domestic Product and Implicit Price Deflator.

\section{Consumption}

1970 forward: Energy Information Administration, State Energy Data System, electricity generated from consumption of wood and waste. 



\section{Section 6. Consumption Adjustments for Calculating Expenditures}

Expenditures developed in the State Energy Price and Expenditure Data System (SEPEDS) and published in this report are calculated by using consumption estimates from the EIA State Energy Data System (SEDS). Since almost all aspects of energy production, processing, and distribution consume energy as an inherent part of those activities, SEDS industrial and transportation sector consumption estimates include energy consumed in the process of providing energy to the end-use consumer. Familiar examples include drilling for oil and gas, refining crude oil into finished petroleum products, transporting natural gas and petroleum by pipeline, and generating and delivering electricity to end users. Energy that is consumed to make energy more useable to the end user is called "process fuel." Energy products that are subsequently incorporated into another energy product for end-use consumption are called "intermediate products." Motor gasoline blending components are familiar examples of intermediate products that are consumed as part of the finished motor gasoline sold at service stations and other outlets.

Process fuel and intermediate products are not purchased by the end user and, therefore, do not have prices. Although the end user does not consume either process fuel or intermediate products directly, he does pay for them, because the cost to the processor or distributor is passed on to the end user in the price of the final end-user product. Hydroelectric power generated by industries and consumed onsite is another form of energy consumption that has no price to the consumer. To estimate energy expenditures, the consumption of process fuel, intermediate products, and industrial hydroelectricity are not assigned prices and are subtracted from the end-use sector in which they are included in SEDS, either the industrial or transportation sector. If their use was left in the consumption estimates and was assigned prices, the expenditures would be counted twice, first as paid by the "processor" (producer, processor, or transporter) and again as included in the price to the end user.
Process fuel consumption adjustments include:

1. Fuel (petroleum, natural gas, coal) and electricity consumed at refineries

2. Crude oil lease, plant, and pipeline fuel

3. Natural gas lease and plant fuel

4. Natural gas pipeline fuel

5. Hydroelectricity generated and used within the industrial sector

6. Electrical system energy losses (i.e., energy consumed in the generation, transmission, and distribution of electricity).

Intermediate product consumption adjustments include:

1. Aviation gasoline blending components

2. Motor gasoline blending components

3. Natural gasoline (1970 through 1983)

4. Pentanes plus (1984 forward)

5. Plant condensate (1970 through 1983)

6. Unfinished oils

7. Unfractionated stream (1970 through 1983).

Starting in 1984, natural gasoline (including isopentane) and plant condensate are reported together as the new product, pentanes plus, and the components of unfractionated stream are reported separately under liquefied petroleum gases.

Table A49 shows estimates of the quantities of energy consumed by State as process fuel and intermediate products in 1993. Estimates for 1970 through 1993 are available on the State Energy Price and Expenditure Data System personal computer diskettes.

Table A50 shows the adjustments made to SEDS national consumption estimates for 1970 through 1993 to derive the net consumption data used to calculate the expenditures in this report.

\section{Adjustment Procedures}

Refinery Fuel. Refinery fuel consumption of distillate fuel, residual fuel, liquefied petroleum gases, 


\begin{tabular}{|c|c|c|c|c|c|c|c|c|}
\hline & \multicolumn{8}{|c|}{ Refinery Use } \\
\hline & Distillate & Residual & LPG & $\begin{array}{c}\text { Other } \\
\text { Potroleum a }\end{array}$ & Natural Gas & Coal & Electricity $b$ & Total \\
\hline AK ................... & 108 & 673 & 18 & 18,380 & 23,029 & - & 98 & 42.306 \\
\hline 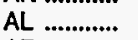 & 30 & 1,161 & 9 & 14,667 & 26,180 & $=$ & 9,415 & $\begin{array}{l}42,400 \\
51,463\end{array}$ \\
\hline AR ................. & 25 & 327 & 8 & 6,628 & 16,595 & - & 3,890 & 27,474 \\
\hline A2 ................... & 605 & $3,42 \overline{0}$ & $18,34 \overline{0}$ & 173,968 & 57,320 & $\overline{-}$ & $100 \overline{0}$ & 264602 \\
\hline 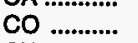 & 2 & 37 & 115 & $\begin{array}{r}7,305 \\
7,300\end{array}$ & $\begin{array}{r}5,320 \\
6,952\end{array}$ & $\overline{-}$ & $\begin{array}{r}10,969 \\
1,152\end{array}$ & $\begin{array}{r}264,622 \\
15,562\end{array}$ \\
\hline CT .................... & - & - & - & 1,730 & - 0,002 & $\overline{-}$ & 1,102 & $\begin{array}{r}15,7302 \\
1,730\end{array}$ \\
\hline 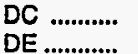 & $\overline{16}$ & $22 \overline{-}$ & $\overline{42}$ & $179 \overline{8}$ & $\overline{-3}$ & $\bar{\varepsilon}$ & - & - \\
\hline 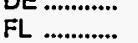 & 10 & 2,226 & 42 & 3,871 & 872 & 46 & 298 & $\begin{array}{r}21,418 \\
3971\end{array}$ \\
\hline GA .................. & 163 & 3,556 & 493 & 4,807 & $7,4 \overline{0}$ & 454 & $2.53 \bar{\sigma}$ & $\begin{array}{r}3,871 \\
19,450\end{array}$ \\
\hline 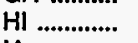 & 31 & 2,378 & 1,404 & 11,385 & 1,440 & 404 & 2, 736 & $\begin{array}{l}15,450 \\
15,935\end{array}$ \\
\hline IA ................... & - & - & - & 1,087 & - & - & - & 1,087 \\
\hline 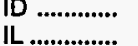 & $\overline{95}$ & $1,22 \overline{2}$ & $1 . \overline{3}$ & 101.020 & $14 . \overline{-}$ & $\overline{51}$ & $35 \overline{9}$ & 101805 \\
\hline IN ...................... & 64 & 5,811 & $\begin{array}{r}1,145 \\
508\end{array}$ & 52,670 & $\begin{array}{l}12,616 \\
12,563\end{array}$ & $\begin{array}{l}51 \\
59\end{array}$ & $\begin{array}{l}3,599 \\
3,524\end{array}$ & $\begin{array}{r}121,805 \\
75,199\end{array}$ \\
\hline KS ................... & 68 & 691 & 1,832 & 25,967 & 9,105 & 2 & , $7,0<4$ & 38.442 \\
\hline KY .................. & 70 & 762 & 474 & $\begin{array}{l}20,907 \\
34,915\end{array}$ & $\begin{array}{l}\quad, 105 \\
3,914\end{array}$ & $\begin{array}{l}2 \\
34\end{array}$ & 3.248 & $\begin{array}{l}38,442 \\
43,416\end{array}$ \\
\hline 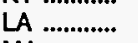 & 80 & 466 & 325 & 288,171 & 162,401 & - & 8,774 & 460,217 \\
\hline 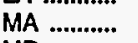 & - & - & - & 2,110 & 02,401 & $\overline{-}$ & 0,714 & $\begin{array}{r}46,2110 \\
2,110\end{array}$ \\
\hline 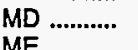 & - & - & - & 1,518 & - & - & - & 1,518 \\
\hline 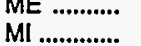 & $\overline{60}$ & $2.20 \overline{2}$ & $1,1 \overline{7}$ & 18.918 & $15 . \overline{-}$ & $\overline{4}$ & 2783 & $A 060^{\circ}$ \\
\hline MN ….............. & 77 & $\begin{array}{l}2,545 \\
2,545\end{array}$ & 581 & $\begin{array}{l}74,984 \\
24,918\end{array}$ & $\begin{array}{r}1,605 \\
4,656\end{array}$ & $\begin{array}{l}44 \\
14\end{array}$ & $\begin{array}{l}2,733 \\
2,180\end{array}$ & $\begin{array}{l}40,680 \\
35,037\end{array}$ \\
\hline MO ................. & - & & & $\begin{array}{r}24,504 \\
7,963\end{array}$ & & 14 & & $\begin{array}{r}35,063 \\
7,963\end{array}$ \\
\hline MS ........... & 24 & 387 & 10 & 34,009 & 13,996 & - & 4,390 & 52,817 \\
\hline MT ........... & 1 & 2,252 & 135 & 17,400 & 1,123 & - & 957 & 21,869 \\
\hline NC ................ & $\overline{-}$ & - & - & 5,457 & - & - & - & 5,457 \\
\hline ND ............ & 39 & 874 & 72 & 5,691 & 717 & 52 & 170 & 7,615 \\
\hline $\begin{array}{l}\mathrm{NE} \text {............. } \\
\text { NH .......... }\end{array}$ & $\overline{-}$ & $=$ & $\overline{-}$ & $\begin{array}{l}364 \\
101\end{array}$ & $\bar{z}$ & - & - & 364 \\
\hline NJ ....................... & 88 & 3,317 & $38 \overline{-}$ & 83.434 & $8.47 \overline{0}$ & 59 & 1273 & $\begin{array}{r}101 \\
97077\end{array}$ \\
\hline NM …................... & 10 & 273 & 52 & 9880 & $\begin{array}{l}8,0410 \\
9,055\end{array}$ & - & $\begin{array}{l}1,2 / 3 \\
1,486\end{array}$ & $\begin{array}{c}97,027 \\
20775\end{array}$ \\
\hline NV .................. & 179 & 226 & 274 & 492 & 1,895 & $=$ & $\begin{array}{l}1,400 \\
1,402\end{array}$ & $\begin{array}{r}20,155 \\
4,468\end{array}$ \\
\hline NY ................. & - & & & 7,415 & & - & $1,40 \mathrm{c}$ & 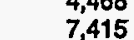 \\
\hline $\mathrm{OH}$ & 87 & 4,792 & 1,464 & 59,587 & 14,797 & 57 & 6,154 & 86,938 \\
\hline $\begin{array}{l}\text { OK ........... } \\
\text { OR }\end{array}$ & 42 & 1,614 & 645 & 37,378 & 13,435 & 15 & 1,046 & 54,176 \\
\hline PA ..................... & $2 \overline{0}$ & $5,45 \overline{6}$ & $42 \overline{8}$ & $74,81 \overline{3}$ & $11,02 \overline{-}$ & $1,13 \overline{7}$ & $39 \overline{19}$ & 97038 \\
\hline $\mathrm{RI}$ & 200 & 0,450 & 428 & $/ 4,813$ & 11,025 & 1,137 & 3,919 & 97,038 \\
\hline SC ................. & - & - & - & 4,178 & $\overline{-}$ & $\bar{z}$ & $\overline{-}$ & $4,17 \overline{8}$ \\
\hline SD .................. & $\overline{15}$ & $\overline{-}$ & $\overline{-1}$ & & - & - & - & \\
\hline TN ................ & 45 & 1,093 & 131 & 23,305 & 6,063 & 56 & 3,891 & 34,584 \\
\hline TX & 146 & $\begin{array}{r}3,660 \\
768\end{array}$ & 1,975 & $\begin{array}{l}532,252 \\
\quad 1341\end{array}$ & 286,099 & - & 26,821 & 850,953 \\
\hline UT ................. & 1 & 768 & 44 & 13,471 & 4,342 & - & 1,020 & 19,647 \\
\hline 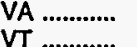 & 126 & 3,482 & 367 & 9,757 & 3,348 & 756 & 1,516 & 19,352 \\
\hline WA ........................ & & $1 . \overline{10}$ & & 85737 & 7070 & - & 7138 & 194270 \\
\hline WI ..................... & 64 & 2,410 & $\begin{array}{l}2,0039 \\
303\end{array}$ & 4287 & 6.385 & 25 & $\begin{array}{l}, 7188 \\
10914\end{array}$ & $\begin{array}{r}104,379 \\
15388\end{array}$ \\
\hline wo ................... & 116 & $\begin{array}{l}2,410 \\
629\end{array}$ & 237 & $\begin{array}{r}4,207 \\
31,544\end{array}$ & $\begin{array}{l}6,3050 \\
2,760\end{array}$ & 631 & $\begin{array}{l}1,914 \\
888\end{array}$ & $\begin{array}{r}15,388 \\
36,806\end{array}$ \\
\hline WY ................. & 1 & 237 & 106 & 11,842 & 5,792 & 1 & $\begin{array}{r}1,208 \\
1,208\end{array}$ & $\begin{array}{r}39,186 \\
19,180\end{array}$ \\
\hline US ............. & 2,907 & 60,858 & 35,378 & $1,872.377$ & 757.685 & 3.491 & 119.123 & 2851819 \\
\hline
\end{tabular}

See footnotes at end of table.

other petroleum (crude oil, petroleum coke, and still gas), natural gas, coal, and electricity is individually estimated for each source and subtracted from its State Energy Data System (SEDS) industrial sector total. Because crude oil consumption is not an individual fuel in SEDS for 1970 through 1982, the small amounts of crude oil that were used at refineries during those years were allocated to residual and distillate fuels consumed at refineries. The allocation from crude oil refinery use to residual and distillate fuels refinery use was made according to each fuel's share of the total crude oil used directly (including losses) as residual and distillate fuels (Petroleum Supply Annual Volume 1, Table 2). Over this period, residual fuel ranged from a low share of 75 percent in 1971 to a high share of 92 percent in 1976. Refinery consumption of still gas, excluding still gas consumed as petrochemical feedstocks, is subtracted 
Table A49. Estimates of Energy Consumed as Process Fuel and Intermediate Products, 1993 (Continued)

(Billion Btu)

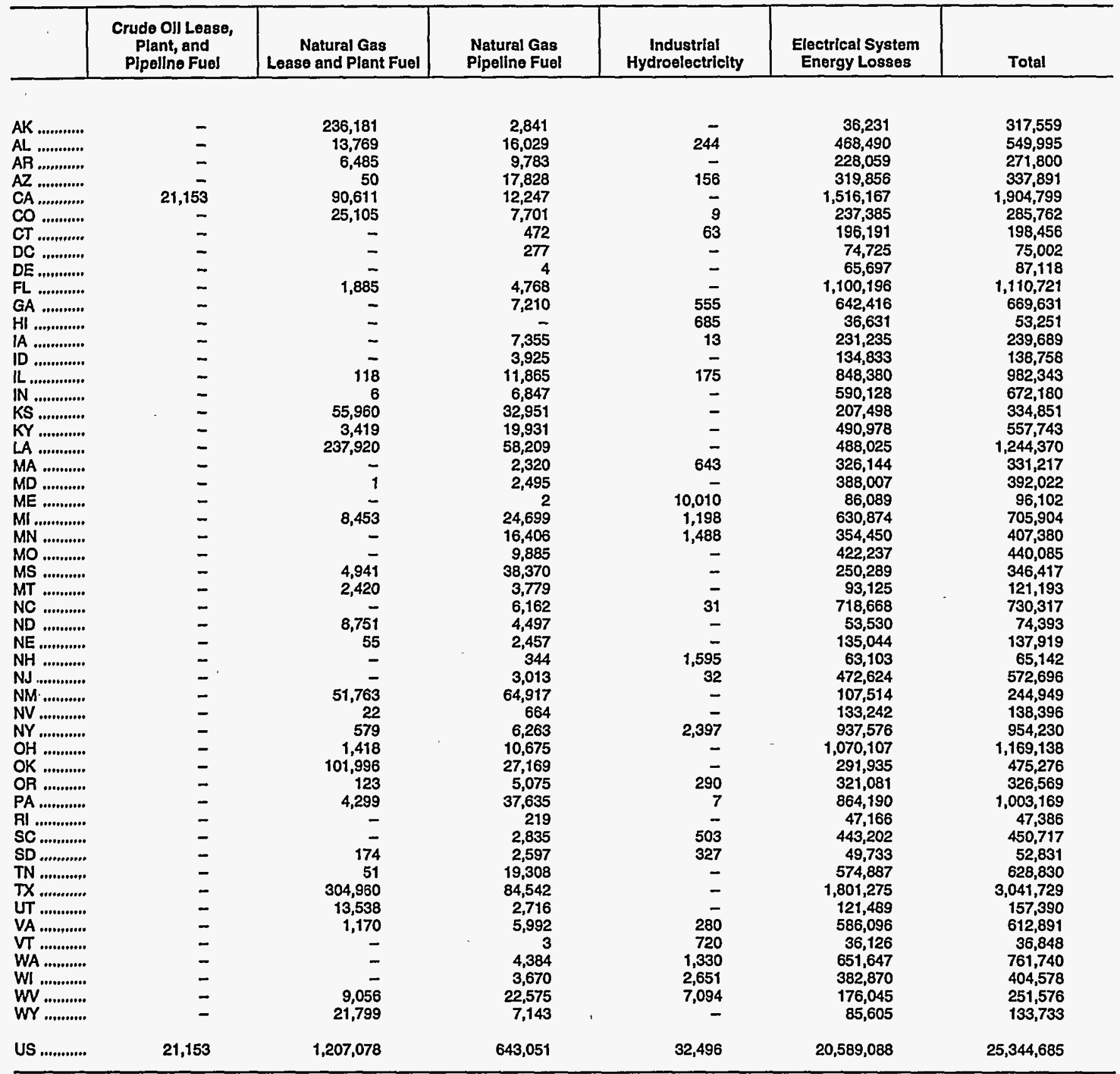

a In this table, "other petroleum" consists of: crude oil, petroleum coke, and stlll gas consumed as process fuel; and aviation gasoline blending components, motor gasoline blending components, pentanes plus, and unfinished olls used as intermediate products. b Electricity is converted at the rate of 3,412 Btu per kilowatthour. -No consumption.

Sources: State Energy Price and Expenditure Data System 1993. from the SEDS industrial sector total for 1970 through 1985. Beginning in 1986, ELA data series no longer report refinery fuel and feedstock use separately, and all SEDS industrial still gas consumption is removed from SEPER.
Refinery fuel consumption data are available in the data sources by Petroleum Administration for Defense District (PADD) (1981 forward) and by State or group of States (1970 through 1980). Where Statelevel consumption data are not available, the State-level estimates are derived by allocating the 
Table A50. Energy Consumption Adjustments, 1970-1993 (Trillion Btu)

\begin{tabular}{|c|c|c|c|c|c|c|c|c|c|}
\hline \multirow[b]{2}{*}{ Year } & \multirow[b]{2}{*}{$\begin{array}{c}\text { Total } \\
\text { (Gross) } \\
\text { Consumption }\end{array}$} & \multicolumn{7}{|c|}{ Adjustments } & \multirow[b]{2}{*}{$\begin{array}{c}\text { Net } \\
\text { Consumption }\end{array}$} \\
\hline & & $\begin{array}{c}\text { Refinery } \\
\text { Use }\end{array}$ & $\begin{array}{c}\text { Crude Oil } \\
\text { Lease, Plant, } \\
\text { and } \\
\text { Plpeline Fuel }\end{array}$ & $\begin{array}{c}\text { Natural } \\
\text { Gas } \\
\text { Lease and } \\
\text { Plant Fuel } \\
\end{array}$ & $\begin{array}{l}\text { Natural } \\
\text { Gas } \\
\text { Plpeline } \\
\text { Fuel }\end{array}$ & $\begin{array}{l}\text { Industrial } \\
\text { Hydro- } \\
\text { electriclty }\end{array}$ & $\begin{array}{l}\text { Electrical } \\
\text { System } \\
\text { Energy } \\
\text { Losses }\end{array}$ & Total & \\
\hline $\begin{array}{l}1970 . . . . . . . . . \\
1971 \ldots . . . . . . \\
1972 . . . . . . . . . \\
1973 . . . . . . . . \\
1974 . . . . . . . .\end{array}$ & $\begin{array}{l}66,334 \\
67,789 \\
71,275 \\
74,351 \\
72,528\end{array}$ & $\begin{array}{l}2,464 \\
2,442 \\
2,576 \\
2,690 \\
2,683\end{array}$ & $\begin{array}{l}- \\
- \\
- \\
-\end{array}$ & $\begin{array}{l}1,442 \\
1,456 \\
1,497 \\
1,539 \\
1,520\end{array}$ & $\begin{array}{l}740 \\
761 \\
786 \\
745 \\
684\end{array}$ & $\begin{array}{l}34 \\
34 \\
34 \\
35 \\
33\end{array}$ & $\begin{array}{l}11,517 \\
12,127 \\
13,110 \\
13,999 \\
14,198\end{array}$ & $\begin{array}{l}16,197 \\
16,820 \\
18,003 \\
19,007 \\
19,119\end{array}$ & $\begin{array}{l}50,138 \\
50,968 \\
53,273 \\
55,344 \\
53,409\end{array}$ \\
\hline $\begin{array}{l}1975 . . . . . . . . . . \\
1976 \ldots . . . . . . . . \\
1977 \ldots . . . . . . . . \\
1978 \ldots . . . . . . . \\
1979 . . . . . . . . .\end{array}$ & $\begin{array}{l}70,569 \\
74,392 \\
76,317 \\
78,158 \\
78,920\end{array}$ & $\begin{array}{l}2,571 \\
2,590 \\
2,687 \\
2,623 \\
2,742\end{array}$ & $\begin{array}{l}- \\
- \\
- \\
-\end{array}$ & $\begin{array}{l}1,434 \\
1,679 \\
1,706 \\
1,694 \\
1,534\end{array}$ & $\begin{array}{l}\mathbf{5 9 5} \\
\mathbf{5 5 9} \\
\mathbf{5 4 4} \\
\mathbf{5 4 1} \\
\mathbf{6 1 3}\end{array}$ & $\begin{array}{l}32 \\
33 \\
33 \\
32 \\
34\end{array}$ & $\begin{array}{l}14,384 \\
15,255 \\
16,060 \\
16,850 \\
17,063\end{array}$ & $\begin{array}{l}19,017 \\
20,116 \\
21,030 \\
21,740 \\
21,985\end{array}$ & $\begin{array}{l}51,553 \\
54,276 \\
55,287 \\
56,419 \\
56,935\end{array}$ \\
\hline 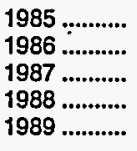 & $\begin{array}{l}74,054 \\
74,290 \\
76,840 \\
80,269 \\
81,317\end{array}$ & $\begin{array}{l}2,046 \\
2,286 \\
2,485 \\
2,696 \\
2,711\end{array}$ & $\begin{array}{r}127 \\
103 \\
72 \\
85 \\
59\end{array}$ & $\begin{array}{r}1,001 \\
954 \\
1,194 \\
1,134 \\
1,103\end{array}$ & $\begin{array}{l}521 \\
501 \\
538 \\
633 \\
650\end{array}$ & $\begin{array}{l}33 \\
33 \\
33 \\
33 \\
33\end{array}$ & $\begin{array}{l}18,631 \\
18,593 \\
19,156 \\
19,887 \\
20,256\end{array}$ & $\begin{array}{l}22,359 \\
22,470 \\
23,479 \\
24,468 \\
24,811\end{array}$ & $\begin{array}{l}51,694 \\
51,820 \\
53,361 \\
55,802 \\
56,506\end{array}$ \\
\hline $\begin{array}{l}1990 \ldots . . . . . . . \\
1991 \ldots . . . . . . . \\
1992 \ldots . . . . . . \\
1993 . . . . . . . .\end{array}$ & $\begin{array}{l}81,143 \\
81,099 \\
82,128 \\
83,958\end{array}$ & $\begin{array}{l}2,803 \\
2,668 \\
2,954 \\
2,852\end{array}$ & $\begin{array}{l}51 \\
39 \\
27 \\
21\end{array}$ & $\begin{array}{l}1,269 \\
1,164 \\
1,209 \\
1,207\end{array}$ & $\begin{array}{l}682 \\
622 \\
608 \\
643\end{array}$ & $\begin{array}{l}33 \\
33 \\
33 \\
32\end{array}$ & $\begin{array}{l}20,221 \\
20,473 \\
20,101 \\
20,589\end{array}$ & $\begin{array}{l}25,059 \\
24,998 \\
24,932 \\
25,345\end{array}$ & $\begin{array}{l}56,084 \\
56,101 \\
57,195 \\
58,613\end{array}$ \\
\hline
\end{tabular}

Note: Totals may not equal sum of components due to independent rounding.

Sources: Total (Gross) Consumption-EIA, State Energy Data Report 1993, Consumption Estimates, (SEDR) DOE/EIA-0214(93) (Washington, DC, July, 1995), p. 21, column titled, "Total."

Refinery Use-State Energy Price and Expenditure Data System 1993 (SEPEDS).

Crude Oll Lease, Plant, and Plpeline Fuel-SEPEDS.
Natural Gas Lease and Plant Fuel-SEPEDS.

Natural Gas Pipeline Fuel-EIA, SEDR, p. 25, column titled, "Natural Gas." Industrial Hydroelectricity-EIA, SEDR, p. 24, column titled, "Industrial Hydropower." (Difference due to rounding of 32.469 trillion Btu.)

Electrical System Energy Losses-EIA, SEDR, Tables 12-15, pp. 22-25, sum of four end-use sectors' column tilled, "Electrical System Energy Losses.

Net Consumption-SEPEDS. district's or group's total consumption to the individual States within the district or group to the States that had operating refineries in a given year. Individual fuels are allocated to the refining States according to each State's share of the refining States' total industrial sector fuel consumption during the year. In some instances, estimated refinery fuel consumption exceeds the SEDS estimate for total industrial consumption of a fuel within a State. When this occurs, the excess refinery fuel consumption is reallocated as shown in Table A51.

Intermediate Products. Aviation gasoline blending components, motor gasoline blending components, natural gasoline (1970 through 1983), pentanes plus 1984 forward), plant condensate (1970 through 1983), unfinished oils, and unfractionated stream (1970 through 1983) are used at refineries and blending plants to make end-use petroleum products, particularly motor gasoline. Accordingly, consumption of these products is completely removed from SEPER.
Crude Oil Lease, Plant, and Pipeline Fuel. Industrial crude oil remaining after refinery fuel consumption is removed is assumed to be used as lease, plant, and pipeline fuel. Since these are process fuel uses, the remaining crude oil is removed from SEDS industrial sector consumption.

Natural Gas Lease and Plant Fuel. Natural gas consumed as lease and plant fuel is process fuel and is subtracted from SEDS industrial sector natural gas totals by State and year.

Natural Gas Pipeline Fuel. Transportation sector natural gas consumption shown in SEDS is used to power pipelines. As such, it is process fuel and is completely removed from the SEPER expenditure calculations.

Industrial Hydroelectricity. Hydroelectricity generated by industries and consumed at the industrial sites has no fuel cost. Operation and maintenance 
Table A51. Reallocations of Excess Refinery Fuel Consumption

\begin{tabular}{|c|c|c|c|c|}
\hline Year & Fuel & $\begin{array}{c}\text { Thousand } \\
\text { Barrels }\end{array}$ & $\begin{array}{c}\text { Excess } \\
\text { In: }\end{array}$ & $\begin{array}{c}\mathrm{Re}- \\
\text { allocated }\end{array}$ \\
\hline 1971 & $\begin{array}{l}\text { Residual } \\
\text { Fuel }\end{array}$ & 294 & Kansas & Oklahoma \\
\hline 1973 & $\begin{array}{l}\text { Residual } \\
\text { Fuel }\end{array}$ & 45 & $\begin{array}{l}\text { Group 4: } \\
\text { Kentucky, } \\
\text { Tennessee }\end{array}$ & Illinois \\
\hline 1979 & LPG & 173 & Montana & Wyoming \\
\hline 1983 & $\begin{array}{l}\text { Petroleum } \\
\text { Coke }\end{array}$ & 3,091 & PADD I & PADD II \\
\hline 1984 & $\begin{array}{l}\text { Crude Oil } \\
\text { Petroleum } \\
\text { Coke }\end{array}$ & $\begin{array}{r}80 \\
992\end{array}$ & $\begin{array}{l}\text { PADD II } \\
\text { PADD I }\end{array}$ & $\begin{array}{l}\text { PADD III } \\
\text { PADD II }\end{array}$ \\
\hline \multirow[t]{3}{*}{1985} & $\begin{array}{l}\text { Petroleum } \\
\text { Coke }\end{array}$ & 80 & PADD II & PADD III \\
\hline & Crude Oil & 719 & PADD I & PADD II \\
\hline & $\begin{array}{l}\text { Residual } \\
\text { Fuel }\end{array}$ & 212 & PADD IV & PADD V \\
\hline \multirow[t]{2}{*}{1986} & $\begin{array}{l}\text { Petroleum } \\
\text { Coke }\end{array}$ & 1,297 & PADD I & PADD II \\
\hline & $\begin{array}{l}\text { Residual } \\
\text { Fuel }\end{array}$ & 403 & PADD IV & PADD V \\
\hline 1987 & $\begin{array}{l}\text { Residual } \\
\text { Fuel }\end{array}$ & 497 & PADD IV & PADD V \\
\hline 1988 & $\begin{array}{l}\text { Crude Oil } \\
\text { Residual } \\
\text { Fuel }\end{array}$ & $\begin{array}{r}8 \\
305\end{array}$ & $\begin{array}{l}\text { PADD III } \\
\text { PADD IV }\end{array}$ & $\begin{array}{l}\text { PADD V } \\
\text { PADD V }\end{array}$ \\
\hline \multirow[t]{2}{*}{1989} & $\begin{array}{l}\text { Crude Oil } \\
\text { Crude Oil }\end{array}$ & $\begin{array}{r}9 \\
15\end{array}$ & $\begin{array}{l}\text { PADD I } \\
\text { PADD III }\end{array}$ & $\begin{array}{l}\text { PADD V } \\
\text { PADD V }\end{array}$ \\
\hline & $\begin{array}{l}\text { Residual } \\
\text { Fuel }\end{array}$ & 381 & PADD IV & PADD V \\
\hline 1990 & $\begin{array}{l}\text { Residual } \\
\text { Fuel }\end{array}$ & 332 & PADD IV & PADD V \\
\hline \multirow[t]{2}{*}{1991} & $\begin{array}{l}\text { Petroleum } \\
\text { Coke }\end{array}$ & 595 & PADD I & PADD II \\
\hline & $\begin{array}{l}\text { Residual } \\
\text { Fuel }\end{array}$ & 374 & PADD IV & PADD V \\
\hline \multirow[t]{3}{*}{1992} & Crude Oil & 3 & PADD II & PADD V \\
\hline & $\begin{array}{l}\text { Crude Oil } \\
\text { Petroleum } \\
\text { Coke }\end{array}$ & $\begin{array}{r}1 \\
838\end{array}$ & $\begin{array}{l}\text { PADD III } \\
\text { PADD I }\end{array}$ & $\begin{array}{l}\text { PADD V } \\
\text { PADD II }\end{array}$ \\
\hline & $\begin{array}{l}\text { Residual } \\
\text { Fuel }\end{array}$ & 355 & PADD IV & PADD V \\
\hline
\end{tabular}

Source: ElA calculations based on data from the State Energy Data Report and the Petroleum Supply Annual.

costs associated with the hydroelectricity are included indirectly in the prices of the industries' products. Therefore, SEDS industrial hydroelectricity consumption estimates are removed from SEPER expenditure calculations.

Electrical System Energy Losses. The amount of energy lost during generation, transmission, and distribution of electricity (including plant use and unaccounted for electrical energy) is process fuel and is subtracted from sectoral energy consumption estimates used in SEPER. The energy losses are "paid for" when residential, commercial, industrial, and transportation sector consumers buy the electricity produced at electric utilities.

\section{Data Sources}

Capacity of Petroleum Refineries. 1982 forward: Energy Information Administration, Petroleum Supply Annual Volume 1, table titled "Number and Capacity of Operable Petroleum Refineries," column heading, "Crude Capacity, Barrels per Calendar Day, Operating" (1982-1985), "Atmospheric Crude Oil Distillation Capacity, Barrels per Calendar Day, Operating" (1986 forward).

1979-1981: Energy Information Administration, Energy Data Reports, Petroleum Refineries in the United States and U.S. Territories, table titled "Number and Capacity of Petroleum Refineries," column heading, "Crude Capacity, Barrels per Calendar Day, Operating."

1978: Energy Information Administration, Energy Data Reports, Petroleum Refineries in the United States and Puerto Rico, table titled "Number and Capacity of Petroleum Refineries," column heading, "Crude Capacity, Barrels per Calendar Day, Operating."

1970-1977: Bureau of Mines, U.S. Department of the Interior, Mineral Industry Surveys, Petroleum Refineries in the United States and Puerto Rico, table titled "Number and Capacity of Petroleum Refineries," column heading, "Crude Capacity, Barrels per Calendar Day, Operating."

Fuel Consumed at Refineries. 1981 forward: Energy Information Administration, Petroleum Supply Annual Volume 1, table titled "Fuels Consumed at Refineries by PAD District." Data for 1991 are from a separately published ERRATA dated November 10, 1992, GPO Stock No. 061-003-00758-9.

1976-1980: Energy Information Administration, Energy Data Reports, Crude Petroleum, Petroleum Products, and Natural Gas Liquids, table titled "Fuels Consumed for All Purposes at Refineries in the United States, by States."

1970-1975: Bureau of Mines, U.S. Department of the Interior, Mineral Industry Surveys, Crude Petroleum, Petroleum Products, and Natural Gas Liquids, tables 
C titled "Fuels Consumed for All Purposes at RefinerO ies in the United States, by States."

N

1970 forward: Energy Information Administration, State Energy Data System, industrial sector consumption estimates for aviation gasoline blending components, crude oil, motor gasoline blending components, natural gasoline (1970-1983), pentanes plus (1984 forward), petroleum coke, plant condensate (1970-1983), still gas (excluding still gas consumed as petrochemical feedstocks, 1970-1985), unfinished oil, and unfractionated stream (1970-1983).

Natural Gas Lease, Plant, and Pipeline Fuel Use. A 1980 forward: Energy Information Administration,
Natural Gas Annual, Table 10 (1980) and Table 13 (1981, 1982); Natural Gas Annual Volume 1, Table 13 (1983-1987) and Table 16 (1988); Natural Gas Annual, Table 16 (1989 forward).

1977-1979: Energy Information Administration, Energy Data Reports, "Natural Gas Production and Consumption," Table $6(1977,1978)$ and Table 5 (1979).

1970-1976: Bureau of Mines, U.S. Department of the Interior, Mineral Industry Surveys, "Natural Gas Production and Consumption," Table 5 (1970) and Table 6 (1971-1976). 
Appendix B

Metric and Other Physical Conversion Factors 


\section{Metric and Other Physical Conversion Factors}

Data presented in this State Energy Price and Expenditure Report and in other Energy Information Administration publications are expressed predominately in units that historically have been used in the United States, such as British thermal units, barrels, cubic feet, and short tons. However, because U.S. commerce involves other nations, most of which use metric units of measure, the U.S. Government is committed to the transition to the metric system, as stated in the Metric Conversion Act of 1975 (Public Law 94-168), amended by the Omnibus Trade and Competitiveness Act of 1988 (Public Law 100-418), and Executive Order 12770 of July 25, 1991.

The metric conversion factors presented in Table B1 can be used to calculate the metric-unit equivalents of values expressed in U.S. customary units. For example, 500 short tons are the equivalent of 453.6 metric tons ( 500 short tons $\times 0.9071847$ metric tons $/$ short ton $=453.6$ metric tons).

In the metric system of weights and measures, the names of multiples and subdivisions of any unit may be derived by combining the name of the unit with prefixes, such as deka, hecto, and kilo, meaning, respectively, 10, 100, 1,000, and deci, centi, and milli, meaning, respectively, one-tenth, one-hundredth, and one-thousandth. Common metric prefixes can be found in Table B2.

The conversion factors presented in Table B3 can be used to calculate equivalents in various physical units commonly used in energy analyses. For example, 10 barrels are the equivalent of 420 U.S. gallons (10 barrels $\times 42$ gallons $/$ barrel $=420$ gallons). 


\begin{tabular}{|c|c|c|c|c|c|}
\hline Type of Unit & U.S. Unit & $\begin{array}{c}\text { multiplied } \\
\text { by }\end{array}$ & $\begin{array}{c}\text { Conversion } \\
\text { Factor }\end{array}$ & equals & Metric Unit \\
\hline \multirow[t]{5}{*}{ Mass } & short tons $(2,000 \mathrm{lb})$ & $x$ & 0.9071847 & $=$ & metric tons $(t)$ \\
\hline & long tons & $x$ & 1.016047 & $=$ & metric tons $(t)$ \\
\hline & pounds $(\mathrm{lb})$ & $x$ & $0.45359237^{a}$ & $=$ & kilograms (kg) \\
\hline & pounds uranium oxide $\left(\mathrm{lb} \mathrm{U}_{3} \mathrm{O}_{8}\right)$ & $x$ & $0.384647^{b}$ & $=$ & kilograms uranium (kgU) \\
\hline & ounces, avoirdupois (avdp oz) & $x$ & 28.34952 & $=$ & grams $(g)$ \\
\hline \multirow[t]{6}{*}{ Volume } & barrels of oil (bbl) & $x$ & 0.1589873 & $=$ & cubic meters $\left(m^{3}\right)$ \\
\hline & cubic yards $\left(y d^{3}\right)$ & $x$ & 0.764555 & $=$ & cubic meters $\left(\mathrm{m}^{3}\right)$ \\
\hline & cubic feet $\left(\mathrm{ft}^{3}\right)$ & $x$ & 0.02831685 & $=$ & cubic meters $\left(m^{3}\right)$ \\
\hline & U.S. gailons (gal) & $x$ & 3.785412 & $=$ & liters (L) \\
\hline & ounces, fluid (fl oz) & $x$ & 29.57353 & $=$ & milliliters ( $\mathrm{mL}$ ) \\
\hline & cubic inches $\left(\mathrm{in}^{3}\right)$ & $x$ & 16.38706 & $=$ & milliliters (mL) \\
\hline \multirow[t]{4}{*}{ Length } & miles (mi) & $x$ & $1.609344^{a}$ & $=$ & kilometers $(\mathrm{km})$ \\
\hline & yards $(y d)$ & $x$ & $0.9144^{a}$ & $=$ & meters $(m)$ \\
\hline & feet $(\mathrm{ft})$ & $x$ & $0.3048^{a}$ & $=$ & meters $(\mathrm{m})$ \\
\hline & inches (in) & $x$ & $2.54^{a}$ & $=$ & centimeters $(\mathrm{cm})$ \\
\hline \multirow[t]{5}{*}{ Area } & acres & $x$ & 0.40469 & $=$ & hectares (ha) \\
\hline & square miles $\left(\mathrm{mi}^{2}\right)$ & $x$ & 2.589988 & $=$ & square kilometers $\left(\mathrm{km}^{2}\right)$ \\
\hline & square yards $\left(y d^{2}\right)$ & $x$ & 0.8361274 & $=$ & square meters $\left(\mathrm{m}^{2}\right)$ \\
\hline & square feet $\left(\mathrm{ft}^{2}\right)$ & $x$ & $0.09290304^{a}$ & $=$ & square meters $\left(\mathrm{m}^{2}\right)$ \\
\hline & square inches $\left(\mathrm{in}^{2}\right)$ & $x$ & $6.4516^{a}$ & $=$ & square centimeters $\left(\mathrm{cm}^{2}\right)$ \\
\hline Temperature & degrees Fahrenheit $(0 \mathrm{~F})$ & $x$ & $\begin{array}{l}5 / 9 \text { (after subtracting } \\
32)^{a, c}\end{array}$ & $=$ & degrees Celsius $\left({ }^{\circ} \mathrm{C}\right)$ \\
\hline \multirow[t]{3}{*}{ Energy } & British thermal units (Btu) & $x$ & $1,055.05585262^{a, d}$ & $=$ & joules (J) \\
\hline & calories (cal) & $x$ & $4.1868^{a}$ & $=$ & joules (J) \\
\hline & kilowatthours $(\mathrm{kWh})$ & $x$ & $3.6^{\mathrm{a}}$ & $=$ & megajoules (MJ) \\
\hline
\end{tabular}

\footnotetext{
${ }_{b}^{a}$ Exact conversion.

Calculated by the Energy Information Administration.

To convert degrees Celsius ( $\left.{ }^{\circ} \mathrm{C}\right)$ to degrees Fahrenheit $\left({ }^{\circ} \mathrm{F}\right)$ exactly, multiply by $9 / 5$, then add 32.

The Btu used in this table is the International Table Btu adopted by the Fifth International Conference on the Properties of Steam, London, 1956.

Notes: - Spaces have been inserted after every third digit to the right of the decimal for ease of reading. - Most metric units shown belong to the International System of Units (SI), and the liter, hectare, and metric ton are accepted for use with the SI units. For more information about the SI units, contact Dr. Barry Taylor at Bullding 221, Room B160, National Institute of Standards and Technology, Gaithersburg, MD 20899, or on telephone number 301-975-4220.

Sources: - General Services Administration, Federal Standard 376B, Preferred Metric Units for General Use by the Federal Government (Washington, DC, January 27, 1993), pp. 9-11, 13, and 16. - National Institute of Standards and Technology, Special Publications 330,811 , and 814 . - American National Standards Institute/Institute of Electrical and Electronic Engineers, ANSI/IEEE Std. 268-1992, pp. 28 and 29.
} 
Table B2. Metric Preflxes

\begin{tabular}{|c|c|c|c|c|c|}
\hline$\underset{\text { Multiple }}{\text { Unit }}$ & Prefix & Symbol & $\begin{array}{c}\text { Unit } \\
\text { Subdivision }\end{array}$ & Prefix & Symbol \\
\hline $10^{1}$ & deka & da & $10^{-1}$ & deci & $d$ \\
\hline $10^{2}$ & hecto & h & $10^{-2}$ & centi & c \\
\hline $10^{3}$ & kilo & $k$ & $10^{-3}$ & milli & $m$ \\
\hline $10^{6}$ & mega & $M$ & $10^{-6}$ & micro & $\mu$ \\
\hline $10^{9}$ & giga & $\mathbf{G}$ & $10^{-9}$ & nano & $n$ \\
\hline $10^{12}$ & tera & $T$ & $10^{-12}$ & pico & $p$ \\
\hline $10^{15}$ & peta & $P$ & $10^{-15}$ & femto & $f$ \\
\hline $10^{18}$ & exa & $E$ & $10^{-18}$ & atto & $a$ \\
\hline $10^{21}$ & zetta & $z$ & $10^{-21}$ & zepto & $z$ \\
\hline $10^{24}$ & yotta & $Y$ & $10^{-24}$ & yocto & $y$ \\
\hline
\end{tabular}

Source: U.S. Department of Commerce, National Institute of Standards and Technology, The International System of Units (SI), NIST Special Publication 330, 1991 Edition (Washington, DC, August 1991), p. 10.

Table B3. Other Physical Conversion Factors

\begin{tabular}{|c|c|c|c|c|c|}
\hline Energy Source & Original Unit & & Conversion Factor & & Final Unit \\
\hline Petroleum & barrels (bbl) & $x$ & $42^{a}$ & $=$ & U.S. gallons (gal) \\
\hline \multirow[t]{3}{*}{ Coal } & short tons & $x$ & $2,000^{a}$ & $=$ & pounds (lb) \\
\hline & long tons & $x$ & $2,240^{a}$ & $=$ & pounds (lb) \\
\hline & metric tons $(t)$ & $x$ & $1,000^{a}$ & $=$ & kilograms (kg) \\
\hline \multirow[t]{2}{*}{ Wood } & cords (cd) & $x$ & $1.25^{b}$ & $=$ & short tons \\
\hline & cords $(c d)$ & $x$ & $128^{a}$ & $=$ & cubic feet $\left(\mathrm{ft}^{3}\right)$ \\
\hline
\end{tabular}

Exact conversion.

${ }^{6}$ Calculated by the Energy information Administration.

Source: U.S. Department of Commerce, National Institute of Standards and Technology, Specifications, Tolerances and Other Technical Requirements for Weighing and Measuring Devices, NIST Handbook 44, 1994 Edition (Washington, DC, October 1993), pp. B-10, C-17, and C-21. 
Appendix C

Summary of Changes 


\section{Summary of Changes Since the State Energy Price and Expenditure Report 1992}

Revisions that are incorporated into the State Energy Price and Expenditure Data System (SEPEDS) since its previous release in December 1994 are summarized in this appendix. All revisions appearing in the data tables of this edition of the State Energy Price and Expenditure Report (SEPER), which covers selected years, and all revisions to the entire database, which covers 1970 through 1993, are reported here. Users of the SEPEDS personal computer (PC) diskettes, which contain data for all 24 years, may notice data changes that do not appear in the report due to the level of rounding in the published tables.

Price and expenditure revisions occur for several reasons: data sources for prices change; price estimation methodologies are revised or price assignment and estimation procedures are updated; data entries are corrected; or consumption estimates are revised. The first three kinds of changes affect Statelevel and U.S. prices directly. The fourth, a revised consumption value, affects only State prices that are estimated by consumption-weighted averaging of adjacent States and, similarly, affects U.S. average prices. Consumption estimates used in SEPER are taken from the State Energy Data System (SEDS), published as the State Energy Data Report 1993, Consumption Estimates (SEDR), which contains full documentation of the consumption estimation procedures. Consumption revisions are described in SEDR, Appendix G, "Summary of Changes." Since energy expenditure estimates depend on both the price and consumption estimates (including the consumption adjustments for process fuel and intermediate products), revision of either or both may cause revisions to the expenditures series.

\section{Coal}

Revisions to coal prices in the States and end-use sectors detailed below caused U.S. total coal average prices and expenditures to be revised in 1986 through 1992. The revisions made were generally by amounts too small to be seen in the rounded data in
SEPER tables, but they may be noticed by users of the SEPEDS PC diskettes.

Residential Sector, 1986 and 1990 through 1992. Prices of coal consumed in the residential sector of Montana in 1986 and 1990 through 1992 and residential coal prices in North Dakota in 1991 and 1992 were revised by using an estimation methodology that is more consistent with that used in previous years. North Dakota residential prices and expenditures decreased by 62 percent in 1991 and by 68 percent in 1992, causing North Dakota's total coal prices and expenditures to decrease by less than onehalf a percent in 1991 and 1992. Montana residential coal prices and associated expenditures doubled, $r$ tripled, or quadrupled in 1986 and 1990 through 1992. Montana's total coal price, which was also affected by price changes in the industrial and commercial sectors described below, decreased by 2 percent in 1986 and by 1 percent or less in 1987 through 1992. U.S. average residential coal prices and expenditures were affected in 1986 and 1990 through 1992, but by less than 1 percent each year.

Industrial and Commercial Sectors, 1977 and 1986 through 1992. Coal prices are developed for the industrial sector and assigned to the commercial sector; therefore, the same revisions occur in both sectors. The Rhode Island industrial coal price in 1977 was revised from $\$ 2.05$ to $\$ 2.04$ per million Btu and in 1990 from $\$ 2.59$ to $\$ 2.62$ per million Btu to be consistent with the stated methodology. The Montana and Nevada prices, which are estimated by averaging adjacent State prices, were revised for 1986 through 1992 by including additional adjacent State prices in the averages. Montana prices decreased by 10 percent to 16 percent each year, while Nevada prices increased by 2 percent to 6 percent in 1986 through 1992. The estimated price of coal in Alaska's commercial sector (there was no industrial consumption) for 1992 was revised from $\$ 2.29$ to $\$ 3.94$ per million Btu by the use of a new estimation methodology that is used for subsequent years. Proportional revisions occurred in each State's industrial and commercial coal expenditures that were calculated 
by using the revised prices. U.S. average prices and expenditures in the commercial and industrial sectors were affected slightly by the State revisions, generally by amounts too small to show in the published report but visible in the full precision data in the SEPEDS PC diskettes. The one exception is the U.S. average commercial coal price in 1992, which reflected the Alaska price increase and changed from $\$ 1.58$ to $\$ 1.68$ per million Btu. U.S. commercial coal expenditures accordingly increased by 6 percent in 1992.

\section{Petroleum}

\section{Distillate Fuel}

Transportation Sector, 1991. The price of diesel fuel in New Hampshire in 1991 was revised from $\$ 8.75$ to $\$ 8.77$ per million Btu by incorporating revised tax data. This revision was too small to cause a change in the New Hampshire total average distillate fuel price or in the U.S. average distillate fuel prices for the transportation sector and total distillate at the rounded level in the published report. However, users of the SEPEDS PC diskettes may see small revisions in the full precision data.

Residential, Commercial, and Industrial Sectors, 1992. The U.S. Department of Commerce, Bureau of the Census, revised 1992 reported taxes for California and Minnesota from 6.5 percent to 6 percent. In both States, distillate fuel prices for the residential, commercial, and industrial sectors decreased by $\$ 0.02$ or $\$ 0.03$ per million Btu. The prices for California and Minnesota are used in the estimation of prices for 13 other States in the commercial sector and 15 other States in the residential and industrial sectors. All the revisions in those States and in the U.S. average price for the three sectors are too small to be seen in the published tables but may be noticed in the SEPEDS PC diskettes. Proportional revisions occurred in the expenditures of the affected States and the United States.

\section{Kerosene}

Residential, Commercial, and Industrial Sectors, 1992. Because kerosene prices are estimated by using distillate fuel prices in the residential and industrial sectors, and the industrial prices are assigned to the commercial sector, the revisions to California and Minnesota taxes as explained in the Distillate Fuel section above are proportionally reflected in the 1992 kerosene price estimates in the

same 13 to 15 States. Some additional States' prices in all three sectors were revised in the last decimal place in the full precision data on the SEPEDS data diskettes due to different rounding procedures used in the price estimation spreadsheets. Only the California and Minnesota price decreases of between $\$ 0.01$ and $\$ 0.05$ per million Btu in the three sectors and the State totals are large enough to be seen in the published report. Expenditures for kerosene calculated with the revised prices were proportionally changed in the three sectors as well as in the States' total expenditures and U.S. sectoral and total expenditures.

\section{Liquefied Petroleum Gases}

Industrial Sector Expenditures, 1992. Revisions to the estimated consumption of liquefied petroleum gases (LPG) in the industrial sectors of Virginia and West Virginia in 1992, as described in SEDR Appendix $G$, caused revisions in SEPEDS LPG expenditures. Virginia's industrial sector LPG expenditures decreased from $\$ 76.6$ million to $\$ 52.2$ million, causing total LPG expenditures for the State to revise from $\$ 185.9$ million to $\$ 161.5$ million. Industrial LPG expenditures in West Virginia increased from $\$ 11.1$ million to $\$ 40.4$ million in 1992 , causing total LPG expenditures for the State to increase from $\$ 33.7$ million to $\$ 63.0$ million. The U.S. average industrial LPG expenditures increased by $\$ 5$ million, and small increases occurred in the U.S. total LPG expenditures and the U.S. average prices for industrial sector and total LPG.

\section{Lubricants}

Transportation and Industrial Sectors, 1989 through 1992. Lubricants prices are developed for the industrial sector and assigned to the transportation sector; therefore, identical price and expenditure revisions occur in both sectors. Lubricants prices for 1989 through 1991 were recalculated by using a revised thermal conversion factor. Prices for all States in all 3 years increased by 9 percent. In 1992, lubricants prices increased by 9 percent, not only because of the conversion factor but also because of the incorporation of more current data from the data source.

\section{Other Petroleum Products}

Miscellaneous Petroleum Products, 1976 through 1979 and 1990 through 1992. The incorporation of 1992 data from the U.S. Department of Commerce, Bureau of the Census, to estimate prices from 1990 
forward caused a review of the data used in estimating miscellaneous petroleum products prices for all years. Different Census data series were selected to estimate the 1976-through-1979 prices to be more consistent with the data used in the other years. A U.S. average price is calculated and assigned to all States in each year. The prices and resulting expenditures for miscellaneous petroleum products increased by 13 percent in each State each year in the 1976-through-1979 period. Prices and expenditures in 1990 through 1992 decreased by 6 percent in every State each year.

\section{Residual Fuel}

Commercial and Industrial Sectors, 1992. The U.S. Department of Commerce, Bureau of the Census, revised 1992 reported taxes for California and Minnesota from 6.5 percent to 6 percent. A data entry error for the Tennessee tax was corrected. These tax changes caused the commercial and industrial residual fuel prices in California and Minnesota to decrease by $\$ 0.01$ per million Btu and the prices in Tennessee to increase by $\$ 0.02$ per million Btu. The comparable commercial and industrial expenditures for residual fuel in the three States reflected the changes in quantities that are too small to be seen in the published report but may be seen in the SEPEDS PC diskettes. The U.S.-level average prices and expenditures were also revised by amounts too small to be seen in SEPER.

Transportation Sector, 1992. The U.S. average residual fuel price used in estimating bunker fuel prices was revised in the source document for 1992. That revision caused small changes (by 0.3 percent or less) in the residual fuel transportation prices in most of the 31 States reporting bunker fuel use. The two exceptions were Oregon, where the price decreased from $\$ 2.55$ to $\$ 1.84$ per million Btu, and Washington, where the price dropped from $\$ 5.43$ to $\$ 1.84$ per million Btu due to revisions in the electric utility sector price used in the estimation.

Electric Utilities, 1992. The price of residual fuel (i.e., heavy oil) paid by electric utilities in Washington in 1992 was revised from $\$ 6.19$ per million Btu to $\$ 2.18$ per million Btu by assigning the correct Census division price. Corresponding expenditures decreased 65 percent, although both previous and revised quantities are too small to show in SEPER tables. This price revision caused a small change in the Washington electric utility average price for all fuels that cannot be seen in the SEPER tables and caused expenditures for fuels to decrease from $\$ 182.7$ million to $\$ 182.5$ million.
Electricity

S
M
$M$
A
$R$
$Y$
O
$F$
C
$H$
A
$N$
$G$
$E$
S

Transportation and Commercial Sectors, 1992. Electricity prices for the commercial sector and expenditures for the transportation and commercial sectors in 1992 were revised due to the routine replacement of preliminary consumption values used in the price estimations for the District of Columbia and the 17 States with transportation electricity use. The consumption revisions are documented in SEDR, Appendix G. Most of the commercial sector price revisions are not visible in the published tables due to the level of rounding but may be noticed in the data on the SEPEDS PC diskettes. In the published SEPER, the prices in California, the District of Columbia, and Pennsylvania rose by $\$ 0.01$ per million Btu, while prices in Illinois and New Jersey decreased by $\$ 0.01$ per million Btu.

Residential and Industrial Sectors, 1992. Electricity prices for the residential and industrial sectors in 1992 were revised in the source document. The Oklahoma residential electricity price increased by less than $\$ 0.01$ per million Btu and can only be noticed on the SEPEDS PC diskettes. The price paid for electricity by the industrial sectors of Alabama, Kentucky, and Mississippi decreased by less than $\$ 0.01$ per million Btu in 1992, while prices in New York and Tennessee increased by $\$ 0.01$ per million Btu or less. Electricity expenditures for those States and sectors were revised proportionally by the change in prices.

\section{Blomass Fuels for Generation of Electricity}

Electric Utilities, 1992. Average prices for wood and waste burned at electric utilities in Washington and Wisconsin were revised by the inclusion of more data from additional electric utilities. The average price paid by Washington electric utilities decreased from $\$ 0.83$ to $\$ 0.78$ per million Btu in 1992 . In Wisconsin, the electric utility average price for biomass fuels increased from $\$ 0.24$ to $\$ 0.76$ per million Btu that year. Proportional increases occurred in the two States' expenditures and, together, they caused a 5-percent increase in the national average price and expenditures for biomass fuels in 1992. 
S Consumption Adjustments for Calculat$U$ ing Expenditures

Changes in consumption estimates of process fuel A and intermediate products are caused by revisions to $\mathbf{R}$ industrial and transportation sector consumption es$\mathbf{Y}$ timates in SEDS. In this edition of SEPER, revisions were caused by the industrial sector liquefied petro- leum gases consumption revisions in SEDS as described in the Liquefied Petroleum Gases section above and in SEDR, Appendix G. These revisions to Virginia and West Virginia consumption affected the two States' allocations of liquefied petroleum gases consumed by refineries. Although the consumption adjustments for process fuel and intermediate products in 1993 are shown in Table A49, the revisions to 1992 data are noticeable only in the data included on the SEPEDS PC diskettes. 
Glossary 


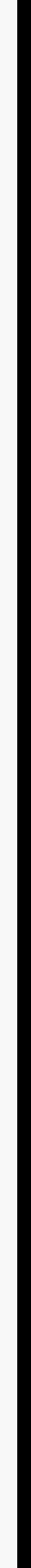




\section{Glossary}

Asphalt: A dark-brown-to-black cement-like material containing bitumens as the predominant constituents obtained by petroleum processing. The definition includes crude asphalt as well as the following finished products: cements, fluxes, the asphalt content of emulsions (exclusive of water), and petroleum distillates blended with asphalt to make cutback asphalts.

ASTM: The American Society for Testing and Materials.

Aviation Gasoline Blending Components: Naphthas that are used for blending or compounding into finished aviation gasoline (e.g., straight-run gasoline, alkylate, and reformate). Excludes oxygenates (alcohols and ethers), butane, and pentanes plus.

Aviation Gasoline: All special grades of gasoline for use in aviation reciprocating engines, as given in ASTM Specification D910 and Military Specification MIL-G-5572. Aviation gasoline includes blending components.

Barrel (petroleum): A unit of volume equal to 42 U.S. gallons.

Biomass Fuels: Wood, waste, and alcohol fuels are the primary examples of biomass fuels. The term "biomass fuels" comprises biomass energy sources and biofuels. Biomass energy sources are essentially. unprocessed; they are burned, as received, to produce thermal energy. Examples are wood, garbage, and corn. Biofuels result from the processing of biomass energy sources. In general, biofuels have a greater energy density and are more easily transported and used. Examples are wood chips, pellets, briquettes, and methanol (made from wood); refusederived fuel (made from garbage); and ethanol (made from corn).

British Thermal Unit (Btu): The quantity of heat needed to raise the temperature of 1 pound of water by $1^{\circ} \mathrm{F}$ at or near $39.2^{\circ} \mathrm{F}$. See Heat Content of a Quantity of Fuel, Gross and Heat Content of a Quantity of Fuel, Net.
Butane: A normally gaseous straight-chain or branched-chain hydrocarbon $\left(\mathrm{C}_{4} \mathrm{H}_{10}\right)$. It is extracted from natural gas or refinery gas streams. It includes isobutane and normal butane and is designated in ASTM Specification D1835 and Gas Processors Association Specifications for commercial butane.

- Isobutane: A normally gaseous branched-chain hydrocarbon. It is a colorless paraffinic gas that boils at a temperature of $10.9^{\circ} \mathrm{F}$. It is extracted from natural gas or refinery gas streams.

- Normal Butane: A normally gaseous straightchain hydrocarbon. It is a colorless paraffinic gas that boils at a temperature of $31.1^{\circ} \mathrm{F}$. It is extracted from natural gas or refinery gas streams.

Butylene: An olefinic hydrocarbon $\left(\mathrm{C}_{4} \mathrm{H}_{8}\right)$ recovered from refinery processes.

Coal: Includes all ranks of coal-anthracite, bituminous coal, subbituminous coal, and ligniteconforming to ASTM Specification D388.

- Coking Coal: Coal that meets the requirements for making coal coke. It must be low in ash and sulfur and form a coke that is capable of supporting the charge of iron ore and limestone in a blast furnace. Coking coal is usually a blend of two or more bituminous coals.

- Steam Coal: In this report, steam coal represents all noncoking coal.

Coal Coke: A hard, porous product made from baking bituminous coal in ovens at temperatures as high as $2,000^{\circ} \mathrm{F}$. It is used both as a fuel and as a reducing agent in smelting iron ore in a blast furnace.

Coke Plants: Plants where coal is carbonized in slot or beehive ovens for the manufacture of coke.

\section{Coking Coal: See Coal.}

Commercial Sector: The commercial sector, as defined economically, consists of business establishments that are not engaged in transportation or in manufacturing or other types of industrial 
activity (agriculture, mining, or construction). Commercial establishments include hotels, motels, restaurants, wholesale businesses, retail stores, laundries, and other service enterprises; religious and nonprofit organizations; health, social, and educational institutions; and Federal, State, and local governments. Street lights, pumps, bridges, and public services are also included if the establishment operating them is considered commercial.

Constant Dollars: Amounts expressed in constant dollars have been adjusted to remove the effect of changes in the purchasing power of the dollar. Prices expressed in constant dollars usually reflect buying power relative to a base year.

Conversion Factor: A number that translates units of one system into corresponding values of another system. Conversion factors can be used to translate physical units of measure for various fuels into Btu equivalents.

Crude Oil Used Directly: Crude oil consumed as fuel by crude oil pipelines and on crude oil leases.

Cubic Foot (natural gas): A unit of volume equal to 1 cubic foot at a pressure base of 14.73 pounds standard per square inch absolute and a temperature base of $60^{\circ} \mathrm{F}$.

Current Dollars: Amounts expressed in current dollars have not been adjusted to remove the effect of changes in the purchasing power of the dollar.

Degree-Day Normals: Simple arithmetic averages of monthly or annual degree-days over a long period of time (the 30-year period 1951 through 1980 is used for the estimates in this report). The averages may be simple degree-day normals or populationweighted degree-day normals. Monthly, State-level simple averages are used for this report.

Degree-Days, Cooling (CDD): The number of degrees per day that the daily average temperature is above $65^{\circ} \mathrm{F}$. The daily average temperature is the mean of the maximum and minimum temperatures for a 24-hour period.

Degree-Days, Heating (HDD): The number of degrees per day that the daily average temperature is below $65^{\circ} \mathrm{F}$. The daily average temperature is the mean of the maximum and minimum temperatures for a 24-hour period.

Degree-Days, Population-Weighted: Heating or cooling degree-days weighted by the population of the area in which the degree-days are recorded. To compute State population-weighted degree-days, each State is divided into from one to nine climatically homogeneous divisions, which are assigned weights based on the ratio of the population of the division to the total population of the State. Degreeday readings for each division are multiplied by the corresponding population weight for each division and those products are then summed to arrive at the State population-weighted degree-day figure. To compute national population-weighted degree-days, the Nation is divided into nine Census regions, each comprising from three to eight States, which are assigned weights based on the ratio of the population of the region to the total population of the Nation. Degree-day readings for each region are multiplied by the corresponding population weight for each region and those products are then summed to arrive at the national population-weighted degree-day figure.

Diesel Fuel: Fuel used for internal combustion in diesel engines; usually that fraction of crude oil that distills after kerosene. (See Distillate Fuel Oil.)

Distillate Fuel Oil: A general classification for one of the petroleum fractions produced in conventional distillation operations. Included are products known as No. 1, No. 2, and No. 4 fuel oils and No. 1, No. 2, and No. 4 diesel fuels. It is used primarily for space heating, on-and off-highway diesel engine fuel (including railroad engine fuel and fuel for agricultural machinery), and electric power generation.

Electrical System Energy Losses: The amount of energy lost during generation, transmission, and distribution of electricity, including plant and unaccounted-for uses.

Electricity Generation: The process of producing electric energy or transforming other forms of energy into electric energy. Also the amount of electric energy produced or expressed in watthours (Wh).

Electricity Sales: The amount of kilowatthours sold in a given period of time; usually grouped by classes of service, such as residential, commercial, industrial, and other. "Other" sales include sales for public street and highway lighting and other sales to public authorities, sales to railroads and railways, and interdepartmental sales.

Electric Power Plant: A station containing prime movers, electric generators, and auxiliary equipment for converting mechanical, chemical, and/or fission energy into electric energy. 
Electric Utility: A corporation, person, agency, authority, or other legal entity or instrumentality that owns and/or operates facilities for the generation, transmission, distribution, or sale of electric energy, primarily for use by the public, and that files forms listed in the Code of Federal Regulations, Title 18, Part 141. Facilities that qualify as cogenerators or small power producers under the Public Utility Regulatory Policies Act are not considered utilities.

Electric Utility Sector: The electric utility sector consists of privately and publicly owned establishments that generate, transmit, distribute, or sell electricity primarily for use by the public and that meet the definition of an electric utility. Nonutility power producers are not included in the electric utility sector.

End-Use Sectors: The residential, commercial, industrial, and transportation sectors of the economy.

Energy: The capacity for doing work as measured by the capability of doing work (potential energy) or the conversion of this capability to motion (kinetic energy). Energy has several forms, some of which are easily convertible and can be changed to another form useful for work. Most of the world's convertible energy comes from fossil fuels that are burned to produce heat that is then used as a transfer medium to mechanical or other means in order to accomplish tasks. Electrical energy is usually measured in kilowatthours, while heat energy is usually measured in British thermal units.

Ethane: A normally gaseous straight-chain hydrocarbon $\left(\mathrm{C}_{2} \mathrm{H}_{6}\right)$. It is a colorless, paraffinic gas that boils at a temperature of $-127.48^{\circ} \mathrm{F}$. It is extracted from natural gas and refinery gas streams.

Ethylene: An olefinic hydrocarbon $\left(\mathrm{C}_{2} \mathrm{H}_{4}\right)$ recovered from refinery processes or petrochemical processes.

Expenditures: In this report, "expenditures" refers to the money directly spent by consumers to purchase energy. Expenditures equal the amount of energy used by the consumer times the price per unit paid by the consumer. In calculating the amount of energy used, process fuel and intermediate products are not included.

Exports: Shipments of goods from the 50 States and the District of Columbia to foreign countries and to Puerto Rico, the Virgin Islands, and other U.S. possessions and territories.

f.a.s.: See Free Alongside Ship.
Federal Energy Regulatory Commission (FERC): The Federal agency with jurisdiction over interstate electricity sales, wholesale electric rates, hydroelectric licensing, natural gas pricing, oil pipeline rates, and gas pipeline certification. FERC is an independent regulatory agency within the Department of Energy and is the successor to the Federal Power Commission.

Federal Power Commission (FPC): The predeçessor agency of the Federal Energy Regulatory Commission. The Federal Power Commission was created by an Act of Congress under the Federal Water Power Act on June 10, 1920. It was charged originally with regulating the electric power and natural gas industries. It was abolished on September 30, 1977, when the Department of Energy was created. Its functions were divided between the Department of Energy and the Federal Energy Regulatory Commission, an independent regulatory agency.

Fossil Fuel: Any naturally occurring organic fuel, such as petroleum, coal, and natural gas.

Free Alongside Ship (f.a.s.): The value of a commodity at the port of exportation, generally including the purchase price, plus all charges incurred in placing the commodity alongside the carrier at the port of exportation.

Gasohol: A blend of finished motor gasoline (leaded or unleaded) and alcohol (generally ethanol but sometimes methanol) limited to 10 percent by volume of alcohol. Gasohol is included in finished leaded and unleaded motor gasoline.

Geothermal Energy (as used at electric utilities): Hot water or steam extracted from geothermal reservoirs in the Earth's crust and supplied to steam turbines at electric utilities that drive generators to produce electricity.

Gross Domestic Product (GDP): The total value of goods and services produced by labor and property located in the United States. As long as the labor and property are located in the United States, the supplier (that is, the workers and, for property, the owners) may be either U.S. residents or residents of foreign countries.

Gross Domestic Product (GDP) Implicit Price Deflator: The implicit price deflator, published by the U.S. Department of Commerce, Bureau of Economic Analysis, is used to convert nominal figures to real figures. 
Gross Domestic Purchases: The total purchases by U.S. residents for all goods and services wherever produced, including imports but excluding exports. It includes all personal consumption expenditures, gross private domestic investment, and government purchases (Federal, State, and local).

Gross Domestic Purchases Price Index: This index measures prices paid by U.S. residents. It is derived from the prices of the components included in gross domestic purchases.

Heat Content of a Quantity of Fuel, Gross: The total amount of heat released when a fuel is burned. Coal, crude oil, and natural gas all include chemical compounds of carbon and hydrogen. When those fuels are burned, the carbon and hydrogen combine with oxygen in the air to produce carbon dioxide and water. Some of the energy released in burning goes into transforming the water into steam and is usually lost. The amount of heat spent in transforming the water into steam is counted as part of gross heat content but is not counted as part of net heat content. Also referred to as the higher heating value. Btu conversion factors typically used in EIA represent gross heat content.

Heat Content of a Quantity of Fuel, Net: The amount of useable heat energy released when a fuel is burned under conditions similar to those in which it is normally used. Also referred to as the lower heating value. Btu conversion factors typically used in EIA represent gross heat content.

Heavy Oil: The fuel oils remaining after the lighter oils have been distilled off during the refining process. Except for start-up and flame stabilization, virtually all petroleum used in steam-electric power plants is heavy oil.

Hydroelectric Power: The production of electricity from the kinetic energy of falling water.

Imports: Receipts of goods into the 50 States and the District of Columbia from foreign countries and from Puerto Rico, the Virgin Islands, and other U.S. possessions and territories.

Industrial Sector: The industrial sector comprises manufacturing industries, which make up the largest part of the sector, along with mining, construction, agriculture, fisheries, and forestry. Establishments in the sector range from steel mills, to small farms, to companies assembling electronic components.
Jet Fuel: The term includes kerosene-type jet fuel and naphtha-type jet fuel. Kerosene-type jet fuel is a kerosene-quality product used primarily for commercial turbojet and turboprop aircraft engines. Naphtha-type jet fuel is a fuel in the heavy naphthas range used primarily for military turbojet and turboprop aircraft engines.

Kerosene: A petroleum distillate that has a maximum distillation temperature of $401^{\circ} \mathrm{F}$ at the 10-percent recovery point, a final boiling point of $572^{\circ} \mathrm{F}$, and a minimum flash point of $100^{\circ} \mathrm{F}$. Included are the two grades designated in ASTM D3699 (No. 1-K and No. 2-K) and all grades of kerosene called range or stove oil. Kerosene is used in space heaters, cook stoves, and water heaters; it is suitable for use as an illuminant when burned in wick lamps.

Lease and Plant Fuel: Natural gas used in well, field, and lease operations (such as gas used in drilling operations, heaters, dehydrators, and field compressors), and as fuel in natural gas processing plants.

Light Oil: Lighter fuel oils distilled off during the refining process. Virtually all petroleum used in internal combustion and gas-turbine engines is light oil.

Liquefied Petroleum Gases (LPG): Ethane, ethylene, propane, propylene, normal butane, butylene, and isobutane produced at refineries or natural gas processing plants, including plants that fractionate new natural gas plant liquids.

Lubricants: Substances used to reduce friction between bearing surfaces or as process materials either incorporated into other materials used as processing aids in the manufacturing of other products or as carriers of other materials. Petroleum lubricants may be produced either from distillates or residues. Other substances may be added to impart or improve certain required properties. Excluded are byproducts of lubricating oil refining, such as aromatic extracts derived from solvent extraction or tars derived from deasphalting. Included are all grades of lubricating oils from spindle oil to cylinder oil and those used in greases. Lubricant categories are paraffinic and naphthenic.

Miscellaneous Petroleum Products: All finished petroleum products not classified elsewhere-for example, petrolatum, lube refining byproducts (aromatic extracts and tars), absorption oils, ram-jet fuel, 
petroleum rocket fuels, synthetic natural gas feedstocks, and specialty oils.

Motor Gasoline: A complex mixture of relatively volatile hydrocarbons, with or without small quantities of additives, obtained by blending appropriate refinery streams to form a fuel suitable for use in spark-ignition engines. Motor gasoline includes both leaded and unleaded grades of finished motor gasoline, blending components, and gasohol.

Motor Gasoline Blending Components: Naphthas that will be used for blending or compounding into finished motor gasoline (e.g., straight-run gasoline, alkylate, reformate, benzene, toluene, and xylene). Excluded are oxygenates (alcohols and ethers), butane, and pentanes plus.

Natural Gas: A mixture of hydrocarbons (principally methane) and small quantities of various nonhydrocarbons existing in the gaseous phase or in solution with crude oil in underground reservoirs.

Natural Gasoline: A mixture of hydrocarbons, mostly pentanes and heavier, extracted from natural gas that meets specifications for natural gasoline set by the Gas Processors Association. Includes isopentane.

Nominal Price: The price paid for a product or service at the time of the transaction. The nominal price, which is expressed in current dollars, is not adjusted to remove the effect of changes in the purchasing power of the dollar.

Nuclear Electric Power: Electricity generated by an electric power plant whose turbines are driven by steam generated in a reactor by heat from the fissioning of nuclear fuel.

Nuclear Electric Power Plant: A single-unit or multiunit facility in which heat produced in one or more reactors by the fissioning of nuclear fuel is used to drive one or more steam turbines.

Nuclear Fuel: Fissionable materials that have been enriched to a composition that, when placed in a nuclear reactor, will support a self-sustaining fission chain reaction, producing heat in a controlled manner for process use.

Pentanes Plus: A mixture of hydrocarbons, mostly pentanes and heavier, extracted from natural gas. Includes isopentane, natural gasoline, and plant condensate.
Petrochemical Feedstocks: Chemical feedstocks derived from petroleum principally for the manufacture of chemicals, synthetic rubber, and a variety of plastics. The categories reported are naphthas less than $401^{\circ} \mathrm{F}$ endpoint and other oils equal to or greater than $401^{\circ} \mathrm{F}$ endpoint.

Petroleum: A generic term applied to oil and oil products in all forms, such as crude oil, lease condensate, unfinished oils, petroleum products, natural gas plant liquids, and nonhydrocarbon compounds blended into finished petroleum products.

Petroleum Coke: A residue that is the final product of the condensation process in cracking. The product is either marketable petroleum coke or catalyst petroleum coke.

Petroleum Coke, Catalyst: The carbonaceous residue that is deposited on and deactivates the catalyst used in many catalytic operations (e.g., catalytic cracking). Carbon is deposited on the catalyst, thus deactivating the catalyst. The catalyst is reactivated by burning off the carbon, which is used as a fuel in the refining process. That carbon or coke is not recoverable in a concentrated form.

Petroleum Coke, Marketable: Those grades of coke produced in delayed or fluid cokers that may be recovered as relatively pure carbon. Marketable petroleum coke may be sold as is or further purified by calcining.

Petroleum Products: Products obtained from the processing of crude oil (including lease condensate), natural gas, and other hydrocarbon compounds. Petroleum products include unfinished oils, liquefied petroleum gases, pentanes plus, aviation gasoline, motor gasoline, naphtha-type jet fuel, kerosene-type jet fuel, kerosene, distillate fuel oil, residual fuel oil, petrochemical feedstocks, special naphthas, lubricants, waxes, petroleum coke, asphalt, road oil, still gas, and miscellaneous products.

Photovoltaic and Solar Thermal Energy (as used at electric utilities): Energy radiated by the sun as electromagnetic waves (electromagnetic radiation) that is converted at electric utilities into electricity by means of solar (photovoltaic) cells or concentrating (focusing) collectors.

Plant Condensate: One of the natural gas liquids, mostly pentanes and heavier hydrocarbons, recovered and separated as liquids at gas inlet separators or scrubbers in processing plants. 
Primary Energy: All energy consumed by end users, excluding electricity but including the energy consumed at electric utilities to generate electricity. (In estimating energy expenditures, there are no fuel-associated expenditures for hydroelectric power, geothermal energy, solar energy, or wind energy, and the quantifiable expenditures for process fuel and intermediate products are excluded.)

Process Fuel: All energy consumed in the acquisition, processing, and transportation of energy. Quantifiable process fuel includes three categories: natural gas lease and plant operations, natural gas pipeline operations, and oil refinery operations.

Propane: A normally gaseous straight-chain hydrocarbon $\left(\mathrm{C}_{3} \mathrm{H}_{8}\right)$. It is a colorless paraffinic gas that boils at a temperature of $-43.67^{\circ} \mathrm{F}$. It is extracted from natural gas or refinery gas streams. It includes all products designated in ASTM Specification D1835 and Gas Processors Association Specifications for commercial propane and HD-5 propane.

Propylene: An olefinic hydrocarbon $\left(\mathrm{C}_{3} \mathrm{H}_{6}\right)$ recovered from refinery or petrochemical processes.

Real Price: A price that has been adjusted to remove the effect of changes in the purchasing power of the dollar. Real prices, which are expressed in constant dollars, usually reflect buying power relative to a base year.

Refinery (petroleum): An installation that manufactures finished petroleum products from crude oil, unfinished oils, natural gas liquids, other hydrocarbons, and alcohol.

Residential Sector: The residential sector is considered to consist of all private residences, whether occupied or vacant, owned or rented, including single-family homes, multifamily housing units, and mobile homes. Secondary homes, such as summer homes, are also included. Institutional housing, such as school dormitories, hospitals, and military barracks, generally are not included in the residential sector; they are included in the commercial sector.

Residual Fuel Oil: The heavier oils that remain after the distillate fuel oils and lighter hydrocarbons are distilled away in refinery operations and that conform to ASTM Specifications D396 and 975. Included are No. 5, a residual fuel oil of medium viscosity; Navy Special, for use in steam-powered vessels in government service and in shore power plants; and No. 6, which includes Bunker C fuel oil and is used for commercial and industrial heating, electricity generation, and to power ships. Imports of residual fuel oil include imported crude oil burned as fuel.

Road Oil: Any heavy petroleum oil, including residual asphaltic oil, used as a dust palliative and surface treatment on roads and highways. It is generally produced in six grades, from 0 , the most liquid, to 5 , the most viscous.

Short Ton (coal): A unit of weight equal to 2,000 pounds.

SIC: See Standard Industrial Classification.

Special Naphthas: All finished products within the naphtha boiling range that are used as paint thinners, cleaners, or solvents. Those products are refined to a specified flash point. Special naphthas include all commercial hexane and cleaning solvents conforming to ASTM Specifications D1836 and D484, respectively. Naphthas to be blended or marketed as motor gasoline or aviation gasoline, or that are to be used as petrochemical and synthetic natural gas (SNG) feedstocks are excluded.

Standard Industrial Classification (SIC): A set of codes developed by the Office of Management and Budget which categorizes industries into groups with similar economic activities.

\section{Steam Coal: See Coal.}

Still Gas (refinery gas): Any form or mixture of gas produced in refineries by distillation, cracking, reforming, and other processes. The principal constituents are methane, ethane, ethylene, normal butane, butylene, propane, and propylene: It is used primarily as refinery fuel and petrochemical feedstock.

Total Energy: All energy consumed by end users including electricity but excluding the energy consumed at electric utilities to generate electricity. (In estimating energy expenditures, there are no fuelassociated expenditures for hydroelectric power, geothermal energy, solar power, or wind energy, and the quantifiable expenditures for process fuel and intermediate products are excluded.)

Transportation Sector: The transportation sector consists of private and public vehicles that move people and commodities. Included are automobiles, trucks, buses, motorcycles, railroads and railways (including streetcars), aircraft, ships, barges, and natural gas pipelines. 
Unfinished Oils: All oils requiring further refinery processing, except those requiring only mechanical blending. Included are naphthas and lighter oils, kerosene and light gas oils, heavy gas oils, and residuum.

Unfractionated Streams: Mixtures of unsegregated natural gas liquid components, excluding those in plant condensate. This product is extracted from natural gas.

United States: The 50 States and the District of Columbia.
Waxes: Solid or semisolid materials derived from petroleum distillates or residues. Waxes are lightcolored, more or less translucent crystalline masses, slightly greasy to the touch, consisting of a mixture of solid hydrocarbons in which the paraffin series predominates. Included are all marketable waxes, whether crude scale or fully refined. Waxes are used primarily as industrial coating for surface protection.

Wind Energy (as used at electric utilities): The kinetic energy of wind converted at electric utilities into mechanical energy by wind turbines (i.e., blades rotating from $a$ hub) that drive generators to produce electricity for distribution. 


\section{Publication Order Form \\ State Energy Data Report 1993, Consumption Estimates}

Published: July 1995

Energy Information Administration

GPO Stock No. 061-003-00917-4

Price per copy: $\$ 33.00$

Personal Name or Attention Line:

Company Name, if applicable:

Street Address:

City, State, Zip Code:

Daytime Phone Number (area code first):

Purchase Order No:

May we make your name and address available to other mailers?

yes

no

The State Energy Data Report 1993, Consumption Estimates, presents annual energy consumption estimates for the 50 States, the District of Columbia, and the United States. The estimates are provided by type of energy (refined petroleum, natural gas, coal, and electricity) and by major consuming sectors (residential, commercial, industrial, transportation, and electric utilities) in physical units and in British thermal units for 1960, 1965, and 1970 through 1993. The 518-page report includes technical documentation describing the data sources and estimation procedures used.

Please include payment with this order form. Allow a minimum of 4 weeks for domestic delivery and an additional 6 weeks for international delivery.

Quantity $x \$ 33.00=\$$ (total due). (International customers add 25\%.)

$\square$ Check payable to Superintendent of Documents GPO Deposit Account No. VISA or MasterCard Account No.

Authorizing Signature
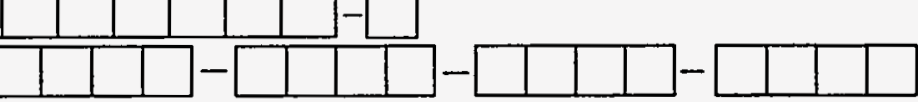

Credit Card Expiration Date

Note: Price includes regular domestic postage and handling. It is subject to change.

Mail order form to: U.S. Government Printing Office

P.O. Box 371954

Pittsburgh, PA 15250-7954

Or fax order form to: $202-512-2250$ 


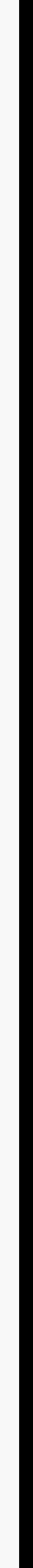




\section{Publication Order Form \\ State Energy Price and Expenditure Report 1993}

Published: December 1995

Energy Information Administration

GPO Stock No. 061-003-00948-4

Price per copy: $\$ 18.00$

Personal Name or Attention Line:

Company Name, if applicable:

Street Address:

City, State, Zip Code:

Daytime Phone Number (area code first):

Purchase Order No:

May we make your name and address available to other mailers?

The State Energy Price and Expenditure Report 1993 presents energy price and expenditure estimates for the 50 States, the District of Columbia, and the United States for 1970, 1975, 1980, 1985 , and 1990 through 1993 . The estimates are provided by energy source (petroleum, natural gas, coal, and electricity) and by major consuming sector (residential, commercial, industrial, transportation, and electric utilities). The 275-page report includes technical documentation describing the data sources and estimation procedures used.

Please include payment with this order form. Allow a minimum of 4 weeks for domestic delivery and an additional 6 weeks for international delivery.

Quantity $x \$ 18.00=\$$ (total due). (International customers add 25\%.)

Check payable to Superintendent of Documents GPO Deposit Account No. VISA or MasterCard Account Authorizing Signature Credit Card Expiration Date

Note: Price includes regular domestic postage and handling. It is subject to change.

Mail order form to: U.S. Government Printing Office

P.O. Box 371954

Pittsburgh, PA 15250-7954

Or fax order form to: $202-512-2250$ 



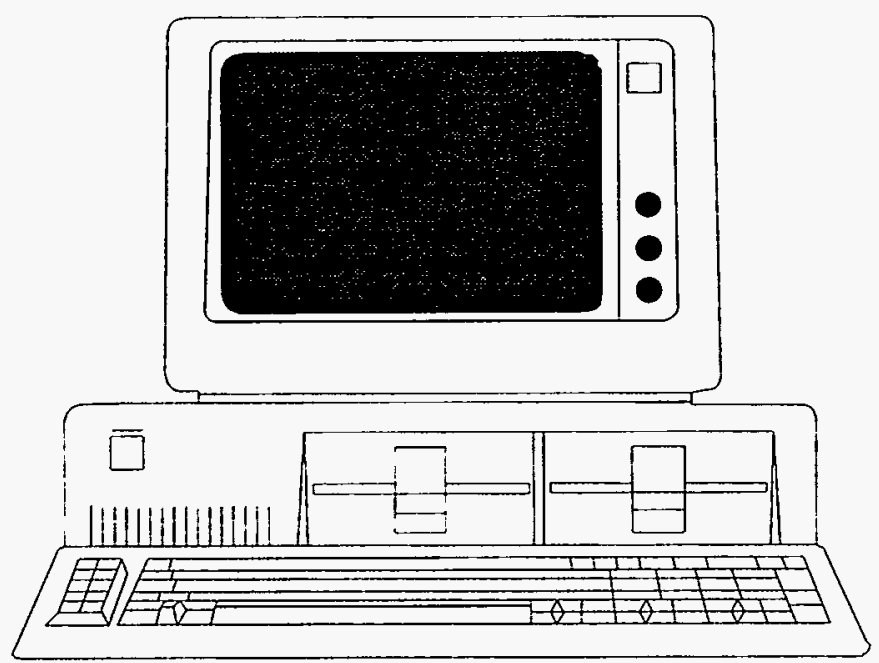

\section{State Energy Data on Diskettes}

\section{Available from GPO and NTIS}

- For IBM-PC and compatible microcomputers

- 5 1/4-inch double-sided high-density diskettes

- ASCII comma-delimited format

- Can easily be imported into Lotus 1-2-3 or dBASE using utilities that are included on the diskettes

State Energy Data System (SEDS) diskettes contain the data for 1960 through 1993 as published in Tables 11 through 322 of the State Energy Data Report 1993, Consumption Estimates. State Energy Price and Expenditure System (SEPEDS) diskettes contain the data for 1970 through 1993 as published in the "Statistical Tables" section of the State Energy Price and Expenditure Report 1993. Although the published tables present data in rounded form, the diskettes contain data in the fullest precision available. Diskettes containing data for all the States within a Census region, the U.S. data, documentation, and utilities can be purchased separately or in a complete set. For prices and more information, contact:

Office of Scientific and Technical Information P.O. Box 62

Oak Ridge, TN 37831

615-576-8401
Order Control National Technical Information Service 5285 Port Royal Road Springfield, VA 22161 703-487-4650 
OFFICIAL BUSINESS

Address Correction Requested

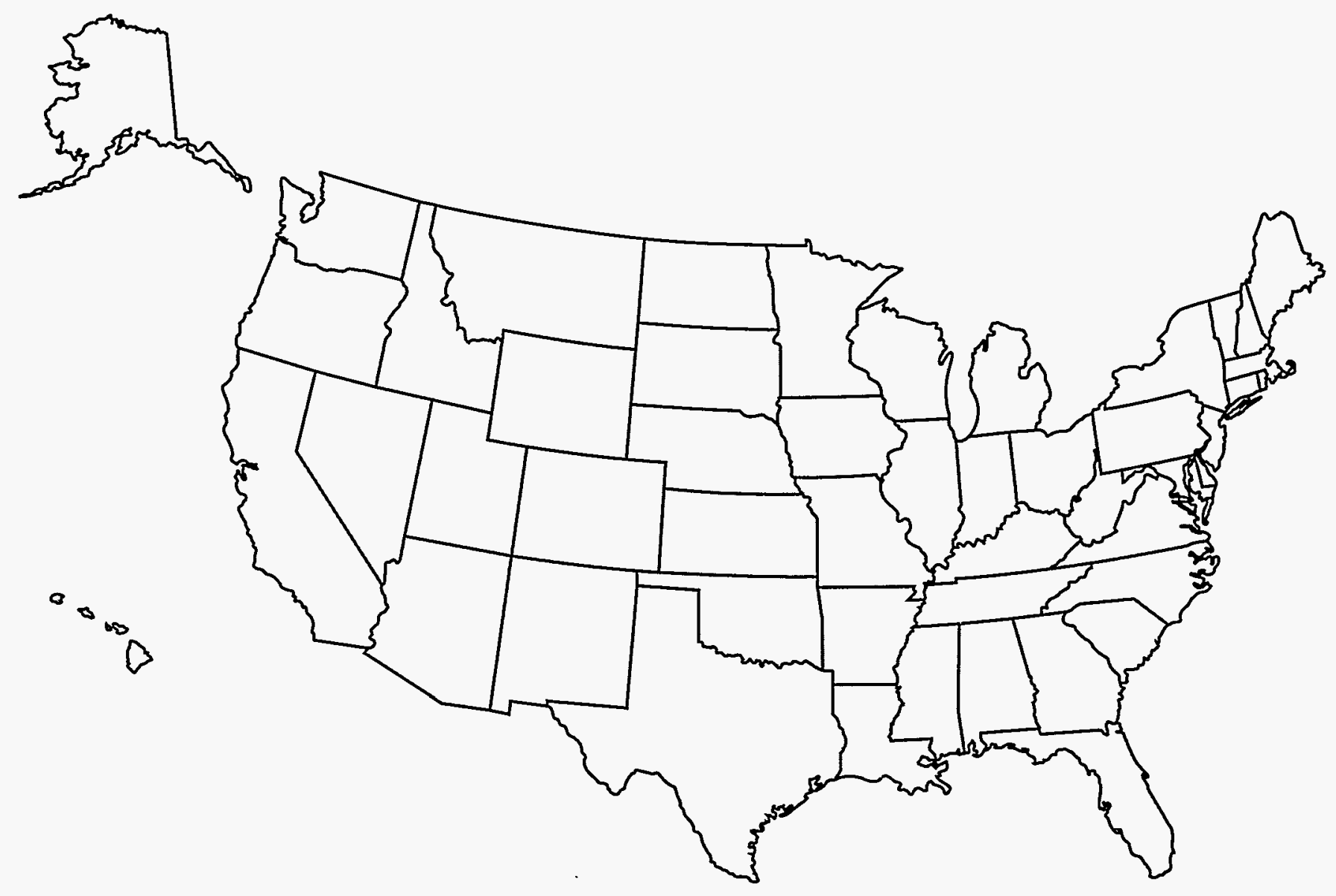

